\title{
STRUCTURAL BEHAVIOUR OF INSULATED FOAM-TIMBER PANELS UNDER GRAVITY AND LATERAL LOADING
}

\author{
by \\ Hassan Abbasi \\ M.A.Sc., University of Windsor, Windsor, ON, Canada, 2009 \\ B.A.Sc., Tehran Azad University, Tehran, Iran, 1992
}

\begin{abstract}
A dissertation
presented to Ryerson University

In partial fulfillment of the

requirements for the degree of

Doctor of Philosophy

in the program of

Civil Engineering
\end{abstract}

Toronto, Ontario, Canada, 2014

(C) HASSAN ABBASI 2014 


\section{AUTHOR'S DECLARATION}

I hereby declare that I am the sole author of this dissertation. This is a true copy of the dissertation, including any required final revisions, as accepted by my examiners.

I authorize Ryerson University to lend this dissertation to other institutions or individuals for the purpose of scholarly research.

I further authorize Ryerson University to reproduce this dissertation by photocopying or by other means, in total or in part, at the request of other institutions or individuals for the purpose of scholarly research.

I understand that my dissertation may be made electronically available to the public. 


\author{
ABSTRACT \\ STRUCTURAL BEHAVIOUR OF INSULATED FOAM-TIMBER PANELS UNDER \\ GRAVITY AND LATERAL LOADING \\ by

\begin{abstract}
Hassan Abbasi
Doctor of Philosophy in Civil Engineering

Civil Engineering Department, Ryerson University, Toronto, ON, Canada, 2014
\end{abstract}

A Structural Insulated Panel (SIP) is a structural element of expanded polystyrene insulation (EPS) core sandwiched between two oriented-strand boards (OSB). This research proposes SIPs in low-rise residential construction (i.e. houses and low-residential building), replacing the traditional conventional joist floors and stud walls. This research investigates (i) developing expressions for flexural, compression, monotonic racking and cyclic lateral load capacities of SIPs as compared to the joist/stud wall construction. In this study, the proposed design of SIPs was based on (i) generally established theory for analysis, (ii) assessment of full-scale SIP panels by a loading tester, and (iii) computer modeling using the finite-element modeling. The research program included (i) testing SIP walls in axial compression and bending, (ii) racking and cyclic testing on SIP shear walls, (iii) development of finite-element computer models of the tested SIP panels and verifying those using experimental findings, (iv) correlation between experimental findings and design equations for strength and serviceability available in the literature and wood design Standards. Modification factors of these equations were developed to allow structural engineers to design SIP panels in residential construction more economically reliably. Experimental results showed that SIP panels are being "as good as" the conventional wood-framing of identical sizes, with respect to flexural, compressive, racking and cyclic loading. Also, results showed SIP walls have a greater ability to dissipate energy under racking and cyclic loading that the stud wall system. Therefore, SIP walls can be used so efficient in seismic zones. Based on cyclic lateral load test results, the values of ductility-related force modification factor $\left(\mathrm{R}_{\mathrm{d}}\right)$ for 
stud wall, short SIP wall and long SIP wall were calculated as $8 \%, 22 \%$ and $14 \%$ lower than the NBCC required value for anchored wall $\left(R_{d}=3.0\right)$, respectively. In addition cyclic lateral load test results showed that the values of over-strength-related force modification factor $\left(\mathrm{R}_{\mathrm{o}}\right)$ for stud wall, short SIP wall and long SIP wall were observed to be $17 \%, 20 \%$ and $14 \%$ higher than the recommended value of $\mathrm{NBCC}\left(\mathrm{R}_{\mathrm{o}}=1.7\right)$ for anchored wall, respectively. So, it is concluded that the over-strength factor indicates a confident reserve of resistance in interconnected wall segments. 


\section{ACKNOWLEDGEMENTS}

I have to thank from my advisor Dr. Khaled Sennah who help me in all of this research. I am also thankful form Mr. Nidal Jaalouk, Mr. Domenic Valle, Mr. Mohammad Aldardari and Mr. Alan Machin whose help me in the experimental test. I appreciate the great cooperation of Thermapan Inc. director and staff for providing specimens for experimental work of this research. I also thank the Ontario Government to support my study through Ontario Graduate Scholarship, Ontario Centers of Excellence Interact Program, Ryerson Graduate Scholarship and Teaching and Research Assistantship. 


\section{DEDICATION}

To my parents, my wife Mina, and my son Arad, whose love and support, have made everything I do possible. 


\section{TABLE OF CONTENTS}

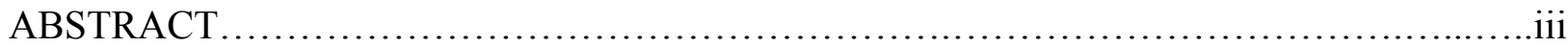

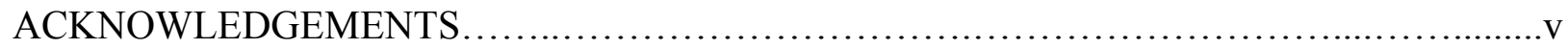

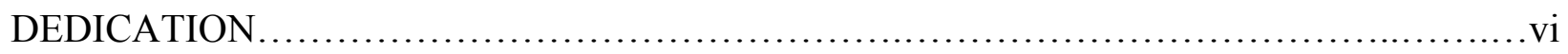

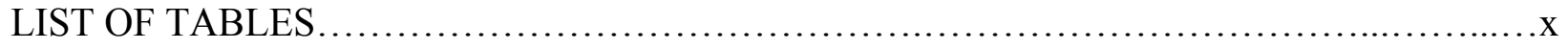

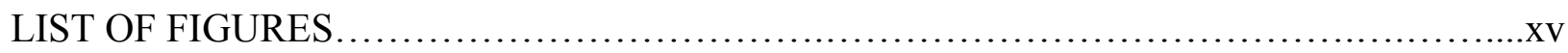

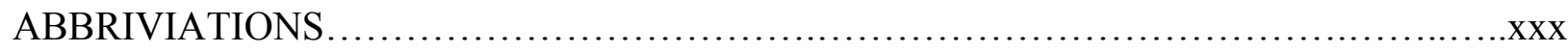

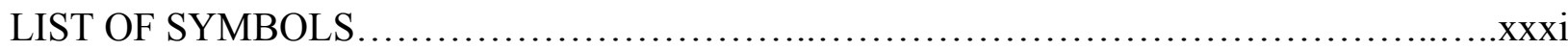

\section{CHAPTERS}

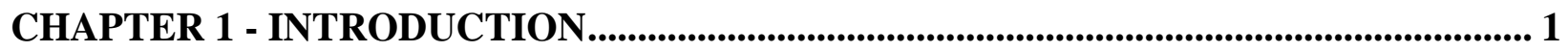

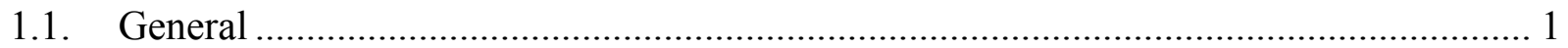

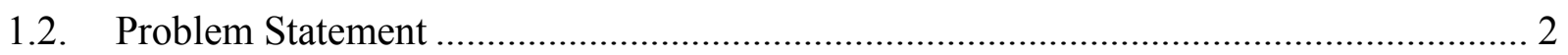

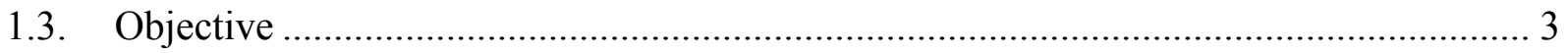

1.4. Scope of Work and Research Methodology …………............................................... 3

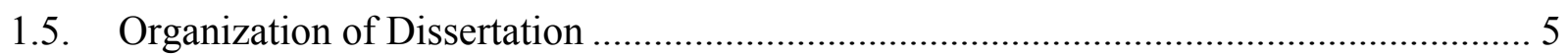

CHAPTER 2 - LITERATURE REVIEW.................................................................................... 6

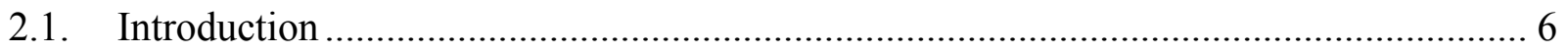

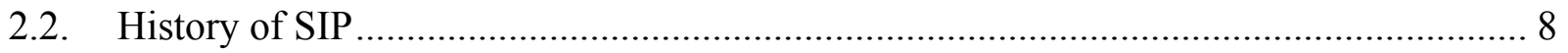

2.3. Advantages and Disadvantages of SIP ...................................................................... 9

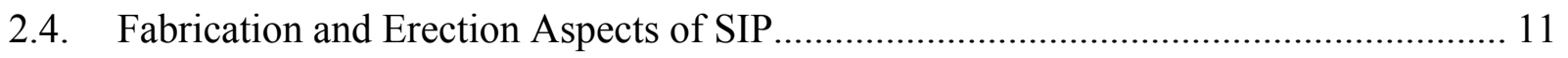

2.5. Structural Analysis and Design of Sandwich Panels ................................................. 13

2.6. Historical Development of Sandwich Theory .............................................................. 14

2.7. Flexural Stresses in Sandwich Panels ........................................................................ 17

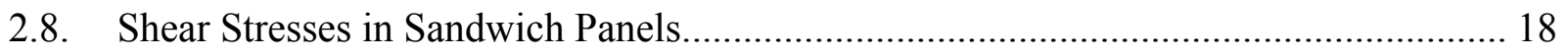

2.9. Buckling in Sandwich Panel Walls ............................................................................ 19

2.10. Elastic Deflection Analysis of Sandwich Panels........................................................... 24

2.11. Theoretical Investigation and Experimental Studies.................................................. 30

2.12. US Acceptance Criteria for Sandwich Panels .............................................................. 35

2.13. Analytical Models for SIP/Stud Wall........................................................................... 37 
2.14. Monotonic and Cyclic Study on Shear Wall ................................................................ 49

2.15. Available Design Methods, Codes, and Guidelines...................................................... 60

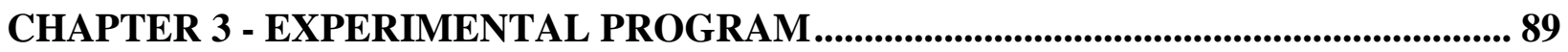

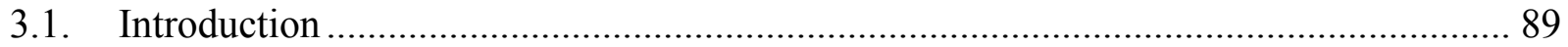

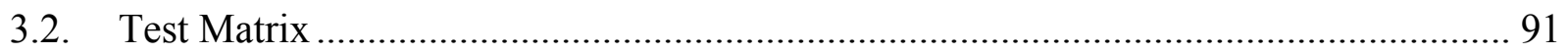

3.3. Stud Wall Descriptions and Material Properties ............................................................ 95

3.4. SIP Walls Descriptions and Material Properties ........................................................... 97

3.5. Material Properties by Experimental Testing................................................................. 99

3.6. Equipments and Instrumentation for Testing ........................................................ 104

3.7. Testing SIP in Flexure (Transverse) Loading ......................................................... 109

3.8. Testing SIP in Axial Compression Loading............................................................... 113

3.9. Testing SIP under Racking and Cyclic Loading ....................................................... 119

3.10. Testing Stud in Flexure (Transverse) Loading........................................................ 127

3.11. Testing Stud Wall in Axial Compression Loading ..................................................... 132

3.12. Testing Stud Wall under Racking and Cyclic Loading............................................. 137

CHAPTER 4 - EXPERIMENTAL RESULTS.......................................................................... 142

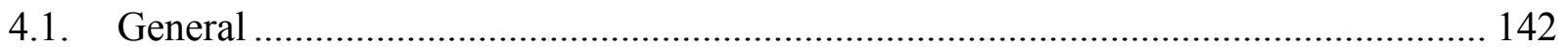

4.2. Test results for axial compression and flexure ........................................................ 142

4.3. Stud Wall under Lateral Loading .......................................................................... 200

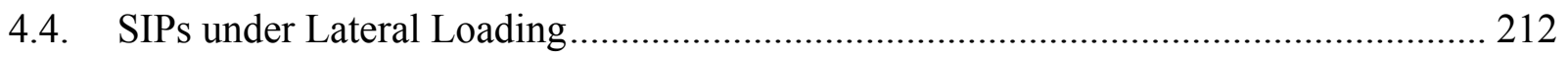

4.5. Equivalent Energy Elastic Plastic (EEEP) …………………………………........ 235

4.6. Strength and Displacement...................................................................................... 237

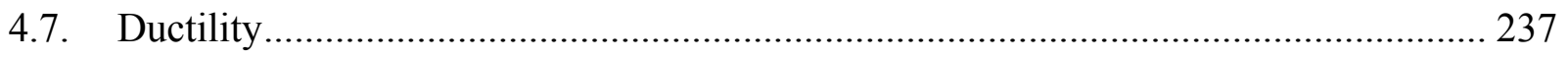

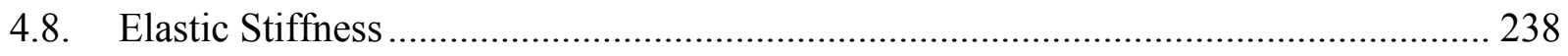

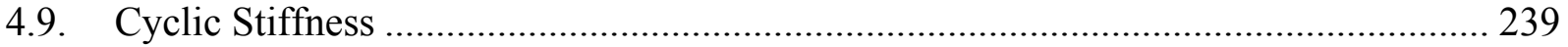

4.10. Ductility Related Force $\left(\mathrm{R}_{\mathrm{d}}\right)$ and Over-strength Related Force Modification Factor $\left(\mathrm{R}_{0}\right)$.

4.11. Comparison of SIP and Stud Panel under Different Load Conditions......................... 252 
CHAPTER 5 - FINITE ELEMENT ANALYSIS, VALIDATION AND PARAMETRIC

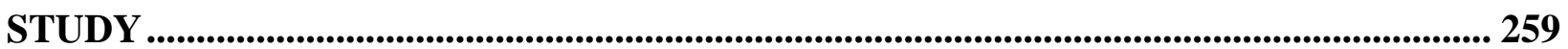

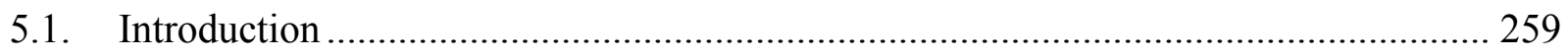

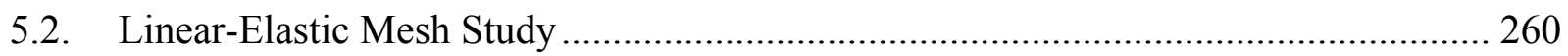

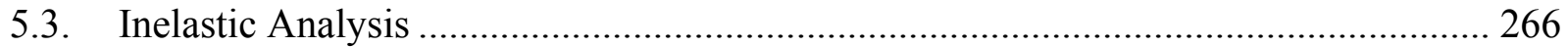

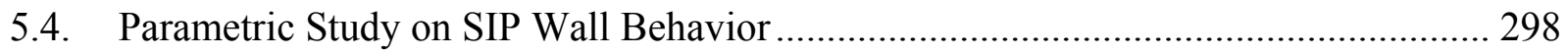

CHAPTER 6 - DEVELOPMENT OF DESIGN TABLES AND EQUATIONS FOR STUD

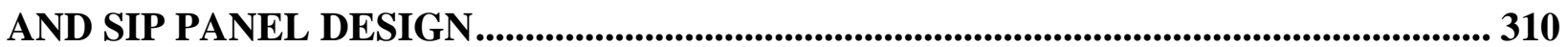

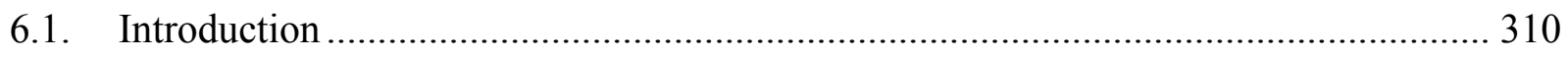

6.2. Correlation of Results from Code Equations and Experimental Data of Stud System 310

6.3. Correlation of Results from Code Equations and Experimental Data of SIP Panel

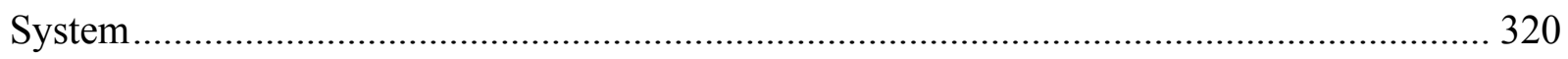

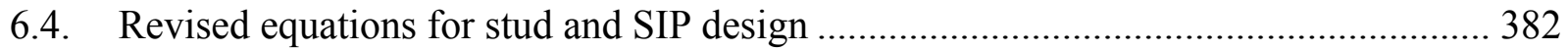

6.5. Design Table for SIP under different load .............................................................. 387

CHAPTER 7 - CONCLUSIONS AND RECOMMENDATIONS........................................... 392

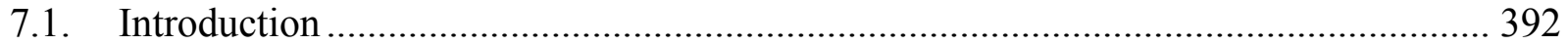

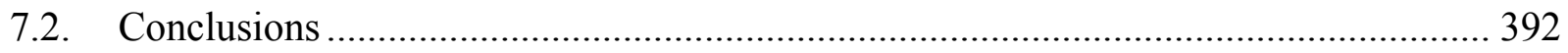

7.3. Recommendations for Future Research ………........................................................ 398

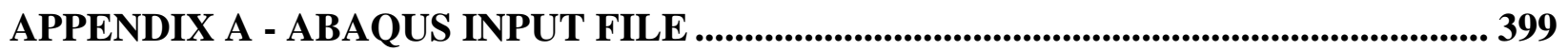

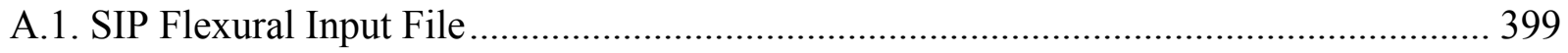

A.2. SIP Compressive Input File ...................................................................................... 430

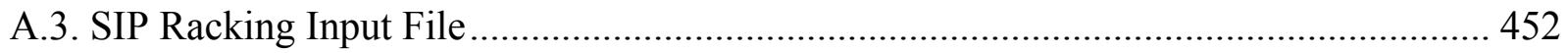

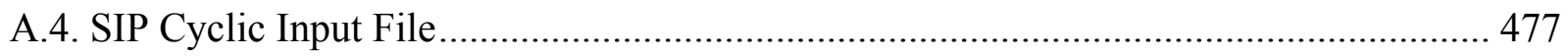

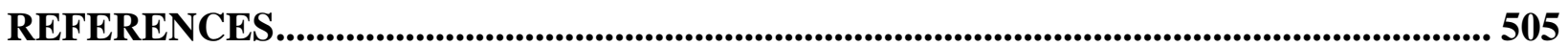




\section{LIST OF TABLES}

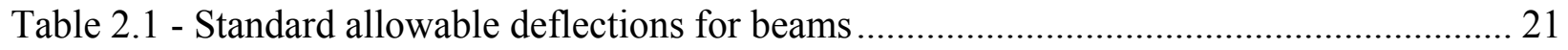

Table 2.2 - Summary of the formulations for thin-walled structure ............................................ 40

Table 2.3 - Summary of analytical studies of wood frame shear wall (Ayoub, 2006) ................. 48

Table 2.4 - CUREE loading sequence for Wood-frame structures (CUREE, 2002) .................... 51

Table 2.5 - SIP Confirmation Test Requirements ........................................................................ 78

Table 2.6 - Performance Requirements for SIP (APA, 2013) .................................................... 79

Table 3.1 - Specimen details for axial compressive load tests ....................................................... 92

Table 3.2 - Specimen details for flexural loading tests .......................................................... 93

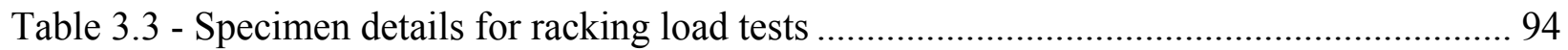

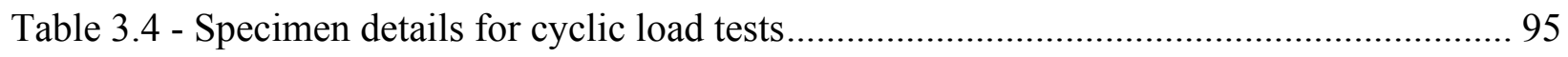

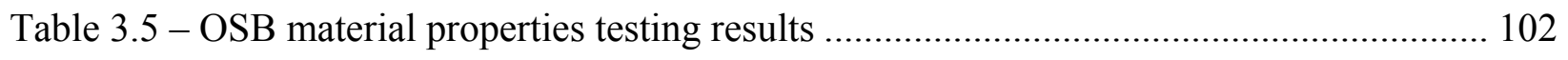

Table 3.6 - EPS modulus of elasticity obtained from flexural tests .......................................... 104

Table 3.7 - Amplitude of primary cycles in Test Method C (ASTM, 2011) .............................. 127

Table 4.1 - Results from axial compressive load tests: mean axial strengths and characteristic

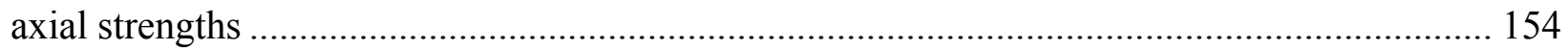

Table 4.2 - Results from axial compressive load tests: Allowable design loads and load at 3-mm

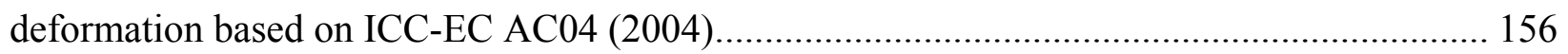
Table 4.3 - Results from flexural load tests: mean resisting moments and characteristic resisting

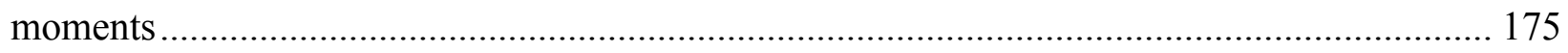

Table 4.4 - Results from flexural load tests: allowable bending moments based on ICC-EC AC04 (2004)

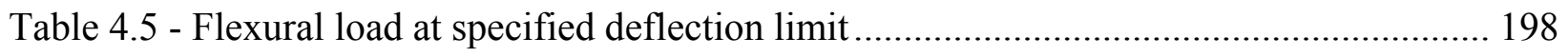

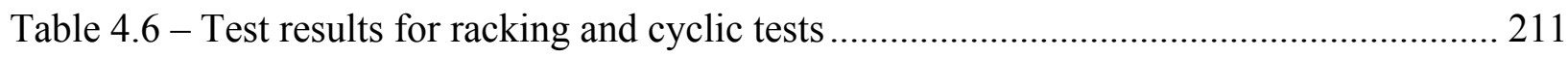

Table 4.7 - Energy dissipated for each cyclic test specimen ...................................................... 237

Table 4.8 - Elastic stiffness, Ke, ductility, $\mu,\left(R_{o}\right)$ and $\left(R_{d}\right)$ for specimen subjected to racking loading Table

Table 4.9 - Elastic stiffness. $K_{e}$, ductility, $\mu,\left(R_{o}\right)$ and $\left(R_{d}\right)$ for specimens subjected to cyclic loading (positive portion). 242 
Table 4.10 - Elastic stiffness. $K_{e}$, ductility, $\mu,\left(R_{o}\right)$ and $\left(R_{d}\right)$ for specimens subjected to cyclic

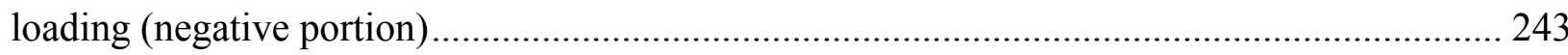

Table 4.11 - Cyclic stiffness for each primary cycle on specimen SW-C-1 …………….......... 244

Table 4.12 - Cyclic stiffness for each primary cycle on specimen SW-C-2 ………................ 244

Table 4.13 - Cyclic stiffness for each primary cycle on specimen SIP-C-1 ………................. 245

Table 4.14 - Cyclic stiffness for each primary cycle on specimen SIP-C-2 ……………….... 245

Table 4.15 - Cyclic stiffness for each primary cycle on specimen SIP-C-3 ………………...... 246

Table 4.16 - Cyclic stiffness for each primary cycle on specimen SIP-C-4 ............................ 246

Table 4.17 - Cyclic stiffness for each primary cycle on specimen SIP-C-5 ………………..... 247

Table 4.18 - Cyclic stiffness for each primary cycle on specimen SIP-C-6 ………………..... 247

Table 4.19 - Mean $R_{d}$ and $R_{0}$ factors for tested wall assemblies ............................................ 252

Table 4.20 - Average stiffness of SIP and stud wall based on results from flexural, compressive

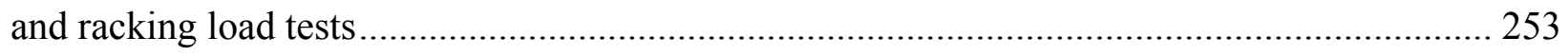

Table 4.21 - Average elastic stiffness and ductility ratio of SIP and stud wall based on results

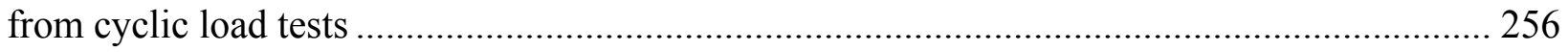

Table 5.1 - Summary of results of linear-elastic mesh study for stud wall.............................. 264 Table 5.2 - Summary of finite-element models for different size of SIP/Stud panel and load

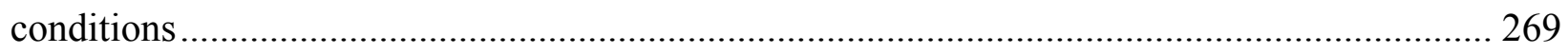

Table 5.3 - Comparisons of results of finite-element analysis and experimental findings ........ 293

Table 5.4 - SIP wall racking capacity under different intensities of gravity loading.................. 300

Table 5.6 - SIP wall capacity for different nail spacing under racking load............................... 305

Table 5.7 - SIP wall capacity for different nail spacing under cyclic load ................................ 307

Table 6.1 - Results from different codes and experimental data for flexural loading on stud

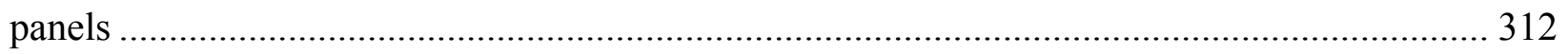

Table 6.2 - Comparison of code results and experimental findings for moment resistance for

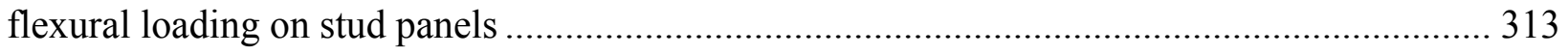

Table 6.3 - Comparison of code results and experimental findings for shear resistance for flexural loading on stud panels ................................................................................................ 313 
Table 6.4 - Comparison of code results and experimental findings for deflection for flexural loading on stud panels

Table 6.5 - Proposed modification factors for moment and shear resistances as well as the deflection of stud panels under flexural loading.

Table 6.6 - Results from different codes and experimental data for compressive load on stud walls

Table 6.7 - Comparison of results from different codes and experimental data for compressive load on stud walls.

Table 6.8 - Results from different codes and experimental data for monotonic racking load on stud walls 316

Table 6.9 - Comparison of results from different codes and experimental data for monotonic racking load resistance of stud walls 317 Table 6.10 - Comparison of results from different codes and experimental data for lateral displacement due to monotonic racking load on stud walls 317 Table 6.11 - Proposed modification factors for racking load and displacement for monotonic lateral load on stud walls.

Table 6.12 - Results from different codes and experimental data for cyclic lateral loading on stud walls 318

Table 6.13 - Comparison of results from different codes and experimental data for lateral resistance due to cyclic lateral loading on stud walls 319 Table 6.14 - Comparison of results from different codes and experimental data for lateral displacement due to cyclic lateral loading on stud walls 319 Table 6.15 - Proposed modification factors for racking load and lateral displacement for cyclic lateral loading on stud walls

Table 6.16 - Comparison of the results from different codes and experimental data for flexural loading on SIP panels...... 321

Table 6.17 - Comparison of the results from different codes and experimental data for bending resistance of SIP panels

Table 6.18 - Comparison of the results from different codes and experimental data for shear resistance of SIP panels 
Table 6.19 - Comparison of the results from different codes and experimental data for deflection of SIP panels under flexural loading.

Table 6.20 - Proposed modification factors for moment and shear resistance as well as the deflection of SIP panels under flexural loading. 358

Table 6.21 - Results from different codes and experimental data for axial compressive load on SIP walls with zero eccentricity. 360

Table 6.22 - Proposed modification factors for axial compressive resistance of SIP walls with zero eccentricity. 366

Table 6.23 - Results from different codes and experimental data for axial compressive load on SIP walls with $\mathrm{t} / 6$ eccentricity 368

Table 6.24 - Proposed modification factors for compressive resistance of SIP panels with t/6 eccentricity..... 372

Table 6.25 - Comparison of the results from different codes and experimental data for monotonic lateral load on SIP walls 374 Table 6.26 - Proposed modification factors for racking resistance and corresponding lateral displacement for SIP walls subjected to racking loading 377

Table 6.27 - Comparison of the results from different codes and experimental data for cyclic lateral load on SIP walls

Table 6.28 - Proposed modification factors for cyclic racking resistance and corresponding lateral displacement for cyclic lateral load on SIP walls 382

Table 6.29 - Proposed resisting moment for stud panels under flexural load..... 383

Table 6.30 - Proposed resisting shear forces for stud panels under flexural load. 383

Table 6.31 - Proposed equations for calculations of stud panel deflection under flexural load 383

Table 6.32 - Proposed resisting moment of SIP panels under flexural load 384

Table 6.33 - Proposed resisting shear force for SIP panels under flexural load 384

Table 6.34 - Proposed deflection of SIP panels under flexural load 384

Table 6.35 - Proposed axial compressive resistance of SIP walls under gravity loading ..... 385

Table 6.36 - Proposed compressive resistance of stud walls subjected to compressive loading at $\mathrm{t} / 6$ eccentricity... 385

Table 6.37 - Proposed compressive resistance of SIP walls subjected to compressive loading at t/6 eccentricity 385 
Table 6.38 - Proposed lateral load resistance of stud walls under monotonic lateral loading ... 386 Table 6.39 - Proposed lateral displacement of stud walls under monotonic lateral loading...... 386 Table 6.40 - Proposed lateral load resistance of SIP walls under monotonic lateral loading .... 386 Table 6.41 - Proposed lateral displacement of SIP walls under monotonic lateral loading....... 386 Table 6.42 - Proposed lateral load resistance of stud walls under cyclic lateral loading........... 386 Table 6.43 - Proposed lateral displacement of stud walls under cyclic lateral loading ............. 387 Table 6.44 - Proposed lateral load resistance of SIP walls under cyclic lateral loading............ 387 Table 6.45 - Proposed lateral displacement SIP walls under cyclic lateral loading................... 387 Table 6.46 - Maximum uniform load based on limiting shear, moment, and deflection obtained

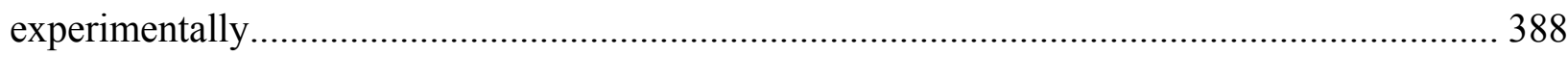
Table 6.47 - Maximum uniform load based on limiting shear, moment, and deflection obtained

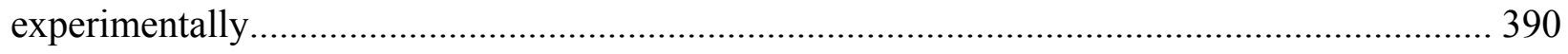
Table 6.48 - Design table for SIP wall under compressive loading with $t / 6$ eccentricity........... 391 


\section{LIST OF FIGURES}

Figure 2.1 - View of a structural insulated panel (Thermapan, 2007) …...................................... 7

Figure 2.2 - Layers in a Structural Insulated Panel (SIPA, 2007) …………………………...... 7

Figure 2.3 - View of residential building construction using SIPs (Kingspan, 2014) .................. 12

Figure 2.4 - Schematic diagram of foam spline and lumber spline detail connections ................ 13

Figure 2.5 - Schematic diagram of sandwich panel member elevation and cross-section (Adapted

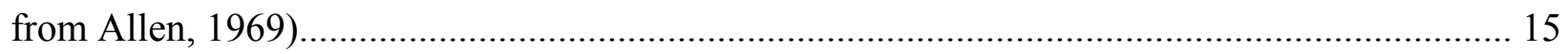

Figure 2.6 - Normal and shear stress profiles of a sandwich beam.......................................... 19

Figure 2.7 - Effect of weak core, neglecting the local bending stiffness of the sheathing (Adapted

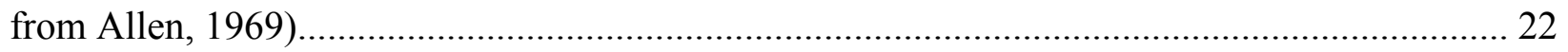

Figure 2.8 - Deflection of an axially loaded pin-ended sandwich strut (Adapted from DIAB,

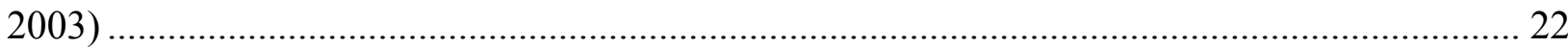

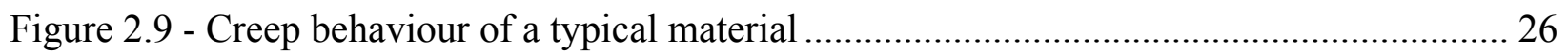

Figure 2.10 - Modes of failure for sandwich walls .............................................................. 37

Figure 2.11 - Sandwich panel configuration (Bazant, 1971) ………………………............... 38

Figure 2.12 - Values of $K_{s}$ in equation for critical shear stress for an infinitely long flat plate with

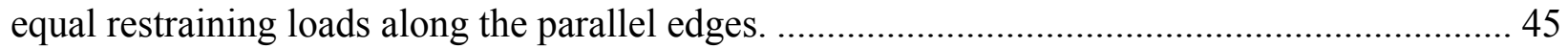

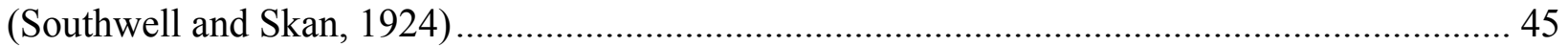

Figure 2.13 - The sheathing area ratio parameters on long shear wall with opening (Dolan and

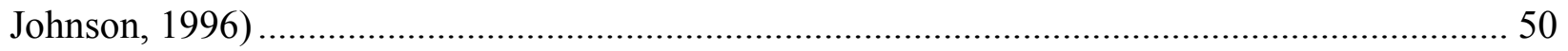

Figure 2.14 - CUREE loading protocol (CUREE, 2002) ………………………………......... 51

Figure 2.15 - Cross section of typical standard shear wall and mid-ply wall ............................... 52

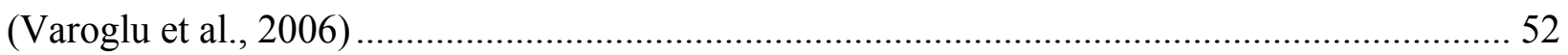

Figure 2.16 - Test assembly and setup (Talbot, 2008)............................................................... 53

Figure 2.17 - The corner test setup of solid model on shake table and seismic mass representing a typical upper floor level and roof system (Van de Lindt, 2008)................................................. 54

Figure 2.18 - Dynamic testing frame with locations of transducers for cyclic shear wall test

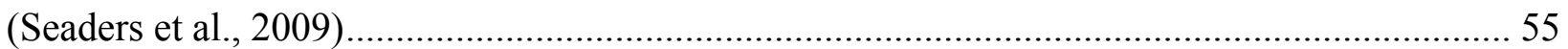

Figure 2.19 - Schematic sketch of shear wall test specimen (White et al., 2009) ………............ 57

Figure 2.20 - Test setup of full-scale cold-formed steel strap-braced wall................................... 58

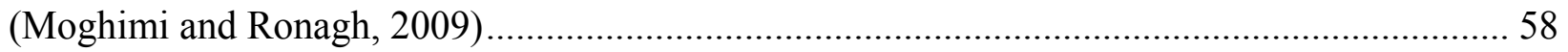


Figure 2.21 - Schematic elevation of the setup for diaphragm under lateral load as well as the Lshape walls (Songlai et al., 2010) .....

Figure 2.22 - Schematic diagram of a stressed skin panel dimensions (Adapted from CAN/CSAO86-09, 2010) 61

Figure 2.23 - Beam under two-point loads with simply-supported conditions ......................... 68

Figure 2.24 - Examples of hold-downs and anchorages (Adapted from CWC, 2010) ............. 71

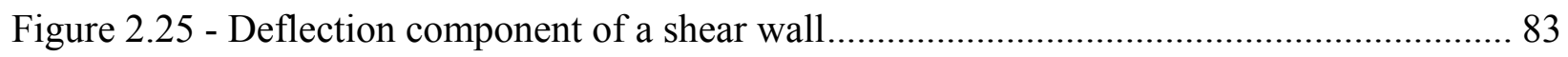

Figure 2.26 - Set of anchor bolts for shear transfer (Adapted from UBC, 2000) ................... 87

Figure 3.1 - Typical floor and basement wall construction using SIPs ................................. 89

Figure 3.2 - Typical section at panel foam-spline connection before and after assembly...........90

Figure 3.3 - Typical section at panel lumber-spline connection before and after assembly........ 91

Figure 3.4 - Schematic diagrams of the tested stud walls ................................................... 96

Figure 3.5 - OSB bending test setup before and after flexural test ...................................... 100

Figure 3.6 - OSB tension test setup before and after tension test ........................................ 100

Figure 3.7 - Typical OSB load-deflection curve obtained from the flexural test .................... 101

Figure 3.8 - Typical OSB load-deflection curve obtained from tension test.......................... 101

Figure 3.9 - EPS bending test setup before and after test .............................................. 103

Figure 3.10 - Typical EPS load-deflection curve obtained from bending test......................... 103

Figure 3.11 - View of hydraulic pump connecting to loading jack..................................... 105

Figure 3.12 - View of hydraulically operated universal loading Jack ................................. 105

Figure 3.13 - View of hydraulic actuator for racking and cyclic loading ............................ 106

Figure 3.14 - View of universal Flat Load Cell model LC411-200k standard high capacity .... 107

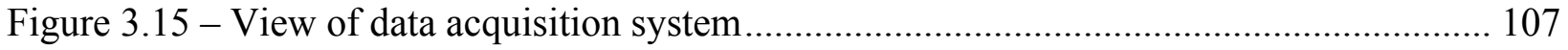

Figure 3.16 - View of computer and data acquisition system in test condition ...................... 107

Figure 3.17 - View of potentiometer used in this study ................................................. 108

Figure 3.18 - View of POTs locations in panel for stud wall specimen ............................... 108

Figure 3.19 - Views of positions of POTs in axial loading condition................................... 109

Figure 3.20 - Schematic plan of the setup for flexure of tests of SIP floor ........................... 110

Figure 3.21 - Typical test setup for flexure of the SIP floor ............................................ 110

Figure 3.22 - Schematic side view of the setup for flexure of the SIP floor ......................... 111 
Figure 3.23 - View of LVDTs at mid-span location of SIP floor 111

Figure 3.24 - Details of test setup for flexure of the SIP floor.....

Figure 3.25- Schematic test setup for the eccentric compressive load test on SIP wall 115

Figure 3.26 - Typical test setup for flexure eccentric compressive load of the SIP wall. 116

Figure 3.27 - Details of the top rail used to distribute the compressive loading over the SIP wall width

Figure 3.28 - Cross-section of the top portion of the SIP wall showing restraint system for lateral movement at top of wall 117

Figure 3.29 - Details of test setup for eccentric compressive load testing on the SIP wall ....... 118 Figure 3.30 - Schematic elevation of the test setup for monotonic and cyclic lateral load for the SIP wall 120

Figure 3.31 - Typical test setup for monotonic and cyclic lateral load testing on the SIP wall. 121 Figure 3.32 - Details of the base and different section of test setup for monotonic and cyclic lateral load for the SIP wall 122

Figure 3.33 - Details of boundary conditions at top and bottom of SIP wall under monotonic and cyclic lateral load. 122

Figure 3.34 - CUREE Protocol for displacement control (Krawinkler et al., 2001) ..... 125

Figure 3.35 - Schematic plan of test setup of flexure for stud panel specimen ..... 129

Figure 3.36 - Typical test setup of flexure for stud panel specimen 130

Figure 3.37 - Schematic side view of the test setup of flexure for stud panel specimen 130

Figure 3.38 - Detail of support condition and POTs position of flexure test for stud panel specimen 131

Figure 3.39 - Schematic test setup for the eccentric compressive load test for stud wall ......... 133

Figure 3.40 - Typical test setup for the eccentric compressive load test for stud wall 134

Figure 3.41 - Details of the top rail used to distribute the compressive loading over the stud wall width 135

Figure 3.42 - Plan cross-section of the top portion of the stud wall showing restraint system for lateral movement at top of wall 135

Figure 3.43 - Details test setup for compressive loading on stud wall 136

Figure 3.44 - Typical test setup of monotonic and cyclic lateral load for the stud wall 138 
Figure 3.45 - Details of top and bottom test setup of monotonic and cyclic lateral load for the stud wall 138

Figure 4.1 - Views of the test setup for wall SIP-A-1 before testing ........................................ 145

Figure 4.2 - Views of the deformed shape of the wall SIP-A-1 after failure ............................. 146

Figure 4.3 - Close-up views of the failure modes of SIP-A-1 specimen .................................... 146

Figure 4.4 - Axial load-axial shortening relationship for specimen SIP-A-1 …....................... 147

Figure 4.5 - Axial load-lateral deflection relationships for specimen SIP-A-1 ......................... 147

Figure 4.6 - Views of the test setup for wall SIP-A-2 before testing........................................ 148

Figure 4.7 - Views of the deformed shape of the wall SIP-A-2 after failure.............................. 149

Figure 4.8 - Close-up views of the failure modes of SIP-A-2 specimen ................................... 149

Figure 4.9 - Axial load-axial shortening relationships for specimen SIP-A-2 ……………...... 150

Figure 4.10 - Axial load-lateral deflection relationship for specimen SIP-A-2 …..................... 150

Figure 4.11 - Views of the test setup for wall SIP-A-3 before testing......................................... 151

Figure 4.12 - Views of the deformed shape of the wall SIP-A-3 after failure.............................. 151

Figure 4.13 - Close-up views of the failure modes of SIP-A-3 specimen ................................. 152

Figure 4.14 - Axial load-axial shortening relationships for specimen SIP-A-3 ……………..... 153

Figure 4.15 - Axial load-lateral deflection relationship for specimen SIP-A-3 ………………... 153

Figure 4.16 - Views of the test setup for stud wall SW-A-1 before testing................................ 157

Figure 4.17 - Views of the deformed shape of the stud wall SW-A-1 after OSB joint opening at

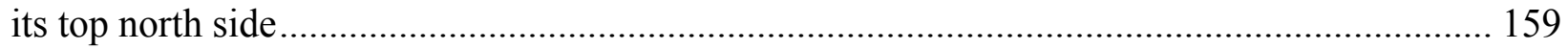

Figure 4.18 - Axial load-axial shortening relationships for stud wall SW-A-1 ......................... 160

Figure 4.19 - Axial load-lateral deflection relationship for stud wall SW-A-1 .......................... 160

Figure 4.20 - Views of the test setup for the stud wall SW-2 before testing ............................... 161

Figure 4.21 - Views of the deformed shape of the stud wall SW-A-2 after failure..................... 162

Figure 4.22 - Rotation of the top rail at failure of stud wall specimen SW-A-2 …………….... 162

Figure 4.23 - Axial load-axial shortening relationships for stud wall SW-A-2 ......................... 163

Figure 4.24 - Axial load-lateral deflection relationships for stud wall SW-A-2 ....................... 163

Figure 4.25 - Views of the test setup for stud wall SW-3 before testing .................................... 164

Figure 4.26 - Views of the deformed shape of stud wall SW-A-3 after failure........................... 165

Figure 4.27 - Axial load-axial shortening relationships for stud wall SW-A-3 .......................... 165 
Figure 4.28 - Axial load-lateral deflection relationships for stud wall SW-A-3 .................... 166

Figure 4.29 - Views of the test setup for specimen SIP-F-1 before testing ........................... 168

Figure 4.30 - View of the deflected shape of specimen SIP-F-1 at support after failure .......... 168

Figure 4.31 - Close-up view of specimen SIP-F-1 showing horizontal shear failure at the top

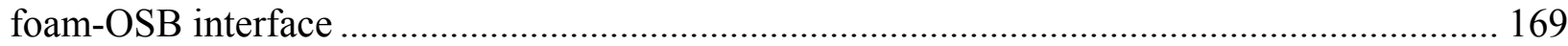

Figure 4.32 - Jacking load-deflection relationship for specimen SIP-F-1 ............................ 169

Figure 4.33 - Views of the test setup for specimen SIP-F-2 before testing ............................ 170

Figure 4.34 - View of the deflected shape of specimen SIP-F-2 after failure ........................ 171

Figure 4.35 - Close-up views of the failure mode of specimen SIP-F-2 …......................... 171

Figure 4.36 - Jacking load-deflection relationship for specimen SIP-F-2 .......................... 172

Figure 4.37 - Views of the test setup for specimen SIP-F-3 before testing ........................... 172

Figure 4.38 - View of the deflected shape of specimen SIP-F-3 after failure ........................ 173

Figure 4.39 - Close-up views of specimen SIP-F-3 showing horizontal shear failure at the top

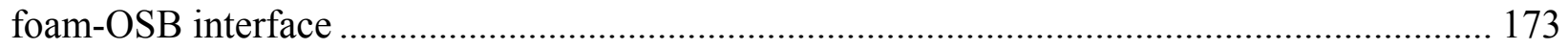

Figure 4.40 - Jacking load-deflection relationship for specimen SIP-F-3 ........................... 174

Figure 4.41 - View of specimen SW-F-1 before testing ................................................. 178

Figure 4.42 - View of deflected shape of specimen SW-F-1 after failure ............................. 179

Figure 4.43 - Close-up view of the flexural failure pattern in the first joist at end of specimen SW-F-1 179

Figure 4.44 - Other view of the deflected shape and flexural failure at mid-span of first joist in specimen SW-F-1. 180

Figure 4.45 - Close-up view from underside of the first joist showing longitudinal and transverse

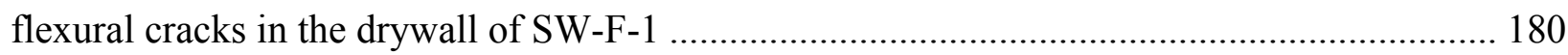

Figure 4.46 - View of the joists after removing the drywall of specimen SW-F-1 ................ 181

Figure 4.47 - Close-up view of the flexural failure longitudinal in joist No. 1 of specimen SW-F-

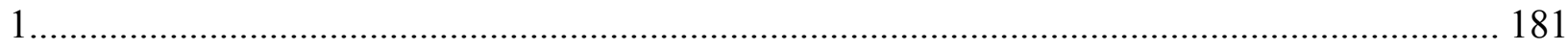

Figure 4.48 - Close-up view of crack in joist No. 2 of specimen SW-F-1 ........................... 181

Figure 4.49 - Axial load-axial shortening relationships for specimen SW-F-1 ..................... 182

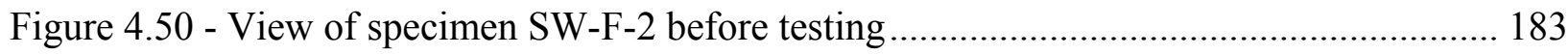

Figure 4.51 - Deflected shape of specimen SW-F-2 after failure ......................................... 183

Figure 4.52 - Close-up view of the deflected shape of specimen SW-F-2 after failure ............ 184 
Figure 4.53 - Close-up view of the flexural failure at the quarter point of joist No. 5 of specimen SW-F-2.

Figure 4.54 - Close-up view of the failure around the quarter point of joist No. 3 of specimen SW-F-2 185

Figure 4.55 - View of drywall break at the quarter point of joist No. 1 of specimen SW-F-2 ... 185

Figure 4.56 - View of the joists after removing the drywall of specimen SW-F-2 186

Figure 4.57 - Close-up view of failure of joists No. 3, 4 and 5 of specimen SW-F-2 after failure 186

Figure 4.58 - Close-up view of failure in joist failure of No. 3 and 4 of specimen SW-F-2 _.... 187

Figure 4.59 - Close-up view of joists 4 and 5 of specimen SW-F-2 _................................ 187

Figure 4.60 - Axial load-axial shortening relationships for specimen SW-F-2 ..................... 187

Figure 4.61 - View of Specimen SW-F-3 before testing .................................................. 188

Figure 4.62 - View of specimen SW-F-3 after failure ................................................... 189

Figure 4.63 - View of the deflected shape of specimen SW-F-3 after failure ......................... 189

Figure 4.64 - View of the permanent deflected shape of specimen SW-F-3 after removing the loading system 190

Figure 4.65 - View of the flexural failure and deformation of joist No. 5 of specimen SW-F-3

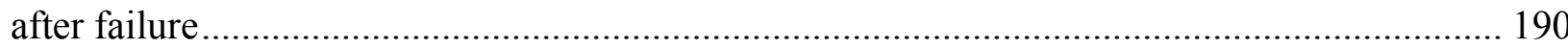

Figure 4.66 - Close-up view of the flexural failure of joist No. 5 in specimen SW-F-3 .......... 191

Figure 4.67 - Front view of the flexural failure of joist No. 5 in specimen SW-F-3 ................ 191

Figure 4.68 - Close-up view of the flexural failure of joists No. 4 and 5 and well as drywall break in specimen SW-F-3

Figure 4.69 - View of the deflected shape of specimen SW-F-3 showing the bearing rotation as well as end stud and OSB sheet separation from joist No. 1

Figure 4.70 - Close-up view of the bearing rotation as well as end stud and OSB sheet separation from joist No. 1 of specimen SW-F-3 after failure. 193

Figure 4.71 - View of the joists after removing the drywall of specimen SW-F-3 ..... 193 Figure 4.72 - Close-up view of the failure in joists No. 4 and 5 in specimen SW-F-3 after removing the drywall 194

Figure 4.73 - Axial load-axial shortening relationship for specimen SW-F-3 194

Figure 4.74 - Test setup of specimen SW-R-1 under racking load. 200 
Figure 4.75 - Global failure of specimen SW-R-1 under racking load showing .................... 201

Figure 4.76 - Detailed failure modes of specimen SW-R-1 under racking loading................ 202

Figure 4.77 - Load vs. lateral deflection response of SW-R-1 (obtained from actuator reading on

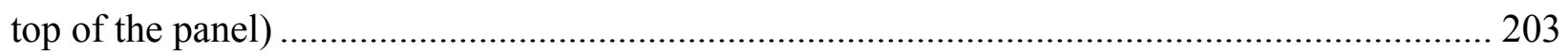

Figure 4.78 - Load vs. lateral deflection response of SW-R-2 (obtained from actuator reading on top of the panel) 203

Figure 4.79 - Load vs. lateral deflection response of SW-R-1 (POTs records up to a load close to failure load) 204

Figure 4.80 - Load vs. lateral deflection response of SW-R-2 (POTs records up to a load close to failure load) 204

Figure 4.81 - Test setup of specimen SW-C-1 under cyclic loading 205

Figure 4.82 - Failure of specimen SW-C-1 under cyclic loading. 206

Figure 4.83 - Detailed failure modes of specimen SW-C-1 under cyclic loading 207

Figure 4.84 - Load vs. lateral deflection response of SW-C-1 (obtained from actuator reading on top of the panel) 208

Figure 4.85 - Load vs. lateral deflection response of SW-C-2 (obtained from actuator reading on top of the panel). 209

Figure 4.86 - Load vs. lateral deflection response of SW-C-1 (POTs records up to a load close to failure load) 209

Figure 4.87 - Load vs. lateral deflection response of SW-C-2 (POTs records up to a load close to failure load) 210

Figure 4.88 - Test setup of specimen SIP-R-1 under racking load. 212

Figure 4.89 - Global failure of specimen SIP-R-1 under racking load showing global shear deformation and relative shear rotation of SIP segments at spline connection 213 Figure 4.90 - Detailed Failure modes of specimen SIP-R-1 under racking load .................... 214

Figure 4.91 - Test setup of specimen SIP-R-4 under racking load ..................................... 215 Figure 4.92 - Global failure of specimen SIP-R-4 under racking load showing global shear deformation and relation shear rotation of SIP segments at spline connection ....................... 216

Figure 4.93 - Detailed Failure modes of specimen SIP-R-4 under racking load ................... 217 Figure 4.94 - Load vs. lateral deflection response of SIP-R-1 (obtained from actuator reading on top of the panel) 218 
Figure 4.95 - Load vs. lateral deflection response of SIP-R-2 (obtained from actuator reading on top of the panel).

Figure 4.96 - Load vs. lateral deflection response of SIP-R-3 (obtained from actuator reading on top of the panel).

Figure 4.97- Load vs. lateral deflection response of SIP-R-4 (obtained from actuator reading on top of the panel)

Figure 4.98 - Load vs. lateral deflection response of SIP-R-5 (obtained from actuator reading on top of the panel).

Figure 4.99 - Load vs. lateral deflection response of SIP-R-6 (obtained from actuator reading on top of the panel).

Figure 4.100 - Load vs. lateral deflection response of SIP-R-1 (POTs records up to a load close to failure load)

Figure 4.101 - Load vs. lateral deflection response of SIP-R-2 (POTs records up to a load close to failure load)

Figure 4.102 - Load vs. lateral deflection response of SIP-R-3 (POTs records up to a load close to failure load)

Figure 4.103 - Load vs. lateral deflection response of SIP-R-4 (POTs records up to a load close to failure load) 222

Figure 4.104 - Load vs. lateral deflection response of SIP-R-5 (POTs records up to a load close to failure load) 223

Figure 4.105 - Load vs. lateral deflection response of SIP-R-6 (POTs records up to a load close to failure load) 223

Figure 4.106 - Test setup of specimen SIP-C-1 under cyclic loading 224

Figure 4.107 - Global failure of specimen SIP-C-1 under cyclic loading showing global shear deformation and relation shear rotation of SIP segments at spline connection ..... 225

Figure 4.108 - Detailed failure modes of specimen SIP-C-1 under cyclic load...................... 226

Figure 4.109 - Test setup of specimen SIP-C-4 under cyclic loading 226

Figure 4.110 - Global failure of specimen SIP-C-4 under cyclic loading showing global shear deformation and relation shear rotation of SIP segments at spline connection ...... 227 Figure 4.111 - Detailed failure modes of specimen SIP-C-4 under cyclic load 228 
Figure 4.112 - Load vs. lateral deflection response of SIP-C-1 (obtained from actuator reading on top of the panel)

Figure 4.113 - Load vs. lateral deflection response of SIP-C-2 (obtained from actuator reading on top of the panel).

Figure 4.114 - Load vs. lateral deflection response of SIP-C-3 (obtained from actuator reading on top of the panel) 230

Figure 4.115 - Load vs. lateral deflection response of SIP-C-4 (obtained from actuator reading on top of the panel)

Figure 4.116 - Load vs. lateral deflection response of SIP-C-5 (obtained from actuator reading on top of the panel).

Figure 4.117 - Load vs. lateral deflection response of SIP-C-6 (obtained from actuator reading on top of the panel) 232

Figure 4.118 - Load vs. lateral deflection response of SIP-C-1 (POTs record up to a load close to the failure load) 232 Figure 4.119 - Load vs. lateral deflection response of SIP-C-2 (POTs record up to a load close to the failure load) 233

Figure 4.120 - Load vs. lateral deflection response of SIP-C-3 (POTs record up to a load close to the failure load) 233 Figure 4.121 - Load vs. lateral deflection response of SIP-C-4 (POTs record up to a load close to the failure load) 234

Figure 4.122 - Load vs. lateral deflection response of SIP-C-5 (POTs record up to a load close to the failure load) 234

Figure 4.123 - Load vs. lateral deflection response of SIP-C-6 (POTs record up to a load close to the failure load) 235

Figure 4.124 - Cyclic load vs. lateral deflection relationship, EEEP curve and envelope of the cyclic load history for Specimen SIP-C-6 . 236

Figure 4.125 - Cyclic stiffness vs. peak displacement relationship for stud walls ..... 248 SW-C-1 and SW-C-2 248

Figure 4.126 - Cyclic stiffness vs. peak displacement relationship for short SIP walls SIP-C-1 to SIP-C-3 248 
Figure 4.127 - Cyclic stiffness vs. peak displacement relationship for long SIP walls SIP-C-4 to SIP-C-6

Figure 4.128 - Hysteretic energy dissipated per cycle vs. displacement of stud wall (SW-C-1 and SW-C-2)

Figure 4.129 - Hysteretic energy dissipated per cycle vs. displacement of short SIP wall (SIP-C1 and SIP-C-3) 250

Figure 4.130 - Hysteretic energy dissipated per cycle vs. displacement of long SIP wall (SIP-C-4 and SIP-C-6) 250

Figure 4.131 - Comparison of load vs. deflection relationship of the SIP specimen SIP-F-1 and stud panel SW-F-1 under flexural loading. 253

Figure 4.132 - Comparison of load vs. axial shortening of the SIP wall SP-A-1 and stud wall SW-A-1 under compressive loading. 254

Figure 4.133 - Comparison of load vs. lateral deflection relationship of the specimen SIP-R-1 and stud wall SW-R-1 under racking load.... 255

Figure 4.134 - Comparison of load vs. lateral deflection history of specimen SIP-C-2 and stud wall SW-C-2 under cyclic loading... 257

Figure 4.135 - Comparison of dissipated energy of SIP and stud wall under cyclic loading ..... 257

Figure 4.136 - Comparison of cyclic stiffness of SIP and stud wall under cyclic loading ......... 258

Figure 5.1 - Views of OSB sheet meshes used for linear elastic mesh study ..... 261

Figure 5.2 - Views of different part of the FEA model in stud panel under compressive loading 262

Figure 5.3 - View of ABAQUS complete FEA model of stud wall frame and OSB sheet for compressive loading. 262

Figure 5.4 - Views of different parts of the FEA model of SIP under compressive loading ..... 263 Figure 5.5 - View of the complete FE model of SIP with OSB sheathing for compressive loading 263

Figure 5.6 - Summary of results of linear elastic mesh study for stud wall. 265

Figure 5.7 - Von Mises stress contour plots from linear elastic mesh study for a stud wall panel 266

Figure 5.8 - Stress-strain relationship of EPS foam sheet obtained from tension test 267 
Figure 5.9 - Engineering stress-strain relationship of OSB sheet obtained from tension test.... 267 Figure 5.10 - Engineering stress-strain relationship of wood in tension obtained from commercial values 268

Figure 5.11 - Surface-to-surface contact and different constraints of drywall and stud frame in ABAQUS model of stud panel ...... 270

Figure 5.12 - Surface-to-surface contact and different constraints of OSB sheet and stud frame in ABAQUS Model of stud panel.

Figure 5.13 - Surface-to-surface contact of OSB sheet with lumber and boundary conditions in ABAQUS Model of SIP panel 272

Figure 5.14 - Surface-to-surface contact of OSB sheet with glue and EPS with Lumber in ABAQUS Model of SIP.

Figure 5.15 - Complete ABAQUS model of stud wall SW-A-1 under compressive loading with eccentricity 273

Figure 5.16 - Complete ABAQUS model of stud wall SW-F-1 under flexural loading 273 Figure 5.17 - Complete ABAQUS model of stud wall SW-R-1 and SW-C-1 under racking or cyclic loading..... 274

Figure 5.18 - Complete ABAQUS model of SIP wall SIP-R-1 and SIP-C-1 under racking or cyclic load 274

Figure 5.19 - Complete ABAQUS model of SIP wall SIP-R-4 and SIP-C-4 under racking or cyclic load 275

Figure 5.20 - Effect of mesh refinement for stud panel wall SW-A-1 under compressive loading 276

Figure 5.21 - Effect of mesh refinement for stud panel floor SW-F-1 under flexural loading .. 276 Figure 5.22 - Deformed configuration of stud wall SW-A-1 loaded in compression. 279

Figure 5.23 - Deformed shape of stud panel SW-F-1 loaded in flexure .................................. 280

Figure 5.24 - Deformed shape of stud wall SW-R-1 under racking load.................................. 281

Figure 5.25 - Deformed shape of stud wall SW-C-1 under cyclic loading $\left(6^{\text {th }}\right.$ cycle $) \ldots \ldots \ldots \ldots . . . . . .283$ Figure 5.26 - Deformed configuration of SIP wall model (SIP-A-1) under axial compressive loading... 284

Figure 5.27 - Deformed configuration of SIP floor model (SIP-F-1) under flexural loading.... 285

Figure 5.28 - Deformed shape of SIP model SIP-R-1 under racking load. 286 
Figure 5.29 - Deformed shape of SIP model SIP-R-4 under racking load................................. 288

Figure 5.30 - Deformed shape of SIP model SIP-C-1 under cyclic load $\left(6^{\text {th }}\right.$ cycle $) \ldots \ldots \ldots \ldots \ldots . . . . . .289$

Figure 5.31 - Deformed shape of SIP model SIP-C-4 under lateral cyclic load $\left(6^{\text {th }}\right.$ cycle $) \ldots . . . .291$

Figure 5.32 - Comparison between the FEA results and experimental findings of stud wall .... 292

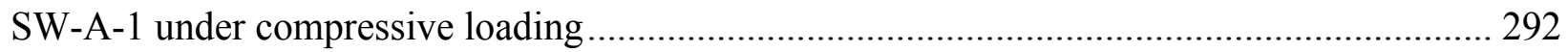

Figure 5.33 - Comparison between the FEA results and experimental findings of stud wall .... 294

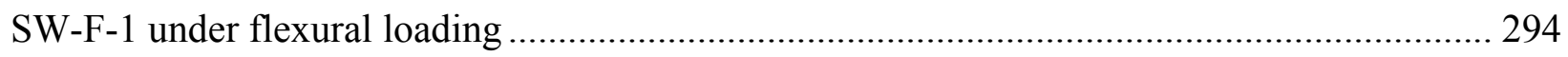

Figure 5.34 - Comparison between the FEA results and experimental findings of stud wall .... 294

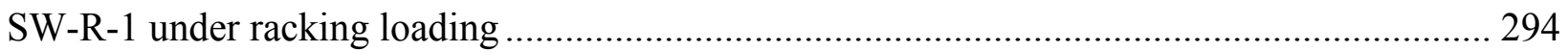

Figure 5.35 - Comparison between the FEA results and experimental findings of stud wall .... 295

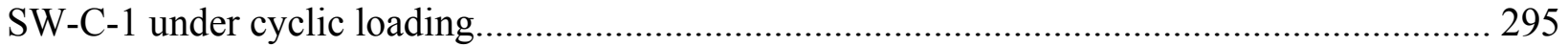

Figure 5.36 - Comparison between the FEA results and experimental findings of stud wall .... 295

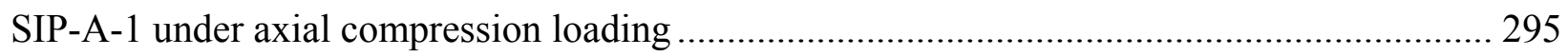

Figure 5.37 - Comparison between the FEA results and experimental findings of stud wall .... 296

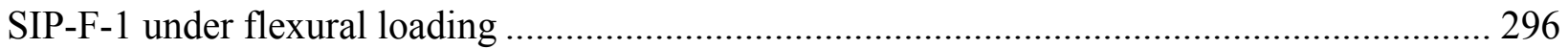

Figure 5.38 - Comparison between the FEA results and experimental findings of SIP wall ..... 296

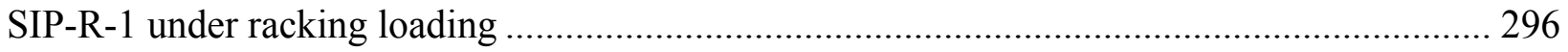

Figure 5.39 - Comparison between the FEA results and experimental findings of SIP wall ..... 297

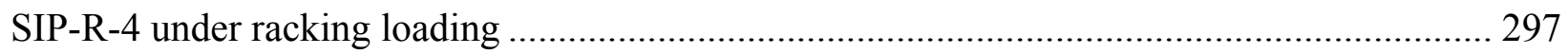

Figure 5.40 - Comparison between the FEA results and experimental findings of SIP wall .... 297

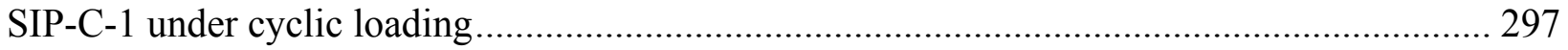

Figure 5.41 - Comparison between the FEA results and experimental findings of SIP wall ..... 298

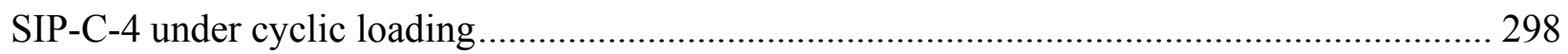

Figure 5.42 - Racking load vs. lateral deflection relationship of SIP wall SIP-R-1 $2440 \times 2750 \times 165 \mathrm{~mm}\left(8^{\prime} \times 9^{\prime} \times 6^{1 / 2} 2^{\prime \prime}\right)$ under racking load and varying gravity loading ................ 300

Figure 5.43 - Racking load vs. lateral deflection relationship of SIP wall SIP-R-4 $3660 \times 2750 \times 165 \mathrm{~mm}\left(12^{\prime} \times 9^{\prime} \times 6^{1 / 2}{ }^{\prime \prime}\right)$ under racking load and varying gravity loading .............. 301

Figure 5.44 - Effect of gravity load on SIP wall racking capacity ............................................. 301

Figure 5.45 - Cyclic load vs. lateral deflection relationship of SIP wall SIP-C-1 $2440 \times 2750 \times 165$

$\mathrm{mm}\left(8^{\prime} \times 9^{\prime} \times 6^{1 / 2} 2^{\prime}\right)$ under cyclic load and varying gravity loading ........................................... 303 
Figure 5.46 - Cyclic load vs. lateral deflection relationship of SIP wall SIP-C-4 $3660 \times 2750 \times 165$ $\mathrm{mm}\left(12^{\prime} \times 9^{\prime} \times 6^{1 / 2} 2^{\prime \prime}\right)$ under cyclic load and varying gravity loading .......................................... 303

Figure 5.47 - Figure 5.43 - Effect of gravity load on SIP wall cyclic capacity ......................... 304 Figure 5.48 - Racking load vs. lateral deflection for SIP wall SIP-R-1 (8'x9'x6 1/2") with

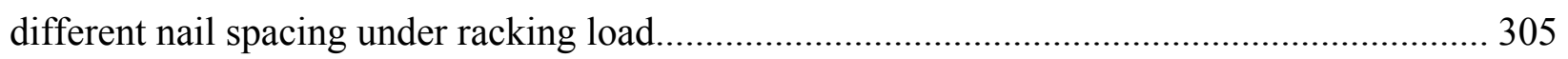

Figure 5.49 - Racking load vs. lateral deflection for SIP wall SIP-R-4 $3660 \times 2750 \times 165 \mathrm{~mm}$

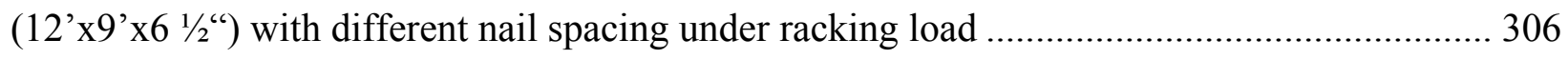

Figure 5.50 - Effect of nail spacing on the racking capacity of SIP walls under racking load .. 306 Figure 5.51 - Cyclic load vs. lateral deflection for SIP wall SIP-C-1 ………………............. 308 $2440 \times 2750 \times 165 \mathrm{~mm}\left(8^{\prime} \times 9\right.$ 'x6 1/2" $)$ with different nail spacing under cyclic load ..................... 308

Figure 5.52 - Cyclic load vs. lateral deflection for SIP wall SIP-C-4 _................................... 308 $3660 \times 2750 \times 165 \mathrm{~mm}\left(12^{\prime} \times 9\right.$ 'x6 $\left.{ }^{1 / 2 “}\right)$ with different nail spacing under cyclic load ................... 308 Figure 5.53 - Effect of nail spacing for different size of SIP wall $2440 \times 2750 \times 165 \mathrm{~mm}$ and $3660 \times 2750 \times 165 \mathrm{~mm}\left(8^{\prime} \times 9^{\prime} \times 6^{1 / 2}\right.$ ' and $8^{\prime} \times 12^{\prime} \times 6^{1 / 2}$ “') under cyclic load 309

Figure 6.1 - Correlation between the resisting moment obtained experimentally and that obtained using CSA-O86-09 along with the values using the proposed modification factor for flexural loading on SIP panels.

Figure 6.2 - Correlation between the resisting moment obtained experimentally and that obtained using DIAB Handbook along with the values using the proposed modification factor for flexural loading on SIP panels.

Figure 6.3 - Correlation between the resisting moment obtained experimentally and that obtained using ANSI/APA along with the values using the proposed modification factor for flexural loading on SIP panels.

Figure 6.4 - Correlation between the resisting shear force obtained experimentally and that obtained using CSA-O86-09 along with the values using the proposed modification factor for flexural loading on SIP panels..... 347

Figure 6.5 - Correlation between the resisting shear force obtained experimentally and that obtained using DIAB Handbook along with the values using the proposed modification factor for flexural loading on SIP panels 347 
Figure 6.6 - Correlation between the resisting shear force obtained experimentally and that obtained using ANSI/APA along with the values using the proposed modification factor for flexural loading on SIP panels 348

Figure 6.7 - Correlation between the deflection obtained experimentally and that obtained using CSA-O86-09 along with the values using the proposed modification factor for flexural loading on SIP panels 357

Figure 6.8 - Correlation between the deflection obtained experimentally and that obtained using DIAB Handbook along with the values using the proposed modification factor for flexural loading on SIP panels...... 357

Figure 6.9 - Correlation between the deflection obtained experimentally and that obtained using ANSI/APA along with the values using the proposed modification factor for flexural loading on SIP panels. 358

Figure 6.10 - Correlation between the axial compressive resistance obtained experimentally and that obtained using NTA IM14 along with the values using the proposed modification factor for compressive loading on SIP walls with zero eccentricity...... 365 Figure 6.11 - Correlation between the axial compressive resistance obtained experimentally and that obtained using DIAB Handbook along with the values using the proposed modification factor for compressive loading on SIP walls with zero eccentricity. 365 Figure 6.12 - Correlation between the axial compressive resistance obtained experimentally and that obtained using ANSI/APA along with the values using the proposed modification factor for compressive loading on SIP walls with zero eccentricity 366 Figure 6.13 - Correlation between the axial compressive resistance obtained experimentally and that obtained using CSA-O86-09 along with the values using the proposed modification factor for compressive loading on SIP walls with $\mathrm{t} / 6$ eccentricity...... 371 Figure 6.14 - Correlation between the axial compressive resistance obtained experimentally and that obtained using DIAB's Handbook along with the values using the proposed modification factor for compressive loading on SIP walls with $\mathrm{t} / 6$ eccentricity ..... 371 Figure 6.15 - Correlation between the axial compressive resistance obtained experimentally and that obtained using ANSI/APA along with the values using the proposed modification factor for compressive loading on SIP walls with $\mathrm{t} / 6$ eccentricity 
Figure 6.16 - Correlation between the monotonic racking resistance obtained experimentally and that obtained using BS 5268-6.2 along with the values using the proposed modification factor for racking loading on SIP walls 376

Figure 6.17 - Correlation between the monotonic lateral displacement obtained experimentally and that obtained using UBC Section 1612.2 along with the values using the proposed modification factor for racking loading on SIP walls 376

Figure 6.18 - Correlation between the monotonic lateral displacement obtained experimentally and that obtained using CSA-O86-09 along with the values using the proposed modification factor for racking loading on SIP walls 377 Figure 6.19 - Correlation between the cyclic racking resistance obtained experimentally and that obtained using BS 5268-6.2 along with the values using the proposed modification factor for cyclic racking loading on SIP walls. 381 Figure 6.20 - Correlation between the cyclic lateral displacement obtained experimentally and that obtained using UBC Section 1612.2 along with the values using the proposed modification factor for cyclic racking loading on SIP walls. 381 Figure 6.21 - Correlation between the cyclic lateral displacement obtained experimentally and that obtained using CSA-O86-09 along with the values using the proposed modification factor for cyclic racking loading on SIP walls 382 


\section{ABBRIVIATIONS}

ACI

APA

ASD

ASTM

BS

CAD

CAM

CCMC

CSA

CWC

DAS

EEEP

EPA

EPS

ETAG

FEA

GWB

HVAC

LVDT

$\mathrm{NBCC}$

NRC

$\mathrm{OBC}$

OSB

SIP

SIPA

SLS

UBC

ULS

XPS
American Concrete Institute

American Panel Association

Allowable Strength Design

American Society for Testing and Materials

British Standard

Computerized Architectural Drawing

Computer Aided Manufacturing

Canadian Construction Materials Commission

Canadian Standard Association

Canadian Wood Council

Data Acquisition System

Equivalent Energy Elastic Plastic

Environmental Protection Agency

Expanded Polystyrene Foam

European technical approval guideline

Finite Element Analysis

Gypsum Wall Board

Heating, Ventilation, and Air Conditioning

Linear Variable Displacement Transducers

National Building Code of Canada

National Research Council Canada

Ontario Building Code

Oriented Strand Boards

Structural Insulated Panel

Structural Insulated Panel Association

Serviceability Limit State

Uniform Building Code

Ultimate Limit State

Extruded Polystyrene Foam 


\section{LIST OF SYMBOLS}

$\bar{y} \quad$ Distance from edge to neutral axis

$\bar{y} \quad$ Mean value of $y$

$(E I)$ Effective stiffness

$(E I) w$ Stiffness of lumber webs

$A \quad$ Total cross-sectional area of the sandwich panel

$A \| \quad$ Total vertical-grain material of stringers and skins

A1 Area of outside skin

A2 Area of inside skin

$A_{1} \quad$ Area of upper skin of section

$A_{2} \quad$ Area of lower skin of section

Ac Core area

Af Flange area

$A i \quad$ Cross-sectional area of each constituent

$A v \quad$ Shear Area of panel for symmetric panel

b Specimen width

$B_{a} \quad$ Specified axial stiffness

Bac Specified axial stiffness of compression flange

Bat Specified axial stiffness of tension flange

$b_{f} \quad$ Width of flange

bf Flange width

$b_{g} \quad$ Contact width between flange and web

$b_{P} \quad$ Width of structural panel

$B v \quad$ Shear-through-thickness rigidity of the sheathing

c Core thickness

$c_{c}$ Distance from neutral axis to compression face

$C_{c r} \quad$ Theoretical skin stress at buckling (wrinkling)

Ce Eccentric load factor

$c_{t} \quad$ Distance from neutral axis to tension face 
$c_{w} \quad$ Greatest distance from neutral axis to outer edge of web

D Sandwich flexural rigidity

d Distance between neutral axis of faces

$d_{a} \quad$ Anchorage slip

$d_{F} \quad$ Nail diameter

$\Delta(t) \quad$ Total time dependent deflection

$\Delta_{0} \quad$ Initial deflection

$\Delta_{a} \quad$ Deflection of the shear wall due to anchorage slip and rotation

$\Delta_{b} \quad$ Bending deflection of the shear wall

$\Delta_{B} \quad$ Mid-span sandwich panel deflection due to bending

$\Delta_{\text {failure }}$ Displacement at failure

$\Delta_{n} \quad$ Deflection of the shear wall due to nail slips (deformation)

$\Delta_{s} \quad$ Storey drift

$\Delta_{S} \quad$ Mid-span sandwich panel deflection due to shear

$\Delta_{s w} \quad$ Deflection of a blocked shear wall segment with $600 \mathrm{~mm}$ stud spacing and nails spaced at $150 \mathrm{~mm}$ on centre around panel edges and $300 \mathrm{~mm}$ on centre along intermediate framing members

$\Delta_{T} \quad$ Total mid-span sandwich panel deflection due to bending

$\Delta_{v} \quad$ Shear deflection of the shear wall

$\Delta_{\text {yield }} \quad$ Displacement at yield

$\mu \quad$ Ductility of structure

E Appropriate modulus of elasticity

E05 Modulus of elasticity for design of compression members

$E b \quad$ Modulus of elasticity for SIP under transverse loading

Ec Modulus of elasticity of core material

Ef Modulus of elasticity of facing material

$E_{i} \quad$ Young's modulus of each layer

en Nail deformation

f Facing thickness

$f_{b} \quad$ Specified strength in bending of webs

$F_{c} \quad$ Allowable facing compressive stress 
Fpeak Peak load on loading history

$F_{V} \quad$ Allowable shear stress in the core

$f_{v} \quad$ Applied shear stress in the core

$f \quad$ Specified strength in shear

$f_{v} \quad$ Specified strength in shear of webs

$G \quad$ Modulus of rigidity of the wood structural panel sheathing

$G \quad$ Modulus of Rigidity of column

$h \quad$ Total height of section

$h_{b} \quad$ thickness of bonded between a bottom placed and

$h_{c} \quad$ thickness of core

$h_{c} \quad$ thickness of top skin

$H_{s} \quad$ Height of shear wall segment

$h t \quad$ Thickness of top sheathing

I Moment of inertia of the uniform cross-section

Ig Gross moment of inertia of panel about neutral axis

Jub Adjustment factor for unblocked shear wall segment

$K_{c} \quad$ Elastic stiffness

$K_{D} \quad$ Load duration factor

$K_{e} \quad$ Effective length factor, elastic stiffness

$K_{F} \quad$ Foundation factor for plywood

$K_{H} \quad$ System factor

$K_{L} \quad$ Lateral stability factor for bending members

$K_{m} \quad$ Product of the material modification factors, service creep factor

$K_{N} \quad$ Notch factor

ks Factor depends on number of test specimens

$K_{S} \quad$ service condition factor for sawn lumber, glued-laminated timber, plywood, construction sheathing OSB, poles, and piling

$K_{S E} \quad$ Service condition factor for modulus of elasticity

$K_{T} \quad$ Treatment factor

$K_{w} \quad$ Product of the wall modification factors 
$K_{z b} \quad$ Size factor for bending for sawn lumber

$K_{z v} \quad$ Size factor in shear

L Span Length

Le Effective length in the plane of the applied moment

Ls $\quad$ Length of shear wall segment

$\ell p \quad$ Span of stressed skin panel

$M \quad$ Applied bending moment on the stressed-skin panel with combined loading

Mf Factored bending moment

mi Test value

$m_{p} \quad$ Specified strength capacity in bending

$M r \quad$ Factored bending moment resistance

$n \quad$ Number of test values

nu Unit lateral nail strength resistance

$P \quad$ Applied axial or concentrated load

$P a \quad$ Allowable axial load on the panel

Pcr Critical axial load

$P_{E} \quad$ Euler buckling load in the plane of the applied moment

$P_{f} \quad$ Factored compressive axial load

$P_{i j} \quad$ Specified uplift restraint force for storey at the bottom of the end stud of a shear wall segment

$p_{p} \quad$ specified strength capacity of flange in axial compression

$\operatorname{Pr} \quad$ Factored compressive load resistance parallel to grain

Q Shear force at the section

$r \quad$ Radius of gyration

$R_{b} \quad$ Basic racking resistance

$\mathrm{R}_{\mathrm{d}} \quad$ Ductility-related force modification factor

$R_{i} \quad$ Ratio of the cross-section area of each layer to the total cross-sectional area

$R_{\text {mech }}$ Over-strength developed when a collapse mechanism is formed

$\mathrm{R}_{\mathrm{o}} \quad$ Over-strength related force modification factor

$R_{s h} \quad$ Over-strength due to strain hardening

$R_{\text {size }} \quad$ Over-strength due to restricted choices for member sizes and dimension rounding 
$R_{\text {yield }}$ Ratio of probable yield strength to minimum specified yield strength with the equivalent energy elastic-plastic bilinear model

$R_{\varphi} \quad$ Over-strength due to the difference between nominal and factored resistance

$s \quad$ Clearance distance between stringers

$S \quad$ Section modulus of stressed-skin panel

$s_{n} \quad$ Nail spacing around panel edge

$s_{y} \quad$ Standard deviation

$\sigma_{\mathrm{c}} \quad$ Normal core stress

$\sigma_{\mathrm{f}} \quad$ Normal facing stress

$\Sigma Q_{W} \quad$ Sum of moments of area of all webs about neutral plane

$t \quad$ Effective thickness of the wood structural panel sheathing

$t_{1} \quad$ Thickness of upper skin of section

$t_{2} \quad$ Thickness of lower skin of section

$T_{f} \quad$ Factored tensile axial load

$t_{p} \quad$ Specified strength capacity of flange in axial tension

$T_{r} \quad$ Factored tensile load resistance parallel to grain

$v \quad$ Maximum shear due to specified loads at the top of the wall

$v_{i} \quad$ Poisson's ratio of each layer

$v_{p f} \quad$ Specified strength capacity in planar shear

$V_{r} \quad$ Factored shear resistance

w Normal uniform load

$w_{1} \quad$ Ordinary bending displacement

$w_{2} \quad$ Additional displacement related to shear deformation of the core

$X_{G} \quad$ Panel geometry reduction factor

$X_{J} \quad$ Stress joint factor

$X_{v} \quad$ Shear modification factor

$y \quad$ Greater value of $y_{t}$ or $y_{c}$

$Y_{c} \quad$ Distance from centroid to the extreme compression fiber

$y_{c} \quad$ Distance from mid of bottom face to neutral axis 
$y_{t} \quad$ Distance from mid of top face to neutral axis

$\mathrm{Z} \quad$ Distance from the neutral axis of the sandwich 


\section{CHAPTER 1}

\section{INTRODUCTION}

\subsection{General}

A Structural Insulated Panel (SIP) is a structural element of expanded polystyrene insulation (EPS) core sandwiched between two oriented-strand boards (OSB) or any other different material. SIPs carry building efficiencies by replacing several components of traditional residential and commercial construction, including: (i) Studs; (ii) Insulation; (iii) Vapor Barrier; and (iv) Air Barrier. The advanced insulation, excellent strength, and fast installation can be offered by a SIP-based structure. In addition, the total construction costs with SIPs are lower than a conventional wood-framed house. The speed of construction, decrease of required HVAC equipment, reduced site waste, reduction construction financing costs, more favorable energyefficient system, and the lower cost of owning a home built with SIPs can be considered as other benefits of these systems. A recent study showed that on a semi-detached home, a SIP-based home consumes about $65 \%$ less energy than a conventionally constructed fiber-glass home of the same size, leading to a long-term investment for home owners. However, the structural adequacy of SIPs is as yet unavailable (Thermapan, 2007).

This research proposes SIPs in low-rise residential construction (i.e. houses and up to threestorey residential building), replacing the traditional conventional stud floors and walls. The structural capacity of sandwich panel system for floor and wall depends on the effectiveness of composite behavior of the foam core and the facings considering the strength and serviceability limit state requirements per Canadian codes for timber design. Strength limit state flexure, axial compression, and combined axial compression, while serviceability limit state includes limiting deflection under operating conditions such as gravity, wind, racking and cyclic loading.

This research investigates (i) developing expressions for flexural, axial compression and shear capacities of SIPs, as well as their combined effect, for ultimate limit states design, and (ii) studying the seismic behavior of SIPs under racking and cyclic lateral loads as compared to the conventional construction using stud walls. 


\subsection{Problem Statement}

The developed structural insulated sandwich timber panels comprise insulated foam glued between two OSB boards. To determine the structural adequacy of the level of adhesion between the foam and the OSB boards and the level of composite action between them, it is felt necessary to conduct experimental testing to-collapse on the developed structural insulated sandwich timber panels. Clause 8.6 of the Canadian Standard for Engineering Design of Wood, CAN/CSA-O86-09 specifies the effective stiffness, bending resistance and shear resistance of stressed skin panels. These stressed skin panels have continuous or spliced longitudinal web members and continuous or spliced panel flanges on one or both panel faces, with the flanges glued to the web members. These strength equations are not applicable to SIPs since they do not address the adequacy of the foam as the main shear carrying element near the supports and the connector between the facings at the maximum moment location. Also, CAN/CSA-O86-09 specifies expressions for the effects of combined axial and bending on the timber stud walls and posts which are applicable to SIPs. However, the available CAN/CSA-O86-09 compressive resistance equations for studs and posts cannot be applied to SIPs as a result of the difference in their structural performance at failure.

The technical guide of Canadian Construction Materials Commission (IRC, 2007) for SIPs (with lumber $1200 \mathrm{~mm}$ o.c. and EPS core) for walls and roof, formed the basis for the experimental testing conducted elsewhere for flexure, axial eccentric and axial concentric loading (Butt, 2008, Mohammed, 2009, Zarghooni, 2009, Seyad Ahmad, 2011), with the ultimate goal of providing enough technical data for strength and serviceability of the developed SIPs. With this database, design tables can be established.

Literature review revealed that racking and seismic behaviour of SIPs is as yet unavailable. CAN/CSA-S406-92, Construction of Preserved Wood Foundations, allows the use of stud wall which is referred to in Part 9 of the National Building Code of Canada (IRC, 2010) and in provincial building codes as applied to buildings not exceeding $557 \mathrm{~m}^{2}$ in building area and not more than two storey high. Building that exceed these limits must be designed according to Standard CAN/CSA-O86-09, Engineering Design on Wood, which is referenced in Part 4 of the NBCC. Clause 4.1.1.4 of the Ontario Building Code (OBC, 2012) specifies that buildings and 
their structural members shall be designed by one of the following methods: (a) Part 4 of this code provides standard procedures and practices and any specifications and standards relate to this code, except in cases that there is a conflict the provisions of the building code shall be used, or (b) Three bases of design are used as following: (i) Generally established theory for analysis, (ii) Assessment of a specified full-scale structure or a sample by a loading tester, and(iii) Studies of model analogues. is procedure ensures a level of safety and performance in design, at least equivalent to that provided and referred to Clause (a) above. Since mechanistic approach for the design of SIPs at ultimate and serviceability limit states is as yet unavailable, the research conducted herein relied on full-scale experimental testing as well as the finite element computer modeling to reach a design procedure for SIPs under various loading conditions.

\subsection{Objective}

The objectives of this study are:

- Develop expressions for the bending moment, shear, axial and combined bending and axial compression for the design of SIPs at the ultimate limit state as well as equation for SIP deflection under loading for transverse serviceability limit state design

- Using the research data obtained from current and previous experimental test results and the finite-element modeling.

- Study using experimental testing and finite element modeling, the structural behavior of SIP walls under seismic loading and establish recommendation for use in seismic-active areas.

- Develop design tables for SIP and stud panel using the experimental results on SIP and stud panel.

\subsection{Scope of Work and Research Methodology}

The scopes of this research work are as follows as based on experimental testing and the finite element modeling:

- Testing selected sizes of conventional stud wall systems to correlate their ultimate strength and serviceability performance with the tested SIPs. The results will be used further to verify and substantiate the finite-element modeling. 
- Constructing finite-element models of the tested SIPs in previous studies and those to be tested in this program. The finite element modeling, along with the available tests data, will be used to develop design procedure at the ULS and SLS for moment, shear, deflection, and combined axial and bending SIPs.

- Conducting experiments on selected SIP and stud wall panels to examine their performance under wind or seismic loading.

The proposed research includes experimental and numerical investigations with ultimate goal of providing research information to develop guidelines for design of SIPs under static and seismic loading conditions. This includes available equations for design of joists, panels, stud walls and SIPs. A review of test methods of SIP panels and stud walls was conducted. The proposed research activities are summarized in the following tasks:

\section{Task (1): Literature Review}

The first step was to review and document foreign and domestic information on the state-of-the art in sandwich panels. Then, an experimental research program was developed, considering practical SIP sizes that can match the commercially available timber joist and stud wall sizes listed in NBCC, OBC and Wood Design Manual (CWC, 2010).

\section{Task (2): Testing SIP walls in axial compression and bending:}

In this task, flexural and axial compression tests were conducted on selected SIP sizes to provide design tables of SIP floor in the form of the factored resisting bending moment, from which the maximum span of joists of roof/floor panels.

\section{Task (3): Racking and Cyclic Testing on SIP Shear Wall}

The SIP walls are tested under lateral load in order to study the effect of shear wall action through wind and seismic loading. The lateral wind load applied to the SIP wall through monotonic loading using a load cell or actuator. The effect of seismic loading was also simulated on SIP through a cyclic loading using an actuator. The selected sizes of conventional stud wall are tested under monotonic and cyclic loading in order to comparing the structural behaviour of SIPs and Stud walls. 


\section{Task (4): FEA of SIP Walls, Validations and Parametric Study}

Due to the high cost of experiments, selected SIP sizes were tested. FEA modeling was developed and verified based on the experimental results. The calibrated finite element models were used to do the parametric study on SIP walls under lateral loading with different parameters. The effect of gravity load on monotonic and cyclic loading on SIP was studied through finite element analysis with different size of the wall. The effect of nail spacing on the SIP wall was also investigated on monotonic and cyclic lateral loading through finite element analysis.

\section{Task (5): Analytical Methods and Design Tables}

The analytical analysis of SIP and stud panel under different load condition is conducted in order to compare with the current and previous experimental results. The equations from different codes and standards were used and the modification factors were developed. The experimental results also provide a database to prepare design tables for SIPs and stud panels under different load conditions.

\subsection{Organization of Dissertation}

The dissertation is divided into seven chapters. Chapter 1 describes the problem statement, objectives, scope of work and research methodology. Chapter 2 provides the detailed literature review on the structural insulated panels. Chapters 3 and 4 provide description of the experimental test program and test results. Chapter 5 describes the finite element modeling of SIP panels as well as validation of the developed FEA models under different load conditions. Chapter 6 describes the analytical methods and design tables developed based on the experimental and theoretical studies. Finally, Chapter 7 provides the conclusion and recommendation for future research on structural insulated panels. 


\section{CHAPTER 2}

\section{LITERATURE REVIEW}

\subsection{Introduction}

Structural insulated panels are produced in different forms, namely: (i) fiber cement faced SIP, (ii) precast concrete sandwich panel, (iii) light weight steel frame panels, and (iv) plywood sandwich panels. There are different approaches to use the sandwich panel such as: (a) wood; (b) corrugated material; (c) honeycomb material; (d) mineral wood and (e) expanded plastics (foam). Also, the sheathing material has a variety such as: (a) thick fiber reinforced composite material like glass fiber, carbon fiber, and aramid fiber etc.; (b) thin metal plates; and (c) profiled plates. The sandwich material components must also be bonded together, using mechanical fastenings or adhesives, in order to act as a composite load-bearing structure.

The structural insulated foam-timber panel (SIP) is a composite material element. The panel is made of an insulating layer of foam which is sandwiched by two layers of structural board. The board is typically oriented strand board (OSB) and the foam either expanded polystyrene foam (EPS), extruded polystyrene foam (XPS) or polyurethane foam. Structural insulated panels (SIP) are used in building walls, ceiling, floors and roofs. According to the Environmental Protection Agency (EPA), the amount of the carbon dioxide $\left(\mathrm{CO}_{2}\right)$ into the atmosphere which is released by U.S. home is about $9980 \mathrm{~kg}(22,000 \mathrm{lb})$ on each year (Rastra, 2009). This amount is almost twice

of the average which vehicle creates. SIPs can widely reduce air leakage in homes and commercial buildings by reducing the amount of energy used for heating and cooling. Figure 2.1 shows a sample of a structural insulated panel. Figure 2.2 also shows layers in the structural insulated panel. 


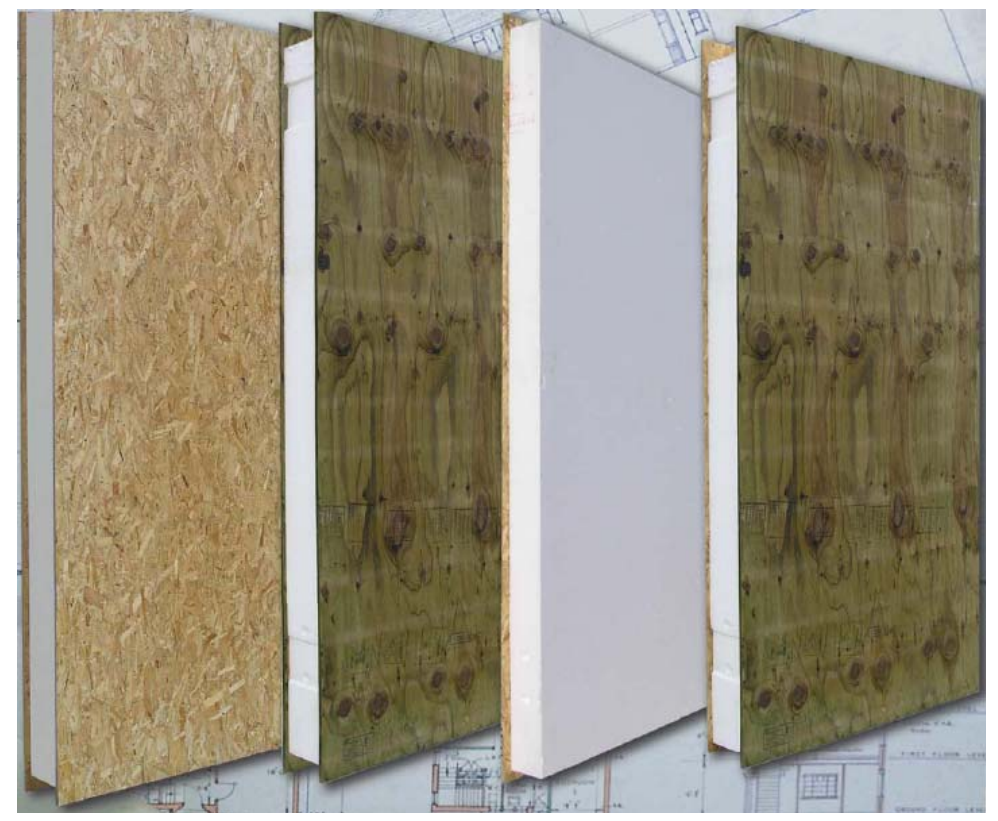

Figure 2.1 - View of a structural insulated panel (Thermapan, 2007)

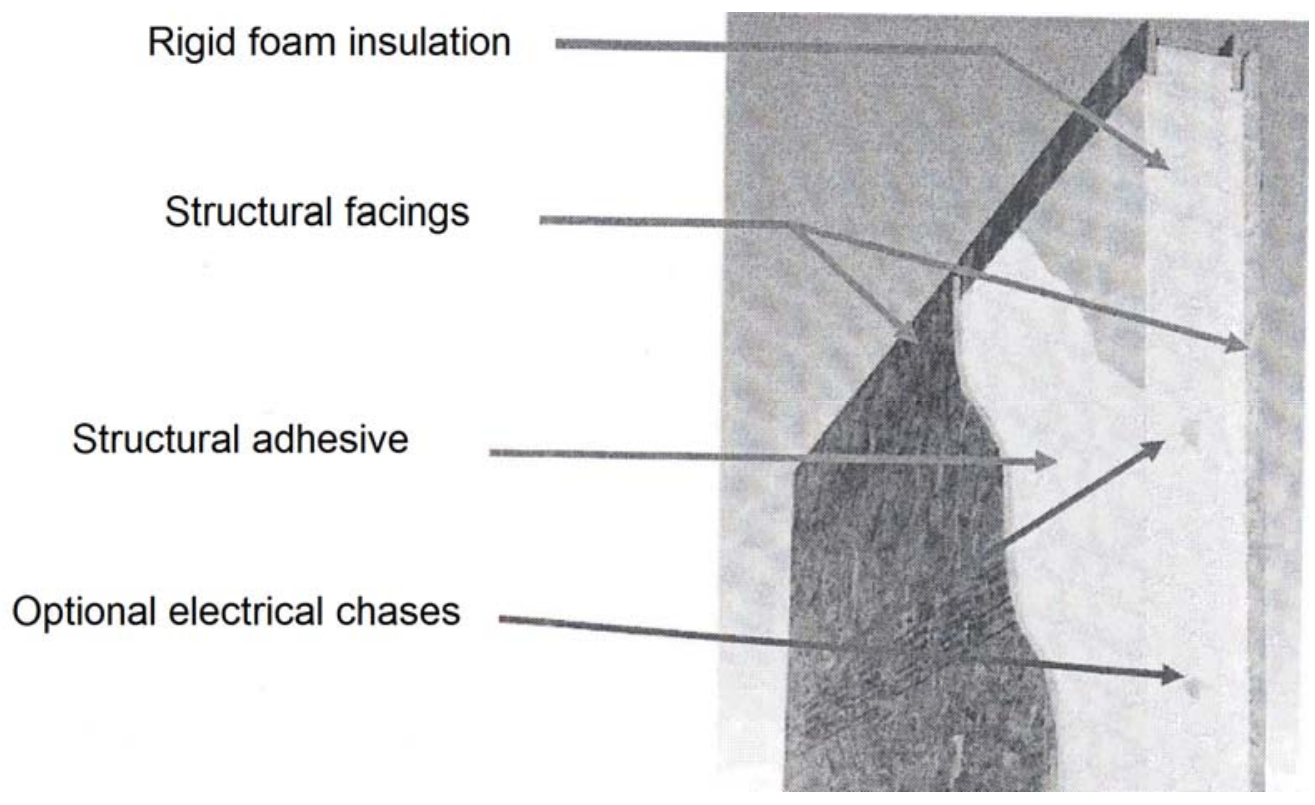

Figure 2.2 - Layers in a Structural Insulated Panel (SIPA, 2007)

The literature review pertained to foam-timber SIPs is presented in the following manner: (i) History of SIPs; (ii) Types of structural insulated sandwich panels; (iii) Structural analysis and design of sandwich panels; (iv) Theoretical investigation and Experimental studies; and (v) Analytical design methods, codes and guidelines. 


\subsection{History of SIP}

In 1935, the Forest Products Laboratory (FPL) in Madison, Wisconsin created the concepts of structural insulated panel. FPL engineers deliberated that plywood and hardboard sheathing could carry a segment of the structural load in wall systems. The prototype structural panels (SIPs) were built using framing members within the panel combined with structural sheathing and insulation. The test homes were made by these panels and monitored for over thirty years. Then, the panels disassembled and re-tested. FLP engineers continued the experimental study on new design and materials during this time period. Famed architect Frank Lloyd Wright built his affordable Usonian houses using structural insulated panels during the 1930's and 1940's Dow, who was one of the Wright's students and son of the founder of the chemical company, produced the first foam core SIP in 1952 and SIPs took an approach rise in technology. The rigid frame insulating products become eagerly accessible in the structural insulated panel production in the 1960's as we found these products today (Sun Spaces, 2010).

The Structural Insulated Panel Association (SIPA) was established in 1990 in US in order to afford support and monitoring for these building technologies. The growth of advanced aided manufacturing (CAM) technology has a positive effect on SIP productions during the 1990's. The computerized architectural drawing (CAD) also provided the compulsory codes to allow automated cutting machines to construct SIPs as a special design of a building. The SIPs manufacturing production modernized with $\mathrm{CAD} / \mathrm{CAM}$ technology to save the labor work and produce accurate flat, straight and true walls (SIPA, 2003).

In response to the need for the industry to devote product documentation, SIPA has cooperated with American Society for Testing and Materials (ASTM) task group to define a standard test method to determine structural capacities of insulated panels (ASTM-E7, 2002). The ASTM standard defines a testing protocol to follow by all manufactures to document the strength and stiffness properties of product to code agencies for product certification. The ASTM standard tests included methods for the following load applications: (i) transverse loads; (ii) axial loads, racking and diaphragm loads; (iii) uplift loads; (iv) creep; (v) combined loading; (vi) impact loading; and (vii) concentrated loading. 


\subsection{Advantages and Disadvantages of SIP}

There are so many advantages in using SIP such as comfortable installation, strength, light weighting, fast construction, and healthy environment, saving money, greater energy savings, straight wall and design friendly.

The different part of a building such as stud and joists, vapour and air barrier and insulation can be replaced in SIP panels. Therefore, SIP framing significantly needs less site labor comparing traditional stud framing. The additional insulation needs deeper size or double farming in wood framing construction. SIP insulation, in comparison with conventional stud frame, takes lower cost. Since the sheathing, fabrication and installation process remains the same, if the panels get thicker, SIPs have the potential for greater quality compared to traditional framing. They are assembled in a controlled factory environment versus the variable "on-site" environment. Panel industry generally facilitates the construction process and makes it more controllable, systematic and quicker (Gagnon and Admas, 1999).

Kermani (2006) stated that in regard to durability, no long-term test program was recorded. However, he mentioned that there are some SIP buildings in the United States that have been used for 50 years. Milner (2003) claimed that a SIP or a quality-manufactured panel could decline or corrupt if they are built improperly, exposed to ultra-violet light, rodents or insects.

The interesting pilot project was launched by Structural Insulated Panel Association (SIPA) in partnership with Oak Ridge National Laboratory (ORNL) when building four "net-zero energy" research home in order to confirm the super airtight and energy efficiency of homes built with SIP products. SIPA (2003) stated in the paper that there is a direct relationship between airtightness and durability. A fundamental part of the SIP building is about appropriate sealed joints. Panel joints must be sealed appropriately in order to prevent moisture, air infiltration and extra-filtration. It causes assurance from entering the building envelope and long-term durability. In a sample project, under blower door testing, using SIP for walls and ceiling, a door, a window, electrical outlets and pre-routed wiring chases showed $90 \%$ less air leakage comparing to the similar room built with 2 by 6 studs, drywall and OSB sheathing, and fiberglass insulation. The 
explanation for the high performance of the SIP test room was appropriate sealed joint which is declared in SIPA's report.

The energy efficiency of SIP is essentially more than a standard frame. The insulating properties of the foam attribute to the efficiency improvement of SIP. The reduction of framing members causes a significant improvement in panels, which can perform as "thermal bridging" (Lee, 1977). SIP panels are highly energy efficient as they create a continuous whole wall system with no thermal consumption and lower energy costs. The main characteristic of energy efficiency of a material is its R-value, which is defined as a measure of the capacity of a material; the greater is its insulating capacity. R-values of SIP panels depend on the type of foam core and its thickness. Eventually, the R-values for a building envelopes built of the SIP panels are much higher than in conventional constructions.

Kosny and Christian (1998) conducted hot-box testing and finite difference computer modeling on clear wall area and wall interface details of the SIP building for thermal performance analysis. The purpose of the project was to show the effect of real world construction techniques on the Rvalues of building system. The analysis indicated the reduction of R-values for clear wall configuration in most wall system. However, the computer modeling and test results shows that the reduction is SIP systems are small and SIPs are reasonably thermal efficient.

SIPs had a rapid growth in USA during early years, but it is used only in about $1 \%$ of new homes. The SIP constructions are growing by $10 \%$ and $23 \%$ in 2004 and 2005, respectively (SIPA, 2003). Mullens et al. (2006) states that several factors limit SIPs growth. They found that the most important factor is probably that the wood-frame construction is less expensive compared to SIP.

Although, the largest cost of SIP cited to material cost, Mullens et al. found that the erection costs could also be higher for SIPs, not to mention that panels did require a construction crane and lift truck. There are some factors such as risk and uncertainty about local building code, durability in long-term, impact of supply chain and construction process which prevent the 
builders of SIP acceptance. Gangon and Adams (1997) concluded that based on the survey results, the information about SIPs can made builders to consider their use in construction.

The unpleasant properties of sandwich materials can be known as follows:

(i) Creep under continuous load with rigid foam cores

(ii) Deformation when one side of sheathing is exposed to extreme heat

(iii)Poor fire resistance with rigid plastic foam cores

The fire assembly testing in construction industry was done for SIP systems. Thus, manufactures across North America have established the performance of SIP system against the fire. Documentation of SIP performance under accurate test standards was published based on the results of this destructive test. SIPs in a similar approach to the other wood-based structures are approved by American National Standards like ASTM E-119 and ASTM E-84 (Kermani, 2006). However, most of buildings higher than three stories need a different kind of regulations due to the loads enforced to the walls and floor systems. While the EPS and Urethane foams (the main core material) are not practically flammable, they will burn when disclosed to flame. So, the use of these materials in high-rise or large public building is limited unless using the extensive fire suppression technology.

\subsection{Fabrication and Erection Aspects of SIP}

SIPs are the production of factories which is offered as a pre-fabricated materials used as floor, roof and wall components on all types of commercial and residential buildings. The connectors, spline, fastener and adhesives are provided by panel manufactures. When the panels are properly assembled and designed, there is no need for frame or skeleton to support them in building. Consequently, the benefits of SIP panels in structures are to provide support and insulation in a single system throughout construction.

Kermani (2006) states two main fabrication methods as following: (i) an industrial adhesive is implemented on pre-cut foam core and the core is cold-pressed between two sheathing (panel boards) until the adhesive is cured or the foam cures to bond the sheathings, and (ii) a single solid building element is produced to provide structural and insulation qualities. The panels are 
manufactured in different sizes and thickness based on application and thermal or structural requirements. The manufacturing process has a major influence on the panel's strength and stiffness, and high quality bonding through is essential. In order to have a strong SIP, there must be no slip between the core material and the outer skins. Adhesive technology is used in order to achieve this purpose. The adhesive used should be able to transfer tensile forces and shear through the interface and strength over time or under moisture effect (Milner, 2003). Figure 2.3 shows a residential building which is constructed using SIPs.

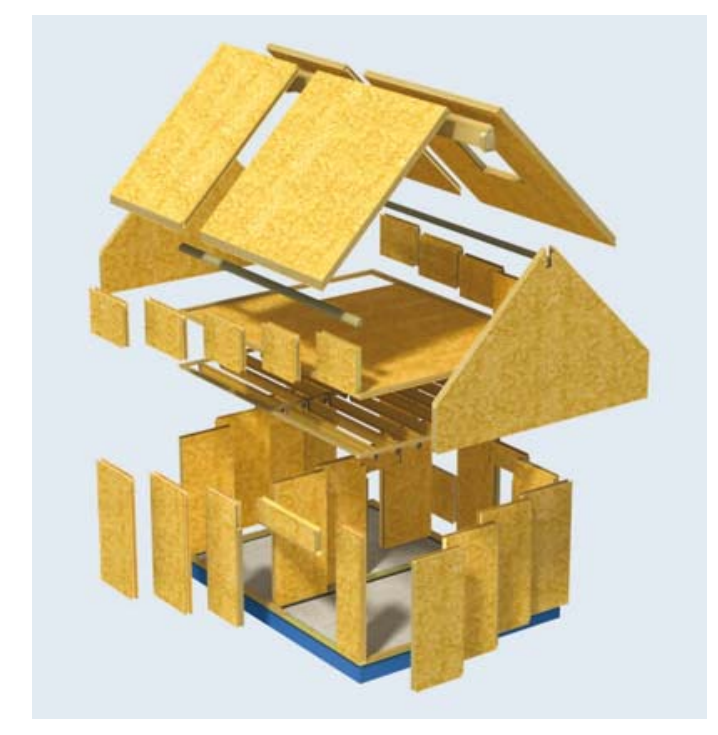

Figure 2.3 - View of residential building construction using SIPs (Kingspan, 2014)

SIP floor and roofs are installed by placing the panels side by side. The connections between the panels in the span direction can be either foam-spline or solid lumber spline. The foam-spline connection is preferred for roof construction to assist in energy efficiency. Butt (2008) describes solid lumber spline connection that is used for floor construction. The foam-spline connection is constructed by providing a recess in the foam core at the long edges of the panels. A foam block with two OSB facings glued to it inserted at the edge of one panel. Then, the adjacent panel is slide over spline. The block OSB facings are then nailed to the OSB of the connected panels to provide structural integrity of the floor or roof. In a solid lumber spline, a recess is formed in the foam core before gluing it to the OSB facings. The width of the insert is usually half the width of the solid sawn lumber. After placing a panel over the walls, a sawn lumber is inserted in the recess along the panel length. Then, the adjacent panel slides over the sawn lumber, followed by 
nailing their OSB facing to the solid lumber. Figure 2.4 shows schematic diagram of foam spline and lumber spline connections.

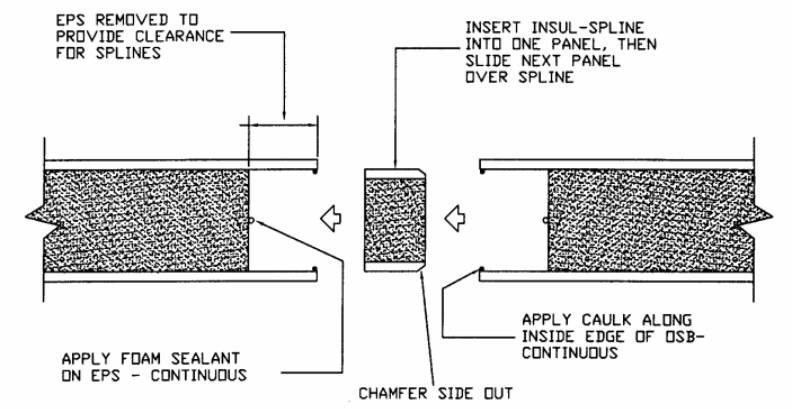

(a) Foam spline connection before assembly

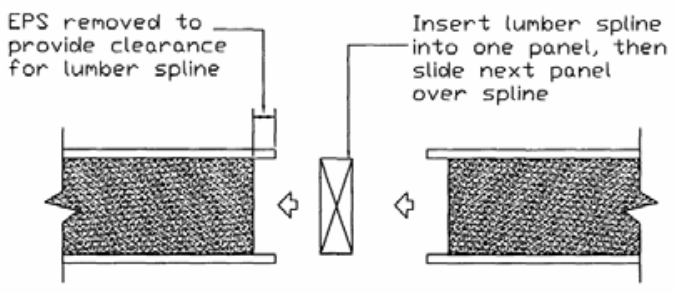

(b) Lumber spline connection before assembly

Figure 2.4 - Schematic diagram of foam spline and lumber spline detail connections

\subsection{Structural Analysis and Design of Sandwich Panels}

In conventional construction, loads are distributed or carried along studs and joists. In case of SIP panels, loads are evenly distributed across the entire panel, allowing greater loads. The core insulation and two sheathing of a SIP are non-structural and weak elements in themselves, but when pressured and laminated together under controlled condition, they will work as a combined action that is much stronger than the sum of its parts. SIPs consume a stressed-skin principle and the total strength of these panels is much greater than the strength of the components. This reduces the necessity of structural framing for these panels.

The comparison of foam panel and I-beam shows the structural similarity. The facing of panels act as the flange and the insulation foam as the web comparing to I-beams. All the components of SIP are stressed under flexural loading. The skins are acting in tension and compression, while the core foam resists against buckling and shear forces. The sheathing of SIP acts as slender columns under in-plane loading. The foam core stabilizes the sheathings and resist against forces from local buckling of the facings. There are three major load components that SIPs are subjected to them:

1. Vertical loads (direct axial compression) 
2. Transverse wind loads (combined axial compression and bending), and

3. In-plane lateral forces imposed by wind and/or seismic loading (racking loading)

The correct design of the details of sandwich construction is as important as the analysis of deflections, stresses and racking loads. These details include nature of the edge members, splices and joints in the core and faces, stiffeners and inserts to distributed concentrated load, type of adhesive, method of fabrication and so fourth. If the temperatures of two faces differ, or if the moistures contents differ (as they may in asbestos cement or hardboard, for example) the differential expansion of the faces may lead to substantial transverse deflections. Special problems arise on acoustic insulation, vapours transmission and fire resistance in building panels. All of the factors mentioned can be very important design considerations but they are beyond the scope of this research.

\subsection{Historical Development of Sandwich Theory}

There are a few papers which have been published about the bending and buckling of sandwich panels with rigid core which cause a considerable bending stiffness and sufficient flexibility against shear deformation on sandwich panel.

Figure 2.5 shows a typical longitudinal and cross-section in a sandwich beam made of a foam core and two facings (i.e. OSB boards). There remains the considerable problem the sandwich with an anti-plane core, one which posses no stiffness in X-Y plane in which the shear stresses $\tau_{z x}$ and $\tau_{y z}$ are constant throughout the depth (i.e. they are independent of $Z$ ). These panels are different from regular homogenous plates since the presence of non-zero shear strains $\left(\varepsilon_{z x}, \varepsilon_{y z}\right)$ in the core and direct strains $\left(\varepsilon_{\mathrm{z}}\right)$ in the core, perpendicular to the faces deteriorate the bending deformation. The shear strain and direct strain in the core are also directly associated with the

possibility of short wavelength instability of the faces (wrinkling). This problem has been the subject of two main methods of analysis, which may be referred to for convenience as the General and the Selective Methods. The following subsections explain briefly each of these methods (Allen, 1969). 


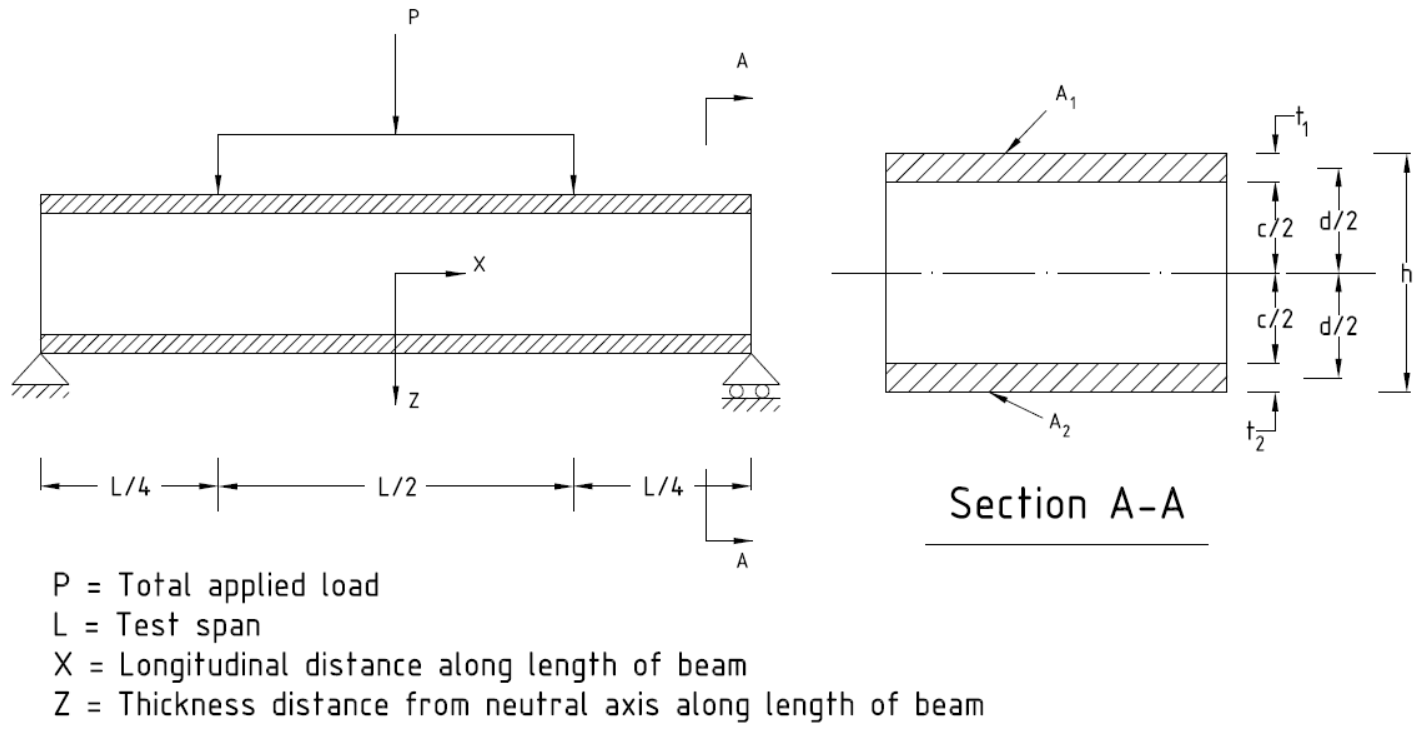

Figure 2.5 - Schematic diagram of sandwich panel member elevation and cross-section (Adapted from Allen, 1969)

\subsubsection{The General Method}

The equations in general methods are arranged to identify the equilibrium of each faces and the core. They also recommend the continuity between the core and the faces. A set of differential equations are resulted and have to be solved in particular cases for flattening of the core, transverse deformation and other equations of interest.

The General Method was investigated by Reissner (1948) in relation to isotropic panels with very thin faces. Although his analysis is not simple, it is possible for Reissner to conclude that the effect of core flexibility in the Z-direction is after all less important than effect of core shear deformation in the transverse planes. Wrinkling instability as such is not discussed. It is only by neglecting the effect of direct transverse core strains that Reissner able to derive a relatively simple differential equation for the transverse displacement, much more recent analysis by Heath (1960) also includes a very similar equation, but sandwich with an orthotropic core. Heath's analysis work based on earlier work by Heath (1948) and is apparently independent of the work of Reissner. 
Raville (1955) applies the General Method to the simply-supported rectangular panel with the uniform transverse and with thin faces. The three displacements of points in the orthotropic antiplane core are expressed as polynomials in z, but the complexity of the analysis again makes it necessary revert to the simplifying assumption of infinite core stiffness in the z-direction. The General Method is the interact-table when applied to sandwich panels, but success has been achieved in relation to sandwich struts and beams. The early works of Williams et al. (1941) and of Cox and Riddell (1945) fall into this category. The first study deals with a sandwich struts with thick faces and an isotropic core (with an extension to orthotropic cores) and the analysis used to form a link between the extreme case of wrinkling instability (no longitudinal displacements of the faces during buckling) and overall Euler type instability, modified for shear deformations in the core (no direct strains in the z-direction). A very thorough analysis of the behavior of the struts isotropic faces and cores was outlined by Goodier (1964) and completed by Goodier and Neou (1951). In the latter paper, the works of Williams and Cox were verified to a degree of accuracy.

\subsubsection{The Selective Method}

In selective method, which has been the basis of this being named (again for convenience) as the bending problem and the wrinkling problem. In the bending problem, it is convenient to assume that the core is not only anti-plane, but also indefinitely stiff in the Z-direction. This excludes the flattening of the core and wrinkling instability, but it does permit the assessment of the effect of core shear deformation on the deflections and stresses in the panel. In the wrinkling problem, the true elastic properties of the core are taken into account but the task is simplified by permitting the middle planes of the faces to deflect in the Z-direction only, not in their own plane.

Most of the published work on sandwich panels refers to the selective method a particular to the bending problem in which core strains in the z-direction is neglected. The assumption that core is weak in the $x-y$ plane leads in any case to the conclusions that the core shear stresses $\tau_{\mathrm{zx}}$ and $\tau_{\mathrm{yz}}$ are independent of $\mathrm{z}$ and a straight line drawn in the unloaded core normal to the faces remains straight after deformation, but is no longer normal to the faces. These assumptions (core weak in $\mathrm{x}-\mathrm{y}$ plane, stiff in z-direction) allow the displacements of the panel to be expressed in terms of only three variables, one of which is the transverse displacement (Allen, 1969). 


\subsection{Flexural Stresses in Sandwich Panels}

Ordinary bending theory is used to define the normal stresses in the faces and core by adopting the composite nature of the cross section, defining the appropriate form of flexural rigidity, D, of the composite section. The stresses in the faces and the core have been defined by Allen (1969) as follows, with dimensional parameters shown in Figure 2.5:

$$
\begin{array}{ccc}
\sigma_{f}=\frac{M z E_{f}}{D} & \text { for } & \frac{c}{2} \leq z \geq \frac{h}{2} \\
\sigma_{c}=\frac{M z E_{c}}{D} & \text { for } & -\frac{c}{2} \leq z \geq \frac{c}{2}
\end{array}
$$

where: $\mathrm{h}=$ specimen height; $\mathrm{c}=$ core thickness; $\mathrm{E}_{\mathrm{f}}=$ modulus of elasticity of facing material; $\mathrm{E}_{\mathrm{c}}$ $=$ modulus of elasticity of core material; $\mathrm{D}=$ sandwich flexural rigidity; $\sigma_{\mathrm{c}}=$ normal core stress; $\sigma_{\mathrm{f}}=$ normal facing stress; $\mathrm{M}=$ bending moment; and $\mathrm{z}=$ distance from the neutral axis of the sandwich.

The flexural rigidity is commonly referred to as D and can be defined as the sum of the flexural rigidities of the faces and the core measured about the neutral axis of sandwich cross-section. Allen (1969) and DIAB (2003) defined the flexural rigidity for a narrow sandwich beam (transverse stresses in the y-direction are assumed to be zero) as follows.

$D=E_{f} \frac{b f^{3}}{6}+E_{f} \frac{b f d^{2}}{2}+E_{c} \frac{b c^{3}}{12}$

where: $\mathrm{c}=$ core thickness; $\mathrm{E}_{\mathrm{f}}=$ modulus of elasticity of facing material; $\mathrm{E}_{\mathrm{c}}=$ modulus of elasticity of core material; $D=$ sandwich flexural rigidity; $b=$ specimen width; $f=$ facing thickness; $d=$ distance between neutral axis of faces $(c+f)$; for equal facing thickness).

The first term may be neglected on the right hand side of the equation in comparison with the second if:

$$
\frac{d}{f}>5.77
$$

If this condition is fulfilled, the local bending stiffness of the faces (bending about their own separate centroidal axes) makes a negligible contribution of the flexural rigidity of the sandwich. 
The third term may be neglected in comparison with the second if:

$$
\frac{E_{f} f d^{2}}{E_{c} c^{2}}>16.7
$$

If this condition is fulfilled, the bending stiffness of the core is negligible.

\subsection{Shear Stresses in Sandwich Panels}

The form of the shear stress $(\tau)$ for a point located at distance $\mathrm{z}$ from the neutral axis homogenous beam can be easily derived by ordinary bending theory and appears as follows.

$$
\tau=\frac{Q S}{I b}
$$

where; $\mathrm{Q}=$ shear force at the section; $\mathrm{I}=$ second moment of area the entire section about its centroid; $\mathrm{b}=$ width at given depth in section; $\mathrm{S}=$ first moment of area of that part of the section up to a point at distance (z) from neutral axis.

For a sandwich beam, the modulus of elasticity of the component part are accounted for bending representing the sum of the products of $\mathrm{S}$ and $\mathrm{E}$ in following equation.

$\tau=\frac{Q S}{D b} \sum(S E)$

The profile of the shear stress through the depth is defined in the below equation.

$\tau(z)=\frac{Q}{D}\left[E_{f} \frac{f d}{2}+\frac{E_{c}}{2}\left(\frac{c^{2}}{4}-z^{2}\right)\right]$

Allen (1969) and DIAB (2003) showed that the above equation may be simplified if the sandwich has a relatively flexible core and if the flexural rigidity of faces about axis of faces is small. It is common to neglect the shear stress of the faces for sandwich cross-section with relatively stiff faces and weak core. Therefore, equation (2.6) can be reduces to the following equation.

$\tau=\frac{Q}{b d}$

The normal and shear stress profiles of a sandwich beam are given in Figure 2.6 where the maximum facing normal stress at the outer fiber is obtained by using $z=h / 2$ in Equation 2.1, the 
minimum facing stress at the interface of the core is obtained by using $\mathrm{z}=\mathrm{c} / 2$, and maximum shear stress in the core is given in Equation 2.9.
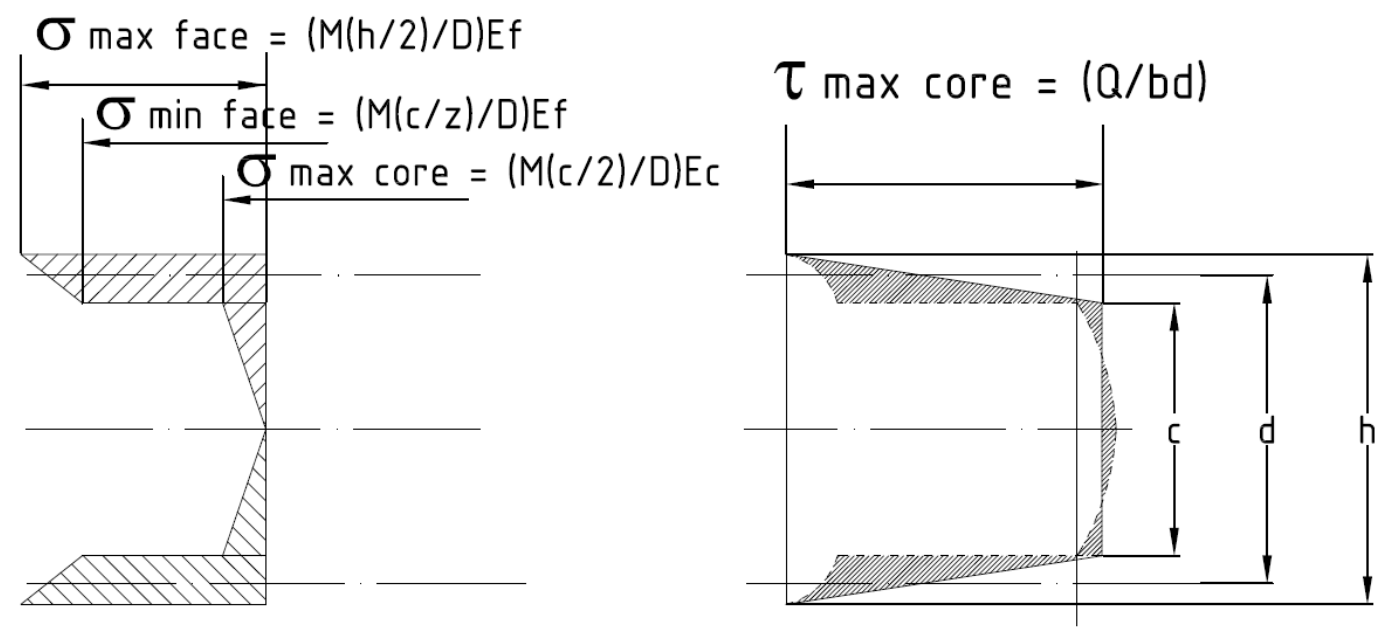

--- Dashed Line $=$ Theoritical Shear Stress Solid Line = Approximated Shear Stress
(a) Normal stress profile
(b) Shear stress profile

Figure 2.6 - Normal and shear stress profiles of a sandwich beam

\subsection{Buckling in Sandwich Panel Walls}

\subsubsection{American Panel Association Standard (APA)}

The APA standard defines a section with trial values of $\mathrm{c}, \mathrm{A}_{1}, \mathrm{~A}_{2}$, and h. Figure 2.8 shows the proposed section of APA. The panel may be checked for all possible modes of failure. The following equation can be used to find the "Neutral Axis" of section. All the parameters are defined in Figure 2.5.

$$
\bar{y}=\frac{A_{1}\left(h-\frac{t_{1}}{2}\right)+A_{2}\left(\frac{t_{2}}{2}\right)}{A_{1}+A_{2}}
$$

where $\bar{y}=$ Distance from edge to neutral axis; $h=$ Total height of section; $t_{1}=$ Thickness of upper skin of section; $t_{2}=$ Thickness of lower skin of section; $A_{1}=$ Area of upper skin of section; $A_{2}=$ Area of lower skin of section 
The moment of inertia and section modulus of composite section is also determined by the following equations:

$$
\begin{aligned}
& I=\frac{A_{1} A_{2}(h+c)^{2}}{4\left(A_{1}+A_{2}\right)} \\
& S_{1}=\frac{I}{h-\bar{y}} ; \quad S_{2}=\frac{I}{\bar{y}}
\end{aligned}
$$

The column buckling load of the section is determined by the following equation:

$$
P_{c r}=\frac{\pi^{2} E I}{(12 L)^{2}\left[1+\frac{\pi^{2} E I}{(12 L)^{2} \times 6(h+c) G_{c}}\right]}
$$

where; $\quad L=$ Span Length

$$
G_{c}=\text { Modulus of Rigidity of column }
$$

The approximate skin stress at the buckling condition is determined from the following equation. For foam or balsa core,

$$
C_{c r}=0.5 \sqrt[3]{E E_{c} G_{c}}
$$

where; $C_{c r}=$ theoretical skin stress at buckling (wrinkling)

For honeycomb core,

$$
C_{c r}=0.82 \sqrt[3]{\frac{E_{c} t}{E_{c}}}
$$

It should be noted that the allowable should be approximately $1 / 3 \mathrm{C}_{\mathrm{cr}}$ for design. Skins are also assumed to be flat. Total deflection including the effects of axial load is approximately equal to

$$
\Delta_{\max }=\frac{\Delta_{T}}{1-\left(P / P_{c r}\right)}
$$

The deflection is usually limited to $\mathrm{L} / 240, \mathrm{~L} / 360$, or some other predetermined allowable amount, such as shown in Table 2.1. 
Table 2.1 - Standard allowable deflections for beams

\begin{tabular}{lc}
\hline \multicolumn{2}{c}{ Floor Beams } \\
\hline Live load only & $\mathrm{L} / 360$ \\
Dead plus live load & $\mathrm{L} / 240$ \\
\hline \multicolumn{2}{c}{ Roof Beams } \\
\hline Live load only & $\mathrm{L} / 240$ \\
Dead plus live load & $\mathrm{L} / 180$ \\
\hline
\end{tabular}

\subsubsection{Analytical Method}

Allen (1969) and DIAB (2003) proposed an analytical method for buckling of sandwich struts. He stated his method based on the standard analysis of struts with thick and thin core. In case of thin faces, the standard analysis of the stability of a uniform axially loaded pin-ended elastic strut indicates that the strut is unstable when the axial thrust is equal to the Euler load, $P_{E}$, where;

$P_{E}=\frac{\pi^{2} D}{L^{2}}$

The flexural rigidity is defined as $(D)$ in this equation. The term $\left(P_{E}\right)$ represents the smallest force at which the member will not return to its straight condition after being given some lateral displacement.

In the case of a sandwich panel, it is possible for shear deformations to occur in the core. These decrease the stiffness of the sandwich panel and the critical load is also less than the Euler load defined by equation (2.17). Consider a sandwich with an anti-plane core and thin faces, the local bending stiffness of which can be ignored. The flexural rigidity is therefore given by:

$D_{1}=D_{2}=\frac{E_{f} b t d^{2}}{2}$

When the strut bends the shear stress distribution is similar to that in Figure 2.7. 


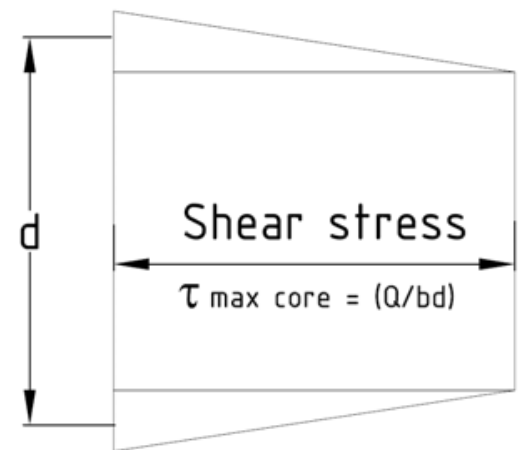

Figure 2.7 - Effect of weak core, neglecting the local bending stiffness of the sheathing (Adapted from Allen, 1969)

At the critical value of the axial thrust $(P)$ there occur two super-imposed displacement, $w_{l}$ (the ordinary bending displacement) and $w_{2}$ (an additional displacement related to shear deformation of the core). Figure 2.8 shows the deflection of an axially loaded pin-ended sandwich strut. At a typical section ( $\mathrm{x}$ ) from support, the bending moment is:

$M=P\left(w_{1}+w_{2}\right)=-D_{1} w_{1}^{\prime \prime}$

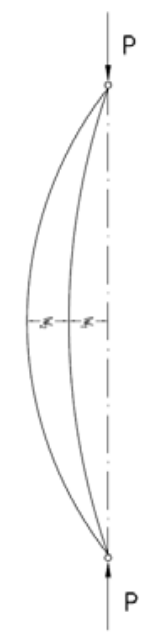

(a) Buckling of strut

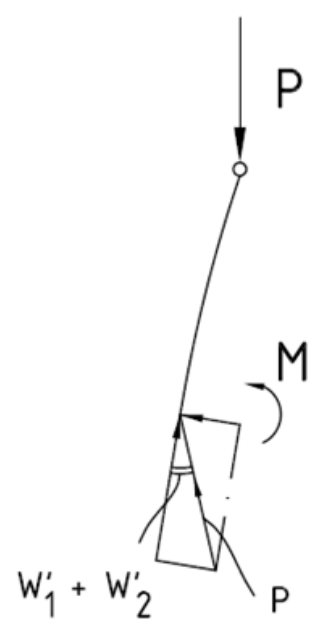

(b) Section of strut

Figure 2.8 - Deflection of an axially loaded pin-ended sandwich strut (Adapted from DIAB, 2003)

The horizontal thrust $(P)$ at the section $(x)$ has a component $P\left(w_{1}^{\prime}+w_{2}^{\prime}\right)$ acting perpendicular to the axis of the panel. This represents shear force and equation (2.20) shows the relationship between shear force and the shear deflection $\left(w_{2}\right)$ : 
$\frac{d w_{2}}{d x}=\gamma \frac{c}{d}=\frac{Q}{G b d} \cdot \frac{c}{d}=\frac{Q}{A G}$

where; $Q=$ Shear force; $\left(A=b d^{2} / c\right)$.

So, the differentiation of shear deflection is presented as following equation:

$w_{2}^{\prime}=\frac{P\left(w_{1}^{\prime}+w_{2}^{\prime}\right)}{A G}$

If the term ( $\left.w_{2}^{\prime}\right)$ differentiated and eliminated from equation (2.19) and equation (2.21), it yields a differential equation for $\left(w_{1}\right)$ as following:

$w_{1}^{\prime \prime \prime}+\alpha^{2} w_{1}^{\prime}=0$

where

$\alpha^{2}=\frac{P}{D_{1}\{(1-(P / A G)\}}$

The solution is of the form

$w_{1}=C_{1} \sin \alpha x+C_{2} \cos \alpha x+C_{3}$

The total deflection, $w_{1}+w_{2}$, may be obtained from equation (2.19) by differentiating equation (2.24) twice and inserting the result in the right-hand side. Thus:

$w_{1}+w_{2}=-\frac{D_{1}}{P}\left\{-C_{1} \alpha^{2} \sin \alpha x-C_{2} \alpha^{2} \cos \alpha x\right\}=\frac{C_{1} \sin \alpha x+C_{2} \cos \alpha x}{1+(P / A G)}$

The boundary condition at $x=0$ and $x=L$ require to apply to find the constant $C_{1}$ and $C_{2}$. The function $(\sin \alpha L)$ vanishes only when $(\alpha L=n \pi)$ or, from equation (2.23).

$P=\frac{P_{E}}{1+\left(P_{E} / A G\right)} ; \quad P_{E}=\frac{\pi^{2} D_{1}}{L^{2}}$

This is the critical load of the sandwich strut; equation (2.26) is essentially the same as the standard result for the critical load of a lattice column. It is often expressed in the equivalent form:

$\frac{1}{P}=\frac{1}{P_{E}}+\frac{1}{A G}$

When $(G)$ is finite, the critical load is less than Euler load; when $(G)$ is infinite, the critical load is equal to the Euler load; and when $(G)$ is small, the critical load approaches the value AG. 


\subsubsection{Canadian Standard (CAN/CSA-O86)}

The Canadian standard CAN/CSA-O86 in clause 7.5.6 stated that the factored compressive resistance parallel to a laterally supported panel edge shall be taken as the following equation.

$$
P_{r}=\phi P_{P} b_{P}
$$

where; $\phi=0.95 ; b_{P}=$ width of structural panel, $\mathrm{mm}$;

$P_{P}=P_{P}\left(K_{D} K_{S} K_{T}\right)$

where; $P_{P}=$ specified strength capacity in axial compression (plywood - Tables 7.3A and \& 7.3B; OSB - Tables 7.3C and 7.3D of CAN/CSA-O86-09), N/mm

The constants $K_{D}, K_{S}$ and $K_{T}$ can be found from clause 4.3.2 to 4.3.4 in CAN/CSA-O86. Clause 5.5.10 of CSA-O86 declares that members subordinate to combine bending and compressive or tensile axial loads must be considered to satisfy the appropriate interaction equation:

$$
\frac{P_{f}}{P_{r}}+\frac{M_{f}}{M_{r}} \leq 1.0
$$

or

$$
\frac{T_{f}}{T_{r}}+\frac{M_{f}}{M_{r}} \leq 1.0
$$

where; $P_{f}=$ factored compressive axial load; $P_{r}=$ factored compressive load resistance parallel to grain calculated in accordance with the requirements of Clause 5.5.6; $M_{f}=$ factored bending moment, taking into account end moments and amplified moments due to axial loads in laterally loaded members; $M_{r}$ = factored bending moment resistance calculated in accordance with the requirements of Clause 5.5.4; $T_{f}=$ factored tensile axial load; $T_{r}=$ factored tensile load resistance parallel to grain calculated in accordance with the requirements of Clause 5.5.9. This clause can be applied to SIP in combined bending and axial load.

\subsection{Elastic Deflection Analysis of Sandwich Panels}

Equations defining the instantaneous elastic mid-span deflection of uniformly loaded simply supported sandwich beams (with relatively thin, stiff faces and thick weak cores) are well known and widely cited. The plywood design specification supplement, entitled "Design and 
Fabrication of Plywood Sandwich Panels" (APA, 1990) and (DIAB, 2003) simplifies the total sandwich beam mid-span deflection, $\Delta_{T}$, to the sum of bending and shear deflection as follows:

$\Delta_{T}=\Delta_{B}+\Delta_{S}$

where: $\Delta_{B}=$ mid-span sandwich panel deflection due to bending; $\Delta_{S}=$ mid-span sandwich panel deflection due to shear

The form of the elastic bending deflection for a simply supported homogenous beam of uniform cross section in quarter point loading is easily derived by ordinary bending theory as follow:

$\Delta_{B}=\frac{11 P L^{3}}{384 E I}$

where: $P=$ total applied load; $L=$ beam span; $E=$ modulus of elasticity of beam material; $I=$ moment of inertia of the uniform cross-section.

When defining the deflection of a sandwich beam, the flexural rigidity (EI) must define in terms of its component materials and their position in the cross section. Allen (1969) and DIAB (2003) also showed that for thin faces (local bending of faces in negligible) negligible core bending stiffness, constant shear stress throughout the core, and negligible shear stress in the skin material, the displacement $\left(\mathrm{w}_{2}\right)$ defines as the shear deformation of the core can be determined by integrating following equation.

$\int \frac{d w_{2}}{d x}=\frac{Q}{A G}$

where; $A=b d^{2} / c$ and $(A G)$ is referred to as the shear stiffness; $Q=$ shear force $=P / 2$ for quarterpoint loading; $G=$ core shear modulus; $X=$ distance from the reaction in shear zone of beam; $w_{2}$ $=$ displacement at $(x)$ from support.

By applying the boundary condition for the simply supported quarter point beam, the maximum shear deflection (at $x=L / 4$ ) associated with the shear deformation of the sandwich loaded at quarter points is defined by below equation. The total sandwich beam deflection reflecting the bending and shear component is presented as follows.

$$
\begin{aligned}
& \Delta_{s}=w_{2 \max }=\frac{P L}{8 A G} \\
& \Delta_{T}=\frac{11 P L^{3}}{384 D}+\frac{P L}{8 A G}
\end{aligned}
$$


In 1996, ASTM included creep loading as an official protocol addressing SIP performance. At this point engineers and designers need validated techniques to define SIP creep performance to consumers and code officials. The National Design Specification for Wood, NDS, (NFPA, 2005) provided convenient method for calculating total deflection for structural wood products subject to long term loading:

$\Delta_{\text {Total }}=K\left(\Delta_{\text {long-term }}\right)+\Delta_{\text {short-term }}$

where; $\Delta_{\text {long-term }}=$ immediate deflection under dead load + long-term portion of live load; $\Delta_{\text {short- }}$ term $=$ deflections under short-term portions of design load; $K=$ constant to calibrate the longterm effects of dead load and live load

The log-term deflection constant, $K$, ranges in magnitude from 1.5 for seasoned lumber and glulum timbers to 2 for green lumber. There is a great need in SIP industry to develop a similar relationship for long-term SIP behavior. This creep can be defined by experimental testing. Figure 2.9 shows a typical diagram of creep behavior of a typical material. The first region shows the instantaneous deflection-time relationship as the member reaches its immediate deflection. The next region defines primary creep where deflection increases at a decreasing rate. The secondary creep region defines primary creep where deflection increasing at a nearly constant rate and finally, the tertiary stage, the deflection is immediately reduced; the elastic deflection will be fully recovered for viscoelastic material and the structure continues to recover its creep deflection.

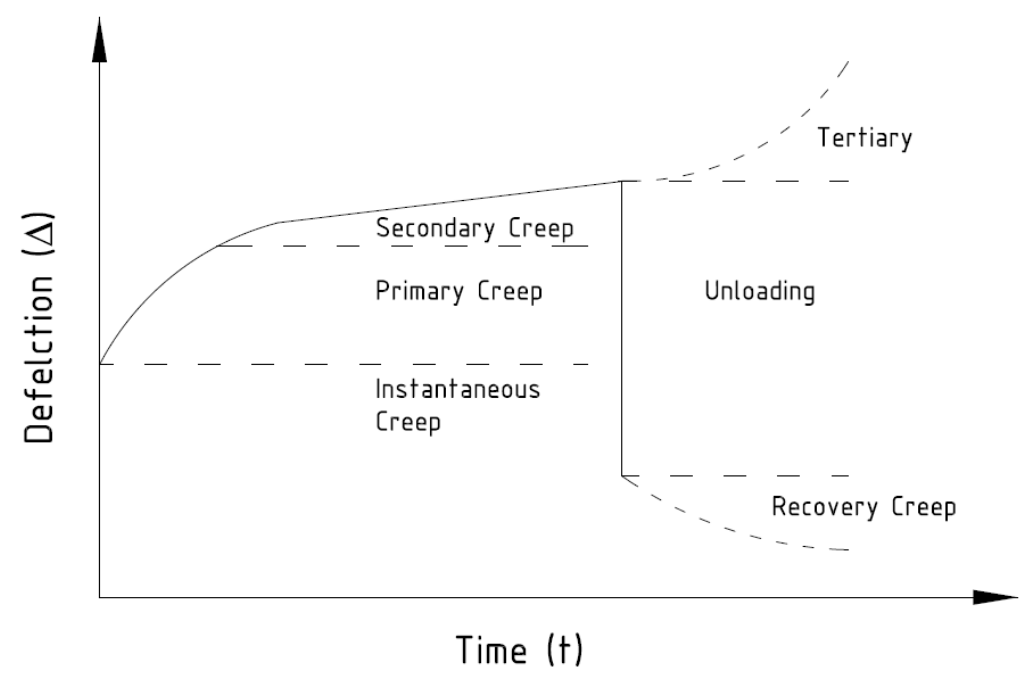

Figure 2.9 - Creep behaviour of a typical material 
The parabolic shape of models engaged a good achievement to illustrate the primary and secondary creep deflection of wood and rigid foam materials. These models can also used in metal skinned sandwich panels (e.g. Davies (1987), Haung and Gibson (1990, 1991), Gerhards (2000) and Hoyle et al. (1985)). Equation (2.37) presents the full form of this model which is called as "Power Model".

$\Delta(t)=\Delta_{0}+A_{1} \cdot t_{2}^{(A)}$

where; $\Delta(t)=$ Total time dependent deflection; $\Delta_{0}=$ Initial deflection; $A_{1}, A_{2}=$ Creep parameters.

The creep behavior of wood on wood (OSB faced solid-sawn wood stud core) has been studied by Wong et al. (1988) for three month load duration. Davies (1986) summarized research predicting the influence of creep on urethane and EPS core metal panels for ten year load duration. Huang and Gibson (1990) reported results on the creep metal faced urethane core panels. Other work by Huang and Gibson (1991) defined parameters for polyurethane foam cores from shear creep tests are recommended by ASTM C237-61.

Taylor (1996) conducted a series of creep testing on OSB/foam structural insulated panels to measure the three month mid-span creep deflections due to sustained loading on quarter points. Four manufactures were incorporated in the experimental study (two EPS and two urethane core manufactures). The EPS designates the expanded polystyrene core type. The results suggested the use of a fractional deflection factor, $K$, the calibration of long-term deflection as 1.5 for EPS core and 2.0 for urethane core for cumulative deflection duration up to three months in the NDS long-term equation.

The ratio of creep to elastic strain is of a great interest to designers. It is defined as Creep Coefficient and denoted by $C_{(t)}$. The American Concrete Institute Standard (ACI 318-2008) states that the effect of creep and shrinkage deflection shall be multiplied by the initial deflection by the creep factor.

$C_{(t)}=\frac{\text { Creep Plus Shrinkage Strains }}{\text { Elastic Strain }} \leq 2.0$ 
And it is taken as (2.0) for 5 years or more; (1.4) for 12 months; (1.2) for 6 months; and (1.0) for 3 months.

Creep is the deformation under sustained load over time as shown in Fig. 2.16a. It is also timedependent parameter which can be quantified as creep compliance (specific creep) and relative creep (creep coefficient) which both are parameters for temperature function, and moisture in case of wood, Fig. 2.16b. The creep rate is increased by the increase of the temperature and/or humidity. Initial strain due to the loading is the major difference for creep for different panel sizes.

Creep is clarified in ACI 209R-92 (ACI, 2008), Predication of Creep, Shrinkage, and Temperature Effects in Concrete Structures, standard. Creep is presented as a constant stress under conditions of steady relative humidity and temperature, supposing the strain at loading (nominal elastic strain) as the immediate strain at any time. Creep consists of three different types of behaviour in wood which are not easy to separate because they can all activate concurrently. These are mechano-sorptive (moisture-change) creep, time-dependent (viscoelastic) creep and pseudo-creep and recovery which have been recognized to differential shrinkage and swelling (Hunt, 1999).

Creep-strain response for wood-based structure is viscoelastic, where represented by elastic spring and viscous dashpot. Viscous flow to ideal fluid requires rate of strain with respect to time be proportional to the applied stress, while plastic deformation is due irreversible changes of position, where strain does not change when the stress is removed.

$$
\begin{aligned}
& \frac{d \varepsilon}{d t} \propto \sigma \\
& \varepsilon=F(\sigma, t)
\end{aligned}
$$

Rheological models (Wu, 2009) are illustrated by Kelvin-Voigt (solid) and Maxwell (fluid). Maxwell body contains of a spring and dashpot in series, while Kelvin-Voigt body contains those in parallel. Maxwell and Kelvin-Voigt are special cases of Kelvin. Kelvin body is determined by inverse LA-PLACE Transform through the following relation, the two parameters 
$(E)$ and $(\lambda)$ can be determined by the using of Marquart-Levenberg algorithm (Least Squares Regression) in connection with experimental data (Betten, 2008).

$$
\varepsilon_{b}(t)=\frac{\sigma_{0}}{E}[1-\exp (-t / \lambda)]
$$

For long-term creep, "Maxwell model" dashpot strain will scale linearly, and by time the spring strain contributes less. Model is not well used to discuss the creep behaviour. Kelvin-Voigt model (Thomson, 1865; and Voigt, 1892) shortly called Kelvin consists of one linear spring (Hooke) and one linear dashpot (Newton), connected in parallel, also known as the three element model and the standard linear solid model (SLS) (Wineman and Rajagopal, 2001). The model assumes full recovery after stress removing, due to the negative stress exerted by the spring. The model is limited and used for short term and primary creep deflection.

The four element parameter model is Kelvin body model in series with dashpot to study the effect of elastic, viscous flow and the retarded elastic. It is called Burger Model (Burgers, 1939). Fridley et al. (1992) developed the five element model to predict the effect of load and environment. It is the four element model with spring to enhance the model from linear to nonlinear model.

Few authors conducted research work on the structural behavior related to sandwich panels. Among them, Liu and Zhao (2007) performed a research on the effect of soft honeycomb core on the flexural vibration of sandwich panel. They use a low and high order shear deformation models in their work. Aviles and Carlson (2006) had an experimental study on the sandwich panels with glass/epoxy faces sheets over a range of $\mathrm{PVC}$ foam cores with in-plane compressive failure. Their work also covers the balsa wood core with one or two square or circular interfacials debond in sandwich panels. The failure due to local buckling of the deboneded face sheet is occurred in most specimens. It is followed by rapid debond development near the panel edges, perpendicular to the applied load.

Meyer-Piening (2006) studied the linear static and buckling analysis in an asymmetric square sandwich plate. The plate has orthotropic stiffness properties in the face layers. 
The experimental and theoretical study on behavior of sandwich structured composites with syntactic foam core material under three-point bending loading conditions is performed by Gupta and Woldesenbet and Gupta et al. (2002). They presented a method of analysis for this type of sandwich structures. An engineering method to estimate the impact effect and damage of flat sandwich panels is also offered by Olsson (2002). The research focused on local core crushing, delamination and large face sheet deflections.

The experimental study on the non-linear behavior of sandwich panels made of thermoplastic foam core and carbon/epoxy fabric faces is conducted by Yoon et al. (2002). The results of experimental study were compared with finite element analysis and predicted results from a proposed analytical method. The finite prism strip modeling is presented by Tham et al. (1982) to study the axial compressive and flexural behavior of prefabricated architectural sandwich panels made of light gauge cold-formed metal facing and foam-in-place rigid urethane cores. Hossain and Wright (2004) performed a similar study on sandwich panel with plain core concrete.

\subsection{Theoretical Investigation and Experimental Studies}

The structural insulated panels (SIP) become more common as an alternative system for structures with conventional framing in light commercial and residential buildings to date. While, there is a little independent data on behaviour and structural performance of these systems. Kermani (2006) states that there are no current SIPs design standards. The only available standard about wood-based sandwich panels is American Plywood Association supplement No. 4 which includes some restricted design information on the transverse, uniform and combined loading cases. A draft European code prEN 14509 CEN/TC 128: Self supporting double skin metal faced insulated sandwich panels" is considered partly appropriate for the design of SIPs. Currently, a European technical approval guideline (ETAG) for product

certification for "Prefabricated wood-based load bearing stressed skin panels" is being drafted but no formal acceptance of this is as yet released. 
Kermani (2006) conducted comprehensive research study on SIPs at Napier University in the United Kingdom and subsequently published several papers in which presented the experimental program and its results. The performance under combined bending and axial compression and the effect of medium-loading on panel integrity for use as load-bearing walls and columns was studied in the series of tests. Panels of different lengths $600 \mathrm{~mm}$ and $400 \mathrm{~mm}$ wide with OSB facing $11 \mathrm{~mm}$ thick and insulating core of $95 \mathrm{~mm}$ were subjected to uniform axial compression in the first test series and to combined bending and axial compression in the second series of tests. To determine the effects of medium-term loading on the deformation characterises (creep effect) of SIPs under axial compression and to examine the possibility of debonding or bulging within the sandwich panel tests were carried out using universal testing machine. Based on the results, Kermani (2006) presented design charts for estimating compressive strength with respect of wall height and also for combined bending and direct compression for $2400 \mathrm{~mm}$ high walls.

Another experimental research program conducted by Kermani (2006) studied the structural performance of wall diaphragms with and without opening, for windows and doors, under the racking loads. Nineteen walls of $2400 \mathrm{~mm}$ x $2400 \mathrm{~mm}$ were tested to estimate the racking resistance of the SIP wall under vertical load applied along the header and to determine the effects of size and position of openings (for windows and doors) on stiffness of SIP walls and the racking strength. Kermani (2006) demonstrated that SIP walls provide higher racking resistance to comparable conventional stud wall. However, the racking resistance of SIP wall is directly related to the openings size. The racking resistance of the wall decrease sharply with an increase in opening size. Dolan and Johnson (1996) and Enjily and Griffiths (1996) previously studied the racking strength reduction and stiffness with respect to the level of opening.

Kermani (2006) conducted a series of additional tests to determine the effect of tensile loading in SIPs. The purpose of these tests was to indicate the structural integrity of SIPs through study of glue bonds between the core and skins. The result of this research indicated that all failures occurred in the polystyrene when the SIPs subjected to skewed or eccentric loading (in-plane shear) and loading (perpendicular to the plane of a panel). The glue lines remained intact and show that suitable strong bonding techniques are available. 
Very few experimental studies were conducted on connections in SIPs. Kermani (2006) carried out the experimental study to evaluate the strength of a glue bonded polystyrene insulated core to OSB manufactured under normal conditions. In order to examine the possible effects of discontinuity in the core material, a number of panel specimens were tested with an unglued joint between the polystyrene core blocks at the mid-height of the panel. In these panels failure was initiated predominantly at that point where up to $20 \%$ reduction in strength was noted. This highlighted the importance of continuity of the core material in providing an adequate composite action over the full loading range.

Butt (2008) performed an extensive experimental program on flexural and creep behaviour when tested 53 full-size structural insulated panels with the aim to evaluate their potential use in lowrise residential building acting as a floor or roof members. The panels were divided into 15 groups that generally differed in size of the panels, thickness of the OSB sheathing and panel connections. The panel width was $1200 \mathrm{~mm}$, the OSB thickness was in most of the panel samples $11 \mathrm{~mm}$ and the thickness of the EPS foam core was constant for all the panels. Butt's research work was focused on structural performance of SIP member. Except of flexural behaviour of panels under static 4-points loading, he also studied the creep behaviour of the SIP panels by testing four sample panels under log term uniform loading.

Butt (2008) also compared the behaviour of the panels with respect to different connections of the adjacent panels that could have either lumber connection or foam-spline connection. Butt (2008) presented the discussion on adequacy of the tested floor or roof SIP panels for their use in residential construction with emphasis on code requirements for ultimate and serviceability limit states.

The correct design of the details of sandwich panels is at least as the analysis of deflections, stresses and backing loads. These details include nature of the edge members, splices and joints in the core and faces, stiffeners and inserts to distributed or concentrated load, type of adhesive, method of fabrication and so forth. If the temperatures of the two faces differ, or if the moisture contents differ the differential expansion of the faces may lead to substantial transverse deflections (Butt, 2008). 
Mohammed (2009) studied on 32 full-size panels under axial and bending loads to evaluate the effect of foam core in composite action of SIP. These tests provide the serviceability and strength requirements of SIPs. The tests were done in structural lab of Ryerson University and the specimens loading were continued till collapse. The results of the tests showed the load carrying capacity of the SIP wall subjected to axial loading or combined axial loading and bending moment. The failure modes in panels under axial concentric and eccentric load were happened in the connection between the OSB or plywood facing and quarter point area of the wall height. The load carrying capacity of the panel has more effected by foam-spline connection rather than the lumber-spline connection. The failure mode of panels under flexural load was happened due to shear failure at one area of the panel end-quarters. Also, a design table for roof and wall was developed based on different types of building. The effect of the nail spacing on preserved SIP for basement foundation is also studied.

Zarghooni (2009) investigated on six panels for short and long term creep under sustained loading. The results of the tests are used to develop applicable test methods. The structural adequacy of the tested panel is shown in test results of short-term creep test, while the total deflection of the panel is increased with time in the long-term creep test. The ultimate load tests were shown that the capacity of the SIPs was "as good as" the conventional wood-frame structures. He tested on 8 and 16 feet long panel under both short-term creep and recovery based on the criteria set forth by NCR/CCMC technical guide of 2007. The creep constant (K) was established a conservative value of 0.74 for total deflection determination due to dead, live and sustained loading for 8 and 16 feet long panel. The live load or snow load on the panel have to limited to $1.9 \mathrm{kPa}$ and $1.63 \mathrm{kPa}$ for 8 and 16 feet panels to qualify for long-term creep. The foam diagonal and horizontal shears between OSB facing and foam core location was the failure mode of 8 feet long panel. While the tensile fracture of the bottom OSB facing or crushing of the OSB top facing was the failure mode in the 16 feet long panels. The specified snow or live load have to limited to 1.76 and $1.32 \mathrm{kPa}$ for 8 and 16 feet long panels in order to qualify the tested panel for ultimate limit state design. The factor of safety for panels was considered as 3 .

Benadova (2009) conducted 18 full-size structural insulated header panels with timber flange and Oriented Strand Board (OSB) webs to determine the behaviour of the beams under gravity load. 
The tests were done in order to meet both serviceability and strength limit state design requirements as per Canadian Standard for timber design. The flexure and shear included in strength requirements, while limiting deflection under operating conditions considered in serviceability. The experimental test results showed the capacity of SIP headers can be "as good as" of conventional wood-frame constructions. The failure mode in the headers was happened due to nail bending and nails holes tear-out at the interface between the top and bottom flange and OSB facing near the support and at the end of OSB web. She recommended that the design of the header should be revised in order to extend the OSB facing to the end of vertical wall stud to establish continuity in web shear. The flexural shear failure in the OSB facing at the quarter point load location also observed in some headers and it is related to discontinuity of the OSB facing at the support. The design table was developed for the maximum roof joist served by selected header sizes. The flexural resistance, shear resistance and flexural stiffness of a boxbeam section specified in CAN/CSA-O86-09 were exceeded for the determined values from experimental results. The reason may be related to discontinuity of the OSB facing of the header at the interface with the supporting wall.

Seyad Ahmad (2011) studied on long-term creep behaviour of selected PWF's sizes under sustained soil pressure. The pressure applied to panel over eight months to determine the axial compressive strength of panels. The structural capacity of the tested panel were compared with conventional wood-frame building and showed that the SIPs are "as good as" the conventional system. The compressive and flexural resistance of the panels based on the experimental results used to establish design tables for wall panels under gravity loading and soil pressure and compare with available Canadian code for force-moment interaction equation. The recorded temperature on creep tests was between $22-25^{\circ} \mathrm{C}$ and the relative humidity was between $20-70 \%$. The cyclic change in temperature and relative humidity over time did not increase with the time on experimental long-term creep deflection. But, the deflection of panel increased about 38 and 35\% for different group of panels after 8 months of sustained sol pressure. The configuration of the tested SIP showed a sufficient short-term serviceability limit state design of permanent wood foundation per CAN/CSA-O86-09. The experimental instantaneous deflection of stress-skinned panel on CSA-O8609 and DIAB's equation showed correlation between the results of SIPs with lumber stud connection. As such, the flexural and shear deflection of SIPs with lumber stud connection was ignored the foam 
core are in shear deformation according to CSA-O86-09 for stress-skinned panel. The presented creep model of this study had a good agreement with experimental data. The logarithmic expression model for prediction of creep deflection appears overestimate values at low time period and underestimates the values at the end of 8-month loading. While, the power model has shown a significant increase in total deflection and was unacceptable for long-term creep deflection. It is also presented different model for predication of short and long term creep. All the presented models predicated an increase in deflection in short and long term creep deflection.

The compressive load test on SIPs showed that failure mode of different panel was almost identical. The failure of panels under eccentric compressive load contain of crushing of OSB face, fracture of lumber-spline, delamination of OSB-foam interface, complete separation of plywood face and foam from OSB facing and delamination of OSB-foam interface between the panel footer and mid-height of the panel. The flexural load tests on SIPs showed that the failure mode of panel has happened at the interface between the top plywood faces and foam core due to shear failure. The sudden failure of panel has occurred in the quarter point location due to delamination (debonding) of the top foam-plywood interface and foam core. The design tables were developed for supported joist length of SIP basement wall for single, double and triple-storey residential building using CAN/CSA-O86.01 code. The characteristic 5-percentile value was calculated based on BS-EN 14358 (BS, 2006) and provided more conservative value comparing to basic average value which is provided by ICC AC-04.

\subsection{US Acceptance Criteria for Sandwich Panels}

According to ICC Acceptance Criteria (ICC, 2004), the load-bearing for sandwich panels shall be determined based on axial loading accompanied by 1/6 of the panel thickness eccentricity. ICC states that the ultimate axial compressive load to be divided by a safety factor (usually 3 ) to determine the allowable axial load based on the allowable stress design method. The resultant normal stresses on the core and face do not have the same linear relationship, and found to be constant throughout each by the following equation.

$$
\sigma_{c}=\frac{E_{c} \cdot P}{A_{c} \cdot E_{C}+A_{f} \cdot E_{f}}
$$


$\sigma_{f}=\frac{E_{f} \cdot P}{A_{c} \cdot E_{C}+A_{f} \cdot E_{f}}$

Where $A_{c}$ and $A_{f}$ are the core area and the flange area, respectively, $E_{c}$ and $E_{f}$ are the modulus of elasticity of the foam and the faces, respectively.

Since the skin faces resist higher level of normal stress than the core (foam), the skin faces fail due to axial compressive load. APA Plywood Design Specification Supplement $4-$ Design \& Fabrication of Plywood Sandwich Panels, (APA, 1990) specifies the following design equations for SIPs under compression loading.

APA specifies that the compression strength of panel under axial loading, $P_{e}$, should satisfy the following:

$P \leq P_{e} \quad$ where $\quad P_{e}=C_{e} \cdot F_{c} \cdot A_{f}$

The eccentric load factor, $C_{e}$, considering the minimum eccentricity equal to not less than $\mathrm{t} / 6$ (e $\geq$ $\mathrm{t} / 6)$ is expressed as follows:

$$
C_{e}=\frac{1}{1+\frac{e \cdot y_{c}}{r^{2}} \sec \left[\frac{12 L}{2 r} \sqrt{\frac{3 P}{A_{f} \cdot E_{b}}}\right]+\frac{3 P \cdot e \cdot y_{c}}{2 A_{v} \cdot G \cdot I}}
$$

where; $A_{f}=$ Area of face; $A_{v}=$ Shear Area of panel for symmetric panel; $C_{e}=$ Eccentric load factor; $E_{b}=$ Modulus of elasticity for SIP under transverse loading (psi); $F_{c}=$ Allowable facing compressive stress (psi); $G=$ SIP shear modulus (psi); $\quad I=\mathrm{SIP}$ moment of inertia (in ${ }^{4} / \mathrm{ft}$ ); $L$ $=$ Span length $(\mathrm{ft}) ; P=$ Applied axial or concentrated load $(\mathrm{lb} / \mathrm{ft}) ; P_{c r}=$ Allowable axial load $(\mathrm{lb} / \mathrm{ft}) ; r=$ Radius of gyration (in); $Y_{c}=$ Distance from centroid to the extreme compression fiber (in).

The global buckling load of a pinned-pinned column under axial loading in critical condition is expressed in equation (2.46) and can be considered as $P$ in equation (2.45). 


$$
P \leq P_{c r} \text { where } P_{c r}=\frac{\pi^{2} E_{b} I}{3\left(12 L^{2}\right)\left[1+\frac{\pi^{2} E_{b} I}{\frac{\pi^{2} E_{b} I}{(12 L) 2 A_{v} G}}\right]}
$$

Figure 2.10 shows the failure modes of a sandwich wall under axial and flexural loads. The failure mode starts from skin and then extended to core of an insulated panel. These failure modes include (a) failure of the face, (b) wrinkling of the face, (c) dimpling of the face, (d) shear failure of the core materials, (e) shear crimping of the core materials, (f) overall buckling, (g) delamination of the interface between the core and the face, (h) long-term creep, (i) overall deflection and (j) local deflection.

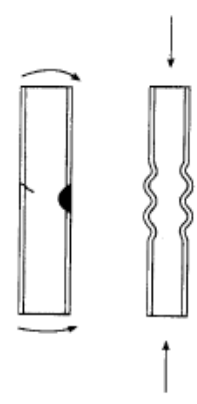

(a)

(b)

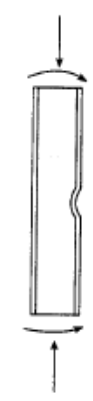

(c)

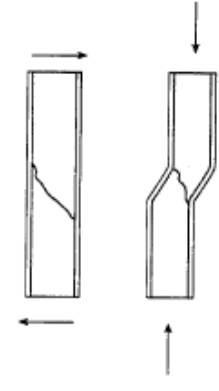

(d)

(e)

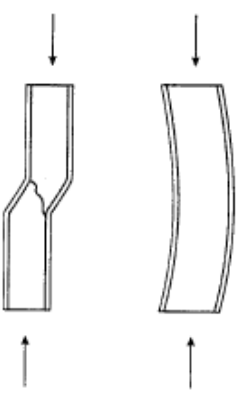

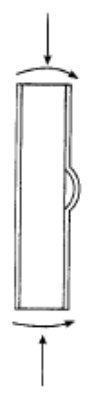

(g)

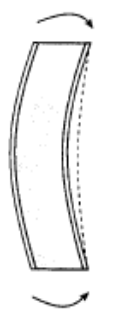

(h)

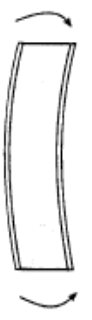

(i)

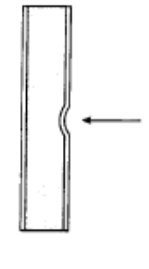

(j)

Figure 2.10 - Modes of failure for sandwich walls

(a) failure of the face; yielding or fracture, (b) wrinkling of the face, (c) dimpling of the face, (d) shear failure of the core materials, (e) shear crimping of the core materials, (f) overall buckling, (g) delamination of the interface between the core and the face (h) long-term creep (i) overall deflection and (j) local deflection

(Straalen et al., 2010)

\subsection{Analytical Models for SIP/Stud Wall}

\subsubsection{Axial Loading}

The structure of a sandwich panel is demonstrated in Figure 2.11. The thickness of core is noted as $\left(h_{c}\right)$ and bonded to and placed between a bottom and top sheathing with thickness of $\left(h_{b}\right)$ and $\left(h_{t}\right)$, respectively. The common boundaries of the sheathings and the core implemented with a 
perfect bonding. Figure 2.11 also shows the local Cartesian coordinates in each layer with subscripts (b), (t) and (c) denoting the bottom sheathing, the top sheathing and the core, respectively. The displacements of the top face sheet, the core, and the bottom face sheet are denoted by (u), (v), and (w) with subscripts $(t),(c)$, and (b), respectively, in the $(x),(y)$, and $(z)$ directions, respectively.

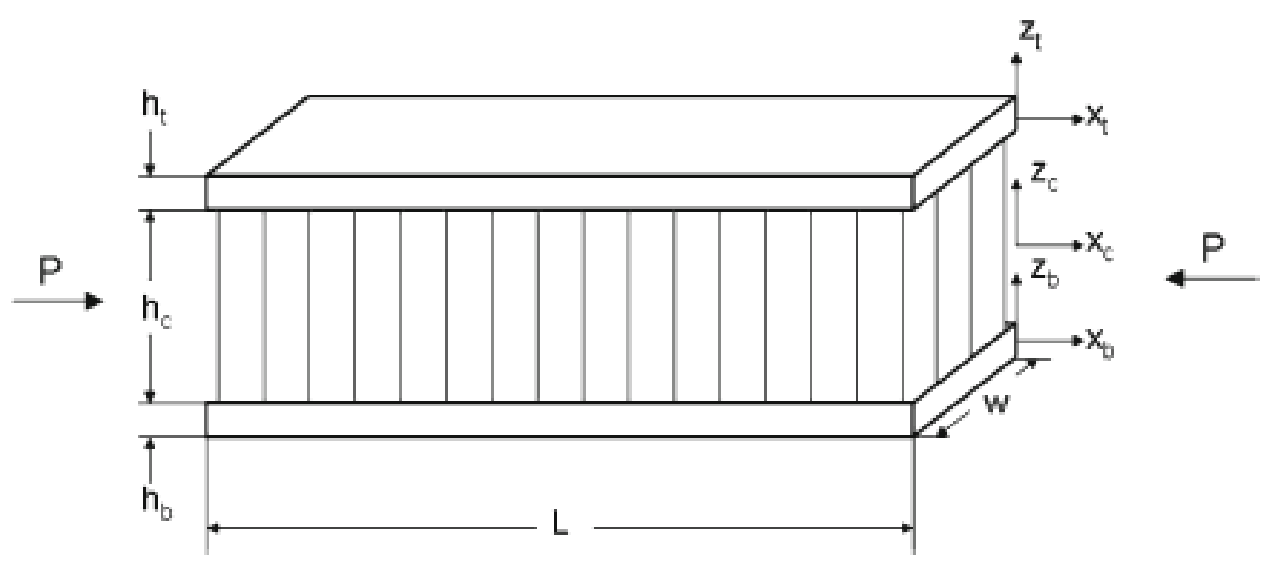

Figure 2.11 - Sandwich panel configuration (Bazant, 1971)

A plane strain deformation in the $x z$-plane in sandwich beam happens when a compressive load in the $x$-direction is applied. This load can enforce through smooth rigid end platens which are parallel to the $y z$-plane and move in the $x$-direction.

The general equation of a solid body in a slightly disturbed state from an initially strained state, governs the incremental stresses. The equations in this condition states as follows (Bazant, 1971):

$$
\begin{aligned}
& \frac{\partial \sigma_{x x}^{*}}{\partial x}+\frac{\partial \sigma_{x y}^{*}}{\partial y}+\frac{\partial \sigma_{x z}^{*}}{\partial z}=0 \\
& \frac{\partial \sigma_{y x}^{*}}{\partial x}+\frac{\partial \sigma_{y y}^{*}}{\partial y}+\frac{\partial \sigma_{y z}^{*}}{\partial z}=0 \\
& \frac{\partial \sigma_{z x}^{*}}{\partial x}+\frac{\partial \sigma_{z y}^{*}}{\partial y}+\frac{\partial \sigma_{z z}^{*}}{\partial z}=0
\end{aligned}
$$

where 
$\sigma_{i j}^{*}=\sigma_{i j}^{(m)}+\sigma_{k j}^{o} u_{i, k}-\left(1-\frac{m}{2}\right)\left(\sigma_{i k}^{o} e_{k j}+\sigma_{j k}^{o} e_{k i}\right)$

and $e_{i j}=1 / 2\left(u_{i, j}+u_{j, i}\right)$.

The quantities with the initial position of equilibrium denotes with a superscript ' 0 ' while quantities arising due to the disturbance is identified with a superscript. The particular pair of work-conjugate stress and strain measure is indicated by superscripts ' $m$ ' which needs to be used.

A general unified formulation for the class of Doyle-Erickson strain tensors, $\varepsilon_{i j}{ }^{(m)}$ and the corresponding constitutive stiffness $C_{i j k l}^{(m)}$ tensor is presented by Bazant (1971) in the form of the following equations:

$$
\begin{aligned}
& \varepsilon_{i j}^{(m)}=\varepsilon_{i j}-\left(1-\frac{m}{2}\right) e_{k i} e_{k j} \\
& C_{i j k l}^{(m)}=C_{i j k l}+\frac{1}{4}(2-m)\left(\sigma_{i k}^{o} \delta_{j l}+\sigma_{j k}^{o} \delta_{i l}+\sigma_{i l}^{o} \delta_{j k}+\sigma_{j l}^{o} \delta_{i k}\right)
\end{aligned}
$$

where the value of $(m)$ represents the different formulations. For example, $m=2$ define the second order Green-Lagrange strain, $\varepsilon_{i j}$, while $\mathrm{m}=1$ conform to the Biot strain measure (Biot, 1939). When $m=0$, the second order Biezeno-Hencky (1929) strain is acquired. The correct pair of incremental strain and incremental stress, and the equivalent fundamental model with the same value of $(\mathrm{m})$ has used during the problem solution phase, because the strain and the equivalent stress measure are actively associate to each other (Bazant, 1971, 2006) and (Hill, 1968).

The formulation of the sandwich beam buckling problem is considered in four different types. The approximations specialized to thin walled structures is presented in Case 1 and Case 2, while different values of ' $\mathrm{m}$ ' in a finite strain setting are considered in Case 3 and Case 4. A thinwalled structure cannot be taken into account for a sandwich beam when the core has a considerable transverse shear stress.

The purpose of selecting Case 1 and Case 2 are not to validate a model for sandwich beam with thin-walled structure. These cases show the differences when the inappropriate formulation is 
considered for the sandwich beam buckling problem. The other four cases show the different kind of approximation for each type of formulation for buckling problem with an orthotropic material such as sandwich panel core, honeycomb core and fiber reinforced composite panel.

Table 2.2 - Summary of the formulations for thin-walled structure

\begin{tabular}{|l|l|}
\hline Case 1 & $\sigma_{i j}^{*}=\sigma_{i j}^{\prime(0)}+\sigma_{k j}^{o} \omega_{i k}^{\prime} ; \quad \varepsilon_{i j}^{(0)} \approx \frac{1}{2}\left(u_{i, j}+u_{i, j}\right)$ \\
& $C_{i j k l}^{(0)}=C_{i j k l}$ \\
\hline Case 2 & $\sigma_{i j}^{*}=\sigma_{i j}^{\prime(0)}+\sigma_{k j}^{o} \omega_{i k}^{\prime} ; \quad \varepsilon_{i j}^{(0)} \approx \frac{1}{2}\left(u_{i, j}+u_{j, i}\right)$ \\
& $C_{i j k l}^{(0)}=C_{i j k l}+\frac{1}{2}\left(\sigma_{i k}^{0} \delta_{j l}+\sigma_{j k}^{0} \delta_{i l}+\sigma_{i l}^{0} \delta_{j k}+\sigma_{j l}^{0} \delta_{i k}\right)$ \\
\hline Case 3 & $\sigma_{i j}^{*}=\sigma_{i j}^{(0)}+\sigma_{k j}^{o} u_{i, k}-\left(\sigma_{i k}^{0} e_{k j}+\sigma_{j k}^{0} e_{k i}\right) \quad ; \quad \varepsilon_{i j}^{(0)} \approx \frac{1}{2}\left(u_{i, j}+u_{j, i}\right)$ \\
& $C_{i j k l}^{(0)}=C_{i j k l}+\frac{1}{2}\left(\sigma_{i k}^{0} \delta_{j l}+\sigma_{j k}^{0} \delta_{i l}+\sigma_{i l}^{0} \delta_{j k}+\sigma_{j l}^{0} \delta_{i k}\right)$ \\
\hline Case 4 & $\sigma_{i k}^{0}=\sigma_{i j}^{(2)}+\sigma_{k j}^{o} u_{i, k} \quad ; \quad \varepsilon_{i j}^{(0)} \approx \frac{1}{2}\left(u_{i, j}+u_{j, i}\right)$ \\
& $C_{i j k l}^{(2)}=C_{i j k l}$ \\
\hline
\end{tabular}

Case 1. Formulation of thin-walled structures considering the estimation of constant stiffness tensor. The value of $(\mathrm{m}=0)$ (instead of $\mathrm{m}=2)$ in Eq. 2.50 .

Case 2. Formulation of thin-walled structures considering the estimation of strain by ignoring axial deformation compared to rotation. The value of $(\mathrm{m}=0)$ in Eq. 2.52.

Case 3. Biezeno-Hencky formulation. The value of $(\mathrm{m}=0)$.

Case 4. Trefftz formulation. The value of $(\mathrm{m}=2)$.

Case 1 and Case 2 are used in special cases of $(m=0)$ in formulations. The axial strain components (eij) are often disregarded in comparison to the rotational effects $\left(\omega_{i j}^{\prime}\right)$ when the rotation are so larger than the axial deformations in case of buckling deformation of a thinwalled structure. In this case (Case 1 and Case 2), Eq. 2.52 is defined as follows: 
$\sigma_{i j}^{*}=\sigma_{i j}^{\prime(m)}+\sigma_{k j}^{o} \omega_{i k}^{\prime}$

where $\omega_{i j}^{\prime}=\frac{1}{2}\left(u_{i, j}-u_{j, i}\right)$.

The constant modulus $\left(C_{i j k l}^{(2)}\right)$ approximation is employed in Case 1 which is not acceptable according to constitutive model related with Eq. 2.53. The results of Case 1 and Case 2 are compared in order to determine the effect of the inappropriate use of the $(\mathrm{m}=0)$ formulation. The buckling problem of a thin-walled structure is defined by Biezeno-Hencky formulation without any simplifying approximation in Case 3. An assessment of the other cases in different analytical discussions is presented in Trefftz formulation (Case 4).

Bazant and Beghini (2005) discussed the Green-Lagrange strain measure for sandwich type structure with small strain and constant modulus of elasticity. Trefftz (1993) presented the differential field equations in Green-Lagrange finite strain measure. Table 2.2 summarizes the different approximation of incremental stress, strain and constitutive relation subjected to the buckling problem of a sandwich beam.

The sandwich beam has uniform strain in the x-direction as presented in Eq. 2.50. The prebuckling axial stress in each layer is $\sigma_{x x}^{0}=-\sigma_{i}$, and not considered in mentioned equation. The axial pre-buckling strain is the same in each layer $\left(\sigma_{i}\right)$ because of prefect bonding in layers. The pre-buckling load $(P)$ is defined in following equation:

$$
\sigma_{i}=\frac{P \hat{E}_{i}}{\hat{E}_{t} A_{t}+\hat{E}_{c} A_{c}+\hat{E}_{b} A_{b}}
$$

or

$\sigma_{i}=\frac{\sigma \hat{E}_{i}}{\hat{E}_{t} R_{t}+\hat{E}_{c} R_{c}+\hat{E}_{b} R_{b}}$

where; $\hat{E}_{i}=E_{i} /\left(1-v_{i}^{2}\right) ; E_{i}=$ Young's modulus of each layer; $v_{i}=$ Poisson's ratio of each layer; $A_{i}=$ cross-sectional area of each constituent; $A=$ total cross-sectional area of the sandwich panel; $R_{i}=$ ratio of the cross-section area of each layer to the total cross-sectional area $A ; \sigma=$ Stress $=$ $P / A$. 
The uniformly strained state can be acquired by substitution of Eq. 2.50 into Eq. 2.47, 2.48 and 2.49. The incremental stresses resulted due to the disturbance are governed the field equations. The following equilibrium equations for the different cases are presented in following equations and need to satisfy with each constituent.

Case 1 and Case 2:

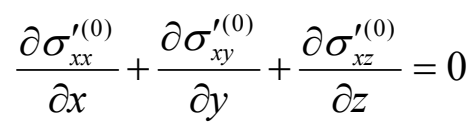

$\frac{\partial}{\partial x}\left[\sigma_{x y}^{\prime(0)}-\omega_{x y}^{\prime} \sigma_{x x}^{0}\right]+\frac{\partial \sigma_{y y}^{(0)}}{\partial y}+\frac{\partial \sigma_{y z}^{(0)}}{\partial z}=0$

$\frac{\partial}{\partial x}\left[\sigma_{x z}^{\prime(0)}-\omega_{x z}^{\prime} \sigma_{x x}^{0}\right]+\frac{\partial \sigma_{y z}^{\prime(0)}}{\partial y}+\frac{\partial{\sigma_{z z}^{\prime(0)}}_{\partial z}}{\partial z}=0$

Case 3:

$\frac{\partial}{\partial x}\left[\sigma_{x x}^{\prime(0)}-\sigma_{x x}^{0} e_{x x}\right]+\frac{\partial}{\partial y}\left[\sigma_{x y}^{\prime(0)}-\sigma_{x x}^{0} e_{x y}\right]+\frac{\partial}{\partial z}\left[\sigma_{x z}^{\prime(0)}-\sigma_{x x}^{0} e_{x z}\right]=0$

$\frac{\partial}{\partial x}\left[\sigma_{x y}^{\prime(0)}-\omega_{x y}^{\prime} \sigma_{x x}^{0}\right]+\frac{\partial{\sigma_{y y}^{\prime(0)}}_{\partial y}}{\partial y} \frac{\partial{\sigma_{y z}^{\prime(0)}}_{\partial z}}{\partial z}=0$

$\frac{\partial}{\partial x}\left[\sigma_{x z}^{\prime(0)}-\omega_{x z}^{\prime} \sigma_{x x}^{0}\right]+\frac{\partial \sigma_{y z}^{\prime(0)}}{\partial y}+\frac{\partial \sigma_{z z}^{(0)}}{\partial z}=0$

Case 4:

$\frac{\partial}{\partial x}\left[\sigma_{x x}^{\prime(2)}-\sigma_{x x}^{0} \frac{\partial u}{\partial x}\right]+\frac{\partial \sigma_{x y}^{\prime(2)}}{\partial y}+\frac{\partial \sigma_{x z}^{\prime(2)}}{\partial z}=0$

$\frac{\partial}{\partial x}\left[\sigma_{x y}^{\prime(2)}-\sigma_{x x}^{0} \frac{\partial v}{\partial x}\right]+\frac{\partial \sigma_{y y}^{\prime(2)}}{\partial y}+\frac{\partial \sigma_{y z}^{\prime(2)}}{\partial z}=0$

$\frac{\partial}{\partial x}\left[{\sigma_{x z}^{\prime(2)}}^{\prime}-\sigma_{x x}^{0} \frac{\partial w}{\partial x}\right]+\frac{\partial \sigma_{y z}^{\prime(2)}}{\partial y}+\frac{\partial \sigma_{z z}^{\prime(2)}}{\partial z}=0$ 


\subsubsection{Bending}

Figure 2.5 shows a typical sandwich beam with different layers. The materials of the faces are identical. The density, Young's modulus and shear modulus of face are denoted as $\rho_{f}, E_{f}$ and $G_{f}$, respectively. The density, Young's modulus and shear modulus of core are also denoted as $\rho_{c}, E_{c}$ and $G_{c}$, respectively. The thickness of each face is shown as $t_{i}(i=1,2)$ and the thickness of core is presented as $c$. The width of beam is $b$ and the span length is $L$.

The normal stress in the faces and core were denoted as $\sigma_{f i}(i=1,2)$ and $\sigma_{c}$ at a distance $z$ from the neutral axis, respectively and it is presented in the following equation (DIAB, 2003):

$$
\begin{aligned}
& \sigma_{f i}=\frac{M_{z} E_{f}}{D} ; \quad i=1,2, \\
& \sigma_{c}=\frac{M_{Z} E_{c}}{D}
\end{aligned}
$$

where, $D=$ the equivalent flexural rigidity of the sandwich beam.

In the case of equal faces $\left(t_{l}=t_{2}=t\right)$, the value of $D$ is defined by following equation (DIAB, 2003):

$$
D=\frac{E_{f} b t^{3}}{6}+\frac{E_{f} b t d^{2}}{2}+\frac{E_{c} b c^{3}}{12}
$$

where;

$$
d=c+\frac{t_{1}}{2}+\frac{t_{2}}{2}=c+t
$$

It is considered that the core has insignificant effect on the bending moment and the thickness of the faces is so thin comparing to the core. So, the flexural rigidity is defined by the following equation (DIAB, 2003).

$$
D \cong E_{f} \frac{b t d^{2}}{2}
$$

The stress distribution in the external faces of beam is not consistent and it is shown in Eq. 2.65. If the thickness of the faces compare to the core is insignificant, the stresses in the faces when $(z$ $=d / 2$ ) are estimated by the following equation:

$$
\sigma_{f} \cong \frac{M}{b t d}
$$


The maximum normal stress in the core can be developed from Eq. 2.66 and 2.67 as follows:

$$
\sigma_{c} \cong \frac{M_{C} E_{C}}{b t d^{2} E_{f}}
$$

The shear stress of the sandwich beam in a distance $z$ above the centroid of the cross-section is given as follows (DIAB, 2003):

$$
\tau=\frac{Q \sum\left(S_{i} E_{i}\right)}{D b}
$$

where; $Q=$ shear force of the section; $S_{i} E_{i}$, = sum of the products of the first moment of area of the part of the section above $z$ and Young's modulus for each component of the beam.

The shear stress varies through the core and has a parabolic shape which is shown in Figure 2.6. When the faces are equal and $z=0$, the equation 2.72 is written as following (DIAB, 2003):

$$
\tau_{c}=\frac{Q}{D}\left\{\frac{E_{f} d t}{2}+\frac{E_{c} c^{2}}{8}\right\}
$$

The shear stress is considered constant through the core if the faces are stiffer and thinner than the core. This function is approximated by a linear distribution which is changing from zero at the outer surface of the face and has a value at the core ends as depicted in Figure 2.6. Hence, the mean shear stress in the core and the face is presented in the following form (DIAB, 2003).

$$
2 \tau_{f} \cong \tau_{c} \cong \frac{Q}{b d}
$$

\subsubsection{Lateral Loading}

The first exact solution for the shear buckling of an infinitely long isotropic plate was given by Southwell and Skan (1924), from the governing differential equation.

$$
D\left[\frac{\partial^{4} W}{\partial x^{4}}+2\left[\frac{\partial^{4} W}{\partial x^{2} \partial y^{2}}\right]+\frac{\partial^{4} W}{\partial y^{4}}\right]+2 \tau d \frac{\partial^{2} W}{\partial x \partial y}=0
$$

A modal form for the buckling displacements was assumed as following function:

$$
W=Y_{(y)} \exp (i k x / b)
$$


where $Y$ is an unknown function in the transverse $y$ direction which satisfies the boundary conditions on the long edges. An exact solution for the critical shear stress, $\tau_{c r}$ and the critical longitudinal wavelength parameter, $k$, can be obtained by an iterative technique.

The critical shear stress, $\tau_{c r}$ were given for simply supported and clamped long edges and the respective buckling coefficients, $K_{s}$, were 5.35 and 8.98 , respectively as follows:

$\tau_{c r}=K_{s} \pi^{2} D / d b^{2}=\left(N_{x y}\right)_{c r} / d$

where, $N_{x y}=$ shear stress resultant; $d=$ plate thickness; $b=$ plate width in $y$ direction; $D=$ $E d^{3} / 12\left(1-v^{2}\right)=$ plate rigidity.

Southwell and Skan (1924) investigated plates with equal elastic restraints against rotation along the long edges. Their work was extended by Stowell (1943). Figure 2.12 shows the ratio of the buckling half-wave length to the plate width $b$ and the dependence of $K_{s}$, on $(2 / \mathrm{b})$ for different values of the edge restraint parameter $\varepsilon$. The extreme cases of simply-supported and clamped long edges are defined with different value of $\varepsilon$ as $(\varepsilon=0)$ and $(\varepsilon=\alpha)$.

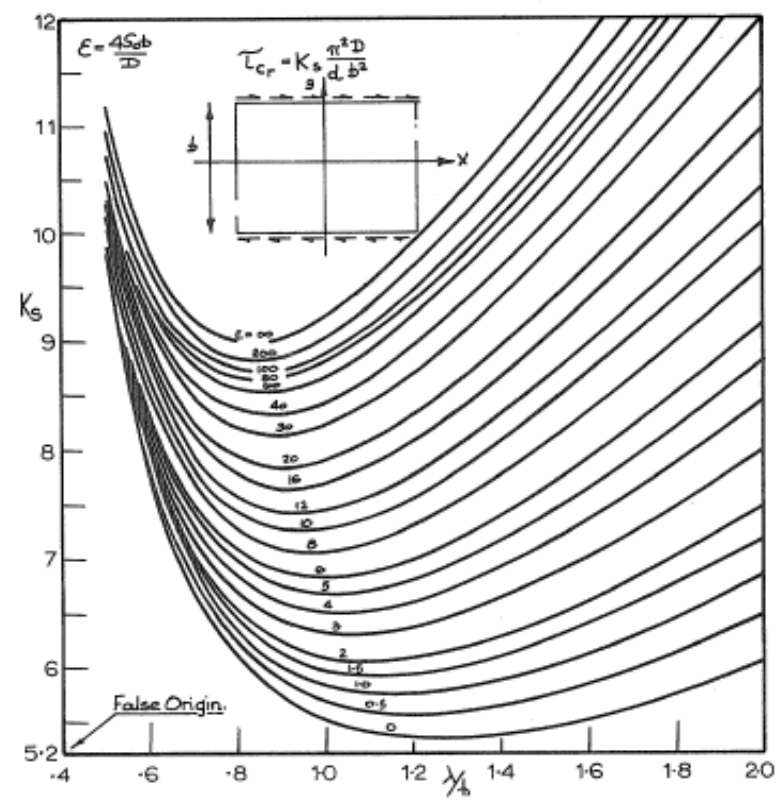

Figure 2.12 - Values of $K_{s}$ in equation for critical shear stress for an infinitely long flat plate with equal restraining loads along the parallel edges.

(Southwell and Skan, 1924) 
The curves shown in Figure 2.12 were obtained from a few exact solution results supplemented by those resulting from an alternative energy method of analysis, $\left(\varepsilon=4 S_{o} b / D\right)$, where, $S_{o}$, is the stiffness per unit length of the elastic restraining medium or the moment required to rotate a unit length of the medium through one-fourth of a radian.

Southwell and Skan (1924) suggested an approximate equation as follows when the value of $\varepsilon$ is different along opposite long edges.

$K_{s}=\left[K_{1} K_{2}\right]^{\frac{1}{2}}$

where $K_{l}$ and $K_{2}$ are the values of $K_{s}$ for equal values of $\varepsilon_{l}$ and $\varepsilon_{2}$, respectively. Thus, when one long edge is simply-supported and the other is clamped, equation 2.77 yields $K_{s}=6.92$ whereas the exact value is 7.07 .

Huber (1922) presented the theory and differential equations of bending of anisotropic plates which is used for shear buckling of orthotropic plates. The shear buckling of a general orthotropic plate is governed by differential equation as follows:

$D_{1} \frac{\partial^{4} W}{\partial x^{4}}+2 D_{3} \frac{\partial^{4} W}{\partial x^{2} \partial y^{2}}+D_{2} \frac{\partial^{4} W}{\partial y^{4}}+2 N_{x y} \frac{\partial^{2} W}{\partial x \partial y}+4 D_{13} \frac{\partial^{4} W}{\partial x^{3} \partial y}+4 D_{23} \frac{\partial^{4} W}{\partial x \partial y^{3}}=0$

The last two terms of the equation are related to the orthotropic coupling. The principle elastic axes are not orthogonal with the plate geometric axes and that is resulted from orthotropic coupling. The special case of the equation is 'special orthotropic' plate $\left(D_{13}=D_{23}=0\right)$.

Bergmann and Reissner $(1932,1948)$ examined the problem of the stability of orthotropic plates due to shear. They considered an infinite long plate in $\mathrm{x}$-direction. The bending rigidity in this direction is neglected. So, the governing differential equation can defined as follows:

$2 D_{3} \frac{\partial^{4} W}{\partial x^{2} \partial y^{2}}+D_{2} \frac{\partial^{4} W}{\partial y^{4}}+2 N_{x y} \frac{\partial^{2} W}{\partial x \partial y}=0 ; \quad D_{1}=0$

A similar method of analysis for exact solution of a plate simply-supported along the long edges is presented as follow:

$N_{x y} b^{2} \cdot 4\left(D_{2} D_{3}\right)^{\frac{1}{2}}=11.71$ 
Wood-shear wall is also modeled by several studies, but each study has the limitation in modeling such as inhomogeneous and anisotropic, and nonlinear connection of sheathing to frame which demonstrate strength and stiffness humiliation under cyclic loading. The complexity of modeling process is caused by combination of these characteristics and considerable degrees of redundancy. Therefore, some techniques are used to reduce this complexity for wood shear wall and building models. Ayoub (2006) summarized a comprehensive review of wood frame numerical models as presented in Table 2.3.

Folz and Filiatrault (2001) studied an inclusive research on wood frame shear walls subjected to seismic ground motions. A numerical model with the ability to capture interaction between framing member and connectors under cyclic loading of wood frame shear wall is developed by them. They intended to present the load sharing and interaction between components of wood shear wall under cyclic loading, while the previous studies mostly concentrated on the influence of fastener type, panel size, gypsum wall board contribution and effect of hold-downs on the response.

Dolan and Madsen (1992) studied the wood shear wall with dowel-type connector and stated that dowel-type connector is highly nonlinear under monotonic loading and demonstrates a pinched hysteretic behavior with strength and cyclic degradation. The degrading pinched hysteresis plot is similar to the stiffness and strength degradation characteristics in the hysteresis curve of a shear wall with sheathing to framing connectors under cyclic loading. The correlation of hysteresis response in wood-frame structure is greater through cyclic loading than monotonic loading. A specific hysteretic model based on a minimum number of path-following rules prevents the need of test on full-scale shear wall. The shear modulus of sheathing panels and cyclic test data of sheathing to framing connector reduces the data which is needed for numerical analysis. 
Table 2.3 - Summary of analytical studies of wood frame shear wall (Ayoub, 2006)

\begin{tabular}{|c|c|c|}
\hline Researcher(s) & Year & Analytical Study \\
\hline Chehab & 1982 & $\begin{array}{l}\text { A model for two-story building with linear elastic material } \\
\text { properties was created }\end{array}$ \\
\hline $\begin{array}{l}\text { Itani and Cheung } \\
\text { Falk and Itani }\end{array}$ & $\begin{array}{l}1984 \\
1989\end{array}$ & $\begin{array}{l}\text { Elastic plane stress model was developed with nonlinear joint } \\
\text { element and beam element. }\end{array}$ \\
\hline Gupta and Kua & $\begin{array}{l}1985 \\
1987\end{array}$ & $\begin{array}{l}\text { Seven "super-element" and nine global DOF model were } \\
\text { developed. }\end{array}$ \\
\hline $\begin{array}{l}\text { Stewart } \\
\text { Filiatrault } \\
\text { Kasal and Leichti }\end{array}$ & $\begin{array}{l}1987 \\
1990 \\
1992\end{array}$ & $\begin{array}{l}\text { Development of a single degree of freedom (SDOF) model } \\
\text { using pinching, strength and stiffness degradation. }\end{array}$ \\
\hline Kasal et al. & 1997 & $\begin{array}{l}\text { Modeling of one-story building using ANSYS finite element } \\
\text { program. }\end{array}$ \\
\hline $\begin{array}{l}\text { Dolan } \\
\text { Dolan and Foschi } \\
\text { White and Dolan }\end{array}$ & $\begin{array}{l}1989 \\
1991 \\
1995\end{array}$ & $\begin{array}{l}\text { Development of a model with four sub-elements } \\
\text { - } \quad \text { elastic beam elements for studs } \\
\text { - } \quad \text { elastic orthotropic element for plywood shear wall } \\
\text { - } \quad \text { nonlinear spring for nails } \\
\text { bilinear compression spring for gap between walls }\end{array}$ \\
\hline Davenne et al. & 1998 & 3D modeling of nail strength degradation \\
\hline Foschi & $\begin{array}{l}1995 \\
2000\end{array}$ & 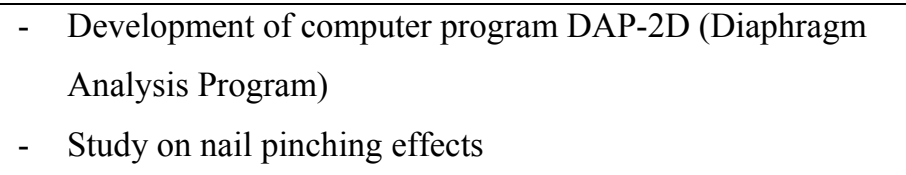 \\
\hline He et al. & 2001 & $\begin{array}{l}\text { Developing of the computer program LightFrame 3D } \\
\text { - Thin plate elements for plywood, beam elements for studs, } \\
\text { nonlinear spring elements } \\
\text { - Demonstration of nail shear and pullout characteristics }\end{array}$ \\
\hline Folz and Filiatrault & 2001 & Computer programs CASHEW and SAWS were developed. \\
\hline Ayoub & 2006 & $\begin{array}{l}\text { Developed SDOF pinched hysteretic model which represented } \\
\text { four types of degradation, namely, strength, unloading stiffness, } \\
\text { accelerated stiffness, and cap deterioration were developed. }\end{array}$ \\
\hline $\begin{array}{l}\text { Pei and van de } \\
\text { Lindt }\end{array}$ & 2007 & $\begin{array}{l}\text { SAPWood computer program capable of time domain analysis } \\
\text { for structural and loss analysis was developed. }\end{array}$ \\
\hline
\end{tabular}

Folz and Filiatrault (2001) presented a model for equivalent SDOF zero-height shear wall spring elements. These elements infinitely connect in-plane stiff diaphragms and it is defined by 10 parameters that assume a shear wall is connected to a rigid frame using a pin connection, 
sheathing member with elastic deformation and non-linear sheathing to framing connectors. The CASHEW (Cyclic Analysis of SHEar Wall) computer program was used to integrate the data and the stiffness degrading hysteresis and strength of wood frame shear wall was reflected by calibrated spring elements. The SDOF system which is presented by Folz and Filliatrault (2001) was utilized to predict the load displacement response of a shear wall under lateral loading. The pinched, strength and stiffness degrading, hysteresis response in experimental study were accurately represented by equivalent shear spring element. Results showed that the computer program were accurately defined the same characteristics in the load-displacement diagram of the experimental model.

Folz and Filiatrault (2004) extended the research on wood-frame building under quasi-static and seismic loads in order to predict the dynamic characteristics and present a simple numerical model. They also presented the implementation and verification of the SAWS (Seismic Analysis of Wood-frame Structures) model to predict the dynamic behaviour of a building.

\subsection{Monotonic and Cyclic Study on Shear Wall}

Dolan and Johnson (1996) performed a considerable monotonic test on one-third scale models and short full size wall to verify the perforated shear wall method. This research gave supplementary information on performance of full-size and long shear wall under monotonic and cyclic loads. The conservative estimate of performance in seismic region was provided by cyclic tests on walls. The aim of this research was establish the effects of opening on full-size wood frame shear walls, shown in Figure 2.13 under monotonic and cyclic tests. 


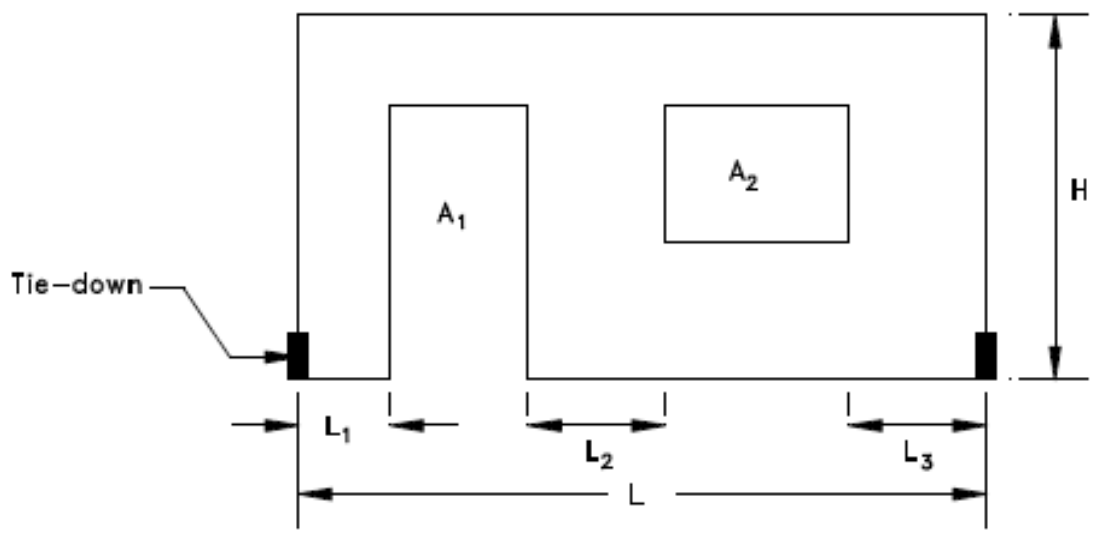

Figure 2.13 - The sheathing area ratio parameters on long shear wall with opening (Dolan and Johnson, 1996)

Jamison (1997) conducted four static one-dimensional ramp tests, and eight static load-controlled tests on SIP shear walls according to ASTM E564. Also eleven cyclic tests are done with Sequential Phased Displacement (SDP) testing procedure by Poter (1987). Structural Engineers Association of Southern California (SEAOSC) presented a fully reserved cyclic loading test method to define test frequencies and removal stabilization cycles for nail fatigue. Jamison (1997) concluded that SIP shear wall performance is related to bottom plate. He stated that the failure in SIP happens in bottom plate connection and the use of tie-down anchors increases stiffness, capacity and wall energy dissipation. He also discussed the use of different configurations of fasteners, contribution of the adhesive, energy dissipation, ductility, damping characteristics which are important in seismic design.

Gatto and Uang (2003) performed several cyclic tests with deformation controlled CUREE protocol. The symmetric initiation, primary and trailing cycles were included in deflection control protocol. The small amplitude load-deformation response was determined by initiation cycles and was consider as a check for equipment and measuring devices. The amplitude of primary cycles was increased and followed by trailing cycles and the amplitude was increased until $75 \%$ of the primary cycle (CUREE, 2002). The CUREE protocol is presented in Table 2.4 and established graphically in Figure 2.14. 
Table 2.4 - CUREE loading sequence for Wood-frame structures (CUREE, 2002)

\begin{tabular}{|c|c|}
\hline Cycle Number & \% $\boldsymbol{\delta}^{(\mathbf{1})}$ \\
\hline 1 to 6 & 5.0 \\
\hline 7 & 7.5 \\
\hline 8 to 13 & 5.6 \\
\hline 14 & 10.0 \\
\hline 15 to 20 & 7.5 \\
\hline 21 & 20.0 \\
\hline 22 to 24 & 15.0 \\
\hline 25 & 30.0 \\
\hline 26 to 28 & 22.5 \\
\hline 29 & 40 \\
\hline 30 to 31 & 30 \\
\hline 32 & 70 \\
\hline 33 to 34 & 52.5 \\
\hline 35 & 100 \\
\hline 36 to 37 & 75 \\
\hline 38 & 150 \\
\hline 39 to 40 & 112.5 \\
\hline
\end{tabular}

(1) $\delta$ is the corresponding displacement related to $80 \%$ of maximum load capacity from monotonic lateral test on shear wall.

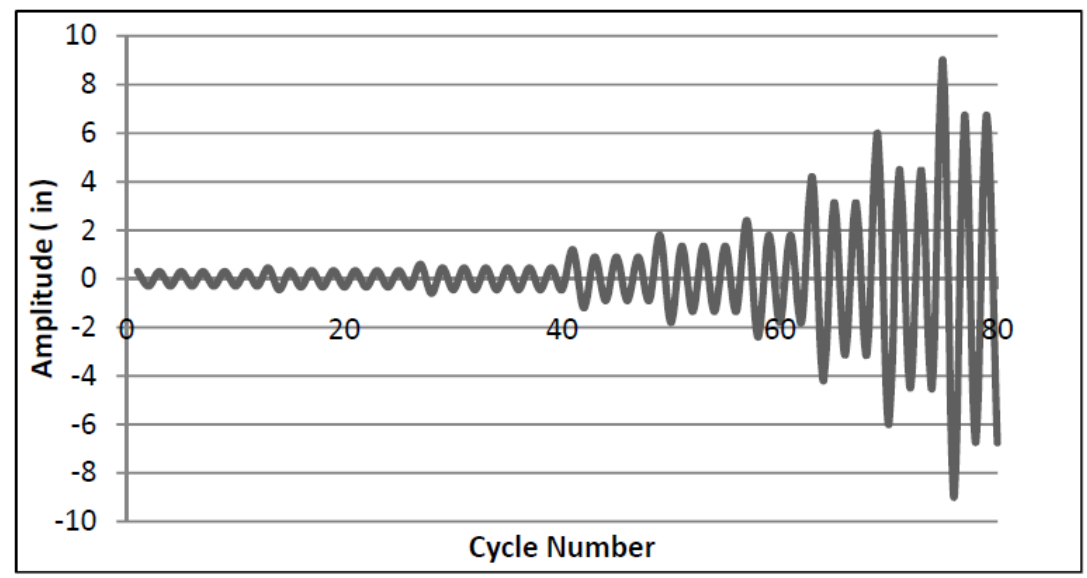

Figure 2.14 - CUREE loading protocol (CUREE, 2002) 
Toothman (2003), Lebeda et al. (2005) and Johnston (2006) studied wood frame shear wall with hold-down anchorage. Each of them concluded that the anchorage has the minimum effect on energy dissipation and stiffness of the wall. However, the anchorage increases the strength and has effect on failure mode of the wall. The configuration of the anchorage was based on ASTM E 2126-09 (2009) and consisted of 18 WS3 wood screw on end stud and sill plate. Johnston et al. (2006) showed that the presence of vertical (gravity) loading increase the energy dissipation and lateral stiffness of the wall.

In comparison with standard shear wall shown in Figure 2.15b, a new concept in shear wall design was presented by Varoglu et al. (2006) as shown in Figure 2.15a. A series of pair of studs with one ply of sheathing material located at the center of the wall was selected. These plates had $90^{\circ}$ rotation and related to this standard shear walls. Because of the location of sheathing panel, this new wall design was called "mid-ply". The static and cyclic loading conditions test were performed to mid-ply wall and compared with standard shear wall under similar loads. The nail connections in mid-ply worked in double shear. However, the nail connections worked in single shear in the standard shear walls. The results of this research had a significant improvement in shear performance of mid-ply walls. The test results showed that the mid-ply has a load carrying capacity more than three times of a similar standard shear wall.

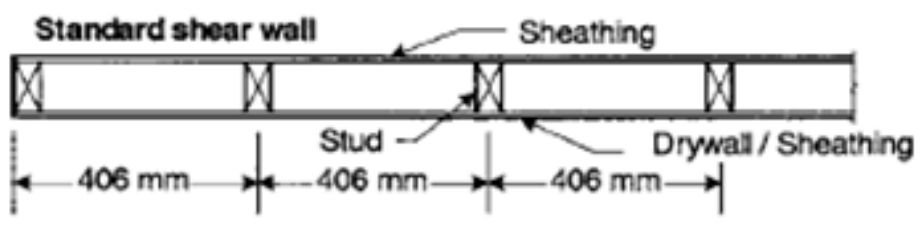

(a) Standard shear wall

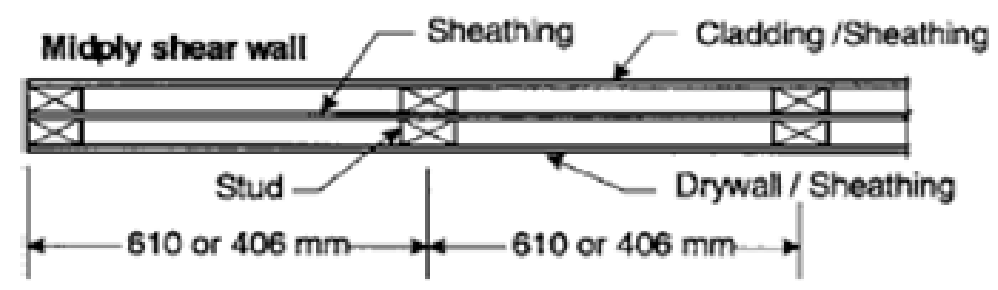

(b) Mid-ply shear wall

Figure 2.15 - Cross section of typical standard shear wall and mid-ply wall (Varoglu et al., 2006) 
Talbot et al. (2008) studied the dynamic cyclic tests of wood shear walls using staples as connectors of sheathing to the studs to determine load and displacement capacities. Enhanced details from standard construction were used to improve the performance of the global system including a double sill plate, a new refined panel corner detail, double staples along blocked edges, and backup bolts for hold-downs. The experimental results showed that the stapled wood shear walls with the enhanced details performed at a level above that allowed by the International Building Code, IBC, (ICC 2006) regarding peak load capacity but slightly less regarding peak drift capacity. Figure 2.16 shows the test assembly and setup of this research.
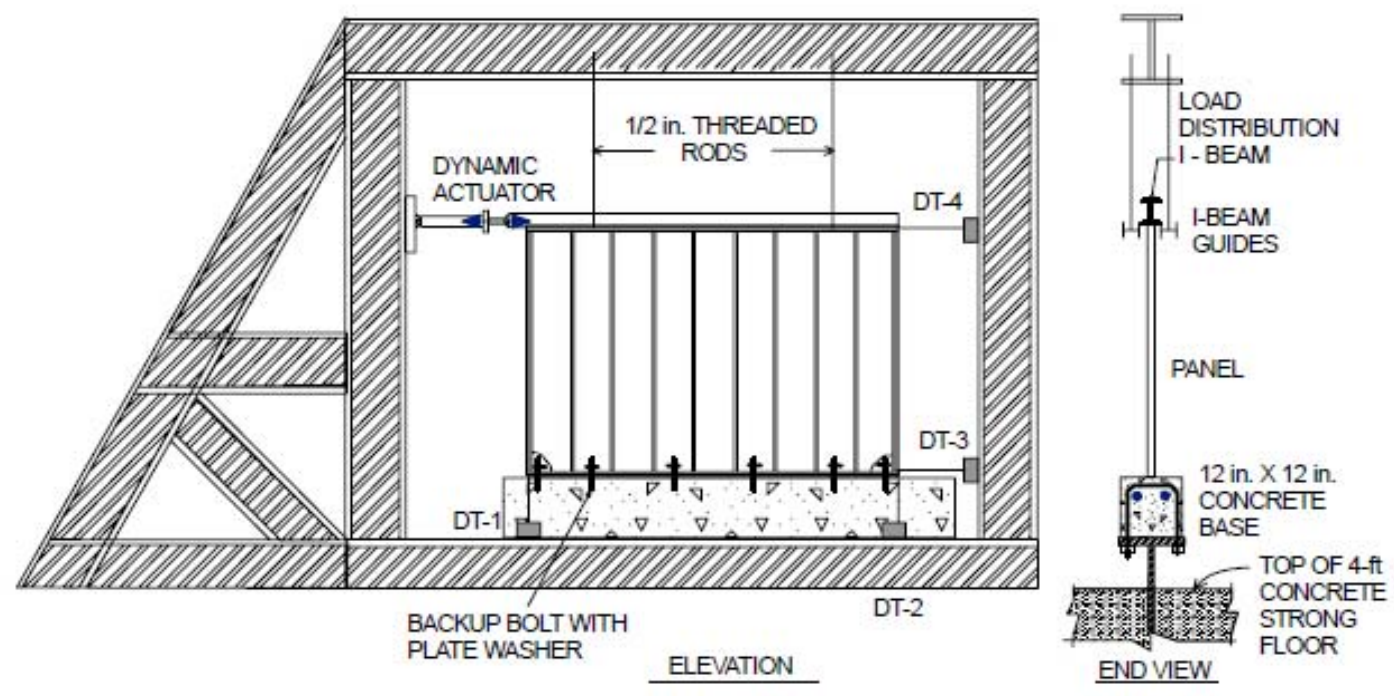

Figure 2.16 - Test assembly and setup (Talbot, 2008)

The test results for material characterization of SIPs and CSIPs (Concrete Structural Insulated Panel) subjected to shear stresses is presented by Mosalam et al. (2008). The main part of the research discussed about racking test results of SIPs using quasi-static and pseudo-dynamic techniques. The validity of pseudo-dynamic testing to characterize the SIPs seismic performance with less sensitivity to test parameters than quasi-static testing was confirmed by test results. Preliminary conclusions included ultimate displacement ductility slightly above 4.0 for SIPs without panel-to-panel connections and logical energy dissipation. The strength of SIP was determined under $100 \%$ of the design basis earthquake (DBE) - $10 \%$ probability of exceedance in 50 years. In addition, the large energy dissipation happened as well as reduction of strength in SIPs for upper-bound earthquake (UBE) - 10\% probability of exceedance in 100 years. 
Van de Lindt (2008) studied the effect of consecutive earthquakes on wood frame structural reliability through experimental investigation. The objective of his research was to examine the performance of wood frame once damaged in the point of view of practicing structural engineers. Six successful earthquake tests on a uniaxial shake table for four different types of specimens were performed namely: (1) isolated shear walls with drywall; (2) isolated shear walls with no drywall; (3) corner assemblies with drywall; and (4) corner assemblies with no drywall. Figure 2.17 indicates the solid schematic model of the test setup including the shake table and seismic mass on the roof. The combination of the test results in this research and engineering logic can be drawn as following:

(1) Comparison of damage of isolated shear wall without drywall and the corner assembly without drywall showed that the strength and stiffness alone are not adequate to reduce the damage of wall;

(2) The strength and stiffness increased when drywall was attached using the fastener;

(3) The whole structures had greater damage than that for the isolated specimens; and

(4) The tested wood frame assemblies exhibited good performance in extreme earthquake simulation.

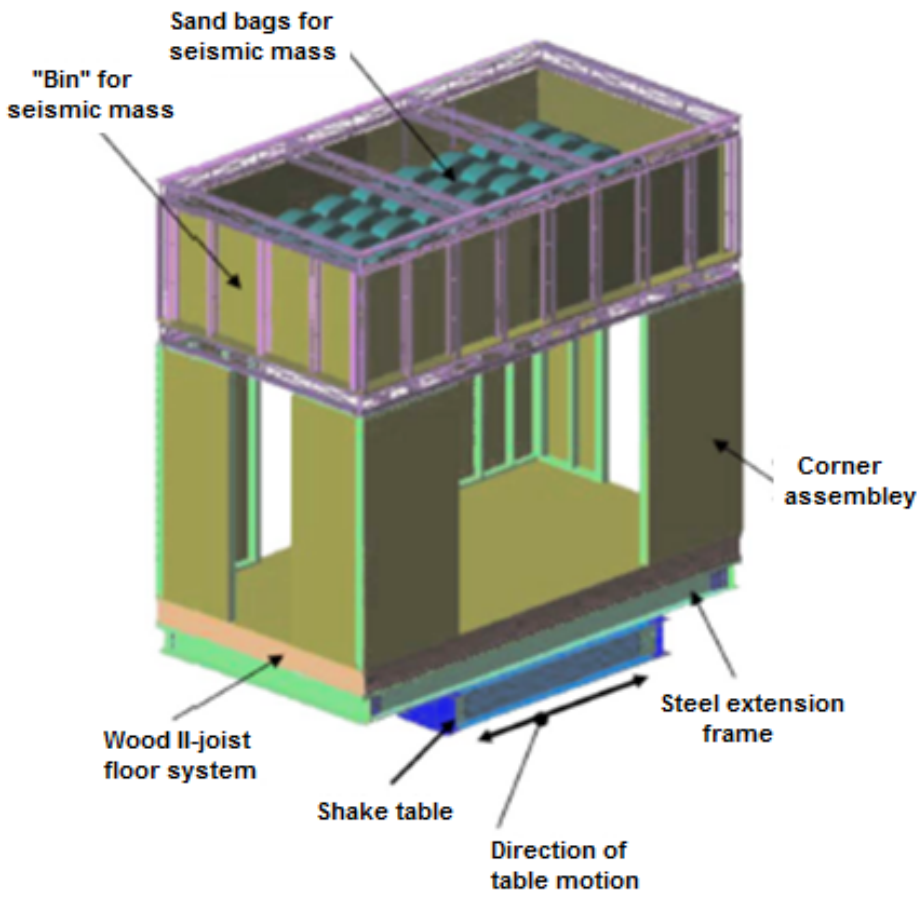

Figure 2.17 - The corner test setup of solid model on shake table and seismic mass representing a typical upper floor level and roof system (Van de Lindt, 2008) 
Seaders et al. (2009) evaluated effects of anchorage, qualitatively and quantitatively, with respect to code-defined performance measures on wood-shear walls under monotonic, cyclic, and earthquake loads to compare performance of the walls under loading protocol. The walls had the size of 2440 by $2440 \mathrm{~mm}$ in elevation and made of 38 x $89 \mathrm{~mm}$ (2' x 4') Douglas-fir studs at 610 $\mathrm{mm}$ on center. The sheathing of the wall was two oriented strand boards (OSB) of $1220 \times 2440 \mathrm{x}$ $11.1 \mathrm{~mm}$ in size. The other side of the wall was 12-mm thick gypsum wallboard (GWB) installed vertically. Two historical reduction zone ground motions scaled to a 10 percent in 50 year probability of exceedance for Seattle, with a $4545 \mathrm{~kg}$ seismic mass were used to test eight walls. Each earthquake time history was applied to two partially anchored and two fully anchored walls. The peak strength and initial stiffness in earthquake tests were lower than the monotonic similar test on walls. The load-deflection envelopes were asymmetrical shape under various ground motions. Six out of eight tests on walls showed that the maximum transient drift of wall exceeded three percent of the limit for collapse which was mentioned to prevent performance in ASCE/SEI 41-06 (ASCE, 2007). The maximum drift on fully and partially anchored walls was similar. Figure 2.18 shows the dynamic testing frame with location of transducers on shear wall.

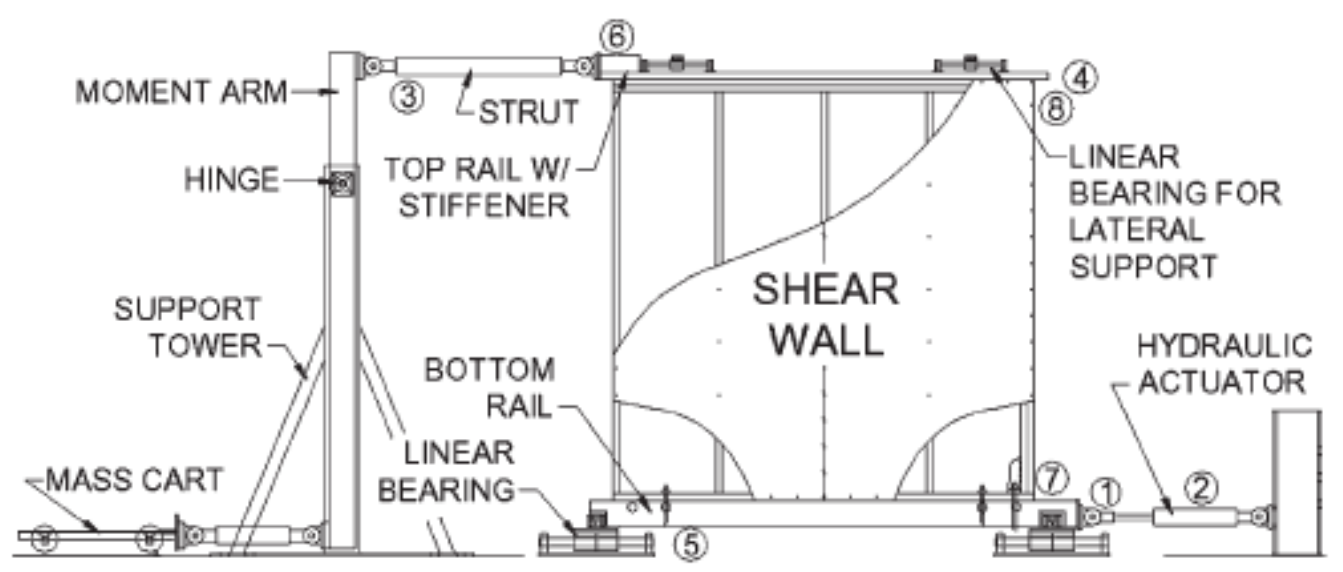

Figure 2.18 - Dynamic testing frame with locations of transducers for cyclic shear wall test (Seaders et al., 2009)

Serrette and Nolan (2009) performed an experimental program to evaluate the performance coldformed steel frame shear walls sheathed with wood panels. The pneumatic driven steel pins were used to attach wood panels. Several valuable characteristics were selected to compare overall 
performance of specific pin-type walls with other shear wall configurations. The results of a series of seven CUREE-based reversed cyclic tests of cold-formed steel shear walls with pneumatically driven steel pins attached to wood structural panels were presented.

The series of tests included different steel thicknesses, sheathing types, fastener schedules, and stud on-center distances. The displacement capacity of the walls was significant according to the test results. The drift requirements in the building code govern the design values of tests. A set of parameters that related to pre- and post-peak response of the wall represent of the overall performance of the tested walls. The average ratio of nominal strength to Allowable Strength Design (ASD) strength of the tested walls was 2.76. The average peak strength to ASD strength was 2.88 . The maximum usable wall displacement was approximately 1.38 times the peak strength displacement and the average peak strength to ASD strength displacement was 9.29. Results showed that shear walls were qualified as high-deformability elements in accordance with the deformability classification provisions in ASCE/SEI 7 (ASCE, 2010).

Memari et al. (2009) studied the monotonic and cyclic loading performance of wood-stud and steel-stud wall specimens is studied through experiments. Both faces of the walls were sheathed with gypsum wall board (GWB). The joint compound at GWB joints and over screw heads were used in the specimens. Their research provided data on load-displacement relationship under monotonic and cyclic hysteresis. Several aspects of failure modes were distinguished based on the visual inspection of the specimens during the tests. The envelope curves of the hysteresis cycles provided the drift and shear-load capacity. The envelope curves of steel-stud and woodstud specimens were compared and the relative behaviors of these wall system including measures of ductility, capacity and energy dissipation were studied. Finally, the effect of GWB joints on the shear capacity of the wall was provided.

White et al. (2009) studied the earthquake performances of wood-frame shear walls under different loading conditions. The monotonic, cyclic, and earthquake loads were applied to fully and partially anchored walls and compared with code measures. The walls were made of Douglas-fir studs and 2440-mm square area. Two gypsum wallboard and two oriented strand board (OSB) were installed on both side of the walls. Two anchor bolts on the sill plate were 
used on partially-anchored walls. The hold-down anchors were also installed at the ends of fullyanchored walls. Figure 2.19 shows the schematic sketch of dynamic test frame which was used in this research. Four time histories were tested namely: three subduction zone ground motions and a strike-slip fault, all scaled to the Seattle design level. For fully-anchored walls, subduction zone tests had capacities, energy dissipation, and failure modes most similar to cyclic tests. Results showed that wall displacement at maximum load was underestimated by cyclic and overestimated by monotonic tests. For partially-anchored walls, subduction zone and strike-slip earthquake tests had capacity, displacement at maximum load, initial stiffness, and ductility most similar to cyclic tests. Energy dissipation was most similar to monotonic tests, and failure modes were consistent with monotonic and cyclic tests. Partially-anchored walls had lower capacity, displacement at maximum load, energy dissipation, and stiffness as compared with fullyanchored walls.

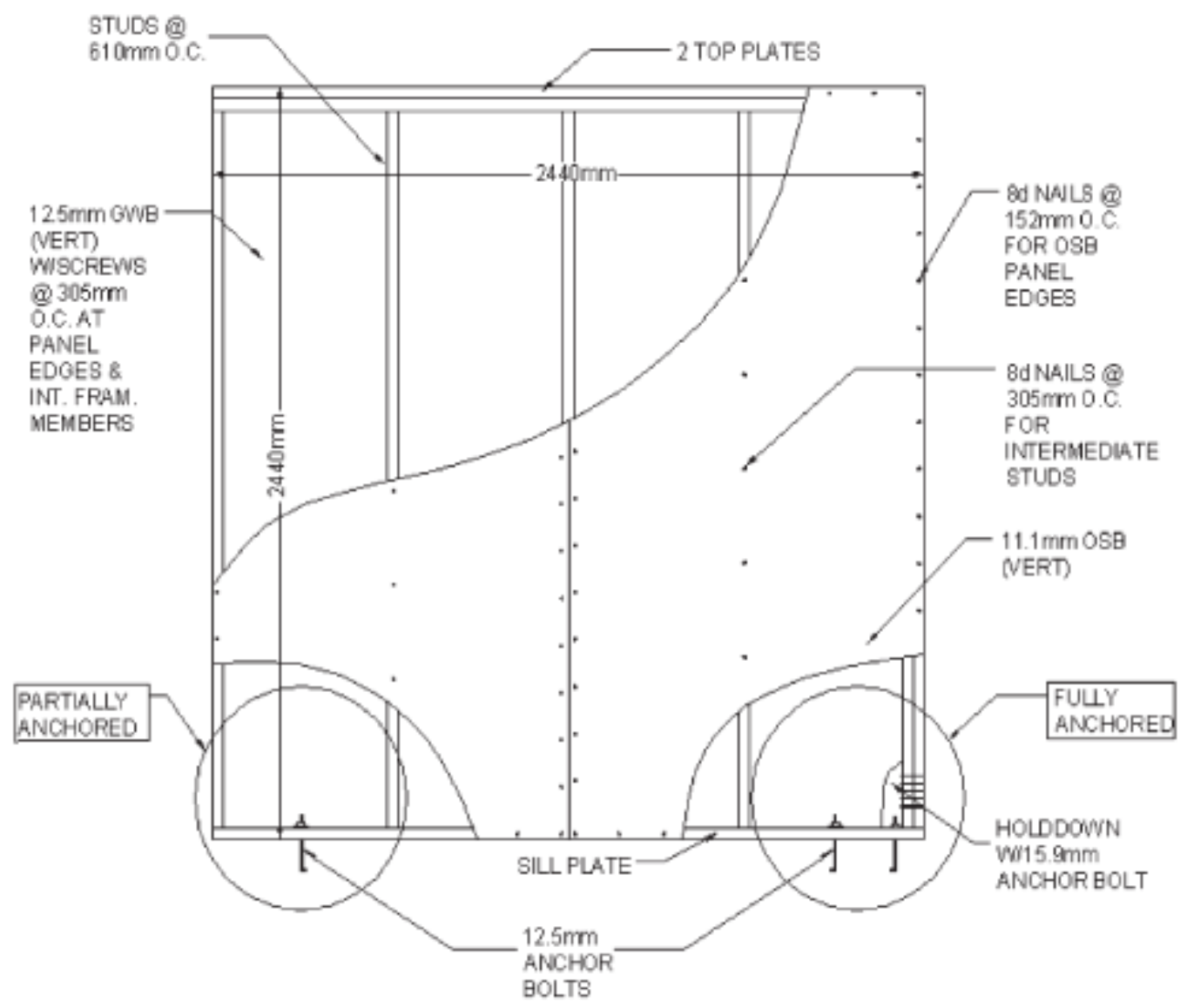

Figure 2.19 - Schematic sketch of shear wall test specimen (White et al., 2009) 
Moghimi and Ronagh (2009) performed an experimental test on full-scale cold-formed steel (CFS) strap-braced walls of $2400 \mathrm{~mm}$ length and $2400 \mathrm{~mm}$ height to examine their behavior. Cyclic loading was applied to twenty full-scale walls with different strap arrangements. Results showed that the performance of cold-formed steel frame shear walls for each arrangement was affected by several factors including brackets at four corners, straps, placing four $\mathrm{C}$-section cutoffs in the track at the four corners of the frame, and hold-downs inside the frame. Figure 2.20 shows the test setup of full-scale cold-formed steel (CFS) strap-braced wall in this research.

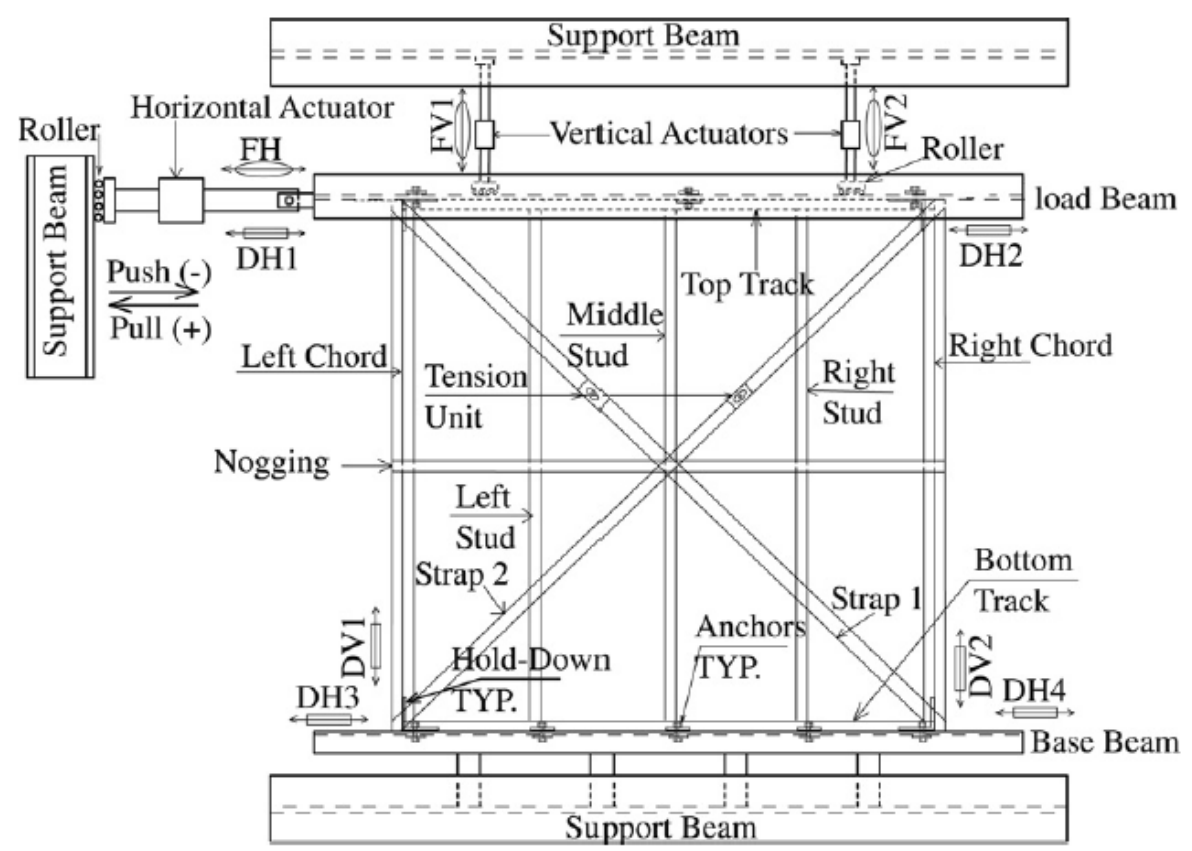

Figure 2.20 - Test setup of full-scale cold-formed steel strap-braced wall (Moghimi and Ronagh, 2009)

Trentiuk (2009) conducted 21 tests on $2400 \times 2400 \mathrm{~mm}$ SIP shear wall under monotonic and cyclic loading. The panels made of 87-mm thick expanded polystyrene (EPS) core and 11-mm thick (OSB) facings. He stated that the nail combination and OSB spline type had an effect on ductility, load carrying capacity, seismic capacity and resistance under fatigue loading. He also mentioned that the primary mode of failure happened in the fastener. The variation of spline type was observed to have no effect on wall performance. The results of monotonic tests were not conservative compared to those for cyclic loading. 
Balckwood (2009) studied on wood frame shear wall models using experimental testing. Five sets of experimental data and a 10-parameter hysteresis model were used to calibrate the models. The systematic scaling and the SAPWood Nail Pattern analysis tool were engaged to develop additional variations. The full-scale shear wall experimental data were compared with resultant hysteresis models. Songlai et al. (2010) conducted a series of tests on full-scale L-shape light wood frame under lateral load which was applied through gasbag. The research showed that shear wall spread out to each other and behaved as continuous shear wall. Test results showed that the light frame wood construction after repeated lateral cyclic load did not show any reduction in racking stiffness while discontinuous and larger amplitude cycling caused residual deformation and reduction in racking stiffness. The high racking resistance and rigidity of the floor diaphragm allowed the light frame wood building remain undamaged under ultimate lateral load. The drift restriction for lateral load design of the structures was recommended by inertstory percentage of the frame wood building. It was also used to calculate racking capacity of the frame with gypsum sheathings. The results of study were recommended to be used for engineering practice in light frame wood structures in seismic regions. Figure 2.21 shows the setup for diaphragm under lateral load as well as the L-shape walls.

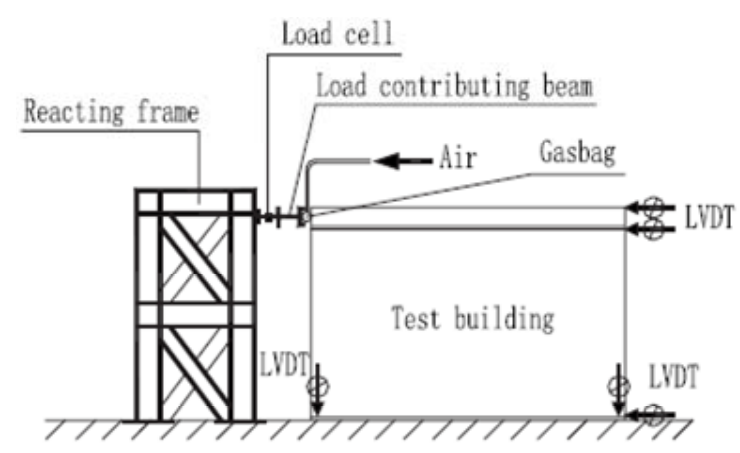

(a) The side of setup

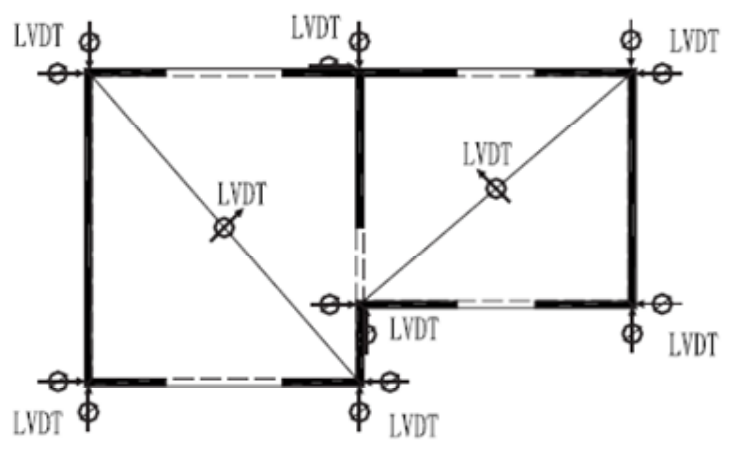

(b) The plan of setup

Figure 2.21 - Schematic elevation of the setup for diaphragm under lateral load as well as the Lshape walls (Songlai et al., 2010)

Mosalam and Gunay (2012) presented a research on seismic performance of energy-efficient structural insulated panels. This study included a test program of hybrid simulation and cyclic tests on seven SIPs and a conventional wood panel. The force-displacement results and local 
displacement measurements were presented in this research. The comparison of SIPs and conventional wood panels was done using heat transfer analyses. The results of this research showed the similarity in structural performance of conventional wood panels and tested SIPs. It was also mentioned the important effect of nail spacing on the response of SIPs. The hybrid simulation was also helped to evaluate the structural performance of SIPs. The superior thermal insulation of SIPs was observed in heat transfer analyses. However, vertical studs in conventional wood panels showed a negative effect on thermal insulation.

Huifeng et al. (2012) studied five half-size structural insulated panel shear walls under low cyclic horizontal loading. The panel consisted of plywood facing with polystyrene foam board core. Different wall depth-to-width ratios and opening size were selected for test specimens. The bearing capacity, failure modes, lateral stiffness, failure mechanics and ductility of each specimen were presented in detail. The test results indicated that the hysteresis curve of each

specimen had a reversed s-shape envelope. Also, the test results showed that the opening dimensions in shear wall and depth-to-width ratio had considerable effects on ductility, load bearing capacity and elastic stiffness. The fasteners slip behaviour of the spline connection along the bottom of specimens has a significant effect on performance of the SIP shear wall. The load bearing capacity decreased no more than $15 \%$ due to the opening in SIP shear wall. The ductility of the SIP shear wall with opening was observed to have no significant change, while a notable decrease in the elastic stiffness of the wall was observed. This study concluded that the performance of the studied SIP shear wall in timber structures was good in seismic region.

\subsection{Available Design Methods, Codes, and Guidelines}

The Structural qualifications of the SIPs have been assessed based on:

1- CSA Standard CAN/CSA-086-09, Engineering Design of Wood;

2- CSA Standard CAN/CSA-S406-92, Construction of Preserved Wood Foundations, (CSA, 1992) and the National Building Code of Canada (NBCC, 2005).

3- Acceptance Criteria for Sandwich Panels (ICC-AC04)

4- American Panel Association (ANSI/APA)

5- British Standard (BS) 
6- Uniform Building Code (UBC)

7- Sandwich Panel Concepts (Diab's Handbook)

8- The evaluation criteria set forth in the NRC/CCMC Technical Guide which focuses on SIPs as being "as good as" the conventional wood-frame buildings with respect to strength and serviceability.

The following section described the available methods and guidelines for SIPs and stud panels under different load conditions.

\subsubsection{CSA - Engineering Design of Wood (CAN/CSA-086-09)}

\subsubsection{Analytical Methods for Stressed Skin Panel (Stud Panel)}

\section{(a) General}

According to CAN/CSA-O86-09 (2010), a stressed skin panel shall have continuous or spliced longitudinal web members and continuous or spliced panel flanges on one or both panel faces, with the flanges glued to the web members as shown in Figure 2.22.

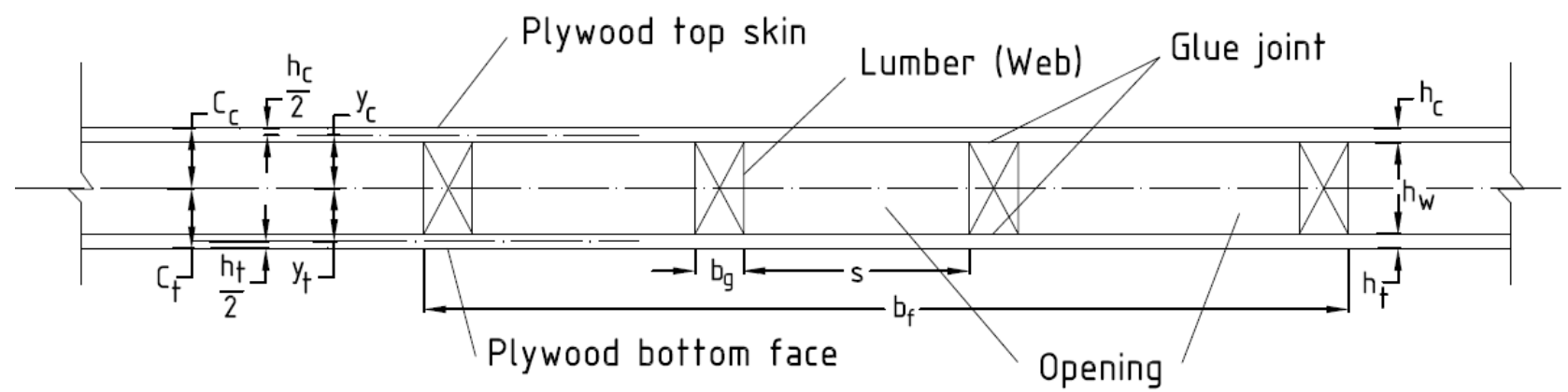

Figure 2.22 - Schematic diagram of a stressed skin panel dimensions (Adapted from CAN/CSA-

O86-09, 2010)

Flat panels with stressed plywood skins and spaced lumber stringers act like a series of built-up I-beams, with the plywood skins taking most of the bending stresses as well as performing as a sheathing function, while the lumber stringers take shear stresses. Since stress-skin panels are usually relatively shallow, any shear deformation between skins and webs would contribute materially to deflection. For maximum stiffness, therefore, a rigid connection is required 
between the plywood and the lumber. Thus, all panels considered in this design method are assumed to be assembled with glue (APA, 1996).

\section{(b) Effective Stiffness}

According to CAN/CSA-O86-09, the effective stiffness, $(E I)_{e}$, of a stressed skin panel shall be taken as follows:

$(E I)_{e}=(E I)_{w} K_{S E}+b_{f}\left(B_{a t} y_{t}{ }^{2}+B_{a c} y_{c}{ }^{2}\right) K_{S}$

where; $(E I)_{w}=$ stiffness of lumber webs, N.mm $; B_{a t}=$ specified axial stiffness of tension flange, $\mathrm{N} / \mathrm{mm}$ ( $B_{a}$ for appropriate panel thickness in Tables 7.3A to 7.3C of CSA-O86); $B_{a c}=$ specified axial stiffness of compression flange, $\mathrm{N} / \mathrm{mm}$ (Tables 7.3A to 7.3C of CSA-O86); $b_{f}, y_{t}, y_{c}=$ panel dimensions, mm, in accordance with Figure 2.22; $K_{S E}=$ service condition factor for modulus of elasticity; $K_{S}=$ service condition factor for sawn lumber, glued-laminated timber, plywood, construction sheathing OSB, poles, and piling (Clause 4.3.3 of CSA-O86).

\section{(c) Bending Resistance}

\section{(c.1) Bending along stressed skin panel span}

According to CAN/CSA-O86-09, the factored bending moment resistance, $M_{r}$, along the direction of the webs of a stressed skin panel shall be the least of the factored resistances of the tension or compression flanges or the web, taken as follows:

\section{(c.1.1) Tension flange:}

$M_{r}=\phi T_{p} X_{J} X_{G} \frac{(E I)_{e}}{B_{a} K_{S} c_{t}}$

where; $\phi=0.95$

$T_{p}=t_{p}\left(K_{D} K_{S} K_{T}\right)$

where; $t_{p}=$ specified strength capacity of flange in axial tension, $\mathrm{N} / \mathrm{mm}$ (Tables 7.3A to 7.3C); $X_{J}$ $=$ stress joint factor (Clause 8.3 of CSA-O86); $X_{G}=$ panel geometry reduction factor (Clause 8.6.3.2 of CSA-O86); $B_{a}=$ specified axial stiffness, $\mathrm{N} / \mathrm{mm}$ (Tables 7.3A to 7.3C of CSA-O86); $c_{t}$ $=$ distance from neutral axis to tension face; $K_{D}=$ load duration factor (Clause 4.3.2 and Table 4.3.2.2 of CSA-O86); $K_{S}=$ service condition factor for sawn lumber, glued-laminated timber, 
plywood, construction sheathing OSB, poles, and piling (Clause 4.3 .3 of CSA-O86); $K_{T}=$ treatment factor (Clause 4.3.4of CSA-O86)

\section{(c1.2) Compression flange}

$M_{r}=\phi P_{p} X_{J} X_{G} \frac{(E I)_{e}}{B_{a} K_{S} c_{c}}$

where; $\phi=0.95$

$P_{p}=p_{p}\left(K_{D} K_{S} K_{T}\right)$

where; $p_{p}=$ specified strength capacity of flange in axial compression, $\mathrm{N} / \mathrm{mm}$ (Tables $7.3 \mathrm{~A}$ to 7.3C of CSA-O86); $c_{c}=$ distance from neutral axis to compression face.

\section{(c1.3) Web}

$M_{r}=\phi F_{b} K_{z b} K_{L} X_{G} \frac{(E I)_{e}}{E K_{S E} c_{w}}$

where; $\phi=0.95 ; K_{S E}=$ service condition factor for modulus of elasticity of flange; $K_{L}=$ lateral stability factor for bending members (Clause 4.3.7 of CSA-O86); $K_{z b}=$ size factor for bending for sawn lumber.

$F_{b}=f_{b}\left(K_{D} K_{s b} K_{T} K_{H}\right)$

where; $f_{b}=$ specified strength in bending of webs, MPa (Tables 5.3.1A to 5.3.1D, 5.3.2 and 5.3.3 for swan lumber); $E=$ modulus of elasticity of web, MPa (Tables 5.3.1A to 5.3.1D, 5.3.2 and 5.3.3 for swan lumber); $c_{w}=$ greatest distance from neutral axis to outer edge of web, $\mathrm{mm} ; K_{H}=$ system factor (Clause 4.3.5 of CSA-O86).

\section{(c.2) Bending perpendicular to panel span}

According to CAN/CSA-O86-09, the factored bending resistance of the compression flange between web members shall be calculated using Clause 7.5.2. This clause stated that, the factored bending resistance, $M_{r}$, of a structural panel in the plane perpendicular to the plane of the panel shall be taken as follows:

$M_{r}=\phi M_{p} b_{p}$

where; $\phi=0.95$

$M p=m_{p}\left(K_{D} K_{S} K_{T} K_{F}\right) \quad$ for plywood 


$$
=m_{p}\left(K_{D} K_{S} K_{T}\right) \quad \text { for OSB }
$$

where; $m_{p}=$ specified strength capacity in bending, N.mm $/ \mathrm{mm}$ (Tables 7.3A and 7.3B for plywood and Table 7.3C for OSB in CSA-O86); $b_{p}=$ width of panel, $\mathrm{mm} ; K_{F}=$ foundation factor for plywood (Clause 7.4.5 of CSA-O86)

\section{(c.3) Buckling of Compression Flange}

According to CAN/CSA-O86-09, the compression flange of a stressed skin panel shall be designed according to principles of engineering mechanics to prevent elastic buckling failure. If a detailed analysis is not made, such a condition shall be assumed to be met if

(a) $s \leq 50 h_{c}$ for panels with their major axis parallel to the span $\left(\ell_{p}\right)$; or (b) $s \leq 40 h_{c}$ for panels with their major axis perpendicular to the $\operatorname{span}\left(\ell_{p}\right)$

where $s=$ clearance distance between stringers as shown in Figure 2.22; $h_{c}=$ thickness of top skin as shown in Figure 2.22; $\ell_{p}=$ span of stressed skin panel, $\mathrm{mm}$.

\section{(d) Shear Resistance}

\section{(d.1) Shear in plane of plies}

According to CAN/CSA-O86-09, the factored planar shear resistance, $V_{r}$, of the compression flange in a stressed skin panel shall be calculated in accordance with Clause 8.6.3.6. The factored shear resistance at the neutral plane of a stressed skin panel shall be taken as follows:

$$
V_{r}=\phi F_{v} K_{N} K_{Z v} \frac{(E I)_{e} \sum b_{g}}{E K_{S E} \sum Q_{w}+B_{a} K_{S} b_{f} y}
$$

where; $\phi=0.9$

$F_{v}=f_{v}\left(K_{D} K_{s v} K_{T} K_{H}\right)$

Where; $f_{v}=$ specified strength in shear of webs, MPa (Tables 5.3.1 and 5.3.2 of CSA-O86 for swan lumber); $K_{N}=$ notch factor (Clauses 5.5.5.4 and 6.5.7.2.2 of CSA-O86); $K_{Z v}=$ size factor in shear (Clauses 5.4.5 of CSA-O86); $b_{g}=$ contact width between flange and web (Figure 2.22); $E=$ modulus of elasticity of web, MPa (Tables 5.3.1A to 5.3.1.D, 5.3.2 and 5.3.3 of CSA-O86 for 
swan lumber); $\Sigma Q_{W}=$ sum of moments of area of all webs about neutral plane, $\mathrm{mm}^{3} ; B_{a}=$ specified axial stiffness, $\mathrm{N} / \mathrm{mm}$ (Tables 7.3A to 7.3C of CSA-O86); $b_{f}=$ width of flange, $\mathrm{mm} ; y=$ the greater value of $y_{t}$ or $y_{c}, \mathrm{~mm}$.

\section{(d.2) Flange-web shear}

According to CAN/CSA-O86-09, the factored shear resistance, $V_{r p}$, of the glued area between the flange and the web of a stressed skin panel shall be taken as the lesser of the shear capacities based on flange or web components, taken as follows:

$V_{r p}=\phi V_{g} \frac{(E I)_{e} \sum\left(b_{g} X_{v}\right)}{B_{a} K_{S} b_{p} y}$

Where; $B_{a}=$ specified axial stiffness, $\mathrm{N} / \mathrm{mm}$ (Tables 7.3A to 7.3C of CSA-O86); $y=$ the greater value of $y_{t}$ or $y_{c}, \mathrm{~mm} ; X_{v}=$ shear modification factor (Figure 8.5.5 of CSA-O86).

\section{(d.2.1) For flange:}

$\phi=0.95$

$V_{g}=v_{p f}\left(K_{D} K_{s} K_{T}\right)$

Where; $v_{p f}=$ specified strength capacity in planar shear, MPa (Tables 7.3A to 7.3C of CSA-O86).

\section{(d.2.2) For web:}

$\phi=0.9$

$V_{g}=f_{v}\left(K_{D} K_{s} K_{T}\right)$

Where; $f_{v}=$ specified strength in shear, MPa (Clauses 5.3.1 and 5.3.2 for swan lumber in CSAO86); $X_{v}=2.00$.

\section{(e) Combined Axial Load and Moment Resistance}

According to CAN/CSA-O86-09, the effect of axial load and moment on Swan Lumber and Glued-laminated timber (Glulam) is described in clause 5.5.10 and 6.5.12, respectively.

\section{(e.1) Swan Lumber}

According to CAN/CSA-O86-09, members subject to combined bending and compressive or tensile axial loads shall be designed to satisfy the appropriate interaction equation as follows: 
$\left(\frac{P_{f}}{P_{r}}\right)^{2}+\frac{M_{f}}{M_{r}}\left[\frac{1}{1-\frac{P_{f}}{P_{E}}}\right] \leq 1$

or

$\frac{T_{f}}{T_{r}}+\frac{M_{f}}{M_{r}} \leq 1$

Where; $P_{f}=$ factored compressive axial load; $P_{r}=$ factored compressive load resistance parallel to grain calculated in accordance with Clause 5.5.6 of CSA-O86; $M_{f}=$ factored bending moment; $M_{r}=$ factored bending moment resistance calculated in accordance with Clause 5.5.4 of CSAO86;

$P_{E}=$ Euler buckling load in the plane of the applied moment

$$
=\frac{\pi^{2} E_{o 5} K_{S E} K_{T} I}{L_{e}^{2}}
$$

Where; $E_{05}=$ modulus of elasticity for design of compression members, $\mathrm{MPa}$, as specified in CSA-O86; $I=$ moment of inertia in the plane of the applied moment, $\mathrm{mm}^{4} ; L_{e}=$ effective length in the plane of the applied moment $=K_{e} L$

Where; $K_{e}=$ the effective length factor given in Clause A.5.5.6.1 of CSA-O86; $T_{f}=$ factored tensile axial load; $T_{r}=$ factored tensile load resistance parallel to grain calculated in accordance with Clause 5.5.9 of CSA-O86.

\section{(e.2) Glulam}

According to CAN/CSA-O86-09 (2010), members subject to combined bending and compressive or tensile axial loads shall be designed to satisfy the appropriate interaction equation as follows:

$\left(\frac{P_{f}}{P_{r}}\right)^{2}+\frac{M_{f}}{M_{r}}\left[\frac{1}{1-\frac{P_{f}}{P_{E}}}\right] \leq 1$

or 
$\frac{T_{f}}{T_{r}}+\frac{M_{f}}{M_{r}} \leq 1$

where; $P_{f}=$ factored compressive axial load; $P_{r}=$ factored compressive load resistance parallel to grain calculated in accordance with Clause 6.5.8.4 using

$F_{c b}=f_{c b}\left(K_{D} K_{H} K_{S c} K_{T}\right)$

$M_{f}=$ factored bending moment; $M_{r}=$ factored bending moment resistance calculated in accordance with Clause 6.5.6.5.1 of CSA-O86; $T_{f}=$ factored tensile axial load; $T_{r}=$ factored tensile load resistance parallel to grain calculated in accordance with Clause 6.5.11 of CSA-O86. $P_{E}=$ Euler buckling load in the plane of the applied moment

$$
=\frac{\pi^{2} E_{o 5} K_{S E} K_{T} I}{L_{e}^{2}}
$$

where; $L_{e}=K_{e} L$ and $K_{e}=$ the effective length factor given in Clause A.5.5.6.1 of CSA-O86.

\section{(f) Deflection}

\section{(f.1) Deflection under Transverse Loading}

According to CAN/CSA-O86-09, the deflection of stressed skin panels shall be calculated using the effective stiffness, $(E I)_{e}$, determined in accordance with Clause 8.6.2 (Section 5.2.2), multiplied by the panel geometry reduction factor, $X_{G}$, determined in accordance with Clause 8.6.3.2. This Clause specifies that the panel geometry reduction factor, $X_{G}$, shall be taken as follows:

$$
X_{G}=1-4.8\left(\frac{s}{\ell_{p}}\right)^{2}
$$

Where; $s=$ clear spacing between longitudinal, $\mathrm{mm} ; \ell_{p}=$ span of stressed skin panel, $\mathrm{mm}$

This formula accounts for shear lag and is valid for values of $\left(s / \ell_{p}\right)$ ranging from 0.05 to 0.25 . The displacement of a beam under two-point loads with simply-supported condition can be calculated from the following formula. Figure 2.23 shows the loads, shear and moment diagrams of the beam. 


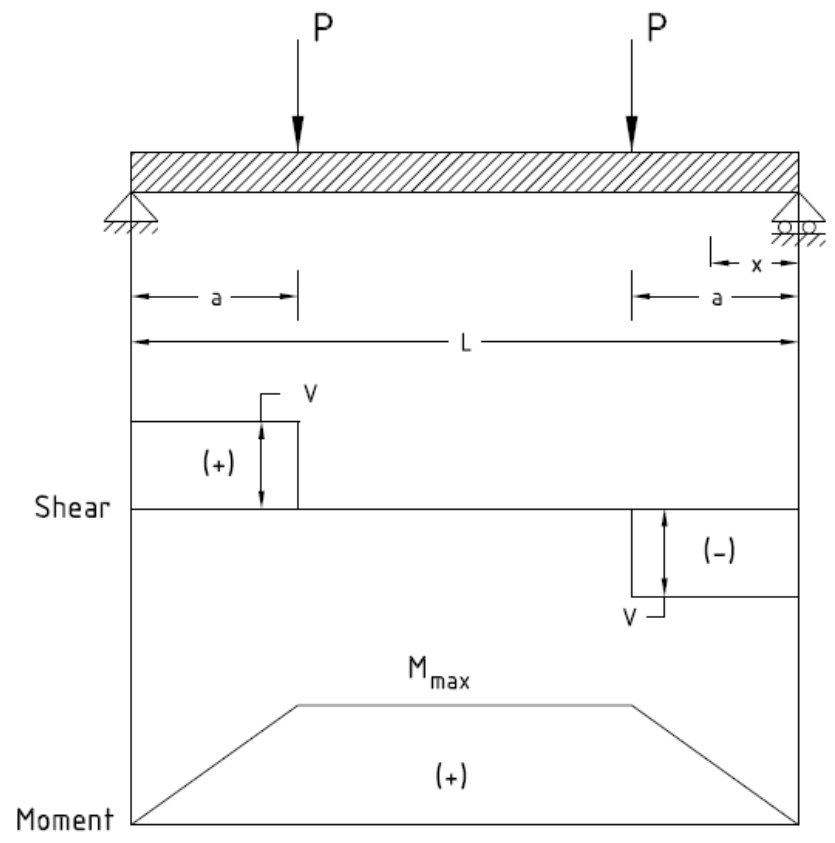

Figure 2.23 - Beam under two-point loads with simply-supported conditions

$\Delta_{\text {max.(center) }}=\frac{P a}{E I}\left(\frac{l^{2}}{8}-\frac{a^{2}}{6}\right)+\frac{P a}{G A^{\prime}}$

Where; $P=$ Load value; $a=$ distance from the edge of beam to the concentrated load location; $l=$ length of beam; $E=$ modulus of Elasticity of beam material; $G=$ shear modulus of beam material; $A^{\prime}=$ shear area of section.

\section{(f.2) Lateral Deflection under Axial Loading}

According to CAN/CSA-O86-09, the lateral deflection of stressed skin panels subjected to compressive axial loading shall be calculated based on Clause A.4.5.2. This clause limits the displacement to $1 / 180$ of the lengths for members subjected to dead, live, wind and snow loads. The permanent deflection of the system is also limited to $1 / 360$ of length.

The lateral deflection of the column with an eccentric load $(e=M / P)$ is calculated from following equation (Gramoll, 2010):

$$
v_{\max }=e\left(\sec \frac{K L}{2}-1\right)
$$


Where; $K=$ stiffness factor which is related to support condition; $L=$ initial length of column.

There is another equation for the calculation of lateral deflection of a column with eccentricity as follows (Beer et al., 2012):

$$
v_{\max }=e\left(\sec \left(\frac{\pi}{2} \sqrt{\frac{P}{P_{c r}}}\right)-1\right)
$$

where;

$P_{c r}=$ critical load $=\frac{\pi^{2} E I}{L_{e}{ }^{2}}$

Where; $L_{e}=$ effective length of column; $e=$ eccentricity of load;

\section{(f.3) Lateral Load Resisting Systems}

Clause 9 of CSA-O86-09 covers the design of structural systems and assemblies subjected to lateral loads, e.g., wind, seismic, or earth pressure loads. Clauses 9.3 to 9.5 apply to the materials specified in Clause 5 for lumber, Clause 6 for glued-laminated timber, Clause 7 for structural panels, and Clause 10 for fastenings. Clauses 9.3 to 9.5 also apply to OSB and wafer board manufactured to meet the requirements of CSA O437.0 and gypsum wallboard conforming to Type X (fire-rated) in ASTM C1396 (CAN/CSA-O86, 2010).

Design of shear walls and diaphragms can be performed using the following methods:

\section{(f.3.1) Standard Methods}

Provisions in Clause 9.3 apply to shear walls and diaphragms constructed with nailed shear panels using structural wood panels, gypsum wallboard, and diagonal lumber sheathing.

\section{(f.3.2) Alternative Methods}

Alternative methods of analysis using the factored lateral strength resistance of nails, spikes, or bolts to achieve ductility and the factored resistance of materials specified in this Standard may be used, provided that it can be demonstrated that such alternative methods of analysis are based on recognized principles of mechanics. 


\section{(f.3.3) Resistance to Overturning}

\section{- Shear wall segments with hold-downs}

Where the factored dead loads are not sufficient to prevent overturning, hold-down connections, shown in Figure 2.24a, shall be designed to resist the factored uplift forces and transfer the forces through a continuous load path to the foundation.

\section{- Shear wall segments without hold-downs}

Where the factored dead loads are not sufficient to prevent overturning and hold-down connections are not used, anchorage, shown in Figure 2.24b, on the bottom plate within $300 \mathrm{~mm}$ from both ends of the shear wall segment shall transfer the uplift force specified in Clause 9.4.5.2 of CSA-O86, to the supporting structure (i.e. to the top plate of the shear wall below or to the foundation).
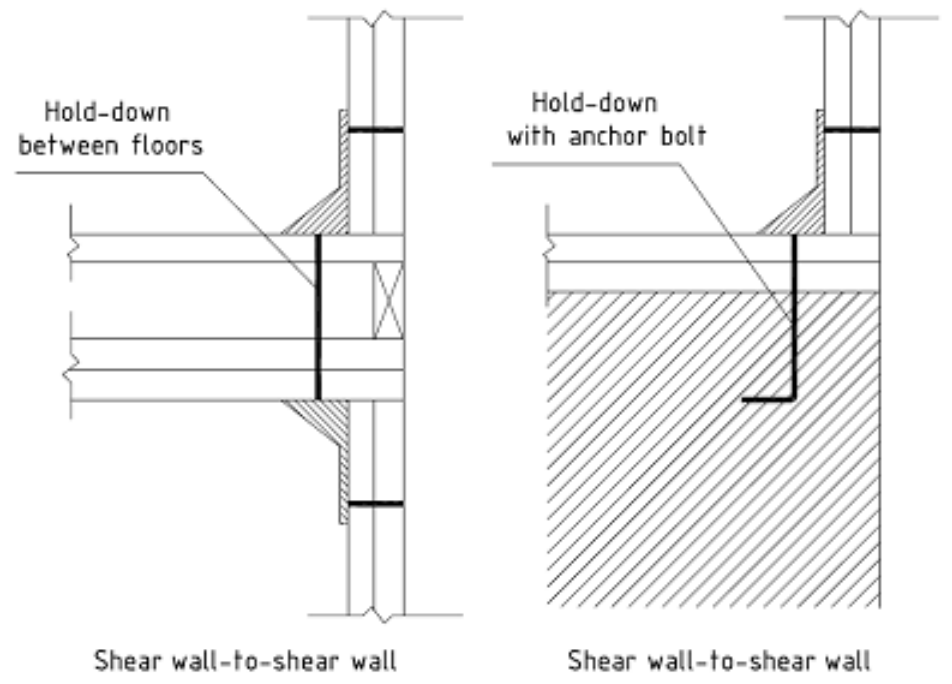

(a) Hold-downs 


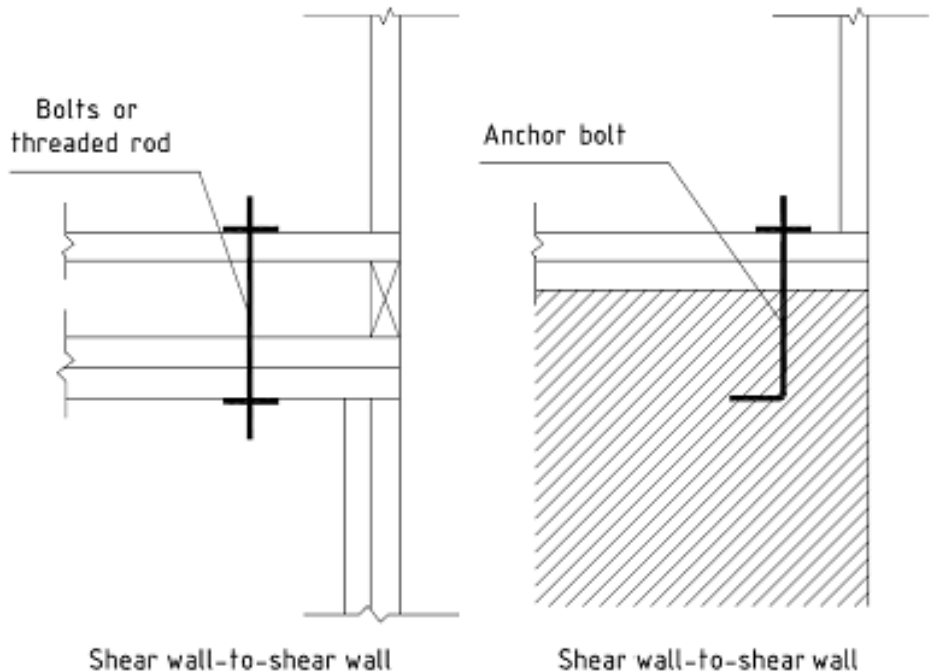

(b) Anchorages

Figure 2.24 - Examples of hold-downs and anchorages (Adapted from CWC, 2010)

\section{(g) Racking Deflection of Panel}

\section{(g.1) Blocked shear wall segments}

According to CAN/CSA O86-09, the static racking deflection at the top of the wall, $\Delta_{s w}$ in mm, of a blocked shear wall segment with wood-based panels constructed in accordance with Clauses 9.5.3 to 9.5.5 may be taken as follows:

$\Delta_{s w}=\frac{2 v H_{s}{ }^{3}}{3 E A L_{s}}+\frac{v H_{s}}{B_{v}}+0.0025 H_{s} e_{n}+\frac{H_{s}}{L_{s}} d_{a}$

Where; $v=$ maximum shear due to specified loads at the top of the wall, $\mathrm{N} / \mathrm{mm} ; H_{s}=$ height of shear wall segment, mm; $E$ = elastic modulus of boundary element (vertical member at shear wall segment boundary), $\mathrm{N} / \mathrm{mm}^{2} ; A=$ cross-sectional area of the boundary member, $\mathrm{mm}^{2} ; L_{s}=$ length of shear wall segment, $\mathrm{mm} ; B_{v}=$ shear-through-thickness rigidity of the sheathing, N/mm (Tables 7.3A, 7.3B, and 7.3C of CSA-O86); $e_{n}=$ nail deformation, mm (Clause A.9.7 of CSAO86); $d_{a}=$ total vertical elongation of the wall anchorage system (including fastener slip, device elongation, anchor or rod elongation, etc.) at the induced shear load.

For shear wall segments without hold-downs, the total vertical elongation, $d_{a}$, may be taken as follows: 
$d_{a}=2.5 d_{F} K_{m}\left[\frac{\left(v H_{s}-P_{i j}\right) \frac{s_{n}}{L_{s}}}{n_{u}}\right]^{1.7}$

Where; $d_{F}=$ nail diameter, $\mathrm{mm} ; K_{m}=$ service creep factor (Table A.10.9.3.2 of CSA-O86); $v=$ maximum shear due to specified load at the top of the wall, $\mathrm{N} / \mathrm{mm} ; H_{s}=$ height of shear wall segment, mm; $P_{i j}=$ specified uplift restraint force for storey I at the bottom of the end stud of a shear wall segment $\mathrm{j}, \mathrm{N} ; s_{n}=$ nail spacing around panel edge, $\mathrm{mm} ; L_{s}=$ length of shear wall segment, $\mathrm{mm} ; n_{u}=$ unit lateral nail strength resistance, $\mathrm{N}$ (Clause 10.9.4 of CSA-O86)

\section{(i.2) Unblocked shear wall segments}

The racking deflection of unblocked shear wall segments with wood-based panels, $\Delta_{u b}$, may be taken as follows:

$$
\Delta_{u b}=\frac{\Delta_{s w}}{J_{u b}}
$$

Where; $\Delta_{s w}=$ deflection of a blocked shear wall segment with $600 \mathrm{~mm}$ stud spacing and nails spaced at $150 \mathrm{~mm}$ on centre around panel edges and $300 \mathrm{~mm}$ on centre along intermediate framing members, calculated in accordance with Clause 9.7.1.1 of CSA-O86; $\mathrm{J}_{\mathrm{ub}}=$ adjustment factor for unblocked shear wall segment (Table 9.4.4 of CSA-O86)

\subsubsection{Construction of Preserved Wood Foundations, CSA-S406, and National Building Code of Canada, NBCC}

Based on NBCC and CAN/CSA-S406, the following loads and load factors can be used to examine the structural adequacy of the panels for serviceability and ultimate limit states design: dead load factor $=1.25$; live load factor $=1.50$; dead load for roofs $=0.5 \mathrm{kPa}$; dead load for floors $=0.47 \mathrm{kPa}$; the intensity of the triangular lateral soil pressure $=4.7 \mathrm{kN} / \mathrm{m}^{2}$; live load for residential construction $=1.9 \mathrm{kPa}$; snow load for residential construction $=$ calculated based on location if the building is in Canada; deflection limit for serviceability (live load effect) = span / 360; deflection limit for serviceability (total load effect: dead and live loads) $=\operatorname{span} / 180$; deflection limit for serviceability (column drift) $=$ span/180. 


\subsubsection{Acceptance Criteria for Sandwich Panels (ICC-AC04)}

The characteristic deflection and ultimate load carrying capacity of each SIP panel are basically the average of three identical panels tested experimentally in accordance with test criteria set forth in this standard. Further, when the results of one of the tested panels vary more than $15 \%$ from the average of the three panels, one of the following two actions can be chosen: (i) the smallest value of those for the three panels; or (ii) the average of results for a minimum of five identical panels. Furthermore, the average of two tests can be used when the higher value is not greater than $5 \%$ of lower value and the lower value will be used with obligatory safety factor.

The safety factor of SIP under ultimate load carrying capacity is depends on followings: (i) the range of test results, (ii) consistency of materials, and (iii) the load-deformation characteristics of the panel. AC04 provides factor of safety for uniform transverse loading as follows:

F.S. $=3.0 \quad$ for ultimate load at shear failure for all loading conditions.

F.S. $=2.5$ for ultimate reaction at failure for all loading conditions.

F.S. $=2.5 \quad$ for ultimate load determined by bending (facing buckling) failure under allowable snow loads.

F.S. $=2.0 \quad$ for ultimate load determined by bending (facing buckling) failure under allowable live loads up to $0.958 \mathrm{kPa}$ (20 lb per square foot).

In case of wall panel axial load tests, AC04 standard specifies that an eccentricity of $1 / 6$ of the panel thickness is considered for a wall panel under axial loading. Also, AC04 specifies that the factored design resisting axial load is determined from the experimental axial load at a net axial deformation of $3.18 \mathrm{~mm}\left(1 / 8^{\prime \prime}\right)$ or the ultimate load divided by a factor of safety determined in accordance with those specified for transverse load testing mentioned above, whichever is lower.

The structural insulated panel (SIP) can be used in building when the building is located on seismic design categories D, E and F (ASCE, 2010). The manufacturer evaluation report has to demonstrate that the SIP can be used in these areas. Section R613 of International Residential Code, IRC, states that the SIP wall construction has to be limited in application when the building is located on seismic design categories A, B and C. However, section R301.1.3 of the 
International Residential Code (IRC) allows using SIP walls in a building which has acceptable engineering practice and formed of structural elements with perspective limits in the code. Also, Section R104.11 enables the use of SIP walls further than the limits which is mentioned in Section R613.2. Additional information from SIP manufacturer can fulfill the IRC Section 104.11 (SIPA, 2011).

The use of SIPs in seismic Design Categories D, E and F can be demonstrated through experimental testing in accordance with test and evaluation criteria established by a recognized agency such as ICC-ES or NTA (ASCE, 2010). The evaluation report of ICC-ES is published in fulfillment with ICC-ES AC04 Acceptance Criteria for Sandwich Panels. AC04 Appendix A, Section 4.5.1 states that shear walls made of structural insulated panels can be used for all seismic design categories used if the requirements of Appendix A are met. The IRC Section R104.11 is used as a base for Appendix A. The use of SIPs in Seismic Design Categories D, E, and $\mathrm{F}$ is also demonstrated in NTA IM 14 SEP01 evaluation report which is published by NTA. As a result, the use of SIP in seismic design categories D, E and F is recommended if the manufacturer achieves an acceptable code report from ICC-ES or NTA (SIPA, 2011).

\subsubsection{American Panel Association (ANSI/APA)}

\subsubsection{Trial Section}

The American Panel Association (APA) defines a "Trial Section" for flexural panel design. Due to the structural efficiency possible with stressed-skin panels, whereby relatively shallow panels prove adequate for strength, the design is likely to be controlled by the allowable deflection. As such the first aspect of the assumed section to be checked is deflection. Moment resistant requirement will be checked next, and shear resistance required is the last, since it is the least likely to control. Shear will, however, sometimes govern when one or both skins are thick and the panel span is short. Stressed-skin panels are designed by a "cut and try" method. A trial section must first be assumed and then checked its ability to do the intended job. The whole $1200-\mathrm{mm}$ wide panel is usually designed as a unit, in order to allow for edge conditions. The equations in the following sections are based on 1200-mm wide panels. They will require adjustment for any other panel width (APA, 1996). 


\subsubsection{Vertical load Formula}

According to APA - Supplement 3 (APA, 1996), few practical end joints for stressed-skin panels will provide any appreciable degree of fixity. Under vertical load, therefore, panels will behave as pin-ended columns. The pin-ended column equations reduce to:

$P_{a}=\frac{3.619 E I_{g}}{144 L^{2}} \quad$ or $\quad F_{C} A_{\|} \quad$ whichever $\quad$ is $\quad$ less

Where; $P_{a}=$ allowable axial load on the panel (lb); $E=$ appropriate modulus of elasticity (see below) (psi); $I_{g}=$ gross moment of inertia of panel about neutral axia (in $\left.{ }^{4}\right) ; L=$ unsupported vertical height of panel (ft); $F_{c}=$ allowable compressive stress (axial) for plywood skins (psi); $A_{\|}$ $=$ total vertical-grain material of stringers and skins $\left(\mathrm{in}^{2}\right)$

Where the modulus of elasticity of skins and stringers are nearly alike, the modulus of elasticity of skins may be used. Otherwise, $E I_{g}$ can be calculated as mentioned in APA standard for use in this equation.

\subsubsection{Combined Axial Load and Moment Resistance}

The American Panel Association Standard defines an interaction equation for the combination of bending and axial load. When designing wall panels subject to wind loads or any other panels where both bending and axial stresses are present, the following combined-load equation can be used.

$\frac{P / A}{F_{a}} \pm \frac{M / S}{F_{b}} \leq 1, \quad$ reduced to the following:

$\frac{P}{P_{a}} \pm \frac{M / S}{F_{C}} \leq 1.0$

Where; $P=$ applied axial load on the panel with combined loading (lb); $P_{a}=$ allowable axial load on the panel if axial load only existed (lb); $M=$ applied bending moment on the stressed-skin panel with combined loading (in-lb); $S=$ section modulus of stressed-skin panel (compression side $)=\mathrm{I}_{\mathrm{n}} / \mathrm{c}\left(\mathrm{in}^{3}\right) ; F_{C}=$ allowable stress in compression parallel to grain from Plywood Design Standard (PDS) Table 3 (psi). Values for $P_{a}$ and $F_{C}$ can be adjusted for duration of load as specified in Plywood Design Standard Section 3.3 (APA, 1997). 


\subsubsection{Shear Stress}

The applied shear stress in a structural insulated panel (SIP) is determined from the following formula:

$$
f_{V}=\frac{w L}{(h+c) 12} \leq F_{V}
$$

where; $w=$ normal uniform load; $L=$ span length; $h=$ panel depth; $c=$ core depth; $f_{v}=\operatorname{applied}$ shear stress in the core; $F_{V}=$ allowable shear stress in the core. In addition to the structural design, there are also numerous architectural details which must be considered, such as connections, joint details, and finishes (APA, 1993).

\subsubsection{Panel Design for Combined Loading}

The determination of core thickness is based on requirements for insulation in many cases instead of strength. Therefore, the following design method is used and will be checked for all possible failure modes under design load. This method is called as "Trial Section" and the following procedure should be done to check it (APA, 1993):

\section{(a) Find Approximate Plywood/OSB Area}

Having the design flexural uniform load, $w$, and axial load, $P$, the required parallel-grain plywood/OSB area is determined by following formula:

$$
A_{1}+A_{2}=\frac{P}{F_{C}}
$$

where; $A_{1}=$ area of outside skin; $A_{2}=$ area of inside skin; $F_{C}=$ allowable compressive stress in parallel plys of plywood.

\section{(b) Find Neutral Axis}

A typical section of a sandwich panel is shown in Figure 2.5. The neutral axis in a composite section can be found from following equation:

$$
y=\frac{A_{1}\left(h-\frac{t_{1}}{2}\right)+A_{2}\left(h-\frac{t_{2}}{2}\right)}{A_{1}+A_{2}}
$$




\section{(c) Find Moment of Inertia (I) and Section Modulus (S)}

The moment of inertia and section modulus for a sandwich panel section can be found from following equations, respectively:

$$
\begin{aligned}
& I=\frac{A_{1} A_{2}(h+c)^{2}}{4\left(A_{1}+A_{2}\right)} \\
& S_{1}=\frac{I}{h-y} \quad, \quad S_{2}=\frac{I}{y}
\end{aligned}
$$

\section{(d) Column Buckling}

In order to find the column buckling load, the Euler's formula can be used based on composite section characteristics as following:

$$
P_{c r}=\frac{\pi^{2} E I}{(12 L)^{2}\left[1+\frac{\pi^{2} E I}{(12 L)^{2} \times 6(h+c) G_{c}}\right]}
$$

\section{(e) Skin Buckling}

The skin buckling of the section can be found from following equation.

$$
\begin{aligned}
C_{c r} & =0.5 \sqrt[3]{E E_{c} G_{c}} \quad \text { for foam or balsa core } \\
C_{c r} & =0.82 \sqrt{E_{C} t / E_{c}} \quad \text { forhoneycomb core }
\end{aligned}
$$

The allowable stress should be approximated as $1 / 3 C_{c r}$ for design purpose. The skins are assumed to be flat.

\section{(f) Maximum Bending Stress}

The maximum bending of the panel stress includes the bending due to the axial load acting through the initial transverse load deflection, $\Delta_{\max }$, and is determined by following equation:

$$
f_{b \max }=\frac{1.5 w L^{2}+P \Delta_{\max }}{S_{1}}
$$




\section{(g) Maximum Combined Stress}

The maximum combined stress in sandwich panel will occur at mid-length or mid-height of the panel and can be calculated as the sum of the applied axial stress and the compressive bending stress in the concave side of the panel.

$$
f_{c \max }=\frac{P}{A_{1}+A_{2}}+f_{b \max }
$$

APA specifies that the maximum combined stress $\left(f_{c \max }\right)$ must be less than $F_{C}$, and less than $1 / 3$ $\mathrm{C}_{\mathrm{cr}}$.

\subsubsection{Standard for Performance-Rated SIPs in Wall Applications}

ANSI/APA PRS 610.1 (APA, 2013) provides requirements and test methods for the qualification and quality assurance of performance-rated SIPs which are manufactured with a foam plastic insulation core bonded between two wood structural panel facings intended for use in wall applications. Table 2.5 shows a limited number of full-scale SIP tests. The performance of the component material specified in this standard is required to verify with SIP specimens. The performance of the SIP has been evaluated by full-scale testing in accordance to APA PRS 610.1 - Section 6. APA provided table 2.6 as the minimum performance required for SIPs evaluate using experiments or approved theoretical design method. SIPs shall meet or exceed these values listed in table 2.6 when the tests are done in accordance with methods mentioned in APA PRS 610.1 - Section 6.

Table 2.5 - SIP Confirmation Test Requirements

\begin{tabular}{|l|c|c|}
\hline \multicolumn{1}{|c|}{ Requirements } & $\begin{array}{c}\text { Minimum number } \\
\text { of tests }\end{array}$ & Test method \\
\hline Axial load & 3 & ASTM E 1803 \\
\hline Transverse load & 3 & ASTM E 1803 \\
\hline Racking resistance & 3 & ASTM E 1803 \\
\hline Bond strength - tension & 5 & ASTM D7446 \\
\hline Bond strength - shear & 5 & ASTM D7446 \\
\hline
\end{tabular}


Table 2.6 - Performance Requirements for SIP (APA, 2013)

\begin{tabular}{|c|c|c|c|c|c|c|}
\hline \multirow{3}{*}{ Property } & \multirow{3}{*}{$\begin{array}{c}\text { SIP configuration } \\
\text { (Thickness } \mathrm{x} \text { length } \mathrm{x} \\
\text { height) }\end{array}$} & \multirow{3}{*}{$\begin{array}{l}\text { Min. } \\
\text { No. } \\
\text { of } \\
\text { tests }\end{array}$} & \multirow{3}{*}{$\begin{array}{c}\text { Test } \\
\text { method }\end{array}$} & \multicolumn{3}{|c|}{ Minimum test value } \\
\hline & & & & \multirow{2}{*}{$\begin{array}{l}\text { Peak } \\
\text { load }\end{array}$} & \multicolumn{2}{|c|}{$\begin{array}{c}\text { Load at } \\
\text { deformation } \\
\text { limit }\end{array}$} \\
\hline & & & & & $\begin{array}{l}\text { Def. } \\
\text { limit } \\
\text { (in.) }\end{array}$ & $\begin{array}{l}\text { Test } \\
\text { load }\end{array}$ \\
\hline \multirow{4}{*}{$\begin{array}{c}\text { Axial load } \\
\text { capacity }\end{array}$} & $4-1 / 2$ in. $x 4 \mathrm{ft} \times 8 \mathrm{ft}$ & 3 & \multirow{4}{*}{$\begin{array}{l}\text { ASTM } \\
\text { E } 1803\end{array}$} & $\begin{array}{l}9,600 \\
\mathrm{lbf} / \mathrm{ft}\end{array}$ & 0.125 & $\begin{array}{l}3,200 \\
1 \mathrm{bf} / \mathrm{ft}\end{array}$ \\
\hline & $4-1 / 2$ in. $\times 4 \mathrm{ft} \times 8 \mathrm{ft}$ & 3 & & $\begin{array}{l}9,300 \\
\mathrm{lbf} / \mathrm{ft}\end{array}$ & 0.125 & $\begin{array}{l}3,100 \\
\mathrm{lbf} / \mathrm{ft}\end{array}$ \\
\hline & $6-1 / 2$ in. $x 4 \mathrm{ft} \times 10 \mathrm{ft}$ & 3 & & $\begin{array}{l}9,600 \\
\mathrm{lbf} / \mathrm{ft}\end{array}$ & 0.125 & $\begin{array}{c}3,200 \\
\mathrm{lbf} / \mathrm{ft}\end{array}$ \\
\hline & $6-1 / 2$ in. $x 4 \mathrm{ft} \times 10 \mathrm{ft}$ & 3 & & $\begin{array}{l}9,300 \\
\mathrm{lbf} / \mathrm{ft}\end{array}$ & 0.125 & $\begin{array}{l}3,100 \\
\mathrm{lbf} / \mathrm{ft}\end{array}$ \\
\hline \multirow{4}{*}{$\begin{array}{c}\text { Transverse } \\
\text { load } \\
\text { capacity }\end{array}$} & $4-1 / 2$ in. $x 4 \mathrm{ft} \times 8 \mathrm{ft}$ & 3 & \multirow{4}{*}{$\begin{array}{l}\text { ASTM } \\
\text { E } 1803\end{array}$} & $\begin{array}{c}85 \\
\mathrm{lbf} / \mathrm{ft}^{2}\end{array}$ & 0.400 & $28 \mathrm{lbf} / \mathrm{ft}^{2}$ \\
\hline & $4-1 / 2$ in. $x 4 \mathrm{ft} \times 8 \mathrm{ft}$ & 3 & & $\begin{array}{c}60 \\
\mathrm{lbf} / \mathrm{ft}^{2}\end{array}$ & 0.500 & $20 \mathrm{lbf} / \mathrm{ft}^{2}$ \\
\hline & $6-1 / 2$ in. $x 4 \mathrm{ft} \times 10 \mathrm{ft}$ & 3 & & $\begin{array}{c}85 \\
\mathrm{lbf} / \mathrm{ft}^{2}\end{array}$ & 0.400 & $28 \mathrm{lbf} / \mathrm{ft}^{2}$ \\
\hline & $6-1 / 2$ in. $x 4 \mathrm{ft} \times 10 \mathrm{ft}$ & 3 & & $\begin{array}{c}60 \\
\mathrm{lbf} / \mathrm{ft}^{2}\end{array}$ & 0.500 & $20 \mathrm{lbf} / \mathrm{ft}^{2}$ \\
\hline \multirow{4}{*}{$\begin{array}{l}\text { Racking } \\
\text { resistance }\end{array}$} & $4-1 / 2$ in. $x 4 \mathrm{ft} \times 8 \mathrm{ft}$ & 3 & \multirow{4}{*}{$\begin{array}{l}\text { ASTM } \\
\text { E } 1803\end{array}$} & \multirow{4}{*}{$\begin{array}{c}945 \\
\mathrm{lbf} / \mathrm{ft}\end{array}$} & מר & \multirow{4}{*}{$315 \mathrm{lbf} / \mathrm{ft}$} \\
\hline & $6-1 / 2$ in. $x 4 \mathrm{ft} \times 8 \mathrm{ft}$ & 3 & & & & \\
\hline & $4-1 / 2$ in. $x 4 \mathrm{ft} \times 10 \mathrm{ft}$ & 3 & & & $0 \Omega$ & \\
\hline & $6-1 / 2$ in. $\times 4 \mathrm{ft} \times 10 \mathrm{ft}$ & 3 & & & $0.2 J$ & \\
\hline
\end{tabular}




\subsubsection{British Standard (BS5268-6.2)}

\subsubsection{Determination of Racking Capacity}

According to BS 5268-6.2 (2001), resistance to horizontal wind forces (racking resistance) should be provided by stiffening elements in the plane of the wall. These should consist of timber frames that are sheathed with board materials or diagonally braced or constructed with moment connections. The racking strength and stiffness of timber frame wall panels should be determined by one of the methods described in following subsections:

\section{(a) Assessment method}

The basic racking resistances given in Table 2 of BS 5268-6.2 should be modified by application of material modification factors and wall modification factors as appropriate. The racking resistance of a wall should be calculated as follows:

Racking resistance of wall $=R_{b} L K_{m} K_{w}$

Where; $R_{b}=$ basic racking resistance in $k N / m$, Table 2 of BS 5268-6.2; $L=$ wall length in meters (m); $K_{m}=$ product of the material modification factors, $K_{201} K_{202} K_{203} ; K_{w}=$ product of the wall modification factors, $K_{204} K_{205} K_{206} K_{207}$.

\section{(b) Load testing}

$2400 \times 2400 \mathrm{~mm}$ square panels should be tested in accordance with BS EN 594 (2011) and the results interpreted in accordance with clauses of BS 5268-6.1 (2001), clause 5 to find the basic test racking resistance of a particular combination of materials and construction. In all respects, the panel should be representative of the construction to be used in the design. The basic test racking resistance values derived from load testing should be substituted for the values given in Table 2 of BS 5268-6.2 and modified by the wall modification factors described in section 6.9. As load testing refers to a specific combination of materials and their fixings, the material modification factors given in section 6.8 of BS standard (i.e. $K_{201}, K_{202}$ and $K_{203}$ ) should not be applied to basic test racking resistance. The racking resistance of a wall should be calculated as following: 
Racking resistance of wall $=R_{b} L K_{w}$

Where; $R_{b}=$ basic test racking resistance in $\mathrm{kN} / \mathrm{m}$ as derived from load testing; $L=$ wall length in meters.

The additional contribution values of a secondary layer of category 1, 2 or 3 materials as provided in Table 2 of BS 5268-6.2 should only be used where the basic test racking resistance of the primary board material does not exceed $2.1 \mathrm{kN} / \mathrm{m}$. In all other cases, the additional contribution should be quantified by load testing the primary board material.

\section{(c) Load testing of full-sized walls}

The walls should be tested in the form in which they are to be used, the permissible racking resistance for the wall derived in accordance with BS EN 594 and the results interpreted in accordance with Clause 5 of BS 5268-6.1 (2001). Material and wall modification factors ( $K_{201}$ to $\left.K_{207}\right)$ should not be applied to wall racking test data derived in this manner.

\section{(d) Detailed analytical methods outside the scope of this British Standard}

The material modification factors given in section 4.8 and wall modification factors given in section 4.9 of BS 5268-6.2 should not be applied to designs carried out independently of this British Standard.

\subsubsection{Determination of Racking Deflection}

According to BS 5268-6.2 (2001), the permissible racking deflection should be within limits appropriate to the type of construction, having particular regard to the possibility of damage to surface materials, ceilings, partitions, doors, windows and finishing. The basic racking resistances given in Table 2 of BS 5268-6.2 are based upon a maximum deflection limit of 0.003 times the panel height. Although it is acceptable to reduce the values given in this table in respect of a smaller deflection limit, it is not acceptable to increase the values given in the table to take account of a higher limit. 


\subsubsection{Uniform Building Code (UBC 23-2, 1997)}

\subsubsection{Deflection of shear wall}

The UBC 23-2 (1997) limits the amount of shear wall deflection for serviceability reasons, for example sufficient to limit cracking in wall coverings. When the building's fundamental period is less than 0.7 second, which is typical for wood buildings, UBC specifies that the maximum inelastic response displacement $\left(\Delta_{M}\right)$ is limited to 0.025 times the shear wall height. If the fundamental period is 0.7 second or greater, the limit on $\left(\Delta_{M}\right)$ reduces to 0.020 times the shear wall height. UBC defines $\Delta_{M}$ as a strength-level inelastic (non-recoverable) displacement that happens when the building is subjected to design earthquake ground motion. $\Delta_{M}$ is determined by amplifying the static storey drift $\left(\Delta_{s}\right)$, by the response modification factor (R) defined in UBC Table 16-N. The storey drift is also needed to determine if a diaphragm is considered flexible or rigid (UBC, 1997).

UBC Section 1630.9.1 requires strength-level load combinations to be used in shear wall deflection calculations, even when allowable stress design (ASD) is being used. The reason for this is that the Code limiting shear wall deflection is in terms of the maximum inelastic response displacement, which is defined at the strength level, not at service or allowable load levels. The complete set of strength-level load combinations are provided in UBC Section 1612.2, but those pertinent to shear wall deflections are dominated by either wind or seismic forces as follows:

$1.2 \mathrm{D}+1.3 \mathrm{~W}+\mathrm{f}_{1} \mathrm{~L}+0.5\left(\mathrm{~L}_{\mathrm{r}}\right.$ or $\left.\mathrm{S}\right)$

$1.2 \mathrm{D}+1.0 \mathrm{E}+\mathrm{f}_{1} \mathrm{~L}+\mathrm{f}_{2} \mathrm{~S}$

where $f_{1}=1.0$ for floors in places of public assembly, for live loads in excess of

$100 \mathrm{psf}$, and for garage live load

$=0.5$ for other live loads

$f_{2}=0.7$ for roof configurations that do not shed snow off the structure

(e.g., saw-tooth roof systems)

$=0.2$ for other roof configurations.

Other strength-level load combinations are provided in the UBC; However, these are the primary lateral load combinations to use for shear wall deflection calculations. Often, these combinations 
can be reduced to simpler expressions when no live, roof live or snow loads are present. UBC 23-2 provides a method for calculating the storey drift of a shear wall. The method accounts for bending and shear in the wall assembly, as well as nail deformation and anchorage slip as depicted in Figure 2.25. Such storey drift can be calculated as follows:

$\Delta_{s}=\Delta_{b}+\Delta_{v}+\Delta_{n}+\Delta_{a}$

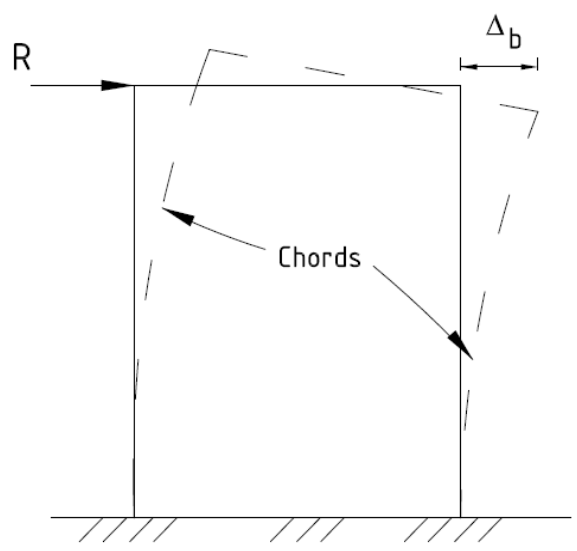

(a)

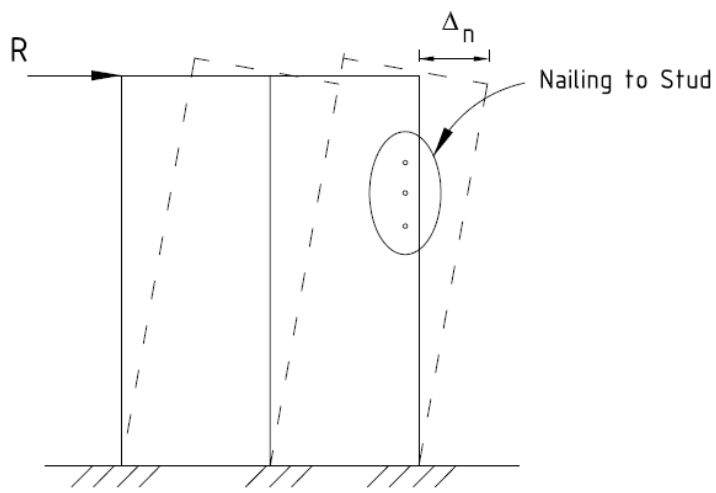

(c)

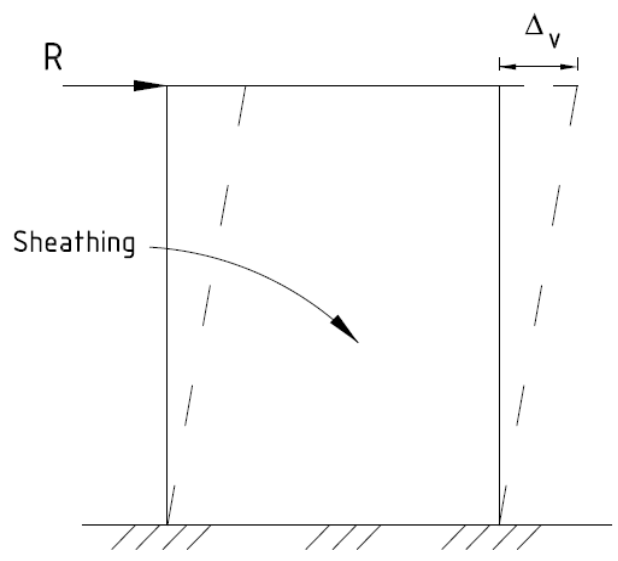

(b)

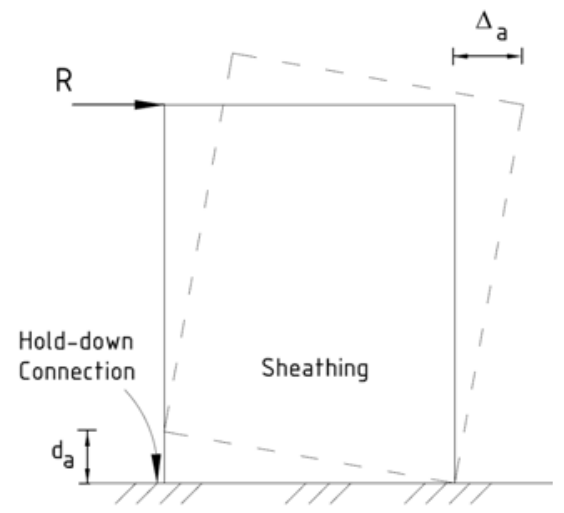

(d)

Figure 2.25 - Deflection component of a shear wall

(a) Bending deformation with lateral deflection $\Delta_{\mathrm{b}}$. (b) Shear deformation with lateral deflection $\Delta_{\mathrm{v}}$. (c) Nail slip deflection $\Delta_{\mathrm{n}}$. (d) Anchorage slip deflection $\Delta_{\mathrm{a}}$ (Adapted from UBC, 1997)

Where; $\Delta_{b}=$ bending deflection of the shear wall; $\Delta_{v}=$ shear deflection of the shear wall; $\Delta_{n}=$ deflection of the shear wall due to nail slips (deformation); $\Delta_{a}=$ deflection of the shear wall due to anchorage slip and rotation. 
A shear wall acts similar to a cantilevered I-beam. The chords resist the moment analogous to the flanges of an I-beam, and axial elongation and shortening of the chords causes deflection. Therefore, the bending rigidity of a shear wall is related to the axial rigidity of the chords EA. The bending deflection of a shear wall is then determined from

$$
\Delta_{b}=\frac{8 v h^{3}}{E A b}
$$

Where; $v=$ shear force at the top of the wall $(\mathrm{lb} / \mathrm{ft}) ; h=$ height of the wall $(\mathrm{ft}) ; b=$ width of the wall (ft); $E=$ modulus of elasticity of the chord (psi);A= cross-sectional area of chord (in. ${ }^{2}$.

While the chords resist the bending moment, the sheathing in a shear wall acts like the web of an I-beam and resists the applied shear. The shear deflection is then related to the shear rigidity $(G)$ of the sheathing:

$$
\Delta_{v}=\frac{v h}{G t}
$$

where; $G=$ modulus of rigidity of the wood structural panel sheathing in psi; $t=$ effective thickness of the wood structural panel sheathing in inch.

If the sheathing is rigidly attached to the studs and if the shear wall is rigidly attached to the foundation, the bending and shear terms $\Delta_{b}$ and $\Delta_{v}$, respectively, would account for all the shear wall deflection. Neither of these conditions exist in typical wood shear walls, and therefore the two additional deflection terms $\Delta_{n}$ and $\Delta_{a}$ must be accounted for in design. The prediction of these deflections relies on experimental data and empirical relationships.

Slip in the nailed connections between the sheathing and framing members reduces the effectiveness of the sheathing in resisting shear by allowing individual sheathing panels to slip relative to each other. The deflection due to nail slip $\left(\Delta_{n}\right)$ is estimated from the relationship

$$
\Delta_{n}=\frac{3}{4} h e_{n}
$$

where; $\mathrm{e}_{n}=$ nail deformation (in.). 
The nail deformation $e_{n}$ is the slip resulting between the stud and the sheathing at the design load. It is dependent on fastener type, minimum penetration, maximum fastener load $V_{n}$, and whether green or dried (seasoned) lumber is used at the time of fabrication. Whether the lumber is initially green or dry, it is assumed that the shear wall will be dry in service and the lumber will dry in place (dry use). The nail deformation $e_{n}$ can be determined either from tables such as UBC Table 23-2-K or from the following equations:

For $6 \mathrm{~d}$ common nail (1-1/4 in. minimum penetration, $180 \mathrm{lb}$ maximum fastener load):

$$
\text { Green lumber (dry use) Dry (seasoned) lumber (dry use) }
$$

$$
e_{n}=\left(\frac{V_{n}}{434}\right)^{2.314} \quad e_{n}=\left(\frac{V_{n}}{456}\right)^{3.144}
$$

For $8 \mathrm{~d}$ common nail (1-7/16 in. minimum penetration, $220 \mathrm{lb}$ maximum fastener load):

Green lumber (dry use) Dry (seasoned) lumber (dry use)

$$
e_{n}=\left(\frac{V_{n}}{857}\right)^{1.869} \quad e_{n}=\left(\frac{V_{n}}{616}\right)^{3.018}
$$

For $10 \mathrm{~d}$ common nail (1-5/8 in. minimum penetration, $260 \mathrm{lb}$ maximum fastener load):

Green lumber (dry use) Dry (seasoned) lumber (dry use)

$$
e_{n}=\left(\frac{V_{n}}{979}\right)^{1.894} \quad e_{n}=\left(\frac{V_{n}}{769}\right)^{3.276}
$$

For 14-ga staple (1 to $2 \mathrm{in}$. minimum penetration, $140 \mathrm{lb}$ maximum fastener load):

Green lumber (dry use) Dry (seasoned) lumber (dry use)

$$
e_{n}=\left(\frac{V_{n}}{902}\right)^{1.464} \quad e_{n}=\left(\frac{V_{n}}{596}\right)^{1.999}
$$

For 14-ga staple ( 2 in. minimum penetration, $170 \mathrm{lb}$ maximum fastener load):

Green lumber (dry use) Dry (seasoned) lumber (dry use)

$$
e_{n}=\left(\frac{V_{n}}{674}\right)^{1.873} \quad e_{n}=\left(\frac{V_{n}}{461}\right)^{2.776}
$$


The fastener load $V_{n}$ is determined by dividing the maximum unit shear force $v$ by the number of nails per foot at the interior panel edges. The equations listed are for Structural I wood structural panels. If the sheathing is not structural, then the resulting nail deformation $e_{n}$ should be increased by 20 percent.

The values listed in UBC Table 23-2-K and those resulting from the use of the above equations are not in full agreement. UBC Table is generated from the equations given in different references and provided here for green lumber (dry use). If dry (seasoned) lumber is used, the footnote of Table 23-2-K indicates the value of $e_{n}$ should be decreased by 50 percent. By inspection, the dry (seasoned) lumber equations do not represent a 50 percent reduction of the green lumber equations. The approach used in the UBC is conservative and appropriate for design use.

Slip or rotation in the anchorage detail permits rigid body rotation of the shear wall. The calculation of the deflection of the shear wall due to slip or rotation in the anchorage detail $\Delta_{a}$ is similar to the calculation of the shear wall deflection due to nail slip. A design slip is estimated for the connection and the deflection of the shear wall is proportioned to the height-to-width ratio of the wall

$\Delta_{a}=\frac{h}{b} d_{a}$

where $d_{a}=$ anchorage slip (in.).

The anchorage slip $d_{a}$ is the total slip between the chord and the anchor bracket as well as the anchor bracket and the foundation at the design load. For many prefabricated anchor brackets, manufacturers provide information regarding slip. For example, $d_{a}=0.125 \mathrm{in}$. is a common value for low-slip anchors which are nailed or lag bolted to the chord.

In summary, the total story drift of a shear wall is calculated from following equation:

$$
\Delta_{s}=\Delta_{b}+\Delta_{v}+\Delta_{n}+\Delta_{a}=\frac{8 v h^{3}}{E A b}+\frac{v h}{G t}+\frac{3}{4} h e_{n}+\frac{h}{b} d_{a}
$$




\subsubsection{Anchorage for Panel Shear (UBC 1806.6)}

According to UBC 1806.6 (2000), the anchor bolt requirement can be determined by first assuming a size of anchor bolt and determining the allowable load per bolt. If the allowable load per anchor bolt is Z' (parallel-to-grain design value), and the total lateral force parallel to the

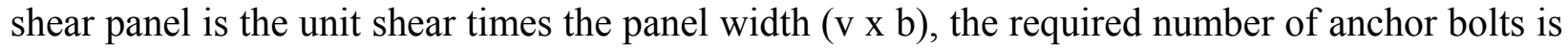
presented in following equation.

$$
N=\frac{v \times b}{Z^{\prime}}
$$

Figure 2.26 shows a separate set of anchor bolts which are provided for shear transfer. These anchor bolts are in addition to those provided for the chord uplift connections.

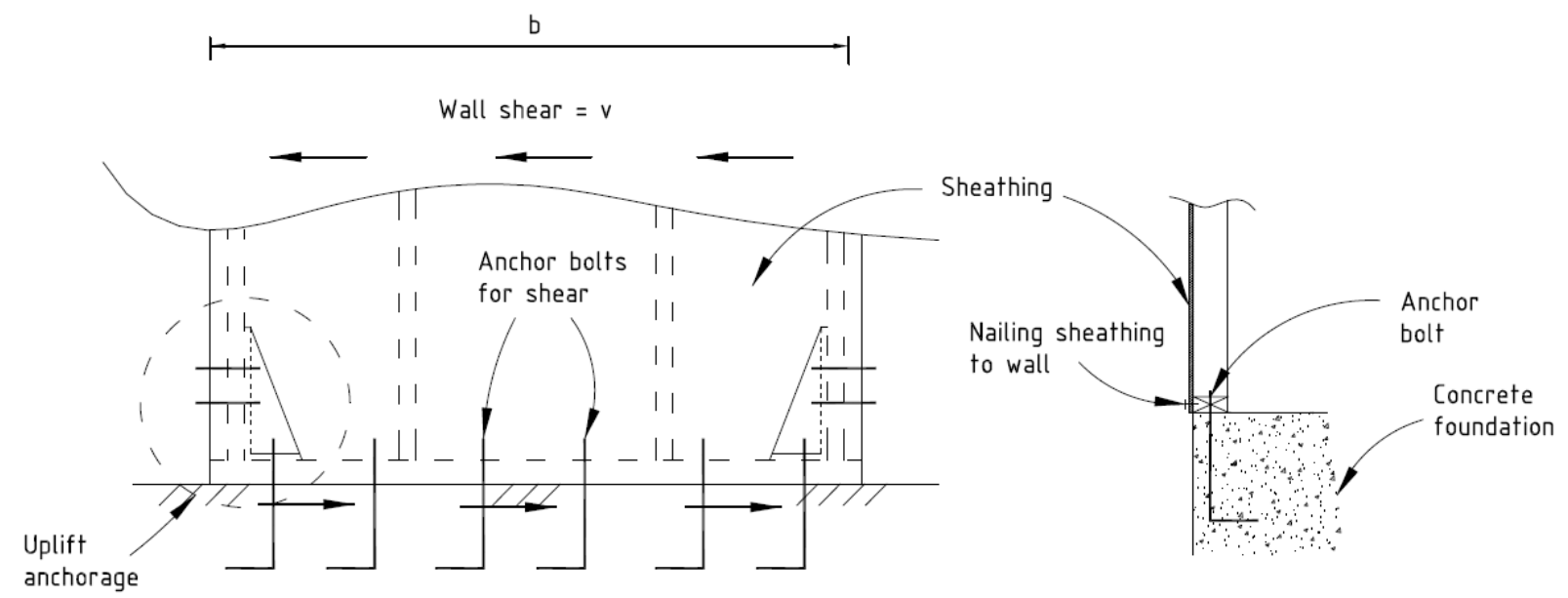

Figure 2.26 - Set of anchor bolts for shear transfer (Adapted from UBC, 2000)

The average spacing is approximately calculated from following equation:

Spacing $=\frac{b}{N}=\frac{b Z^{\prime}}{v b}=\frac{Z^{\prime}}{v}$

This spacing is approximate because starting and ending anchor bolts must be set in from the panel ends a sufficient distance to clear the chords and tie-down brackets. The code minimum anchor bolt requirement for wood-frame walls is given in UBC Chapter 18, Sec. 1806.6 as follows:

Seismic zones 0,1,2 and 3: $\quad$ 1/ 2-in. diameter anchor bolts at $6 \mathrm{ft}-0$ in. o.c.

Seismic zone 4:

5/ 8-in. diameter anchor bolts at $6 \mathrm{ft}-0$ in. o.c. 
Additionally, in seismic zones 3 and 4, a minimum $2 \times 2 \times 3 / 16$ inch thick steel plate washer must be used on each anchor bolt. A minimum of two anchor bolts is required per wall plate, and one bolt is required within 12 in. of the end of each plate piece. Anchor bolts for shear walls are usually larger and more closely spaced than the code minimum. 


\section{CHAPTER 3}

\section{EXPERIMENTAL PROGRAM}

\subsection{Introduction}

The SIPs which have been used in the experimental study, are produced by Thermapan Inc. (Thermapan, 2007) in standard sizes of $1.2 \mathrm{~m}$ wide and lengths of 2.43, 2.72, 3.05, 3.66, 4.27 and $4.90 \mathrm{~m}$. SIPs can be used in used for many different applications, such as interior and exterior walls, roofs, floors, foundations, timber frame, additions, and renovations. Thermapan SIPs are composed of thick layer of expanded polystyrene insulation (EPS) board laminated between two sheets of oriented strand board (OSB), as shown in Figure 3.1. The facing of these developed panels is made of two faces of Oriented Strand Board (OSB), $11 \mathrm{~mm}\left(7 / 16^{\prime \prime}\right)$ thickness, holding a foam core for floor and wall construction.

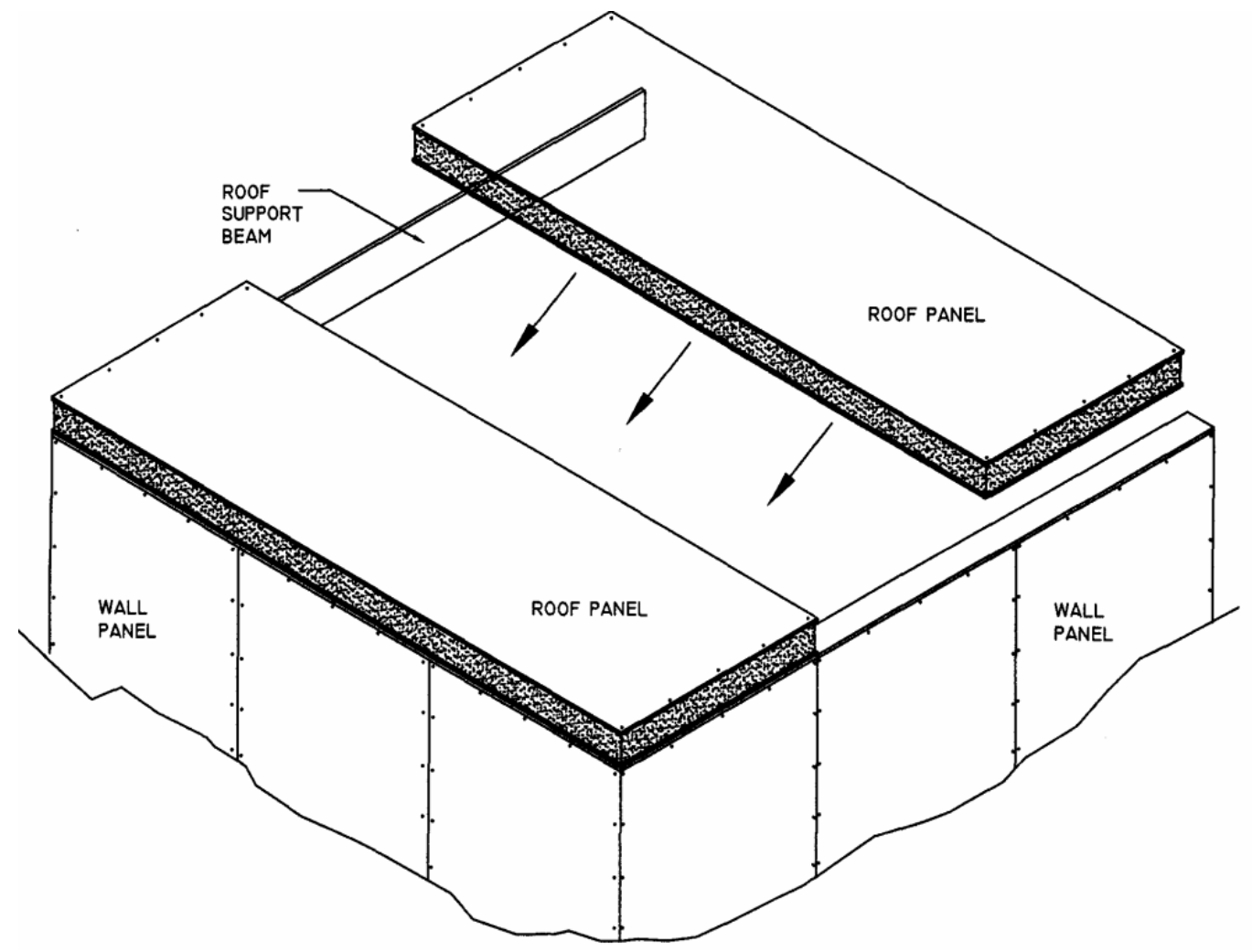

Figure 3.1 - Typical floor and basement wall construction using SIPs 
SIP floors and roofs are installed by placing the panels side by side as shown in Figure 3.1. The joint between the panels in the span direction can be either foam-spline connection or lumberspline connection. In the foam-spline connection, shown in Figure 3.2, a recess is formed in the foam core at the long edges of the panels. A foam block, with two OSB facings glued to it, is inserted at the edge of one panel. Then, the other panel is slide over the spline. The block OSB facings are then nailed to the OSB of the connected panels which provides structural integrity to the floor or wall. Figure 3.2 shows a typical section of foam-spline connection before and after assembly. The width of the insert for the foam-spline connection is usually half the width of the solid sawn lumber. It is preferred for roof construction to assist in energy efficiency. In case of lumber-spline joint, shown in Figure 3.3, a recess is formed along the longitudinal edges of the foam during manufacturing. After placing the panel over the wall, a sawn lumber is inserted in the recess along the panel length. Then, the adjacent panel slides over the sawn lumber, followed by nailing the OSB facings to the solid lumber. Figure 3.3 shows a schematic diagram of the solid lumber-spline connection before and after assembly.
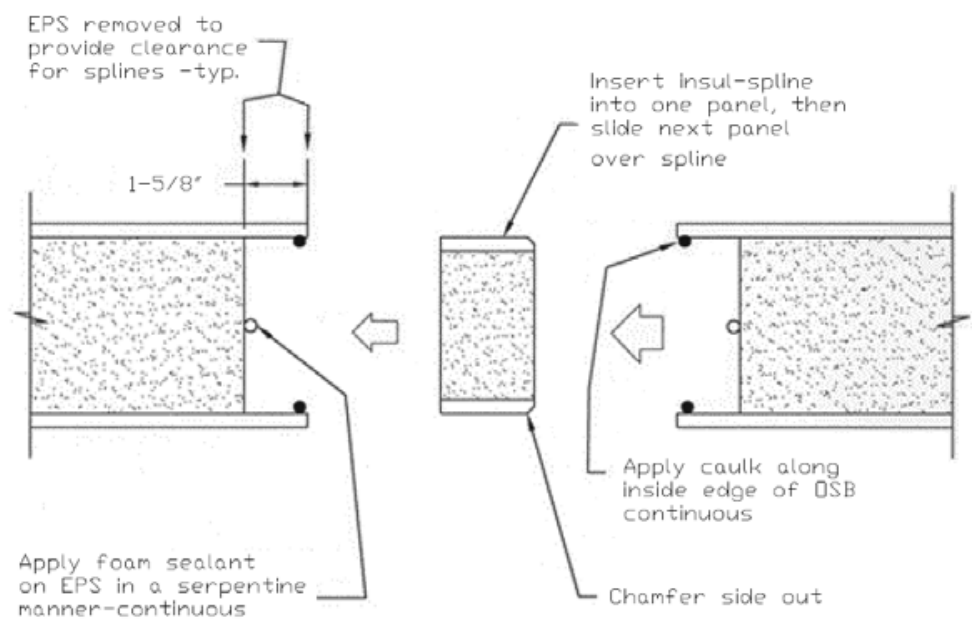

(a) Before assembly

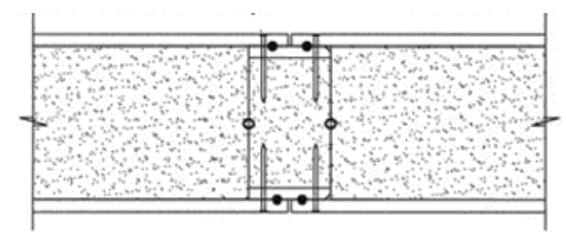

(b) After assembly

Figure 3.2 - Typical section at panel foam-spline connection before and after assembly 


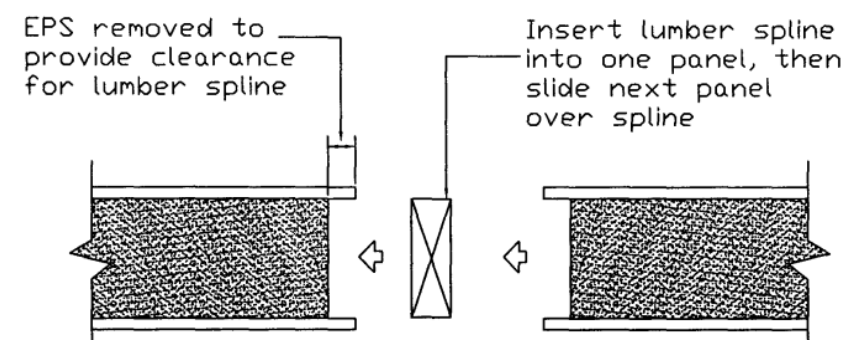

(a) Before assembly

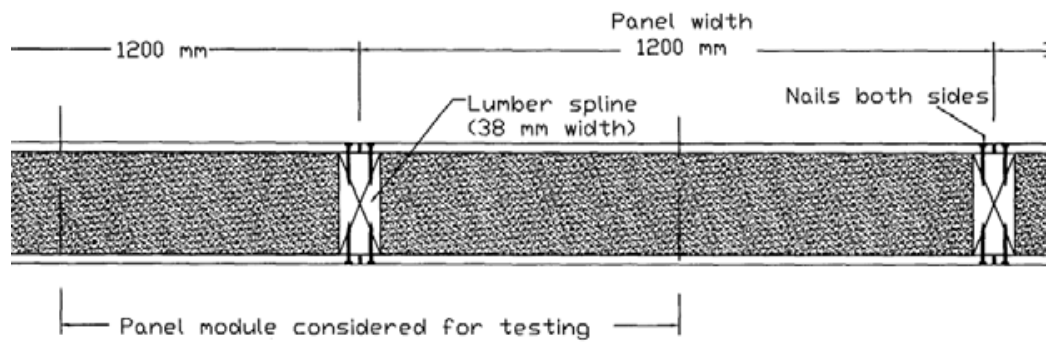

(b) After assembly

Figure 3.3 - Typical section at panel lumber-spline connection before and after assembly

This chapter is divided into the following sections:

1. Test matrix

2. Stud wall descriptions and material properties

3. SIP wall descriptions and material properties

4. Material properties by experimental testing

5. Equipments and instrumentation for testing

6. Testing SIP in flexure (transverse) loading

7. Testing SIP wall in axial compression loading

8. Testing SIP wall under racking and cyclic loading

9. Testing stud panel in flexure (transverse) loading

10. Testing stud wall in axial compression loading

11. Testing stud wall under racking and cyclic loading

\subsection{Test Matrix}

The experimental research program aimed at developing a better understanding of the structural behaviour of these timber sandwich panels at service and ultimate loading conditions when they 
act as walls in residential construction. Twenty eight tests were performed on different panel sizes at the structures laboratory of Ryerson University to provide experimental data that would then be evaluated for building code compliance. The tested specimens were divided into 4 groups based on the type of wall and loading conditions. These test groups were conducted on conventional stud wall systems and SIP wall systems of identical dimensions to qualify the SIP wall to be "as good as" the conventional wood-frame buildings with respect to (i) axial capacity and deformation, (ii) flexural capacity and deformation, an (iii) racking and cyclic load capacity and deformation. Tables 3.1 and 3.2 summarize the tested specimens for axial compressive loading and flexural loading, respectively. Tables 3.3 and 3.4 summarize the tested specimens for racking and cyclic loading, respectively. Three identical specimens were considered in each group to better present the structural performance per the test method. The following sections describe the specimen geometry and material properties in addition to the test method, test setup and test procedure.

Table 3.1 - Specimen details for axial compressive load tests

\begin{tabular}{|c|c|c|c|c|}
\hline Test No. & Test type & $\begin{array}{c}\text { Panel size } \\
(\mathbf{l} \times \mathbf{w} \times \mathbf{t}), \\
\text { mm (ft-inches) }\end{array}$ & $\begin{array}{c}\text { Thickness of } \\
\text { sheathing (OSB), } \\
\text { mm (inches) }\end{array}$ & Specimen type \\
\hline SW-A-1 & $\begin{array}{c}\text { Axial loading } \\
\text { (at } \mathrm{t} / 6)\end{array}$ & $\begin{array}{c}2450 \times 2750 \times 165 \mathrm{~mm} \\
\left(8^{\prime} \times 9^{\prime} \times 6.5^{\prime \prime}\right)\end{array}$ & $\begin{array}{l}11 \mathrm{~mm} \\
(7 / 16 ")\end{array}$ & $\begin{array}{c}\text { Conventional } \\
\text { stud panel }\end{array}$ \\
\hline SW-A-2 & $\begin{array}{l}\text { Axial loading } \\
\text { (at } \mathrm{t} / 6)\end{array}$ & $\begin{array}{c}2450 \times 2750 \times 165 \mathrm{~mm} \\
\left(8^{\prime} \times 9^{\prime} \times 6.5^{\prime \prime}\right)\end{array}$ & $\begin{array}{l}11 \mathrm{~mm} \\
(7 / 16 ”)\end{array}$ & $\begin{array}{c}\text { Conventional } \\
\text { stud panel }\end{array}$ \\
\hline SW-A-3 & $\begin{array}{l}\text { Axial loading } \\
\text { (at } \mathrm{t} / 6 \text { ) }\end{array}$ & $\begin{array}{c}2450 \times 2750 \times 165 \mathrm{~mm} \\
\left(8^{\prime} \times 9^{\prime} \times 6.5^{\prime \prime}\right)\end{array}$ & $\begin{array}{l}11 \mathrm{~mm} \\
\left(7 / 16^{\prime \prime}\right)\end{array}$ & $\begin{array}{c}\text { Conventional } \\
\text { stud panel }\end{array}$ \\
\hline SIP-A-1 & $\begin{array}{c}\text { Axial loading } \\
\text { (at t/6) }\end{array}$ & $\begin{array}{c}1220 \times 2750 \times 165 \mathrm{~mm} \\
\left(4^{\prime} \times 9^{\prime} \times 6.5^{\prime \prime}\right)\end{array}$ & $\begin{array}{l}11 \mathrm{~mm} \\
\left(7 / 16^{\prime \prime}\right)\end{array}$ & SIP \\
\hline SIP-A-2 & $\begin{array}{c}\text { Axial loading } \\
\text { (at } \mathrm{t} / 6)\end{array}$ & $\begin{array}{c}1220 \times 2750 \times 165 \mathrm{~mm} \\
\left(4^{\prime} \times 9^{\prime} \times 6.5^{\prime \prime}\right)\end{array}$ & $\begin{array}{l}11 \mathrm{~mm} \\
\left(7 / 16^{\prime \prime}\right)\end{array}$ & SIP \\
\hline SIP-A-3 & $\begin{array}{c}\text { Axial loading } \\
\text { (at } \mathrm{t} / 6)\end{array}$ & $\begin{array}{c}1220 \times 2750 \times 165 \mathrm{~mm} \\
\left(4^{\prime} \times 9^{\prime} \times 6.5^{\prime \prime}\right)\end{array}$ & $\begin{array}{l}11 \mathrm{~mm} \\
(7 / 16 ”)\end{array}$ & SIP \\
\hline
\end{tabular}


Table 3.2 - Specimen details for flexural loading tests

\begin{tabular}{|c|c|c|c|c|}
\hline Test No. & Test type & $\begin{array}{c}\text { Panel size } \\
(\mathbf{l} \times \mathbf{w} \times \mathbf{t}), \\
\text { mm (ft-inches) }\end{array}$ & $\begin{array}{c}\text { Thickness of } \\
\text { sheathing (OSB), } \\
\text { mm (inches) }\end{array}$ & Specimen type \\
\hline SW-F-1 & Flexural loading & $\begin{array}{c}2450 \times 2750 \times 165 \mathrm{~mm} \\
\left(8^{\prime} \times 9^{\prime} \times 6.5^{\prime \prime}\right)\end{array}$ & $\begin{array}{l}11 \mathrm{~mm} \\
\left(7 / 16^{\prime}\right)\end{array}$ & $\begin{array}{c}\text { Conventional } \\
\text { stud panel }\end{array}$ \\
\hline SW-F-2 & Flexural loading & $\begin{array}{c}2450 \times 2750 \times 165 \mathrm{~mm} \\
\left(8^{\prime} \times 9^{\prime} \times 6.5^{\prime \prime}\right)\end{array}$ & $\begin{array}{l}11 \mathrm{~mm} \\
\left(7 / 16^{\prime \prime}\right)\end{array}$ & $\begin{array}{c}\text { Conventional } \\
\text { stud panel }\end{array}$ \\
\hline SW-F-3 & Flexural loading & $\begin{array}{c}2450 \times 2750 \times 165 \mathrm{~mm} \\
\left(8^{\prime} \times 9^{\prime} \times 6.5^{\prime \prime}\right)\end{array}$ & $\begin{array}{l}11 \mathrm{~mm} \\
(7 / 16 ”)\end{array}$ & $\begin{array}{c}\text { Conventional } \\
\text { stud panel }\end{array}$ \\
\hline SIP-F-1 & Flexural loading & $\begin{array}{c}1220 \times 2750 \times 165 \mathrm{~mm} \\
\left(4^{\prime} \times 9^{\prime} \times 6.5^{\prime \prime}\right)\end{array}$ & $\begin{array}{l}11 \mathrm{~mm} \\
(7 / 16 ”)\end{array}$ & SIP \\
\hline SIP-F-2 & Flexural loading & $\begin{array}{c}1220 \times 2750 \times 165 \mathrm{~mm} \\
\left(4^{\prime} \times 9^{\prime} \times 6.5^{\prime \prime}\right)\end{array}$ & $\begin{array}{l}11 \mathrm{~mm} \\
\left(7 / 16^{\prime \prime}\right)\end{array}$ & SIP \\
\hline SIP-F-3 & Flexural loading & $\begin{array}{c}1220 \times 2750 \times 165 \mathrm{~mm} \\
\left(4^{\prime} \times 9^{\prime} \times 6.5^{\prime \prime}\right)\end{array}$ & $\begin{array}{l}11 \mathrm{~mm} \\
\left(7 / 16^{\prime \prime}\right)\end{array}$ & SIP \\
\hline
\end{tabular}


Table 3.3 - Specimen details for racking load tests

\begin{tabular}{|c|c|c|c|c|}
\hline Test No. & Test type & $\begin{array}{c}\text { Panel size } \\
(\mathbf{l} \times \mathbf{w} \times \mathbf{t}), \\
\text { mm (ft-inches) }\end{array}$ & $\begin{array}{c}\text { Thickness } \\
\text { of sheathing } \\
\text { (OSB), } \\
\text { mm (inch) }\end{array}$ & Specimen type \\
\hline SW-R-1 & Racking Loading & $\begin{array}{c}2450 \times 2750 \times 165 \mathrm{~mm} \\
\left(8^{\prime} \times 9^{\prime} \times 6.5^{\prime \prime}\right)\end{array}$ & $\begin{array}{l}11 \mathrm{~mm} \\
\left(7 / 16^{\prime \prime}\right)\end{array}$ & $\begin{array}{c}\text { Conventional } \\
\text { stud panel }\end{array}$ \\
\hline SW-R-2 & Racking Loading & $\begin{array}{c}2450 \times 2750 \times 165 \mathrm{~mm} \\
\left(8^{\prime} \times 9^{\prime} \times 6.5^{\prime \prime}\right)\end{array}$ & $\begin{array}{l}11 \mathrm{~mm} \\
\left(7 / 16^{\prime \prime}\right)\end{array}$ & $\begin{array}{c}\text { Conventional } \\
\text { stud panel }\end{array}$ \\
\hline SIP-R-1 & Racking Loading & $\begin{array}{c}2450 \times 2750 \times 165 \mathrm{~mm} \\
\left(8^{\prime} \times 9^{\prime} \times 6.5^{\prime \prime}\right)\end{array}$ & $\begin{array}{l}11 \mathrm{~mm} \\
(7 / 16 ”)\end{array}$ & SIP \\
\hline SIP-R-2 & Racking Loading & $\begin{array}{c}2450 \times 2750 \times 165 \mathrm{~mm} \\
\left(8^{\prime} \times 9^{\prime} \times 6.5^{\prime \prime}\right)\end{array}$ & $\begin{array}{l}11 \mathrm{~mm} \\
\left(7 / 16^{\prime \prime}\right)\end{array}$ & SIP \\
\hline SIP-R-3 & Racking Loading & $\begin{array}{c}2450 \times 2750 \times 165 \mathrm{~mm} \\
\left(8^{\prime} \times 9^{\prime} \times 6.5^{\prime \prime}\right)\end{array}$ & $\begin{array}{l}11 \mathrm{~mm} \\
\left(7 / 16^{\prime \prime}\right)\end{array}$ & SIP \\
\hline SIP-R-4 & Racking Loading & $\begin{array}{c}3660 \times 2750 \times 165 \mathrm{~mm} \\
\left(12^{\prime} \times 9^{\prime} \times 6.5^{\prime \prime}\right)\end{array}$ & $\begin{array}{l}11 \mathrm{~mm} \\
(7 / 16 ”)\end{array}$ & SIP \\
\hline SIP-R-5 & Racking Loading & $\begin{array}{c}3660 \times 2750 \times 165 \mathrm{~mm} \\
\left(12^{\prime} \times 9^{\prime} \times 6.5^{\prime \prime}\right)\end{array}$ & $\begin{array}{l}11 \mathrm{~mm} \\
\left(7 / 16^{\prime \prime}\right)\end{array}$ & SIP \\
\hline SIP-R-6 & Racking Loading & $\begin{array}{c}3660 \times 2750 \times 165 \mathrm{~mm} \\
\left(12^{\prime} \times 9^{\prime} \times 6.5^{\prime \prime}\right)\end{array}$ & $\begin{array}{l}11 \mathrm{~mm} \\
\left(7 / 16^{\prime \prime}\right)\end{array}$ & SIP \\
\hline
\end{tabular}


Table 3.4 - Specimen details for cyclic load tests

\begin{tabular}{|c|c|c|c|c|}
\hline Test No. & Test type & $\begin{array}{c}\text { Panel Size } \\
(\mathbf{l} \times \mathrm{w} \times \mathrm{t}) \text {, } \\
\text { mm (ft-inch) }\end{array}$ & $\begin{array}{c}\text { Thickness } \\
\text { of sheathing } \\
\text { (OSB), } \\
\text { mm (inch) }\end{array}$ & Specimen type \\
\hline SW-C-1 & Cyclic Loading & $\begin{array}{c}2450 \times 2750 \times 165 \mathrm{~mm} \\
\left(8^{\prime} \times 9^{\prime} \times 6.5^{\prime \prime}\right)\end{array}$ & $\begin{array}{l}11 \mathrm{~mm} \\
\left(7 / 16^{\prime \prime}\right)\end{array}$ & $\begin{array}{c}\text { Conventional } \\
\text { stud panel }\end{array}$ \\
\hline SW-C-2 & Cyclic Loading & $\begin{array}{c}2450 \times 2750 \times 165 \mathrm{~mm} \\
\left(8^{\prime} \times 9^{\prime} \times 6.5^{\prime \prime}\right)\end{array}$ & $\begin{array}{l}11 \mathrm{~mm} \\
\left(7 / 16^{\prime \prime}\right)\end{array}$ & $\begin{array}{l}\text { Conventional } \\
\text { stud panel }\end{array}$ \\
\hline SIP-C-1 & Cyclic Loading & $\begin{array}{c}2450 \times 2750 \times 165 \mathrm{~mm} \\
\left(8^{\prime} \times 9^{\prime} \times 6.5^{\prime \prime}\right)\end{array}$ & $\begin{array}{l}11 \mathrm{~mm} \\
\left(7 / 16^{\prime \prime}\right)\end{array}$ & SIP \\
\hline SIP-C-2 & Cyclic Loading & $\begin{array}{c}2450 \times 2750 \times 165 \mathrm{~mm} \\
\left(8^{\prime} \times 9^{\prime} \times 6.5^{\prime \prime}\right)\end{array}$ & $\begin{array}{l}11 \mathrm{~mm} \\
\left(7 / 16^{\prime \prime}\right)\end{array}$ & SIP \\
\hline SIP-C-3 & Cyclic Loading & $\begin{array}{c}2450 \times 2750 \times 165 \mathrm{~mm} \\
\left(8^{\prime} \times 9^{\prime} \times 6.5^{\prime \prime}\right)\end{array}$ & $\begin{array}{l}11 \mathrm{~mm} \\
\left(7 / 16^{\prime \prime}\right)\end{array}$ & SIP \\
\hline SIP-C-4 & Cyclic Loading & $\begin{array}{c}3660 \times 2750 \times 165 \mathrm{~mm} \\
\left(12^{\prime} \times 9^{\prime} \times 6.5^{\prime \prime}\right)\end{array}$ & $\begin{array}{l}11 \mathrm{~mm} \\
\left(7 / 16^{\prime \prime}\right)\end{array}$ & SIP \\
\hline SIP-C-5 & Cyclic Loading & $\begin{array}{c}3660 \times 2750 \times 165 \mathrm{~mm} \\
\left(12^{\prime} \times 9^{\prime} \times 6.5^{\prime \prime}\right)\end{array}$ & $\begin{array}{l}11 \mathrm{~mm} \\
\left(7 / 16^{\prime \prime}\right)\end{array}$ & SIP \\
\hline SIP-C-6 & Cyclic Loading & $\begin{array}{c}3660 \times 2750 \times 165 \mathrm{~mm} \\
\left(12^{\prime} \times 9^{\prime} \times 6.5^{\prime \prime}\right)\end{array}$ & $\begin{array}{l}11 \mathrm{~mm} \\
\left(7 / 16^{\prime \prime}\right)\end{array}$ & SIP \\
\hline
\end{tabular}

\subsection{Stud Wall Descriptions and Material Properties}

All stud panels were manufactured for conventional wall construction with timber stud and 11 mm (7/16") thick OSB boards for the outside facing (i.e. loading face) and $12.7 \mathrm{~mm}(1 / 2$ ") drywall board for the inside facing. Stud wall panels consisted of 3 identical panels of $2.45 \mathrm{~m}$ (8') width and $2.73 \mathrm{~m}\left(9^{\prime}\right)$ length. These panels are listed in Table 3.1 as SW-A-1, SW-A-2 and SW-A-3. 38x140 mm (2"x6") timber studs were used between the OSB and drywall facings at spacing of $600 \mathrm{~mm}$ (24") centre-to-centre. It should be noted that the applied compressive load was eccentric to the mid-thickness of the panel. An eccentricity of $\mathrm{t} / 6$ was considered, where $t$ is the thickness of the panel. Stud floor panels consisted of 3 identical panels to those tested under compressive loading and are listed as SW-F-1, SW-F-2 and SW-F-3 in Table 3.2. As for racking load tests, two identical specimens made of conventional stud walls were constructed and labeled 
as SW-R-1 and SW-R-2 as depicted in Table 3.3. While other two identical stud wall specimens, labeled SW-C-1, and SW-C-2 as shown in Table 3.4. Each stud wall specimen had a length of $2.45 \mathrm{~m}$, height of $2.75 \mathrm{~m}$ and total thickness of $165 \mathrm{~mm}$ as depicted in Figure 3.4.

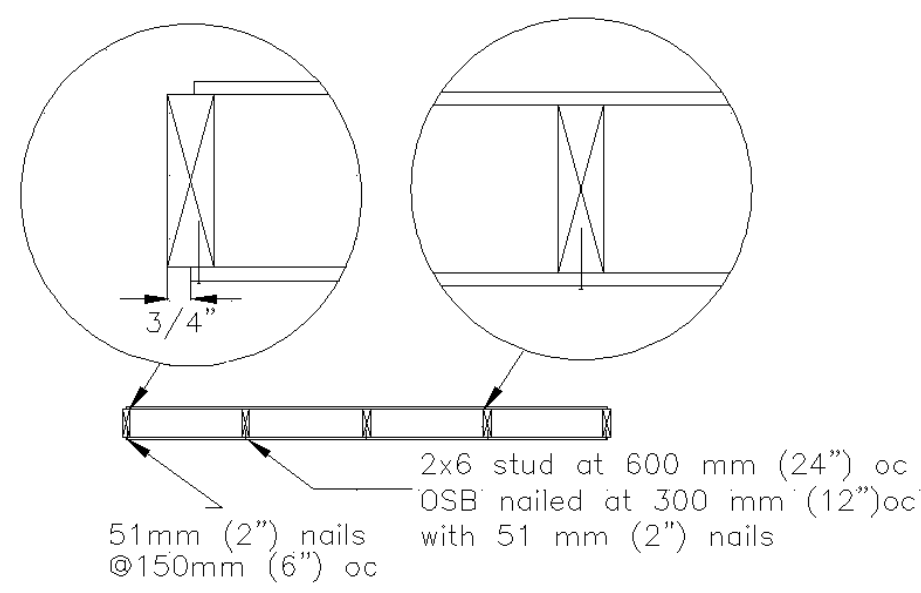

(a) Detail of lumber-spline connection

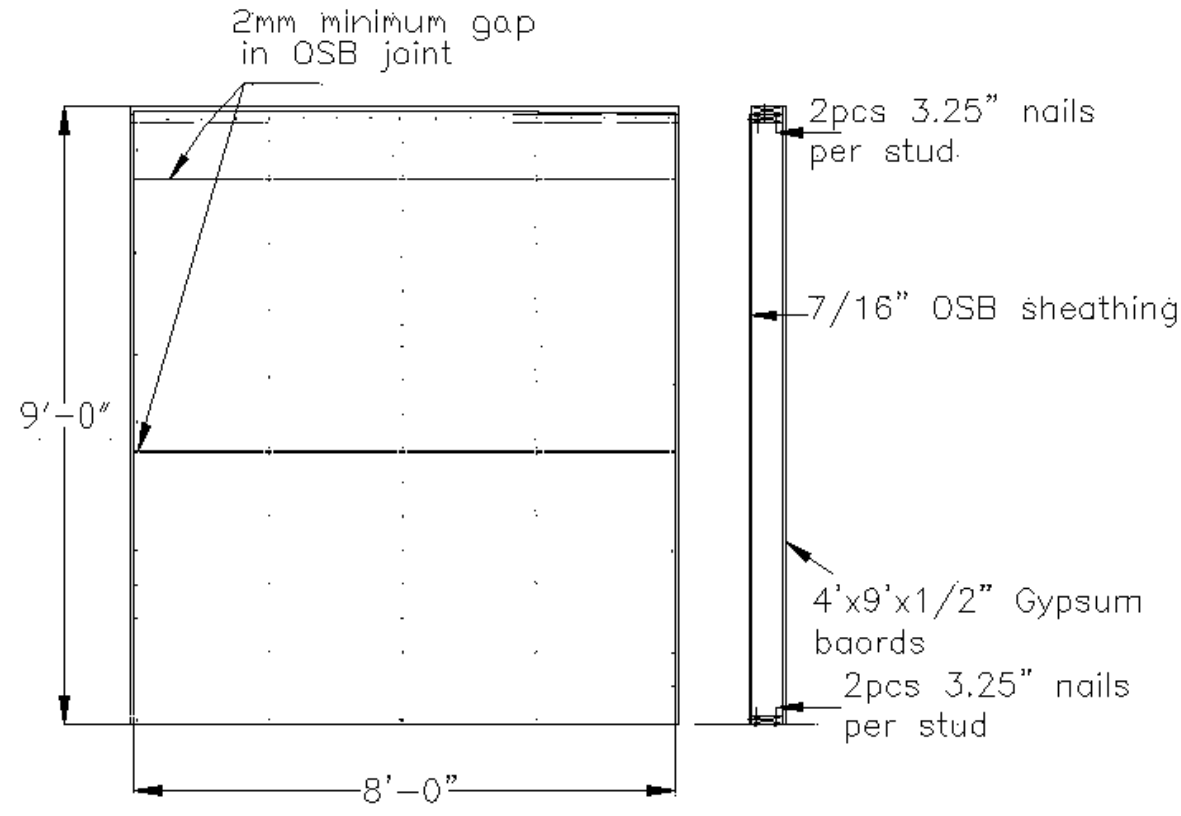

(b) Elevation and view of stud wall

Figure 3.4 - Schematic diagrams of the tested stud walls

For all panels, 51x $2.4 \mathrm{~mm}$ (2"x0.095") diameter, hot-dipped galvanized, gun nails were used to connect the facings to the studs at $300 \mathrm{~mm}$ (12") spacing. With $20 \mathrm{~mm}(3 / 4$ ") facing edge distance, the facing edges were connected to the exterior studs using similar nails but at $150 \mathrm{~mm}$ 
(6") spacing. It should be noted that the OSB facings were nailed to the studs in three segments with a $2 \mathrm{~mm}$ gap at the facing horizontal joints as depicted in Figure 3.2. The drywall facing was nailed to the studs in two vertical segments. The studs were Spruce-Pine-Fir species combination with No. 2 grade (i.e. SPF No. 2). Per the Canadian Standard of Engineering Design of Wood, CAN/CSA-O86-09, the following are the material properties of the tested studs.

Flexural strength $=11.8 \mathrm{MPa}$; shear strength $=1.5 \mathrm{MPa}$; compressive strength parallel to the grains $=11.5 \mathrm{MPa}$; modulus of elasticity $=9500 \mathrm{MPa}$.

\subsection{SIP Walls Descriptions and Material Properties}

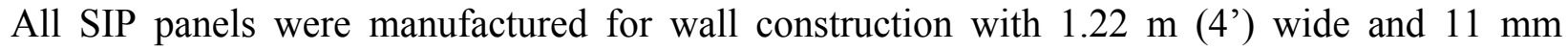
(7/16") thick OSB boards for the side facings. SIP wall specimens consisted of 3 identical panels of $1.22 \mathrm{~m}$ (4') length, $165 \mathrm{~mm}\left(6^{1 / 2}\right.$ ”) total depth, and foam-spline connection. It should be noted that the foam core depth is simply the difference between the total depth and the thickness of the two OSB facings. It should be noted that the applied compressive load was eccentric to the midthickness of the panel. An eccentricity of ( $t / 6)$ was considered, where $(t)$ is the thickness of the panel. SIP floor specimens consisted of 3 identical panels to those in SIP wall specimens except they were subjected to flexural loading. These SIP specimens are listed as SIP-F-1, SIP-F-2 and SIP-F-3 in Table 3.2. As for racking and cyclic load tests, two SIP panel groups were considered in this study. Specimens in these groups are identical except that the specimen length was $2.45 \mathrm{~m}$ in the first group while it was $3.66 \mathrm{~m}$ in the second group. As for racking load tests for the first group, three identical specimens made of SIPs were constructed and labeled as SIP-R-1, SIP-R-2 and SIP-R-3 as depicted in Table 3.3. While other three identical SIP specimens, labeled SIP-C1, SIP-C-2 and SIP-C-3 as shown in Table 3.4. Each SIP wall specimen in the first group had a length of $2.45 \mathrm{~m}$, height of $2.75 \mathrm{~m}$ and total thickness of $165 \mathrm{~mm}$. As for racking load tests for the second group, three identical specimens made of SIPs were constructed and labeled as SIPR-1, SIP-R-2 and SIP-R-3 as depicted in Table 3.3. While other three identical SIP specimens, labeled SIP-C-1, SIP-C-2 and SIP-C-3 as shown in Table 3.4. Each SIP wall specimen in the second group had a length of $3.66 \mathrm{~m}$, height of $2.75 \mathrm{~m}$ and total thickness of $165 \mathrm{~mm}$. It should be noted that SIP panels of 2.45-m length were made of two prefabricated SIP walls of $1.22 \mathrm{~mm}$ length jointed together with lumber-spline connection as depicted in Fig. 3.3. On the other hand, 
the SIP panel of $3.66 \mathrm{~m}$ length was assembled of three prefabricated SIP walls of $1.22 \mathrm{~mm}$ length with lumber-spline connection.

The exterior faces of the SIP specimens were oriented strand board (OSB) manufactured and grade stamped as per APA (APA 1990). The OSB board fabricate panels had 1R24/EF16/W24 panel mark with $11 \mathrm{~mm}$ thickness construction sheathing. The material properties for OBS boards are specified as follows:

Modulus of elasticity: $5515 \mathrm{MPa}(800,000 \mathrm{psi})$ in the span direction

$1551 \mathrm{MPa}(225,000 \mathrm{psi})$ in the direction normal to the span direction

Modulus of rupture: $28.955 \mathrm{MPa}$ (4200 psi) in the span direction

12.409 $\mathrm{MPa}(1800 \mathrm{psi})$ in the direction normal to the span direction

However, material characteristics as specified in the OSB Design Manual (SBA, 2004) for the 1R24/EF16/W24 panel are as follows:

Bending resistance, $\mathrm{M}_{\mathrm{r}}$

Bending stiffness, EI

Axial stiffness, EA

Axial tensile resistance, $\mathrm{T}_{\mathrm{r}}$

Axial compressive resistance, $\mathrm{P}_{\mathrm{r}}$

Shear through thickness resistance, $\mathrm{V}_{\mathrm{r}}=44 \mathrm{~N} / \mathrm{mm}$

Shear through thickness rigidity, $\mathrm{G}=11,000 \mathrm{~N} / \mathrm{mm}$

$$
\begin{aligned}
& =228 \mathrm{~N} \cdot \mathrm{mm} / \mathrm{mm} \\
& =730,000 \mathrm{~N} \cdot \mathrm{mm}^{2} / \mathrm{mm} \\
& =38,000 \mathrm{~N} / \mathrm{mm} \\
& =57 \mathrm{~N} / \mathrm{mm} \\
& =67 \mathrm{~N} / \mathrm{mm}
\end{aligned}
$$

When expanded polystyrene (EPS) exposed to steam, it provides a uniform closed cell with high resistant to heat and moisture. This process called in-plant expansion process and it is fused into blocks. Blocks are cured for dimensional stability and cut into boards. The expanded polystyrene (EPS) core type 1 was used to fabricate the panels. The priority density demonstrates a load failure of $172.36 \mathrm{kPa}$ (25 psi) when tested as per ASTM C297. The EPS core material must meet the standard CAN/ULC-S701 and demonstrate the following characteristics:

$$
\begin{array}{ll}
\text { Nominal density } & =16 \mathrm{~kg} / \mathrm{m}^{3}\left(1.0 \mathrm{lbs} / \mathrm{ft}^{3}\right) \\
\text { Flexural strength } & =172 \mathrm{kPa}(25 \mathrm{psi}) \\
\text { Tensile strength } & =103 \mathrm{kPa}(15 \mathrm{psi}) \\
\text { Compressive strength } & =70 \mathrm{kPa}(10 \mathrm{psi})
\end{array}
$$


Shear strength $\quad=83 \mathrm{kPa}(12 \mathrm{psi})$

Shear modulus $\quad=2758 \mathrm{kPa}(400 \mathrm{psi})$

The urethane adhesive used to connect the EPS core to OSB facings must meet the following standards:

ASTM D-2294: 7 Day High Temperature Creep Test

ASTM C-297 : Tension Test of Flat Sandwich Construction in a Flat-wise Plane

ASTM D-1877: Resistance of Adhesive to Cyclic Laboratory Aging Conditions

ASTM D-905 : Block Shear Test Using Plywood

ASTM D-1002: Strength Properties of Adhesive Bonds in Shear by Tension Loading

For all panels, 51x2.4 mm (2"x0.095") diameter, hot-dipped galvanized, gun nails at $200 \mathrm{~mm}$ (8") spacing with $20 \mathrm{~mm}(3 / 4 ")$ minimum edge distance to connect the OSB sheets to the foam splines and lumber splines. Also, this nail arrangement was used to connect the panel facings to the lumber plates at the top and bottom of the walls.

\subsection{Material Properties by Experimental Testing}

The objective of bending and tension tests was to obtain experimentally the OSB modulus of elasticity, modulus of rupture, and axial tensile resistance and correlate them with the commercially available data. Test procedure specified in ASTM Standards D1037-06a (2006), C203-05A (2012) and C273-00 (2000) were used to determine these structural material quantities. The following subsections explain the test method, test procedure and results in case of bending, tension and shear.

\subsubsection{OSB Bending and Tension Tests}

OSB specimen was tested in flexure using the three-point flexural test method. The specimen size was $315 \times 77 \times 11 \mathrm{~mm}$. Figure 3.5 shows views of the OSB specimen before and after the flexural test. Figure 3.6 also shows views of the OSB specimen and test setup before and after tension test. As for the tension test, the specimen size was $254 \times 51 \times 11 \mathrm{~mm}$. Figure 3.7 depicts the applied jacking load-deflection relationship for OSB specimen obtained from bending test. Figure 3.8 also shows the applied jacking load-deflection relationship for OSB specimen obtained from tension test. Table 3.5 summarizes the results in the form of modulus of elasticity 
and modulus or rupture of 10 identical OSB specimens. Based on the average values of samples, it was observed that the OSB average modulus of elasticity and modulus of rupture of the specimens were $3.85 \mathrm{GPa}$ and $32.63 \mathrm{MPa}$ compared to $3.50 \mathrm{GPa}$ and $29.00 \mathrm{MPa}$ obtained from the available OSB data sheet, respectively. Also, the average tensile resistance of the OSB specimens was obtained $10.20 \mathrm{~N} / \mathrm{mm}^{2}$ experimentally, compared to $9.40 \mathrm{~N} / \mathrm{mm}^{2}$ obtained from the available OSB data sheet. Given the apparent changes in the experimental and commerciallyavailable values, it was decided to conduct analysis in this research using the available OSB manufacturer's data as used by design engineers. One may observe the significant scatter of data obtained for the 10 identical OSB specimens shown in Table 3.5. This may be attributed to the fact that the OSB sheet is an engineered product with random wood strands glued together under heat and pressure leading to non-homogenous mechanical properties within a small width of 77 or 51 for the tested specimens. So, it is recommended to conduct sensitivity study on OSB specimen of different width ranging from 51 to $200 \mathrm{~mm}$ under bending or axial tension to determine the proper specimen width that would lead to more uniform mechanical properties for tested 10 specimens.

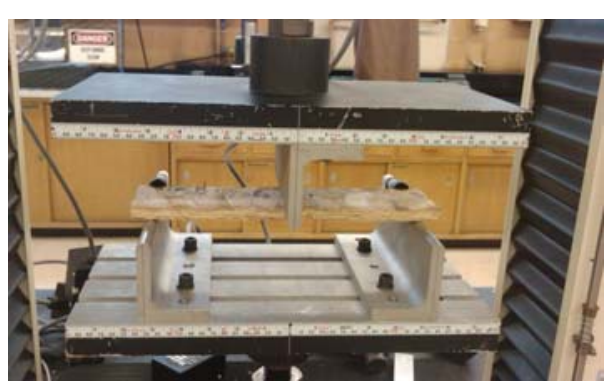

(a) Test setup before bending test

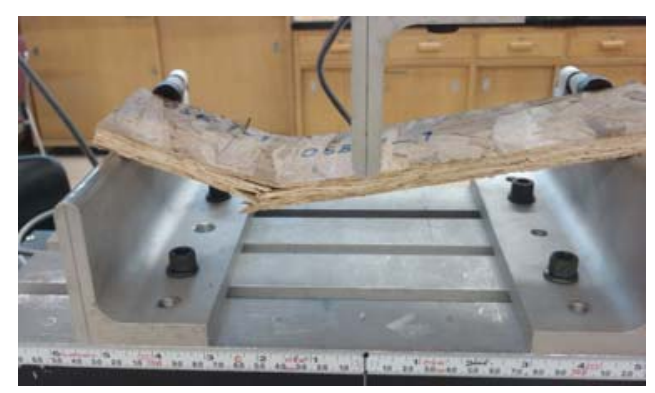

(b) Failure after bending test

Figure 3.5 - OSB bending test setup before and after flexural test

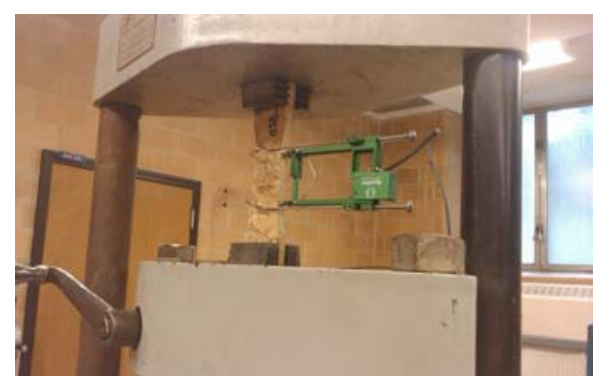

(a) Test setup before tension test

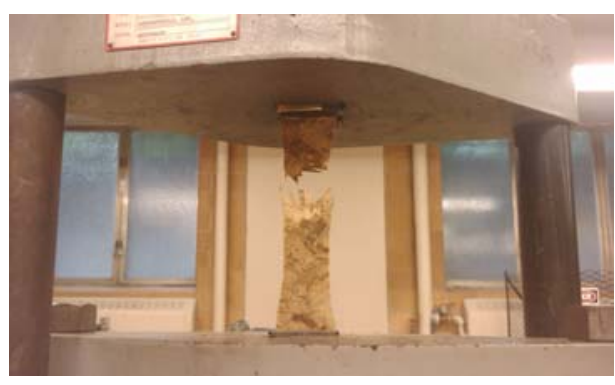

(b) Failure after tension test

Figure 3.6 - OSB tension test setup before and after tension test 


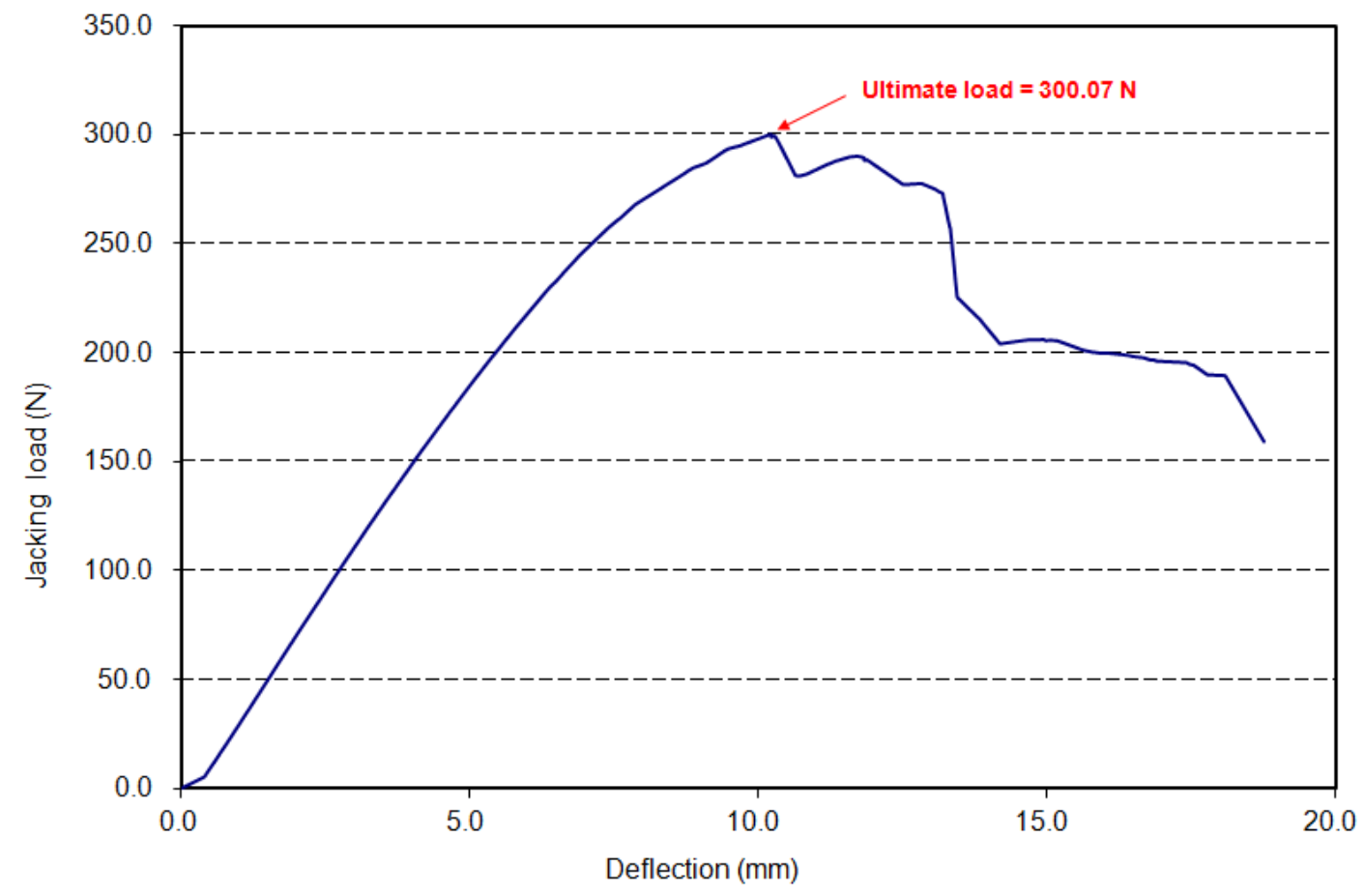

Figure 3.7 - Typical OSB load-deflection curve obtained from the flexural test

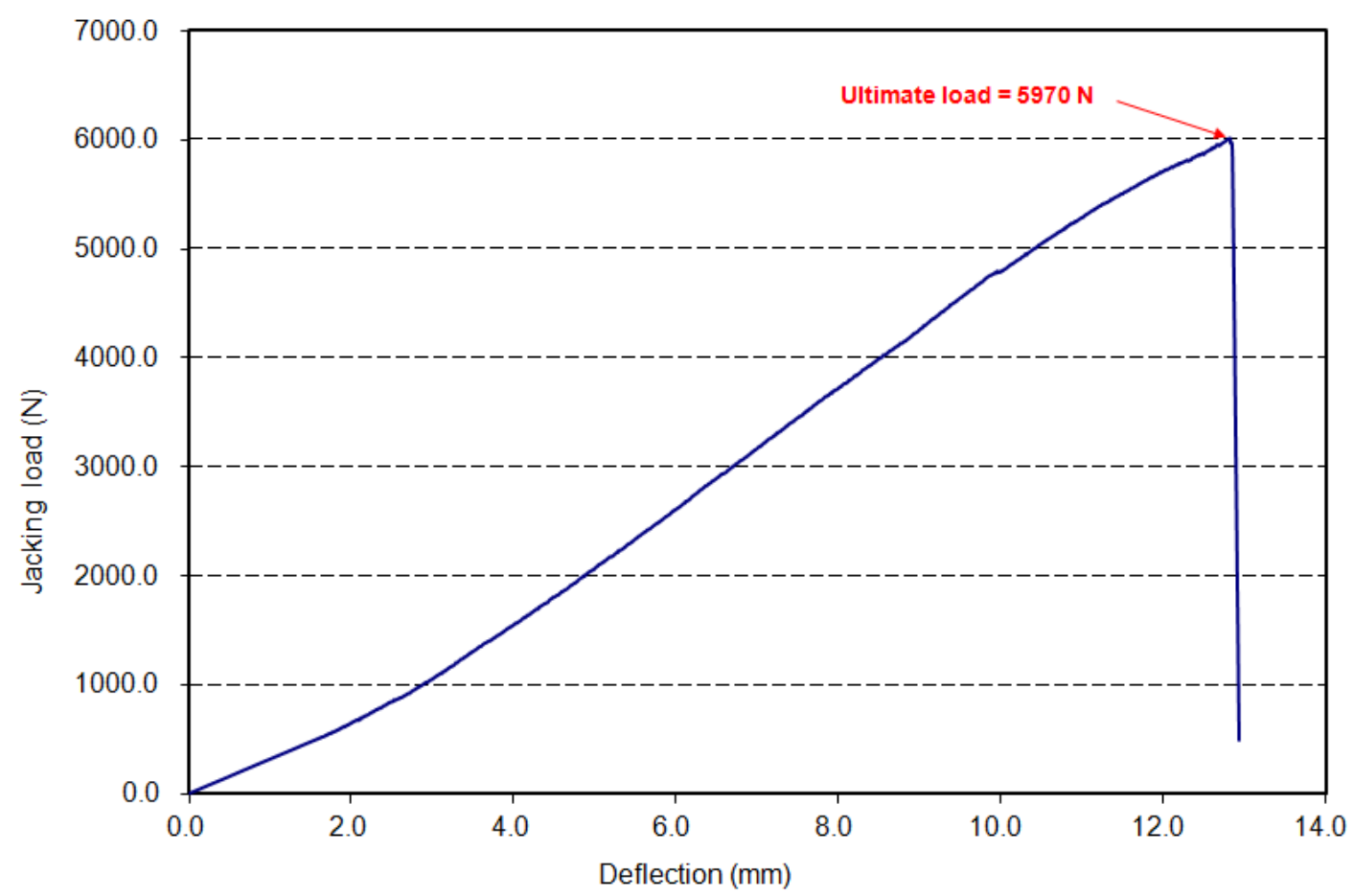

Figure 3.8 - Typical OSB load-deflection curve obtained from tension test 
Table 3.5 - OSB material properties testing results

\begin{tabular}{|c|c|c|c|c|c|}
\hline \multirow{2}{*}{$\begin{array}{c}\text { Specimen } \\
\text { No. }\end{array}$} & \multicolumn{2}{|c|}{ Modulus of elasticity } & \multicolumn{2}{c|}{ Modulus of rupture } & $\begin{array}{c}\text { Tensile } \\
\text { strength }\end{array}$ \\
\cline { 2 - 6 } & $\mathbf{( G P a )}$ & $\mathbf{( P s i )}$ & $\mathbf{( M P a )}$ & $\mathbf{( P s i )}$ & $\mathbf{( N / \mathbf { m m } ^ { 2 } )}$ \\
\hline 1 & 4.69 & 680,227 & 20.79 & 3015 & 8.43 \\
\hline 2 & 2.46 & 356,793 & 26.44 & 3835 & 10.26 \\
\hline 3 & 3.39 & 491,678 & 21.34 & 3095 & 9.95 \\
\hline 4 & 4.60 & 667,174 & 23.51 & 3409 & 14.38 \\
\hline 5 & 2.82 & 409,006 & 31.15 & 4518 & 14.95 \\
\hline 6 & 4.50 & 652,670 & 20.72 & 3005 & 7.85 \\
\hline 7 & 4.69 & 680,227 & 49.57 & 7189 & 12.34 \\
\hline 8 & 3.91 & 567,098 & 38.57 & 5594 & 11.60 \\
\hline 9 & 3.69 & 535,189 & 57.10 & 8281 & 3.44 \\
\hline 10 & 3.76 & 545,342 & 37.10 & 5381 & 8.83 \\
\hline Avg. & $\mathbf{3 . 8 5}$ & $\mathbf{5 5 8 , 5 4 0}$ & $\mathbf{3 2 . 6 3}$ & $\mathbf{4 7 3 2}$ & $\mathbf{1 0 . 2 0}$ \\
\hline St. Dev. & $\mathbf{0 . 7 9}$ & $\mathbf{1 1 4 , 7 9 6}$ & $\mathbf{1 2 . 8 0}$ & $\mathbf{1 8 5 6}$ & $\mathbf{3 . 3 8}$ \\
\hline COV & $\mathbf{0 . 2 0 6}$ & $\mathbf{0 . 2 0 6}$ & $\mathbf{0 . 3 9 2}$ & $\mathbf{0 . 3 9 2}$ & $\mathbf{0 . 3 3 1}$ \\
\hline
\end{tabular}

\subsubsection{EPS Bending Tests}

EPS specimen was tested in flexure using the three-point flexural test method. The specimen size was $300 \times 100 \times 25 \mathrm{~mm}$. Figure 3.9 shows views of the EPS specimen test setup before and after the flexural test. Figure 3.10 also depicts the applied jacking load-deflection relationship for EPS specimen obtained from bending test. Table 3.6 summarizes the results in the form of modulus of elasticity and modulus or rupture of 10 identical EPS specimens. Based on the average values of samples, it was observed that the EPS average modulus of elasticity of the specimens were 2.53 MPa compared to $2.48 \mathrm{MPa}$ obtained from the available EPS manufacturer's data sheet, respectively. Given the apparent changes in the experimental and commercially-available values, it was decided to conduct analysis in this research using the available EPS data as used by design engineers. 


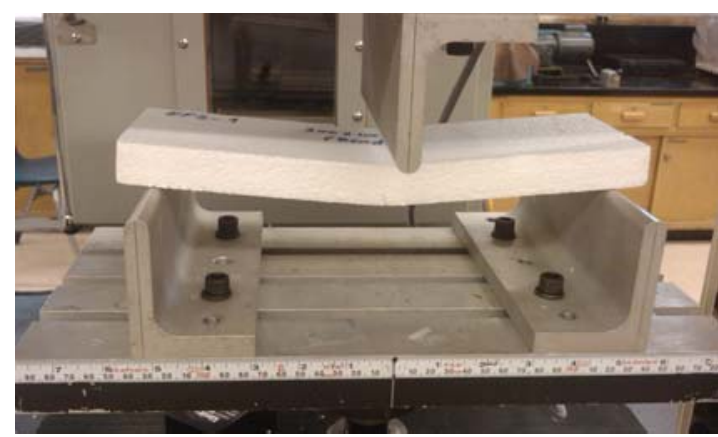

(a) Before bending test

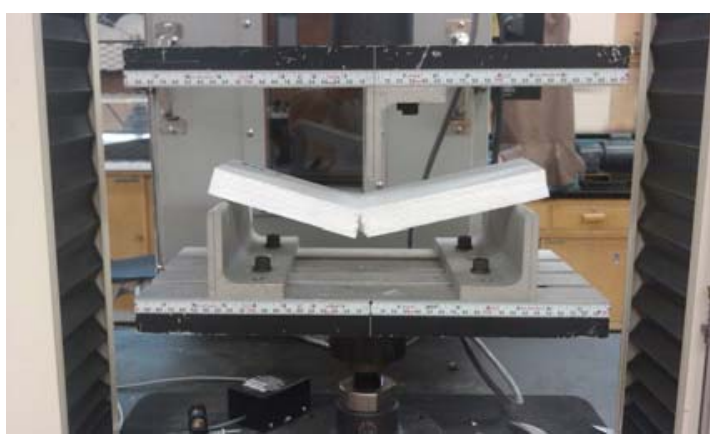

(b) After bending test

Figure 3.9 - EPS bending test setup before and after test

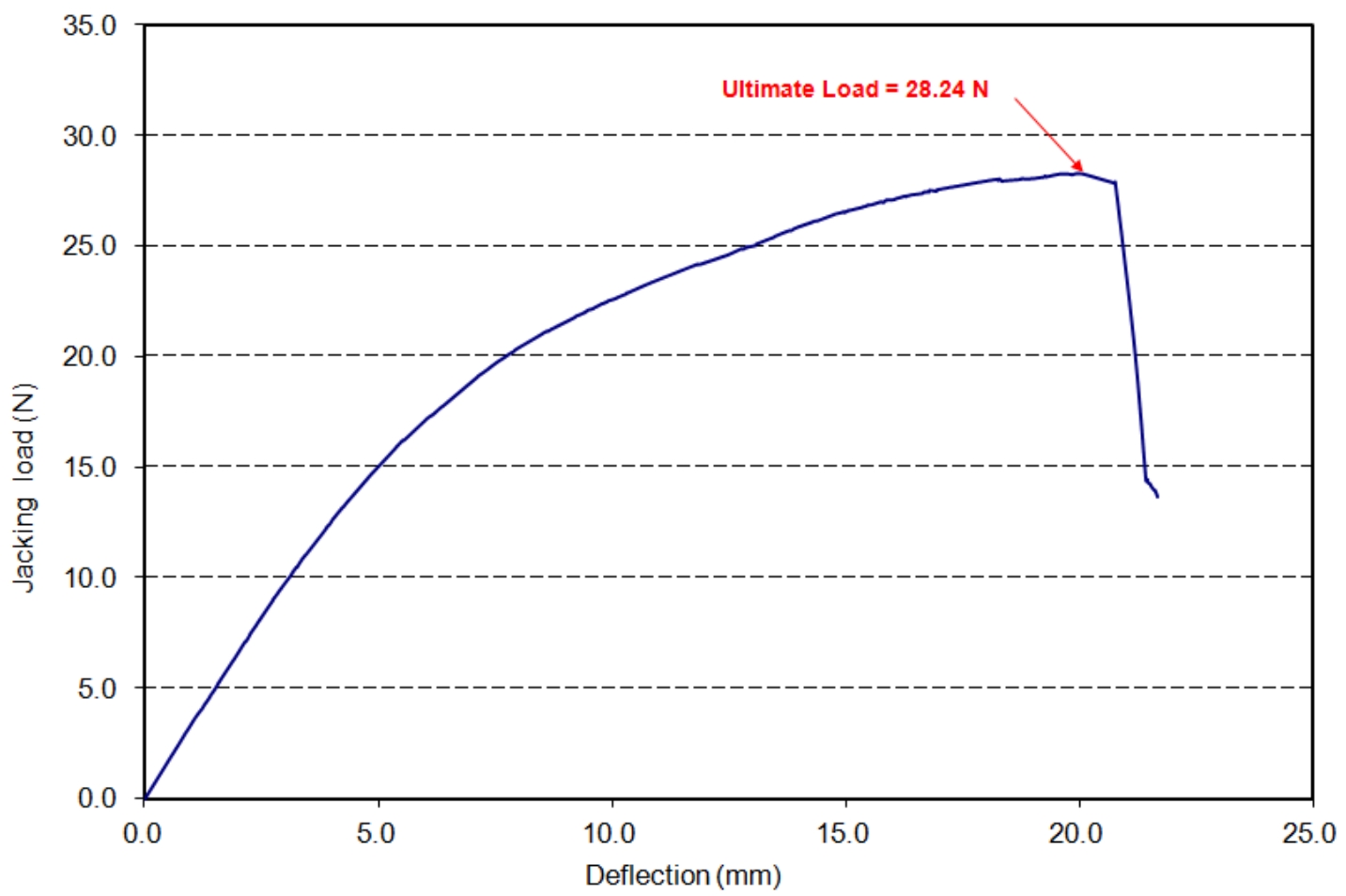

Figure 3.10 - Typical EPS load-deflection curve obtained from bending test 
Table 3.6 - EPS modulus of elasticity obtained from flexural tests

\begin{tabular}{|c|c|c|}
\hline $\begin{array}{c}\text { Specimen } \\
\text { No. }\end{array}$ & \multicolumn{2}{|c|}{ Modules of elasticity } \\
\cline { 2 - 3 } & $\mathbf{( G P a )}$ & $\mathbf{( P s i )}$ \\
\hline 1 & 2.59 & 375.65 \\
\hline 2 & 2.08 & 301.68 \\
\hline 3 & 2.59 & 375.65 \\
\hline 4 & 2.32 & 336.49 \\
\hline 5 & 1.67 & 242.21 \\
\hline 6 & 2.25 & 326.33 \\
\hline 7 & 2.75 & 398.85 \\
\hline 8 & 2.96 & 429.31 \\
\hline 9 & 2.98 & 432.21 \\
\hline 10 & 3.06 & 443.82 \\
\hline Avg. & $\mathbf{2 . 5 3}$ & $\mathbf{3 6 6 . 2 2}$ \\
\hline St. Dev. & $\mathbf{0 . 4 5}$ & $\mathbf{6 4 . 6 5}$ \\
\hline COV & $\mathbf{0 . 1 7 7}$ & $\mathbf{0 . 1 7 7}$ \\
\hline
\end{tabular}

\subsection{Equipments and Instrumentation for Testing}

The following instruments are being used for the experimental program:

- Hydraulically operated universal loading jack

- Hydraulic actuator for applying lateral loading

- Universal Flat Load Cell (50 Kips or $222 \mathrm{kN}$ )

- Potentiometer (POT)

- Data Acquisition System (DAS)

\subsubsection{Hydraulically Operated Universal Loading Jack}

The hydraulic loading jack is worked typical oil and it is using a pressurized hydraulic fluid. There is a piston with a rod inside the hydraulic jack that moves up and down. Both end of the cylinder are closed and there is a cap end in the bottom. The piston rod comes out from the cylinder where the jack head is located. There is two parts in the cylinder which is called as cap end and rod end which is divided inside by piston. The linear work and motion of the piston is 
provided by hydraulic pressure. The actuator or motor side of the system is working with a hydraulic cylinder. (CRC Inc., 2012)

The hydraulic pump provides a regulated or fixes flow of oil into the system. The cylinder and object or machine components are connected by piston rod. The piston rod starts are moved upward by pumping hydraulic oil to the bottom side of the hydraulic cylinder. The oil is pushed in the other back to the reservoir by piston. The pressure in the cylinder times the piston area are equaled to the force on the piston rod if the oil pressure in the piston rod chamber is considered as zero. Figure 3.11 and 3.12 show a hydraulic pump and hydraulic loading jack which were used in plate test, respectively. A 10 gallon capacity hydraulic pump and a $900 \mathrm{kN}$ (200 kips) capacity universal loading jack both made by ENERPAC company were used in this study.

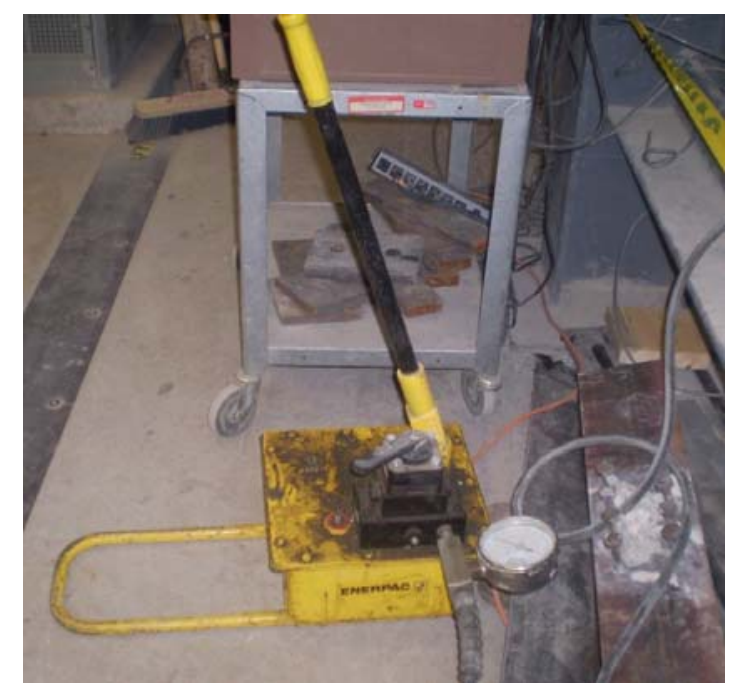

Figure 3.11 - View of hydraulic pump connecting to loading jack

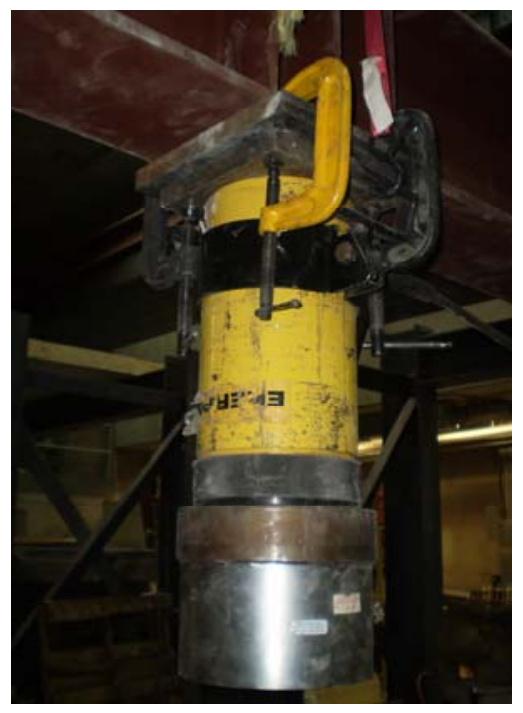

Figure 3.12 - View of hydraulically operated universal loading Jack

\subsubsection{Hydraulic Actuator}

A hydraulic actuator is a cylindrical motor with fluid which converts hydraulic power to mechanical force. The linear, rotary or oscillatory is produced by this mechanical motion. The actuator has different operation like mechanical stiffness, dynamic response and high power per unit weight and volume. (Sclater, 2007) 
The cylindrical actuator is used in this research which provides a fixed length of straight-line motion. A tight-fitting piston moving in a closed cylinder is usually used in this actuator. One end of the cylinder attaches to a rod in piston to make a mechanical output. Each end of the cylinder has a port with double action cylinder to admit or return hydraulic fluid. A valve function with four-way direction connects one cylinder port to the hydraulic system and other parts to return the actuator depending on power stroke direction. Figure 3.13 shows a hydraulic actuator that is used for applying racking and cyclic loads.

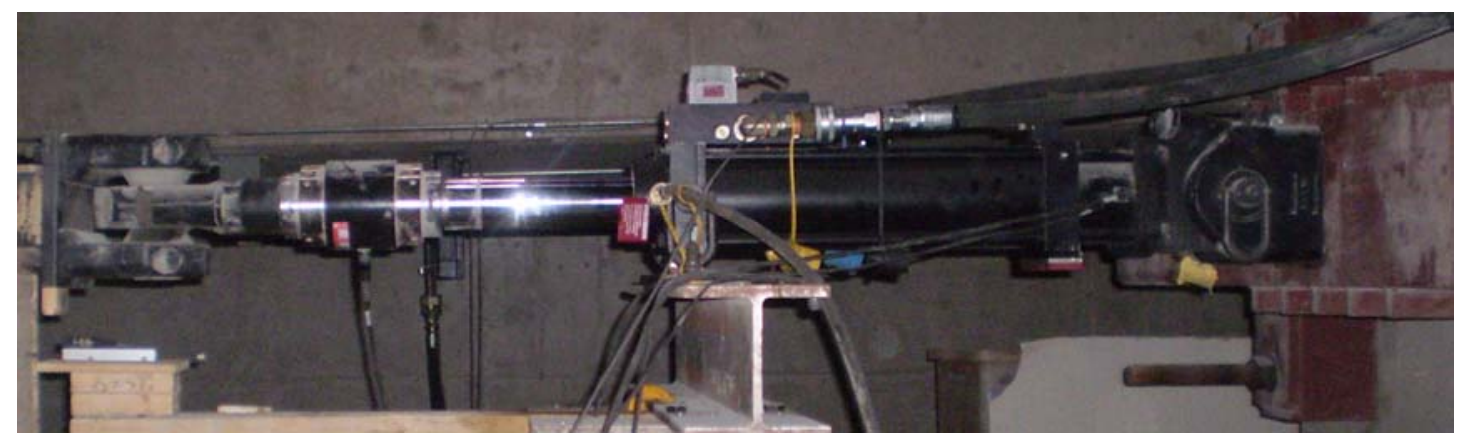

Figure 3.13 - View of hydraulic actuator for racking and cyclic loading

\subsubsection{Universal Flat Load Cell}

A load cell is a device which converts a force to an electrical signal. The conversion of force is happens in mechanical and electrical stages. The force applies to a strain gauge through mechanical effect. The deformation (strain) is converted through strain gauge to electrical signals. The electrical output of signal is in "Millivolts" and an instrumentation amplifier is required for conversion. An algorithm is used to calculate the applied force from output of the transducer (OMEGA Inc., 2003).

The load cell has a spring-like behavior in the stem. This spring has to deform in order to measure the loads. Sometimes, there is some oscillating data pattern is happened in load cell and damp out with a control system. Figure 3.14 shows a universal flat load cell which is used in plate test. The capacity of load cell (model LC-411) is $900 \mathrm{KN}$ (200 kips) in compression and tension. 


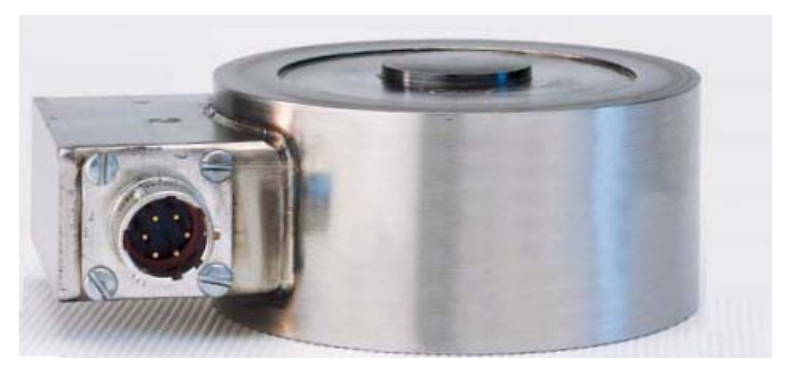

Figure 3.14 - View of universal Flat Load Cell model LC411-200k standard high capacity

\subsubsection{Data Acquisition System (DAS)}

Data acquisition system which is sometimes called as DAQ or DAS is an instrument to generate data from different sources and controlled by a computer. The system contains sensors to convert any measurement parameter to an electrical signal and recorded by a special hardware. Acquired data will store on a computer using supplied software which is prepared by various different language such as Basic, C, FORTRAN, etc. There is some especial software which is designed for large scale data acquisition system like LabVIEW and StrainSmart which provides graphical tools and libraries for data acquisition and analysis (T\&M World Mag., 2006). Figure 3.15 and 3.16 show the data acquisition system (SYSTEM 8000) and computer connections which were used for the current experimental study, respectively. The maximum scanning rate that can be achieved by this DAS is one sample per second.

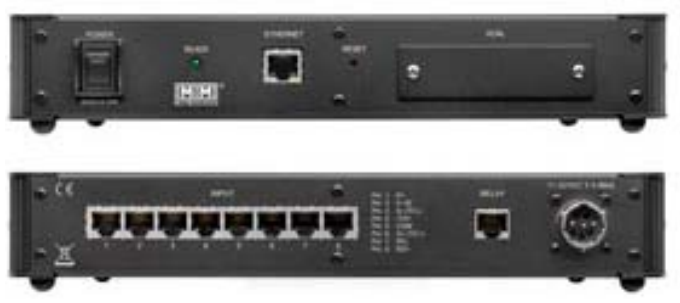

Figure 3.15 - View of data acquisition system

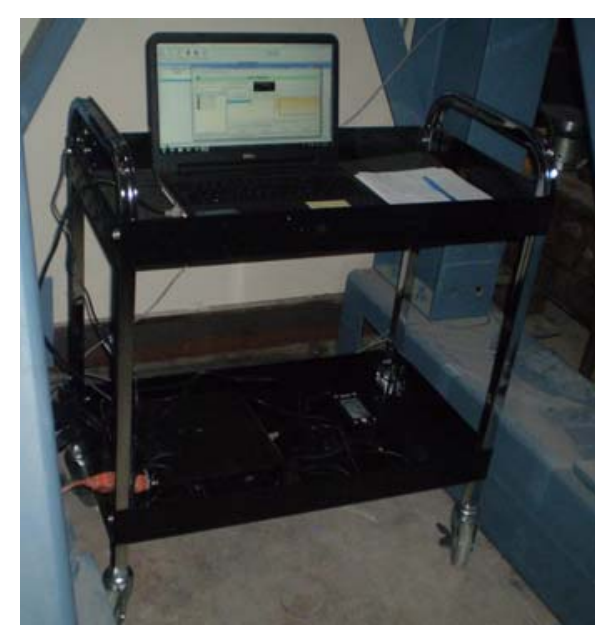

Figure 3.16 - View of computer and data acquisition system in test condition 
The data acquisition system was made ready before applying any load. The data acquisition system used StrainSmart software to append the output data for the different instruments which were used in the test program. Some of the data were in millivolts, millimeter or other units which the user set the program for that. All the required channels were set to one instrument and calibrate in order to record the correct data using offset and scale factor.

\subsubsection{Potentiometer (POT)}

A potentiometer which is called as "POT" is a component and three-terminal resistor with a sliding contact which forms a changeable voltage separator. It is also acts as a variable resistor or rheostat with two terminals which is connected one to the end and the other to wiper. A potentiometer is a voltage divider used for measuring of electrical potential (voltage). Potentiometer normally used as position transducers for measuring deflection variation. The calibration for each POT has done before the test was performed. Figure 3.17 shows the typical potentiometer which is used in this research. Figure 3.18 also show the location of the POTs in test condition. Figures 3.19(a) and 3.19(b) show the detail position of each POT in axial loading condition.

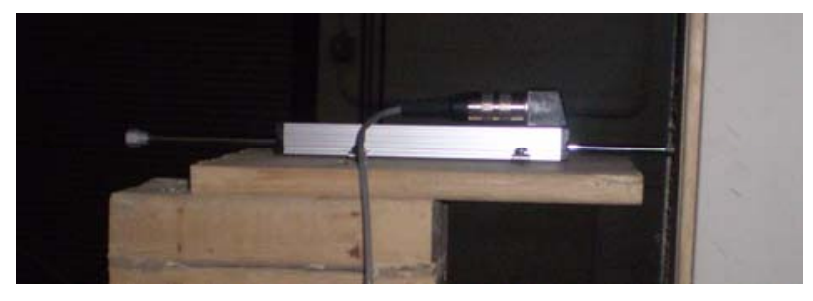

Figure 3.17 - View of potentiometer used in this study

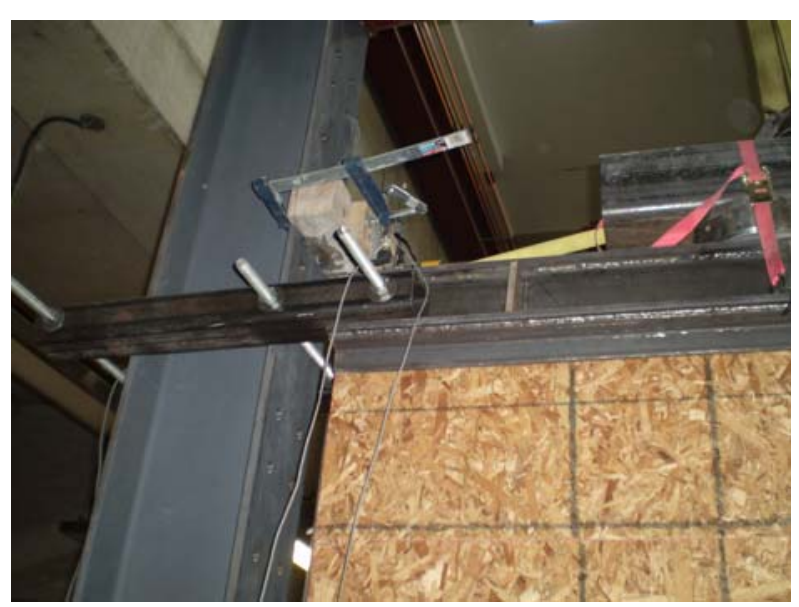

Figure 3.18 - View of POTs locations in panel for stud wall specimen

As a result, as soon as the panel loaded, the readings of the POT were affected and in some cases, the POT either stopped working or the POTs slipped off. 


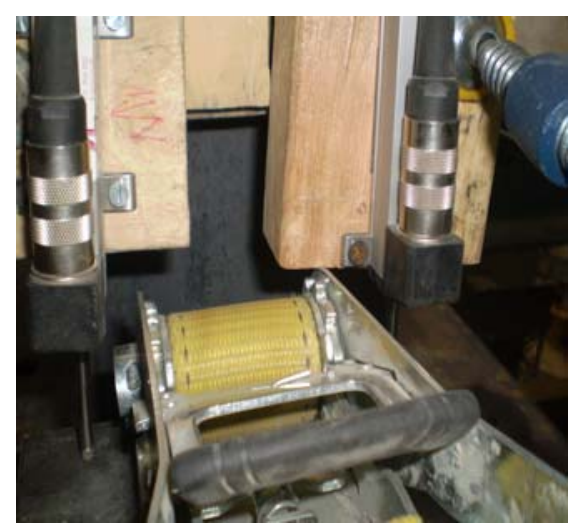

(a) POT 1 and POT 2 in panel

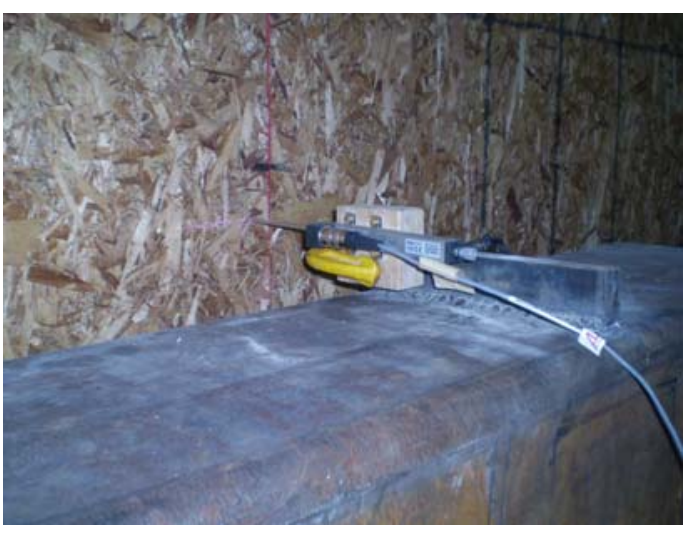

(b) POT 3 location in back of the panel

Figure 3.19 - Views of positions of POTs in axial loading condition

\subsection{Testing SIP in Flexure (Transverse) Loading}

\subsubsection{Test Method for SIP in Flexure}

The objective of this test was to establish the factored design flexural capacity of selected panels that would further be uses with the obtained factored design flexural load to apply the flexural equation to determine either the factored flexural load or factored bending moment that can safely be applied on the floor panels. Bending qualification tests on the panels were conducted as specified in the method described in the ASTM E1803-06, Standard Test Methods for Determining Strength Capacities of Structural Insulated Panels, ASTM E2322-03, Standard Test Method for Conducting Transverse and Concentrated Load Tests on Panels used in Floor and Roof Construction and ASTM E72-10, Standard Test Methods of Conducting Strength Tests of Panels for Building Construction. ASTM E72-10 specifies at least three identical specimens for each test group.

\subsubsection{Test Setup for SIP in Flexure}

Each tested panel was supported over two $25.4 \mathrm{~mm}$ steel rollers at each side in the short direction. $1200 \times 150 \times 12 \mathrm{~mm}$ steel plates were inserted between the steel rollers and the supporting steel pedestal resting on the laboratory strong floor. Other similar-size steel plates were inserted between the supporting roller and the panel bottom facing. A $150 \times 150 \times 12.7 \mathrm{~mm}$ HSS beam of $1200 \mathrm{~mm}$ length used to transfer the applied jacking load to a $102 \times 1020 \times 6.4 \mathrm{~mm}$ HSS beam that was laid transversally over the top panel facing at the quarter points to spread the 
load over the panel width. Steel roller and plate assembly similar to that used to support the panel over the steel pedestals was used to support the $1200 \mathrm{~mm}$ length HSS beam over the two 1200 $\mathrm{mm}$ length HSS spread beams at the quarter points. The weight of this loading system is $2.0 \mathrm{kN}$. Figure 3.20 shows the schematic plan of the setup for flexure test on the SIP specimens SIP-F-1 to SIP-F-3. Figure 3.21 also shows the typical test setup for flexure of the SIP floor on the structural lab. The location of POTs in these specimens was in the middle below of the specimen in order to determine the deflection in middle and both sides of the panel. Figure 3.22 also shows schematic side view of the setup for flexure test on stud wall specimens.

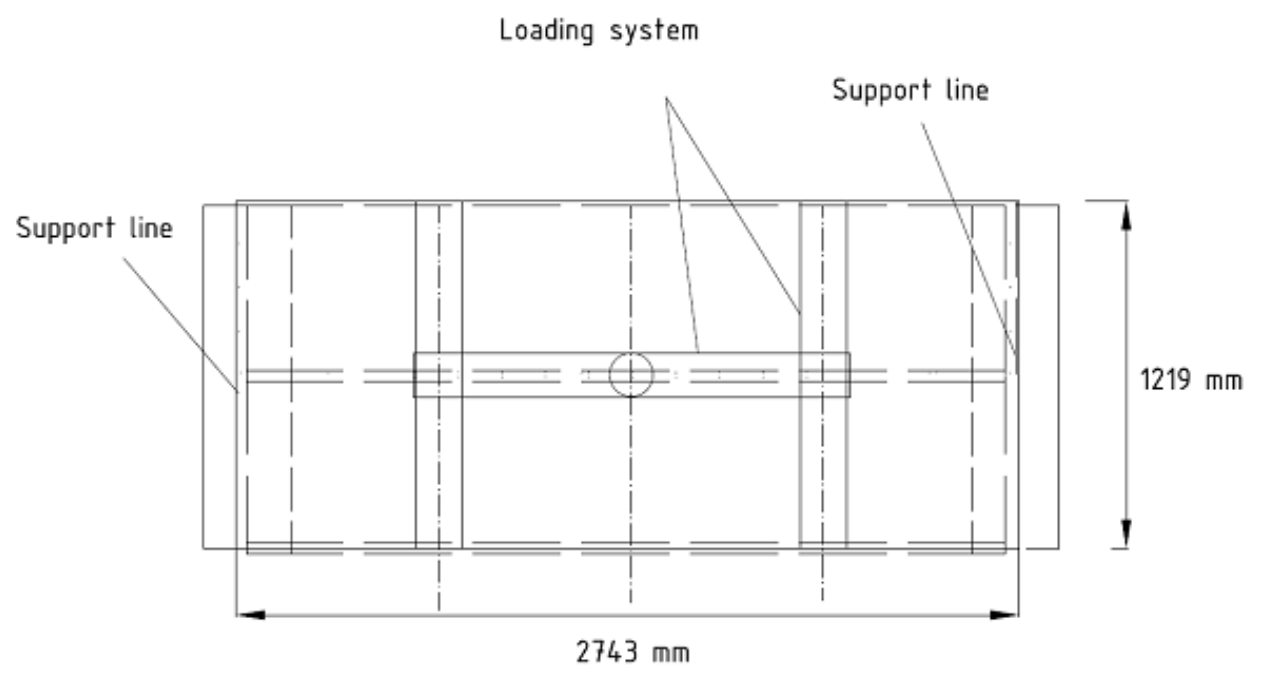

Figure 3.20 - Schematic plan of the setup for flexure of tests of SIP floor

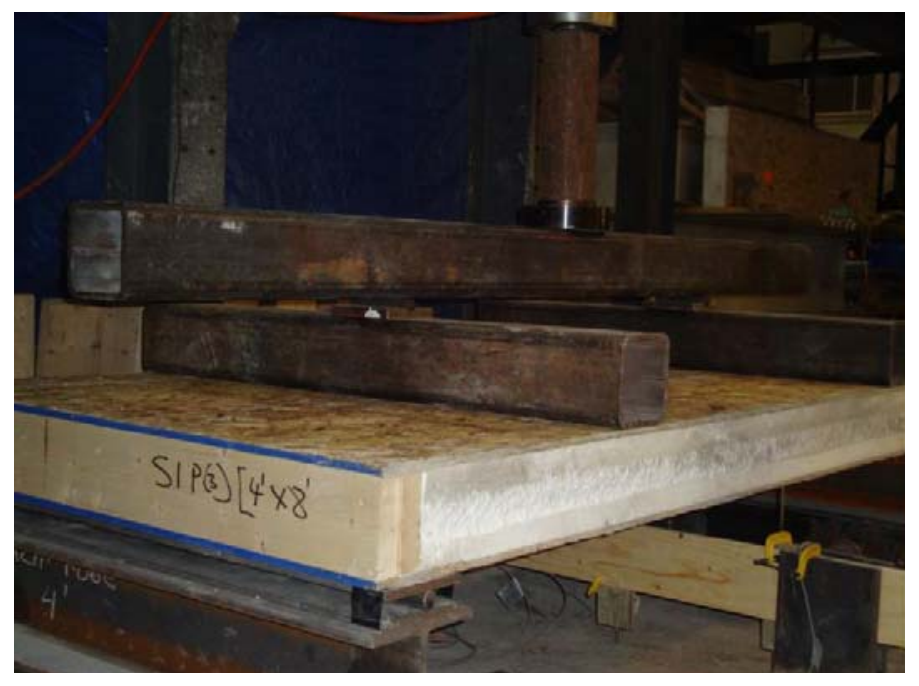

Figure 3.21 - Typical test setup for flexure of the SIP floor 
Mid-span deflection was measured using four linear variable displacement transducers (LVDTs). These LVDTs were positioned underneath the panel, with two LVDTs were located at $25 \mathrm{~mm}$ from the panel free edges and other two LVDTs located at the third points of the panel width. Figure 3.23 shows view of the LVDTs installed at mid-span location under the panel. Figure 3.24 also shows the detail of test setup for flexure of the SIP floor. The load was applied through a jacking load system with a universal flat load cell of $222 \mathrm{kN}(50,000 \mathrm{lb})$ capacity. During each test, the process for collecting and converting data captured by the LVDTs and load cell was done using test control software (TCS) with a SYSTEM 5000 data acquisition unit which was adjusted to sample the data at rate of 10 reading per second during the loading test.

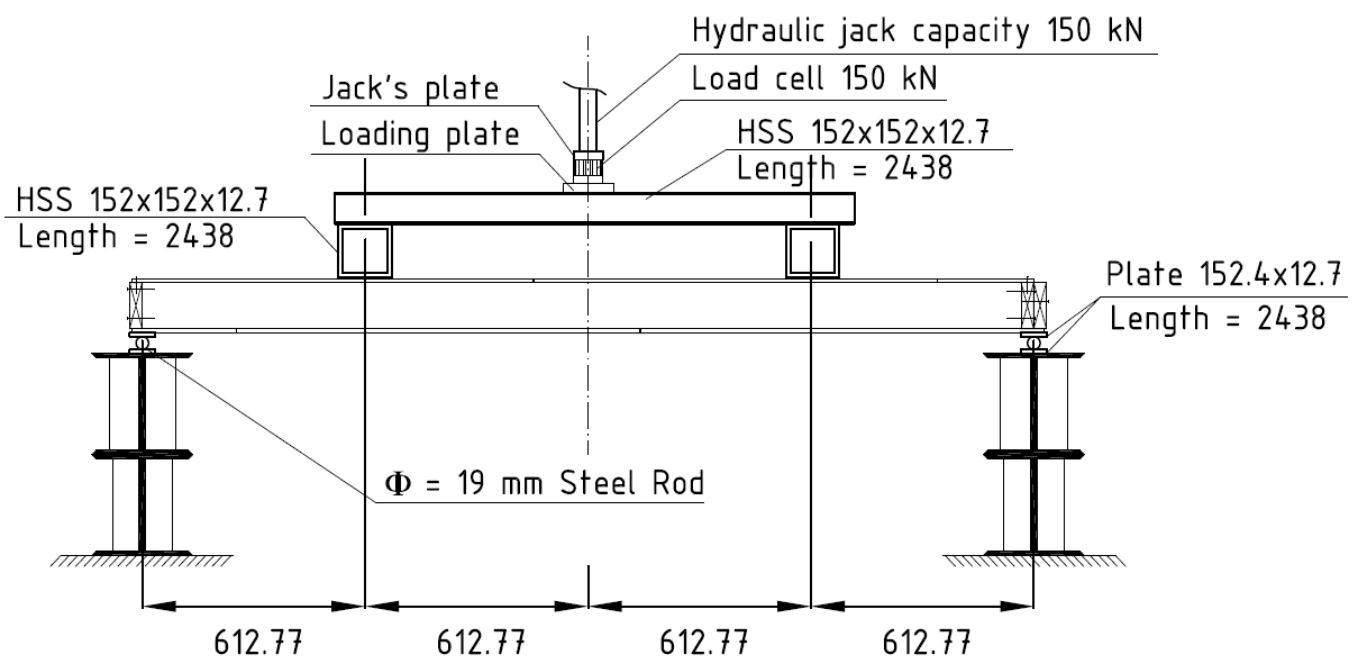

Note: All dimensions are in milimeter.

Figure 3.22 - Schematic side view of the setup for flexure of the SIP floor

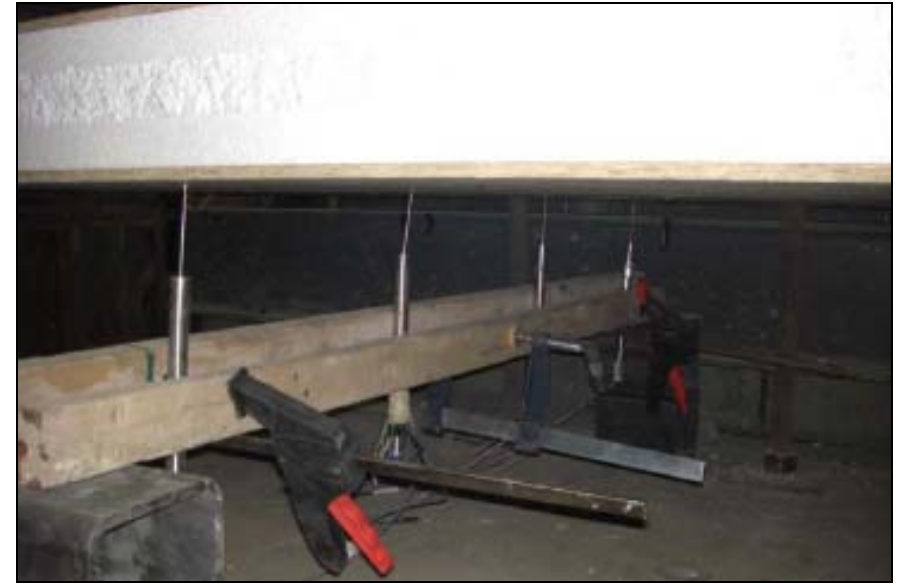

Figure 3.23 - View of LVDTs at mid-span location of SIP floor 


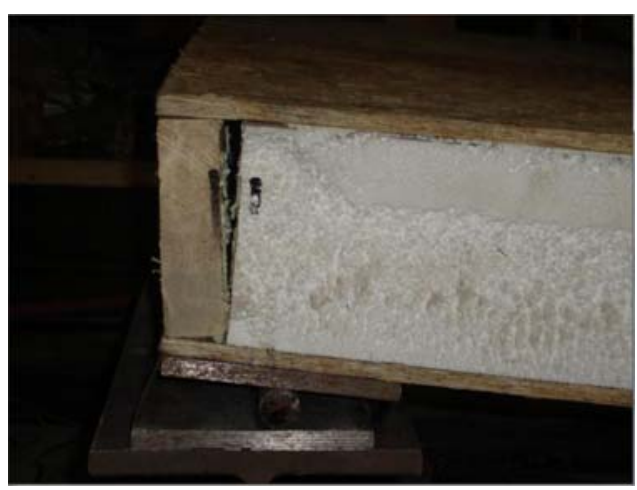

(a) Support condition

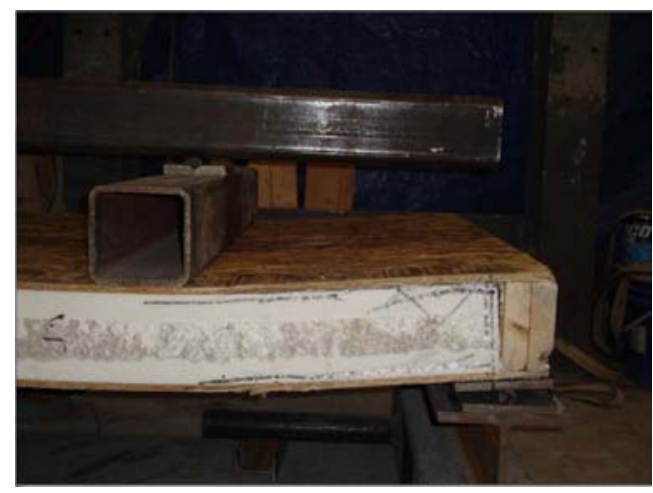

(b) Loading system on panel

Figure 3.24 - Details of test setup for flexure of the SIP floor

\subsubsection{Test Procedure for SIP in Flexure}

ASTM E72-10 (2010) recommends using "two-point" loading for transverse load tests. The specimen should be tested as a simple beam on a $150 \mathrm{~mm}$ span (6 inch) less than the specimen length. The load is applied on two equal portions toward the middle of the span and at a distance of one quarter of the span from support. The weight of specimen between the supports shall be considered when the wall specimens tested horizontally. The transverse loads to the outside of the face for each specimen is applied. The number of the three specimens should be considered for transverse load test.

ASTM E2322-03 (2009) also provides the following consideration for apply the load to each individual specimen in increments so that a sufficient number of readings will be obtained to determine definitely the load-deformation curve using the following sequence:

1. A small initial load about $5 \%$ smaller than the expected ultimate load capacity should apply, hold for $5 \mathrm{~min}$ and then release before starting the test. All the devices for measuring should set to zero before the beginning of the test.

2. The initial load and deformation of the specimen should be recorded and then the load on the specimen will increase to the first pre-determined increment.

3. The load and deformation reading is recorded and the load will release back to the initial load. Then the new set of recording should be started. 
4. The load will be increased to the next increment and the information will be recorded as indicated in the above steps. The reading of all load increments will be recoded up to final load capacity.

5. The load level should maintain as constant as possible for a period of 5 min after each load increment.

6. The initial and 5-min readings should plot in the form of load-deformation curves.

ASTM E1803-06 (2006) describes that following modification on ASTM E72-10, Section 11 for SIP under transverse load:

1. The support condition should be considered as close as to actual construction.

2. The applied load to specimens should be incremental.

3. The deflection of the specimen must be recorded at initial load and after each increment of load.

4. The load must be decreased to the initial load after $0.75,1.50$ and 2.0 times of the expected design load is accomplished. The incremental load is continued and the deflection at each load should be recorded until there is no risk of measuring device damage.

5. The load is continuously increased until the maximum load is achieved.

Flexural tests were performed in the structures laboratory of Ryerson University. The test set-up was prepared for each test as explained earlier. For each panel, jacking load was applied in increments so that visual inspection could be performed to record any change in structural integrity of the sandwich panel. The tests were terminated after panel failure when the jacking load was not increasing while panel deflection was increasing by continuous pressing of the pump handle. At that stage, failure mode was observed and test data was then used to draw the load-deflection relationships for each panel.

\subsection{Testing SIP in Axial Compression Loading}

\subsubsection{Test Method for SIP in Compression}

The objective of this set of testing is to provide design tables of wall panels in the form of factored design resisting line load. These design tables will assist in establishing the maximum 
span of joists of roof/floor panels or the maximum span of SIP floor or roof served by SIP wall as based on different snow load values (i.e. 1.0, 1.5, 2.0, 2.5 and $3.0 \mathrm{kPa}$ for example). For the purpose of structural qualifications of SIPs, the Canadian Construction Materials Commission (CCMC) produced a technical guide (IRC, 2007) in collaboration with the National Research Council Canada (NRC) to describe the technical requirements and performance criteria for the assessment of stressed skin panels (with lumber $1200 \mathrm{~mm}$ o.c. and EPS core) for walls and roofs. In this guide, the performance of the stressed skin panels for walls and roofs, have been evaluated, as an alternative solution, with respect to Part 4, Structural Design, and Part 9, Housing and Small Buildings, of the National Building Code of Canada (NBCC, 2005).

The technical guide focuses on structurally qualifying the stressed skin composite panels to be as good as the structural capacity of the conventional wood-frame buildings. CCMC published a report to complete this technical guide through a successful evaluation. Only the products have the proper identification number of CCMC's evaluation number can be applied in published CCMC evaluation report. This NRC/IRC/CCMC Technical Guide specifies test methods for SIPs which is similar to those specified in ASTM E72-10, Standard Test Methods for Conducting Strength Tests of Panels for Building Construction, (ASTM, 2010) as well as ICC AC04, Acceptance Criteria for Sandwich Panels, (2004). The ICC AC04 acceptance criteria are based on ASTM E72-10 standard test methods. The ANSI/APA PRS-610.1 (2013), Standard for Performance-Rated Structural Insulated Panels in Wall Applications, published by APA - The Engineered Wood Association in USA, provides similar structural qualification procedure and criteria for the performance-rated SIPs to those in ASTM E72-10 and ASTM E1803-06, Test Methods for Determining Structural Capacities of Insulated Panels. ASTM E72-10 specifies at least three identical specimens for each test group.

\subsubsection{Test Setup for SIP in Compression}

The SIP wall specimen was aligned vertically and supported directly over the laboratory's floor to prepare the test setup. A uniformly distributed line load was applied on the top side over the $1220 \mathrm{~mm}$ width using a loading assembly. This loading assembly was composed of a $1220 \times 350 \times 12.7 \mathrm{~mm}$ steel base plate resting over the top side of the panel. A $150 \times 150 \times 12.7 \mathrm{~mm}$ HSS box beam of length $1220 \mathrm{~mm}$ was welded to the top side of the steel base plate to transfer 
the applied jacking load over the panel width. Two $125 \times 125 \times 12.7 \mathrm{~mm}$ steel angles of $1220 \mathrm{~mm}$ length were welded to the steel base plate, one on each side of the wall panel to stabilize the loading assembly during the test. The weight of the loading assembly was calculated as $3.34 \mathrm{kN}$. Figure 3.25 indicates the schematic test setup for the eccentric compressive load test which was used for specimen SIP-A-1 to SIP-A-3. Figure 3.26 also shows the typical test setup for flexure eccentric compressive load of the SIP wall in structural lab. The location of POTs in these specimens was in top two sides of the specimen and in the middle of the panel in order to determine the deflection in top and middle of the panel.

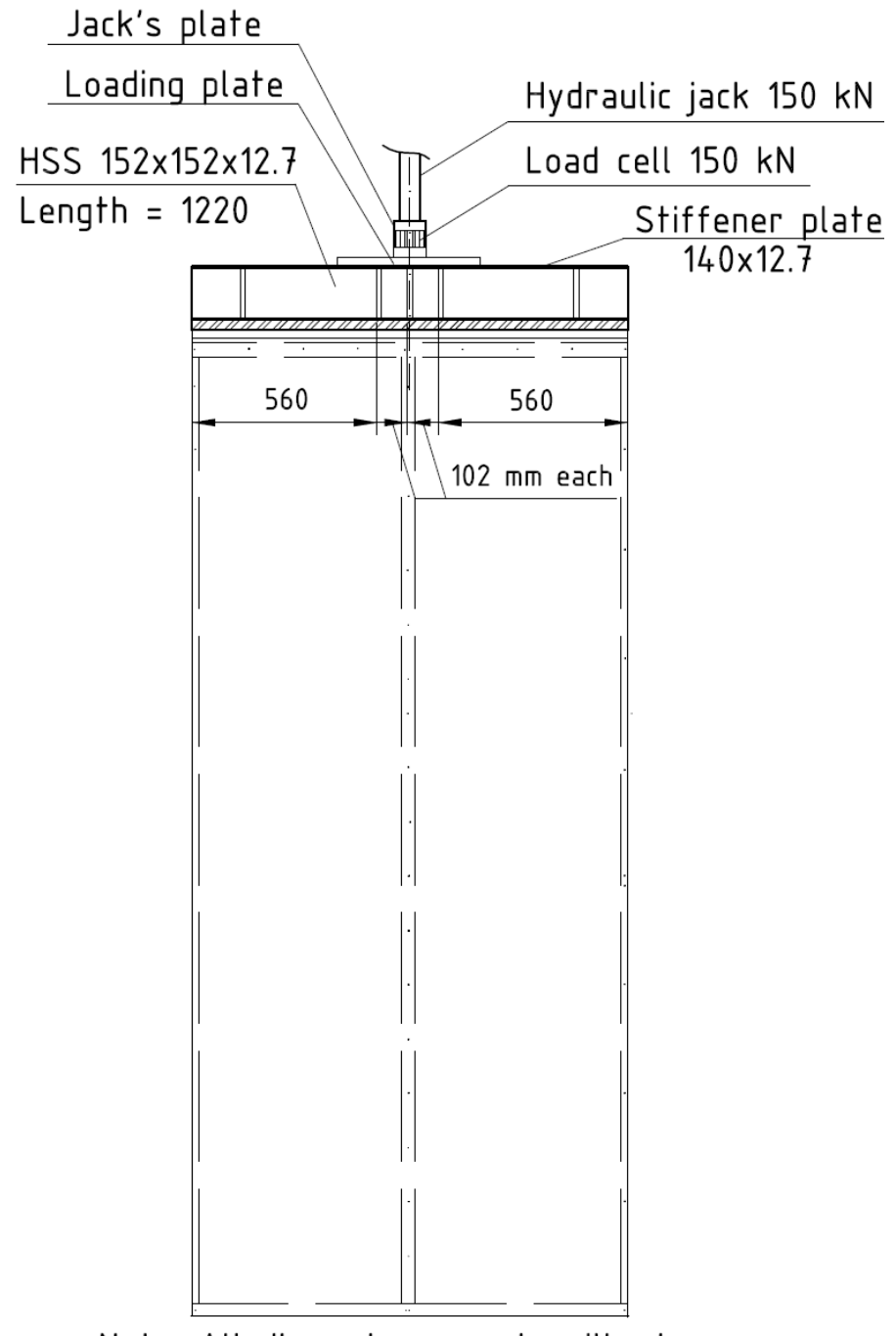

Note: All dimensions аге in milimeter.

(a) Elevation

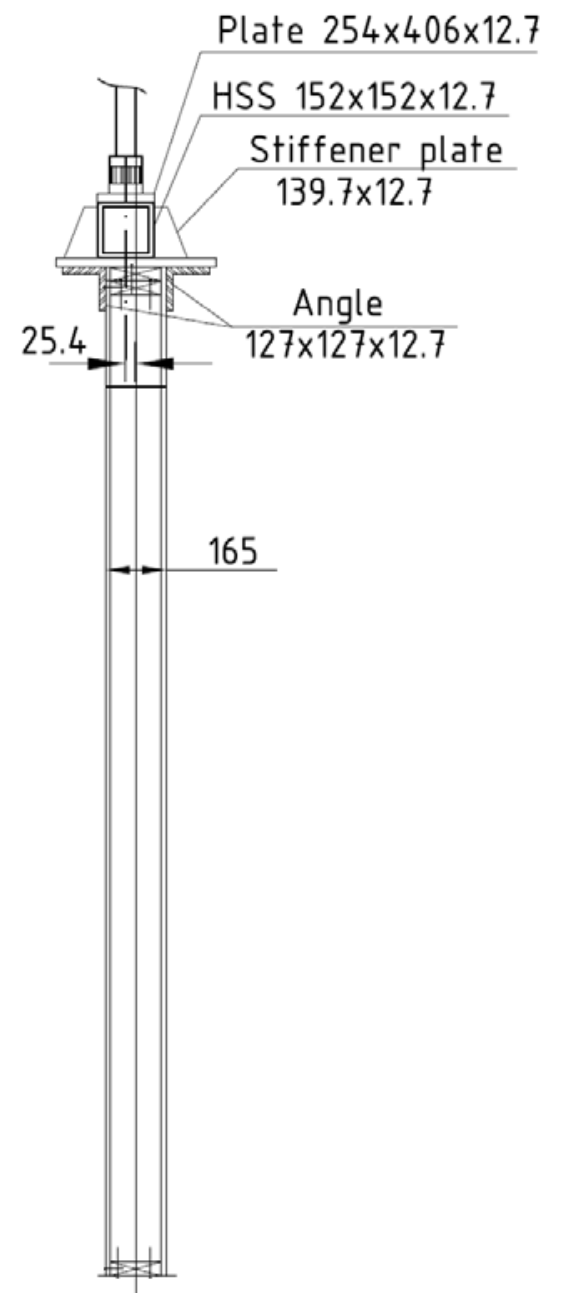

(b) Cross-section

Figure 3.25- Schematic test setup for the eccentric compressive load test on SIP wall 


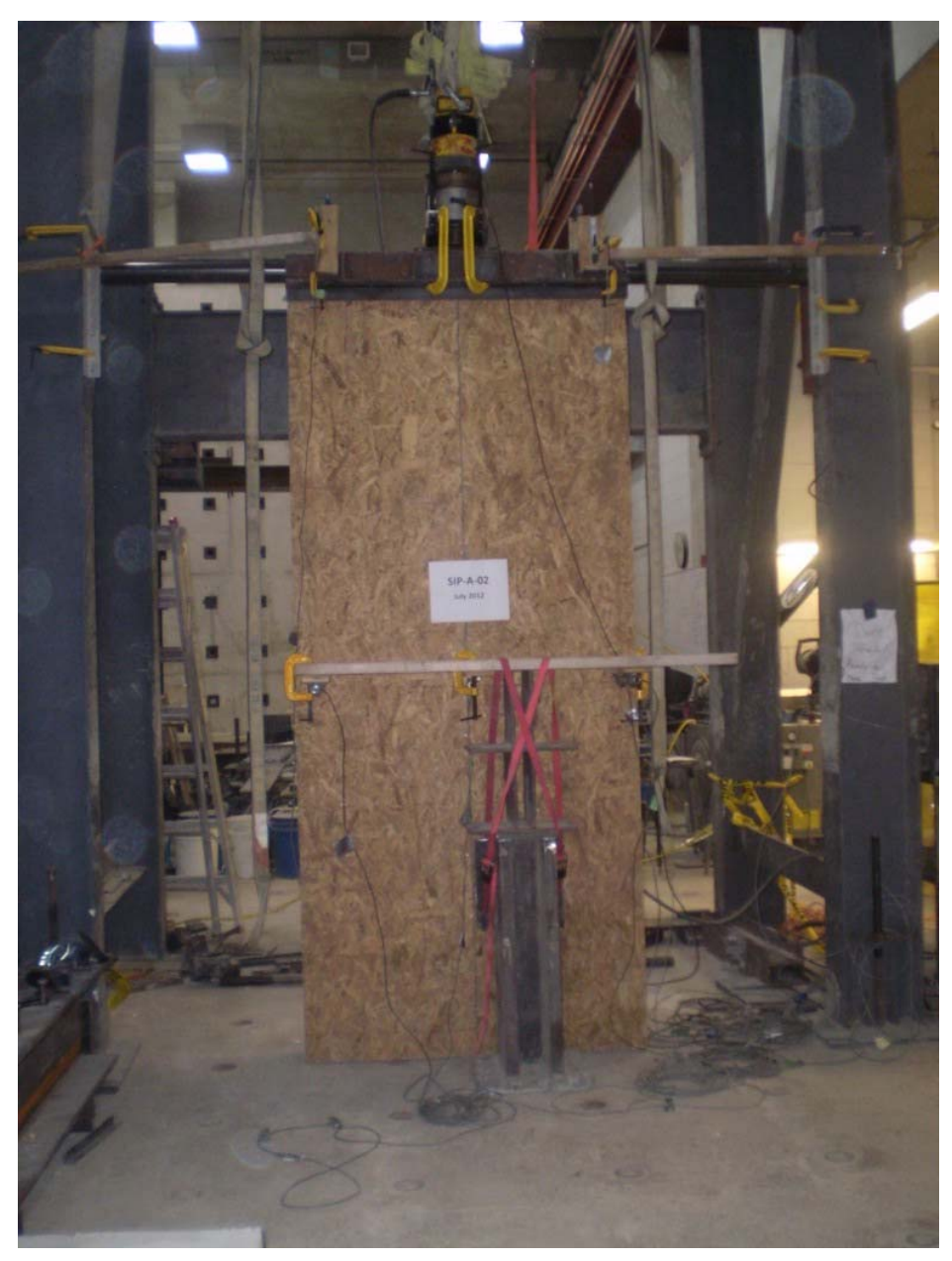

Figure 3.26 - Typical test setup for flexure eccentric compressive load of the SIP wall

Figure 3.27 shows the details of the top rail used to distribute the compressive loading over the SIP wall width. Figure 3.28 also shows the top portion of the SIP wall showing restraint system for lateral movement at top of the stud wall.

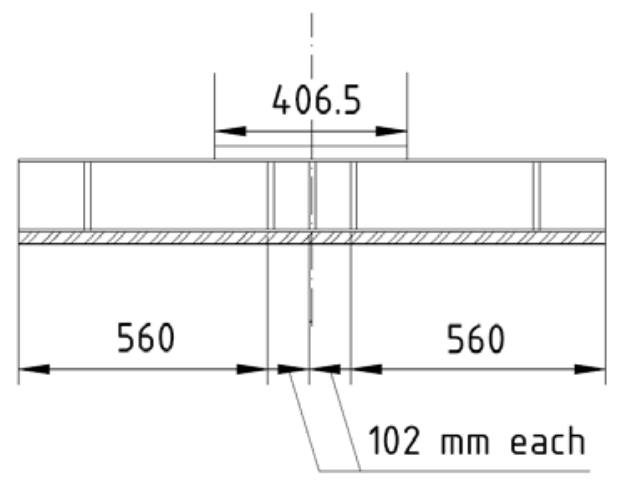

Note: All dimensions are in milimeter.

(a) Top rail elevation 


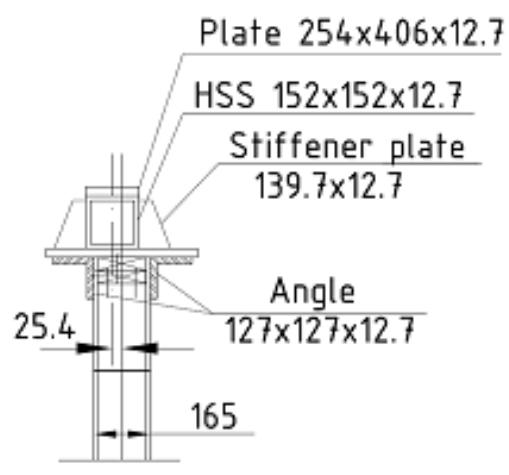

(b) Cross-section in top rail

Figure 3.27 - Details of the top rail used to distribute the compressive loading over the SIP wall width

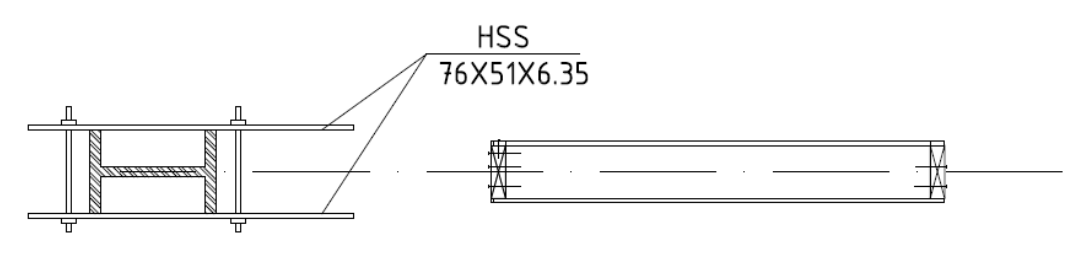

Note: All dimensions аге in milimeter.

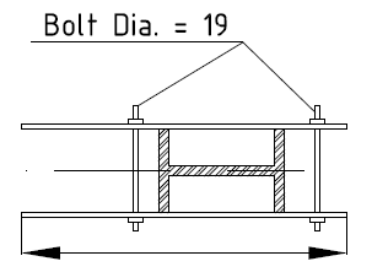

863.5

Figure 3.28 - Cross-section of the top portion of the SIP wall showing restraint system for lateral movement at top of wall

Three potentiometers (POTs) were used to measure horizontal displacement at the mid-height of the specimen. Four potentiometers (POTs) were installed vertically over the four corners on the top side of the specimen to record axial shortening of the wall panel under load. Figure 3.29 shows the detail of test setup for compressive eccentric load on the SIP wall. The compressive load was applied through a jacking load system with a universal flat load cell of $444 \mathrm{kN}(100,000$ lb) capacity to measure the jacking load. During testing, the process for collecting and converting data captured by the LVDT's, POTs and load cell were done using a test control software (TCS) with SYSTEM 5000 data acquisition unit which was adjusted to sample the data at rate of 10 reading per second during the test. 


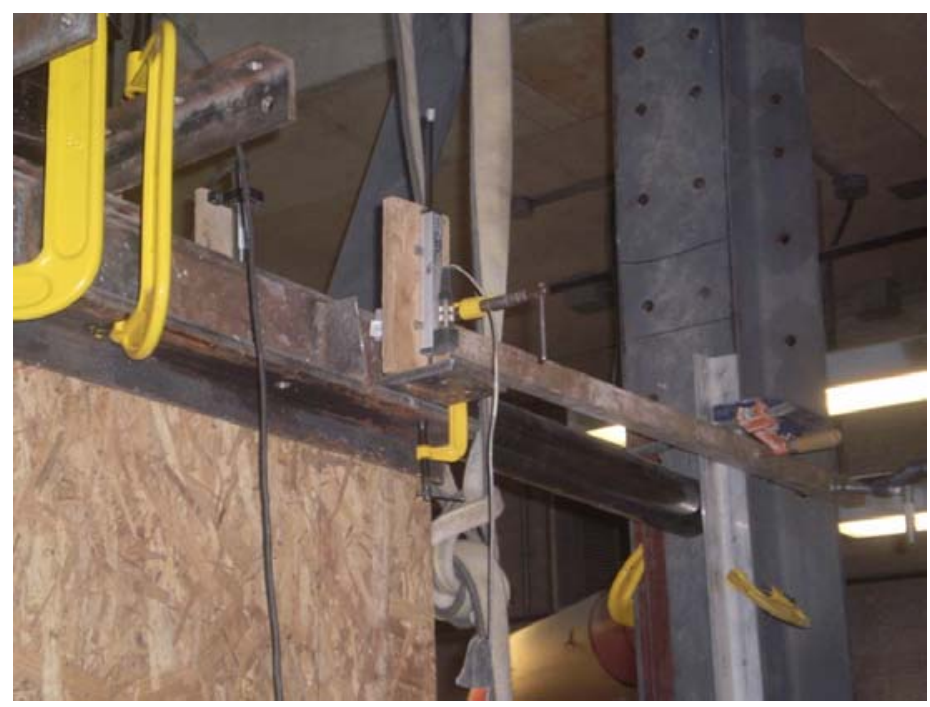

(a) Top corner of the panel

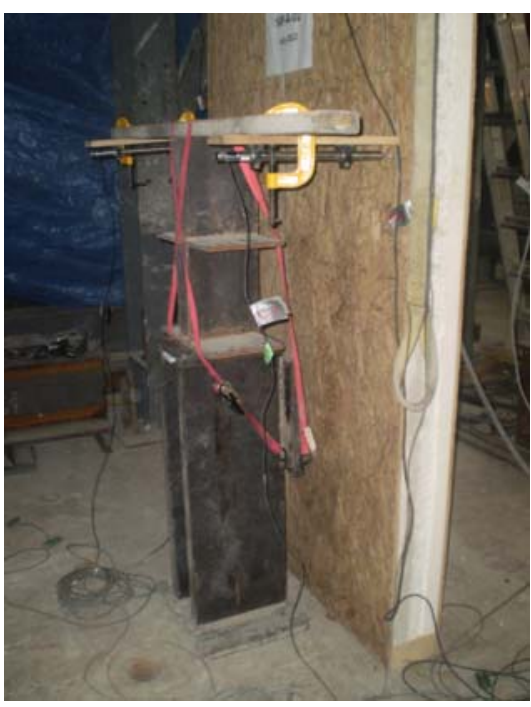

(b) Bottom and POT condition

Figure 3.29 - Details of test setup for eccentric compressive load testing on the SIP wall

\subsubsection{Test Procedure for SIP in Compression}

ASTM E72-10 (2010) recommends that compression test on specimen as column should be done on a flat end at the bottom. The compressive load should apply to a steel plate at the upper end of the specimen. The load has to apply uniformly along a line parallel to the inside face of specimen. The load must apply on one-third of the thickness of the specimen from the inside face. The movement of the testing machine crosshead should be nominally $0.8 \mathrm{~mm} / \mathrm{min}(0.03$ inch/min) for load rate.

ASTM E1803-06 (2006) describes that following modification on ASTM E72-10, Section 11 for SIP under axial compressive load:

1. The manufacturer's requirements shall be applied for bearing condition at the bottom of the SIP wall.

2. A minimum of one gage shall be mounted for axial displacement measurement at midwidth of specimen. It is also required to mount a minimum of one gage for transverse displacement at mid-height of specimen.

The tests were performed in the structures laboratory of Ryerson University. The test set-up was prepared for each test which included installing the POTs at the predetermined locations. For 
each specimen, the jacking load was continuously at a slow rate. Visual inspection was continuously conducted during the test record any change in the structural integrity of the wall specimen. Each test was terminated after the wall specimen failure. Failure of the specimen was considered when the recorded jacking load was not increasing or when the specimen could not absorb more loads while recorded axial shortening was increasing by continuously pressing the pump handle. Mode of failure was recorded and test data was then used to draw the loaddeflection and load-axial shortening relationships for each panel.

\subsection{Testing SIP under Racking and Cyclic Loading}

\subsubsection{Test Method for SIP in Racking and Cyclic}

The objective of this test was to establish the factored design lateral capacity of selected wall panels that would further be uses with the obtained factored design lateral load to determine either the factored lateral load can safely be applied on the wall panels. Racking load qualification tests on the panels were conducted as specified in the method described in the ASTM E564-06, Standard Practice for Static Load Test for Shear Resistance of Framed Walls for Buildings, ASTM E72-10, Standard Test Methods of Conducting Strength Tests of Panels for Building Construction, and AC 130-07, Acceptance Criteria for Prefabricated Wood Shear Panels. Cyclic load qualification tests on the panels were conducted as specified in the method described in the ASTM E2126-11, Standard Test Methods for Cyclic (Reversed) Load Test for Shear Resistance of Vertical Elements of the Lateral Force Resisting Systems for Buildings. These standards specify at least three identical specimens for each test group.

\subsubsection{Test Setup for SIP in Racking and Cyclic}

Figure 3.30 shows the schematic diagram of the test setup for the lateral load test which will use for specimen SIP-R-1 to SIP-R-3, SIP-R-4 to SIP-R-6, SIP-C-1 to SIP-C-3 and SIP-C-4 to SIPC-6. Figure 3.31 also shows the typical test setup for monotonic and cyclic lateral load on the SIP wall. These tests will conduct under monotonic (racking) and cyclic loading condition. The location of POTs in these specimens will be in top two sides of the specimen and in the middle of the panel in order to determine the deflection in top and middle of the panel. Figure 3.32 shows the details of the base and different section of test setup for monotonic and cyclic lateral 
load for the SIP wall. Figure 3.33 also shows the boundary conditions of top and bottom of SIP wall under monotonic and cyclic lateral load.

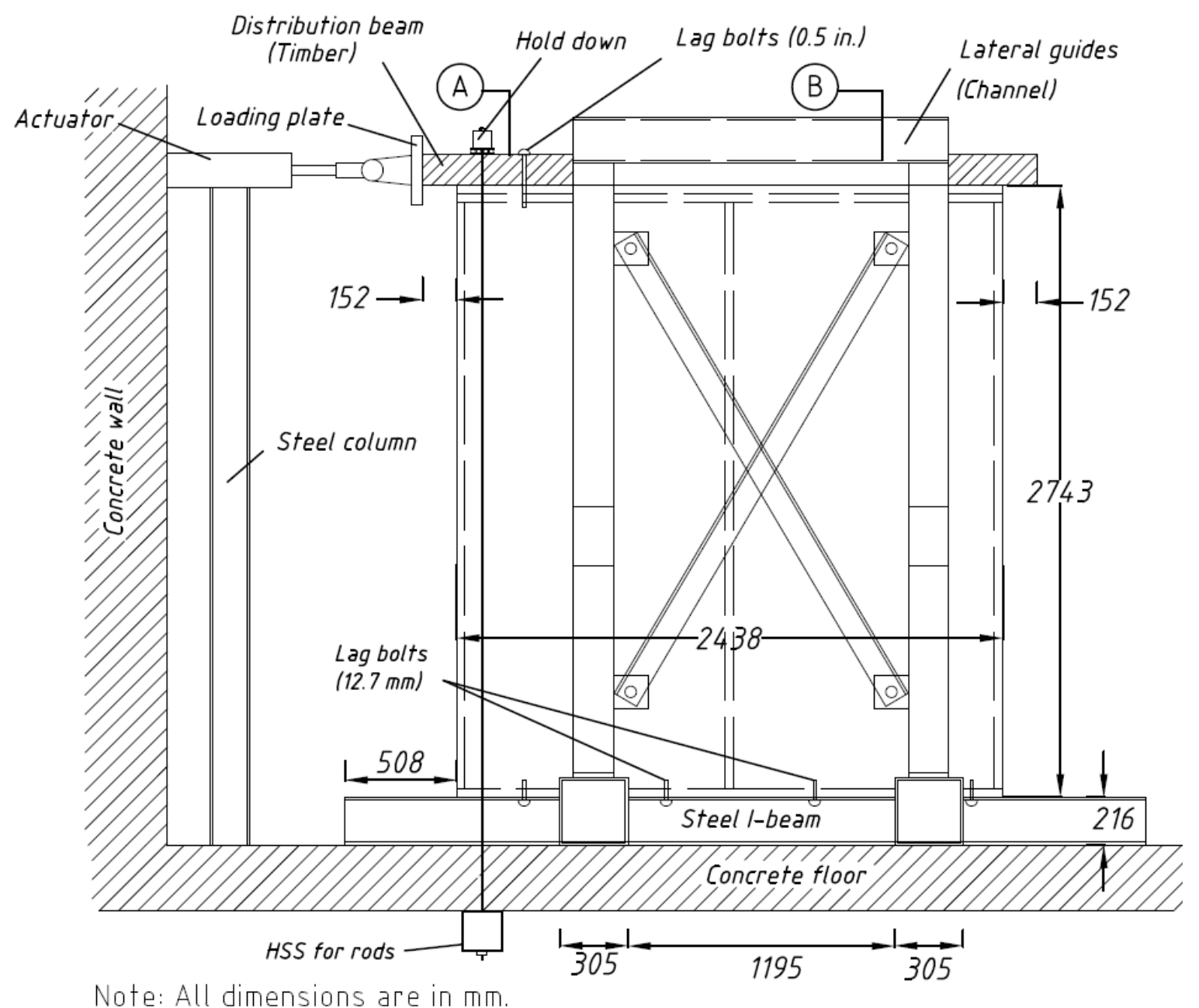

Figure 3.30 - Schematic elevation of the test setup for monotonic and cyclic lateral load for the SIP wall 


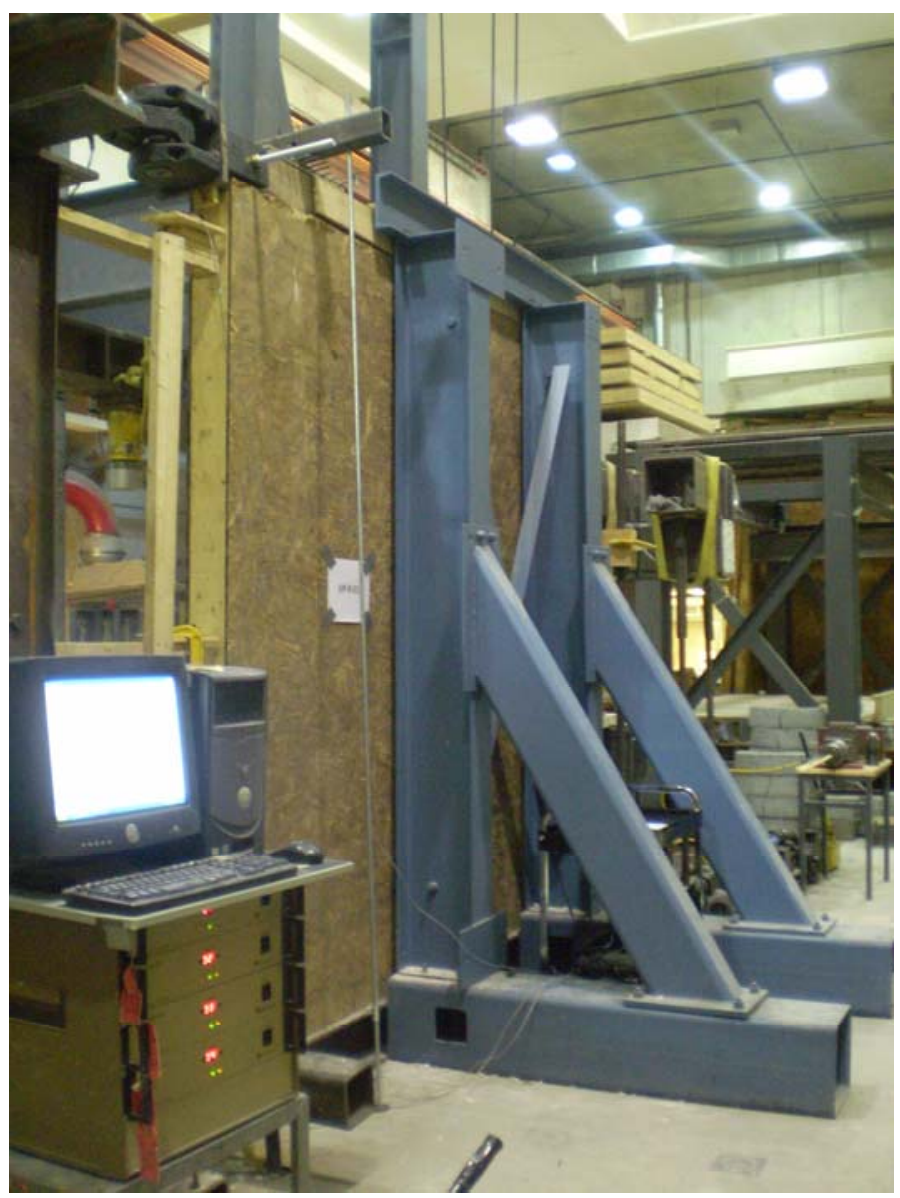

Figure 3.31 - Typical test setup for monotonic and cyclic lateral load testing on the SIP wall

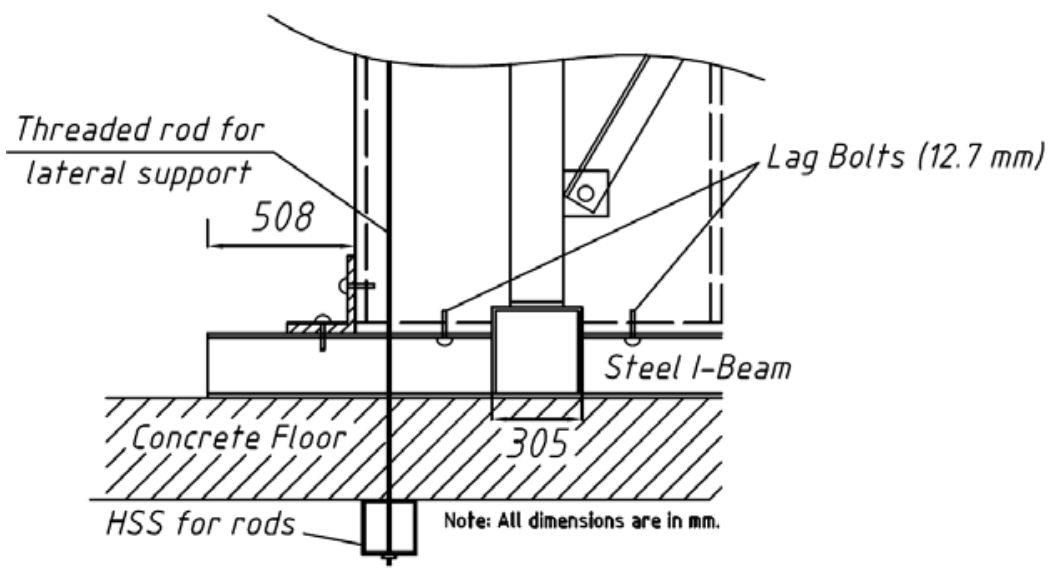

(a) Detail of support 

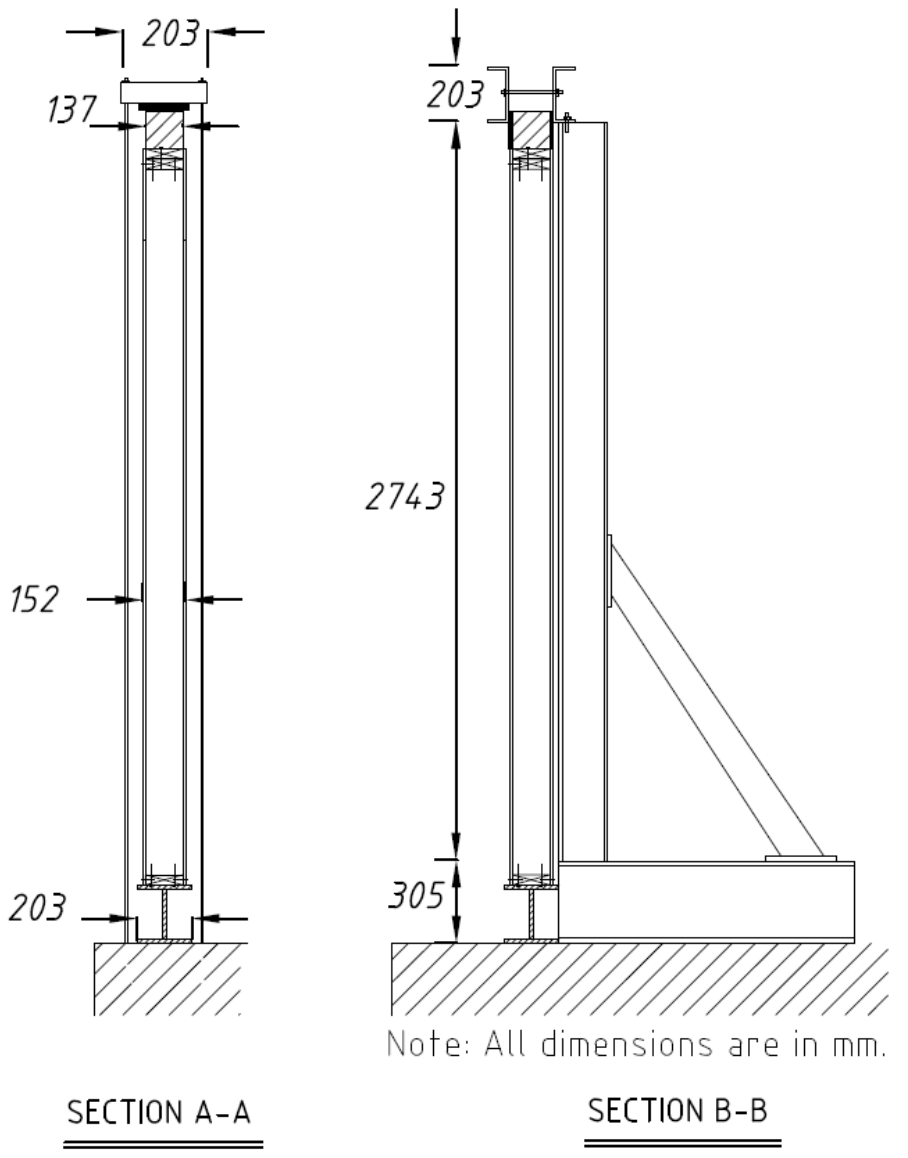

(b) Test setup cross-sections

Figure 3.32 - Details of the base and different section of test setup for monotonic and cyclic lateral load for the SIP wall

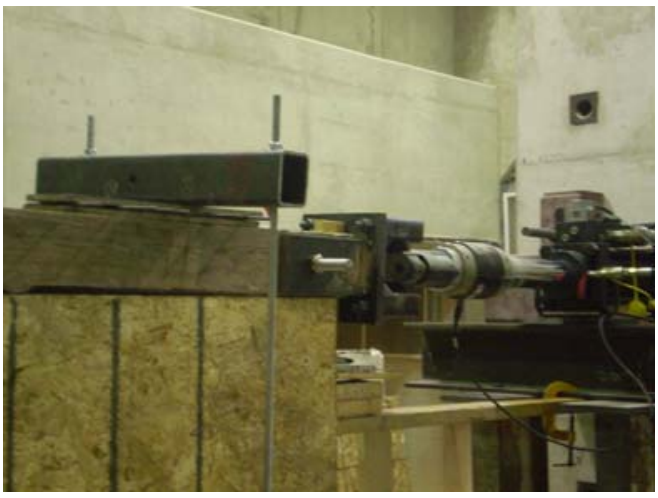

(a) Boundary condition on top of wall

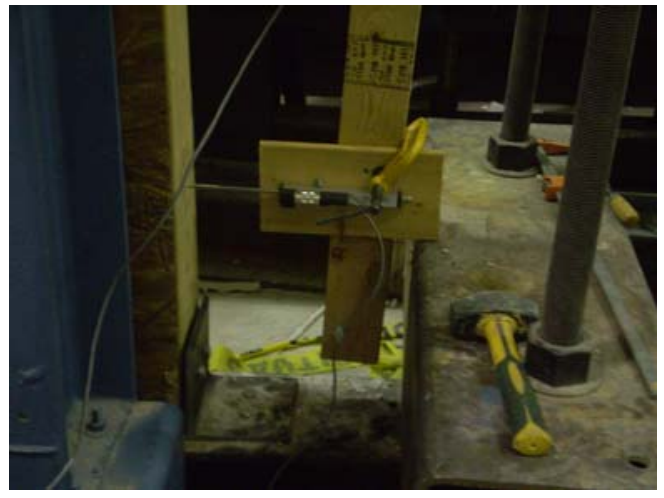

(b) Steel angle at base of the wall

Figure 3.33 - Details of boundary conditions at top and bottom of SIP wall under monotonic and cyclic lateral load 


\subsubsection{Test Procedure for SIP in Racking and Cyclic}

\subsubsection{Monotonic (Racking) Load}

Monotonic tests are conducted according to ASTM E564-06 (ASTM, 2006) test protocol. The ASTM E564-06 protocol needs that ultimate load be reached in no less than 5 minutes. The following steps must be done during the racking load test:

Step 1: The frame stiffness should determine prior to the application of the web elements. The frame is loaded in a manner similar to that intended for the completed panel. If the frame is a "standard" frame, the stiffness of the frame should be determined.

Step 2: The procedure for complete panel loading is performed. The loads apply to the panel parallel to and the top of the wall though a beam which is attached to the top plate of panel. The rate of load has to choose such that the anticipated full design load level will be reached in not less than $10 \mathrm{~min}$.

Step 3: In order to establish the load-deformation curve prior to failure, at least ten sets of uniformly spaced deflection readings should provided. Load and deformation reading must be recorded in a proper rate. Hydraulic jacks or actuator can apply the load to specimen and have been calibrated prior to test. The load also can apply by any other suitable types of loading apparatus. If the weight of loading system affects the results, it should be separately consider.

Step 4: Dial gages or other suitable devices are measured the deformation to provide an acceptable load-deformation curve. The deflection should be recorded to the nearest 0.25 $\mathrm{mm}(0.01$ in. $)$.

Step 5: Load-measuring apparatus shall be record the load and deflection of top of the wall. When approximately reaching to one third and two thirds of the estimated ultimate load, the load can be removed and the panel recovery should record after $5 \mathrm{~min}$.

Step 6: Maintain a record of the total length of time the panel is under load.

ASTM E72-10 (2010) recommends the several considerations during the monotonic lateral loading on stud wall. The size of the specimens should be $2400 \times 2400 \mathrm{~mm}$ (8x8 feet) and the load has to apply to the panel through a constructed frame as it mentioned in test setup section. The minimum of three specimens should be used for the racking test. When the panel is assembled 
the minimum moisture contact shall be between 12 and $15 \%$ and the variation shall not be more than $3 \%$ from the initial moisture content.

ASTM E72-10 (2010) also recommends that the load on specimen should apply continuously at a uniform rate of motion of the loading device. The speed of loading is recommended in a way that the total load of $3.5 \mathrm{kN}$ (790 lbf) shall be applied more than 2 minute from starting of the test. The interval load rate of $3.5 \mathrm{kN}$ shall apply to the specimen in different values of 7.5 to 10.5 $\mathrm{kN}$ (1570 to $2360 \mathrm{lbf}$ ) total load and to failure of the specimen. Therefore, load on specimen will apply in three stages to 3.5, 7.0 and $10.5 \mathrm{kN}(790,1570$ and $2360 \mathrm{lbf})$ total load at a uniform rate. After each stage, the load on specimen should be removed and all the residual deflection shall return to zero, then the next stage should be started. After these cycles of loading and unloading on $3.5,7.0$ and $10.5 \mathrm{kN}$, the specimen shall be loaded until the failure or $100 \mathrm{~mm}$ (4 inch) of total deflection is happened.

ASTM E1803-06 (2006) has some modification for SIP wall in accordance with ASTM E72-10, Section 14 as following:

1. The attaching the SIPs together and to the top and bottom plate shall be followed by manufacturer's method. The load shall be applied through a load beam to the top plate of the SIP.

2. The minimum size of the SIP specimens shall be $2400 \times 2400 \mathrm{~mm}(8 \times 8$ feet $)$ consisting of a minimum of two $1200 \times 2400 \mathrm{~mm}(4 \times 8$ feet $)$ SIPs.

3. The type and spacing of fastener or adhesives shall be the same as actual construction and are required by the manufacturer. The ends of SIP panel shall be assembled per manufacturer's requirement and act as boundary members.

\subsubsection{Cyclic Loading}

CUREE protocol for ordinary ground motions is used on cyclic tests. This protocol was provided by CUREE-Caltech Wood frame project (Krawinkler, et al., 2001) as shown in Figure 3.34. The CUREE protocol consider a set of primary cycles which displacement amplitudes followed by 2 to 5 trailing cycles at $75 \%$ of the primary cycle amplitude. The reference displacement $\left(\Delta_{\text {ref }}\right)$ is determined from monotonic testing. The displacement amplitude of each cycle is proportional to 
this reference displacement. The $80 \%$ of the ultimate load is selected and $60 \%$ of the displacement after ultimate is calculated as reference displacement. The post peak behavior of the test specimen is depended on reference displacement. Langlois et al. (2004) conducted several tests and using the CUREE test protocol to show insensible small changes in the reference displacement.

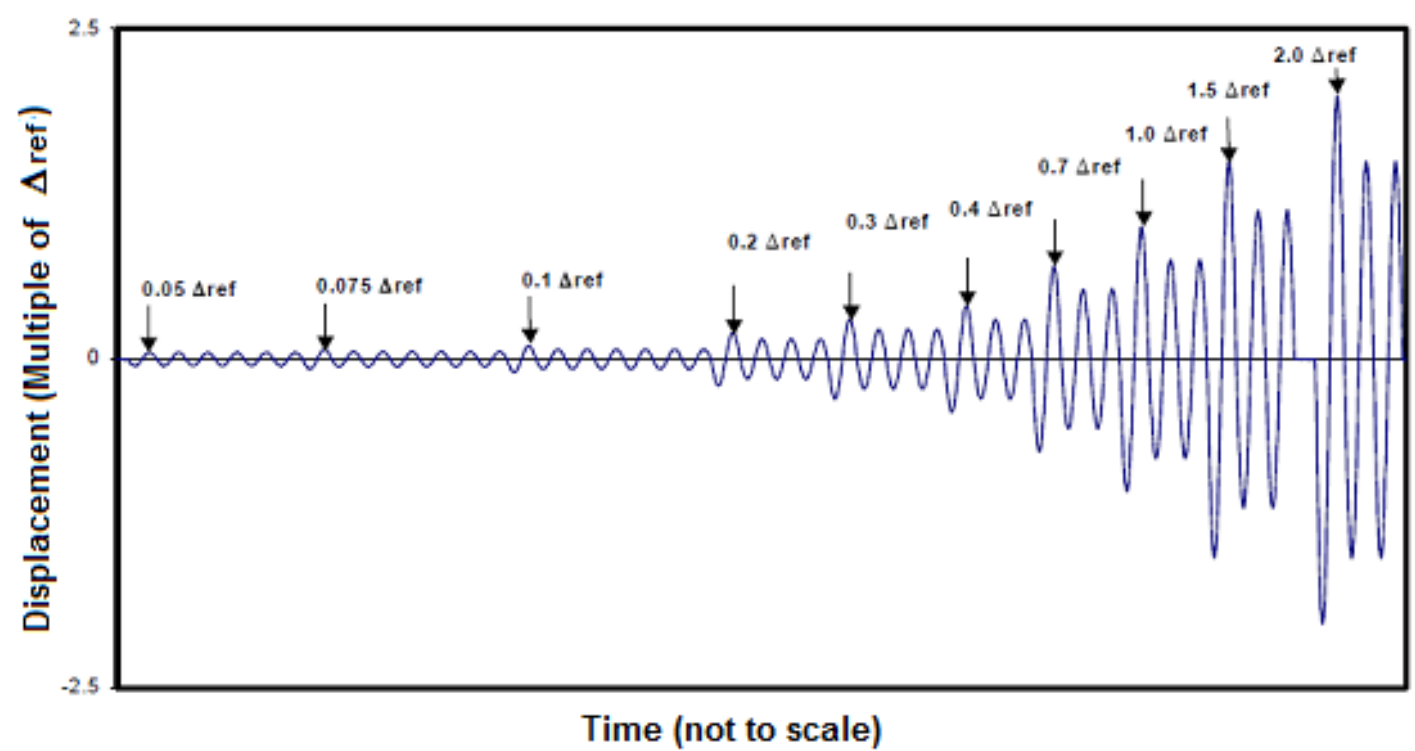

Figure 3.34 - CUREE Protocol for displacement control (Krawinkler et al., 2001)

The displacement amplitudes have a sequence of increasing value. It is started gradually up to $0.4 \Delta_{\text {ref }}$ and then changing to larger steps. The statistical analysis of inelastic time histories can provide the increment of larger step. These steps are selected in order to model the structural behavior of a panel during an actual seismic event (ASTM, 2011). The cyclic load is applied to specimens up to a certain level of displacement and increasing at $0.5 \Delta_{\text {ref }}$ per increment. The amplitude is increasing continuously up to $1.5 \Delta_{\text {ref. }}$ Each group of cycles has to separate by a short pause of 15 seconds to control the stop of the test when a wall collapses.

ASTM E2126-11 (2011) recommend three method for cyclic load on shear wall namely, Method A, B and C. The Test Method C was used in this research and it has presented as following:

1. The loading procedure shall be applied with displacement control and involved the group of displacement cycles at incremental increasing displacement level. The six series initiation cycles of equal amplitude is started in loading history. In addition, a primary 
cycle with amplitude as a percent of the reference deformation and consequent trailing cycles with $75 \%$ amplitude of the primary one is applied on each phase of loading history.

2. The reference deformation is an estimation of maximum displacement which is appropriate with $0.8 P_{\text {peak }}$ load in a primary cycle.

3. The reference deformation is described in Table 3 and shall be less than 0.025 times the height of wall. Additional phases of cycles will be applied to the specimen if the panel has not failed at the end of Phase 8 of Table 3.7. An increase in amplitude of $\alpha(\alpha \# 0.5)$ over the previous primary cycle shall be considered on a primary cycle and two trailing cycles with $75 \%$ amplitude of the primary one are pursued.

4. A constant cyclic frequency or constant rate of displacement can be chosen for actuator displacement. The displacement rate must be between 0.04 and $2.5 \mathrm{in} . / \mathrm{s}$ (1.0 and 63.5 $\mathrm{mm} / \mathrm{s})$. In order to prevent inertial effects of the mass of the wall the cyclic frequency must range from 0.2 to $0.5 \mathrm{~Hz}$ during cyclic loading. The procedure of loading shall follow until the applied load reduces more than $0.2 P_{\text {peak }}$ or failure limit state occurs.

5. The displacement measurement device must have a resolution of 0.005 inch $(0.13 \mathrm{~mm})$ and the sampling rate of displacement shall be 100 reading per cycle. 
Table 3.7 - Amplitude of primary cycles in Test Method C (ASTM, 2011)

\begin{tabular}{|c|c|c|c|}
\hline Pattern & Step & $\begin{array}{c}\text { Minimum No. of } \\
\text { cycles }\end{array}$ & $\begin{array}{c}\text { Amplitude of primary } \\
\text { cycles }(\% \mathbf{~} \boldsymbol{\Delta})\end{array}$ \\
\hline 1 & 1 & 6 & 5 \\
\hline 2 & 2 & 7 & 7.5 \\
\cline { 2 - 4 } & 3 & 7 & 10 \\
\hline \multirow{2}{*}{3} & 4 & 4 & 20 \\
\cline { 2 - 4 } & 5 & 4 & 30 \\
\hline \multirow{3}{*}{4} & 6 & 3 & 40 \\
\cline { 2 - 4 } & 7 & 3 & 70 \\
\cline { 2 - 4 } & 8 & 3 & 100 \\
\cline { 2 - 4 } & 9 & 3 & $100 \alpha$ (until specimen failure) \\
\cline { 2 - 4 } & 10 & 3 & Additional increments of \\
& & &
\end{tabular}

Note: $\alpha<=0.5$

\subsection{Testing Stud in Flexure (Transverse) Loading}

\subsubsection{Test Method for Stud in Flexure}

The objective of this set of testing is to experimental data for the ultimate load carrying capacity and serviceability limits for correlation with those obtained from SIP tests to investigate whether SIPs can perform as good as stud panels as loading carrying elements in residential construction. As such, identical test setup to that used for SIP testing was utilized in stud panel testing. The main difference was that the stud wall specimens were of $2400 \mathrm{~mm}\left(8^{\prime}\right)$ width. Flexural tests on the specimens were conducted as specified in the method described in the ASTM E72-10, Transverse Load Test, and ASTM E2322-03, Standard Test Method for Conducting Transverse and Concentrated Load Tests on Panels used in Floor and Roof Construction. ASTM E72-10 specifies at least three identical specimens for each test group. 


\subsubsection{Test Setup for Stud in Flexure}

Each tested panel was supported over two $25.4 \mathrm{~mm}$ steel rollers at each side in the short direction. $2400 \times 150 \times 12 \mathrm{~mm}$ steel plates were inserted between the steel rollers and the supporting steel pedestal resting on the laboratory strong floor. Other similar-size steel plates were inserted between the supporting roller and the panel bottom facing. A $150 \times 150 \times 12.7 \mathrm{~mm}$ HSS beam of $2400 \mathrm{~mm}$ length used to transfer the applied jacking load to a $102 \times 1020 \times 6.4 \mathrm{~mm}$ HSS beam that was laid transversally over the top panel facing at the quarter points to spread the load over the panel width. Steel roller and plate assembly similar to that used to support the panel over the steel pedestals was used to support the $2400 \mathrm{~mm}$ length HSS beam over the two 2400 mm length HSS spread beams at the quarter points. The weight of this loading system is $2.0 \mathrm{kN}$. Figure 3.35 shows the schematic plan of the setup of flexure test for stud panel specimens SW-F1 to SW-F-3. Figure 3.36 also shows the typical test setup of flexure for stud panel specimen on structural lab. The location of POTs in these specimens was in the middle below of the specimen in order to determine the deflection in middle and both sides of the panel. Figure 3.37 shows schematic side view of the test setup of flexure test for stud panel specimens. Figure 3.38 also shows detail of support condition and POTs position of flexure test for stud panel specimen. 


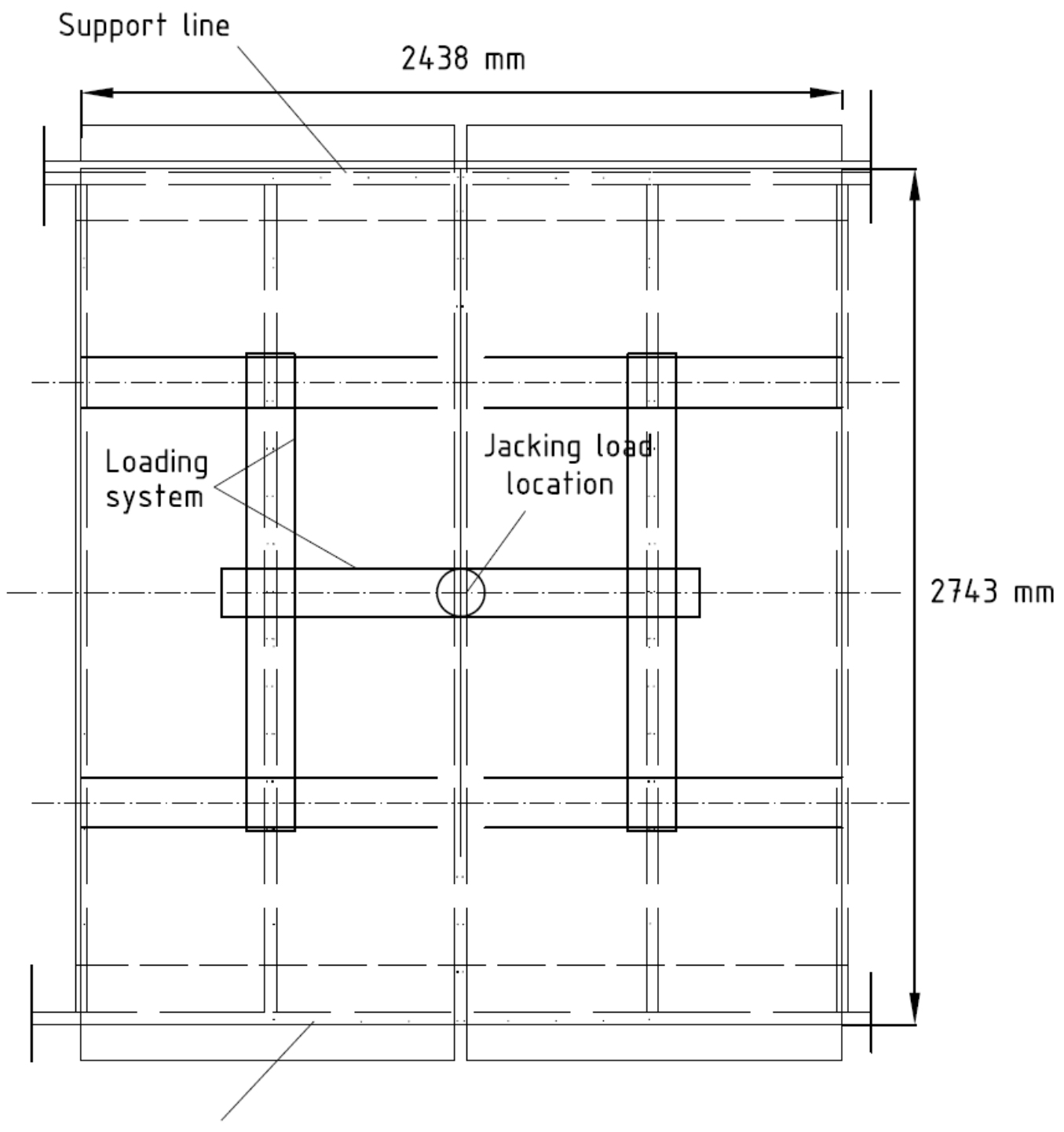

Support line

Figure 3.35 - Schematic plan of test setup of flexure for stud panel specimen 


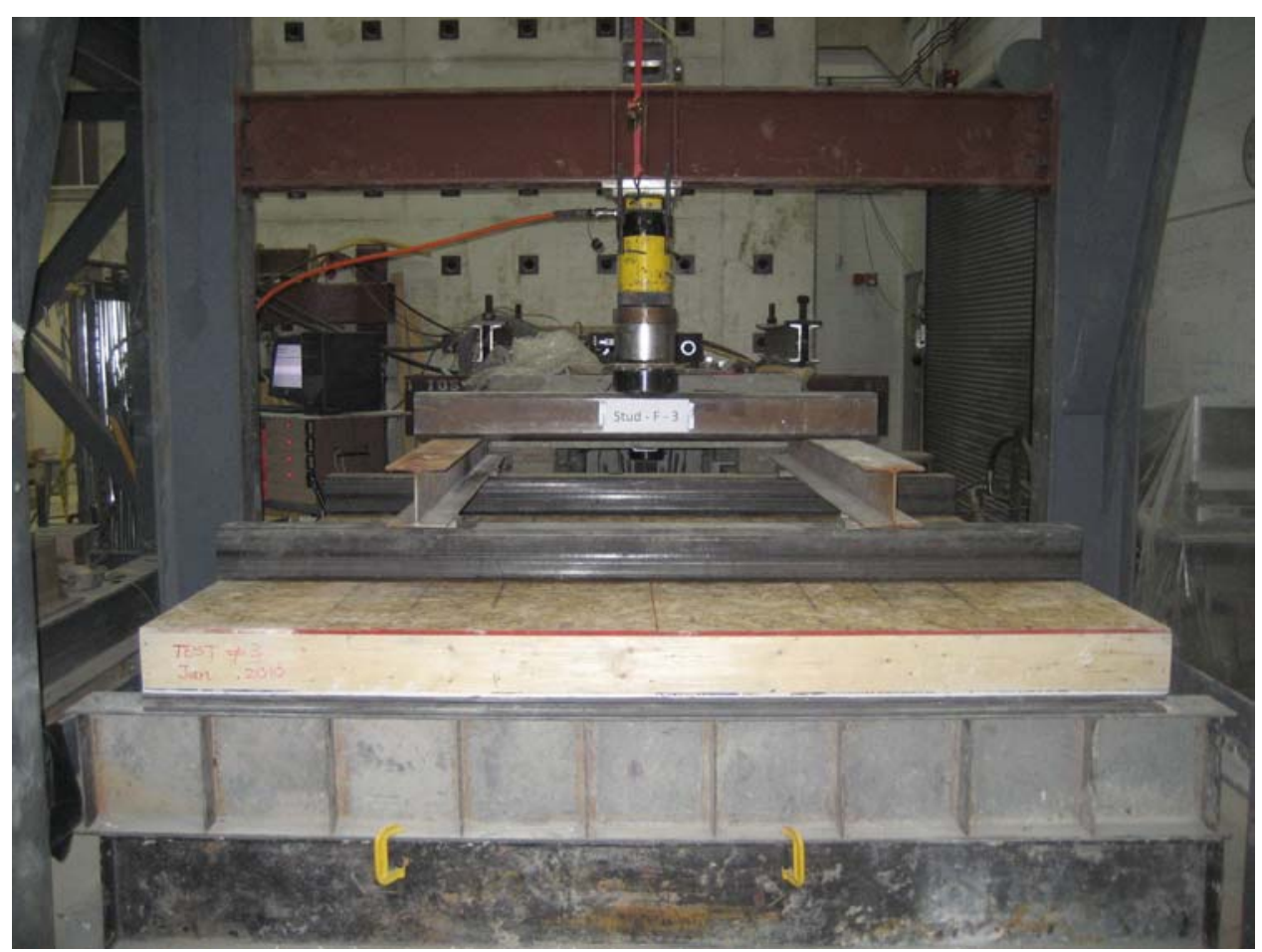

Figure 3.36 - Typical test setup of flexure for stud panel specimen

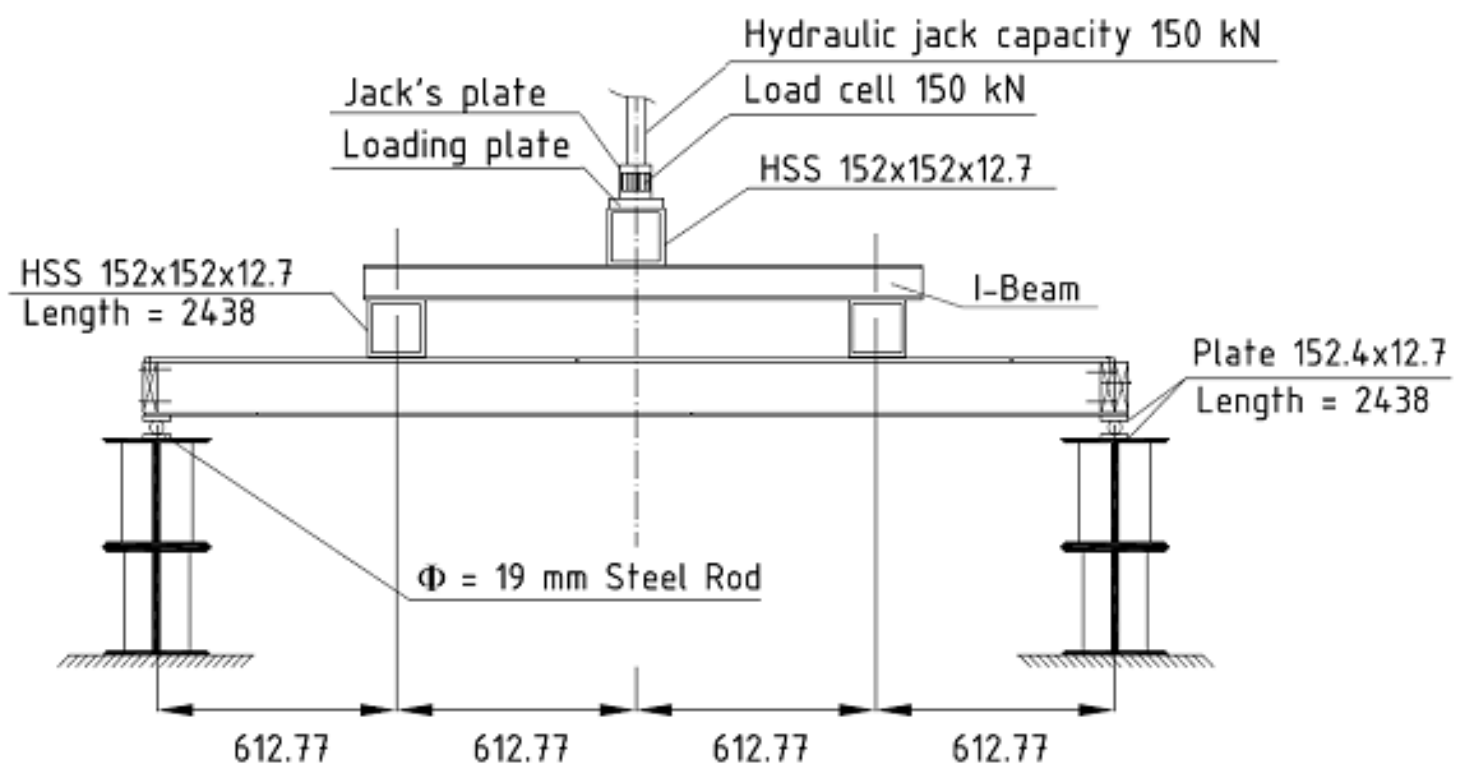

Note: All dimensions are in milimeter.

Figure 3.37 - Schematic side view of the test setup of flexure for stud panel specimen 


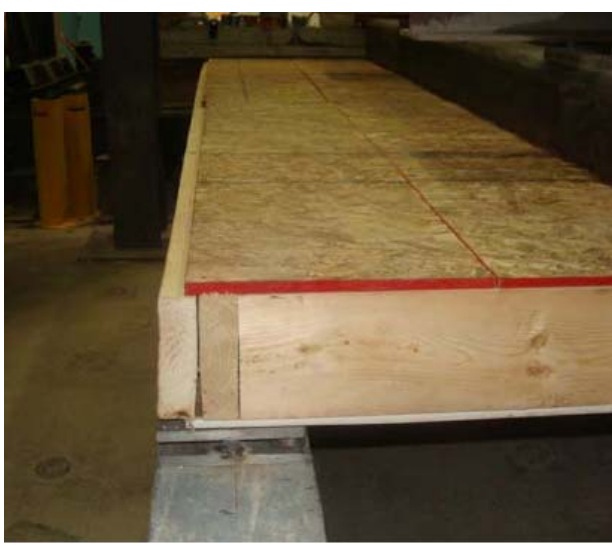

(a) Support condition

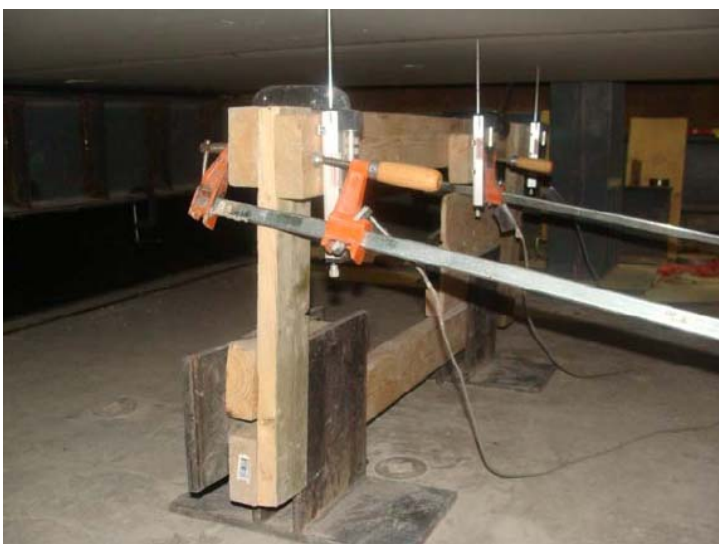

(b) POTs position under the panel

Figure 3.38 - Detail of support condition and POTs position of flexure test for stud panel specimen

\subsubsection{Test Procedure for Stud in Flexure}

ASTM E72-10 (2010) recommends using "two-point" loading for transverse load tests. The specimen should be tested as a simple beam on a $150 \mathrm{~mm}$ span (6 inch) less than the specimen length. The load is applied on two equal portions toward the middle of the span and at a distance of one quarter of the span from support. The weight of specimen between the supports shall be considered when the wall specimens tested horizontally. The transverse loads to the outside of the face for each specimen is applied. The number of the three specimens should be considered for transverse load test.

ASTM E2322-03 (2009) also provides the following consideration for apply the load to each individual specimen in increments so that a sufficient number of readings will be obtained to determine definitely the load-deformation curve using the following sequence:

1. A small initial load about 5\% smaller than the expected ultimate load capacity should apply, hold for $5 \mathrm{~min}$ and then release before starting the test. All the devices for measuring should set to zero before the beginning of the test.

2. The initial load and deformation of the specimen should be recorded and then the load on the specimen will increase to the first pre-determined increment.

3. The load and deformation reading is recorded and the load will release back to the initial load. Then the new set of recording should be started. 
4. The load will be increased to the next increment and the information will be recorded as indicated in the above steps. The reading of all load increments will be recoded up to final load capacity.

5. The load level should maintain as constant as possible for a period of 5 min after each load increment.

6. The initial and 5-min readings should plot in the form of load-deformation curves.

Flexural tests were performed in the structures laboratory of Ryerson University. The test setup was prepared for each test as explained earlier. For each panel, jacking load was applied in increments so that visual inspection could be performed to record any change in structural integrity of the sandwich panel. The tests were terminated after panel failure when the jacking load was not increasing while panel deflection was increasing by continuous pressing of the pump handle. At that stage, failure mode was observed and test data was then used to draw the load-deflection relationships for each panel.

\subsection{Testing Stud Wall in Axial Compression Loading}

\subsubsection{Test Method for Stud Wall in Compression}

The objective of this set of testing is to obtain experimental data for the ultimate load carrying capacity and serviceability limits for correlation with those obtained from SIP tests to investigate whether SIPs can perform as good as stud walls as loading carrying elements in residential construction. As such, identical test method to that used for SIP testing was utilized in stud wall testing. The main difference was that the stud wall specimens were of $2400 \mathrm{~mm}\left(8^{\prime}\right)$ width.

\subsubsection{Test Setup for Stud Wall in Compression}

The stud wall specimen was aligned vertically and supported directly over the laboratory's floor to prepare the test setup. A uniformly distributed line load was applied on the top side over the $2440 \mathrm{~mm}$ width using a loading assembly. This loading assembly was composed of a $2440 \times 350 \times 12.7 \mathrm{~mm}$ steel base plate resting over the top side of the panel. A $150 \times 150 \times 12.7 \mathrm{~mm}$ HSS box beam of length $2440 \mathrm{~mm}$ was welded to the top side of the steel base plate to transfer the applied jacking load over the panel width. Two $125 \times 125 \times 12.7 \mathrm{~mm}$ steel angles of $2440 \mathrm{~mm}$ length were welded to the steel base plate, one on each side of the wall panel to stabilize the 
loading assembly during the test. The weight of the loading assembly was calculated as $3.34 \mathrm{kN}$. Figure 3.39 indicates the test setup for the eccentric compressive load test which was used for specimen SW-A-1 to SW-A-3. Figure 3.40 also shows typical test setup for the eccentric compressive load test for stud wall specimens. The location of POTs in these specimens was in top two sides of the specimen and in the middle of the panel in order to determine the deflection in top and middle of the panel.

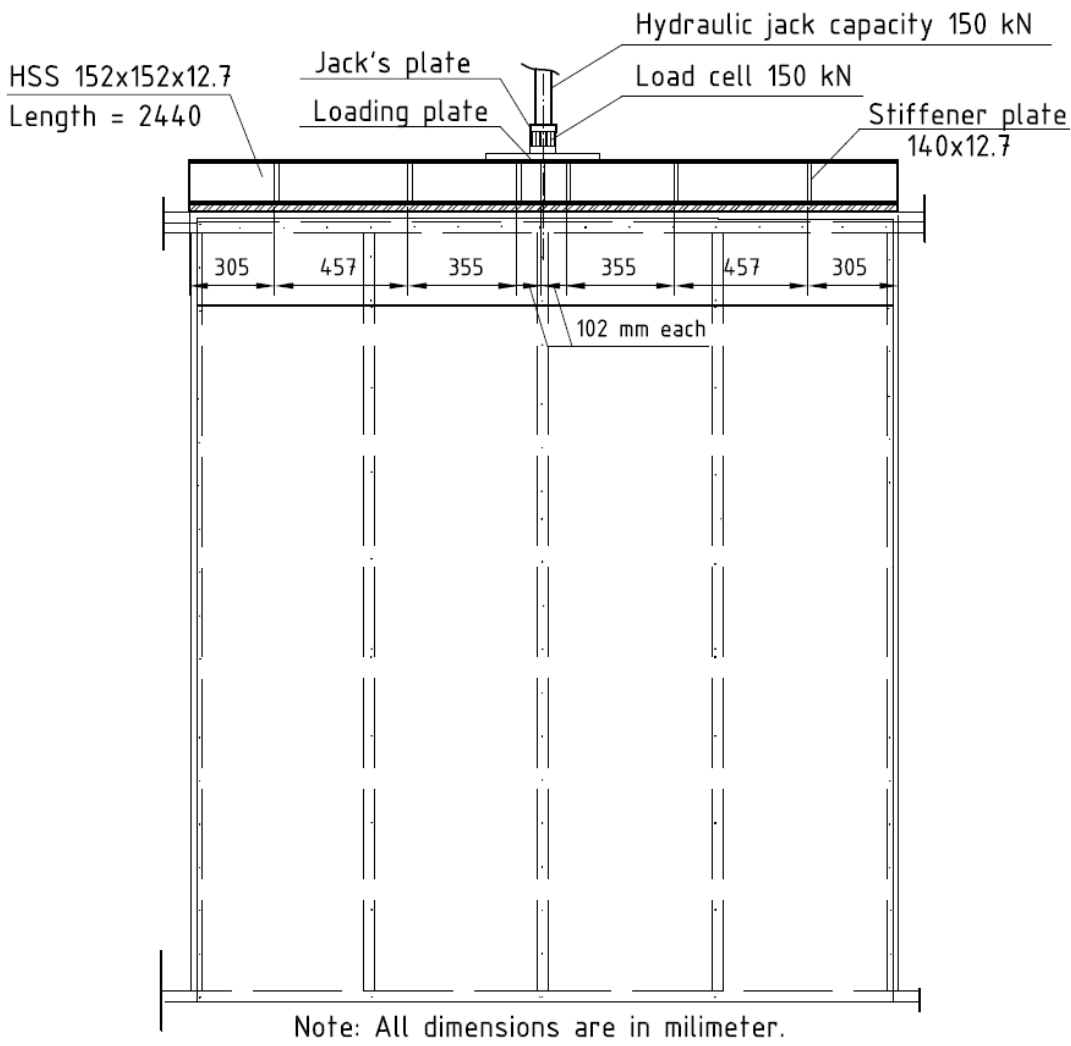

(a) Elevation

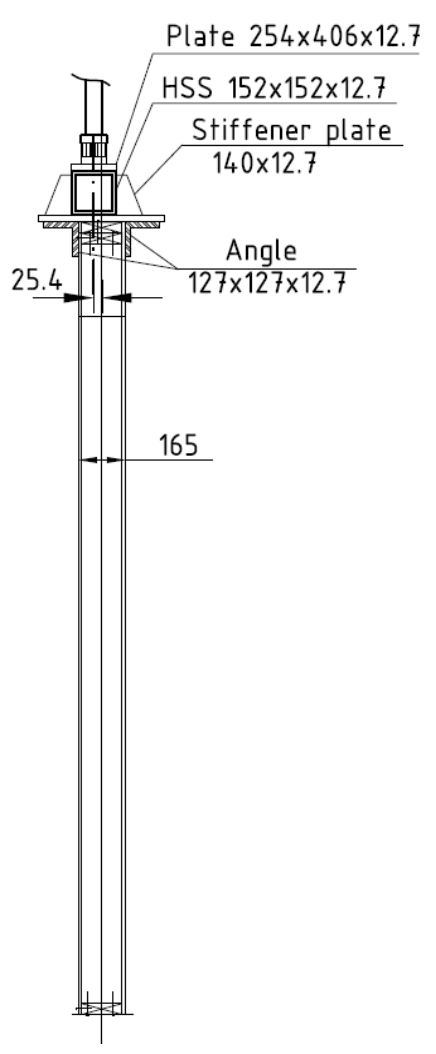

(b) Cross-section

Figure 3.39 - Schematic test setup for the eccentric compressive load test for stud wall 


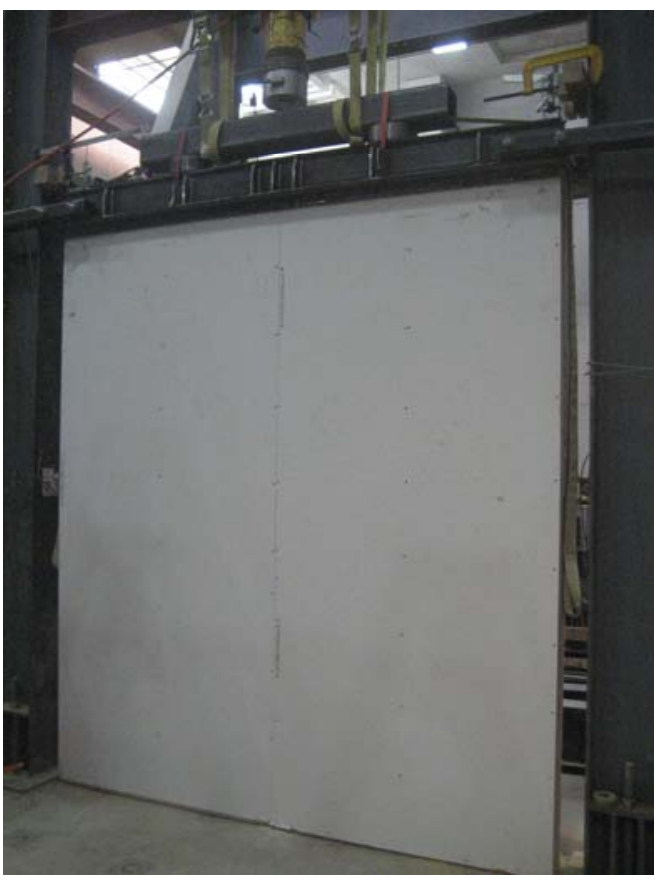

(a) Drywall side of the panel

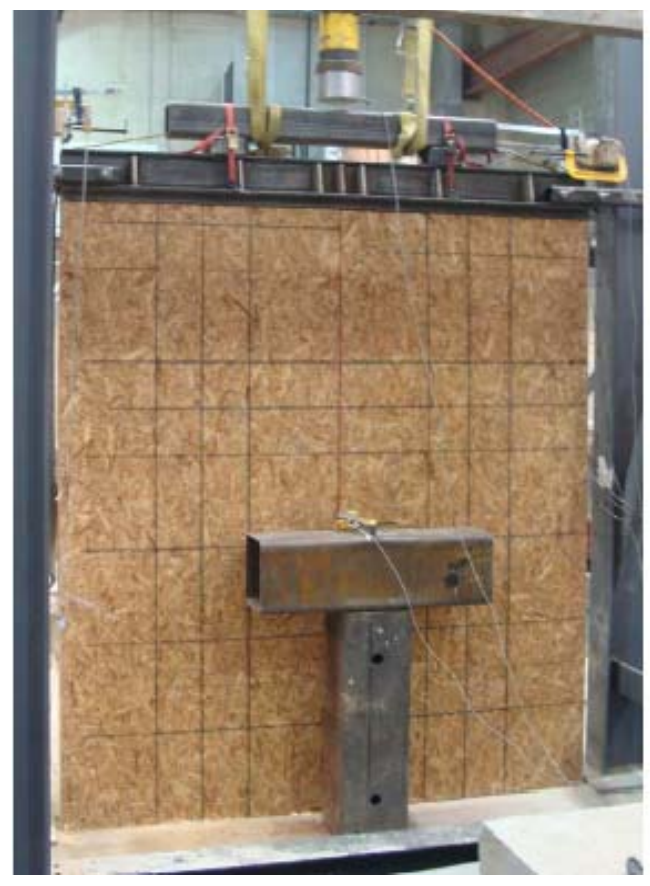

(b) OSB side of the panel

Figure 3.40 - Typical test setup for the eccentric compressive load test for stud wall

Figure 3.41 shows the details of the top rail used to distribute the compressive loading over the stud wall width. Figure 3.42 also shows the top portion of the stud wall showing restraint system for lateral movement at top of the stud wall.

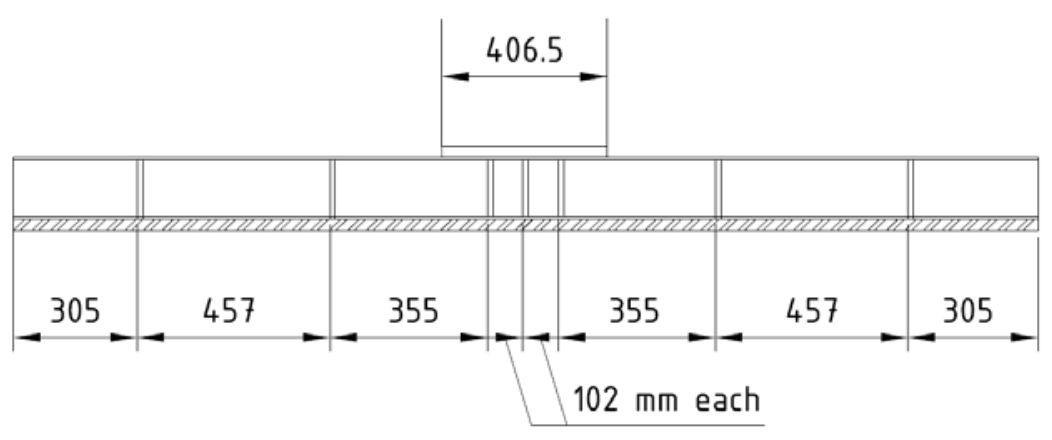

Note: All dimensions are in milimeter.

(a) Top rail elevation 


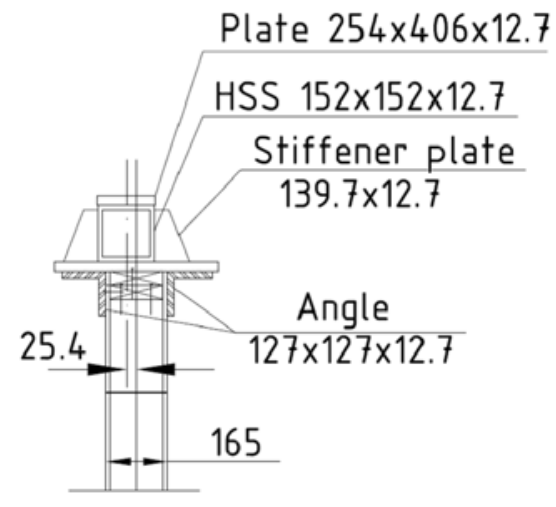

(b) Cross-section in top rail

Figure 3.41 - Details of the top rail used to distribute the compressive loading over the stud wall width

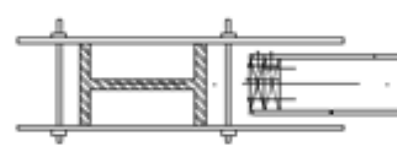

Note: All dimensions are in milimeter.

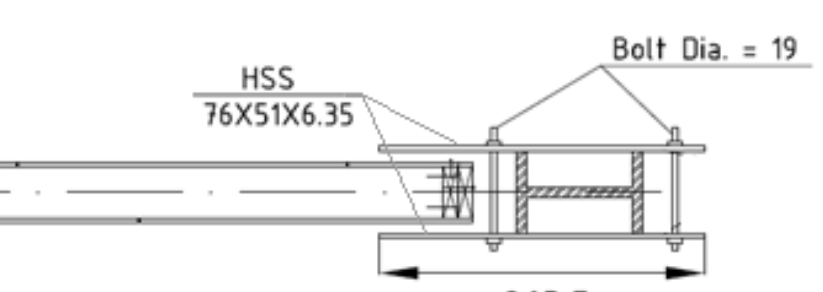

863.5

Figure 3.42 - Plan cross-section of the top portion of the stud wall showing restraint system for lateral movement at top of wall

Three potentiometers (POTs) were used to measure horizontal displacement at the mid-height of the specimen. Four potentiometers (POTs) were installed vertically over the four corners on the top side of the specimen to record axial shortening of the wall panel under load. Figure 3.43 shows the details test setup for compressive loading on stud wall. The compressive load was applied through a jacking load system with a universal flat load cell of $444 \mathrm{kN}(100,000 \mathrm{lb})$ capacity to measure the jacking load. During testing, the process for collecting and converting data captured by the LVDT's, POTs and load cell were done using a test control software (TCS) with SYSTEM 5000 data acquisition unit which was adjusted to sample the data at rate of 10 reading per second during the test. 


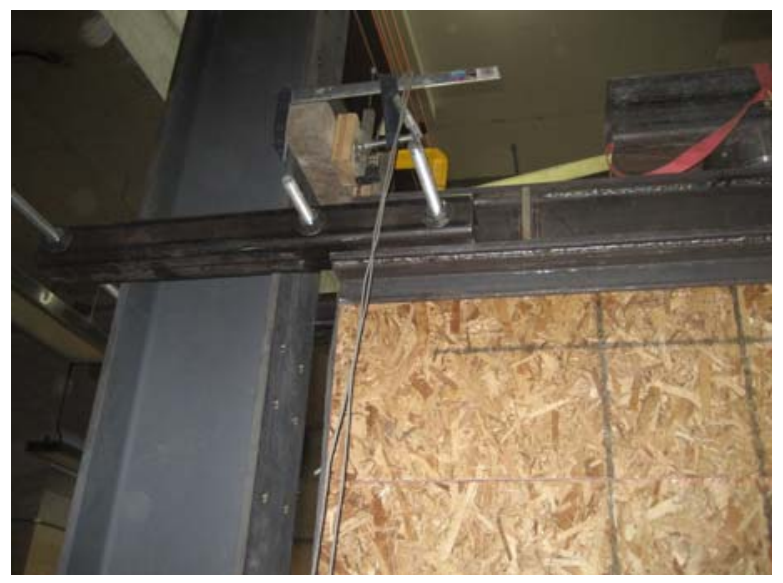

(a) Top corner of the panel

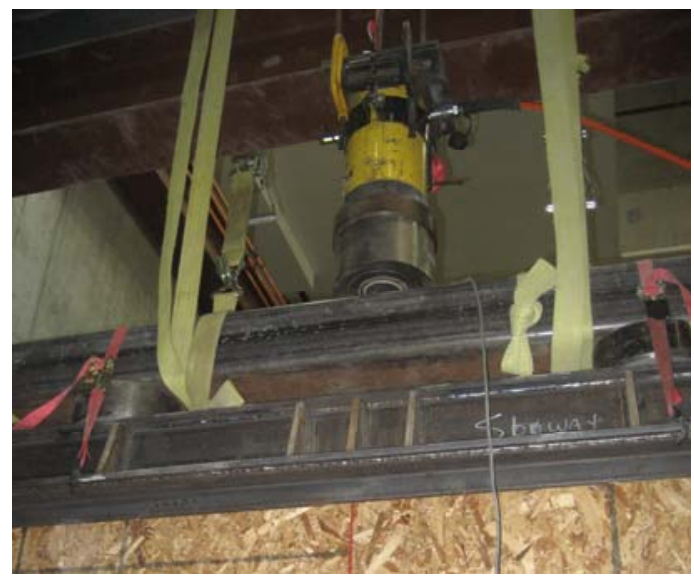

(b) Load cell and loading system

Figure 3.43 - Details test setup for compressive loading on stud wall

\subsubsection{Test Procedure for Stud Wall in Compression}

ASTM E72-10 (2010) recommends that compression test on specimen as column should be done on a flat end at the bottom. The compressive load should apply to a steel plate at the upper end of the specimen. The load has to apply uniformly along a line parallel to the inside face of specimen. The load must apply on one-third of the thickness of the specimen from the inside face. The movement of the testing machine crosshead should be nominally $0.8 \mathrm{~mm} / \mathrm{min}(0.03$ $\mathrm{inch} / \mathrm{min}$ ) for load rate.

The tests were performed in the structures laboratory of Ryerson University. The test setup was prepared for each test which included installing the POTs at the predetermined locations. For each specimen, the jacking load was continuously at a slow rate. Visual inspection was continuously conducted during the test record any change in the structural integrity of the wall specimen. Each test was terminated after the wall specimen failure. Failure of the specimen was considered when the recorded jacking load was not increasing or when the specimen could not absorb more loads while recorded axial shortening was increasing by continuously pressing the pump handle. Mode of failure was recorded and test data was then used to draw the loaddeflection and load-axial shortening relationships for each panel. 


\subsection{Testing Stud Wall under Racking and Cyclic Loading}

\subsubsection{Test Method for Stud Wall in Racking and Cyclic}

The objective of this set of testing is to experimental data for the ultimate load carrying capacity and serviceability limits for correlation with those obtained from SIP tests to investigate whether SIPs can perform as good as stud walls as loading carrying elements in residential construction. As such, identical test method to that used for SIP testing was utilized in stud wall testing. Racking load qualification tests on the panels were conducted as specified in the method described in the ASTM E564-06, Standard Practice for Static Load Test for Shear Resistance of Framed Walls for Buildings, ASTM E72-10, Standard Test Methods of Conducting Strength Tests of Panels for Building Construction, and AC 130-07, Acceptance Criteria for Prefabricated Wood Shear Panels. Cyclic load qualification tests on the panels were conducted as specified in the method described in the ASTM E2126-11, Standard Test Methods for Cyclic (Reversed) Load Test for Shear Resistance of Vertical Elements of the Lateral Force Resisting Systems for Buildings. These standards specify at least three identical specimens for each test group.

\subsubsection{Test Setup for Stud Wall in Racking and Cyclic}

The identical test setup to that used for SIP wall in racking and cyclic lateral load was utilized in stud wall testing. The specimens are denoted as SW-R-1 to SW-R-3 and SW-C-1 to SW-C-3 for racking and cyclic test, respectively. Figure 3.44 also shows the typical test setup of monotonic and cyclic lateral load for the stud wall at structural lab. The location of POTs in these specimens will be in top two sides of the specimen and in the middle of the panel in order to determine the deflection in top and middle of the panel. Figure 3.45 also depicts the details of top and bottom test setup of monotonic and cyclic lateral load for the stud wall. 


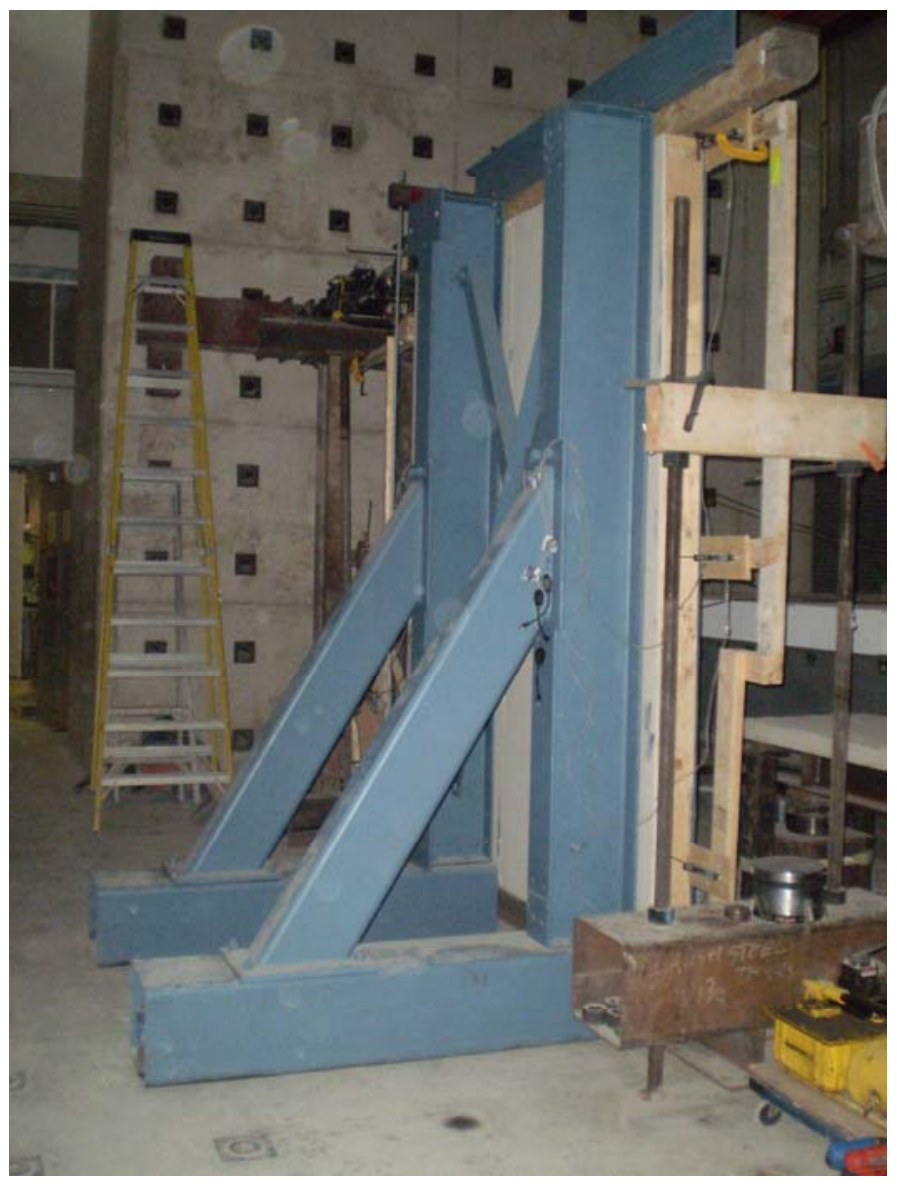

Figure 3.44 - Typical test setup of monotonic and cyclic lateral load for the stud wall

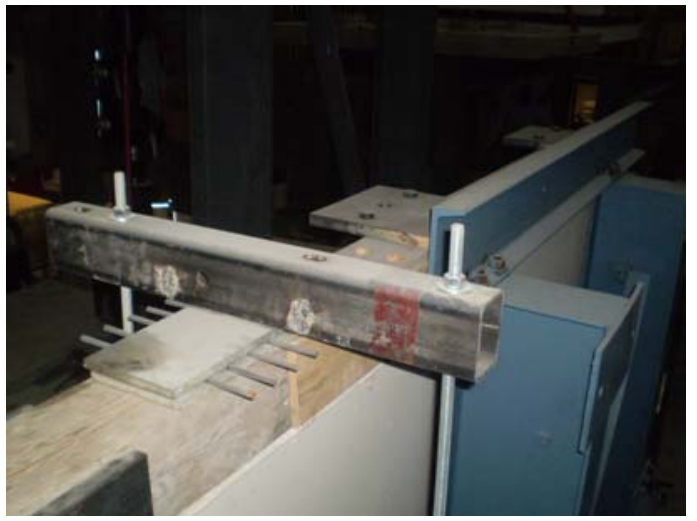

(a) Top of the panel with lateral support

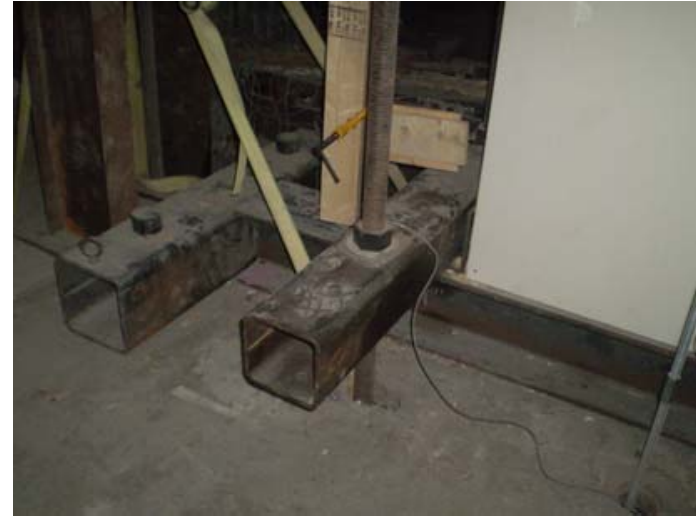

(b) Bottom support condition

Figure 3.45 - Details of top and bottom test setup of monotonic and cyclic lateral load for the stud wall 


\subsubsection{Test Procedure for Stud Wall in Racking and Cyclic}

\subsubsection{Monotonic (Racking) Load}

Monotonic tests are conducted according to ASTM E564-06 (ASTM, 2006) test protocol. The ASTM E564-06 protocol needs that ultimate load be reached in no less than 5 minutes. The following steps must be done during the racking load test:

Step 1: The frame stiffness should determine prior to the application of the web elements. The frame is loaded in a manner similar to that intended for the completed panel. If the frame is a "standard" frame, the stiffness of the frame should be determined.

Step 2: The procedure for complete panel loading is performed. The loads apply to the panel parallel to and the top of the wall though a beam which is attached to the top plate of panel. The rate of load has to choose such that the anticipated full design load level will be reached in not less than $10 \mathrm{~min}$.

Step 3: In order to establish the load-deformation curve prior to failure, at least ten sets of uniformly spaced deflection readings should provided. Load and deformation reading must be recorded in a proper rate. Hydraulic jacks or actuator can apply the load to specimen and have been calibrated prior to test. The load also can apply by any other suitable types of loading apparatus. If the weight of loading system affects the results, it should be separately consider.

Step 4: Dial gages or other suitable devices are measured the deformation to provide an acceptable load-deformation curve. The deflection should be recorded to the nearest 0.25 $\mathrm{mm}(0.01$ in. $)$.

Step 5: Load-measuring apparatus shall be record the load and deflection of top of the wall. When approximately reaching to one third and two thirds of the estimated ultimate load, the load can be removed and the panel recovery should record after $5 \mathrm{~min}$.

Step 6: Maintain a record of the total length of time the panel is under load.

ASTM E72-10 (2010) recommends the several considerations during the monotonic lateral loading on stud wall. The size of the specimens should be $2400 \times 2400 \mathrm{~mm}$ ( $8 \times 8$ feet) and the load has to apply to the panel through a constructed frame as it mentioned in test setup section. The minimum of three specimens should be used for the racking test. When the panel is assembled 
the minimum moisture contact shall be between 12 and $15 \%$ and the variation shall not be more than $3 \%$ from the initial moisture content.

ASTM E72-10 (2010) also recommends that the load on specimen should apply continuously at a uniform rate of motion of the loading device. The speed of loading is recommended in a way that the total load of $3.5 \mathrm{kN}$ (790 lbf) shall be applied more than 2 minute from starting of the test. The interval load rate of $3.5 \mathrm{kN}$ shall apply to the specimen in different values of 7.5 to 10.5 $\mathrm{kN}$ (1570 to $2360 \mathrm{lbf}$ ) total load and to failure of the specimen. Therefore, load on specimen will apply in three stages to 3.5, 7.0 and $10.5 \mathrm{kN}(790,1570$ and $2360 \mathrm{lbf})$ total load at a uniform rate. After each stage, the load on specimen should be removed and all the residual deflection shall return to zero, then the next stage should be started. After these cycles of loading and unloading on $3.5,7.0$ and $10.5 \mathrm{kN}$, the specimen shall be loaded until the failure or $100 \mathrm{~mm}$ (4 inch) of total deflection is happened.

\subsubsection{Cyclic Loading}

The identical protocol to that used for SIP wall in cyclic lateral load was utilized in stud wall. CUREE protocol for ordinary ground motions is used on cyclic tests. The CUREE protocol consider a set of primary cycles which displacement amplitudes followed by 2 to 5 trailing cycles at $75 \%$ of the primary cycle amplitude. The reference displacement $\left(\Delta_{\text {ref }}\right)$ is determined from monotonic testing. The displacement amplitude of each cycle is proportional to this reference displacement. The $80 \%$ of the ultimate load is selected and $60 \%$ of the displacement after ultimate is calculated as reference displacement.

The displacement amplitudes have a sequence of increasing value. It is started gradually up to $0.4 \Delta_{\text {ref }}$ and then changing to larger steps. The statistical analysis of inelastic time histories can provide the increment of larger step. These steps are selected in order to model the structural behavior of a panel during an actual seismic event (ASTM 2001). The cyclic load is applied to specimens up to a certain level of displacement and increasing at $0.5 \Delta_{\text {ref }}$ per increment. The

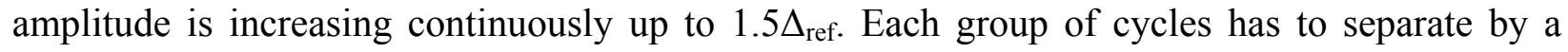
short pause of 15 seconds to control the stop of the test when a wall collapses. 
ASTM E2126-11 (2011) recommend three method for cyclic load on shear wall namely, Method $\mathrm{A}, \mathrm{B}$ and $\mathrm{C}$. The Test Method $\mathrm{C}$ was used in this research and it has presented as following:

1. The loading procedure shall be applied with displacement control and involved the group of displacement cycles at incremental increasing displacement level. The six series initiation cycles of equal amplitude is started in loading history. In addition, a primary cycle with amplitude as a percent of the reference deformation and consequent trailing cycles with $75 \%$ amplitude of the primary one is applied on each phase of loading history.

2. The reference deformation is an estimation of maximum displacement which is appropriate with $0.8 P_{\text {peak }}$ load in a primary cycle.

3. The reference deformation was already described in SIP wall loading and shall be less than 0.025 times the height of wall. Additional phases of cycles will be applied to the specimen if the panel has not failed at the end of Phase 8 of cycles. An increase in amplitude of $\alpha(\alpha \# 0.5)$ over the previous primary cycle shall be considered on a primary cycle and two trailing cycles with $75 \%$ amplitude of the primary one are pursued.

4. A constant cyclic frequency or constant rate of displacement can be chosen for actuator displacement. The displacement rate must be between 0.04 and 2.5 in./s (1.0 and 63.5 $\mathrm{mm} / \mathrm{s}$ ). In order to prevent inertial effects of the mass of the wall the cyclic frequency must range from 0.2 to $0.5 \mathrm{~Hz}$ during cyclic loading. The procedure of loading shall follow until the applied load reduces more than $0.2 P_{\text {peak }}$ or failure limit state occurs.

5. The displacement measurement device must have a resolution of 0.005 inch $(0.13 \mathrm{~mm})$ and the sampling rate of displacement shall be 100 reading per cycle. 


\section{CHAPTER 4}

\section{EXPERIMENTAL RESULTS}

\subsection{General}

This Chapter discusses the results obtained from testing actual-size structural insulated foamtimber panels (SIPs) and conventional stud panels (STUDs) under flexural, compressive, racking, and seismic lateral loading. The experimental results for all panels are presented in sequence for each test type. The structural adequacy of the tested SIP panels for possible use in residential construction is presented at the end of the Chapter.

\subsection{Test results for axial compression and flexure}

This section presents the experimental findings on selected SIP panels to investigate their structural behavior when used as floors and walls in residential construction. The results of axial load and flexural tests performed in this study established a database that can be used further to develop design tables of SIP floors and walls subjected to flexural, axial compressive loading or combined axial compressive loading and lateral bending moment resulting from wind loading. A set of STUD panels of identical geometry to the tested SIP panels were tested to correlate their results with those obtained from SIP panel tests. In this chapter, experimental findings are used to investigate whether the tested SIPs are "as good as" the conventional wood-frame STUD system in structural design.

\subsubsection{Code Requirements for the Structural Qualification of the SIPs}

The Structural qualifications of the SIPs are assessed in this Chapter based on:

1- The general design principles provided in CSA Standard CAN/CSA-086.09, Engineering Design of Wood;

2- The evaluation criteria set forth in the NRC/CCMC Technical Guide which focuses on SIPs as being "as good as" the conventional wood-frame buildings with respect to strength and serviceability; and 
3- CSA Standard CAN/CSA-S406-92, Construction of Preserved Wood Foundations, (1992) and the National Building Code of Canada (NBCC 2005).

Based on NBCC and CAN/CSA-S406, the following loads and load factors can be used to examine the structural adequacy of the panels for serviceability and ultimate limit states design:

Dead load factor $\quad=1.25$

Live load factor $\quad=1.50$

Dead load for roofs $\quad=0.5 \mathrm{kPa}$

Dead load for floors $=0.47 \mathrm{kPa}$

The intensity of the triangular lateral soil pressure $=4.7 \mathrm{kN} / \mathrm{m}^{2}$

Live load for residential construction $=1.9 \mathrm{kPa}$

Snow load for residential construction $=1.9 \mathrm{kPa}$ (for simplification of comparison)

Deflection limit for serviceability (live load effect) $=$ span / 360

Deflection limit for serviceability (total load effect, dead + live $)=$ span $/ 180$

The average deflection and ultimate load carrying capacity of each specimen group are basically the average of those for the three panels in each panel group as per the Acceptance criteria for SIPs set forth in ICC-ES AC04 (2004). Further, when the results of one of the tested panel vary more than $15 \%$ from the average of the three panels, one of the following two actions was chosen: (i) the lowest test value may be used; or (ii) the average result based on a minimum of five tests may be used regardless of the variations. Moreover, the results from two tests could be used when the higher value does not exceed the lower value by more than $5 \%$ and the lower value is used with the required factors of safety. Factor of safety for ultimate load carrying capacity of SIPs is dependent on the followings: (i) consistency of materials, (ii) the range of test results, and (iii) the load-deformation characteristics of the panel. AC04 generally applies a factor of safety of 3 to the ultimate load based on the average of three tests which called in this research as panel group. However, for the case of the tested panels in this research, AC04 provides the following factors of safety applicable to uniform transverse loads:

F.S. $=3.0$ for ultimate load at shear failure for all loading conditions. F.S. $=2.5$ for ultimate reaction at failure for all loading conditions 
F.S. $=2.5$ for ultimate load determined by bending (facing buckling) failure under allowable snow loads.

F.S. $=2$ for ultimate load determined by bending (facing buckling) failure under allowable live loads up to $0.958 \mathrm{kPa}$ (20 Lb per square foot).

In case of wall panel axial load tests, AC04 specifies that wall panels shall support an axial loading applied with an eccentricity of 1/6 the panel thickness. Also, AC04 specifies that the factored design resisting axial load is determined from the experimental axial load at a net axial deformation of $3 \mathrm{~mm}\left(1 / 8^{\prime \prime}\right)$ or the ultimate load divided by a factor of safety determined in accordance with those specified for transverse load testing mentioned above, whichever is lower.

\subsubsection{Results of SIP Walls for Axial Loading}

In this group, three identical SIP walls (SIP-A-1, SIP-A-2, and SIP-A-3) were tested to complete collapse under uniformly distributed axial compression load with $t / 6$ eccentricity where $t$ is the total thickness of the wall. Each Wall was of $2750 \mathrm{~mm}$ height, $1220 \mathrm{~mm}$ wide and $165 \mathrm{~mm}$ thick, with foam-spline connection. Per ASTM method, each wall was loaded using incremental loading. The wall was loaded up to $20 \mathrm{kN}$, followed by releasing the load to zero. Then, the load was applied to reach $40 \mathrm{kN}$, followed by releasing the load. This incremental loading was followed till failure of the wall occurred.

Figure 4.1 shows view of panel SIP-A-1 before testing, while Figures 4.2 and 4.3 show views of the permanent deformed shape of the wall after failure as well as close-up views of the local damage and deformation, respectively. It was observed that the failure mode in wall SIP-A-1 was due to OSB facing crushing at one side of its bottom plate leading to global permanent lateral deformation of the wall as shown in Figure 4.2. This led to foam delamination at the foam-OSB sheet interface as shown in Figure 4.3. In addition, bending deformation of the wall let to shear failure of the foam at the bottom of the wall in the form of diagonal crack as depicted in Figure 4.3-b. During the test, nail slip occurred along the row of nails connecting the OSB sheet and bottom plates attached to the lab floor. This resulted in large shear force at the interface between the OSB sheet and the foam, leading to foam-OSB debonding. The failure of the wall was abrupt causing a sudden drop in the applied jacking load as depicted in the load-axial shortening 
relationship in Figure 4.4. It was observed that linear elastic behaviour was maintained till failure. Figure 4.5 depicts the change on lateral deflection of the wall at its mid-height with increase in applied load. Given the nature of the load-deformation relationship and the observed failure modes shown in Figures 4.2 and 4.3, one may conclude that the traditional Euler buckling failure mode did not exist in this case.
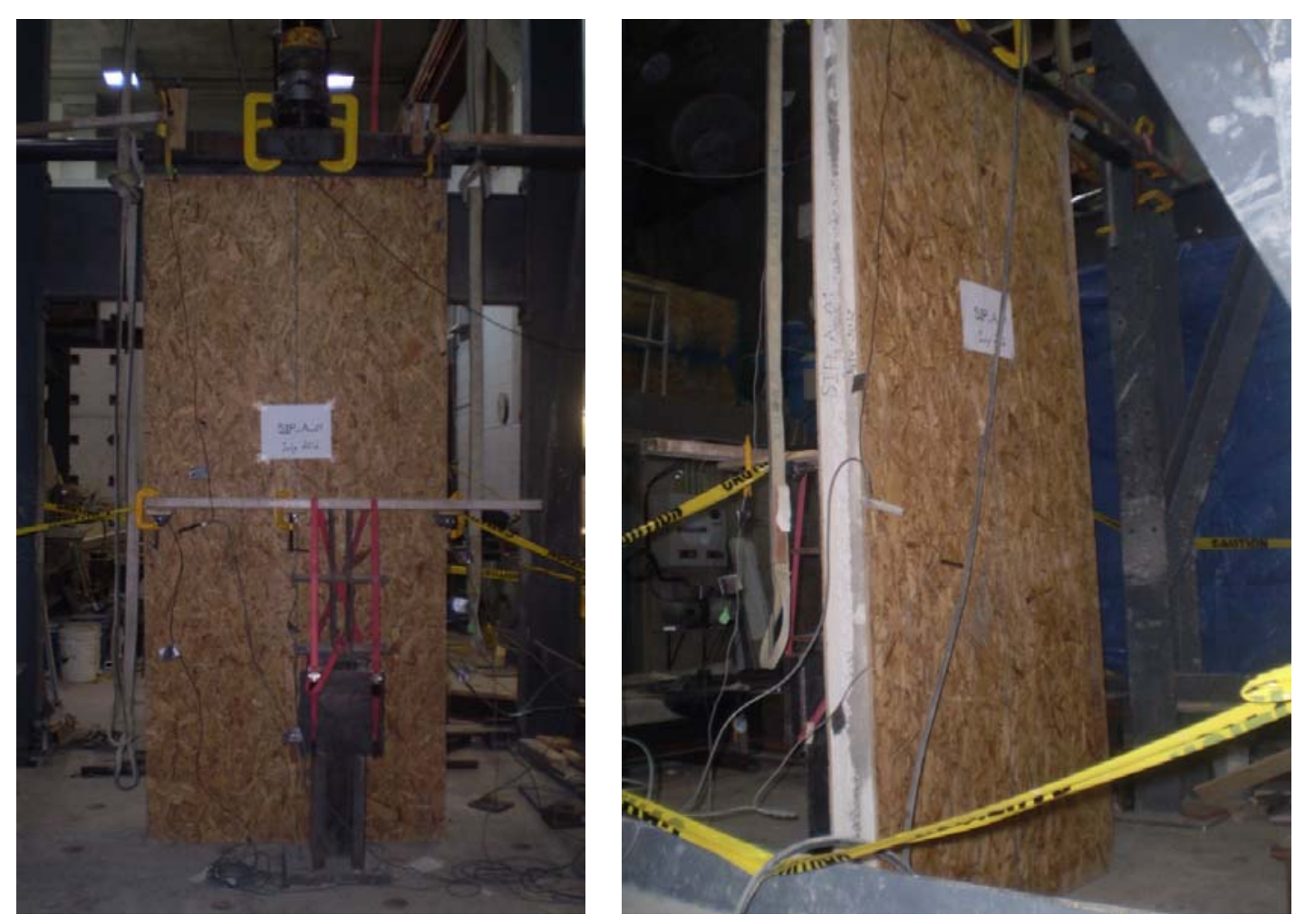

Figure 4.1 - Views of the test setup for wall SIP-A-1 before testing 


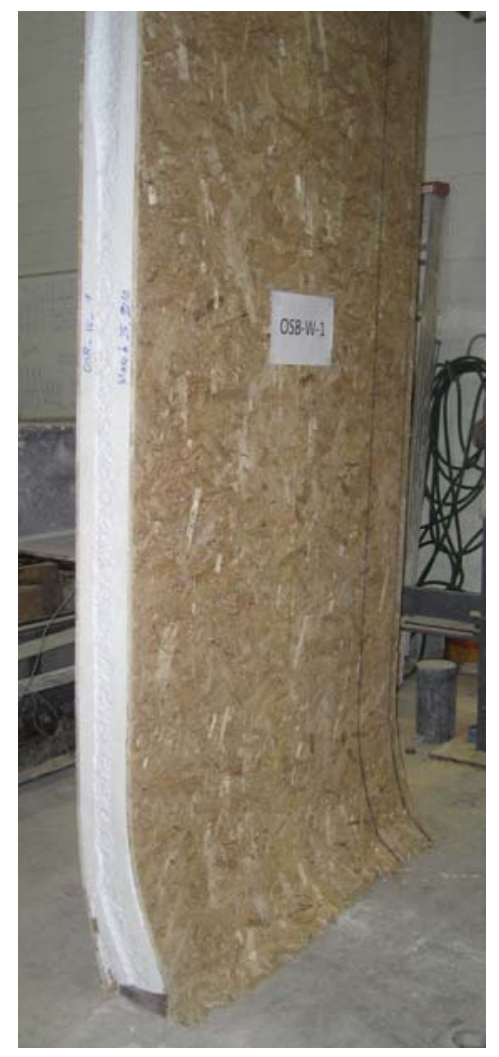

(a) Left side

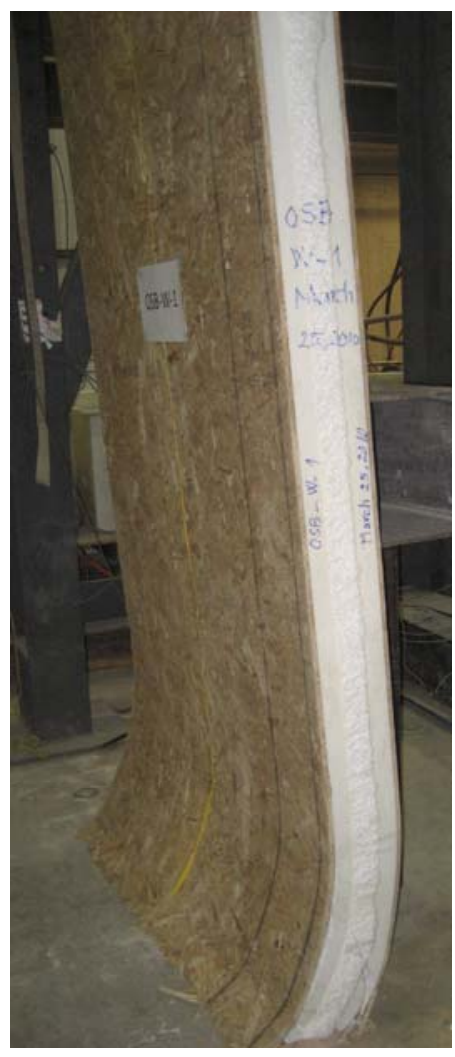

(b) Right side

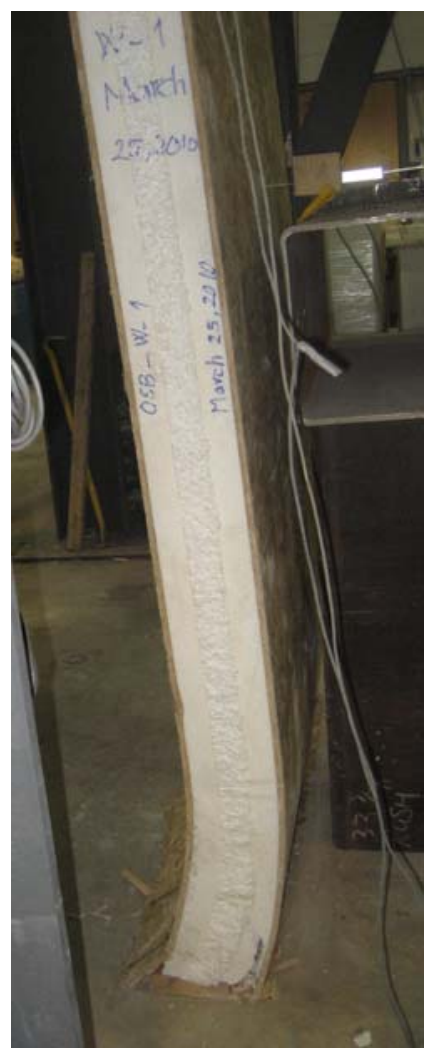

(c) Elevation

Figure 4.2 - Views of the deformed shape of the wall SIP-A-1 after failure

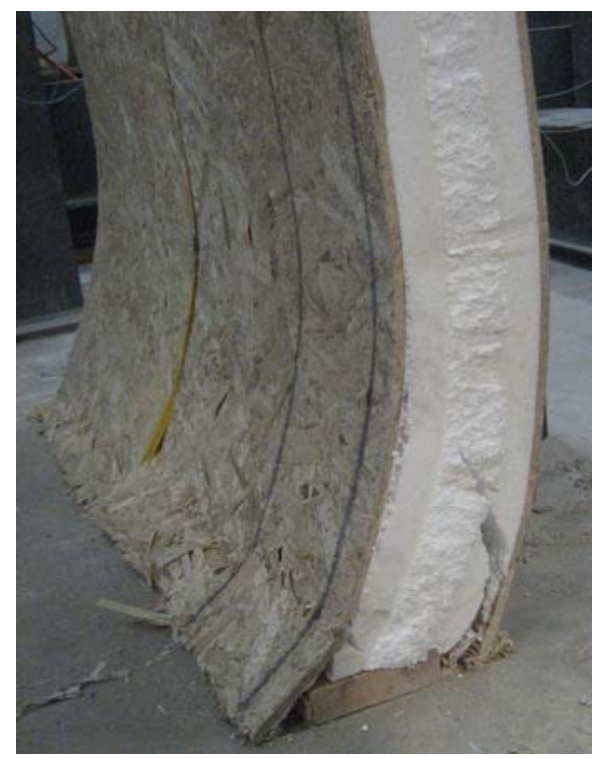

(a) Right side of the wall specimen

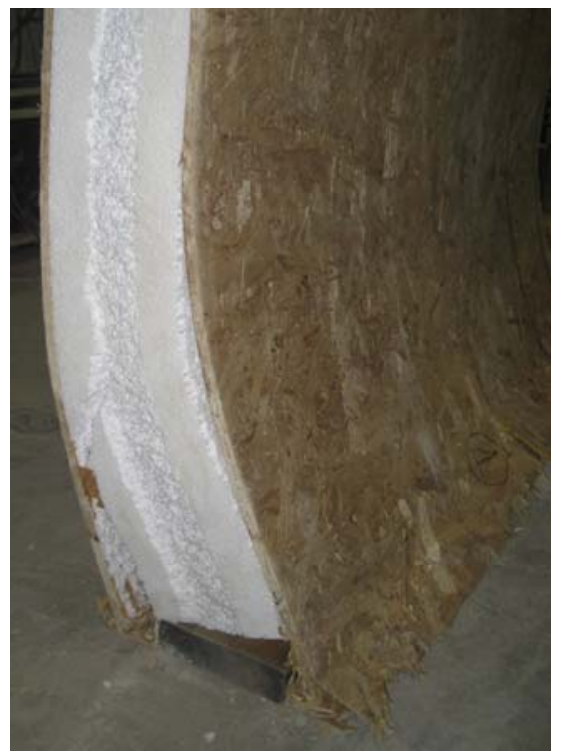

(b) Left side of the wall specimen

Figure 4.3 - Close-up views of the failure modes of SIP-A-1 specimen 


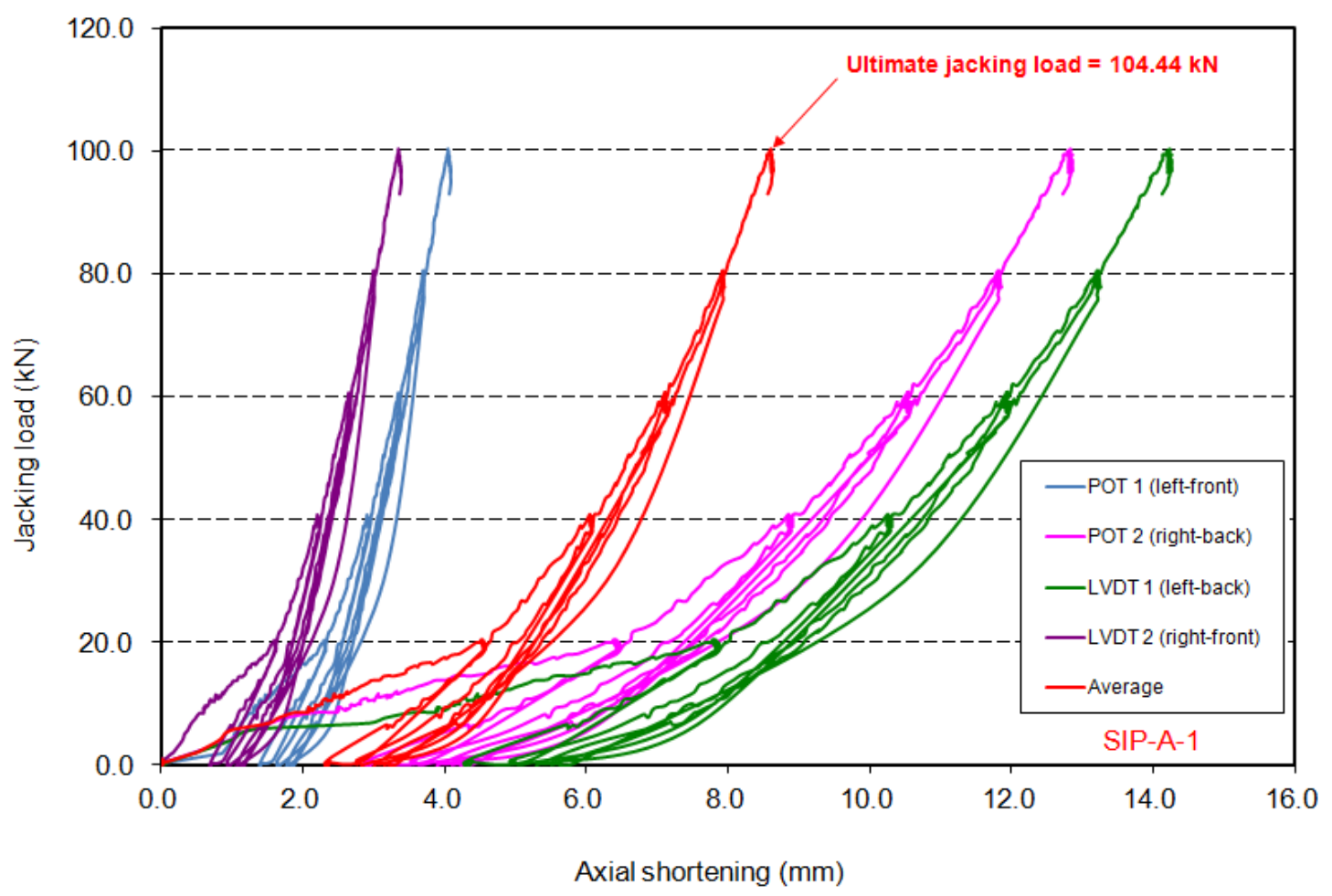

Figure 4.4 - Axial load-axial shortening relationship for specimen SIP-A-1

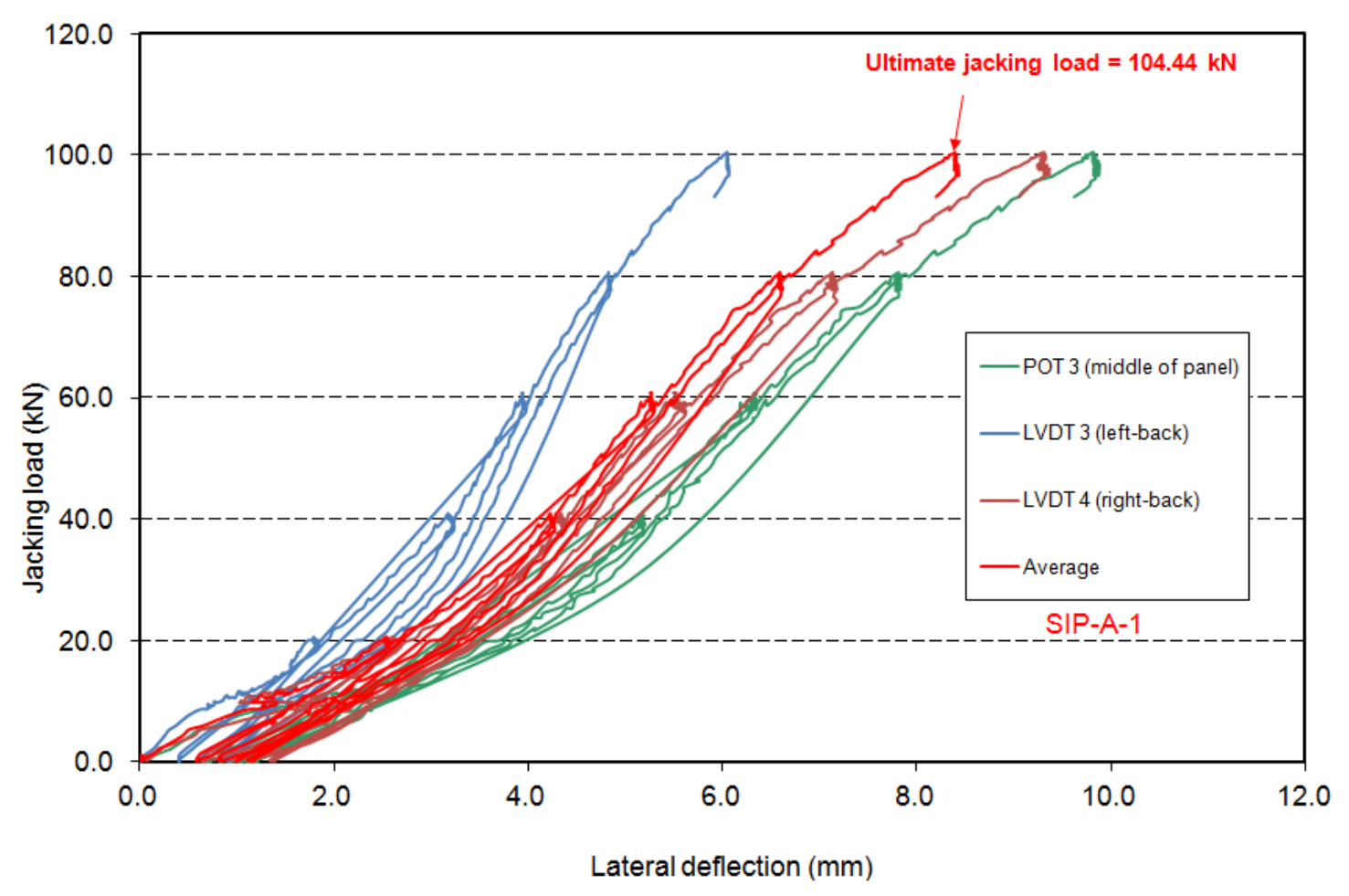

Figure 4.5 - Axial load-lateral deflection relationships for specimen SIP-A-1 
Similar behavior to wall SIP-A-1 was observed for walls SIP-A-2 and SIP-A-3. Figure 4.6 shows view of wall SIP-A-2 before testing, while Figures 4.7 and 4.8 show views of the permanent deformed shape of the wall after failure as well as close-up views of the local damage and deformation in the OSB facing and foam core, respectively. Figure 4.9 depicts the load-axial shortening relationship, while Figure 4.10 shows the load-lateral deflection relationship for specimen SIP-A-2. Figure 4.11 shows view of wall SIP-A-3 before testing, while Figs. 4.12 and 4.13 show views of the permanent deformed shape of the wall after failure as well as close-up views of the local damage and deformation in the OSB facing, respectively. Figure 4.14 depicts the load-axial shortening relationship for this wall, while Figure 4.15 shows the load-lateral deflection relationship for specimen SIP-A-3.

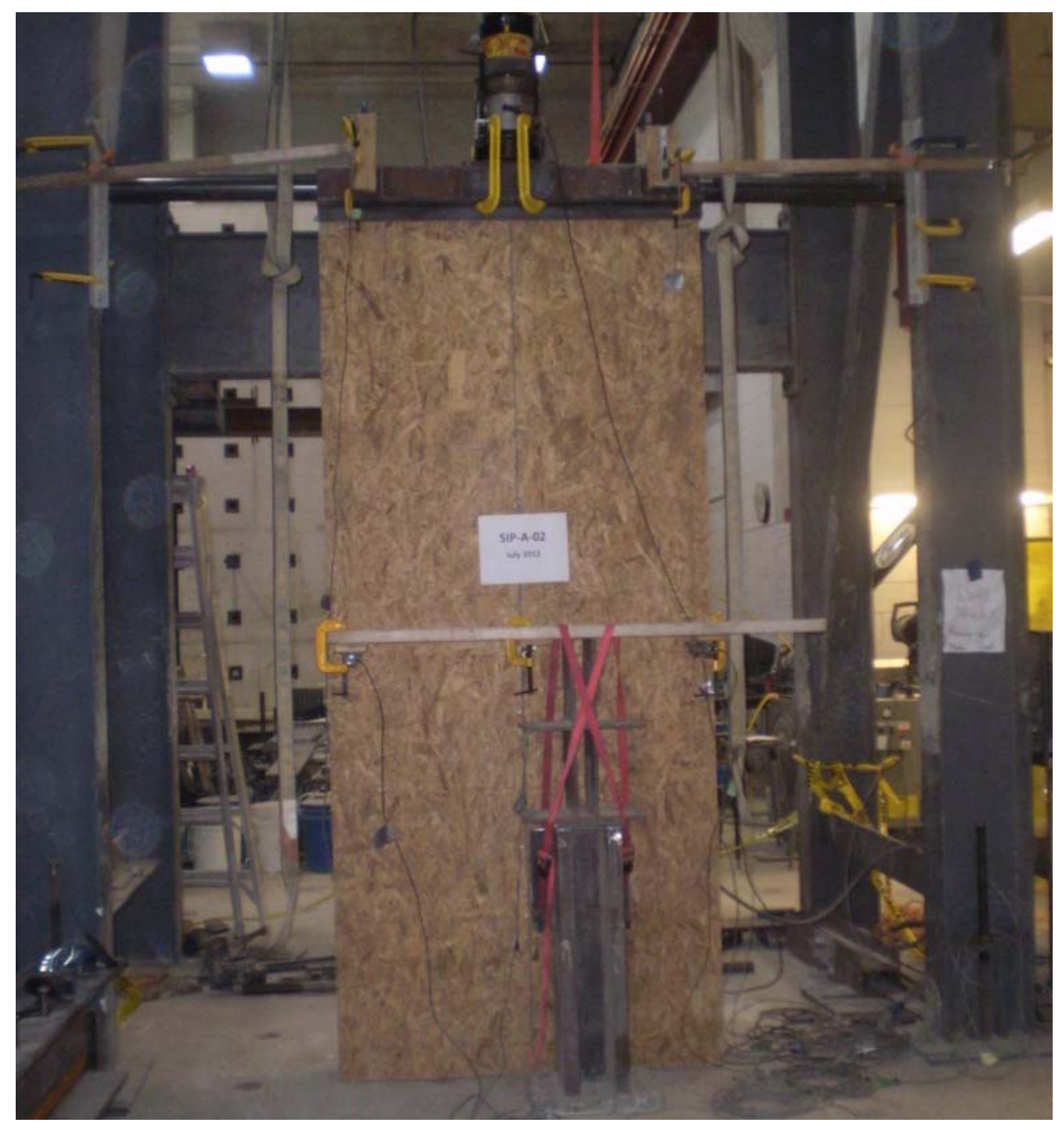

Figure 4.6 - Views of the test setup for wall SIP-A-2 before testing 


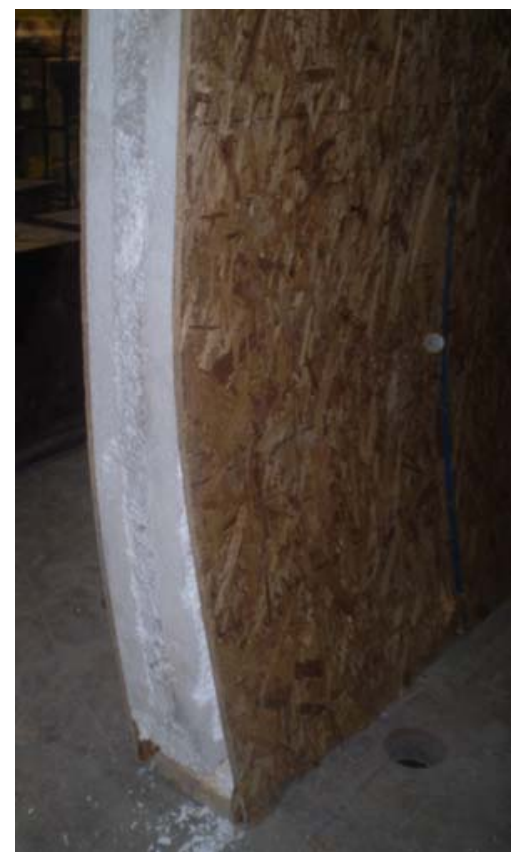

(a) Left side

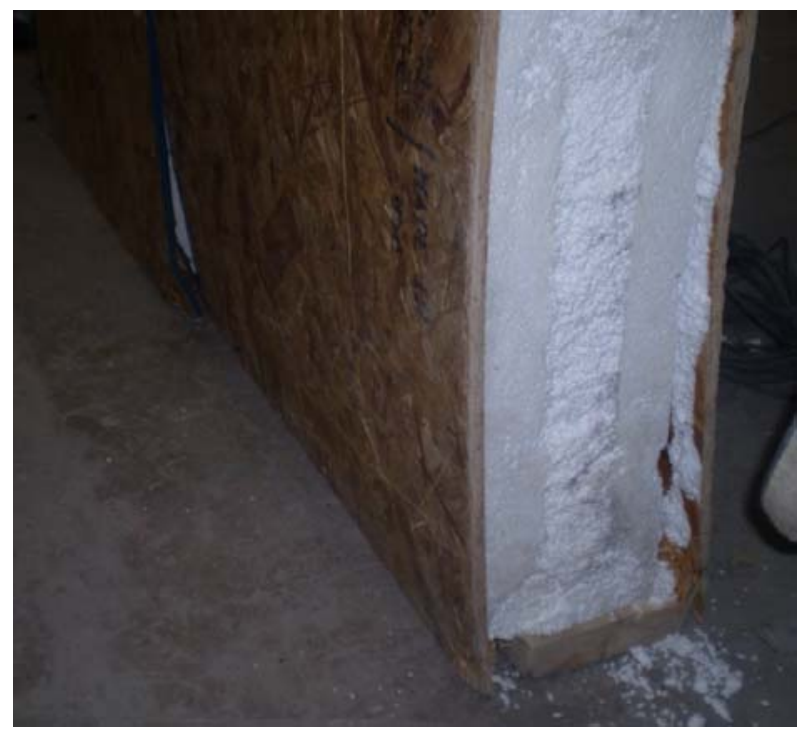

(b) Left side and middle portion of the panel

Figure 4.7 - Views of the deformed shape of the wall SIP-A-2 after failure

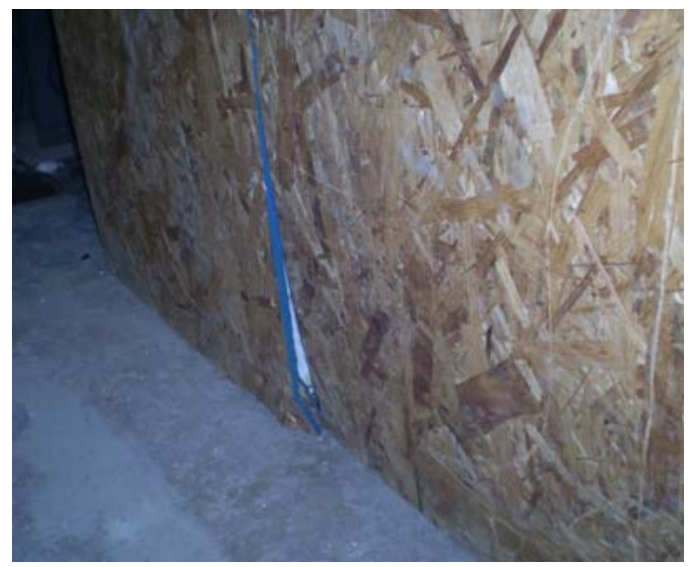

(a)Crushing of OSB at bottom of wall

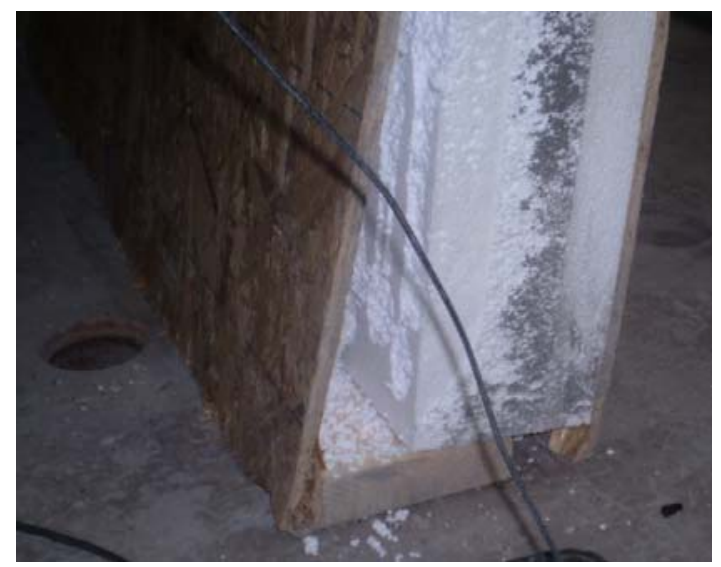

(b) Foam-OSB debonding and OSB crushing

Figure 4.8 - Close-up views of the failure modes of SIP-A-2 specimen 


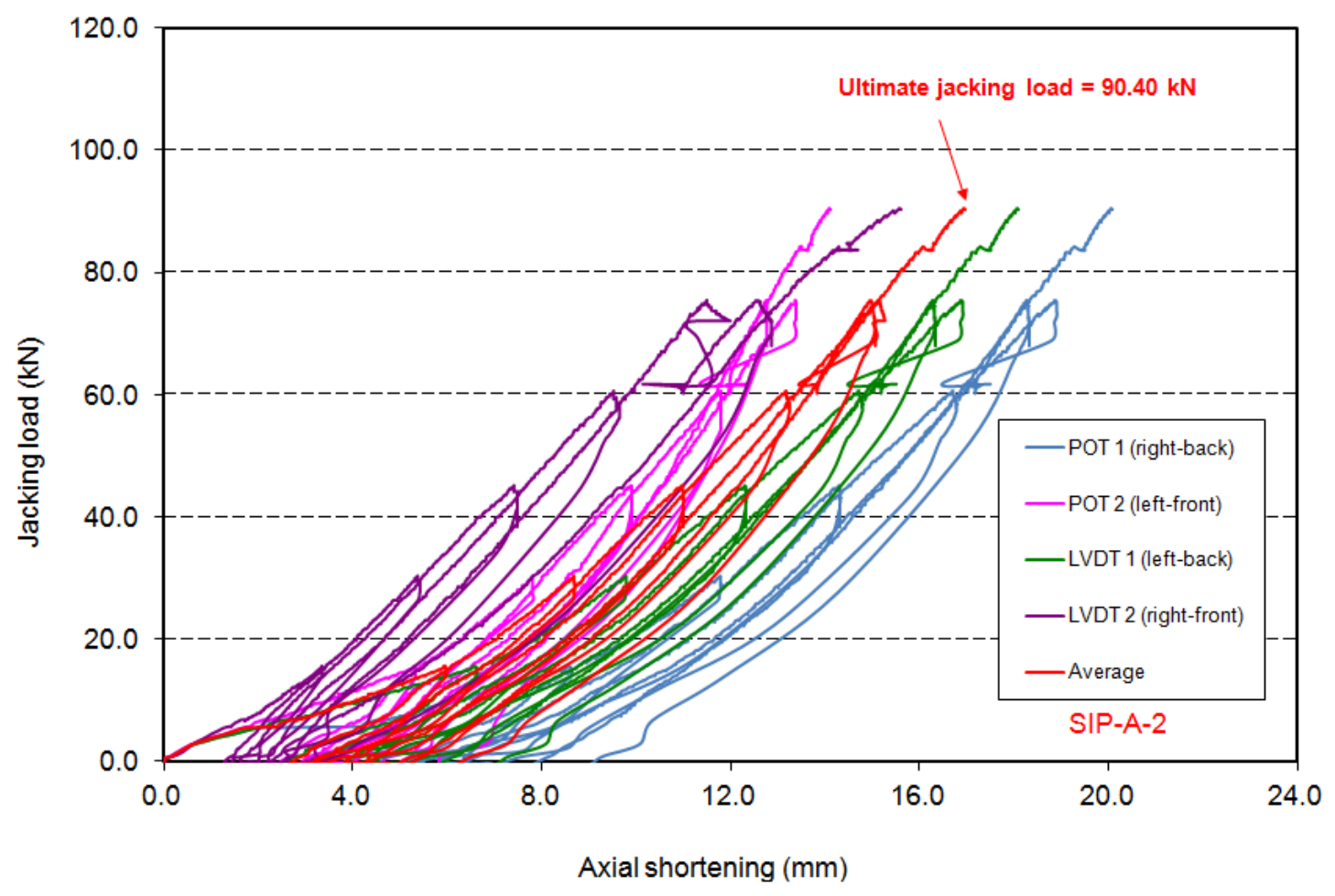

Figure 4.9 - Axial load-axial shortening relationships for specimen SIP-A-2

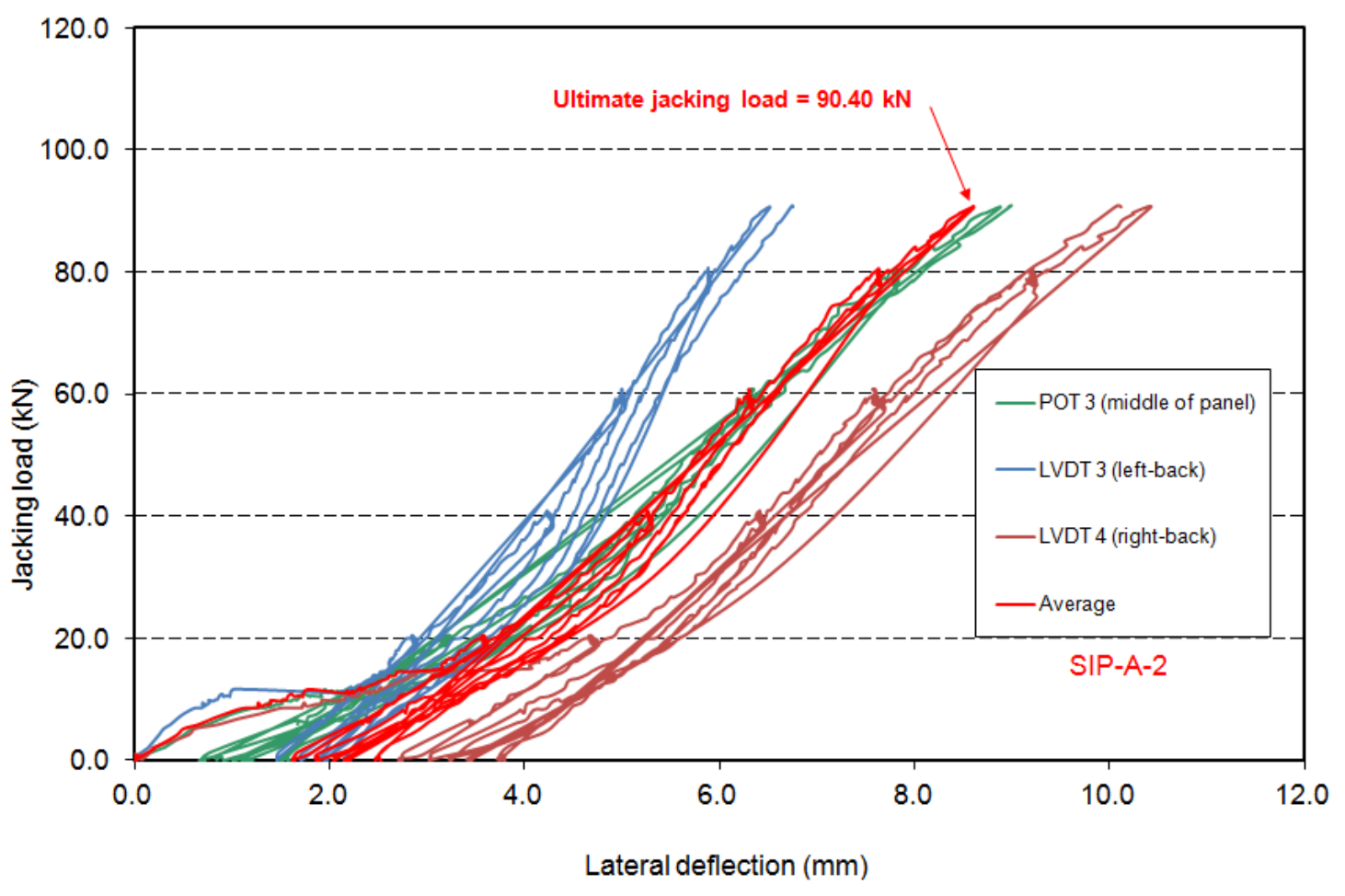

Figure 4.10 - Axial load-lateral deflection relationship for specimen SIP-A-2 

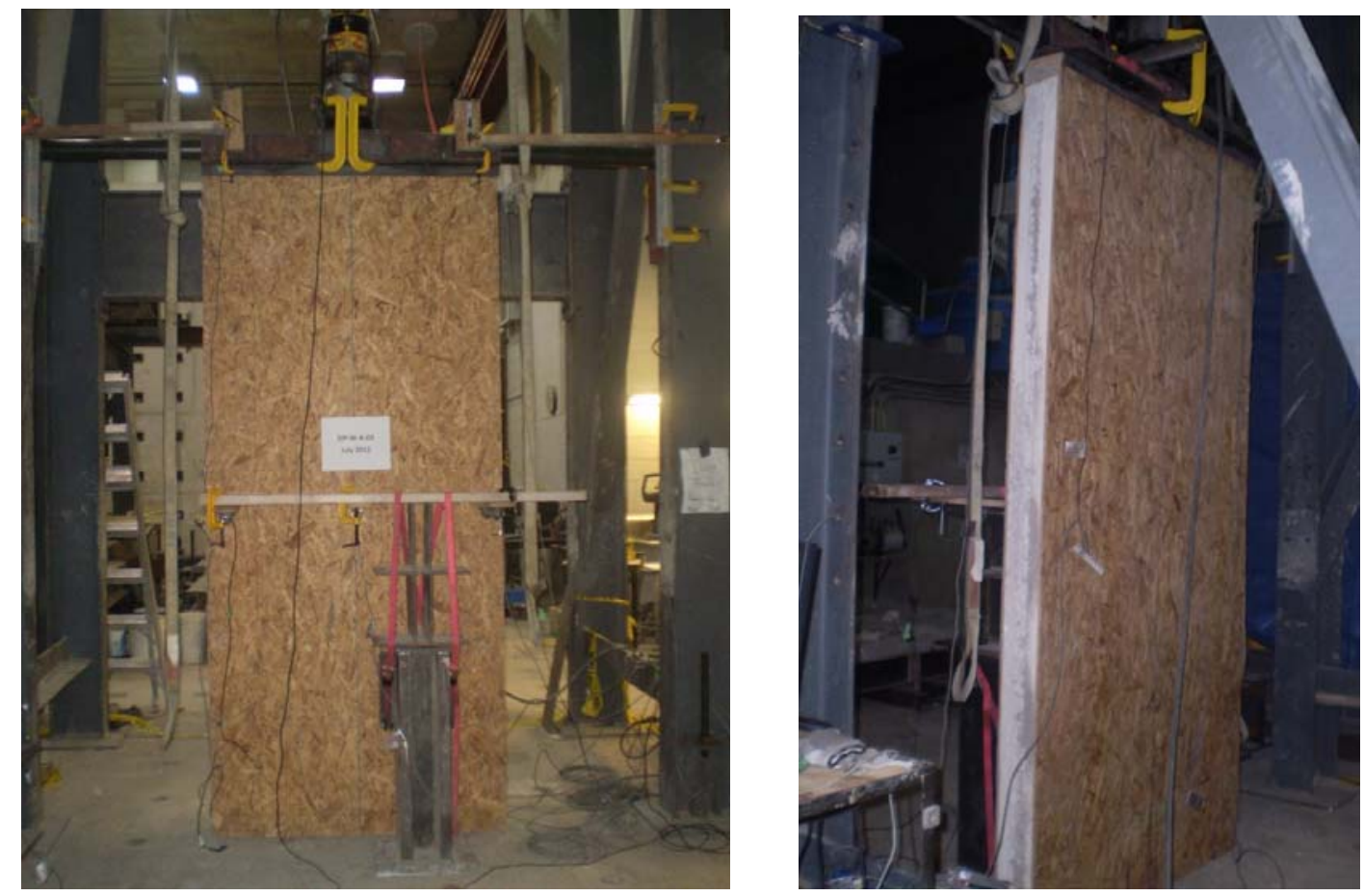

Figure 4.11 - Views of the test setup for wall SIP-A-3 before testing
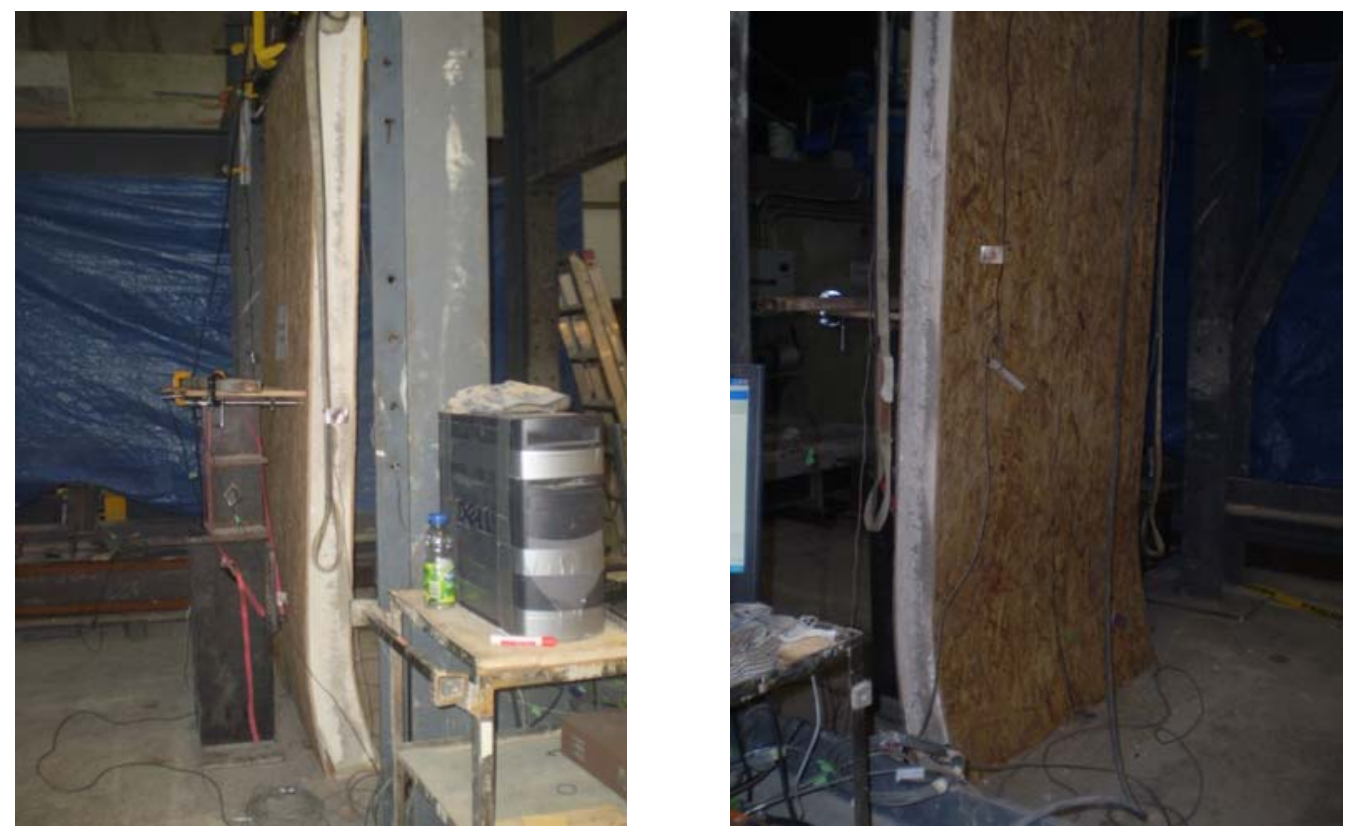

Figure 4.12 - Views of the deformed shape of the wall SIP-A-3 after failure 


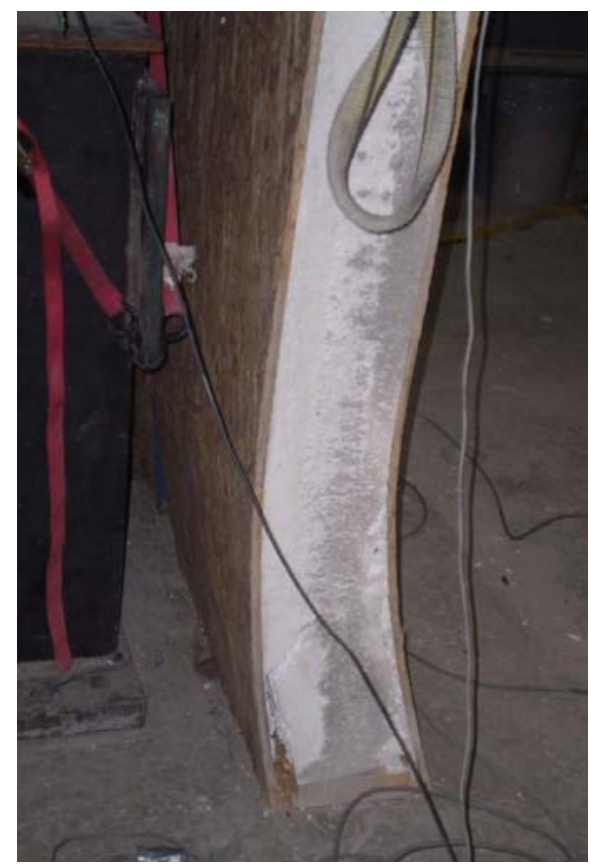

(a) Deflected shape from left side

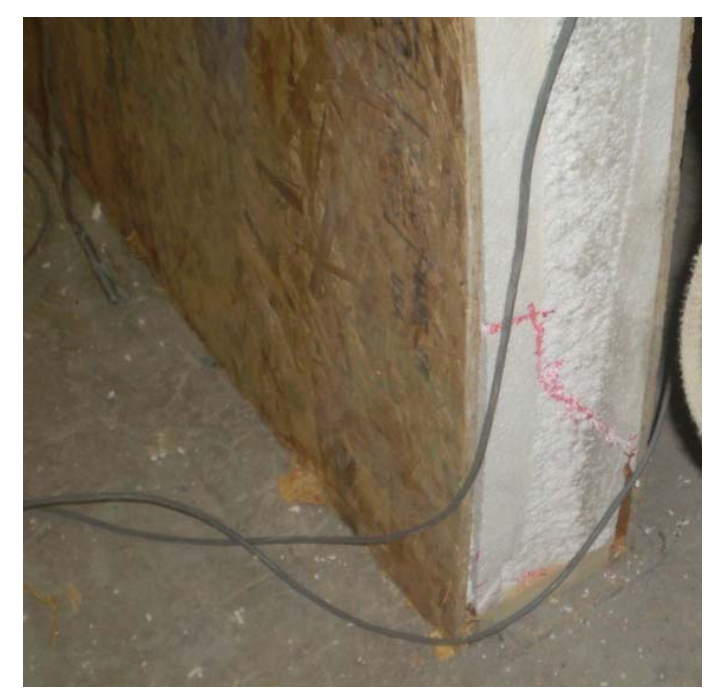

(c) Shear crack in foam at right side

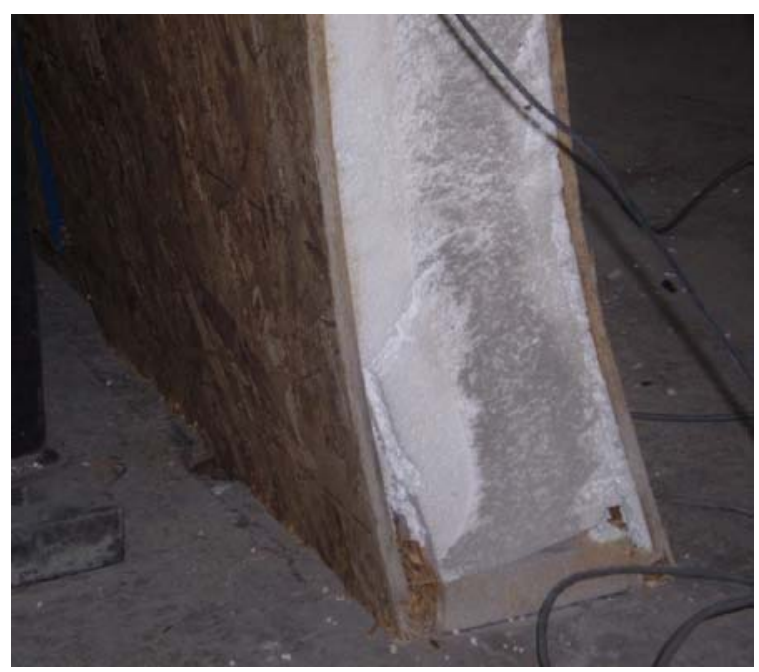

(b) Foam-OSB debonding and shear crack in foam

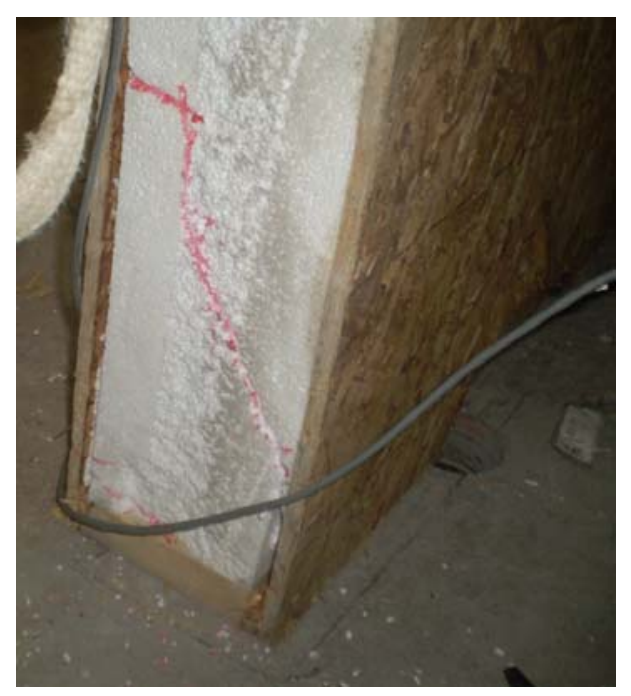

(d) Foam-OSB debonding and OSB crushing

Figure 4.13 - Close-up views of the failure modes of SIP-A-3 specimen 


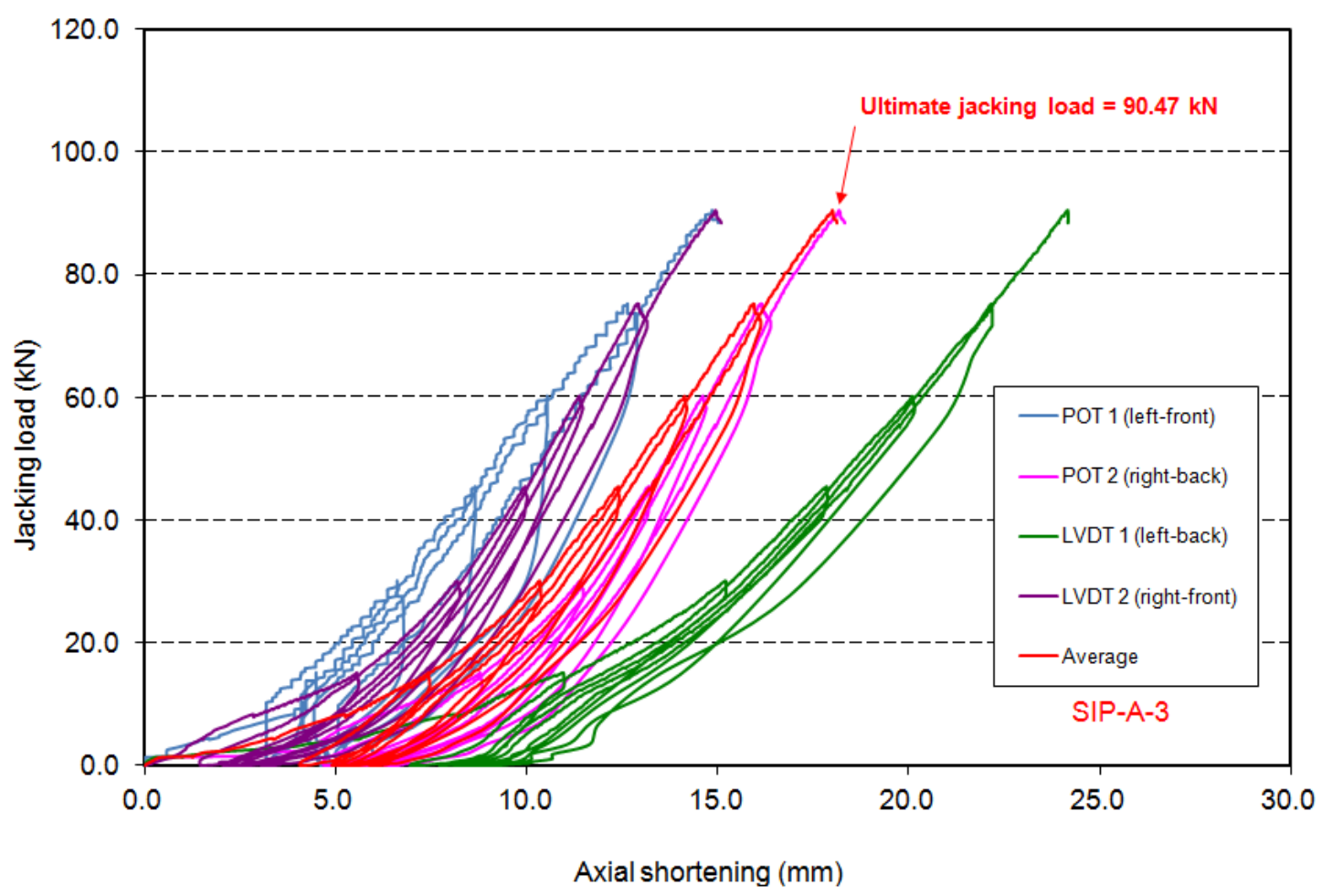

Figure 4.14 - Axial load-axial shortening relationships for specimen SIP-A-3

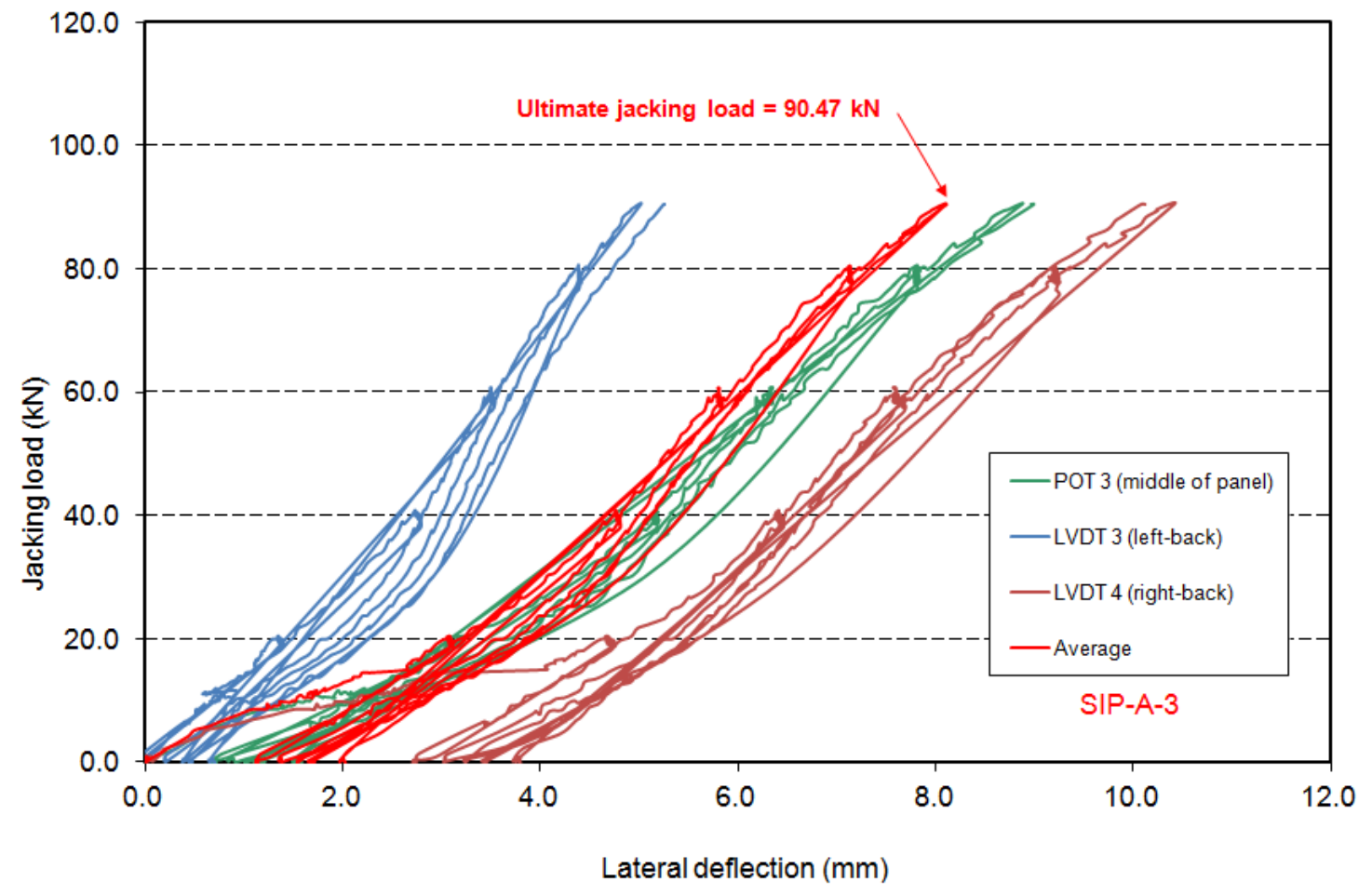

Figure 4.15 - Axial load-lateral deflection relationship for specimen SIP-A-3 
Table 4.1 shows that the experimental ultimate jacking loads were 104.44, 90.40, and $90.47 \mathrm{kN}$ for walls SIP-A-1, SIP-A-2 and SIP-A-3, respectively. By adding the $1.25 \mathrm{kN}$ weight of the loading system over the wall, the adjusted experimental ultimate jacking loads were 105.69, 91.65 and $91.72 \mathrm{kN}$ for walls SIP-A-1, SIP-A-2 and SIP-A-3, respectively. This leads to an average experimental ultimate compressive load of $96.35 \mathrm{kN}$ for this SIP wall as shown in Table 4.1.

Table 4.1- Results from axial compressive load tests: mean axial strengths and characteristic axial strengths

\begin{tabular}{|c|c|c|c|c|c|c|c|c|}
\hline Group & $\begin{array}{l}\text { Test } \\
\text { No. }\end{array}$ & Test type & $\begin{array}{l}\text { Panel size: } \\
\mathrm{I} \times \mathrm{w} \times \mathrm{t} \\
(\text { inch) }\end{array}$ & $\begin{array}{l}\text { Connect. } \\
\text { type }\end{array}$ & $\begin{array}{l}\text { Experim. } \\
\text { ultimate } \\
\text { jacking } \\
\text { load }(\mathbf{k N})\end{array}$ & $\begin{array}{l}\text { Adjusted } \\
\text { experim. } \\
\text { ultimate } \\
\text { jacking } \\
\text { load (kN) }\end{array}$ & $\begin{array}{l}\text { Mean } \\
\text { axial } \\
\text { strength } \\
(\mathbf{k N})^{(3)}\end{array}$ & $\begin{array}{l}\text { Charac. } \\
\text { axial } \\
\text { strength } \\
(\mathbf{k N})^{(3)}\end{array}$ \\
\hline \multirow[t]{3}{*}{ SIP } & SIP-A-1 & $\begin{array}{l}\text { Axial } \\
\text { load at } t / 6\end{array}$ & $\begin{array}{l}1220 \times 2743 \times 165 \\
\left(4^{\prime} \times 99^{\prime} \times 6^{1 / 2}{ }^{\prime \prime}\right)\end{array}$ & $\begin{array}{l}\text { Foam } \\
\text { spline }\end{array}$ & $104.44 *$ & $105.69^{(\mathbf{1})}$ & \multirow[t]{3}{*}{96.35} & \multirow[t]{3}{*}{45.07} \\
\hline & SIP-A-2 & $\begin{array}{l}\text { Axial } \\
\text { load at } t / 6\end{array}$ & $\begin{array}{l}1220 \times 2743 \times 165 \\
\left(4^{\prime} \times 99^{\prime} \times 6^{1 / 2 \prime}{ }^{\prime \prime}\right)\end{array}$ & $\begin{array}{l}\text { Foam } \\
\text { spline }\end{array}$ & $90.40^{*}$ & $91.65^{(\mathbf{1})}$ & & \\
\hline & SIP-A-3 & $\begin{array}{l}\text { Axial } \\
\text { load at } t / 6\end{array}$ & $\begin{array}{l}1220 \times 2743 \times 165 \\
\left(4^{\prime} \times 9 \times \times 6^{\prime} \stackrel{\prime}{\prime}{ }^{\prime \prime}\right)\end{array}$ & $\begin{array}{l}\text { Foam } \\
\text { spline }\end{array}$ & $90.47 *$ & $91.72^{(\mathbf{1})}$ & & \\
\hline \multirow[t]{3}{*}{$\begin{array}{l}\text { SIP } \\
\text { (Mohamed, 2009) }\end{array}$} & W7 & $\begin{array}{l}\text { Axial } \\
\text { load at } t / 6\end{array}$ & $\begin{array}{l}1220 \times 2743 \times 165 \\
\left(4^{\prime} \times 99^{\prime} \times 6{ }^{\prime}{ }^{\prime \prime}{ }^{\prime \prime}\right)\end{array}$ & $\begin{array}{l}\text { Foam } \\
\text { spline }\end{array}$ & $199.58^{*}$ & $200.83^{(1)}$ & \multirow[t]{3}{*}{214.64} & \multirow[t]{3}{*}{104.21} \\
\hline & W8 & $\begin{array}{l}\text { Axial } \\
\text { load at } t / 6\end{array}$ & $\begin{array}{l}1220 \times 2743 \times 165 \\
\left(4^{\prime} \times 99^{\prime} \times 6^{1 / 2 \prime}\right)\end{array}$ & $\begin{array}{l}\text { Foam } \\
\text { spline }\end{array}$ & $172.36^{*}$ & $173.61^{(1)}$ & & \\
\hline & W9 & $\begin{array}{l}\text { Axial } \\
\text { load at } \mathrm{t} / 6\end{array}$ & $\begin{array}{l}1220 \times 2743 \times 165 \\
\left(4^{\prime} \times 9^{\prime} \times 6{ }^{\prime}{ }^{\prime \prime}{ }^{\prime \prime}\right)\end{array}$ & $\begin{array}{l}\text { Foam } \\
\text { spline }\end{array}$ & $268.23^{*}$ & $269.48^{(1)}$ & & \\
\hline \multirow[t]{3}{*}{ STUD } & SW-A-1 & $\begin{array}{l}\text { Axial } \\
\text { load at } \mathrm{t} / 6\end{array}$ & $\begin{array}{l}2440 \times 2743 \times 165 \\
\left(8^{\prime} \times 9^{\prime} \times 6 \frac{1}{\prime \prime}{ }^{\prime \prime}\right)\end{array}$ & 2x6@2’ & $253.51 * *$ & $102.74^{(2)}$ & \multirow[t]{3}{*}{100.05} & \multirow[t]{3}{*}{48.49} \\
\hline & SW-A-2 & $\begin{array}{l}\text { Axial } \\
\text { load at } \mathrm{t} / 6\end{array}$ & $\begin{array}{l}2440 \times 2743 \times 165 \\
\left(8^{\prime} \times 9^{\prime} \times 6 \frac{1}{2} 2^{\prime \prime}\right)\end{array}$ & 2x6@2’ & $297.23 * *$ & $120.23^{(2)}$ & & \\
\hline & SW-A-3 & $\begin{array}{l}\text { Axial } \\
\text { load at } \mathrm{t} / 6\end{array}$ & $\begin{array}{l}2440 \times 2743 \times 165 \\
\left(8^{\prime} \times 9^{\prime} \times 6 \frac{1}{2}{ }^{\prime \prime}\right)\end{array}$ & 2x6@2’ & $189.60 * *$ & $77.18^{(2)}$ & & \\
\hline
\end{tabular}

* Did not include $1.25 \mathrm{kN}$ weight of the loading system.

** Did not include $3.34 \mathrm{kN}$ weight of the loading system.

(1) Included $1.25 \mathrm{kN}$ weight of the loading system.

(2) Included $3.34 \mathrm{kN}$ weight of the loading system. Values are divided by 2 for $1.2 \mathrm{~m}$ panel width and multiplied by 0.8 for using 5 studs rather than 4 in the tested wall.

${ }^{(3)}$ Values are per panel width of $1.2 \mathrm{~m}$. 
In engineering design, the experimental failure load of a group of specimens is affected by the number of identical specimens considered for testing along with the deviation of the strength value of each specimen as compared to the mean value. The European Standard EN 14358:2006 (EN, 2006) specified the following equations to determine the characteristic value of experimental data, $m_{k}$, for timber structures. It is assumed that the characteristic value of a material parameter or a resistance shall be determined at a confidence level of $\alpha=75 \%$, where the confidence level $\alpha$ is defined as the probability of which the characteristic value is greater that than the estimator on the characteristic value.

$$
\begin{aligned}
& m_{k}=\exp \left(\bar{y}-k_{s} s_{y}\right) \\
& \bar{y}=\frac{1}{n} \sum_{i=1}^{n} \ln m_{t} \\
& s_{y}=\sqrt{\frac{1}{n-1} \sum_{i=1}^{n}\left(\ln m_{t}-\bar{y}\right)^{2}}
\end{aligned}
$$

where;

$\bar{y}=$ Mean value

$k_{s}=$ factor depends on number of test specimens $\left(k_{s}=3.15\right.$ for 3 specimens $)$.

$s_{y}=$ Standard deviation

$n=$ Number of test values

$m_{i}=$ test value

Using equation 4.1 to 4.3 , the characteristic ultimate compressive resisting load of the SIP panel group was calculated as $45.07 \mathrm{kN}$.

As per AC-04, the allowable design compressive load is the experimental ultimate load divided by 2.5. Thus, the allowable design compressive loads are calculated as 42.28, 36.66 and 36.69 $\mathrm{kN}$ for walls SIP-A-1, SIP-A-2 and SIP-A-3, respectively. Since the obtained design values of the three identical walls are less than $15 \%$ difference with the average value of the three values, the allowable design compressive load for this SIP group is $38.54 \mathrm{kN}$, as the average of the three values. This value is recorded in Table 4.2. Table 4.2 shows that the jacking loads at 3-mm (1/8”) axial shortening were 36.30, 28.08 and 25.10 kN for walls SIP-A-1, SIP-A-2 and SIP-A-3, respectively. Since the obtained design values of the three values are more than $15 \%$ difference 
with the average value of the three walls, the allowable design compressive load per the $3 \mathrm{~mm}$ $\left(1 / 8^{\prime \prime}\right)$ axial shortening criteria for this SIP group is $29.93 \mathrm{kN}$, as the least value of the three. This value is reported in summary Tables 4.2. Based on the results recorded in Tables 4.1 and 4.2, it can be concluded that the allowable design compressive load for this SIP wall group is $38.54 \mathrm{kN}$.

Table 4.2 - Results from axial compressive load tests: Allowable design loads and load at 3-mm deformation based on ICC-EC AC04 (2004)

\begin{tabular}{|c|c|c|c|c|c|c|c|c|c|}
\hline Group & $\begin{array}{l}\text { Test } \\
\text { No. }\end{array}$ & $\begin{array}{l}\text { Test } \\
\text { type }\end{array}$ & $\begin{array}{l}\text { Panel size: } \\
\mathrm{l} \times \mathrm{w} \times \mathrm{t} \\
(\mathrm{ft}-\mathrm{inch})\end{array}$ & $\begin{array}{l}\text { Connec. } \\
\text { type }\end{array}$ & $\begin{array}{l}\text { Adjusted } \\
\text { experim. } \\
\text { ultimate } \\
\text { jacking } \\
\text { load } \\
(k N)^{(4)}\end{array}$ & $\begin{array}{l}\text { Allowable } \\
\text { jacking } \\
\text { load } \\
(\mathrm{kN})^{(4)}\end{array}$ & $\begin{array}{l}\text { Mean } \\
\text { allowable } \\
\text { jacking } \\
\text { load } \\
(\mathrm{kN})^{(4)}\end{array}$ & $\begin{array}{l}\text { Jacking } \\
\text { load at } \\
\text { 3-mm } \\
\text { axial } \\
\text { shortening } \\
\text { limit } \\
(\mathrm{kN})^{(4)}\end{array}$ & $\begin{array}{l}\text { Mean load } \\
\text { at 3-mm } \\
\text { axial } \\
\text { shortening } \\
(\mathrm{kN})^{(4)}\end{array}$ \\
\hline \multirow[t]{3}{*}{ SIP } & SIP-A-1 & $\begin{array}{l}\text { Axial } \\
\text { (at } t / 6)\end{array}$ & $\begin{array}{l}1220 \times 2743 \times 165 \\
\left(4^{\prime} \times 9^{\prime} \times 6^{1 / 2 \prime}{ }^{\prime \prime}\right)\end{array}$ & $\begin{array}{l}\text { Foam } \\
\text { spline }\end{array}$ & $105.69^{(1)}$ & $42.28^{(3)}$ & \multirow[t]{3}{*}{38.54} & 36.60 & \multirow[t]{3}{*}{29.93} \\
\hline & SIP-A-2 & $\begin{array}{l}\text { Axial } \\
(\text { at } t / 6)\end{array}$ & $\begin{array}{l}1220 \times 2743 \times 165 \\
\left(4^{\prime} \times 9^{\prime} \times 6^{1 / 2 \prime}{ }^{\prime \prime}\right)\end{array}$ & $\begin{array}{l}\text { Foam } \\
\text { spline }\end{array}$ & $91.65^{(\mathbf{1})}$ & $36.66^{(3)}$ & & 28.08 & \\
\hline & SIP-A-3 & $\begin{array}{l}\text { Axial } \\
\text { (at } t / 6)\end{array}$ & $\begin{array}{l}1220 \times 2743 \times 165 \\
\left(4^{\prime} \times 9^{\prime} \times 6^{1 / 2 \prime}{ }^{\prime \prime}\right)\end{array}$ & $\begin{array}{l}\text { Foam } \\
\text { spline }\end{array}$ & $91.72^{(1)}$ & $36.69^{(3)}$ & & 25.10 & \\
\hline \multirow{3}{*}{$\begin{array}{l}\text { SIP } \\
\text { (Mohamed, } \\
\text { 2009) }\end{array}$} & W7 & $\begin{array}{l}\text { Axial } \\
\text { (at } \mathrm{t} / 6 \text { ) }\end{array}$ & $\begin{array}{l}1220 \times 2743 \times 165 \\
\left(4^{\prime} \times 9^{\prime} \times 6{ }^{1 / 2} 2^{\prime \prime}\right)\end{array}$ & $\begin{array}{l}\text { Foam } \\
\text { spline }\end{array}$ & $200.83^{(1)}$ & $80.33^{(3)}$ & \multirow[t]{3}{*}{69.44} & 86.66 & \multirow[t]{3}{*}{56.61} \\
\hline & W8 & $\begin{array}{l}\text { Axial } \\
\text { (at } t / 6)\end{array}$ & $\begin{array}{l}1220 \times 2743 \times 165 \\
\left(4^{\prime} \times 9^{\prime} \times 6 \frac{1}{2} 2^{\prime \prime}\right)\end{array}$ & $\begin{array}{l}\text { Foam } \\
\text { spline }\end{array}$ & $173.61^{(\mathbf{1})}$ & $69.44^{(3)}$ & & 56.61 & \\
\hline & W9 & $\begin{array}{l}\text { Axial } \\
\text { (at } t / 6)\end{array}$ & $\begin{array}{l}1220 \times 2743 \times 165 \\
\left(4^{\prime} \times 9^{\prime} \times 6^{1 / 2 \prime}{ }^{\prime \prime}\right)\end{array}$ & $\begin{array}{l}\text { Foam } \\
\text { spline }\end{array}$ & $269.48^{(\mathbf{1})}$ & $107.79^{(3)}$ & & 87.82 & \\
\hline \multirow[t]{3}{*}{ STUD } & SW-A-1 & $\begin{array}{l}\text { Axial } \\
\text { (at } t / 6)\end{array}$ & $\begin{array}{l}2440 \times 2743 \times 165 \\
\left(8^{\prime} \times 9^{\prime} \times 6 \frac{1}{1} 2^{\prime \prime}\right)\end{array}$ & 2x6@2' & $102.74^{(2)}$ & $41.10^{(3)}$ & \multirow[t]{3}{*}{30.87} & 58.97 & \multirow[t]{3}{*}{$25.06^{(5)}$} \\
\hline & SW-A-2 & $\begin{array}{l}\text { Axial } \\
\text { (at } \mathrm{t} / 6 \text { ) }\end{array}$ & $\begin{array}{l}2440 \times 2743 \times 165 \\
\left(8^{\prime} \times 9^{\prime} \times 6 \frac{1}{2} 2^{\prime \prime}\right)\end{array}$ & 2x6@2’ & $120.23^{(2)}$ & $48.09^{(3)}$ & & 62.98 & \\
\hline & SW-A-3 & $\begin{array}{l}\text { Axial } \\
\text { (at } t / 6)\end{array}$ & $\begin{array}{l}2440 \times 2743 \times 165 \\
\left(8^{\prime} \times 9^{\prime} \times 6^{1 / 2 \prime}{ }^{\prime \prime}\right)\end{array}$ & 2x6@2’ & $77.18^{(2)}$ & $30.87^{(3)}$ & & 65.99 & \\
\hline
\end{tabular}

(1) Included $1.25 \mathrm{kN}$ weight of the loading system.

(2) Included $3.34 \mathrm{kN}$ weight of the loading system.

(3) Considering safety factor of 2.5 .

(4) Values are per panel width of $1.2 \mathrm{~m}$.

${ }^{(5)}$ Values are divided by 2 for $1.2 \mathrm{~m}$ panel width and multiplied by 0.8 for using 5 studs rather than 4 in the tested wall. 


\subsubsection{Results of Stud Group for Axial Loading}

In this group, three identical stud wall specimens, namely: SW-A-1, SW-A-2, and SW-A-3, were tested to complete collapse under uniformly distributed axial compression load with $\mathrm{t} / 6$ eccentricity. Each specimen was of $2450 \mathrm{~mm}$ width contract to $1220 \mathrm{~mm}$ width considered for SIP specimens. Figure 4.16 shows view of stud specimen SW-A-1 before testing, while Figure 4.17 shows views of the permanent deformed shape of the panel after failure as well as close-up views of the local damage and deformation. It was observed that the failure mode in stud wall SW-A-1 was due to global bending of the studs. This is shown through the bending deformation of the wall from the drywall side as shown in Figure 4.17-d and the widening of the gap between OSB segments near the top of the wall as shown in Figs. 4.17-a through 4.17-c. Failure of the stud wall was considered reached with the specimen did not absorb any more loading while deformation was increasing. It should be noted that the top spread beam loading system was considerably rotating at failure.

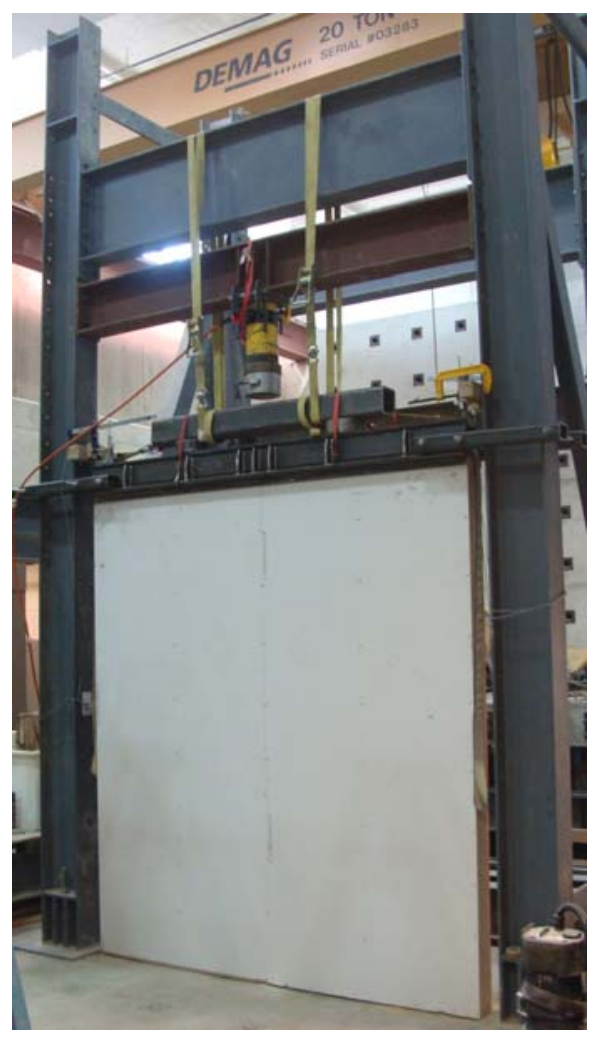

(a) Dry wall side

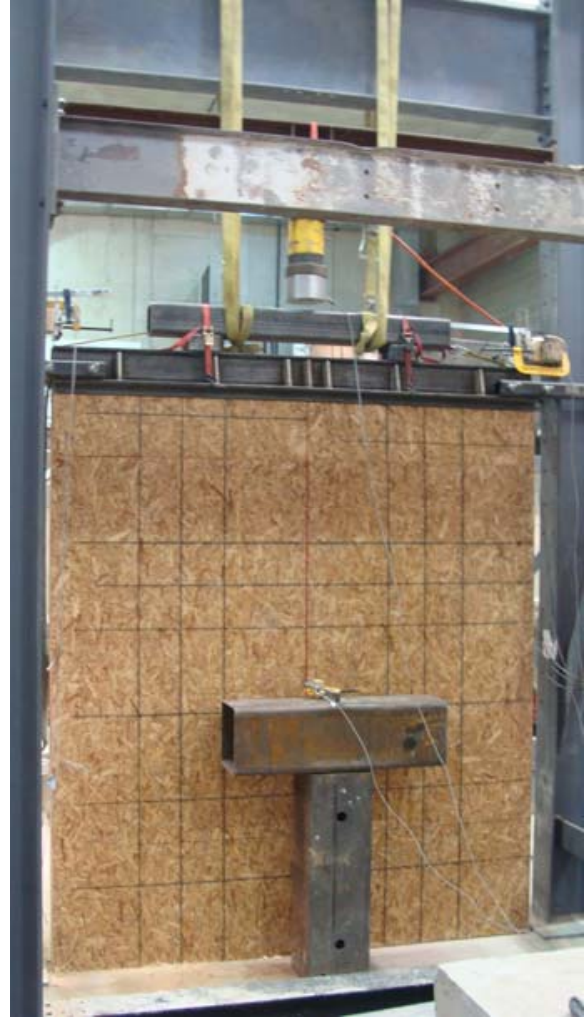

(b) OSB sheet side

Figure 4.16 - Views of the test setup for stud wall SW-A-1 before testing 


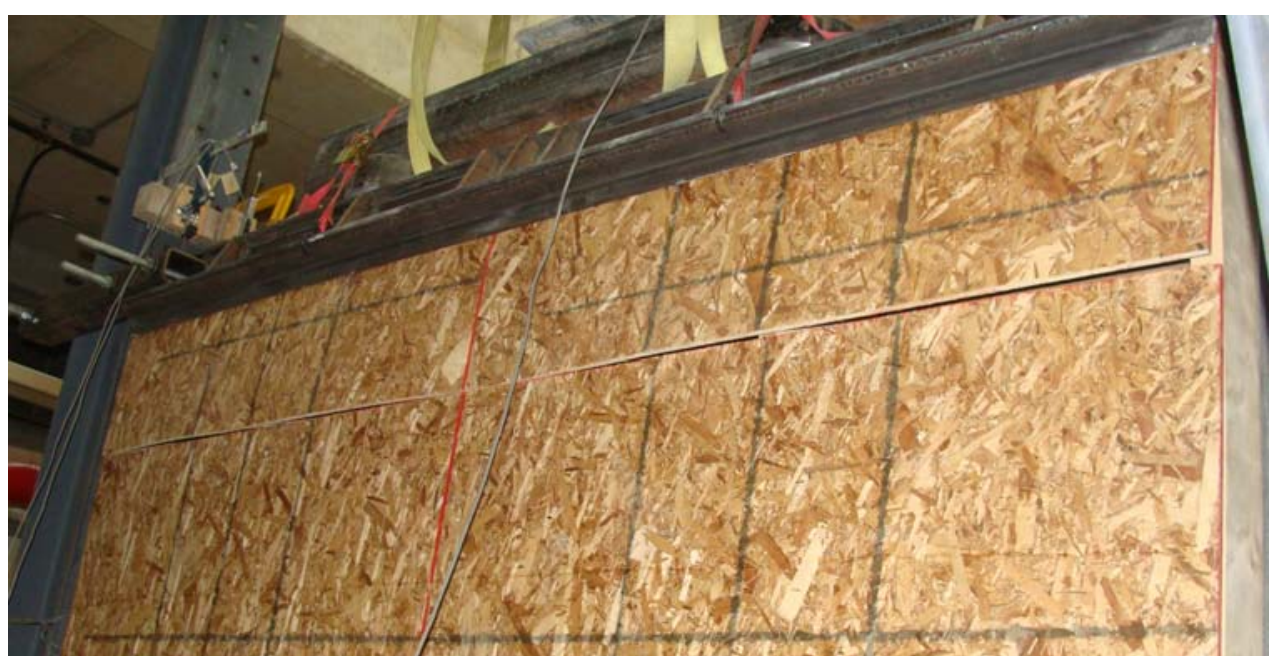

(a) View of the panel after failure showing OSB sheet joint widening near the top of wall

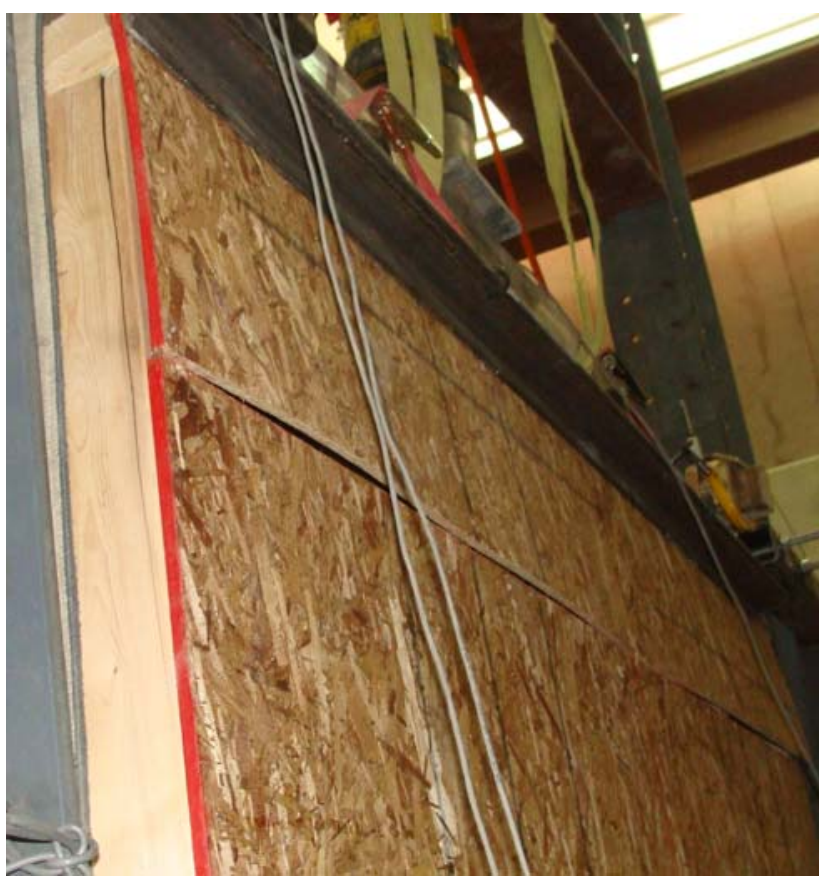

(b) View of the OSB sheet joint widening from left side sheet joint

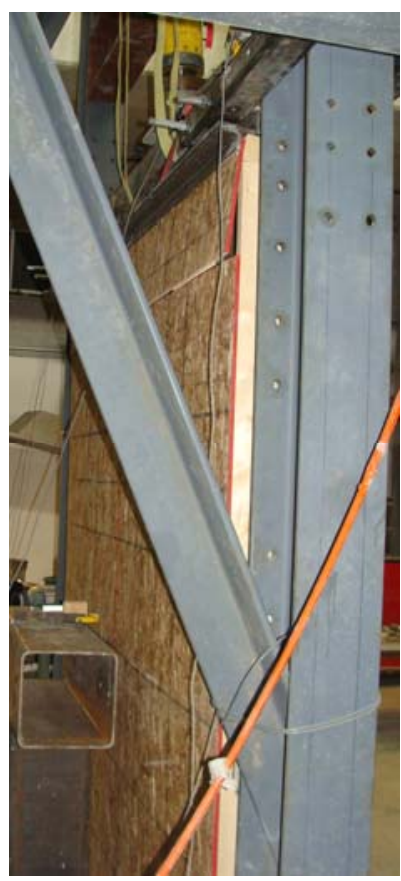

(c) View of the OSB widening from the right side 


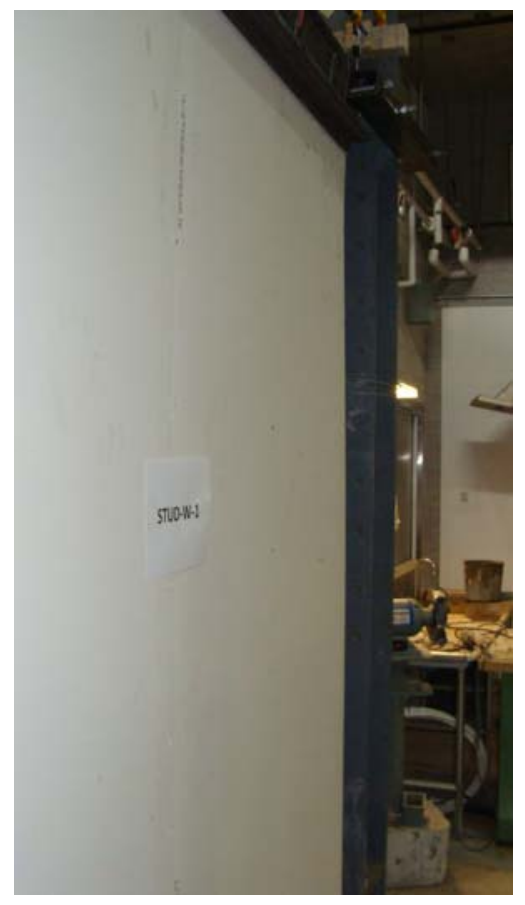

(d) View of the wall from the dry wall side showing the lateral deflection of the wall Figure 4.17 - Views of the deformed shape of the stud wall SW-A-1 after OSB joint opening at its top north side

Figure 4.18 depicts the load-axial shortening relationship, while Figure 4.19 depicts the loadlateral deflection relationship at the mid-height of the wall. It can be observed that at the beginning of the test, the wall axial shortening did not increase linearly with load increase till about $50 \mathrm{kN}$, then the system started to pick up the load leading to linear increase in the elastic shortening with load increase through a considerable portion of the load history, followed by nonlinear increase in axial shortening while approaching failure. 


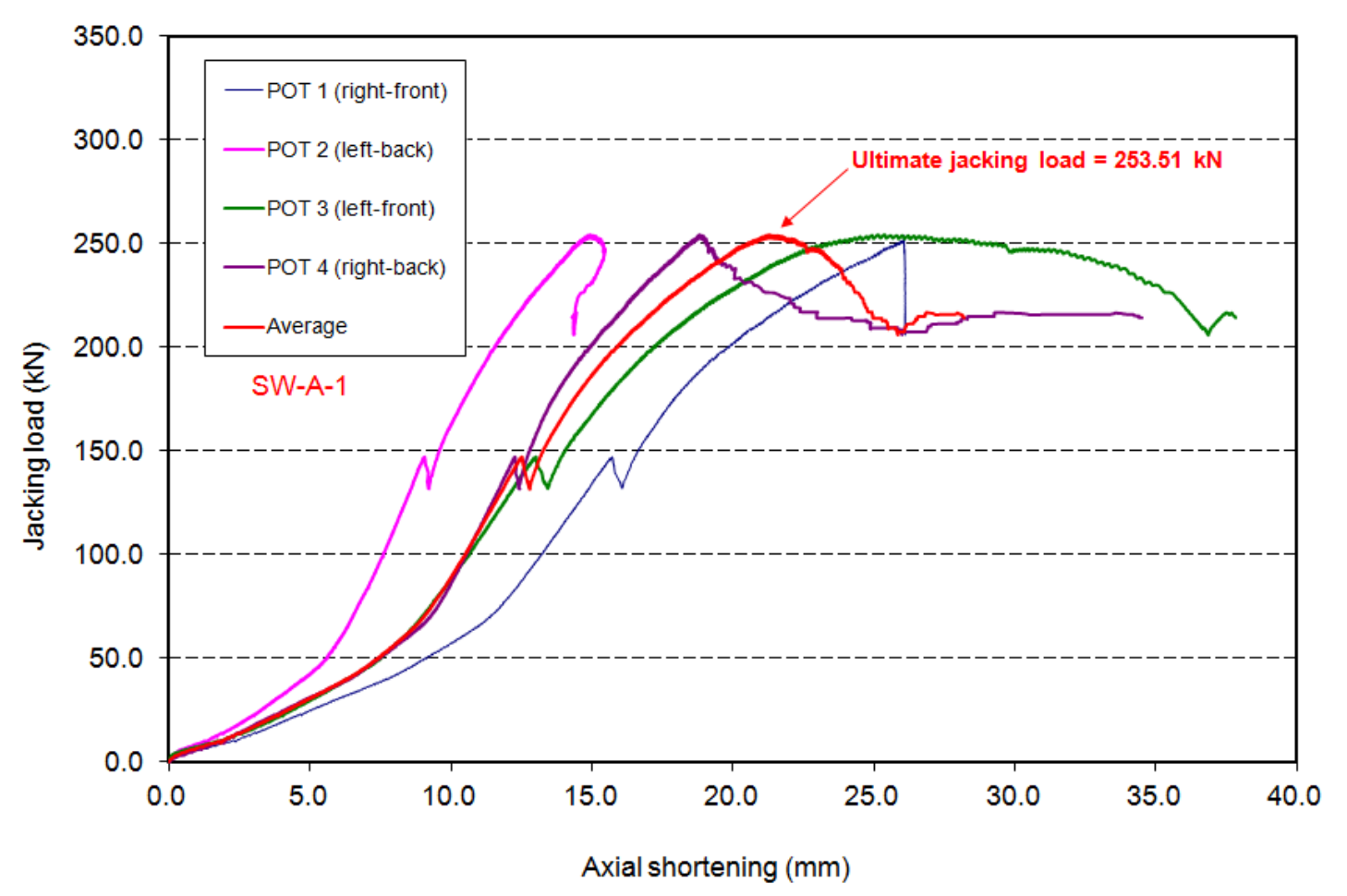

Figure 4.18 - Axial load-axial shortening relationships for stud wall SW-A-1

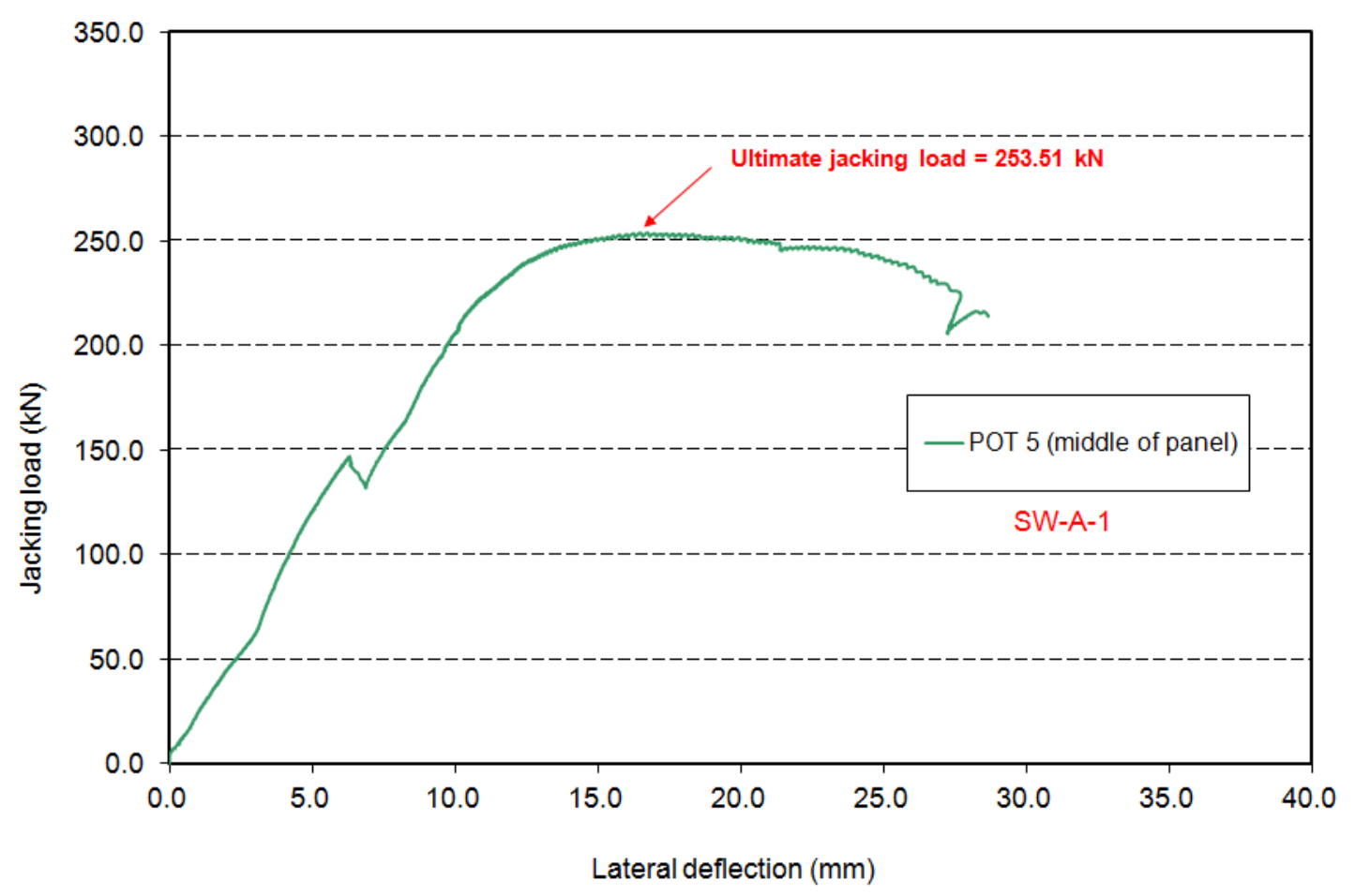

Figure 4.19 - Axial load-lateral deflection relationship for stud wall SW-A-1 
Similar behavior to wall SW-A-1 was observed for the identical walls SW-A-2 and SW-A-3. Figure 4.20 shows view of stud wall specimen SW-A-2 before testing, while Figs. 4.21(a) and 4.21(b) show views of the permanent deformed shape of the wall after failure that was mainly flexural. Also, Figure 4.22 shows view of the rotated top loading system at failure. Figure 4.23 depicts the load-axial shortening relationship for this wall, while Figure 4.24 depicts the axial load-lateral deflection relationship for wall SW-A-2. Figure 4.25 shows view of panel SW-A-3 before testing, while Figure 4.26 shows views of the permanent deformed shape of the stud wall after failure which is similar to those for specimens SW-F-1 and SW-F-2. Figure 4.27 depicts the load-axial shortening relationship for the model. It can be observed that lateral deflection increased with increase in the applied load. This may be attributed to the lateral bending of the wall due to the applied eccentric loading as well as the non-symmetric section as a result of using two different facing materials (i.e. OSB from one side and drywall from the other side).

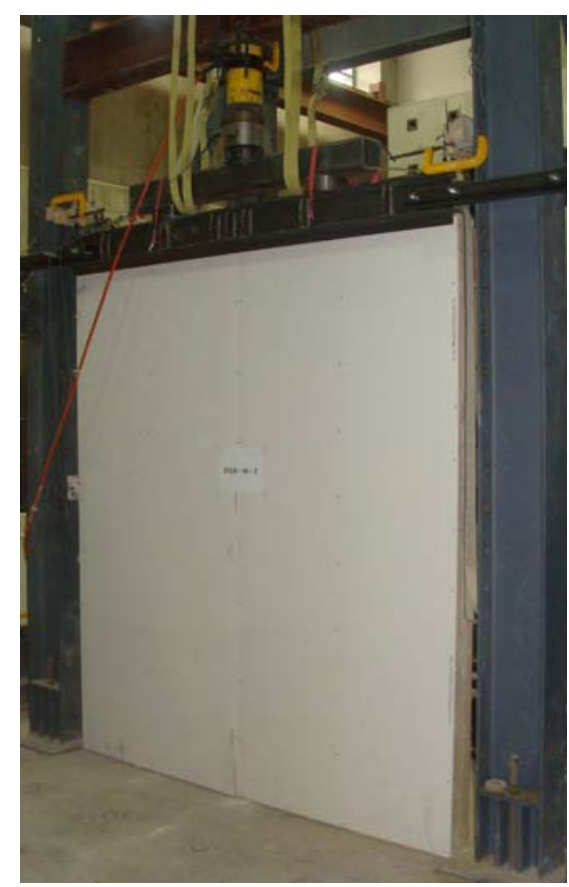

(a) Dry wall side

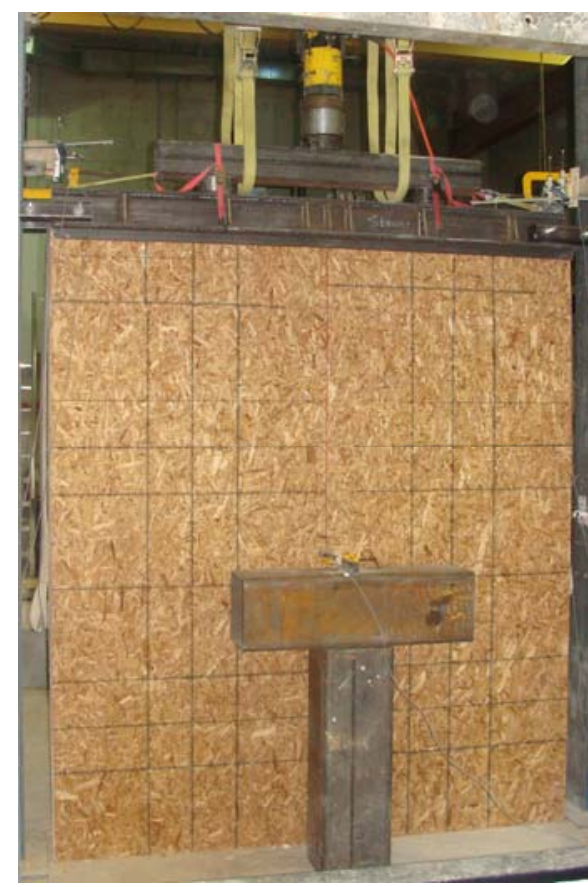

(b) OSB sheet side

Figure 4.20 - Views of the test setup for the stud wall SW-2 before testing 


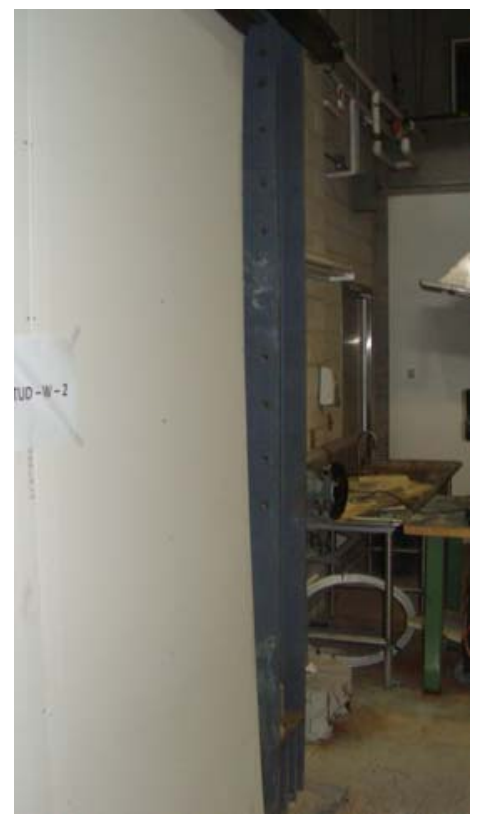

(a) Lateral deflected shape of the drywall side of the wall

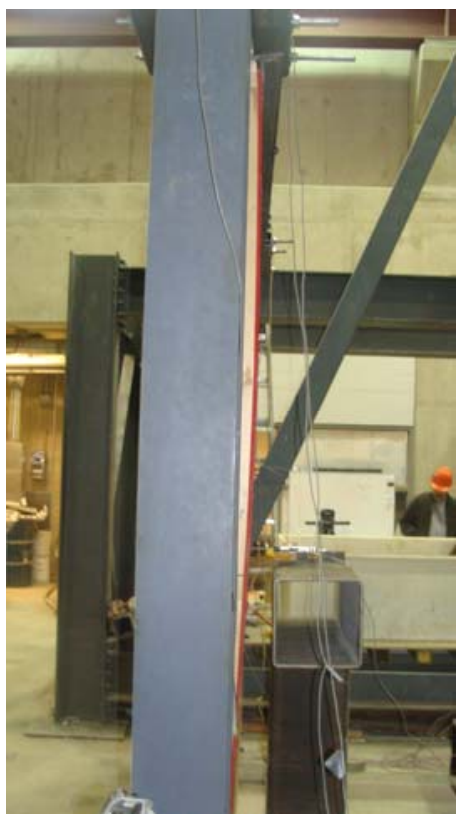

(b) Lateral deflection shape of the OSB sheet side of the wall

Figure 4.21 - Views of the deformed shape of the stud wall SW-A-2 after failure

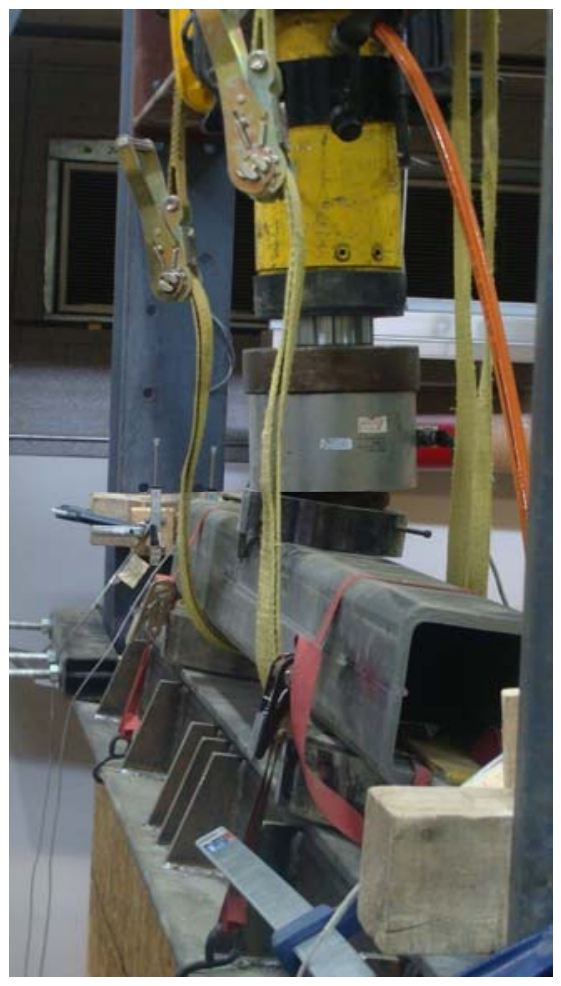

Figure 4.22 - Rotation of the top rail at failure of stud wall specimen SW-A-2 


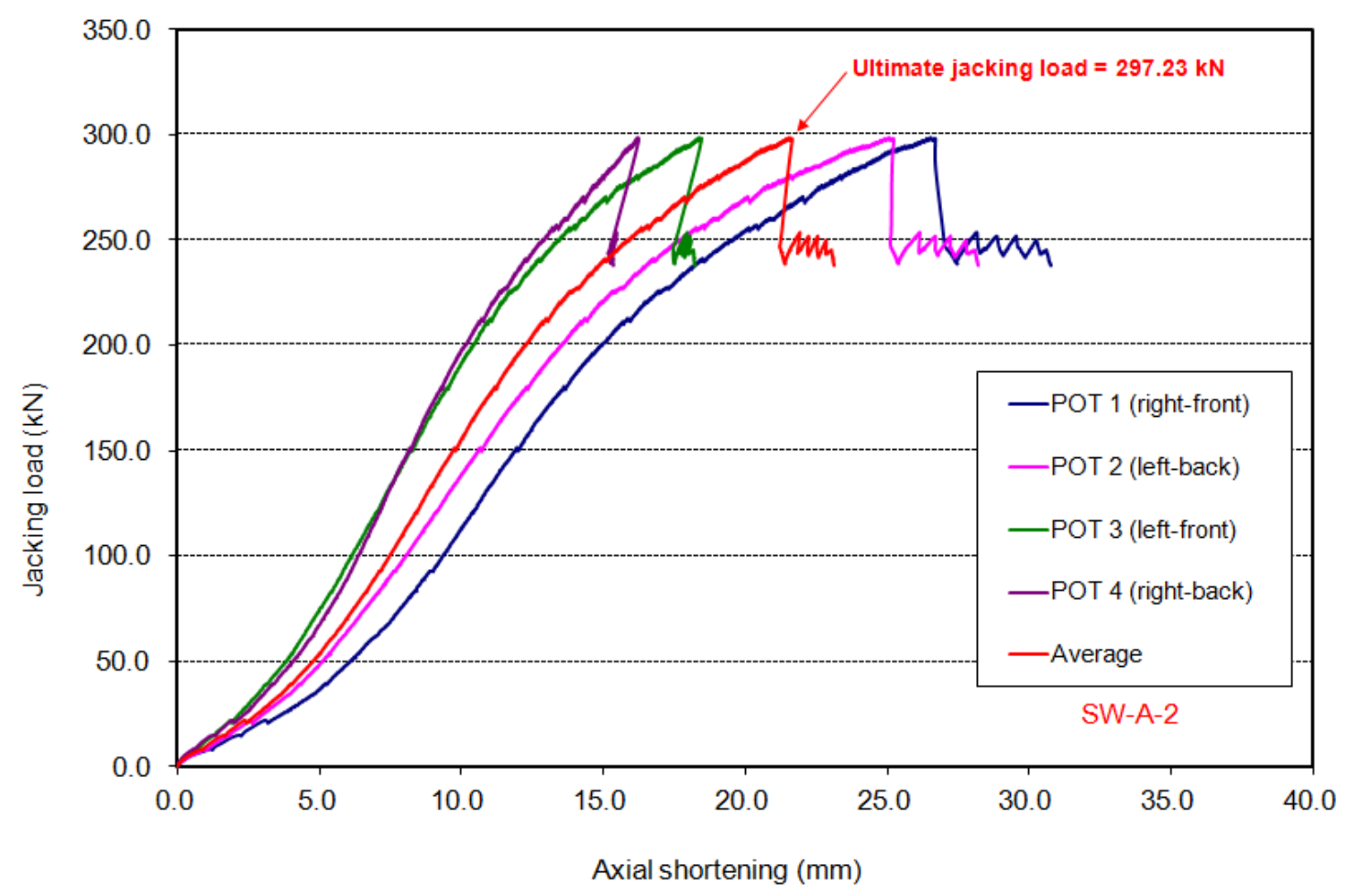

Figure 4.23 - Axial load-axial shortening relationships for stud wall SW-A-2

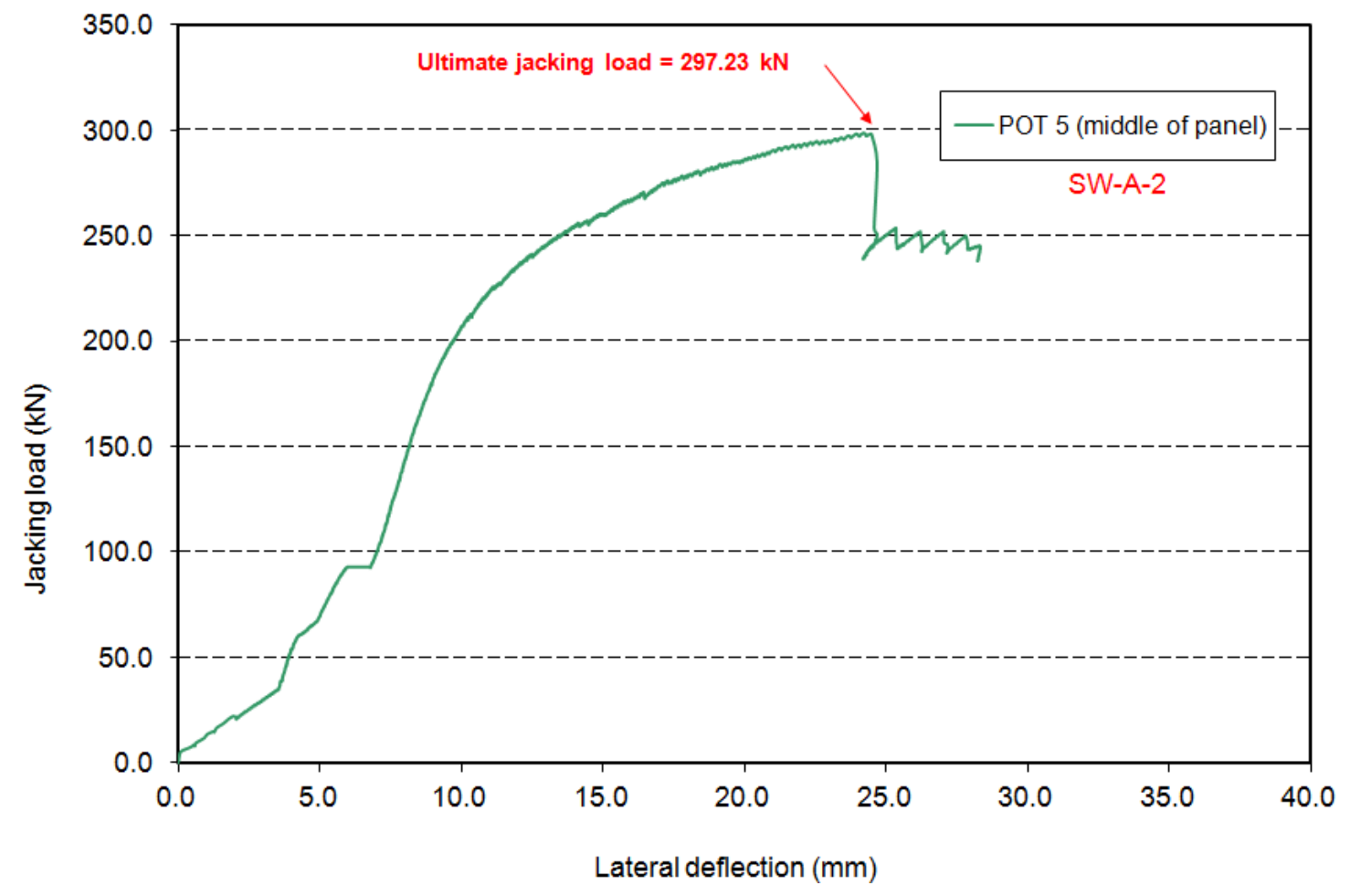

Figure 4.24 - Axial load-lateral deflection relationships for stud wall SW-A-2 


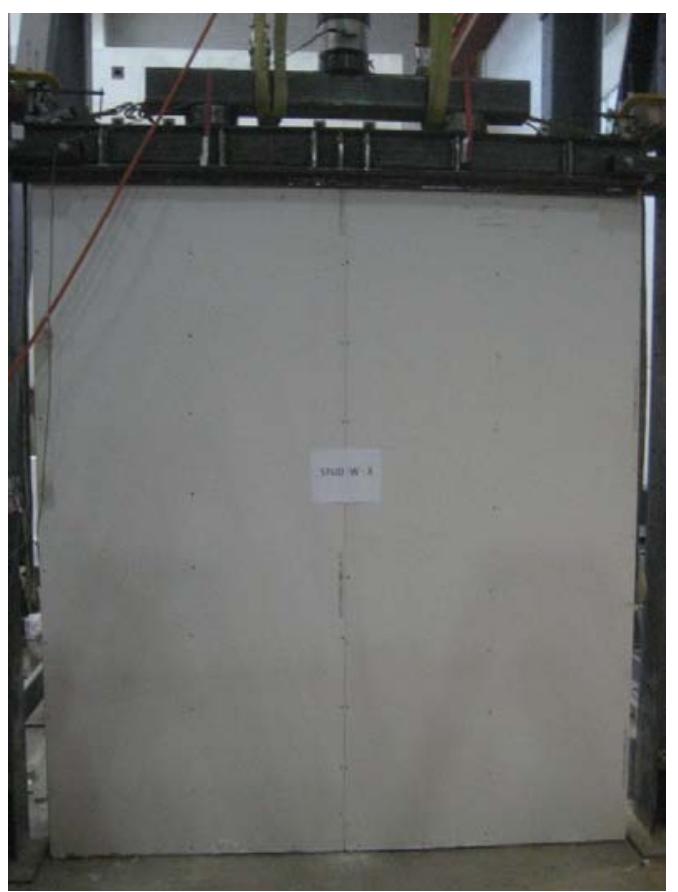

(a) Dry wall side

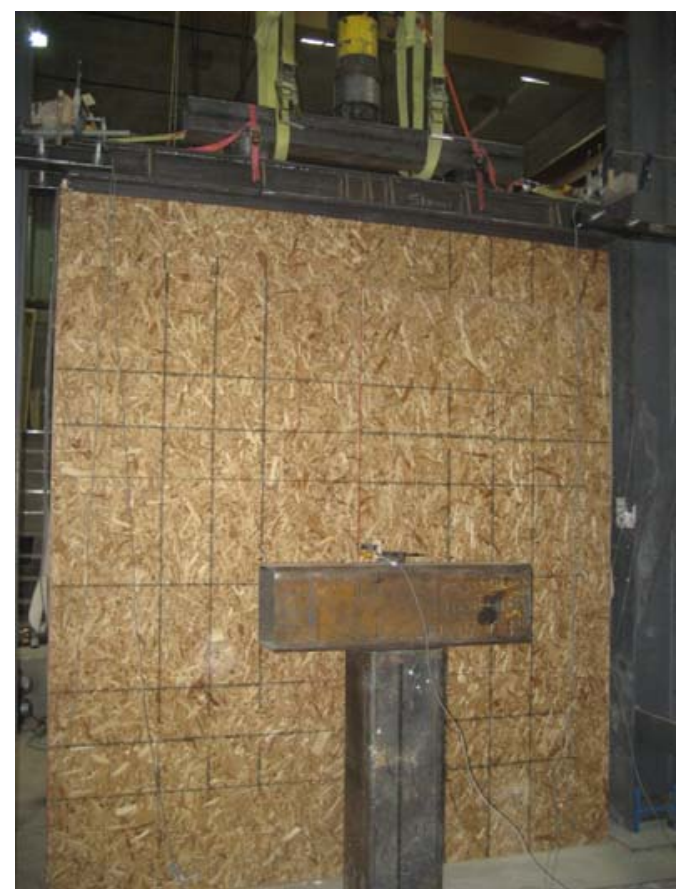

(b) OSB sheet side

Figure 4.25 - Views of the test setup for stud wall SW-3 before testing

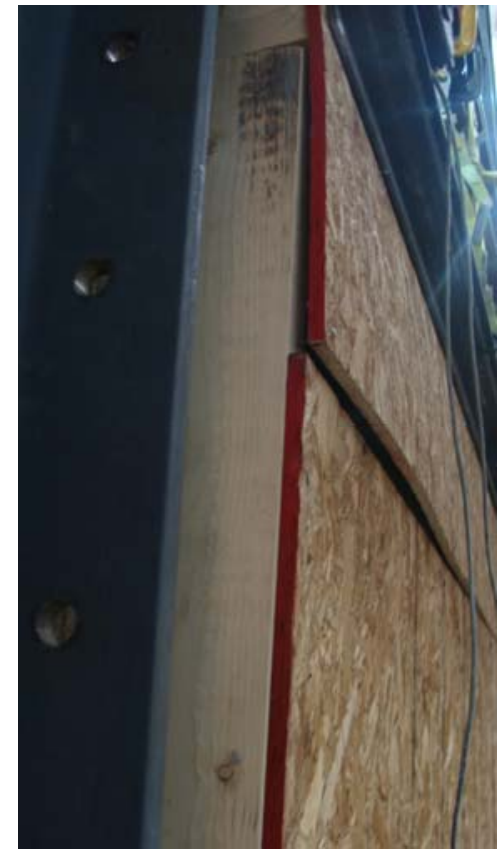

(a) View of OSB sheet joint opening in the side of the wall

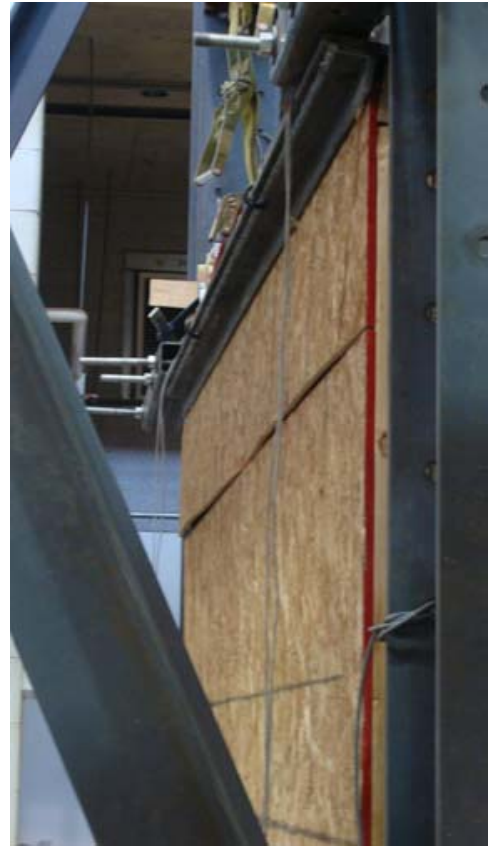

(b) View of OSB sheet joint opening in left right side of the wall 


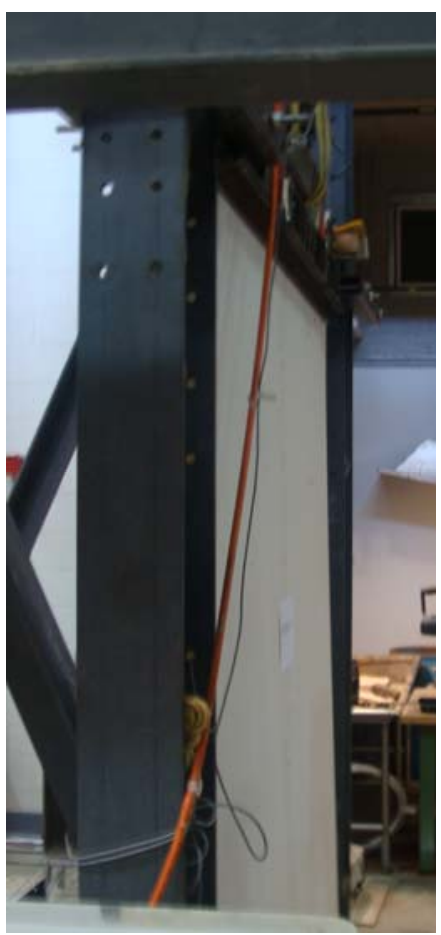

(c) View of the deflected shape of the wall from the drywall side

Figure 4.26 - Views of the deformed shape of stud wall SW-A-3 after failure

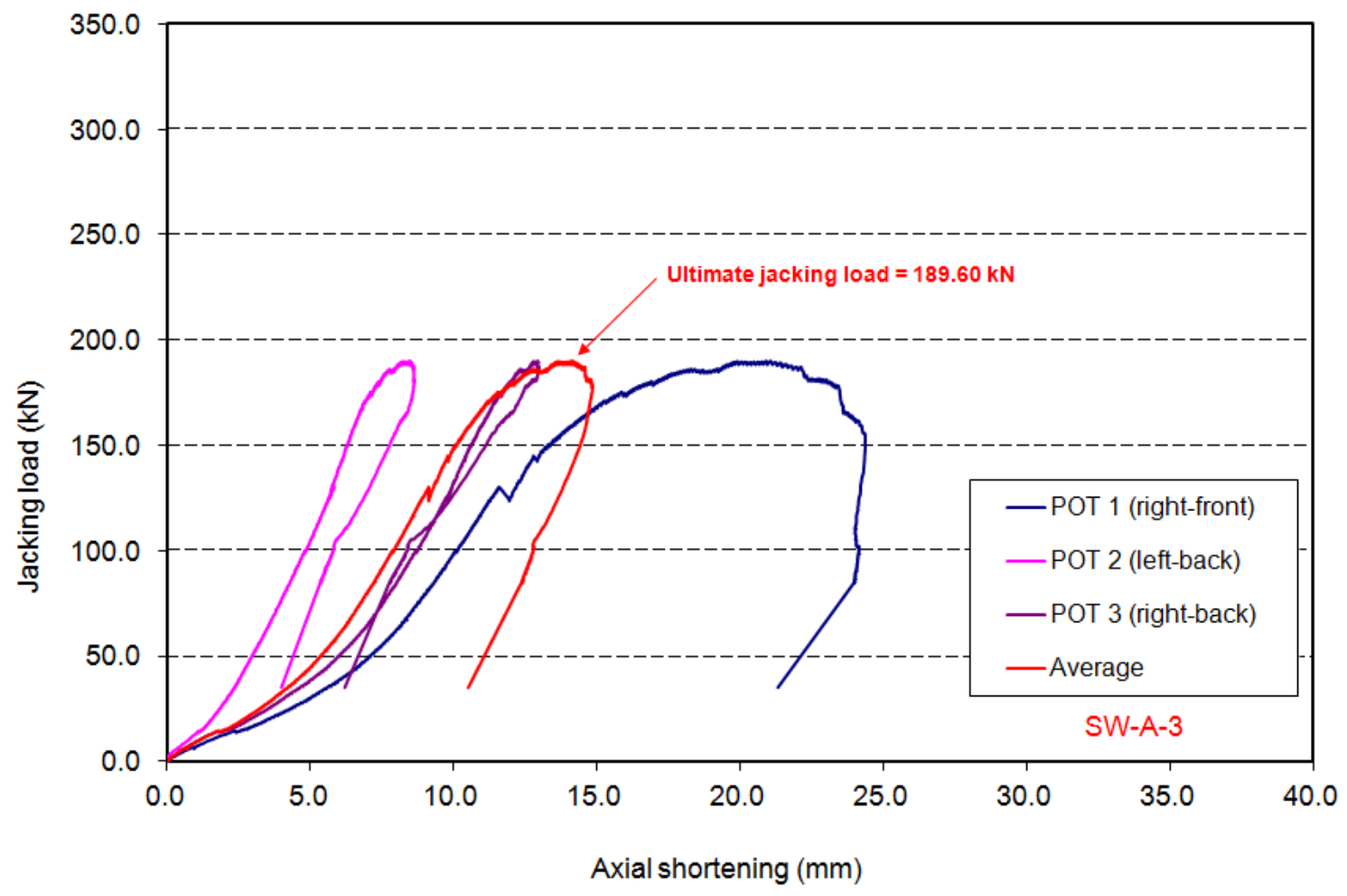

Figure 4.27 - Axial load-axial shortening relationships for stud wall SW-A-3 


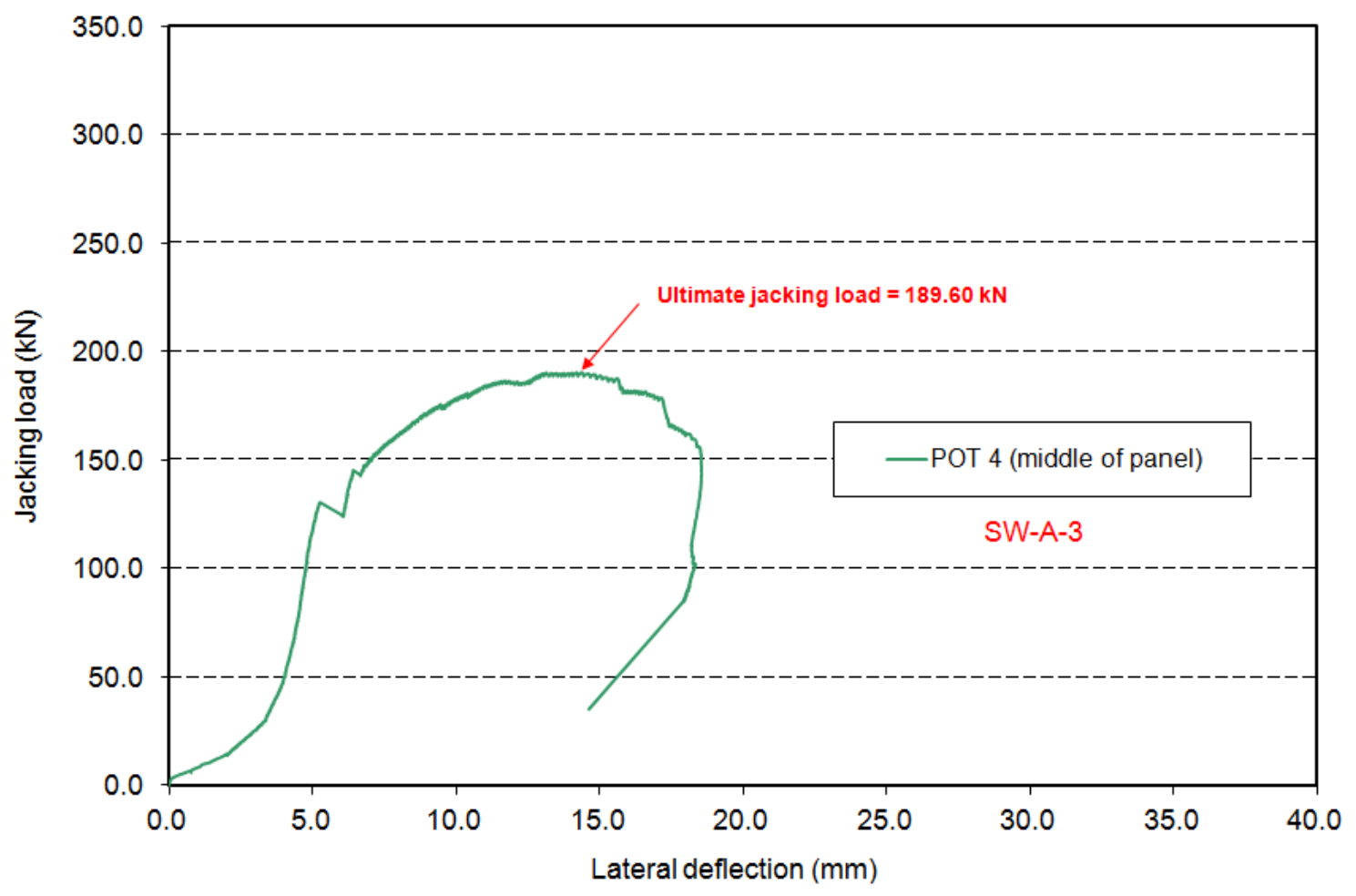

Figure 4.28 - Axial load-lateral deflection relationships for stud wall SW-A-3

Table 4.1 shows that the experimental ultimate jacking loads were $253.51,297.23$ and $189.60 \mathrm{kN}$ for walls SW-A-1, SW-A-2 and SW-A-3, respectively. These values were adjusted by adding the 3.34-kN self-weight of the loading system. Also these values were divided by 2 so that the design load would be for 1220 -mm wall width as that for SIP walls. In addition, the resulting value was multiplied by 0.8 to take into account the use of 5 studs in the $2450-\mathrm{mm}$ wide wall rather than 4 studs. Thus, the experimental ultimate loads were adjusted to be 102.74, 120.23 and $77.18 \mathrm{kN}$ for walls SW-A-1, SW-A-2 and SW-A-3, respectively. This leads to an average experimental ultimate compressive load of $100.05 \mathrm{kN}$ for this STUD wall as shown in Table 4.1.

AC04 specifies that the allowable design compressive load is the experimental ultimate load divided by 2.5 . Thus, the allowable design compressive loads were calculated per stud wall width of $1220 \mathrm{~mm}$ as 41.10, 48.09, and $20.8 \mathrm{kN}$ for walls SW-A-1, SW-A-2 and SW-A-3, respectively. Since one of the obtained design values of the three identical walls is more than $15 \%$ difference with the average value of the three specimens, the allowable design compressive load for this STUD wall is $30.87 \mathrm{kN}$, as the least value of the three. 
Table 4.1 shows that the jacking loads at 3-mm (1/8“) axial shortening as 58.97, 62.98, and 65.99 $\mathrm{kN}$ for walls SW-A-1, SW-A-2 and SW-A-3, respectively. Since the obtained design values are within $15 \%$ difference with the average value of the three walls, the allowable design compressive load per the 3-mm axial shortening criteria for this STUD group is taken as 62.65 $\mathrm{kN}$, as the average of the three values. By dividing this value over 2 to obtain the resistance for $1,220 \mathrm{~mm}$ wide stud wall and by 0.8 due to using 5 studs in the wall rather than 4 over $2450 \mathrm{~mm}$ width, the allowable design compressive load to at $3-\mathrm{mm}$ axial shortening is taken as $25.06 \mathrm{kN}$ as reported in Table 4.2. Based on the results recorded in Table 4.2, it can be concluded that the allowable design compressive load for this STUD wall group is $25.06 \mathrm{kN}$.

\subsubsection{Results of SIP Panels for Flexural Loading}

In this group, three identical panels were tested to-collapse under flexure load. Each panel was made of $2750 \mathrm{~mm}$ length, $1220 \mathrm{~mm}$ wide and $165 \mathrm{~mm}$ thick, with foam-spline connection. Per ASTM method, each panel was loaded in flexural at the quarter points using incremental loading. The panel was first loaded up to $5 \mathrm{kN}$ jacking load, followed by releasing the load to zero. Then, the load was applied to reach $10 \mathrm{kN}$, followed by releasing the load. This incremental loading was followed till failure of the panel occurred.

Figure 4.29 shows views of panel SIP-F-1 before testing, while Figure 4.30 shows view of the permanent deformed shape of the panel after failure. The failure mode was due to horizontal shear failure at the interface between the foam and top OSB facing. This failure occurred between the top surface of the foam and the adhesive over a panel length between the support and the quarter-point line, causing top foam-OSB delamination (debonding) over the supports. Shear failure was sudden causing a sudden drop in the applied jacking load as depicted in the load-deflection history shown in Figure 4.32. Figure 4.31 shows close-up view of this type of failure. It has been noted that noise was heard when approaching failure load and the shear failure was abrupt causing a sudden drop in the applied jacking load. It was observed that linear

elastic behaviour was maintained along a significant portion of the load history before failure as depicted in Figure 4.32. 


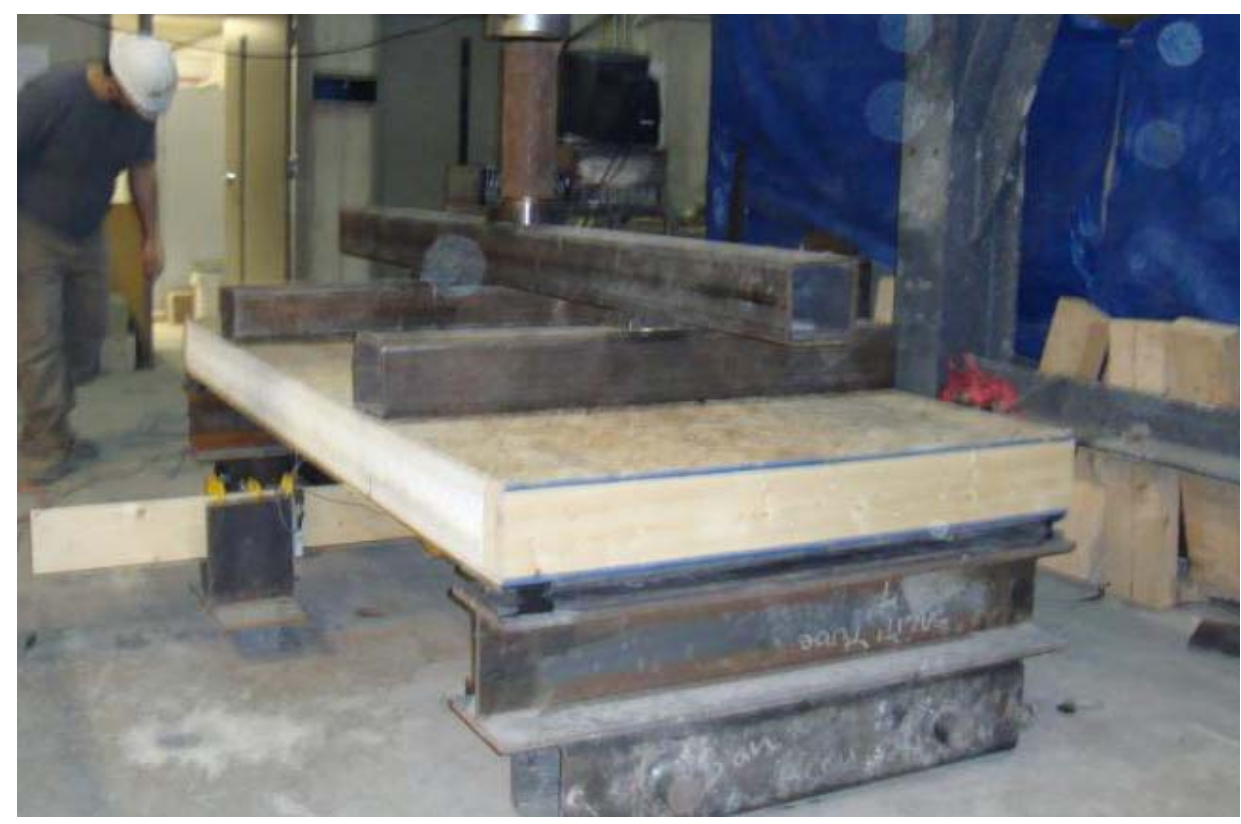

Figure 4.29 - Views of the test setup for specimen SIP-F-1 before testing

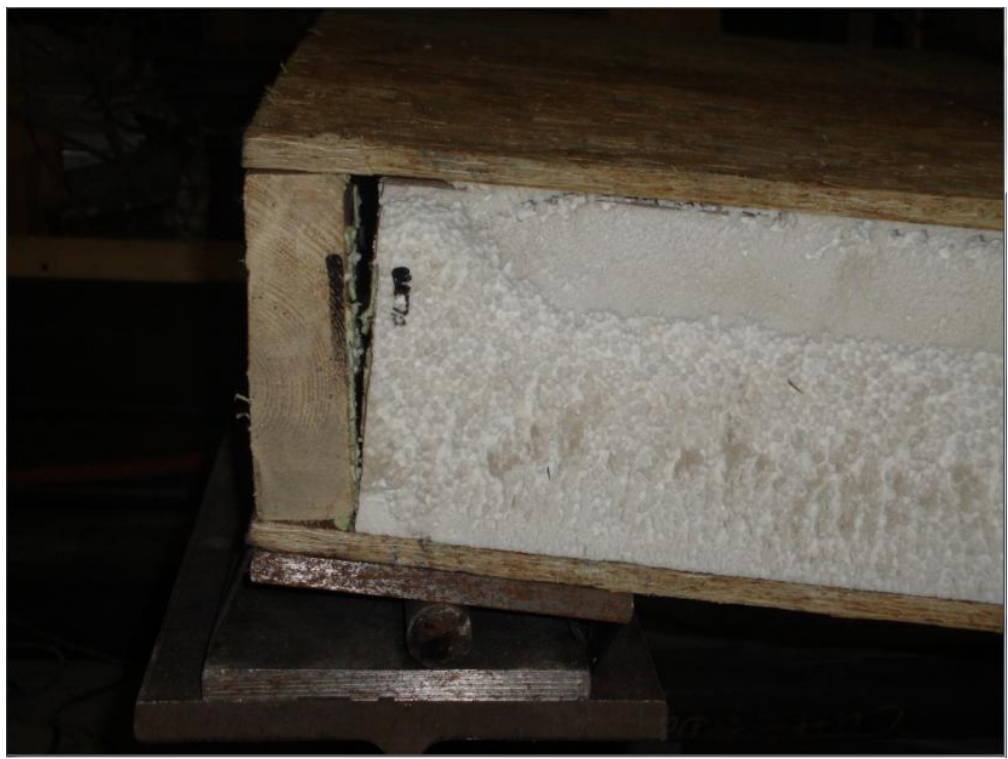

Figure 4.30 - View of the deflected shape of specimen SIP-F-1 at support after failure 


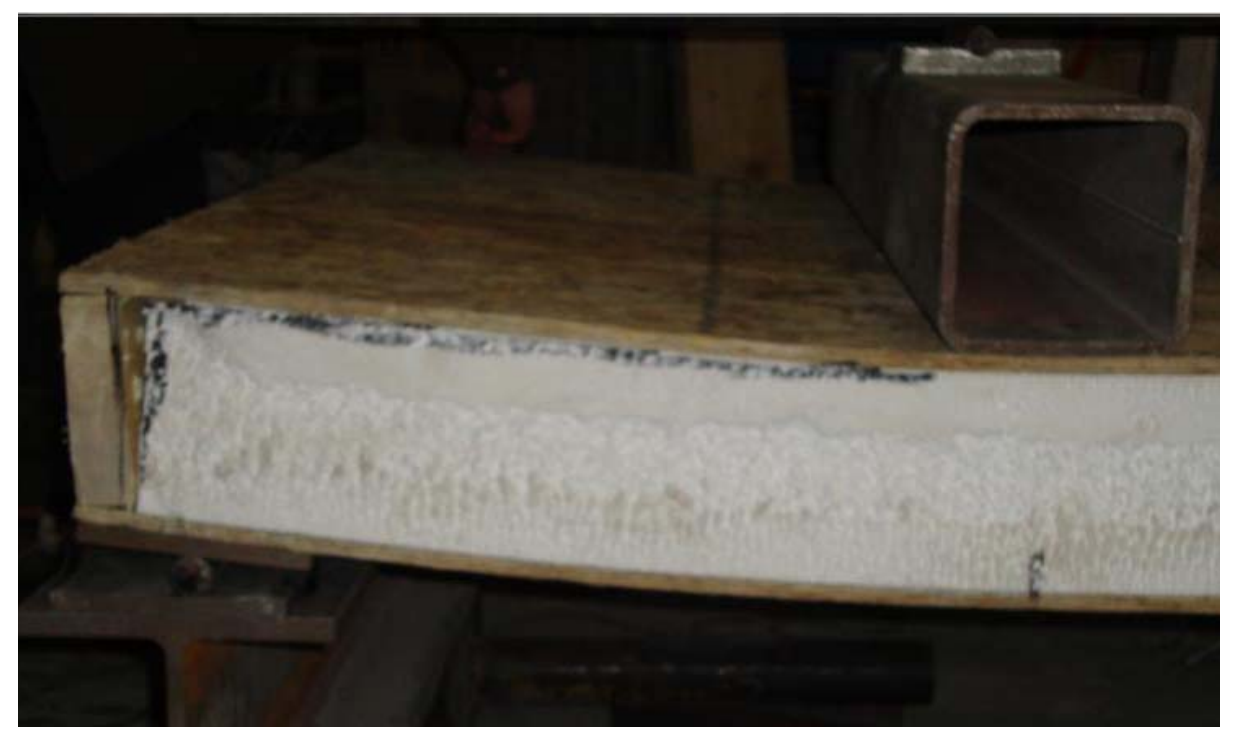

Figure 4.31 - Close-up view of specimen SIP-F-1 showing horizontal shear failure at the top foam-OSB interface

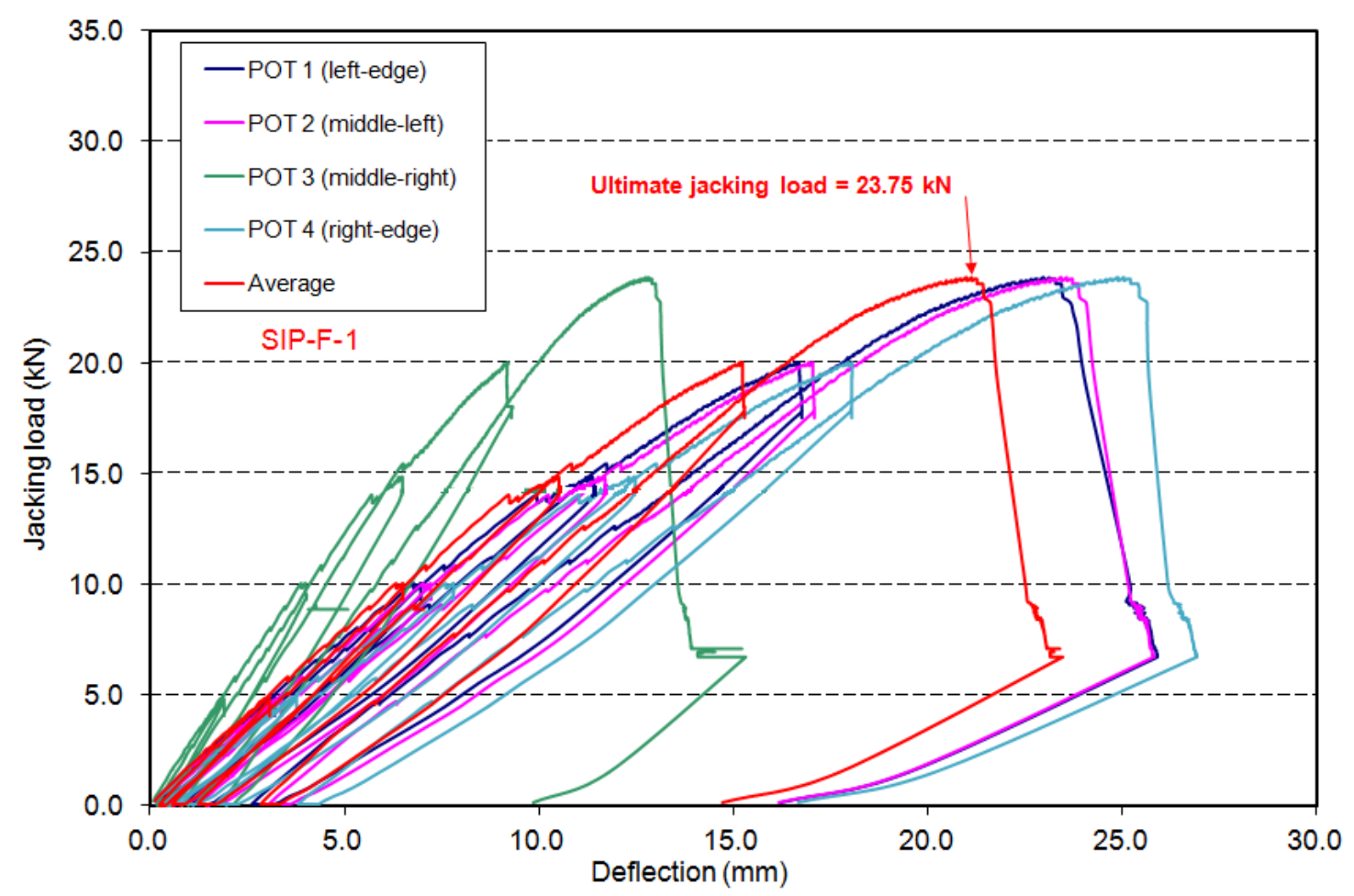

Figure 4.32 - Jacking load-deflection relationship for specimen SIP-F-1

Similar behavior to SIP-F-1 was observed for panels SIP-F-2 and SIP-F-3. Figures 4.33 and 4.34 show views of panel SIP-F-2 before and after testing, respectively. While Figure 4.35 showed 
close-up view of the shear failure at the OSB-foam interface. Figure 4.36 depicts the jacking load-deflection relationship for panel SIP-F-2. It can be observed from deflection readings that linear-elastic behavior was dominant along a significant portion of the load history irrespective of the incremental loading. Figures 4.37 and 4.38 show views of panel SIP-F-3 before and after testing, respectively. While Figure 4.39 showed close-up view of the shear failure at the OSBfoam interface. Figure 4.40 depicts the jacking load-deflection relationship for panel SIP-F-2.

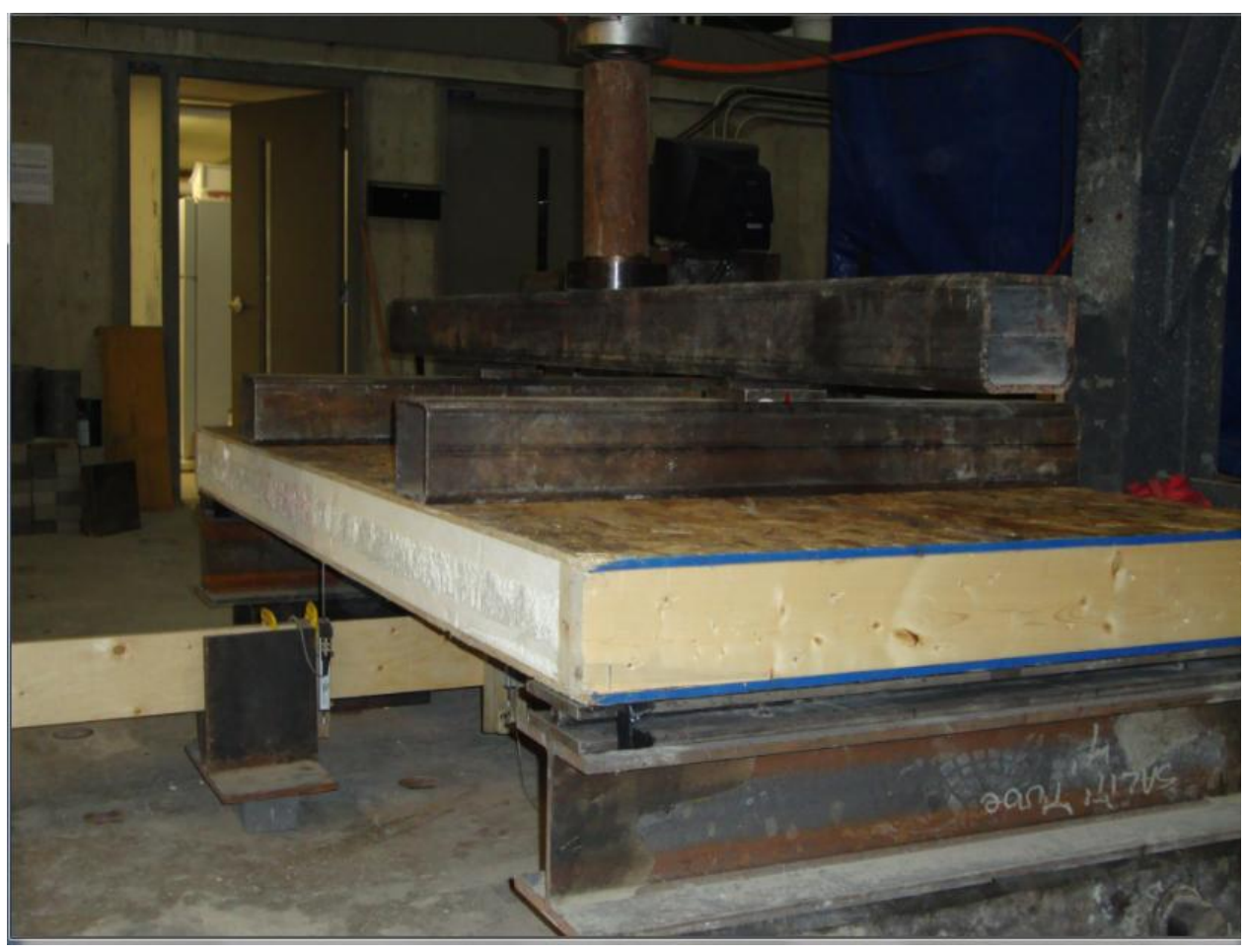

Figure 4.33 - Views of the test setup for specimen SIP-F-2 before testing 


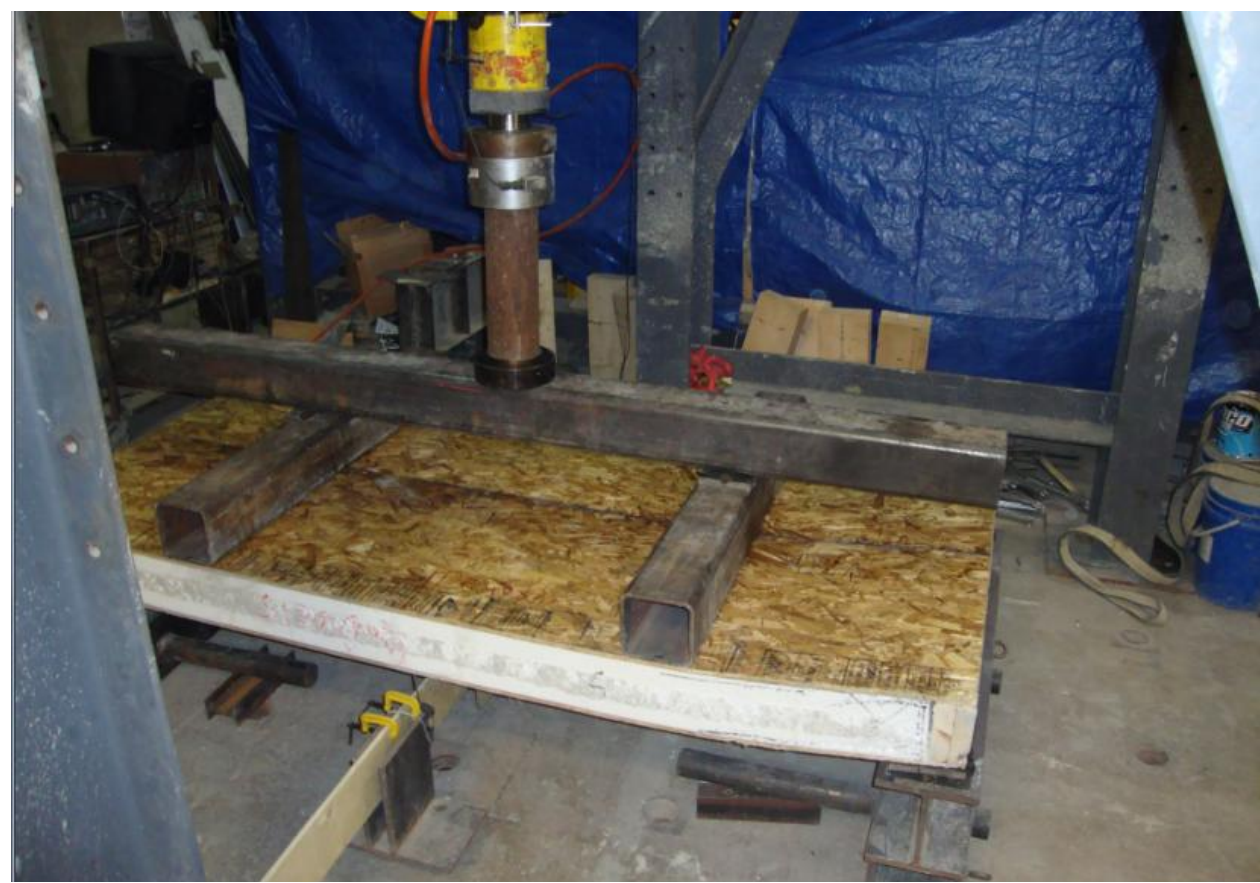

Figure 4.34 - View of the deflected shape of specimen SIP-F-2 after failure

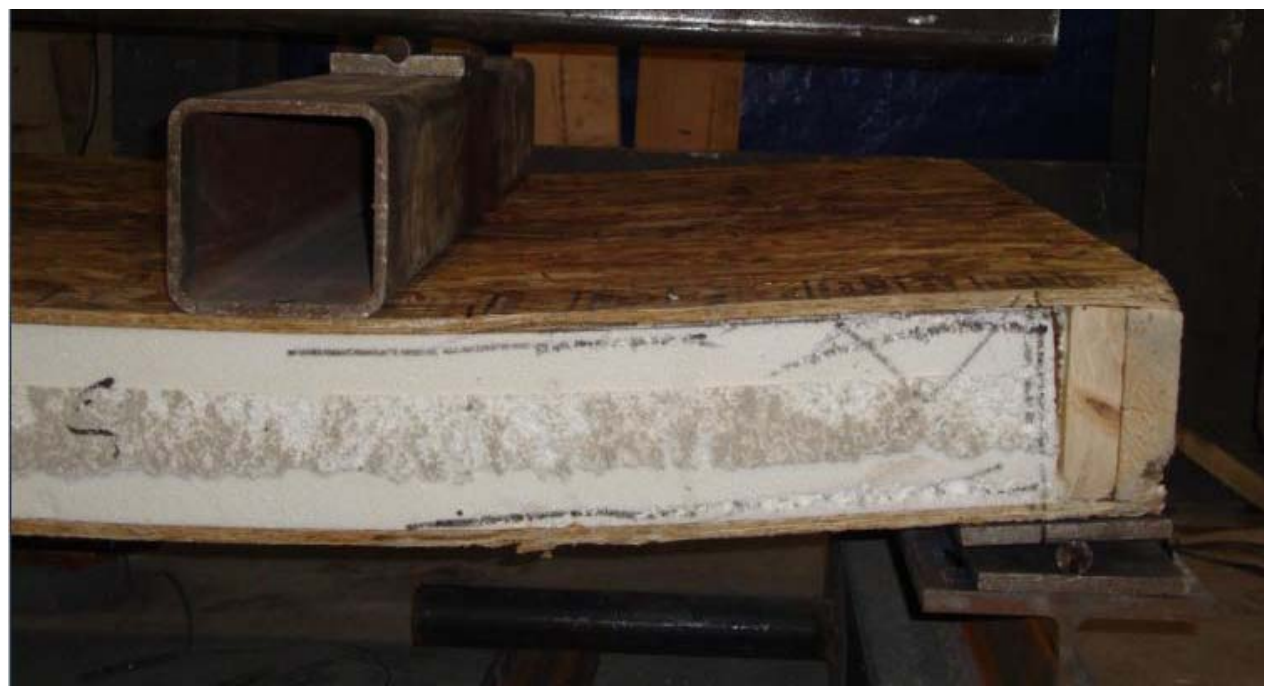

Figure 4.35 - Close-up views of the failure mode of specimen SIP-F-2 


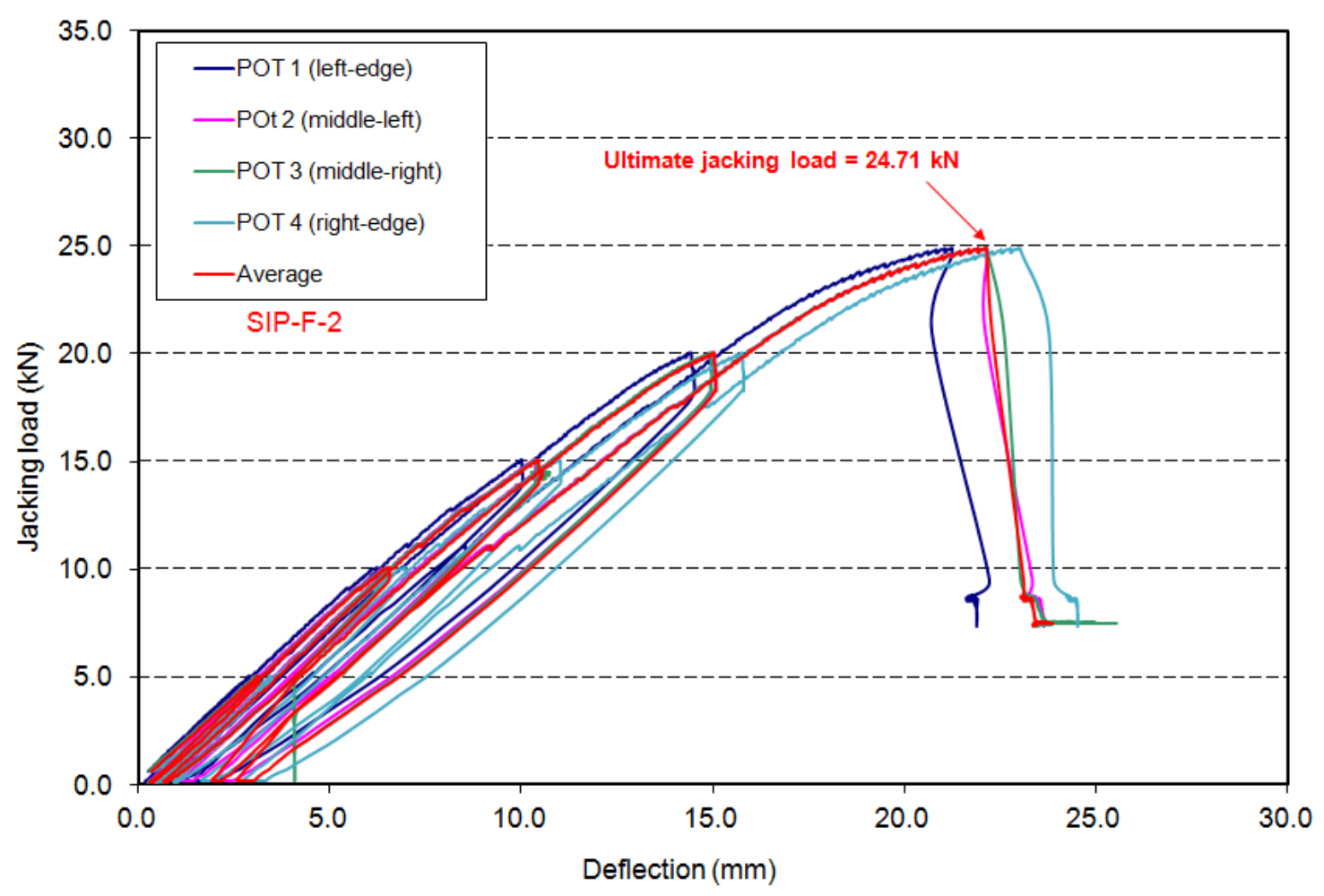

Figure 4.36 - Jacking load-deflection relationship for specimen SIP-F-2

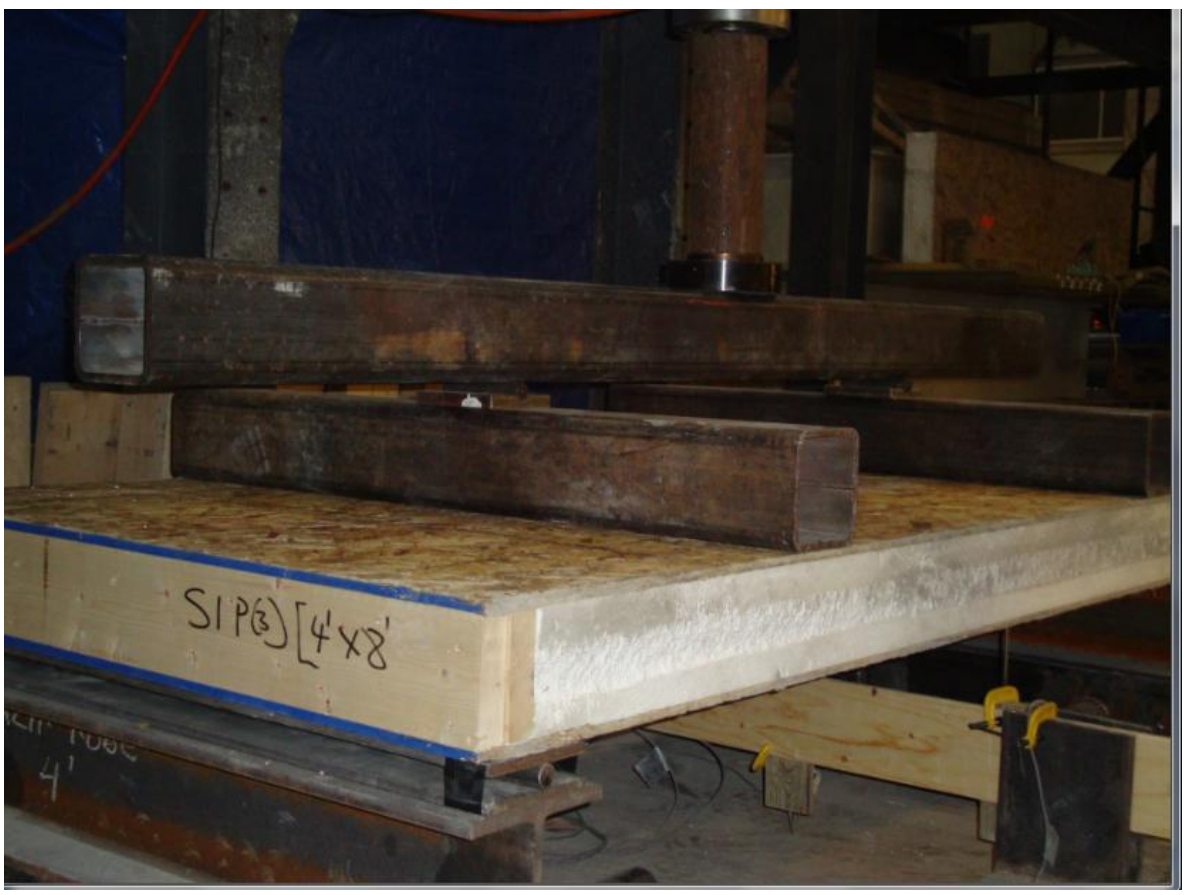

Figure 4.37 - Views of the test setup for specimen SIP-F-3 before testing 


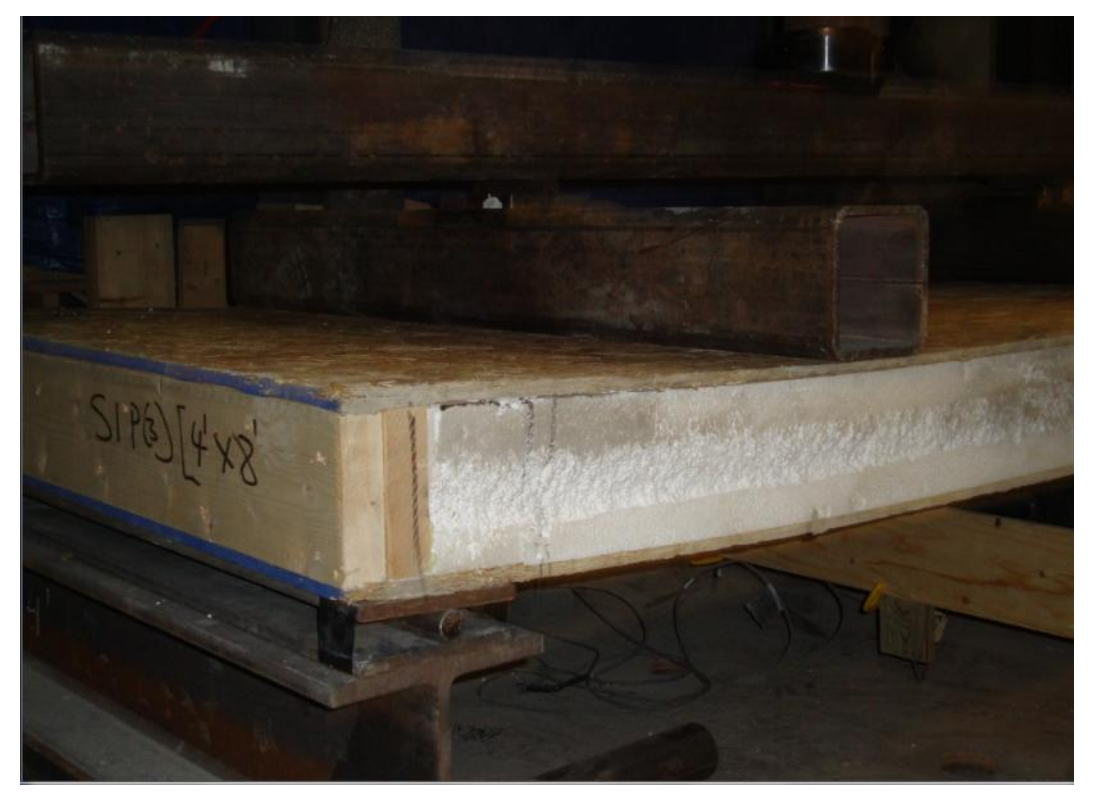

Figure 4.38 - View of the deflected shape of specimen SIP-F-3 after failure

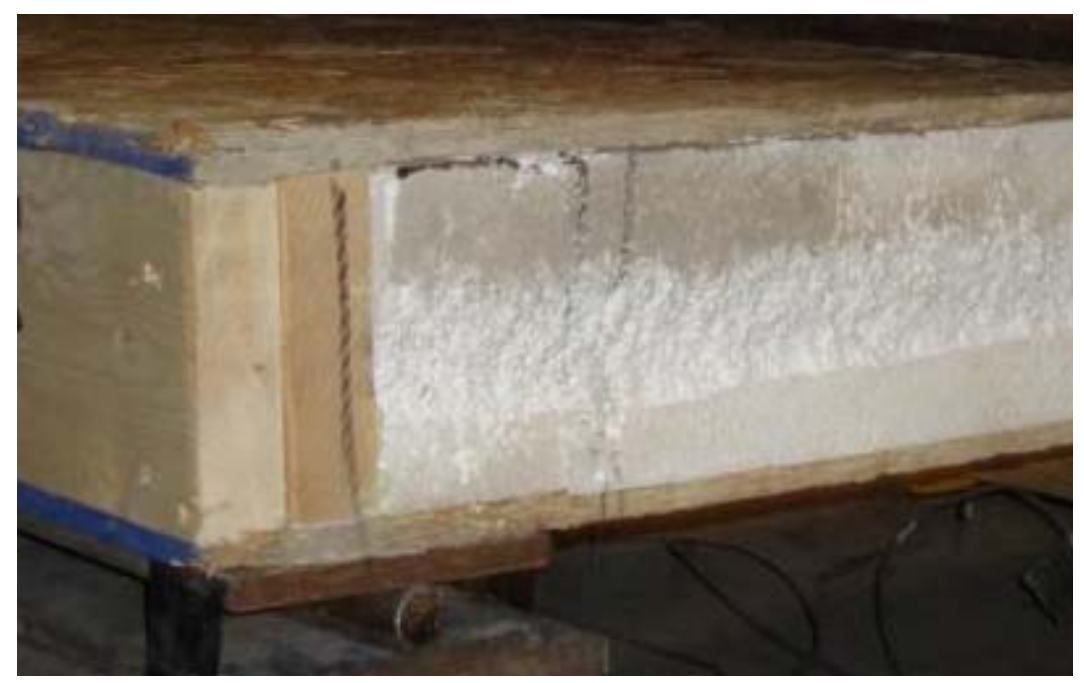

Figure 4.39 - Close-up views of specimen SIP-F-3 showing horizontal shear failure at the top foam-OSB interface 


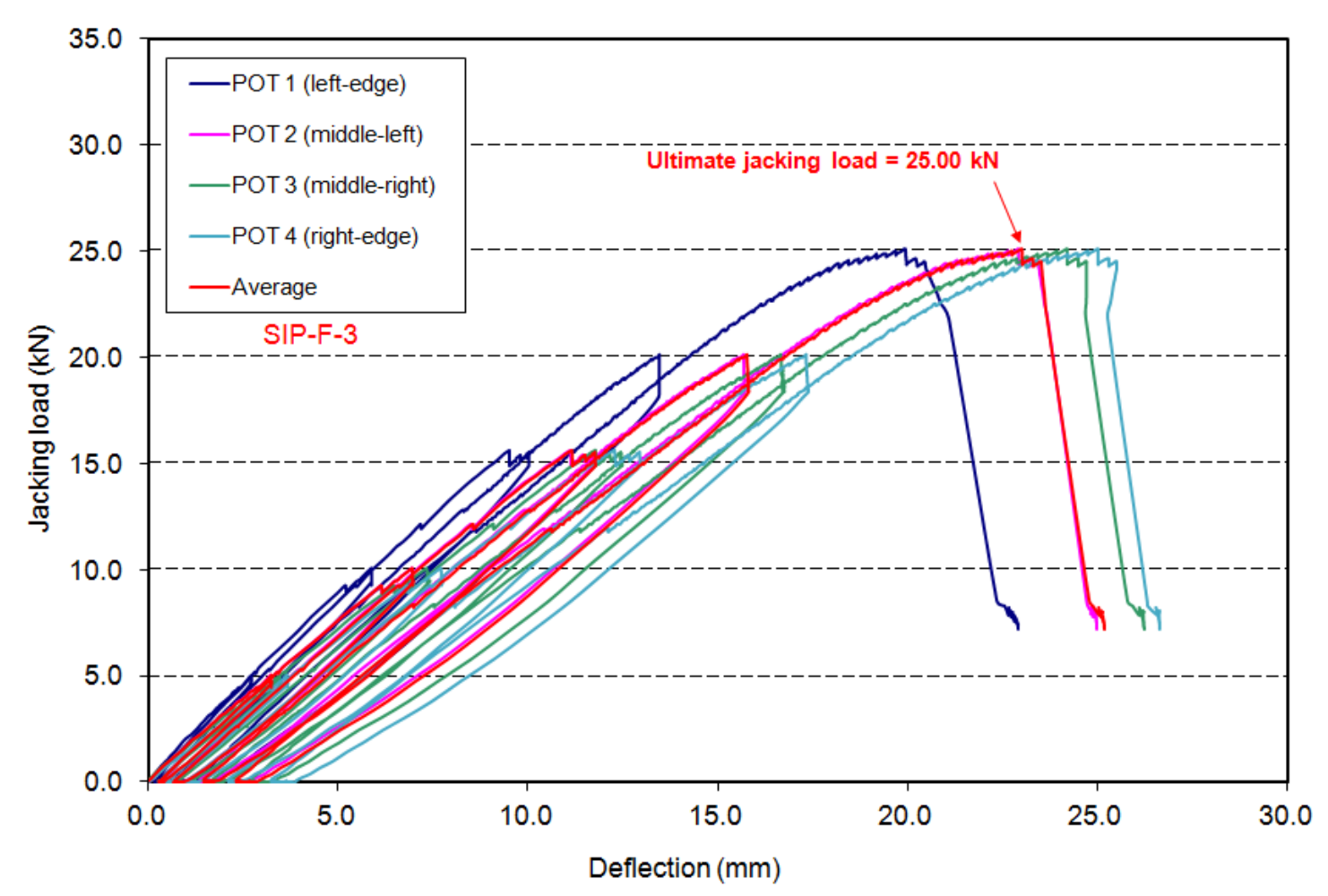

Figure 4.40 - Jacking load-deflection relationship for specimen SIP-F-3

Table 4.3 shows a summary of panel configurations along with the ultimate jacking load for the SIP group. It can be observed that the ultimate jacking loads were $23.75,25.00$, and $24.71 \mathrm{kN}$ for panels SIP-F-1, SIP-F-2, and SIP-F-3, respectively. By adding $2 \mathrm{kN}$ weight of the loading system and multiplying it by $\mathrm{L} / 8$, where $\mathrm{L}=2590.8 \mathrm{~mm}$, the adjusted experimental ultimate resisting moment were calculated as $8.33,8.74$, and $8.65 \mathrm{kN}-\mathrm{m}$. This leads to an average experimental ultimate resisting moment of the SIP group of $8.57 \mathrm{kN}-\mathrm{m}$. 
Table 4.3 - Results from flexural load tests: mean resisting moments and characteristic resisting moments

\begin{tabular}{|c|c|c|c|c|c|c|c|c|}
\hline Group & $\begin{array}{l}\text { Test } \\
\text { No. }\end{array}$ & Test typ & $\begin{array}{l}\text { Panel size: } \\
\mathrm{l} \times \mathrm{w} \times \mathrm{t} \\
(\mathrm{mm}, \mathrm{ft}-\mathrm{inch})\end{array}$ & $\begin{array}{l}\text { Connection } \\
\text { type }\end{array}$ & $\begin{array}{l}\text { Experim. } \\
\text { Ultimate } \\
\text { jacking } \\
\text { load }(\mathrm{kN})\end{array}$ & $\begin{array}{l}\text { Adjusted } \\
\text { Experim. } \\
\text { Ultimate } \\
\text { moment } \\
(\mathrm{kN}-\mathrm{m})^{(3)}\end{array}$ & $\begin{array}{l}\text { Mean } \\
\text { resisting } \\
\text { moment } \\
(\mathrm{kN}-\mathrm{m})^{(3)}\end{array}$ & $\begin{array}{l}\text { Char. } \\
\text { resisting } \\
\text { moment } \\
(\mathrm{kN}-\mathrm{m})^{(3)}\end{array}$ \\
\hline \multirow[t]{3}{*}{ SIP } & SIP-F-1 & Flexural & $\begin{array}{l}1220 \times 2743 \times 165 \\
\left(4^{\prime} \times 9^{\prime} \times 6 \frac{1}{\prime \prime} "\right)\end{array}$ & Foam spline & $23.75^{*}$ & $8.33^{(1)}$ & \multirow[t]{3}{*}{8.57} & \multirow[t]{3}{*}{7.26} \\
\hline & SIP-F-2 & Flexural & $\begin{array}{l}1220 \times 2743 \times 165 \\
\left(4^{\prime} \times 9^{\prime} \times 6 \frac{1}{2} 2^{\prime \prime}\right)\end{array}$ & Foam spline & $25.00^{*}$ & $8.74^{(1)}$ & & \\
\hline & SIP-F-3 & Flexural & $\begin{array}{l}1220 \times 2743 \times 165 \\
\left(4^{\prime} \times 9^{\prime} \times 6 \frac{1}{\prime \prime} "\right)\end{array}$ & Foam spline & $24.71^{*}$ & $8.65^{(\mathbf{1})}$ & & \\
\hline \multirow{5}{*}{$\begin{array}{l}\text { SIP } \\
\text { (Mohamed, } \\
\text { 2009) }\end{array}$} & WS19 & Flexural & $\begin{array}{l}1220 \times 2743 \times 165 \\
\left(4^{\prime} \times 9^{\prime} \times 6 \frac{1}{\prime \prime} "\right)\end{array}$ & Foam spline & $27.22 *$ & $9.46^{(\mathbf{1})}$ & \multirow{5}{*}{9.42} & \multirow{5}{*}{8.55} \\
\hline & WS20 & Flexural & $\begin{array}{l}1220 \times 2743 \times 165 \\
\left(4^{\prime} \times 9^{\prime} \times 6 \frac{1}{\prime \prime} "\right)\end{array}$ & Foam spline & $27.77^{*}$ & $9.64^{(1)}$ & & \\
\hline & WS21 & Flexural & $\begin{array}{l}1220 \times 2743 \times 165 \\
\left(4^{\prime} \times 9^{\prime} \times 6 \frac{1}{2} 2^{\prime \prime}\right)\end{array}$ & Foam spline & $24.99^{*}$ & $8.74^{(\mathbf{1})}$ & & \\
\hline & WS22 & Flexural & $\begin{array}{l}1220 \times 2743 \times 165 \\
\left(4^{\prime} \times 9^{\prime} \times 6 \frac{1}{2} 2^{\prime \prime}\right)\end{array}$ & Foam spline & $28.77^{*}$ & $9.96^{(\mathbf{1})}$ & & \\
\hline & WS23 & Flexural & $\begin{array}{l}1220 \times 2743 \times 165 \\
\left(4^{\prime} \times 9^{\prime} \times 6 \frac{1}{2} 2^{\prime \prime}\right)\end{array}$ & Foam spline & $26.77^{*}$ & $9.32^{(\mathbf{1})}$ & & \\
\hline \multirow[t]{3}{*}{ STUD } & SW-F-1 & Flexural & $\begin{array}{l}2440 \times 2743 \times 165 \\
\left(8^{\prime} \times 9^{\prime} \times 6 \frac{1}{1} 2^{\prime \prime}\right)\end{array}$ & 2x6@2'-0" & $59.68^{* *}$ & $8.23^{(2)}$ & \multirow[t]{3}{*}{8.91} & \multirow[t]{3}{*}{7.19} \\
\hline & SW-F-2 & Flexural & $\begin{array}{l}2440 \times 2743 \times 165 \\
\left(8^{\prime} \times 9^{\prime} \times 6 \frac{1}{\prime \prime} "\right)\end{array}$ & 2x6@ 2'-0" & $67.68^{* *}$ & $9.26^{(2)}$ & & \\
\hline & SW-F-3 & Flexural & $\begin{array}{l}2440 \times 2743 \times 165 \\
\left(8^{\prime} \times 9^{\prime} \times 6 \frac{1}{2 \prime} "\right)\end{array}$ & 2x6@2’-0" & $67.56^{* *}$ & $9.25^{(2)}$ & & \\
\hline
\end{tabular}

* Did not include $2 \mathrm{kN}$ weight of the loading system.

** Did not include $3.82 \mathrm{kN}$ weight of the loading system.

1) Included $2 \mathrm{kN}$ weight of loading system and then multiplied by $\mathrm{L} / 8$ to obtain bending moment $(\mathrm{L}=2.5908 \mathrm{~m})$

(2) Included $3.82 \mathrm{kN}$ weight of the loading system. Values divided by 2 for $1.2 \mathrm{~m}$ panel width and multiplied by 0.8 for using 5 studs rather than 4 in the tested wall and then multiplied by $\mathrm{L} / 8$ to obtain bending moment $(\mathrm{L}=2.5908$ $\mathrm{m})$.

${ }^{(3)}$ Values are per panel width of $1.2 \mathrm{~m}$. 
Table 4.3 shows a summary of SIP panel results conforming to AC04 design criteria. It can be observed that the ultimate jacking loads were 23.75, 25.00, and $24.71 \mathrm{kN}$ for panels SIP-F-1, SIP-F-2, and SIP-F-3, respectively. By adding $2 \mathrm{kN}$ weight of the loading system, the ultimate applied loads were 25.75, 27.00, and $26.71 \mathrm{kN}$ for panels SIP-F-1, SIP-F-2, and SIP-F-3, respectively. It is worth mentioning that the ultimate jacking load for each panel is within $15 \%$ difference from the average jacking load of the three panels. Thus, the allowable design jacking load is taken as the average experimental ultimate jacking load divided by a factor of safety of 3 per AC04 design criteria (i.e. $26.49 / 3=8.83 \mathrm{kN}$ ). This makes the allowable design bending moment resistance of this SIP group as $2.86 \mathrm{kN}-\mathrm{m}$ for $1220 \mathrm{~mm}$ panel width as reported in Table 4.4 . 
Table 4.4 - Results from flexural load tests: allowable bending moments based on ICC-EC AC04 (2004)

\begin{tabular}{|c|c|c|c|c|c|c|c|c|}
\hline Group & $\begin{array}{l}\text { Test } \\
\text { No. }\end{array}$ & Test type & $\begin{array}{l}\text { Panel size: } \\
\mathrm{l} \times \mathrm{w} \times \mathrm{t} \\
(\mathrm{mm}, \mathrm{ft}-\mathrm{inch})\end{array}$ & $\begin{array}{l}\text { Connection } \\
\text { type }\end{array}$ & $\begin{array}{l}\text { Experim. } \\
\text { ultimate } \\
\text { jacking } \\
\text { load } \\
(\mathrm{kN})\end{array}$ & $\begin{array}{l}\text { Adjusted } \\
\text { experim. } \\
\text { ultimate } \\
\text { load } \\
\text { (kN) }\end{array}$ & $\begin{array}{l}\text { Mean } \\
\text { allow. } \\
\text { jacking } \\
\text { load } \\
(k N)^{(5)}\end{array}$ & $\begin{array}{l}\text { Mean } \\
\text { allow. } \\
\text { moment } \\
(\mathrm{kN}-\mathrm{m})^{(5)}\end{array}$ \\
\hline \multirow[t]{3}{*}{ SIP } & SIP-F-1 & Flexural & $\begin{array}{l}1220 \times 2743 \times 165 \\
\left(4^{\prime} \times 9^{\prime} \times 6^{\prime}{ }^{\prime}{ }^{\prime \prime}\right)\end{array}$ & Foam spline & $23.75^{*}$ & $25.75^{(\mathbf{1})}$ & \multirow[t]{3}{*}{$8.83^{(2)}$} & \multirow[t]{3}{*}{$2.86^{(3)}$} \\
\hline & SIP-F-2 & Flexural & $\begin{array}{l}1220 \times 2743 \times 165 \\
\left(4^{\prime} \times 9^{\prime} \times 6^{1}{ }^{\prime \prime} "\right)\end{array}$ & Foam spline & $25.00^{*}$ & $27.00^{(1)}$ & & \\
\hline & SIP-F-3 & Flexural & $\begin{array}{l}1220 \times 2743 \times 165 \\
\left(4^{\prime} \times 9^{\prime} \times 6^{1 / 2 \prime}{ }^{\prime \prime}\right)\end{array}$ & Foam spline & $24.71^{*}$ & $26.71^{(1)}$ & & \\
\hline \multirow[t]{5}{*}{$\begin{array}{l}\text { SIP } \\
\text { (Mohamed, 2009) }\end{array}$} & WS19 & Flexural & $\begin{array}{l}1220 \times 2743 \times 165 \\
\left(4^{\prime} \times 9^{\prime} \times 6 \frac{1}{1} 2^{\prime \prime}\right)\end{array}$ & Foam spline & $27.22 *$ & $29.22^{(1)}$ & \multirow{5}{*}{$9.70^{(2)}$} & \multirow{5}{*}{$3.14^{(3)}$} \\
\hline & WS20 & Flexural & $\begin{array}{l}1220 \times 2743 \times 165 \\
\left(4^{\prime} \times 9^{\prime} \times 6 \frac{1}{2} 2^{\prime \prime}\right)\end{array}$ & Foam spline & $27.77^{*}$ & $29.77^{(1)}$ & & \\
\hline & WS21 & Flexural & $\begin{array}{l}1220 \times 2743 \times 165 \\
\left(4^{\prime} \times 9^{\prime} \times 6 \frac{1}{2}{ }^{\prime \prime}\right)\end{array}$ & Foam spline & $24.99 *$ & $26.99^{(1)}$ & & \\
\hline & WS22 & Flexural & $\begin{array}{l}1220 \times 2743 \times 165 \\
\left(4^{\prime} \times 9^{\prime} \times 6^{1 / 2 \prime}{ }^{\prime \prime}\right)\end{array}$ & Foam spline & $28.77^{*}$ & $30.77^{(1)}$ & & \\
\hline & WS23 & Flexural & $\begin{array}{l}1220 \times 2743 \times 165 \\
\left(4^{\prime} \times 9^{\prime} \times 6 \quad \frac{1}{2} 2^{\prime \prime}\right)\end{array}$ & Foam spline & $26.77^{*}$ & $28.77^{(1)}$ & & \\
\hline \multirow[t]{3}{*}{ STUD } & SW-F-1 & Flexural & $\begin{array}{l}2440 \times 2743 \times 165 \\
\left(8^{\prime} \times 9^{\prime} \times 6 \frac{1}{2} 2^{\prime \prime}\right)\end{array}$ & 2x6@2’-0" c & $59.68 * *$ & $63.50^{(1)}$ & \multirow[t]{3}{*}{$22.93^{(2)}$} & \multirow[t]{3}{*}{$2.97^{(4)}$} \\
\hline & SW-F-2 & Flexural & $\begin{array}{l}2440 \times 2743 \times 165 \\
\left(8^{\prime} \times 9^{\prime} \times 6 \frac{1}{2}{ }^{\prime \prime}\right)\end{array}$ & 2x6@2’-0"c & $67.68^{* *}$ & $71.50^{(1)}$ & & \\
\hline & SW-F-3 & Flexural & $\begin{array}{l}2440 \times 2743 \times 165 \\
\left(8^{\prime} \times 9^{\prime} \times 6^{1 / 2 \prime}{ }^{\prime \prime}\right)\end{array}$ & 2x6@2’-0”c & $67.56^{* *}$ & $71.38^{(\mathbf{1})}$ & & \\
\hline
\end{tabular}

* Did not include $2 \mathrm{kN}$ weight of the loading system.

** Did not include $3.82 \mathrm{kN}$ weight of the loading system.

(1) Include weight of the loading system.

(2) Considering safety factor of 3

${ }^{3}$ Multiplied by L/8 to obtain bending moment $(\mathrm{L}=2.5908 \mathrm{~m})$

(4) Values divided by 2 for $1.2 \mathrm{~m}$ panel width and multiplied by 0.8 for using 5 studs rather than 4 in the tested wall and then multiplied by $\mathrm{L} / 8$ to obtain bending moment $(\mathrm{L}=2.5908 \mathrm{~m})$.

${ }^{(5)}$ Values are per panel width of $1.2 \mathrm{~m}$. 


\subsubsection{Results of Stud Group for Flexural Loading}

In this group, three identical specimens, namely: SW-F-1, SW-F-2, and SW-F-3, were tested tocomplete-collapse under flexure loading. Each panel was of 2450-mm, 2750-mm length and 165$\mathrm{mm}$ total thickness. Figure 4.41 shows views of panel SW-F-1 before testing, while Figure 4.42 shows view of the permanent deformed shape of the stud specimen after failure. The failure mode was due to pure flexural at the mid-span location. The flexural failure occurred in the first stud in the west side of the specimen as shown in Figs. 4.43 and 4.44. Tensile fracture in both the stud and the bottom drywall was suddenly occurred at failure. View of the tensile fracture of the drywall under the first stud is observed in Figure 4.45. After the test, the stud specimen was removed from the test setup and then the drywall was removed to observe other damage in internal studs, as shown in Figure 4.46. Figure 3.47 shows close-up view of the flexural failure of stud No.1 on the west side of the specimen. While Figure 4.48 shows horizontal splitting in the second stud from the west side of the specimen between the mid-span and quarter point. The failure was sudden and noisy causing a sudden drop in the applied jacking load as depicted in the load-deflection history shown in Figure 4.49. The load-deflection history shows linear-elastic relationship till failure.

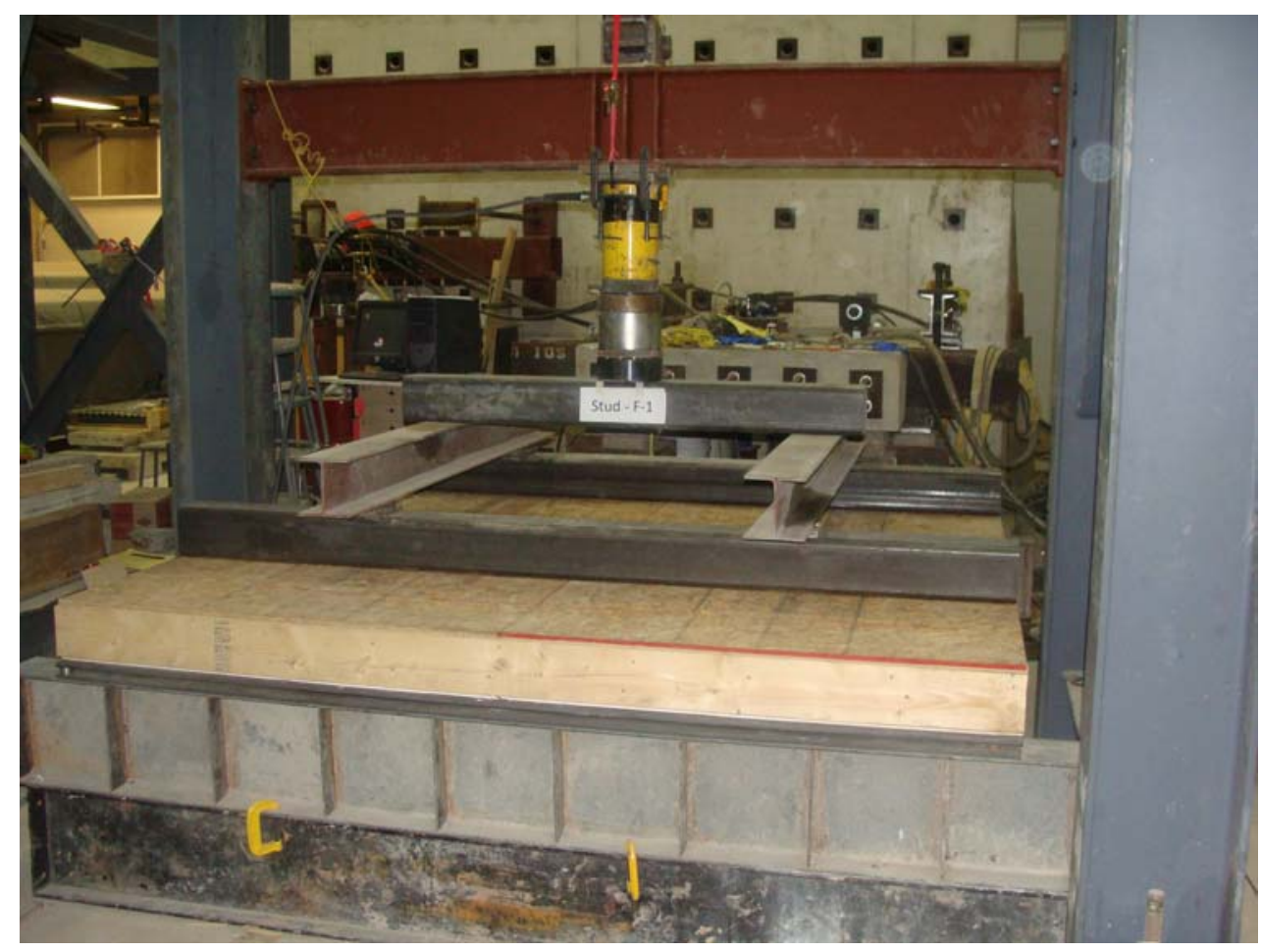

Figure 4.41 - View of specimen SW-F-1 before testing 


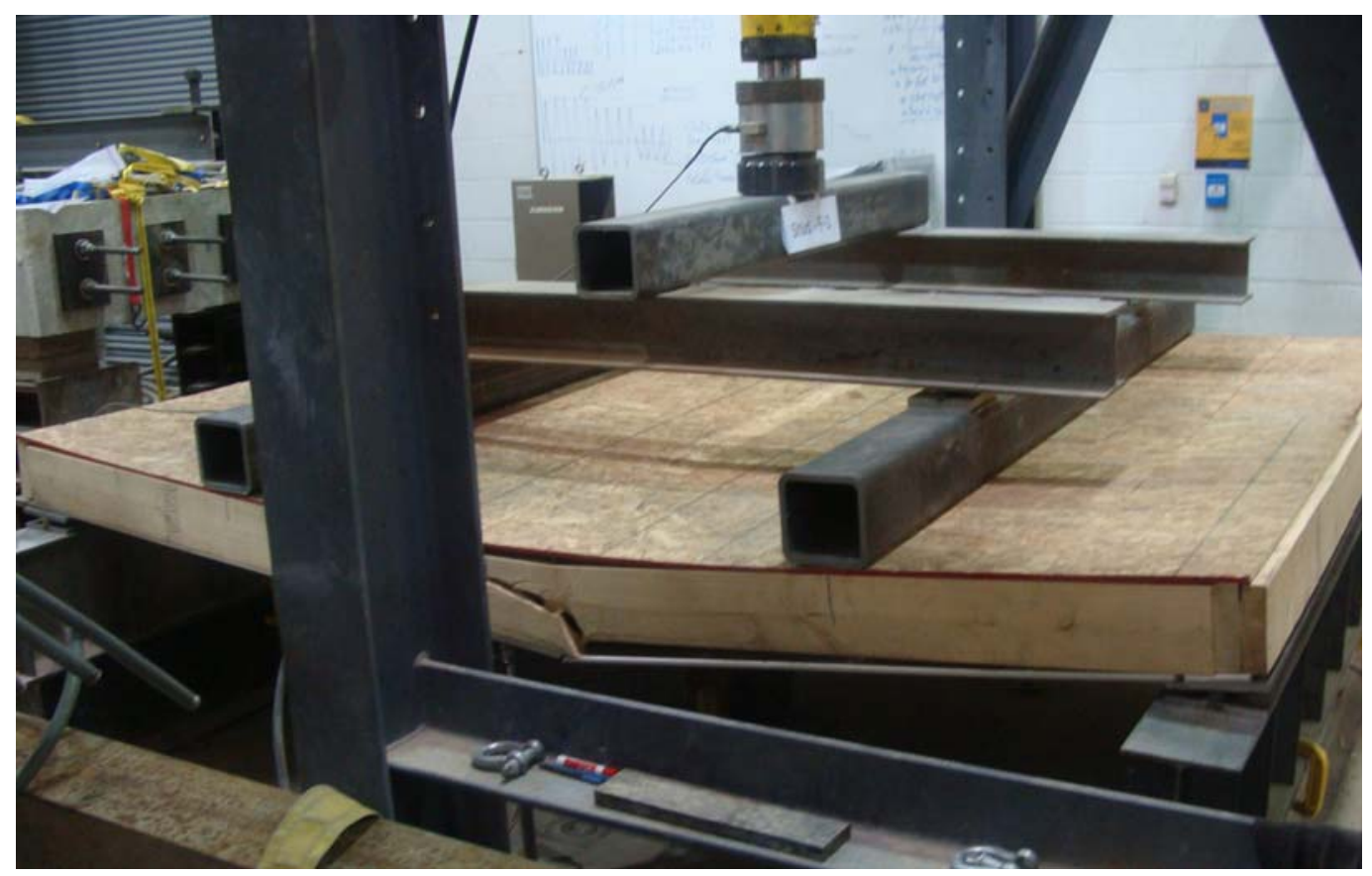

Figure 4.42 - View of deflected shape of specimen SW-F-1 after failure

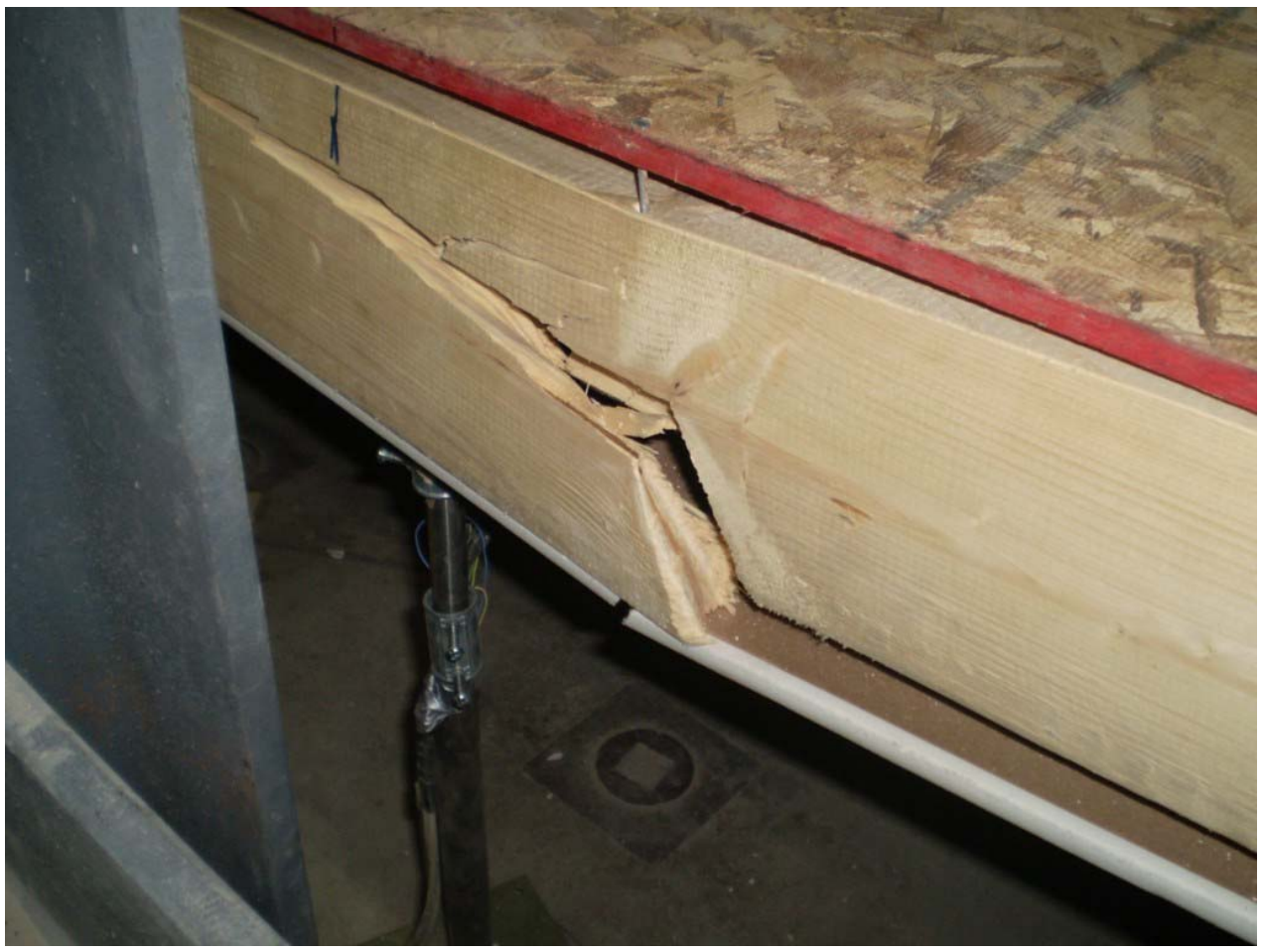

Figure 4.43 - Close-up view of the flexural failure pattern in the first joist at end of specimen SW-F-1 


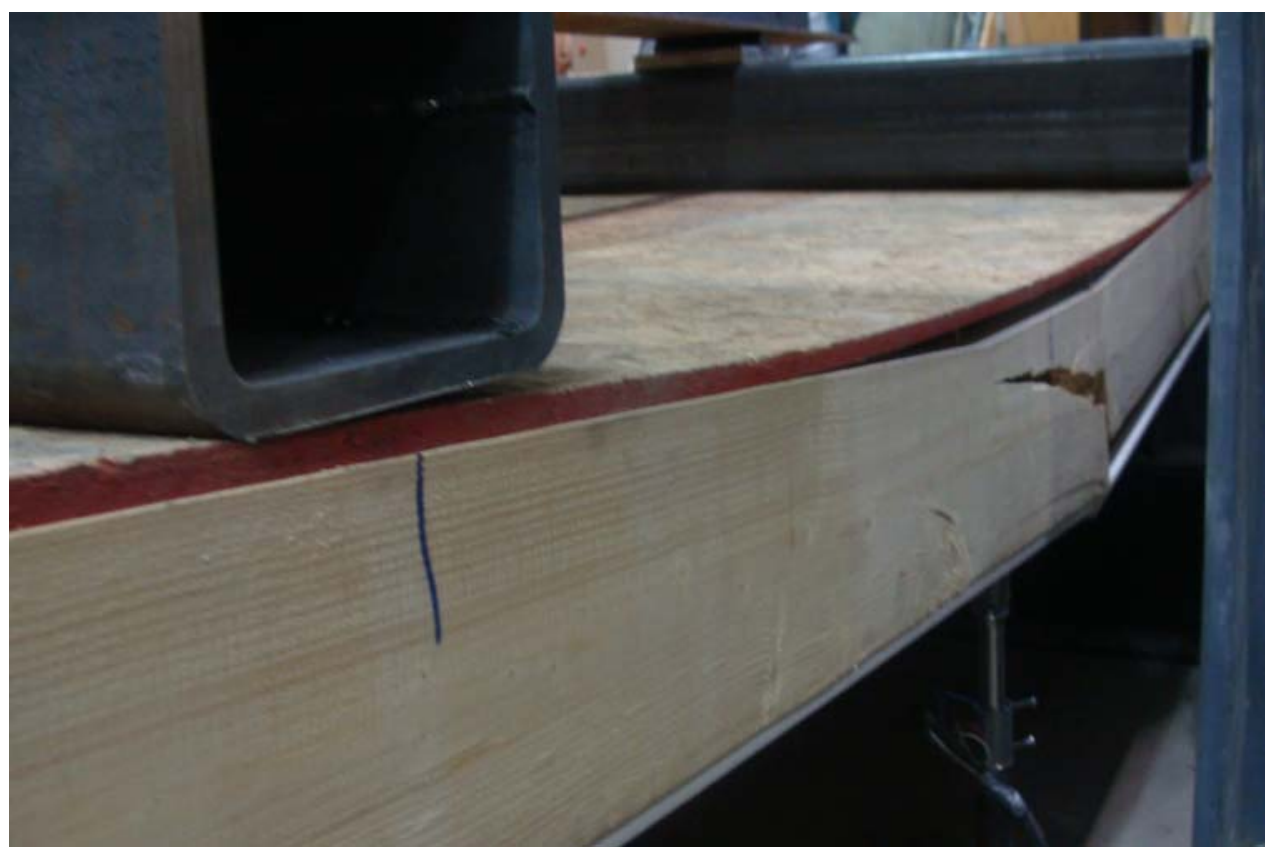

Figure 4.44 - Other view of the deflected shape and flexural failure at mid-span of first joist in specimen SW-F-1

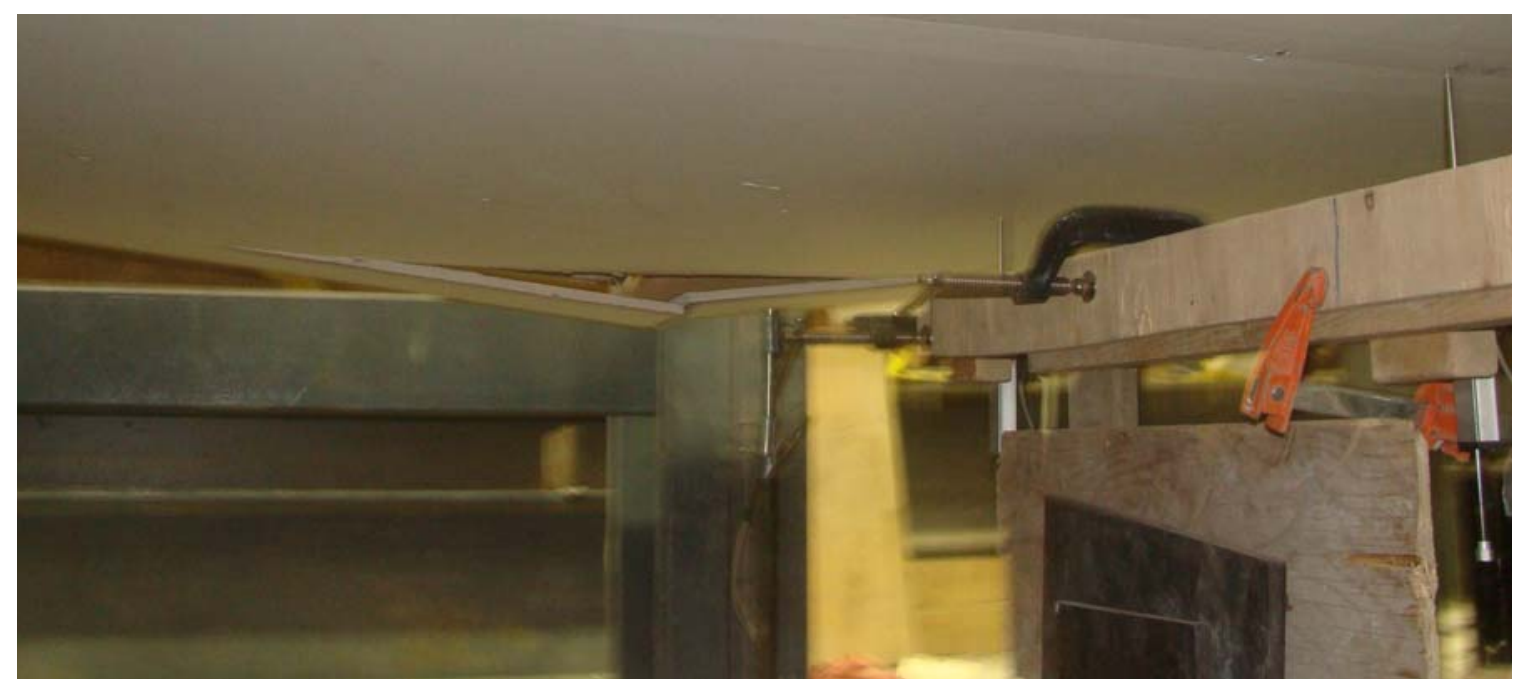

Figure 4.45 - Close-up view from underside of the first joist showing longitudinal and transverse flexural cracks in the drywall of SW-F-1 


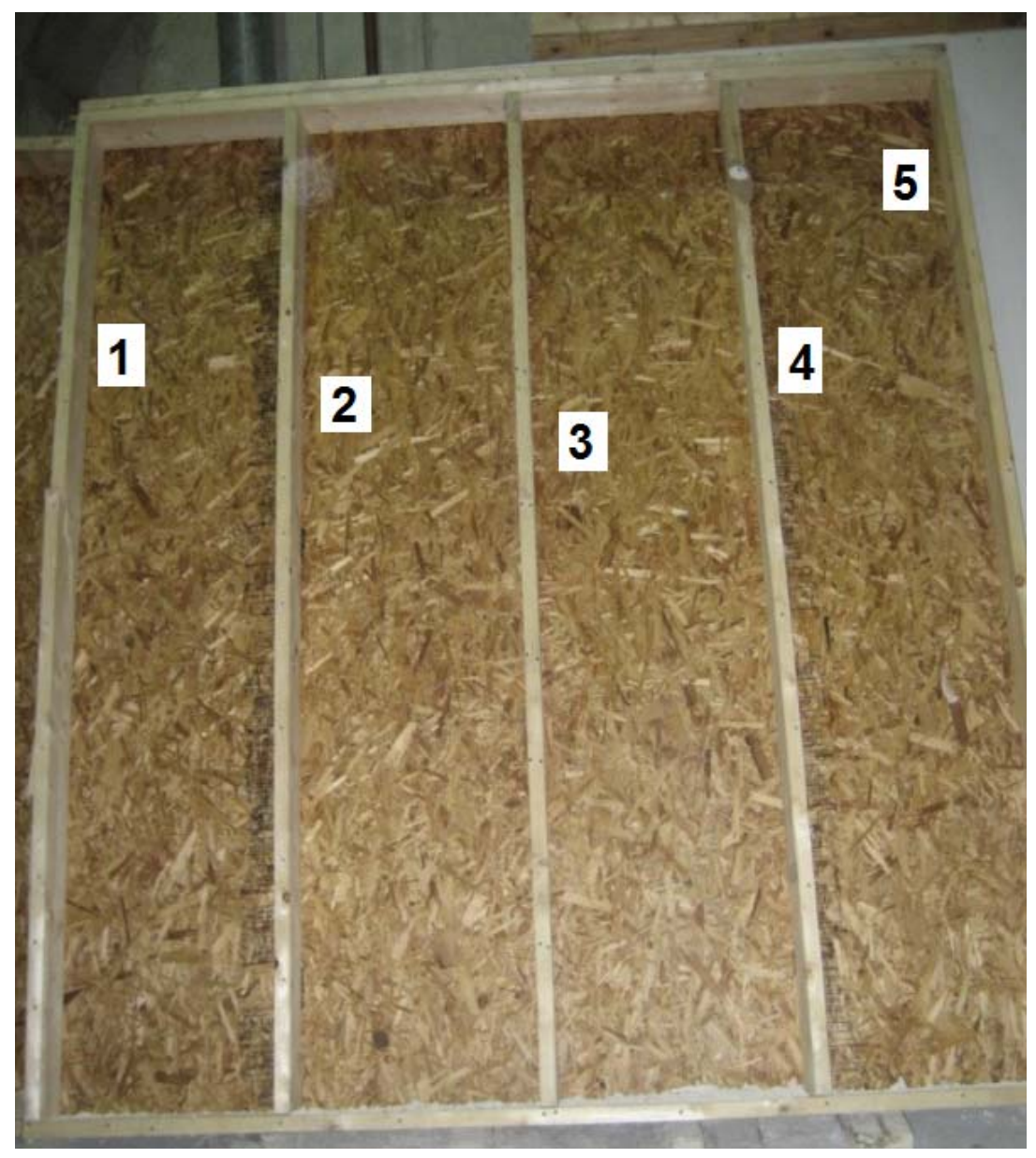

Figure 4.46 - View of the joists after removing the drywall of specimen SW-F-1

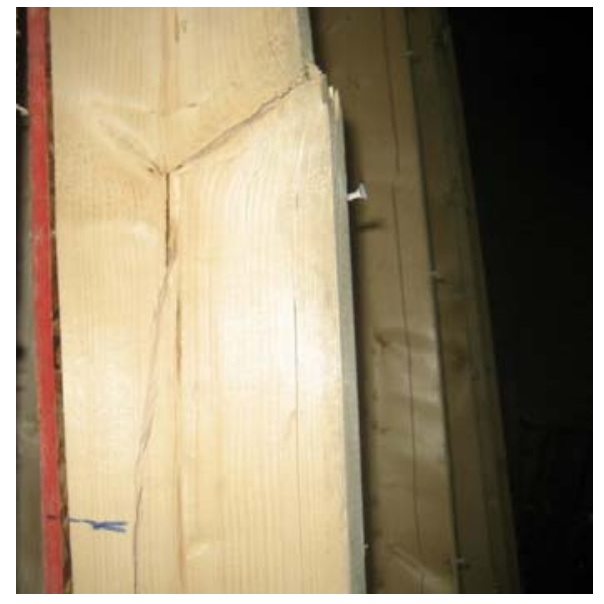

Figure 4.47 - Close-up view of the flexural failure longitudinal in joist No. 1 of specimen SW-F-1

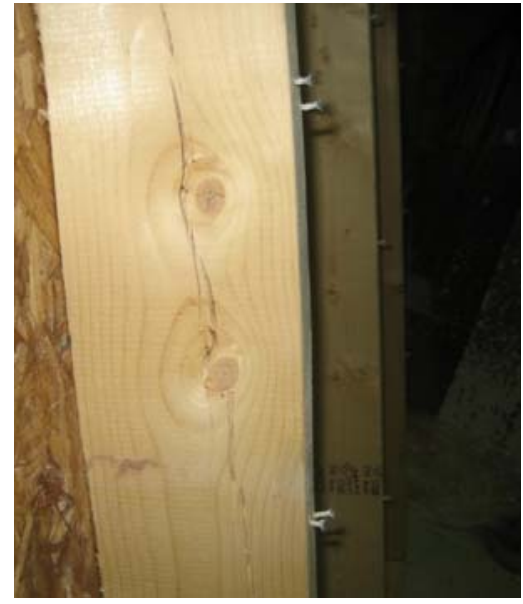

Figure 4.48 - Close-up view of crack in joist No. 2 of specimen SW-F-1 


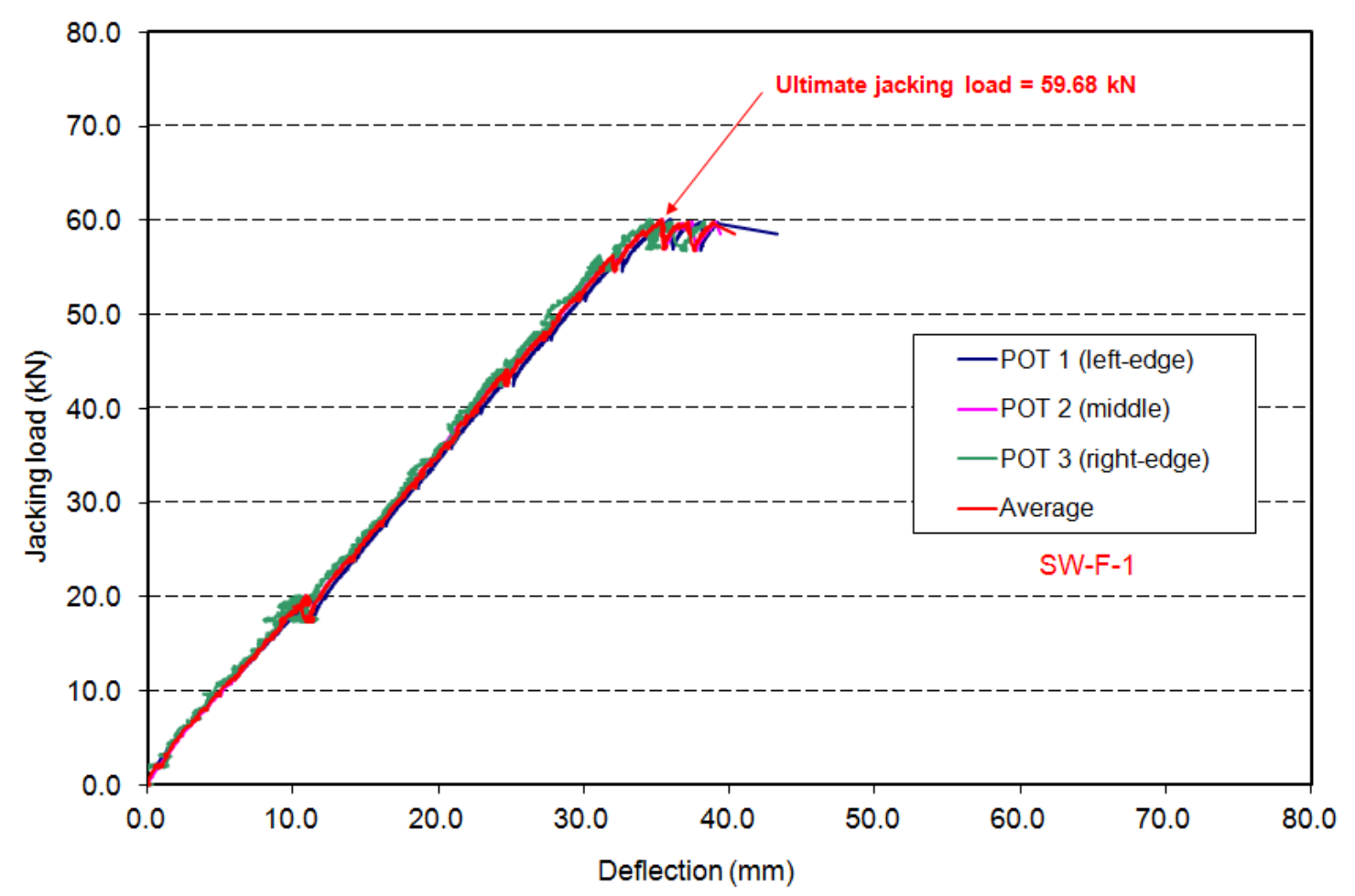

Figure 4.49 - Axial load-axial shortening relationships for specimen SW-F-1

Similar behaviour to Stud panel SW-F-1 was observed in the other two specimens, SW-F-2 and SW-F-3. Figure 4.50 shows views of panel SW-F-1 before testing, while Figures 4.51 and 4.52 show views of the permanent deformed shape of the stud specimen after failure. The failure mode was due to combined flexure and shear at the quarter point location, just under the HSS spread beam. Figure 4.53 shows the failure pattern of stud No. 5, just at the east side of the specimen. This combined flexural-shear failure occurred at the quarter point region and extends through the web towards the support. Tensile fracture of the drywall was also observed in Figs. 4.53 just under stud No. 5 and extends to adjacent studs as shown in Figure 4.54 toward stud No. 4 and Figure 4.55 for studs No. 1, 2 and 3. After the test, the stud specimen was removed from the test setup and then the drywall was removed to observe other damage in internal studs, as shown in Figure 4.56. Figures 4.57, 4.58 and 4.59 show close-up views of the failure mode of studs No. 3, 4 and 5 of specimen SW-F-2. In addition to the failure in stud No. 5, stud No. 4 also failed at the quarter point due to tensile fracture at the bottom fibres, extending through the web towards the stud mid-span. Stud No. 3 shows signed of less severe failure through web fracture extending between the mid span and the mid-length between the quarter point and the support ad 
depicted in Figure 4.58. The failure was sudden and noisy causing a sudden drop in the applied jacking load as depicted in the load-deflection history shown in Figure 4.60. The load-deflection history shows linear-elastic relationship till failure.

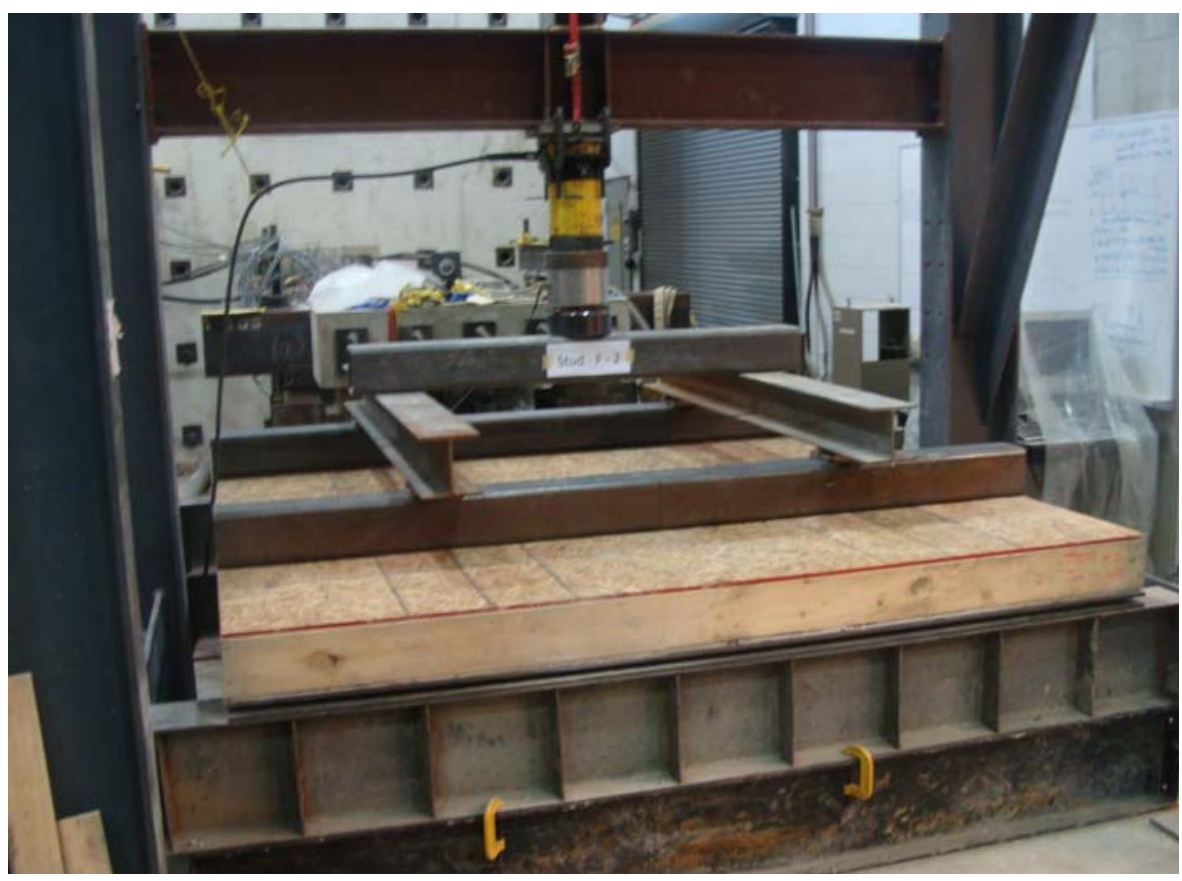

Figure 4.50 - View of specimen SW-F-2 before testing

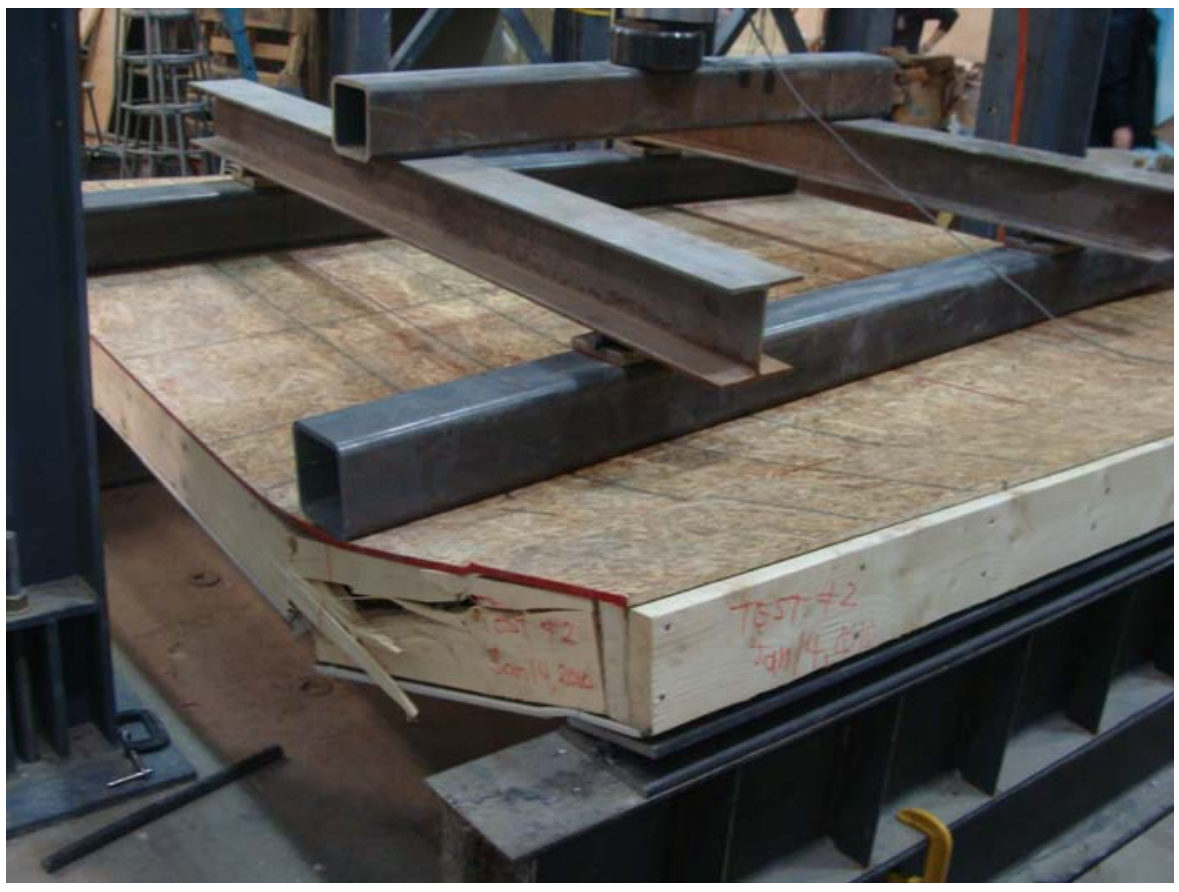

Figure 4.51 - Deflected shape of specimen SW-F-2 after failure 


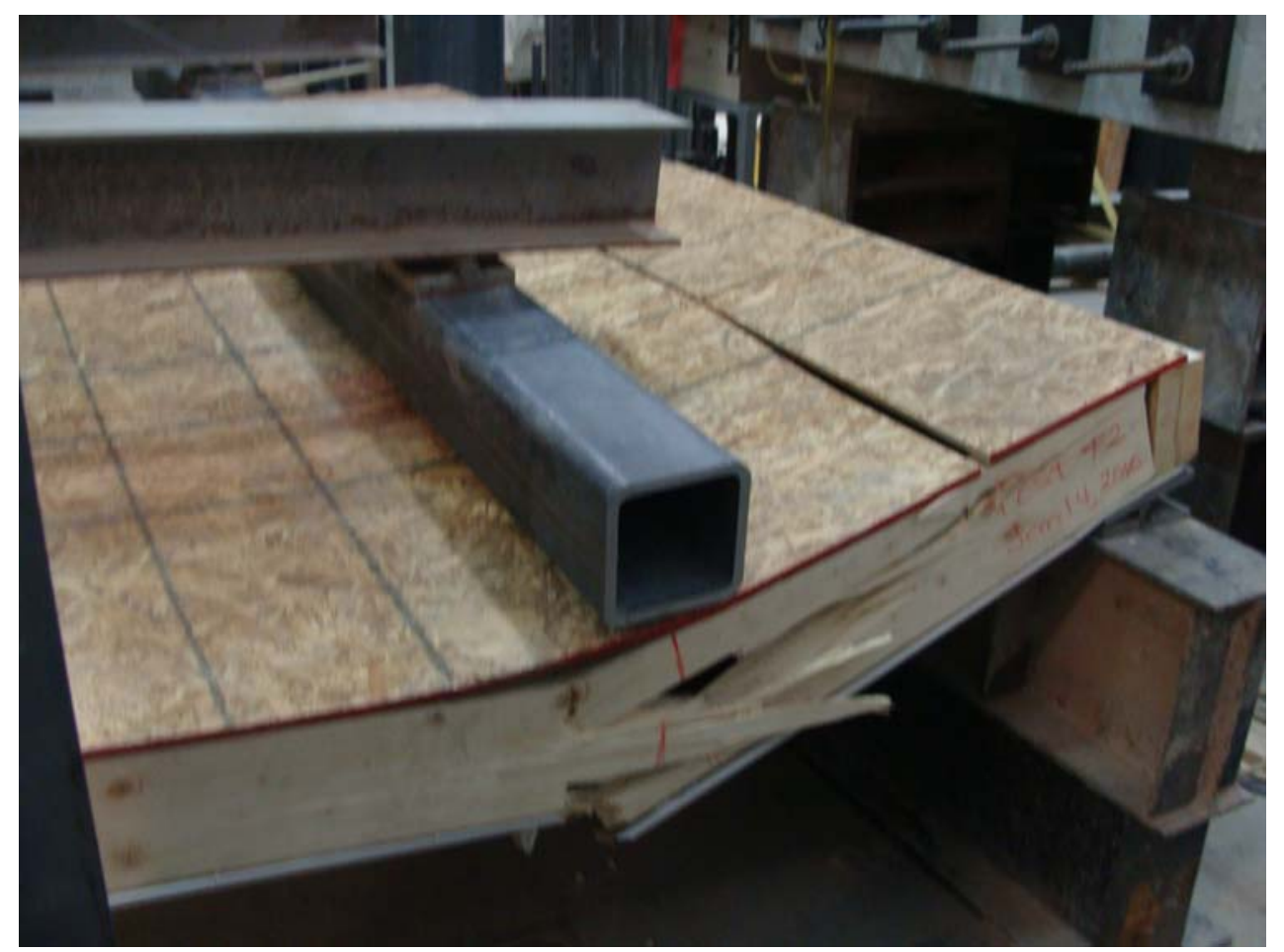

Figure 4.52 - Close-up view of the deflected shape of specimen SW-F-2 after failure

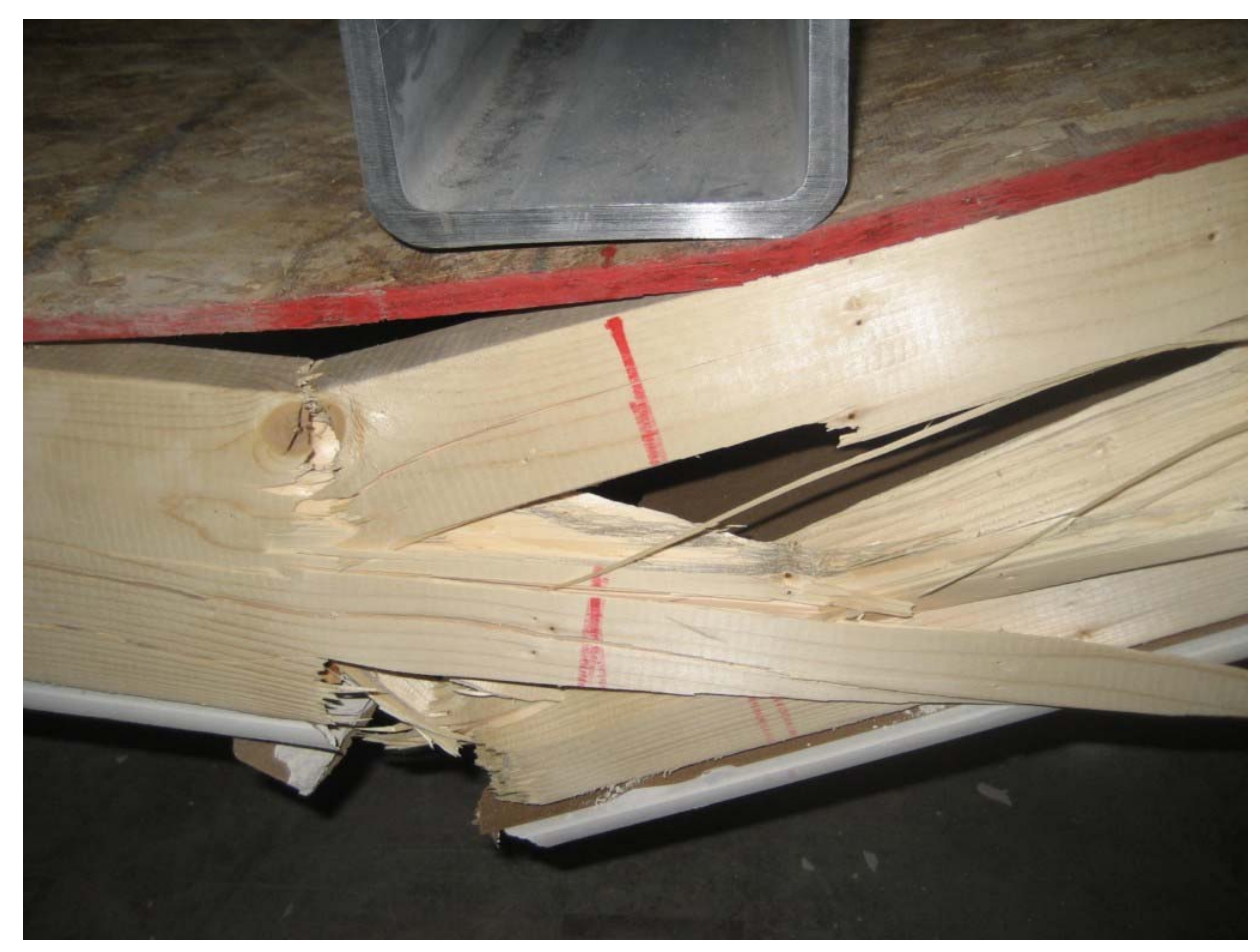

Figure 4.53 - Close-up view of the flexural failure at the quarter point of joist No. 5 of specimen SW-F-2 


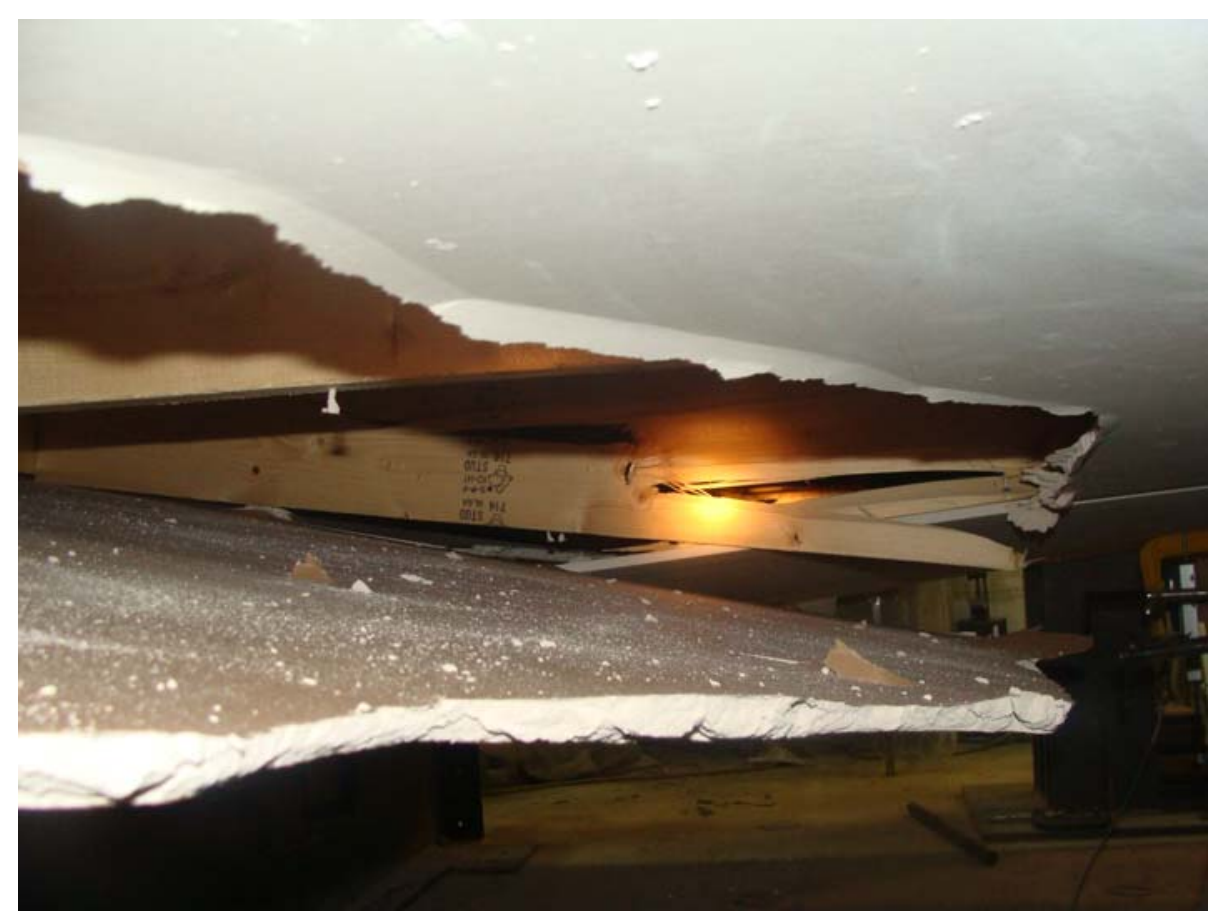

Figure 4.54 - Close-up view of the failure around the quarter point of joist No. 3 of specimen SW-F-2

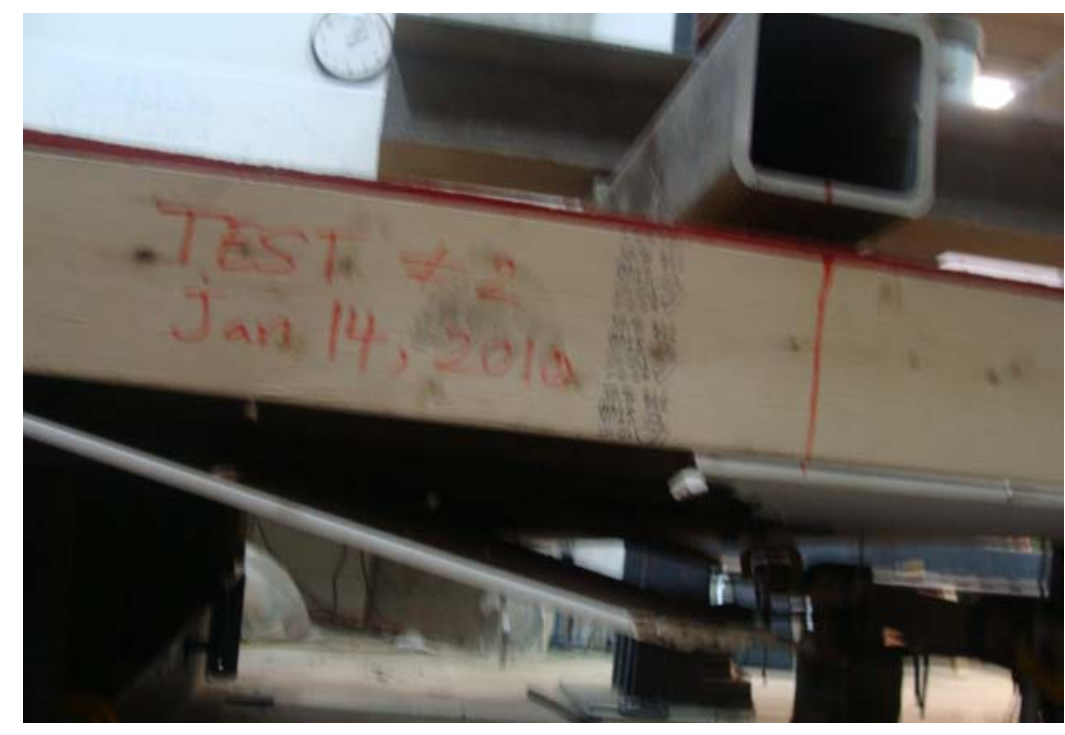

Figure 4.55 - View of drywall break at the quarter point of joist No. 1 of specimen SW-F-2 


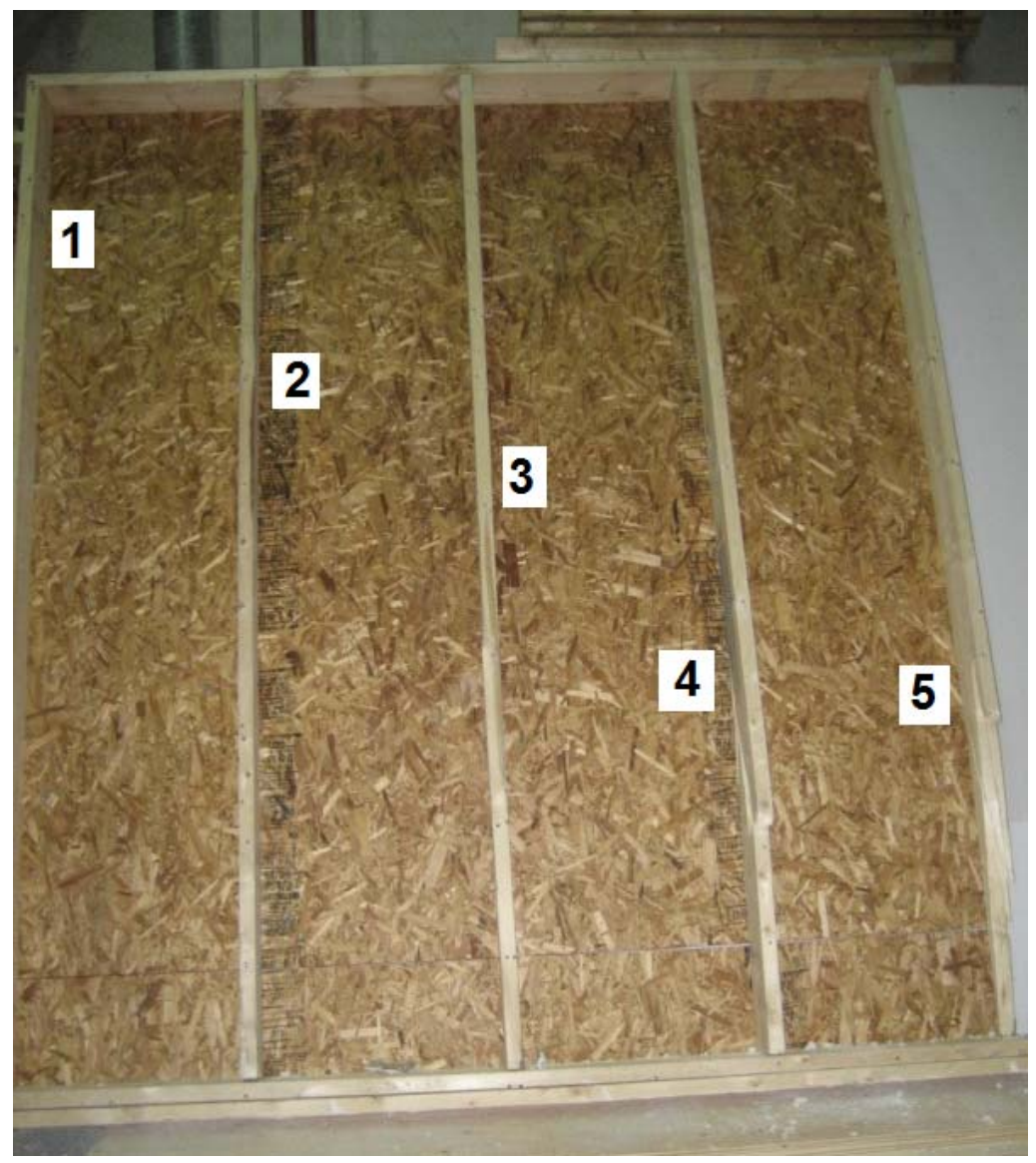

Figure 4.56 - View of the joists after removing the drywall of specimen SW-F-2

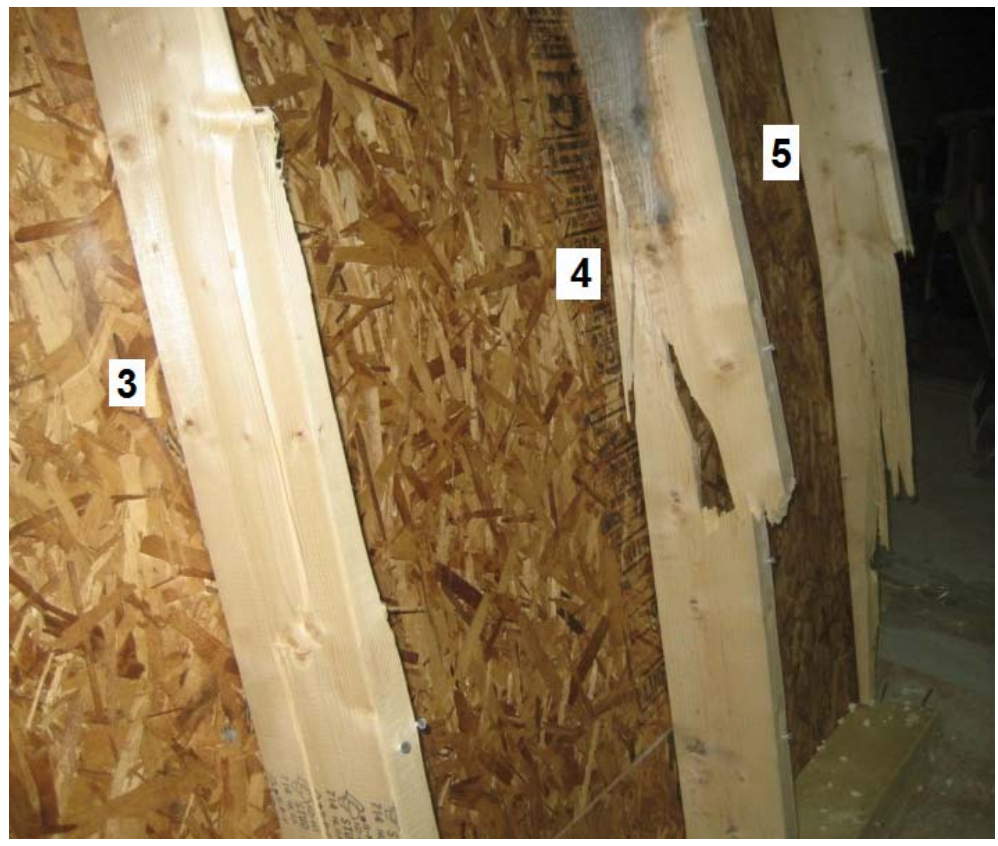

Figure 4.57 - Close-up view of failure of joists No. 3, 4 and 5 of specimen SW-F-2 after failure 


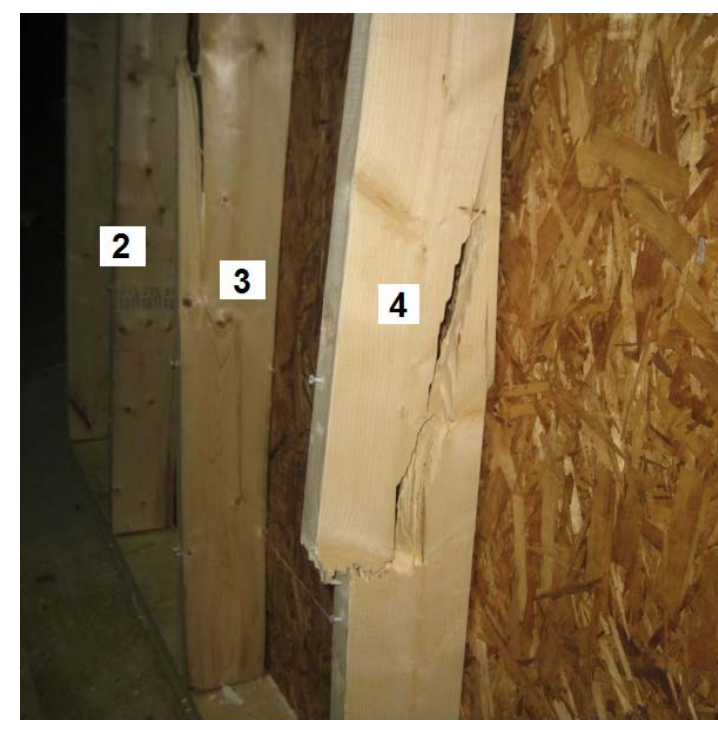

Figure 4.58 - Close-up view of failure in joist failure of No. 3 and 4 of specimen

\section{SW-F-2}

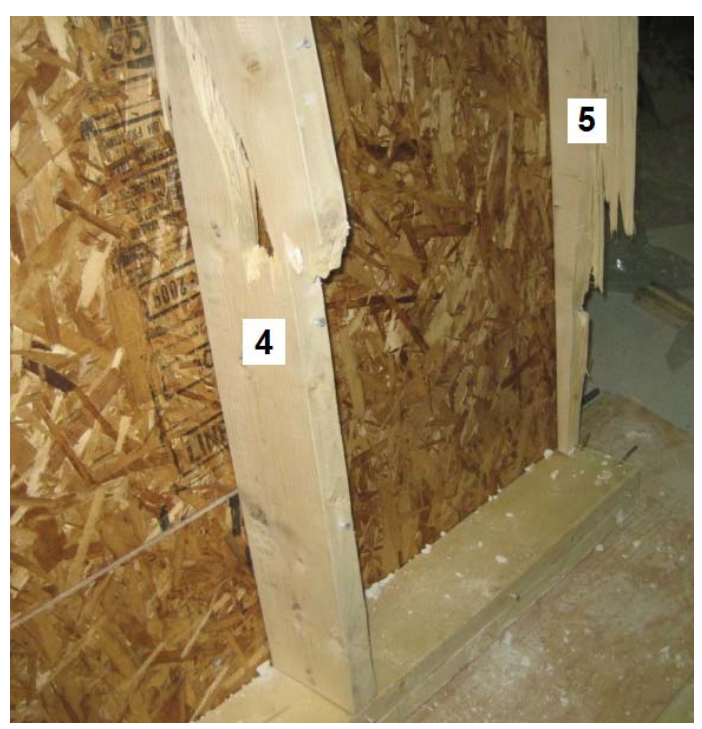

Figure 4.59 - Close-up view of joists 4 and 5 of specimen SW-F-2

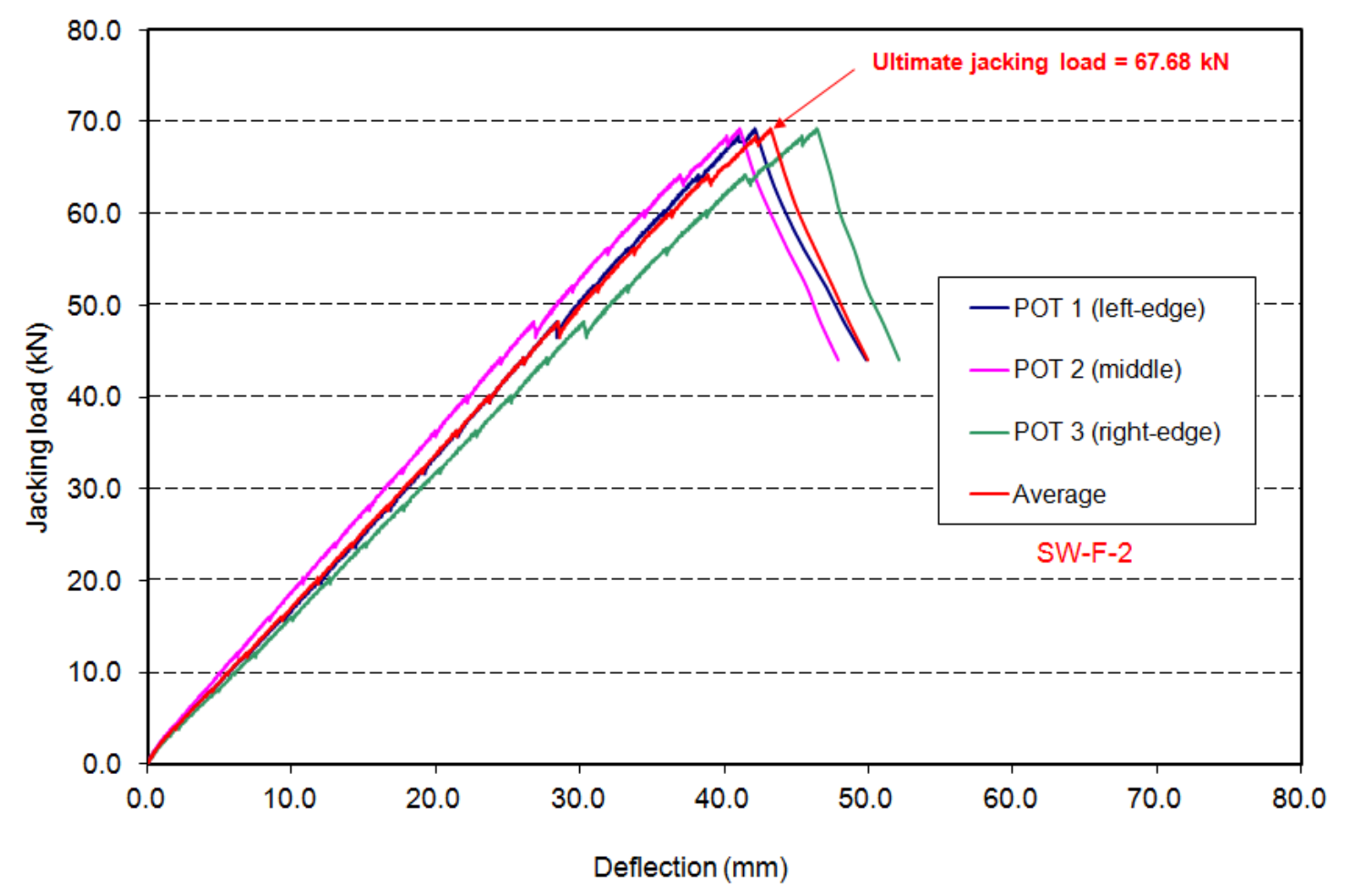

Figure 4.60 - Axial load-axial shortening relationships for specimen SW-F-2 
Figure 4.61 shows view of panel SW-F-3 before testing, while Figs. 4.62 and 4.63 shows view of the permanent deformed shape of the stud specimen after failure. View of the permanent deformed shape of the specimen after removing the loading system is shown in Figure 4.64. The failure mode was pure flexural in stud No 5. In the east side of the specimen, at about $200 \mathrm{~mm}$ off the mid-span location as shown in Figs. 4.65 through 4.6. Figure 4.68 shows that tensile fracture also occurred in stud No. 4 and the bottom drywall was also ruptured. Figures 4.69 and 4.70 show observed large deformation in the support bearing and splitting of the end stud plates connected to the end of stud No. 1. After the test, the stud specimen was removed from the test setup and then the drywall was removed to observe other damage in internal studs, as shown in Figure 4.71. Figure 4.72 shows close-up view of the flexural failure of studs No.4 and 5 on the east side of the specimen. No signs of failure were observed in studs No. 1, 2 and 3 . The failure was sudden and noisy causing a sudden drop in the applied jacking load as depicted in the loaddeflection history shown in Figure 4.73. The load-deflection history shows linear-elastic relationship till failure.

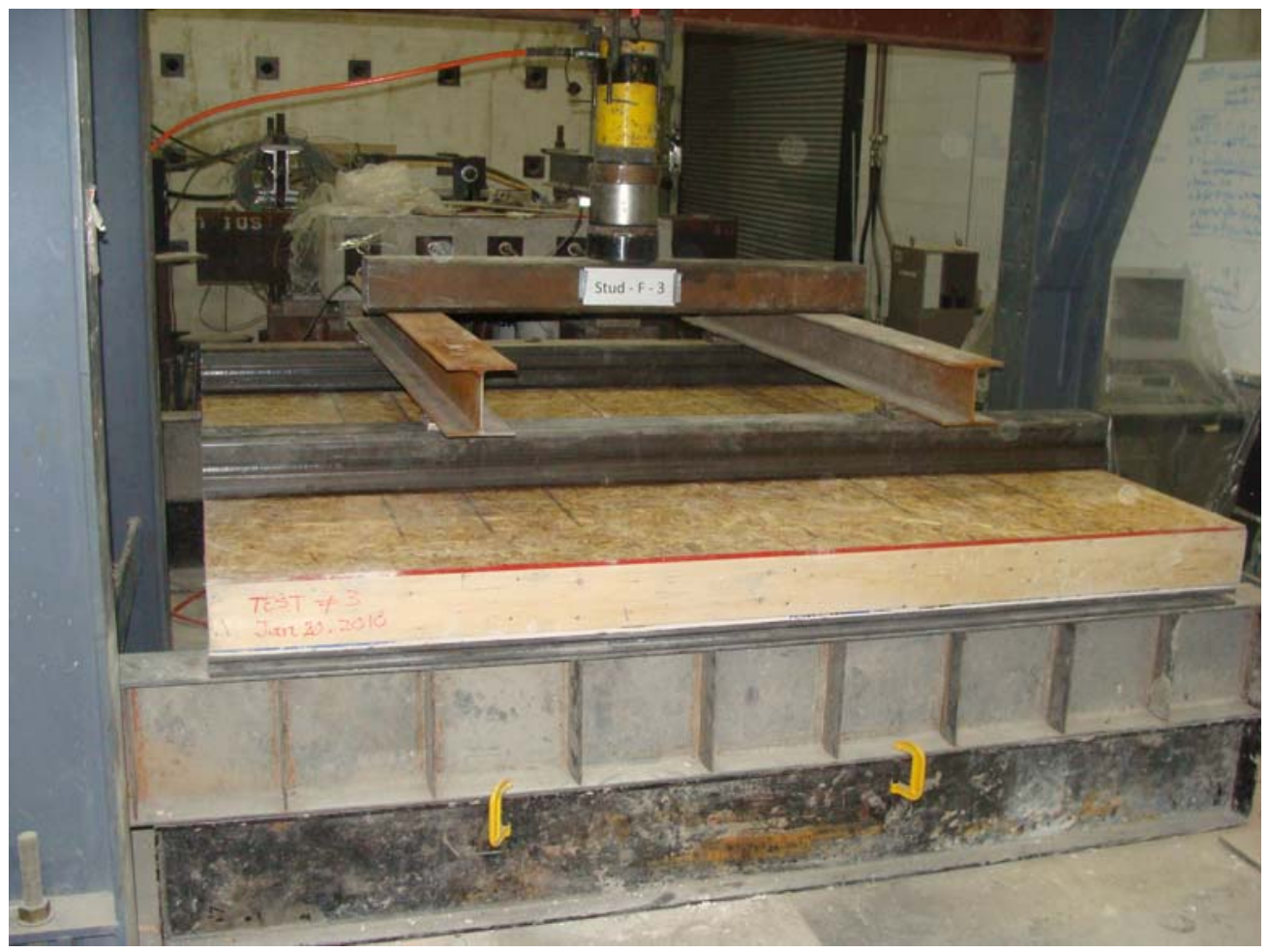

Figure 4.61 - View of Specimen SW-F-3 before testing 


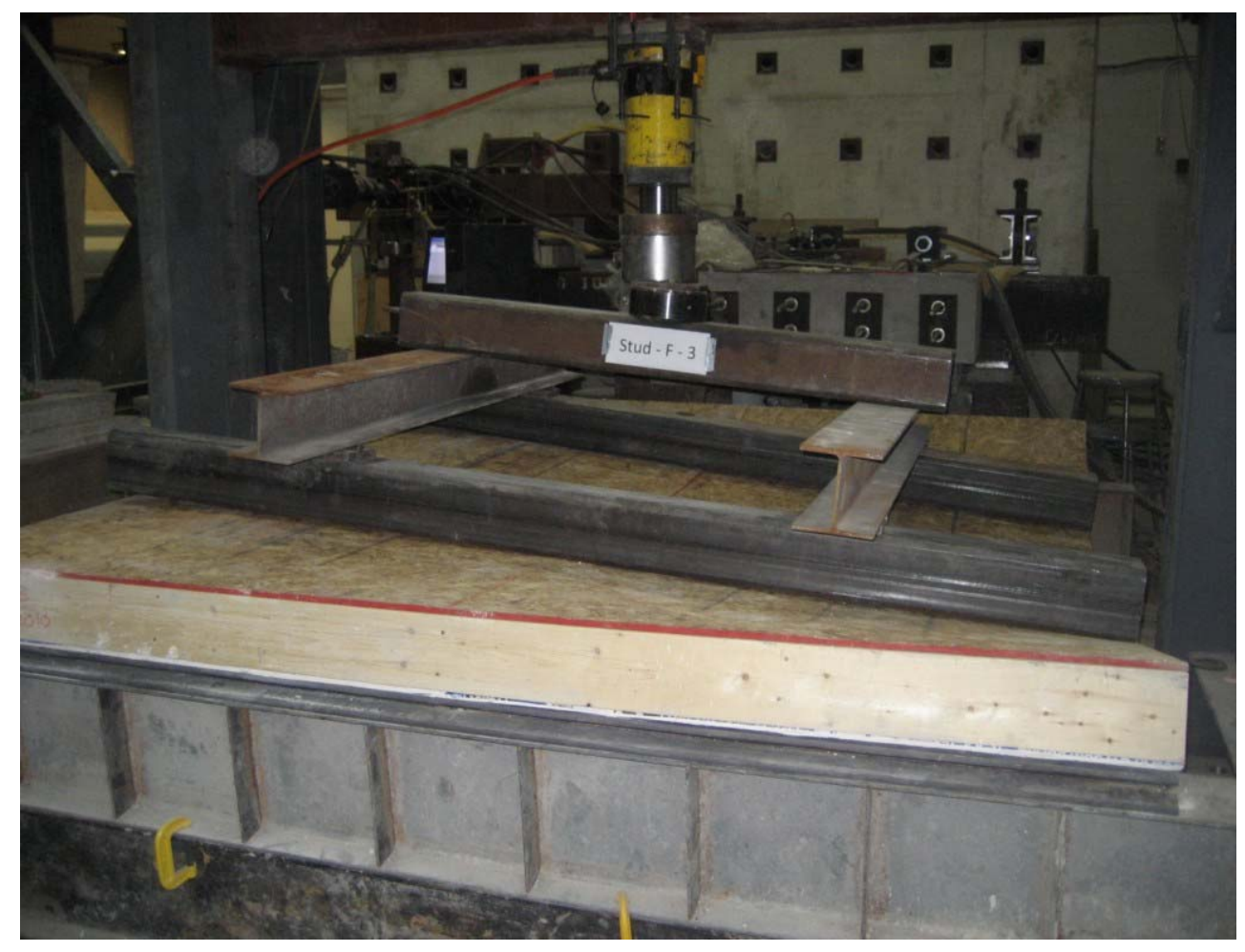

Figure 4.62 - View of specimen SW-F-3 after failure

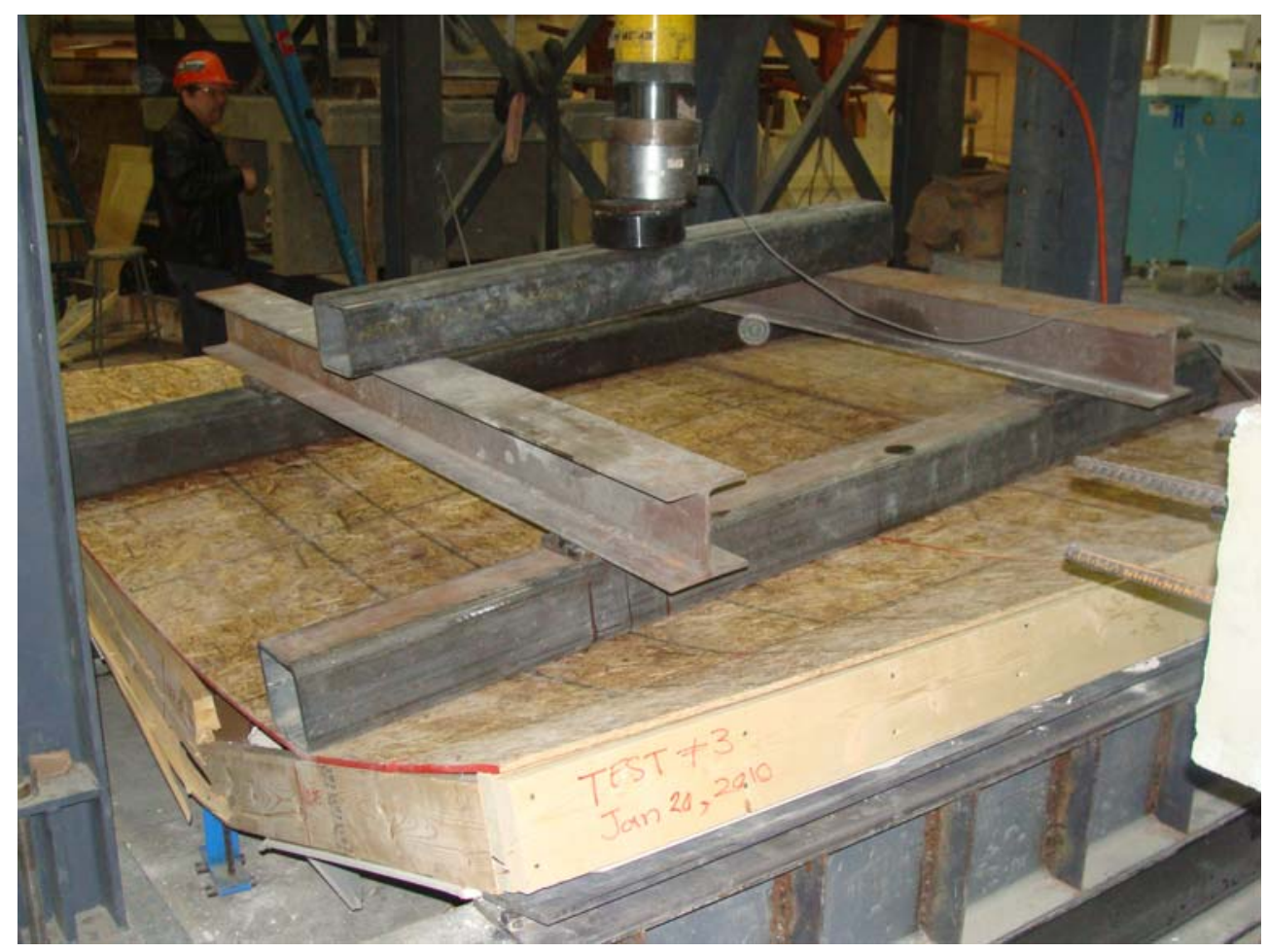

Figure 4.63 - View of the deflected shape of specimen SW-F-3 after failure 


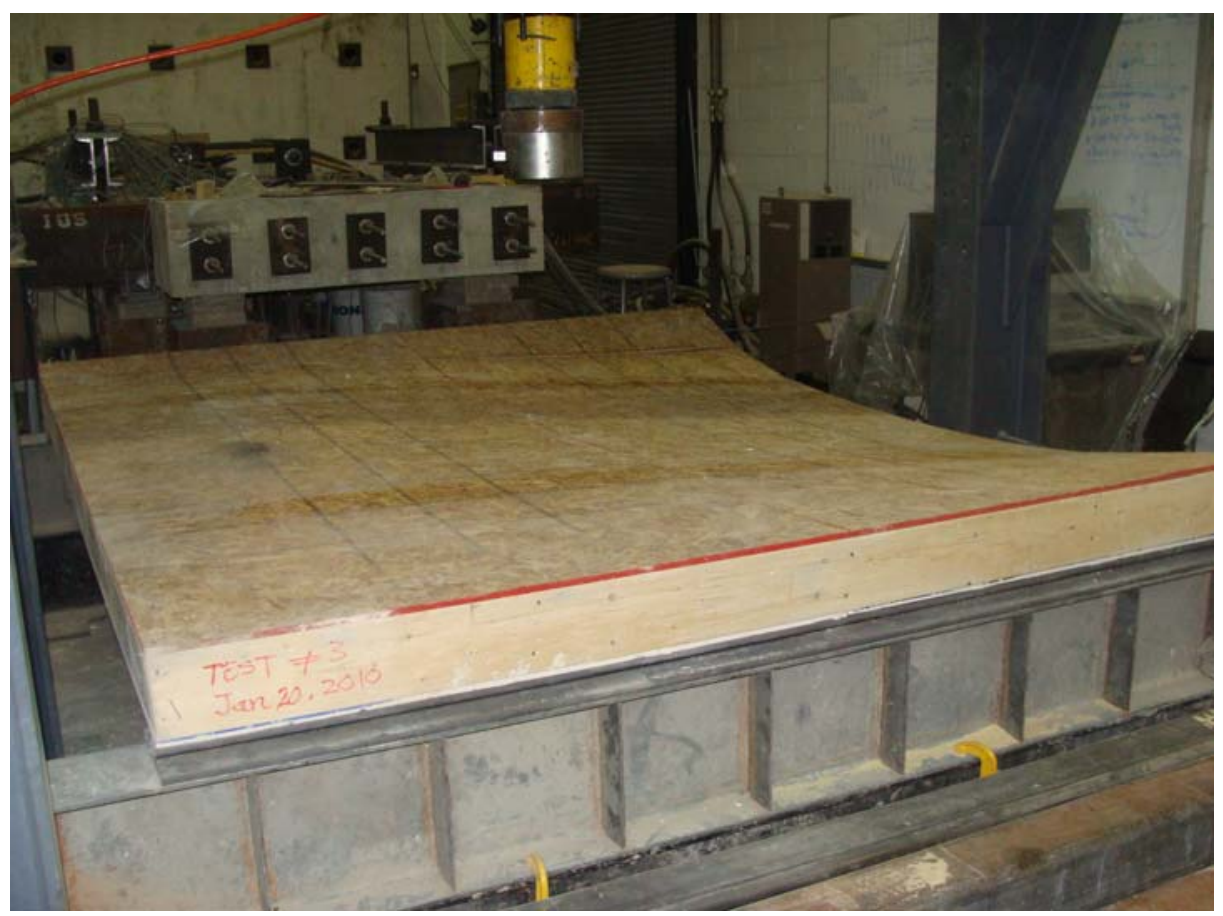

Figure 4.64 - View of the permanent deflected shape of specimen SW-F-3 after removing the loading system

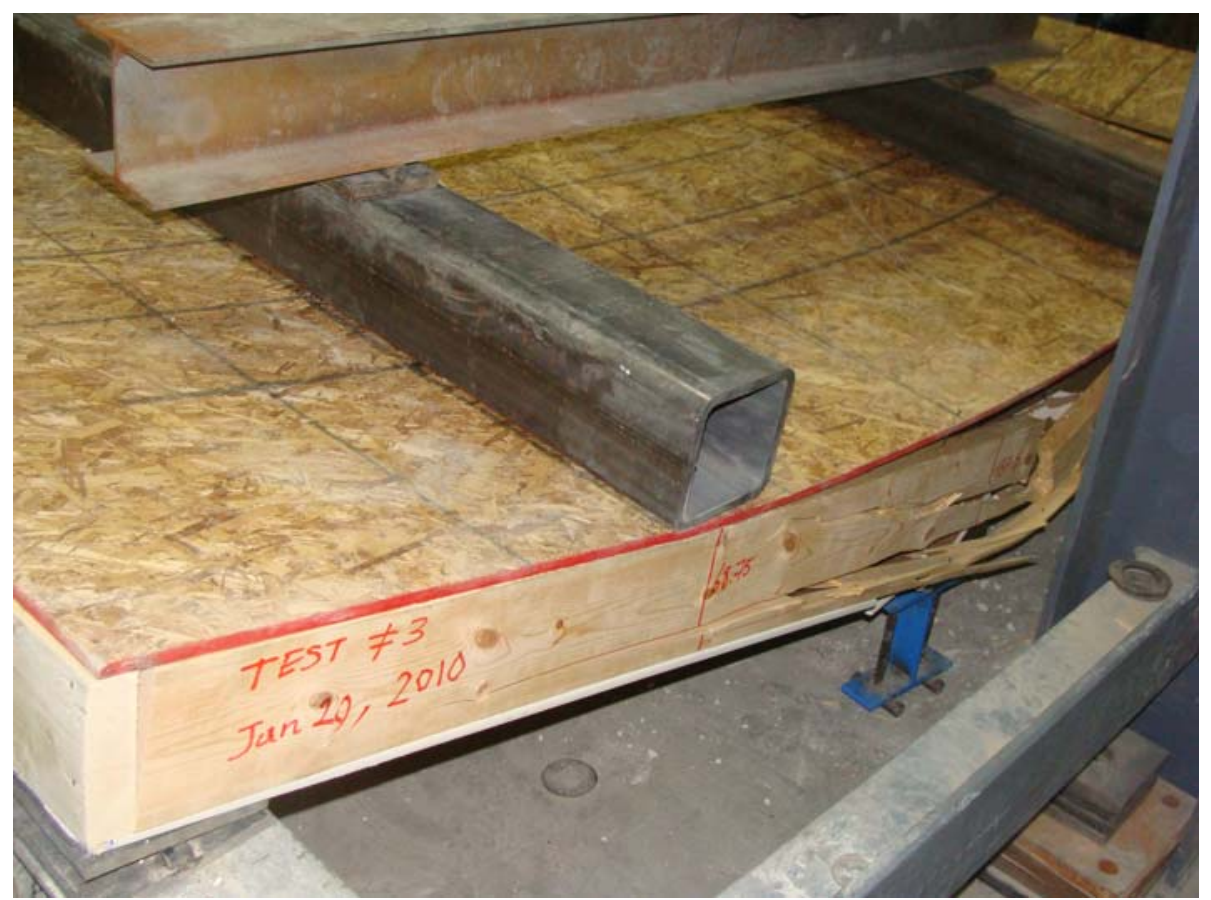

Figure 4.65 - View of the flexural failure and deformation of joist No. 5 of specimen SW-F-3 after failure 


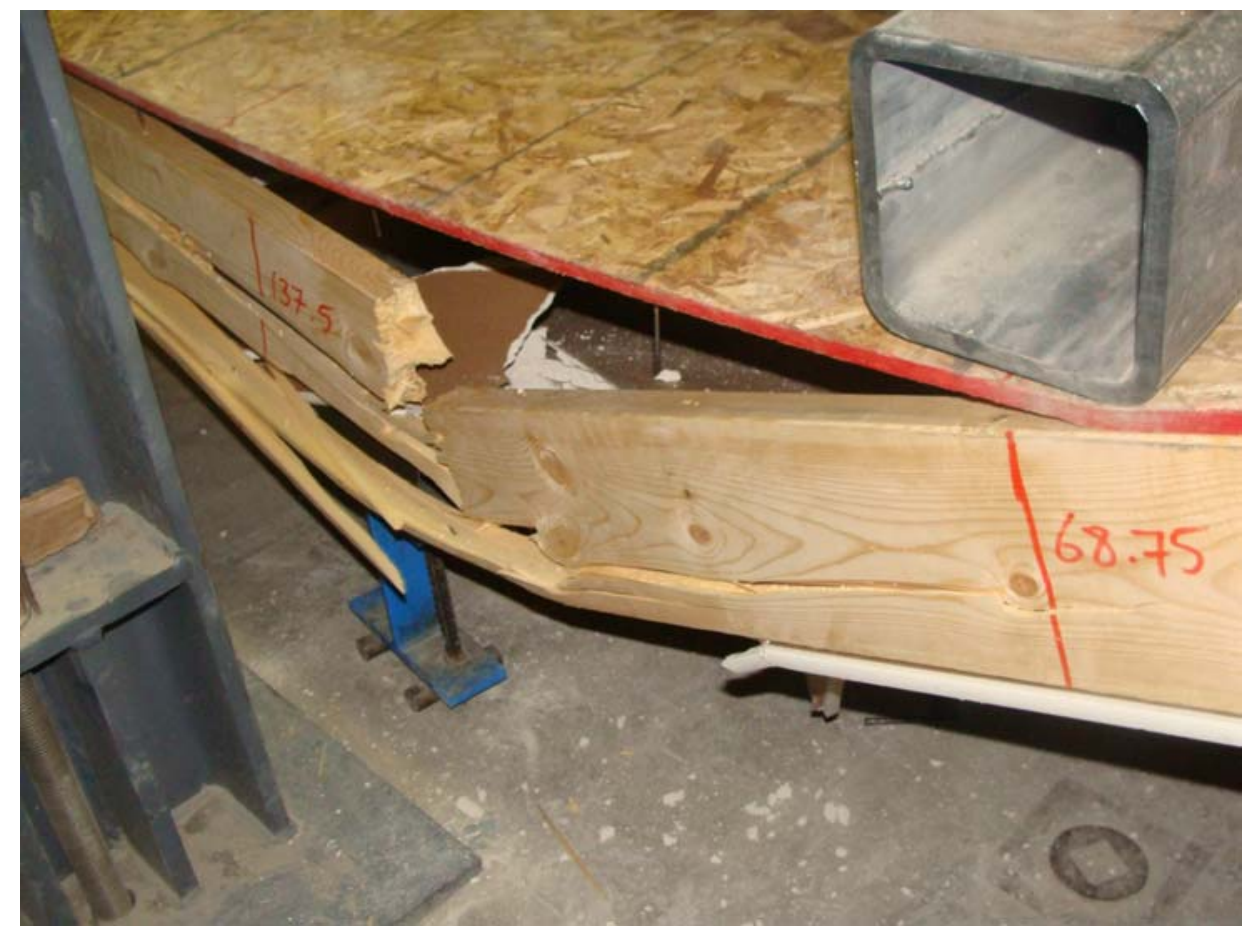

Figure 4.66 - Close-up view of the flexural failure of joist No. 5 in specimen SW-F-3

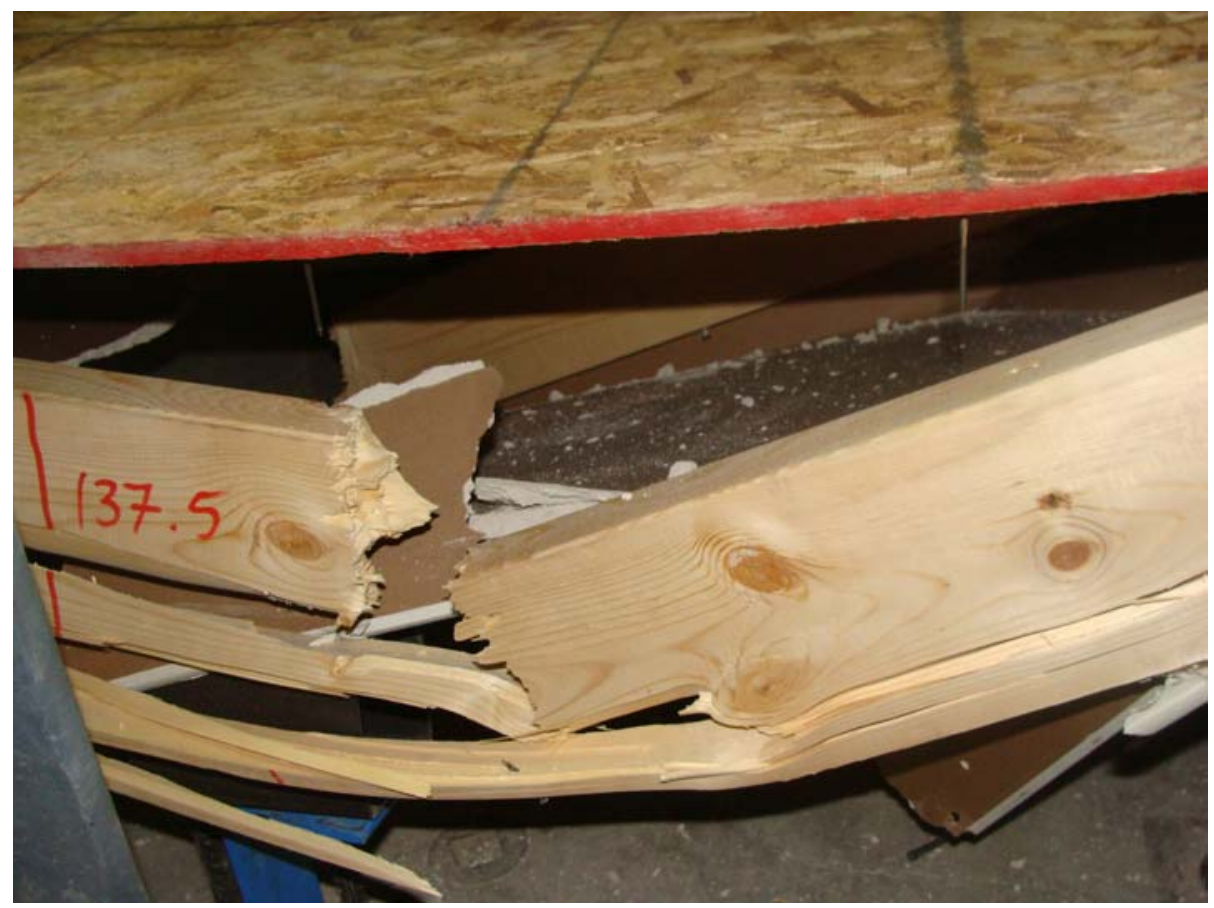

Figure 4.67 - Front view of the flexural failure of joist No. 5 in specimen SW-F-3 


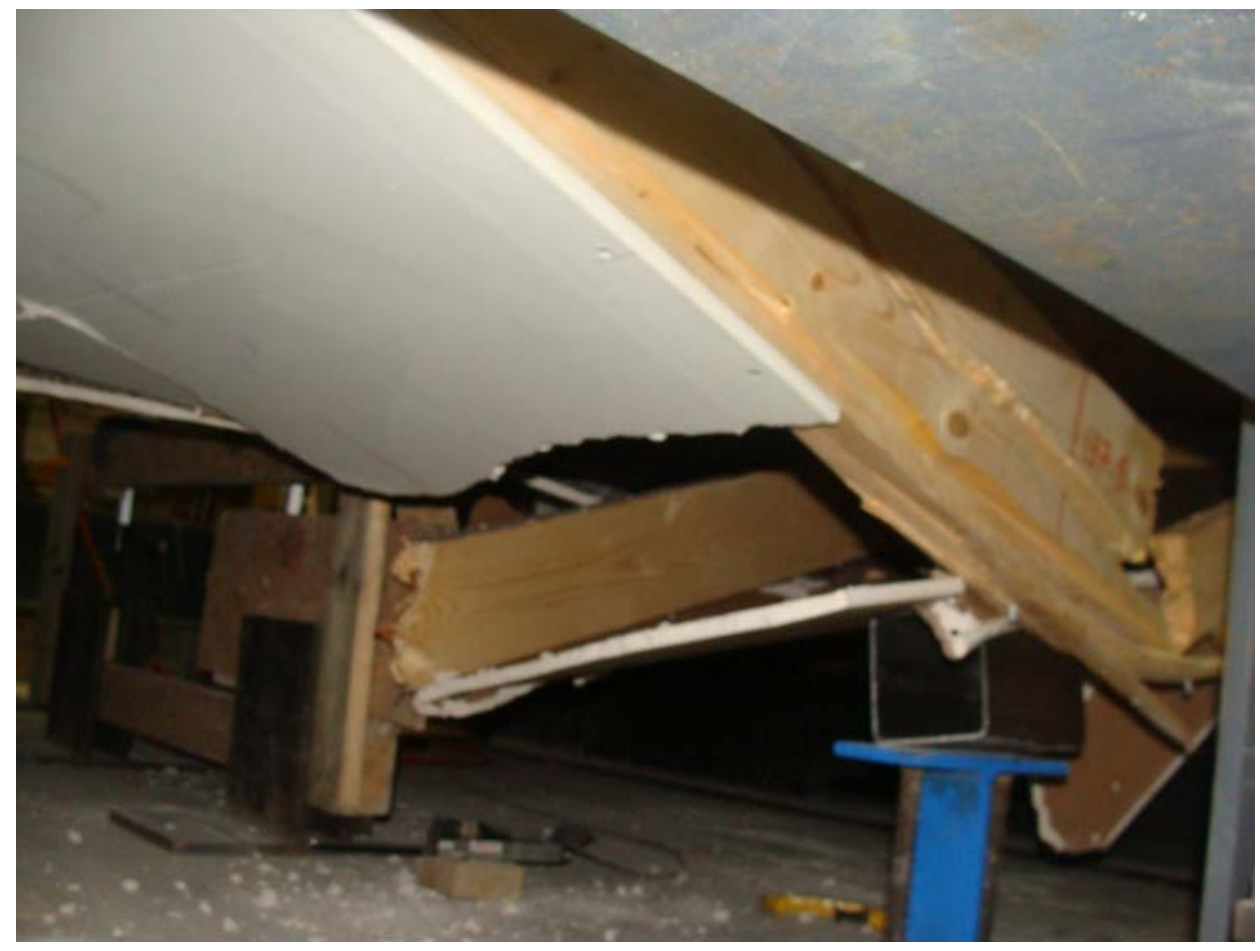

Figure 4.68 - Close-up view of the flexural failure of joists No. 4 and 5 and well as drywall break in specimen SW-F-3

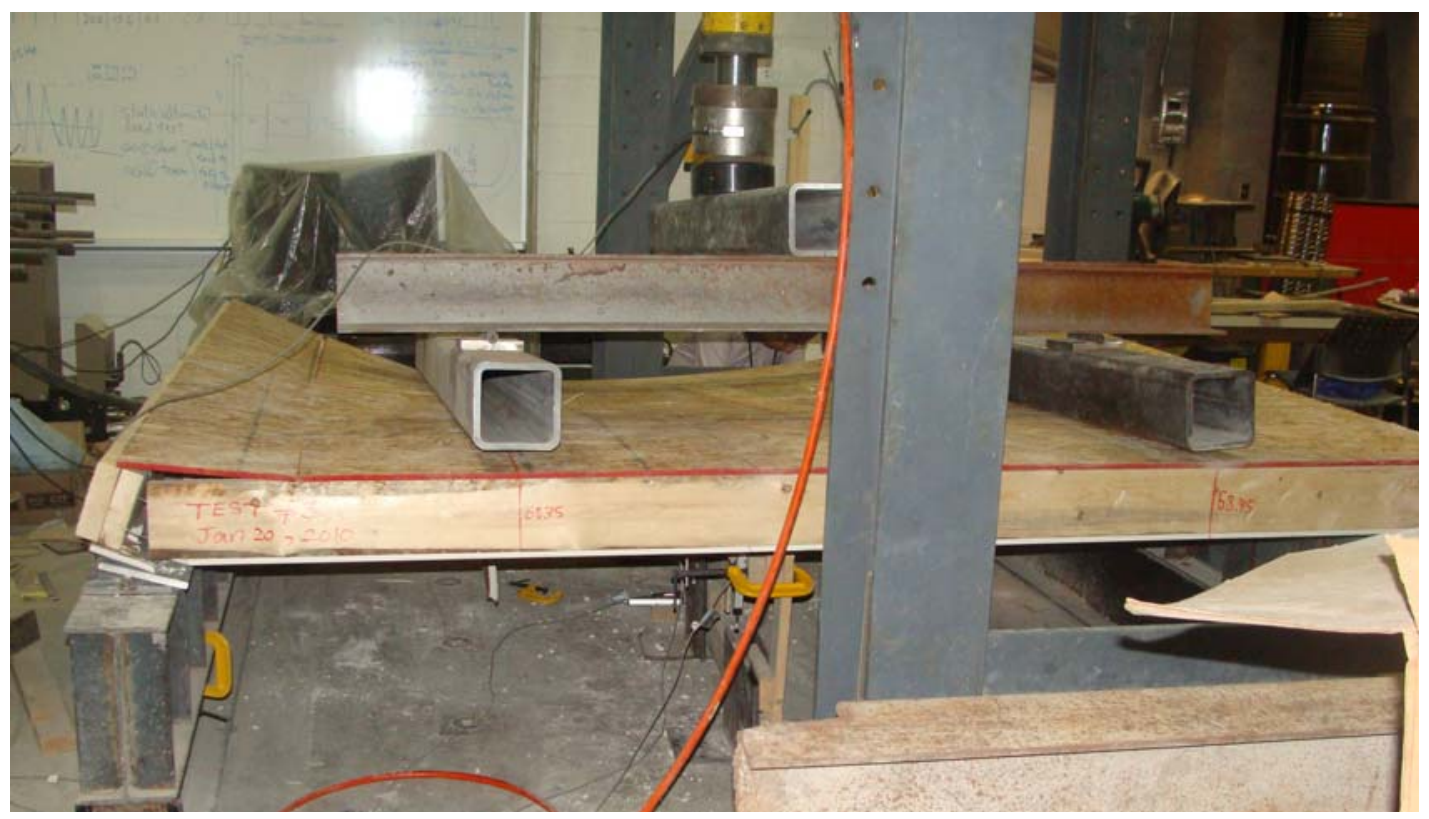

Figure 4.69 - View of the deflected shape of specimen SW-F-3 showing the bearing rotation as well as end stud and OSB sheet separation from joist No. 1 


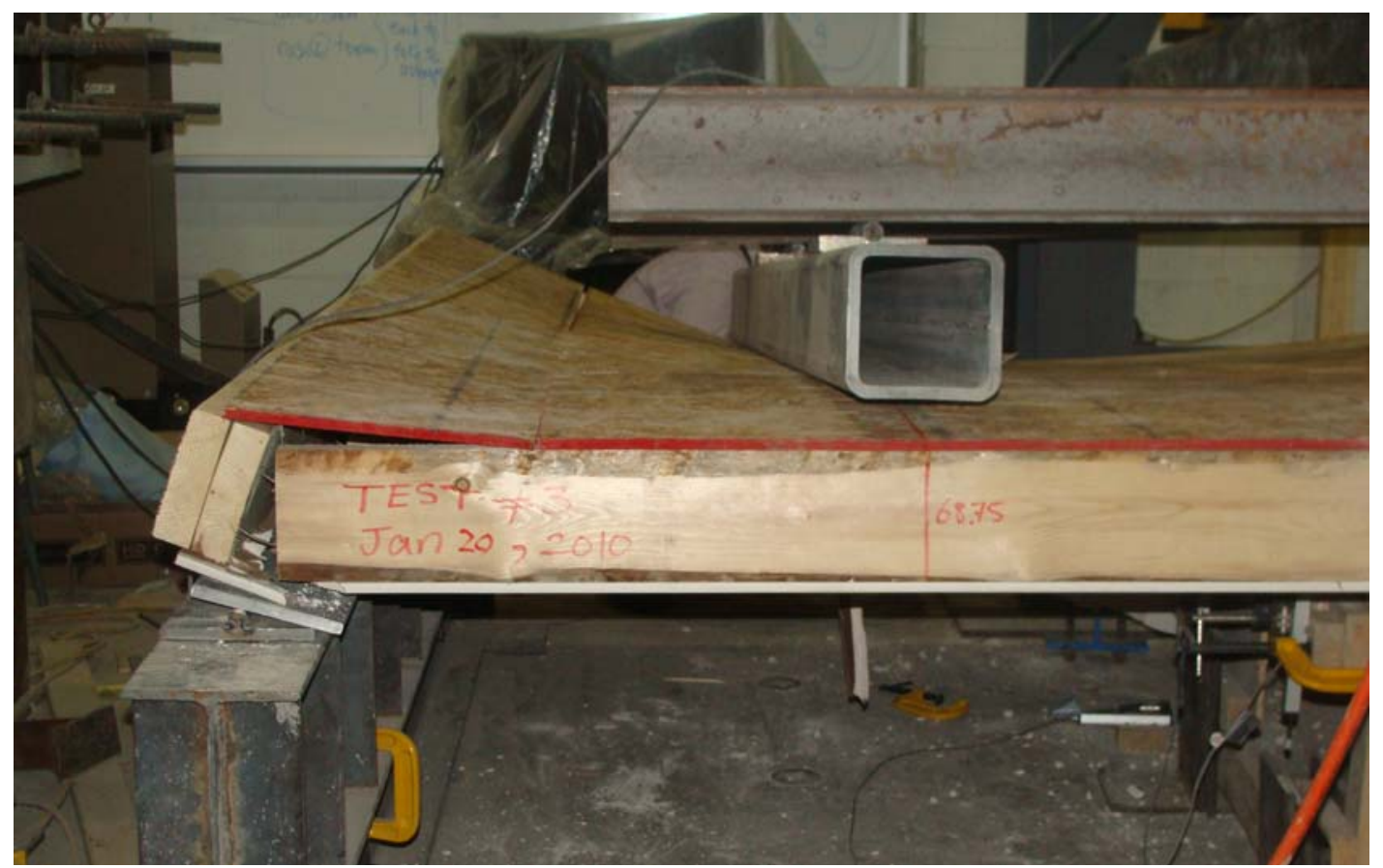

Figure 4.70 - Close-up view of the bearing rotation as well as end stud and OSB sheet separation from joist No. 1 of specimen SW-F-3 after failure

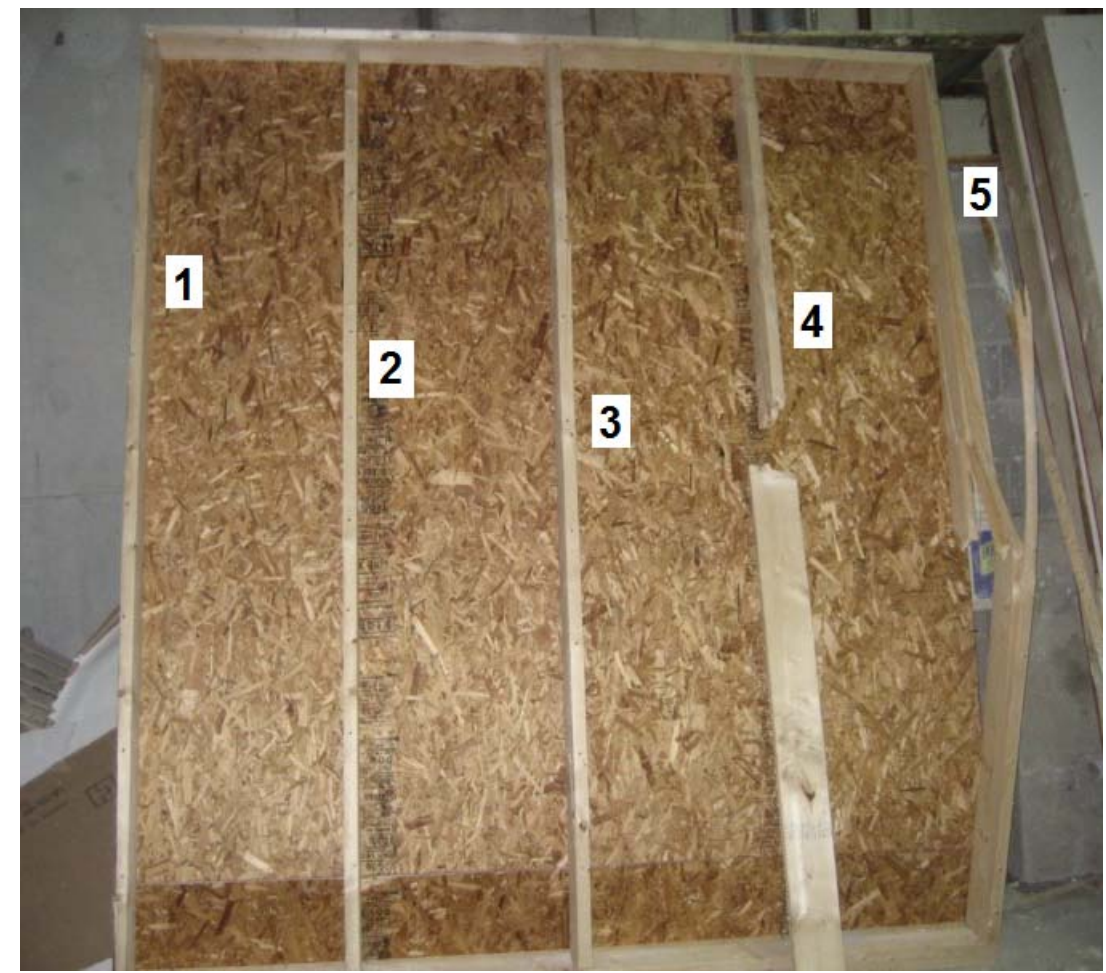

Figure 4.71 - View of the joists after removing the drywall of specimen SW-F-3 


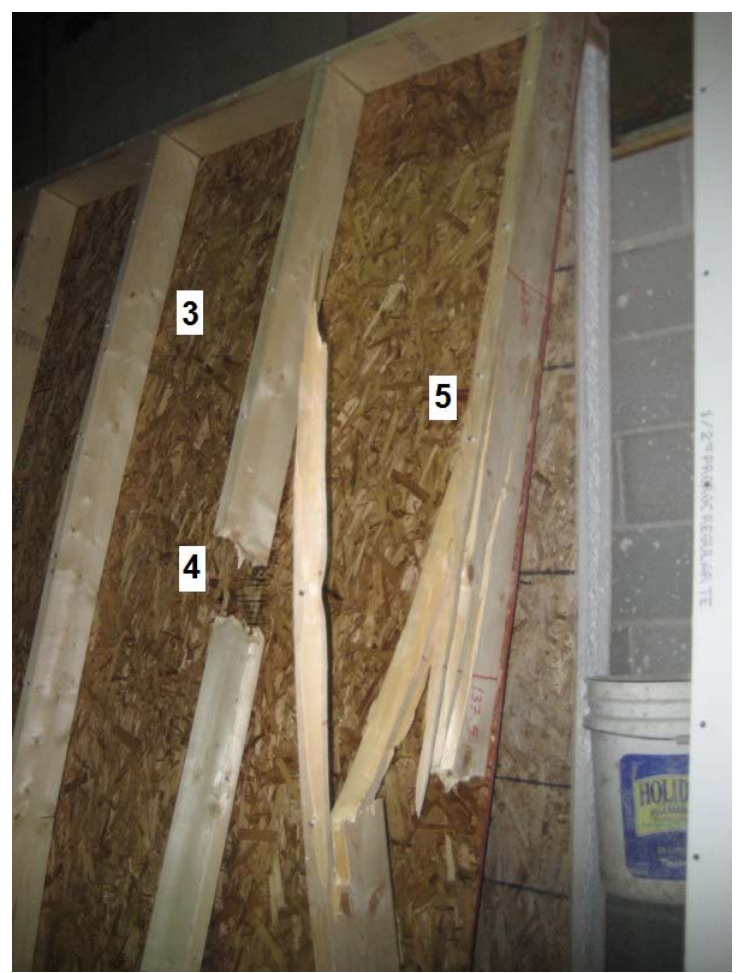

Figure 4.72 - Close-up view of the failure in joists No. 4 and 5 in specimen SW-F-3 after removing the drywall

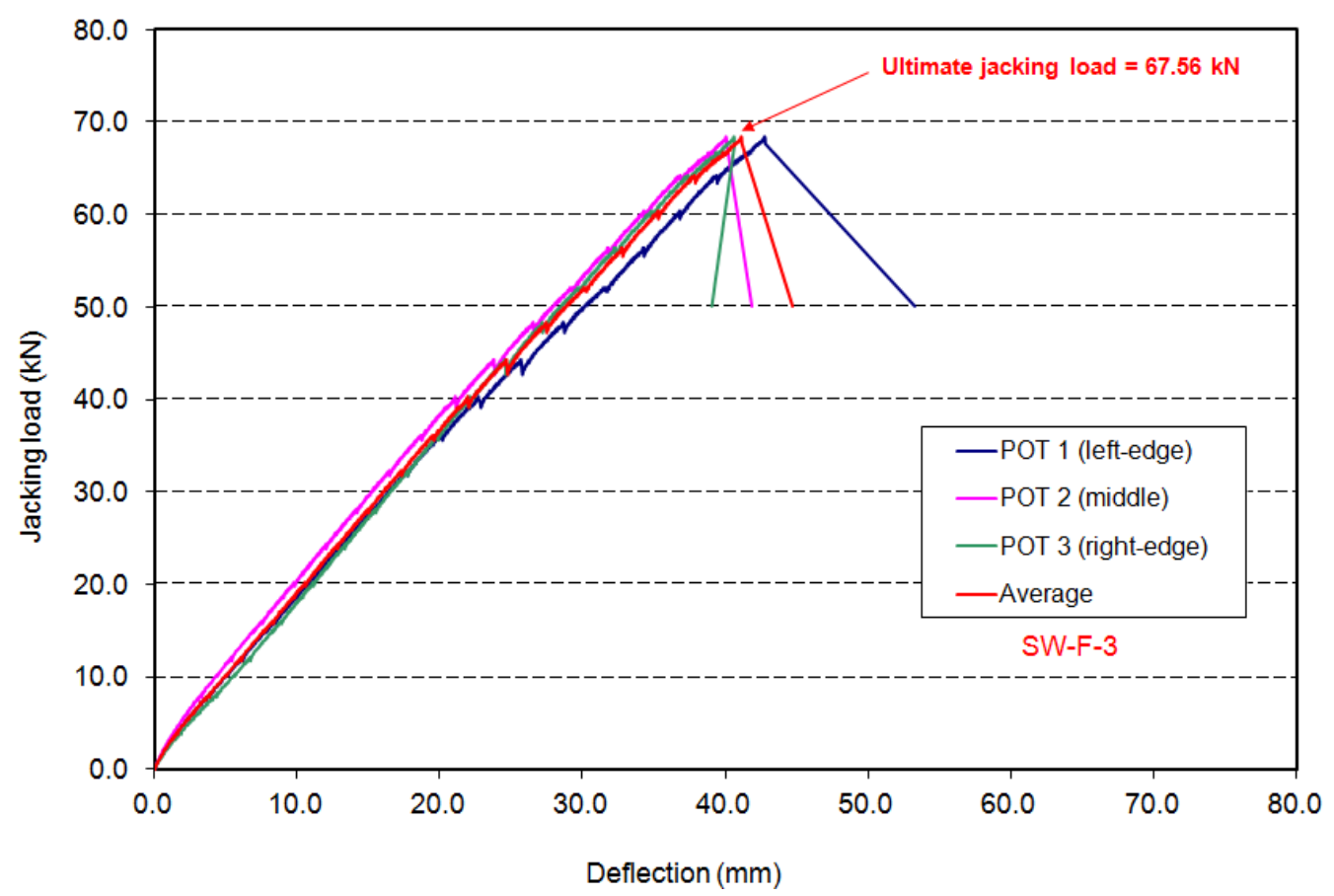

Figure 4.73 - Axial load-axial shortening relationship for specimen SW-F-3 
Table 4.3 shows a summary of stud specimen configurations along with the ultimate jacking load for the stud panel group. It can be observed that the ultimate jacking loads were 59.68, 67.68 and $67.56 \mathrm{kN}$ for specimens SW-F-1, SW-F-2 and SW-F-3, respectively. By adding the weight of the loading system of $3.82 \mathrm{kN}$ and multiplying each load by $\mathrm{L} / 8$, where $\mathrm{L}=2.5808 \mathrm{~mm}$, the adjusted experimental ultimate moment of each panel can be obtained. However, each value was multiplied by 0.8 for using 5 studs rather than 4 in each tested panel. Thus, the adjusted experimental resisting moments were calculated as $8.23,9.26$ and $9.25 \mathrm{kN}-\mathrm{m}$ as shown in Table 4.3. Thus, the average value of the tested panels was taken as $8.91 \mathrm{kN}-\mathrm{m}$.

Table 4.3 shows a summary of stud specimen configurations and results per AC04 design criteria. It can be observed that the ultimate jacking loads were 59.68, 67.68 and $67.56 \mathrm{kN}$ for specimens SW-F-1, SW-F-2 and SW-F-3, respectively. By adding the weight of the loading system of $3.82 \mathrm{kN}$, the ultimate loads were calculated as $63.50,71.50$ and $71.38 \mathrm{kN}$ for specimens SW-F-1, SW-F-2 and SW-F-3, respectively. Thus, the allowable design load applied to this stud panel was taken as the average experimental ultimate load divided by a factor of safety of 3 (i.e. $68.79 / 3=22.935 \mathrm{kN}$ ). By multiplying this value by $\mathrm{L} / 8$, where $\mathrm{L}=2.5808 \mathrm{~mm}$, and dividing it by 2 the allowable design bending moment for this studs group can be obtained for 1220 -mm panel width. However, each value was multiplied by 0.8 for using 5 studs rather than 4 in each tested panel. Thus, the allowable design bending moment of this studs group was calculated as $2.97 \mathrm{kN}-\mathrm{m}$.

\subsubsection{Correlation of Test Results for SIPs and Stud Wall Specimens for Axial Capacity and Deformation}

Based on the aforementioned sections, Tables 4.1 through 4.5 were prepared to examine whether the structural qualification of SIPs is being "as good as" the structural capacity of the conventional wood-frame buildings. There tables present the maximum values for moment and axial compressive force based on the maximum jacking load obtained experimentally. It should be noted that the tested SIP panels in this study had a $2 \times 6$ bottom plate connected to the bottom of the OSB sheet with one row of nails. However, Mohamed (2009) tested identical SIP panels with two $2 \times 6$ bottom plates connected to the bottom of the OSB sheet with two rows of nails. The later panels expected to have greater load carrying capacities that those considered in this 
study given the fact that the recorded failures was mainly at during excessive bearing on the nails at the bottom of the OSB sheet. Tables 4.1 and 4.2 summarize the test results reported by Mohamed (2009).

For the acceptance criteria of SIPs, Clause 5.2.1 of the CCMC technical guide (IRC, 2007) specifies that the stressed skin panel's mean axial strength and characteristic axial strength shall exceed the conventional wall panel's mean and characteristic axial strengths. In addition, the stressed skin panel loads at 3-mm deformation shall exceed the loads on the conventional panel at 3-mm deformation for all cases.

As it can be observed from Table 4.1 that the characteristic ultimate resisting compressive load for SIPs with one bottom plate and one row of nails $(45.07 \mathrm{kN})$ is less than that for stud wall specimens $(48.49 \mathrm{kN})$ by $7 \%$. Also, Table 4.1 shows that the characteristic ultimate resisting compressive load for SIPs with two bottom plates and two rows of nails $(104.21 \mathrm{kN})$ is greater than that for stud wall specimens $(48.49 \mathrm{kN})$ by $115 \%$.

Based on AC04 design criteria, Table 4.2 shows that the allowable compressive load based on failure loads for SIPs with one bottom plate and one row of nails $(38.54 \mathrm{kN})$ is more than that for stud wall specimens $(30.87 \mathrm{kN})$ by $25 \%$. Also, Table 4.2 shows that the allowable compressive load based on the 3-mm axial deformation for SIPs with one bottom plates and one rows of nails $(29.93 \mathrm{kN})$ is greater than that for stud wall specimens $(25.06 \mathrm{kN})$ by $119 \%$.

Table 4.2 shows that the allowable compressive load based on failure loads for SIPs with two bottom plate and two row of nails $(69.44 \mathrm{kN})$ is more than that for stud wall specimens (30.87 $\mathrm{kN}$ ) by $125 \%$. Also, Table 4.2 shows that the allowable compressive load based on the $3-\mathrm{mm}$ axial deformation for SIPs with two bottom plates and two rows of nails $(56.61 \mathrm{kN})$ is greater than that for stud wall specimens $(25.06 \mathrm{kN})$ by $126 \%$.

Based on the above-mentioned comparison, SIPs are being "as good as" the conventional stud wood-framing with respect to axial capacity and deformation per AC04 design criteria irrespective whether one or two plates were used at the bottom of the wall. As for the 
characteristic ultimate compressive load, SIPs with two bottom plates and two rows of nails are being "as good as" the stud wall system. However, SIPs with one bottom plate and one row of nails underestimates the stud wall capacity by $7 \%$.

\subsubsection{Correlation of Test Results for SIPs and Stud Floor Specimens for Bending Capacity and Deformation}

Based on the aforementioned sections, Tables 4.3, 4.4 and 4.5 were prepared to examine whether the structural qualification of SIPs is being "as good as" the structural capacity of the conventional wood-frame buildings. Table 4.3 present the characteristic ultimate resisting moment for the tested panels, while Table 4.4 presents the allowable bending moments based on AC04 criteria. Table 4.5 presents the total load applied to the panel to meet the deflection criteria. 
Table 4.5 - Flexural load at specified deflection limit

\begin{tabular}{|c|c|c|c|c|c|c|c|c|}
\hline Group & $\begin{array}{l}\text { Test } \\
\text { No. }\end{array}$ & $\begin{array}{l}\text { Panel size: } \\
\mathrm{l} \times \mathrm{w} \times \mathrm{t} \\
(\mathrm{mm}, \mathrm{ft}-\mathrm{inch})\end{array}$ & $\begin{array}{l}\text { Flexural } \\
\text { load at } \\
\text { L/360 } \\
\text { deflection } \\
(\mathrm{kN})\end{array}$ & $\begin{array}{l}\text { Adjusted } \\
\text { flexural } \\
\text { load at } \\
\text { L/360 } \\
\text { Deflection } \\
(k N)\end{array}$ & $\begin{array}{l}\text { Mean } \\
\text { Flexural } \\
\text { load at } \\
\text { L/360 } \\
\text { Deflection } \\
(k N)\end{array}$ & $\begin{array}{l}\text { Flexural } \\
\text { load at } \\
\text { L/180 } \\
\text { deflection } \\
(\mathrm{kN})\end{array}$ & $\begin{array}{l}\text { Adjusted } \\
\text { flexural } \\
\text { load at } \\
\text { L/180 } \\
\text { deflection } \\
(k N)\end{array}$ & $\begin{array}{l}\text { Mean } \\
\text { Flexural } \\
\text { Load at } \\
\text { L/180 } \\
\text { Deflection } \\
(k N)^{(1)}\end{array}$ \\
\hline \multirow[t]{3}{*}{ SIP } & SIP-F-1 & $\begin{array}{l}1220 \times 2743 \times 165 \\
\left(4^{\prime} \times 9^{\prime} \times 6 \frac{1}{2}{ }^{\prime}\right)\end{array}$ & 11.61 & 11.61 & \multirow[t]{3}{*}{11.39} & 20.05 & 20.05 & \multirow[t]{3}{*}{19.83} \\
\hline & SIP-F-2 & $\begin{array}{l}1220 \times 2743 \times 165 \\
\left(4^{\prime} \times 9^{\prime} \times 6^{1 / 2 \prime}{ }^{\prime \prime}\right)\end{array}$ & 11.47 & 11.47 & & 20.12 & 20.12 & \\
\hline & SIP-F-3 & $\begin{array}{l}1220 \times 2743 \times 165 \\
\left(4^{\prime} \times 9^{\prime} \times 6 \frac{1}{2}{ }^{\prime \prime}\right)\end{array}$ & 11.10 & 11.10 & & 19.31 & 19.31 & \\
\hline \multirow[t]{5}{*}{$\begin{array}{l}\text { SIP } \\
\text { (Mohamed, 2009) }\end{array}$} & WS19 & $\begin{array}{l}1220 \times 2743 \times 165 \\
\left(4^{\prime} \times 9^{\prime} \times 6 \frac{1}{2}{ }^{\prime \prime}\right)\end{array}$ & 11.07 & 11.07 & \multirow{5}{*}{10.83} & 19.14 & 19.14 & \multirow{5}{*}{18.95} \\
\hline & WS20 & $\begin{array}{l}1220 \times 2743 \times 165 \\
\left(4^{\prime} \times 9^{\prime} \times 6^{1 / 2 \prime}{ }^{\prime \prime}\right)\end{array}$ & 10.63 & 10.63 & & 19.04 & 19.04 & \\
\hline & WS21 & $\begin{array}{l}1220 \times 2743 \times 165 \\
\left(4^{\prime} \times 9^{\prime} \times 6^{1} \stackrel{1}{\prime}{ }^{\prime \prime}\right)\end{array}$ & 11.00 & 11.00 & & 18.77 & 18.77 & \\
\hline & WS22 & $\begin{array}{l}1220 \times 2743 \times 165 \\
\left(4^{\prime} \times 9^{\prime} \times 6 \frac{1}{2}{ }^{\prime \prime}\right)\end{array}$ & 10.70 & 10.70 & & 18.60 & 18.60 & \\
\hline & WS23 & $\begin{array}{l}1220 \times 2743 \times 165 \\
\left(4^{\prime} \times 9^{\prime} \times 6 \frac{1}{2} 2^{\prime \prime}\right)\end{array}$ & 10.75 & 10.75 & & 19.19 & 19.19 & \\
\hline \multirow[t]{3}{*}{ STUD } & SW-F-1 & $\begin{array}{l}2440 \times 2743 \times 165 \\
\left(8^{\prime} \times 9^{\prime} \times 6 \frac{1}{2}{ }^{\prime \prime}\right)\end{array}$ & 13.11 & $5.24 *$ & \multirow[t]{3}{*}{5.23} & 24.18 & $9.67 *$ & \multirow[t]{3}{*}{9.92} \\
\hline & SW-F-2 & $\begin{array}{l}2440 \times 2743 \times 165 \\
\left(8^{\prime} \times 9^{\prime} \times 6^{1 / 2 \prime}{ }^{\prime \prime}\right)\end{array}$ & 12.29 & $4.92 *$ & & 23.75 & 9.50* & \\
\hline & SW-F-3 & $\begin{array}{l}2440 \times 2743 \times 165 \\
\left(8^{\prime} \times 9^{\prime} \times 6 \frac{1}{2}{ }^{\prime \prime}\right)\end{array}$ & 13.81 & $5.52 *$ & & 26.50 & $10.60^{*}$ & \\
\hline
\end{tabular}

* Values divided by 2 for $1.2 \mathrm{~m}$ panel width and multiplied by 0.8 for using 5 studs rather than 4 in the tested wall ${ }^{(1)}$ Values are per panel width of $1.2 \mathrm{~m}$.

Clause 5.2.2 of the CCMC technical guide (IRC, 2007) states that the (i) ultimate bending strength and (ii) loads up to L/180 (i.e. L/360, L/240 and L/180) bending deflection of all panels shall be recorded. For the acceptance criteria of SIPs, Clause 5.2.2 of the CCMC technical guide specifies that the stressed skin panel's mean bending strength and characteristic bending strength shall exceed the conventional wall panel's mean and characteristic bending strengths. 
In addition, the stressed skin panel loads at L/360 deflection shall exceed the loads on the conventional panel at L/360 deflection for all cases.

Table 4.3 shows that the characteristic resisting bending moment for SIPs with one end plate $(7.26 \mathrm{kN}-\mathrm{m})$ is greater than that for the stud wall specimens $(7.19 \mathrm{kN}-\mathrm{m})$ by $1 \%$. As for SIPs with two end plates, the characteristic resisting bending moment $(8.55 \mathrm{kN}-\mathrm{m})$ is greater than that for the stud wall specimens $(7.19 \mathrm{kN}-\mathrm{m})$ by $19 \%$. As for AC0 4 design criteria, Table 4.4 shows that the allowable bending moment for SIPs with one end plate $(2.86 \mathrm{kN}-\mathrm{m})$ is smaller than that for the stud wall specimens $(2.97 \mathrm{kN}-\mathrm{m})$ by $4 \%$. As for SIPs with two end plates, the allowable bending moment $(3.14 \mathrm{kN}-\mathrm{m})$ is greater than that for the stud wall specimens $(2.97 \mathrm{kN}-\mathrm{m})$ by $6 \%$.

With respect to deflection acceptance criteria, Table 4.5 summarizes the total flexural load applied to the panels at the deflection limits of $\mathrm{L} / 360$ and L/180 for the SIP and stud wall groups. It can be observed that the flexural load of SIP panels with one end plate at L/360 deflection limit $(11.39 \mathrm{~mm})$ is greater than that for stud wall specimen $(5.23 \mathrm{~mm})$ by $214 \%$. Also, it can be observed that the flexural load of SIP panels with one end plate at L/180 deflection limit (19.83 $\mathrm{mm})$ is greater than that for stud wall specimen $(9.92 \mathrm{~mm})$ by $91 \%$. Table 4.5 reports the deflection values at L/360 deflection limits of 11.39 and $10.83 \mathrm{~mm}$ for SIPs with one and two end plates, respectively. Also, Table 4.5 reports the deflection values at L/180 deflection limits of 19.83 and $18.95 \mathrm{~mm}$ for SIPs with one and two end plates, respectively. This leads to the conclusion that using one or two end plates in SIP panels has insignificant effect on the deflection values at the deflection limits.

Based on the above-mentioned comparison, SIPs are being "as good as" the conventional stud wood-framing with respect to characteristic ultimate resisting moment irrespective of whether one or two plates exist at panel end. Per AC04 design criteria, SIPs with two bottom plates and two rows of nails are being "as good as" the stud wall system. However, SIPs with one bottom plate and one row of nails underestimates the stud wall capacity by $4 \%$. The flexural load at L/360 deflection on SIP panel with one or two end plates exceeds the loads on the conventional stud panel at $\mathrm{L} / 360$ deflection. 


\subsection{Stud Wall under Lateral Loading}

\subsubsection{Racking Load}

Two identical stud walls, namely SW-R-1, and SW-R-2, were selected for racking load test. The wall was of $2450 \mathrm{~mm}$ length, $2750 \mathrm{~mm}$ height and $165 \mathrm{~mm}$ total thickness, with 2x6 studs every $600 \mathrm{~mm}$. The wall has sheathing made of OSB sheets from one side and drywall from the other side. The wall was placed over a W200 I-beam that rest on the laboratory floor. The bottom plates of the wall were connected to the I-beam using eight lag bolts in every $304.8 \mathrm{~mm}(1 \mathrm{ft})$ in order to provide a fully fixed support to the wall base. Tie-down system was used to hold the wall in position while applying the racking load at the top of the wall using an actuator as depicted in Fig. 4.74.

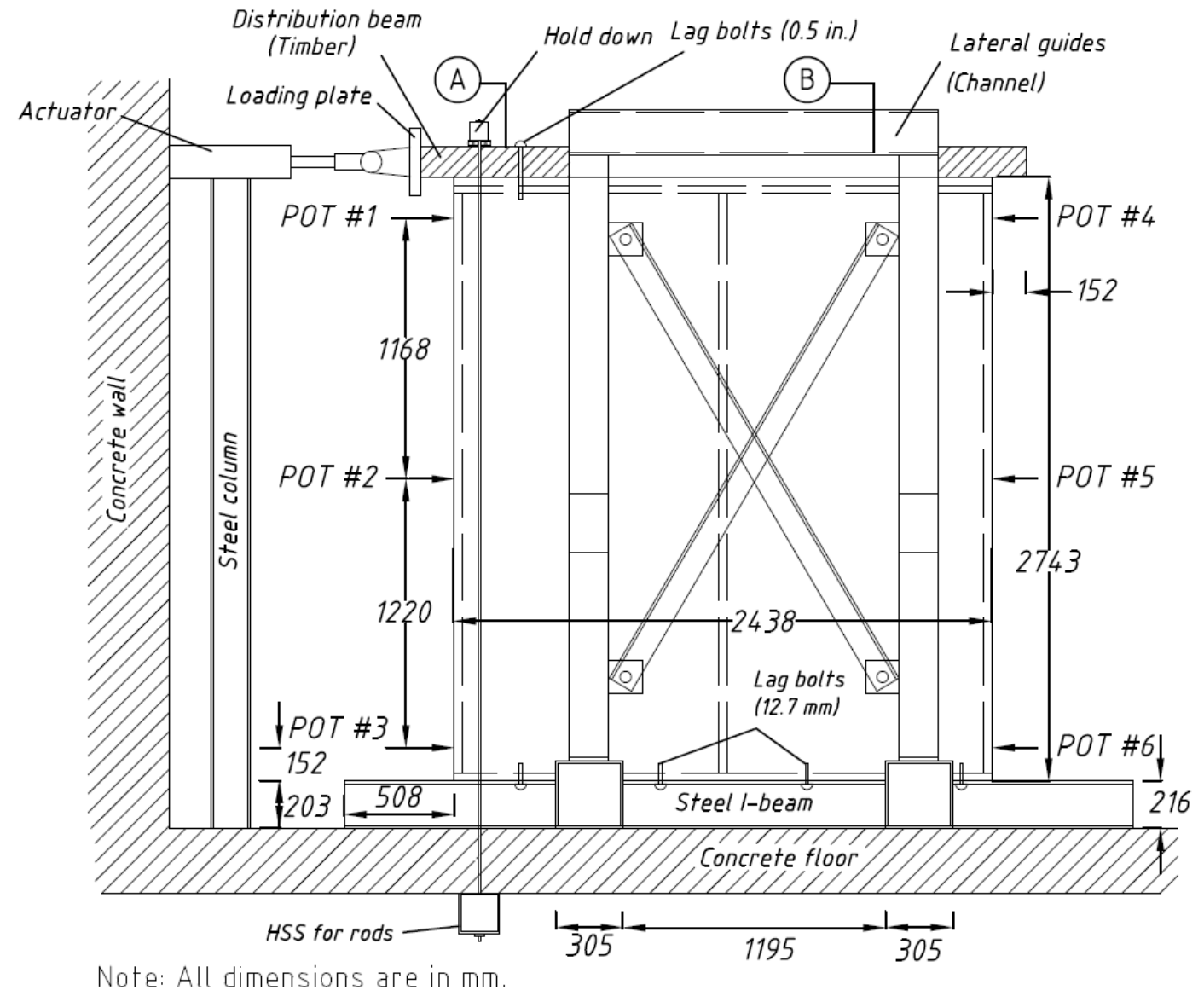

Figure 4.74 - Test setup of specimen SW-R-1 under racking load

The test started with applying load up to $3.5 \mathrm{kN}$, followed by releasing the load, after 5 minute of releasing the load, the jacking load is applied up to $7.5 \mathrm{kN}$ and releasing to zero level. After 5 
minute of releasing the load, the jacking load is applied up to $10 \mathrm{kN}$, then releasing the load and applied the ultimate load up to failure of specimen. Figure 4.75 shows the general failure mode of wall SW-R-1 which was shear deformation and sheathing separation from the studs due to nail slip.

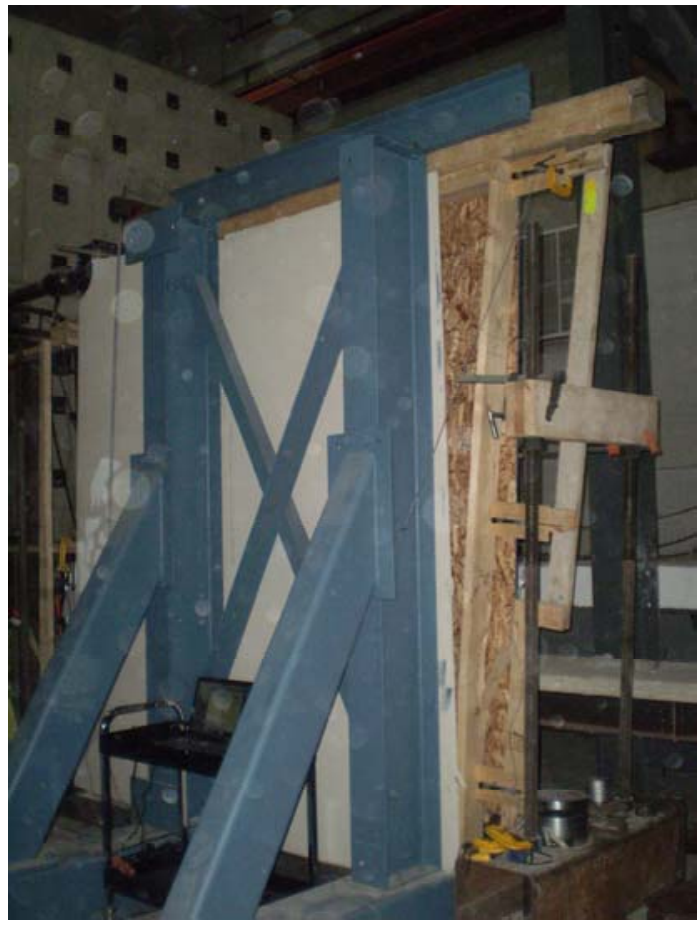

(a) Large shear deformation on the right side of the wall

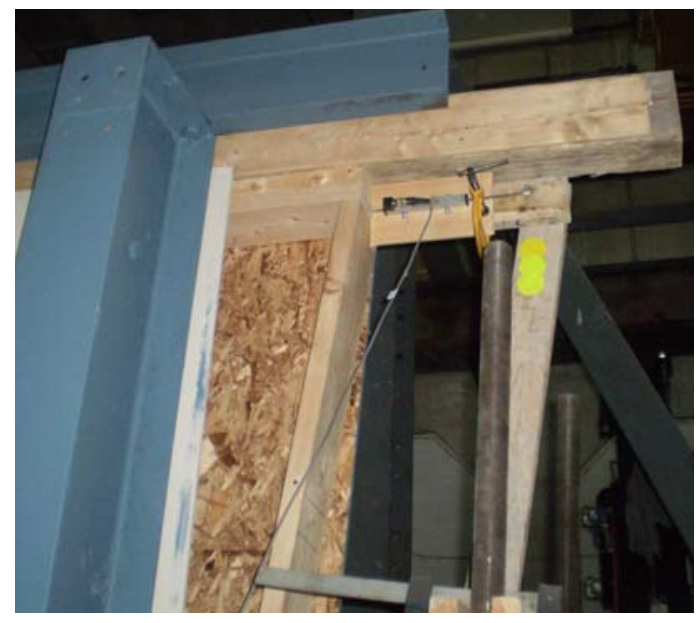

(b) Close-up view of the separation of OSB and drywall from the stud at top of wall

Figure 4.75 - Global failure of specimen SW-R-1 under racking load showing

The racking load was applied to the top left side of the wall with increasing load to failure. While approaching failure, shear deformation was observed through the individual rotations of the OSB sheets against each other as depicted in Fig. 4.76(c). Also, nail slip was observed and separation of the dry wall and OSB sheets from the studs as depicted in Figs. 4.76(a) and 4.76(b), respectively. Damage of the gypsum board was observed at the top of the wall on the back side of the load location as shown in Fig. 4.76(a). Separation and crushing of OSB sheet at spline connection and movement of OSB sheet in horizontal direction was observed at failure as depicted in Fig. 4.76(d). No wall uplift was observed at failure which means that the provided anchorage was adequate. Similar failure mode was observed in wall SW-R-2. Figures 4.77 and 
4.78 depict the load vs. lateral deflection relationships for walls SW-R-1 and SW-R-2, respectively. It can be observed that the wall behaved elastically along a significant portion of the failure load. The failure loads for walls SW-R-1 and SW-R-2 were 13.70 and $9.74 \mathrm{kN}$, respectively. The maximum load reached at approximately $50 \mathrm{~mm}$ for the two walls. Figures 4.79 and 4.80 show the change in wall lateral deflection with increase in the racking loading at different POT locations shown in Fig. 4.74.

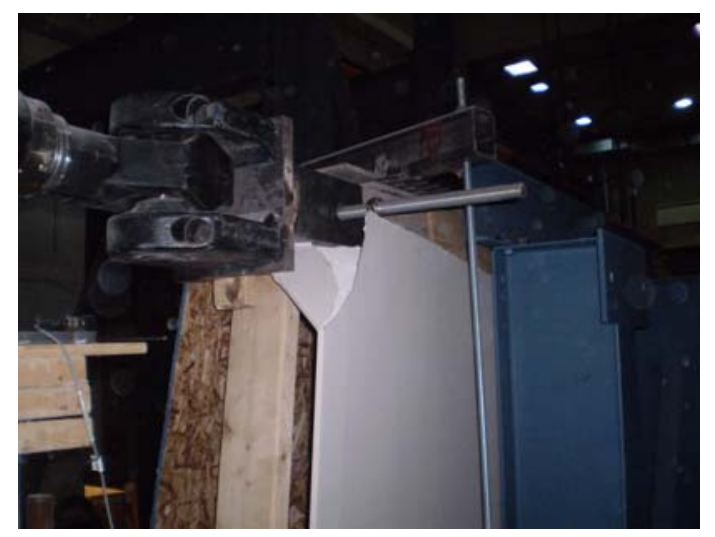

(a) Failure of gypsum board on top

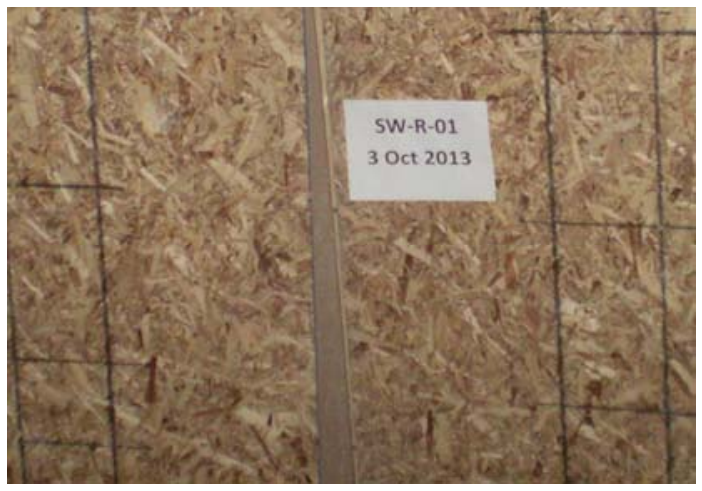

(c) Separation and crushing of OSB sheet at spline connection

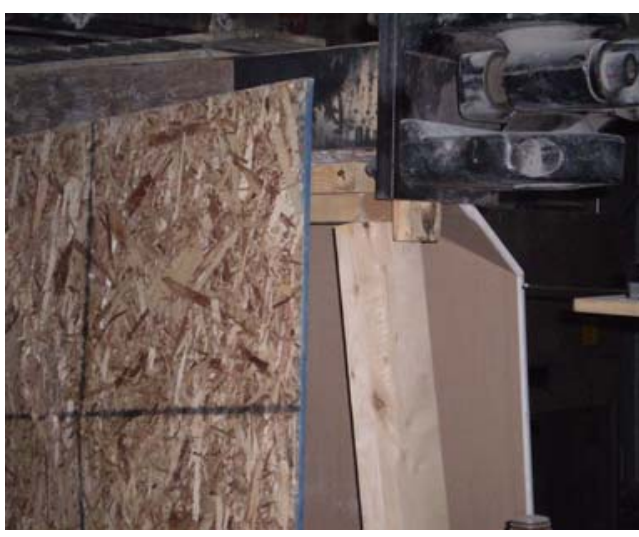

(b) Separation of OSB sheet from stud frame

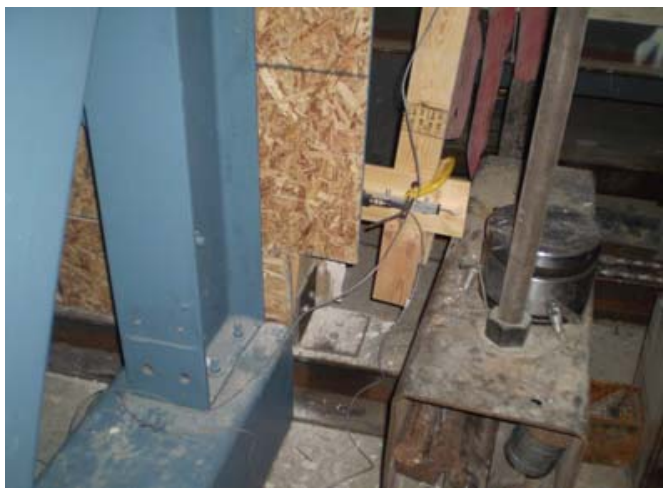

(d) Movement of OSB sheet in horizontal direction

Figure 4.76 - Detailed failure modes of specimen SW-R-1 under racking loading 


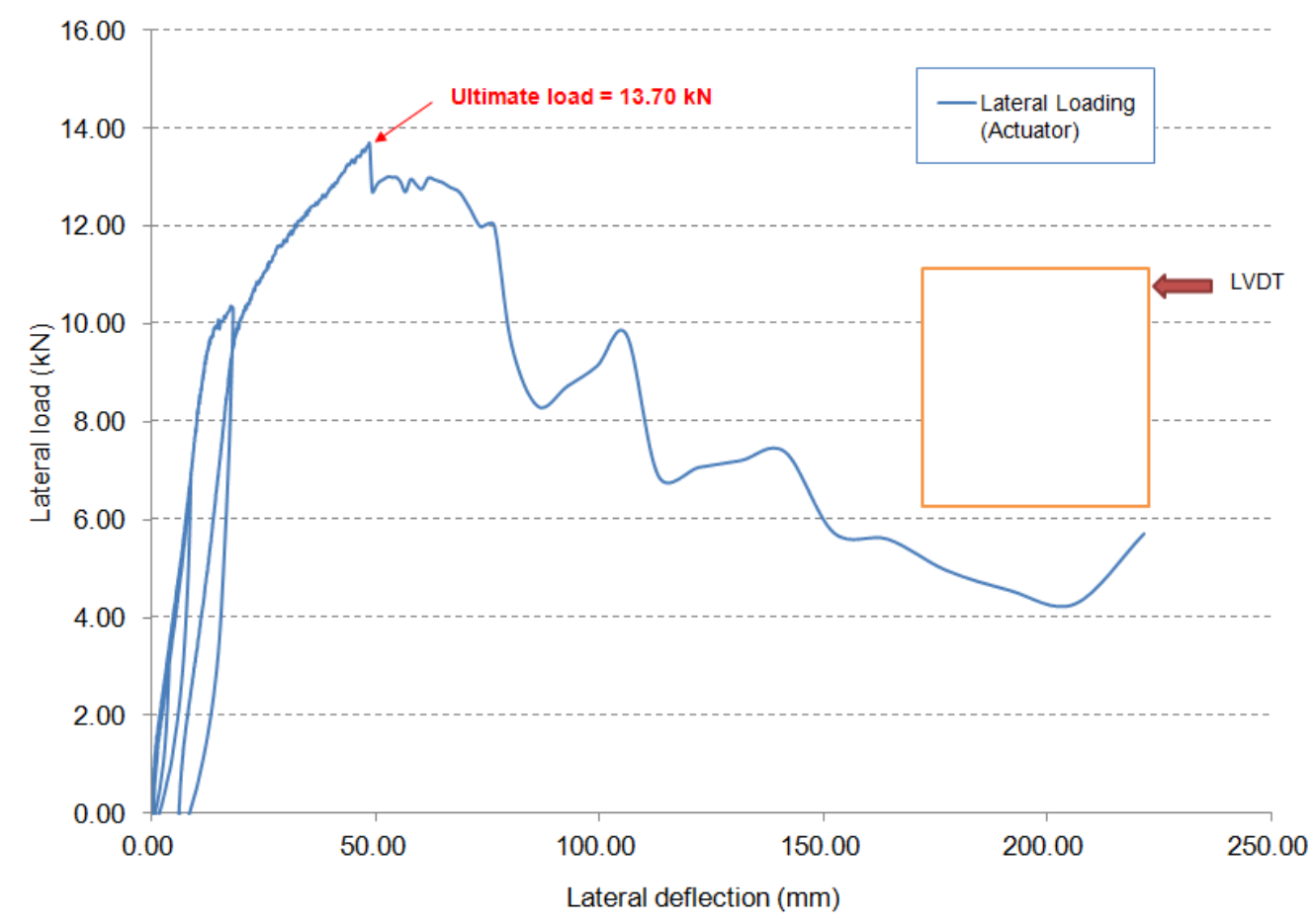

Figure 4.77 - Load vs. lateral deflection response of SW-R-1 (obtained from actuator reading on top of the panel)

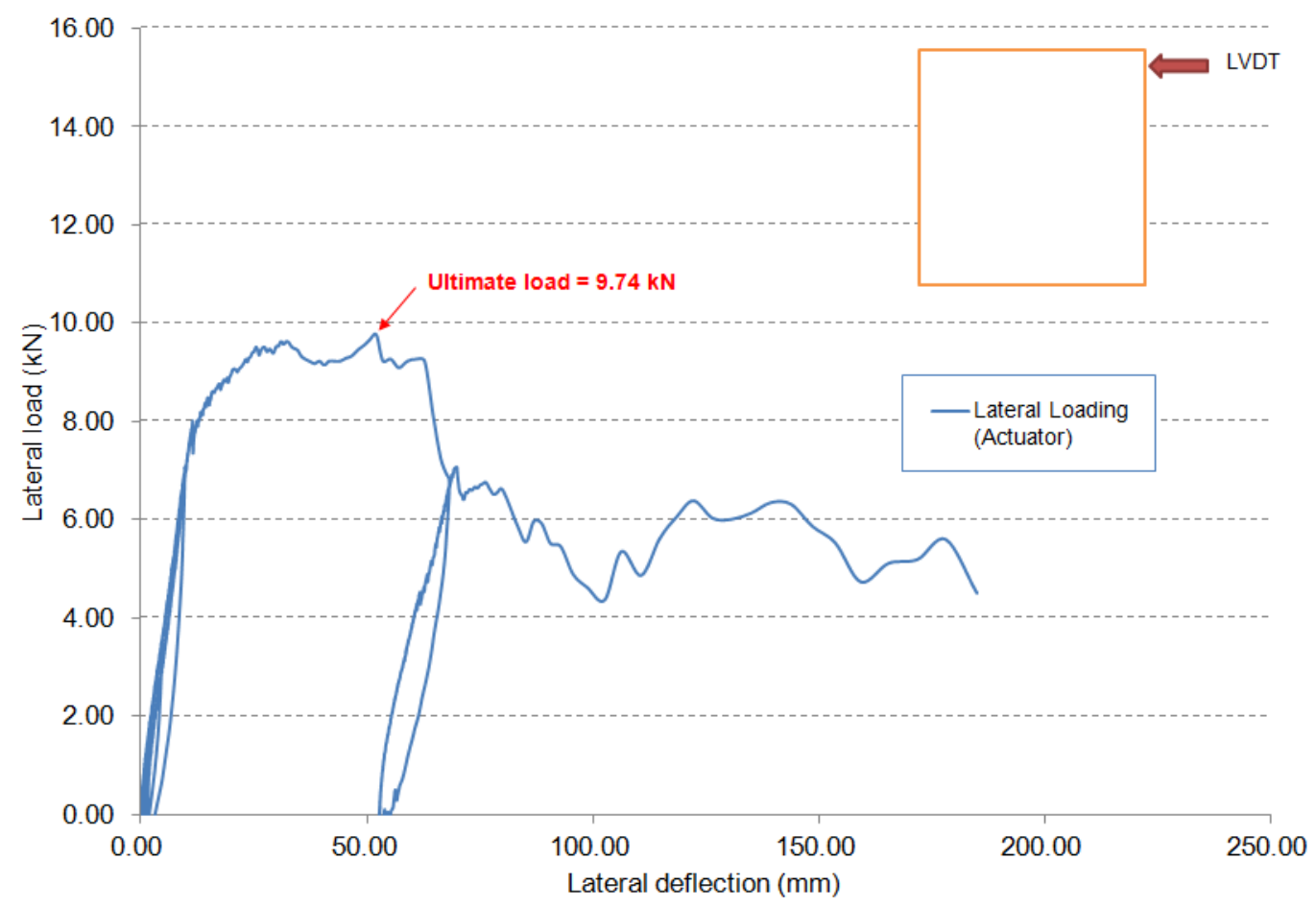

Figure 4.78 - Load vs. lateral deflection response of SW-R-2 (obtained from actuator reading on top of the panel) 


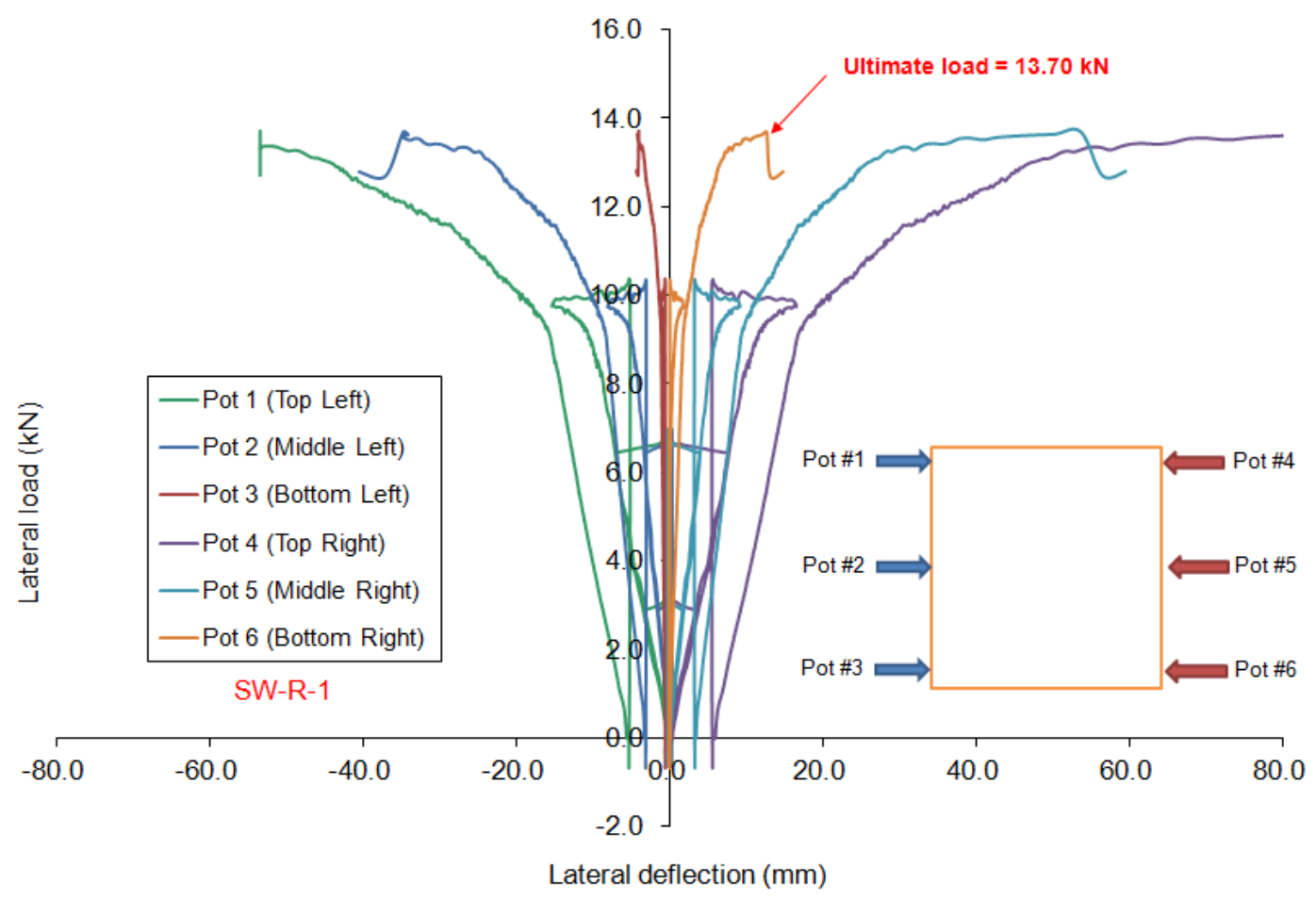

Figure 4.79 - Load vs. lateral deflection response of SW-R-1 (POTs records up to a load close to failure load)

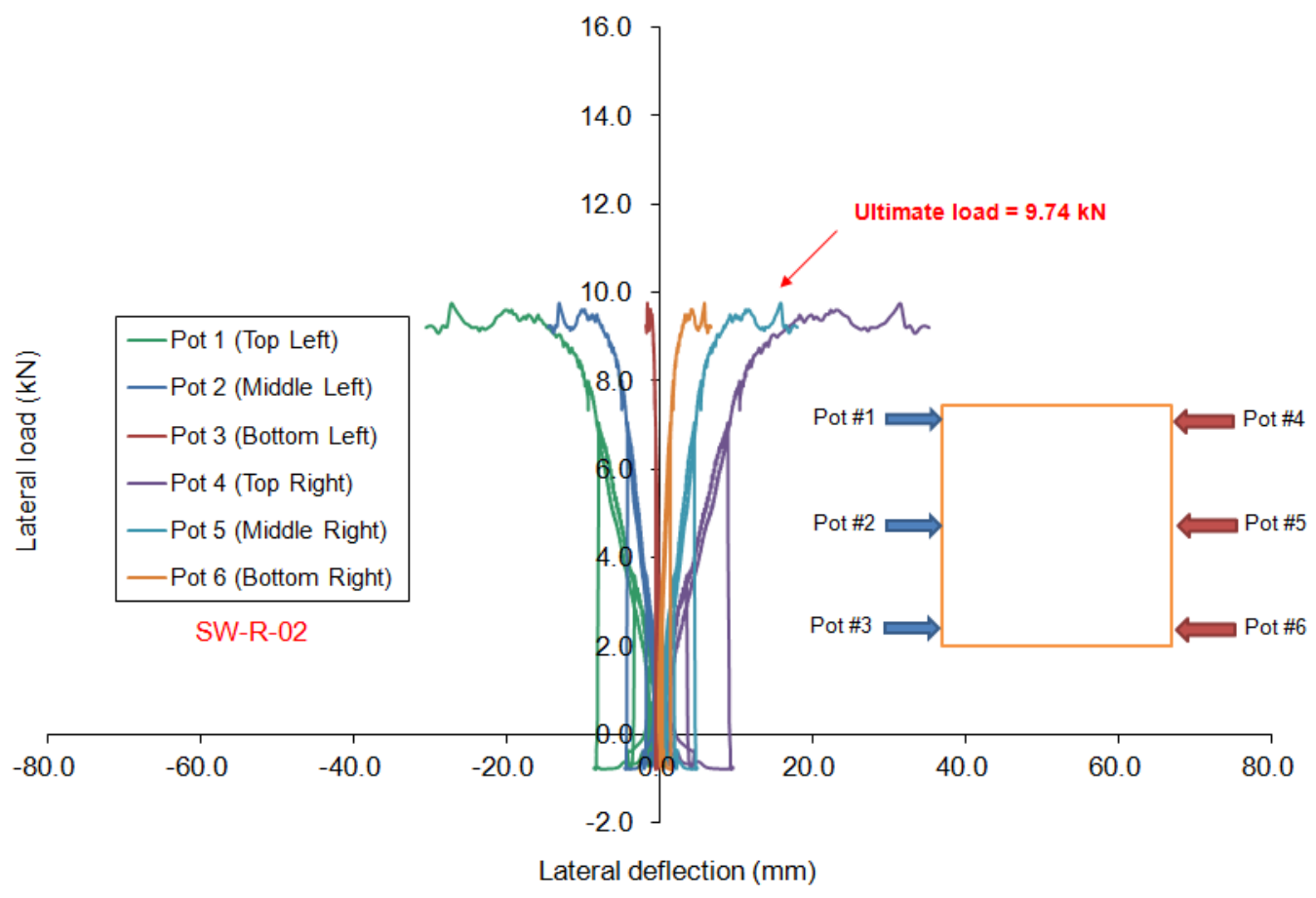

Figure 4.80 - Load vs. lateral deflection response of SW-R-2 (POTs records up to a load close to failure load) 


\subsubsection{Cyclic Loading}

Two identical stud walls, namely SW-C-1, and SW-C-2, were selected for cyclic load test. The wall was of $2450 \mathrm{~mm}$ length, $2750 \mathrm{~mm}$ height and $165 \mathrm{~mm}$ total thickness, with $2 \times 6$ studs every $600 \mathrm{~mm}$. The wall has sheathing made of OSB sheets from one side and drywall from the other side. The wall was placed over a W200 I-beam that rest on the laboratory floor. The bottom plates of the wall were connected to the I-beam using eight lag bolts in every $304.8 \mathrm{~mm}(1 \mathrm{ft})$ in order to provide a fully fixed support to the wall base. Tie-down system was used to hold the wall in position while applying the cyclic load at the top of the wall using an actuator as depicted in Fig. 4.81. The CUREE protocol for displacement control explained in Fig. 3.34 was used in this cyclic load test. Figure 4.82 shows the general failure mode of wall SW-C-1 which was global shear deformation of the wall and drywall separation from the studs due to nail slip and bearing failure of the drywall at nail location.

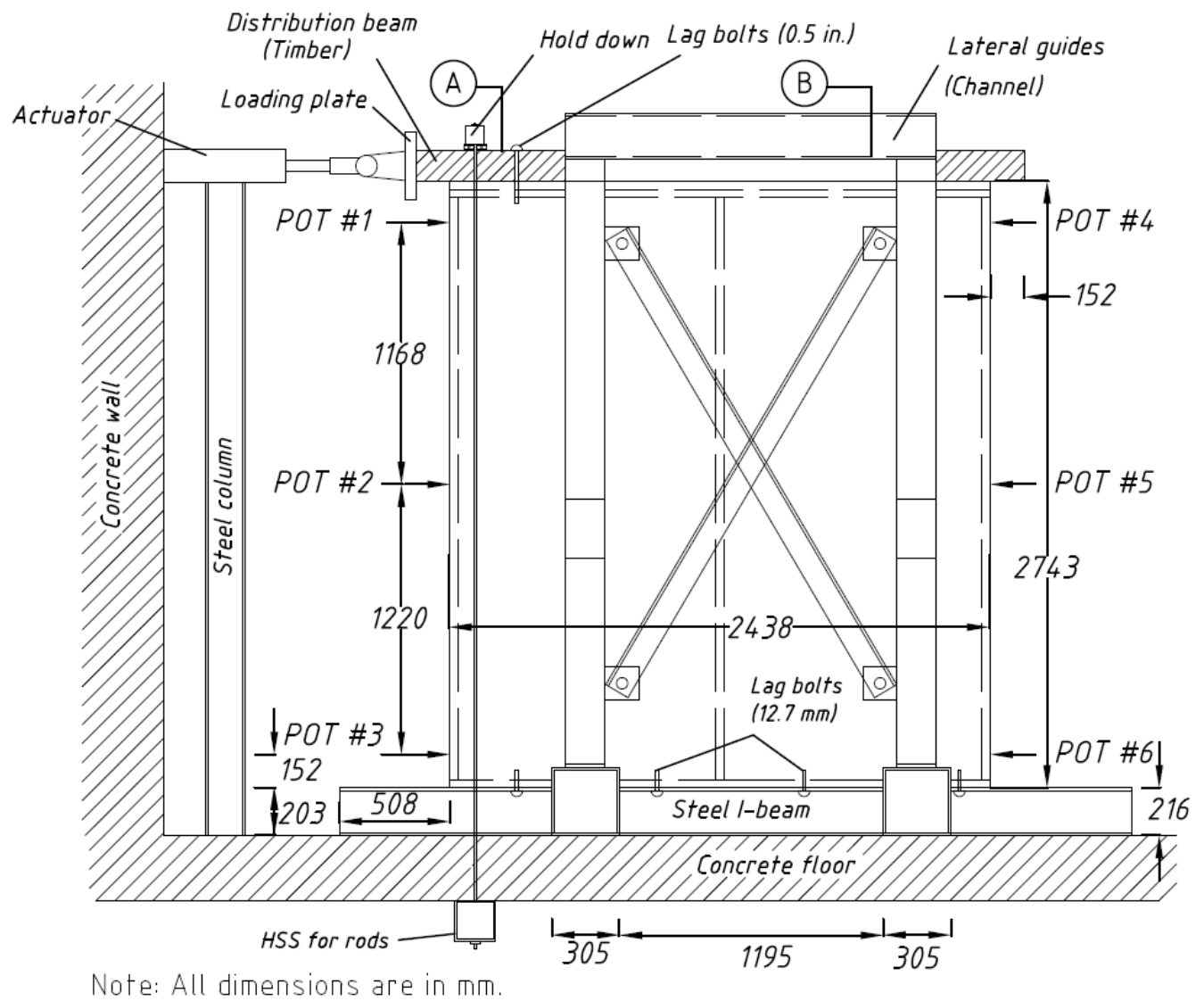

Figure 4.81 - Test setup of specimen SW-C-1 under cyclic loading 


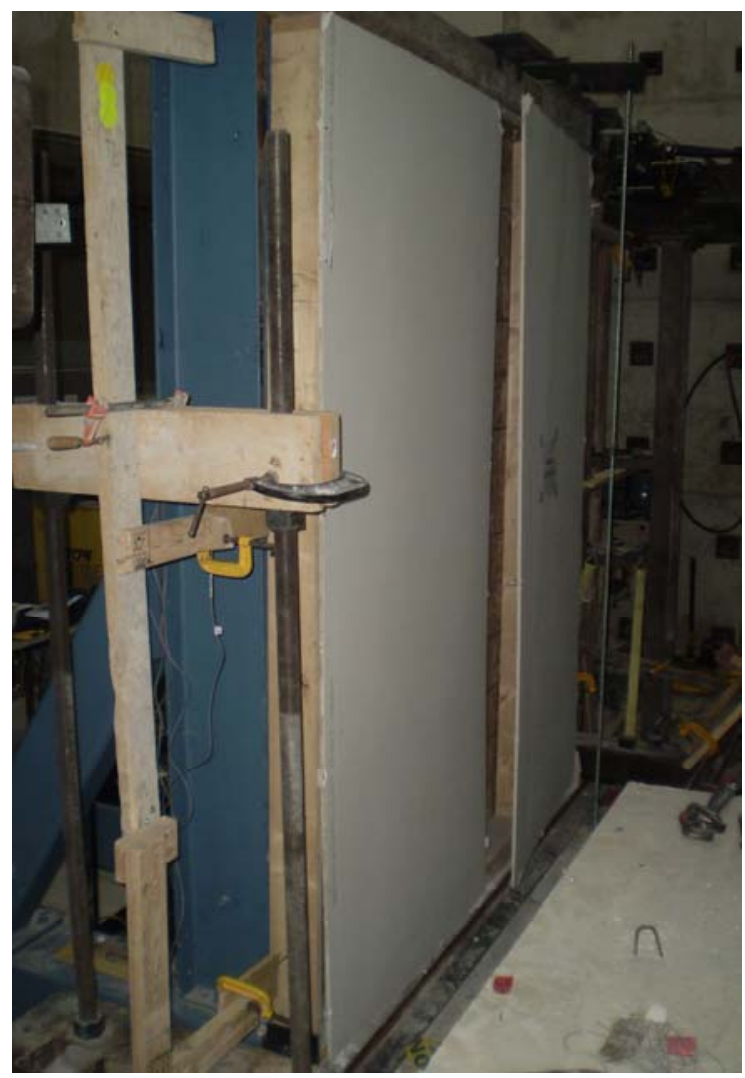

(a) Separation of dry wall

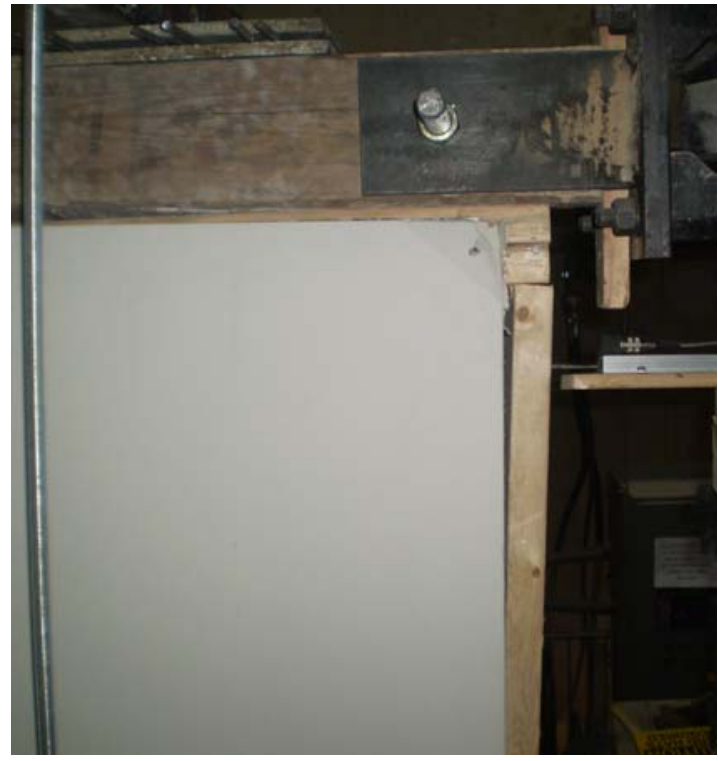

(c) Shear deformation of the wall at the

$$
\text { drywall side }
$$

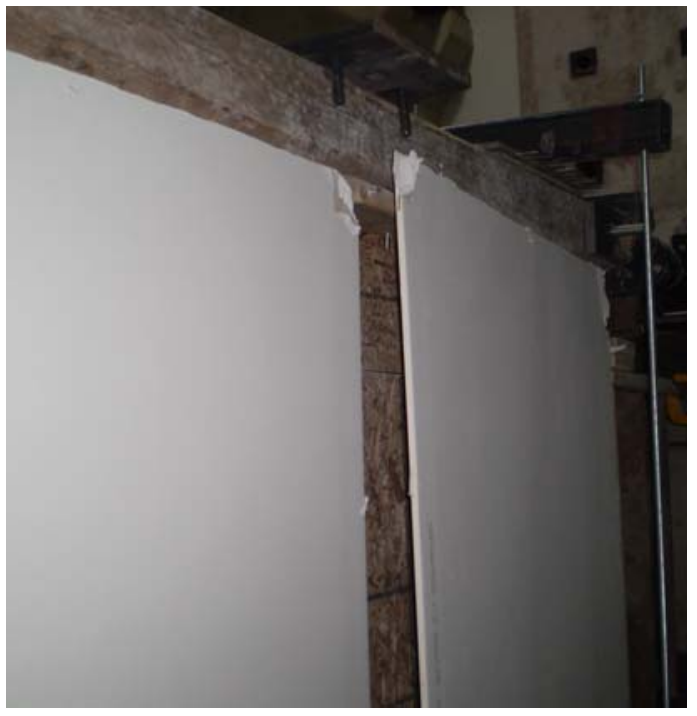

(b) Separation of dry wall from the stud

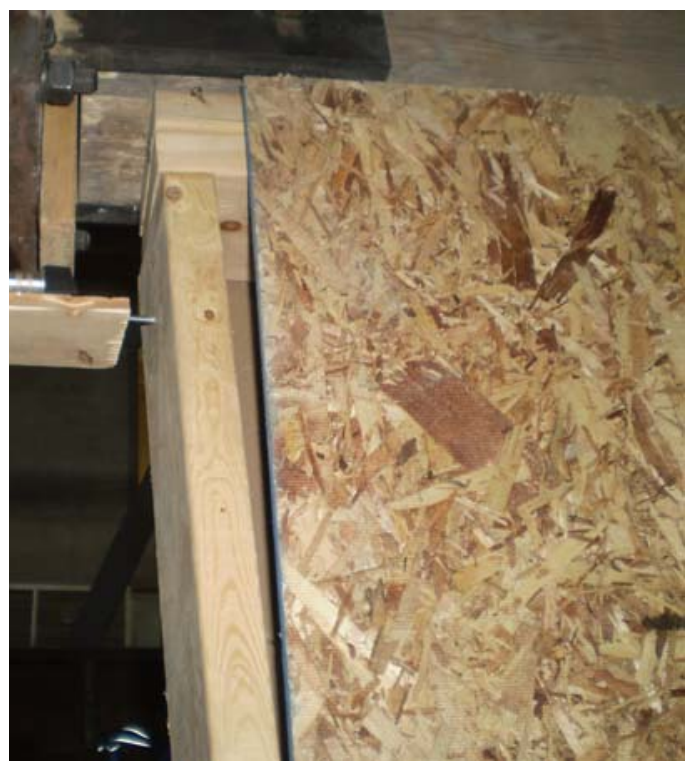

(d) Shear deformation of the wall at the OSB side

Figure 4.82 - Failure of specimen SW-C-1 under cyclic loading 
The cyclic load was applied to the top left side of the wall with applied increasing displacement to failure. While approaching failure, shear deformation was observed through the rotations of the studs as depicted in Figs. 4.82(c) and 4.82(d). Figure 4.83(a) shows view of the OSB sheet separation at the joint, while Fig. 4.83(b) shows view of the crushing of gypsum board at nail location. Figure 4.83(c) shows view of the separation of OSB sheet and gypsum board from at the stud location, while Fig. 4.83(d) shows view of bending of the nail and OSB crushing. No wall uplift was observed at failure which means that the provided anchorage was adequate. Similar failure mode was observed in wall SW-R-2.

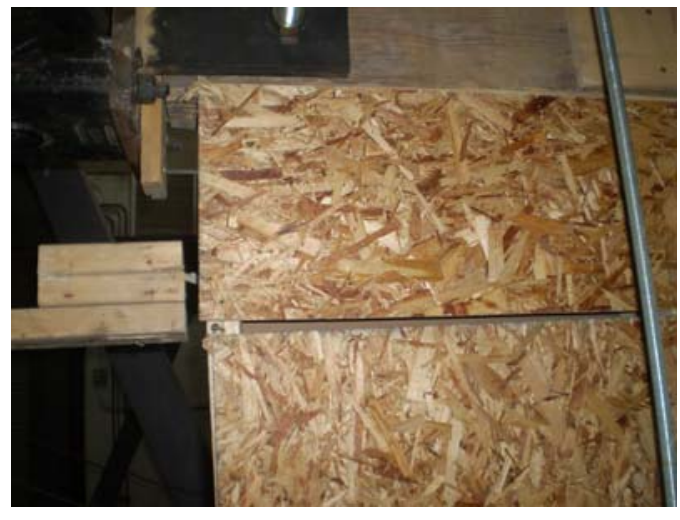

(a) Separation of OSB sheet on joint

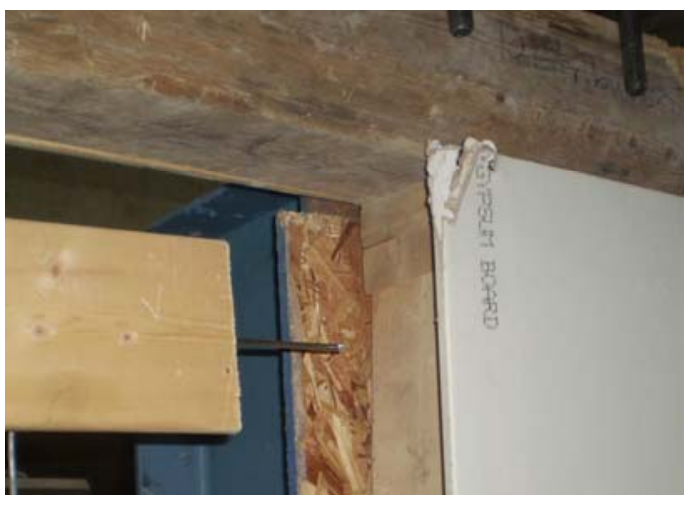

(c) Separation of OSB sheet and gypsum board from stud frame at top end

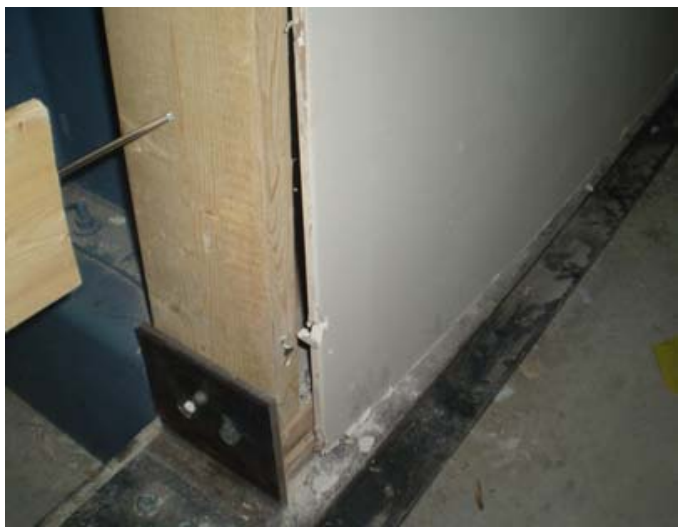

(b) Crushing of gypsum board in nail location

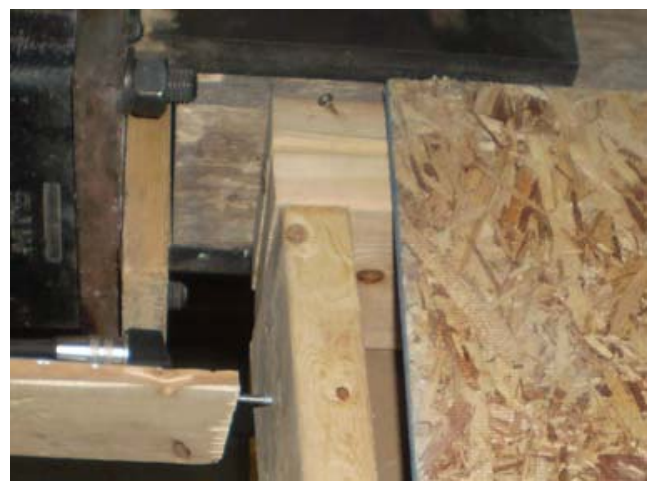

(d) Bending of the nail and OSB crushing in nail connection

Figure 4.83 - Detailed failure modes of specimen SW-C-1 under cyclic loading 
Figures 4.84 and 4.85 depict the load vs. lateral deflection responses for walls SW-R-1 and SWR-2, respectively. It can be observed that the failure loads for walls SW-R-1 and SW-R-2 were 12.30 and $14.42 \mathrm{kN}$, respectively. Figures 4.86 and 4.87 show the change in wall lateral deflection with increase in the cyclic loading at different POT locations shown in Fig. 4.74. Table 4.6 summarizes the failure loads for each wall due to racking and cyclic loads.

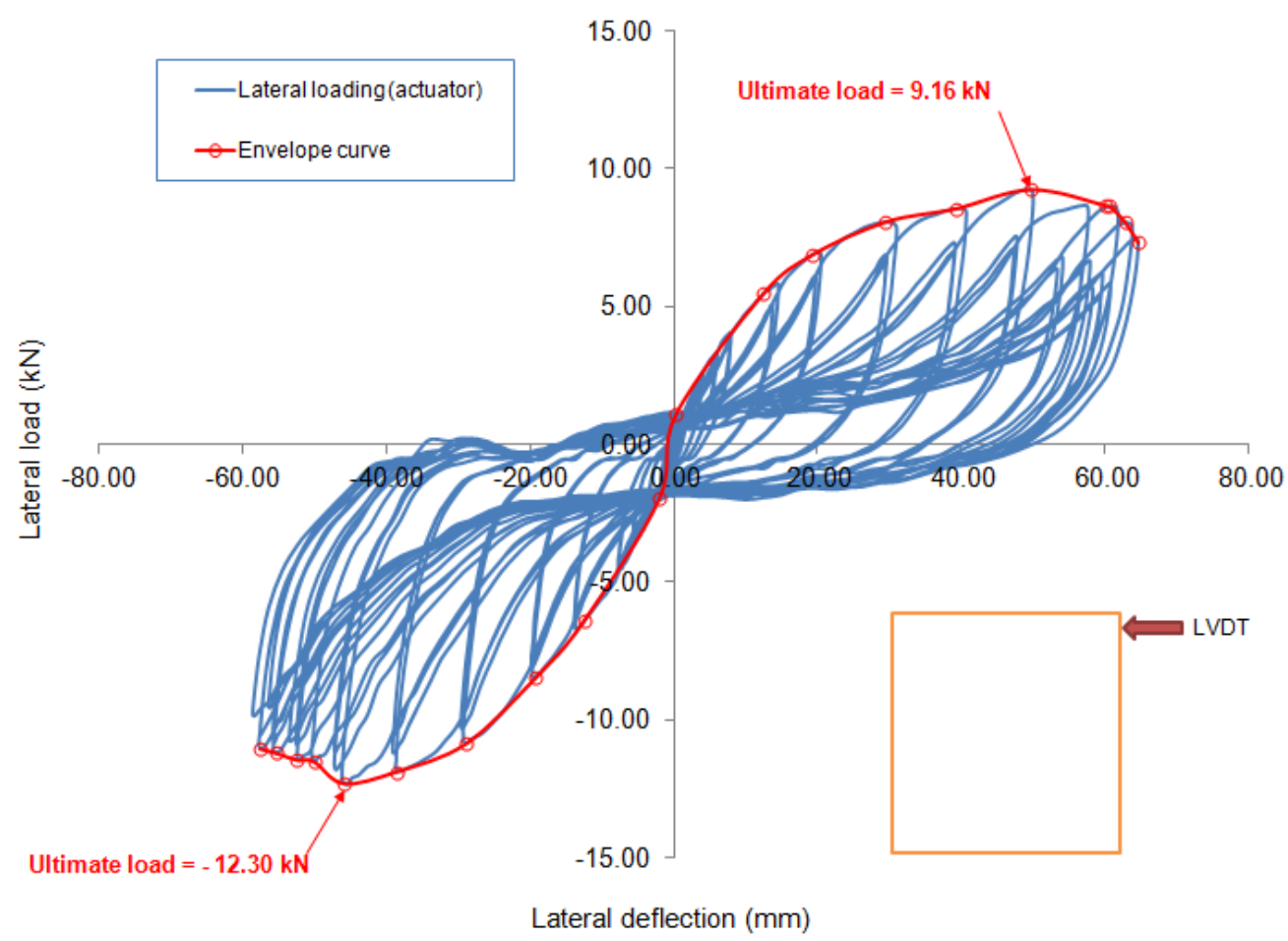

Figure 4.84 - Load vs. lateral deflection response of SW-C-1 (obtained from actuator reading on top of the panel) 


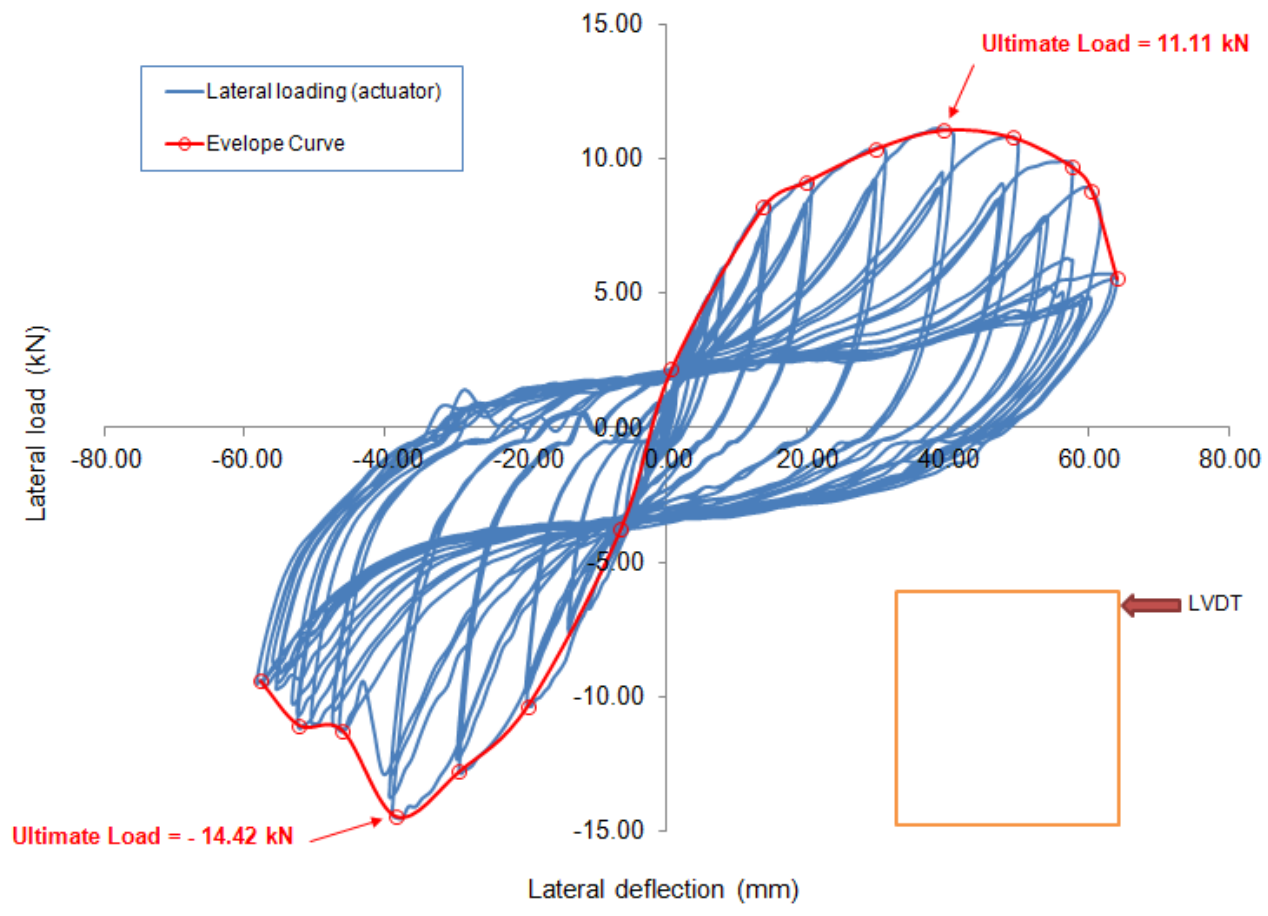

Figure 4.85 - Load vs. lateral deflection response of SW-C-2 (obtained from actuator reading on top of the panel)

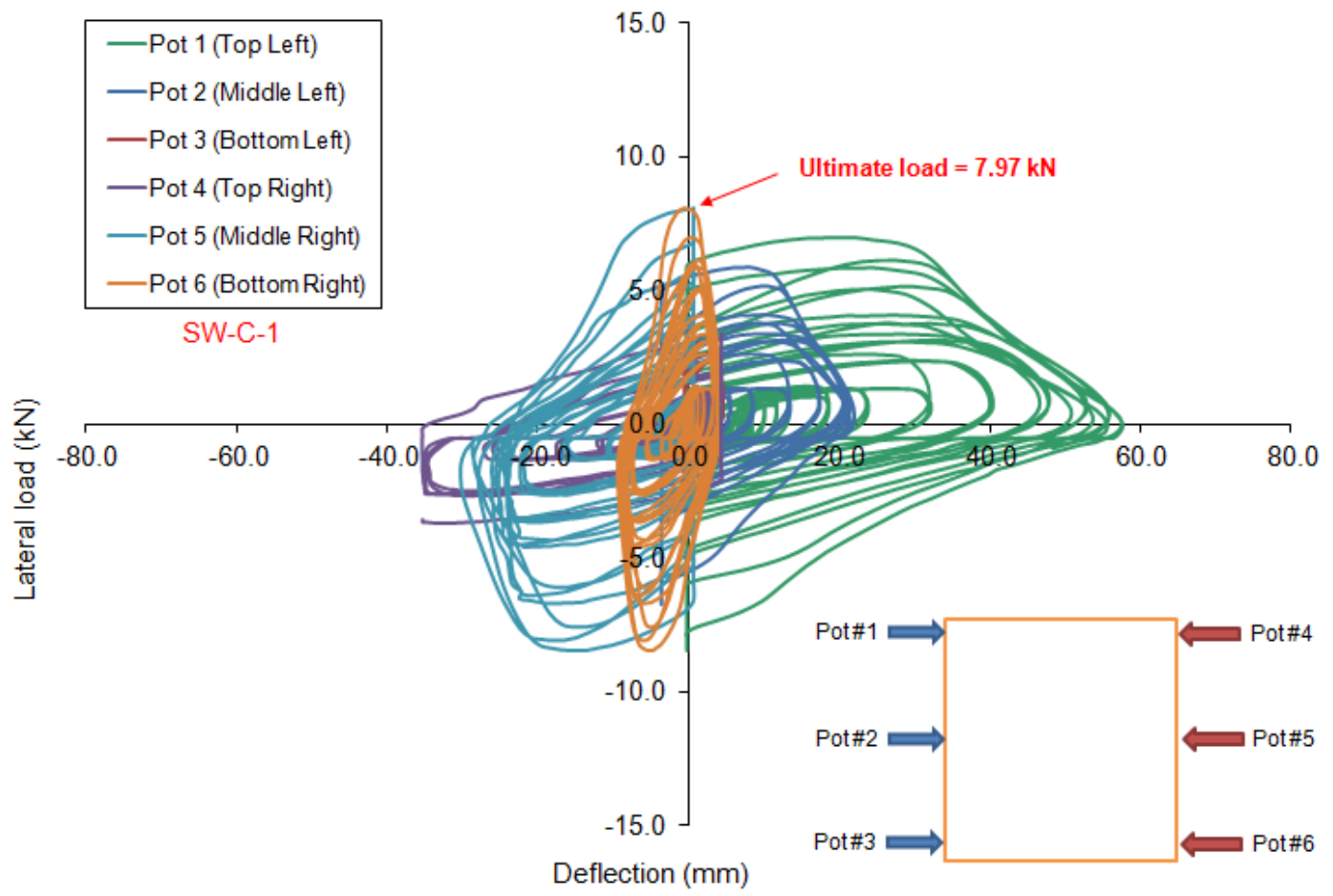

Figure 4.86 - Load vs. lateral deflection response of SW-C-1 (POTs records up to a load close to failure load) 


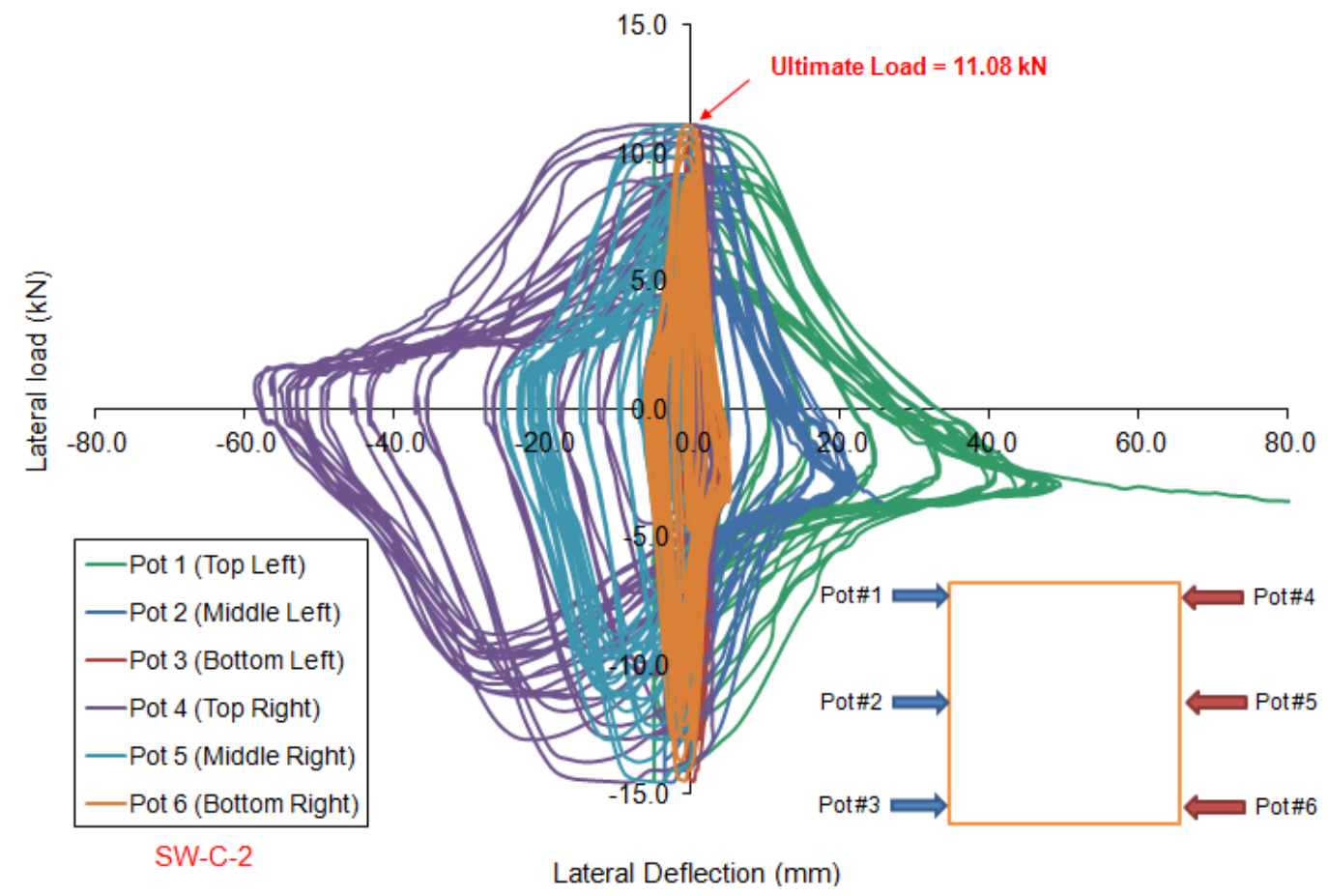

Figure 4.87 - Load vs. lateral deflection response of SW-C-2 (POTs records up to a load close to failure load) 
Table 4.6 - Test results for racking and cyclic tests

\begin{tabular}{|c|c|c|c|c|c|c|}
\hline \multicolumn{7}{|c|}{ Racking load test results } \\
\hline $\begin{array}{l}\text { Test } \\
\text { No. }\end{array}$ & $\begin{array}{l}\text { Test } \\
\text { type }\end{array}$ & \multicolumn{2}{|c|}{$\begin{array}{c}\text { Panel Size } \\
\text { (length } \times \text { width } \times \text { thickness }) \text {, } \\
\text { mm }\end{array}$} & $\begin{array}{l}\text { Specimen } \\
\text { type }\end{array}$ & $\begin{array}{c}\text { Ultimate } \\
\text { racking } \\
\text { load } \\
\text { (kN) }\end{array}$ & $\begin{array}{l}\text { Average } \\
\text { ultimate } \\
\text { racking } \\
\text { load }(k N)\end{array}$ \\
\hline SW-R-1 & Racking & \multicolumn{2}{|c|}{$2450 \times 2750 \times 165 \mathrm{~mm}$} & stud panel & 13.70 & \multirow{2}{*}{11.72} \\
\hline SW-R-2 & Racking & \multicolumn{2}{|c|}{$2450 \times 2750 \times 165 \mathrm{~mm}$} & stud panel & 9.74 & \\
\hline SIP-R-1 & Racking & \multicolumn{2}{|c|}{$2450 \times 2750 \times 165 \mathrm{~mm}$} & SIP & 25.32 & \multirow{3}{*}{26.21} \\
\hline SIP-R-2 & Racking & \multicolumn{2}{|c|}{$2450 \times 2750 \times 165 \mathrm{~mm}$} & SIP & 30.87 & \\
\hline SIP-R-3 & Racking & \multicolumn{2}{|c|}{$2450 \times 2750 \times 165 \mathrm{~mm}$} & SIP & 22.45 & \\
\hline SIP-R-4 & Racking & \multicolumn{2}{|c|}{$3660 \times 2750 \times 165 \mathrm{~mm}$} & SIP & 40.86 & \multirow{3}{*}{40.14} \\
\hline SIP-R-5 & Racking & \multicolumn{2}{|c|}{$3660 \times 2750 \times 165 \mathrm{~mm}$} & SIP & 38.94 & \\
\hline SIP-R-6 & Racking & \multicolumn{2}{|c|}{$3660 \times 2750 \times 165 \mathrm{~mm}$} & SIP & 40.64 & \\
\hline \multicolumn{7}{|c|}{ Cyclic load test results } \\
\hline $\begin{array}{l}\text { Test } \\
\text { No. }\end{array}$ & $\begin{array}{l}\text { Test } \\
\text { Type }\end{array}$ & $\begin{array}{c}\text { Panel Size } \\
\text { (Length } \times \text { Width } \times \\
\text { Thickness), } \\
\text { mm }\end{array}$ & $\begin{array}{c}\text { Specimen } \\
\text { type }\end{array}$ & \multicolumn{2}{|c|}{$\begin{array}{l}\text { Ultimate cyclic load } \\
\qquad(\mathrm{kN})\end{array}$} & $\begin{array}{c}\text { Average } \\
\text { ultimate } \\
\text { cyclic } \\
\text { load } \\
(k N)\end{array}$ \\
\hline SW-C-1 & Cyclic & $2450 \times 2750 \times 165 \mathrm{~mm}$ & stud panel & \multicolumn{2}{|c|}{$+9.16 /-12.30$} & \multirow{2}{*}{11.75} \\
\hline SW-C-2 & Cyclic & $2450 \times 2750 \times 165 \mathrm{~mm}$ & stud panel & \multicolumn{2}{|c|}{$+11.11 /-14.42$} & \\
\hline SIP-C-1 & Cyclic & $2450 \times 2750 \times 165 \mathrm{~mm}$ & SIP & \multicolumn{2}{|c|}{$+18.52 /-40.03$} & \multirow{3}{*}{29.31} \\
\hline SIP-C-2 & Cyclic & $2450 \times 2750 \times 165 \mathrm{~mm}$ & SIP & \multicolumn{2}{|c|}{$+22.14 /-33.40$} & \\
\hline SIP-C-3 & Cyclic & $2450 \times 2750 \times 165 \mathrm{~mm}$ & SIP & \multicolumn{2}{|c|}{$+22.40 /-39.37$} & \\
\hline SIP-C-4 & Cyclic & $3660 \times 2750 \times 165 \mathrm{~mm}$ & SIP & \multicolumn{2}{|c|}{$+32.01 /-25.40$} & \multirow{3}{*}{30.16} \\
\hline SIP-C-5 & Cyclic & $3660 \times 2750 \times 165 \mathrm{~mm}$ & SIP & \multicolumn{2}{|c|}{$+27.57 /-28.82$} & \\
\hline SIP-C-6 & Cyclic & $3660 \times 2750 \times 165 \mathrm{~mm}$ & SIP & \multicolumn{2}{|c|}{$+30.79 /-36.37$} & \\
\hline
\end{tabular}




\subsection{SIPs under Lateral Loading}

\subsubsection{Racking Load}

Three identical SIP walls, namely SIP-R-1, SIP-R-2 and SIP-R-3, were selected for racking load test. The wall was of $2450 \mathrm{~mm}$ length, $2750 \mathrm{~mm}$ height and $165 \mathrm{~mm}$ total thickness, with two SIP panels connected together using foam-spline connection. The wall has sheathing made of OSB sheets on each side of the wall. The wall was placed over a W200 I-beam that rest on the laboratory floor. The bottom plates of the wall were connected to the I-beam using eight lag bolts in every $304.8 \mathrm{~mm}(1 \mathrm{ft})$ in order to provide a fully fixed support to the wall base. Tie-down system was used to hold the wall in position while applying the racking load at the top of the wall using an actuator as depicted in Fig. 4.88. The test started with applying load up to $3.5 \mathrm{kN}$, followed by releasing the load, after 5 minute of releasing the load, the jacking load is applied up to $7.5 \mathrm{kN}$ and releasing to zero level. After 5 minute of releasing the load, the jacking load is applied up to $10 \mathrm{kN}$, then releasing the load and applied the ultimate load up to failure of specimen. Figure 4.89 shows the general failure mode of wall SIP-R-1 which was global shear deformation and relative shear rotation of SIP segments at spline connection.

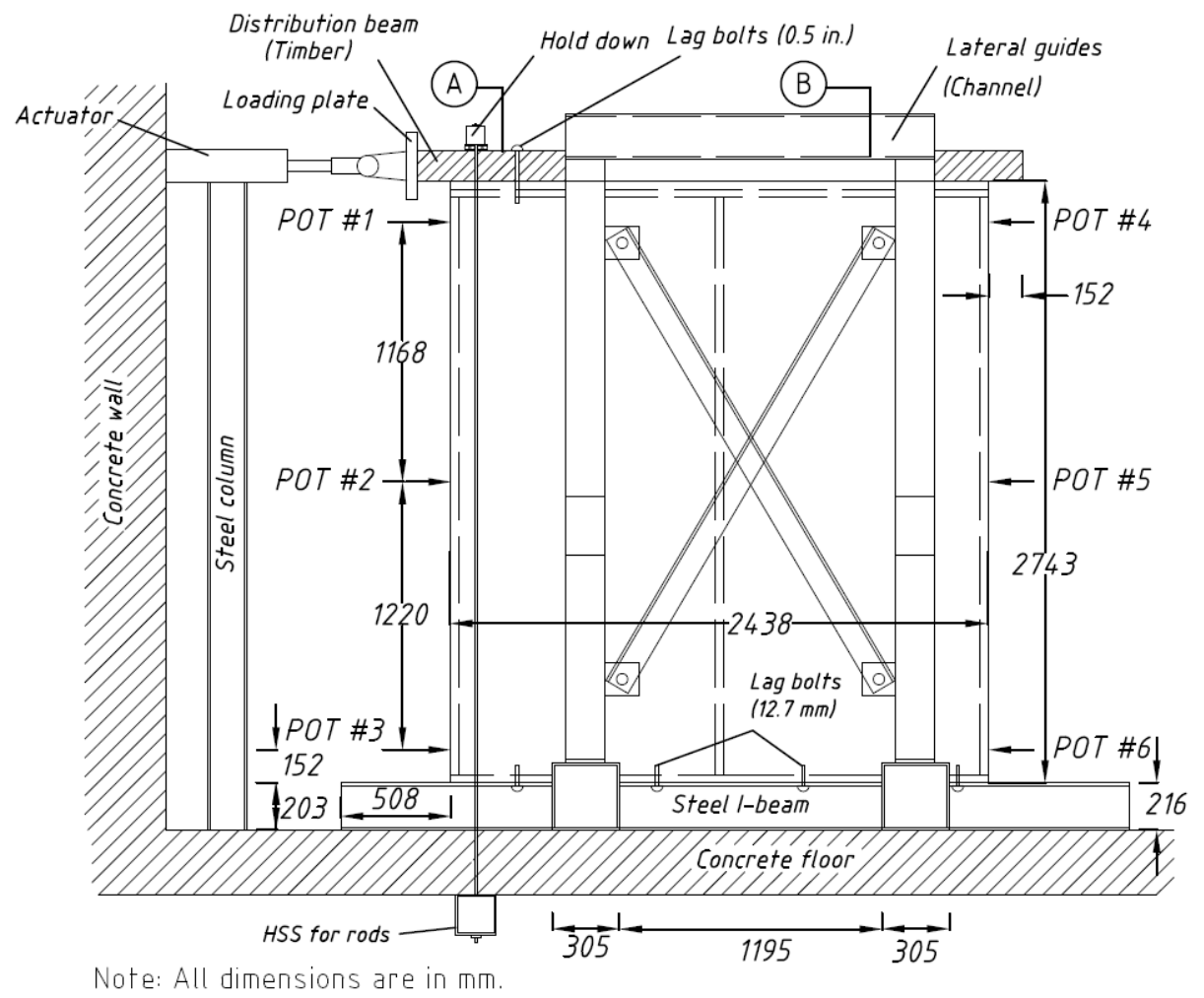

Figure 4.88 - Test setup of specimen SIP-R-1 under racking load 


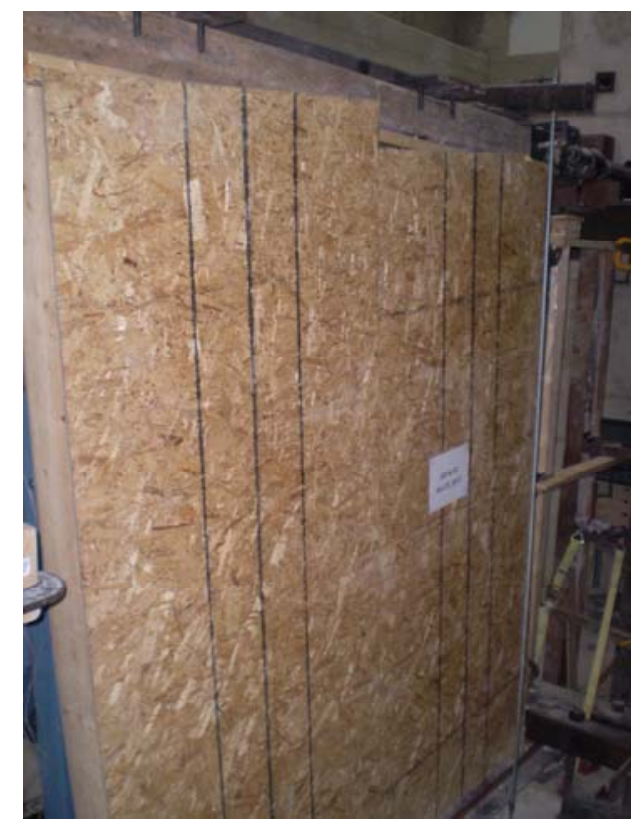

(a) Front side

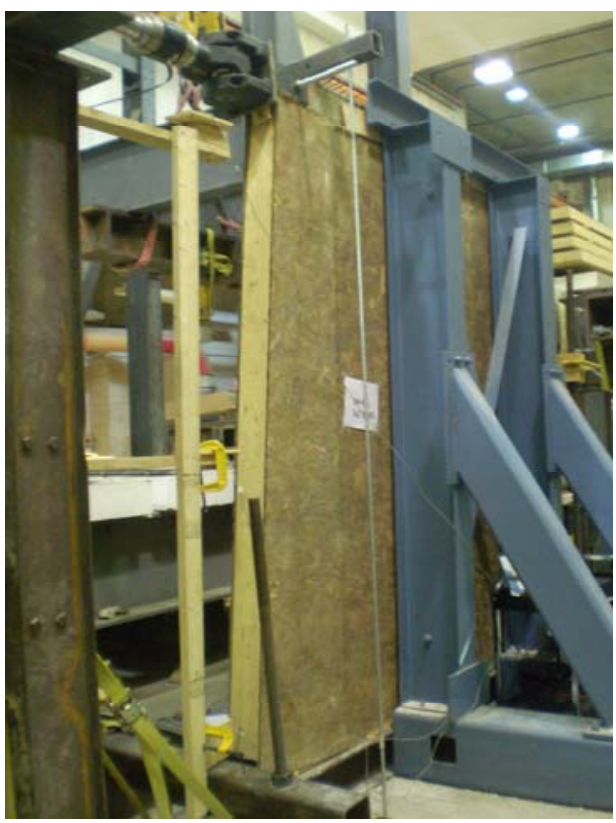

(b) Back side

Figure 4.89 - Global failure of specimen SIP-R-1 under racking load showing global shear deformation and relative shear rotation of SIP segments at spline connection

In addition to the global shear deformation depicted in Fig. 4.89, uplift at the bottom of wall panel in one side of the panel, as shown in Fig. 4.90(a). Also, OSB crushing at the same location on the other side of the wall and Nail bending and OSB crushing at the top and bottom of the wall were observed as shown in Fig. 4.90(b) due to the rotation of OSB sheathing against the top and bottom plates nailed to. It should be noted that at the uplift location depicted in Fig. 4.90(a), the bottom of OSB sheet tear away from the bottom plate at the nail locations. Figure 4.90(c) shows separation of the OSB sheet from the top plate due to shear rotation. Also, Fig. 4.90(d) shows close-up view of the shear rotation of the OSB sheets against each other, crushing of the front side of the back OSB sheet and uplift and separation of the back side of the front OSB sheet from the bottom plate. It was observed that few of the nails at the spline connection and connection to bottom and top plates no rupture but they were pulled out totally and partially from their location. Similar failure mode was observed for identical panels SIP-R-2 and SIP-R-3. 


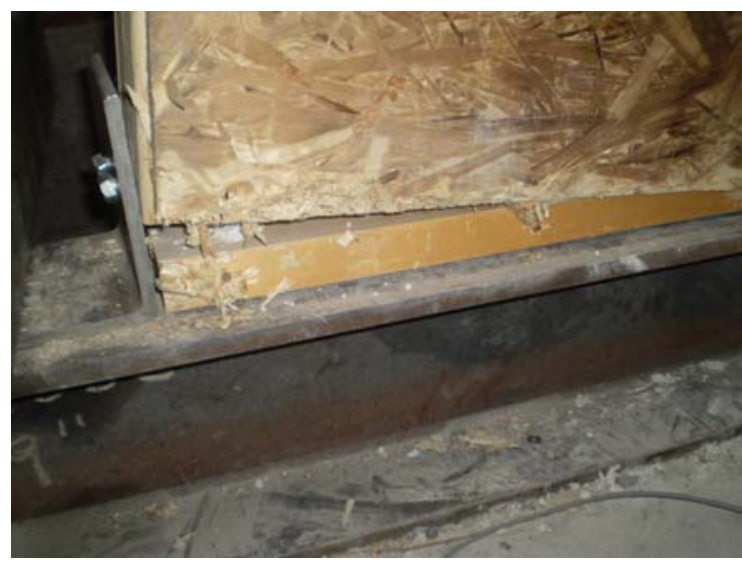

(a) Uplift at the bottom and panel crushing

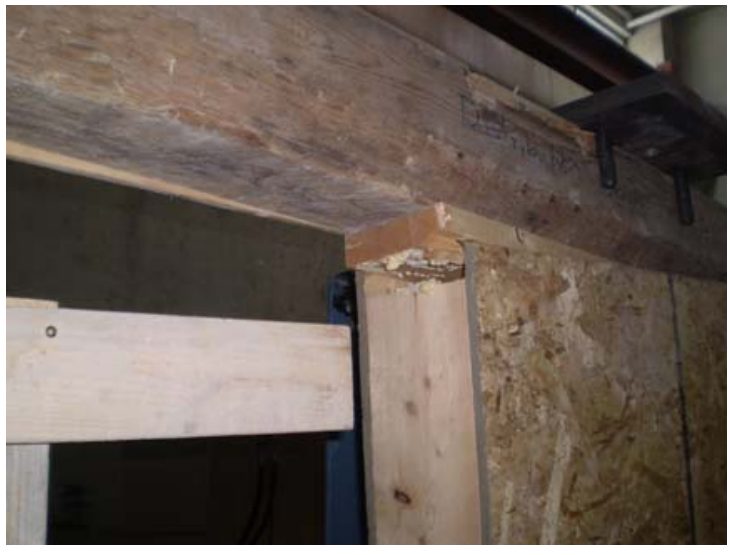

(c) Separation of the end plate and OSB

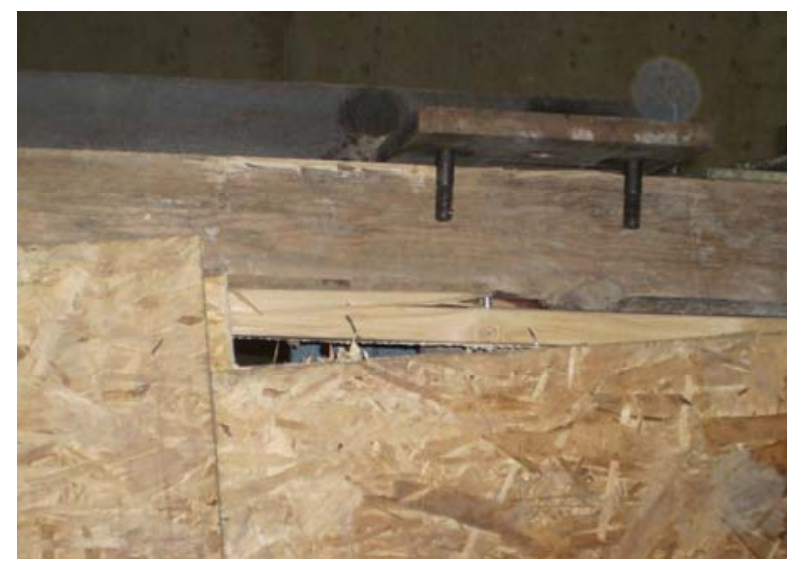

(b) Nail bending and OSB crushing

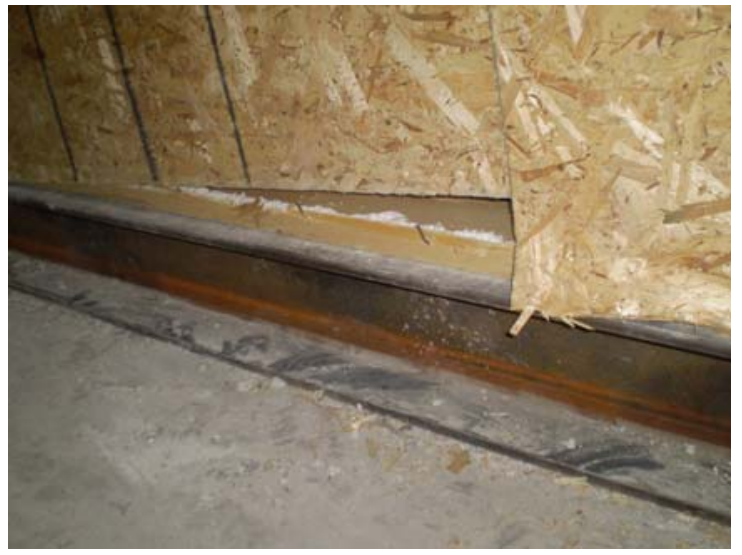

(d) Rotation of OSB sheet and nail bending

Figure 4.90 - Detailed Failure modes of specimen SIP-R-1 under racking load

A Second set of SIP walls was tested under racking loading. In this set, three identical SIP walls, namely SIP-R-4, SIP-R-5 and SIP-R-6, were selected for racking load test. The wall was of 3660 $\mathrm{mm}$ length, $2750 \mathrm{~mm}$ height and $165 \mathrm{~mm}$ total thickness, with three SIP panels connected together using foam-spline connections. The wall has sheathing made of OSB sheets on each side of the wall. The wall was placed over a W200 I-beam that rest on the laboratory floor. The bottom plates of the wall were connected to the I-beam using twelve lag bolts in every $304.8 \mathrm{~mm}$ ( $1 \mathrm{ft}$ ) in order to provide a fully fixed support to the wall base. Tie-down system was used to hold the wall in position while applying the racking load at the top of the wall using an actuator as depicted in Fig. 4.91. Figure 4.92 shows the general failure mode of wall SIP-R-4 which was global shear deformation and relative shear rotation of SIP segments at spline connection. 
Figure 4.93(a) shows view of the uplift of the rear side of the wall along with tearing and cracking of end vertical lumber at its fixation. Figure 4.93(b) shows view of bending of nails and crushing or tearing of OSB sheet at nail location, and separation of OSB sheet from the bottom plate. The nails in SIP had no rupture but they were pulled out totally and partially from their location. The uplift of the panel also happened and the end lumbers were tearing in the location of side screws. Similar observation is shown in Fig. 4.93(c) at the top plate location. Figure 4.93(d) shows close-up view of the nail pullout at the middle of the panel at its connection to the top plate. Similar failure mode was observed for identical panels SIP-R-5 and SIP-R-6.

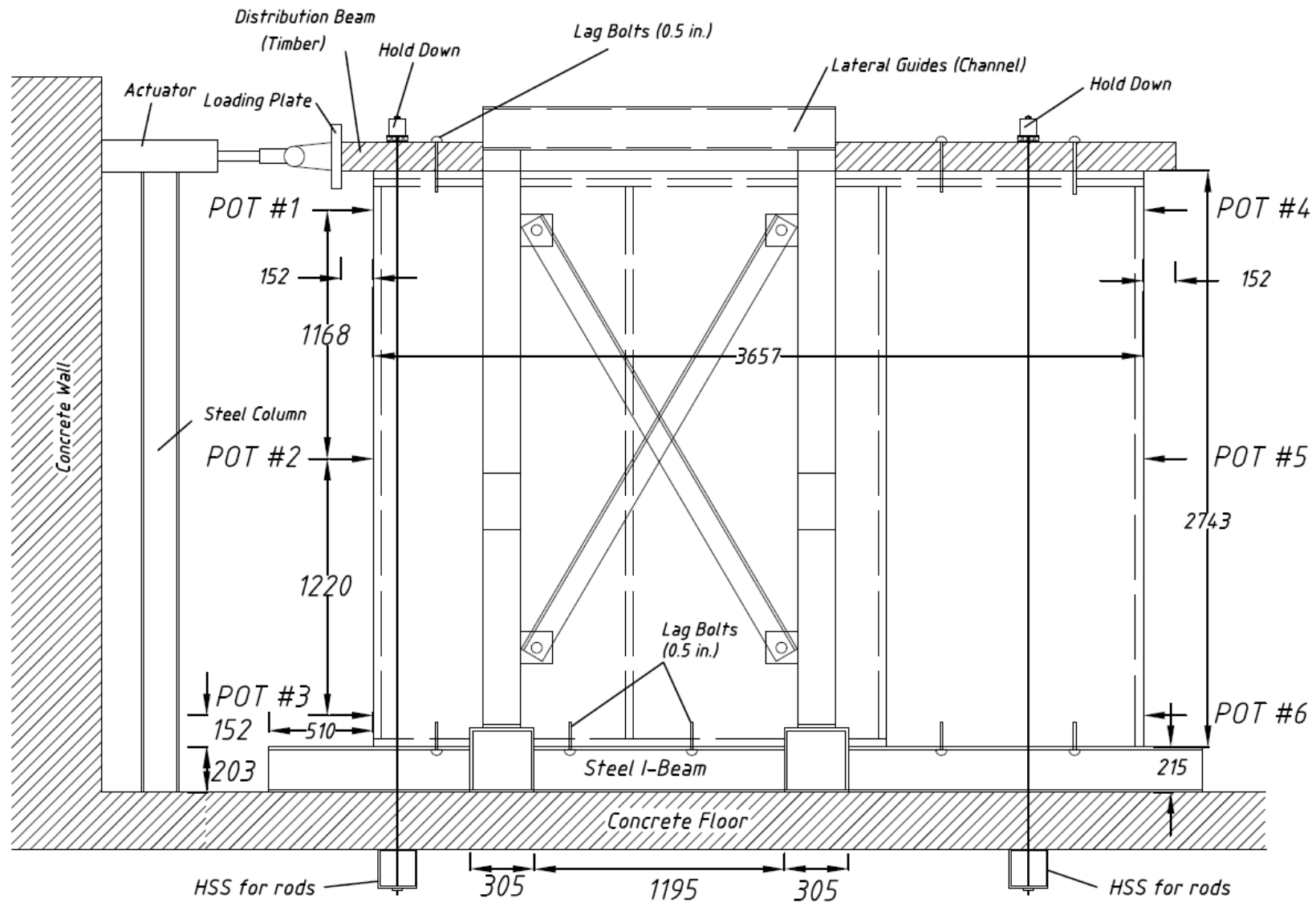

Figure 4.91 - Test setup of specimen SIP-R-4 under racking load 


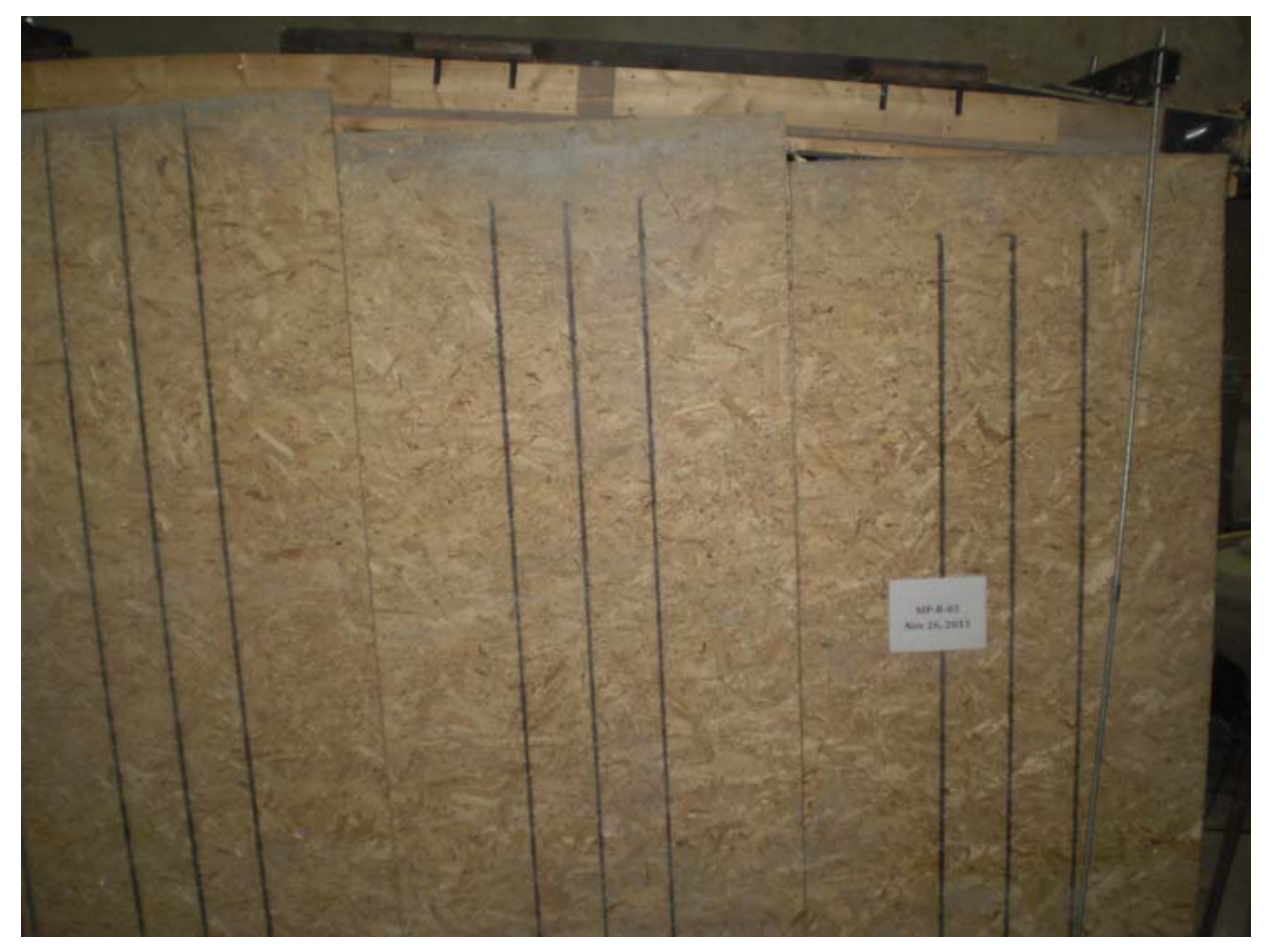

Figure 4.92 - Global failure of specimen SIP-R-4 under racking load showing global shear deformation and relation shear rotation of SIP segments at spline connection

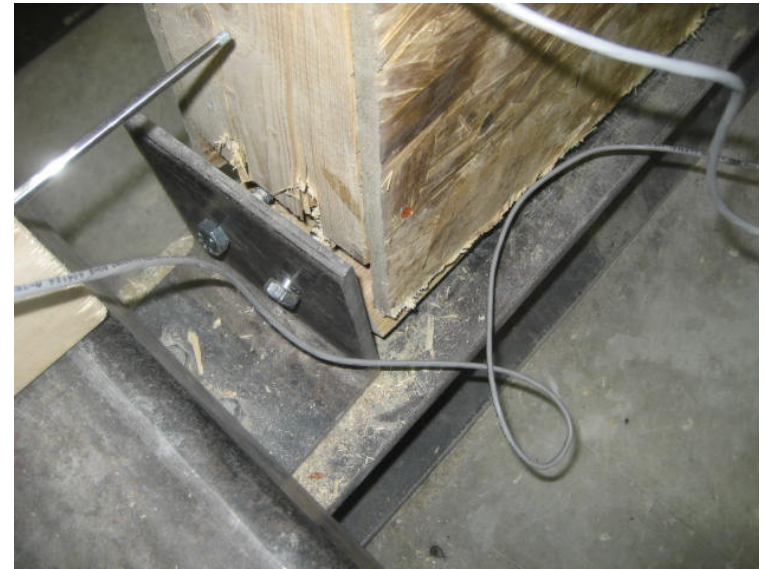

(a) Tearing and cracking of end lumber at the bottom of panel

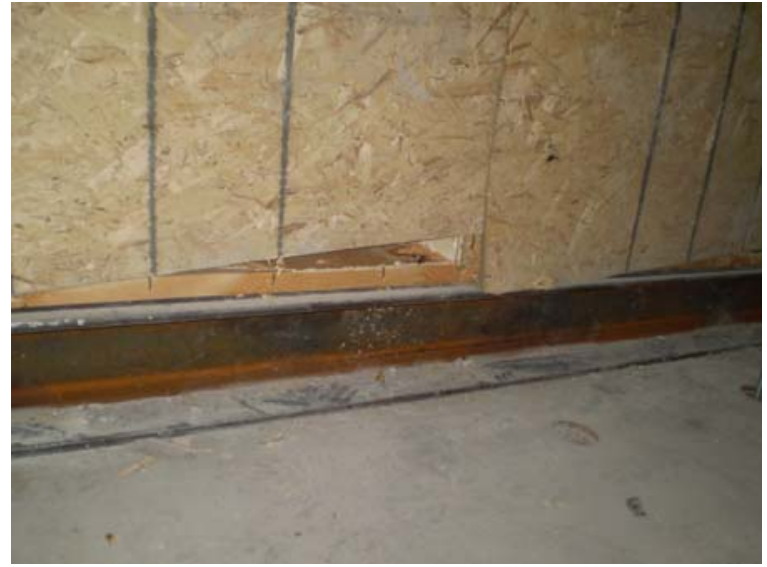

(b) Bending of nail and crushing of OSB sheet at nail location 


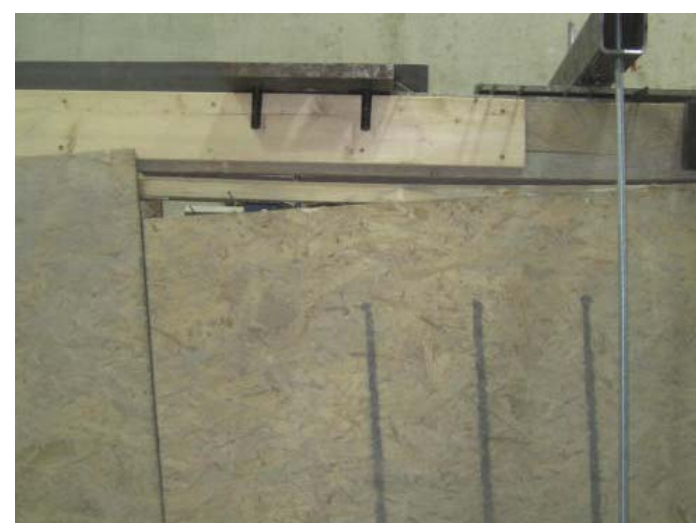

(c) Separation top plate from OSB

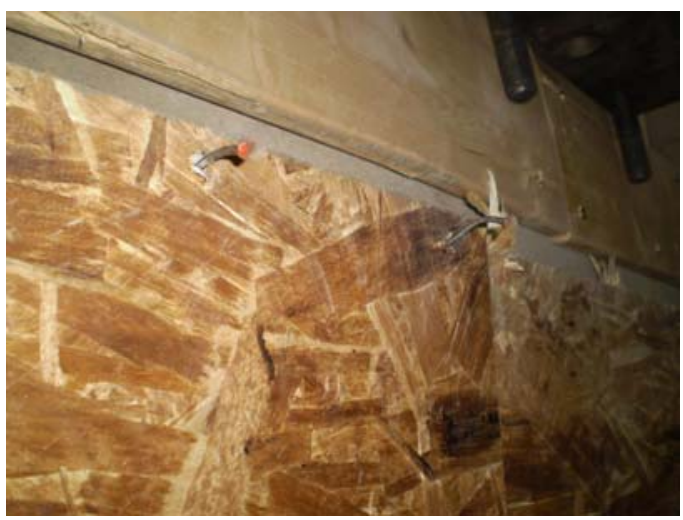

(d) Nail pull out at the middle of panel

Figure 4.93 - Detailed Failure modes of specimen SIP-R-4 under racking load

Figures 4.94, 4.95 and 4.96 depict the racking load vs. lateral deflection relationships for at the top of walls SIP-R-1, SIP-R-2 and SIP-R-3, respectively. However, Figs. 4.100, 4.101 and 4.102 show the racking load vs. lateral deflection at different heights of walls SIP-R-1, SIP-R-2 and SIP-R-3, respectively. Results show that the ultimate racking loads for walls SIP-R-1, SIP-R-2 and SIP-R-3, were 25.32, 30.87 and $22.45 \mathrm{kN}$, respectively. An average value, shown in Table 4.6, of $26.21 \mathrm{kN}$ showed superior performance of SIPs compared to stud wall system of identical geometry and average ultimate racking load of $11.72 \mathrm{kN}$. Figures 4.97, 4.98 and 4.99 depict the racking load vs. lateral deflection relationships for at the top of walls SIP-R-4, SIP-R-5 and SIPR-6, respectively. However, Figs. 4.103, 4.104 and 4.105 show the racking load vs. lateral deflection at different heights of walls SIP-R-4, SIP-R-5 and SIP-R-6, respectively. Results show that the ultimate racking loads for walls SIP-R-4, SIP-R-5 and SIP-R-6, were 40.86, 38.94, and $40.64 \mathrm{kN}$, respectively. An average value, shown in Table 4.6, of $40.14 \mathrm{kN}$ showed that with increase in wall length from $2440 \mathrm{~mm}\left(8^{\prime}\right)$ to $3660 \mathrm{~mm}\left(12^{\prime}\right)$, the average racking load increased by $53 \%$. 


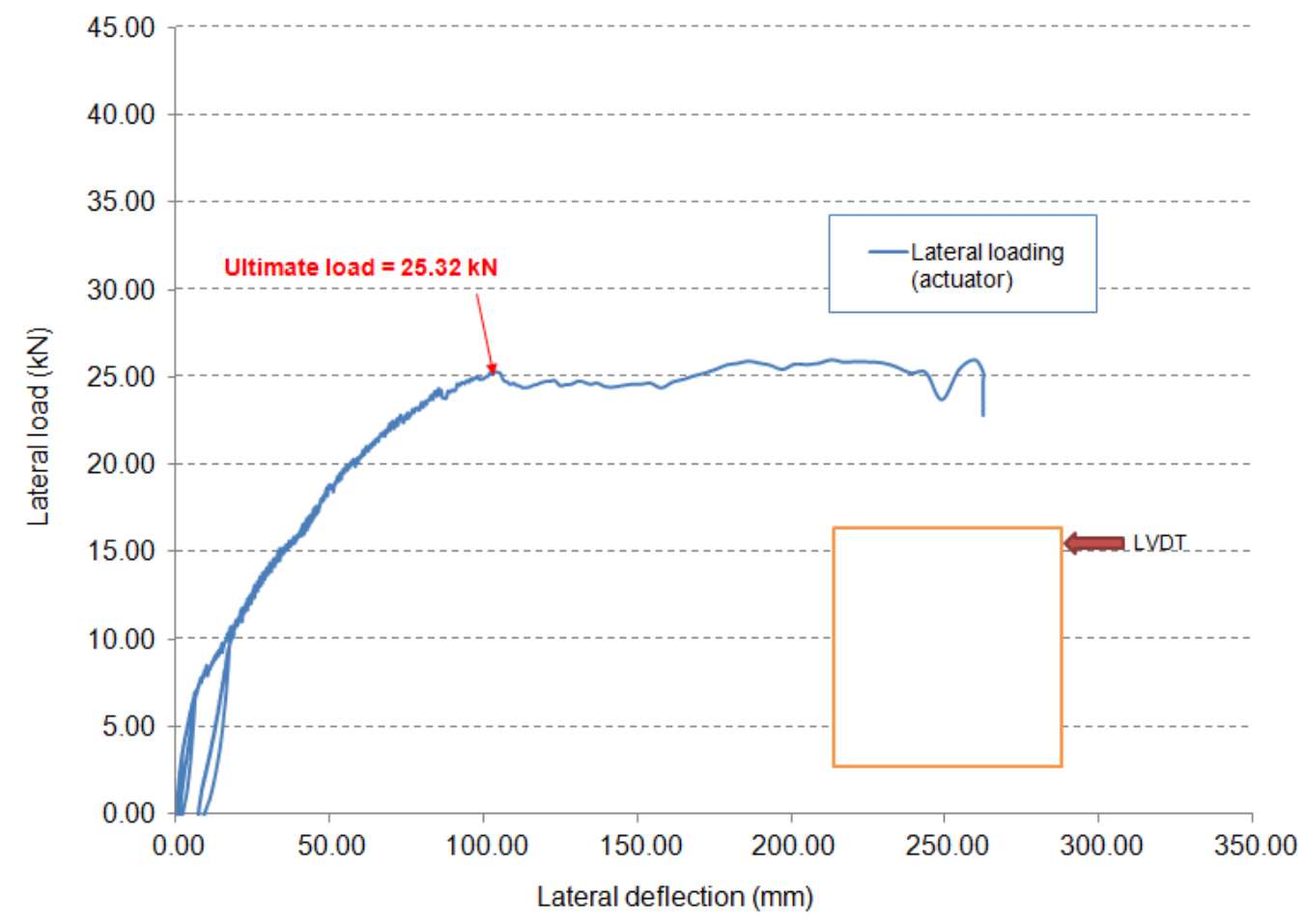

Figure 4.94 - Load vs. lateral deflection response of SIP-R-1 (obtained from actuator reading on top of the panel)

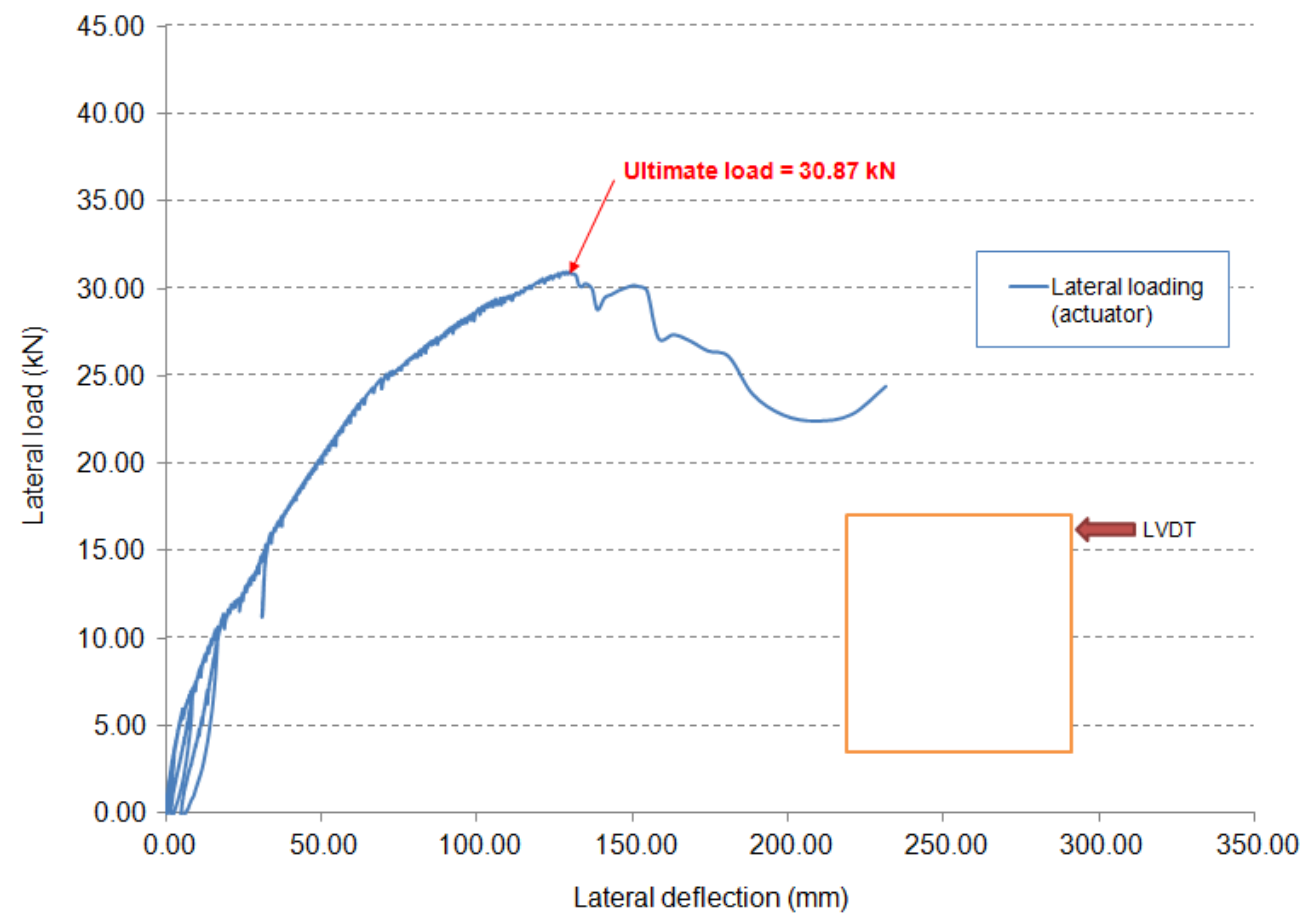

Figure 4.95 - Load vs. lateral deflection response of SIP-R-2 (obtained from actuator reading on top of the panel) 


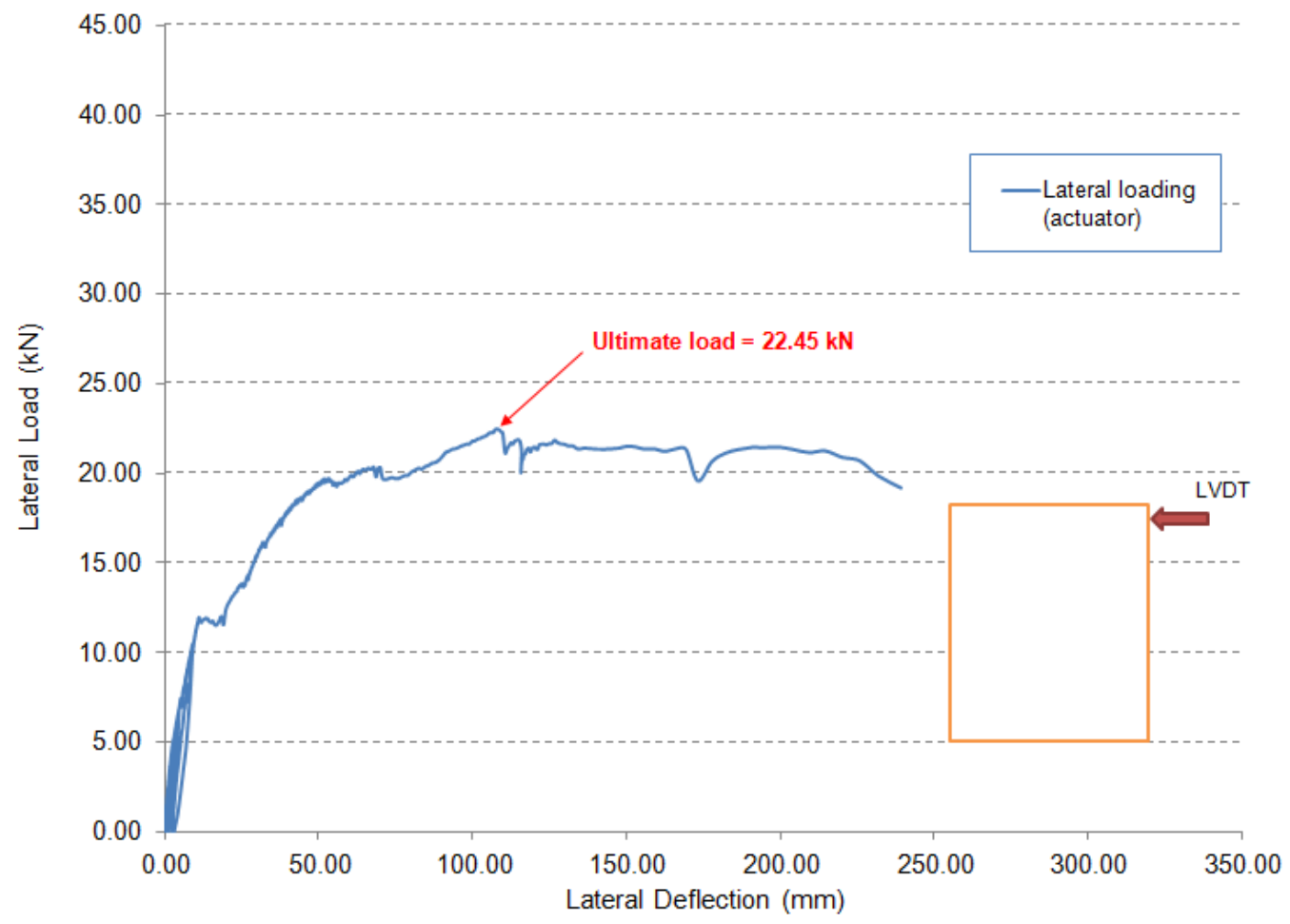

Figure 4.96 - Load vs. lateral deflection response of SIP-R-3 (obtained from actuator reading on top of the panel)

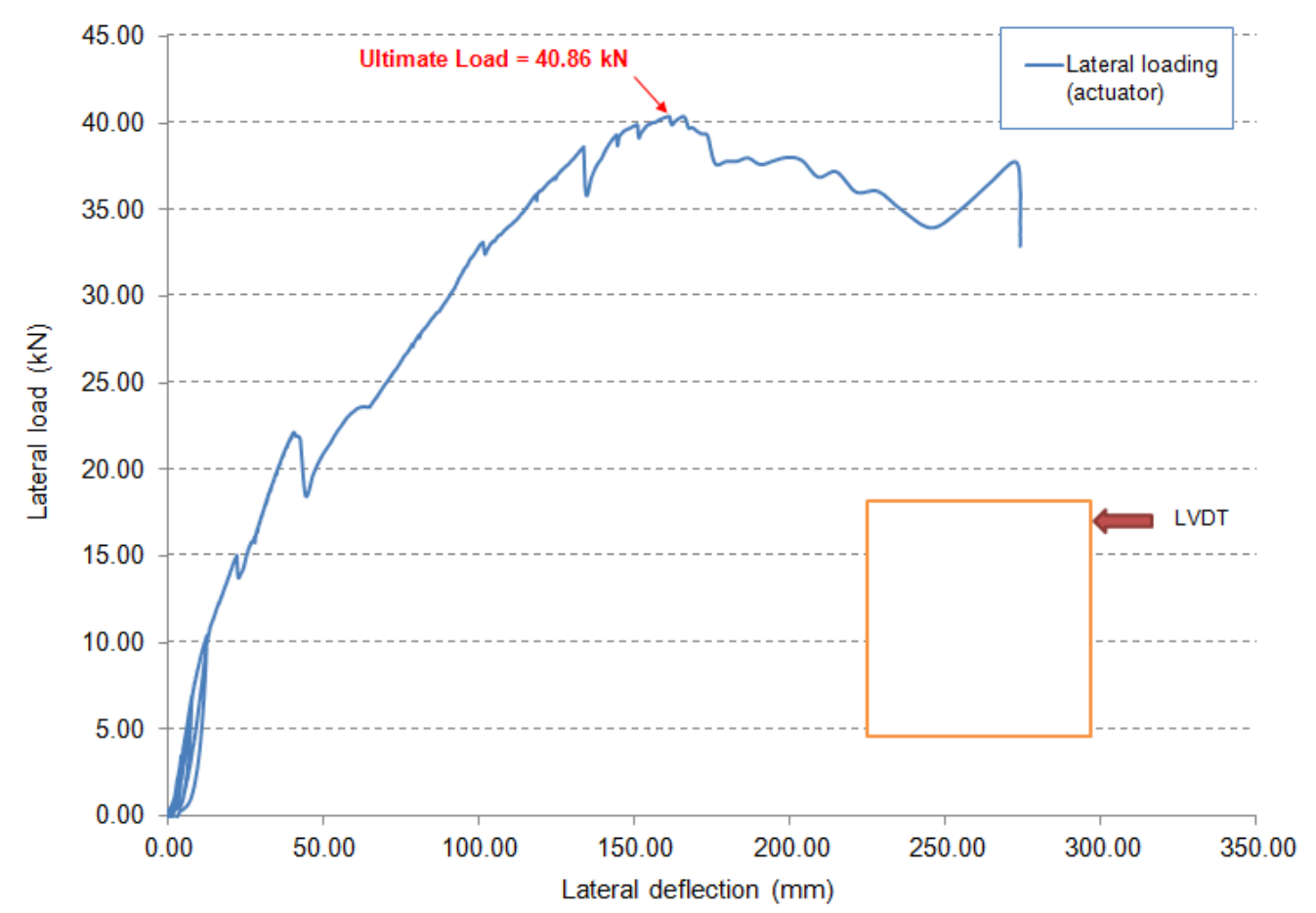

Figure 4.97- Load vs. lateral deflection response of SIP-R-4 (obtained from actuator reading on top of the panel) 


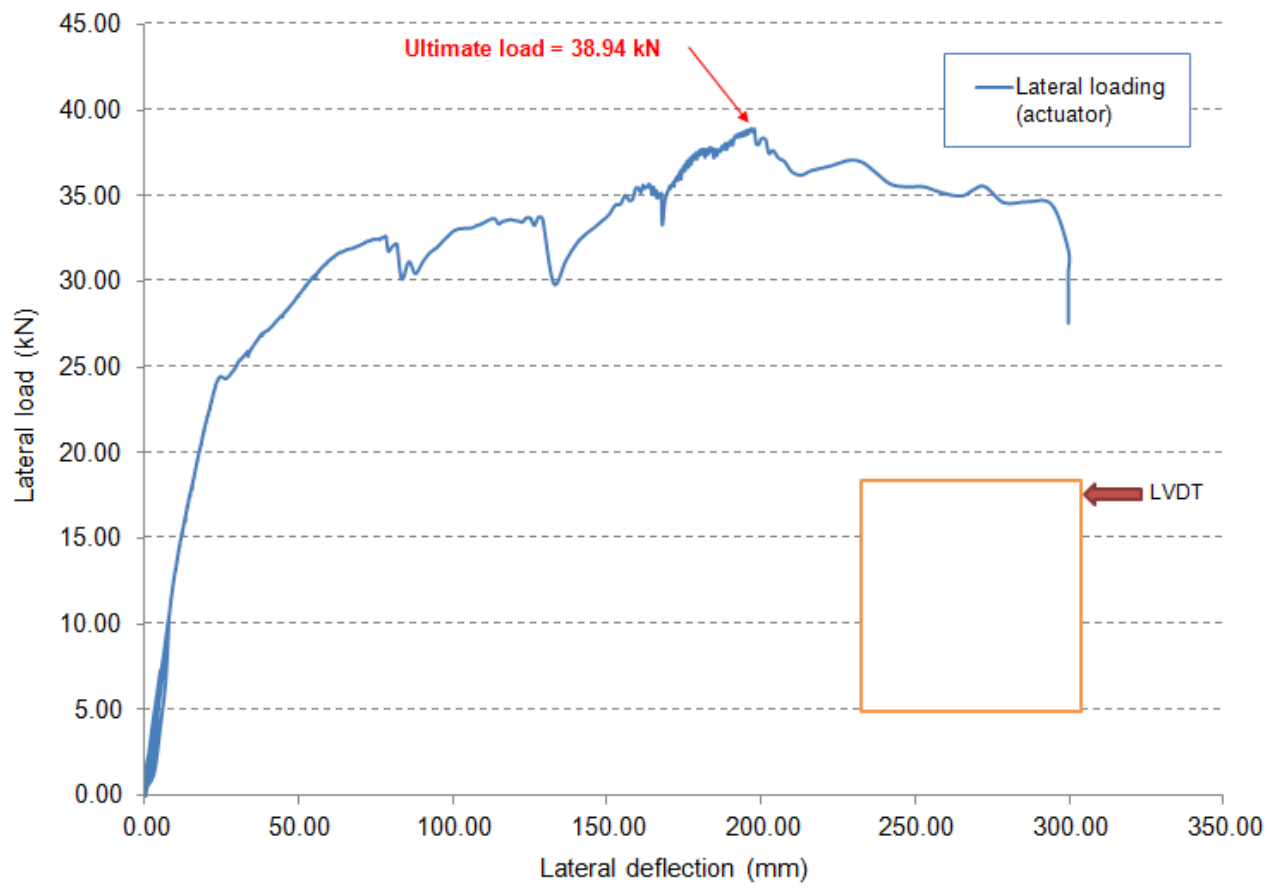

Figure 4.98 - Load vs. lateral deflection response of SIP-R-5 (obtained from actuator reading on top of the panel)

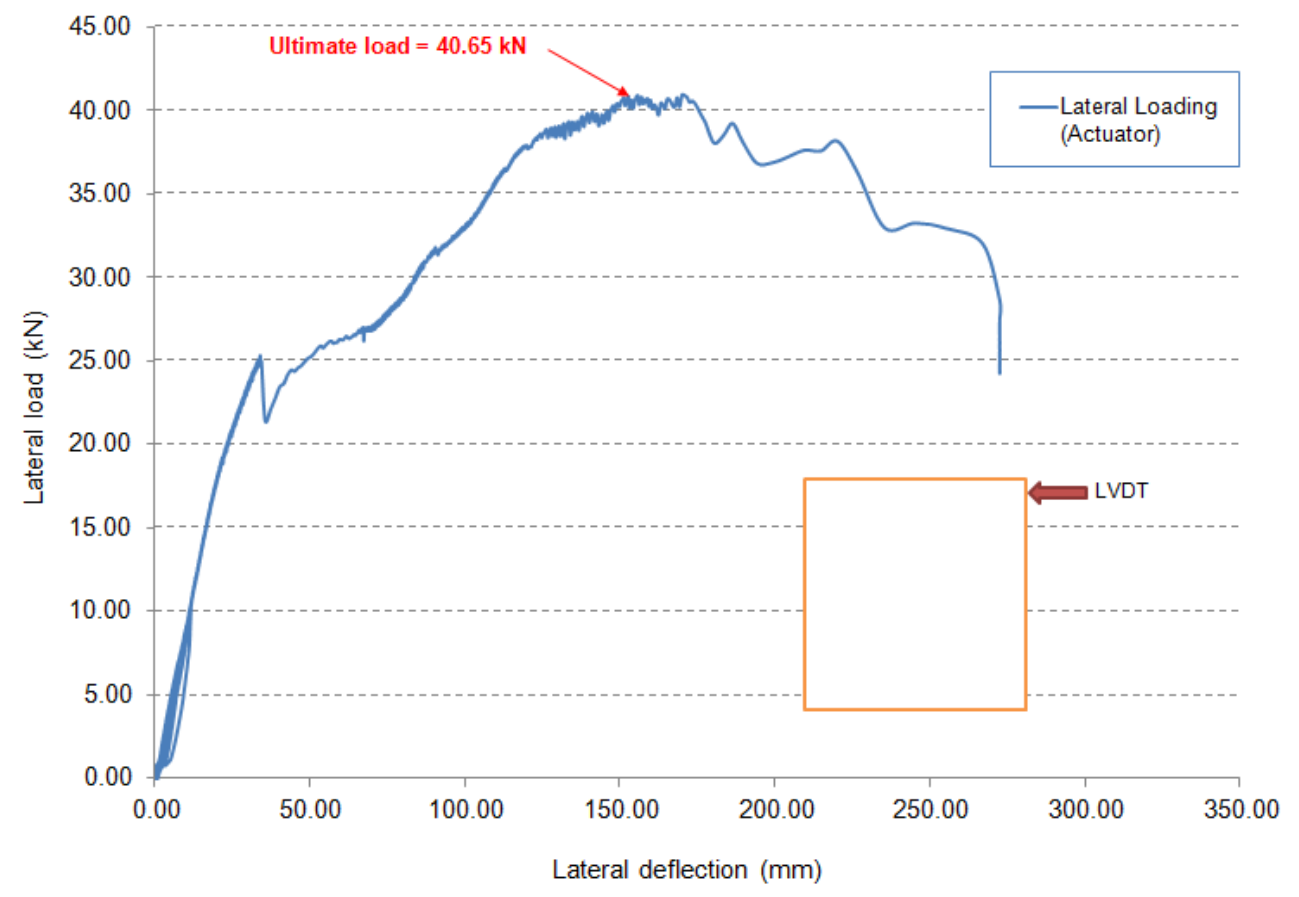

Figure 4.99 - Load vs. lateral deflection response of SIP-R-6 (obtained from actuator reading on top of the panel) 


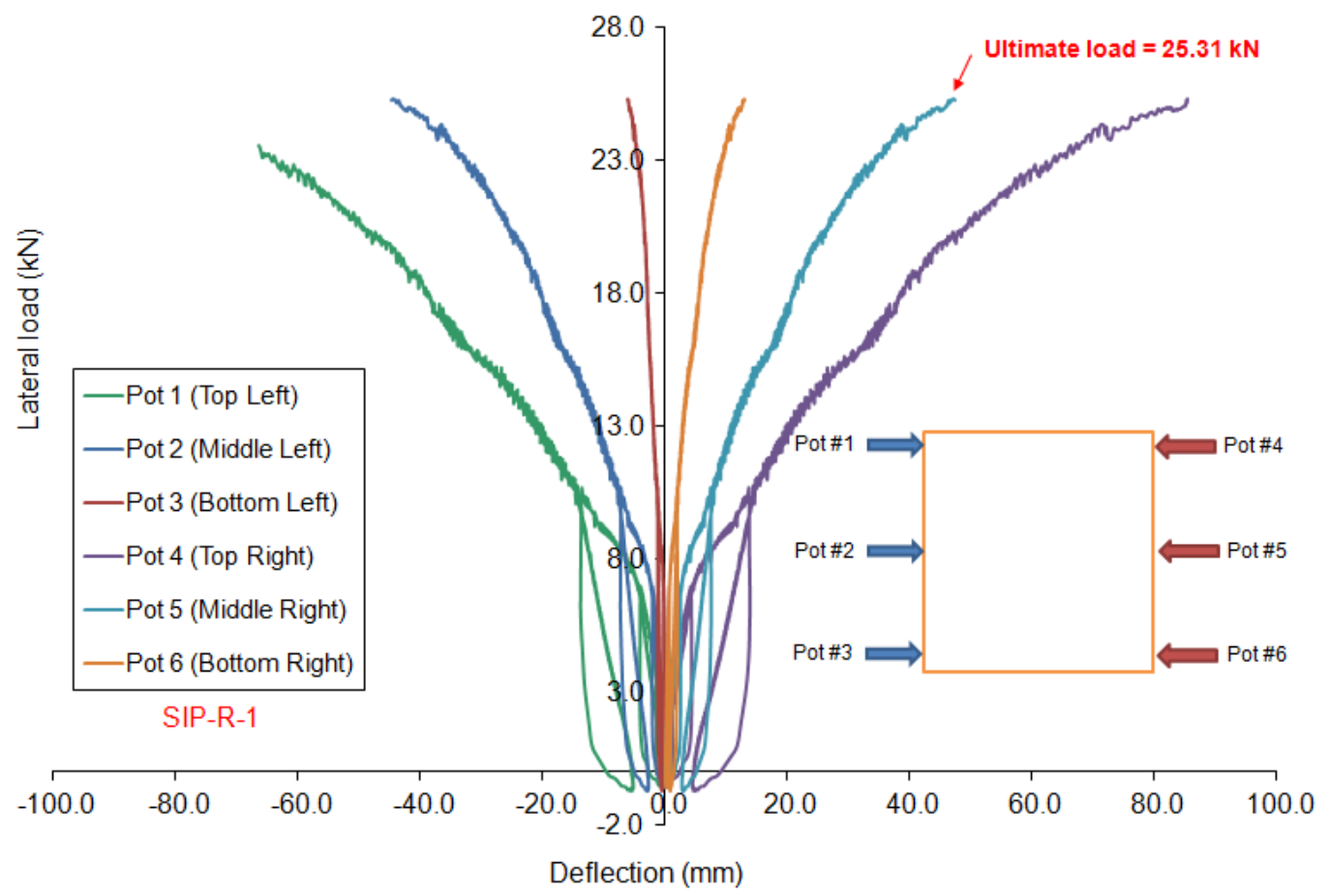

Figure 4.100 - Load vs. lateral deflection response of SIP-R-1 (POTs records up to a load close to failure load)

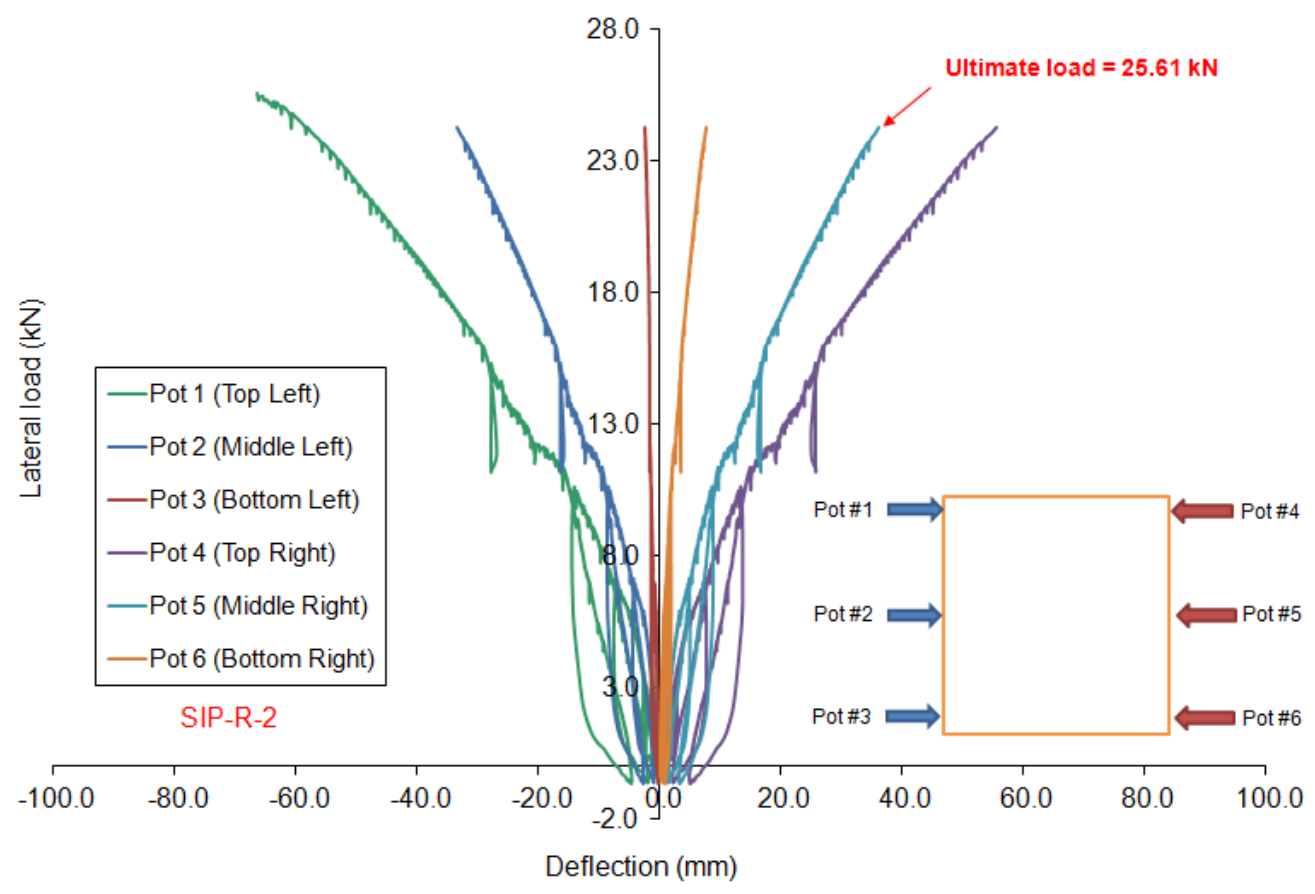

Figure 4.101 - Load vs. lateral deflection response of SIP-R-2 (POTs records up to a load close to failure load) 


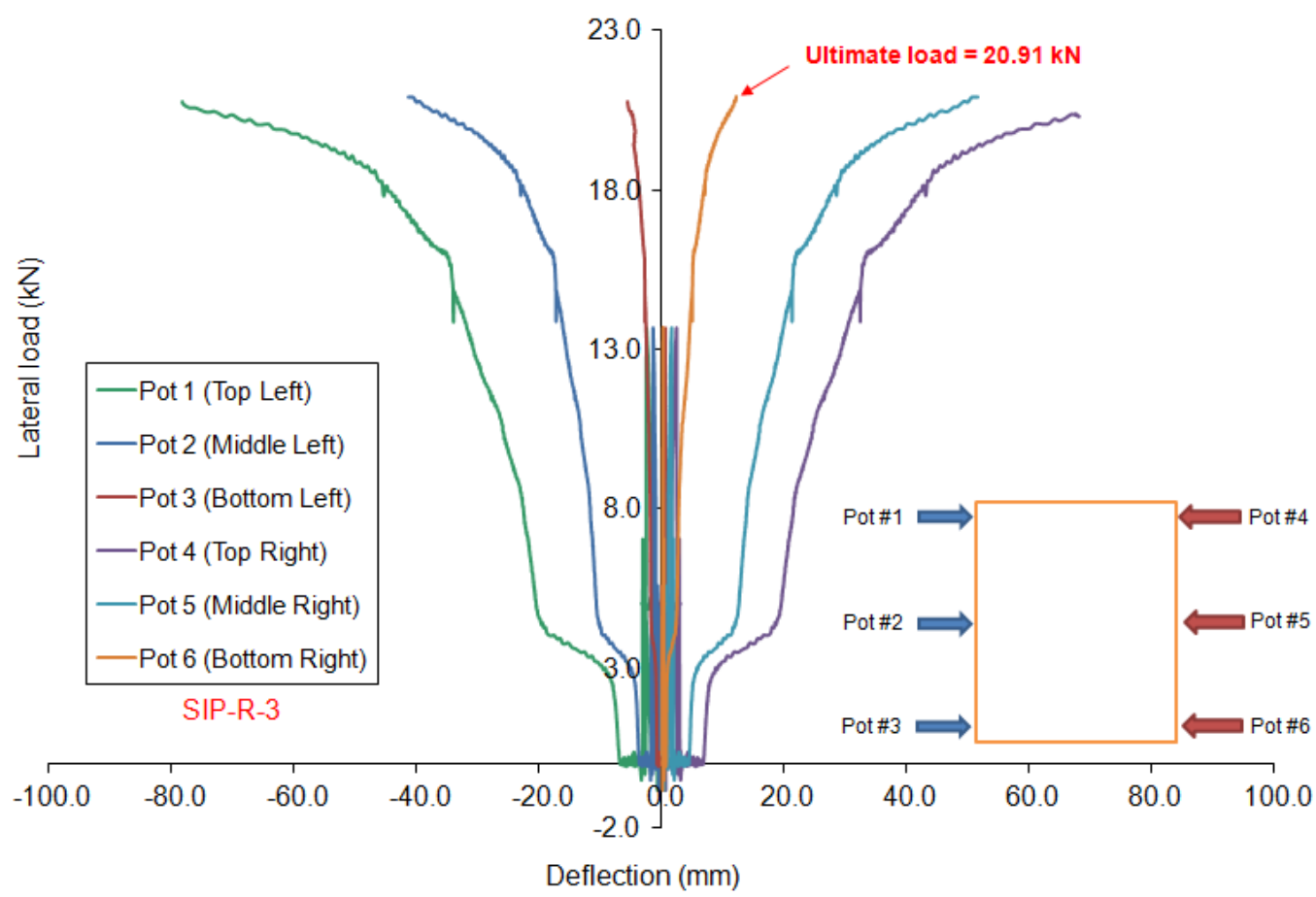

Figure 4.102 - Load vs. lateral deflection response of SIP-R-3 (POTs records up to a load close to failure load)

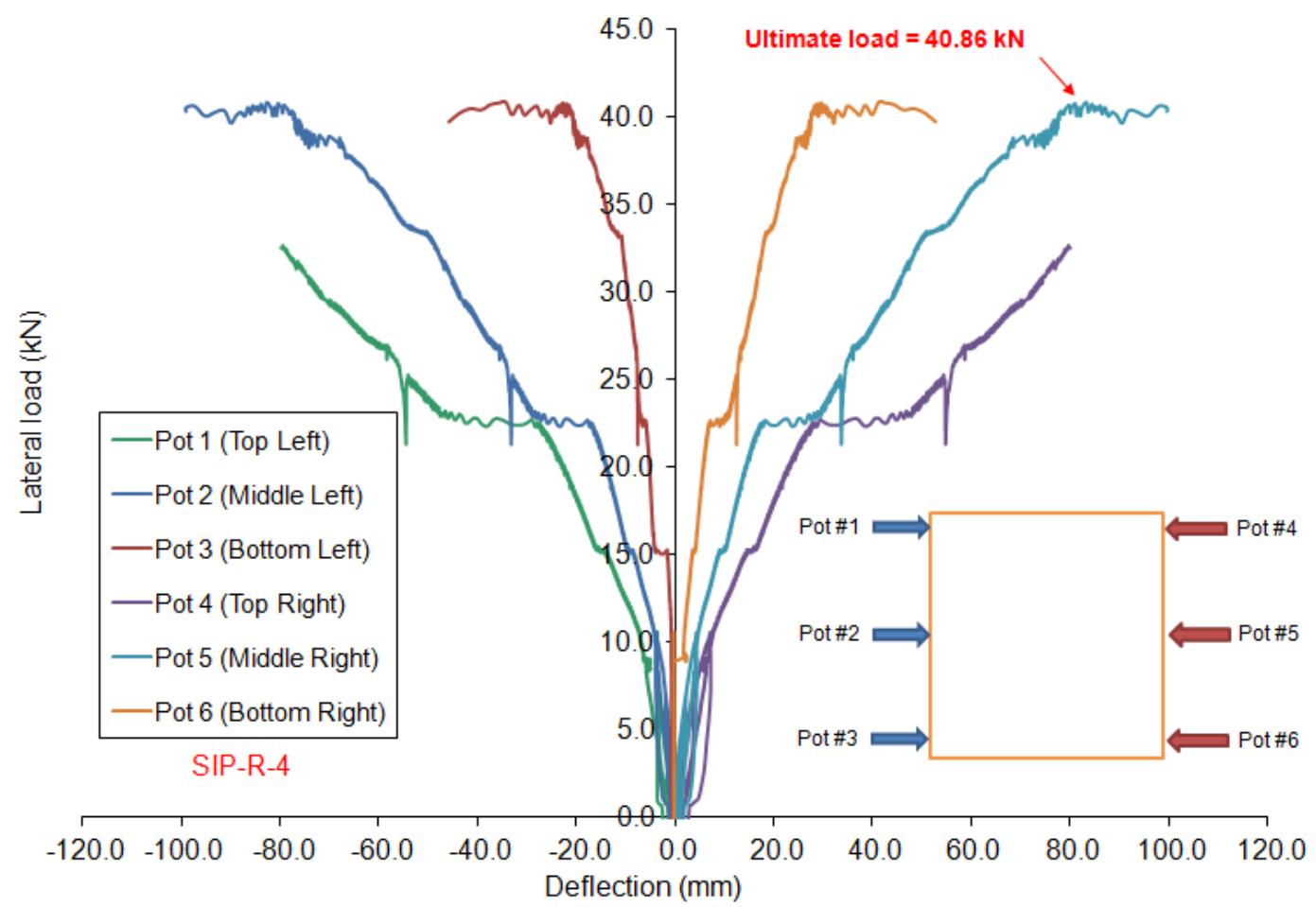

Figure 4.103 - Load vs. lateral deflection response of SIP-R-4 (POTs records up to a load close to failure load) 


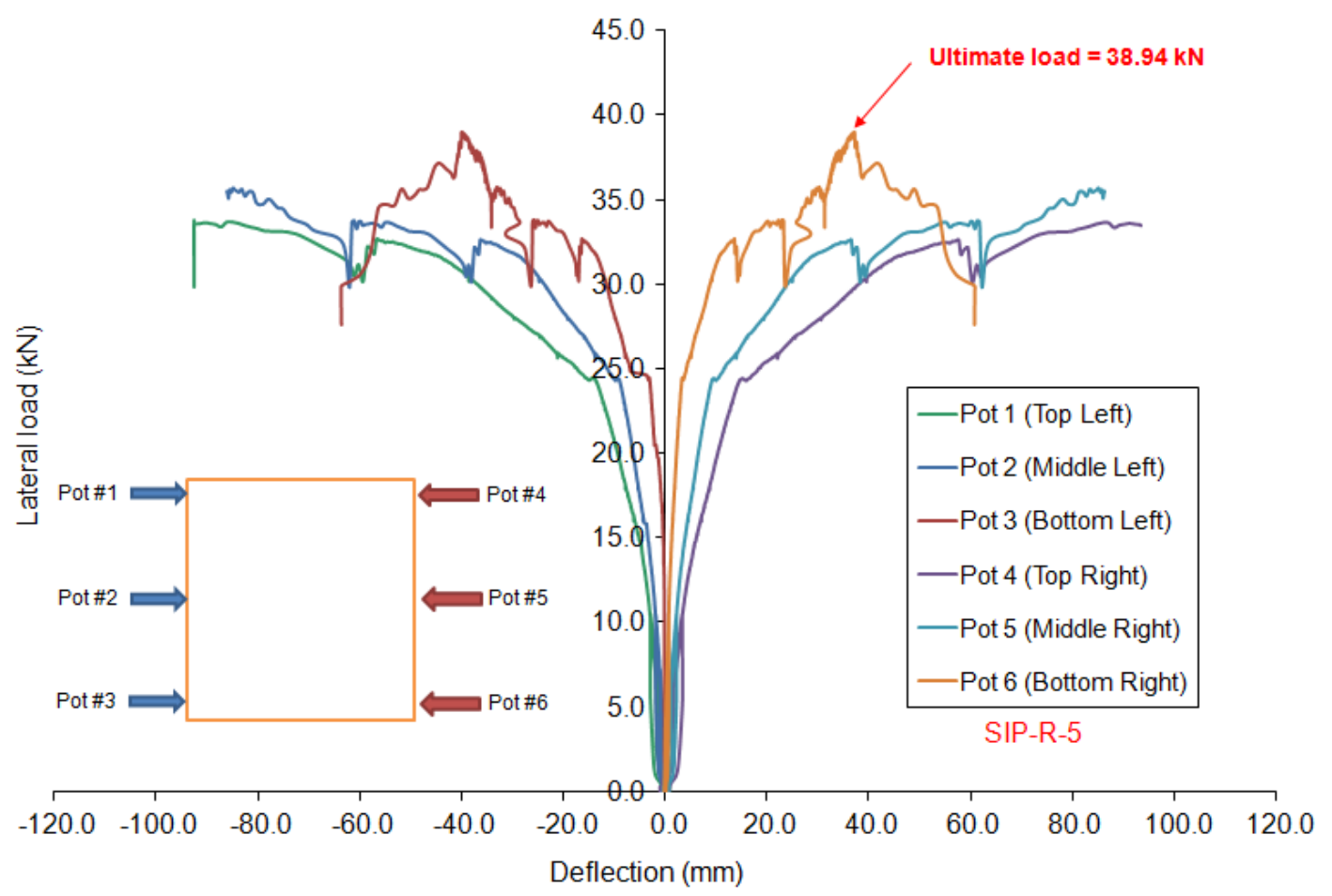

Figure 4.104 - Load vs. lateral deflection response of SIP-R-5 (POTs records up to a load close to failure load)

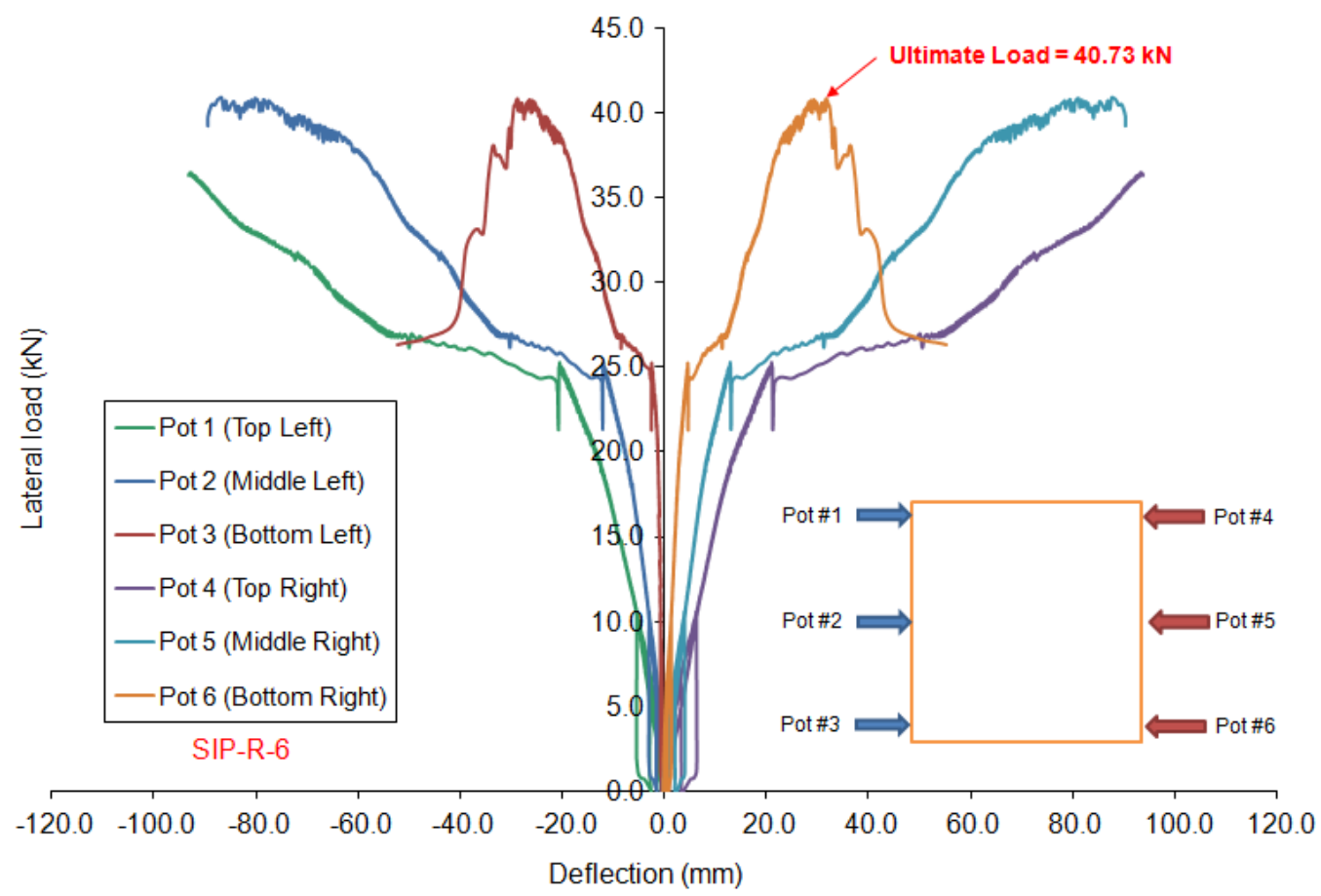

Figure 4.105 - Load vs. lateral deflection response of SIP-R-6 (POTs records up to a load close to failure load) 


\subsubsection{Cyclic Loading}

Three identical SIP walls, namely SIP-C-1, SIP-C-2 and SIP-C-3, were selected for cyclic load test to correlate the results with those obtained for stud walls of identical geometry. The wall was of $2450 \mathrm{~mm}$ length, $2750 \mathrm{~mm}$ height and $165 \mathrm{~mm}$ total thickness, with two SIP panels connected together using foam-spline connection. The wall has sheathing made of OSB sheets on each side of the wall. The wall was placed over a W200 I-beam that rest on the laboratory floor. The bottom plates of the wall were connected to the I-beam using eight lag bolts in every $304.8 \mathrm{~mm}$ ( $1 \mathrm{ft}$ ) in order to provide a fully fixed support to the wall base. Tie-down system was used to hold the wall in position while applying the racking load at the top of the wall using an actuator as depicted in Fig. 4.106. Figure 4.107 shows the general failure mode of wall SIP-R-1 which was global shear deformation and relative shear rotation of SIP segments at spline connection.

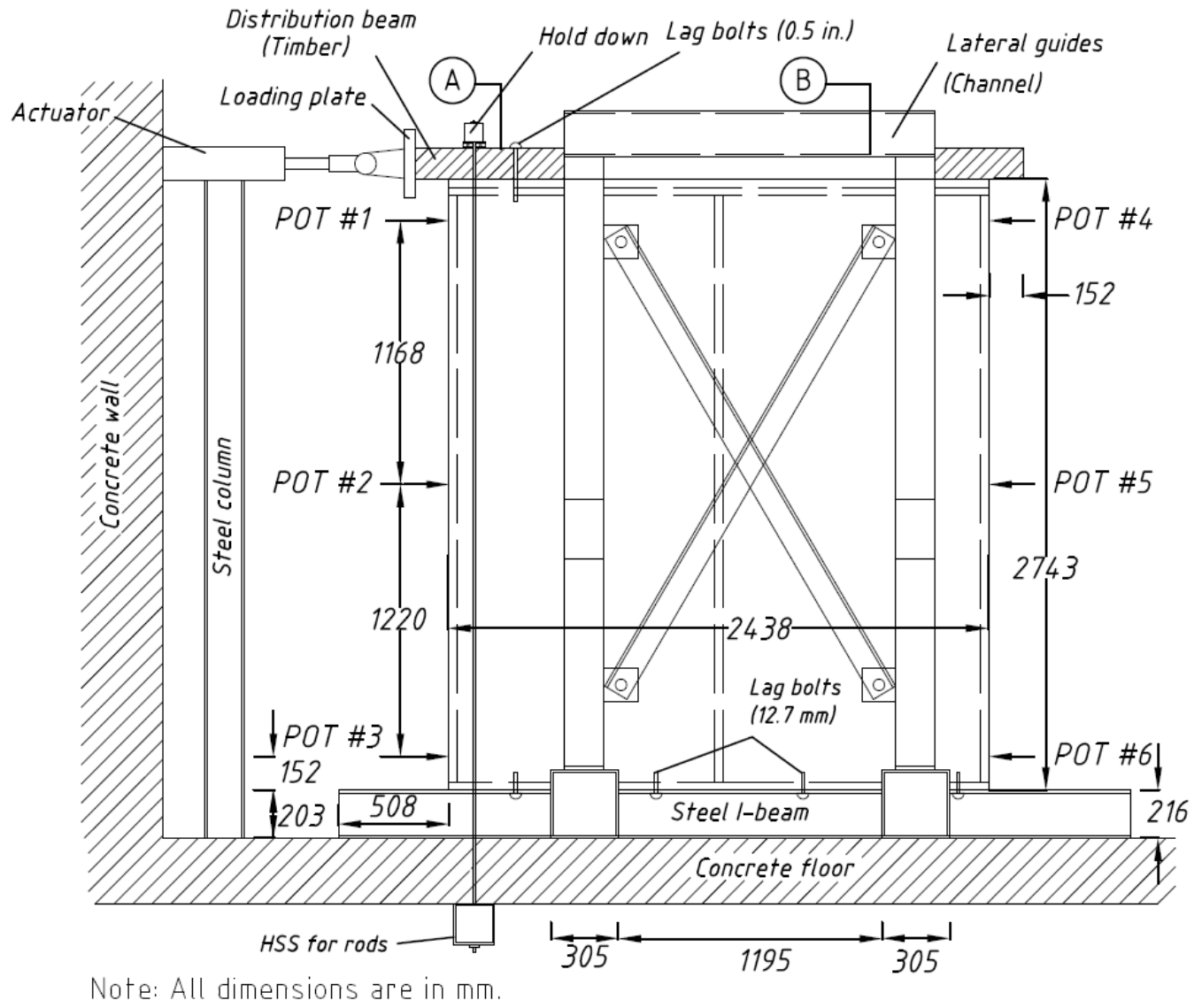

Figure 4.106 - Test setup of specimen SIP-C-1 under cyclic loading 
In addition to the global shear deformation depicted in Fig. 4.107, uplift at the bottom of wall panel in one side of the panel, as shown in Fig. 4.108(a). Figure 108(b) shows view of OSB sheet and spline separation at the middle of the panel due to shear deformation of the wall. Figure 4.108(c) shows rotation of the bottom of OSB sheets at spline location, leading to OSB crushing and nail bending. Figure 108(d) shows close-up view of lumber separation from the OSB sheet at top of the panel. Similar failure mode was observed for identical panels SIP-C-2 and SIP-C-3.

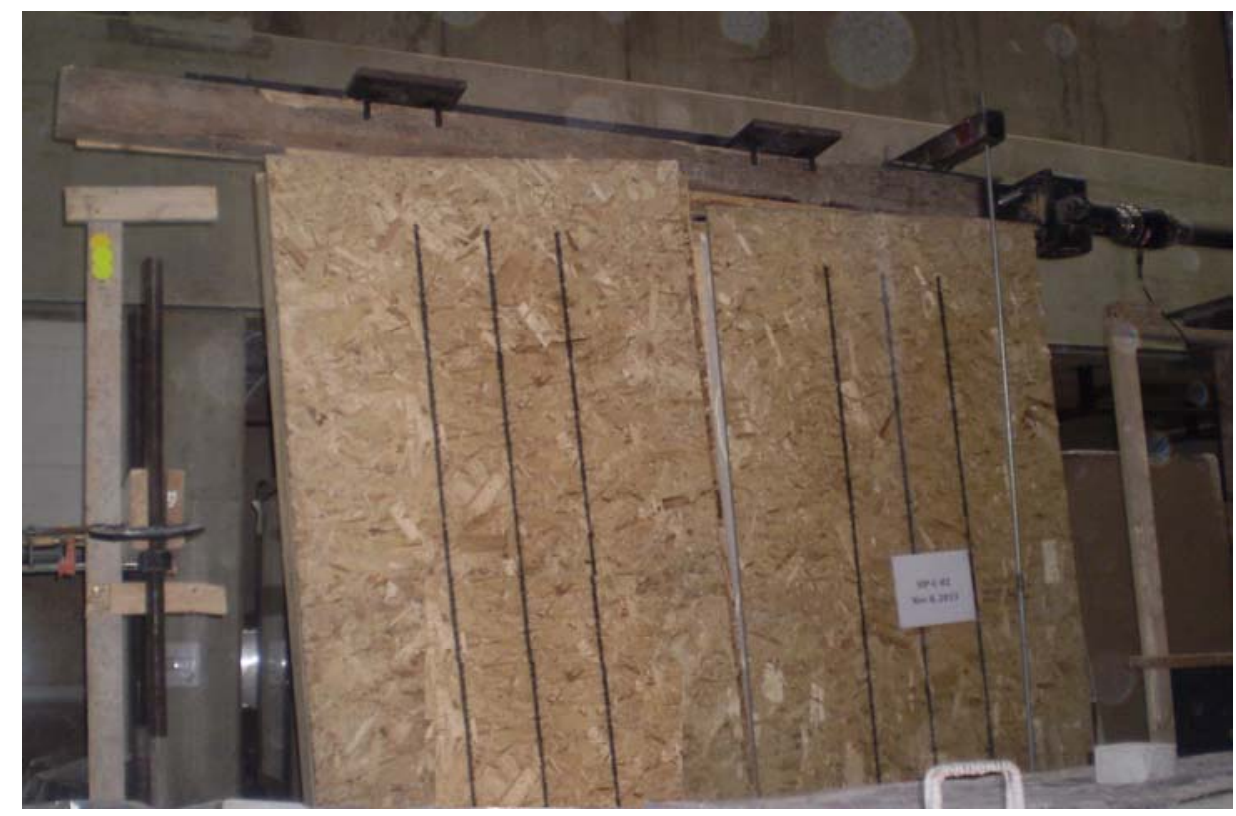

Figure 4.107 - Global failure of specimen SIP-C-1 under cyclic loading showing global shear deformation and relation shear rotation of SIP segments at spline connection

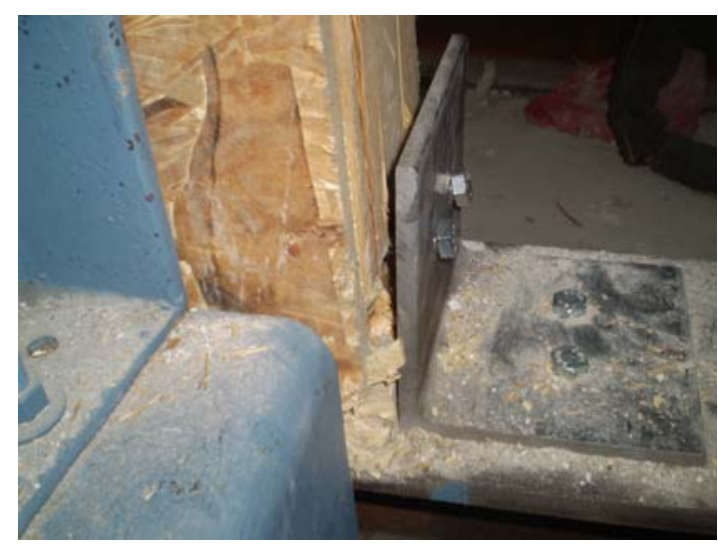

(a) Uplift at the bottom and lumber cracking at nail location

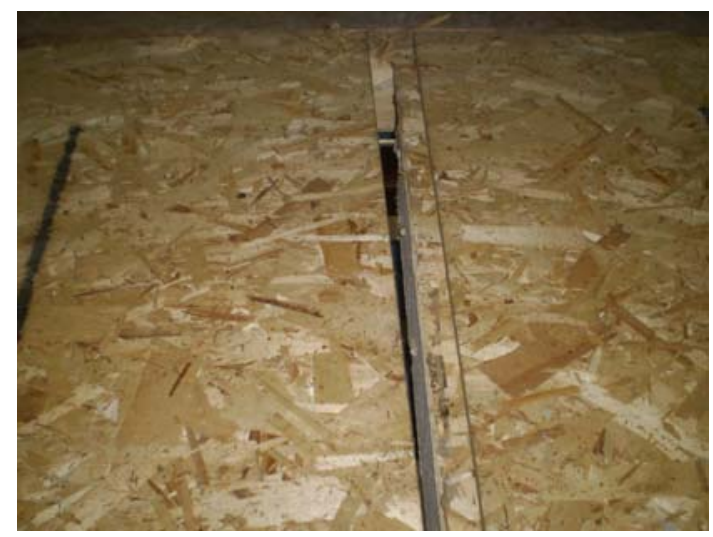

(b) OSB and spline separation at the middle of the panel 


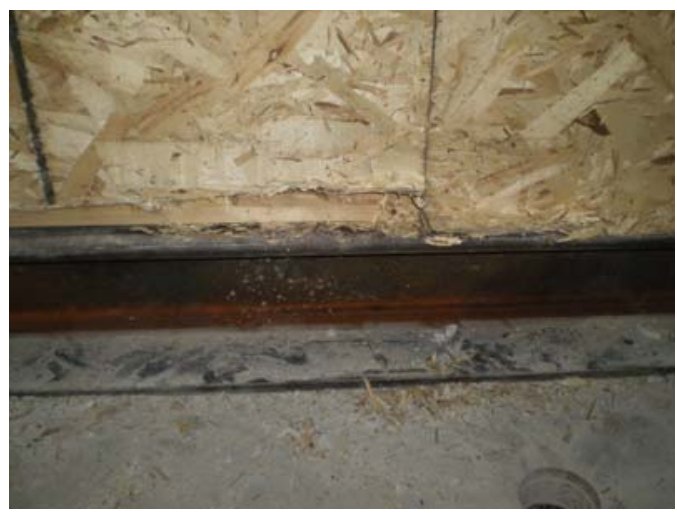

(c) Rotation of OSB sheet, nail bending and OSB crushing at nail location

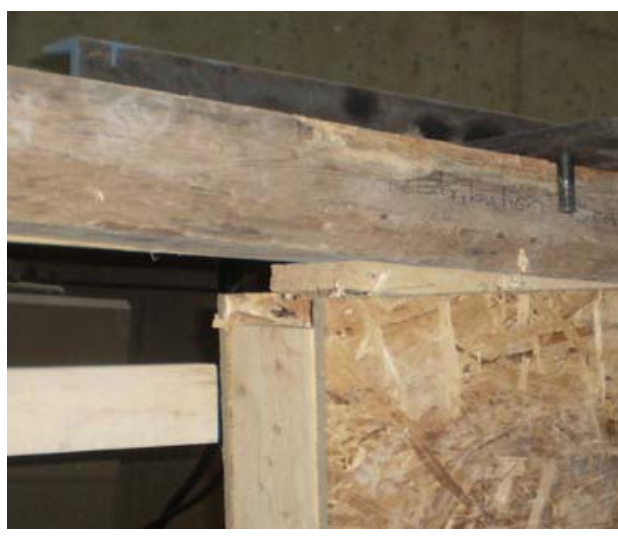

(d) Separation of lumbers at connection at top of the panel

Figure 4.108 - Detailed failure modes of specimen SIP-C-1 under cyclic load

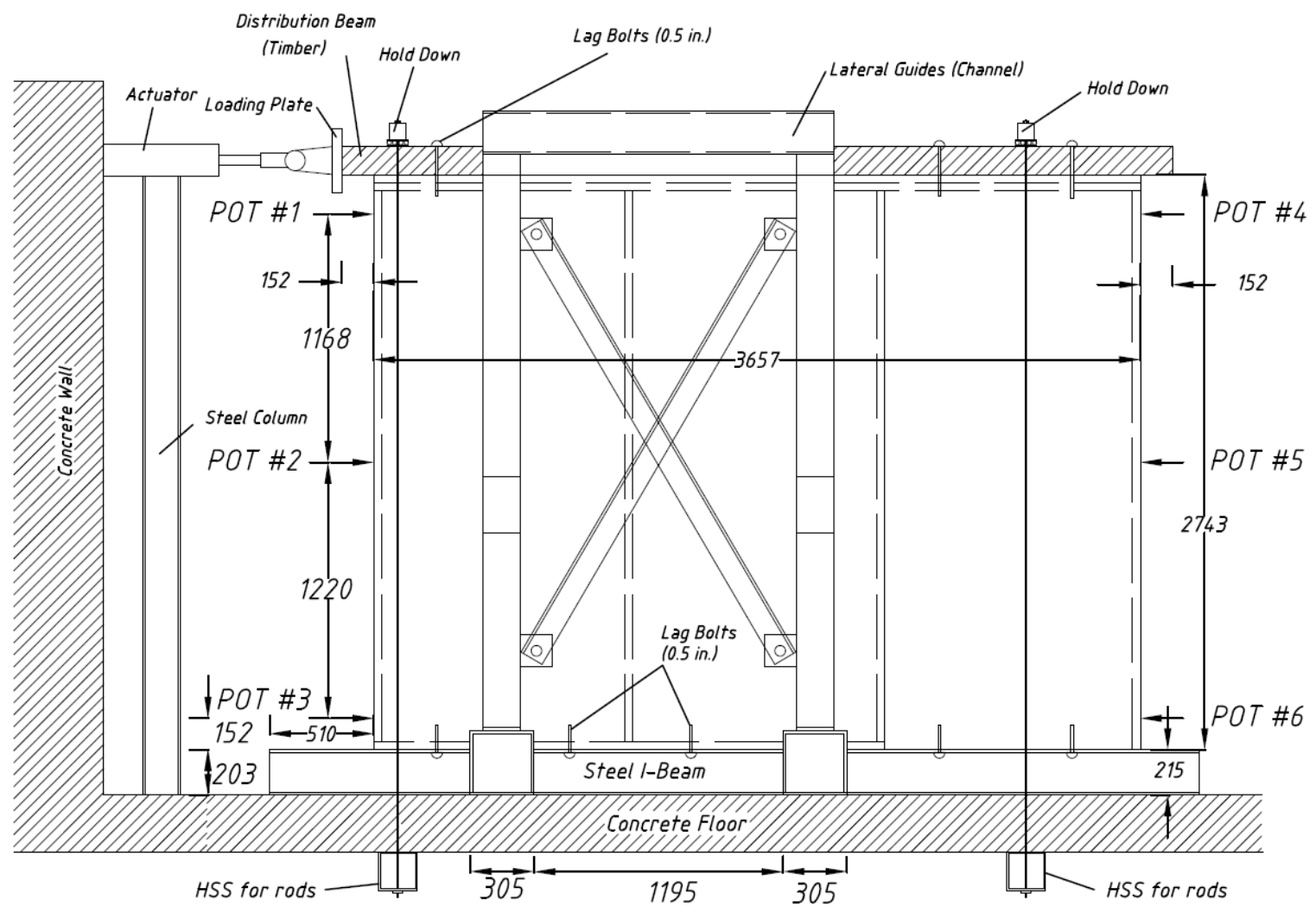

Figure 4.109 - Test setup of specimen SIP-C-4 under cyclic loading

A Second set of SIP walls was tested under cyclic loading. In this set, three identical SIP walls, namely SIP-C-4, SIP-C-5 and SIP-C-6, were selected for cyclic load test. The wall was of 3660 
$\mathrm{mm}$ length, $2750 \mathrm{~mm}$ height and $165 \mathrm{~mm}$ total thickness, with three SIP panels connected together using foam-spline connections. The wall has sheathing made of OSB sheets on each side of the wall. The wall was placed over a W200 I-beam that rest on the laboratory floor. The bottom plates of the wall were connected to the I-beam using twelve lag bolts in every $304.8 \mathrm{~mm}$ ( $1 \mathrm{ft}$ ) in order to provide a fully fixed support to the wall base. Tie-down system was used to hold the wall in position while applying the cyclic load at the top of the wall using an actuator as depicted in Fig. 4.109. Figure 4.110 shows the general failure mode of wall SIP-C-4 which was global shear deformation and relative shear rotation of SIP segments at spline connection.

Figure 4.111(a) shows view of the uplift of the rear side of the wall along with tearing and cracking of end vertical lumber at its fixation. Figure 4.111(b) shows view of bending of nails and crushing or tearing of OSB sheet at nail location, and separation of OSB sheet from the bottom plate. The relative rotation of the panel was also observed in vertical direction, causing crushing at top of the OSB sheet as depicted in Fig. 4.111(c). Figure 4.111(d) shows close-up view of the nail pullout at the middle of the panel at its connection to the top plate. Similar failure mode was observed for identical panels SIP-C-5 and SIP-C-6.

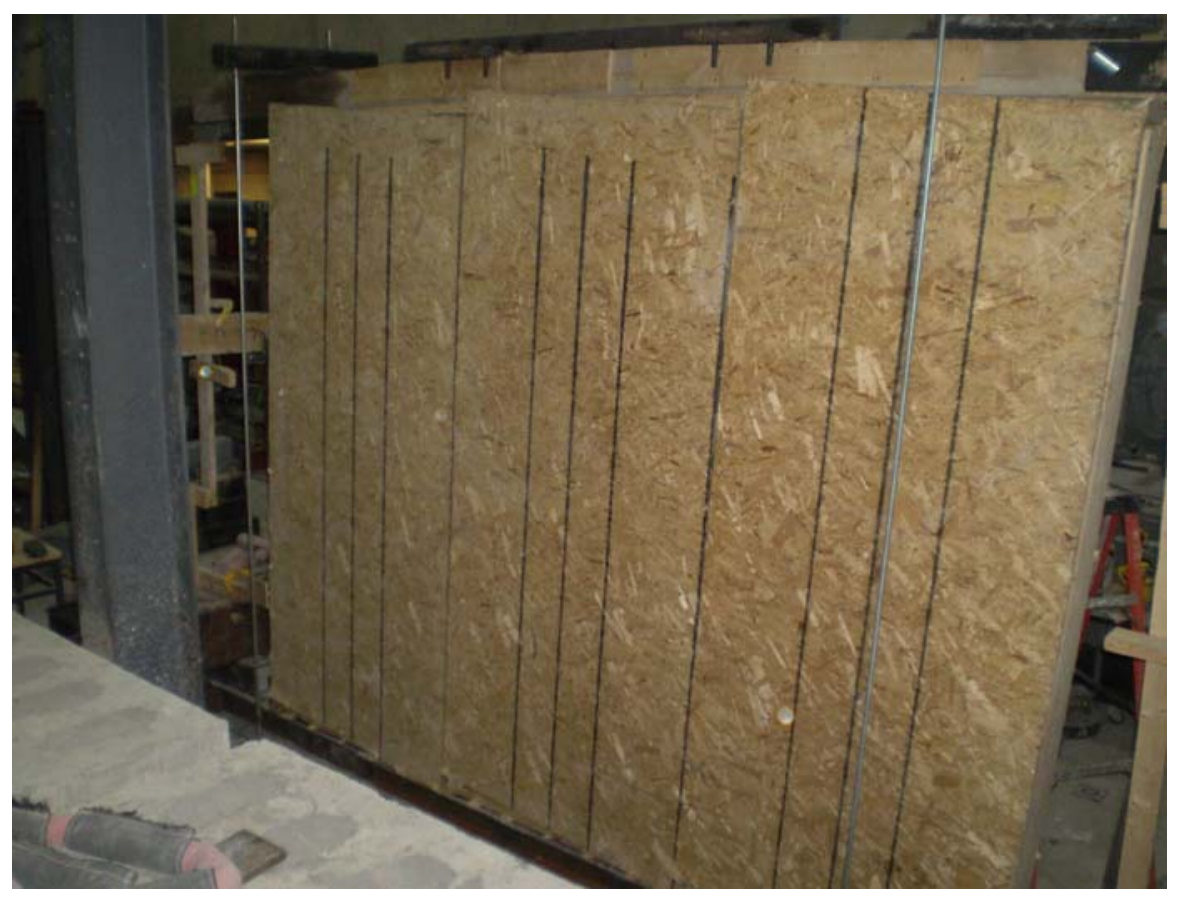

Figure 4.110 - Global failure of specimen SIP-C-4 under cyclic loading showing global shear deformation and relation shear rotation of SIP segments at spline connection 


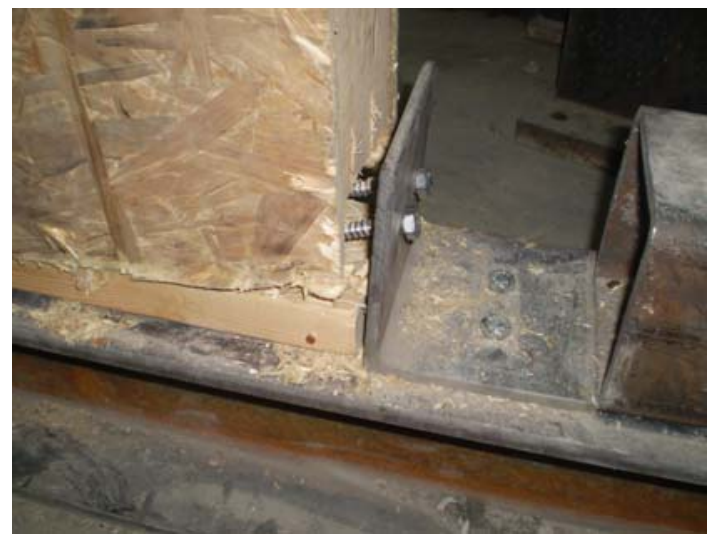

(a)Uplift, tearing and cracking of lumber at the bottom of the panel

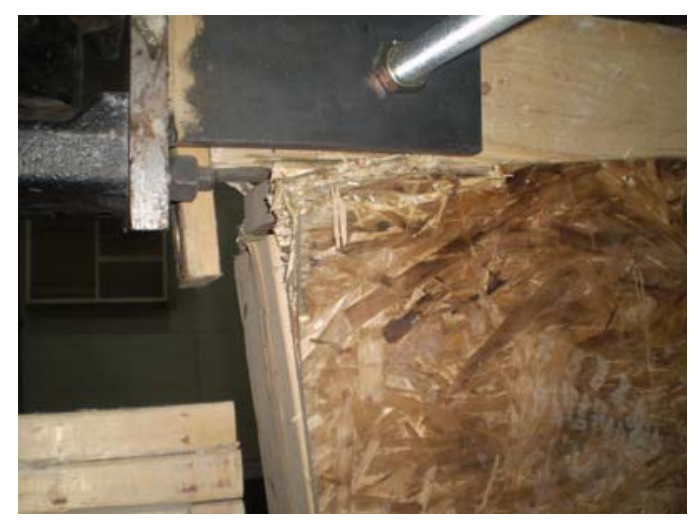

(c)Crushing OSB sheet at top of the panel

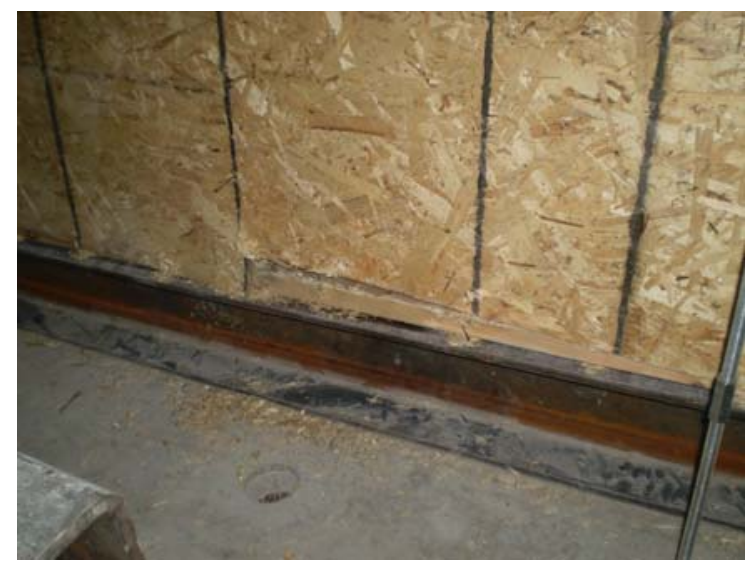

(b)Bending of nail and crushing of OSB sheet in nail location

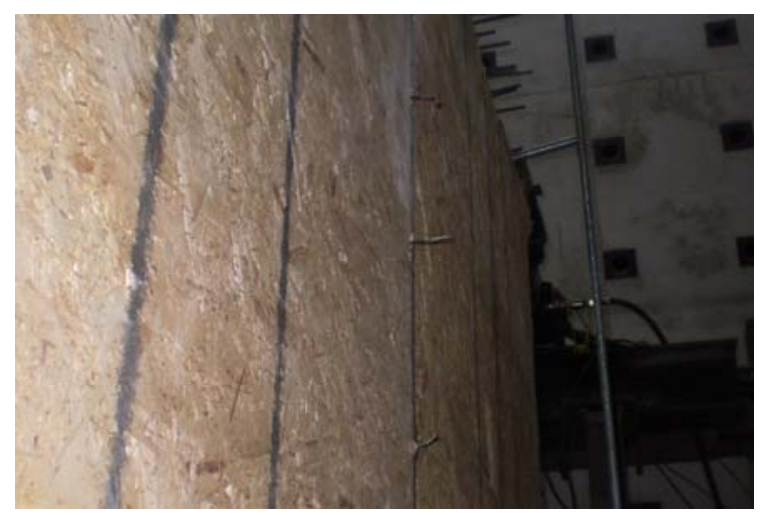

(d)Nail pull out at the middle of panel

Figure 4.111 - Detailed failure modes of specimen SIP-C-4 under cyclic load

Figures 4.112, 4.113 and 4.114 depict the racking load vs. lateral deflection relationships for at the top of walls SIP-C-1, SIP-C-2 and SIP-C-3, respectively. However, Figs. 4.118, 4.119 and 4.120 show the cyclic load vs. lateral deflection at different heights of walls SIP-C-1, SIP-C-2 and SIP-C-3, respectively. Results show that the ultimate racking loads for walls SIP-C-1, SIPC-2 and SIP-C-3, were 40.03, 33.40 and $39.37 \mathrm{kN}$, respectively. An average value, shown in Table 4.6, of $29.31 \mathrm{kN}$ showed superior performance of SIPs compared to stud wall system of identical geometry and average ultimate cyclic load of $11.75 \mathrm{kN}$. Figures 4.115, 4.116 and 4.117 depict the cyclic load vs. lateral deflection relationships for at the top of walls SIP-C-4, SIP-C-5 and SIP-C-6, respectively. However, Figs. 4.121, 4.122 and 4.123 show the cyclic load vs. lateral deflection at different heights of walls SIP-C-4, SIP-C-5 and SIP-C-6, respectively. Results show that the ultimate cyclic loads for walls SIP-C-4, SIP-C-5 and SIP-C-6, were 32.01, 28.82, and 
$36.37 \mathrm{kN}$, respectively. An average value, shown in Table 4.6, of $30.16 \mathrm{kN}$ showed that with increase in wall length from $2440 \mathrm{~mm}\left(8^{\prime}\right)$ to $3660 \mathrm{~mm}\left(12^{\prime}\right)$, the average cyclic load increased by $2.9 \%$.

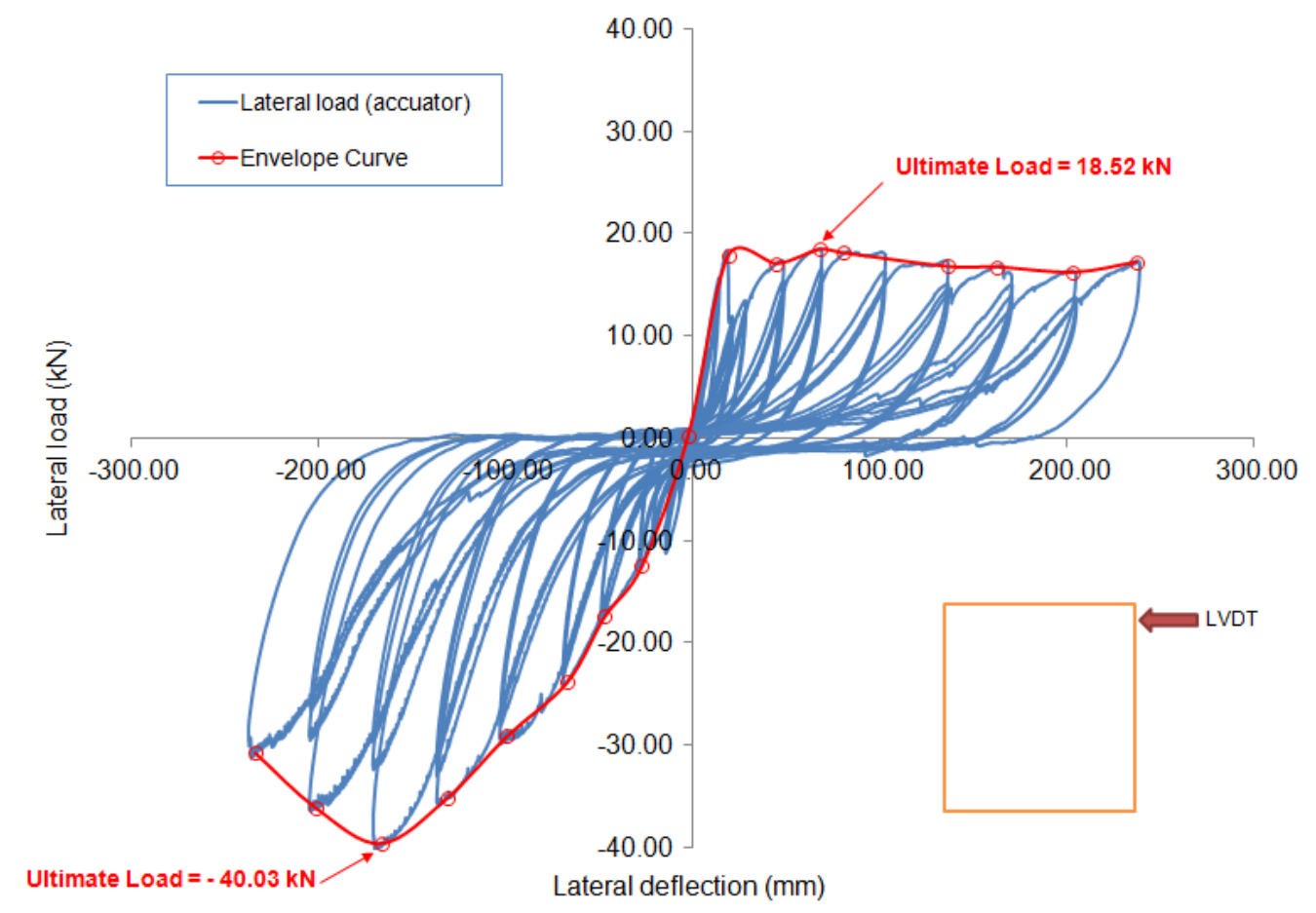

Figure 4.112 - Load vs. lateral deflection response of SIP-C-1 (obtained from actuator reading on top of the panel) 


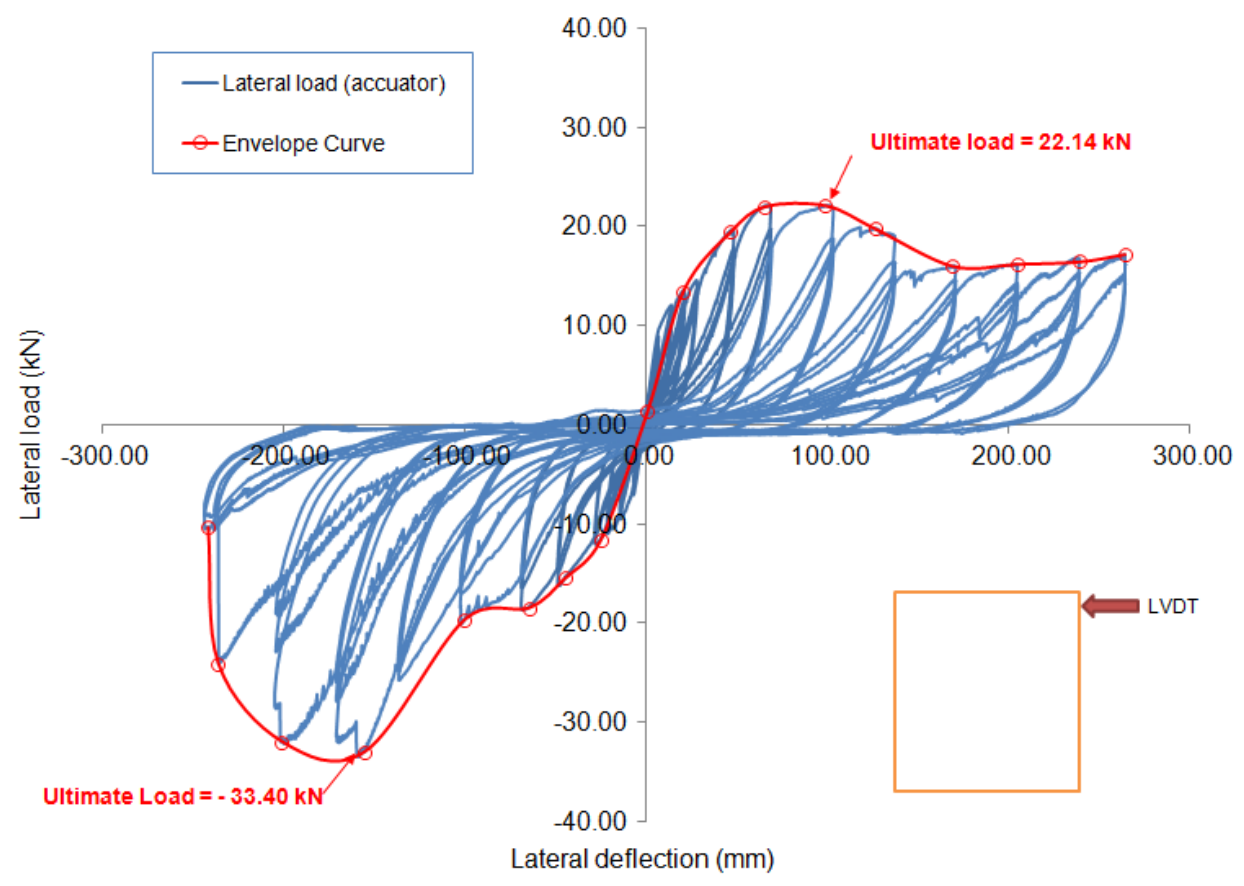

Figure 4.113 - Load vs. lateral deflection response of SIP-C-2 (obtained from actuator reading on top of the panel)

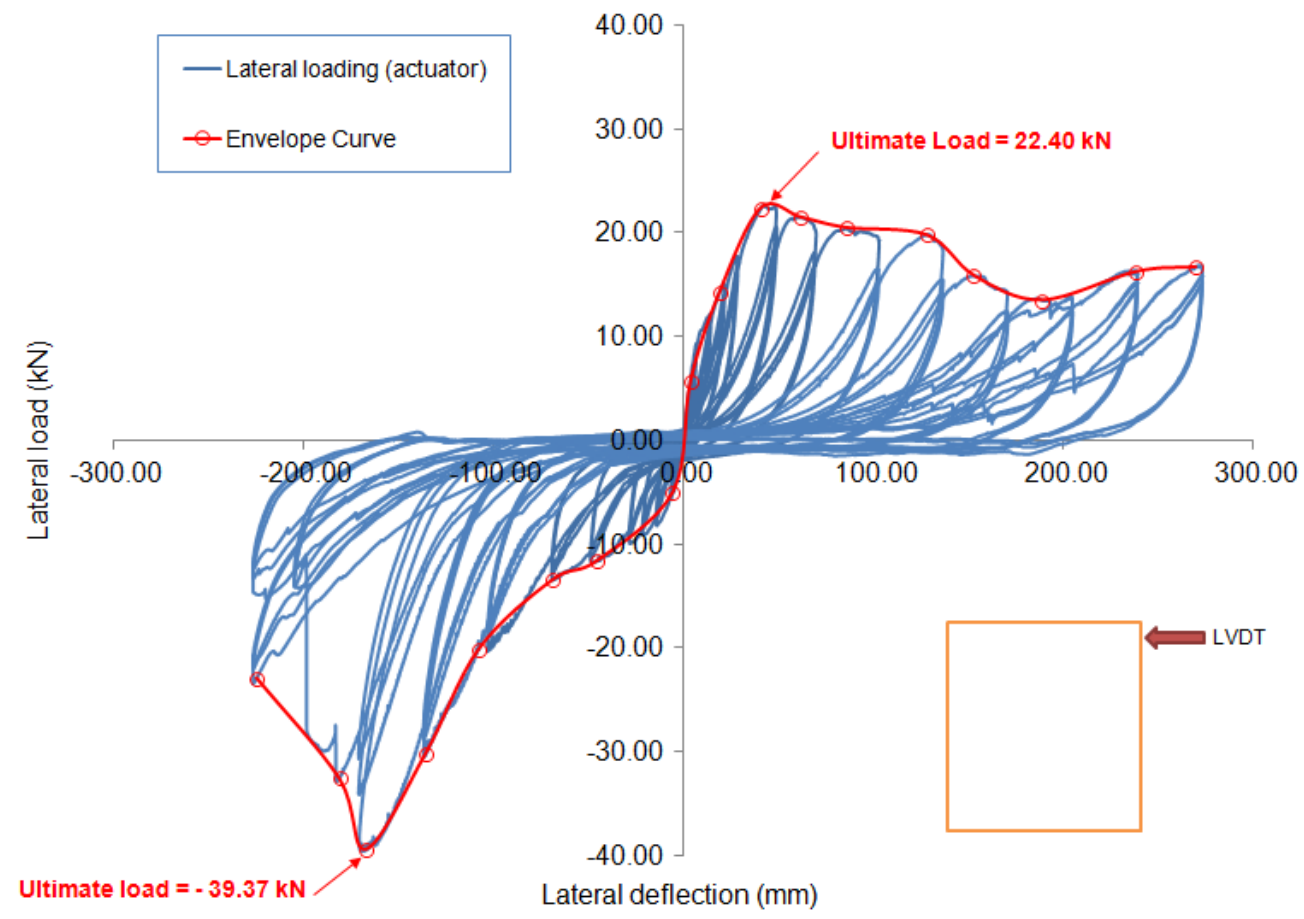

Figure 4.114 - Load vs. lateral deflection response of SIP-C-3 (obtained from actuator reading on top of the panel) 


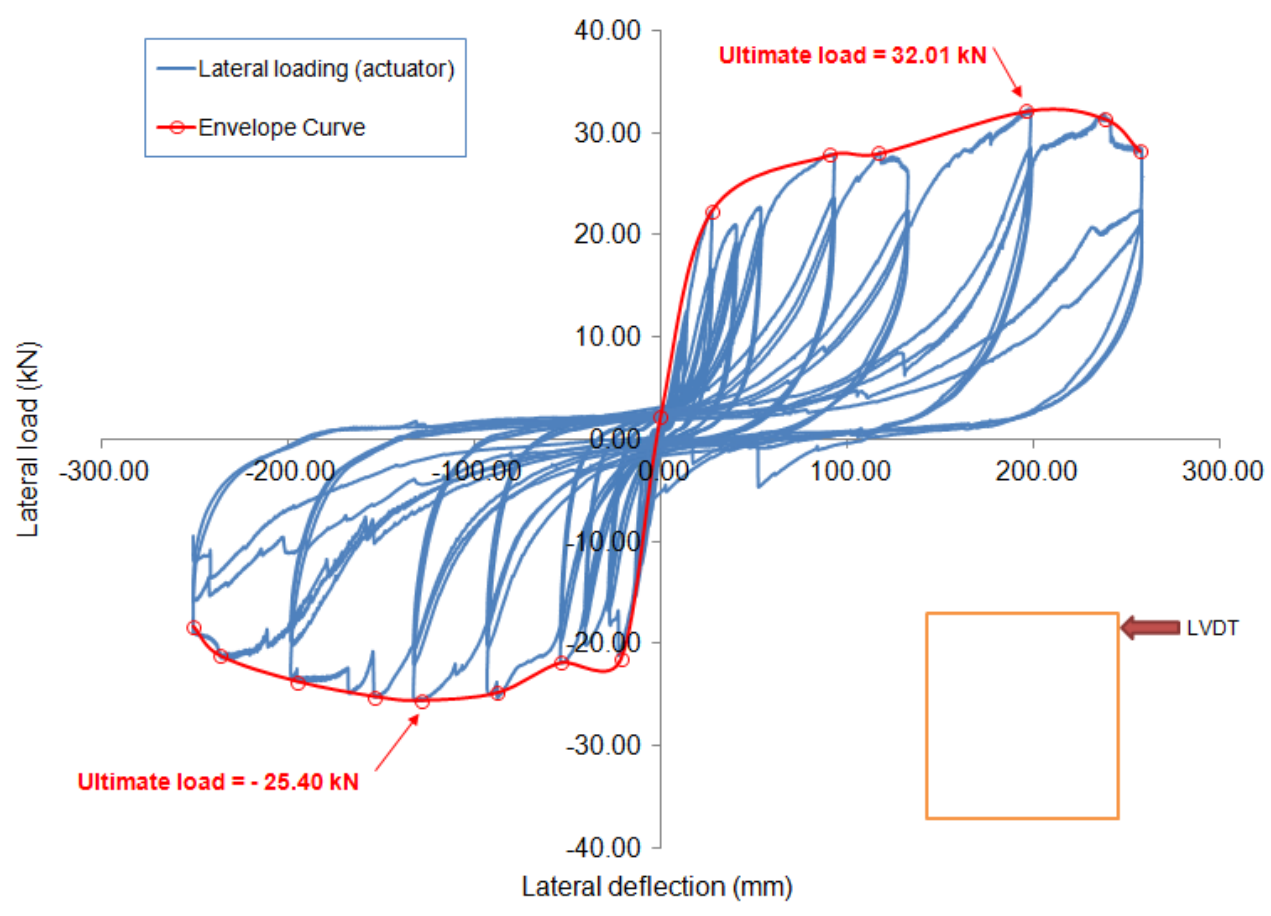

Figure 4.115 - Load vs. lateral deflection response of SIP-C-4 (obtained from actuator reading on top of the panel)

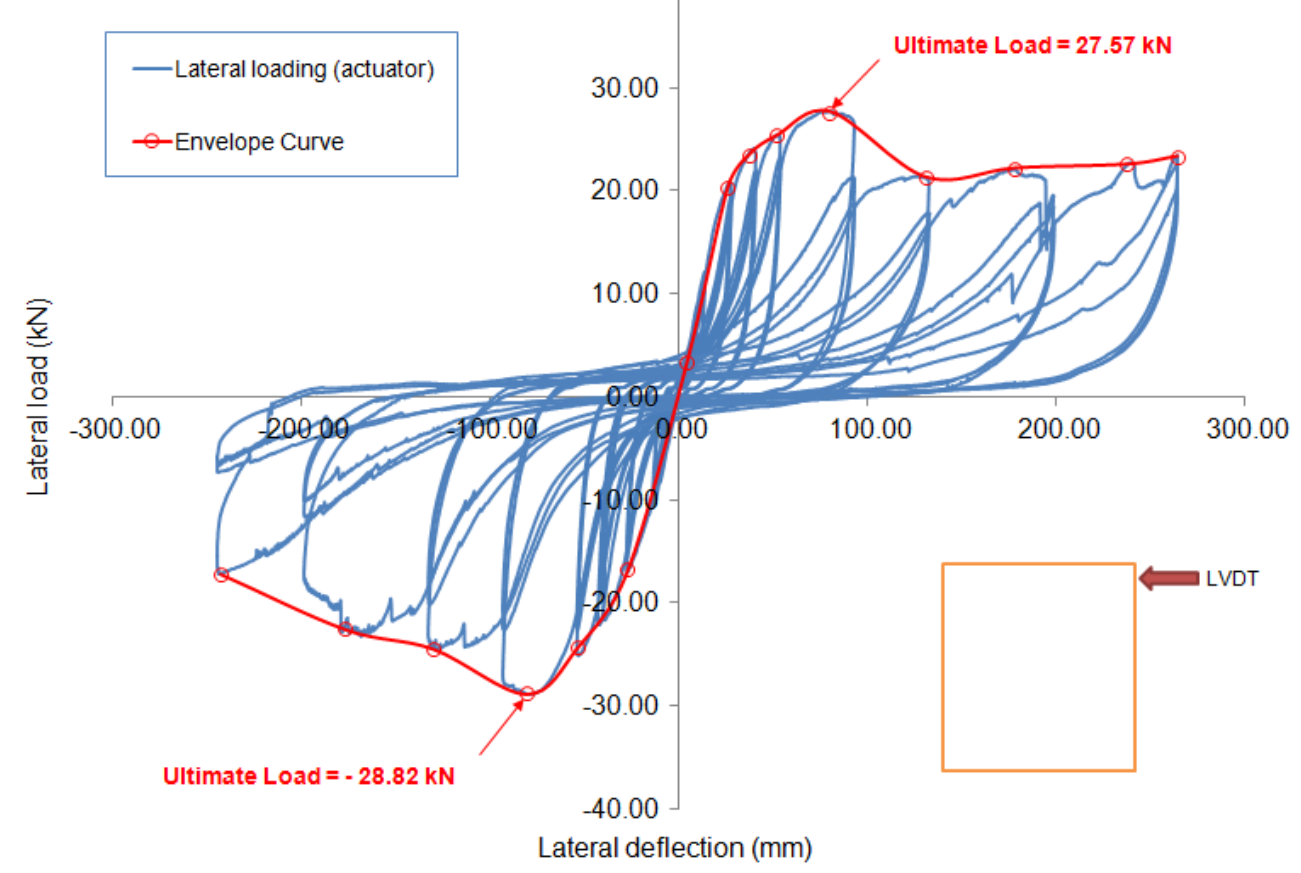

Figure 4.116 - Load vs. lateral deflection response of SIP-C-5 (obtained from actuator reading on top of the panel) 


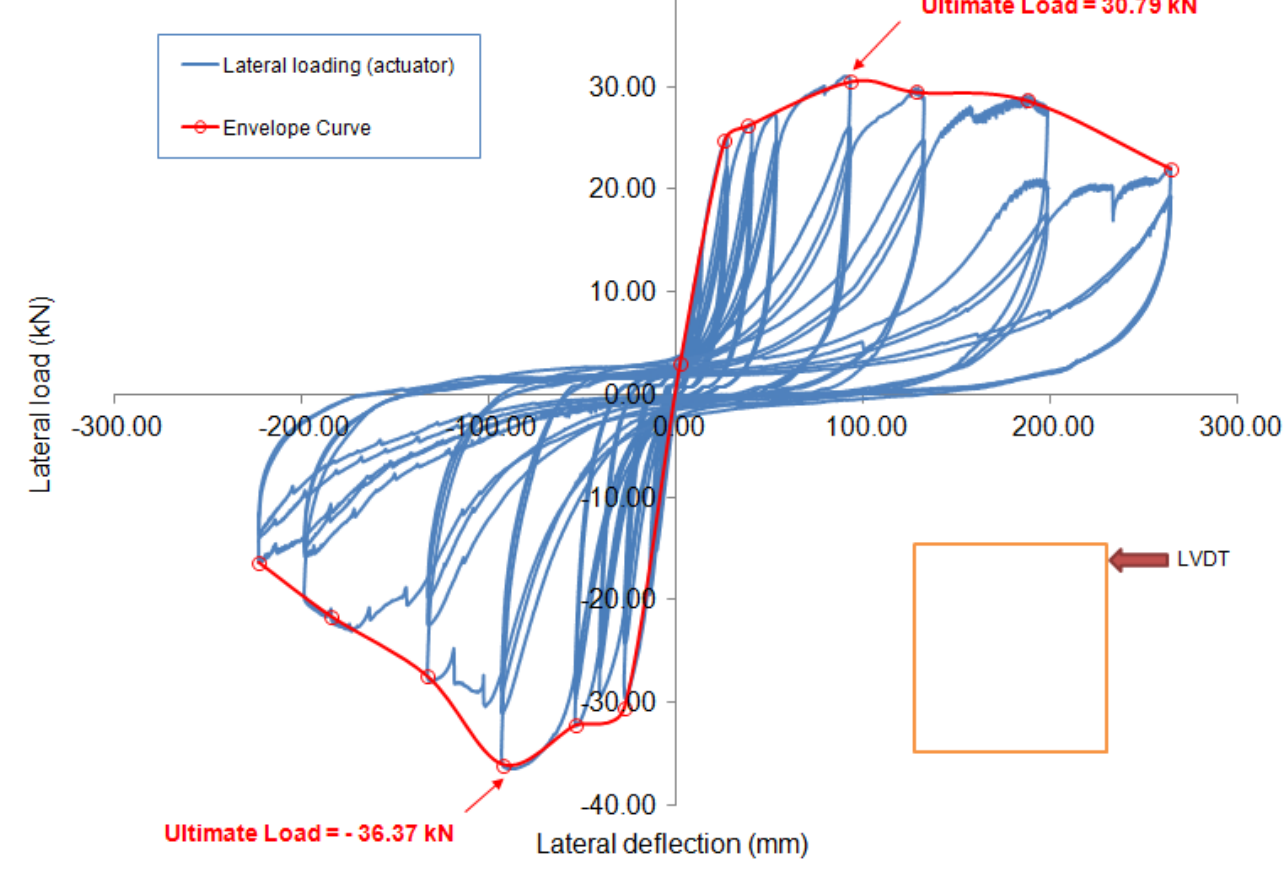

Figure 4.117 - Load vs. lateral deflection response of SIP-C-6 (obtained from actuator reading on top of the panel)
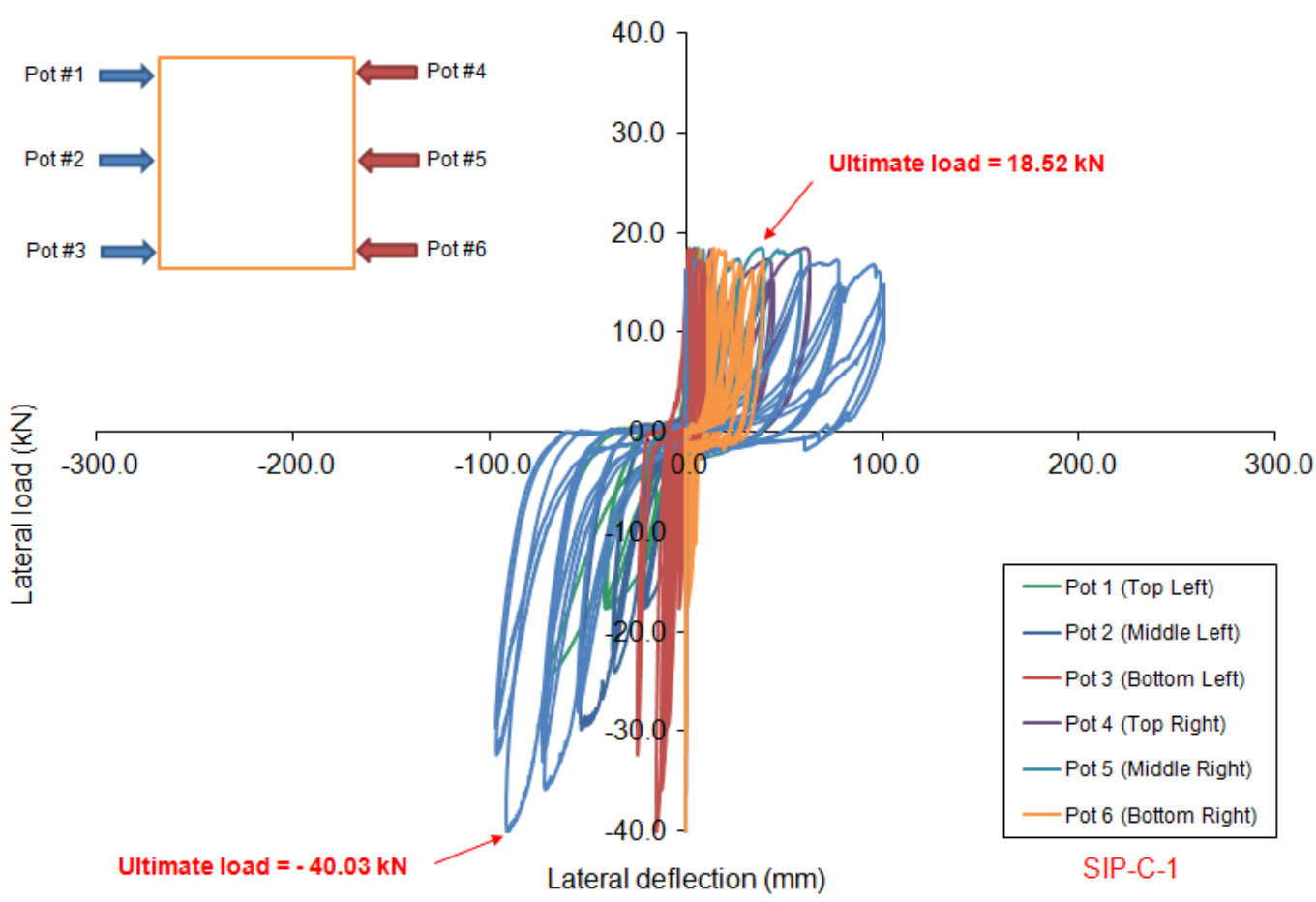

Figure 4.118 - Load vs. lateral deflection response of SIP-C-1 (POTs record up to a load close to the failure load) 


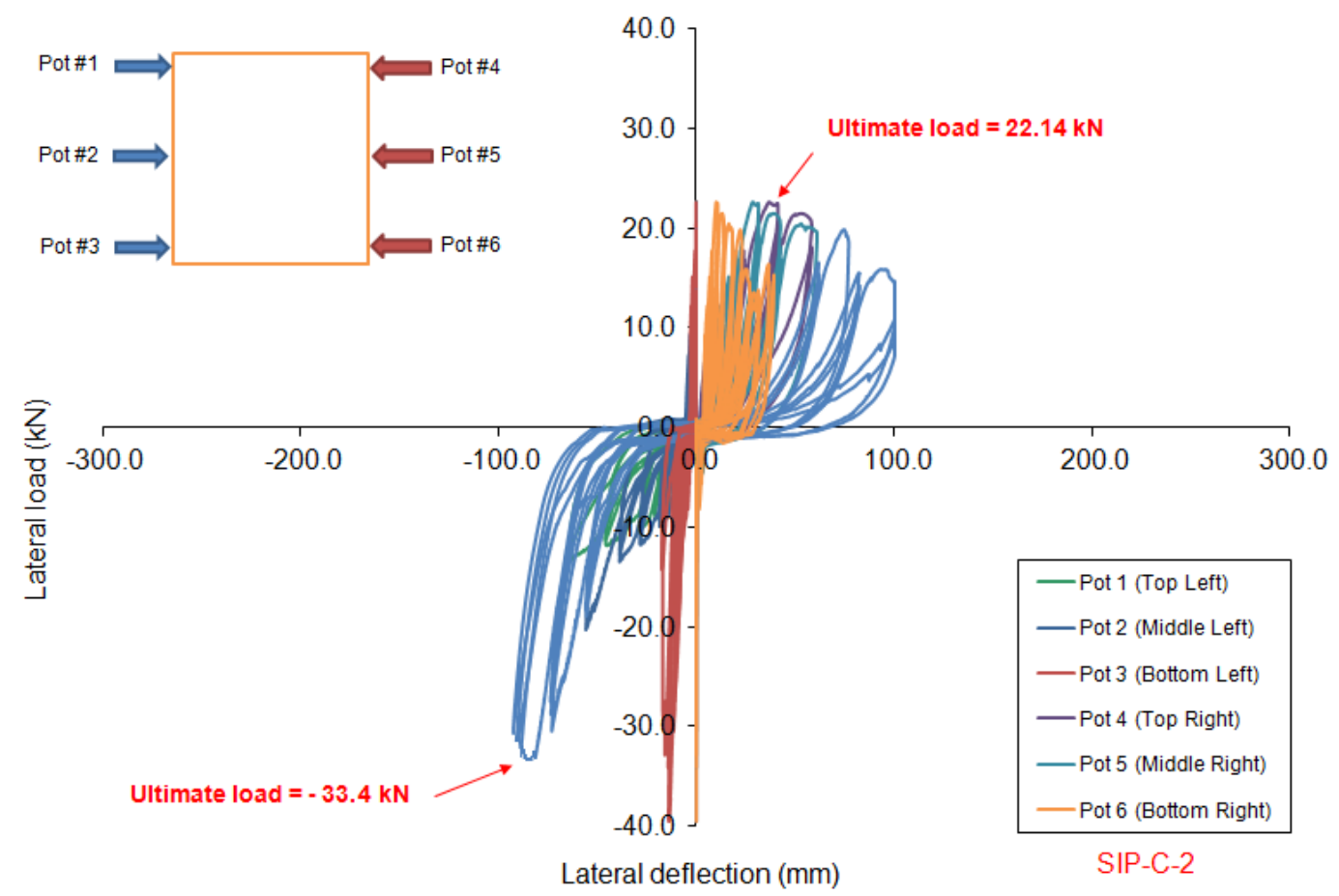

Figure 4.119 - Load vs. lateral deflection response of SIP-C-2 (POTs record up to a load close to the failure load)
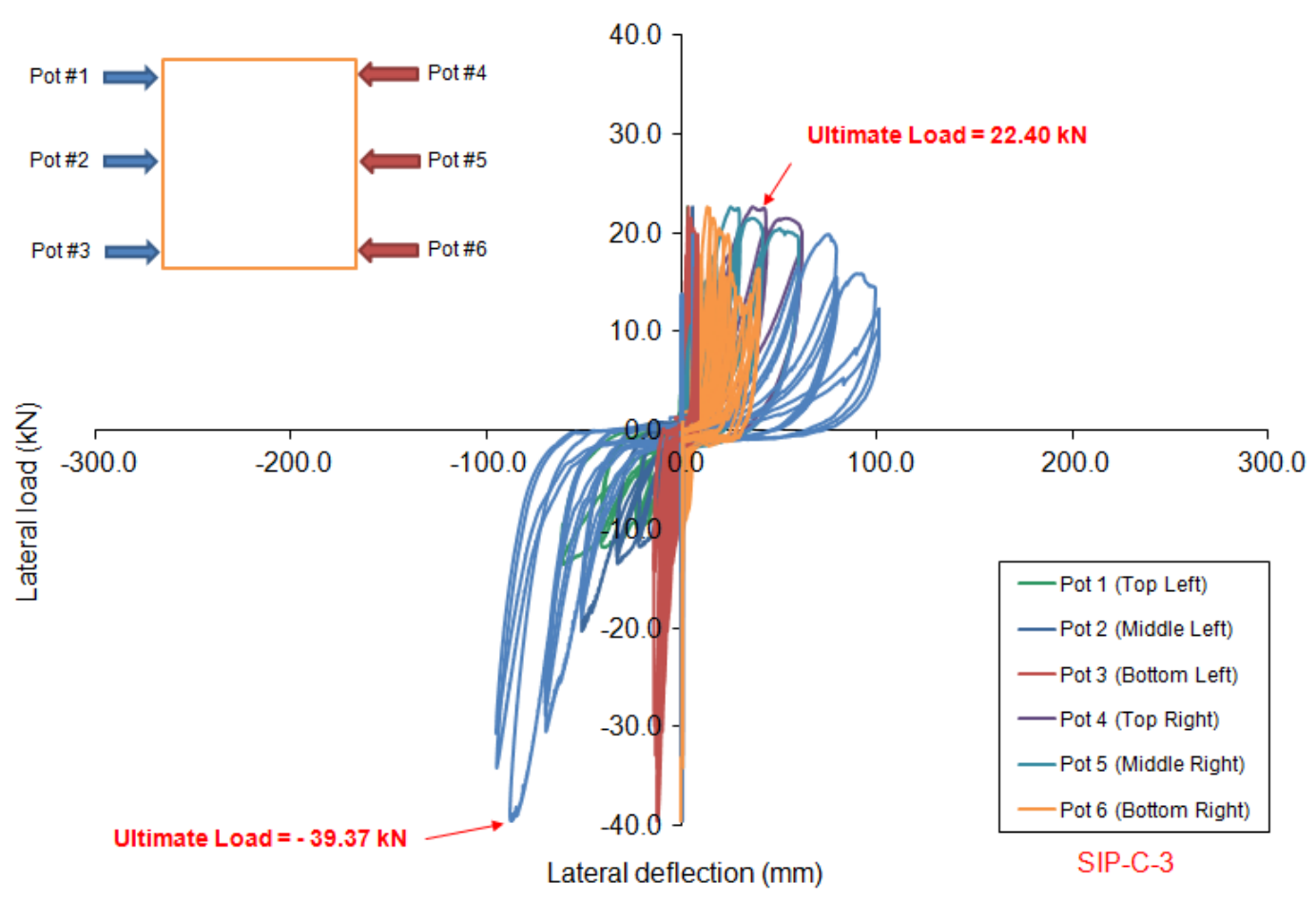

Figure 4.120 - Load vs. lateral deflection response of SIP-C-3 (POTs record up to a load close to the failure load) 


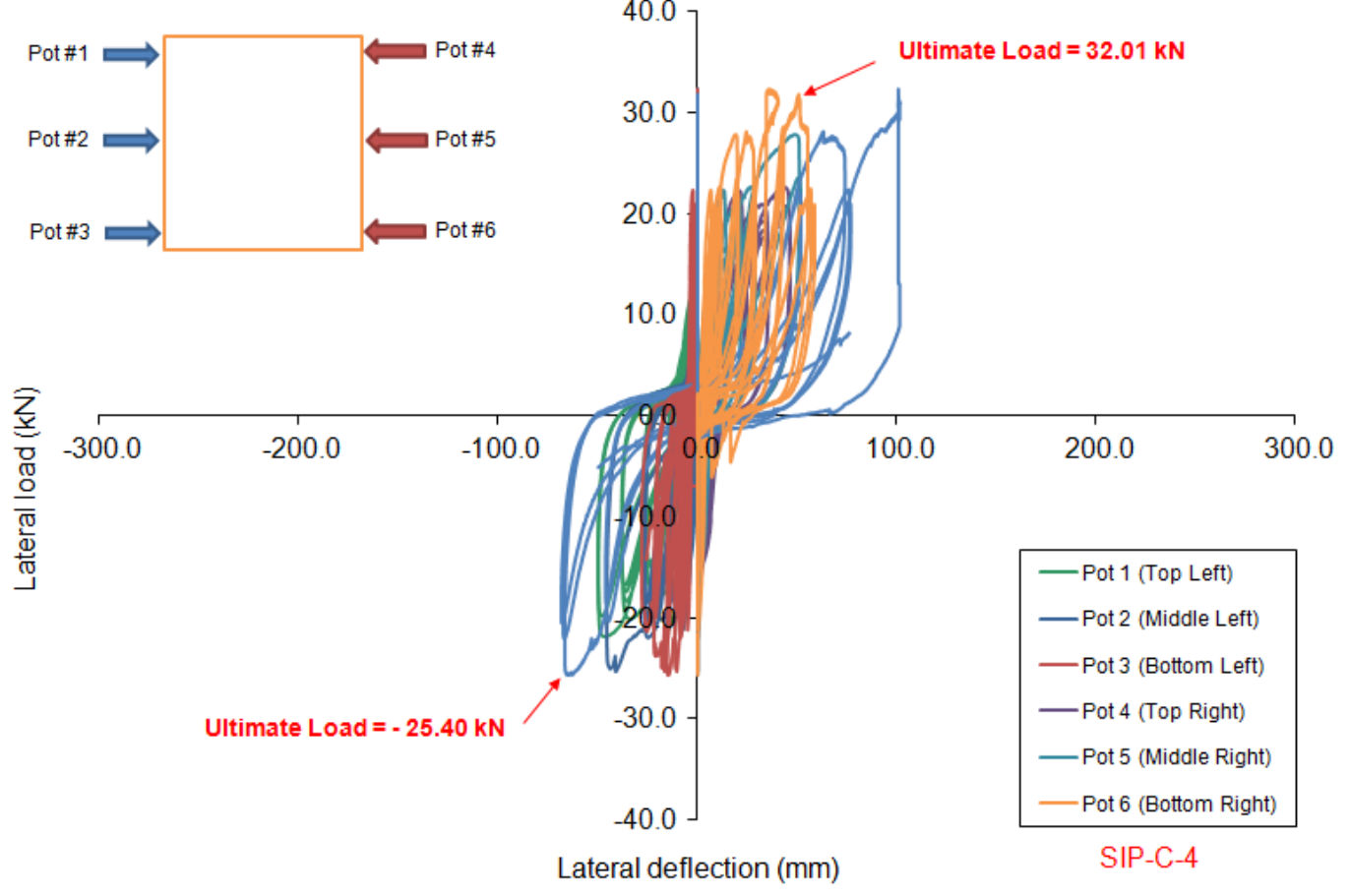

Figure 4.121 - Load vs. lateral deflection response of SIP-C-4 (POTs record up to a load close to the failure load)
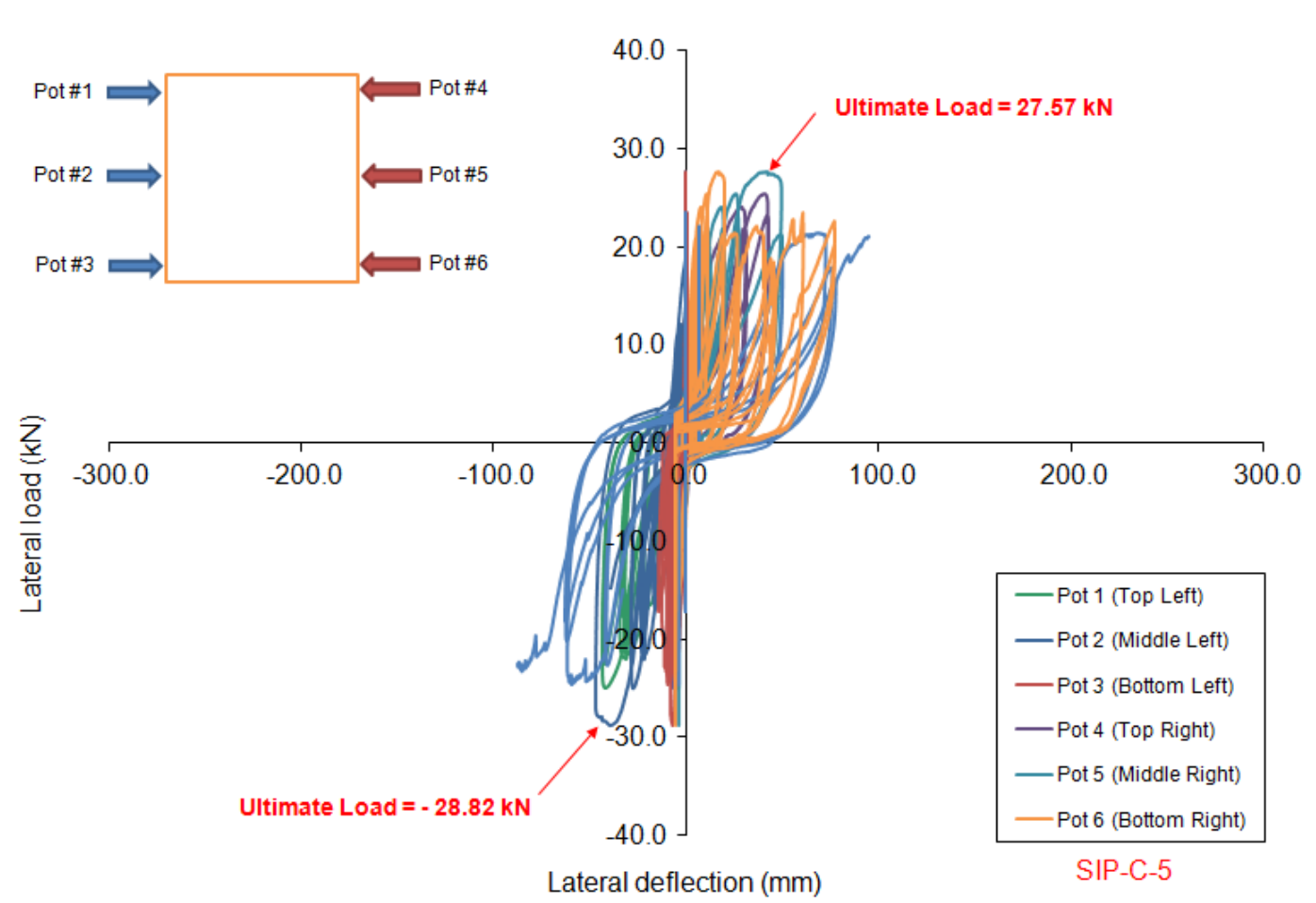

Figure 4.122 - Load vs. lateral deflection response of SIP-C-5 (POTs record up to a load close to the failure load) 

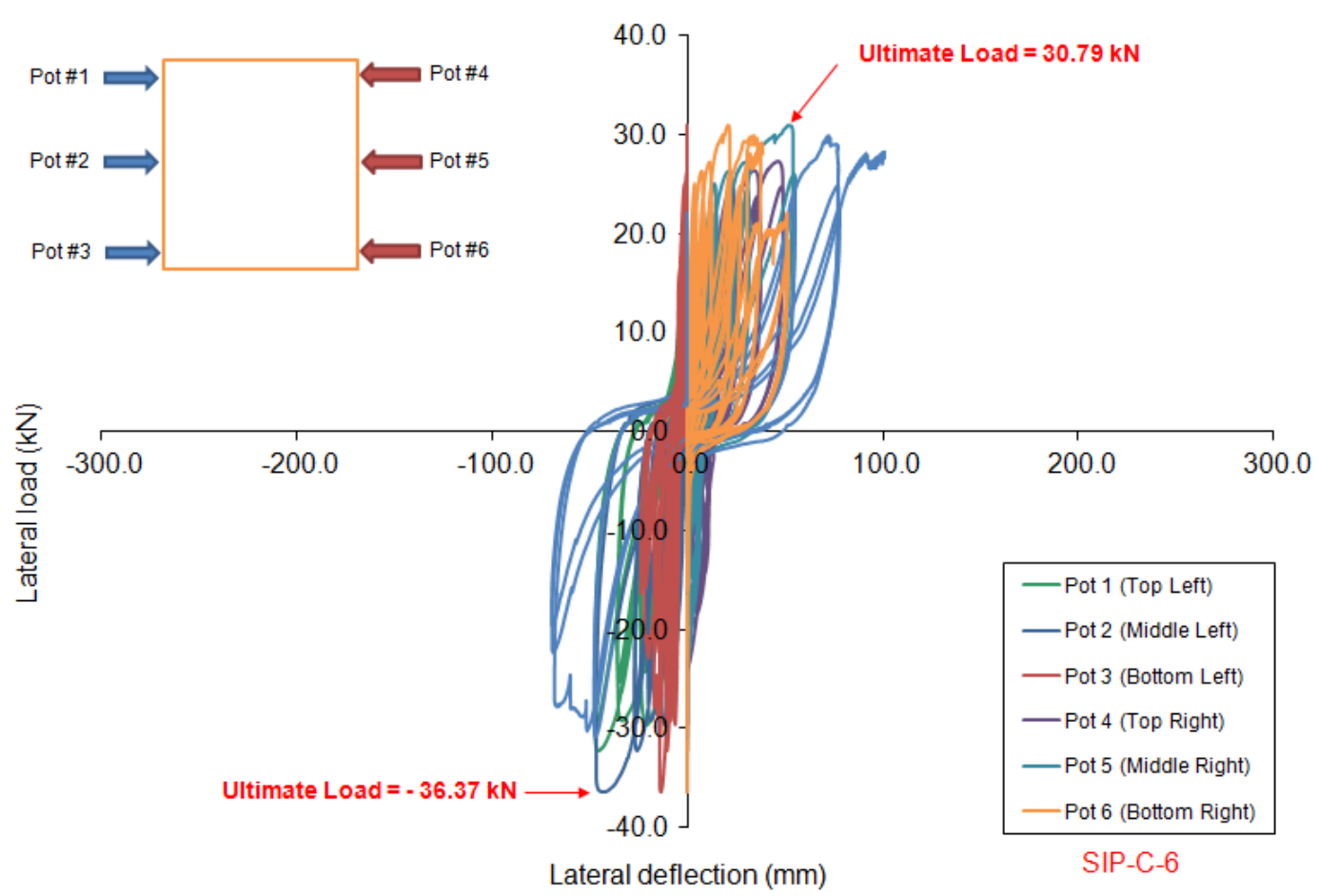

Figure 4.123 - Load vs. lateral deflection response of SIP-C-6 (POTs record up to a load close to the failure load)

\subsection{Equivalent Energy Elastic Plastic (EEEP)}

The Equivalent Energy Elastic Plastic (EEEP) method was presented by Porter (1987). In this method, a nonlinear inelastic system is transformed to a bilinear idealized elastic-plastic system. The load vs. deformation graph of cyclic test is used to determine the parameters identifying the EEEP bilinear curve. The two lines of this curve can be drawn in a way that the area under the curve of the actual system is equal to the area under these two lines. The area under the actual cyclic load history is determined with an imaginary vertical line projected up (or down) from 0.8 $F_{\text {peak }}$ on the degradation segment of the curve where $F_{\text {peak }}$ is the ultimate cyclic load reached experimentally. Therefore, the amount of energy dissipated of the actual nonlinear system is equal to the elastic-plastic system. The typical EEEP curve, cyclic load vs. deformation and envelope curve of the cyclic test for specimen SIP-C-6 are shown in Figure 4.124. 


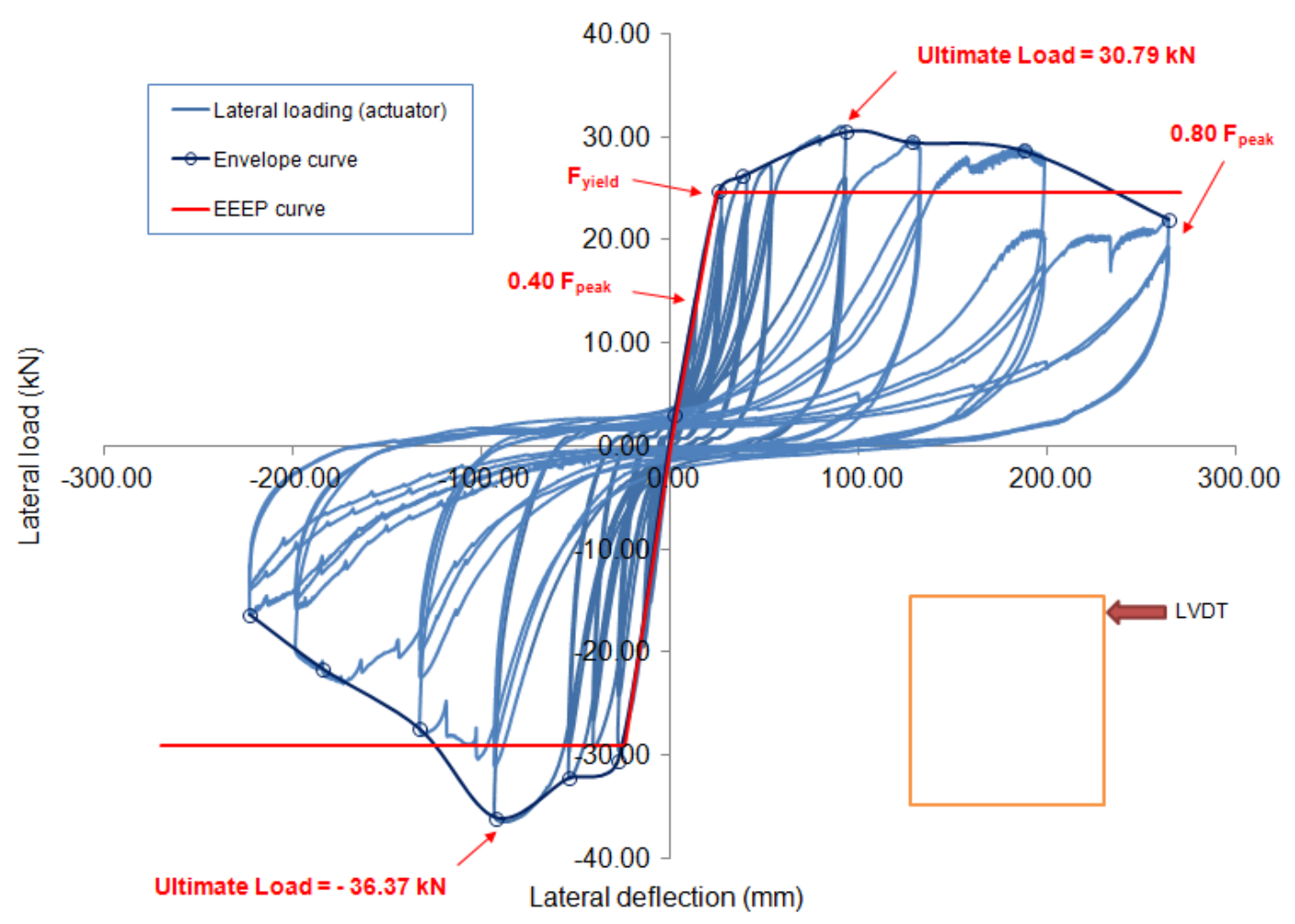

Figure 4.124 - Cyclic load vs. lateral deflection relationship, EEEP curve and envelope of the cyclic load history for Specimen SIP-C-6

The straight part of the EEEP curve represents the elastic properties of the system. It is drawn in a way that it passes through the point of $0.4 \mathrm{~F}_{\text {peak }}$ on the nonlinear curve of the actual system. The horizontal part of the EEEP curve represents the plastic properties of the system and it is located in a way that the energies dissipated by this curve and the actual cyclic load-deformation history of the wall are identical. The intersection of these two EEEP lines indicates the displacement and yield load, $F_{\text {yield, }}$ of the system. It should be noted that the actual energy of the system is much greater than the area under the envelope curve because many hysteresis of the cyclic test overlap on another. However, the EEEP method is considered an effective method to determine the performance characteristics of nonlinear and inelastic systems. It should also be mentioned that both negative and positive cyclic load values are produced in a cyclic test as depicted in Fig. 4.124. Table 4.7 shows the amount of energy dissipated on each cyclic test specimen. It can be observed that the average energy dissipated in the positive portion of the cyclic load history was $0.51,3.45$ and $6.13 \mathrm{kN}-\mathrm{m}$ for the stud wall, the short SIP wall and the long SIP wall, respectively. On the other hand, the average energy dissipated in the negative portion of the 
cyclic load history was $0.55,6.37$ and $4.23 \mathrm{kN}-\mathrm{m}$ for the stud wall, the short SIP wall and the long SIP wall, respectively. Results show that SIP's ability to dissipate energy during cyclic loading is in the range of 6 to 11 times that of the stud wall system.

Table 4.7 - Energy dissipated for each cyclic test specimen

\begin{tabular}{|c|c|c|c|c|c|c|c|c|}
\hline Specimen & $\begin{array}{c}\text { F }_{\text {peak }} \\
(\text { Pos.) } \\
(\mathbf{k N})\end{array}$ & $\begin{array}{c}0.8 \\
F_{\text {peak }} \\
(\text { Pos.) } \\
(\mathrm{kN})\end{array}$ & $\begin{array}{c}0.4 \text { F }_{\text {peak }} \\
(\text { Pos.) } \\
(\mathbf{k N})\end{array}$ & $\begin{array}{c}F_{\text {peak }} \\
(\mathrm{Neg} .) \\
(\mathbf{k N})\end{array}$ & $\begin{array}{c}0.8 \\
F_{\text {peak }} \\
(\mathrm{Neg} .) \\
(\mathrm{kN})\end{array}$ & $\begin{array}{c}0.4 \mathrm{~F}_{\text {peak }} \\
(\mathrm{Neg.}) \\
(\mathrm{kN})\end{array}$ & $\begin{array}{c}\text { Energy } \\
\text { (Pos.) } \\
(\mathrm{kN}-\mathrm{m})\end{array}$ & $\begin{array}{c}\text { Energy } \\
(\mathrm{Neg} .) \\
(\mathrm{kN}-\mathrm{m})\end{array}$ \\
\hline SW-C-1 & 9.16 & 7.33 & 3.66 & -12.30 & -9.84 & -4.92 & 0.473 & 0.574 \\
\hline SW-C-2 & 11.11 & 8.89 & 4.44 & -14.42 & -11.54 & -5.77 & 0.538 & 0.522 \\
\hline SIP-C-1 & 18.52 & 14.82 & 7.41 & -40.03 & -32.02 & -16.01 & 3.630 & 7.366 \\
\hline SIP-C-2 & 22.14 & 17.71 & 8.86 & -33.40 & -26.72 & -13.36 & 4.227 & 5.878 \\
\hline SIP-C-3 & 22.40 & 17.92 & 8.96 & -39.37 & -31.50 & -15.75 & 2.515 & 5.862 \\
\hline SIP-C-4 & 32.01 & 25.61 & 12.80 & -25.40 & -20.32 & -10.16 & 6.587 & 5.084 \\
\hline SIP-C-5 & 27.57 & 22.06 & 11.03 & -28.82 & -23.06 & -11.53 & 5.759 & 4.124 \\
\hline SIP-C-6 & 30.79 & 24.63 & 12.32 & -36.37 & -29.10 & -14.55 & 6.035 & 3.492 \\
\hline
\end{tabular}

\subsection{Strength and Displacement}

Load capacity of the wall defines the strength of the panel as the peak load $\left(\mathrm{F}_{\text {peak }}\right)$ in the cyclic load-deformation history. Given the wall ductility, when the load capacity of the wall reaches $0.8 \mathrm{~F}_{\text {peak }}$ on the degradation portion of the envelope curve, the wall is supposed to have failed. The yield strength of the system is calculated from the EEEP curve and the actual yield strength typically happens around $0.4 \mathrm{~F}_{\text {peak }}$. The lateral deflection of the wall can be expressed in the form of wall elastic stiffness, cyclic stiffness and ductility. In this research, the failure load is considered by multiplying peak load by 0.8 . The following sections explain the calculations of the wall ductility, elastic stiffness and cyclic stiffness for the sake of comparison between SIP walls and the conventional stud wall system.

\subsection{Ductility}

One of the most important of a structure to resist under seismic load is ductility. The ability of structure to deform and resist load without sudden failure is defined as ductility of structure. In 
this research, the ratio of the displacement at failure $\left(\Delta_{\text {failure }}\right)$ to the displacement at yield $\left(\Delta_{\text {yield }}\right)$ is presented as the ductility of the system through following equation:

$$
\mu=\frac{\Delta_{\text {failure }}}{\Delta_{\text {yield }}}
$$

Ductility ratio shows that the structure will not fail suddenly and the failure will not be brittle. However, the ductility factor may not be a direct indication of structure subjected to seismic loads. It is found that walls with no hold-down resistant system and with large ductility have large deformation due to large rigid body rotation. Thus, $\left(\Delta_{\text {failure }}\right)$ intensifies while $\left(\Delta_{\text {yield }}\right)$ has a low rate, leading to an increase in the ductility factor of the system. These types of walls do not have a good performance under seismic loads because they dissipate only minimum energy. Table 4.8 shows the calculations for the ductility ratio of the SIP and stud walls based on results from racking tests. While, Tables 4.9 and 4.10 show ductility calculations on the positive and negative portions, respectively, of cyclic load history of each specimen.

Based on the experimental racking test results, the average value of ductility ratio was calculated as 6.92 for the stud wall system, 6.38 for the short SIP wall and 5.27 for the long SIP wall. These values show that the ductility of the stud wall is slightly greater than that for SIP wall of the same size ( $8.4 \%$ difference). Also, it can be observed that with increase in length of SIP wall, ductility ratio decreases.

Based on cyclic load test results, the average value of ductility ratio was calculated as 4.19 for the stud wall, 4.35 for the short SIP wall and 4.14 for the long SIP wall. These values were calculated based on the ultimate load reached experimentally under cyclic loading. These values

show that the ductility ratio of the stud wall is slightly less than that for the SIP wall of the same size (3.8\% difference). Also, it can be observed that with increase in length of SIP wall, ductility ratio decreases.

\subsection{Elastic Stiffness}

The slope of the elastic line portion of the EEEP curve is called "Elastic stiffness" and is denoted as $\left(K_{e}\right)$. In fact, the origin and $0.4 \mathrm{~F}_{\text {peak }}$ on the envelope curve is used for secant stiffness 
determination. A good approximation of the elastic limit of a light-frame shear wall can be obtained from the load at $0.4 \mathrm{~F}_{\text {peak }}$. The corresponding displacement to $0.4 \mathrm{Fpeak}$ is considered and once the displacement is exceeded, the inelastic behavior of wall is reached, leading to permanent deformations of the wall. The following equation defines the elastic stiffness of the shear wall:

$$
K_{e}=\frac{0.4 F_{\text {peak }}}{\Delta_{0.4 F p e a k}}
$$

Table 4.8 shows the calculations for the wall elastic stiffness based on results from racking tests. While Tables 4.9 and 4.10 show the elastic stiffness calculations on the positive and negative portions, respectively, of cyclic load history of each specimen.

Based on racking load test results, the average value of elastic stiffness was calculated as 0.77 $\mathrm{kN} / \mathrm{mm}$ for the stud wall, $0.79 \mathrm{kN} / \mathrm{mm}$ for the short SIP wall and $0.99 \mathrm{kN} / \mathrm{mm}$ for the long SIP wall. These values show that the elastic stiffness of the stud wall is slightly less than that for SIP wall of the same size ( $2 \%$ difference). Also, it can be observed that with increase in length of SIP

wall, elastic stiffness increases. Based on cyclic load test results in the positive portion of the load history, the average value of elastic stiffness was calculated as $0.23 \mathrm{kN} / \mathrm{mm}$ for the stud wall, $0.55 \mathrm{kN} / \mathrm{mm}$ for the short SIP wall and $0.69 \mathrm{kN} / \mathrm{mm}$ for the long SIP wall. However, these values were calculated in the negative portion of the load history as $0.42 \mathrm{kN} / \mathrm{mm}$ for the stud wall, $0.29 \mathrm{kN} / \mathrm{mm}$ for the short SIP wall and $0.54 \mathrm{kN} / \mathrm{mm}$ for the long SIP wall. Such discrepancies in the results between the positive and negative portions of the load history lead to the conclusion that the results from racking testing are more reliable.

\subsection{Cyclic Stiffness}

The cyclic stiffness can define for each cycle as $\left(\mathrm{k}_{\mathrm{c}}\right)$. The stiffness of each cycle is considered the slope of the line passing through the point of the peak load and the origin on the load vs. displacement graph. Each cycle has two slopes and peak loads in positive and negative direction and is averaged in a cyclic test procedure. The ability of the structure to sustain loads at various amounts of displacement is provided by this parameter. It should be noted that the nail slip after elastic limit and crushing of the fibers in sheathing and framing around the sheathing nail are the results of stiffness degradation of shear wall. Table 4.11 to 4.18 shows the values of the cyclic 
stiffness for 10 cycles of the load history for each specimen. Figures 4.125 to 4.127 show the cyclic stiffness vs. peak displacement for stud wall, short and long SIP specimen, respectively. These graphs clearly indicate the degradation of the stiffness in structure under increasing cyclic loading. Figures 4.128 to 4.130 show the hysteretic energy dissipated per cycle vs. displacement for stud wall, short and long SIP specimen, respectively. Results show SIP panels has great ability to dissipate energy under cyclic loading compared to the stud wall system. For example, the maximum value of energy dissipated per cycle is about $550 \mathrm{kN}-\mathrm{mm}$ for the stud wall, 7200 $\mathrm{kN}-\mathrm{mm}$ for the short SIP wall and $7500 \mathrm{kN}-\mathrm{mm}$ for the long SIP wall. This indicates that the energy dissipated by SIP wall in seismic zone is about 12 times greater than that for the stud wall. Therefore, the SIP wall can be used so efficient in seismic zones. 
Table 4.8 - Elastic stiffness, Ke, ductility, $\mu,\left(\mathrm{R}_{\mathrm{o}}\right)$ and $\left(\mathrm{R}_{\mathrm{d}}\right)$ for specimen subjected to racking loading

\begin{tabular}{|c|c|c|c|c|c|c|c|c|c|c|c|c|c|}
\hline Specimen & $\begin{array}{l}F_{\text {peak }} \\
(\mathbf{k N})\end{array}$ & $\begin{array}{l}\Delta_{\text {peak }} \\
(\mathrm{mm})\end{array}$ & $\begin{array}{l}F_{\text {yield }} \\
(\mathrm{kN})\end{array}$ & $\begin{array}{l}\Delta_{\text {yield }} \\
(\mathrm{mm})\end{array}$ & $\begin{array}{c}0.4 F_{\text {peak }} \\
(\mathbf{k N})\end{array}$ & $\begin{array}{c}\Delta @ 0.4 F_{\text {peak }} \\
(\mathbf{m m})\end{array}$ & $\begin{array}{c}0.8 F_{\text {peak }} \\
(\mathrm{kN})\end{array}$ & $\begin{array}{c}\Delta @ 0.8 F_{\text {peak }} \\
(\mathbf{m m})\end{array}$ & $\begin{array}{l}\Delta_{\text {failure }} \\
(\mathrm{mm})\end{array}$ & $\begin{array}{c}\mathrm{K}_{\mathrm{e}} \\
(\mathrm{kN} / \mathrm{mm})\end{array}$ & $\begin{array}{c}\text { Ductility } \\
\text { Ratio } \\
(\mu)\end{array}$ & $\mathbf{R}_{\mathbf{0}}$ & $\mathbf{R}_{\mathbf{d}}$ \\
\hline SW-R-1 & 13.70 & 48.28 & 11.23 & 7.65 & 5.48 & 6.76 & 10.96 & 24.41 & 58.41 & 0.811 & 7.64 & 2.11 & 3.78 \\
\hline SW-R-2 & 9.74 & 52.08 & 8.96 & 7.96 & 3.90 & 5.33 & 7.79 & 11.15 & 49.25 & 0.731 & 6.19 & 1.88 & 3.37 \\
\hline SIP-R-1 & 25.32 & 103.88 & 21.02 & 22.74 & 10.13 & 16.19 & 20.26 & 57.13 & 157.62 & 0.626 & 6.93 & 2.08 & 3.59 \\
\hline SIP-R-2 & 30.87 & 130.73 & 27.47 & 50.44 & 12.35 & 24.21 & 24.70 & 68.48 & 225.86 & 0.510 & 4.48 & 1.94 & 2.82 \\
\hline SIP-R-3 & 22.45 & 107.96 & 18.18 & 30.94 & 8.98 & 7.29 & 17.96 & 40.29 & 239.23 & 1.232 & 7.73 & 2.14 & 3.80 \\
\hline SIP-R-4 & 40.86 & 161.47 & 36.77 & 50.39 & 16.34 & 28.92 & 32.69 & 99.48 & 275.81 & 0.565 & 5.47 & 1.92 & 3.15 \\
\hline SIP-R-5 & 38.94 & 195.57 & 34.27 & 55.67 & 15.58 & 13.09 & 31.15 & 59.52 & 294.08 & 1.190 & 5.28 & 1.97 & 3.09 \\
\hline SIP-R-6 & 40.65 & 155.28 & 36.99 & 54.82 & 16.26 & 13.5 & 32.52 & 98.7 & 276.81 & 1.204 & 5.05 & 1.90 & 3.02 \\
\hline
\end{tabular}


Table 4.9 - Elastic stiffness. $K_{e}$, ductility, $\mu,\left(R_{o}\right)$ and $\left(R_{d}\right)$ for specimens subjected to cyclic loading (positive portion)

\begin{tabular}{|c|c|c|c|c|c|c|c|c|c|c|c|c|}
\hline Specimen & $\begin{array}{l}F_{\text {peak }} \\
(\mathbf{k N})\end{array}$ & $\begin{array}{l}\Delta_{\text {peak }} \\
(\mathrm{mm})\end{array}$ & $\begin{array}{l}F_{\text {yield }} \\
(\mathrm{kN})\end{array}$ & $\begin{array}{l}\Delta_{\text {yield }} \\
\text { (mm) }\end{array}$ & $\begin{array}{c}0.4 F_{\text {peak }} \\
(\mathbf{k N})\end{array}$ & $\begin{array}{c}\Delta @ 0.4 F_{\text {peak }} \\
(\mathrm{mm})\end{array}$ & $\begin{array}{c}0.8 F_{\text {peak }} \\
(\mathbf{k N})\end{array}$ & $\begin{array}{c}\Delta @ 0.8 F_{\text {peak }} \\
(\mathrm{mm})\end{array}$ & $\begin{array}{c}\mathrm{K}_{\mathrm{e}} \\
(\mathrm{kN} / \mathrm{mm})\end{array}$ & $\begin{array}{c}\text { Ductility } \\
\text { Ratio } \\
(\mu)\end{array}$ & $\mathbf{R}_{\mathbf{0}}$ & $\mathbf{R}_{\mathbf{d}}$ \\
\hline SW-C-1 & 9.16 & 59.94 & 7.79 & 13.80 & 3.66 & 15.44 & 7.33 & 65.27 & 0.237 & 4.73 & 2.04 & 2.91 \\
\hline SW-C-2 & 11.11 & 39.38 & 10.00 & 14.12 & 4.44 & 18.49 & 8.89 & 60.49 & 0.240 & 4.28 & 1.92 & 2.75 \\
\hline SIP-C-1 & 18.52 & 80.73 & 15.93 & 6.22 & 7.41 & 11.07 & 14.82 & 12.80 & 0.669 & 2.06 & 2.01 & 1.77 \\
\hline SIP-C-2 & 22.14 & 98.76 & 20.15 & 8.70 & 8.86 & 17.36 & 17.71 & 18.50 & 0.510 & 2.13 & 1.90 & 1.80 \\
\hline SIP-C-3 & 22.40 & 40.44 & 19.26 & 10.28 & 8.96 & 19.47 & 17.92 & 29.46 & 0.460 & 2.87 & 2.01 & 2.18 \\
\hline SIP-C-4 & 32.01 & 198.30 & 28.17 & 18.92 & 12.80 & 14.45 & 25.61 & 68.94 & 0.886 & 3.64 & 1.97 & 2.51 \\
\hline SIP-C-5 & 27.57 & 79.76 & 25.36 & 12.49 & 11.03 & 21.77 & 22.06 & 39.76 & 0.507 & 3.18 & 1.88 & 2.32 \\
\hline SIP-C-6 & 30.79 & 92.88 & 26.79 & 8.16 & 12.32 & 18.23 & 24.63 & 25.72 & 0.676 & 3.15 & 1.99 & 2.30 \\
\hline
\end{tabular}


Table 4.10 - Elastic stiffness. $K_{e}$, ductility, $\mu,\left(R_{o}\right)$ and $\left(R_{d}\right)$ for specimens subjected to cyclic loading (negative portion)

\begin{tabular}{|c|c|c|c|c|c|c|c|c|c|c|c|c|}
\hline Specimen & $\begin{array}{l}\mathbf{F}_{\text {peak }} \\
(\mathbf{k N})\end{array}$ & $\begin{array}{l}\Delta_{\text {peak }} \\
(\mathrm{mm})\end{array}$ & $\begin{array}{l}F_{\text {yield }} \\
(\mathbf{k N})\end{array}$ & $\begin{array}{l}\Delta_{\text {yield }} \\
(\mathbf{m m})\end{array}$ & $\begin{array}{c}0.4 F_{\text {peak }} \\
(\mathbf{k N})\end{array}$ & $\begin{array}{c}\Delta @ 0.4 F_{\text {peak }} \\
(\mathrm{mm})\end{array}$ & $\begin{array}{c}0.8 F_{\text {peak }} \\
(\mathbf{k N})\end{array}$ & $\begin{array}{c}\Delta @ 0.8 F_{\text {peak }} \\
(\mathrm{mm})\end{array}$ & $\begin{array}{c}K_{e} \\
(\mathbf{k N} / \mathbf{m m})\end{array}$ & $\begin{array}{c}\text { Ductility } \\
\text { Ratio } \\
(\mu)\end{array}$ & $\mathbf{R}_{\mathbf{0}}$ & $\mathbf{R}_{\mathbf{d}}$ \\
\hline SW-C-1 & -12.30 & -45.83 & -3.69 & -6.18 & -4.92 & -11.64 & -9.84 & -22.49 & 0.423 & 3.64 & 2.11 & 2.51 \\
\hline SW-C-2 & -14.42 & -38.49 & -4.33 & -9.52 & -5.77 & -13.70 & -11.54 & -45.12 & 0.421 & 4.74 & 1.90 & 2.91 \\
\hline SIP-C-3 & -39.37 & -166.88 & -11.81 & -30.88 & -15.75 & -95.63 & -31.50 & -145.21 & 0.165 & 4.70 & 1.99 & 2.90 \\
\hline SIP-C-4 & -25.40 & -132.60 & -7.62 & -13.29 & -10.16 & -20.19 & -20.32 & -61.29 & 0.503 & 4.61 & 1.88 & 2.87 \\
\hline SIP-C-5 & -28.82 & -80.92 & -8.65 & -12.18 & -11.53 & -25.36 & -23.06 & -58.74 & 0.455 & 4.82 & 2.01 & 2.94 \\
\hline
\end{tabular}


Table 4.11 - Cyclic stiffness for each primary cycle on specimen SW-C-1

\begin{tabular}{|c|c|c|c|c|}
\hline $\begin{array}{c}\text { Primary } \\
\text { Cycle No. }\end{array}$ & $\begin{array}{c}\text { Avg. Disp. } \\
\mathbf{( m m})\end{array}$ & $\begin{array}{c}\text { Avg. Load } \\
\mathbf{( k N )}\end{array}$ & $\begin{array}{c}\text { Work per cycle } \\
\mathbf{( k N}-\mathbf{m})\end{array}$ & $\begin{array}{c}\text { Cyclic Stiff. } \\
\mathbf{( k N / m m )}\end{array}$ \\
\hline 1 & 6.52 & 3.05 & 0.020 & 0.468 \\
\hline 2 & 13.73 & 5.15 & 0.071 & 0.375 \\
\hline 3 & 20.48 & 6.68 & 0.137 & 0.326 \\
\hline 4 & 30.98 & 7.84 & 0.243 & 0.253 \\
\hline 5 & 40.65 & 8.68 & 0.353 & 0.214 \\
\hline 6 & 49.94 & 8.99 & 0.449 & 0.180 \\
\hline 7 & 57.64 & 8.56 & 0.493 & 0.149 \\
\hline 8 & 57.59 & 8.47 & 0.488 & 0.147 \\
\hline 9 & 61.7 & 7.96 & 0.491 & 0.129 \\
\hline 10 & 64.57 & 7.32 & 0.473 & 0.113 \\
\hline
\end{tabular}

Table 4.12 - Cyclic stiffness for each primary cycle on specimen SW-C-2

\begin{tabular}{|c|c|c|c|c|}
\hline $\begin{array}{c}\text { Primary } \\
\text { Cycle No. }\end{array}$ & $\begin{array}{c}\text { Avg. Disp. } \\
\mathbf{( m m )}\end{array}$ & $\begin{array}{c}\text { Avg. Load } \\
\mathbf{( k N )}\end{array}$ & $\begin{array}{c}\text { Work per cycle } \\
\mathbf{( k N}-\mathbf{m})\end{array}$ & $\begin{array}{c}\text { Cyclic Stiff. } \\
\mathbf{( k N / m m )}\end{array}$ \\
\hline 1 & 7.65 & 5.73 & 0.044 & 0.749 \\
\hline 2 & 13.71 & 8.22 & 0.113 & 0.600 \\
\hline 3 & 19.76 & 9.14 & 0.181 & 0.463 \\
\hline 4 & 29.79 & 10.38 & 0.309 & 0.348 \\
\hline 5 & 39.94 & 11.08 & 0.443 & 0.277 \\
\hline 6 & 49.27 & 10.80 & 0.532 & 0.219 \\
\hline 7 & 56.31 & 9.89 & 0.557 & 0.176 \\
\hline 8 & 59.53 & 8.94 & 0.532 & 0.150 \\
\hline 9 & 62.47 & 5.71 & 0.357 & 0.091 \\
\hline 10 & 64.07 & 5.54 & 0.355 & 0.086 \\
\hline
\end{tabular}


Table 4.13 - Cyclic stiffness for each primary cycle on specimen SIP-C-1

\begin{tabular}{|c|c|c|c|c|}
\hline $\begin{array}{c}\text { Primary } \\
\text { Cycle No. }\end{array}$ & $\begin{array}{c}\text { Avg. Disp. } \\
\mathbf{( m m})\end{array}$ & $\begin{array}{c}\text { Avg. Load } \\
\mathbf{( k N )}\end{array}$ & $\begin{array}{c}\text { Work per cycle } \\
\mathbf{( k N}-\mathbf{m})\end{array}$ & $\begin{array}{c}\text { Cyclic Stiff. } \\
\mathbf{( k N / m m )}\end{array}$ \\
\hline 1 & 13.32 & 7.62 & 0.101 & 0.572 \\
\hline 2 & 29.64 & 12.42 & 0.368 & 0.419 \\
\hline 3 & 46.66 & 16.93 & 0.790 & 0.363 \\
\hline 4 & 71.76 & 23.49 & 1.686 & 0.327 \\
\hline 5 & 101.22 & 29.55 & 2.991 & 0.292 \\
\hline 6 & 133.47 & 35.51 & 4.740 & 0.266 \\
\hline 7 & 166.66 & 39.43 & 6.571 & 0.237 \\
\hline 8 & 203.07 & 36.19 & 7.349 & 0.178 \\
\hline 9 & 204.64 & 28.81 & 5.896 & 0.141 \\
\hline 10 & 235.88 & 30.63 & 7.225 & 0.130 \\
\hline
\end{tabular}

Table 4.14 - Cyclic stiffness for each primary cycle on specimen SIP-C-2

\begin{tabular}{|c|c|c|c|c|}
\hline $\begin{array}{c}\text { Primary } \\
\text { Cycle No. }\end{array}$ & $\begin{array}{c}\text { Avg. Disp. } \\
\mathbf{( m m})\end{array}$ & $\begin{array}{c}\text { Avg. Load } \\
\mathbf{( k N )}\end{array}$ & $\begin{array}{c}\text { Work per cycle } \\
\mathbf{( k N}-\mathbf{m})\end{array}$ & $\begin{array}{c}\text { Cyclic Stiff. } \\
\mathbf{( k N / m m )}\end{array}$ \\
\hline 1 & 29.41 & 11.65 & 0.343 & 0.396 \\
\hline 2 & 48.81 & 16.12 & 0.787 & 0.330 \\
\hline 3 & 68.97 & 18.56 & 1.280 & 0.269 \\
\hline 4 & 102.05 & 20.08 & 2.049 & 0.197 \\
\hline 5 & 135.16 & 25.55 & 3.453 & 0.189 \\
\hline 6 & 157.67 & 32.86 & 5.181 & 0.208 \\
\hline 7 & 199.38 & 31.85 & 6.350 & 0.160 \\
\hline 8 & 204.62 & 22.37 & 4.577 & 0.109 \\
\hline 9 & 234.46 & 23.39 & 5.484 & 0.100 \\
\hline 10 & 242.81 & 10.46 & 2.540 & 0.043 \\
\hline
\end{tabular}


Table 4.15 - Cyclic stiffness for each primary cycle on specimen SIP-C-3

\begin{tabular}{|c|c|c|c|c|}
\hline $\begin{array}{c}\text { Primary } \\
\text { Cycle No. }\end{array}$ & $\begin{array}{c}\text { Avg. Disp. } \\
\mathbf{( m m})\end{array}$ & $\begin{array}{c}\text { Avg. Load } \\
\mathbf{( k N )}\end{array}$ & $\begin{array}{c}\text { Work per cycle } \\
\mathbf{( k N}-\mathbf{m})\end{array}$ & $\begin{array}{c}\text { Cyclic Stiff. } \\
\mathbf{( k N / m m )}\end{array}$ \\
\hline 1 & 23.42 & 7.15 & 0.167 & 0.305 \\
\hline 2 & 42.23 & 10.23 & 0.432 & 0.242 \\
\hline 3 & 68.00 & 12.70 & 0.864 & 0.187 \\
\hline 4 & 105.25 & 18.95 & 1.994 & 0.180 \\
\hline 5 & 136.49 & 30.39 & 4.148 & 0.223 \\
\hline 6 & 169.96 & 39.15 & 6.654 & 0.230 \\
\hline 7 & 182.66 & 32.75 & 5.982 & 0.179 \\
\hline 8 & 226.45 & 23.05 & 5.220 & 0.102 \\
\hline 9 & 225.49 & 14.65 & 3.303 & 0.065 \\
\hline 10 & 222.05 & 11.14 & 2.474 & 0.050 \\
\hline
\end{tabular}

Table 4.16 - Cyclic stiffness for each primary cycle on specimen SIP-C-4

\begin{tabular}{|c|c|c|c|c|}
\hline $\begin{array}{c}\text { Primary } \\
\text { Cycle No. }\end{array}$ & $\begin{array}{c}\text { Avg. Disp. } \\
\mathbf{( m m})\end{array}$ & $\begin{array}{c}\text { Avg. Load } \\
\mathbf{( k N )}\end{array}$ & $\begin{array}{c}\text { Work per cycle } \\
\mathbf{( k N}-\mathbf{m})\end{array}$ & $\begin{array}{c}\text { Cyclic Stiff. } \\
\mathbf{( k N / m m )}\end{array}$ \\
\hline 1 & 25.98 & 22.00 & 0.572 & 0.847 \\
\hline 2 & 38.08 & 20.65 & 0.786 & 0.542 \\
\hline 3 & 50.45 & 22.25 & 1.123 & 0.441 \\
\hline 4 & 90.24 & 27.82 & 2.510 & 0.308 \\
\hline 5 & 116.71 & 28.07 & 3.276 & 0.241 \\
\hline 6 & 129.82 & 21.95 & 2.850 & 0.169 \\
\hline 7 & 196.65 & 32.15 & 6.322 & 0.163 \\
\hline 8 & 237.50 & 31.27 & 7.427 & 0.132 \\
\hline 9 & 253.86 & 22.27 & 5.653 & 0.088 \\
\hline 10 & 249.92 & 19.22 & 4.803 & 0.077 \\
\hline
\end{tabular}


Table 4.17 - Cyclic stiffness for each primary cycle on specimen SIP-C-5

\begin{tabular}{|c|c|c|c|c|}
\hline $\begin{array}{c}\text { Primary } \\
\text { Cycle No. }\end{array}$ & $\begin{array}{c}\text { Avg. Disp. } \\
\mathbf{( m m})\end{array}$ & $\begin{array}{c}\text { Avg. Load } \\
\mathbf{( k N )}\end{array}$ & $\begin{array}{c}\text { Work per cycle } \\
\mathbf{( k N}-\mathbf{m})\end{array}$ & $\begin{array}{c}\text { Cyclic Stiff. } \\
\mathbf{( k N / m m )}\end{array}$ \\
\hline 1 & 14.10 & 9.42 & 0.133 & 0.668 \\
\hline 2 & 27.41 & 16.74 & 0.459 & 0.611 \\
\hline 3 & 42.80 & 21.83 & 0.934 & 0.510 \\
\hline 4 & 52.48 & 24.99 & 1.311 & 0.476 \\
\hline 5 & 84.81 & 28.38 & 2.407 & 0.335 \\
\hline 6 & 129.16 & 23.93 & 3.091 & 0.185 \\
\hline 7 & 172.78 & 22.81 & 3.941 & 0.132 \\
\hline 8 & 198.45 & 18.43 & 3.657 & 0.093 \\
\hline 9 & 243.45 & 17.19 & 4.185 & 0.071 \\
\hline 10 & 244.32 & 7.02 & 1.715 & 0.029 \\
\hline
\end{tabular}

Table 4.18 - Cyclic stiffness for each primary cycle on specimen SIP-C-6

\begin{tabular}{|c|c|c|c|c|}
\hline $\begin{array}{c}\text { Primary } \\
\text { Cycle No. }\end{array}$ & $\begin{array}{c}\text { Avg. Disp. } \\
\mathbf{( m m})\end{array}$ & $\begin{array}{c}\text { Avg. Load } \\
\mathbf{( k N )}\end{array}$ & $\begin{array}{c}\text { Work per cycle } \\
\mathbf{( k N}-\mathbf{m})\end{array}$ & $\begin{array}{c}\text { Cyclic Stiff. } \\
\mathbf{( k N / m m )}\end{array}$ \\
\hline 1 & 13.32 & 14.21 & 0.189 & 1.067 \\
\hline 2 & 26.82 & 25.11 & 0.673 & 0.936 \\
\hline 3 & 38.96 & 26.32 & 1.025 & 0.676 \\
\hline 4 & 51.18 & 27.09 & 1.386 & 0.529 \\
\hline 5 & 90.19 & 31.00 & 2.796 & 0.344 \\
\hline 6 & 129.79 & 29.22 & 3.792 & 0.225 \\
\hline 7 & 154.80 & 27.68 & 4.285 & 0.179 \\
\hline 8 & 187.51 & 28.71 & 5.383 & 0.153 \\
\hline 9 & 263.91 & 22.14 & 5.843 & 0.084 \\
\hline 10 & 262.83 & 18.71 & 4.918 & 0.071 \\
\hline
\end{tabular}




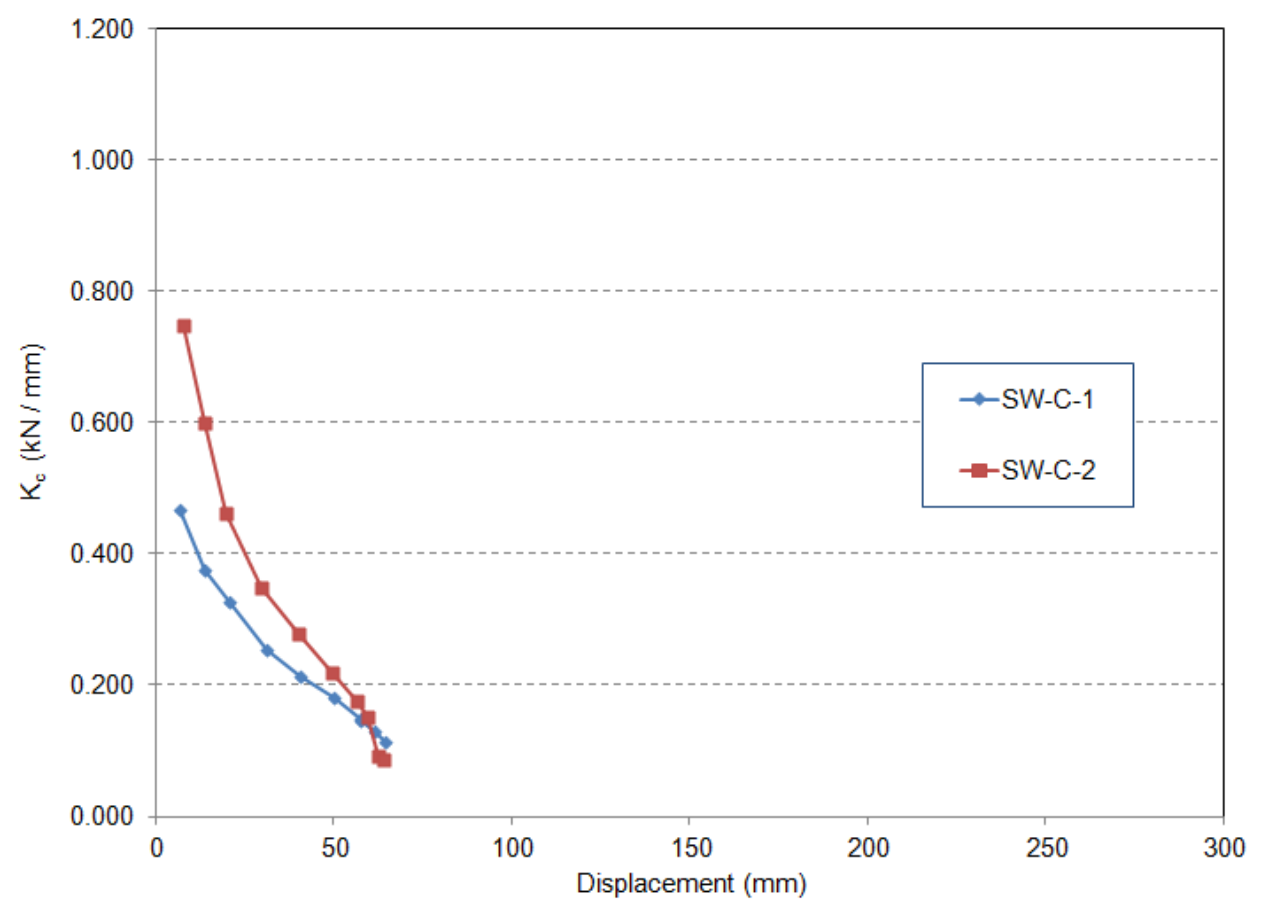

Figure 4.125 - Cyclic stiffness vs. peak displacement relationship for stud walls SW-C-1 and SW-C-2

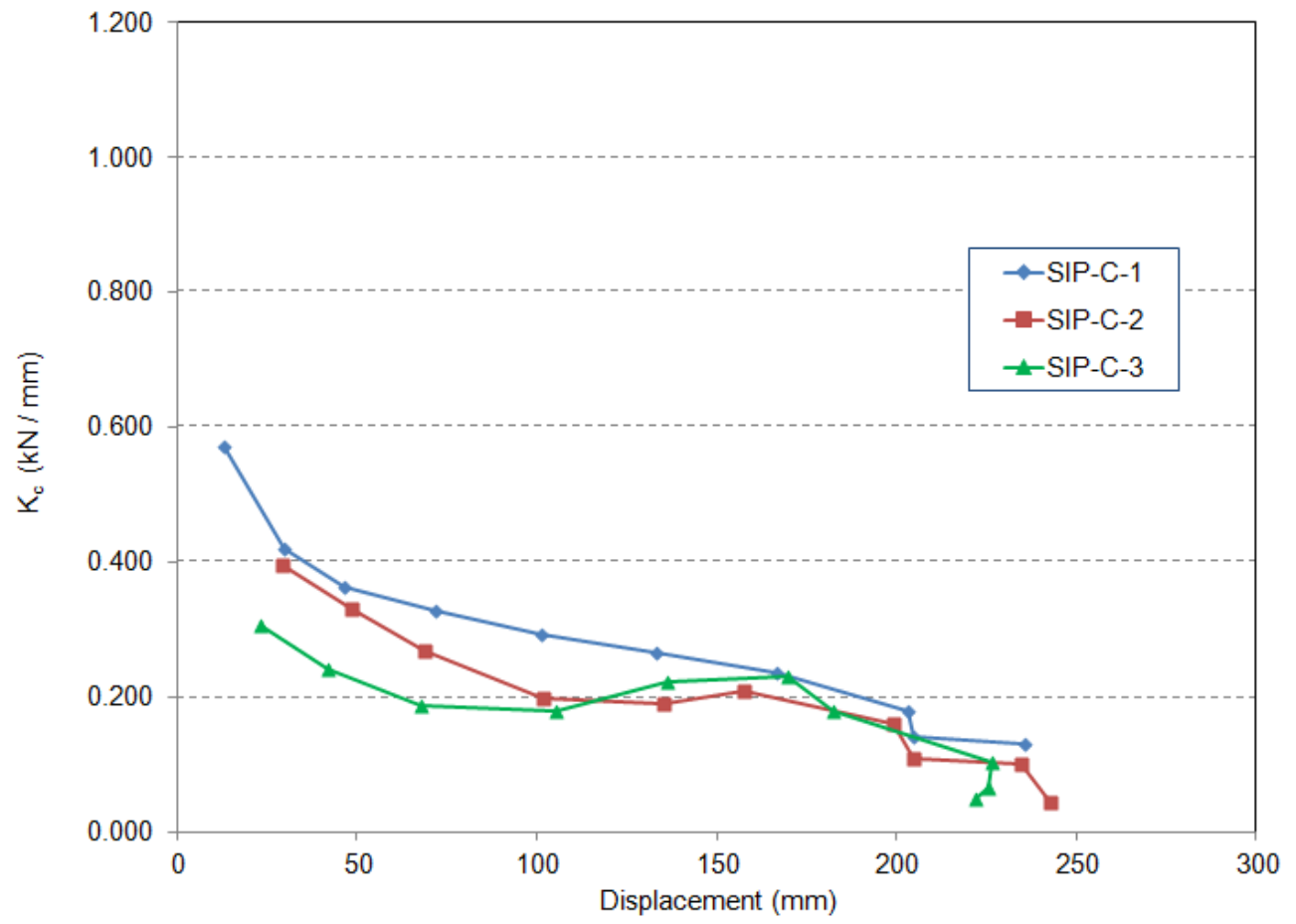

Figure 4.126 - Cyclic stiffness vs. peak displacement relationship for short SIP walls SIP-C-1 to SIP-C-3 


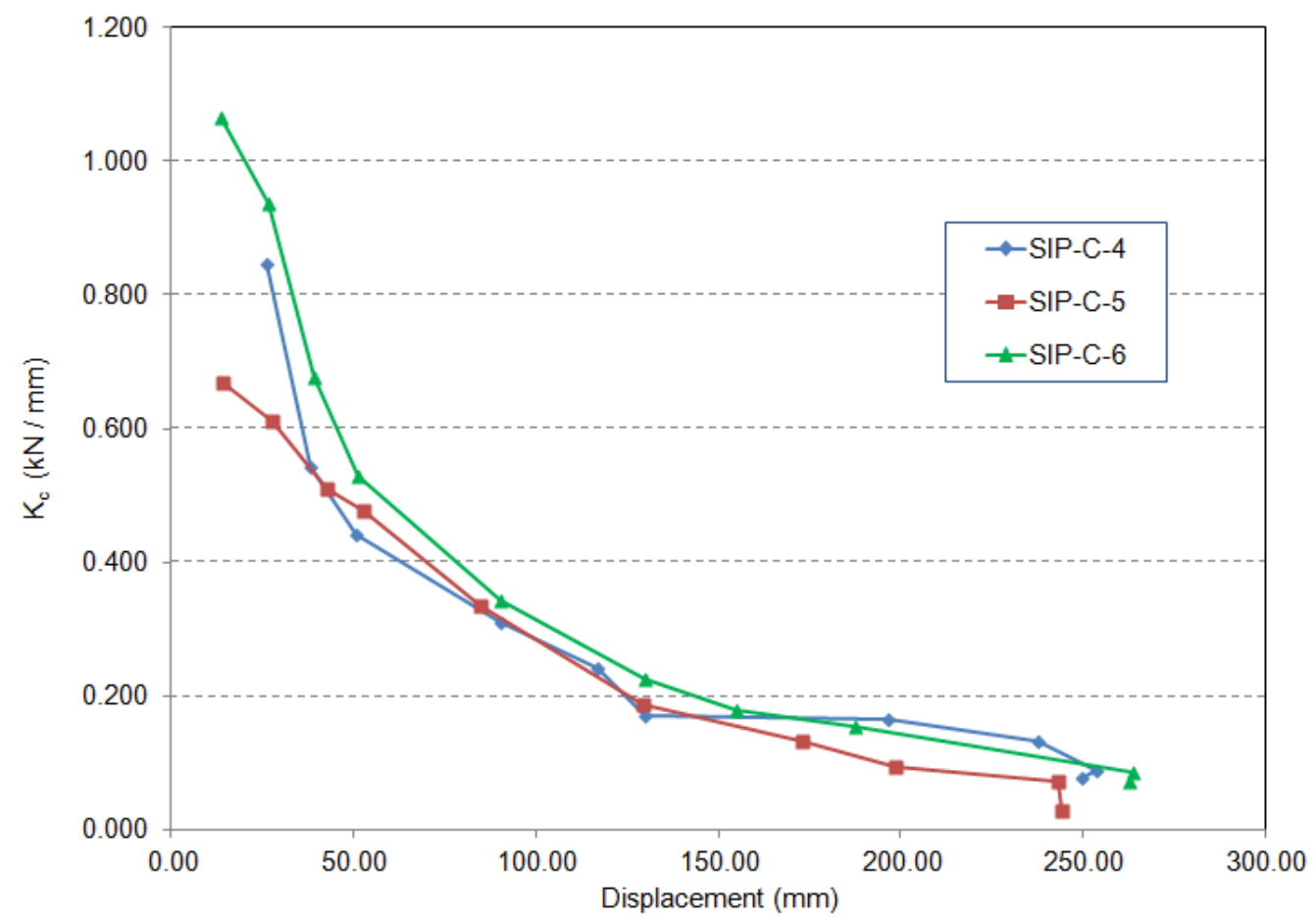

Figure 4.127 - Cyclic stiffness vs. peak displacement relationship for long SIP walls SIP-C-4 to SIP-C-6

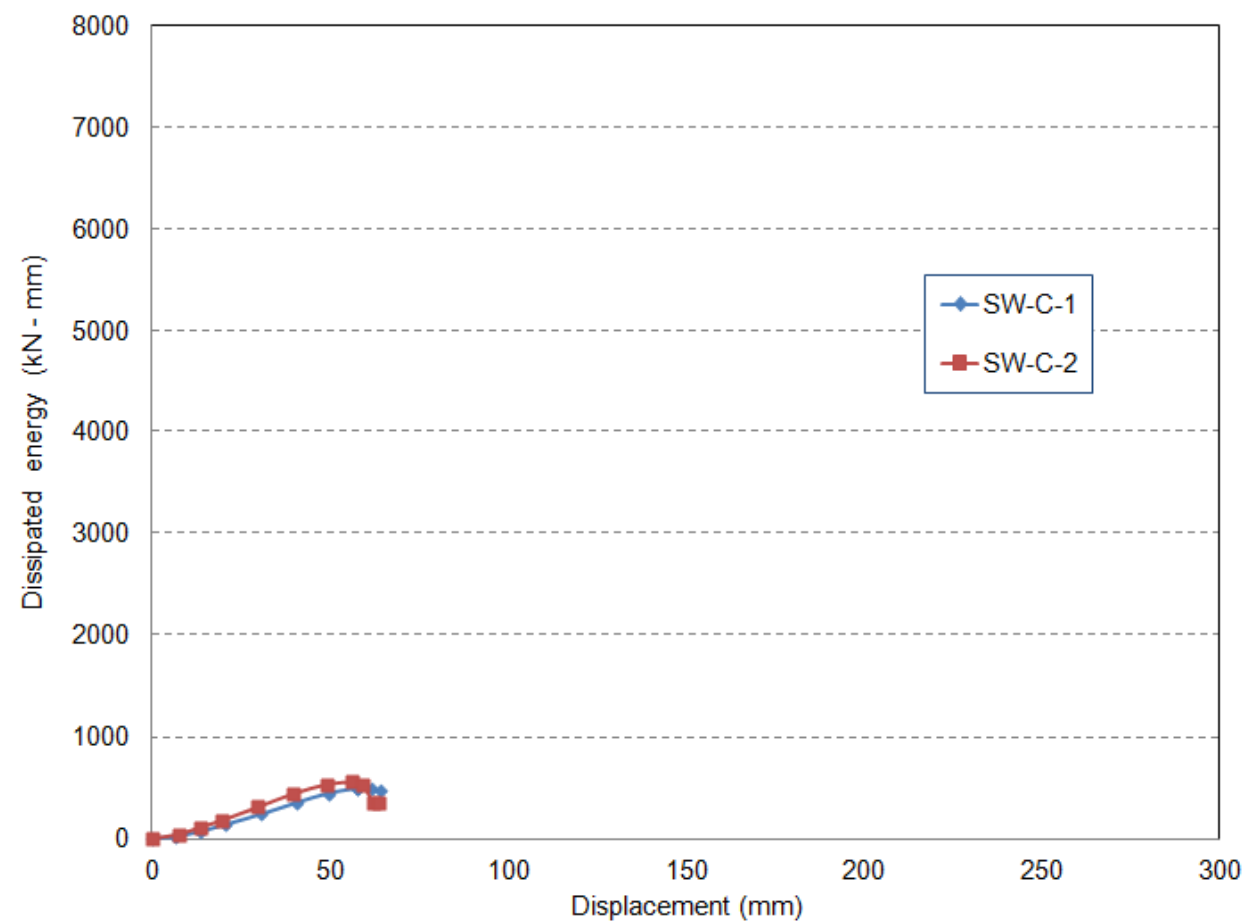

Figure 4.128 - Hysteretic energy dissipated per cycle vs. displacement of stud wall (SW-C-1 and SW-C-2) 


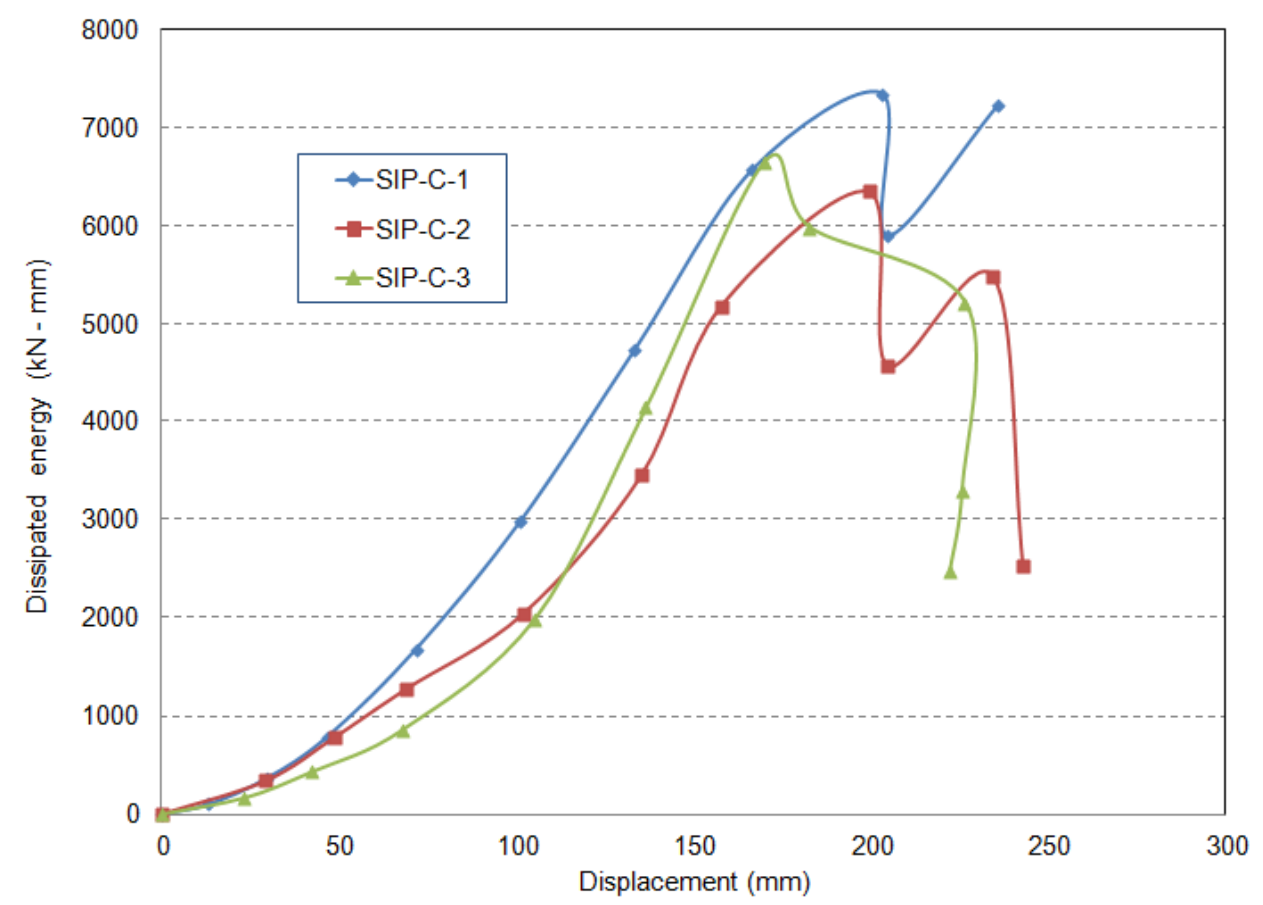

Figure 4.129 - Hysteretic energy dissipated per cycle vs. displacement of short SIP wall (SIP-C1 and SIP-C-3)

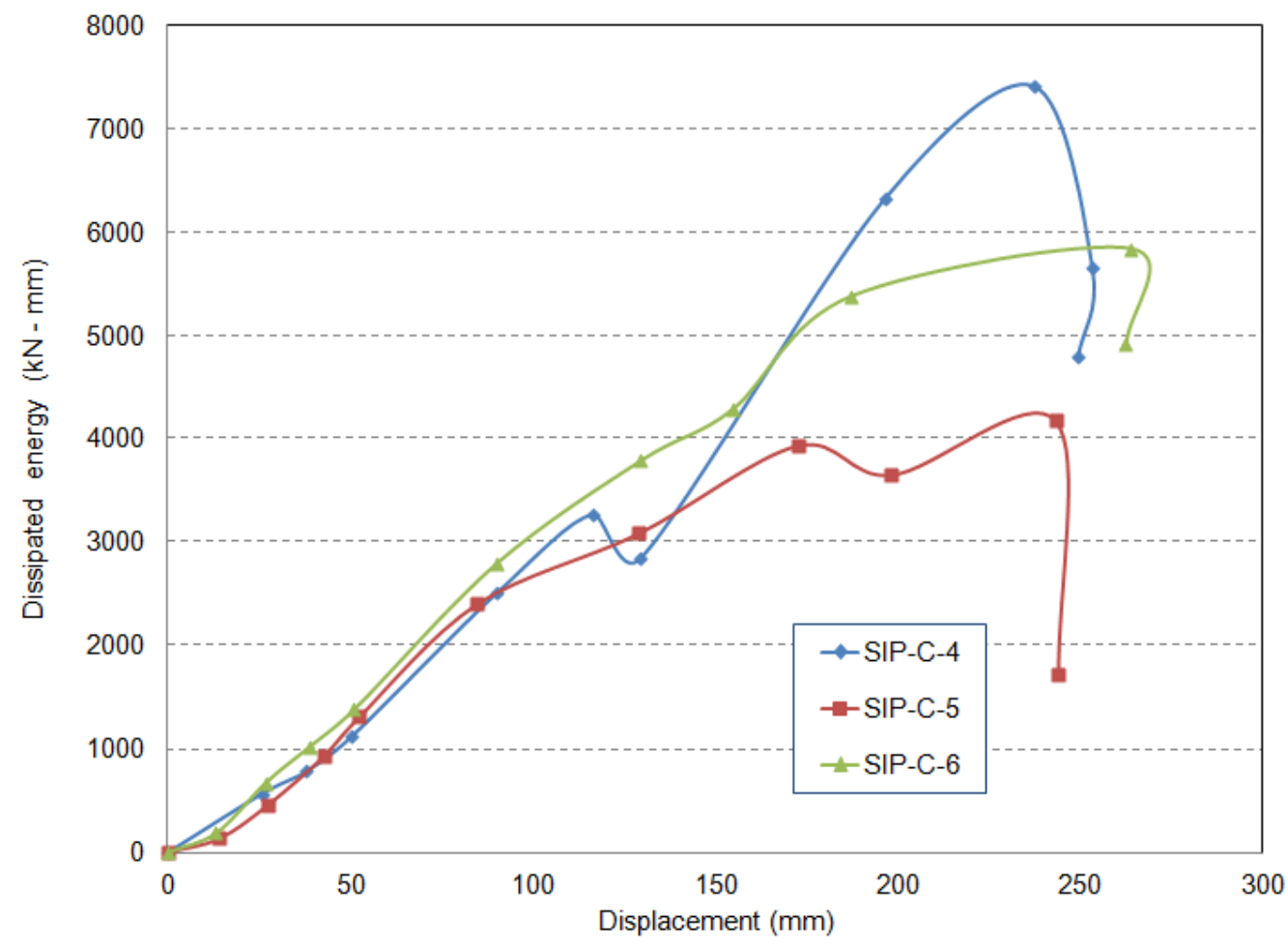

Figure 4.130 - Hysteretic energy dissipated per cycle vs. displacement of long SIP wall (SIP-C-4 and SIP-C-6) 


\subsection{Ductility Related Force $\left(R_{d}\right)$ and Over-strength Related Force Modification Factor}

$\left(\mathbf{R}_{\mathbf{0}}\right)$

The verification of NBCC-2010 requirements for ductility-related force modification factor $\left(\mathrm{R}_{\mathrm{d}}\right)$ and the over-strength related force modification factor $\left(R_{0}\right)$ are done with ductility ratios, maximum capacities and yield loads. The values of $R_{d}=3.0$ and $R_{o}=1.7$ for nailed shear walls with wood based panels is recommended in Table 4.1.8.9 of NBCC (NBCC, 2010).

Boudreault et al. (2007) presented the Equation (4.6) for force modification factor $\left(\mathrm{R}_{\mathrm{d}}\right)$ calculations as follows:

$R_{d}=\sqrt{2 \mu-1}$

Where $\mu$ is defined as the ductility ratio calculated earlier in this chapter. This equation can be used for structures composed of light-framed shear walls with the natural period of vibration $(\mathrm{T})$ between 0.1 and $0.5 \mathrm{sec}$ (Boudreault et al., 2007).

Mitchell et al. (2003) also suggested an equation for calculation of the over-strength related force modification factor $\left(\mathrm{R}_{\mathrm{o}}\right)$ as follows:

$R_{0}=R_{\text {size }} \cdot R_{\phi} \cdot R_{\text {yield }} \cdot R_{\text {sh }} \cdot R_{\text {mech }}$

Where,

$R_{\text {size }}=1.15$, over-strength due to restricted choices for member sizes and dimension rounding (Mitchell et al., 2003);

$R_{\phi}=1.43$, over-strength due to the difference between nominal and factored resistance $\left(\mathrm{R}_{\phi}=\right.$ $1 / \phi$, with $\phi$ as the resistance factor) (Mitchell et al., 2003);

$R_{\text {yield }}=$ Ratio of probable yield strength to minimum specified yield strength with the equivalent energy elastic-plastic bilinear model, EEEP (Boudreault et al., 2007);

$R_{s h}=1.05$, over-strength due to strain hardening (Mitchell et al., 2003); and

$R_{\text {mech }}=1.00$, over-strength developed when a collapse mechanism is formed (Mitchell et al., 2003).

Based on the above equations, the mean values of $R_{d}$ and $R_{o}$ are calculated and presented in the Table 4.19 based on results reported in Tables 4.8 through 4.10 for racking and cyclic load tests 
on stud walls, short SIP walls and long SIP walls. The values of $R_{d}$ for stud wall, short SIP wall and long SIP wall are observed to be 19\%, 13\% and 3\% higher than the NBCC required value for anchored wall $\left(\mathrm{R}_{\mathrm{d}}=3.0\right)$ based on the racking load test results, respectively. On the other hand, the values of $R_{d}$ for stud wall, short SIP wall and long SIP wall are observed to be $8 \%$, $22 \%$ and $14 \%$ lower than the NBCC required value for anchored wall $\left(\mathrm{R}_{\mathrm{d}}=3.0\right)$ based on cyclic load test results, respectively. The values of factor $\mathrm{R}_{\mathrm{o}}$ presented in Table 4.19 for stud wall, short SIP wall and long SIP wall are observed to be $17 \%, 20 \%$ and $14 \%$ higher than the recommended value of NBCC $\left(R_{o}=1.7\right)$ for anchored wall, respectively. So, it is concluded that the overstrength factor indicates a confident reserve of resistance in interconnected wall segments.

Table 4.19 - Mean $\mathrm{R}_{\mathrm{d}}$ and $\mathrm{R}_{\mathrm{o}}$ factors for tested wall assemblies

\begin{tabular}{|c|c|c|c|c|c|c|}
\hline \multirow{2}{*}{$\begin{array}{c}\text { Force modification } \\
\text { factor }\end{array}$} & \multicolumn{3}{|c|}{ Racking load } & \multicolumn{3}{c|}{ Cyclic load } \\
\cline { 2 - 7 } & $\begin{array}{c}\text { Stud } \\
\text { wall, }\end{array}$ & $\begin{array}{c}\text { Short SIP } \\
\text { wall, } \\
\text { SW-R }\end{array}$ & $\begin{array}{c}\text { Long SIP } \\
\text { wall, } \\
\text { SIP-R }\end{array}$ & $\begin{array}{c}\text { Stud } \\
\text { wall, } \\
\text { SIP-R }\end{array}$ & $\begin{array}{c}\text { Short SIP } \\
\text { wall, } \\
\text { SIP-R }\end{array}$ & $\begin{array}{c}\text { Long SIP } \\
\text { wall, } \\
\text { SIP-R }\end{array}$ \\
\hline $\mathbf{R}_{\mathbf{d}}$ & 3.58 & 3.40 & 3.09 & 2.77 & 2.34 & 2.59 \\
\hline $\mathbf{R}_{\mathbf{0}}$ & 2.00 & 2.05 & 1.93 & 1.99 & 2.02 & 1.96 \\
\hline
\end{tabular}

\subsection{Comparison of SIP and Stud Panel under Different Load Conditions}

\subsubsection{SIP vs. Stud Panel under Flexural Loading}

In addition to the correlations of ultimate loads and deflection values at the deflection limits mentioned earlier, this section discusses both the elastic stiffness and ductility ratio of the test panels. Figure 4.131 shows the comparison of the load vs. deflection relationship of specimen SIP-F-1 and stud panel SW-F-1 under flexural loading. The calculated elastic stiffness of the stud and SIP panels based on the load vs. deflection curves are 0.87 and $1.26 \mathrm{kN} / \mathrm{mm}$, respectively. While the calculated ductility ratio of the Stud and SIP panels are 1.21 and 1.16, respectively. So, it is concluded that the flexural stiffness of SIP is more than that of the stud panel, while the ductility ratio of the stud panel is more than that of the SIP panel of the same size. Table 4.20 tabulates the results presented here. 


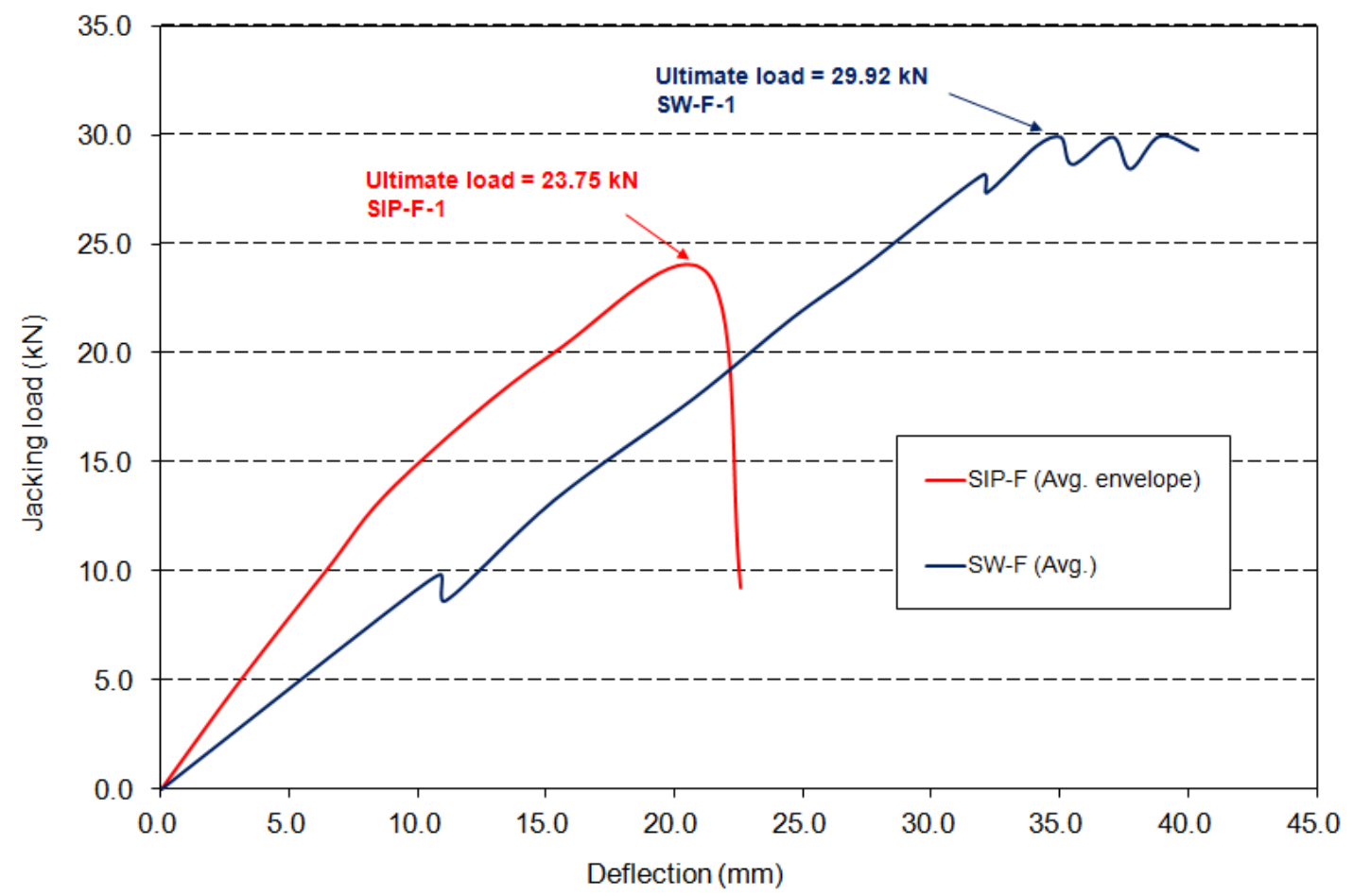

Figure 4.131 - Comparison of load vs. deflection relationship of the SIP specimen SIP-F-1 and stud panel SW-F-1 under flexural loading

Table 4.20 - Average stiffness of SIP and stud wall based on results from flexural, compressive and racking load tests

\begin{tabular}{|c|c|c|c|}
\hline \multirow{2}{*}{ Load type } & Wall type & Average $\mathbf{K}_{\mathbf{e}}(\mathbf{k N} / \mathbf{m m})$ & $\begin{array}{c}\text { Average ductility } \\
\text { ratio }\end{array}$ \\
\hline \multirow{2}{*}{ Flexural } & Stud & 0.87 & 1.21 \\
\cline { 2 - 4 } & SIP & 1.26 & 1.16 \\
\hline \multirow{2}{*}{ Compression } & Stud & 6.15 & 1.21 \\
\cline { 2 - 4 } & SIP & 12.85 & 1.18 \\
\hline \multirow{2}{*}{ Racking } & Stud & 1.70 & 0.64 \\
\cline { 2 - 4 } & SIP & 1.36 & 0.51 \\
\hline
\end{tabular}

\subsubsection{SIP vs. Stud Panel under Compressive Loading}

In addition to the correlations of ultimate loads and axial shortening design criteria mentioned earlier, this section discusses both the elastic stiffness and ductility ratio of the test walls. Figure 
4.132 shows the comparison of the load vs. axial shortening relationship of specimen SIP-A-1 and stud wall SW-A-1 under compressive loading. The calculated elastic stiffness of the stud and SIP walls based on the load vs. axial shortening curves are 6.15 and $12.85 \mathrm{kN} / \mathrm{mm}$, respectively. While, the calculated ductility ratio of the Stud and SIP walls are 1.21 and 1.18, respectively. So, it is concluded that the flexural stiffness of SIP wall is more than that of the stud wall, while the ductility ratio of the stud wall is more than that of the SIP wall of the same size. Table 4.19 tabulates the results presented here.

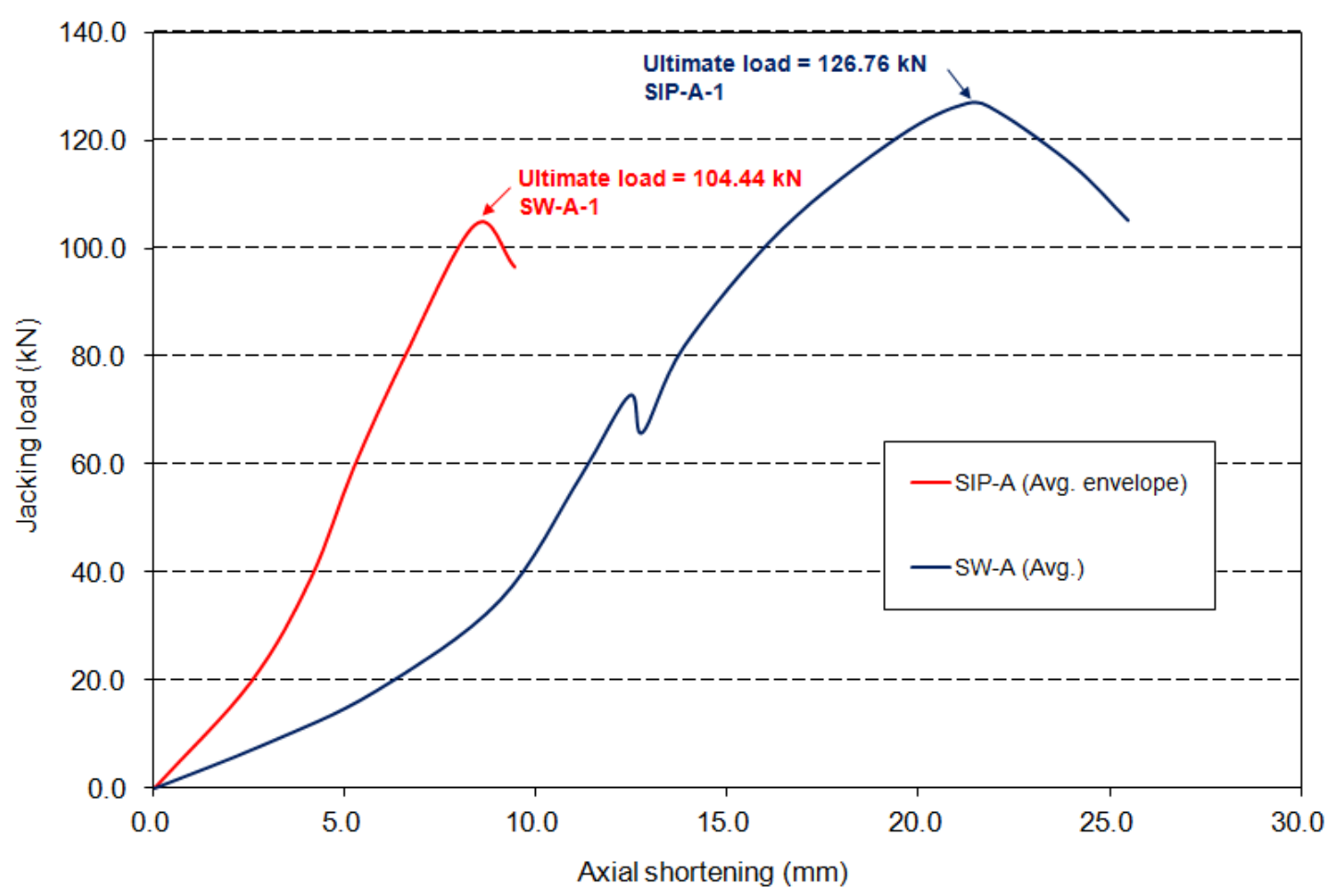

Figure 4.132 - Comparison of load vs. axial shortening of the SIP wall SP-A-1 and stud wall SW-A-1 under compressive loading

\subsubsection{SIP vs. Stud Panel under Racking Load}

In addition to the correlations of ultimate racking loads mentioned earlier, this section discusses both the elastic stiffness and ductility ratio of the test walls. Figure 4.133 shows the comparison of the load vs. lateral deflection relationship of specimen SIP-R-1 and stud wall SW-R-1 under racking loading. The calculated elastic stiffness of the stud and SIP walls based on the load vs. lateral deflection curves are 1.70 and $1.36 \mathrm{kN} / \mathrm{mm}$, respectively. While, the calculated ductility ratio of the Stud and SIP walls are 0.64 and 0.51, respectively. So, it is concluded that for this 
panel wall configuration, both the racking stiffness and ductility ratio of SIP are less than those of the stud panel of the same size. Table 4.20 tabulates the results presented here.

As shown in Fig. 4.133, the amount of the area below the load vs. lateral deflection curve is assumed to be the dissipated energy of the system. This energy is determined by Equivalent Energy Elastic Plastic (EEEP) method which is presented earlier in this chapter. It can be concluded that the amount of energy dissipated by SIP wall is greater than that of the stud wall of the same size. Therefore, SIP walls can provide better performance than stud walls in energy efficiency in wood building construction.

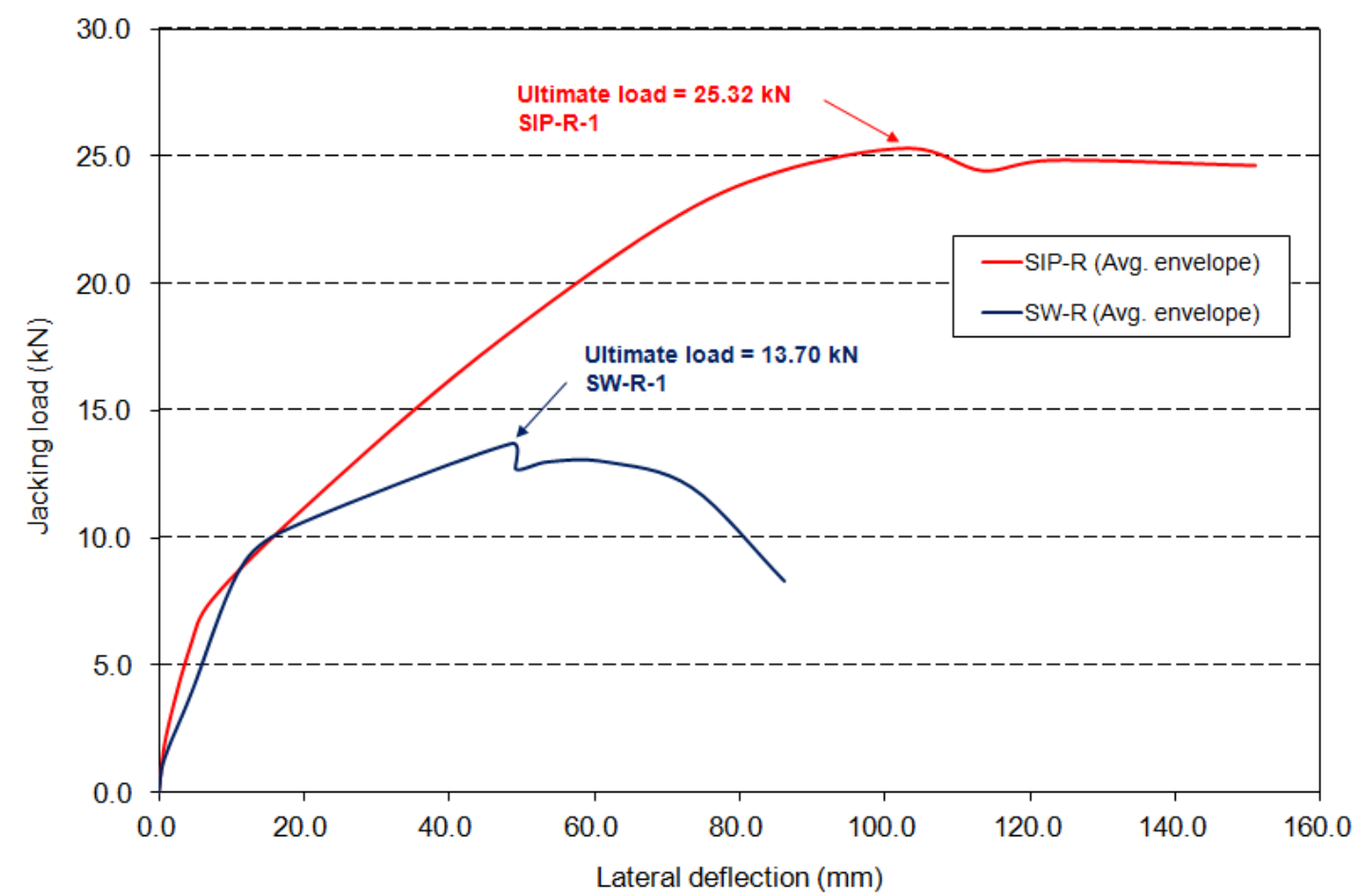

Figure 4.133 - Comparison of load vs. lateral deflection relationship of the specimen SIP-R-1 and stud wall SW-R-1 under racking load

\subsubsection{SIP vs. Stud Panel under Cyclic Loading}

In addition to the correlation of ultimate load obtained for stud and SIP panels under cyclic loading, this section emphasize on these findings in summary. As shown in Fig. 4.134, the amount of the area below the load vs. lateral deflection envelope is assumed to be the dissipated energy of the system. This figure shows such cyclic load-deflection history envelopes for walls 
SIP-C-1 and SW-C-1. In addition, Fig. 4.135 depicts the correlation between the dissipated energy of the SIP and stud walls with increase in lateral deflection under cyclic loading. Comparisons of the dissipated energy per cycle revealed a higher capacity (about 14 times greater) for SIPS walls compared to the conventional stud wall system. It can be concluded that the amount of energy dissipated by SIP wall is greater than that of the stud wall of the same size. Therefore, SIP walls can provide better performance than stud walls in energy efficiency in wood building construction. Figure 4.136 shows the comparison of cyclic stiffness energy of for walls SIP-C-1 and SW-C-1 under cyclic loading. It can be observed that the cyclic stiffness of SIP wall is about $20 \%$ more than the stud wall with the same size. Table 4.21 summarizes the average elastic stiffness and ductility ratio of SIP and stud wall based on results from cyclic load tests discussed earlier in this chapter.

Table 4.21 - Average elastic stiffness and ductility ratio of SIP and stud wall based on results from cyclic load tests

\begin{tabular}{|c|c|c|c|c|c|c|}
\hline Specimen & $\begin{array}{c}\text { Avg. } K_{e} \\
(\mathrm{kN} / \mathrm{mm}) \text {, } \\
\text { negative } \\
\text { portion }\end{array}$ & $\begin{array}{c}\text { Avg. } K_{\mathrm{e}} \\
(\mathrm{kN} / \mathrm{mm}) \text {, } \\
\text { positive } \\
\text { portion }\end{array}$ & $\begin{array}{c}\text { Avg. } \\
\text { Ductility } \\
\text { ratio, } \\
\text { negative } \\
\text { portion }\end{array}$ & $\begin{array}{c}\text { Avg. } \\
\text { Ductility } \\
\text { ratio, } \\
\text { positive } \\
\text { portion }\end{array}$ & $\begin{array}{c}\text { Average } \\
K_{\mathrm{e}} \\
(\mathrm{kN} / \mathrm{mm})\end{array}$ & $\begin{array}{c}\text { Average } \\
\text { ductility } \\
\text { ratio }\end{array}$ \\
\hline Stud Wall & 0.42 & 0.23 & 4.19 & 4.51 & 0.33 & 4.35 \\
\hline Short SIP & 0.29 & 0.55 & 4.35 & 2.35 & 0.42 & 3.35 \\
\hline Long SIP & 0.54 & 0.69 & 4.14 & 3.32 & 0.62 & 3.73 \\
\hline
\end{tabular}




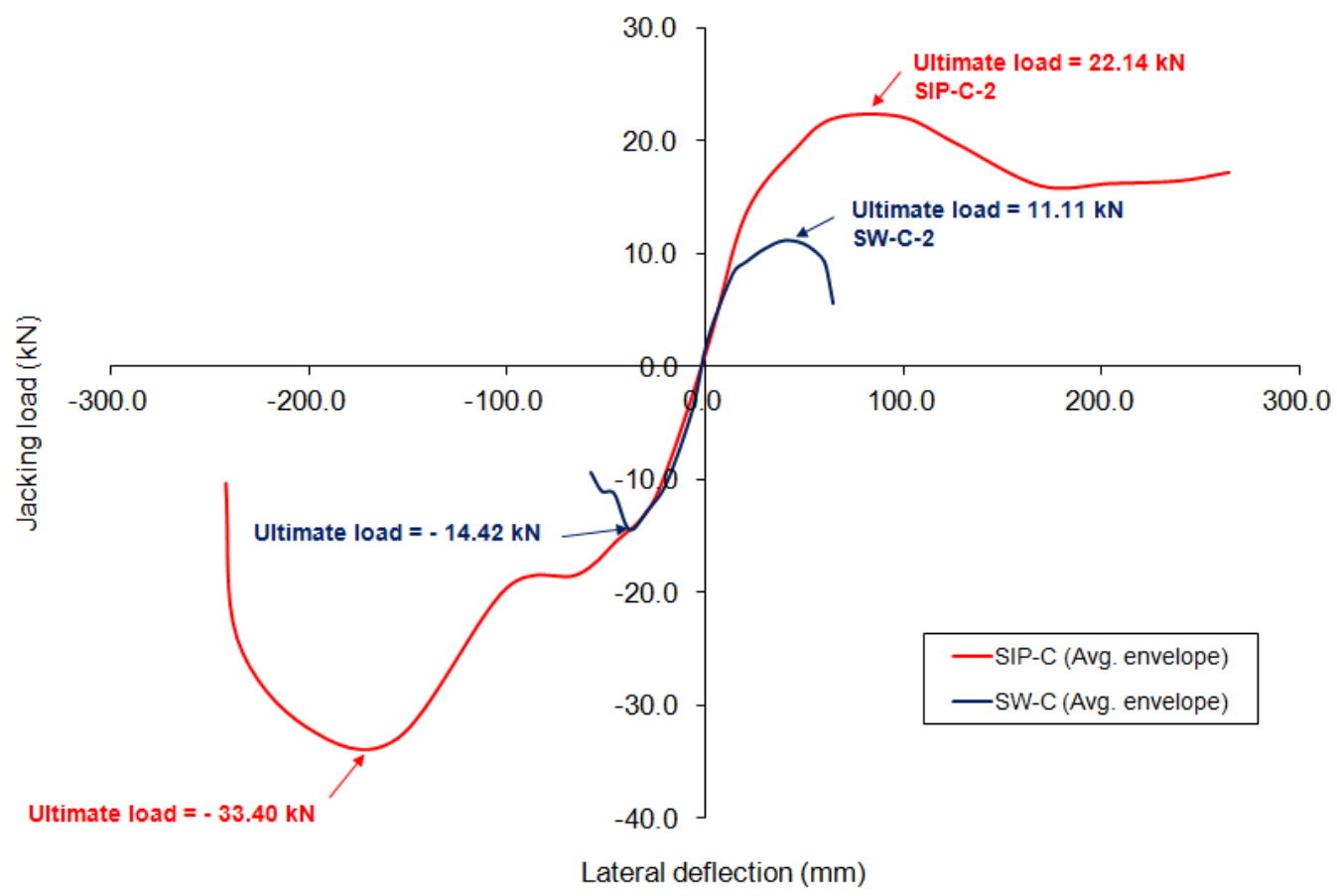

Figure 4.134 - Comparison of load vs. lateral deflection history of specimen SIP-C-2 and stud wall SW-C-2 under cyclic loading

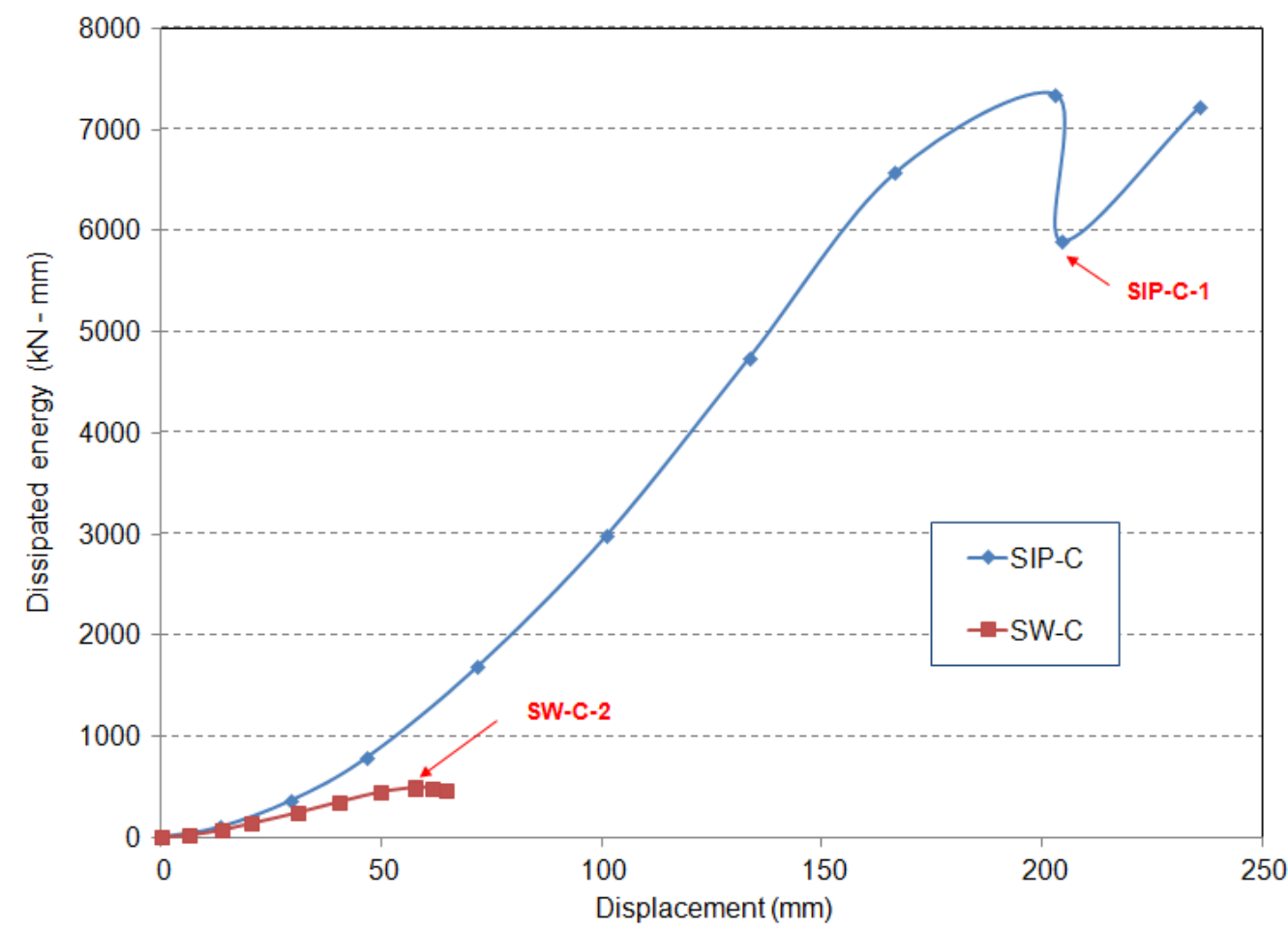

Figure 4.135 - Comparison of dissipated energy of SIP and stud wall under cyclic loading 


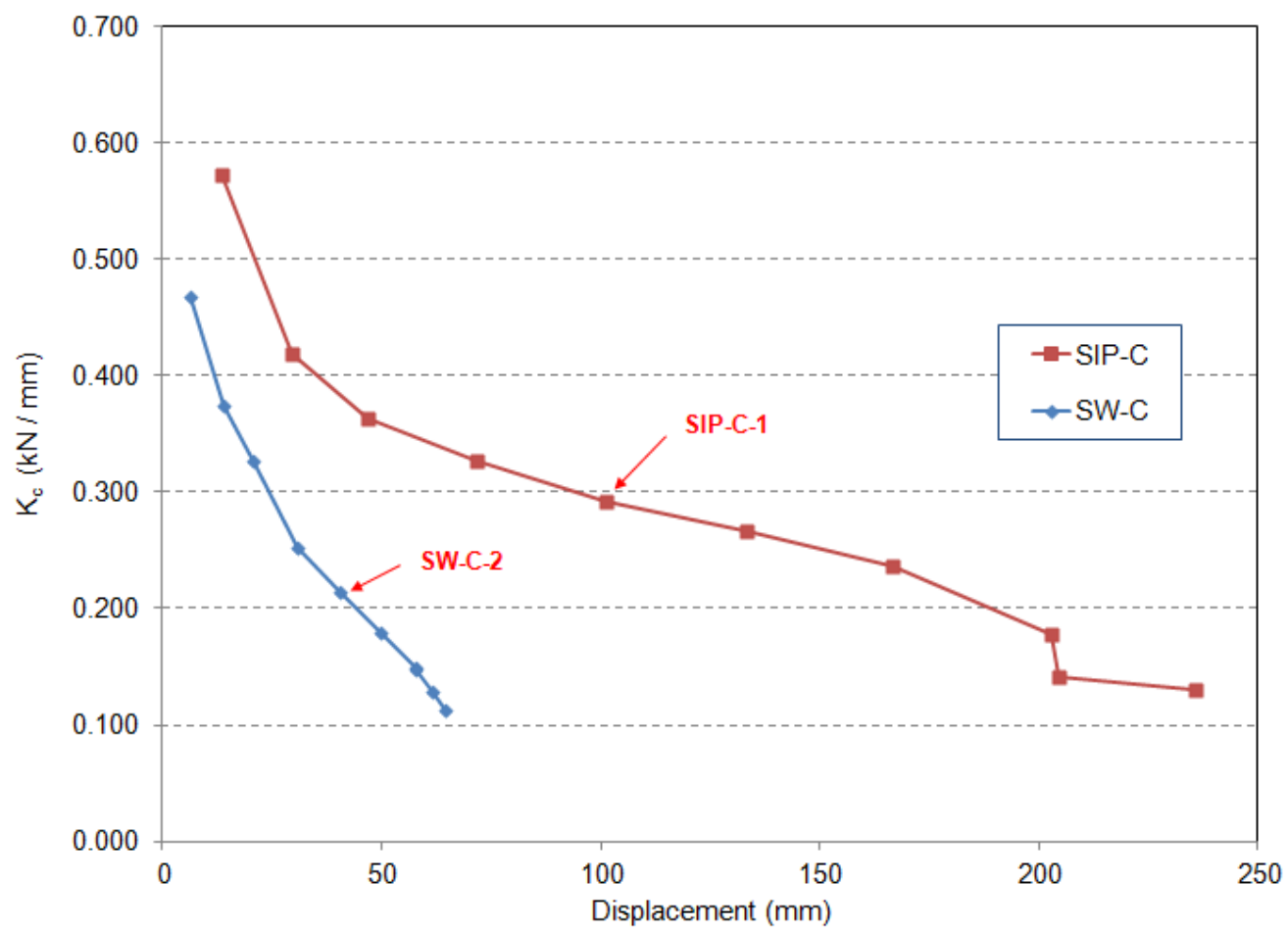

Figure 4.136 - Comparison of cyclic stiffness of SIP and stud wall under cyclic loading 


\section{CHAPTER 5}

\section{FINITE ELEMENT ANALYSIS, VALIDATION}

AND PARAMETRIC STUDY

\subsection{Introduction}

In this chapter, numerical investigation of the behavior of structural insulated panels with OSB sheathing, performed using the finite-element (FE) program ABAQUS (2010), is presented. The investigation comprised of four phases. In the first phase, finite-element models were developed to predict the behavior of structural insulated panels under monotonic compressive loading. In this phase, the developed FE models were validated using the experimental data for SIP and stud wall panels under monotonic compressive loading. In second phase of the investigation, the developed FE models were verified using experimental data obtained from flexural tests. The third phase investigated the applicability of the developed FE models to predict the behavior under monotonic and cyclic lateral (racking) loading. In the last phase, a parametric study was conducted to examine the effects of gravity loading as well as nail spacing on the response of SIP walls subjected to racking loading.

The basic steps involved at the beginning of this investigation included:

(1) A linear-elastic mesh study of SIP and stud panels to establish the level of mesh refinement and convergence in straining actions.

(2) Incorporating the inelastic behavior while considering mesh refinement, material strain hardening and contact surfaces between foam and OSB sheets.

Preliminary sensitivity study reveals that the 8-node brick element (designed as C3D8I in ABAQUS element library) is a good choice to model OSB sheets, studs, foam core and top, bottom and side plates in case of flexural and compressive loading. On the other hand the study revealed that the 4-node plane stress/strain element (designed as CPS4I in ABAQUS element library) is a good choice to model all elements forming either the stud wall or SIP wall with the 
presence of spring element representing the nail behavior as well as the contact element to represent the glue between the OSB sheet and the foam core.

\subsection{Linear-Elastic Mesh Study}

The main purpose of the linear-elastic mesh study was to determine the level of mesh refinement required to ensure convergence. In this step, four different finite-element meshes were used to model the tested panels in case of compressive and flexural loading (see Figure 5.1). For each mesh, solid element with 8-node and incompatible mode (C3D8I), available in ABAQUS element library, was used to model the stud wall frame and OSB sheathing. Linear elastic material properties were assigned to studs and OSB sheets. These elements can simulate the bending behavior of the pure displacement problem. The incompatible mode of these elements formulates low-order enhanced strain elements. The incompatible modes can simulate a surprising behavior in linear and nonlinear analysis problem.

The modulus of elasticity used in the linear-elastic material was based on properties obtained from material tests presented in Chapter 3. The rotational and translational degrees of freedom at the nodes along the connected edge of stud frame and OSB were applied based on actual boundary and restraint conditions considered in the tested panels. The load was applied in the finite-element model through a reference node away from the panel. This reference node is also used for applying the desired displacement to model. Figure 5.2 show views of the FE model for different parts of stud panel, namely: the stud framing, the OSB sheet and the drywall. While, Figure 5.3 shows view of complete FE model of stud wall frame and OSB sheet for compressive load analysis. Figure 5.4 shows views of the components forming the SIP panel, namely: the OSB sheet, the EPS foam core and the lumber framing. While, Figure 5.5 shows View of the complete FE model of SIP with OSB sheathing for compressive load analysis. 


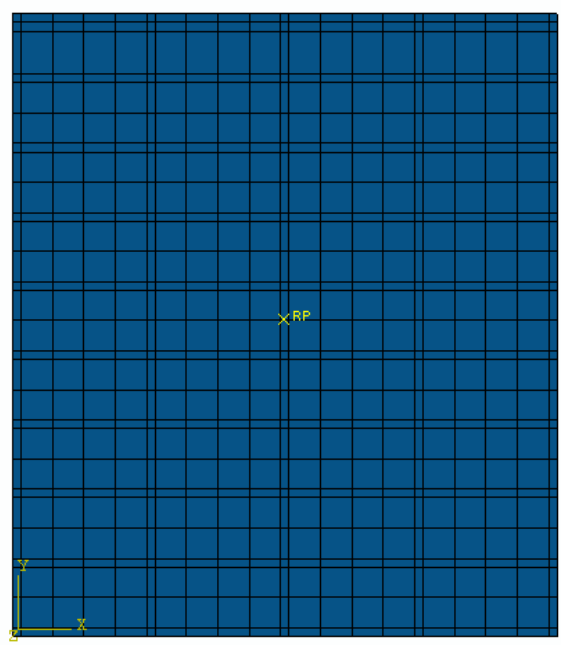

(a) Mesh 1

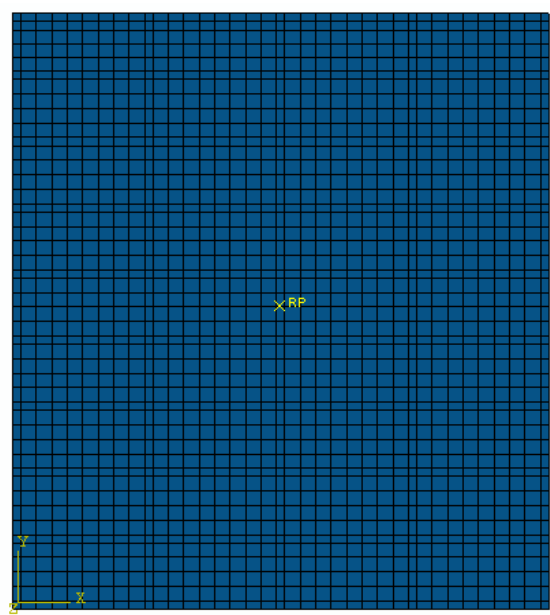

(c) Mesh 3

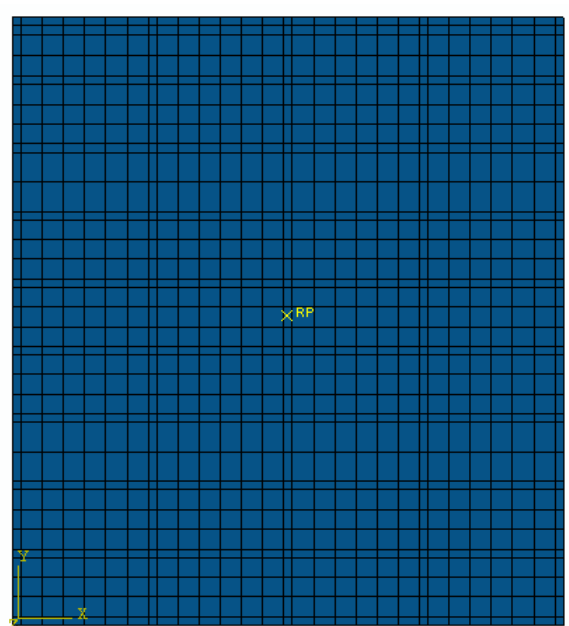

(b) Mesh 2

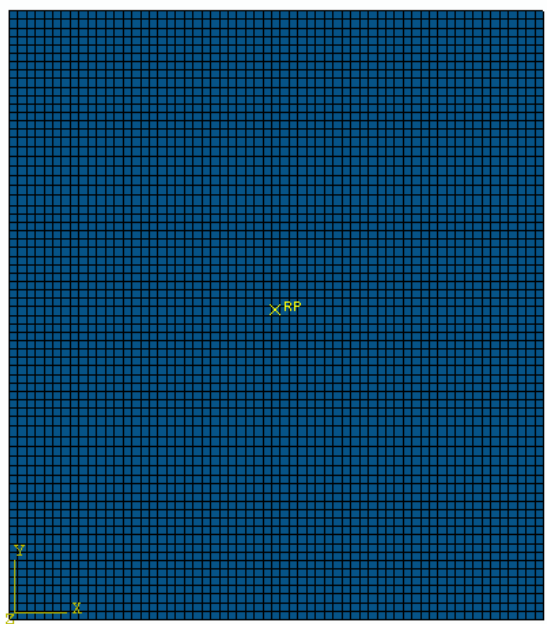

(d) Mesh 4

Figure 5.1 - Views of OSB sheet meshes used for linear elastic mesh study 


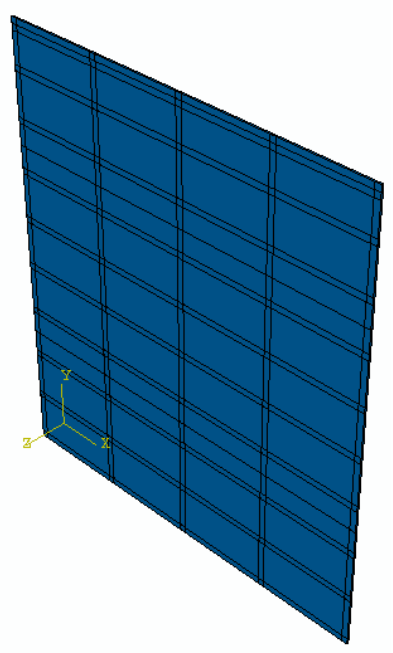

(a) OSB sheet

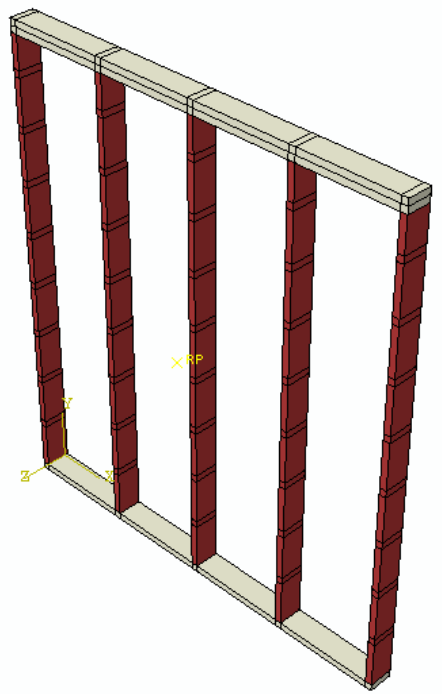

(b) Stud frame

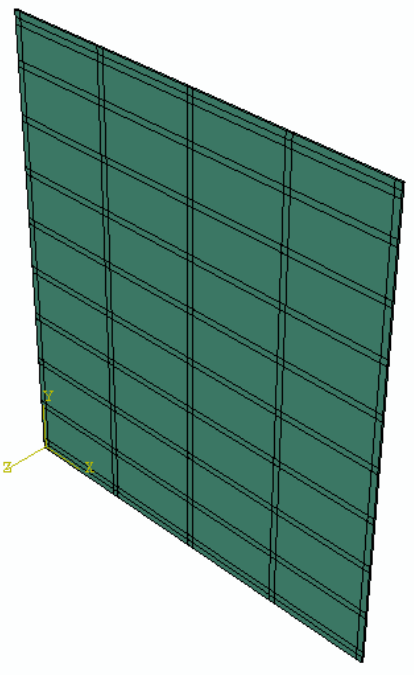

(c) Drywall sheet

Figure 5.2 - Views of different part of the FEA model in stud panel under compressive loading

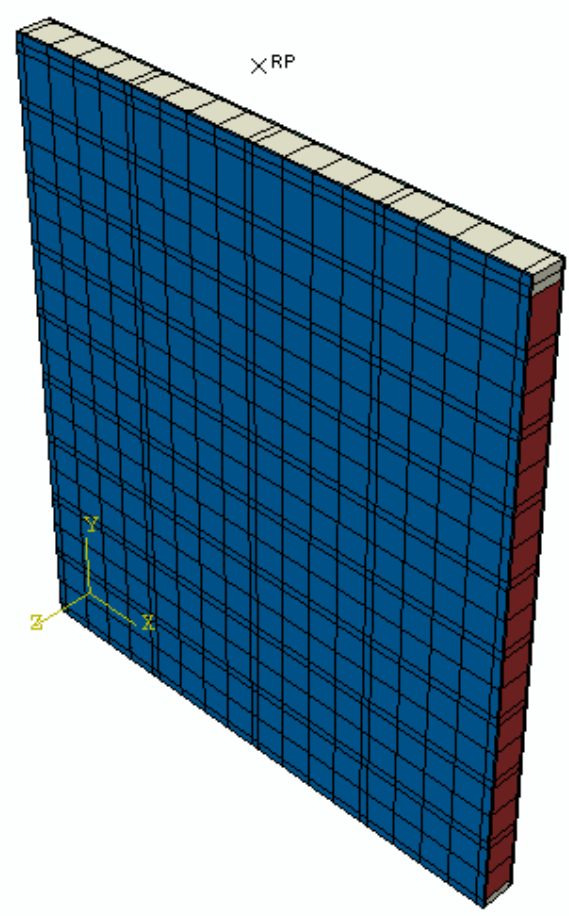

Figure 5.3 - View of ABAQUS complete FEA model of stud wall frame and OSB sheet for compressive loading 


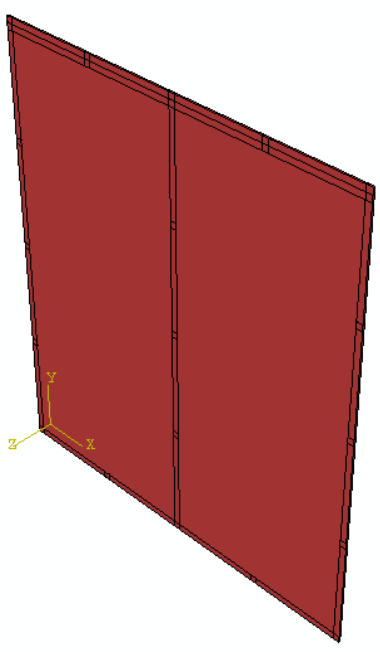

(a) OSB sheet

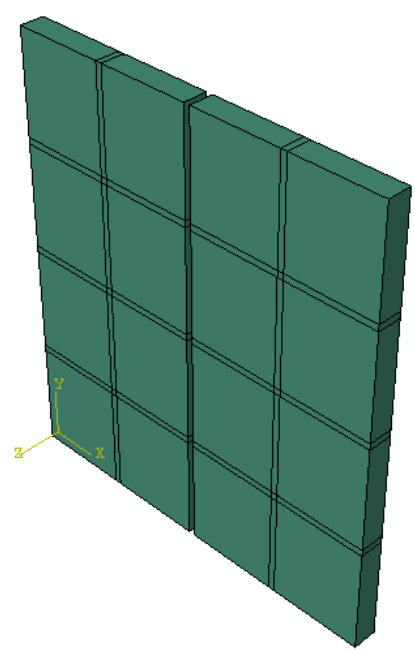

(b) EPS core

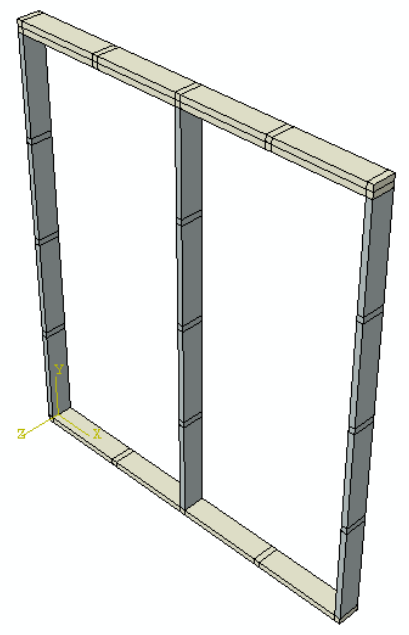

(c) Lumber frame

Figure 5.4 - Views of different parts of the FEA model of SIP under compressive loading

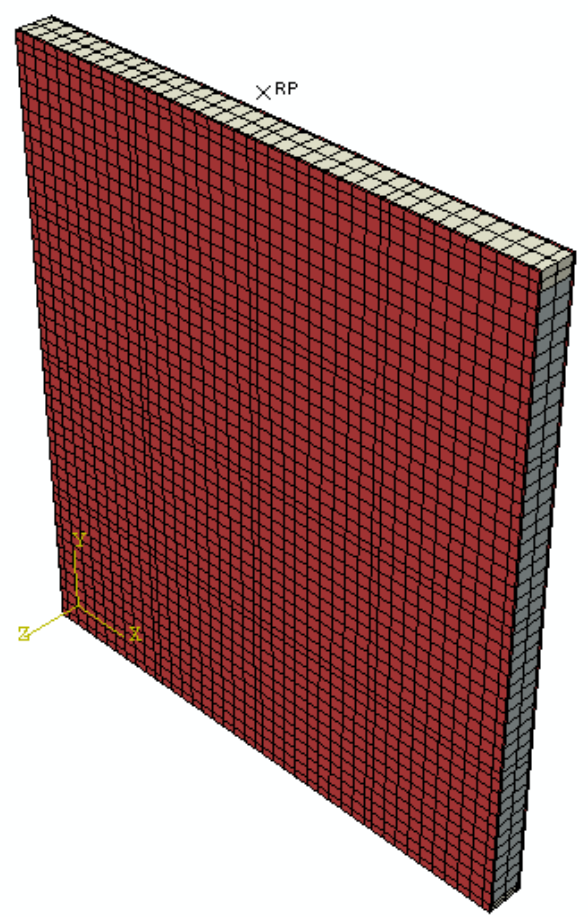

Figure 5.5 - View of the complete FE model of SIP with OSB sheathing for compressive loading

A mesh study was conducted using the developed linear-elastic FE model to investigate the level of mesh refinement that would be required for modeling the panels under monotonic loading. For the linear-elastic mesh study, a predetermined load in elastic range was applied to each FE 
model. Axial shortening and Von Mises stress output at the element in the middle of the panel was compared for the various meshes. However, axial shortening output was used primarily in selecting the sufficient level of mesh refinement. Four different FE meshes with the number of solid elements ranging from 1364 to 8972 were investigated. Table 5.1 and Figure 5.6 compare the axial shortening, U (in-plane), and Von Mises stress, VM (max), for four levels of mesh refinement. Results show that wall axial shortening was illustrated to converge to exact solution as the level of mesh refinement increased. It can be observed that the third finest mesh, named as Mesh 3 in Figure 5.1(c), appears to be adequate for predicting displacements in the elastic range. Refinement beyond this point appears to have little effect on in-plane displacement. Figure 5.7 shows views of the Von Mises stress contour plots for each level of mesh refinement for a stud wall subjected to eccentric compressive loading. The greatest Von Mises stresses for the studied FE meshes were plotted in Figure 5.6, indicating that the rate of change of these stresses decrease with increase in total number of finite-elements incorporated in the model. Subsequent to the investigation of mesh refinement, the third finest mesh (Mesh 3 in Figure 5.1) was adopted and used in all other panels.

Table 5.1 - Summary of results of linear-elastic mesh study for stud wall

\begin{tabular}{|c|c|c|c|c|}
\hline $\begin{array}{c}\text { Finite-element } \\
\text { model }\end{array}$ & Mesh number* & $\begin{array}{c}\text { Number of } \\
\text { elements }\end{array}$ & $\begin{array}{c}\text { Von Mises } \\
\text { stress, VM } \\
(\mathrm{MPa})\end{array}$ & $\begin{array}{c}\text { In-plane } \\
\text { displacement, } \\
\text { U (mm) }\end{array}$ \\
\hline MS1 & Mesh 1 & 1364 & 84.11 & 7.30 \\
\hline MS2 & Mesh 2 & 2630 & 93.57 & 6.84 \\
\hline MS3 & Mesh 3 & 3960 & 95.10 & 6.68 \\
\hline MS4 & Mesh 4 & 8972 & 103.61 & 6.37 \\
\hline
\end{tabular}

* Mesh numbers correspond to OSB sheet meshes shown in Figure 5.1. 


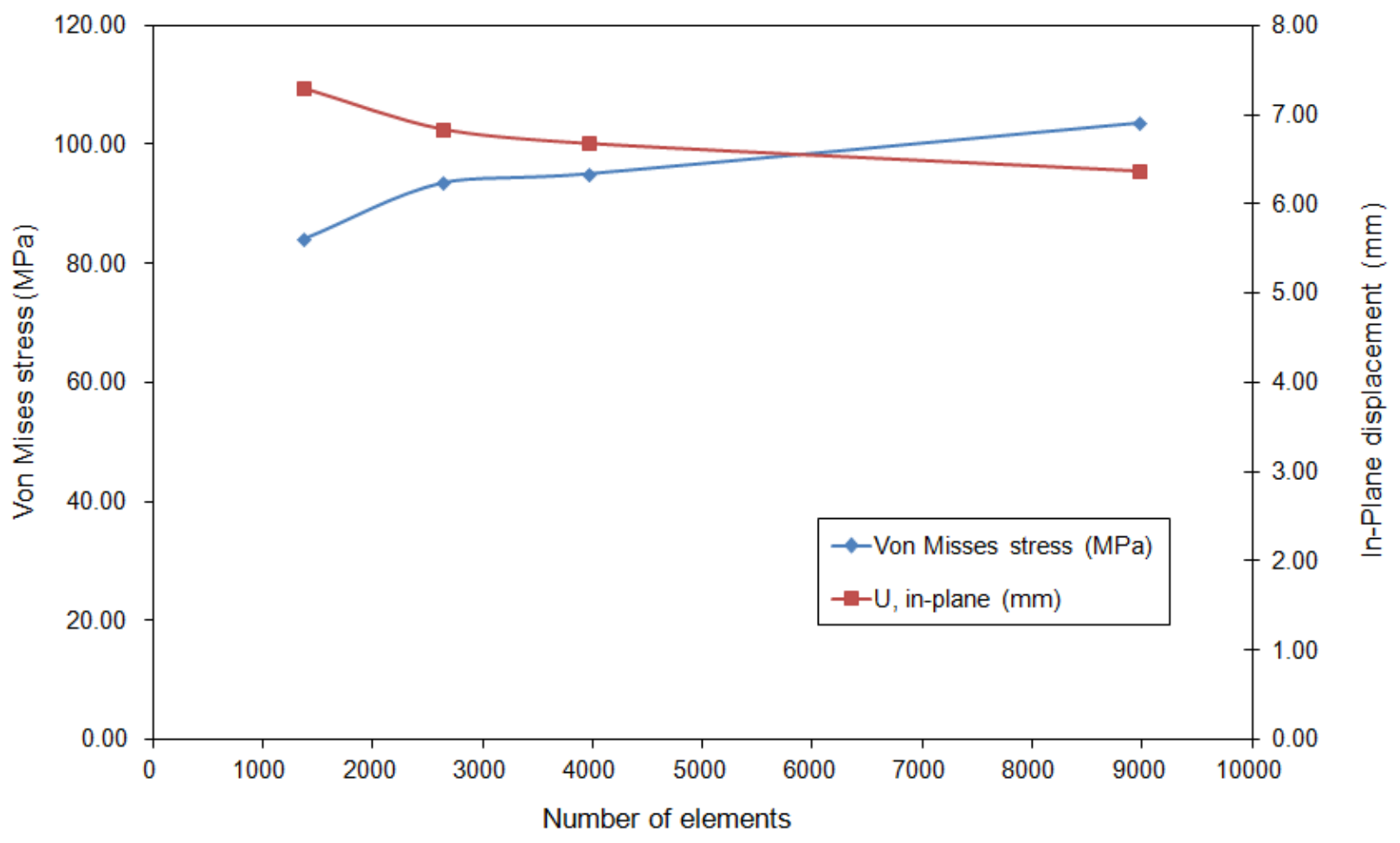

Figure 5.6 - Summary of results of linear elastic mesh study for stud wall

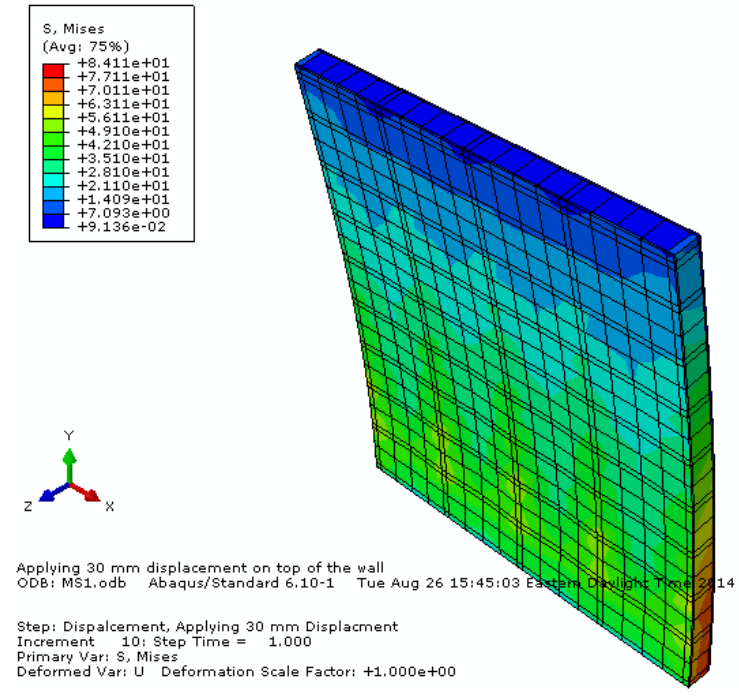

(a) Mesh 1

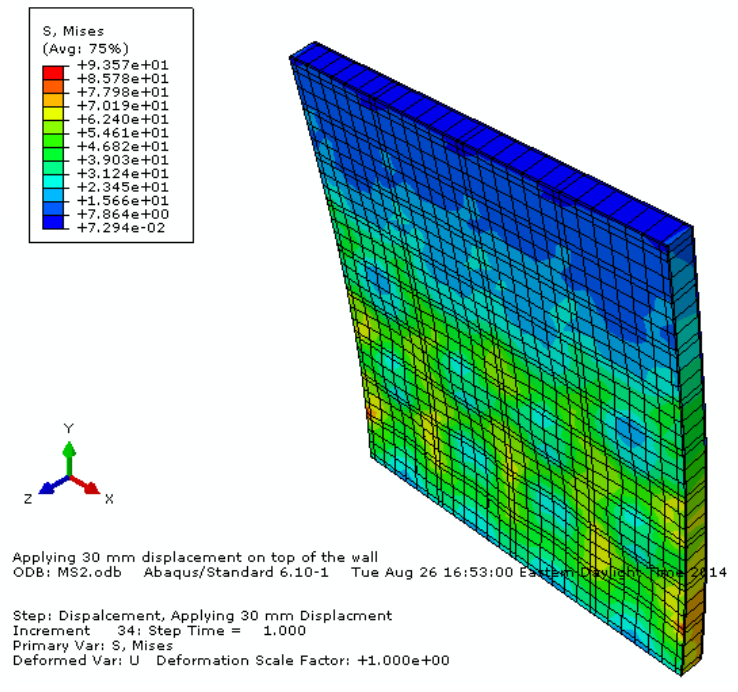

(b) Mesh 2 


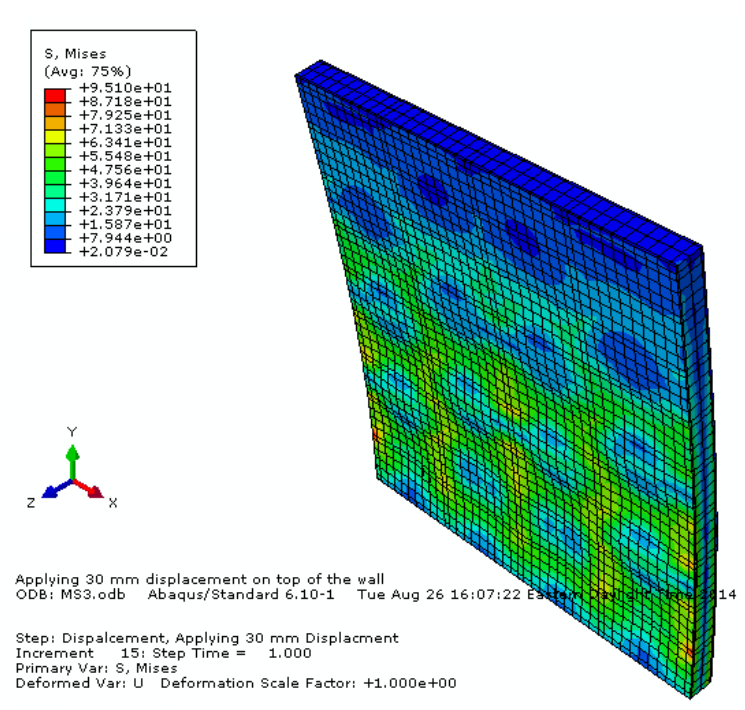

(c) Mesh 3

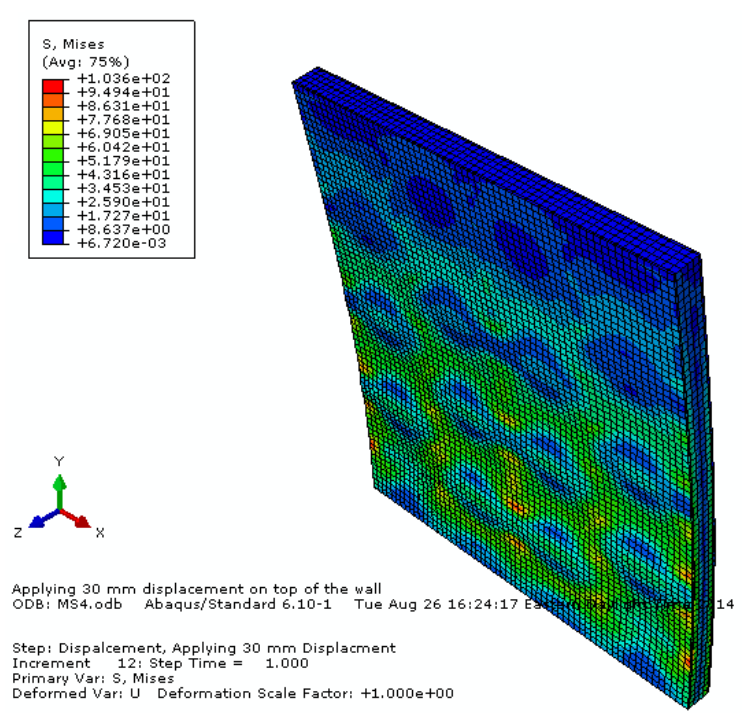

(d) Mesh 4

Figure 5.7 - Von Mises stress contour plots from linear elastic mesh study for a stud wall panel

\subsection{Inelastic Analysis}

For this portion of the analysis, nonlinear material behavior was included in the FE models to investigate the behavior of SIP panels beyond the elastic range. Anisotropic strain hardening materials model was developed based on the results from material tests conducted on OSB and EPS samples as reported in chapter 3. True stress versus plastic strain material properties were used in the FE models. Figures 5.8, 5.9 and 5.10 show the experimental stress versus strain relationships of EPS, OSB and wood materials, respectively, based on tension tests reported in chapter 3. Table 5.2 presents that the panels that were considered in the sensitivity study for mesh convergence. It should be noted that FE model designations in this table were considered identical to the specimen designations shown in chapters 3 and 4 . 


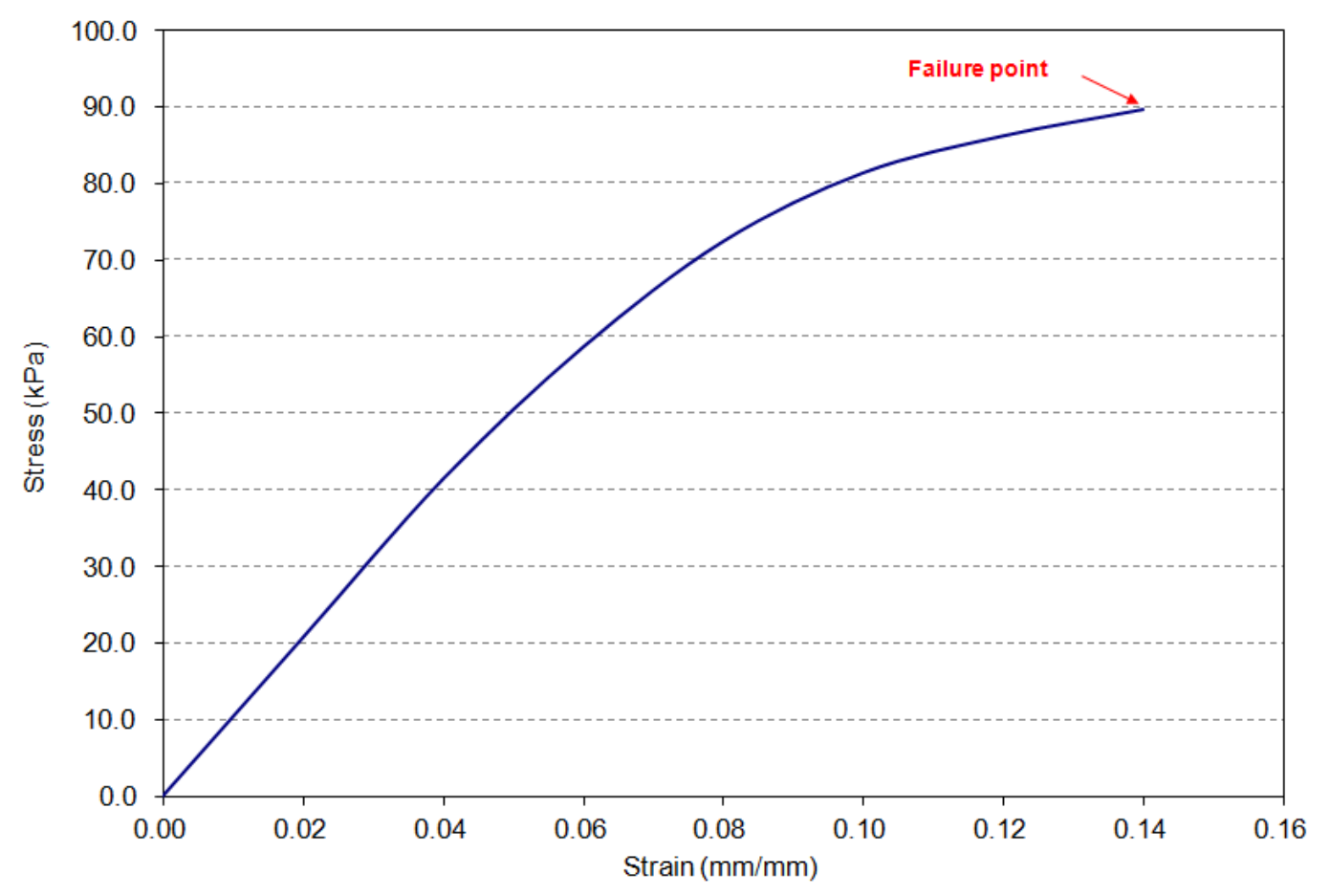

Figure 5.8 - Stress-strain relationship of EPS foam sheet obtained from tension test

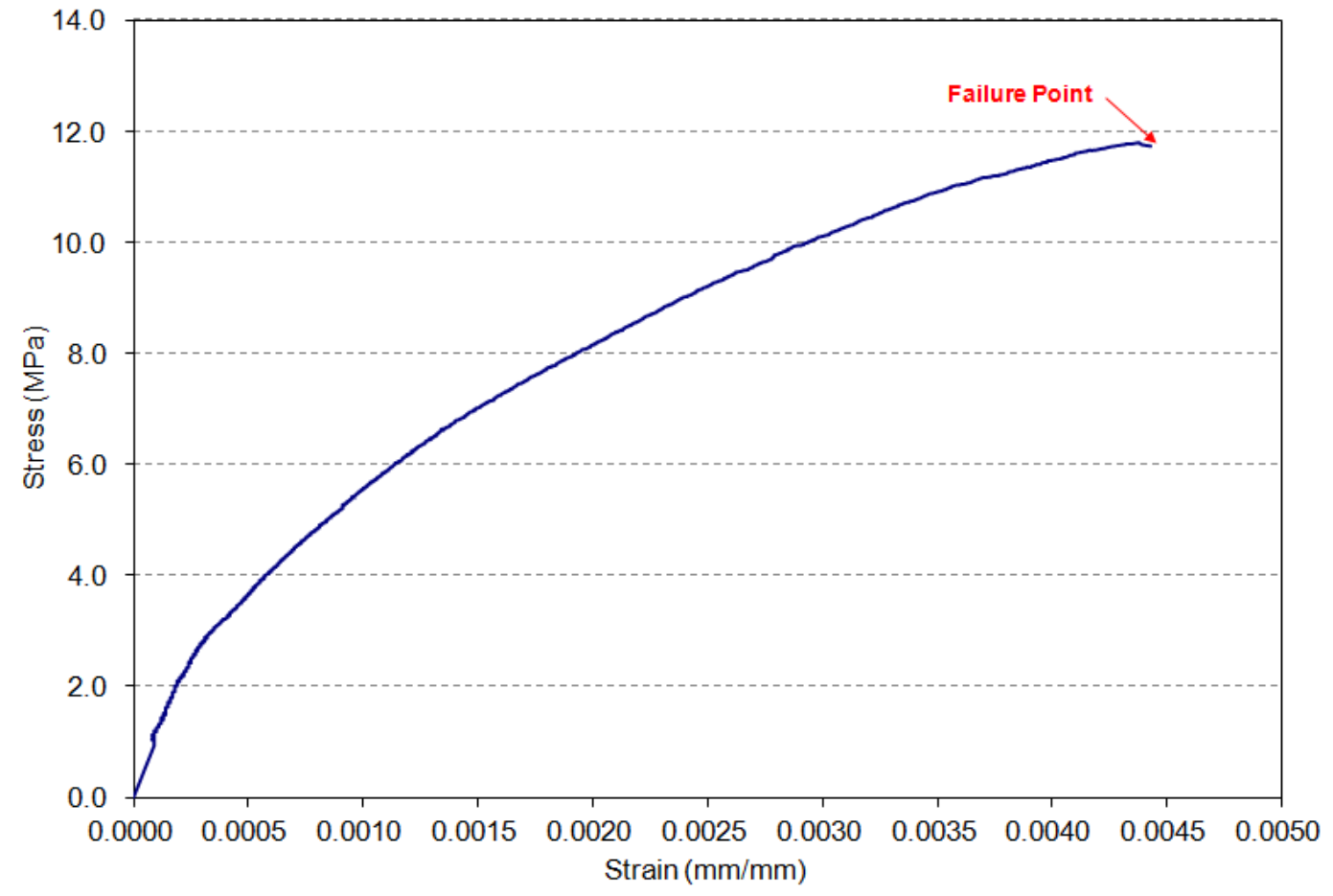

Figure 5.9 - Engineering stress-strain relationship of OSB sheet obtained from tension test 


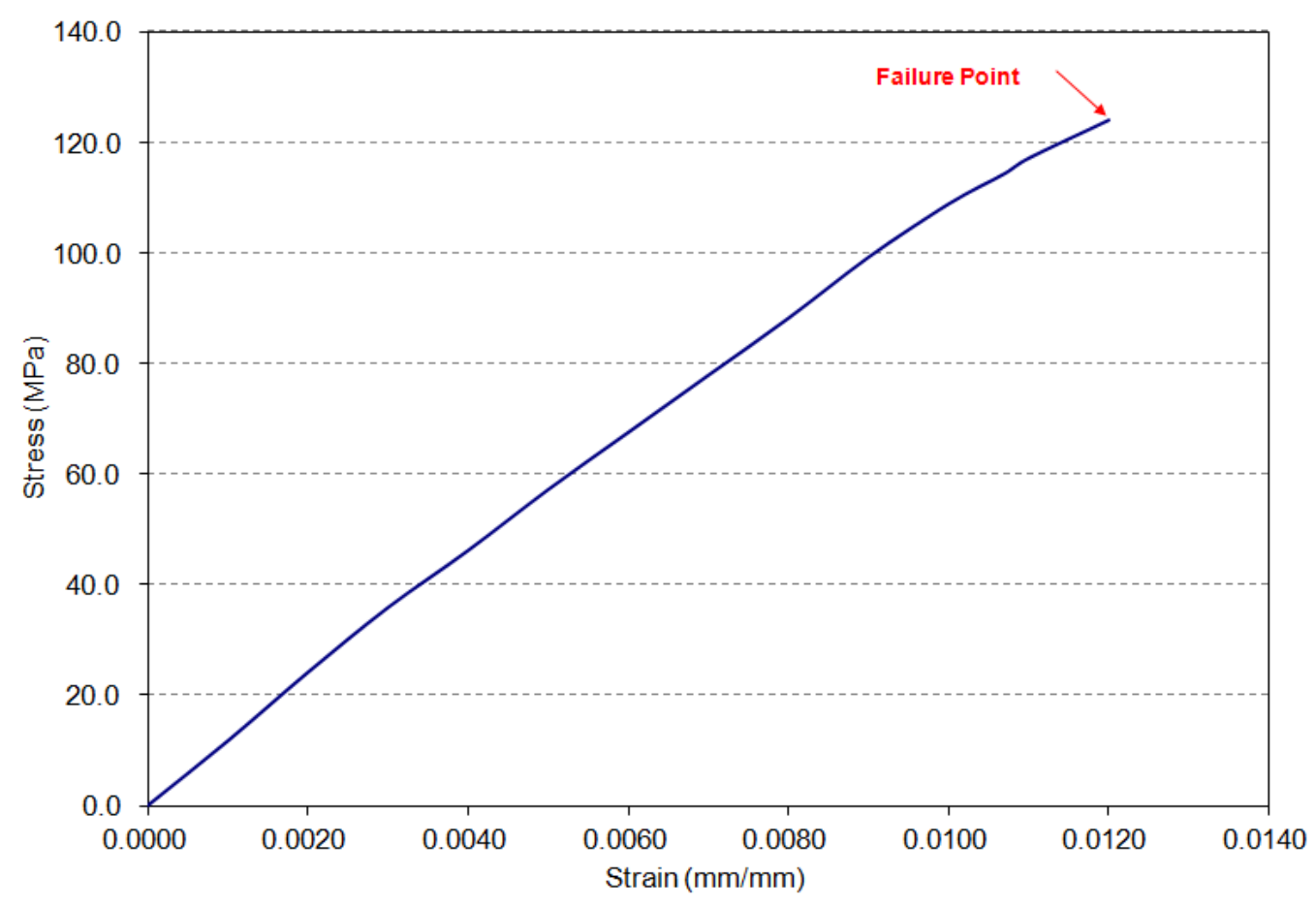

Figure 5.10 - Engineering stress-strain relationship of wood in tension obtained from commercial values 
Table 5.2 - Summary of finite-element models for different size of SIP/Stud panel and load conditions

\begin{tabular}{|c|c|c|c|c|}
\hline Test No. & Test Type & $\begin{array}{c}\text { Panel Size } \\
(\mathbf{l} \times \mathbf{w} \times \mathbf{t}) \\
\text { mm (ft-inches) }\end{array}$ & $\begin{array}{c}\text { Thickness } \\
\text { of sheathing } \\
\text { (OSB), } \\
\text { mm (inch) }\end{array}$ & $\begin{array}{c}\text { Specimen } \\
\text { type }\end{array}$ \\
\hline SW-A-1 & $\begin{array}{c}\text { Axial loading } \\
\text { (at } \mathrm{t} / 6)\end{array}$ & $\begin{array}{c}2440 \times 2750 \times 165 \mathrm{~mm} \\
\left(8^{\prime} \times 9^{\prime} \times 6^{1 / 2 \prime}\right)\end{array}$ & $\begin{array}{l}11 \mathrm{~mm} \\
\left(7 / 16^{\prime \prime}\right)\end{array}$ & $\begin{array}{c}\text { Conventional } \\
\text { stud }\end{array}$ \\
\hline SW-F-1 & Flexural Test & $\begin{array}{c}2440 \times 2750 \times 165 \mathrm{~mm} \\
\left(8^{\prime} \times 9^{\prime} \times 6 \frac{1 / 2 "}{2}\right)\end{array}$ & $\begin{array}{l}11 \mathrm{~mm} \\
\left(7 / 16^{\prime \prime}\right)\end{array}$ & $\begin{array}{c}\text { Conventional } \\
\text { stud }\end{array}$ \\
\hline SW-R-1 & Racking Loading & $\begin{array}{c}2440 \times 2750 \times 165 \mathrm{~mm} \\
\left(8^{\prime} \times 9^{\prime} \times 6^{1 / 2 \prime}\right)\end{array}$ & $\begin{array}{l}11 \mathrm{~mm} \\
(7 / 16 ")\end{array}$ & $\begin{array}{c}\text { Conventional } \\
\text { stud }\end{array}$ \\
\hline SW-C-1 & Cyclic Loading & $\begin{array}{c}2440 \times 2750 \times 165 \mathrm{~mm} \\
\left(8^{\prime} \times 9^{\prime} \times 6^{1 / 2 ”}\right)\end{array}$ & $\begin{array}{l}11 \mathrm{~mm} \\
\left(7 / 16^{\prime \prime}\right)\end{array}$ & $\begin{array}{c}\text { Conventional } \\
\text { stud }\end{array}$ \\
\hline SIP-A-1 & $\begin{array}{c}\text { Axial loading } \\
\text { (at } \mathrm{t} / 6)\end{array}$ & $\begin{array}{c}1120 \times 2750 \times 165 \mathrm{~mm} \\
\left(4^{\prime} \times 9^{\prime} \times 6^{1}{ }^{\prime \prime}\right)\end{array}$ & $\begin{array}{l}11 \mathrm{~mm} \\
(7 / 16 ")\end{array}$ & SIP \\
\hline SIP-F-1 & Flexural Test & $\begin{array}{c}1220 \times 2750 \times 165 \mathrm{~mm} \\
\left(4^{\prime} \times 9^{\prime} \times 6^{\prime}{ }^{\prime \prime \prime}\right)\end{array}$ & $\begin{array}{l}11 \mathrm{~mm} \\
\left(7 / 16^{\prime \prime}\right)\end{array}$ & SIP \\
\hline SIP-R-1 & Racking Loading & $\begin{array}{c}2440 \times 2750 \times 165 \mathrm{~mm} \\
\left(8^{\prime} \times 9^{\prime} \times 6^{1 / 2 \prime}{ }^{\prime \prime}\right)\end{array}$ & $\begin{array}{l}11 \mathrm{~mm} \\
\left(7 / 16^{\prime \prime}\right)\end{array}$ & SIP \\
\hline SIP-R-4 & Racking Loading & $\begin{array}{c}3660 \times 2750 \times 165 \mathrm{~mm} \\
\left(12^{\prime} \times 9^{\prime} \times 6 \frac{1}{\prime \prime} "\right)\end{array}$ & $\begin{array}{l}11 \mathrm{~mm} \\
\left(7 / 16^{\prime \prime}\right)\end{array}$ & SIP \\
\hline SIP-C-1 & Cyclic Loading & $\begin{array}{c}2440 \times 2750 \times 165 \mathrm{~mm} \\
\left(8^{\prime} \times 9^{\prime} \times 6^{1 / 2 ”}\right)\end{array}$ & $\begin{array}{l}11 \mathrm{~mm} \\
(7 / 16 ")\end{array}$ & SIP \\
\hline SIP-C-4 & Cyclic Loading & $\begin{array}{c}3660 \times 2750 \times 165 \mathrm{~mm} \\
\left(12^{\prime} \times 9^{\prime} \times 6{ }^{1 / 2 \prime}\right)\end{array}$ & $\begin{array}{l}11 \mathrm{~mm} \\
\left(7 / 16^{\prime \prime}\right)\end{array}$ & SIP \\
\hline
\end{tabular}

The contact between OSB/Drywall sheets and stud frame in the stud wall system was simulated by surface-to-surface contact available in ABAQUS software. This surface-to-surface contact was considered between the studs and the top and bottom plates. However, the constraints available in ABAQUS software were considered to represent the effect of the nail on connecting the OSB/Drywall sheet and the studs. This tie constraint was developed between the outer 
peripheral surface of the stud frame and inner peripheral surface of the sheet. There is also a rigid body constraint in the model which defines the virtual connection of reference point to the top part of wall in order to apply a distributed load to panel. Figures 5.11 and 5.12 show the different contacts and constraints on a stud wall system.

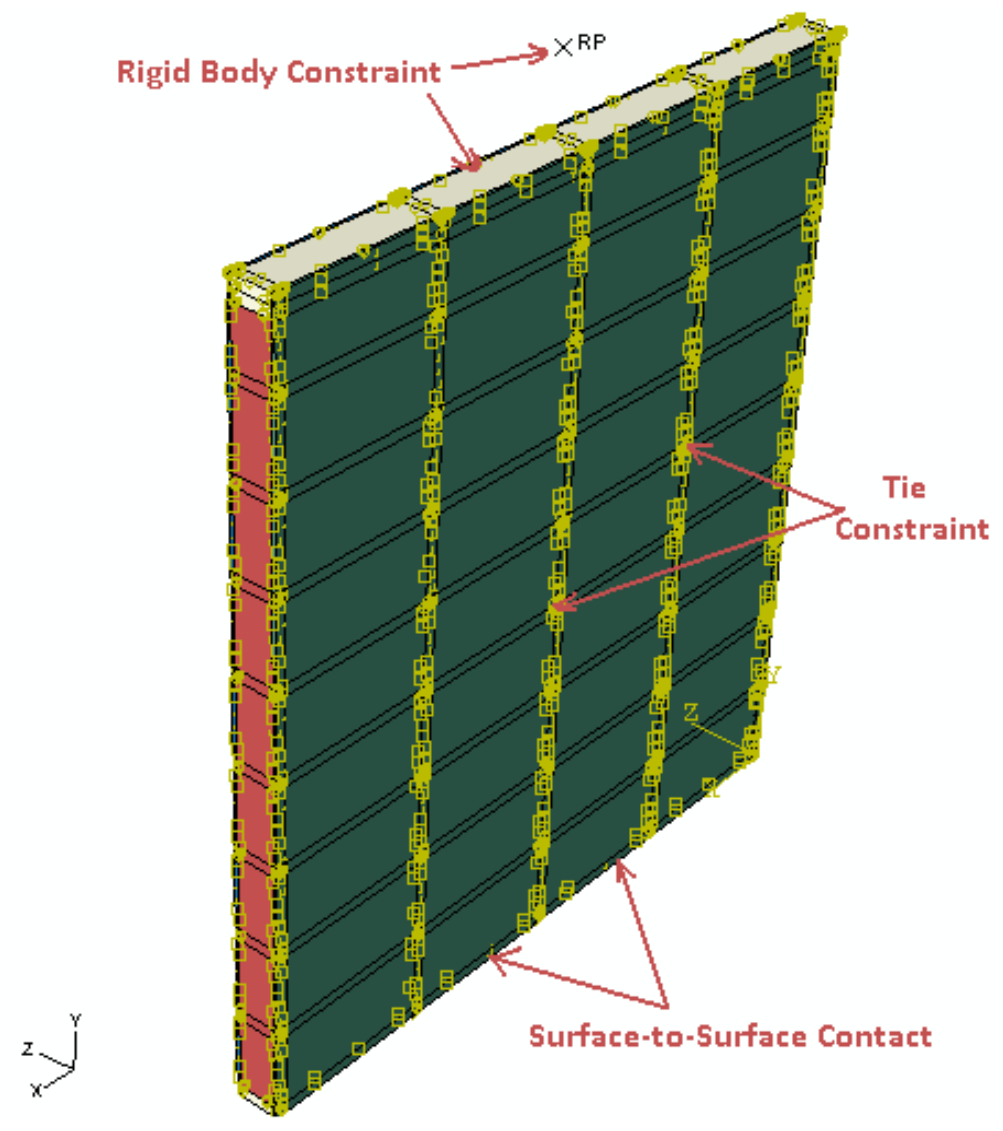

Figure 5.11 - Surface-to-surface contact and different constraints of drywall and stud frame in ABAQUS model of stud panel 


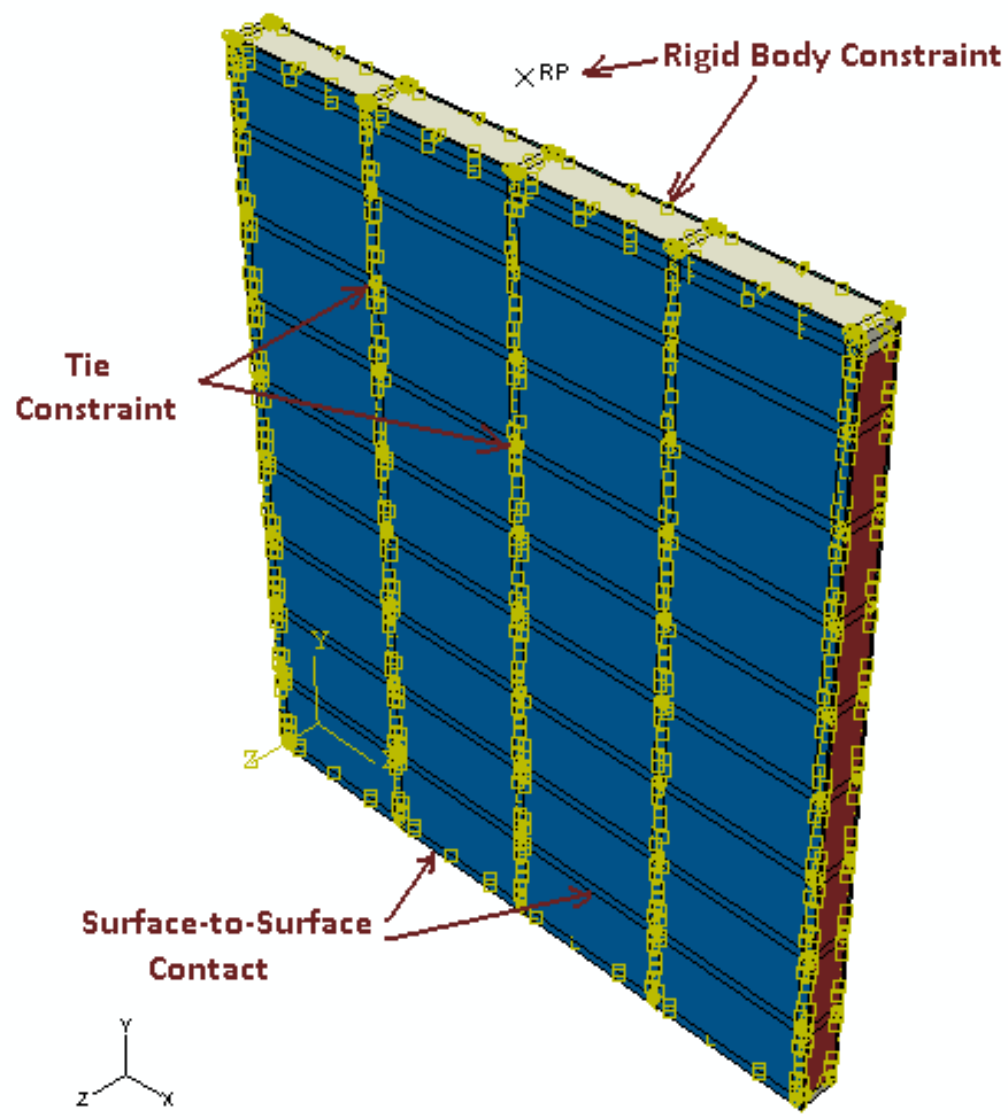

Figure 5.12 - Surface-to-surface contact and different constraints of OSB sheet and stud frame in ABAQUS Model of stud panel

The contact between OSB sheets and EPS core foam was simulated by surface-to-surface contact. This surface-to-surface contact represents that hard or glue contact between the OSB sheet and the foam core. The effect of the nails on connecting the OSB sheet and lumber was defined by tie constraint which was developed between the outer peripheral surface of frame and inner peripheral surface of the sheet. This constraint was also used for foam-spline connection between panel segments. The FE model included a rigid body constraint which defines the virtual connection of reference point to the loading surface in order to apply a distributed load to the top of the panel. Figure 5.13 shows the different constraints on SIP model, while Figure 5.14 also shows the surface-to-surface contacts of OSB sheet with EPS foam core representing the glue and between the EPS foam core and the lumber. Figures 5.15 to 5.19 show views of finite element model of SIP and stud panels considered in this study. 


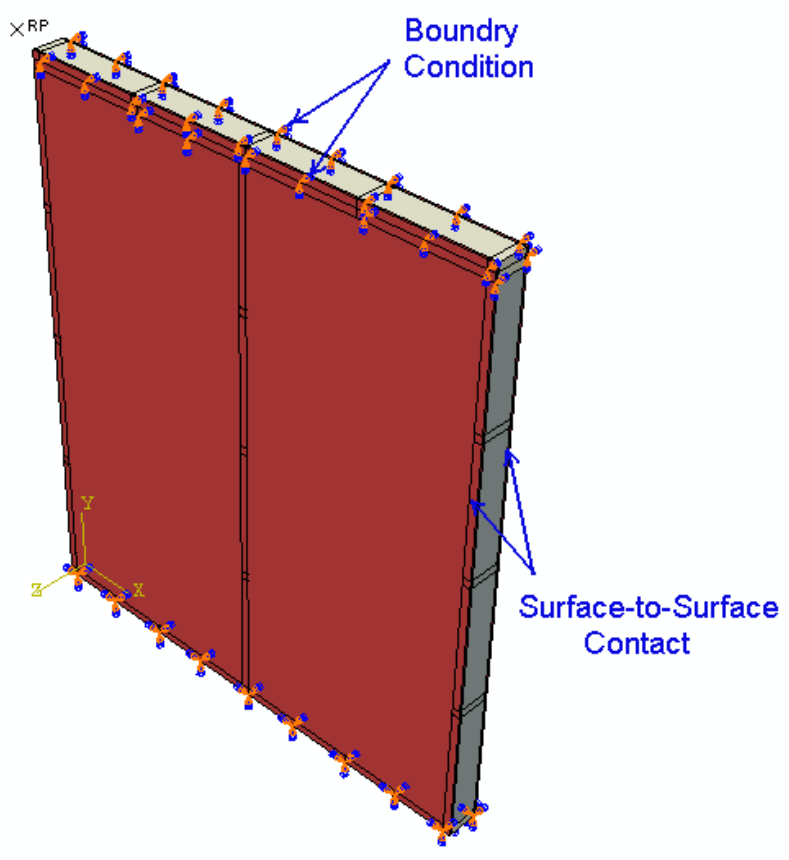

Figure 5.13 - Surface-to-surface contact of OSB sheet with lumber and boundary conditions in ABAQUS Model of SIP panel

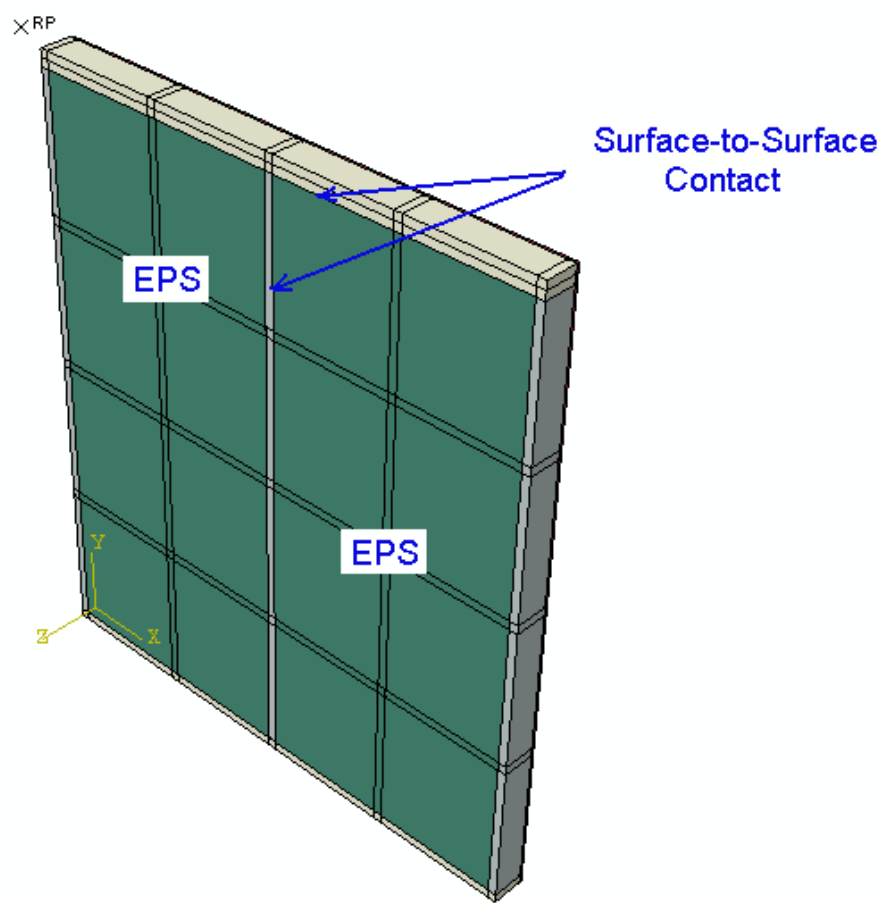

Figure 5.14 - Surface-to-surface contact of OSB sheet with glue and EPS with Lumber in ABAQUS Model of SIP 


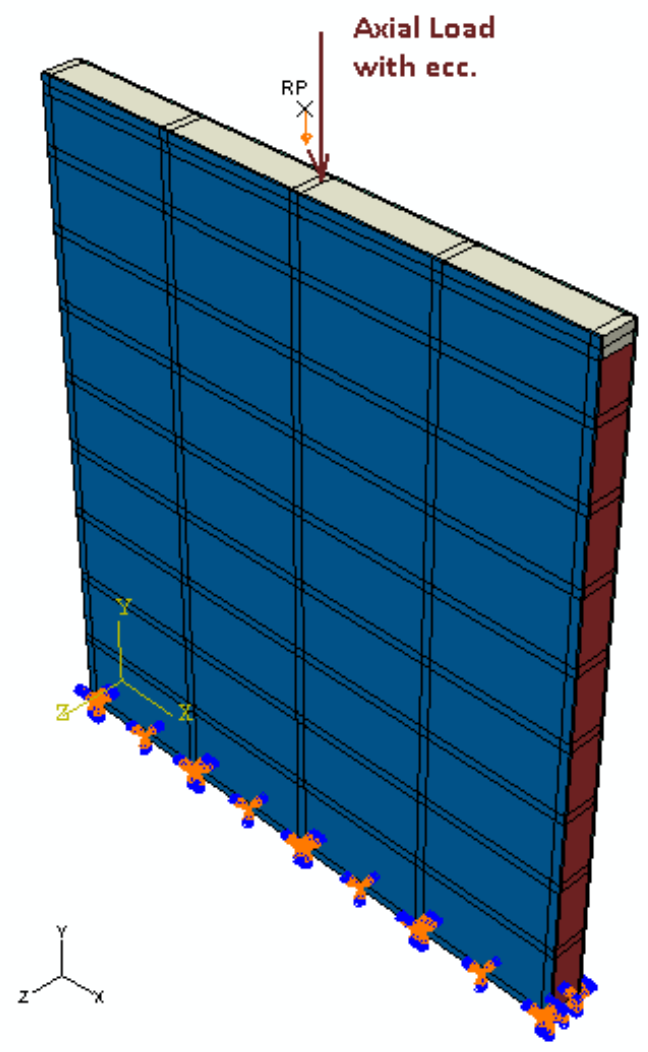

Figure 5.15 - Complete ABAQUS model of stud wall SW-A-1 under compressive loading with eccentricity

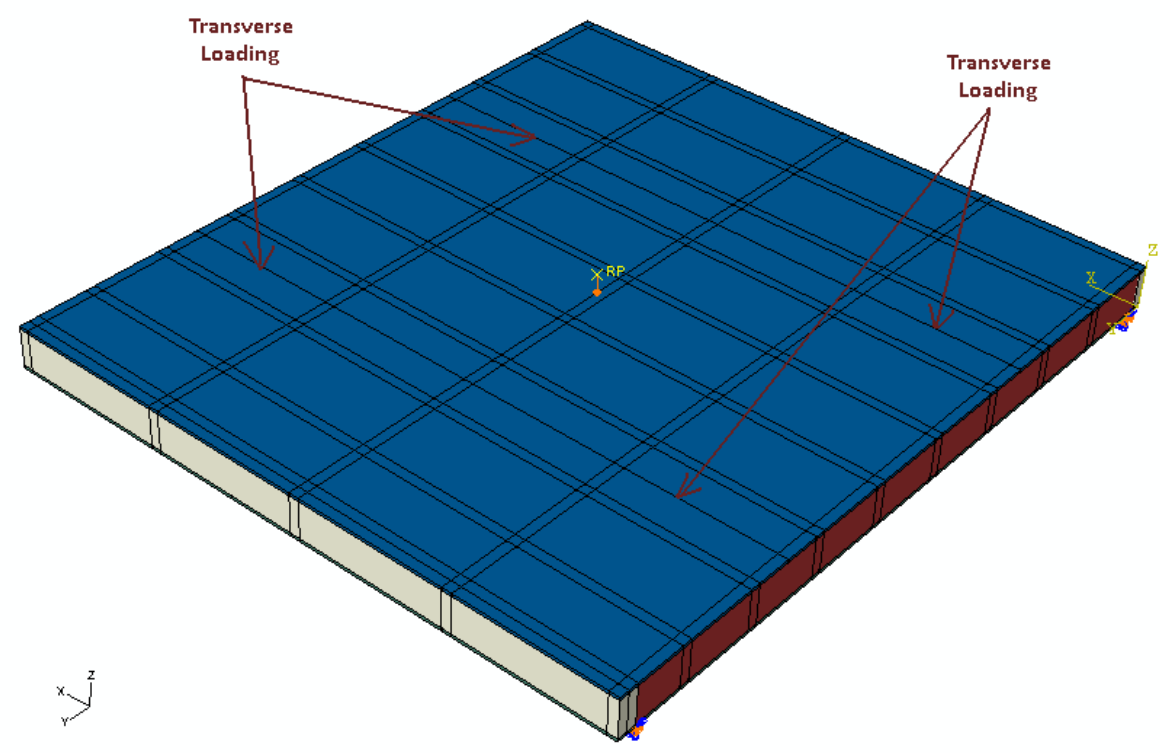

Figure 5.16 - Complete ABAQUS model of stud wall SW-F-1under flexural loading 

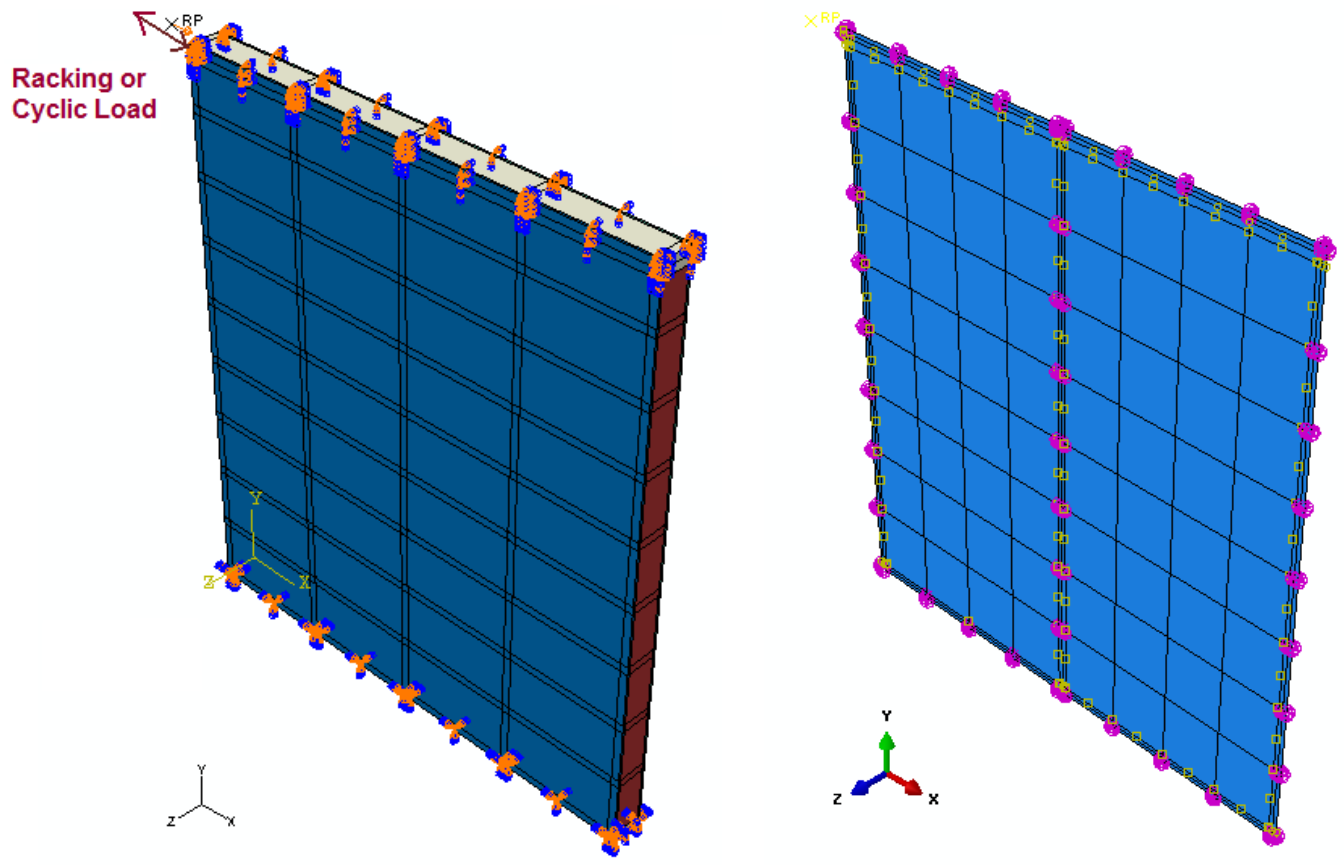

(a) FEM with Nails (Tie-Contact)

(b) FEM with Nail (Oriented Springs)

Figure 5.17 - Complete ABAQUS model of stud wall SW-R-1 and SW-C-1 under racking or cyclic loading
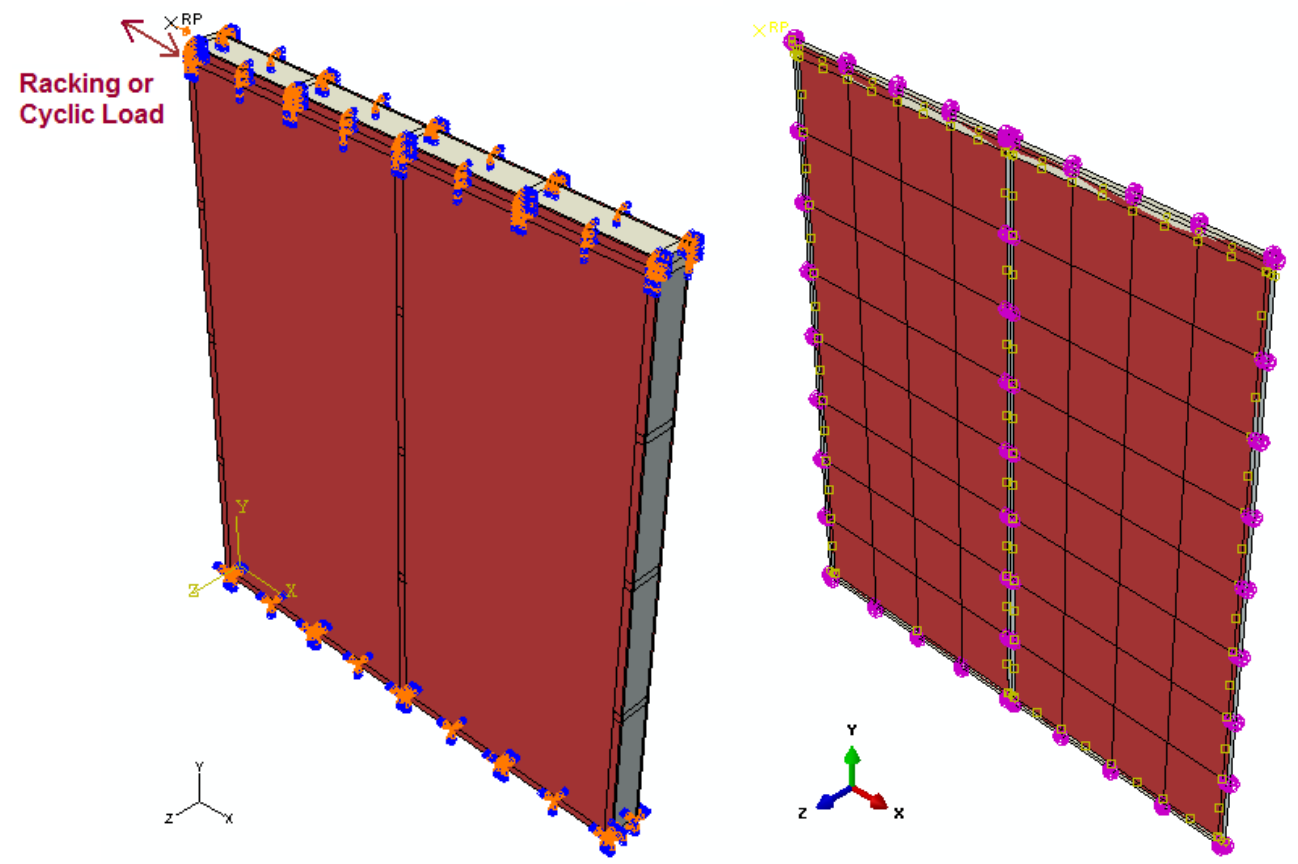

(a) FEM with nails (Tie-Contact)

(b) FEM with nails (Oriented Springs)

Figure 5.18 - Complete ABAQUS model of SIP wall SIP-R-1 and SIP-C-1 under racking or cyclic load 


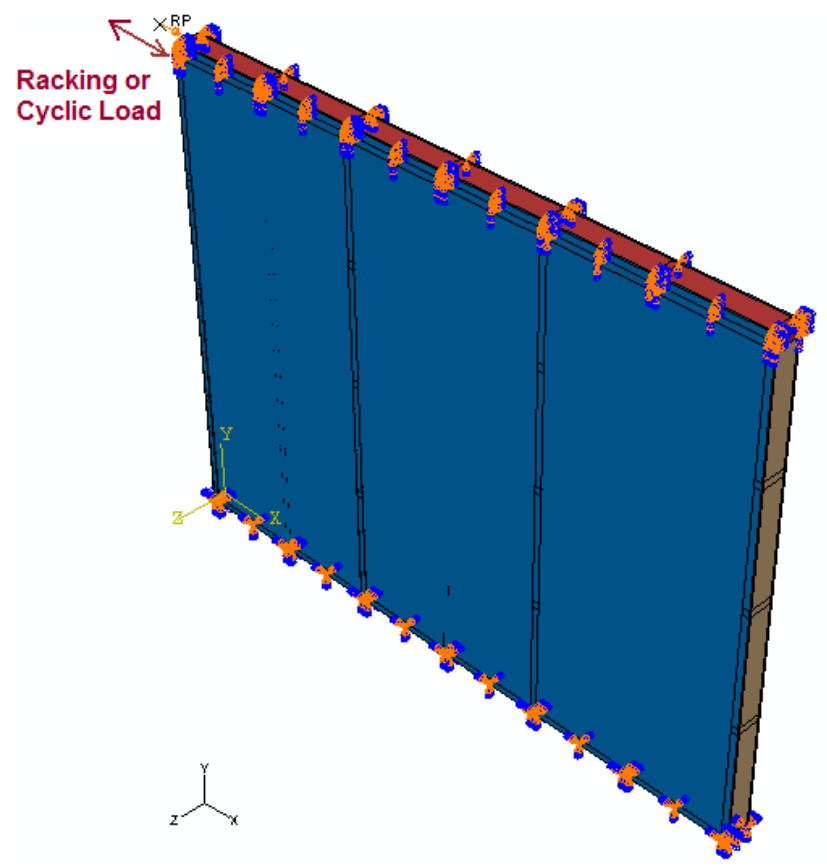

(a) FEM with nails (Tie-Contact)

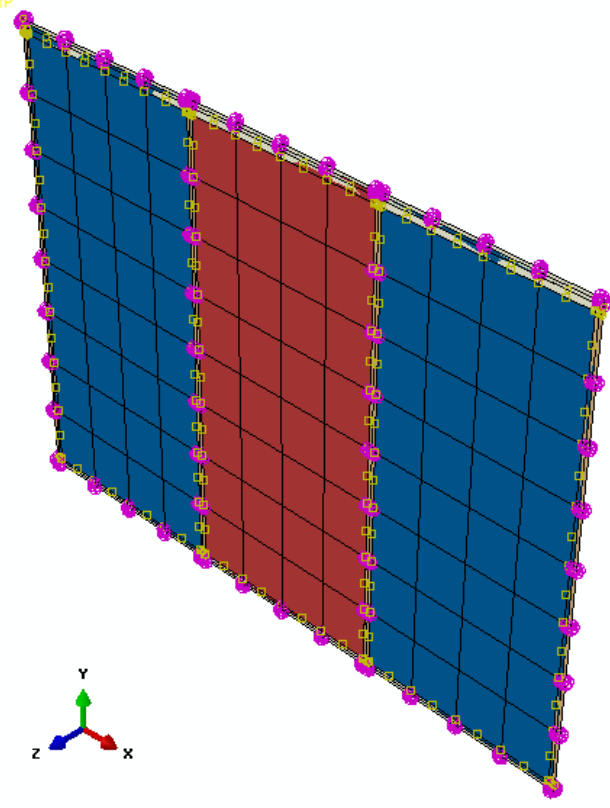

b) FEM with nails (Oriented Springs)

Figure 5.19 - Complete ABAQUS model of SIP wall SIP-R-4 and SIP-C-4 under racking or cyclic load

The effect of FE mesh refinement on the non-linear behavior of stud wall under compressive loading was investigated. Figure 5.20 depicts the load-axial shortening relationships for different FE mesh sizes. It appears that the change in nonlinear behavior decreases with increase in mesh size. The difference between subsequent refinements appears to diminish as the mesh is refined. Based on the results shown in Figure 5.20, the third finest mesh, Mesh 3 in Figure 5.1(c), was selected for the remaining compressive load analysis. Figure 5.21 shows the change in loaddeflection relationship for as stud system under flexural loading with the change in FE mesh size. Again, FE mesh 3 showed better convergence that FE meshes 1 and 3 . As such, it has subsequently been used in further modeling of the stud system. 


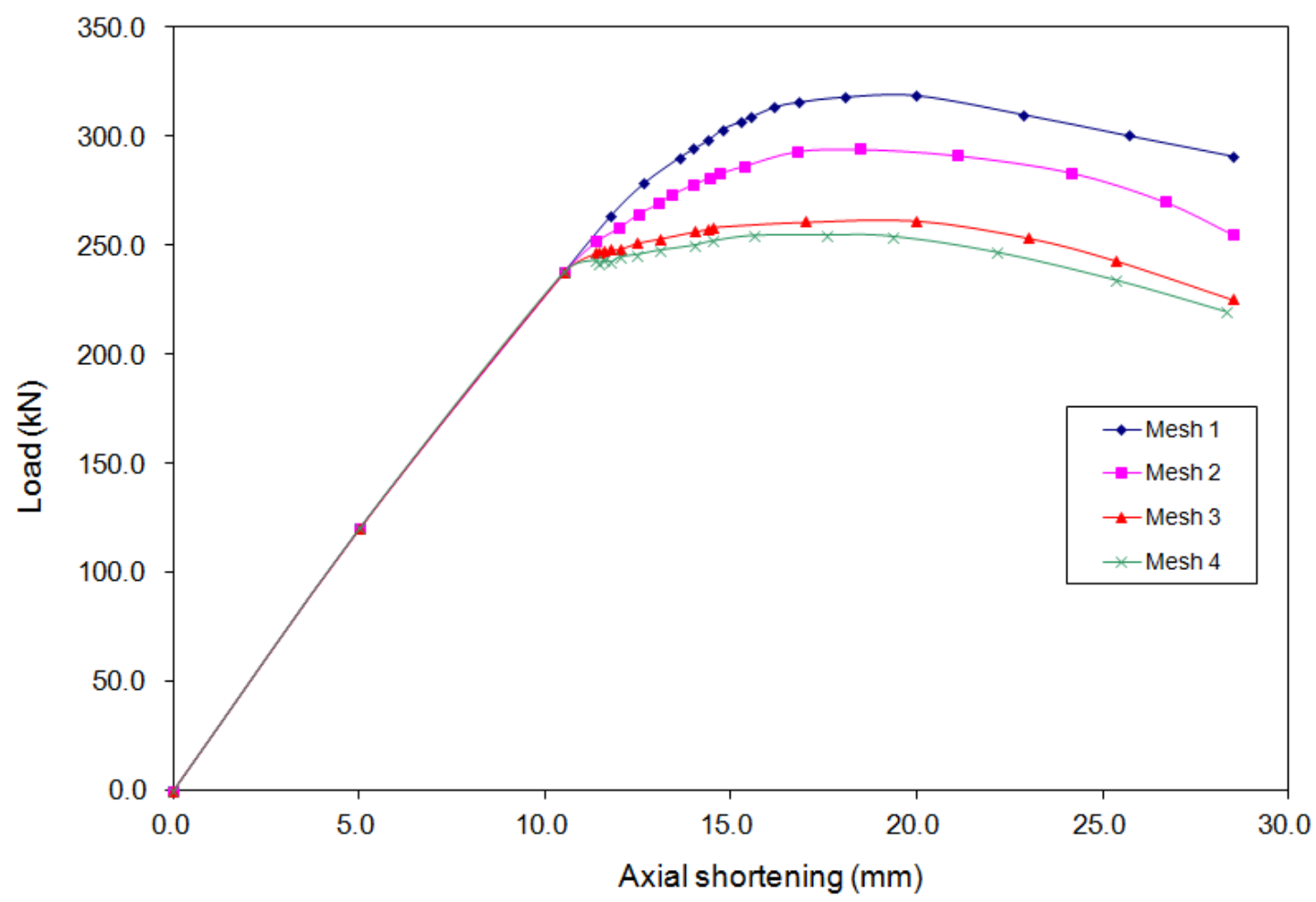

Figure 5.20 - Effect of mesh refinement for stud panel wall SW-A-1 under compressive loading

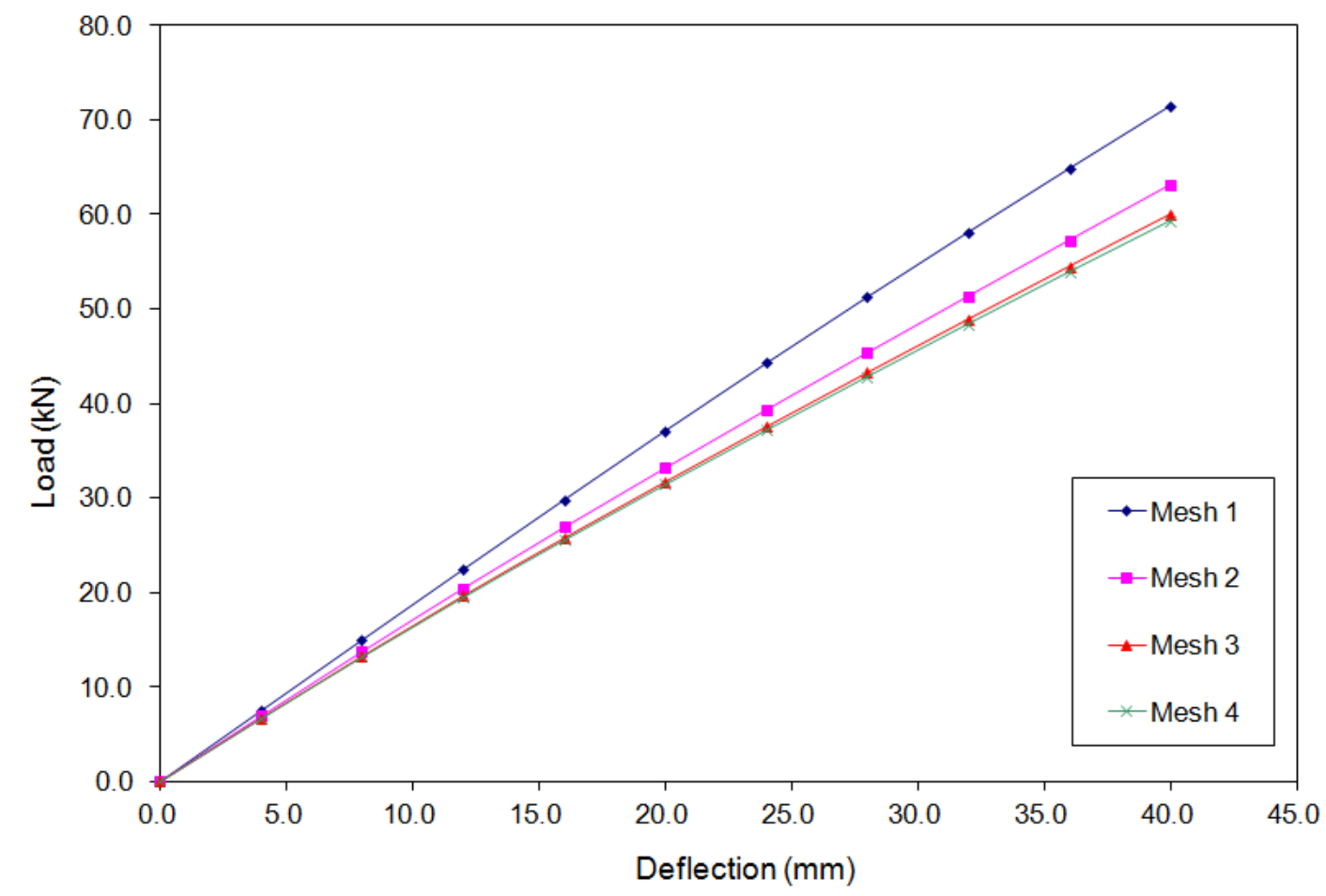

Figure 5.21 - Effect of mesh refinement for stud panel floor SW-F-1 under flexural loading 
As mentioned earlier, the 8-node brick element (designed as C3D8I in ABAQUS element library) was selected to model OSB sheets, studs, foam core and top, bottom and side plates in case of flexural and compressive loading. A sensitivity study was undertaken to examine the correlation of the failure modes obtained experimentally and those obtained from the FE modeling. Figure 5.22 shows view of the contour lines of stresses and deformed shape in stud wall model SW-A-1 along with the deformed shape at failure in the tested stud wall. Similar failure mode was observed. Figure 5.23 shows view of the contour lines of stresses and deformed shape in stud wall model SW-F-1 along with the deformed shape at failure in the tested stud wall subjected to flexural loading. Similar failure mode was observed in the form of high stresses at the mid-span location leading to failure due to bending.

As for racking and cyclic loading on stud and SIP panels, it was mentioned earlier that the 4node plane stress/strain element (designed as CPS4I in ABAQUS element library) was used to model all elements forming either the stud wall or SIP wall with the presence of spring element representing the nail behavior as well as the contact element to represent the glue between the OSB sheet and the foam core. To examine the effect of the modeling of the contact between the OSB sheet and the studs and top and bottom plates in stud wall system, two types of constraints were considered. The first one is "tie contact" which provides rigid link between the nodes of the OSB sheet and the corresponding nodes in the studs and lumber plates, irrespective of the intensity of the applied load. The second constraint is "oriented spring" to represent the nail connecting the OSB sheet to the studs and lumber plates. Two perpendicular springs were included at the nail location to allow for deformation of the nail on the plane of the OSB sheet with a spring stiffness of $450 \mathrm{kN} / \mathrm{mm}$. To reach this value of spring stiffness, different values were considered in the model and by comparing the resulting load-lateral deflection relationships. Then, it was decided to use $450 \mathrm{kN} / \mathrm{mm}$ that showed the best convergence for deflection values through trial and error approach.

Figure 5.24 shows views of the FE deformed shapes and contour lines of stresses of stud model SW-R-1 subjected to racking loading. It can be observed that the use of tie contact constraint between the OSB sheet and the studs led to buckling of the sheet as depicted in Figure 5.24(a). However, the use of oriented spring constraint let to relative shear deformation between the 
adjacent OSB sheet and stress concentration at the top and bottom of the wall along with stress concentration at nail location at failure as depicted in Figures 5.24(b) and (c). Such failure mode depicted with the use of spring constraints matched the failure mode observed experimentally as shown in Figure 5.24(d) and as reported in chapter 4. Similar observations were noticed in case of stud wall SW-C-1 under cyclic loading as depicted in Figure 5.25. Figure 5.26 and 5.27 also show the SIP walls subjected to axial compressive and flexural loading and deformation configuration of these panels, respectively. In addition, identical observations were noticed in case of SIP walls subjected to racking loading for the short panel, SIP-R-1, and long panel, SIPR-4, as depicted in Figures 5.28 and 5.29, respectively. Moreover, similar observations were noticed in case of SIP walls subjected to cyclic loading for the short panel, SIP-C-1, and long panel, SIP-C-4, as depicted in Figures 5.30 and 5.31, respectively. As such, it can be concluded that nail connection has a significant effect in panel failure mode and intensity of panel lateral deflection under racking and cyclic loading.
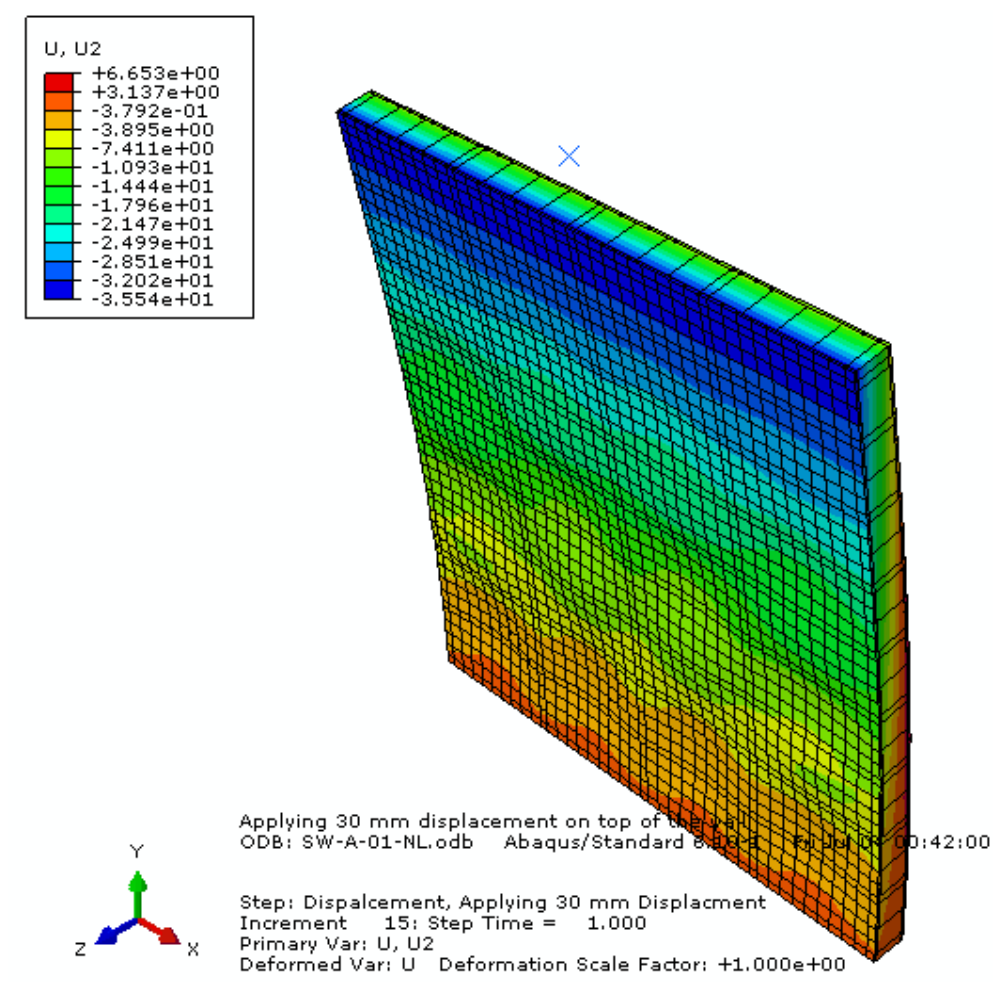

(a) View of the finite-element model of stud wall SW-A-01showing contour lines under compressive loading 


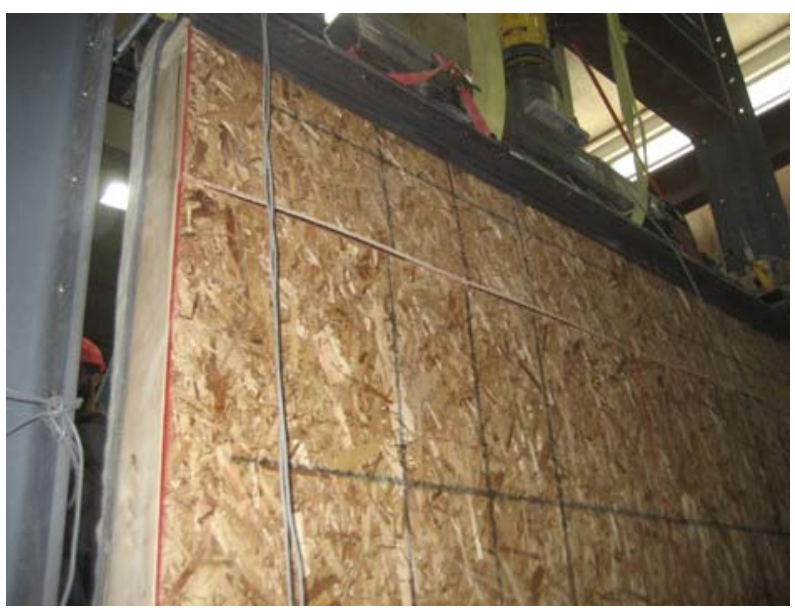

(b) View of deformation and failure of specimen SW-A-1

Figure 5.22 - Deformed configuration of stud wall SW-A-1 loaded in compression

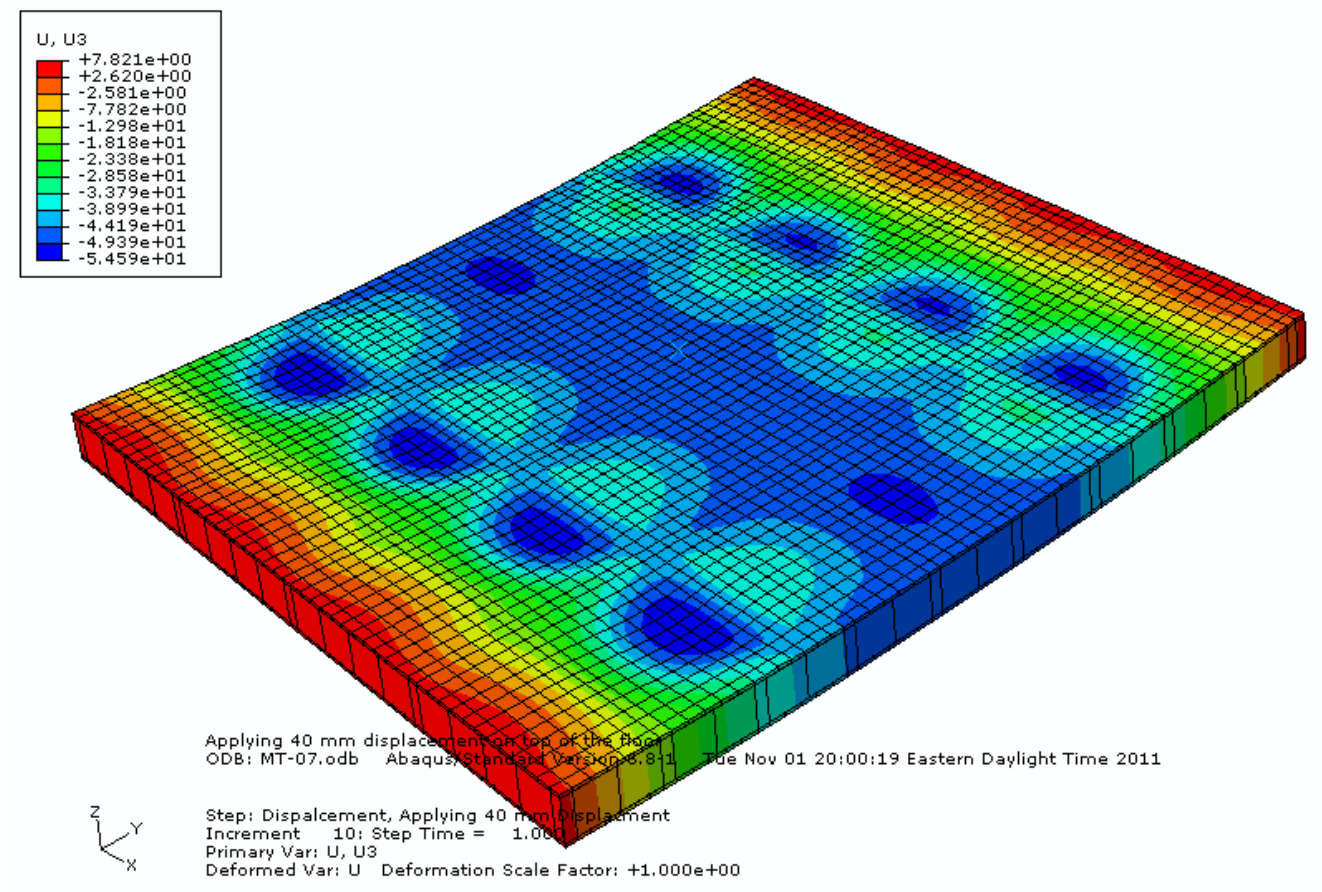

(a) View of the finite-element model of stud panel SW-F-1 showing contour lines under flexural loading 


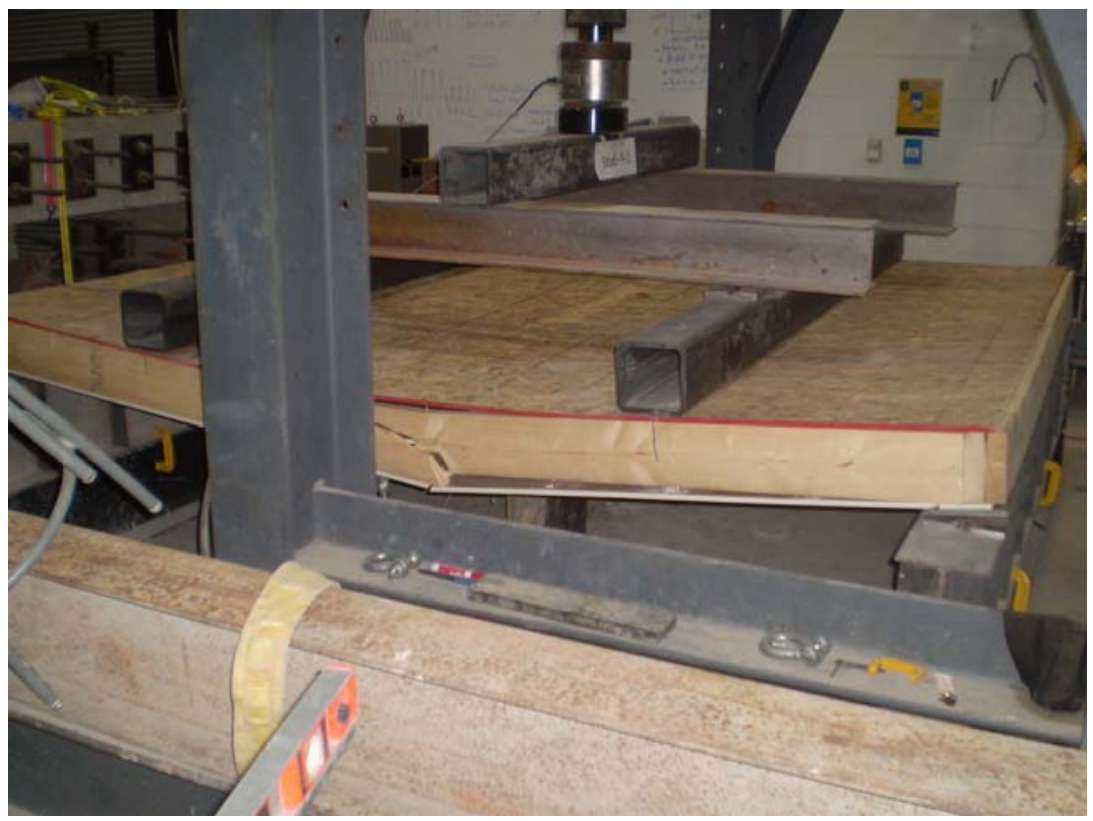

(b) Deformation and failure of specimen SW-F-1

Figure 5.23 - Deformed shape of stud panel SW-F-1 loaded in flexure
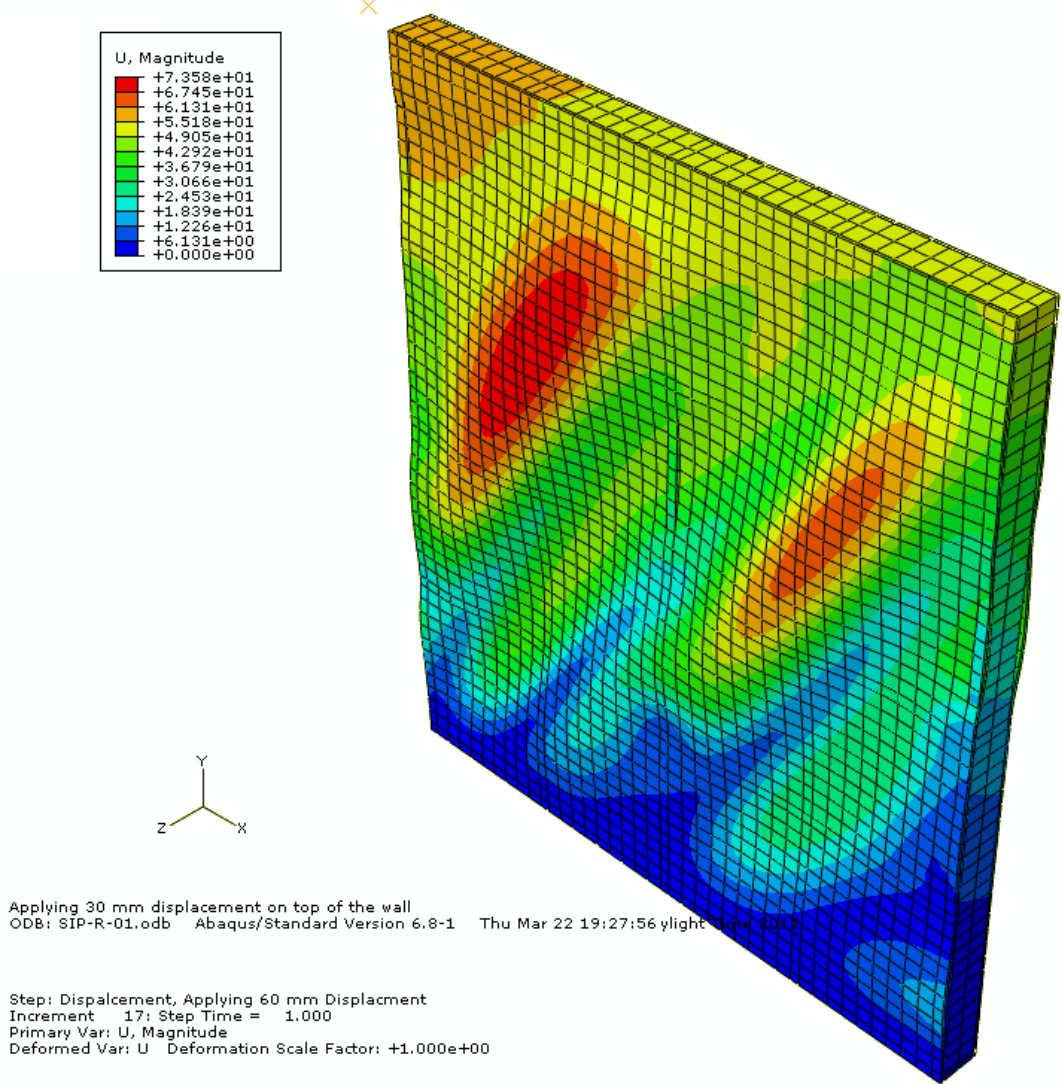

(a) Finite element model of SW-R-1 (Nail - Tie Contact) 


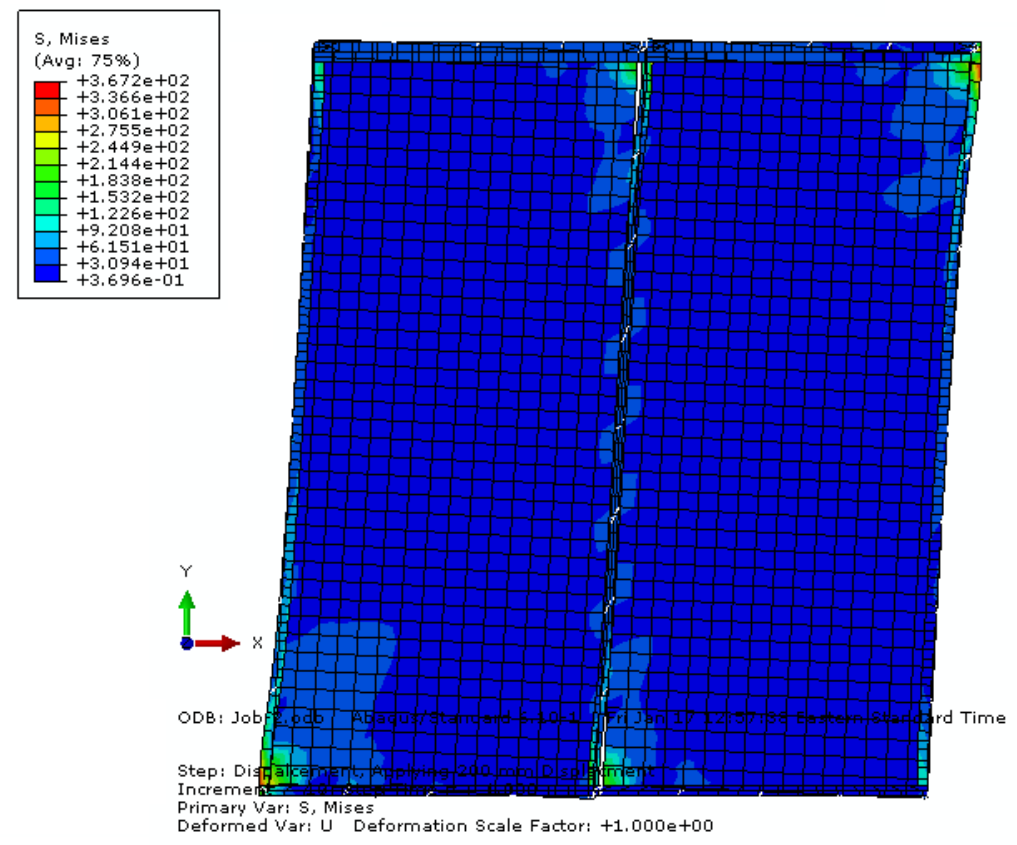

(b) Finite element model of SW-R-1-N (Nail - Oriented Spring)

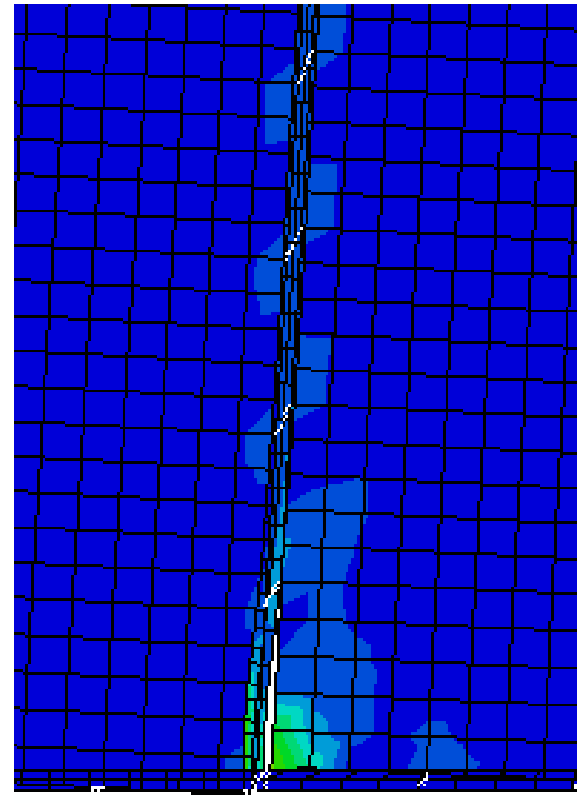

(c) Stress concentration on nails

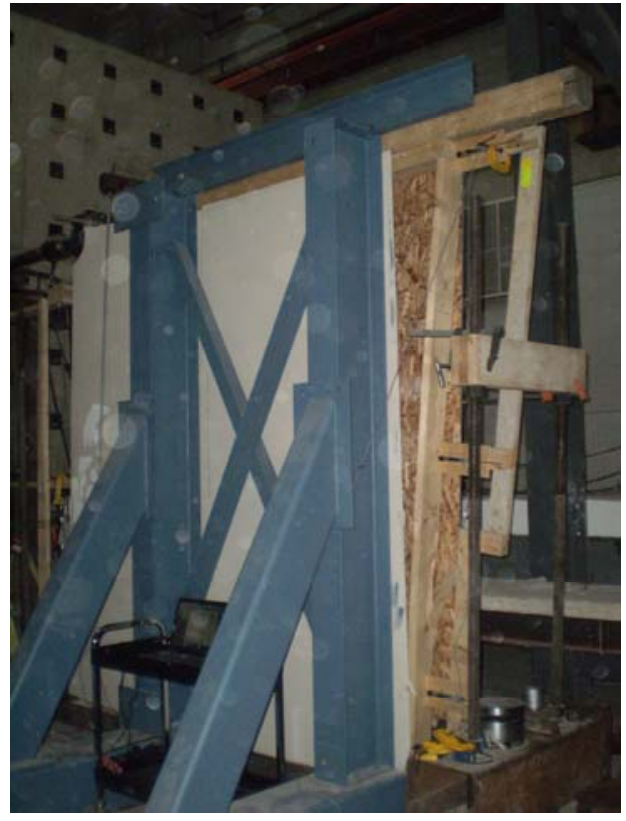

(d) Deformation at failure

Figure 5.24 - Deformed shape of stud wall SW-R-1 under racking load 

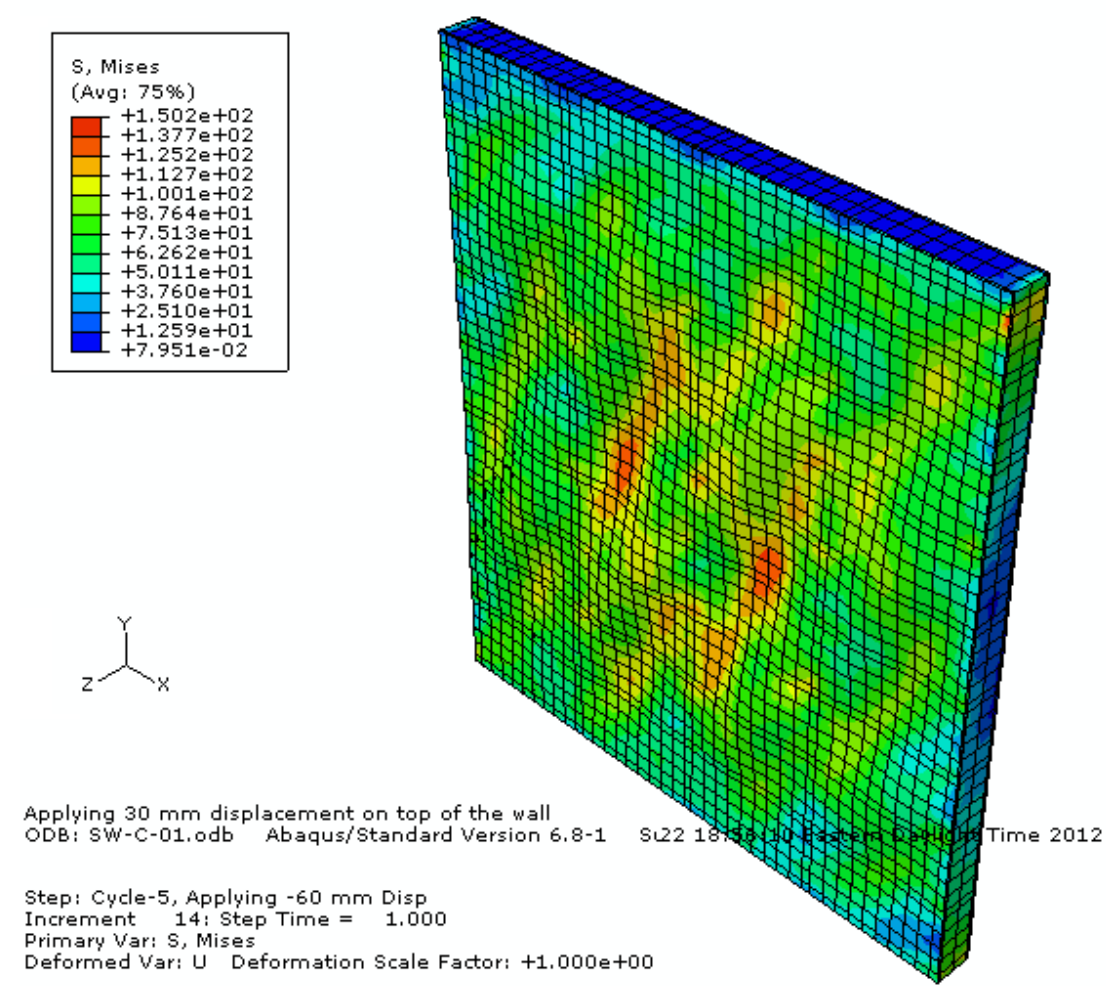
Applying $30 \mathrm{~mm}$ displacement on top of the wall
ODB: Sw-C-01.0db Abaqus/Standard Version $6.8-1$

Step: Cycle-5, Applying $-60 \mathrm{~mm}$ Disp

Increment $14:$ Step Time $=1.000$

Deformed Var: U Deformation Scale Factor: $+1.000 e+00$

(a) Finite element model of SW-C-1 (Nail - Tie Contact)
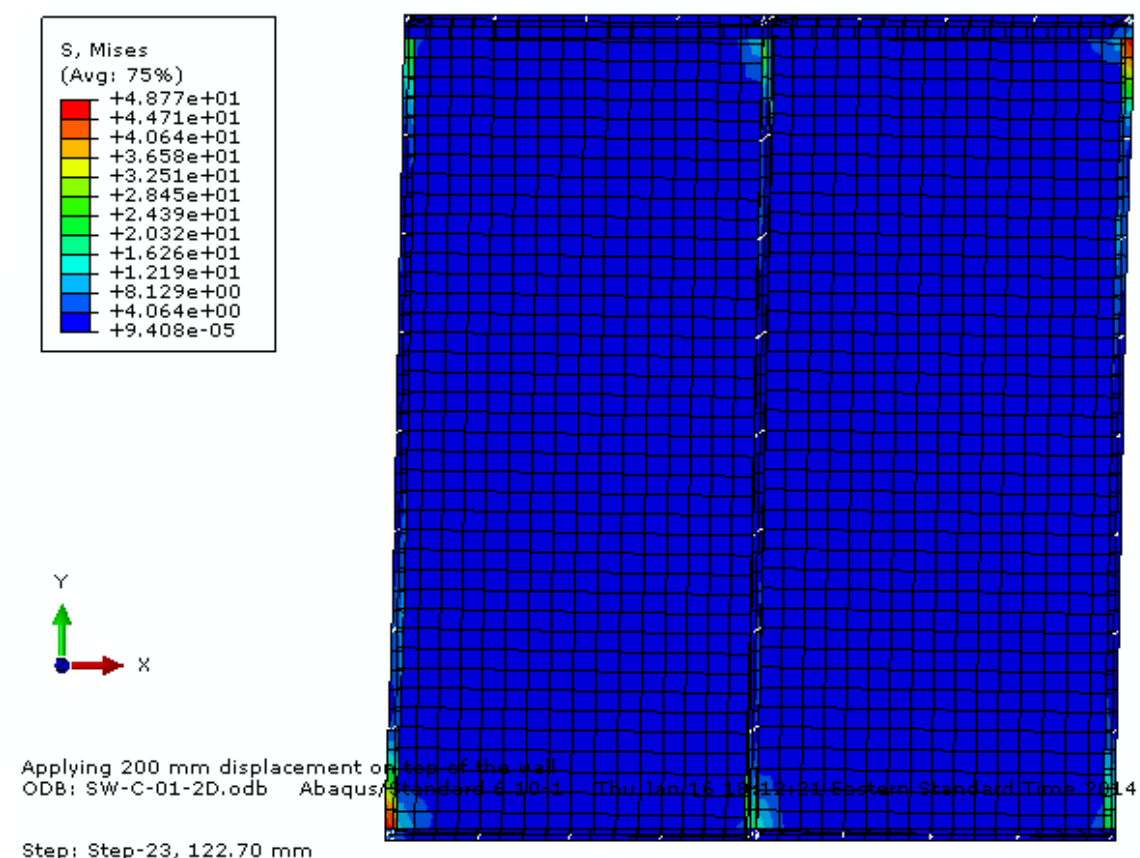

Step: Step-23, $122.70 \mathrm{~mm}$

Increment 10: Step Time $=1.000$

Primary Var: S, Mises

Deformed Var: U Deformation Scale Factor: $+1.000 e+00$

(b) Finite element model of SW-C-1 (Nail - Oriented Spring) 


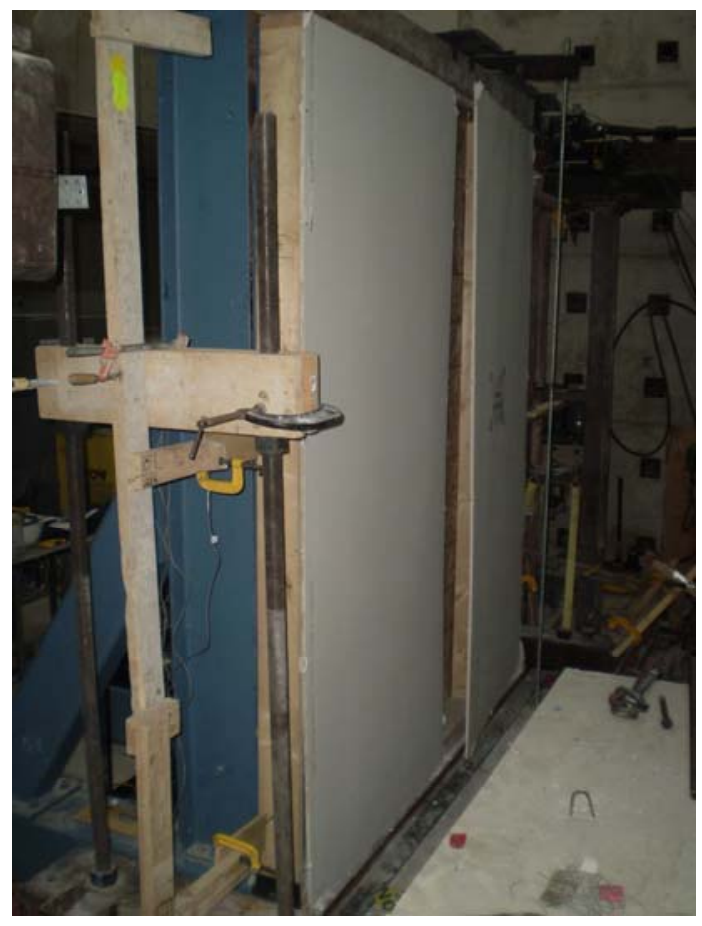

(c) Deformation at failure

Figure 5.25 - Deformed shape of stud wall SW-C-1 under cyclic loading $\left(6^{\text {th }}\right.$ cycle)
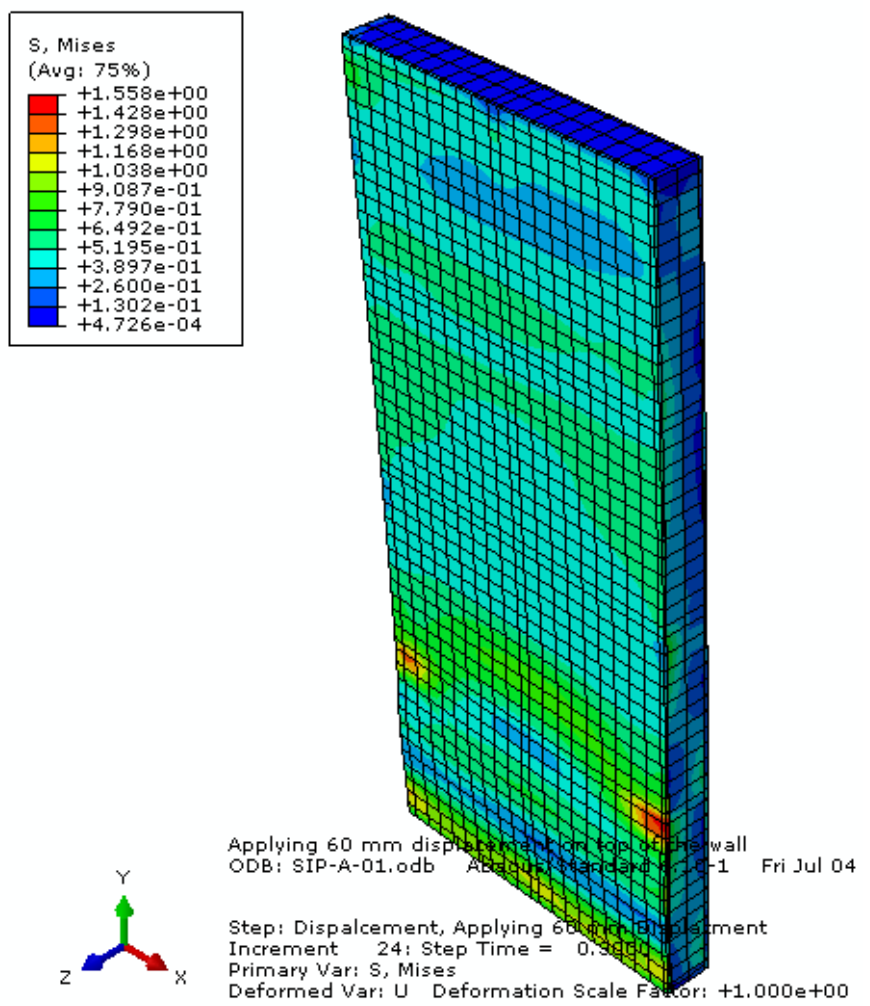

(a) Finite element model of SIP-A-1 


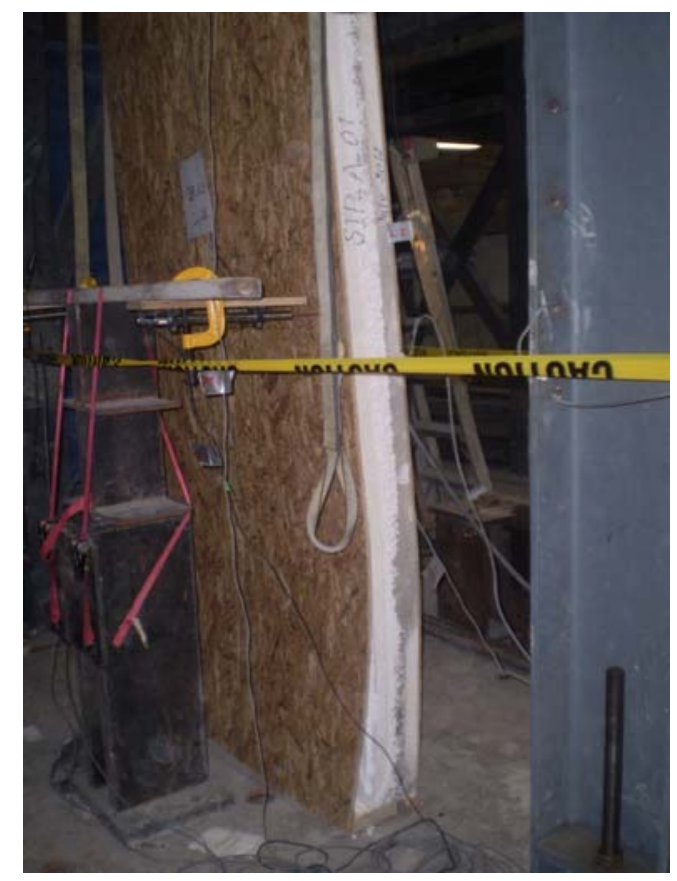

(b) Deformation and failure in specimen SIP-A-1

Figure 5.26 - Deformed configuration of SIP wall model (SIP-A-1) under axial compressive loading

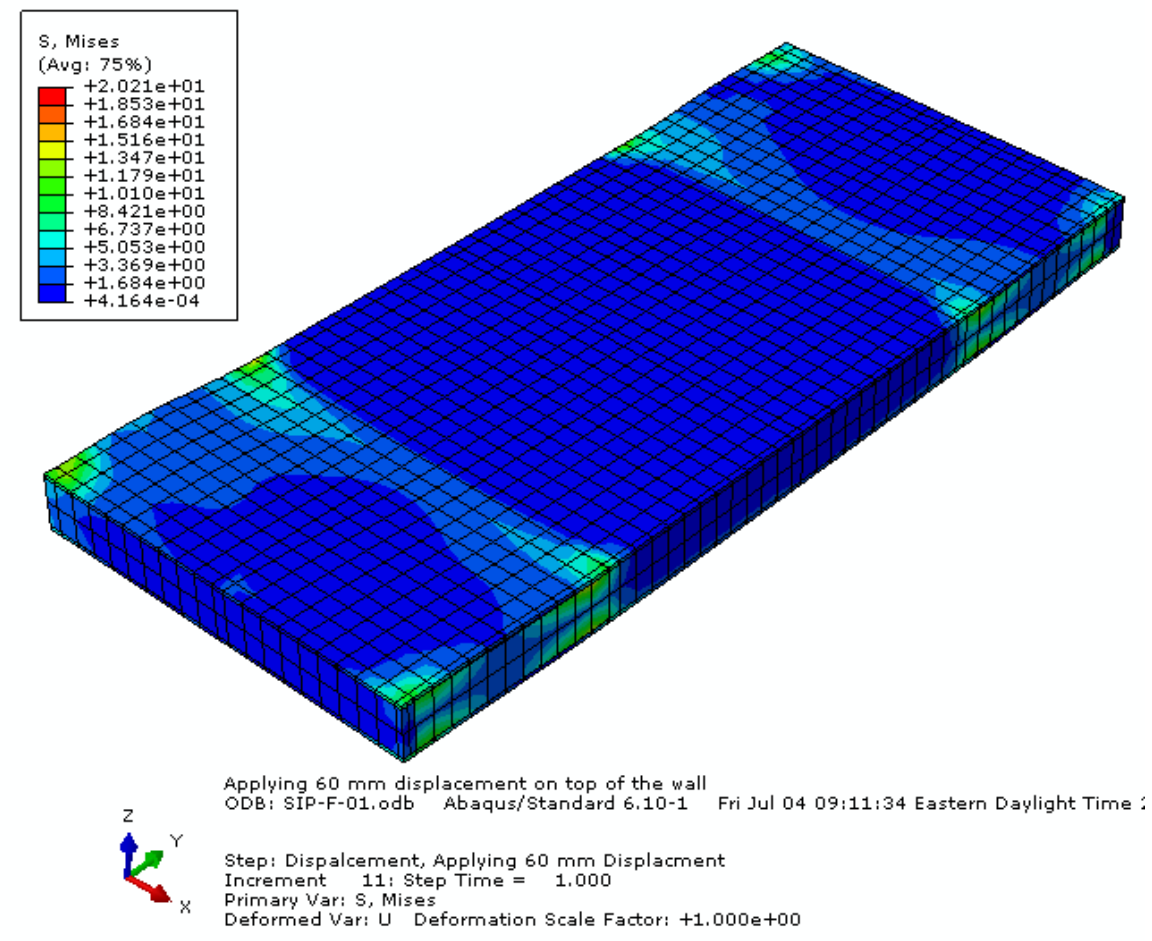

(a) Finite element model of SIP-F-1 


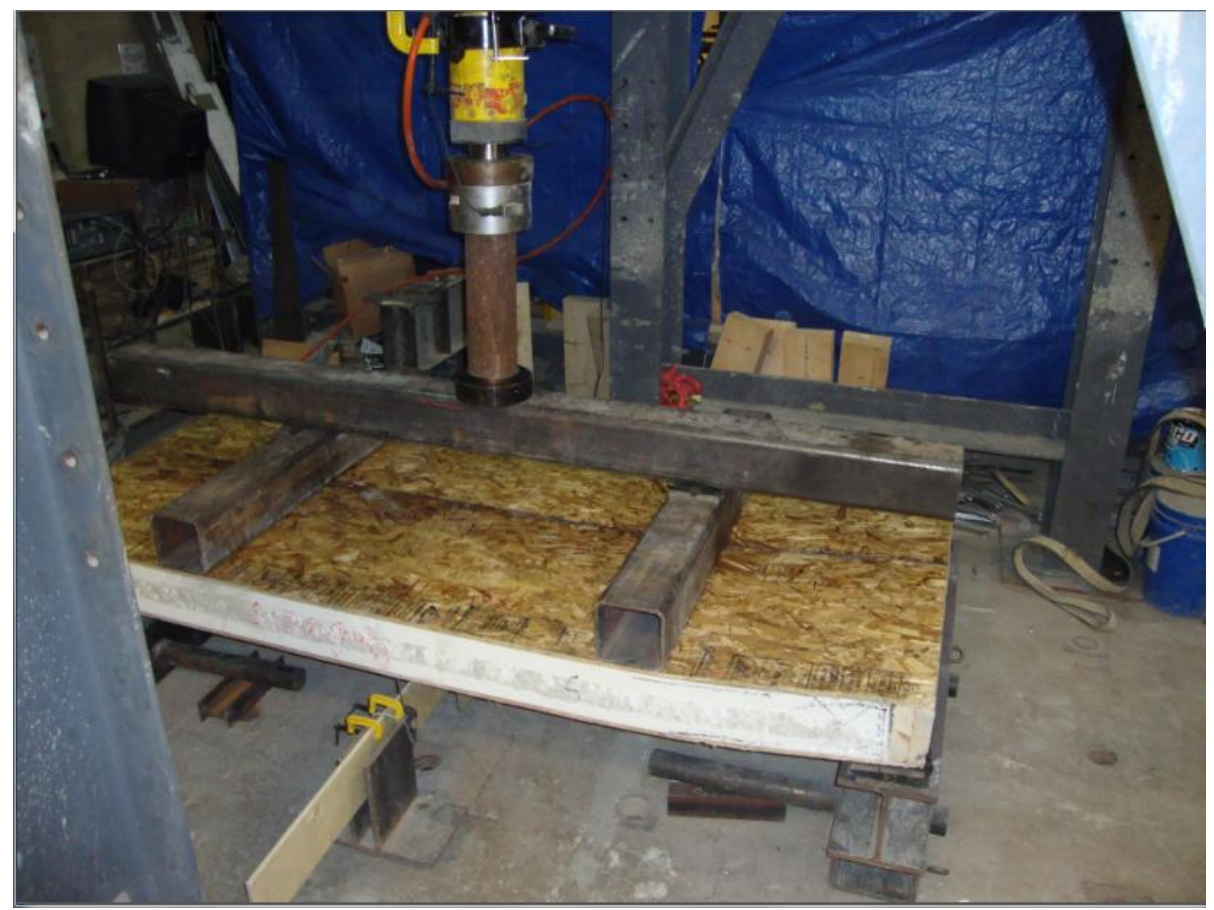

(b) Deformation and failure in specimen SIP-F-1

Figure 5.27 - Deformed configuration of SIP floor model (SIP-F-1) under flexural loading
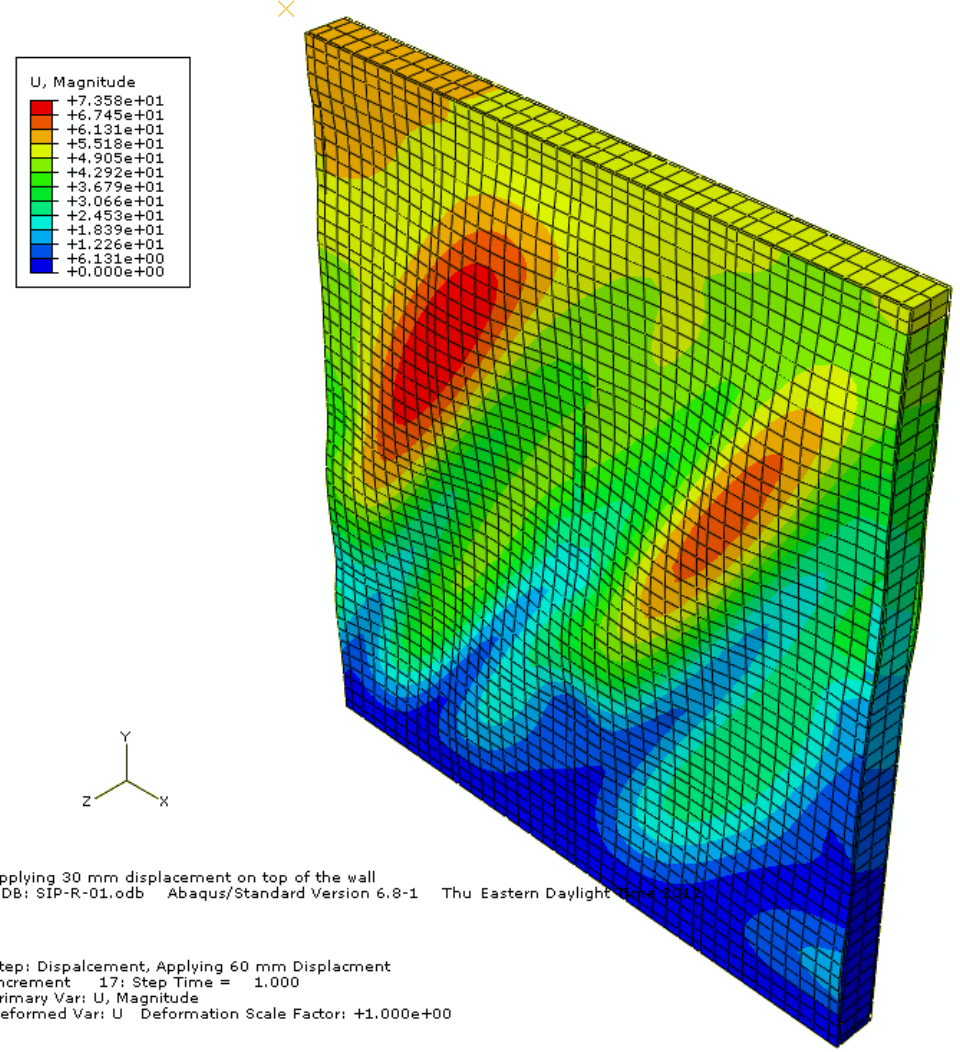

(a) Finite element model of SIP-R-1 (Nail - Tie Contact) 


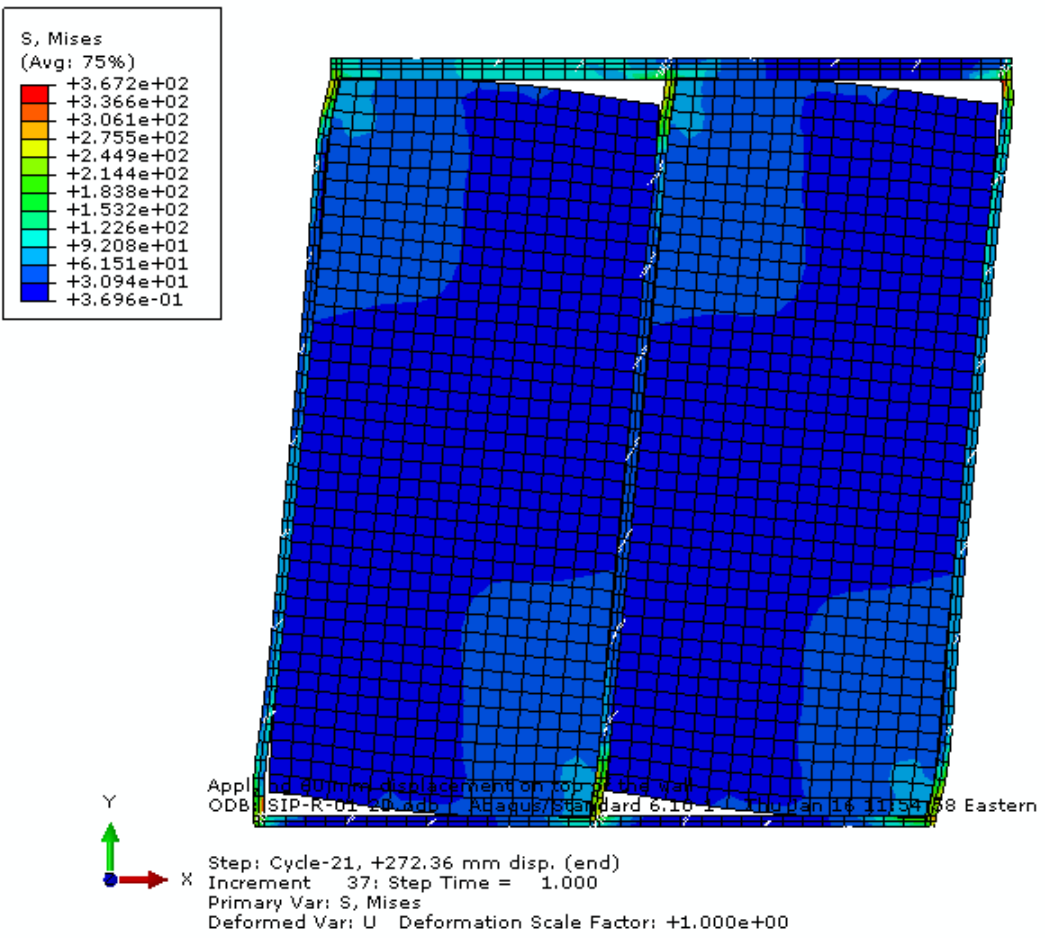

(b) Finite element model of SIP-R-1-N (Nail - Oriented Spring)

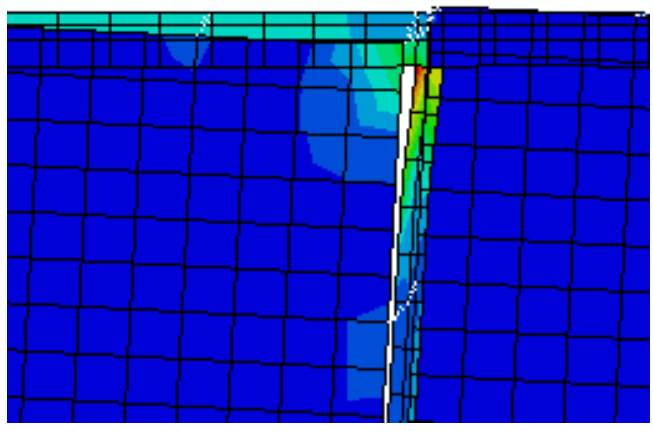

(c) Stress concentration on nails

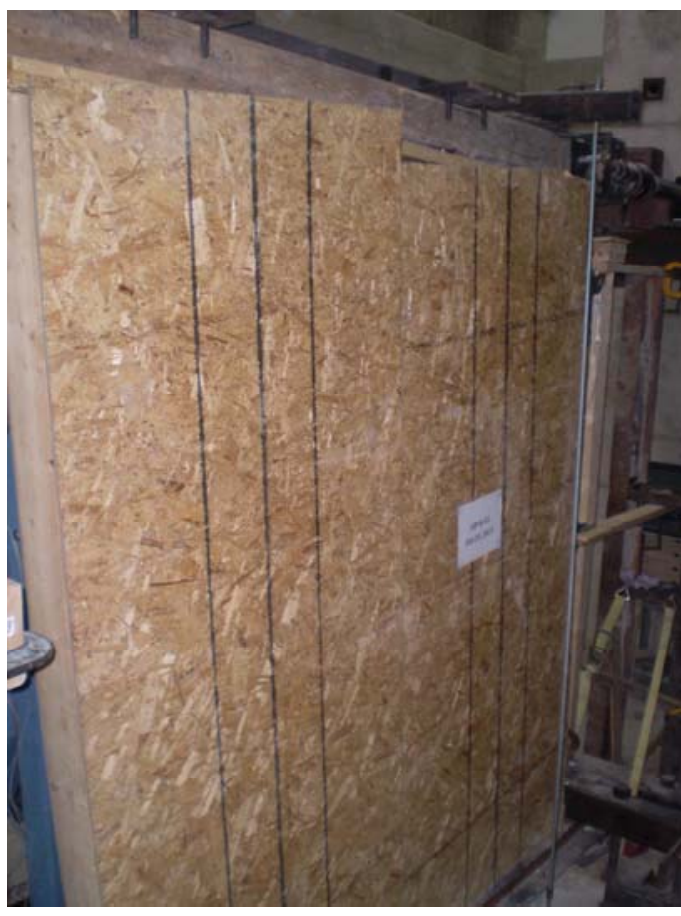

(d) Deformation at failure

Figure 5.28 - Deformed shape of SIP model SIP-R-1 under racking load 

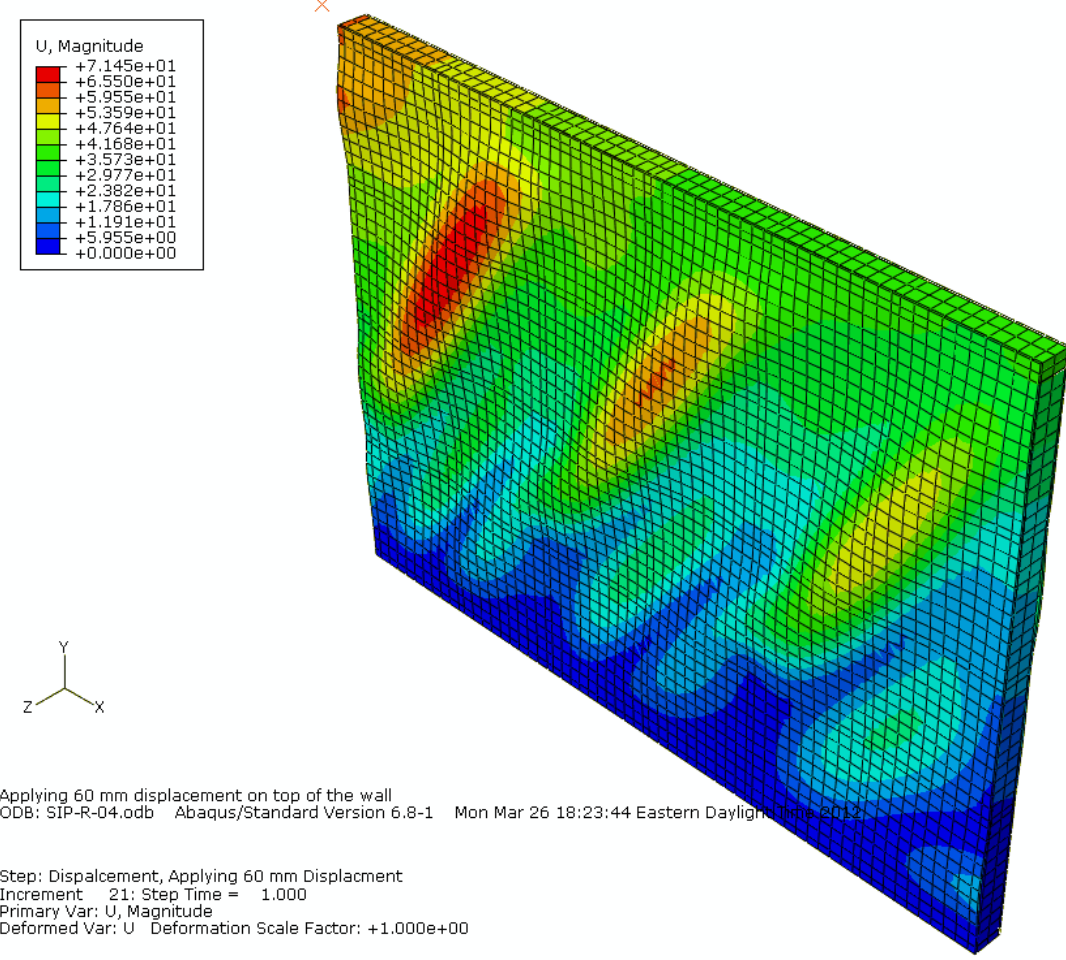

(a) Finite element model of SIP-R-4 (Nail - Tie Contact)
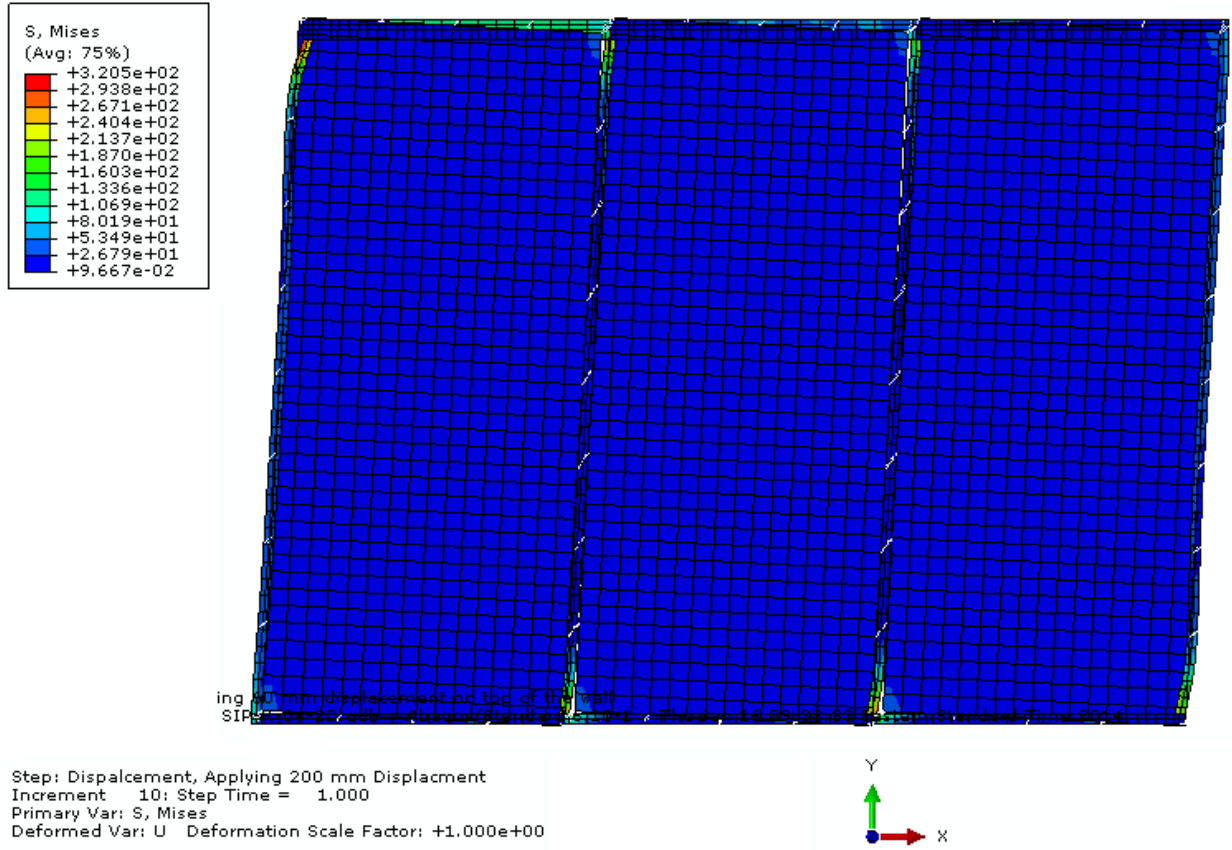

Step: Dispalcement, Applying $200 \mathrm{~mm}$ Displacment Increment $10:$ Step Time $=1.000$

Deformed Var: U Deformation Scale Factor: $+1.000 e+00$

(b) Finite element model of SIP-R-4-N (Nail - Oriented Spring) 


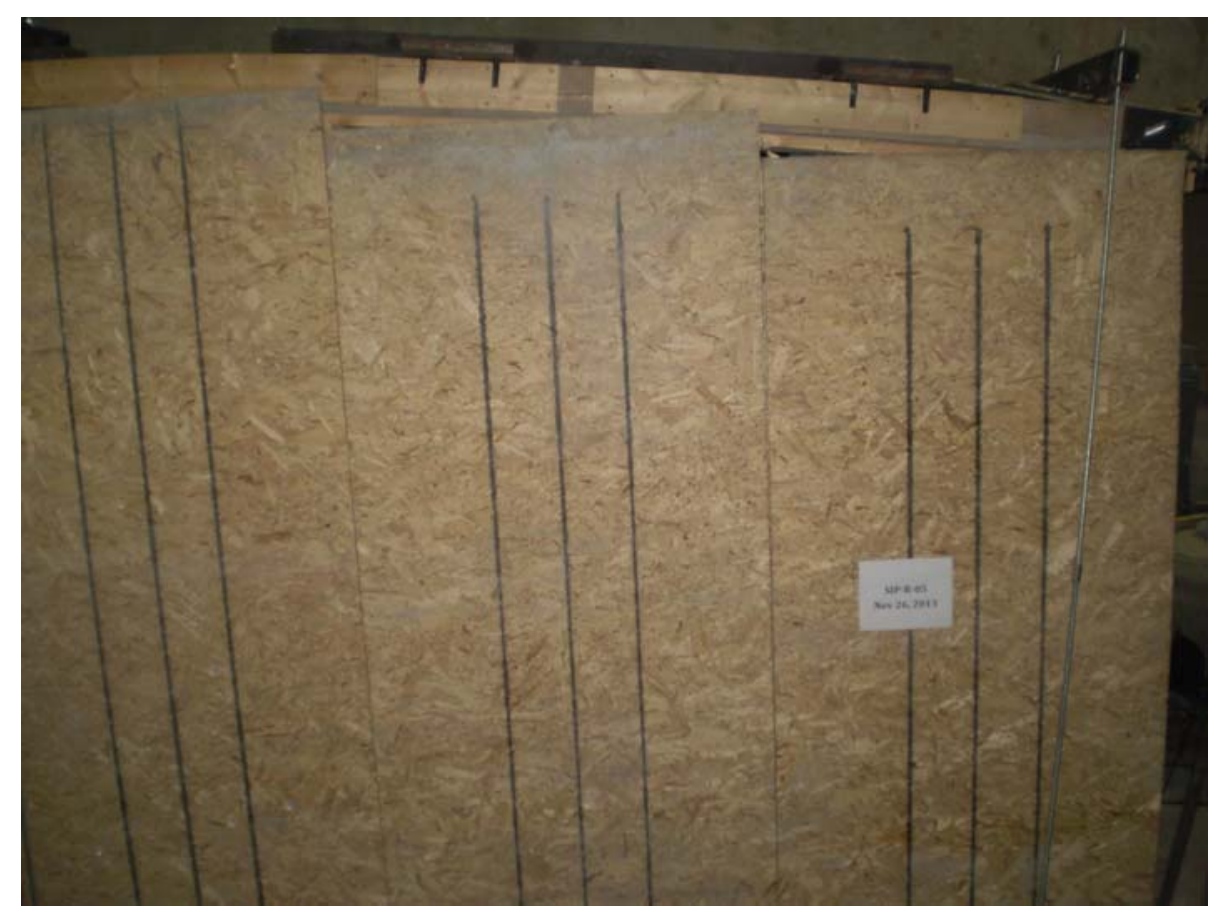

(c) Deformation at failure of specimen SIP-R-4

Figure 5.29 - Deformed shape of SIP model SIP-R-4 under racking load
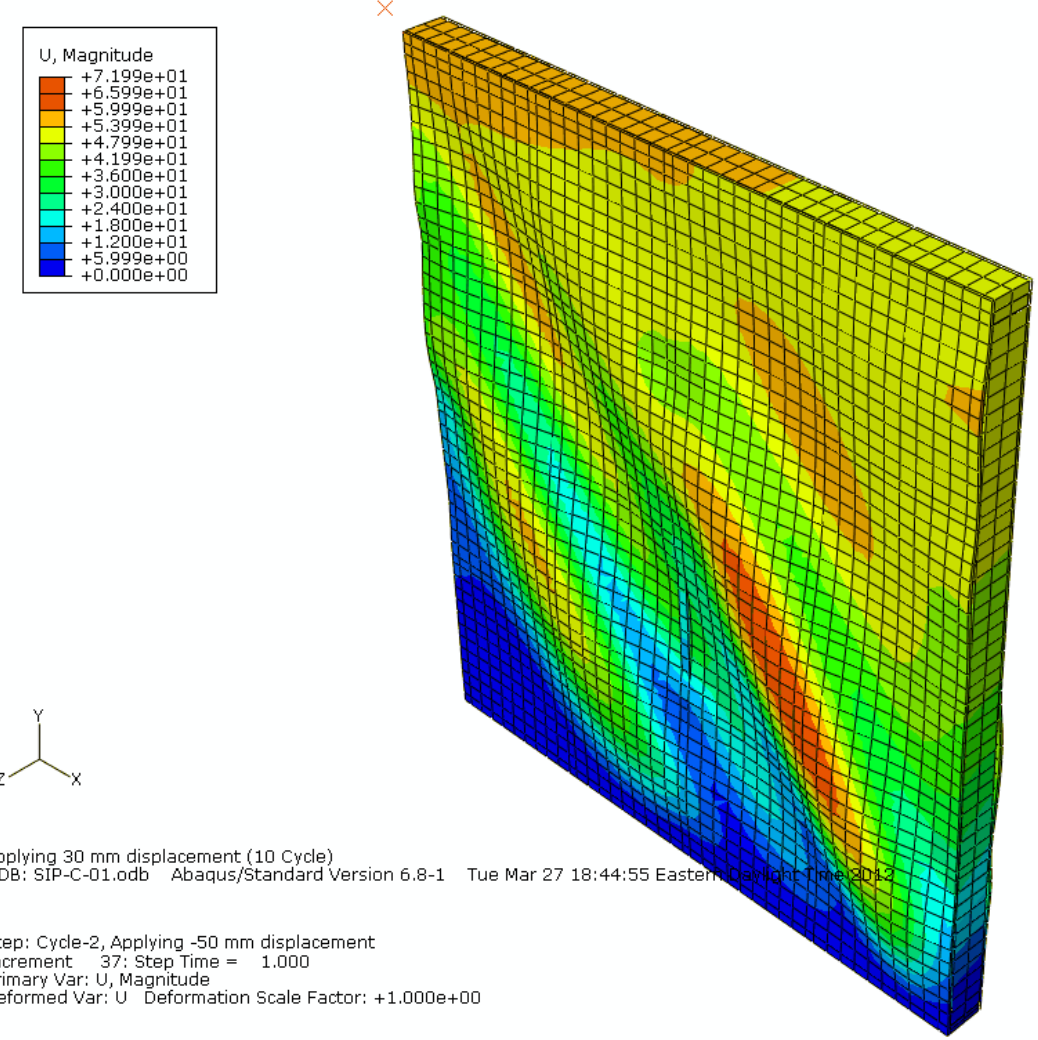

(a) Finite element model of SIP-C-1 (Nail - Tie Contact) 


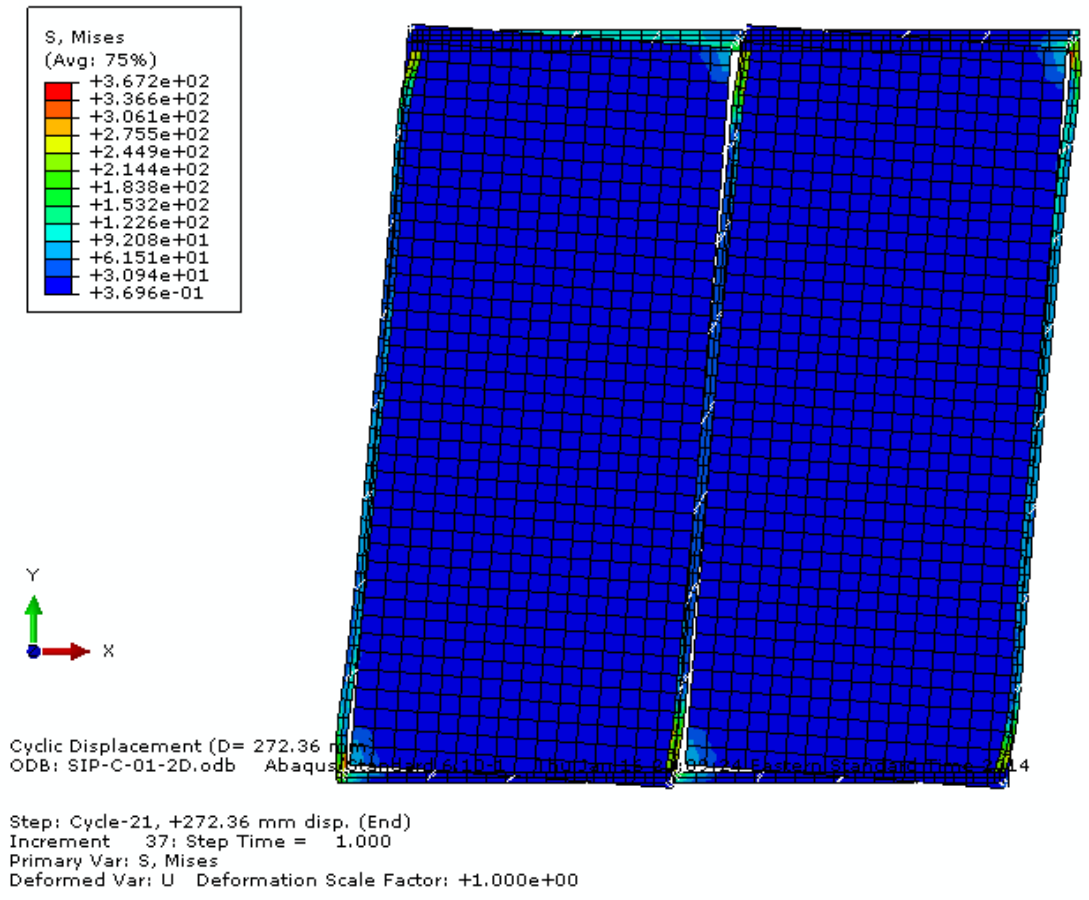

(b) Finite element model of SIP-C-1-N (Nail - Oriented Spring)

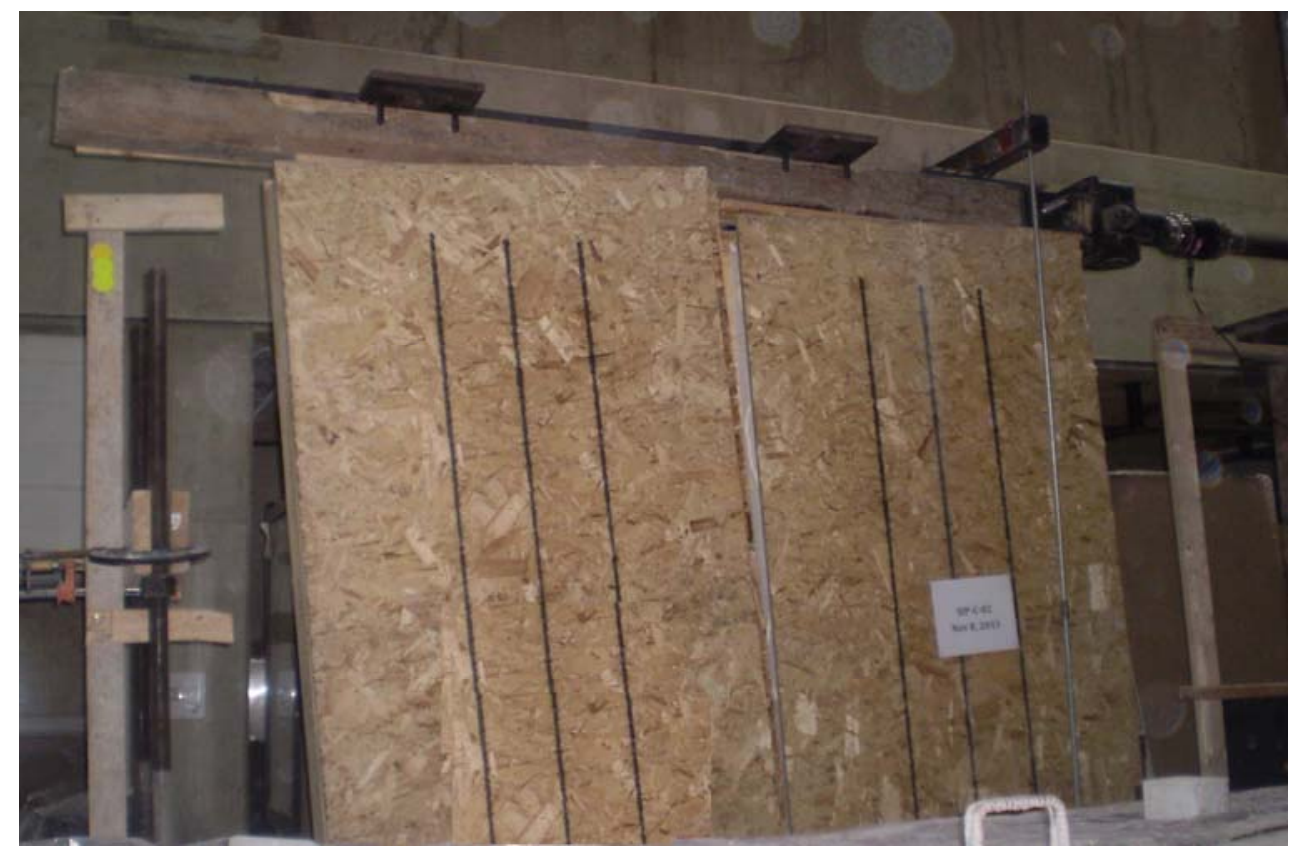

(c) Deformation at failure of specimen SIP-C-1

Figure 5.30 - Deformed shape of SIP model SIP-C-1 under cyclic load $\left(6^{\text {th }}\right.$ cycle) 

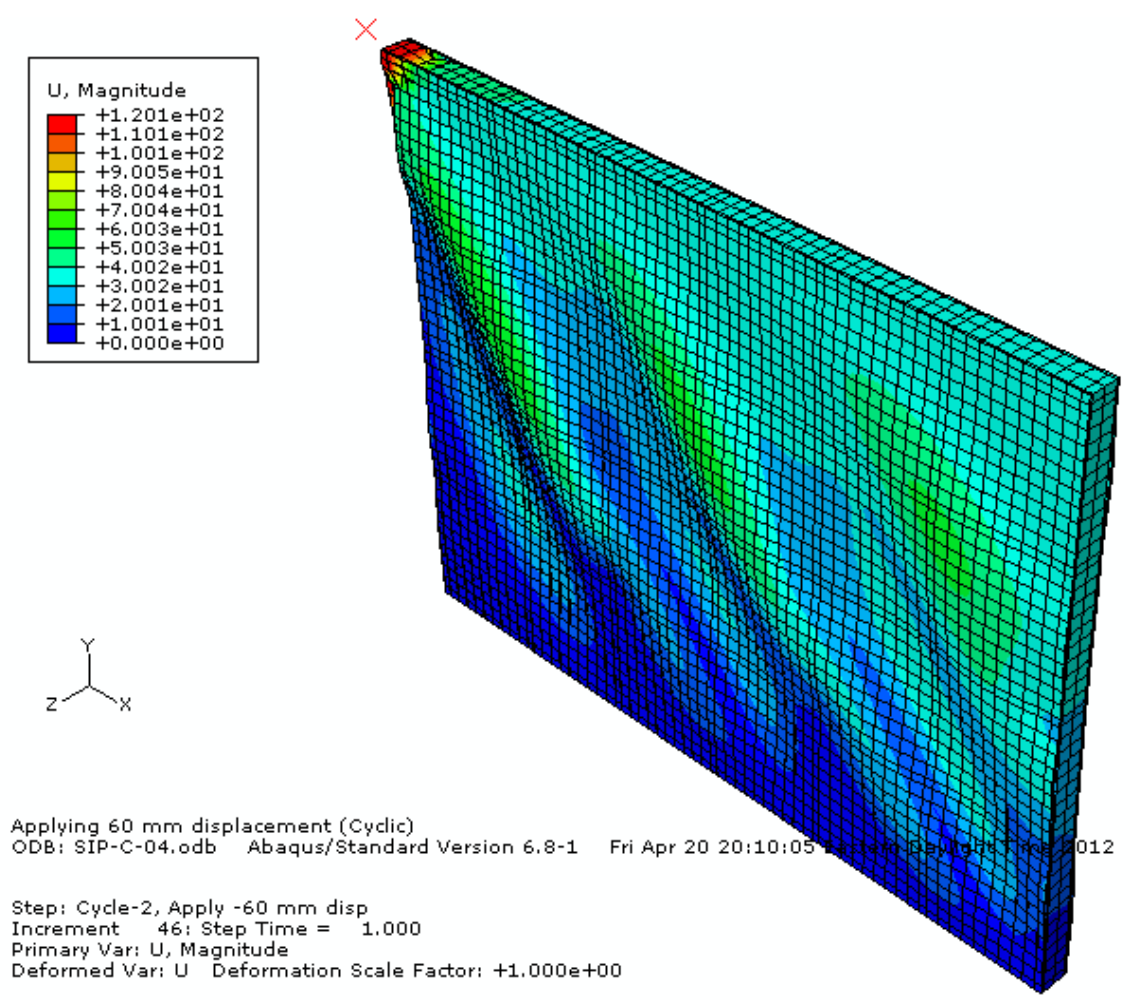

Applying $60 \mathrm{~mm}$ displacement (Cyclic)

ODB: SIP-C-04.odb Abaqus/Standard Version 6.8-1 Fri Apr 20 20:10:0

Step: Cycle-2, Apply $-60 \mathrm{~mm}$ disp

Increment 46: Step Time $=1.000$

Primary Var: U, Magnitude

Deformed Var: U Deformation Scale Factor: +1.000 e+00

(a) Finite element model of SIP-C-4 (Nail - Tie Contact)

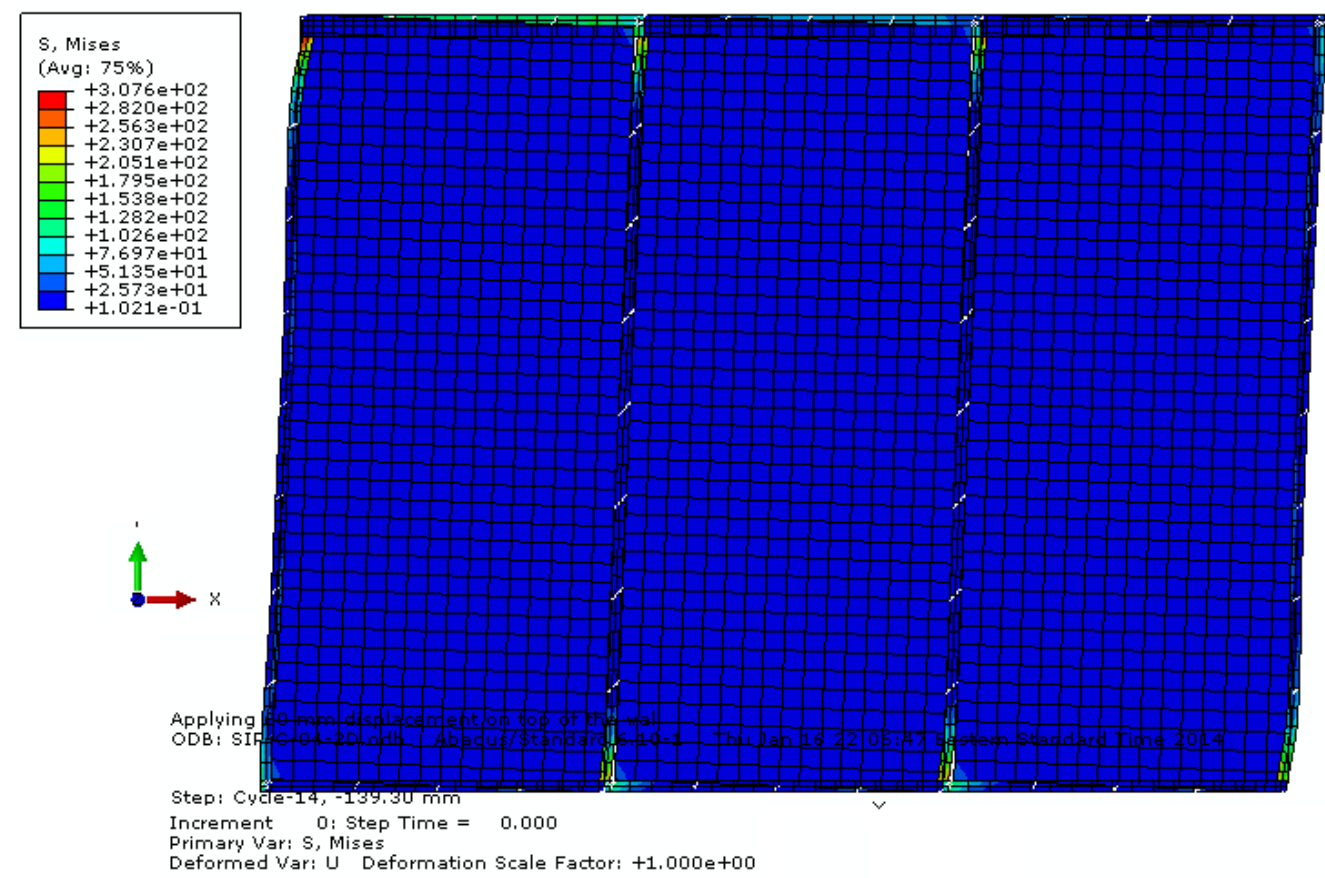

(b) Finite element model of SIP-C-4-N (Nail - Oriented Spring) 


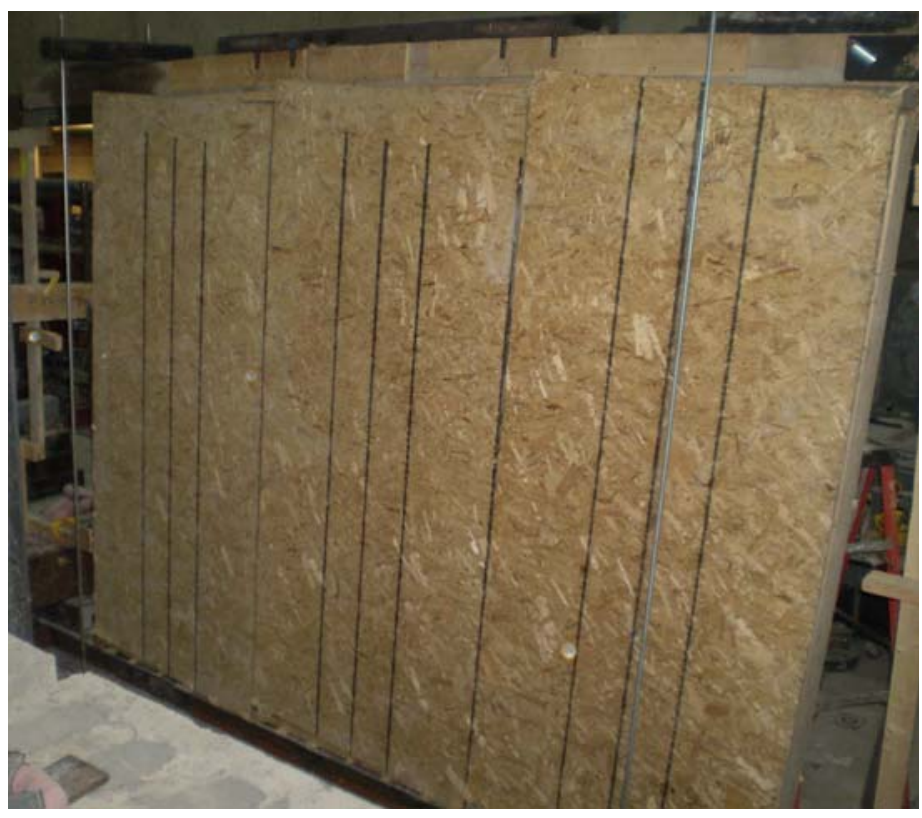

(c) Deformation at failure of specimen SIP-C-4

Figure 5.31 - Deformed shape of SIP model SIP-C-4 under lateral cyclic load $\left(6^{\text {th }}\right.$ cycle)

Table 5.3 compares ultimate loads obtained experimentally and from the FE modeling for panels listed in Table 5.2 using FE mesh 3 that showed good convergence in FE results. It can be observed that the ratio of FE ultimate load to the experimental ultimate load varies from 1.02 to 1.17. Thus, it can be concluded that FE mesh 3 resulted in good agreement between the experimental findings and the results from FE modeling. To present the ultimate load values shown in Table 5.3, Figures 5.32 through 5.41 were produced. In these figures, correlation between the experimental findings and the results from FE modeling along the load history was conducted. Good agreement was observed between the experimental findings and FE results in the elastic and the nonlinear range of flexural, compressive and racking load histories. However, fair agreement was observed between the FE results and the experimental findings in case of cyclic loading history. One may observe that there was a kink in the linear elastic stage of grantee loading on stud wall at a jacking load of about $150 \mathrm{kN}$ shown in Figure 5.32 based on experimental results. This was attributed to the axial shortening in the studs that closed the $2 \mathrm{~mm}$ gap between OSB facing orientated horizontally as depicted in Figure 3.4. However, the behaviour was not observed in finite element modeling since the OSB sheets were modeled without the presence of the $2 \mathrm{~mm}$ gap. 
One may notice in Figure 5.35 that the finite element modeling did not simulate the stiffness degradation of the hysteresis curves obtained experimentally for stud wall under cyclic loading. It can be observed that the softening of the load-deflection relationship in each cycle experimental finding in contrast to the hardening of the load-deflection relationship in each cycle. In addition, the finite element modeling did not show load degradation before failure compared to that observed in the experimental findings. This may be attributed to the fact that the change in nail behaviour under cyclic loading was not fully modeled. Similar observation was noticed in case of SIP walls as depicted in Figure 5.40 and 5.41.

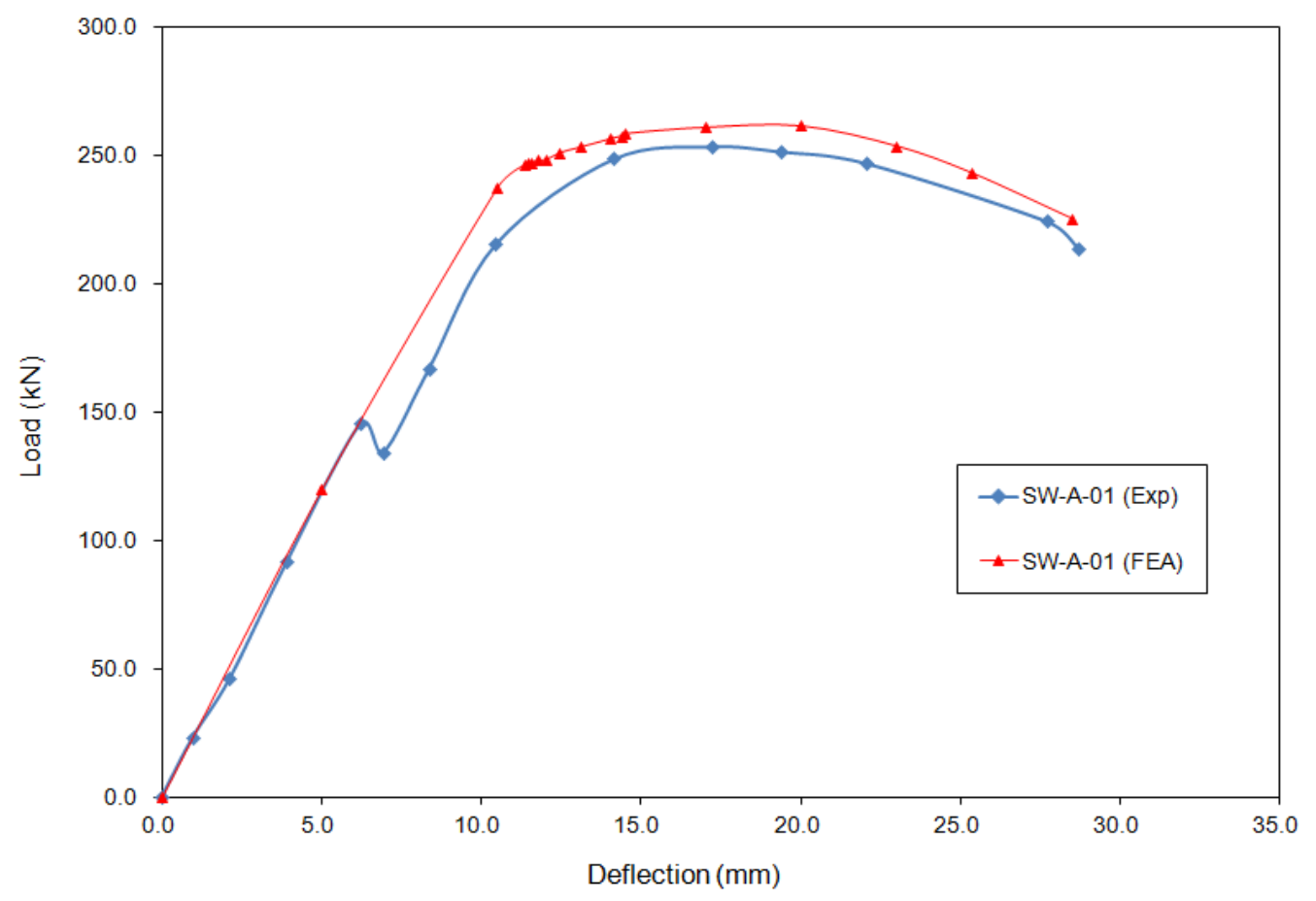

Figure 5.32 - Comparison between the FEA results and experimental findings of stud wall SW-A-1 under compressive loading 
Table 5.3 - Comparisons of results of finite-element analysis and experimental findings

\begin{tabular}{|c|c|c|c|c|c|}
\hline $\begin{array}{c}\text { Test } \\
\text { Specimen }\end{array}$ & Panel Size & Panel Material & $\begin{array}{l}\text { Experimental } \\
\text { capacity }(\mathrm{kN})\end{array}$ & $\begin{array}{c}\text { FEA } \\
\text { ultimate } \\
\text { capacity } \\
(\mathrm{kN})\end{array}$ & $\begin{array}{l}\text { FEA/test } \\
\text { load ratio }\end{array}$ \\
\hline SW-A-1 & $\begin{array}{c}2440 \times 2750 \times 165 \mathrm{~mm} \\
\left(8^{\prime} \times 9^{\prime} \times 6^{1 / 2 \prime}{ }^{\prime \prime}\right)\end{array}$ & $\begin{array}{c}\text { Conventional } \\
\text { stud }\end{array}$ & 253.51 & 261.40 & 1.03 \\
\hline SW-F-1 & $\begin{array}{c}2440 \times 2750 \times 165 \mathrm{~mm} \\
\left(8^{\prime} \times 9^{\prime} \times 6^{1 / 2 \prime}{ }^{\prime \prime}\right)\end{array}$ & $\begin{array}{c}\text { Conventional } \\
\text { stud }\end{array}$ & 58.80 & 59.99 & 1.02 \\
\hline SW-R-1 & $\begin{array}{c}2440 \times 2750 \times 165 \mathrm{~mm} \\
\left(8^{\prime} \times 9^{\prime} \times 6^{1 / 2 \prime}{ }^{\prime \prime}\right)\end{array}$ & $\begin{array}{c}\text { Conventional } \\
\text { stud }\end{array}$ & 13.70 & 14.46 & 1.05 \\
\hline SW-C-1 & $\begin{array}{c}2440 \times 2750 \times 165 \mathrm{~mm} \\
\left(8^{\prime} \times 9^{\prime} \times 6^{1 / 2 \prime}\right)\end{array}$ & $\begin{array}{c}\text { Conventional } \\
\text { stud }\end{array}$ & 9.94 & 11.63 & 1.17 \\
\hline SIP-A-1 & $\begin{array}{c}1220 \times 2750 \times 165 \mathrm{~mm} \\
\left(4^{\prime} \times 9^{\prime} \times 6^{1 / 2 \prime}\right)\end{array}$ & SIP & 100.09 & 95.21 & 0.95 \\
\hline SIP-F-1 & $\begin{array}{c}1220 \times 2750 \times 165 \mathrm{~mm} \\
\left(4^{\prime} \times 9^{\prime} \times 6^{\prime 1 / 2 \prime}\right)\end{array}$ & SIP & 23.82 & 27.53 & 1.15 \\
\hline SIP-R-1 & $\begin{array}{c}2440 \times 2750 \times 165 \mathrm{~mm} \\
\left.\left(8^{\prime} \times 9^{\prime} \times 6^{1 / 2 \prime}\right)^{\prime}\right)\end{array}$ & SIP & 25.33 & 26.02 & 1.03 \\
\hline SIP-R-4 & $\begin{array}{c}3660 \times 2750 \times 165 \mathrm{~mm} \\
\left(12^{\prime} \times 9^{\prime} \times 6^{1 / 2}{ }^{\prime \prime}\right)\end{array}$ & SIP & 39.92 & 40.78 & 1.02 \\
\hline SIP-C-1 & $\begin{array}{c}2440 \times 2750 \times 165 \mathrm{~mm} \\
\left(8^{\prime} \times 9^{\prime} \times 6^{1 / 2 \prime}{ }^{\prime \prime}\right)\end{array}$ & SIP & 22.09 & 25.40 & 1.15 \\
\hline SIP-C-4 & $\begin{array}{c}3660 \times 2750 \times 165 \mathrm{~mm} \\
\left(12^{\prime} \times 9^{\prime} \times 6^{1 / 2 \prime}\right)\end{array}$ & SIP & 21.92 & 22.48 & 1.03 \\
\hline
\end{tabular}




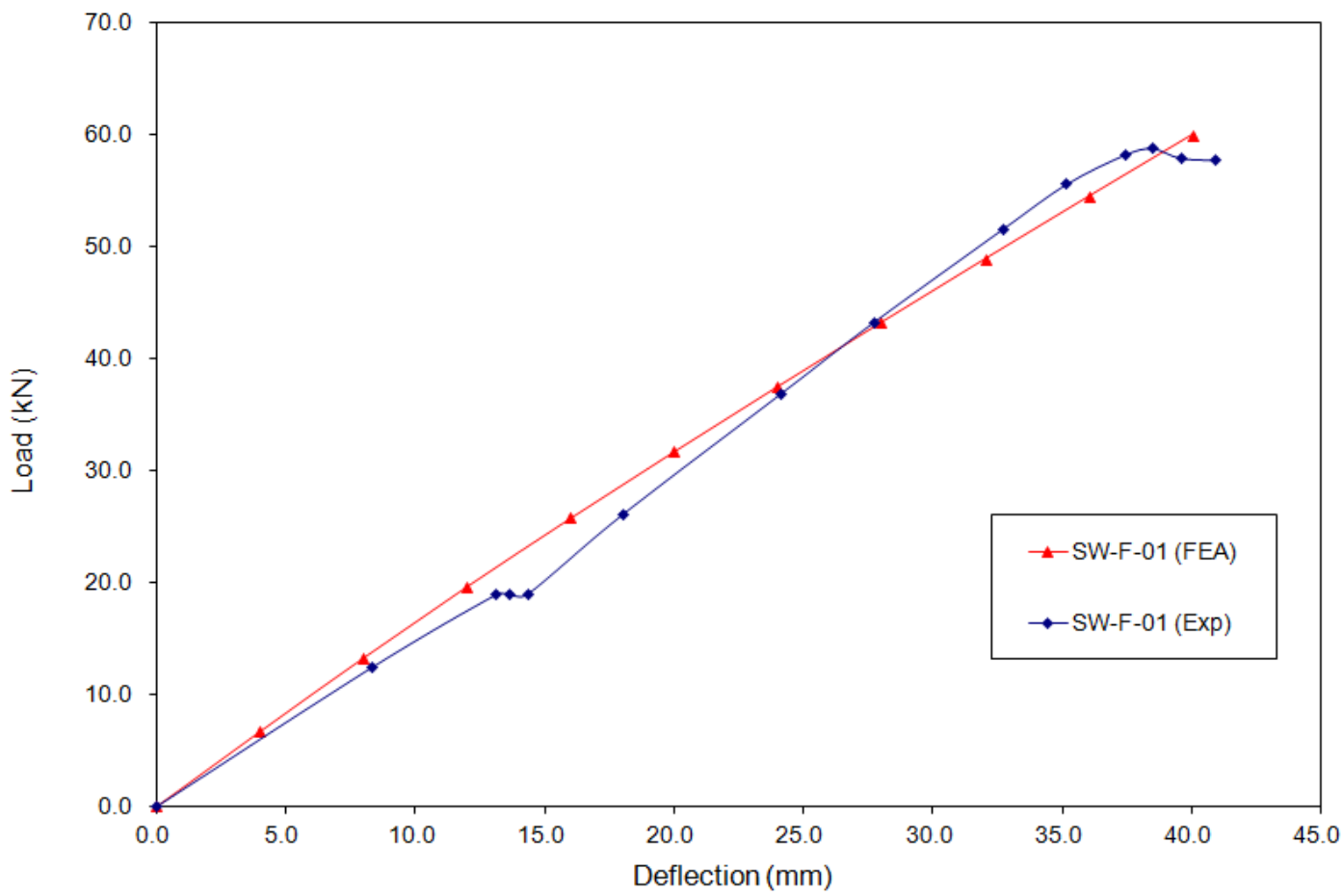

Figure 5.33 - Comparison between the FEA results and experimental findings of stud wall SW-F-1 under flexural loading

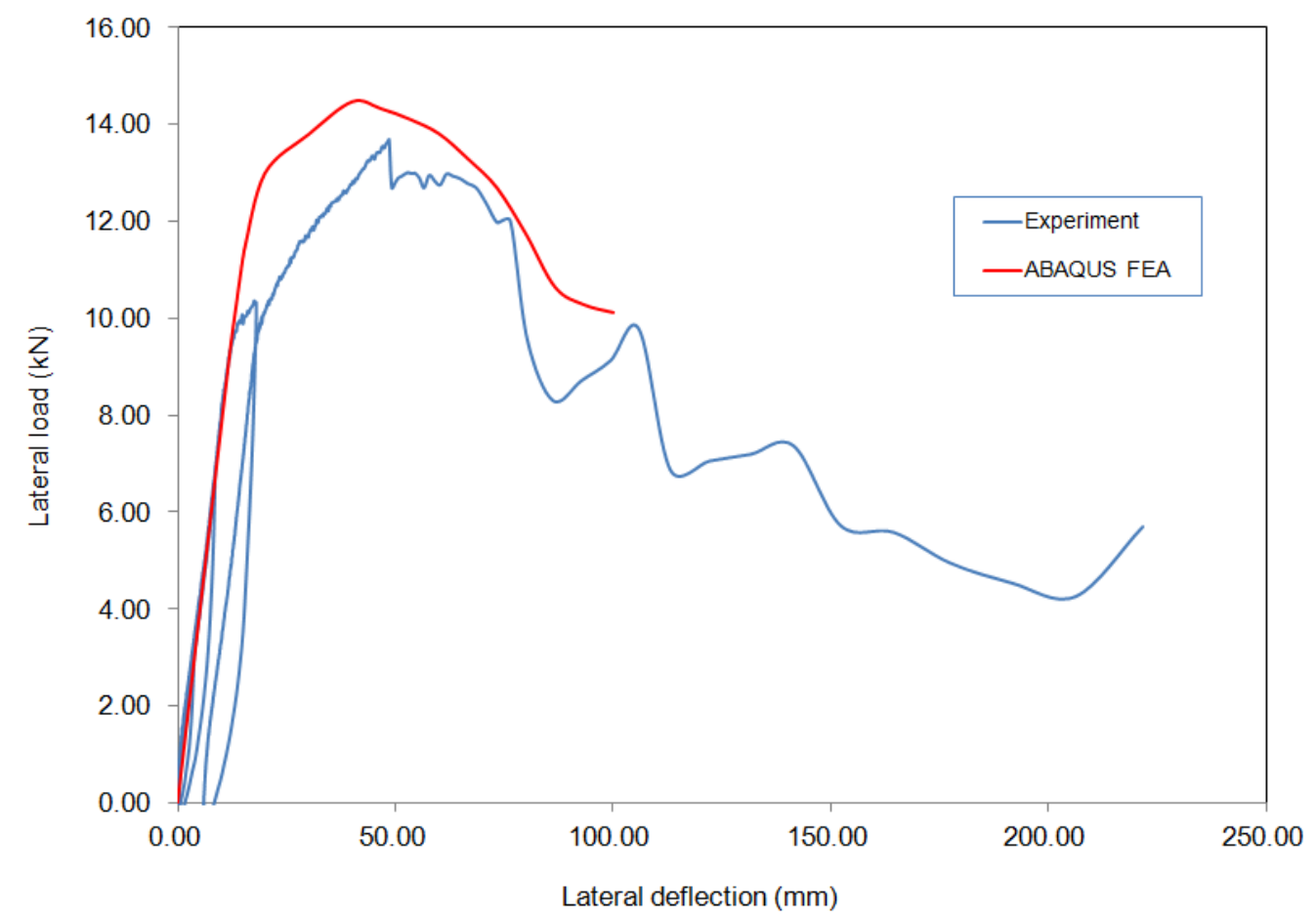

Figure 5.34 - Comparison between the FEA results and experimental findings of stud wall SW-R-1 under racking loading 


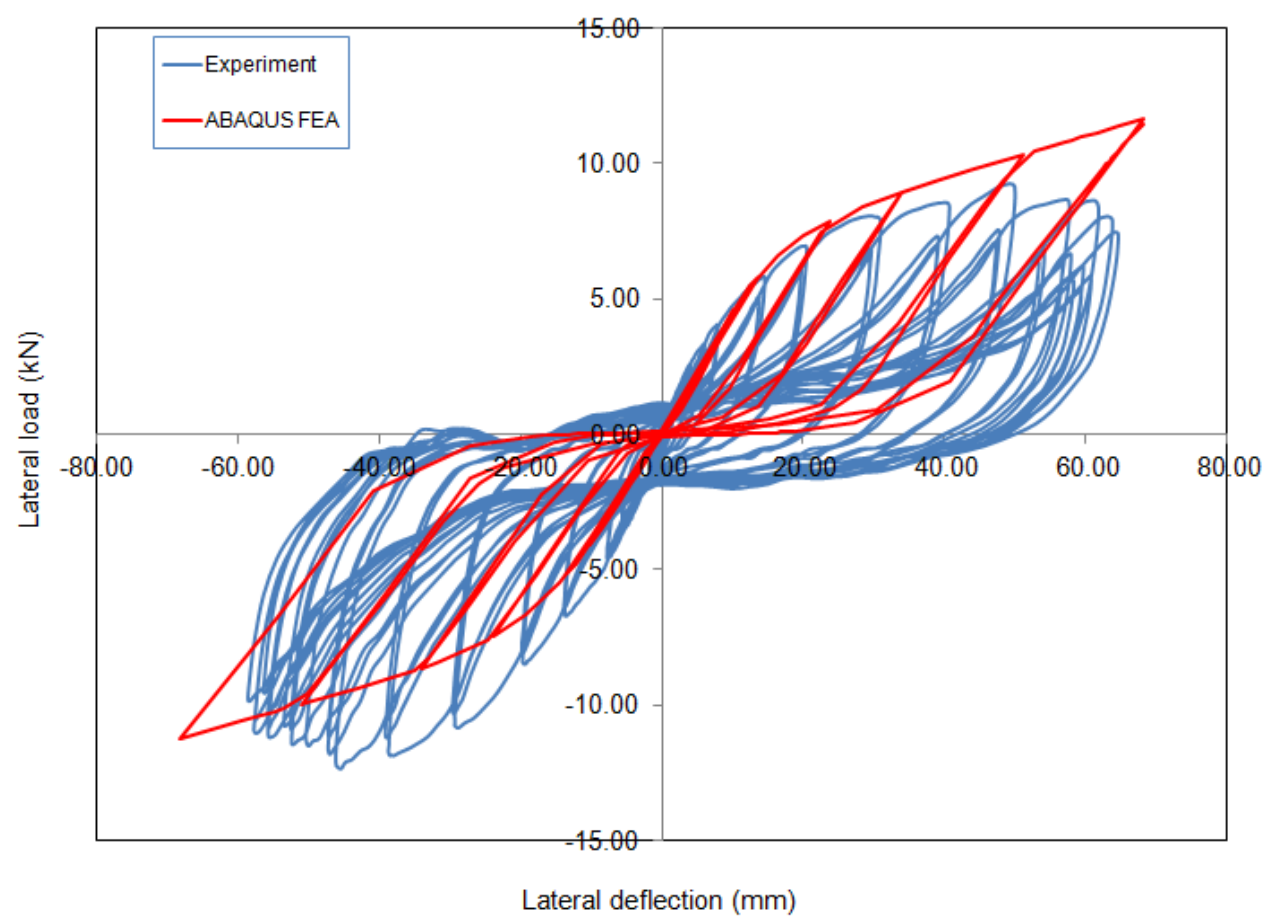

Figure 5.35 - Comparison between the FEA results and experimental findings of stud wall SW-C-1 under cyclic loading

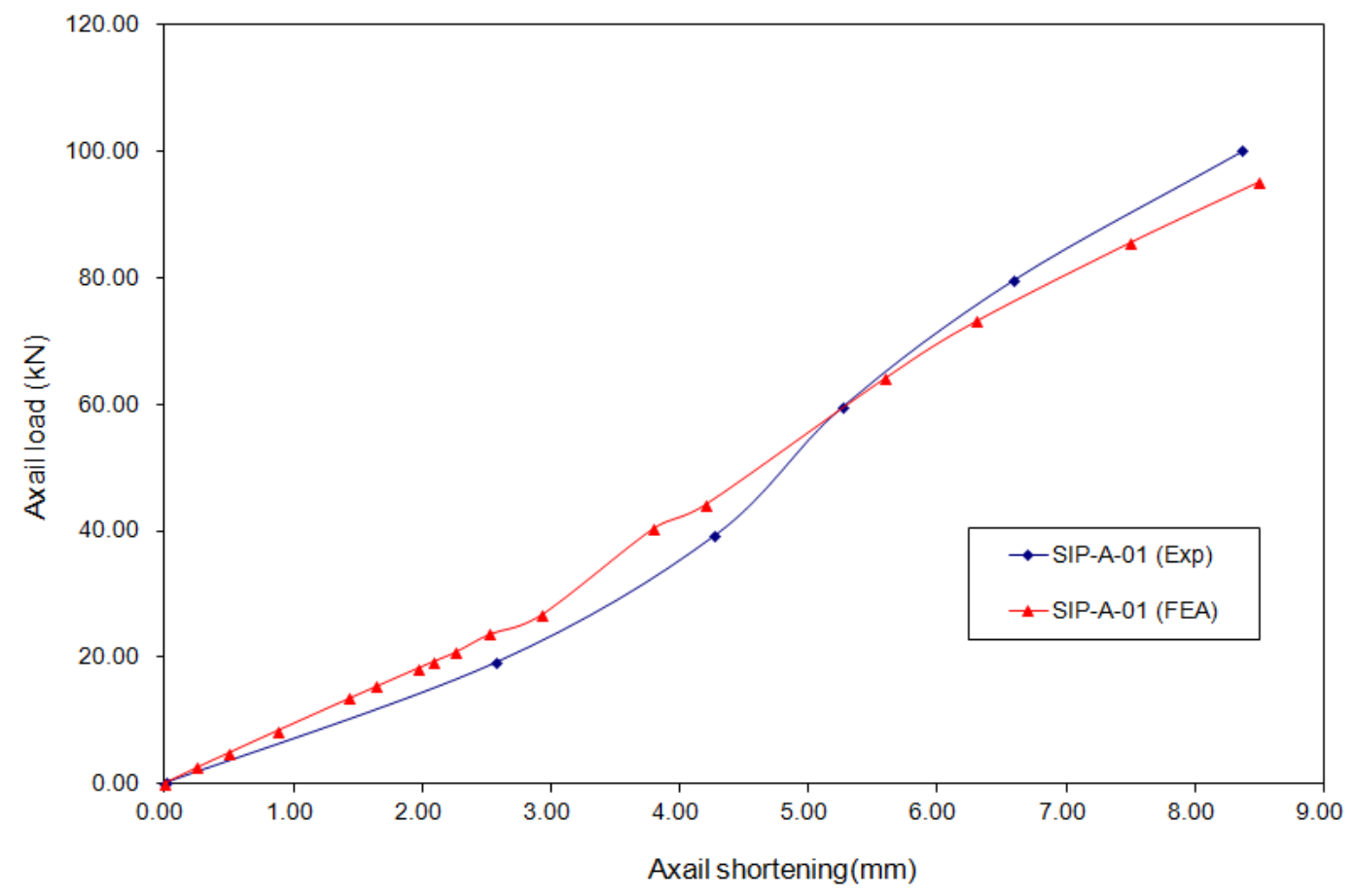

Figure 5.36 - Comparison between the FEA results and experimental findings of stud wall SIP-A-1 under axial compression loading 


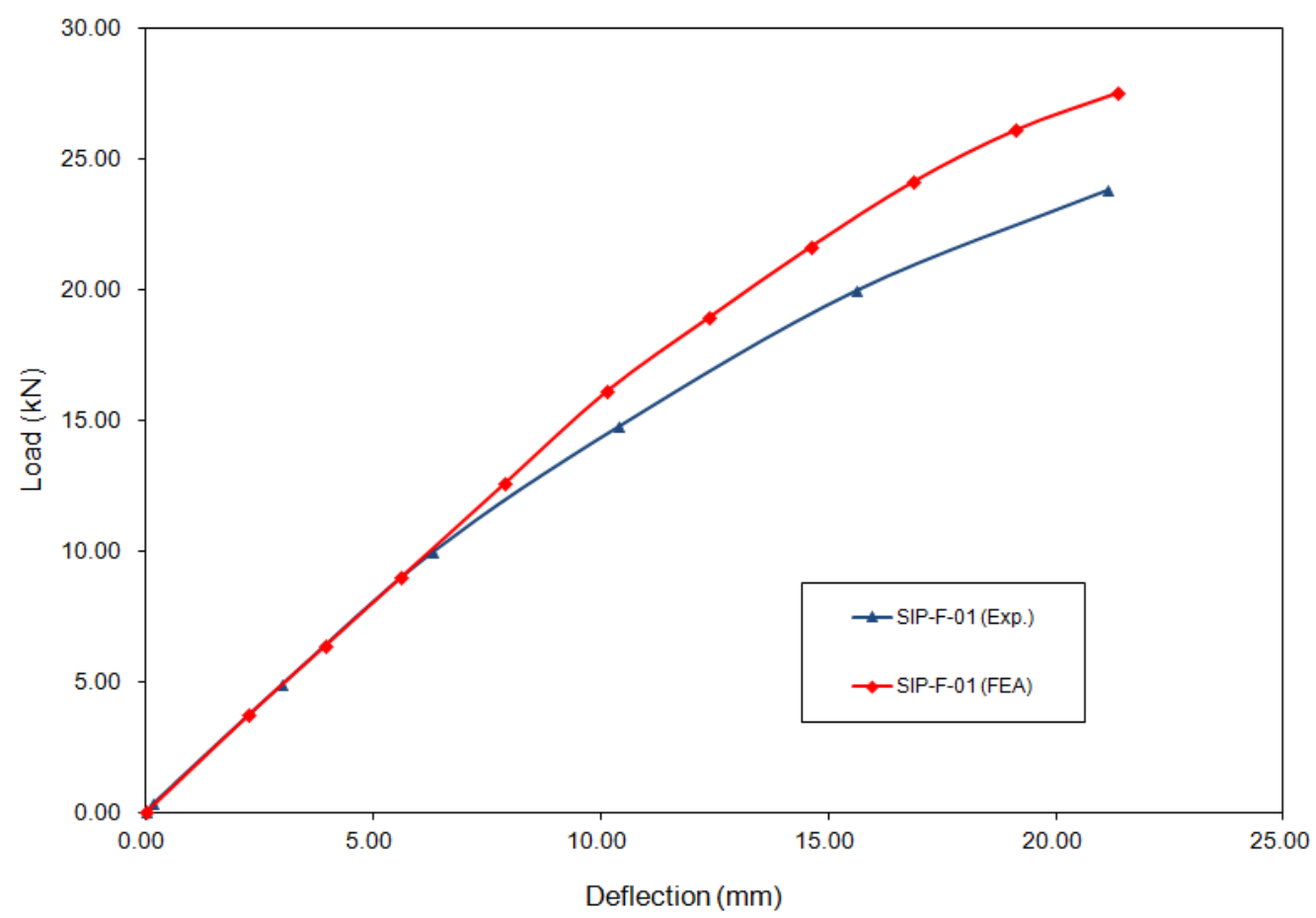

Figure 5.37 - Comparison between the FEA results and experimental findings of stud wall SIP-F-1 under flexural loading

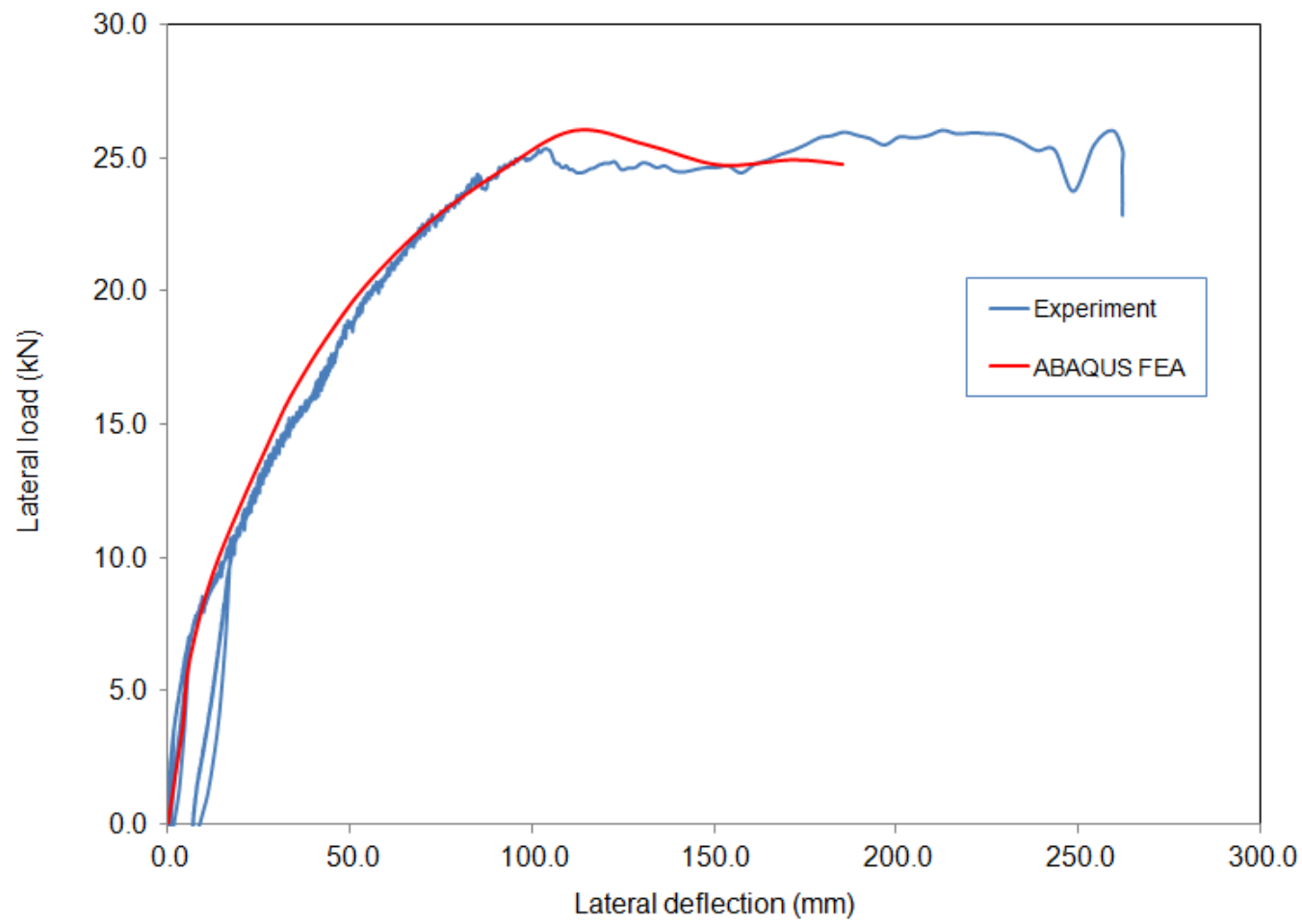

Figure 5.38 - Comparison between the FEA results and experimental findings of SIP wall SIP-R-1 under racking loading 


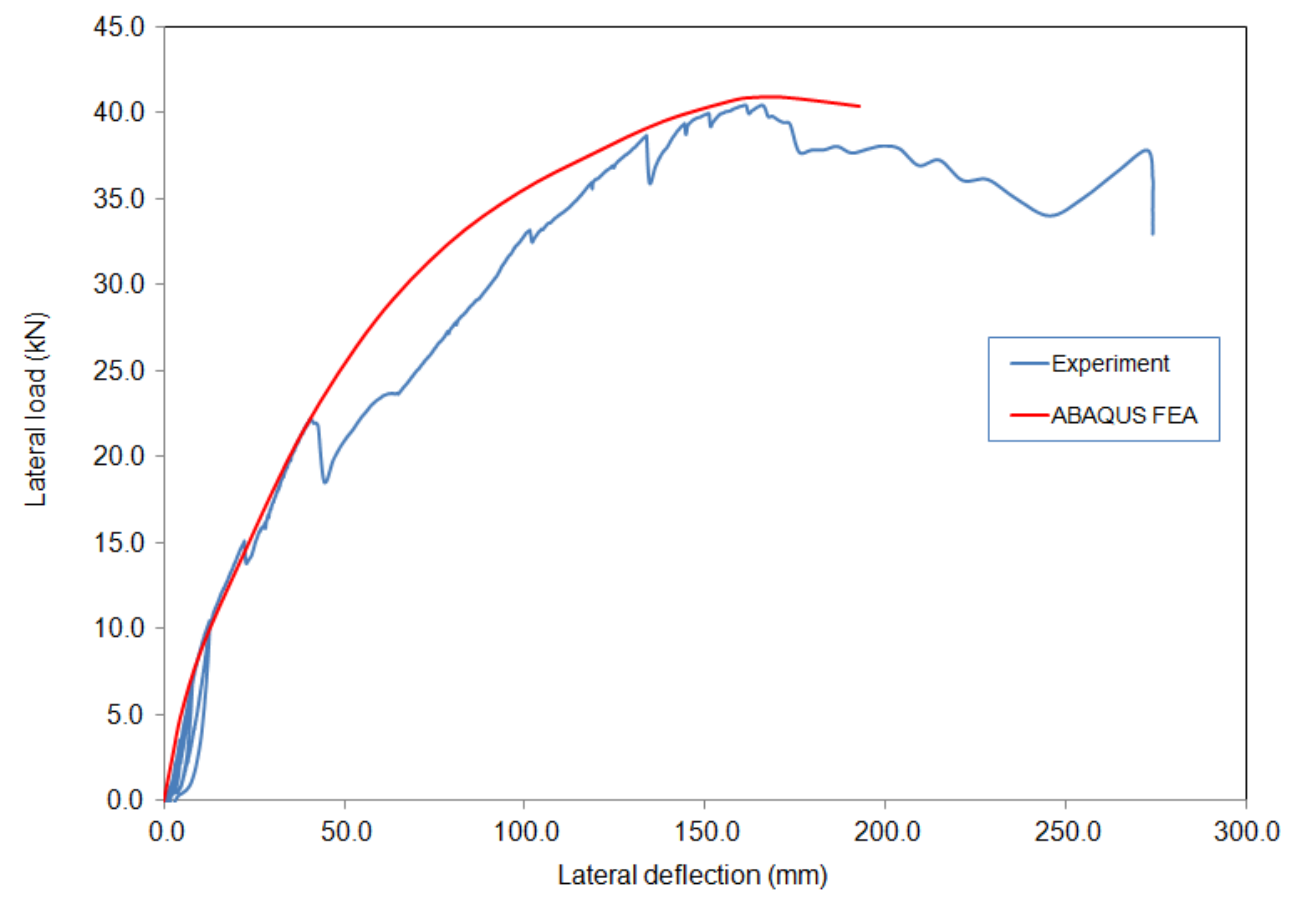

Figure 5.39 - Comparison between the FEA results and experimental findings of SIP wall SIP-R-4 under racking loading

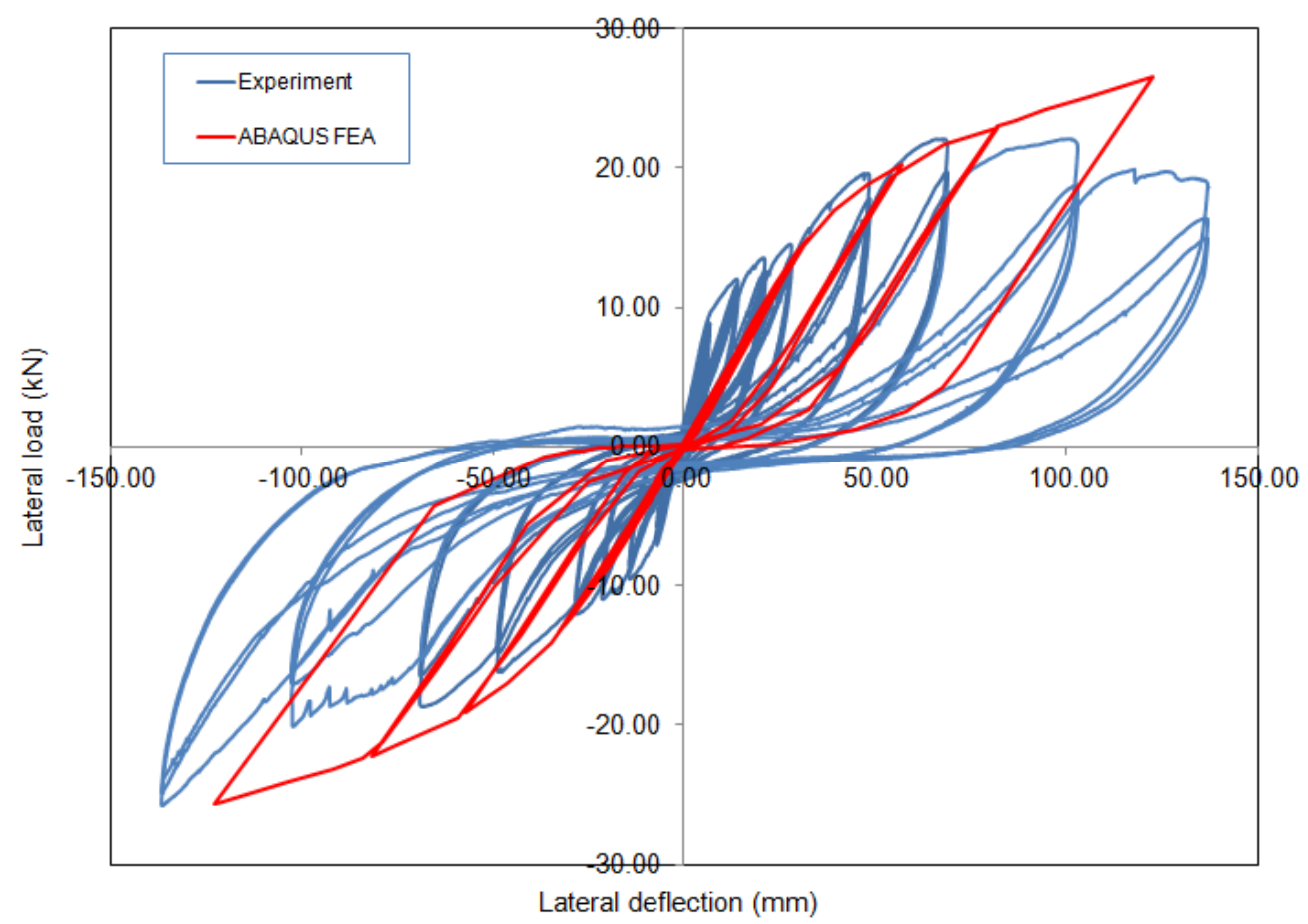

Figure 5.40 - Comparison between the FEA results and experimental findings of SIP wall SIP-C-1 under cyclic loading 


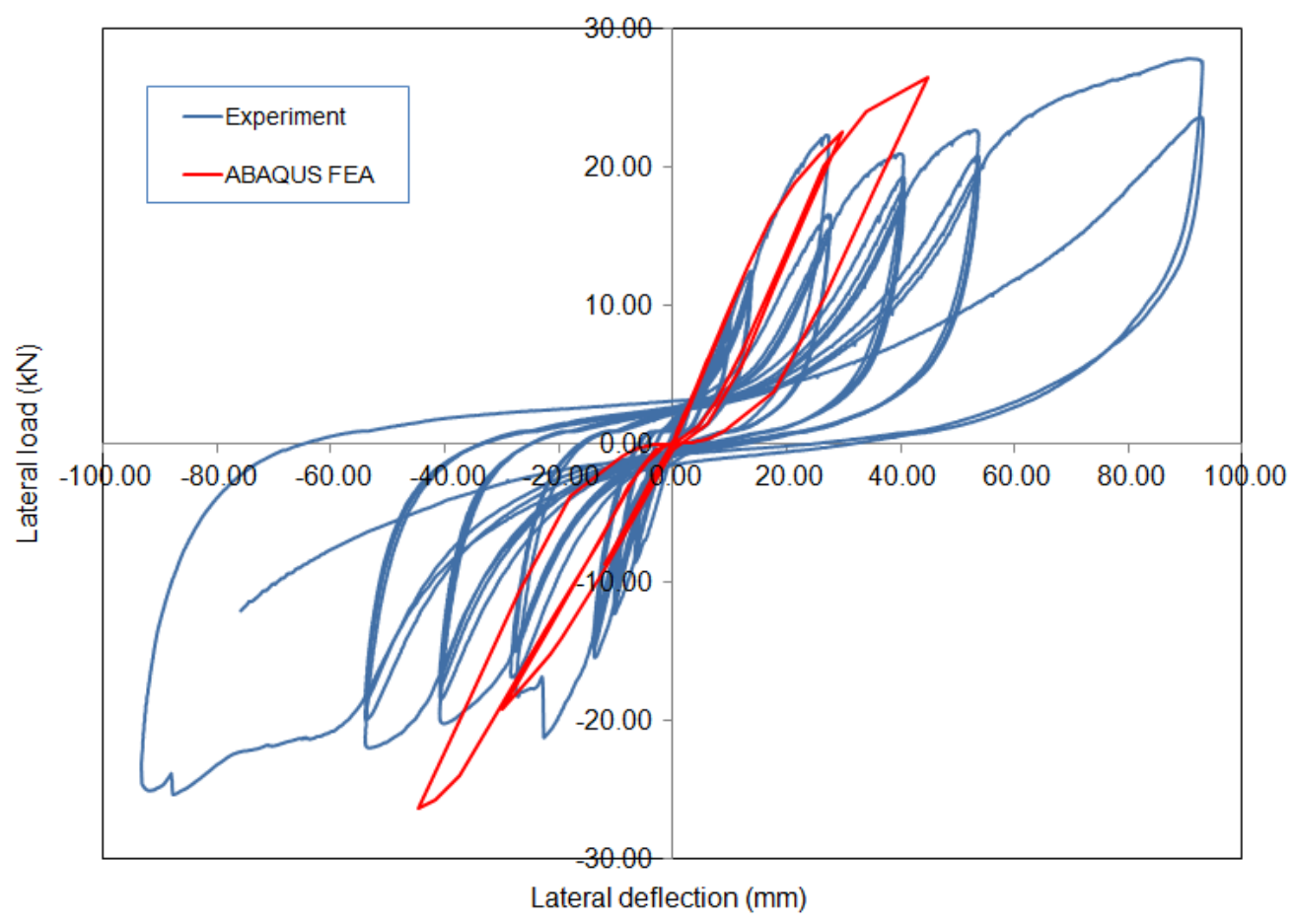

Figure 5.41 - Comparison between the FEA results and experimental findings of SIP wall SIP-C-4 under cyclic loading

\subsection{Parametric Study on SIP Wall Behavior}

After verifying and substantiating the developed FE models for the SIP walls, a parametric study was conducted to examine the effect of intensity of gravity loading spread on top of the wall on the racking and cyclic capacity of the wall. Also, the effect of nail spacing on the structural performance of SIP wall was investigated. The following subsections present details of this study.

\subsubsection{Effect of Gravity Loading on Racking and Cyclic Response}

\section{(a) Effect of Gravity Load on Racking Response}

In order to apply gravity load on SIP walls, different load combination were considered. The location of the wall in the building indicates the amount of the dead load transferring from roof and floor(s) to the wall. In this study, the amount of the dead load for the roof was assumed as $0.5 \mathrm{kPa}$. Live load and snow load for the wall was considered as $2.0 \mathrm{kPa}$, respectively. Considering distances between exterior and interior walls as $4.9 \mathrm{~m}$, the served width of the joists by the exterior wall was assumed $2.7 \mathrm{~m}$ and the tributary width for the interior wall was taken as 
$4.9 \mathrm{~m}$. According to NBCC (2010), the load combinations for the exterior wall resulting from roof loading are as follows:

Case (1): $\quad 1.25 \mathrm{D}+0.5 \mathrm{~S}=4.39 \mathrm{kN} / \mathrm{m}$

Case (2): $\quad 1.25 \mathrm{D}+1.5 \mathrm{~S}=9.79 \mathrm{kN} / \mathrm{m}$

According to NBCC (2010), the load combinations for the exterior wall resulting from roof loading and one floor loading are as follows:

Case (1): $\quad 1.25 \mathrm{D}+0.5 \mathrm{~S}+1.5 \mathrm{~L}=12.49 \mathrm{kN} / \mathrm{m}$

Case (2): $\quad 1.25 \mathrm{D}+1.5 \mathrm{~S}+0.5 \mathrm{~L}=12.49 \mathrm{kN} / \mathrm{m}$

The load combinations for the interior wall resulting from roof loading are as follows:

Case (1): $\quad 1.25 \mathrm{D}+0.5 \mathrm{~S}=8.78 \mathrm{kN} / \mathrm{m}$

Case (2): $\quad 1.25 \mathrm{D}+1.5 \mathrm{~S}=19.58 \mathrm{kN} / \mathrm{m}$

While the load combinations for the interior wall, resulting from roof loading and one floor loading are as follows:

Case (1): $\quad 1.25 \mathrm{D}+0.5 \mathrm{~S}+1.5 \mathrm{~L}=24.98 \mathrm{kN} / \mathrm{m}$

Case (2): $\quad 1.25 \mathrm{D}+1.5 \mathrm{~S}+0.5 \mathrm{~L}=24.98 \mathrm{kN} / \mathrm{m}$

Based on the above information, gravity loads of 4.39, 9.79, 12.49, 19.58 and $24.98 \mathrm{kN} / \mathrm{m}$ were applied to the top of the wall while applying racking loading. Table 5.4 shows the racking capacities obtained from FE modeling under different intensities of gravity loading. Also, Figures 5.42 and 5.43 show the load vs. lateral deflection relationships for the short and long SIP walls, respectively, under racking load as affected by the presence of gravity loading on the wall. In addition, Figure 5.44 depicts the change in racking capacities with the increase in the intensity of gravity loading. It can be observed that the racking capacity of the wall decreases with increase in the intensity of the gravity loading. 
Table 5.4-SIP wall racking capacity under different intensities of gravity loading

\begin{tabular}{|c|c|c|c|c|}
\hline $\begin{array}{c}\text { Intensity of } \\
\text { gravity loading } \\
(\mathbf{k N} / \mathbf{m})\end{array}$ & $\begin{array}{c}2440 \times 2750 \\
\times 165 \mathrm{~mm} \\
\left(8^{\prime} \times 9 \text { 'x6 } 1 / 2 \text { ”) SIP }\right. \\
\text { racking capacity } \\
(\mathrm{kN})\end{array}$ & $\begin{array}{c}2440 \times 2750 \\
\times 165 \mathrm{~mm} \\
\left(8^{\prime} \times 9 ’ \times 6 \text { 1/2”) SIP }\right. \\
\text { racking load per } \\
\text { unit width } \\
(\mathrm{kN} / \mathrm{m})\end{array}$ & $\begin{array}{c}3660 \times 2750 \\
\times 165 \mathrm{~mm} \\
(12 \text { 'x9'x6 1/2”) SIP } \\
\text { racking capacity } \\
(\mathrm{kN})\end{array}$ & $\begin{array}{c}3660 \times 2750 \\
\times 165 \mathrm{~mm} \\
\left(12^{\prime} \times 9 ’ \times 6 \text { 1/2”) }\right. \\
\text { SIP racking } \\
\text { capacity per unit } \\
\text { width }(\mathrm{kN} / \mathrm{m})\end{array}$ \\
\hline 0 & 26.02 & 10.62 & 40.94 & 11.22 \\
\hline 4.39 & 25.34 & 10.34 & 38.07 & 10.45 \\
\hline 9.79 & 23.23 & 9.48 & 35.61 & 9.76 \\
\hline 12.49 & 21.49 & 8.81 & 34.71 & 9.51 \\
\hline 19.58 & 17.09 & 7.00 & 32.64 & 8.94 \\
\hline 24.98 & 14.31 & 5.86 & 29.83 & 8.17 \\
\hline
\end{tabular}

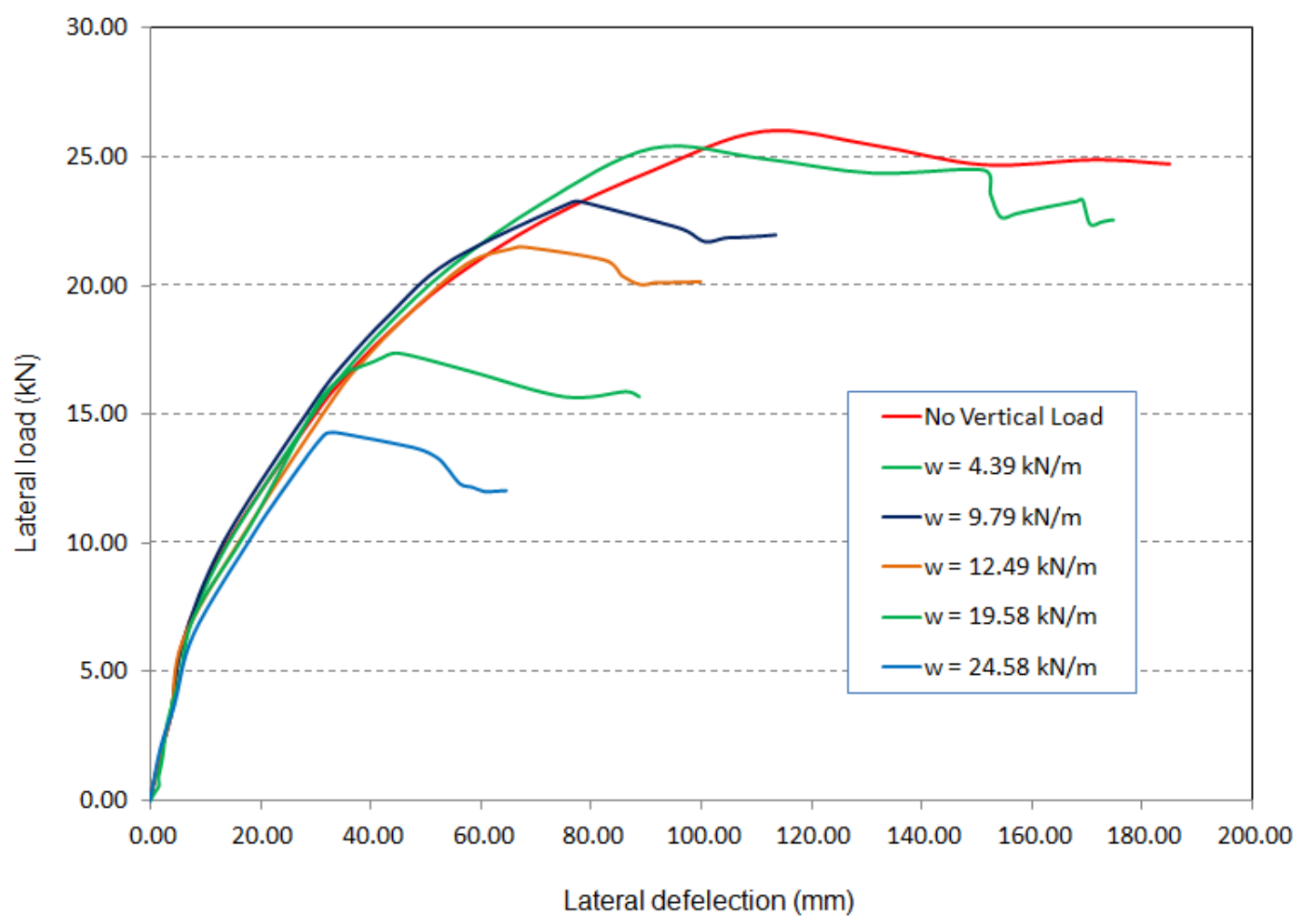

Figure 5.42 - Racking load vs. lateral deflection relationship of SIP wall SIP-R-1 $2440 \times 2750 \times 165 \mathrm{~mm}\left(8^{\prime} \times 9^{\prime} \times 6^{1 / 2}{ }^{\prime \prime}\right)$ under racking load and varying gravity loading 


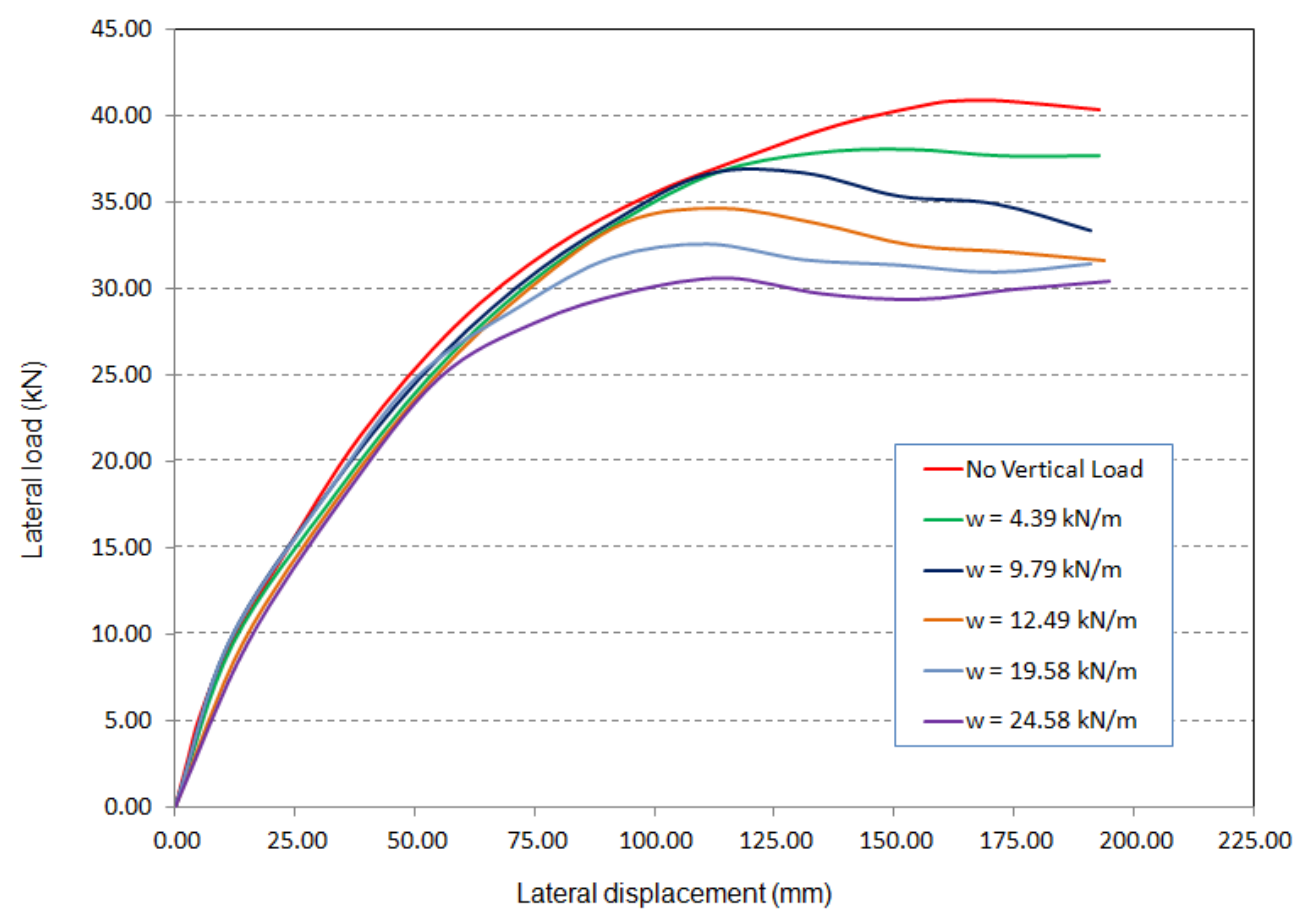

Figure 5.43 - Racking load vs. lateral deflection relationship of SIP wall SIP-R-4 $3660 \times 2750 \times 165 \mathrm{~mm}\left(12^{\prime} \times 9^{\prime} \times 6^{1 / 2} 2^{\prime \prime}\right)$ under racking load and varying gravity loading

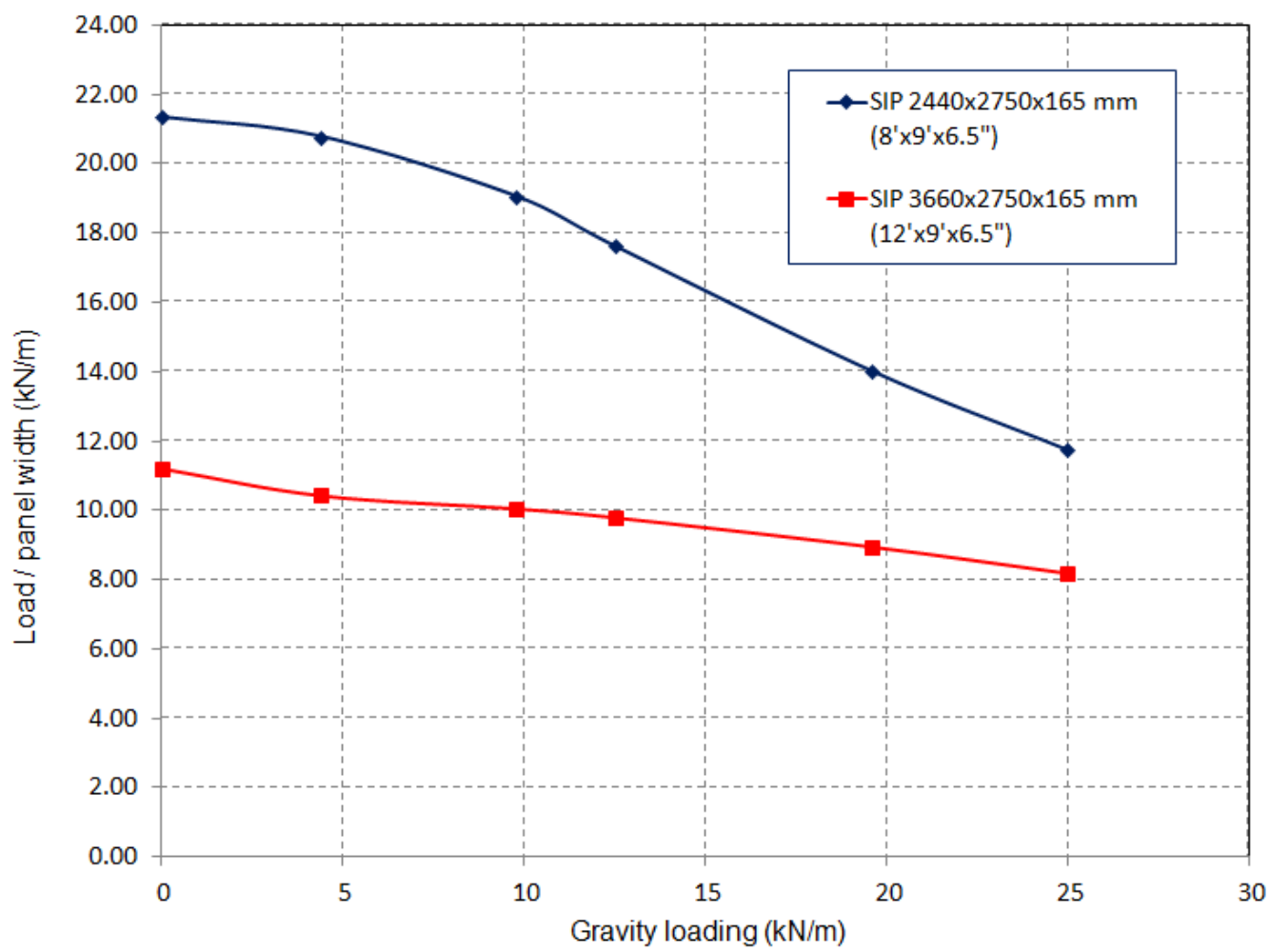

Figure 5.44 - Effect of gravity load on SIP wall racking capacity 


\section{(b) Effect of Gravity Load on Cyclic Response}

Based on the above information, gravity loads of 4.39, 9.79, 12.49, 19.58 and $24.98 \mathrm{kN} / \mathrm{m}$ were applied to the top of the wall while applying cyclic lateral loading. Table 5.5 shows the cyclic load capacities obtained from FE modeling under different intensities of gravity loading. Also, Figures 5.45 and 5.46 show the cyclic load vs. lateral deflection relationships for the short and long SIP walls, respectively, under cyclic load as affected by the presence of gravity loading on the wall. In addition, Figure 5.47 depicts the change in cyclic lateral load capacities with the increase in the intensity of gravity loading. It can be observed that the cyclic capacity of the wall decreases with increase with increase in the intensity of the gravity loading.

Table 5.5 - SIP wall cyclic capacity under different intensities of gravity loading

\begin{tabular}{|c|c|c|c|c|}
\hline $\begin{array}{c}\text { Intensity } \\
\text { of } \\
\text { gravity } \\
\text { loading } \\
\text { (kN/m) }\end{array}$ & $\begin{array}{c}\text { 2440×2750 } \\
\text { ×165 mm } \\
\text { (8’x9'x6 1/2”) } \\
\text { SIP cyclic } \\
\text { capacity (kN) }\end{array}$ & $\begin{array}{c}2440 \times 2750 \\
\times 165 \text { mm } \\
\text { (8’x9’x6 1/2”) SIP } \\
\text { cyclic load per } \\
\text { unit width } \\
(\mathrm{kN} / \mathrm{m})\end{array}$ & $\begin{array}{c}3660 \times 2750 \\
\times 165 \mathrm{~mm} \\
(12 \text { ’x9’x6 1/2”) } \\
\text { SIP cyclic } \\
\text { capacity (kN) }\end{array}$ & $\begin{array}{c}3660 \times 2750 \\
\times 165 \mathrm{~mm} \\
(12 ’ \times 9 ’ x 6 \text { 1/2”) } \\
\text { SIP cyclic } \\
\text { capacity per unit } \\
\text { width }(\mathrm{kN} / \mathrm{m})\end{array}$ \\
\hline 0 & 26.55 & 10.84 & 26.42 & 7.24 \\
\hline 4.39 & 24.47 & 9.98 & 21.62 & 5.92 \\
\hline 9.79 & 22.12 & 9.03 & 18.29 & 5.01 \\
\hline 12.49 & 19.74 & 8.09 & 15.84 & 4.34 \\
\hline 19.58 & 17.28 & 7.08 & 14.85 & 4.07 \\
\hline 24.98 & 16.87 & 6.91 & 13.99 & 3.83 \\
\hline
\end{tabular}




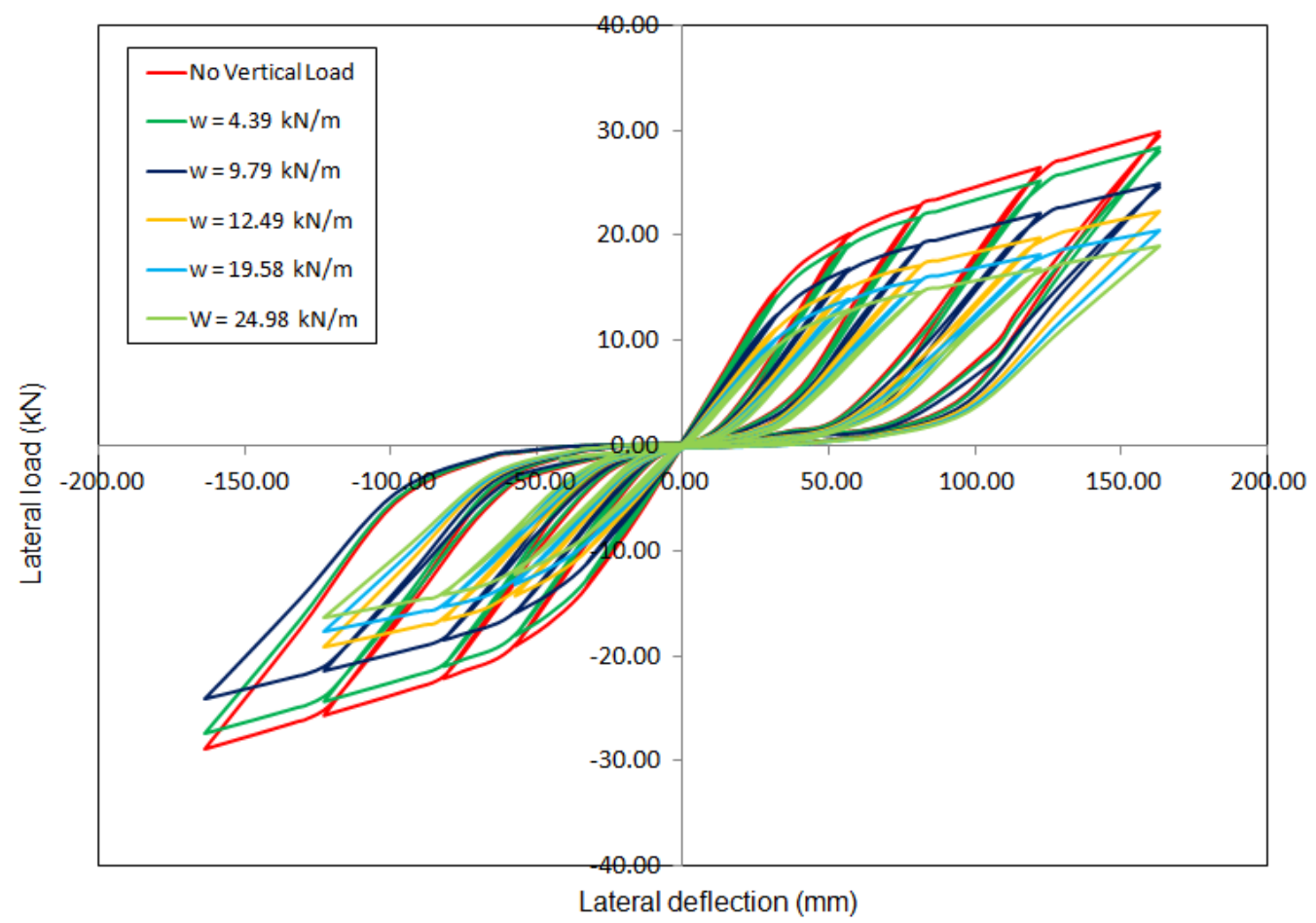

Figure 5.45 - Cyclic load vs. lateral deflection relationship of SIP wall SIP-C-1 $2440 \times 2750 \times 165$ $\mathrm{mm}\left(8^{\prime} \times 9^{\prime} \times 6^{1 / 2}{ }^{\prime}\right)$ under cyclic load and varying gravity loading

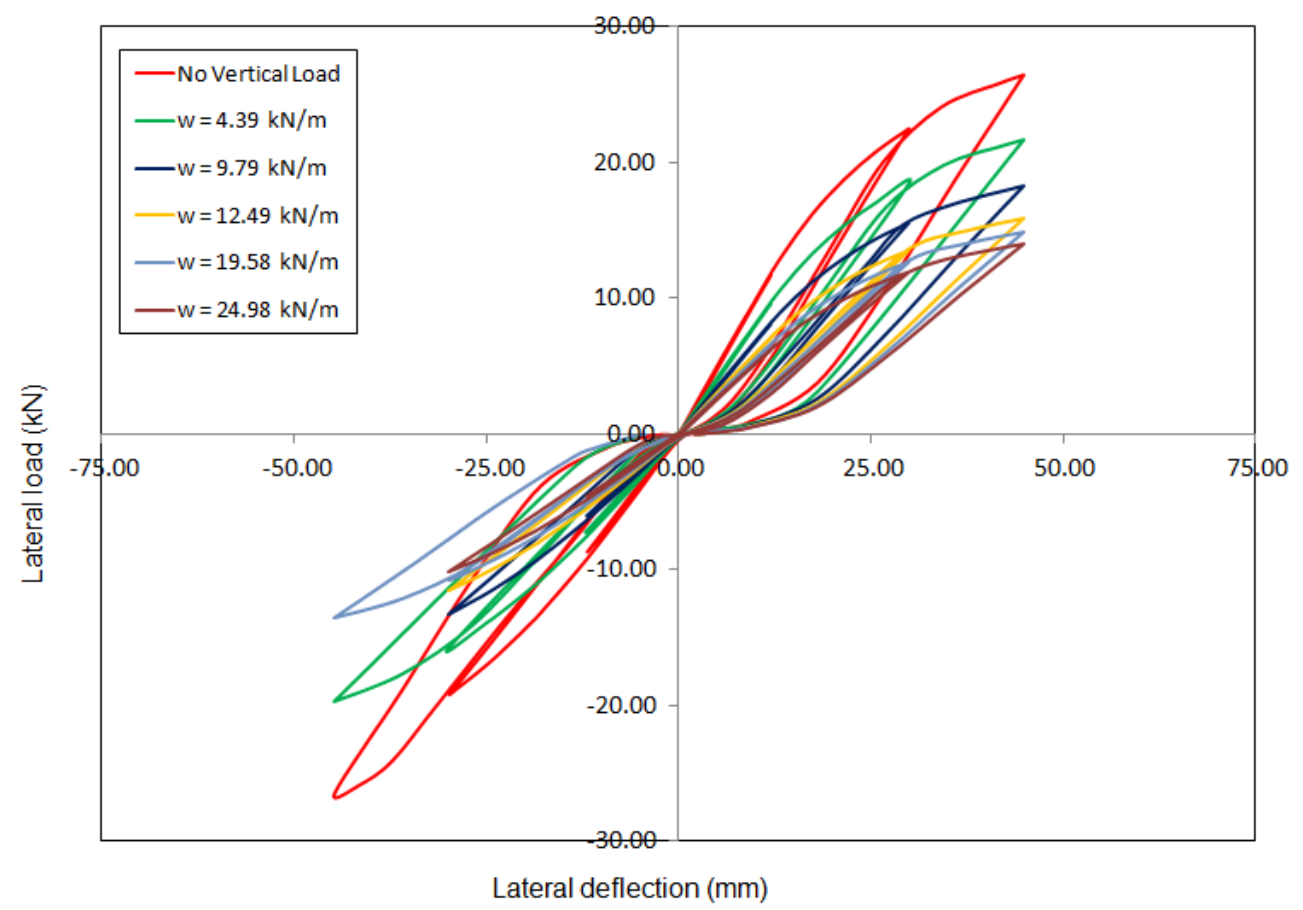

Figure 5.46 - Cyclic load vs. lateral deflection relationship of SIP wall SIP-C-4 3660×2750×165 $\mathrm{mm}\left(12^{\prime} \times 9^{\prime} \times 6^{1 / 2} \mathbf{2}^{\prime}\right)$ under cyclic load and varying gravity loading 


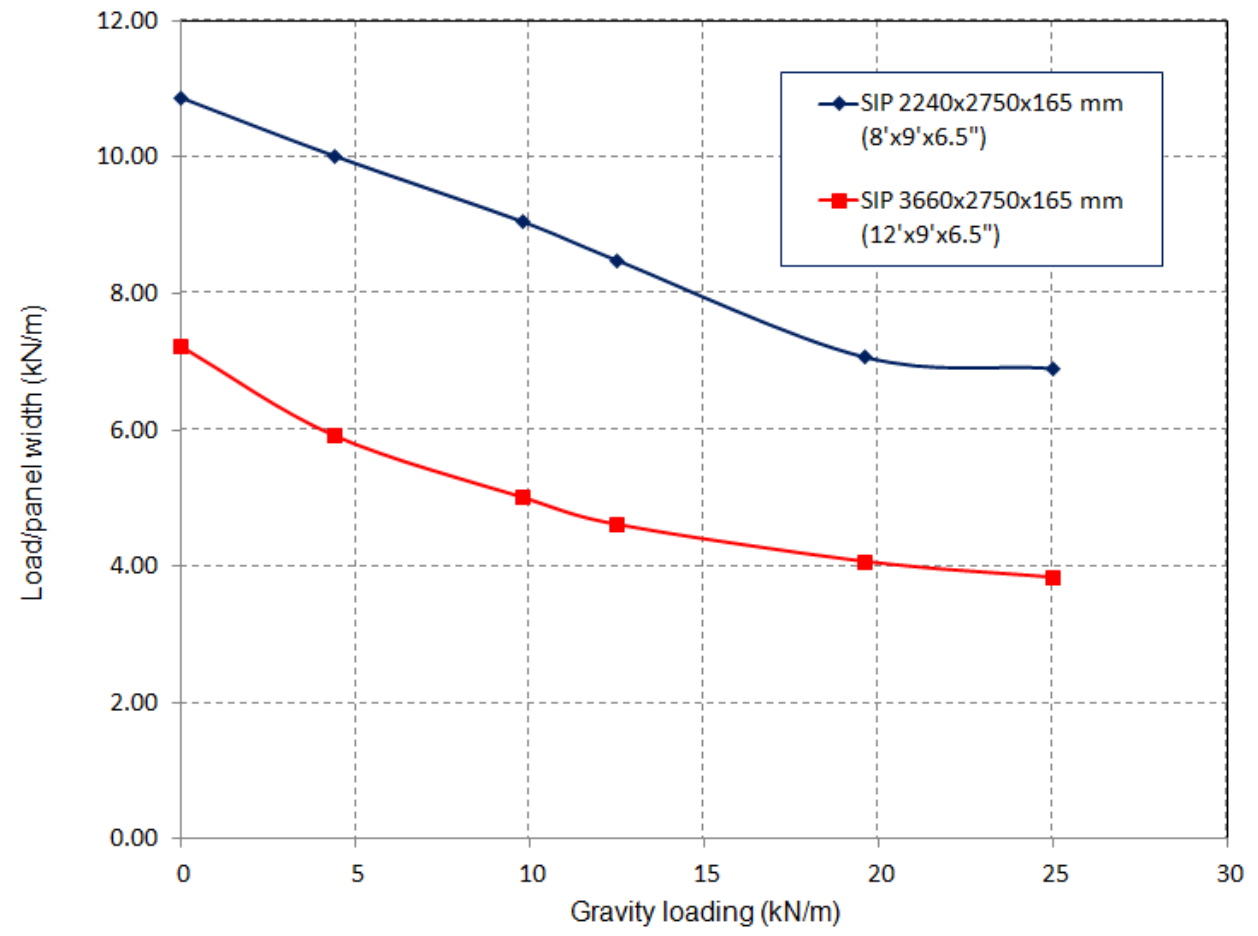

Figure 5.47 - Figure 5.43 - Effect of gravity load on SIP wall cyclic capacity

\subsubsection{Effect of Nail Spacing on Racking and Cyclic Response of SIP panels}

\section{(a) Effect of Nail Spacing on Racking Response of SIP Panels}

Nails were used to connect the ends of the OSB sheets of SIP panel segments to each other through the foam-spline connections. Also, nails were used to connect the ends of the OSB sheets forming SIP panels to the top and bottom lumber plates. As mentioned earlier, nails were defined as oriented springs at the nail location. The distances between nails along the connection lines were considered 165, 330 and $660 \mathrm{~mm}$ to study the effect of nail spacing. Table 5.6 shows the racking capacities of the wall with different nail spacing. While Figures 5.48 and 5.49 depict the racking load vs. lateral deflection of the short and long SIP walls, respectively, under racking load with different nail spacing. Figure 5.50 shows the effect of different nail spacing on the racking capacities of SIP walls.

Results show that SIP wall racking capacity increases with decrease in nail spacing. For example, racking capacity of SIP-R-1 wall changed from 26.02 to $28.46 \mathrm{kN}$ with the change of nail spacing from 330 to $165 \mathrm{~mm}$ spacing (an increase of $9.4 \%$ for doubling the number of nails). 
However, such effect is less in case of longer SIP panels. For example, racking capacity of SIPR-4 wall changed from 40.94 to $41.88 \mathrm{kN}$ with the change of nail spacing from 330 to $165 \mathrm{~mm}$ spacing (an increase of only $2.3 \%$ for doubling the number of nails).

Table 5.6 - SIP wall capacity for different nail spacing under racking load

\begin{tabular}{|c|c|c|}
\hline Nail Spacing (mm) & $\begin{array}{c}2440 \times 2750 \times 165 \mathrm{~mm} \\
\left(8^{\prime} \times 9 ’ \times 6 \text { 1/2”) }\right. \\
\text { SIP racking capacity } \\
(\mathrm{kN})\end{array}$ & $\begin{array}{c}3660 \times 2750 \times 165 \mathrm{~mm} \\
(12 ’ \times 9 ’ \times 6 \text { 1/2”) } \\
\text { SIP racking capacity } \\
(\mathrm{kN})\end{array}$ \\
\hline 660 & 25.59 & 38.36 \\
\hline 330 & 26.02 & 40.94 \\
\hline 165 & 28.46 & 41.88 \\
\hline
\end{tabular}

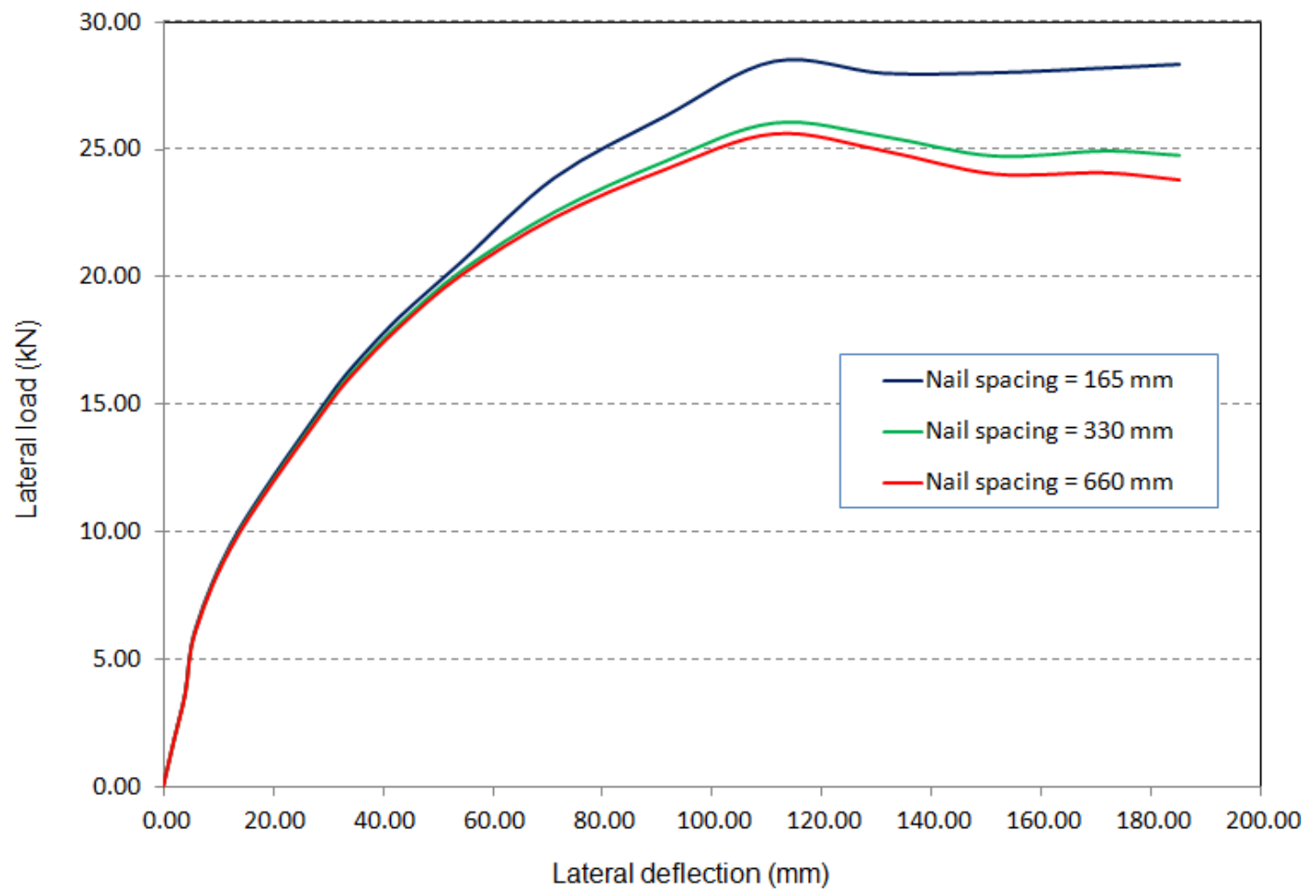

Figure 5.48 - Racking load vs. lateral deflection for SIP wall SIP-R-1 (8'x9'x6 1/2“) with different nail spacing under racking load 


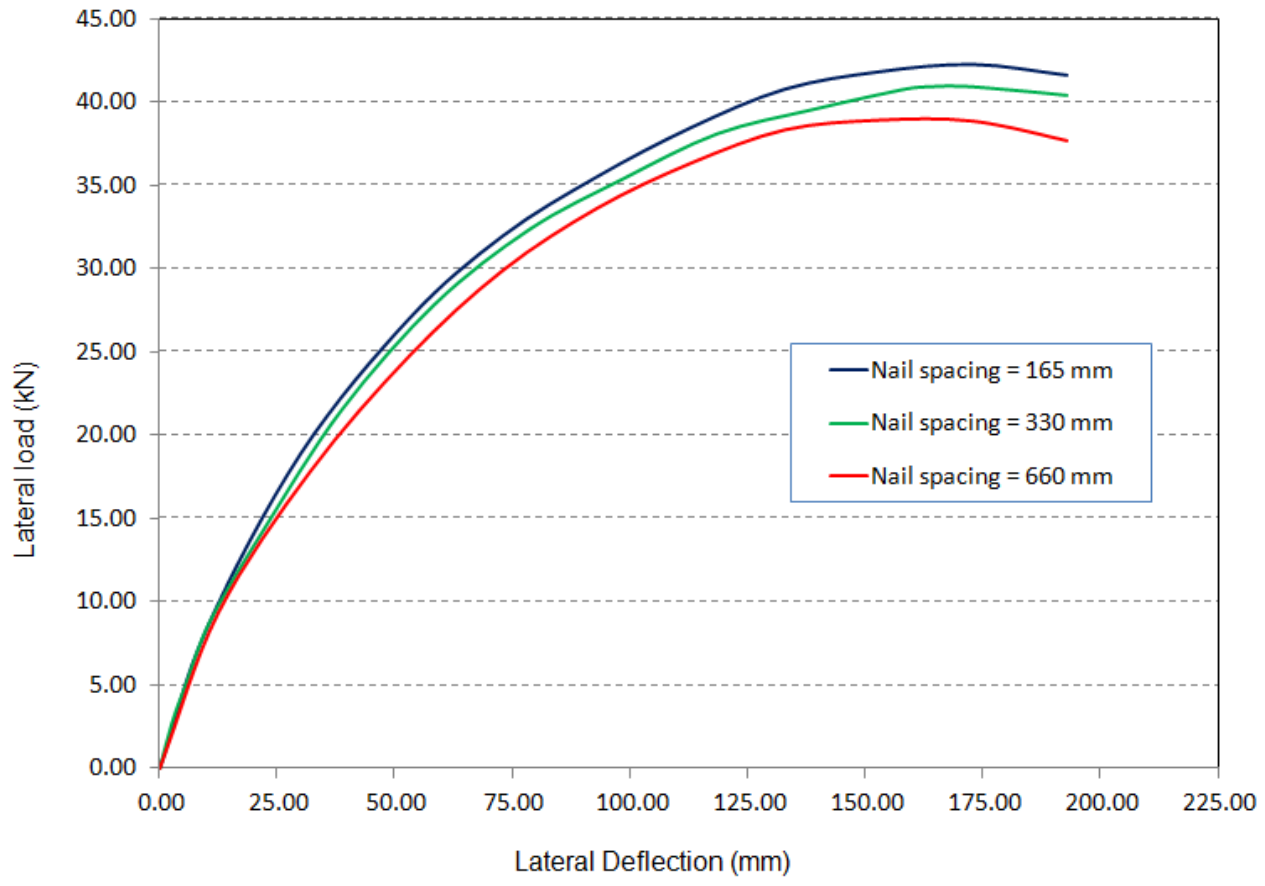

Figure 5.49 - Racking load vs. lateral deflection for SIP wall SIP-R-4 3660×2750×165 mm (12'x9'x6 1/2“) with different nail spacing under racking load

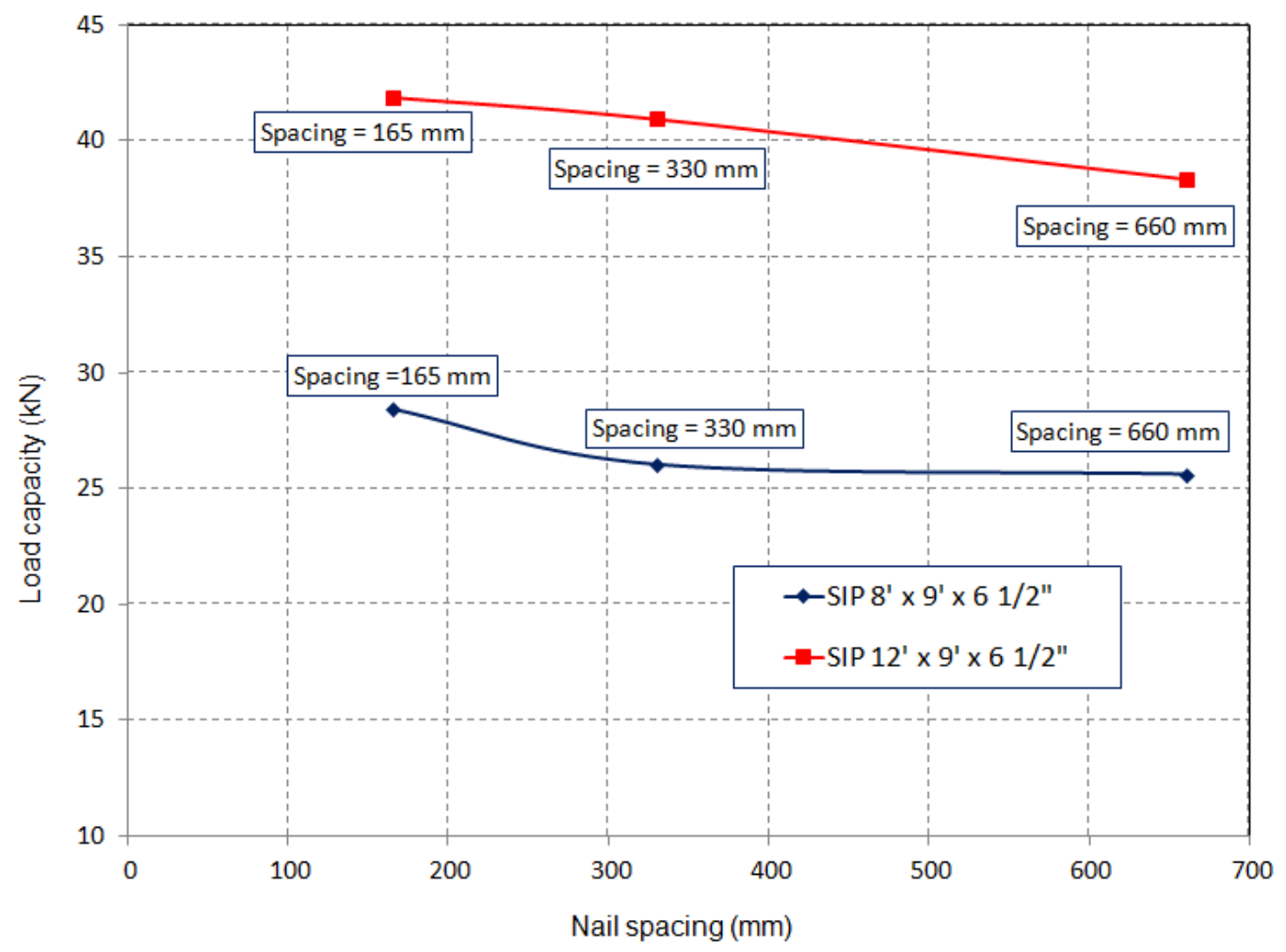

Figure 5.50 - Effect of nail spacing on the racking capacity of SIP walls under racking load 


\section{(b) Effect of Nail Spacing on Cyclic Response}

To study the effect of nail spacing on the cyclic lateral load capacity of SIP wall, the distances between nails along the connection lines were considered 165, 330 and $660 \mathrm{~mm}$. Table 5.7 shows the cyclic capacities of the wall with different nail spacing. While Figures 5.51 and 5.52 depict the cyclic load vs. lateral deflection of the short and long SIP walls, respectively, under cyclic load with different nail spacing. Figure 5.53 shows the effect of different nail spacing on the cyclic capacities of SIP walls. Results show that SIP wall cyclic capacity increases with decrease in nail spacing. For example, the cyclic capacity of SIP-C-1 wall changed from 23.12 to 25.42 $\mathrm{kN}$ with the change of nail spacing from 330 to $165 \mathrm{~mm}$ spacing (an increase of $9.95 \%$ for doubling the number of nails). However, such effect is less in case of longer SIP panels. For example, the cyclic capacity of SIP-C-4 wall changed from 25.78 to $26.59 \mathrm{kN}$ with the change of nail spacing from 330 to $165 \mathrm{~mm}$ spacing (an increase of only $3.1 \%$ for doubling the number of nails).

Table 5.7 - SIP wall capacity for different nail spacing under cyclic load

\begin{tabular}{|c|c|c|}
\hline Nail spacing (mm) & $\begin{array}{c}2440 \times 2750 \times 165 \mathrm{~mm} \\
(8 ' \times 9 ’ \times 6 \text { 1/2”) } \\
\text { SIP cyclic capacity } \\
(\mathrm{kN})\end{array}$ & $\begin{array}{c}3660 \times 2750 \times 165 \mathrm{~mm} \\
\left(12^{\prime} \times 99^{\prime} \times 6^{1 / 2} 2^{\prime}\right) \\
\text { SIP cyclic capacity } \\
(\mathrm{kN})\end{array}$ \\
\hline 660 & 22.18 & 23.20 \\
\hline 330 & 23.12 & 25.78 \\
\hline 165 & 25.42 & 26.59 \\
\hline
\end{tabular}




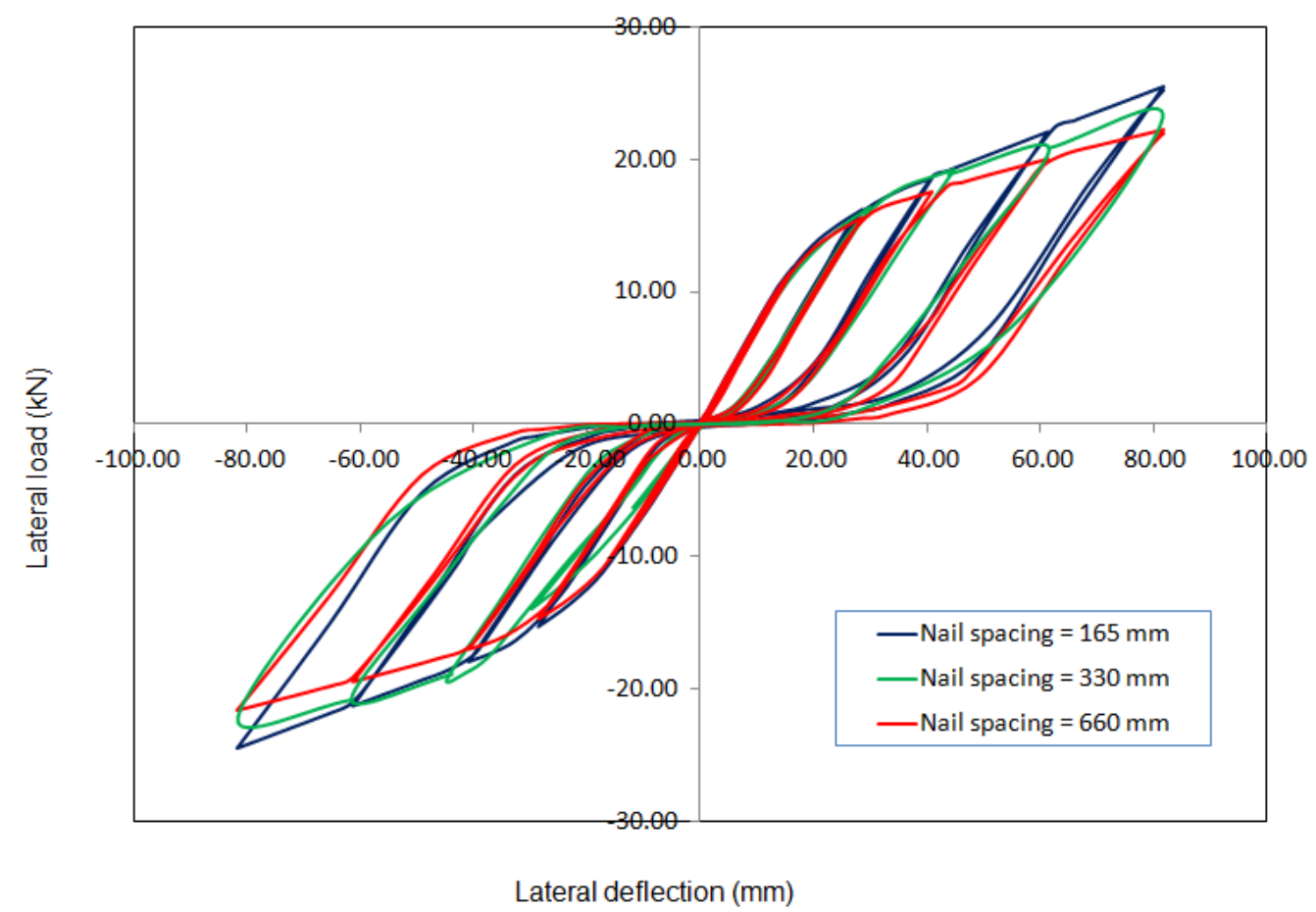

Figure 5.51 - Cyclic load vs. lateral deflection for SIP wall SIP-C-1

$2440 \times 2750 \times 165 \mathrm{~mm}\left(8^{\prime} \times 9^{\prime} \times 6^{1 / 2^{\prime c}}\right)$ with different nail spacing under cyclic load

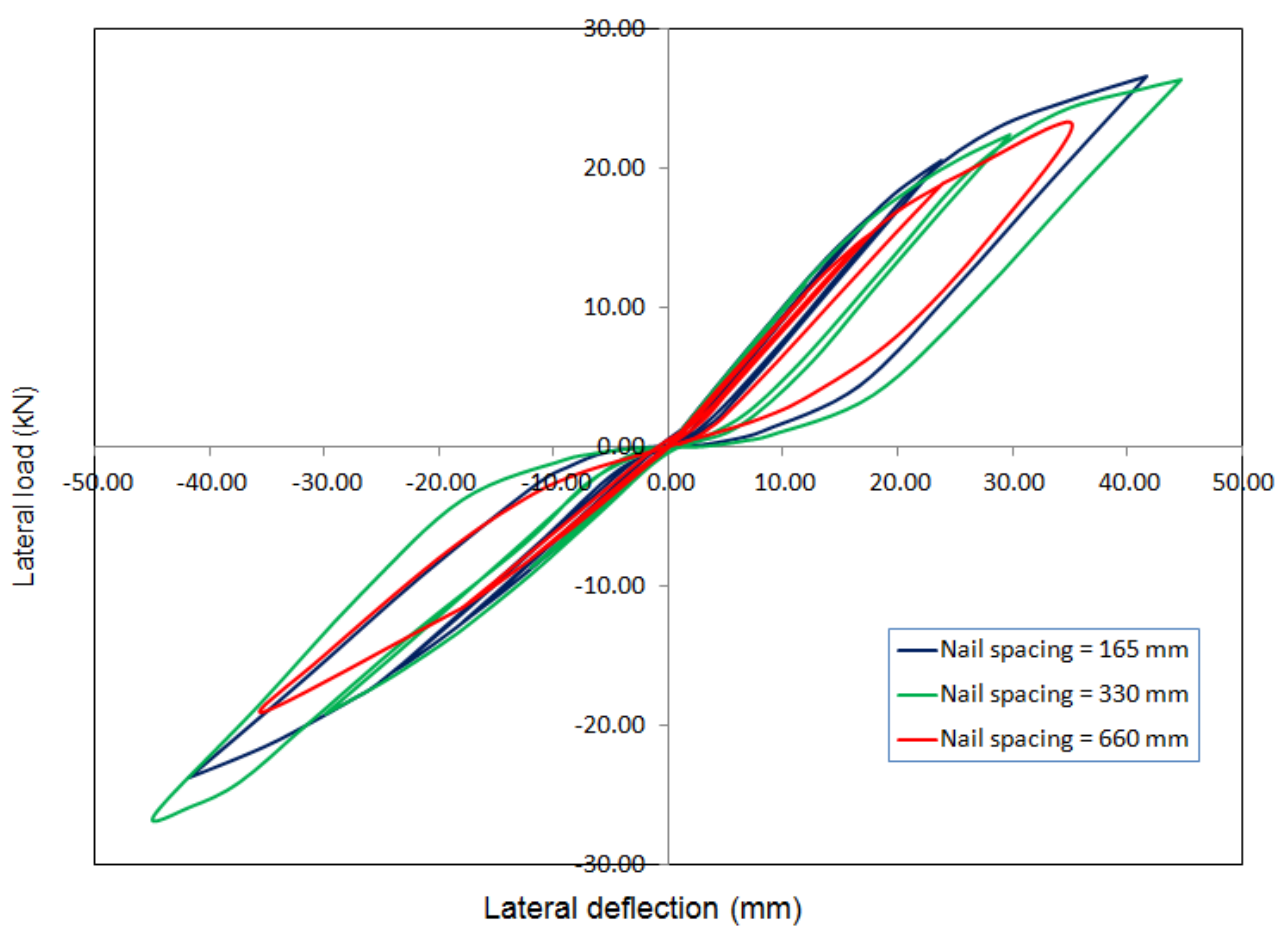

Figure 5.52 - Cyclic load vs. lateral deflection for SIP wall SIP-C-4

$3660 \times 2750 \times 165 \mathrm{~mm}\left(12^{\prime} \times 9^{\prime} \times 6^{1 / 2} 2^{\prime \prime}\right)$ with different nail spacing under cyclic load 


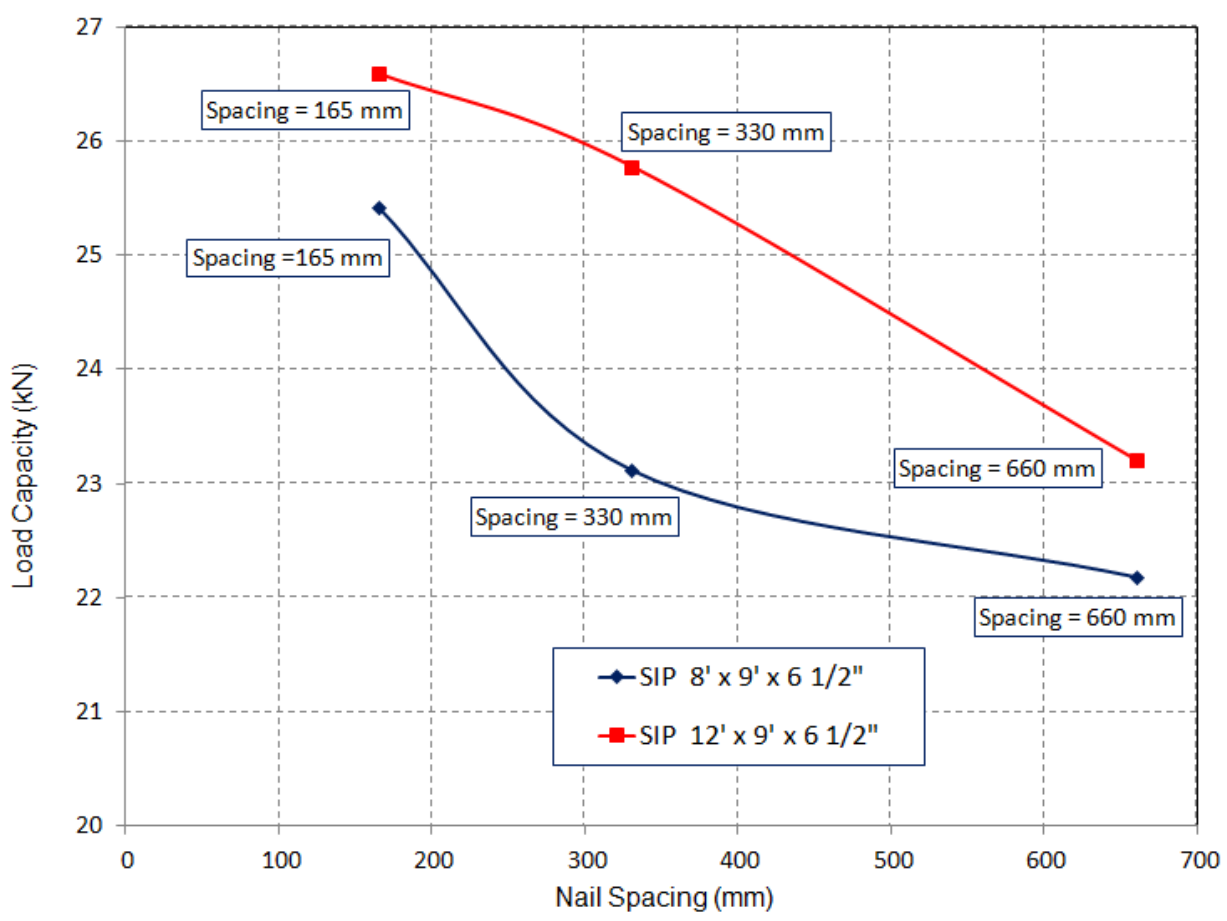

Figure 5.53 - Effect of nail spacing for different size of SIP wall $2440 \times 2750 \times 165 \mathrm{~mm}$ and

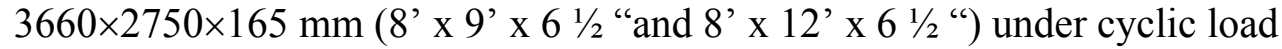




\section{CHAPTER 6}

\section{DEVELOPMENT OF DESIGN TABLES AND EQUATIONS FOR STUD AND SIP PANEL DESIGN}

\subsection{Introduction}

This chapter presents the results of the correlation between the experimental findings of stud and SIP panels and available equations in the literature. Based on this comparison, modification factors for the available design equations were established to have better representation of experimental findings at ultimate and serviceability limit state design. In addition, design tables of the maximum uniform loading in SIP panels under flexural loading as well as under compressive loading were established.

\subsection{Correlation of Results from Code Equations and Experimental Data of Stud System}

\subsubsection{Flexural Loading on Stud Panels}

Manual calculations were performed to determine the moment and shear capacities of the studied stud panels when subjected to flexural loading. Table 6.1 shows the results obtained for bending resistance and shear resisting force of the tested stud panels SW-F-1 through SW-F-3 based on CSA-O86-09 and ANSI/APA standards. Also, Table 6.1 shows the applied experimental load at a panel deflection equal to span/360. The corresponding deflection values obtained using equations available in CSA-O86-09 and ANSI/APA standards were also presented in this table. Tables 6.2 and 6.3 show the ratios between the resisting moments and shear forces, respectively, obtained from code equations and the experimental data. Table 6.4 also shows the comparison of code results and experimental findings for deflection for flexural loading on stud panels. It can be observed that code equations provide moment and shear resistance greater than those obtained experimentally. As such modification factors were proposed to lower the values obtained from code equations. Table 6.5 shows these modification factors as 0.89 and 1.19 for moment and shear resistances, respectively, obtained from CSA-086-09 equations. Also, Table 6.5 shows these modification factors as 0.87 and 1.01 for moment and shear resistances, respectively, 
obtained from APA equations. Similar observations for deflection calculation as depicted in Table 6.5. As such, Table 6.5 proposes the use of modification factors of 0.82 and 0.46 to the available deflection equations in CSA-O86-09 and APA Standard, respectively. 
Table 6.1 - Results from different codes and experimental data for flexural loading on stud panels

\begin{tabular}{|c|c|c|c|c|c|c|c|c|c|c|}
\hline \multicolumn{5}{|c|}{ Experimental Data } & \multicolumn{3}{|c|}{ CSA-O86-09 ${ }^{(3)}$} & \multicolumn{3}{|c|}{ APA Standard ${ }^{(3)}$} \\
\hline Specimen & $\begin{array}{c}\text { Ultimate } \\
\text { Load } \\
(\mathbf{k N})^{(1)}\end{array}$ & $\begin{array}{l}\text { Bending } \\
\text { Moment } \\
\left(\text { kN.m) }{ }^{(2)}\right.\end{array}$ & $\begin{array}{c}\text { Ultimate } \\
\text { shear } \\
\text { Force } \\
\text { (kN) }\end{array}$ & $\begin{array}{l}\text { Flexural } \\
\text { load }(\mathrm{kN}) \\
\text { at L/360 } \\
\text { Deflection } \\
(\mathrm{mm})^{(4)}\end{array}$ & $\begin{array}{c}\text { Bending } \\
\text { Resistance } \\
\text { (kN.m) }\end{array}$ & $\begin{array}{c}\text { Shear } \\
\text { Resistance } \\
\text { (kN) }\end{array}$ & $\begin{array}{c}\text { Deflection } \\
\text { (mm) }\end{array}$ & $\begin{array}{c}\text { Bending } \\
\text { Resistance } \\
\text { (kN.m) }\end{array}$ & $\begin{array}{c}\text { Shear } \\
\text { Resistance } \\
\text { (kN) }\end{array}$ & $\begin{array}{c}\text { Deflection } \\
\text { (mm) }\end{array}$ \\
\hline $\begin{array}{c}\text { SW-F-01 } \\
\text { 2440x2743x165 } \\
\mathrm{mm}\left(8^{\prime} \times 9 \text { 9'x6.5”) }\right.\end{array}$ & 59.68 & 16.47 & 31.75 & $\begin{array}{c}13.10 \\
7.20\end{array}$ & & & & & & \\
\hline $\begin{array}{c}\text { SW-F-02 } \\
\text { 2440x2743x165 } \\
\text { mm (8’x9'x6.5”) }\end{array}$ & 67.68 & 18.52 & 35.75 & $\begin{array}{l}12.29 \\
7.20\end{array}$ & 16.10 & 23.28 & 8.77 & 16.57 & 27.35 & 15.58 \\
\hline $\begin{array}{c}\text { SW-F-03 } \\
2440 \times 2743 \times 165 \\
\mathrm{~mm}\left(8^{\prime} \times 9 \text { 'x6.5”) }\right.\end{array}$ & 67.56 & 18.49 & 35.68 & $\begin{array}{c}13.81 \\
7.20\end{array}$ & & & & & & \\
\hline
\end{tabular}

${ }^{(1)}$ Did not include $3.82 \mathrm{kN}$ weight of the loading system.

(2) Included $3.82 \mathrm{kN}$ weight of the loading system. Values are per 8 ' panel width and multiplied by 0.8 for using 5 studs rather than 4 in the tested wall and then multiplied by $\mathrm{L} / 8$ to obtain bending moment $(\mathrm{L}=2.5908 \mathrm{~m})$.

(3) Values are per 8' panel width and multiplied by 0.8 for using 5 studs rather than 4 in the tested wall and then multiplied by $\mathrm{L} / 8$ to obtain bending moment $(\mathrm{L}=$ $2.5908 \mathrm{~m})$.

(4) The first number of the total load applied to the panel in $\mathrm{kN}$ to get the deflection in mm which is the second number. 
Table 6.2 - Comparison of code results and experimental findings for moment resistance for flexural loading on stud panels

\begin{tabular}{|c|c|c|c|c|c|c|}
\hline \multirow{2}{*}{ Specimen } & \multirow{2}{*}{$\begin{array}{l}M_{r(\text { Exp.) }} \\
\text { (kN-m) }\end{array}$} & \multirow{2}{*}{$\begin{array}{c}\text { Characteristic } \\
\text { value, } M_{\mathbf{r}} \\
\text { (kN-m) }\end{array}$} & \multicolumn{2}{|c|}{$\begin{array}{c}\text { Bending Resistance } \\
\text { (kN-m) }\end{array}$} & \multicolumn{2}{|c|}{ Ratio ( $\mathbf{M}_{\mathrm{r}(\mathrm{code})} / \mathbf{M}_{\mathrm{r}(\exp )}$} \\
\hline & & & $\begin{array}{c}\text { CSA-O86- } \\
09\end{array}$ & $\begin{array}{c}\text { APA } \\
\text { Standard }\end{array}$ & $\begin{array}{c}\text { CSA-O86- } \\
09\end{array}$ & $\begin{array}{c}\text { APA } \\
\text { Standard }\end{array}$ \\
\hline $\begin{array}{c}\text { SW-F-01 } \\
\left(8^{\prime} \times 9 ' x 6.5^{\prime \prime}\right)\end{array}$ & 16.47 & \multirow{3}{*}{14.38} & \multirow{3}{*}{16.10} & \multirow{3}{*}{16.57} & \multirow{3}{*}{1.12} & \multirow{3}{*}{1.15} \\
\hline $\begin{array}{c}\text { SW-F-02 } \\
\left(8^{\prime} \times 9 ' x 6.5 "\right)\end{array}$ & 18.52 & & & & & \\
\hline $\begin{array}{c}\text { SW-F-03 } \\
\left(8^{\prime} \times 9 \text { 9'x 6.5") }\right.\end{array}$ & 18.49 & & & & & \\
\hline
\end{tabular}

Table 6.3 - Comparison of code results and experimental findings for shear resistance for flexural loading on stud panels

\begin{tabular}{|c|c|c|c|c|c|c|}
\hline \multirow[b]{2}{*}{ Specimen } & \multirow{2}{*}{$\begin{array}{c}\mathrm{V}_{\mathbf{r}(\text { Exp. })} \\
(\mathbf{k N})\end{array}$} & \multirow{2}{*}{$\begin{array}{l}\text { Characteristic } \\
\text { value, } V_{\mathbf{r}}(\mathbf{k N})\end{array}$} & \multicolumn{2}{|c|}{ Shear Resistant (kN) } & \multicolumn{2}{|c|}{ Ratio ( $\mathbf{V}_{\mathbf{r}(\text { code })} / \mathbf{V}_{\mathbf{r}(\exp )}$} \\
\hline & & & $\begin{array}{c}\text { CSA- } \\
\text { O86-09 }\end{array}$ & $\begin{array}{c}\text { APA } \\
\text { Standard }\end{array}$ & $\begin{array}{l}\text { CSA- } \\
\text { O86-09 }\end{array}$ & $\begin{array}{c}\text { APA } \\
\text { Standard }\end{array}$ \\
\hline $\begin{array}{c}\text { SW-F-01 } \\
\left(8^{\prime} \times 9 \text { 'x 6.5") }\right.\end{array}$ & 31.75 & \multirow{3}{*}{27.73} & \multirow{3}{*}{23.28} & \multirow{3}{*}{27.35} & \multirow{3}{*}{0.84} & \multirow{3}{*}{0.99} \\
\hline $\begin{array}{c}\text { SW-F-02 } \\
\left(8^{\prime} \times 9 \text { 'x 6.5") }\right.\end{array}$ & 35.75 & & & & & \\
\hline $\begin{array}{c}\text { SW-F-03 } \\
\left(8^{\prime} \times 9 ' x 6.5 "\right)\end{array}$ & 35.68 & & & & & \\
\hline
\end{tabular}


Table 6.4 - Comparison of code results and experimental findings for deflection for flexural loading on stud panels

\begin{tabular}{|c|c|c|c|c|c|c|}
\hline \multirow[b]{2}{*}{ Specimen } & \multirow[b]{2}{*}{$\begin{array}{c}\text { Deflection }_{\text {(Exp.) }} \\
(\mathbf{m m})\end{array}$} & \multirow{2}{*}{$\begin{array}{l}\text { Characteristic } \\
\text { value, } \\
\text { deflection }_{(\exp )} \\
\text { (mm) }\end{array}$} & \multicolumn{2}{|c|}{ Deflection (mm) } & \multicolumn{2}{|c|}{ Ratio (Defl $_{(\text {code) }} /$ Defl $_{(\text {exp) }}$} \\
\hline & & & $\begin{array}{l}\text { CSA- } \\
\text { O86-09 }\end{array}$ & $\begin{array}{c}\text { APA } \\
\text { Standard }\end{array}$ & $\begin{array}{l}\text { CSA- } \\
\text { O86-09 }\end{array}$ & $\begin{array}{c}\text { APA } \\
\text { Standard }\end{array}$ \\
\hline $\begin{array}{c}\text { SW-F-01 } \\
\left(8^{\prime} \times 9 ' x 6.5 "\right)\end{array}$ & 7.20 & \multirow{3}{*}{7.20} & \multirow{3}{*}{8.77} & \multirow{3}{*}{15.58} & \multirow{3}{*}{1.22} & \multirow{3}{*}{2.16} \\
\hline $\begin{array}{c}\text { SW-F-02 } \\
\left(8^{\prime} \times 9 ' x 6.5 "\right)\end{array}$ & 7.20 & & & & & \\
\hline $\begin{array}{c}\text { SW-F-03 } \\
\left(8^{\prime} \times 9 ' \times 6.5^{\prime \prime}\right)\end{array}$ & 7.20 & & & & & \\
\hline
\end{tabular}

Table 6.5 - Proposed modification factors for moment and shear resistances as well as the deflection of stud panels under flexural loading

\begin{tabular}{|c|c|c|c|c|c|c|}
\hline & \multicolumn{2}{|c|}{ Moment resistance } & \multicolumn{2}{c|}{ Shear resistance } & \multicolumn{2}{c|}{ Deflection } \\
\hline $\begin{array}{c}\text { Modification } \\
\text { factor }\end{array}$ & $\begin{array}{c}\text { CSA- } \\
\text { O86-09 }\end{array}$ & $\begin{array}{c}\text { APA } \\
\text { Standard }\end{array}$ & $\begin{array}{c}\text { CSA- } \\
\text { O86-09 }\end{array}$ & $\begin{array}{c}\text { APA } \\
\text { Standard }\end{array}$ & $\begin{array}{c}\text { CSA- } \\
\text { O86-09 }\end{array}$ & $\begin{array}{c}\text { APA } \\
\text { Standard }\end{array}$ \\
\hline $\mathbf{k}$ & 0.89 & 0.87 & 1.19 & 1.01 & 0.82 & 0.46 \\
\hline
\end{tabular}

\subsubsection{Compressive Loading on Stud Walls}

Manual calculations were performed to determine the compressive resistance of the studied stud walls when subjected to compressive loading with eccentricity of $t / 6$ per the test method. Table 6.6 shows the results obtained for compressive resistances for the studied stud walls SW-A-1 through SW-A-3 based on CSA-O86-09 and ANSI/APA standards. Table 6.7 shows the ratios between the resisting compressive forces obtained from code equations and the experimental data. It can be observed that code equations provide moment and shear resistance greater than those obtained experimentally. For example, the ratios between values obtained from CSA-O8609 and APA Standards and the experimental data were 3.69 and 1.78, respectively, as depicted in Table 6.7. As such modification factors were proposed to lower the values obtained from code equations. These modification factors are proposed to be 0.27 and 0.56 for compressive resistance values obtained from CSA-O86-09 and APA equations, respectively. 
Table 6.6 - Results from different codes and experimental data for compressive load on stud walls

\begin{tabular}{|c|c|c|c|c|c|}
\hline \multirow[b]{2}{*}{ Specimen } & \multirow[b]{2}{*}{$\begin{array}{c}\text { Ultimate } \\
\text { compressive } \\
\text { load }(\mathbf{k N})^{(1)}\end{array}$} & \multirow[b]{2}{*}{$\begin{array}{c}\text { Ultimate } \\
\text { compressive } \\
\text { load }(k N)^{(2)}\end{array}$} & \multirow{2}{*}{$\begin{array}{c}\text { Moment due to } \\
\text { load } \\
\text { eccentricity } \\
(\mathrm{kN}-\mathrm{m})\end{array}$} & CSA-086-09 ${ }^{(3)}$ & APA Standard $^{(3)}$ \\
\hline & & & & $\begin{array}{c}\text { Compressive } \\
\text { load resistance } \\
(\mathbf{k N})\end{array}$ & $\begin{array}{c}\text { Compressive } \\
\text { load resistance } \\
(\mathbf{k N})\end{array}$ \\
\hline $\begin{array}{c}\text { SW-A-01 } \\
2440 \times 2743 \times 165 \mathrm{~mm} \\
\left(8^{\prime} \times 99^{\prime} \times 6.5^{\prime \prime}\right)\end{array}$ & 253.51 & 205.48 & 5.65 & & \\
\hline $\begin{array}{c}\text { SW-A-02 } \\
2440 \times 2743 \times 165 \mathrm{~mm} \\
\left(8^{\prime} \times 99^{\prime} \times 6.5^{\prime \prime}\right)\end{array}$ & 297.23 & 240.46 & 6.61 & 358.11 & 172.38 \\
\hline $\begin{array}{c}\text { SW-A-03 } \\
2440 \times 2743 \times 165 \mathrm{~mm} \\
\left(8^{\prime} \times 99^{\prime} \times 6.5^{\prime \prime}\right)\end{array}$ & 189.60 & 154.36 & 4.52 & & \\
\hline
\end{tabular}

(1) Did not include $3.34 \mathrm{kN}$ weight of the loading system.

(2) Included $3.34 \mathrm{kN}$ weight of the loading system. Values are for 8' panel width and multiplied by 0.8 for using 5 studs rather than 4 in the tested wall.

${ }^{(3)}$ Values are for 8 ' panel width and multiplied by 0.8 for using 5 studs rather than 4 in the tested wall.

Table 6.7 - Comparison of results from different codes and experimental data for compressive load on stud walls

\begin{tabular}{|c|c|c|c|c|c|c|}
\hline \multirow{2}{*}{ Specimen } & \multirow{2}{*}{$\begin{array}{c}P_{\mathbf{r}(\exp .)} \\
(\mathbf{k N})\end{array}$} & \multirow{2}{*}{$\begin{array}{c}\text { Characteristic } \\
\text { value, } \mathbf{P}_{\mathbf{r ( e x p . )}} \\
\text { (kN) }\end{array}$} & \multicolumn{2}{|c|}{$\begin{array}{c}\text { Compressive load resistance } \\
\qquad(\mathrm{kN})\end{array}$} & \multicolumn{2}{|c|}{ Ratio $\left(\mathbf{P}_{\mathbf{r}(\text { code })} / \mathbf{P}_{\mathbf{r}(\text { exp.) }}\right)$} \\
\hline & & & CSA-086-09 & $\begin{array}{c}\text { APA } \\
\text { Standard }\end{array}$ & CSA-086-09 & $\begin{array}{c}\text { APA } \\
\text { Standard }\end{array}$ \\
\hline $\begin{array}{l}\text { SW-A-01 } \\
\left(8^{\prime} \times 9 ' \times 6.5^{\prime \prime}\right)\end{array}$ & 205.48 & \multirow{3}{*}{96.97} & \multirow{3}{*}{358.11} & \multirow{3}{*}{172.38} & \multirow{3}{*}{3.69} & \multirow{3}{*}{1.78} \\
\hline $\begin{array}{l}\text { SW-A-02 } \\
\left(8^{\prime} \times 9 ' \times 6.5^{\prime \prime}\right)\end{array}$ & 240.46 & & & & & \\
\hline $\begin{array}{l}\text { SW-A-03 } \\
\left(8^{\prime} \times 9 ' \times 6.5^{\prime \prime}\right)\end{array}$ & 154.36 & & & & & \\
\hline
\end{tabular}




\subsubsection{Racking Load on Stud Walls}

Manual calculations were performed to determine the racking load capacity and associated lateral displacement at the top of the stud walls when subjected to lateral loading at the top of the wall. Table 6.8 shows the results obtained for the tested stud walls SW-R-1 and SW-R-2 based on BS 5268-6.2, UBC Section 1612.2 and CSA-O86-09 Standards. Also, Tables 6.9 and 6.10 show the ratios between the racking resistance and corresponding lateral displacements, respectively, obtained from code equations and the experimental data. It can be observed that BS 5268-6.2 code equation provides a ratio of 0.5. As such, a modification factor of 2.0 was proposed in Table 6.11 to increase value obtained from BS 5268-6.2 code equation. Table 6.10 shows that the ratios between the code equations and experimental data were 0.78 and 1.18 for UBC Section 1612.2 and CSA-O86-09 Standards, respectively. As such, the modification factors of 1.28 and 0.84 were proposed in Table 6.11 for lateral displacement values obtained from UBC Section 1612.2 and CSA-O86-09 Standards, respectively.

Table 6.8 - Results from different codes and experimental data for monotonic racking load on stud walls

\begin{tabular}{|c|c|c|c|c|c|}
\hline & Experimental & Experimental & BS 5268-6.2 & $\begin{array}{c}\text { UBC Section } \\
1612.2\end{array}$ & $\begin{array}{c}\text { CAN/CSA } \\
\text { O86-09 }\end{array}$ \\
\hline Specimen & $\begin{array}{c}\text { ultimate } \\
\text { racking load, } \\
(\mathbf{k N})\end{array}$ & $\begin{array}{c}\text { lateral } \\
\text { displacement } \\
(\mathbf{m m})\end{array}$ & $\begin{array}{c}\text { Racking } \\
\text { resistance, } \\
\text { L }_{\mathbf{r}(\text { Code) }} \\
\text { (kN) }\end{array}$ & $\begin{array}{c}\text { Lateral } \\
\text { displacement } \\
\text { (mm) }\end{array}$ & $\begin{array}{c}\text { Lateral } \\
\text { displacement } \\
\text { (mm) }\end{array}$ \\
\hline $\begin{array}{c}\text { SW-R-01 } \\
2440 \times 2743 \times 165 \mathrm{~mm} \\
\left(8^{\prime} \times 9 ' \times 6.5^{\prime \prime}\right)\end{array}$ & 13.70 & 47.21 & \multirow{2}{*}{5.80} & \multirow{2}{*}{31.35} & \multirow{2}{*}{47.64} \\
\hline $\begin{array}{c}\text { SW-R-02 } \\
2440 \times 2743 \times 165 \mathrm{~mm} \\
\left(8^{\prime} \times 9 ' \times 6.5^{\prime \prime}\right)\end{array}$ & 9.54 & 33.29 & & & \\
\hline
\end{tabular}


Table 6.9 - Comparison of results from different codes and experimental data for monotonic racking load resistance of stud walls

\begin{tabular}{|c|c|c|c|c|}
\hline \multirow{2}{*}{ Specimen } & \multirow{2}{*}{$\begin{array}{c}\text { Experimental } \\
\qquad \mathbf{L}_{\mathbf{r}}(\mathbf{k N})\end{array}$} & \multirow{2}{*}{$\begin{array}{c}\text { Average } \\
\text { value, } \\
\text { Lr(exp.) } \\
\text { (kN) }\end{array}$} & $\begin{array}{c}\text { Racking } \\
\text { resistance }(k N)\end{array}$ & $\begin{array}{c}\text { Ratio } \\
\left(\mathbf{L}_{\mathbf{r}(\text { Code })} / \mathbf{L}_{\mathbf{r}(\text { Exp. })}\right)\end{array}$ \\
\hline & & & BS 5268-6.2 & BS 5268-6.2 \\
\hline SW-R-01 (8'x9'x 6.5") & 13.70 & \multirow{2}{*}{11.62} & \multirow{2}{*}{5.80} & \multirow{2}{*}{0.50} \\
\hline SW-R-02 (8'x9'x 6.5") & 9.54 & & & \\
\hline
\end{tabular}

Table 6.10 - Comparison of results from different codes and experimental data for lateral displacement due to monotonic racking load on stud walls

\begin{tabular}{|c|c|c|c|c|c|c|}
\hline \multirow{2}{*}{ Specimen } & \multirow{2}{*}{$\begin{array}{c}\text { Disp.(exp.) } \\
\text { (mm) }\end{array}$} & \multirow{2}{*}{$\begin{array}{l}\text { Average } \\
\text { Disp.(exp.) }\end{array}$} & \multicolumn{2}{|c|}{ Lateral Displacement (mm) } & \multicolumn{2}{|c|}{ Ratio (Disp.(Code) $/$ Disp.(Exp.) } \\
\hline & & & $\begin{array}{c}\text { UBC Section } \\
1612.2\end{array}$ & $\begin{array}{c}\text { CAN/CSA } \\
\text { 086-09 }\end{array}$ & $\begin{array}{c}\text { UBC } \\
\text { Section } \\
1612.2\end{array}$ & $\begin{array}{c}\text { CAN/CSA } \\
\text { 086-09 }\end{array}$ \\
\hline $\begin{array}{c}\text { SW-R-01 } \\
\left(8^{\prime} \times 9 ' x 6.5^{\prime \prime}\right)\end{array}$ & 47.21 & \multirow{2}{*}{40.25} & \multirow{2}{*}{31.35} & \multirow{2}{*}{47.64} & \multirow{2}{*}{0.78} & \multirow{2}{*}{1.18} \\
\hline $\begin{array}{c}\text { SW-R-02 } \\
\text { (8'x9'x 6.5") }\end{array}$ & 33.29 & & & & & \\
\hline
\end{tabular}

Table 6.11 - Proposed modification factors for racking load and displacement for monotonic lateral load on stud walls

\begin{tabular}{|c|c|c|c|}
\hline \multirow{2}{*}{$\begin{array}{c}\text { Modification } \\
\text { factor }\end{array}$} & $\begin{array}{c}\text { Racking load } \\
\text { resistance }\end{array}$ & \multicolumn{2}{|c|}{ Lateral displacement } \\
\cline { 2 - 4 } & BS 5268-6.2 & UBC Section & $\begin{array}{c}\text { CAN/CSA } \\
\text { 086-09 }\end{array}$ \\
\hline $\mathbf{k}$ & 2.0 & 1.28 & 0.84 \\
\hline
\end{tabular}




\subsubsection{Cyclic Lateral Load on Stud Walls}

Similar tables to those produced for racking load capacity and associated lateral displacement at the top of the stud walls when subjected to lateral loading at the top of the wall were established for cyclic lateral loading. Table 6.12 shows the results obtained for the tested stud walls SW-C-1 and SW-C-2 based on BS 5268-6.2, UBC Section 1612.2 and CSA-O86-09 Standards. Also, Tables 6.13 and 6.14 show the ratios between the racking resistance and corresponding lateral displacements, respectively, obtained from code equations and the experimental data for cyclic loading. It can be observed that BS 5268-6.2 code equation provides a ratio of 0.436 , showing an underestimation in the wall response. As such, a modification factor of 2.30 was proposed in Table 6.15 to increase value obtained from BS 5268-6.2 code equation to match that obtained experimentally. Table 6.12 shows that the ratios between the code equations and experimental data for lateral displacement of the wall were 0.74 and 1.08 for UBC Section 1612.2 and CSA086-09 Standards, respectively. As such, the modification factors of 1.36 and 0.93 were proposed in Table 6.15 for cyclic lateral displacement values obtained from UBC Section 1612.2 and CSA-O86-09 Standards, respectively.

Table 6.12 - Results from different codes and experimental data for cyclic lateral loading on stud walls

\begin{tabular}{|c|c|c|c|c|c|c|c|}
\hline \multirow[b]{2}{*}{ Specimen } & \multicolumn{2}{|c|}{ Peak Load (kN) } & \multicolumn{2}{|c|}{ Lateral Disp. (mm) } & \multirow{2}{*}{$\begin{array}{c}\text { BS 5268-6.2 } \\
\text { Racking } \\
\text { resistance } \\
(\mathrm{kN})\end{array}$} & \multirow{2}{*}{$\begin{array}{c}\text { UBC Section } \\
1612.2 \\
\text { Lateral } \\
\text { displacement } \\
(\mathrm{mm})\end{array}$} & \multirow{2}{*}{$\begin{array}{c}\text { CAN/CSA } \\
\text { O86-09 } \\
\text { Lateral } \\
\text { displacement } \\
(\mathrm{mm})\end{array}$} \\
\hline & Positive & Negative & Positive & Negative & & & \\
\hline $\begin{array}{c}\text { SW-C-01 } \\
2440 \times 2743 \times 165 \\
\mathrm{~mm} \\
(8 ' \times 9 \text { 9'x 6.5") }\end{array}$ & 9.16 & 12.30 & 49.66 & 45.83 & \multirow{2}{*}{5.82} & 28.84 & 42.69 \\
\hline $\begin{array}{c}\text { SW-C-02 } \\
2440 \times 2743 \times 165 \\
\mathrm{~mm} \\
\left(8^{\prime} \times 99^{\prime} \times 6.5 "\right)\end{array}$ & 11.11 & 14.42 & 39.38 & 38.49 & & 32.83 & 47.90 \\
\hline
\end{tabular}


Table 6.13 - Comparison of results from different codes and experimental data for lateral resistance due to cyclic lateral loading on stud walls

\begin{tabular}{|c|c|c|c|c|}
\hline \multirow[t]{2}{*}{ Specimen } & \multirow[t]{2}{*}{ Experimental $L_{\mathbf{r}}(\mathbf{k N})$} & \multirow{2}{*}{$\begin{array}{c}\text { Average } \\
\text { experimental } \\
\text { value } L_{r(\text { exp.) }}\end{array}$} & $\begin{array}{c}\text { Lateral resistance } \\
(\mathbf{k N})\end{array}$ & $\begin{array}{c}\text { Ratio } \\
\left(\mathbf{L}_{\mathbf{r}(\text { Code })} / \mathbf{L}_{\mathbf{r}(\text { Exp. })}\right)\end{array}$ \\
\hline & & & BS 5268-6.2 & BS 5268-6.2 \\
\hline $\begin{array}{c}\text { SW-C-01 } \\
\left(8^{\prime} \times 9 ' \times 6.5^{\prime \prime}\right)\end{array}$ & 12.30 & \multirow{2}{*}{13.36} & \multirow{2}{*}{5.82} & \multirow{2}{*}{0.436} \\
\hline $\begin{array}{c}\text { SW-C-02 (8'x9'x } \\
\left.6.5^{\prime \prime}\right)\end{array}$ & 14.42 & & & \\
\hline
\end{tabular}

Table 6.14 - Comparison of results from different codes and experimental data for lateral displacement due to cyclic lateral loading on stud walls

\begin{tabular}{|c|c|c|c|c|c|c|}
\hline \multirow{2}{*}{ Specimen } & \multirow{2}{*}{$\begin{array}{l}\text { Disp.(exp.) } \\
(\mathbf{m m})\end{array}$} & \multirow{2}{*}{$\begin{array}{c}\text { Average } \\
\text { value } \\
\text { Disp.(exp.) }\end{array}$} & \multicolumn{2}{|c|}{ Lateral Displacement (mm) } & \multicolumn{2}{|c|}{$\begin{array}{c}\text { Ratio } \\
\text { (Disp.(Code) }^{\text {(Disp.(Exp.) })}\end{array}$} \\
\hline & & & $\begin{array}{c}\text { UBC Section } \\
1612.2\end{array}$ & $\begin{array}{c}\text { CAN/CSA } \\
\text { 086-09 }\end{array}$ & $\begin{array}{c}\text { UBC } \\
\text { Section } \\
1612.2\end{array}$ & $\begin{array}{c}\text { CAN/CSA } \\
\text { 086-09 }\end{array}$ \\
\hline $\begin{array}{c}\text { SW-C-01 } \\
\left(8^{\prime} \times 9 ' x 6.5^{\prime \prime}\right)\end{array}$ & 49.66 & \multirow{2}{*}{44.52} & \multirow{2}{*}{32.83} & \multirow{2}{*}{47.9} & \multirow{2}{*}{0.74} & \multirow{2}{*}{1.08} \\
\hline $\begin{array}{c}\text { SW-C-02 } \\
\left(8^{\prime} \times 9^{\prime} \times 6.5^{\prime \prime}\right)\end{array}$ & 39.38 & & & & & \\
\hline
\end{tabular}

Table 6.15 - Proposed modification factors for racking load and lateral displacement for cyclic lateral loading on stud walls

\begin{tabular}{|c|c|c|c|}
\hline \multirow{2}{*}{$\begin{array}{c}\text { Modification } \\
\text { factor }\end{array}$} & $\begin{array}{c}\text { Racking load } \\
\text { resistance }\end{array}$ & \multicolumn{2}{|c|}{ Lateral displacement } \\
\cline { 2 - 4 } & BS 5268-6.2 & $\begin{array}{c}\text { UBC Section } \\
\mathbf{1 6 1 2 . 2}\end{array}$ & $\begin{array}{c}\text { CAN/CSA } \\
\mathbf{0 8 6 - 0 9}\end{array}$ \\
\hline $\mathbf{k}$ & 2.30 & 1.36 & 0.93 \\
\hline
\end{tabular}




\subsection{Correlation of Results from Code Equations and Experimental Data of SIP Panel System}

\subsubsection{Flexural Loading on SIP Panels}

Manual calculations were performed to determine the moment and shear capacities of the SIP panels tested in this research as well as SIP panels tested elsewhere (Syed Ahmed, 2011; Mohamed, 2009; Butt, 2008) when subjected to flexural loading. Table 6.16 shows the results obtained for bending resistance and shear resisting force of these tested SIP panels based on CSA-O86-09, DIAB Handbook and APA Standard. Also, Table 6.16 shows the applied dead and live load and the corresponding panel deflection in the elastic range. The corresponding deflection values obtained using equations available in CSA-O86-09; DIAB Handbook and ANSI/APA standards were also presented in this table. Tables 6.17 and 6.18 show the ratios between the resisting moments and shear forces, respectively, obtained from code equations and the experimental data for SIP panels. It can be observed that code equations provide moment and shear resistance greater than those obtained experimentally. As such modification factors were proposed to lower the values obtained from code equations. Table 6.20 shows these modification factors as $0.46,0.27$ and 0.27 for moment resistances obtained from CSA-O86-09, DIAB and APA equations, respectively. Also, Table 6.20 shows these modification factors as $0.26,0.61$ and 0.53 for shear resistances obtained from CSA-O86-09, DIAB and APA equations.

Similar observations for deflection calculation as depicted in Table 6.19. As such, Table 6.20 proposes the use of modification factors of $0.55,0.36$ and 0.67 to the available deflection equations in CSA-O86-09, DIAB Handbook and APA Standard, respectively. To provide confidence on the proposed modification factors, Figures 6.1, 6.2 and 6.3 depict correlations between the resisting moments obtained experimentally and those obtained using CSA-O86-09, DIAB and APA Standard, respectively. The figures show significant change of the values obtained from code equation when compared to experimental data. Also, it shows good correlation between the obtained values from code equations after applying the proposed modification factors. Similar correlations are presented for shear resistance of SIP panels in Figures 6.4 through 6.6. Moreover, similar correlations are presented for deflection calculations of SIP panels under flexural loading in Figures 6.7 through 6.9. 
Table 6.16 - Comparison of the results from different codes and experimental data for flexural loading on SIP panels

\begin{tabular}{|c|c|c|c|c|c|c|c|c|c|c|c|c|c|}
\hline \multirow[b]{2}{*}{ Specimen } & \multirow{2}{*}{$\begin{array}{c}\text { Exp. } \\
\text { ultimate } \\
\text { load } \\
(\mathbf{k N})^{*}\end{array}$} & \multirow{2}{*}{$\begin{array}{c}\text { Exp. } \\
\text { resisting } \\
\text { bending } \\
\text { moment } \\
(\mathbf{k N} . m)^{(1)}\end{array}$} & \multirow{2}{*}{$\begin{array}{c}\text { Exp. } \\
\text { resist. } \\
\text { shear } \\
\text { force } \\
(\mathbf{k N})^{(2)}\end{array}$} & \multirow[b]{2}{*}{$\begin{array}{l}\text { Load and } \\
\text { deflect. at } \\
(\mathrm{D}+\mathrm{L})^{(3)}\end{array}$} & \multicolumn{3}{|c|}{ CSA-086-09 } & \multicolumn{3}{|c|}{ DIAB Sandwich Handbook } & \multicolumn{3}{|c|}{ APA Standard } \\
\hline & & & & & $\begin{array}{l}\text { Mom. } \\
\text { resist. } \\
\text { (kN.m) }\end{array}$ & $\begin{array}{r}\text { Shear } \\
\text { resist. } \\
(\mathrm{kN})\end{array}$ & $\begin{array}{c}\text { Deflec. } \\
(\mathrm{mm})\end{array}$ & $\begin{array}{l}\text { Mom. } \\
\text { resist. } \\
\text { (kN.m) }\end{array}$ & $\begin{array}{l}\text { Shear } \\
\text { resist. } \\
(\mathrm{kN})\end{array}$ & $\begin{array}{c}\text { Deflec. } \\
(\mathrm{mm})\end{array}$ & $\begin{array}{l}\text { Mom. } \\
\text { resist. } \\
(\mathrm{kN.m})\end{array}$ & $\begin{array}{l}\text { Shear } \\
\text { resist. } \\
(\mathbf{k N})\end{array}$ & $\begin{array}{c}\text { Deflect. } \\
\text { (mm) }\end{array}$ \\
\hline $\begin{array}{c}\text { SIP-F-01 } \\
1220 \times 2743 \times 165 \mathrm{~mm} \\
\left(4^{\prime} \times 9 ' \times 6.5^{\prime \prime}\right)\end{array}$ & 23.75 & 9.69 & 12.88 & $\begin{array}{c}11.61 \\
7.20\end{array}$ & \multirow{3}{*}{8.30} & \multirow{3}{*}{10.27} & \multirow{3}{*}{9.64} & \multirow{3}{*}{12.94} & \multirow{3}{*}{10.87} & \multirow{3}{*}{18.26} & \multirow{3}{*}{8.32} & \multirow{3}{*}{9.32} & \multirow{3}{*}{7.53} \\
\hline $\begin{array}{c}\text { SIP-F-02 } \\
\text { 1220x2440x165 mm } \\
\left(4^{\prime} \times 9 ' \times 6.5^{\prime \prime}\right)\end{array}$ & 24.71 & 8.65 & 13.36 & $\begin{array}{c}11.47 \\
7.20\end{array}$ & & & & & & & & & \\
\hline $\begin{array}{c}\text { SIP-F-03 } \\
\text { 1220x2440x165 mm } \\
\left(4^{\prime} \times 9 \text { 'x6.5") }\right.\end{array}$ & 25.00 & 8.74 & 13.50 & $\begin{array}{c}11.10 \\
7.20\end{array}$ & & & & & & & & & \\
\hline
\end{tabular}

* Did not include $2 \mathrm{kN}$ weight of the loading system.

(1) Included $2 \mathrm{kN}$ weight of loading system and then multiplied by $\mathrm{L} / 8$ to obtain bending moment $(\mathrm{L}=2.5908 \mathrm{~m})$

(2) Included $2 \mathrm{kN}$ weight of loading system

${ }^{3}$ The first number of the total load applied to the panel in $\mathrm{kN}$ to get the deflection in mm which is the second number. 
Table 6.16 - Comparison of the results from different codes and experimental data for flexural loading on SIP panels (continued)

\begin{tabular}{|c|c|c|c|c|c|c|c|c|c|c|c|c|c|}
\hline \multirow[b]{2}{*}{ Specimen } & \multirow{2}{*}{$\begin{array}{c}\text { Exp. } \\
\text { Ultim. } \\
\text { load } \\
(\mathbf{k N})^{*}\end{array}$} & \multirow{2}{*}{$\begin{array}{c}\text { Exp. } \\
\text { resisting } \\
\text { bending } \\
\text { moment } \\
(\mathrm{kN} . \mathrm{m})^{(1)}\end{array}$} & \multirow{2}{*}{$\begin{array}{l}\text { Exp. } \\
\text { resist. } \\
\text { shear } \\
\text { force } \\
(\mathrm{kN})^{(2)}\end{array}$} & \multirow{2}{*}{$\begin{array}{c}\text { Load } \\
\text { and } \\
\text { deflect. } \\
\text { at }(\mathrm{D}+\mathrm{L}) \\
(3)\end{array}$} & \multicolumn{3}{|c|}{ CSA-086-09 } & \multicolumn{3}{|c|}{ DIAB Sandwich Handbook } & \multicolumn{3}{|c|}{ APA Standard } \\
\hline & & & & & $\begin{array}{l}\text { Mom. } \\
\text { resist. } \\
\text { (kN.m) }\end{array}$ & $\begin{array}{c}\text { Shear } \\
\text { resist. } \\
\text { (kN) }\end{array}$ & $\begin{array}{l}\text { Def. } \\
(\mathrm{mm})\end{array}$ & $\begin{array}{l}\text { Mom. } \\
\text { resist. } \\
\text { (kN.m) }\end{array}$ & $\begin{array}{c}\text { Shear } \\
\text { resist. } \\
(\mathbf{k N})\end{array}$ & $\begin{array}{l}\text { Def. } \\
(\mathrm{mm})\end{array}$ & $\begin{array}{l}\text { Mom. } \\
\text { resist. } \\
\text { (kN.m) }\end{array}$ & $\begin{array}{c}\text { Shear } \\
\text { resist. } \\
(\mathbf{k N})\end{array}$ & $\begin{array}{l}\text { Def. } \\
\text { (mm) }\end{array}$ \\
\hline $\begin{array}{c}\mathrm{A}-13 \\
610 \times 2440 \times 165 \mathrm{~mm} \\
(2 ' \times 8 ' \times 6.5 ")\end{array}$ & 21.26 & 6.64 & 11.63 & $\begin{array}{c}3.345 \\
2.89\end{array}$ & \multirow{3}{*}{4.73} & \multirow{3}{*}{14.84} & \multirow{3}{*}{1.54} & \multirow{3}{*}{8.00} & \multirow{3}{*}{6.25} & \multirow{3}{*}{4.17} & \multirow{3}{*}{8.11} & \multirow{3}{*}{7.21} & \multirow{3}{*}{2.20} \\
\hline $\begin{array}{c}\text { A-14 } \\
610 \times 2440 \times 165 \mathrm{~mm} \\
\left(2 ' \times 8 ' \times 6.5^{\prime \prime}\right)\end{array}$ & 21.22 & 6.64 & 11.61 & $\begin{array}{c}2.345 \\
2.90\end{array}$ & & & & & & & & & \\
\hline $\begin{array}{c}\mathrm{A}-15 \\
610 \times 2440 \times 165 \mathrm{~mm} \\
\left(2 ' \times 8 ' \times 6.5^{\prime \prime}\right)\end{array}$ & 11.44 & 3.84 & 6.72 & $\begin{array}{c}2.345 \\
2.98\end{array}$ & & & & & & & & & \\
\hline
\end{tabular}

* Zarghooni (2009), did not include $2 \mathrm{kN}$ weight of the loading system.

(1) Included $2 \mathrm{kN}$ weight of loading system and then multiplied by $\mathrm{L} / 8$ to obtain bending moment $(\mathrm{L}=2.286 \mathrm{~m}$ for the 8 ' long panel and $\mathrm{L}=4.724 \mathrm{~m}$ for the 16 ' long panel)

(2) Included $2 \mathrm{kN}$ weight of loading system

${ }^{3}$ The first number of the total load applied to the panel in $\mathrm{kN}$ to get the deflection in mm which is the second number. 
Table 6.16 - Comparison of the results from different codes and experimental data for flexural loading on SIP panels (continued)

\begin{tabular}{|c|c|c|c|c|c|c|c|c|c|c|c|c|c|}
\hline \multirow[b]{2}{*}{ Specimen } & \multirow{2}{*}{$\begin{array}{c}\text { Exp. } \\
\text { Ultim. } \\
\text { load } \\
(\mathbf{k N})^{*}\end{array}$} & \multirow{2}{*}{$\begin{array}{c}\text { Exp. } \\
\text { resisting } \\
\text { bending } \\
\text { moment } \\
(\mathrm{kN} . \mathrm{m})^{(1)}\end{array}$} & \multirow{2}{*}{$\begin{array}{l}\text { Exp. } \\
\text { resist. } \\
\text { shear } \\
\text { force } \\
(k N)^{(2)}\end{array}$} & \multirow[b]{2}{*}{$\begin{array}{l}\text { Load and } \\
\text { deflect. at } \\
(D+L)^{(3)}\end{array}$} & \multicolumn{3}{|c|}{ CSA-086-09 } & \multicolumn{3}{|c|}{ DIAB Sandwich Handbook } & \multicolumn{3}{|c|}{ APA Standard } \\
\hline & & & & & $\begin{array}{l}\text { Mom. } \\
\text { resist. } \\
\text { (kN.m) }\end{array}$ & $\begin{array}{r}\text { Shear } \\
\text { resist. } \\
(\mathbf{k N})\end{array}$ & $\begin{array}{l}\text { Def. } \\
(\mathrm{mm})\end{array}$ & $\begin{array}{l}\text { Mom. } \\
\text { resist. } \\
\text { (kN.m) }\end{array}$ & $\begin{array}{l}\text { Shear } \\
\text { resist. } \\
(\mathbf{k N})\end{array}$ & $\begin{array}{l}\text { Def. } \\
(\mathrm{mm})\end{array}$ & $\begin{array}{l}\text { Mom. } \\
\text { resist. } \\
\text { (kN.m) }\end{array}$ & $\begin{array}{l}\text { Shear } \\
\text { resist. } \\
(\mathbf{k N})\end{array}$ & $\begin{array}{l}\text { Def. } \\
(\mathbf{m m})\end{array}$ \\
\hline $\begin{array}{c}\text { A-16 } \\
610 \times 4878 \times 165 \mathrm{~mm} \\
\left(2^{\prime} \times 16 ' \times 10.25^{\prime \prime}\right)\end{array}$ & 22.77 & 14.63 & 12.39 & $\begin{array}{c}6.91 \\
17.20\end{array}$ & \multirow{3}{*}{10.40} & \multirow{3}{*}{15.73} & \multirow{3}{*}{27.89} & \multirow{3}{*}{8.00} & \multirow{3}{*}{6.25} & \multirow{3}{*}{48.75} & \multirow{3}{*}{17.94} & \multirow{3}{*}{15.94} & \multirow{3}{*}{17.60} \\
\hline $\begin{array}{c}\text { A-17 } \\
610 \times 4878 \times 165 \mathrm{~mm} \\
\left(2^{\prime} \times 16^{\prime} \times 10.25^{\prime \prime}\right)\end{array}$ & 21.55 & 13.91 & 11.78 & $\begin{array}{c}6.91 \\
17.10\end{array}$ & & & & & & & & & \\
\hline $\begin{array}{c}\text { A-18 } \\
610 \times 4878 \times 165 \mathrm{~mm} \\
\left(2^{\prime} \times 16 ' \times 10.25^{\prime \prime}\right)\end{array}$ & 22.33 & 14.37 & 12.17 & $\begin{array}{c}6.91 \\
16.80\end{array}$ & & & & & & & & & \\
\hline
\end{tabular}

* Zarghooni (2009), did not include $2 \mathrm{kN}$ weight of the loading system.

(1) Included $2 \mathrm{kN}$ weight of loading system and then multiplied by $\mathrm{L} / 8$ to obtain bending moment $(\mathrm{L}=2.286 \mathrm{~m}$ for the 8 ' long panel and $\mathrm{L}=4.724 \mathrm{~m}$ for the 16 ' long panel)

(2) Included $2 \mathrm{kN}$ weight of loading system

${ }^{3}$ The first number of the total load applied to the panel in $\mathrm{kN}$ to get the deflection in mm which is the second number. 
Table 6.16 - Comparison of the results from different codes and experimental data for flexural loading on SIP panels (continued)

\begin{tabular}{|c|c|c|c|c|c|c|c|c|c|c|c|c|c|}
\hline \multirow[b]{2}{*}{ Specimen } & \multirow{2}{*}{$\begin{array}{l}\text { Exp. } \\
\text { Ultim. } \\
\text { load } \\
\text { (kN)* }\end{array}$} & \multirow{2}{*}{$\begin{array}{c}\text { Exp. } \\
\text { resist. } \\
\text { bending } \\
\text { moment } \\
(\mathbf{k N . m )})^{(1)}\end{array}$} & \multirow{2}{*}{$\begin{array}{l}\text { Exp. } \\
\text { resist. } \\
\text { shear } \\
\text { force } \\
(k N)^{(2)}\end{array}$} & \multirow{2}{*}{$\begin{array}{c}\text { Load } \\
\text { and } \\
\text { deflec. } \\
\text { at } \\
\text { (D+L) } \\
\text { (3) }\end{array}$} & \multicolumn{3}{|c|}{ CSA-086-09 } & \multicolumn{3}{|c|}{ DIAB Sandwich Handbook } & \multicolumn{3}{|c|}{ APA Standard } \\
\hline & & & & & $\begin{array}{l}\text { Mom. } \\
\text { resist. } \\
\text { (kN.m) }\end{array}$ & $\begin{array}{r}\text { Shear } \\
\text { resist. } \\
(\mathbf{k N})\end{array}$ & $\begin{array}{c}\text { Deflec. } \\
\text { (mm) }\end{array}$ & $\begin{array}{l}\text { Mom. } \\
\text { resist. } \\
\text { (kN.m) }\end{array}$ & $\begin{array}{l}\text { Shear } \\
\text { resist. } \\
(\mathbf{k N})\end{array}$ & $\begin{array}{c}\text { Deflec. } \\
\text { (mm) }\end{array}$ & $\begin{array}{l}\text { Mom. } \\
\text { resist. } \\
\text { (kN.m) }\end{array}$ & $\begin{array}{l}\text { Shear } \\
\text { resist. } \\
\text { (kN) }\end{array}$ & $\begin{array}{c}\text { Deflec. } \\
\text { (mm) }\end{array}$ \\
\hline $\begin{array}{c}\text { WS-19 } \\
\text { 1220x2743x165 mm } \\
\left(4^{\prime} \times 9 ' \times 6.5^{\prime \prime}\right)\end{array}$ & 27.22 & 9.46 & 14.61 & $\begin{array}{c}11.07 \\
7.20\end{array}$ & \multirow{5}{*}{9.46} & \multirow{5}{*}{11.54} & \multirow{5}{*}{9.12} & \multirow{5}{*}{15.82} & \multirow{5}{*}{12.35} & \multirow{5}{*}{16.32} & \multirow{5}{*}{12.43} & \multirow{5}{*}{11.05} & \multirow{5}{*}{8.74} \\
\hline $\begin{array}{c}\text { WS-20 } \\
\text { 1220x2743x165 mm } \\
\left(4^{\prime} \times 9 ' \times 6.5 "\right)\end{array}$ & 27.77 & 9.64 & 14.89 & $\begin{array}{c}10.63 \\
7.20\end{array}$ & & & & & & & & & \\
\hline $\begin{array}{c}\text { WS-21 } \\
\text { 1220x2743x165 mm } \\
\left(4{ }^{\prime} \times 9 ' \times 6.5 "\right)\end{array}$ & 24.99 & 8.74 & 13.50 & $\begin{array}{c}11.00 \\
7.20\end{array}$ & & & & & & & & & \\
\hline $\begin{array}{c}\text { WS-22 } \\
\text { 1220x2743x165 mm } \\
\left(4{ }^{\prime} \times 9 ' \times 6.5 "\right)\end{array}$ & 28.77 & 9.96 & 15.39 & $\begin{array}{c}10.70 \\
7.20\end{array}$ & & & & & & & & & \\
\hline $\begin{array}{c}\text { WS-23 } \\
1220 \times 2743 \times 165 \mathrm{~mm} \\
\left(4^{\prime} \times 9 ' \times 6.5^{\prime \prime}\right)\end{array}$ & 26.77 & 9.31 & 14.39 & $\begin{array}{c}10.75 \\
7.20\end{array}$ & & & & & & & & & \\
\hline
\end{tabular}

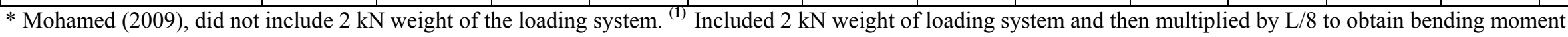
$(\mathrm{L}=2.5908 \mathrm{~m})^{(2)}$ Included $2 \mathrm{kN}$ weight of loading system. ${ }^{(3}$ The first number of the total load applied to the panel in $\mathrm{kN}$ to get the deflection in mm which is the second number. 
Table 6.16 - Comparison of the results from different codes and experimental data for flexural loading on SIP panels (continued)

\begin{tabular}{|c|c|c|c|c|c|c|c|c|c|c|c|c|c|}
\hline \multirow[b]{2}{*}{ Specimen } & \multirow{2}{*}{$\begin{array}{c}\text { Exp. } \\
\text { ultimate } \\
\text { load } \\
(\mathrm{kN})^{*}\end{array}$} & \multirow{2}{*}{$\begin{array}{c}\text { Exp. } \\
\text { resisting } \\
\text { bending } \\
\text { moment } \\
(\mathrm{kN} . \mathrm{m})^{(1)}\end{array}$} & \multirow{2}{*}{$\begin{array}{l}\text { Exp. } \\
\text { resist. } \\
\text { shear } \\
\text { force } \\
(k N)^{(2)}\end{array}$} & \multirow{2}{*}{$\begin{array}{c}\text { Load } \\
\text { and } \\
\text { deflect. } \\
\text { at (L) } \\
\text { (3) }\end{array}$} & \multicolumn{3}{|c|}{ CSA-086-09 } & \multicolumn{3}{|c|}{ DIAB Sandwich Handbook } & \multicolumn{3}{|c|}{ APA Standard } \\
\hline & & & & & $\begin{array}{l}\text { Mom. } \\
\text { resist. } \\
\text { (kN.m) }\end{array}$ & $\begin{array}{c}\text { Shear } \\
\text { resist. } \\
(\mathbf{k N})\end{array}$ & $\begin{array}{l}\text { Deflect } \\
\text {. (mm) }\end{array}$ & $\begin{array}{l}\text { Mom. } \\
\text { resist. } \\
\text { (kN.m) }\end{array}$ & $\begin{array}{c}\text { Shear } \\
\text { resist. } \\
(\mathbf{k N})\end{array}$ & $\begin{array}{l}\text { Deflect } \\
\text {. (mm) }\end{array}$ & $\begin{array}{l}\text { Mom. } \\
\text { resist. } \\
\text { (kN.m) }\end{array}$ & $\begin{array}{c}\text { Shear } \\
\text { resist. } \\
(\mathbf{k N})\end{array}$ & $\begin{array}{l}\text { Deflect } \\
\text {. (mm) }\end{array}$ \\
\hline $\begin{array}{c}\text { B-4 } \\
1220 \times 3048 \times 165 \mathrm{~mm} \\
\left(4{ }^{\prime} \times 10 ' \times 6.5 ”\right)\end{array}$ & 37.98 & 14.47 & 19.99 & $\begin{array}{c}6.708 \\
4.90\end{array}$ & & & & & & & & & \\
\hline $\begin{array}{c}\text { B-5 } \\
1220 \times 3048 \times 165 \mathrm{~mm} \\
\left(4^{\prime} \times 10^{\prime} \times 6.5^{\prime \prime}\right)\end{array}$ & 39.48 & 15.01 & 20.74 & $\begin{array}{c}6.708 \\
4.70\end{array}$ & 15.13 & 18.46 & 1.61 & 25.31 & 19.76 & 6.77 & 19.76 & 17.57 & 4.88 \\
\hline $\begin{array}{c}\text { B-43 } \\
1220 \times 3048 \times 165 \mathrm{~mm} \\
\left(4^{\prime} \times 10^{\prime} \times 6.5^{\prime \prime}\right)\end{array}$ & 40.92 & 15.53 & 21.46 & $\begin{array}{c}6.708 \\
4.85\end{array}$ & & & & & & & & & \\
\hline
\end{tabular}

* Butt (2008), did not include $2 \mathrm{kN}$ weight of the loading system.

(1) Included $2 \mathrm{kN}$ weight of loading system and then multiplied by $\mathrm{L} / 8$ to obtain bending moment $(\mathrm{L}=2.8956 \mathrm{~m})$

(2) Included $2 \mathrm{kN}$ weight of loading system

( 3 The first number of the live load applied to the panel in $\mathrm{kN}$ to get the deflection in mm which is the second number. 
Table 6.16 - Comparison of the results from different codes and experimental data for flexural loading on SIP panels (continued)

\begin{tabular}{|c|c|c|c|c|c|c|c|c|c|c|c|c|c|}
\hline \multirow[b]{2}{*}{ Specimen } & \multirow{2}{*}{$\begin{array}{c}\text { Exp. } \\
\text { ultim. } \\
\text { load } \\
(k N)^{*}\end{array}$} & \multirow{2}{*}{$\begin{array}{c}\text { Exp. } \\
\text { resist. } \\
\text { bending } \\
\text { moment } \\
(\mathrm{kN.m})^{(1)}\end{array}$} & \multirow{2}{*}{$\begin{array}{c}\text { Exp. } \\
\text { resist. } \\
\text { shear } \\
\text { force } \\
(\mathrm{kN})^{(2)}\end{array}$} & \multirow{2}{*}{$\begin{array}{c}\text { Load } \\
\text { and } \\
\text { deflec. } \\
\text { at (L) } \\
\text { (3) }\end{array}$} & \multicolumn{3}{|c|}{ CSA-086-09 } & \multicolumn{3}{|c|}{ DIAB Sandwich Handbook } & \multicolumn{3}{|c|}{ APA Standard } \\
\hline & & & & & $\begin{array}{l}\text { Mom. } \\
\text { resist. } \\
\text { (kN.m) }\end{array}$ & $\begin{array}{c}\text { Shear } \\
\text { resist. } \\
(\mathrm{kN})\end{array}$ & $\begin{array}{c}\text { Deflec. } \\
\text { (mm) }\end{array}$ & $\begin{array}{l}\text { Mom. } \\
\text { resist. } \\
\text { (kN.m) }\end{array}$ & $\begin{array}{c}\text { Shear } \\
\text { resist. } \\
(\mathrm{kN})\end{array}$ & $\begin{array}{c}\text { Deflec. } \\
(\mathrm{mm})\end{array}$ & $\begin{array}{l}\text { Mom. } \\
\text { resist. } \\
\text { (kN.m) }\end{array}$ & $\begin{array}{c}\text { Shear } \\
\text { resist. } \\
(\mathrm{kN})\end{array}$ & $\begin{array}{l}\text { Deflec. } \\
\text { (mm) }\end{array}$ \\
\hline $\begin{array}{c}\text { D-10 } \\
1220 \times 3048 \times 165 \mathrm{~mm} \\
(4 \text { 'x12'x 6.5") }\end{array}$ & 34.88 & 16.16 & 18.44 & $\begin{array}{l}8.12 \\
9.52\end{array}$ & \multirow{3}{*}{9.74} & \multirow{3}{*}{11.88} & \multirow{3}{*}{13.40} & \multirow{3}{*}{19.38} & \multirow{3}{*}{12.72} & \multirow{3}{*}{17.07} & \multirow{3}{*}{20.39} & \multirow{3}{*}{18.12} & \multirow{3}{*}{8.38} \\
\hline $\begin{array}{c}\text { D-11 } \\
1220 \times 3048 \times 165 \mathrm{~mm} \\
\left(4^{\prime} \times 12 ' \times 6.5^{\prime \prime}\right)\end{array}$ & 30.53 & 14.25 & 16.27 & $\begin{array}{c}8.12 \\
10.76\end{array}$ & & & & & & & & & \\
\hline $\begin{array}{c}\text { D-44 } \\
1220 \times 3048 \times 165 \mathrm{~mm} \\
\left(4^{\prime} \times 12^{\prime} \times 6.5^{\prime \prime}\right)\end{array}$ & 34.27 & 15.89 & 18.14 & $\begin{array}{l}8.12 \\
8.73\end{array}$ & & & & & & & & & \\
\hline
\end{tabular}

* Butt (2008), did not include $2 \mathrm{kN}$ weight of the loading system.

(1) Included $2 \mathrm{kN}$ weight of loading system and then multiplied by $\mathrm{L} / 8$ to obtain bending moment $(\mathrm{L}=3.5052 \mathrm{~m}$ for the 12 ' long panel and $\mathrm{L}=2.8956 \mathrm{~m}$ for the 10 ' long panel)

(2) Included $2 \mathrm{kN}$ weight of loading system

(3 The first number of the live load applied to the panel in $\mathrm{kN}$ to get the deflection in mm which is the second number. 
Table 6.16 - Comparison of the results from different codes and experimental data for flexural loading on SIP panels (continued)

\begin{tabular}{|c|c|c|c|c|c|c|c|c|c|c|c|c|c|}
\hline \multirow[b]{2}{*}{ Specimen } & \multirow{2}{*}{$\begin{array}{l}\text { Exp. } \\
\text { ultim. } \\
\text { load } \\
(\mathbf{k N})^{*}\end{array}$} & \multirow{2}{*}{$\begin{array}{c}\text { Exp. } \\
\text { resist. } \\
\text { bending } \\
\text { moment } \\
\text { (kN.m) } \\
\text { (1) }\end{array}$} & \multirow{2}{*}{$\begin{array}{c}\text { Exp. } \\
\text { resist. } \\
\text { shear } \\
\text { force } \\
(\mathbf{k N})^{(2)}\end{array}$} & \multirow{2}{*}{$\begin{array}{c}\text { Load } \\
\text { and } \\
\text { deflec. } \\
\text { at (L) } \\
\text { (3) }\end{array}$} & \multicolumn{3}{|c|}{ CSA-086-09 } & \multicolumn{3}{|c|}{ DIAB Sandwich Handbook } & \multicolumn{3}{|c|}{ APA Standard } \\
\hline & & & & & $\begin{array}{l}\text { Mom. } \\
\text { resist. } \\
\text { (kN.m) }\end{array}$ & $\begin{array}{l}\text { Shear } \\
\text { resist. } \\
\text { (kN) }\end{array}$ & $\begin{array}{c}\text { Deflec. } \\
(\mathrm{mm})\end{array}$ & $\begin{array}{l}\text { Mom. } \\
\text { resist. } \\
\text { (kN.m) }\end{array}$ & $\begin{array}{l}\text { Shear } \\
\text { resist. } \\
\text { (kN) }\end{array}$ & $\begin{array}{c}\text { Deflec. } \\
(\mathrm{mm})\end{array}$ & $\begin{array}{l}\text { Mom. } \\
\text { resist. } \\
\text { (kN.m) }\end{array}$ & $\begin{array}{c}\text { Shear } \\
\text { resist. } \\
(\mathbf{k N})\end{array}$ & $\begin{array}{c}\text { Deflec. } \\
(\mathrm{mm})\end{array}$ \\
\hline $\begin{array}{c}\mathrm{E}-13 \\
1220 \times 3048 \times 165 \mathrm{~mm} \\
\left(4{ }^{\prime} \times 10^{\prime} \times 8.25 "\right)\end{array}$ & 41.81 & 15.86 & 21.41 & $\begin{array}{l}6.71 \\
4.10\end{array}$ & \multirow{3}{*}{15.13} & \multirow{3}{*}{15.02} & \multirow{3}{*}{3.06} & \multirow{3}{*}{19.44} & \multirow{3}{*}{15.68} & \multirow{3}{*}{7.78} & \multirow{3}{*}{21.18} & \multirow{3}{*}{14.61} & \multirow{3}{*}{4.41} \\
\hline $\begin{array}{c}\text { E-14 } \\
1220 \times 3048 \times 165 \mathrm{~mm} \\
(4 ' \times 10 ' x 8.25 ")\end{array}$ & 43.96 & 16.64 & 22.98 & $\begin{array}{l}6.71 \\
3.75\end{array}$ & & & & & & & & & \\
\hline $\begin{array}{c}\text { E-15-1 } \\
1220 \times 3048 \times 165 \mathrm{~mm} \\
\left(4{ }^{\prime} \times 10^{\prime} \times 8.25 "\right)\end{array}$ & 42.57 & 16.13 & 22.29 & $\begin{array}{l}6.71 \\
3.94\end{array}$ & & & & & & & & & \\
\hline
\end{tabular}

* Butt (2008), did not include $2 \mathrm{kN}$ weight of the loading system.

(1) Included $2 \mathrm{kN}$ weight of loading system and then multiplied by $\mathrm{L} / 8$ to obtain bending moment ( $\mathrm{L}=3.5052 \mathrm{~m}$ for the 12 ' long panel and $\mathrm{L}=2.8956 \mathrm{~m}$ for the 10 ' long panel)

(2) Included $2 \mathrm{kN}$ weight of loading system

(3 The first number of the live load applied to the panel in $\mathrm{kN}$ to get the deflection in mm which is the second number. 
Table 6.16 - Comparison of the results from different codes and experimental data for flexural loading on SIP panels (continued)

\begin{tabular}{|c|c|c|c|c|c|c|c|c|c|c|c|c|c|}
\hline \multirow[b]{2}{*}{ Specimen } & \multirow{2}{*}{$\begin{array}{c}\text { Exp. } \\
\text { ultimate } \\
\text { load } \\
(\mathrm{kN})^{*}\end{array}$} & \multirow{2}{*}{$\begin{array}{c}\text { Exp. } \\
\text { resisting } \\
\text { bending } \\
\text { moment } \\
(\mathrm{kN} . \mathrm{m})^{(1)}\end{array}$} & \multirow{2}{*}{$\begin{array}{c}\text { Exp. } \\
\text { resist. } \\
\text { shear } \\
\text { force } \\
(k N)^{(2)}\end{array}$} & \multirow{2}{*}{$\begin{array}{c}\text { Load } \\
\text { and } \\
\text { deflect. } \\
\text { at (L) } \\
\text { (3) }\end{array}$} & \multicolumn{3}{|c|}{ CSA-086-09 } & \multicolumn{3}{|c|}{ DIAB Sandwich Handbook } & \multicolumn{3}{|c|}{ APA Standard } \\
\hline & & & & & $\begin{array}{l}\text { Mom. } \\
\text { resist. } \\
\text { (kN.m) }\end{array}$ & $\begin{array}{c}\text { Shear } \\
\text { resist. } \\
(\mathbf{k N})\end{array}$ & $\begin{array}{c}\text { Deflect. } \\
\text { (mm) }\end{array}$ & $\begin{array}{l}\text { Mom. } \\
\text { resist. } \\
\text { (kN.m) }\end{array}$ & $\begin{array}{c}\text { Shear } \\
\text { resist. } \\
(\mathrm{kN})\end{array}$ & $\begin{array}{c}\text { Deflect. } \\
\text { (mm) }\end{array}$ & $\begin{array}{l}\text { Mom. } \\
\text { resist. } \\
\text { (kN.m) }\end{array}$ & $\begin{array}{c}\text { Shear } \\
\text { resist. } \\
\text { (kN) }\end{array}$ & $\begin{array}{c}\text { Deflect. } \\
\text { (mm) }\end{array}$ \\
\hline $\begin{array}{c}\text { H-22 } \\
\text { 1220x3048x165 mm } \\
\text { (4'x14'x 8.25") }\end{array}$ & 40.37 & 21.79 & 21.19 & $\begin{array}{c}9.53 \\
12.03\end{array}$ & & & & & & & & & \\
\hline $\begin{array}{c}\text { H-23-1 } \\
1220 \times 3048 \times 165 \mathrm{~mm} \\
\left(4^{\prime} \times 14^{\prime} \times 8.25^{\prime \prime}\right)\end{array}$ & 37.00 & 20.06 & 19.50 & $\begin{array}{c}9.53 \\
11.11\end{array}$ & 20.72 & 20.57 & 12.44 & 19.44 & 15.68 & 21.56 & 29.23 & 20.16 & 13.26 \\
\hline $\begin{array}{c}\text { H-24 } \\
1220 \times 3048 \times 165 \mathrm{~mm} \\
\left(4^{\prime} \times 14^{\prime} \times 8.25^{\prime \prime}\right)\end{array}$ & 42.59 & 22.93 & 22.20 & $\begin{array}{r}9.53 \\
11.20\end{array}$ & & & & & & & & & \\
\hline
\end{tabular}

* Butt (2008), did not include $2 \mathrm{kN}$ weight of the loading system.

(1) Included $2 \mathrm{kN}$ weight of loading system and then multiplied by L/8 to obtain bending moment $(\mathrm{L}=4.1148 \mathrm{~m})$

(2) Included $2 \mathrm{kN}$ weight of loading system

( 3 The first number of the live load applied to the panel in $\mathrm{kN}$ to get the deflection in mm which is the second number. 
Table 6.16 - Comparison of the results from different codes and experimental data for flexural loading on SIP panels (continued)

\begin{tabular}{|c|c|c|c|c|c|c|c|c|c|c|c|c|c|}
\hline \multirow[b]{2}{*}{ Specimen } & \multirow{2}{*}{$\begin{array}{c}\text { Exp. } \\
\text { ultimate } \\
\text { load } \\
(\mathbf{k N})^{*}\end{array}$} & \multirow{2}{*}{$\begin{array}{c}\text { Exp. } \\
\text { resisting } \\
\text { bending } \\
\text { moment } \\
(\mathrm{kN} . \mathrm{m})^{(1)}\end{array}$} & \multirow{2}{*}{$\begin{array}{l}\text { Exp. } \\
\text { resist. } \\
\text { shear } \\
\text { force } \\
(\mathbf{k N})^{(2)}\end{array}$} & \multirow{2}{*}{$\begin{array}{c}\text { Load } \\
\text { and } \\
\text { deflect. } \\
\text { at (L) }\end{array}$} & \multicolumn{3}{|c|}{ CSA-086-09 } & \multicolumn{3}{|c|}{ DIAB Sandwich Handbook } & \multicolumn{3}{|c|}{ APA Standard } \\
\hline & & & & & $\begin{array}{l}\text { Mom. } \\
\text { resist. } \\
\text { (kN.m) }\end{array}$ & $\begin{array}{c}\text { Shear } \\
\text { resist. } \\
\text { (kN) }\end{array}$ & $\begin{array}{c}\text { Deflect. } \\
\text { (mm) }\end{array}$ & $\begin{array}{l}\text { Mom. } \\
\text { resist. } \\
\text { (kN.m) }\end{array}$ & $\begin{array}{c}\text { Shear } \\
\text { resist. } \\
(\mathbf{k N})\end{array}$ & $\begin{array}{c}\text { Deflect. } \\
\text { (mm) }\end{array}$ & $\begin{array}{l}\text { Mom. } \\
\text { resist. } \\
\text { (kN.m) }\end{array}$ & $\begin{array}{c}\text { Shear } \\
\text { resist. } \\
(\mathrm{kN})\end{array}$ & $\begin{array}{c}\text { Deflect. } \\
\text { (mm) }\end{array}$ \\
\hline $\begin{array}{c}\mathrm{F}-16 \\
1220 \times 3657 \times 210 \mathrm{~mm} \\
\left(4{ }^{\prime} \times 12^{\prime} \times 8.25^{\prime \prime}\right)\end{array}$ & 47.89 & 21.86 & 24.95 & $\begin{array}{l}8.12 \\
5.57\end{array}$ & & & & & & & & & \\
\hline $\begin{array}{c}\text { F-16-1 } \\
1220 \times 3657 \times 210 \mathrm{~mm} \\
\left(4^{\prime} \times 12^{\prime} \times 8.25^{\prime \prime}\right)\end{array}$ & 36.72 & 16.97 & 19.36 & $\begin{array}{l}8.12 \\
6.94\end{array}$ & & & & & & & & & \\
\hline $\begin{array}{c}\text { F-17 } \\
1220 \times 3657 \times 210 \mathrm{~mm} \\
\left(4^{\prime} \times 12^{\prime} \times 8.25^{\prime \prime}\right)\end{array}$ & 41.63 & 19.12 & 21.82 & $\begin{array}{l}8.12 \\
5.97\end{array}$ & 21.75 & 21.59 & 6.56 & 19.44 & 15.68 & 13.34 & 30.57 & 21.08 & 7.80 \\
\hline $\begin{array}{c}\text { F-18 } \\
1220 \times 3657 \times 210 \mathrm{~mm} \\
\left(4^{\prime} \times 12^{\prime} \times 8.25^{\prime \prime}\right)\end{array}$ & 34.96 & 16.19 & 18.48 & $\begin{array}{l}8.12 \\
6.54\end{array}$ & & & & & & & & & \\
\hline $\begin{array}{c}\text { F-19 } \\
1220 \times 3657 \times 210 \mathrm{~mm} \\
\left(4^{\prime} \times 12^{\prime} \times 8.25^{\prime \prime}\right)\end{array}$ & 52.29 & 23.79 & 23.79 & $\begin{array}{l}8.12 \\
5.26\end{array}$ & & & & & & & & & \\
\hline
\end{tabular}

* Butt (2008), did not include $2 \mathrm{kN}$ weight of the loading system.

(1) Included $2 \mathrm{kN}$ weight of loading system and then multiplied by $\mathrm{L} / 8$ to obtain bending moment $(\mathrm{L}=3.5052 \mathrm{~m})$

(2) Included $2 \mathrm{kN}$ weight of loading system. ${ }^{(3}$ The first number of the live load applied to the panel in $\mathrm{kN}$ to get the deflection in mm which is the second number. 
Table 6.16 - Comparison of the results from different codes and experimental data for flexural load on SIP (continued)

\begin{tabular}{|c|c|c|c|c|c|c|c|c|c|c|c|c|c|}
\hline \multirow[b]{2}{*}{ Specimen } & \multirow{2}{*}{\begin{tabular}{|c} 
Exp. \\
ultimat \\
e load \\
$(\mathrm{kN})^{*}$
\end{tabular}} & \multirow{2}{*}{$\begin{array}{c}\text { Exp. } \\
\text { resisting } \\
\text { bending } \\
\text { moment } \\
(\mathrm{kN} \cdot \mathrm{m})^{(1)}\end{array}$} & \multirow{2}{*}{$\begin{array}{c}\text { Exp. } \\
\text { resist. } \\
\text { shear } \\
\text { force } \\
(\mathrm{kN})^{(2)}\end{array}$} & \multirow{2}{*}{$\begin{array}{c}\text { Load } \\
\text { and } \\
\text { deflect. } \\
\text { at }(L)^{(3)}\end{array}$} & \multicolumn{3}{|c|}{ CSA-086-09 } & \multicolumn{3}{|c|}{ DIAB Sandwich Handbook } & \multicolumn{3}{|c|}{ APA Standard } \\
\hline & & & & & $\begin{array}{l}\text { Mom. } \\
\text { resist. } \\
\text { (kN.m) }\end{array}$ & $\begin{array}{c}\text { Shear } \\
\text { resist. } \\
(\mathbf{k N})\end{array}$ & $\begin{array}{l}\text { Deflect } \\
\text {. (mm) }\end{array}$ & $\begin{array}{l}\text { Mom. } \\
\text { resist. } \\
\text { (kN.m) }\end{array}$ & $\begin{array}{l}\text { Shear } \\
\text { resist. } \\
(\mathbf{k N})\end{array}$ & $\begin{array}{l}\text { Deflect } \\
\text {. (mm) }\end{array}$ & $\begin{array}{l}\text { Mom. } \\
\text { resist. } \\
\text { (kN.m) }\end{array}$ & $\begin{array}{c}\text { Shear } \\
\text { resist. } \\
(\mathrm{kN})\end{array}$ & $\begin{array}{l}\text { Deflect } \\
\text {. (mm) }\end{array}$ \\
\hline $\begin{array}{c}\mathrm{J}-28 \\
1220 \times 3657 \times 260 \mathrm{~mm} \\
\left(4^{\prime} \times 12^{\prime} \times 10.25^{\prime \prime}\right)\end{array}$ & 40.57 & 18.65 & 21.29 & $\begin{array}{l}8.12 \\
4.64\end{array}$ & \multirow{3}{*}{23.49} & \multirow{3}{*}{23.32} & \multirow{3}{*}{3.43} & \multirow{3}{*}{23.60} & \multirow{3}{*}{19.48} & \multirow{3}{*}{11.18} & \multirow{3}{*}{26.41} & \multirow{3}{*}{14.50} & \multirow{3}{*}{3.88} \\
\hline $\begin{array}{c}\mathrm{J}-29 \\
1220 \times 3657 \times 260 \mathrm{~mm} \\
\left(4^{\prime} \times 12^{\prime} \times 10.25^{\prime \prime}\right)\end{array}$ & 42.04 & 19.30 & 22.02 & $\begin{array}{l}8.12 \\
5.04\end{array}$ & & & & & & & & & \\
\hline $\begin{array}{c}\mathrm{J}-30 \\
1220 \times 3657 \times 260 \mathrm{~mm} \\
\left(4^{\prime} \times 12^{\prime} \times 10.25^{\prime \prime}\right)\end{array}$ & 45.17 & 20.67 & 23.59 & $\begin{array}{l}8.12 \\
4.97\end{array}$ & & & & & & & & & \\
\hline
\end{tabular}

* Butt (2008), did not include $2 \mathrm{kN}$ weight of the loading system.

(1) Included $2 \mathrm{kN}$ weight of loading system and then multiplied by $\mathrm{L} / 8$ to obtain bending moment $(\mathrm{L}=3.5052 \mathrm{~m})$

(2) Included $2 \mathrm{kN}$ weight of loading system

${ }^{3}$ The first number of the live load applied to the panel in $\mathrm{kN}$ to get the deflection in $\mathrm{mm}$ which is the second number. 
Table 6.16 - Comparison of the results from different codes and experimental data for flexural loading on SIP panels (continued)

\begin{tabular}{|c|c|c|c|c|c|c|c|c|c|c|c|c|c|}
\hline \multirow[b]{2}{*}{ Specimen } & \multirow{2}{*}{$\begin{array}{c}\text { Exp. } \\
\text { ultimate } \\
\text { load } \\
(\mathbf{k N})^{*}\end{array}$} & \multirow{2}{*}{$\begin{array}{c}\text { Exp. } \\
\text { resisting } \\
\text { bending } \\
\text { moment } \\
(\mathrm{kN} . \mathrm{m})^{(1)}\end{array}$} & \multirow{2}{*}{$\begin{array}{l}\text { Exp. } \\
\text { resist. } \\
\text { shear } \\
\text { force } \\
(\mathbf{k N})^{(2)}\end{array}$} & \multirow{2}{*}{$\begin{array}{c}\text { Load } \\
\text { and } \\
\text { deflect. } \\
\text { at (L) } \\
\text { (3) }\end{array}$} & \multicolumn{3}{|c|}{ CSA-086-09 } & \multicolumn{3}{|c|}{ DIAB Sandwich Handbook } & \multicolumn{3}{|c|}{ APA Standard } \\
\hline & & & & & $\begin{array}{l}\text { Mom. } \\
\text { resist. } \\
\text { (kN.m) }\end{array}$ & $\begin{array}{l}\text { Shear } \\
\text { resist. } \\
\text { (kN) }\end{array}$ & $\begin{array}{c}\text { Deflect. } \\
\text { (mm) }\end{array}$ & $\begin{array}{l}\text { Mom. } \\
\text { resist. } \\
\text { (kN.m) }\end{array}$ & $\begin{array}{l}\text { Shear } \\
\text { resist. } \\
(\mathrm{kN})\end{array}$ & $\begin{array}{c}\text { Deflect. } \\
\text { (mm) }\end{array}$ & $\begin{array}{l}\text { Mom. } \\
\text { resist. } \\
\text { (kN.m) }\end{array}$ & $\begin{array}{l}\text { Shear } \\
\text { resist. } \\
(\mathbf{k N})\end{array}$ & $\begin{array}{c}\text { Deflect. } \\
\text { (mm) }\end{array}$ \\
\hline $\begin{array}{c}\text { K-31 } \\
1220 \times 4267 \times 260 \mathrm{~mm} \\
\left(4^{\prime} \times 14^{\prime} \times 10.25^{\prime \prime}\right)\end{array}$ & 41.89 & 22.57 & 21.95 & $\begin{array}{l}9.53 \\
8.75\end{array}$ & \multirow{3}{*}{27.24} & \multirow{3}{*}{27.05} & \multirow{3}{*}{6.49} & \multirow{3}{*}{23.60} & \multirow{3}{*}{19.48} & \multirow{3}{*}{17.47} & \multirow{3}{*}{30.68} & \multirow{3}{*}{16.85} & \multirow{3}{*}{6.12} \\
\hline $\begin{array}{c}\text { K-32 } \\
1220 \times 4267 \times 260 \mathrm{~mm} \\
\left(4^{\prime} \times 14^{\prime} \times 10.25^{\prime \prime}\right)\end{array}$ & 44.71 & 24.03 & 23.36 & $\begin{array}{l}9.53 \\
8.25\end{array}$ & & & & & & & & & \\
\hline $\begin{array}{c}\mathrm{K}-33 \\
1220 \times 4267 \times 260 \mathrm{~mm} \\
\left(4^{\prime} \times 14^{\prime} \times 10.25^{\prime \prime}\right)\end{array}$ & 39.00 & 21.09 & 20.50 & $\begin{array}{l}9.53 \\
8.13\end{array}$ & & & & & & & & & \\
\hline
\end{tabular}

* Butt (2008), did not include $2 \mathrm{kN}$ weight of the loading system.

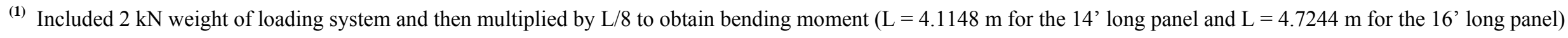

(2) Included $2 \mathrm{kN}$ weight of loading system

( 3 The first number of the live load applied to the panel in $\mathrm{kN}$ to get the deflection in $\mathrm{mm}$ which is the second number. 
Table 6.16 - Comparison of the results from different codes and experimental data for flexural loading on SIP panels (continued)

\begin{tabular}{|c|c|c|c|c|c|c|c|c|c|c|c|c|c|}
\hline \multirow[b]{2}{*}{ Specimen } & \multirow{2}{*}{$\begin{array}{c}\text { Exp. } \\
\text { ultimate } \\
\text { load } \\
(\mathbf{k N})^{*}\end{array}$} & \multirow{2}{*}{$\begin{array}{c}\text { Exp. } \\
\text { resisting } \\
\text { bending } \\
\text { moment } \\
(\mathrm{kN} . \mathrm{m})^{(1)}\end{array}$} & \multirow{2}{*}{$\begin{array}{c}\text { Exp. } \\
\text { resist. } \\
\text { shear } \\
\text { force } \\
(\mathbf{k N})^{(2)}\end{array}$} & \multirow{2}{*}{$\begin{array}{c}\text { Load } \\
\text { and } \\
\text { deflect. } \\
\text { at (L) } \\
\text { (3) }\end{array}$} & \multicolumn{3}{|c|}{ CSA-086-09 } & \multicolumn{3}{|c|}{ DIAB Sandwich Handbook } & \multicolumn{3}{|c|}{ APA Standard } \\
\hline & & & & & $\begin{array}{l}\text { Mom. } \\
\text { resist. } \\
\text { (kN.m) }\end{array}$ & $\begin{array}{c}\text { Shear } \\
\text { resist. } \\
\text { (kN) }\end{array}$ & $\begin{array}{c}\text { Deflect. } \\
\text { (mm) }\end{array}$ & $\begin{array}{l}\text { Mom. } \\
\text { resist. } \\
\text { (kN.m) }\end{array}$ & $\begin{array}{l}\text { Shear } \\
\text { resist. } \\
(\mathbf{k N})\end{array}$ & $\begin{array}{c}\text { Deflect. } \\
\text { (mm) }\end{array}$ & $\begin{array}{l}\text { Mom. } \\
\text { resist. } \\
\text { (kN.m) }\end{array}$ & $\begin{array}{l}\text { Shear } \\
\text { resist. } \\
(\mathbf{k N})\end{array}$ & $\begin{array}{c}\text { Deflect. } \\
\text { (mm) }\end{array}$ \\
\hline $\begin{array}{c}M-37 \\
1220 \times 4877 \times 260 \mathrm{~mm} \\
\left(4^{\prime} \times 16^{\prime} \times 10.25^{\prime \prime}\right)\end{array}$ & 39.65 & 24.60 & 20.83 & $\begin{array}{l}10.94 \\
39.65\end{array}$ & \multirow{3}{*}{30.51} & \multirow{3}{*}{30.29} & \multirow{3}{*}{11.27} & \multirow{3}{*}{23.60} & \multirow{3}{*}{19.48} & \multirow{3}{*}{26.17} & \multirow{3}{*}{34.36} & \multirow{3}{*}{18.87} & \multirow{3}{*}{9.45} \\
\hline $\begin{array}{c}\text { M-38 } \\
1220 \times 4877 \times 260 \mathrm{~mm} \\
\left(4^{\prime} \times 16^{\prime} \times 10.25^{\prime \prime}\right)\end{array}$ & 43.61 & 26.93 & 22.81 & $\begin{array}{l}10.94 \\
43.61\end{array}$ & & & & & & & & & \\
\hline $\begin{array}{c}M-39 \\
1220 \times 4877 \times 260 \mathrm{~mm} \\
\left(4^{\prime} \times 16^{\prime} \times 10.25^{\prime \prime}\right)\end{array}$ & 42.05 & 26.01 & 22.03 & $\begin{array}{l}10.94 \\
42.05\end{array}$ & & & & & & & & & \\
\hline
\end{tabular}

* Butt (2008), did not include $2 \mathrm{kN}$ weight of the loading system.

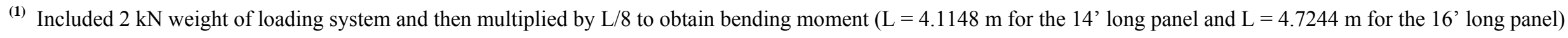

(2) Included $2 \mathrm{kN}$ weight of loading system

( 3 The first number of the live load applied to the panel in $\mathrm{kN}$ to get the deflection in $\mathrm{mm}$ which is the second number. 
Table 6.17 - Comparison of the results from different codes and experimental data for bending resistance of SIP panels

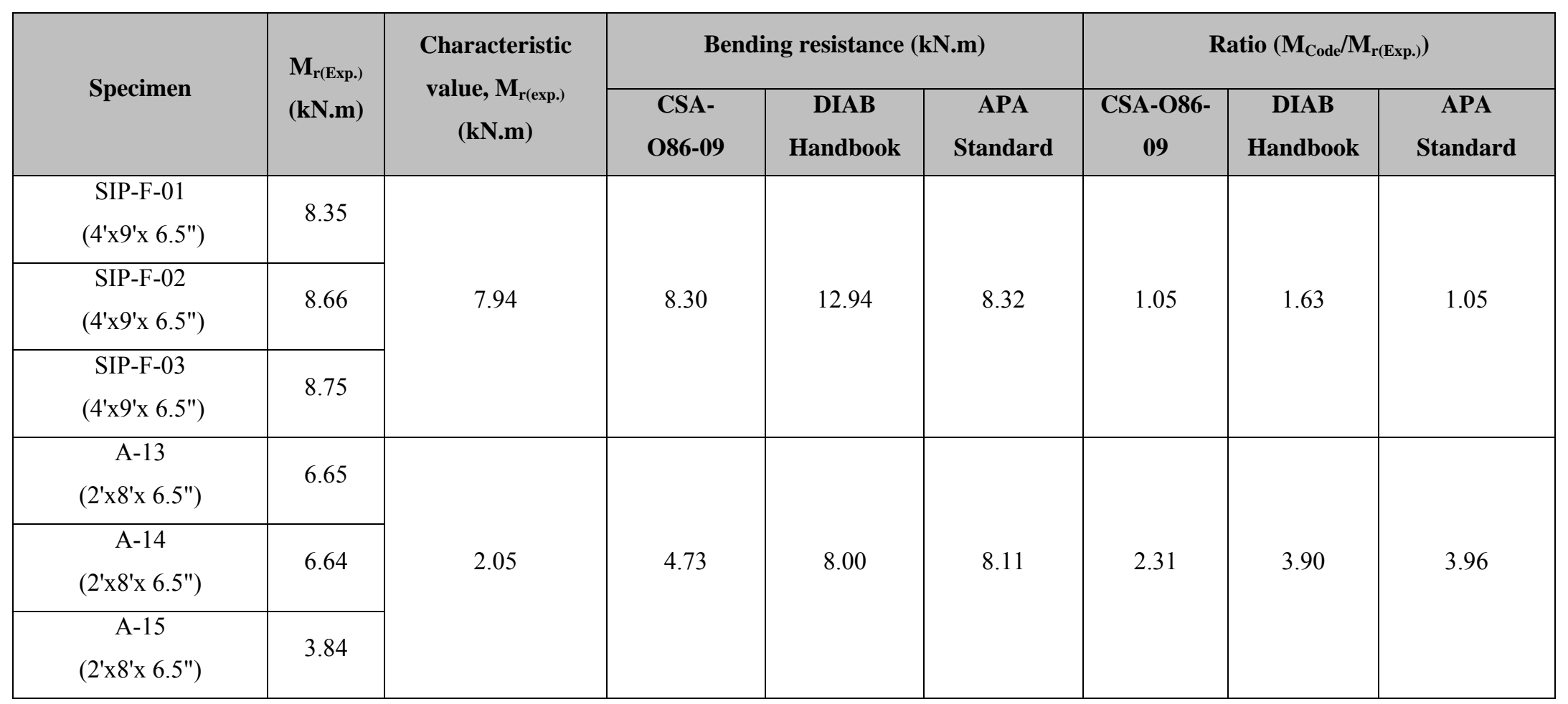


Table 6.17 - Comparison of the results from different codes and experimental data for bending resistance of SIP panels (continued)

\begin{tabular}{|c|c|c|c|c|c|c|c|c|}
\hline \multirow{2}{*}{ Specimen } & \multirow{2}{*}{$\begin{array}{l}M_{r(\text { Exp.) }} \\
\text { (kN.m) }\end{array}$} & \multirow{2}{*}{$\begin{array}{c}\text { Characteristic } \\
\text { value } \mathbf{M}_{\mathbf{r}(\text { exp.) }} \\
\text { (kN.m) }\end{array}$} & \multicolumn{3}{|c|}{ Bending resistance (kN.m) } & \multicolumn{3}{|c|}{ Ratio ( $\mathbf{M}_{\mathbf{r}(\text { code) }} / \mathbf{M}_{\mathbf{r} \text { (Exp.) })}$} \\
\hline & & & $\begin{array}{c}\text { CSA- } \\
\text { O86-09 }\end{array}$ & $\begin{array}{c}\text { DIAB } \\
\text { Handbook }\end{array}$ & $\begin{array}{c}\text { APA } \\
\text { Standard }\end{array}$ & $\begin{array}{c}\text { CSA- } \\
\text { O86-09 }\end{array}$ & $\begin{array}{c}\text { DIAB } \\
\text { Handbook }\end{array}$ & $\begin{array}{c}\text { APA } \\
\text { Standard }\end{array}$ \\
\hline $\begin{array}{c}\mathrm{A}-16 \\
\left(22^{\prime} \times 16 ' \times 10.25^{\prime \prime}\right)\end{array}$ & 14.64 & \multirow{3}{*}{13.20} & \multirow{3}{*}{10.40} & \multirow{3}{*}{8.00} & \multirow{3}{*}{17.94} & \multirow{3}{*}{0.79} & \multirow{3}{*}{0.61} & \multirow{3}{*}{1.36} \\
\hline $\begin{array}{c}\text { A-17 } \\
\left(2^{\prime} \times 16 ' x 10.25^{\prime \prime}\right)\end{array}$ & 13.91 & & & & & & & \\
\hline $\begin{array}{c}\mathrm{A}-18 \\
\left(2^{\prime} \times 16 ' \times 10.25^{\prime \prime}\right)\end{array}$ & 14.38 & & & & & & & \\
\hline $\begin{array}{c}\text { WS-19 } \\
\text { (4'x9'x 6.5") }\end{array}$ & 9.47 & \multirow{5}{*}{8.56} & \multirow{5}{*}{9.46} & \multirow{5}{*}{15.82} & \multirow{5}{*}{12.43} & \multirow{5}{*}{1.11} & \multirow{5}{*}{1.85} & \multirow{5}{*}{1.45} \\
\hline $\begin{array}{c}\text { WS-20 } \\
\left(4^{\prime} \times 9 ' x \quad 6.5^{\prime \prime}\right)\end{array}$ & 9.65 & & & & & & & \\
\hline $\begin{array}{c}\text { WS-21 } \\
\left(4^{\prime} \times 9 ' \times 6.5^{\prime \prime}\right)\end{array}$ & 8.75 & & & & & & & \\
\hline $\begin{array}{c}\text { WS-22 } \\
\text { (4'x9'x 6.5") }\end{array}$ & 9.97 & & & & & & & \\
\hline $\begin{array}{c}\text { WS-23 } \\
\left(4^{\prime} \times 9 ' x 6.5^{\prime \prime}\right)\end{array}$ & 9.33 & & & & & & & \\
\hline
\end{tabular}


Table 6.17 - Comparison of the results from different codes and experimental data for bending resistance of SIP panels (continued)

\begin{tabular}{|c|c|c|c|c|c|c|c|c|}
\hline \multirow{2}{*}{ Specimen } & \multirow{2}{*}{$\begin{array}{l}M_{r(\text { Exp.) }} \\
\text { (kN.m) }\end{array}$} & \multirow{2}{*}{$\begin{array}{l}\text { Characteristic } \\
\text { value } M_{r(\text { exp.) }} \\
\text { (kN.m) }\end{array}$} & \multicolumn{3}{|c|}{ Bending resistance (kN.m) } & \multicolumn{3}{|c|}{ Ratio ( $\mathbf{M}_{\mathbf{r}(\text { code) }} / \mathbf{M}_{\mathbf{r} \text { (Exp.) })}$} \\
\hline & & & $\begin{array}{c}\text { CSA- } \\
\text { O86-09 }\end{array}$ & $\begin{array}{c}\text { DIAB } \\
\text { Handbook }\end{array}$ & $\begin{array}{c}\text { APA } \\
\text { Standard }\end{array}$ & $\begin{array}{c}\text { CSA- } \\
\text { O86-09 }\end{array}$ & $\begin{array}{c}\text { DIAB } \\
\text { Handbook }\end{array}$ & $\begin{array}{c}\text { APA } \\
\text { Standard }\end{array}$ \\
\hline $\begin{array}{c}\text { B-4 } \\
\left(4^{\prime} \times 10^{\prime} \times 6.5 "\right)\end{array}$ & 14.48 & \multirow{3}{*}{13.42} & \multirow{3}{*}{15.13} & \multirow{3}{*}{25.31} & \multirow{3}{*}{19.76} & \multirow{3}{*}{1.13} & \multirow{3}{*}{1.89} & \multirow{3}{*}{1.47} \\
\hline $\begin{array}{c}\text { B-5 } \\
\left(4^{\prime} \times 10^{\prime} \times 6.5 "\right)\end{array}$ & 15.03 & & & & & & & \\
\hline $\begin{array}{c}\text { B-43 } \\
(4 ' \times 10 ' x 6.5 ")\end{array}$ & 15.55 & & & & & & & \\
\hline $\begin{array}{c}\text { D-10 } \\
\left(4^{\prime} \times 12 ' x 6.5 "\right)\end{array}$ & 16.17 & \multirow{3}{*}{12.30} & \multirow{3}{*}{9.74} & \multirow{3}{*}{19.38} & \multirow{3}{*}{20.39} & \multirow{3}{*}{0.79} & \multirow{3}{*}{1.58} & \multirow{3}{*}{1.66} \\
\hline $\begin{array}{c}\text { D-11 } \\
\left(4^{\prime} \times 12 ' x 6.5 "\right)\end{array}$ & 14.26 & & & & & & & \\
\hline $\begin{array}{c}\text { D-44 } \\
\left(4^{\prime} \times 12 ' x 6.5 "\right)\end{array}$ & 16.25 & & & & & & & \\
\hline
\end{tabular}


Table 6.17 - Comparison of the results from different codes and experimental data for bending resistance of SIP panels (continued)

\begin{tabular}{|c|c|c|c|c|c|c|c|c|}
\hline \multirow{2}{*}{ Specimen } & \multirow{2}{*}{$\begin{array}{l}M_{r(\text { Exp.) }} \\
\text { (kN.m) }\end{array}$} & \multirow{2}{*}{$\begin{array}{c}\text { Characteristic } \\
\text { value } \mathbf{M}_{\mathrm{r}(\text { exp.) }} \\
\text { (kN.m) }\end{array}$} & \multicolumn{3}{|c|}{ Bending resistance (kN.m) } & \multicolumn{3}{|c|}{ Ratio ( $\mathbf{M}_{\mathbf{r}(\text { code) }} / \mathbf{M}_{\mathbf{r} \text { (Exp.) })}$} \\
\hline & & & $\begin{array}{c}\text { CSA- } \\
\text { O86-09 }\end{array}$ & $\begin{array}{c}\text { DIAB } \\
\text { Handbook }\end{array}$ & $\begin{array}{c}\text { APA } \\
\text { Standard }\end{array}$ & $\begin{array}{c}\text { CSA- } \\
\text { O86-09 }\end{array}$ & $\begin{array}{c}\text { DIAB } \\
\text { Handbook }\end{array}$ & $\begin{array}{c}\text { APA } \\
\text { Standard }\end{array}$ \\
\hline $\begin{array}{c}\text { E-13 } \\
\left(4^{\prime} \times 10^{\prime} \times 8.25 "\right)\end{array}$ & 15.87 & \multirow{3}{*}{8.72} & \multirow{3}{*}{15.13} & \multirow{3}{*}{19.44} & \multirow{3}{*}{21.18} & \multirow{3}{*}{1.74} & \multirow{3}{*}{2.23} & \multirow{3}{*}{2.43} \\
\hline $\begin{array}{c}\text { E-14 } \\
\left(4^{\prime} \times 10^{\prime} \times 8.25^{\prime \prime}\right)\end{array}$ & 16.65 & & & & & & & \\
\hline $\begin{array}{c}\text { E-15-1 } \\
\left(4^{\prime} \times 10 ' x 8.25 "\right)\end{array}$ & 12.20 & & & & & & & \\
\hline $\begin{array}{c}\text { H-22 } \\
\left.\text { (4'x14'x } 8.25^{\prime \prime}\right)\end{array}$ & 21.81 & \multirow{3}{*}{17.44} & \multirow{3}{*}{20.72} & \multirow{3}{*}{19.44} & \multirow{3}{*}{29.23} & \multirow{3}{*}{1.19} & \multirow{3}{*}{1.11} & \multirow{3}{*}{1.68} \\
\hline $\begin{array}{c}\mathrm{H}-23-1 \\
\left(4^{\prime} \times 14^{\prime} \times 8.25 "\right)\end{array}$ & 20.07 & & & & & & & \\
\hline $\begin{array}{c}\mathrm{H}-24 \\
\left(4^{\prime} \times 14^{\prime} \times 8.25^{\prime \prime}\right)\end{array}$ & 22.95 & & & & & & & \\
\hline
\end{tabular}


Table 6.17 - Comparison of the results from different codes and experimental data for bending resistance of SIP panels (continued)

\begin{tabular}{|c|c|c|c|c|c|c|c|c|}
\hline \multirow{2}{*}{ Specimen } & \multirow{2}{*}{$\begin{array}{l}M_{r(\text { Exp.) }} \\
\text { (kN.m) }\end{array}$} & \multirow{2}{*}{$\begin{array}{c}\text { Characteristic } \\
\text { value } \mathbf{M}_{\mathbf{r}(\text { exp.) }} \\
\text { (kN.m) }\end{array}$} & \multicolumn{3}{|c|}{ Bending resistance (kN.m) } & \multicolumn{3}{|c|}{ Ratio ( $\left.\mathbf{M}_{\mathbf{r ( c o d e})} / \mathbf{M}_{\mathbf{r} \text { (Exp.) }}\right)$} \\
\hline & & & $\begin{array}{l}\text { CSA- } \\
\text { O86-09 }\end{array}$ & $\begin{array}{c}\text { DIAB } \\
\text { Handbook }\end{array}$ & $\begin{array}{c}\text { APA } \\
\text { Standard }\end{array}$ & $\begin{array}{c}\text { CSA- } \\
\text { O86-09 }\end{array}$ & $\begin{array}{c}\text { DIAB } \\
\text { Handbook }\end{array}$ & $\begin{array}{c}\text { APA } \\
\text { Standard }\end{array}$ \\
\hline $\begin{array}{c}\text { F-16 } \\
\left(4^{\prime} \times 12^{\prime} \times 8.25^{\prime \prime}\right)\end{array}$ & 21.87 & \multirow{5}{*}{15.54} & \multirow{5}{*}{21.75} & \multirow{5}{*}{19.44} & \multirow{5}{*}{30.57} & \multirow{5}{*}{1.40} & \multirow{5}{*}{1.25} & \multirow{5}{*}{1.97} \\
\hline $\begin{array}{c}\text { F-16-1 } \\
\left(4^{\prime} \times 12 ' x 8.25 "\right)\end{array}$ & 16.98 & & & & & & & \\
\hline $\begin{array}{c}\text { F-17 } \\
\left(4^{\prime} \times 12^{\prime} \times 8.25^{\prime \prime}\right)\end{array}$ & 19.13 & & & & & & & \\
\hline $\begin{array}{c}\mathrm{F}-18 \\
\left(4^{\prime} \times 12^{\prime} \times 8.25 "\right)\end{array}$ & 16.21 & & & & & & & \\
\hline $\begin{array}{c}\mathrm{F}-19 \\
\left(4{ }^{\prime} \times 12 ' x 8.25 "\right)\end{array}$ & 23.80 & & & & & & & \\
\hline $\begin{array}{c}\mathrm{J}-28 \\
\left(4^{\prime} \times 12 ' x \quad 10.25 "\right)\end{array}$ & 18.66 & \multirow{3}{*}{16.57} & \multirow{3}{*}{23.49} & \multirow{3}{*}{23.60} & \multirow{3}{*}{26.41} & \multirow{3}{*}{1.42} & \multirow{3}{*}{1.42} & \multirow{3}{*}{1.59} \\
\hline $\begin{array}{c}\mathrm{J}-29 \\
\left(4^{\prime} \times 12 ' \mathrm{x} 10.25 "\right)\end{array}$ & 19.31 & & & & & & & \\
\hline $\begin{array}{c}\mathrm{J}-30 \\
\left(4^{\prime} \times 12 ' \times 10.25^{\prime \prime}\right)\end{array}$ & 20.68 & & & & & & & \\
\hline
\end{tabular}


Table 6.17 - Experimental results, characteristic value and ratio of moment resistant for transverse load on SIP (continued)

\begin{tabular}{|c|c|c|c|c|c|c|c|c|}
\hline \multirow{2}{*}{ Specimen } & \multirow{2}{*}{$\begin{array}{l}M_{\text {r(Exp.) }} \\
(\mathbf{k N . m )}\end{array}$} & \multirow{2}{*}{$\begin{array}{c}\text { Characteristic } \\
\text { Value, } \mathbf{M}_{\mathrm{r}(\text { exp.) }} \\
\text { (kN.m) }\end{array}$} & \multicolumn{3}{|c|}{ Bending resistance (kN.m) } & \multicolumn{3}{|c|}{ Ratio ( $\left.\mathbf{M}_{\mathbf{r}(\text { code })} / \mathbf{M}_{\mathbf{r}(\text { Exp.) }}\right)$} \\
\hline & & & $\begin{array}{c}\text { CSA- } \\
\text { O86-09 }\end{array}$ & $\begin{array}{c}\text { DIAB } \\
\text { Handbook }\end{array}$ & $\begin{array}{c}\text { APA } \\
\text { Standard }\end{array}$ & $\begin{array}{c}\text { CSA- } \\
\text { O86-09 }\end{array}$ & $\begin{array}{c}\text { DIAB } \\
\text { Handbook }\end{array}$ & $\begin{array}{c}\text { APA } \\
\text { Standard }\end{array}$ \\
\hline $\begin{array}{c}\text { K-31 } \\
\left(4^{\prime} \times 14^{\prime} \times 10.25^{\prime \prime}\right)\end{array}$ & 22.59 & \multirow{2}{*}{18.36} & \multirow{2}{*}{27.24} & \multirow{2}{*}{23.60} & \multirow{2}{*}{30.68} & \multirow{2}{*}{1.48} & \multirow{2}{*}{1.29} & \multirow{2}{*}{1.67} \\
\hline $\begin{array}{c}\mathrm{K}-33 \\
\left(4^{\prime} \times 14^{\prime} \times 10.25^{\prime \prime}\right)\end{array}$ & 21.10 & & & & & & & \\
\hline $\begin{array}{c}M-37 \\
\left(4^{\prime} \times 16 ' x 10.25^{\prime \prime}\right)\end{array}$ & 24.61 & \multirow{2}{*}{22.37} & \multirow{2}{*}{30.51} & \multirow{2}{*}{23.60} & \multirow{2}{*}{34.36} & \multirow{2}{*}{1.36} & \multirow{2}{*}{1.05} & \multirow{2}{*}{1.54} \\
\hline $\begin{array}{c}\text { M-39 } \\
\left(4^{\prime} \times 16 ' x 10.25^{\prime \prime}\right)\end{array}$ & 26.03 & & & & & & & \\
\hline
\end{tabular}




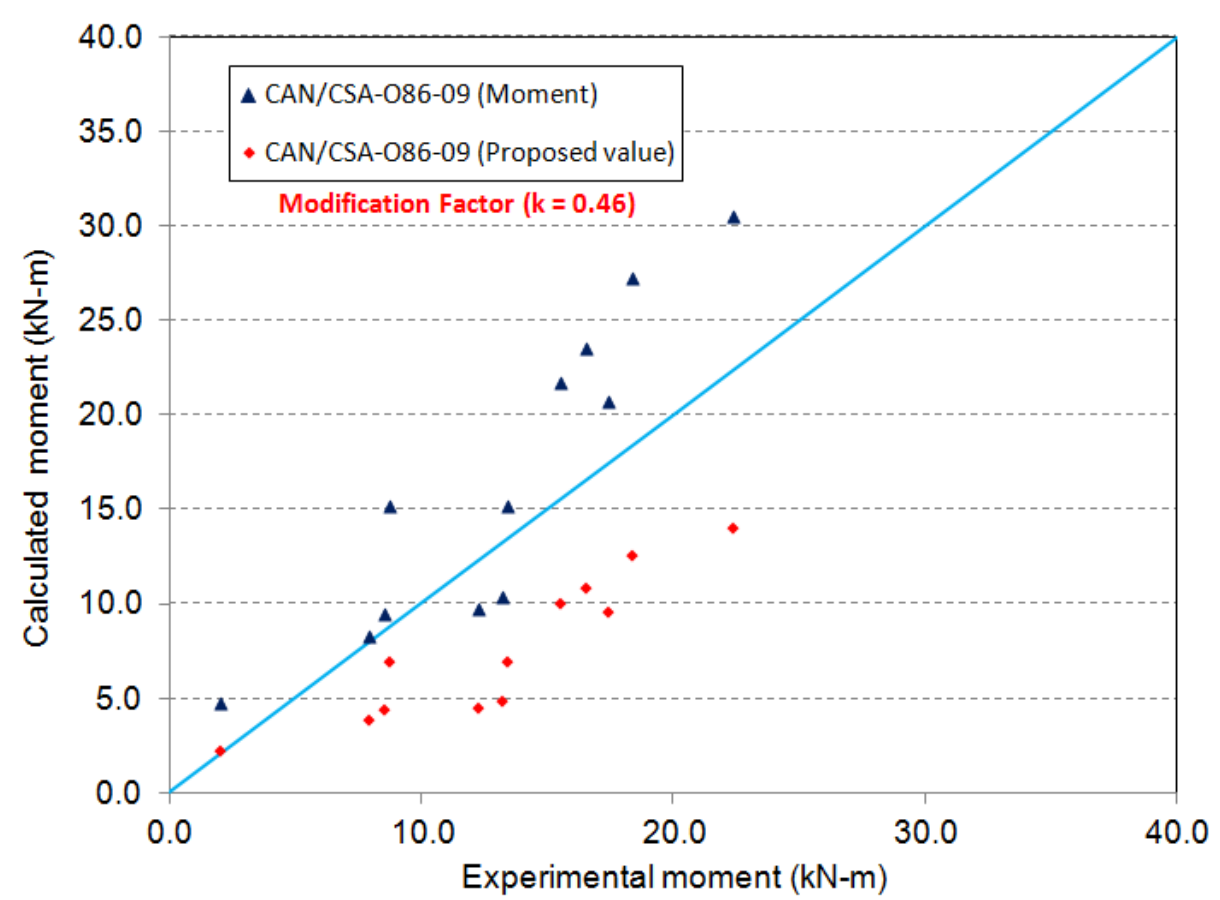

Figure 6.1 - Correlation between the resisting moment obtained experimentally and that obtained using CSA-O86-09 along with the values using the proposed modification factor for flexural

loading on SIP panels

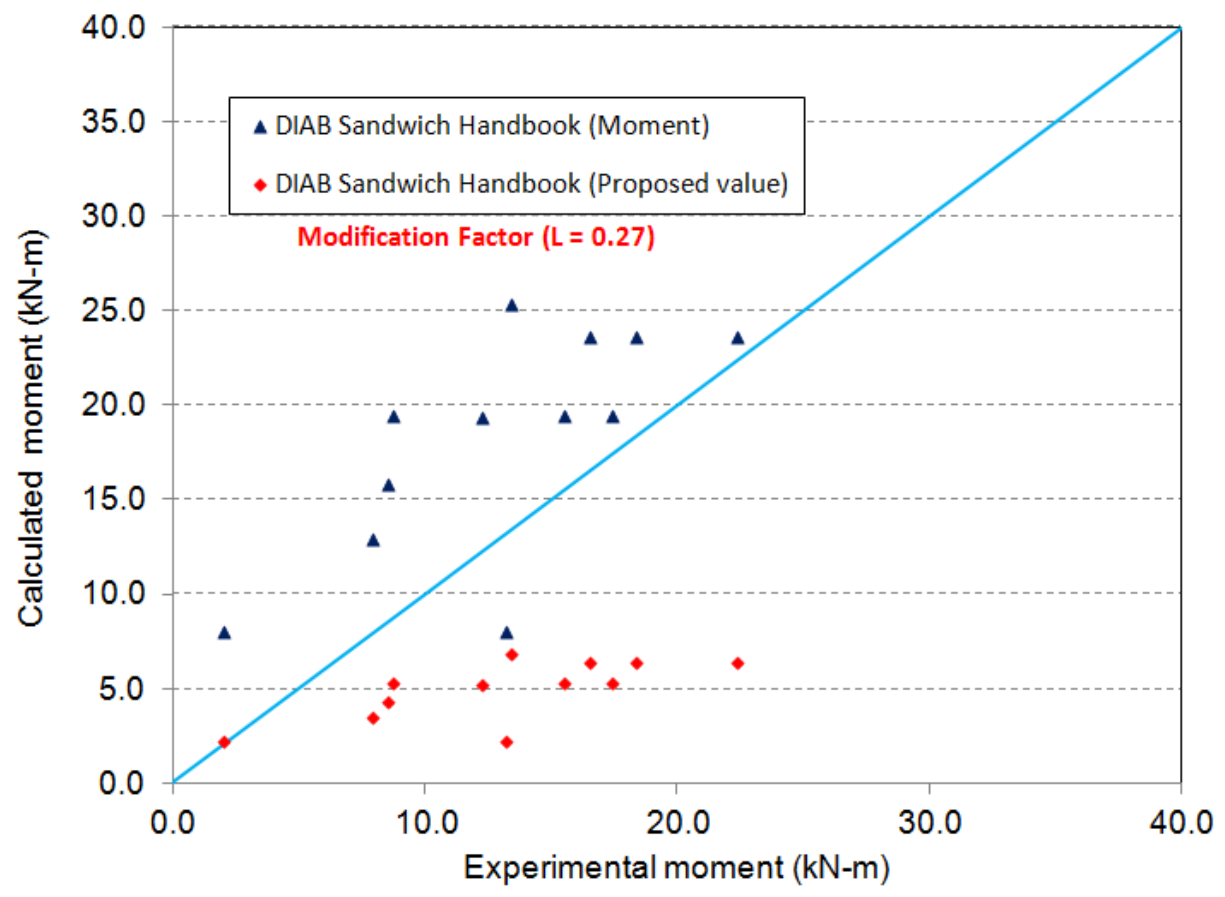

Figure 6.2 - Correlation between the resisting moment obtained experimentally and that obtained using DIAB Handbook along with the values using the proposed modification factor for flexural loading on SIP panels 


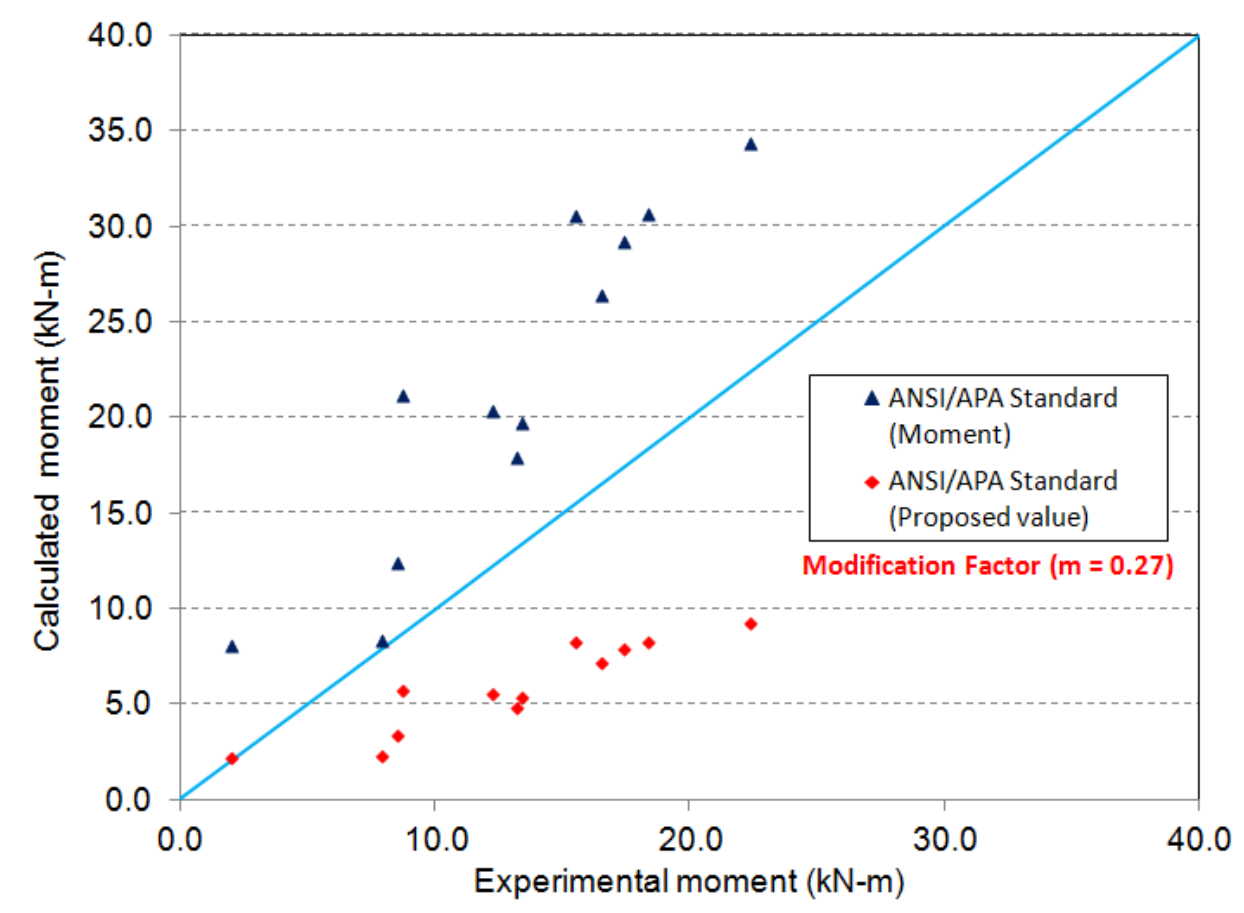

Figure 6.3 - Correlation between the resisting moment obtained experimentally and that obtained using ANSI/APA along with the values using the proposed modification factor for flexural loading on SIP panels 
Table 6.18 - Comparison of the results from different codes and experimental data for shear resistance of SIP panels

\begin{tabular}{|c|c|c|c|c|c|c|c|c|}
\hline \multirow{2}{*}{ Specimen } & \multirow{2}{*}{$\begin{array}{l}\mathrm{V}_{\mathrm{r}(\text { Exp. })} \\
(\mathbf{k N})\end{array}$} & \multirow{2}{*}{$\begin{array}{c}\text { Characteristic } \\
\text { value, } V_{\text {r(exp.) }}(k N)\end{array}$} & \multicolumn{3}{|c|}{ Shear resistance $(\mathrm{kN})$} & \multicolumn{3}{|c|}{ Ratio ( $\left.\mathbf{V}_{\text {Code }} / \mathbf{V}_{\mathbf{r ( E x p . )}}\right)$} \\
\hline & & & $\begin{array}{l}\text { CSA- } \\
\text { O86-09 }\end{array}$ & $\begin{array}{c}\text { DIAB } \\
\text { Handbook }\end{array}$ & $\begin{array}{c}\text { APA } \\
\text { Standard }\end{array}$ & $\begin{array}{c}\text { CSA-O86- } \\
09\end{array}$ & $\begin{array}{c}\text { DIAB } \\
\text { Handbook }\end{array}$ & $\begin{array}{c}\text { APA } \\
\text { Standard }\end{array}$ \\
\hline $\begin{array}{c}\text { SIP-F-01 } \\
\left(4^{\prime} \times 9 \text { 'x } 6.5^{\prime \prime}\right)\end{array}$ & 12.88 & \multirow{3}{*}{12.24} & \multirow{3}{*}{10.27} & \multirow{3}{*}{10.87} & \multirow{3}{*}{9.32} & \multirow{3}{*}{0.84} & \multirow{3}{*}{0.89} & \multirow{3}{*}{0.76} \\
\hline $\begin{array}{c}\text { SIP-F-02 } \\
\left(4^{\prime} \times 9 \text { 'x } 6.5 "\right)\end{array}$ & 13.36 & & & & & & & \\
\hline $\begin{array}{c}\text { SIP-F-03 } \\
\left(4^{\prime} \times 9 \text { 'x } 6.5^{\prime \prime}\right)\end{array}$ & 13.50 & & & & & & & \\
\hline $\begin{array}{c}\mathrm{A}-13 \\
\left(2 \mathrm{\prime} \times 8 \mathrm{\prime} \times 6.5^{\prime \prime}\right)\end{array}$ & 11.63 & \multirow{3}{*}{3.58} & \multirow{3}{*}{14.84} & \multirow{3}{*}{6.25} & \multirow{3}{*}{7.21} & \multirow{3}{*}{4.15} & \multirow{3}{*}{1.75} & \multirow{3}{*}{2.01} \\
\hline $\begin{array}{c}\mathrm{A}-14 \\
(2 ' x 8 \text { 'x 6.5") }\end{array}$ & 11.61 & & & & & & & \\
\hline $\begin{array}{c}\mathrm{A}-15 \\
\left(2^{\prime} \times 8^{\prime} \times 6.5 "\right)\end{array}$ & 6.72 & & & & & & & \\
\hline $\begin{array}{c}\text { A-16 } \\
\left(2^{\prime} \times 16 \text { 'x10.25”) }\right.\end{array}$ & 12.39 & \multirow{3}{*}{11.17} & \multirow{3}{*}{15.73} & \multirow{3}{*}{6.25} & \multirow{3}{*}{15.94} & \multirow{3}{*}{1.41} & \multirow{3}{*}{0.56} & \multirow{3}{*}{1.43} \\
\hline $\begin{array}{c}\mathrm{A}-17 \\
(2 \mathrm{2} \times 16 \mathrm{6} \times 10.25 ”)\end{array}$ & 11.78 & & & & & & & \\
\hline $\begin{array}{c}\text { A-18 } \\
\left(2^{\prime} \times 16 \text { 'x10.25”) }\right.\end{array}$ & 12.17 & & & & & & & \\
\hline
\end{tabular}


Table 6.18 - Comparison of the results from different codes and experimental data for shear resistance of SIP panels (continued)

\begin{tabular}{|c|c|c|c|c|c|c|c|c|}
\hline \multirow{2}{*}{ Specimen } & \multirow{2}{*}{$\begin{array}{c}\mathrm{V}_{\mathbf{r}(\text { Exp.) }} \\
(\mathbf{k N})\end{array}$} & \multirow{2}{*}{$\begin{array}{c}\text { Characteristic } \\
\text { value, } \mathbf{V}_{\mathbf{r}(\text { exp.) }} \\
(\mathbf{k N})\end{array}$} & \multicolumn{3}{|c|}{ Shear resistance $(k N)$} & \multicolumn{3}{|c|}{ Ratio $\left(\mathbf{V}_{\text {Code }} / \mathbf{V}_{\mathbf{r}(\text { Exp. } .}\right)$} \\
\hline & & & $\begin{array}{c}\text { CSA- } \\
\text { O86-09 }\end{array}$ & $\begin{array}{c}\text { DIAB } \\
\text { Handbook }\end{array}$ & $\begin{array}{c}\text { APA } \\
\text { Standard }\end{array}$ & $\begin{array}{c}\text { CSA-086- } \\
09\end{array}$ & $\begin{array}{c}\text { DIAB } \\
\text { Handbook }\end{array}$ & $\begin{array}{c}\text { APA } \\
\text { Standard }\end{array}$ \\
\hline $\begin{array}{c}\text { WS-19 } \\
\left(4^{\prime} \times 9 \text { 'x } 6.5 "\right)\end{array}$ & 14.61 & \multirow{5}{*}{13.20} & \multirow{5}{*}{11.54} & \multirow{5}{*}{12.35} & \multirow{5}{*}{11.05} & \multirow{5}{*}{0.87} & \multirow{5}{*}{0.94} & \multirow{5}{*}{0.84} \\
\hline $\begin{array}{c}\text { WS-20 } \\
\left(4^{\prime} \times 9 \text { 'x } 6.5 "\right)\end{array}$ & 14.89 & & & & & & & \\
\hline $\begin{array}{c}\text { WS-21 } \\
\left(4^{\prime} \times 9 \text { 'x } 6.5 "\right)\end{array}$ & 13.50 & & & & & & & \\
\hline $\begin{array}{c}\text { WS-22 } \\
\left(4^{\prime} \times 9 \text { 'x } 6.5 "\right)\end{array}$ & 15.39 & & & & & & & \\
\hline $\begin{array}{c}\text { WS-23 } \\
\left(4^{\prime} \times 9 \text { 'x } 6.5 "\right)\end{array}$ & 14.39 & & & & & & & \\
\hline $\begin{array}{c}\text { B-4 } \\
\left(4^{\prime} \times 10^{\prime} \times 6.5^{\prime \prime}\right)\end{array}$ & 19.99 & \multirow{3}{*}{18.53} & \multirow{3}{*}{18.46} & \multirow{3}{*}{19.76} & \multirow{3}{*}{17.57} & \multirow{3}{*}{1.00} & \multirow{3}{*}{1.07} & \multirow{3}{*}{0.95} \\
\hline $\begin{array}{c}\text { B-5 } \\
\left(4^{\prime} \times 10^{\prime} \times 6.5 "\right)\end{array}$ & 20.74 & & & & & & & \\
\hline $\begin{array}{c}\text { B-43 } \\
\left(4^{\prime} \times 10^{\prime} \times 6.5^{\prime \prime}\right)\end{array}$ & 21.46 & & & & & & & \\
\hline
\end{tabular}


Table 6.18 - Comparison of the results from different codes and experimental data for shear resistance of SIP panels (continued)

\begin{tabular}{|c|c|c|c|c|c|c|c|c|}
\hline \multirow{2}{*}{ Specimen } & \multirow{2}{*}{$\begin{array}{c}\mathrm{V}_{\mathbf{r}(\text { Exp. })} \\
(\mathbf{k N})\end{array}$} & \multirow{2}{*}{$\begin{array}{c}\text { Characteristic } \\
\text { value, } \mathbf{V}_{\text {r(exp.) }} \\
(\mathbf{k N})\end{array}$} & \multicolumn{3}{|c|}{ Shear resistance $(\mathrm{kN})$} & \multicolumn{3}{|c|}{ Ratio $\left(\mathbf{V}_{\text {Code }} / \mathbf{V}_{\mathbf{r ( E x p . )}}\right)$} \\
\hline & & & $\begin{array}{c}\text { CSA- } \\
\text { O86-09 }\end{array}$ & $\begin{array}{c}\text { DIAB } \\
\text { Handbook }\end{array}$ & $\begin{array}{c}\text { APA } \\
\text { Standard }\end{array}$ & $\begin{array}{c}\text { CSA-086- } \\
09\end{array}$ & $\begin{array}{c}\text { DIAB } \\
\text { Handbook }\end{array}$ & $\begin{array}{c}\text { APA } \\
\text { Standard }\end{array}$ \\
\hline $\begin{array}{c}\text { D-10 } \\
\left(4^{\prime} \times 12 ' x 6.5^{\prime \prime}\right)\end{array}$ & 18.44 & \multirow{3}{*}{14.03} & \multirow{3}{*}{11.88} & \multirow{3}{*}{12.72} & \multirow{3}{*}{18.12} & \multirow{3}{*}{0.85} & \multirow{3}{*}{0.91} & \multirow{3}{*}{1.29} \\
\hline $\begin{array}{c}D-11 \\
\left(4^{\prime} \times 12 ' x 6.5 "\right)\end{array}$ & 16.27 & & & & & & & \\
\hline $\begin{array}{c}\mathrm{D}-44 \\
\left(4^{\prime} \times 12^{\prime} \times 6.5^{\prime \prime}\right)\end{array}$ & 18.54 & & & & & & & \\
\hline $\begin{array}{c}\text { E-13 } \\
(4 ' x 10 ' x 8.25 ")\end{array}$ & 21.91 & \multirow{3}{*}{12.04} & \multirow{3}{*}{15.02} & \multirow{3}{*}{15.68} & \multirow{3}{*}{14.61} & \multirow{3}{*}{1.25} & \multirow{3}{*}{1.30} & \multirow{3}{*}{1.21} \\
\hline $\begin{array}{c}\text { E-14 } \\
\left(4^{\prime} \times 10 ' x 8.25 "\right)\end{array}$ & 22.98 & & & & & & & \\
\hline $\begin{array}{c}\text { E-15-1 } \\
\text { (4'x10'x 8.25") }\end{array}$ & 16.85 & & & & & & & \\
\hline
\end{tabular}


Table 6.18 - Comparison of the results from different codes and experimental data for shear resistance of SIP panels (continued)

\begin{tabular}{|c|c|c|c|c|c|c|c|c|}
\hline \multirow{2}{*}{ Specimen } & \multirow{2}{*}{$\begin{array}{c}\mathrm{V}_{\mathrm{r}(\text { Exp. })} \\
(\mathbf{k N})\end{array}$} & \multirow{2}{*}{$\begin{array}{l}\text { Characteristic } \\
\text { value, } \mathrm{V}_{\text {r(exp.) }} \\
\text { (kN) }\end{array}$} & \multicolumn{3}{|c|}{ Shear resistance $(\mathrm{kN})$} & \multicolumn{3}{|c|}{ Ratio $\left(\mathbf{V}_{\text {Code }} / \mathbf{V}_{\mathbf{r ( E x p . )}}\right)$} \\
\hline & & & $\begin{array}{c}\text { CSA- } \\
\text { O86-09 }\end{array}$ & $\begin{array}{c}\text { DIAB } \\
\text { Handbook }\end{array}$ & $\begin{array}{c}\text { APA } \\
\text { Standard }\end{array}$ & $\begin{array}{c}\text { CSA-086- } \\
09\end{array}$ & $\begin{array}{c}\text { DIAB } \\
\text { Handbook }\end{array}$ & $\begin{array}{c}\text { APA } \\
\text { Standard }\end{array}$ \\
\hline $\begin{array}{c}\text { H-22 } \\
(4 ' x 14 ' x 8.25 ")\end{array}$ & 21.19 & \multirow{3}{*}{16.94} & \multirow{3}{*}{20.57} & \multirow{3}{*}{15.68} & \multirow{3}{*}{20.16} & \multirow{3}{*}{1.21} & \multirow{3}{*}{0.93} & \multirow{3}{*}{1.19} \\
\hline $\begin{array}{c}\text { H-23-1 } \\
\left(4^{\prime} \times 14 \text { 'x 8.25") }\right.\end{array}$ & 19.50 & & & & & & & \\
\hline $\begin{array}{c}\text { H-24 } \\
(4 ' x 14 ' x 8.25 ")\end{array}$ & 22.30 & & & & & & & \\
\hline $\begin{array}{c}\text { F-16 } \\
\left(4^{\prime} \times 12^{\prime} \times 8.25^{\prime \prime}\right)\end{array}$ & 24.95 & \multirow{5}{*}{17.72} & \multirow{5}{*}{21.59} & \multirow{5}{*}{15.68} & \multirow{5}{*}{21.08} & \multirow{5}{*}{1.22} & \multirow{5}{*}{0.88} & \multirow{5}{*}{1.19} \\
\hline $\begin{array}{c}\text { F-16-1 } \\
\left(4^{\prime} \times 12 ' x 8.25 "\right)\end{array}$ & 19.36 & & & & & & & \\
\hline $\begin{array}{c}\mathrm{F}-17 \\
\left(4^{\prime} \times 12^{\prime} \times 8.25 "\right)\end{array}$ & 21.82 & & & & & & & \\
\hline $\begin{array}{c}\text { F-18 } \\
\left(4^{\prime} \times 12 ' \times 8.25^{\prime \prime}\right)\end{array}$ & 18.48 & & & & & & & \\
\hline $\begin{array}{c}\text { F-19 } \\
\left(4^{\prime} \times 12 ' x 8.25^{\prime \prime}\right)\end{array}$ & 27.15 & & & & & & & \\
\hline
\end{tabular}


Table 6.18 - Comparison of the results from different codes and experimental data for shear resistance of SIP panels (continued)

\begin{tabular}{|c|c|c|c|c|c|c|c|c|}
\hline \multirow{2}{*}{ Specimen } & \multirow{2}{*}{$\begin{array}{c}\mathrm{V}_{\mathrm{r}(\text { Exp. })} \\
(\mathbf{k N})\end{array}$} & \multirow{2}{*}{$\begin{array}{c}\text { Characteristic } \\
\text { value, } \mathrm{V}_{\mathrm{r}(\mathrm{exp} .)} \\
(\mathbf{k N})\end{array}$} & \multicolumn{3}{|c|}{ Shear resistance $(\mathrm{kN})$} & \multicolumn{3}{|c|}{ Ratio $\left(\mathbf{V}_{\text {Code }} / \mathbf{V}_{\mathbf{r ( E x p . )}}\right)$} \\
\hline & & & $\begin{array}{c}\text { CSA- } \\
\text { O86-09 }\end{array}$ & $\begin{array}{c}\text { DIAB } \\
\text { Handbook }\end{array}$ & $\begin{array}{c}\text { APA } \\
\text { Standard }\end{array}$ & $\begin{array}{c}\text { CSA-086- } \\
09\end{array}$ & $\begin{array}{c}\text { DIAB } \\
\text { Handbook }\end{array}$ & $\begin{array}{c}\text { APA } \\
\text { Standard }\end{array}$ \\
\hline $\begin{array}{c}\mathrm{J}-28 \\
\left(4^{\prime} \times 12^{\prime} \times 10.25 "\right)\end{array}$ & 21.29 & \multirow{3}{*}{18.89} & \multirow{3}{*}{23.32} & \multirow{3}{*}{19.48} & \multirow{3}{*}{14.50} & \multirow{3}{*}{1.23} & \multirow{3}{*}{1.03} & \multirow{3}{*}{0.77} \\
\hline $\begin{array}{c}J-29 \\
\left(4^{\prime} \times 12^{\prime} \times 10.25 "\right)\end{array}$ & 22.02 & & & & & & & \\
\hline $\begin{array}{c}J-30 \\
\left(4^{\prime} \times 12^{\prime} \times 10.25 "\right)\end{array}$ & 23.59 & & & & & & & \\
\hline $\begin{array}{c}\text { K-31 } \\
\text { (4'x14'x 10.25") }\end{array}$ & 21.95 & \multirow{3}{*}{17.84} & \multirow{3}{*}{27.05} & \multirow{3}{*}{19.48} & \multirow{3}{*}{16.85} & \multirow{3}{*}{1.52} & \multirow{3}{*}{1.09} & \multirow{3}{*}{0.94} \\
\hline $\begin{array}{c}\text { K-32 } \\
\left(4^{\prime} \times 14^{\prime} \times 10.25 "\right)\end{array}$ & 23.36 & & & & & & & \\
\hline $\begin{array}{c}\text { K-33 } \\
\left(4^{\prime} \times 14^{\prime} \times 10.25 "\right)\end{array}$ & 20.50 & & & & & & & \\
\hline
\end{tabular}


Table 6.18 - Comparison of the results from different codes and experimental data for shear resistance of SIP panels (continued)

\begin{tabular}{|c|c|c|c|c|c|c|c|c|}
\hline \multirow{2}{*}{ Specimen } & \multirow{2}{*}{$\begin{array}{c}\mathrm{V}_{\mathrm{r}(\text { Exp. })} \\
(\mathbf{k N})\end{array}$} & \multirow{2}{*}{$\begin{array}{l}\text { Characteristic } \\
\text { value, } \mathbf{V}_{\text {r(exp.) }} \\
\text { (kN) }\end{array}$} & \multicolumn{3}{|c|}{ Shear resistance $(\mathrm{kN})$} & \multicolumn{3}{|c|}{ Ratio $\left(\mathbf{V}_{\text {Code }} / \mathbf{V}_{\mathbf{r ( E x p . )}}\right)$} \\
\hline & & & $\begin{array}{c}\text { CSA- } \\
\text { O86-09 }\end{array}$ & $\begin{array}{c}\text { DIAB } \\
\text { Handbook }\end{array}$ & $\begin{array}{c}\text { APA } \\
\text { Standard }\end{array}$ & $\begin{array}{c}\text { CSA-O86- } \\
09\end{array}$ & $\begin{array}{c}\text { DIAB } \\
\text { Handbook }\end{array}$ & $\begin{array}{c}\text { APA } \\
\text { Standard }\end{array}$ \\
\hline $\begin{array}{c}\text { M-37 } \\
\text { (4'x16'x 10.25") }\end{array}$ & 20.83 & \multirow{3}{*}{18.93} & \multirow{3}{*}{30.29} & \multirow{3}{*}{19.48} & \multirow{3}{*}{18.87} & \multirow{3}{*}{1.60} & \multirow{3}{*}{1.03} & \multirow{3}{*}{1.00} \\
\hline $\begin{array}{c}\mathrm{M}-38 \\
\left(4^{\prime} \times 16^{\prime} \times 10.25 "\right)\end{array}$ & 22.81 & & & & & & & \\
\hline $\begin{array}{c}\text { M-39 } \\
\left(4^{\prime} \times 16^{\prime} \times 10.25^{\prime \prime}\right)\end{array}$ & 22.03 & & & & & & & \\
\hline
\end{tabular}




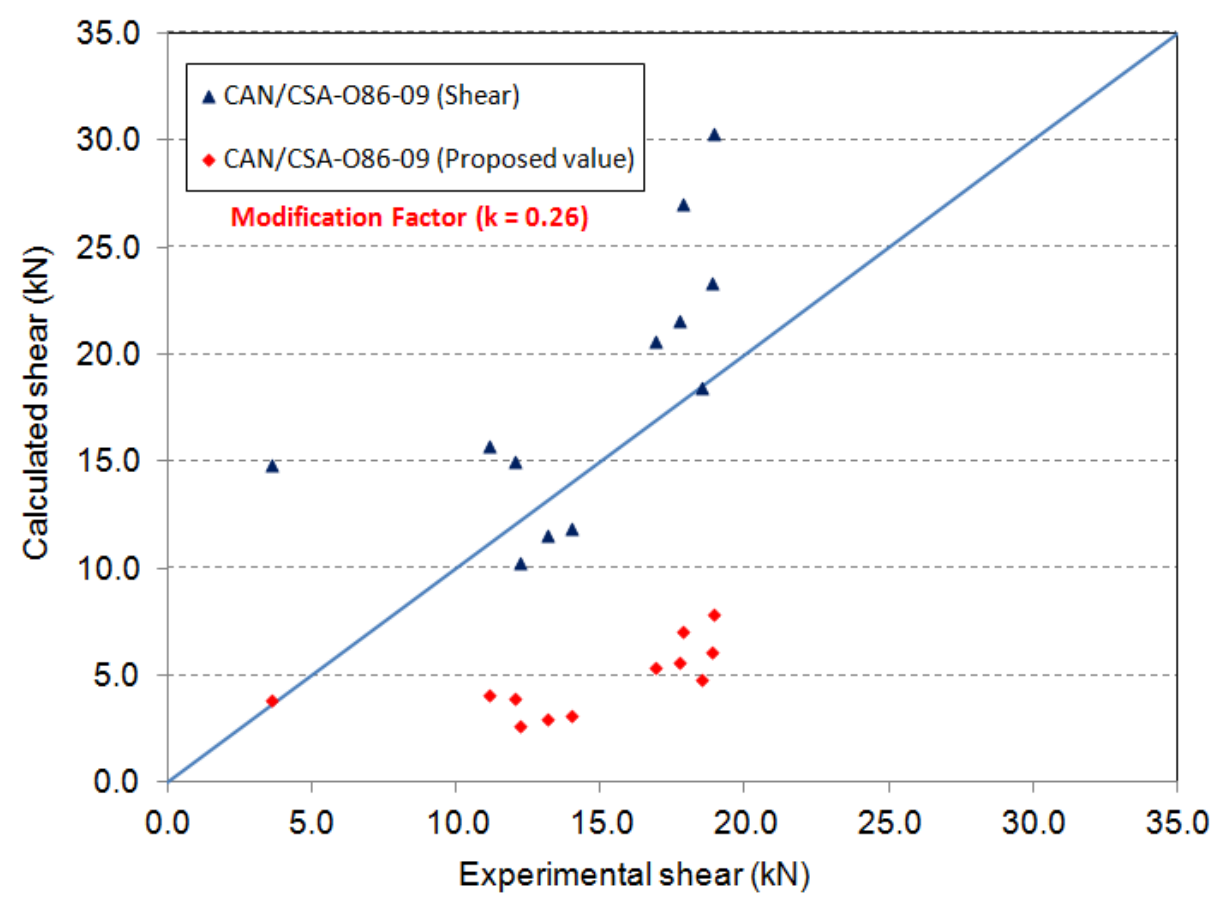

Figure 6.4 - Correlation between the resisting shear force obtained experimentally and that obtained using CSA-O86-09 along with the values using the proposed modification factor for flexural loading on SIP panels

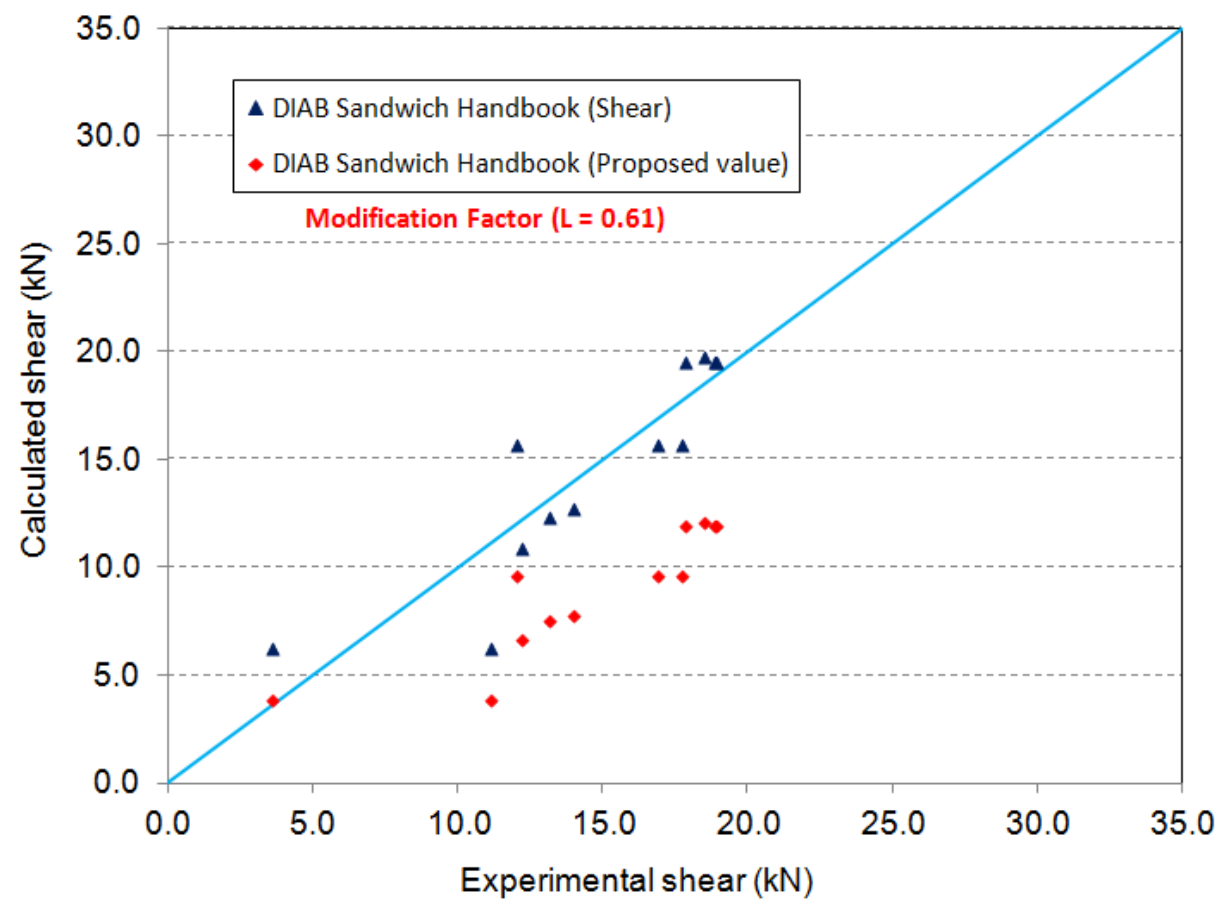

Figure 6.5 - Correlation between the resisting shear force obtained experimentally and that obtained using DIAB Handbook along with the values using the proposed modification factor for flexural loading on SIP panels 


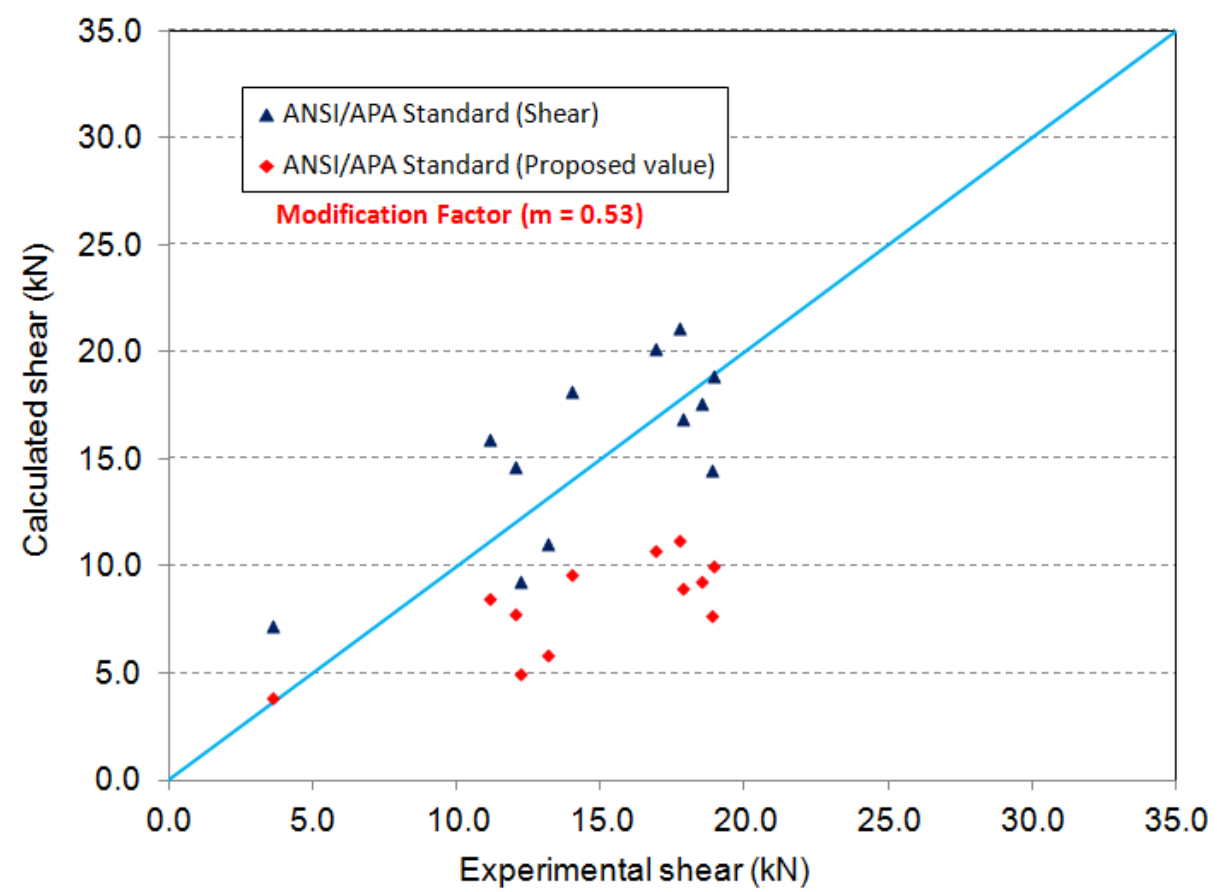

Figure 6.6 - Correlation between the resisting shear force obtained experimentally and that obtained using ANSI/APA along with the values using the proposed modification factor for flexural loading on SIP panels 
Table 6.19 - Comparison of the results from different codes and experimental data for deflection of SIP panels under flexural loading

\begin{tabular}{|c|c|c|c|c|c|c|c|c|}
\hline \multirow{2}{*}{ Specimen } & \multirow{2}{*}{$\begin{array}{l}\text { Deflect.(Exp.) } \\
\text { (mm) }\end{array}$} & \multirow{2}{*}{$\begin{array}{l}\text { Characteristic } \\
\text { value, Deflect.(exp.) } \\
\text { (mm) }\end{array}$} & \multicolumn{3}{|c|}{ Deflection (mm) } & \multicolumn{3}{|c|}{ Ratio (Deflect.Code/ Deflect.(Exp.)) } \\
\hline & & & $\begin{array}{c}\text { CSA- } \\
\text { O86-09 }\end{array}$ & $\begin{array}{c}\text { DIAB } \\
\text { Handbook }\end{array}$ & $\begin{array}{c}\text { APA } \\
\text { Standard }\end{array}$ & $\begin{array}{c}\text { CSA- } \\
\text { O86-09 }\end{array}$ & $\begin{array}{c}\text { DIAB } \\
\text { Handbook }\end{array}$ & $\begin{array}{c}\text { APA } \\
\text { Standard }\end{array}$ \\
\hline $\begin{array}{c}\text { SIP-F-01 } \\
\text { (4'x9'x 6.5") }\end{array}$ & 7.20 & \multirow{3}{*}{7.20} & \multirow{3}{*}{9.64} & \multirow{3}{*}{18.26} & \multirow{3}{*}{7.53} & \multirow{3}{*}{1.34} & \multirow{3}{*}{2.54} & \multirow{3}{*}{1.05} \\
\hline $\begin{array}{c}\text { SIP-F-02 } \\
\left(4^{\prime} \times 9 \text { 'x } 6.5 "\right)\end{array}$ & 7.20 & & & & & & & \\
\hline $\begin{array}{c}\text { SIP-F-03 } \\
\left(4^{\prime} \times 9 \text { 'x } 6.5 "\right)\end{array}$ & 7.20 & & & & & & & \\
\hline $\begin{array}{c}\text { A-13 } \\
\left(2^{\prime} \times 8 \text { 'x 6.5") }\right.\end{array}$ & 2.89 & \multirow{3}{*}{2.77} & \multirow{3}{*}{1.54} & \multirow{3}{*}{4.17} & \multirow{3}{*}{2.20} & \multirow{3}{*}{0.56} & \multirow{3}{*}{1.51} & \multirow{3}{*}{0.79} \\
\hline $\begin{array}{c}\text { A-14 } \\
\left(2^{\prime} \times 8 \text { 'x } 6.5^{\prime \prime}\right)\end{array}$ & 2.90 & & & & & & & \\
\hline $\begin{array}{c}\mathrm{A}-15 \\
\left(2 \mathrm{\prime} \times 8^{\prime} \times 6.5^{\prime \prime}\right)\end{array}$ & 2.98 & & & & & & & \\
\hline
\end{tabular}


Table 6.19 - Comparison of the results from different codes and experimental data for deflection of SIP panels under flexural loading (continued)

\begin{tabular}{|c|c|c|c|c|c|c|c|c|}
\hline \multirow{2}{*}{ Specimen } & \multirow{2}{*}{$\begin{array}{c}\text { Deflect.(Exp.) } \\
\text { (mm) }\end{array}$} & \multirow{2}{*}{$\begin{array}{l}\text { Characteristic } \\
\text { value, } \\
\text { Deflect.(exp.) }(\mathrm{mm})\end{array}$} & \multicolumn{3}{|c|}{ Deflection (mm) } & \multicolumn{3}{|c|}{ Ratio (Deflect.Code / Deflect.(Exp.)) } \\
\hline & & & $\begin{array}{c}\text { CSA- } \\
\text { 086-09 }\end{array}$ & $\begin{array}{c}\text { DIAB } \\
\text { Handbook }\end{array}$ & $\begin{array}{c}\text { APA } \\
\text { Standard }\end{array}$ & $\begin{array}{c}\text { CSA- } \\
\text { 086-09 }\end{array}$ & $\begin{array}{c}\text { DIAB } \\
\text { Handbook }\end{array}$ & $\begin{array}{c}\text { APA } \\
\text { Standard }\end{array}$ \\
\hline $\begin{array}{c}\text { A-16 } \\
(2 ' \times 16 ' \times 10.25 ")\end{array}$ & 17.20 & \multirow{3}{*}{16.39} & \multirow{3}{*}{27.89} & \multirow{3}{*}{48.85} & \multirow{3}{*}{17.60} & \multirow{3}{*}{1.70} & \multirow{3}{*}{2.98} & \multirow{3}{*}{1.07} \\
\hline $\begin{array}{c}\mathrm{A}-17 \\
\left(2^{\prime} \times 16^{\prime} \times 10.25^{\prime \prime}\right)\end{array}$ & 17.10 & & & & & & & \\
\hline $\begin{array}{c}\mathrm{A}-18 \\
\left(2^{\prime} \times 16 ' \times 10.25 ”\right)\end{array}$ & 16.80 & & & & & & & \\
\hline
\end{tabular}


Table 6.19 - Comparison of the results from different codes and experimental data for deflection of SIP panels under flexural loading (continued)

\begin{tabular}{|c|c|c|c|c|c|c|c|c|}
\hline \multirow{2}{*}{ Specimen } & \multirow{2}{*}{$\begin{array}{c}\text { Deflect.(Exp.) } \\
\text { (mm) }\end{array}$} & \multirow{2}{*}{$\begin{array}{c}\text { Characteristic } \\
\text { value, } \\
\text { Deflect.(exp.) }(\mathrm{mm})\end{array}$} & \multicolumn{3}{|c|}{ Deflection (mm) } & \multicolumn{3}{|c|}{ Ratio (Deflect.Code / Deflect.(Exp.) $_{\text {) }}$} \\
\hline & & & $\begin{array}{c}\text { CSA- } \\
\text { O86-09 }\end{array}$ & $\begin{array}{c}\text { DIAB } \\
\text { Handbook }\end{array}$ & $\begin{array}{c}\text { APA } \\
\text { Standard }\end{array}$ & $\begin{array}{c}\text { CSA- } \\
\text { O86-09 }\end{array}$ & $\begin{array}{c}\text { DIAB } \\
\text { Handbook }\end{array}$ & $\begin{array}{c}\text { APA } \\
\text { Standard }\end{array}$ \\
\hline $\begin{array}{c}\text { WS-19 } \\
\left(4^{\prime} \times 9 ' x 6.5 "\right)\end{array}$ & 7.20 & \multirow{5}{*}{7.20} & \multirow{5}{*}{9.12} & \multirow{5}{*}{16.32} & \multirow{5}{*}{8.74} & \multirow{5}{*}{1.27} & \multirow{5}{*}{2.27} & \multirow{5}{*}{1.21} \\
\hline $\begin{array}{c}\text { WS-20 } \\
\left(4^{\prime} \times 9 \text { 'x } 6.5^{\prime \prime}\right)\end{array}$ & 7.20 & & & & & & & \\
\hline $\begin{array}{c}\text { WS-21 } \\
\left(4^{\prime} \times 9 ' x 6.5 "\right)\end{array}$ & 7.20 & & & & & & & \\
\hline $\begin{array}{c}\text { WS-22 } \\
\left(4^{\prime} \times 9 ' x 6.5 "\right)\end{array}$ & 7.20 & & & & & & & \\
\hline $\begin{array}{c}\text { WS-23 } \\
\left(4^{\prime} \times 9 ' x 6.5 "\right)\end{array}$ & 7.20 & & & & & & & \\
\hline
\end{tabular}


Table 6.19 - Comparison of the results from different codes and experimental data for deflection of SIP panels under flexural loading (continued)

\begin{tabular}{|c|c|c|c|c|c|c|c|c|}
\hline \multirow{2}{*}{ Specimen } & \multirow{2}{*}{$\begin{array}{c}\text { Deflect.(Exp.) } \\
\text { (mm) }\end{array}$} & \multirow{2}{*}{$\begin{array}{c}\text { Characteristic } \\
\text { value, Deflect.(exp.) } \\
\text { (mm) }\end{array}$} & \multicolumn{3}{|c|}{ Deflection (mm) } & \multicolumn{3}{|c|}{ Ratio (Deflect.Code / Deflect.(Exp.) } \\
\hline & & & $\begin{array}{c}\text { CSA- } \\
\text { 086-09 }\end{array}$ & $\begin{array}{c}\text { DIAB } \\
\text { Handbook }\end{array}$ & $\begin{array}{c}\text { APA } \\
\text { Standard }\end{array}$ & $\begin{array}{c}\text { CSA- } \\
\text { 086-09 }\end{array}$ & $\begin{array}{c}\text { DIAB } \\
\text { Handbook }\end{array}$ & $\begin{array}{c}\text { APA } \\
\text { Standard }\end{array}$ \\
\hline $\begin{array}{c}\text { B-4 } \\
\left(4^{\prime} \times 10^{\prime} \times 6.5^{\prime \prime}\right)\end{array}$ & 4.90 & \multirow{3}{*}{4.50} & \multirow{3}{*}{1.61} & \multirow{3}{*}{6.77} & \multirow{3}{*}{4.88} & \multirow{3}{*}{0.36} & \multirow{3}{*}{1.50} & \multirow{3}{*}{1.08} \\
\hline $\begin{array}{c}\text { B-5 } \\
\left(4^{\prime} \times 10^{\prime} \times 6.5^{\prime \prime}\right)\end{array}$ & 4.70 & & & & & & & \\
\hline $\begin{array}{c}\text { B-43 } \\
\left(4^{\prime} \times 10^{\prime} \times 6.5^{\prime \prime}\right)\end{array}$ & 4.85 & & & & & & & \\
\hline $\begin{array}{c}\text { D-10 } \\
\left(4^{\prime} \times 12^{\prime} \times 6.5^{\prime \prime}\right)\end{array}$ & 9.52 & \multirow{3}{*}{6.92} & \multirow{3}{*}{13.40} & \multirow{3}{*}{17.07} & \multirow{3}{*}{8.38} & \multirow{3}{*}{1.94} & \multirow{3}{*}{2.47} & \multirow{3}{*}{1.21} \\
\hline $\begin{array}{c}\text { D-11 } \\
\left(4^{\prime} \times 12^{\prime} \times 6.5^{\prime \prime}\right)\end{array}$ & 10.76 & & & & & & & \\
\hline $\begin{array}{c}\mathrm{D}-44 \\
\left(4^{\prime} \times 12^{\prime} \times 6.5^{\prime \prime}\right)\end{array}$ & 8.73 & & & & & & & \\
\hline
\end{tabular}


Table 6.19 - Comparison of the results from different codes and experimental data for SIP deflection under flexural loading (continued)

\begin{tabular}{|c|c|c|c|c|c|c|c|c|}
\hline \multirow[b]{2}{*}{ Specimen } & \multirow[b]{2}{*}{$\begin{array}{c}\text { Deflect.(Exp.) } \\
\text { (mm) }\end{array}$} & \multirow{2}{*}{$\begin{array}{c}\text { Characteristic } \\
\text { value, } \\
\text { Deflect.(exp.) } \\
\text { (mm) }\end{array}$} & \multicolumn{3}{|c|}{ Deflection (mm) } & \multicolumn{3}{|c|}{ Ratio (Deflect.Code / Deflect.(Exp.) } \\
\hline & & & $\begin{array}{c}\text { CSA- } \\
\text { O86-09 }\end{array}$ & $\begin{array}{c}\text { DIAB } \\
\text { Handbook }\end{array}$ & $\begin{array}{c}\text { APA } \\
\text { Standard }\end{array}$ & $\begin{array}{c}\text { CSA- } \\
\text { O86-09 }\end{array}$ & $\begin{array}{c}\text { DIAB } \\
\text { Handbook }\end{array}$ & $\begin{array}{c}\text { APA } \\
\text { Standard }\end{array}$ \\
\hline $\begin{array}{c}\mathrm{E}-13 \\
\left(4^{\prime} \times 10^{\prime} \times 8.25^{\prime \prime}\right)\end{array}$ & 4.10 & \multirow{3}{*}{3.41} & \multirow{3}{*}{3.06} & \multirow{3}{*}{7.78} & \multirow{3}{*}{4.41} & \multirow{3}{*}{0.90} & \multirow{3}{*}{2.28} & \multirow{3}{*}{1.29} \\
\hline $\begin{array}{c}\text { E-14 } \\
\left(4^{\prime} \times 10 ' x 8.25^{\prime \prime}\right)\end{array}$ & 3.75 & & & & & & & \\
\hline $\begin{array}{c}\text { E-15-1 } \\
\left(4^{\prime} \times 10 ' x 8.25^{\prime \prime}\right)\end{array}$ & 3.94 & & & & & & & \\
\hline $\begin{array}{c}\mathrm{H}-22 \\
\left(4^{\prime} \times 14^{\prime} \times 8.25^{\prime \prime}\right)\end{array}$ & 12.03 & \multirow{3}{*}{9.97} & \multirow{3}{*}{12.44} & \multirow{3}{*}{21.56} & \multirow{3}{*}{13.26} & \multirow{3}{*}{1.25} & \multirow{3}{*}{2.16} & \multirow{3}{*}{1.33} \\
\hline $\begin{array}{c}\text { H-23-1 } \\
\left(4^{\prime} \times 14 \text { 'x } 8.25^{\prime \prime}\right)\end{array}$ & 11.11 & & & & & & & \\
\hline $\begin{array}{c}\mathrm{H}-24 \\
\left(4^{\prime} \mathrm{x} 14 \text { 'x } 8.25^{\prime \prime}\right)\end{array}$ & 11.20 & & & & & & & \\
\hline
\end{tabular}


Table 6.19 - Comparison of the results from different codes and experimental data for deflection of SIP panels under flexural loading (continued)

\begin{tabular}{|c|c|c|c|c|c|c|c|c|}
\hline \multirow{2}{*}{ Specimen } & \multirow{2}{*}{$\begin{array}{l}\text { Deflect.(Exp.) } \\
\text { (mm) }\end{array}$} & \multirow{2}{*}{$\begin{array}{l}\text { Characteristic } \\
\text { value, } \\
\text { Deflect.(exp.) } \\
\text { (mm) }\end{array}$} & \multicolumn{3}{|c|}{ Deflection (mm) } & \multicolumn{3}{|c|}{ Ratio (Deflect.Code / Deflect.(Exp.)) } \\
\hline & & & $\begin{array}{c}\text { CSA- } \\
\text { O86-09 }\end{array}$ & $\begin{array}{c}\text { DIAB } \\
\text { Handbook }\end{array}$ & $\begin{array}{c}\text { APA } \\
\text { Standard }\end{array}$ & $\begin{array}{c}\text { CSA- } \\
\text { O86-09 }\end{array}$ & $\begin{array}{c}\text { DIAB } \\
\text { Handbook }\end{array}$ & $\begin{array}{c}\text { APA } \\
\text { Standard }\end{array}$ \\
\hline $\begin{array}{c}\text { F-16 } \\
\left(4^{\prime} \times 12 ' x 8.25^{\prime \prime}\right)\end{array}$ & 5.57 & \multirow{5}{*}{4.94} & \multirow{5}{*}{6.56} & \multirow{5}{*}{13.34} & \multirow{5}{*}{7.80} & \multirow{5}{*}{1.33} & \multirow{5}{*}{2.70} & \multirow{5}{*}{1.58} \\
\hline $\begin{array}{c}\text { F-16-1 } \\
\left(4^{\prime} \times 12^{\prime} \times 8.25 "\right)\end{array}$ & 6.94 & & & & & & & \\
\hline $\begin{array}{c}\text { F-17 } \\
\left(4{ }^{\prime} \times 12^{\prime} \times 8.25 "\right)\end{array}$ & 5.97 & & & & & & & \\
\hline $\begin{array}{c}\text { F-18 } \\
\left(4^{\prime} \times 12 ' x 8.25^{\prime \prime}\right)\end{array}$ & 6.54 & & & & & & & \\
\hline $\begin{array}{c}\text { F-19 } \\
\left(4^{\prime} \times 12 ' x 8.25^{\prime \prime}\right)\end{array}$ & 5.26 & & & & & & & \\
\hline
\end{tabular}


Table 6.19 - Comparison of the results from different codes and experimental data for deflection of SIP panels under flexural loading (continued)

\begin{tabular}{|c|c|c|c|c|c|c|c|c|}
\hline \multirow{2}{*}{ Specimen } & \multirow{2}{*}{$\begin{array}{c}\text { Deflect.(Exp.) } \\
\text { (mm) }\end{array}$} & \multirow{2}{*}{$\begin{array}{c}\text { Characteristic } \\
\text { value, } \\
\text { Deflect.(exp.) } \\
\text { (mm) }\end{array}$} & \multicolumn{3}{|c|}{ Deflection (mm) } & \multicolumn{3}{|c|}{ Ratio (Deflect.Code / Deflect.(Exp.)) } \\
\hline & & & $\begin{array}{c}\text { CSA- } \\
\text { O86-09 }\end{array}$ & $\begin{array}{c}\text { DIAB } \\
\text { Handbook }\end{array}$ & $\begin{array}{c}\text { APA } \\
\text { Standard }\end{array}$ & $\begin{array}{c}\text { CSA- } \\
\text { 086-09 }\end{array}$ & $\begin{array}{c}\text { DIAB } \\
\text { Handbook }\end{array}$ & $\begin{array}{c}\text { APA } \\
\text { Standard }\end{array}$ \\
\hline $\begin{array}{c}\mathrm{J}-28 \\
\left(4^{\prime} \times 12^{\prime} \times 10.25^{\prime \prime}\right)\end{array}$ & 4.64 & \multirow{3}{*}{4.25} & \multirow{3}{*}{3.43} & \multirow{3}{*}{11.18} & \multirow{3}{*}{3.88} & \multirow{3}{*}{0.81} & \multirow{3}{*}{2.63} & \multirow{3}{*}{0.91} \\
\hline $\begin{array}{c}\mathrm{J}-29 \\
\left(4^{\prime} \times 12^{\prime} \times 10.25^{\prime \prime}\right)\end{array}$ & 5.04 & & & & & & & \\
\hline $\begin{array}{c}\mathrm{J}-30 \\
\left(4^{\prime} \times 12^{\prime} \times 10.25^{\prime \prime}\right)\end{array}$ & 4.97 & & & & & & & \\
\hline $\begin{array}{c}\text { K-31 } \\
\left(4^{\prime} \times 14^{\prime} \times 10.25^{\prime \prime}\right)\end{array}$ & 8.75 & \multirow{3}{*}{7.41} & \multirow{3}{*}{6.49} & \multirow{3}{*}{17.47} & \multirow{3}{*}{6.12} & \multirow{3}{*}{0.88} & \multirow{3}{*}{2.36} & \multirow{3}{*}{0.83} \\
\hline $\begin{array}{c}\text { K-32 } \\
\left(4^{\prime} \times 14^{\prime} \times 10.25^{\prime \prime}\right)\end{array}$ & 8.25 & & & & & & & \\
\hline $\begin{array}{c}\text { K-33 } \\
\left(4^{\prime} \times 14^{\prime} \times 10.25^{\prime \prime}\right)\end{array}$ & 8.13 & & & & & & & \\
\hline
\end{tabular}


Table 6.19 - Comparison of the results from different codes and experimental data for deflection of SIP panels under flexural loading (continued)

\begin{tabular}{|c|c|c|c|c|c|c|c|c|}
\hline \multirow{2}{*}{ Specimen } & \multirow{2}{*}{$\begin{array}{c}\text { Deflect. }_{\text {(Exp.) }} \\
\text { (mm) }\end{array}$} & \multirow{2}{*}{$\begin{array}{l}\text { Characteristic } \\
\text { value, } \\
\text { Deflect.(exp.) } \\
\text { (mm) }\end{array}$} & \multicolumn{3}{|c|}{ Deflection (mm) } & \multicolumn{3}{|c|}{ Ratio (Deflect.Code $/$ Deflect.(Exp.) $_{\text {(E) }}$} \\
\hline & & & $\begin{array}{c}\text { CSA- } \\
\text { O86-09 }\end{array}$ & $\begin{array}{c}\text { DIAB } \\
\text { Handbook }\end{array}$ & $\begin{array}{c}\text { APA } \\
\text { Standard }\end{array}$ & $\begin{array}{c}\text { CSA- } \\
\text { O86-09 }\end{array}$ & $\begin{array}{c}\text { DIAB } \\
\text { Handbook }\end{array}$ & $\begin{array}{c}\text { APA } \\
\text { Standard }\end{array}$ \\
\hline $\begin{array}{c}\text { M-37 } \\
\left(4^{\prime} \times 16 \text { 'x 10.25") }\right.\end{array}$ & 39.65 & \multirow{3}{*}{35.88} & \multirow{3}{*}{11.27} & \multirow{3}{*}{26.17} & \multirow{3}{*}{9.45} & \multirow{3}{*}{0.31} & \multirow{3}{*}{0.73} & \multirow{3}{*}{0.26} \\
\hline $\begin{array}{c}\text { M-38 } \\
\left(4^{\prime} \times 16 \text { 'x 10.25") }\right.\end{array}$ & 43.61 & & & & & & & \\
\hline $\begin{array}{c}\text { M-39 } \\
\left(4^{\prime} \times 16 ' x 10.25 "\right)\end{array}$ & 42.05 & & & & & & & \\
\hline
\end{tabular}




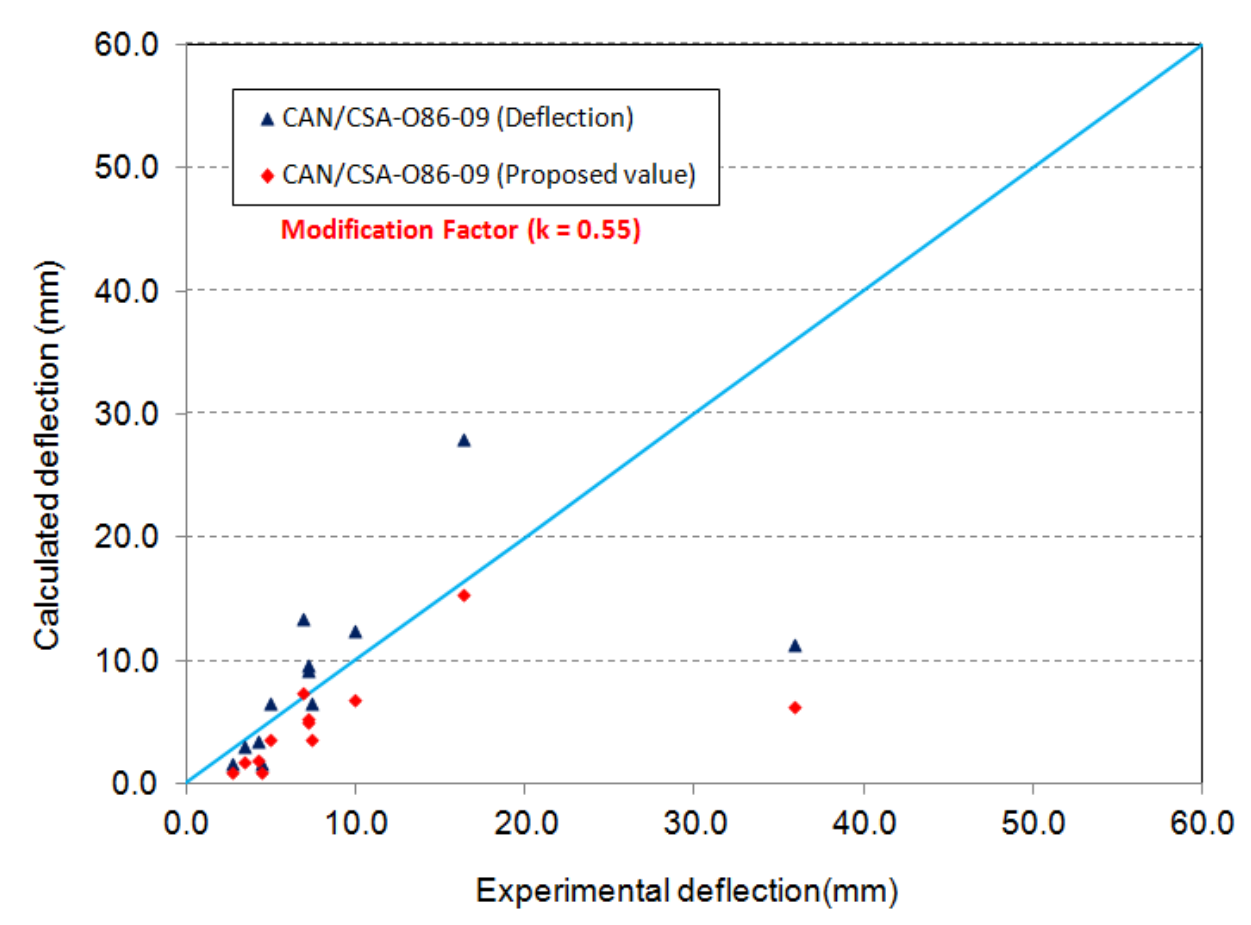

Figure 6.7 - Correlation between the deflection obtained experimentally and that obtained using CSA-O86-09 along with the values using the proposed modification factor for flexural loading on SIP panels

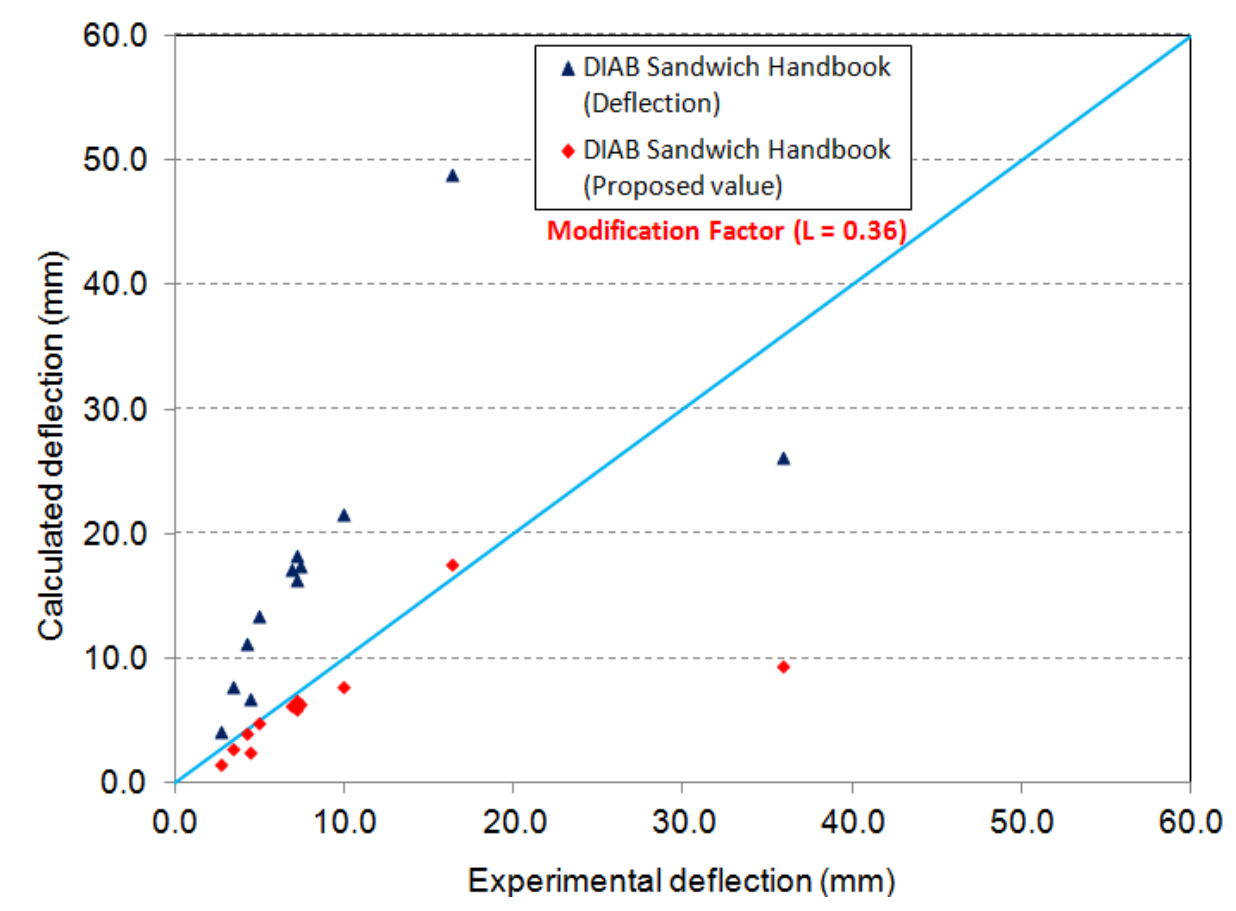

Figure 6.8 - Correlation between the deflection obtained experimentally and that obtained using DIAB Handbook along with the values using the proposed modification factor for flexural loading on SIP panels 


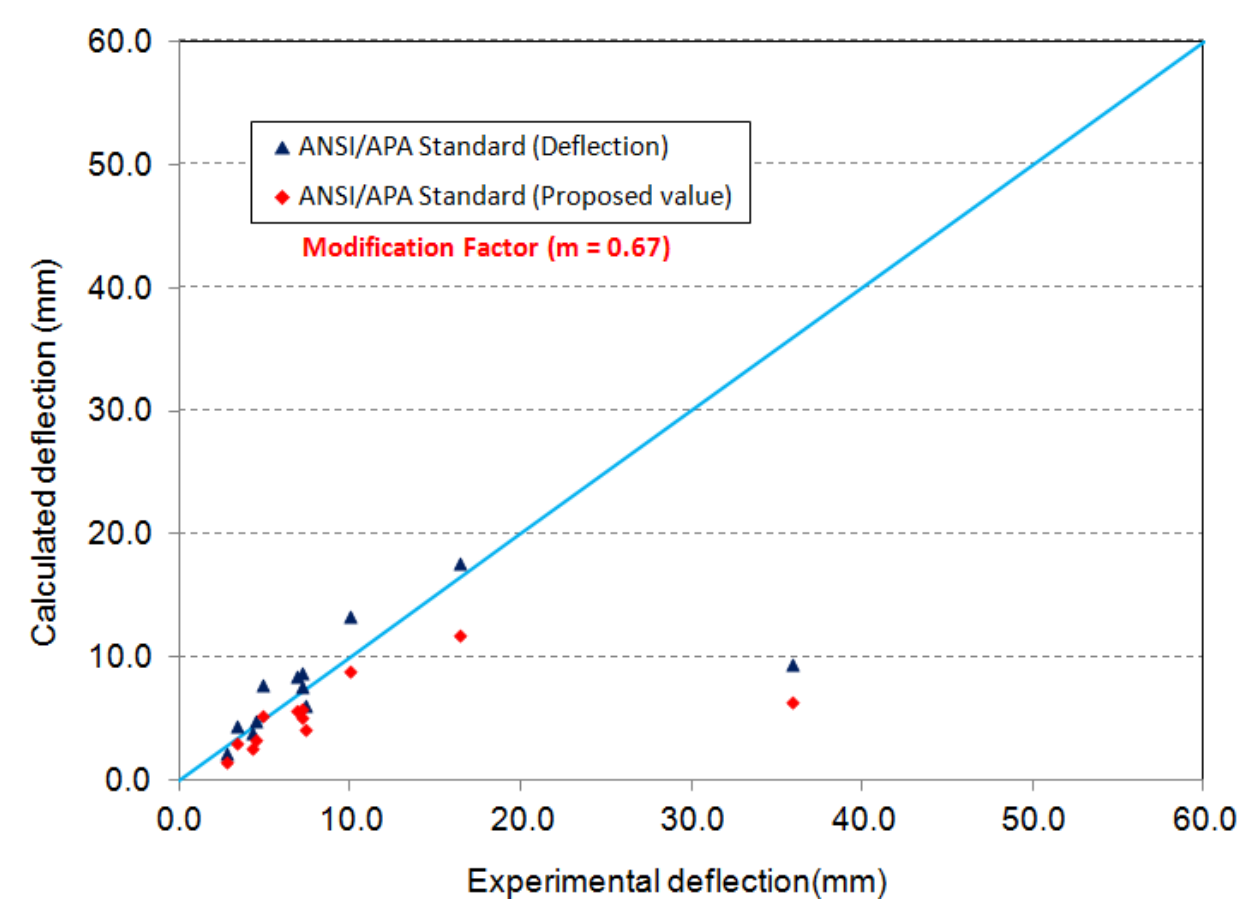

Figure 6.9 - Correlation between the deflection obtained experimentally and that obtained using ANSI/APA along with the values using the proposed modification factor for flexural loading on SIP panels

Table 6.20 - Proposed modification factors for moment and shear resistance as well as the deflection of SIP panels under flexural loading

\begin{tabular}{|c|c|c|c|c|c|c|c|c|c|}
\hline \multirow{2}{*}{$\begin{array}{c}\text { Modification } \\
\text { factor }\end{array}$} & \multicolumn{3}{|c|}{ Moment resistance } & \multicolumn{3}{c|}{ Shear Resistance } & \multicolumn{3}{c|}{ Deflection } \\
\cline { 2 - 10 } & $\begin{array}{c}\text { CSA- } \\
\text { O86-09 }\end{array}$ & $\begin{array}{c}\text { DIAB } \\
\text { Hand. }\end{array}$ & $\begin{array}{c}\text { ANSI/ } \\
\text { APA }\end{array}$ & $\begin{array}{c}\text { CSA- } \\
\text { O86-09 }\end{array}$ & $\begin{array}{c}\text { DIAB } \\
\text { Hand. }\end{array}$ & $\begin{array}{c}\text { ANSI/ } \\
\text { APA }\end{array}$ & $\begin{array}{c}\text { CSA- } \\
\text { O86-09 }\end{array}$ & $\begin{array}{c}\text { DIAB } \\
\text { Hand. }\end{array}$ & $\begin{array}{c}\text { ANSI/ } \\
\text { APA }\end{array}$ \\
\hline $\mathbf{k}$ & 0.46 & 0.27 & 0.27 & 0.26 & 0.61 & 0.53 & 0.55 & 0.36 & 0.67 \\
\hline
\end{tabular}

\subsubsection{Compressive loading on SIP walls}

The compressive resistances of the studied SIP walls when subjected to compressive loading with eccentricity of $t / 6$ per the test method or without load eccentricity were calculated using different codes and experimentally. Table 6.21 shows the results obtained for compressive resistances for the studied SIP walls in this research as well as for SIP panels tested elsewhere (Mohamed, 2009). Three different codes were considered to determine the wall compressive resistance with zero initial eccentricity, namely: NTA IM14 TIP01, DIAB Handbook and ANSI/APA standard. Table 6.21 shows the ratios between the resisting compressive forces 
obtained from code equations and the experimental data considering zero initial eccentricities. It can be observed that the equations in DIAB Handbook and ANSI/APA standards provide compressive resistances less than those obtained experimentally, while NTA IM14 TIP01 Standard provides values of compressive resistance of SIP walls greater than those obtained experimentally. As such, modification factors were proposed, as depicted in Table 6.22, to be 0.33, 0.79 and 0.51 for compressive resistance values obtained from NTA IM14 TIP01, DIAB Handbook and ANSI/APA standard, respectively. To provide confidence on the proposed modification factors, Figures $6.10,6.11$ and 6.12 present the ccorrelation between the axial compressive resistance obtained experimentally and those obtained using NTA IM14, DIAB Handbook and APA Standard, along with the values using the proposed modification factor for compressive loading on SIP walls with zero eccentricity. 
Table 6.21 - Results from different codes and experimental data for axial compressive load on SIP walls with zero eccentricity

\begin{tabular}{|c|c|c|c|c|c|c|c|c|c|}
\hline \multirow[b]{2}{*}{ Specimen $*$} & \multirow{2}{*}{$\begin{array}{c}\text { Exp. } \\
\text { Ultim. } \\
\text { axial } \\
\text { load } \\
(k N)^{(1)}\end{array}$} & \multirow{2}{*}{$\begin{array}{c}\text { Modified } \\
\text { Exp. } \\
\text { Ultimate } \\
\text { axial load } \\
(\mathrm{kN})^{(2)}\end{array}$} & \multirow{2}{*}{$\begin{array}{c}\text { Character. } \\
\text { value, } \\
\text { Pr(exp.) }_{(k N)}\end{array}$} & \multicolumn{3}{|c|}{ Axial compressive resistance $(\mathrm{kN})$} & \multicolumn{3}{|c|}{ Ratio ( $\left.\mathbf{P}_{\mathbf{r}(\text { code })} / \mathbf{P}_{\mathbf{r}(\text { Exp. })}\right)$} \\
\hline & & & & $\begin{array}{c}\text { NTA IM } 14 \\
\text { TIP01 }\end{array}$ & $\begin{array}{c}\text { DIAB's } \\
\text { Handbook }\end{array}$ & $\begin{array}{c}\text { APA } \\
\text { Standard }\end{array}$ & $\begin{array}{c}\text { NTA IM } 14 \\
\text { TIP01 }\end{array}$ & $\begin{array}{c}\text { DIAB's } \\
\text { Handbook }\end{array}$ & $\begin{array}{c}\text { APA } \\
\text { Standard }\end{array}$ \\
\hline $\begin{array}{c}\mathrm{W}-4 \\
1220 \times 2743 \times 165 \mathrm{~mm} \\
\left(4^{\prime} \times 9 ' \times 6.5 "\right)\end{array}$ & 286.35 & 218.79 & & & & & & & \\
\hline $\begin{array}{c}\text { W-5 } \\
1220 \times 2743 \times 165 \mathrm{~mm} \\
\left(4{ }^{\prime} \times 9 ' \times 6.5^{\prime \prime}\right)\end{array}$ & 286.50 & 287.75 & 218.79 & 388.12 & 117.54 & 125.20 & 1.77 & 0.54 & 0.57 \\
\hline $\begin{array}{c}\text { W-6 } \\
1220 \times 2743 \times 165 \mathrm{~mm} \\
\left(4{ }^{\prime} \times 9 ' \times 6.5 "\right)\end{array}$ & 344.64 & 345.89 & & & & & & & \\
\hline
\end{tabular}

* Mohamed (2009)

(1) Did not include $1.25 \mathrm{kN}$ weight of the loading system.

(2) Included $1.25 \mathrm{kN}$ weight of the loading system. 
Table 6.21 - Results from different codes and experimental data for axial compressive load on SIP walls with zero eccentricity (continued)

\begin{tabular}{|c|c|c|c|c|c|c|c|c|c|}
\hline \multirow[b]{2}{*}{ Specimen * } & \multirow{2}{*}{$\begin{array}{c}\text { Exp. } \\
\text { Ultim. } \\
\text { axial } \\
\text { load } \\
(\mathbf{k N})^{(1)}\end{array}$} & \multirow{2}{*}{$\begin{array}{c}\text { Modified } \\
\text { Exp. } \\
\text { Ultimate } \\
\text { axial load } \\
\text { (kN) }^{(2)}\end{array}$} & \multirow[b]{2}{*}{$\begin{array}{c}\text { Character. } \\
\text { value, } \mathbf{P}_{\text {r(exp.) }} \\
\text { (kN) }\end{array}$} & \multicolumn{3}{|c|}{ Axial compressive resistance (kN) } & \multicolumn{3}{|c|}{ Ratio $\left(\mathbf{P}_{\mathbf{r}(\text { code })} / \mathbf{P}_{\mathbf{r}(\text { Exp. })}\right)$} \\
\hline & & & & $\begin{array}{c}\text { NTA IM } 14 \\
\text { TIP01 }\end{array}$ & $\begin{array}{c}\text { DIAB's } \\
\text { Handbook }\end{array}$ & $\begin{array}{c}\text { APA } \\
\text { Standard }\end{array}$ & $\begin{array}{c}\text { NTA IM } 14 \\
\text { TIP01 }\end{array}$ & $\begin{array}{c}\text { DIAB's } \\
\text { Handbook }\end{array}$ & $\begin{array}{c}\text { APA } \\
\text { Standard }\end{array}$ \\
\hline $\begin{array}{c}\mathrm{W}-10 \\
1220 \times 3048 \times 165 \\
\mathrm{~mm}\left(4^{\prime} \times 10 ' \times 6.5 "\right)\end{array}$ & 221.70 & 155.46 & \multirow{3}{*}{155.46} & \multirow{3}{*}{252.73} & \multirow{3}{*}{95.21} & \multirow{3}{*}{121.80} & \multirow{3}{*}{1.63} & \multirow{3}{*}{0.61} & \multirow{3}{*}{0.78} \\
\hline $\begin{array}{c}\mathrm{W}-11 \\
1220 \times 3048 \times 165 \\
\mathrm{~mm}\left(4^{\prime} \times 10 \text { 'x6.5") }\right.\end{array}$ & 215.56 & 216.81 & & & & & & & \\
\hline $\begin{array}{c}\mathrm{W}-12 \\
1220 \times 3048 \times 165 \\
\mathrm{~mm}\left(4^{\prime} \times 10 ' \times 6.5^{\prime \prime}\right)\end{array}$ & 186.19 & 187.44 & & & & & & & \\
\hline
\end{tabular}

* Mohamed (2009)

(1) Did not include $1.25 \mathrm{kN}$ weight of the loading system.

(2) Included $1.25 \mathrm{kN}$ weight of the loading system. 
Table 6.21 - Results from different codes and experimental data for axial compressive load on SIP walls with zero eccentricity (continued)

\begin{tabular}{|c|c|c|c|c|c|c|c|c|c|}
\hline \multirow[b]{2}{*}{ Specimen * } & \multirow{2}{*}{$\begin{array}{c}\text { Exp. } \\
\text { Ultim. } \\
\text { axial } \\
\text { load } \\
(\mathbf{k N})^{(1)}\end{array}$} & \multirow{2}{*}{$\begin{array}{c}\text { Modified } \\
\text { Exp. } \\
\text { Ultimate } \\
\text { axial load } \\
(\mathrm{kN})^{(2)}\end{array}$} & \multirow{2}{*}{$\begin{array}{l}\text { Character. } \\
\text { value, } \mathbf{P}_{\mathbf{r}(\exp .)} \\
\text { (kN) }\end{array}$} & \multicolumn{3}{|c|}{ Axial compressive resistance $(\mathbf{k N})$} & \multicolumn{3}{|c|}{ Ratio $\left(\mathbf{P}_{\mathbf{r}(\text { code })} / \mathbf{P}_{\mathbf{r}(\text { Exp. })}\right)$} \\
\hline & & & & $\begin{array}{l}\text { NTA IM } \\
14 \text { TIP01 }\end{array}$ & $\begin{array}{c}\text { DIAB's } \\
\text { Handbook }\end{array}$ & $\begin{array}{c}\text { APA } \\
\text { Standard }\end{array}$ & $\begin{array}{l}\text { NTA IM } \\
14 \text { TIP01 }\end{array}$ & $\begin{array}{c}\text { DIAB's } \\
\text { Handbook }\end{array}$ & $\begin{array}{c}\text { APA } \\
\text { Standard }\end{array}$ \\
\hline $\begin{array}{c}\mathrm{W}-13 \\
1220 \times 3048 \times 165 \\
\mathrm{~mm}\left(4 ' \mathrm{x} 12 ' \times 6.5^{\prime}\right)\end{array}$ & 259.35 & 260.60 & \multirow{3}{*}{169.26} & \multirow{3}{*}{239.82} & \multirow{3}{*}{66.12} & \multirow{3}{*}{115.25} & \multirow{3}{*}{1.42} & \multirow{3}{*}{0.39} & \multirow{3}{*}{0.68} \\
\hline $\begin{array}{c}\mathrm{W}-14 \\
1220 \times 3048 \times 165 \\
\mathrm{~mm}\left(4^{\prime} \times 12 \text { 'x6.5") }\right.\end{array}$ & 217.34 & 218.59 & & & & & & & \\
\hline $\begin{array}{c}\mathrm{W}-15 \\
1220 \times 3048 \times 165 \\
\mathrm{~mm}\left(4^{\prime} \times 12 ' \times 6.5 "\right)\end{array}$ & 218.30 & 219.55 & & & & & & & \\
\hline * Mohamed (2009) & & & & & & & & & \\
\hline
\end{tabular}


Table 6.21 - Results from different codes and experimental data for axial compressive load on SIP walls with zero eccentricity (continued)

\begin{tabular}{|c|c|c|c|c|c|c|c|c|c|}
\hline \multirow[b]{2}{*}{ Specimen * } & \multirow{2}{*}{$\begin{array}{c}\text { Exp. } \\
\text { Ultim. } \\
\text { axial } \\
\text { load } \\
(\mathbf{k N})^{(1)}\end{array}$} & \multirow{2}{*}{$\begin{array}{c}\text { Modified } \\
\text { Exp. } \\
\text { Ultimate } \\
\text { axial load } \\
(\mathbf{k N})^{(2)}\end{array}$} & \multirow{2}{*}{$\begin{array}{c}\text { Character. } \\
\text { value, } P_{\text {r(exp.) }} \\
\text { (kN) }\end{array}$} & \multicolumn{3}{|c|}{ Axial compressive resistance $(\mathrm{kN})$} & \multicolumn{3}{|c|}{ Ratio ( $\left.\mathbf{P}_{\mathbf{r}(\text { code })} / \mathbf{P}_{\mathbf{r}(\text { Exp. })}\right)$} \\
\hline & & & & $\begin{array}{l}\text { NTA IM } \\
14 \text { TIP01 }\end{array}$ & $\begin{array}{c}\text { DIAB's } \\
\text { Handbook }\end{array}$ & $\begin{array}{c}\text { APA } \\
\text { Standard }\end{array}$ & $\begin{array}{l}\text { NTA IM } \\
14 \text { TIP01 }\end{array}$ & $\begin{array}{c}\text { DIAB's } \\
\text { Handbook }\end{array}$ & $\begin{array}{c}\text { APA } \\
\text { Standard }\end{array}$ \\
\hline $\begin{array}{c}\text { W-16 } \\
1220 \times 4877 \times 165 \\
\text { mm (4'x16'x6.5") }\end{array}$ & 209.64 & 210.89 & & & & & & & \\
\hline $\begin{array}{c}\mathrm{W}-17 \\
1220 \times 4877 \times 165 \\
\mathrm{~mm}\left(4^{\prime} \times 16 ' \times 6.5^{\prime \prime}\right)\end{array}$ & 145.14 & 146.39 & 63.91 & 207.48 & 85.01 & 133.40 & 3.25 & 1.33 & 2.09 \\
\hline $\begin{array}{c}\text { W-18 } \\
1220 \times 4877 \times 165 \\
\mathrm{~mm}(4 \text { 'x16'x6.5") }\end{array}$ & 119.91 & 121.16 & & & & & & & \\
\hline
\end{tabular}

* Mohamed (2009)

(1) Did not include $1.25 \mathrm{kN}$ weight of the loading system.

(2) Included $1.25 \mathrm{kN}$ weight of the loading system. 
Table 6.21 - Results from different codes and experimental data for axial compressive load on SIP walls with zero eccentricity (continued)

\begin{tabular}{|c|c|c|c|c|c|c|c|c|c|}
\hline \multirow[b]{2}{*}{ Specimen * } & \multirow{2}{*}{$\begin{array}{c}\text { Exp. } \\
\text { Ultim. } \\
\text { axial } \\
\text { load } \\
(\mathbf{k N})^{(1)}\end{array}$} & \multirow{2}{*}{$\begin{array}{l}\text { Modified } \\
\text { Exp. } \\
\text { Ultimate } \\
\text { axial load } \\
(\mathrm{kN})^{(2)}\end{array}$} & \multirow{2}{*}{$\begin{array}{c}\text { Character. } \\
\text { value, } \\
\text { P }_{\text {r(exp.) }}(k N)\end{array}$} & \multicolumn{3}{|c|}{ Axial compressive resistance $(\mathrm{kN})$} & \multicolumn{3}{|c|}{ Ratio ( $\left.\mathbf{P}_{\mathbf{r}(\text { code })} / \mathbf{P}_{\mathbf{r}(\text { Exp. })}\right)$} \\
\hline & & & & $\begin{array}{c}\text { NTA IM } 14 \\
\text { TIP01 }\end{array}$ & $\begin{array}{c}\text { DIAB's } \\
\text { Handbook }\end{array}$ & $\begin{array}{c}\text { APA } \\
\text { Standard }\end{array}$ & $\begin{array}{c}\text { NTA IM } 14 \\
\text { TIP01 }\end{array}$ & $\begin{array}{c}\text { DIAB's } \\
\text { Handbook }\end{array}$ & $\begin{array}{c}\text { APA } \\
\text { Standard }\end{array}$ \\
\hline $\begin{array}{c}\mathrm{W}-27 \\
1220 \times 2743 \times 165 \\
\mathrm{~mm}\left(4^{\prime} \times 9 ' \times 8.25 "\right)\end{array}$ & 355.81 & 357.06 & & & & & & & \\
\hline $\begin{array}{c}\mathrm{W}-28 \\
1220 \times 2743 \times 165 \\
\mathrm{~mm}\left(4^{\prime} \times 99^{\prime} \times 8.25^{\prime \prime}\right)\end{array}$ & 317.94 & 319.19 & 243.01 & 336.72 & 195.26 & 137.08 & 1.39 & 0.80 & 0.56 \\
\hline $\begin{array}{c}\mathrm{W}-29 \\
1220 \times 2743 \times 165 \\
\mathrm{~mm}\left(4^{\prime} \times 99^{\prime} \times 8.25^{\prime \prime}\right)\end{array}$ & 408.26 & 409.51 & & & & & & & \\
\hline
\end{tabular}




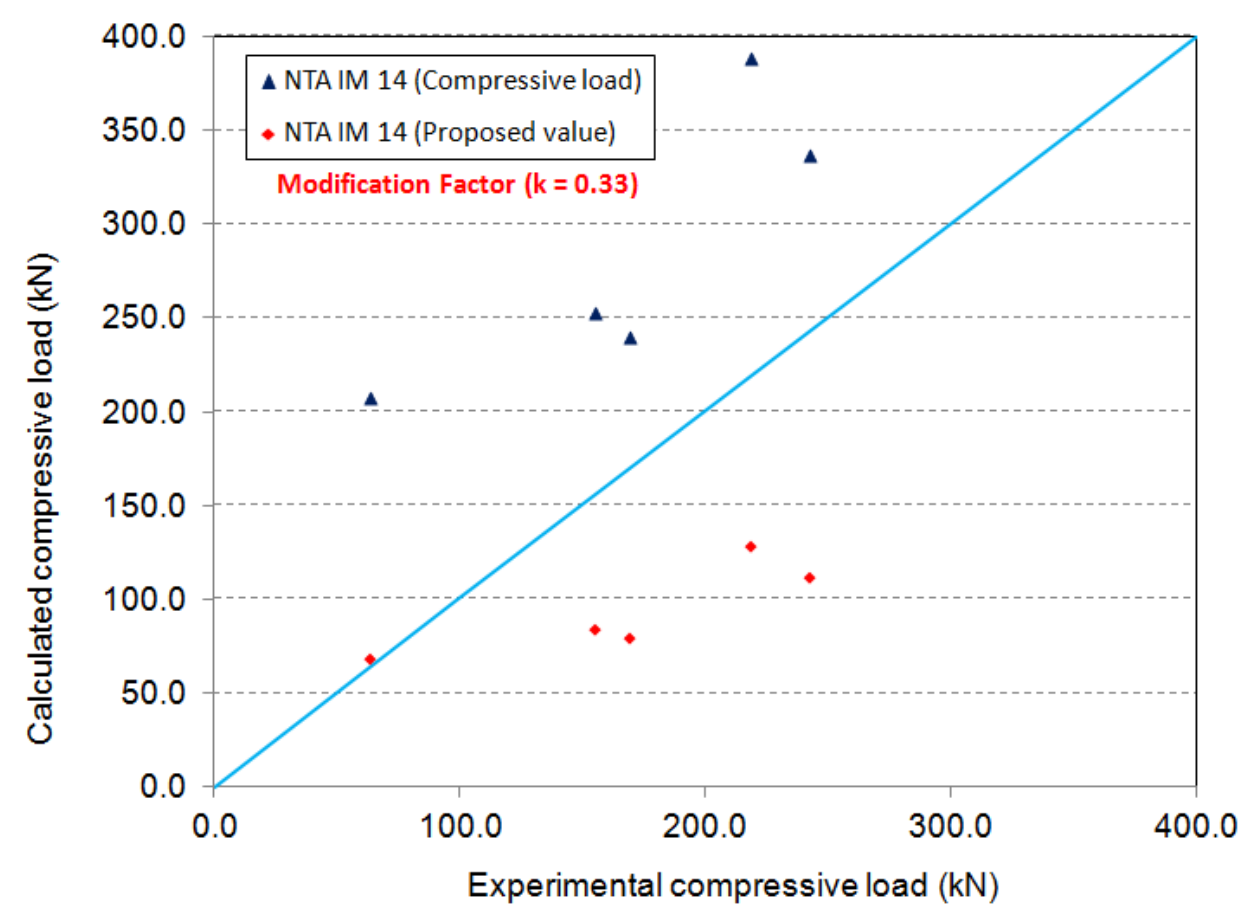

Figure 6.10 - Correlation between the axial compressive resistance obtained experimentally and that obtained using NTA IM14 along with the values using the proposed modification factor for compressive loading on SIP walls with zero eccentricity

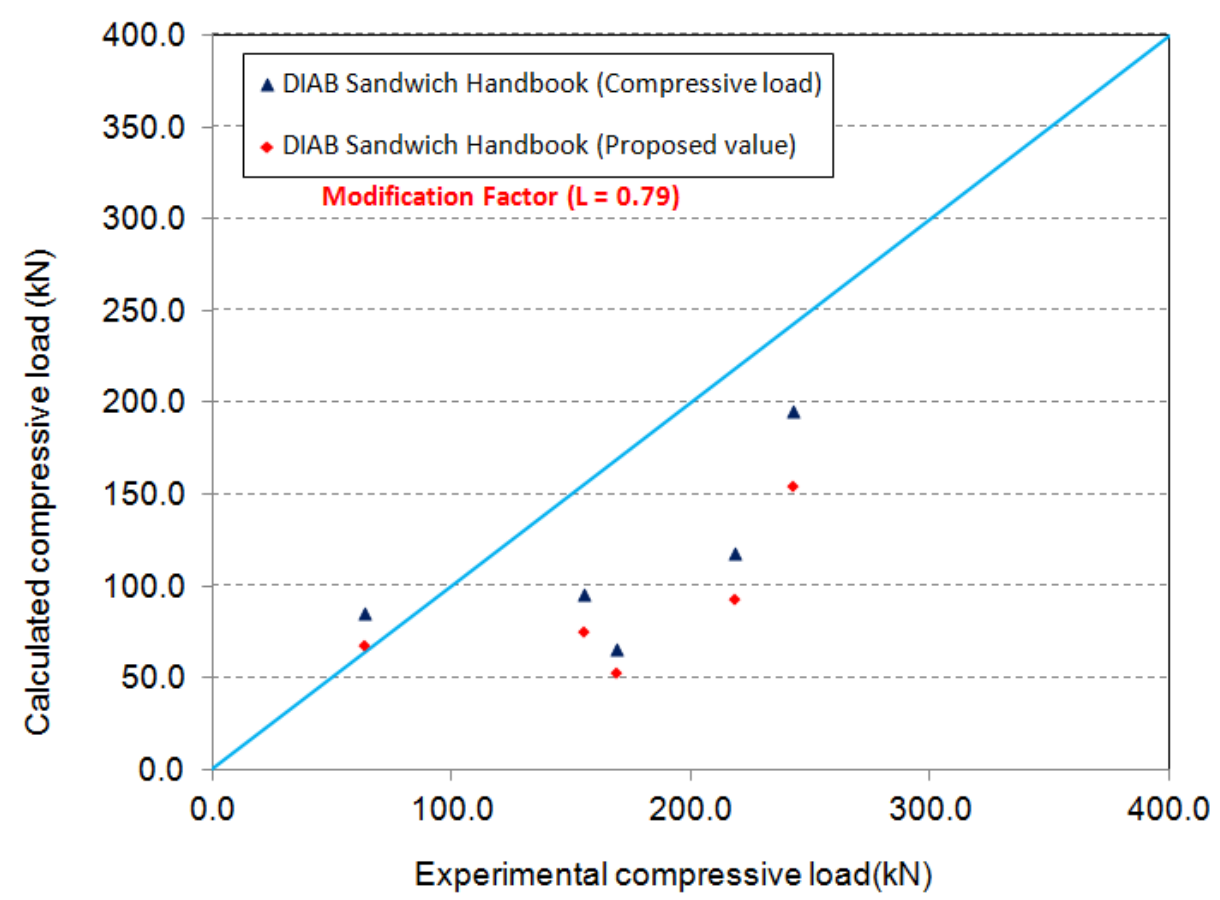

Figure 6.11 - Correlation between the axial compressive resistance obtained experimentally and that obtained using DIAB Handbook along with the values using the proposed modification factor for compressive loading on SIP walls with zero eccentricity 


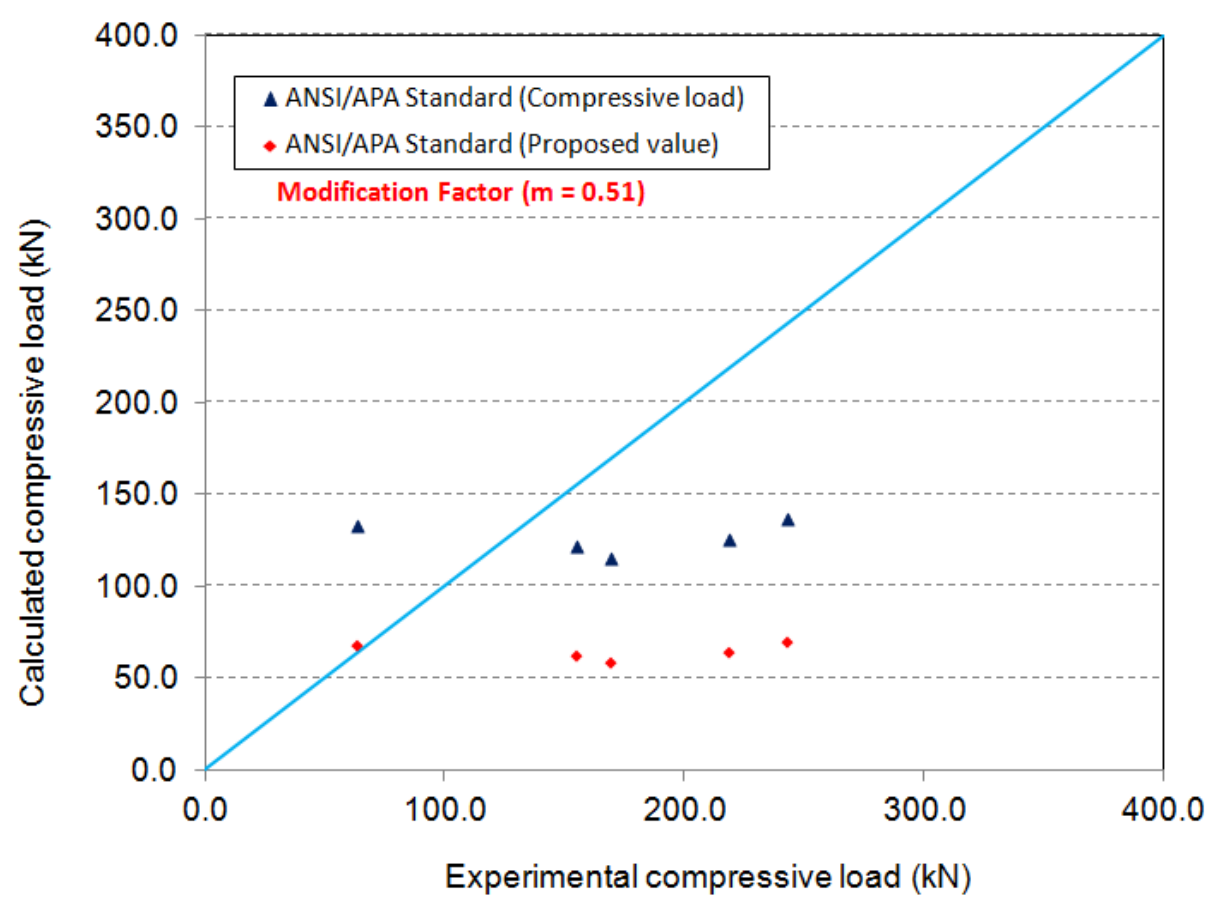

Figure 6.12 - Correlation between the axial compressive resistance obtained experimentally and that obtained using ANSI/APA along with the values using the proposed modification factor for compressive loading on SIP walls with zero eccentricity

Table 6.22 - Proposed modification factors for axial compressive resistance of SIP walls with zero eccentricity

\begin{tabular}{|c|c|c|c|}
\hline \multirow{2}{*}{$\begin{array}{c}\text { Modification } \\
\text { factor }\end{array}$} & \multicolumn{3}{|c|}{ Axial compressive resistance } \\
\cline { 2 - 4 } & NTA IM 14 & $\begin{array}{c}\text { DIAB } \\
\text { Handbook }\end{array}$ & ANSI/APA \\
\hline $\mathbf{k}$ & 0.33 & 0.79 & 0.51 \\
\hline
\end{tabular}

The compressive resistances of the studied SIP walls when subjected to compressive loading with eccentricity of $t / 6$ per the test method were calculated using different codes and experimentally and recorded in Table 6.23. These SIP panels under eccentric compressive loading were tested elsewhere for use in basement wall construction (Sayed Ahmed, 2011; Mohamed, 2009). Three different codes were considered to determine the wall compressive resistance with $\mathrm{t} / 6$ initial eccentricity, namely: CSA-O86-09, DIAB Handbook and ANSI/APA standard. Table 6.23 shows the ratios between the resisting compressive forces obtained from code equations and the experimental data. The corresponding modification factors were 
proposed, as depicted in Table 6.24, to be $0.39,0.47$ and 0.48 for compressive resistance values with $\mathrm{t} / 6$ eccentricity obtained from CSA-O86-09, DIAB Handbook and ANSI/APA standard, respectively. To provide confidence on the proposed modification factors, Figure 6.13 presents the correlation between the compressive resistances obtained experimentally and those obtained using CSA-O86-09 Standard along with the values using the proposed modification factor for compressive loading on SIP walls with t/6 eccentricity. Figures 6.14 and 6.15 also show the correlation between the compressive resistances obtained experimentally and those obtained using DIAB's handbook and ANSI/APA Standard along with the values using the proposed modification factor for compressive loading on SIP walls with $\mathrm{t} / 6$ eccentricity, respectively. 
Table 6.23 - Results from different codes and experimental data for axial compressive load on SIP walls with $\mathrm{t} / 6$ eccentricity

\begin{tabular}{|c|c|c|c|c|c|c|c|c|c|}
\hline \multirow[b]{2}{*}{ Specimen * } & \multirow{2}{*}{$\begin{array}{c}\text { Exp. } \\
\text { Ultimate } \\
\text { axial } \\
\text { load } \\
(\mathbf{k N})^{(1)}\end{array}$} & \multirow{2}{*}{$\begin{array}{c}\text { Modified } \\
\text { Exp. } \\
\text { Ultimate } \\
\text { axial load } \\
(\mathbf{k N})^{(2)}\end{array}$} & \multirow{2}{*}{$\begin{array}{c}\text { Charac. } \\
\text { value, } \\
\text { Pr(exp.) } \\
\text { (kN) }\end{array}$} & \multicolumn{3}{|c|}{ Axial compressive resistance $(\mathrm{kN})$} & \multicolumn{3}{|c|}{ Ratio ( $\left.\mathbf{P}_{\text {r(code) }} / \mathbf{P}_{\text {r(Exp.) }}\right)$} \\
\hline & & & & $\begin{array}{c}\text { CSA- } \\
\text { O86-09 }\end{array}$ & $\begin{array}{c}\text { DIAB's } \\
\text { Handbook }\end{array}$ & ANSI/APA & $\begin{array}{c}\text { CSA- } \\
\text { O86-09 }\end{array}$ & $\begin{array}{c}\text { DIAB's } \\
\text { Handbook }\end{array}$ & ANSI/APA \\
\hline $\begin{array}{c}\text { III-9 } \\
1220 \times 3048 \times 260 \\
m m\left(4^{\prime} \times 10 ' x 10.25 "\right)\end{array}$ & 291.46 & 292.71 & & & & & & & \\
\hline $\begin{array}{c}\text { III-10 } \\
1220 \times 3048 \times 260 \\
\mathrm{~mm}\left(4^{\prime} \times 10 ' \times 10.25 "\right)\end{array}$ & 341.83 & 343.08 & 224.96 & 251.72 & 249.61 & 205.20 & 1.12 & 1.11 & 0.91 \\
\hline $\begin{array}{c}\text { III-11 } \\
1220 \times 3048 \times 260 \\
\text { mm (4'x10'x10.25") }\end{array}$ & 285.47 & 286.72 & & & & & & & \\
\hline
\end{tabular}

* Sayed Ahmed (2011) for SIP basement wall.

(1) Did not include $1.25 \mathrm{kN}$ weight of the loading system.

(2) Included $1.25 \mathrm{kN}$ weight of the loading system. 
Table 6.23 - Results from different codes and experimental data for axial compressive load on SIP walls with t/6 eccentricity (continued)

\begin{tabular}{|c|c|c|c|c|c|c|c|c|c|}
\hline \multirow[b]{2}{*}{ Specimen * } & \multirow{2}{*}{$\begin{array}{c}\text { Exp. } \\
\text { Ultimate } \\
\text { axial } \\
\text { load } \\
(\mathbf{k N})^{(1)}\end{array}$} & \multirow{2}{*}{$\begin{array}{c}\text { Modified } \\
\text { Exp. } \\
\text { Ultimate } \\
\text { axial load } \\
\text { (kN) }^{(2)}\end{array}$} & \multirow{2}{*}{$\begin{array}{c}\text { Charac. } \\
\text { value, } \\
\text { Pr(exp.) }_{\text {(kN) }}\end{array}$} & \multicolumn{3}{|c|}{ Axial compressive resistance $(\mathrm{kN})$} & \multicolumn{3}{|c|}{ Ratio $\left(\mathbf{P}_{\mathbf{r}(\text { code })} / \mathbf{P}_{\mathbf{r}(\text { Exp. })}\right)$} \\
\hline & & & & $\begin{array}{c}\text { CSA- } \\
\text { O86-09 }\end{array}$ & $\begin{array}{c}\text { DIAB's } \\
\text { Handbook }\end{array}$ & ANSI/APA & $\begin{array}{c}\text { CSA- } \\
\text { 086-09 }\end{array}$ & $\begin{array}{c}\text { DIAB's } \\
\text { Handbook }\end{array}$ & ANSI/APA \\
\hline $\begin{array}{c}\text { IV-10 } \\
1220 \times 2743 \times 210 \\
\mathrm{~mm}\left(4^{\prime} \times 99^{\prime} \times 8.25 "\right)\end{array}$ & 173.69 & 174.94 & \multirow{3}{*}{85.82} & \multirow{3}{*}{233.40} & \multirow{3}{*}{195.27} & \multirow{3}{*}{188.10} & \multirow{3}{*}{2.72} & \multirow{3}{*}{2.28} & \multirow{3}{*}{2.19} \\
\hline $\begin{array}{c}\text { IV-11 } \\
1220 \times 2743 \times 210 \\
m m\left(4^{\prime} \times 9 ' x 8.25 "\right)\end{array}$ & 182.86 & 184.11 & & & & & & & \\
\hline $\begin{array}{c}\text { IV-12 } \\
1220 \times 2743 \times 210 \\
\mathrm{~mm}\left(4^{\prime} \times 99^{\prime} \times 8.25^{\prime \prime}\right)\end{array}$ & 292.94 & 294.19 & & & & & & & \\
\hline
\end{tabular}

* Sayed Ahmed (2011) for SIP basement wall.

(1) Did not include $1.25 \mathrm{kN}$ weight of the loading system.

(2) Included $1.25 \mathrm{kN}$ weight of the loading system. 
Table 6.23 - Results from different codes and experimental data for axial compressive load on SIP walls with $t / 6$ eccentricity (continued)

\begin{tabular}{|c|c|c|c|c|c|c|c|c|c|}
\hline \multirow[b]{2}{*}{ Specimen * } & \multirow{2}{*}{$\begin{array}{c}\text { Exp. } \\
\text { Ultim. } \\
\text { axial } \\
\text { load } \\
(k N)^{(1)}\end{array}$} & \multirow{2}{*}{$\begin{array}{c}\text { Modified } \\
\text { Exp. } \\
\text { Ultimate } \\
\text { axial load } \\
(\mathrm{kN})^{(2)}\end{array}$} & \multirow{2}{*}{$\begin{array}{c}\text { Character. } \\
\text { value, } P_{r(\text { exp. })} \\
(k N)\end{array}$} & \multicolumn{3}{|c|}{ Axial compressive resistance $(\mathrm{kN})$} & \multicolumn{3}{|c|}{ Ratio $\left(\mathbf{P}_{\mathbf{r}(\text { code })} / \mathbf{P}_{\mathbf{r}(\text { Exp. })}\right)$} \\
\hline & & & & $\begin{array}{c}\text { CSA- } \\
\text { O86-09 }\end{array}$ & $\begin{array}{c}\text { DIAB's } \\
\text { Handbook }\end{array}$ & ANSI/APA & $\begin{array}{c}\text { CSA- } \\
\text { O86-09 }\end{array}$ & $\begin{array}{c}\text { DIAB's } \\
\text { Handbook }\end{array}$ & ANSI/APA \\
\hline $\begin{array}{c}\mathrm{W}-7 \\
1220 \times 2743 \times 165 \\
\mathrm{~mm}\left(4^{\prime} \times 9 ' \times 6.5^{\prime \prime}\right)\end{array}$ & 199.58 & 200.83 & \multirow{3}{*}{104.21} & \multirow{3}{*}{108.74} & \multirow{3}{*}{132.54} & \multirow{3}{*}{122.20} & \multirow{3}{*}{1.04} & \multirow{3}{*}{1.27} & \multirow{3}{*}{1.17} \\
\hline $\begin{array}{c}\mathrm{W}-8 \\
1220 \times 2743 \times 165 \\
\mathrm{~mm}\left(4^{\prime} \times 9^{\prime} \times 6.5^{\prime \prime}\right)\end{array}$ & 172.36 & 173.61 & & & & & & & \\
\hline $\begin{array}{c}\text { W-9 } \\
1220 \times 2743 \times 165 \\
m m\left(4^{\prime} \times 9 ' \times 6.5^{\prime \prime}\right)\end{array}$ & 268.23 & 269.48 & & & & & & & \\
\hline
\end{tabular}

* Mohamed (2009) for SIP basement wall.

(1) Did not include $1.25 \mathrm{kN}$ weight of the loading system.

(2) Included $1.25 \mathrm{kN}$ weight of the loading system. 


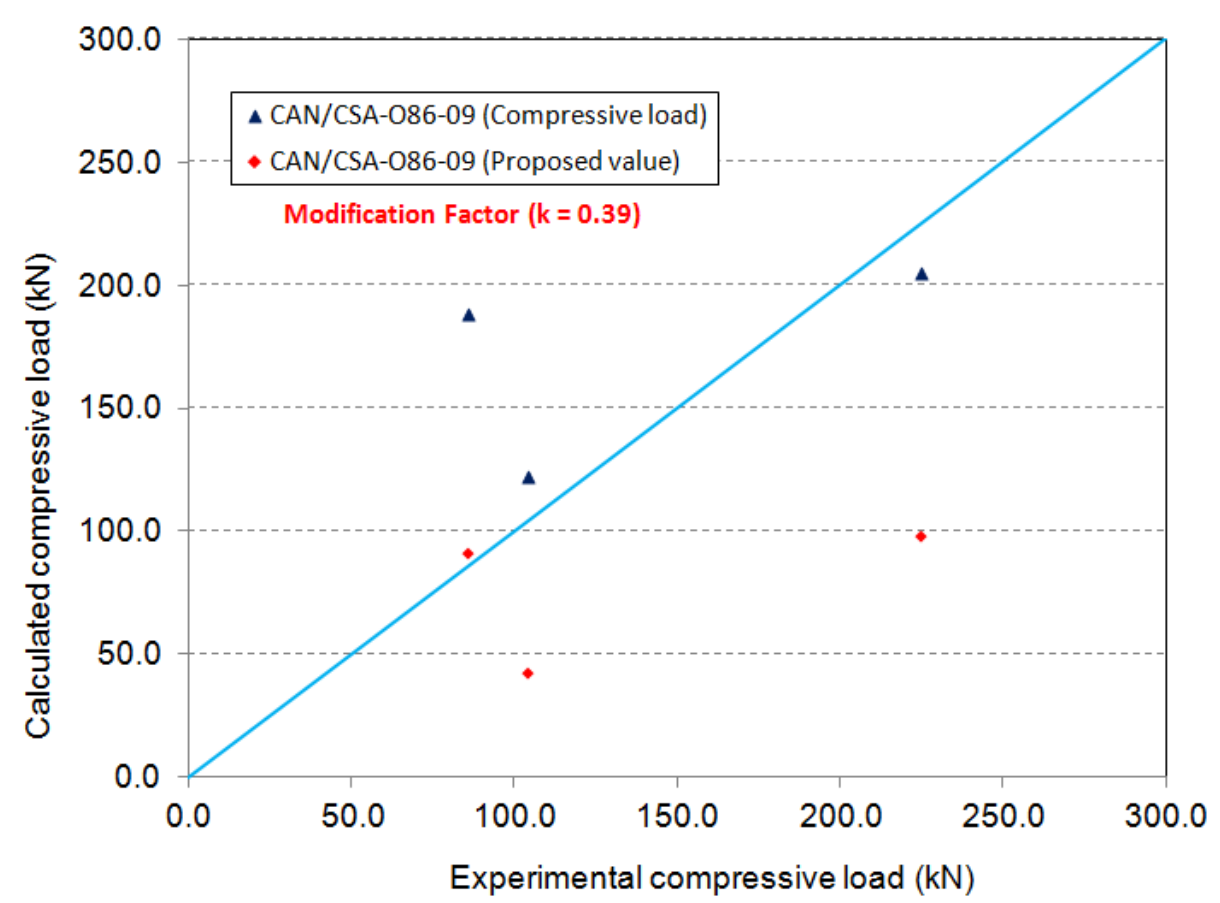

Figure 6.13 - Correlation between the axial compressive resistance obtained experimentally and that obtained using CSA-O86-09 along with the values using the proposed modification factor for compressive loading on SIP walls with $\mathrm{t} / 6$ eccentricity

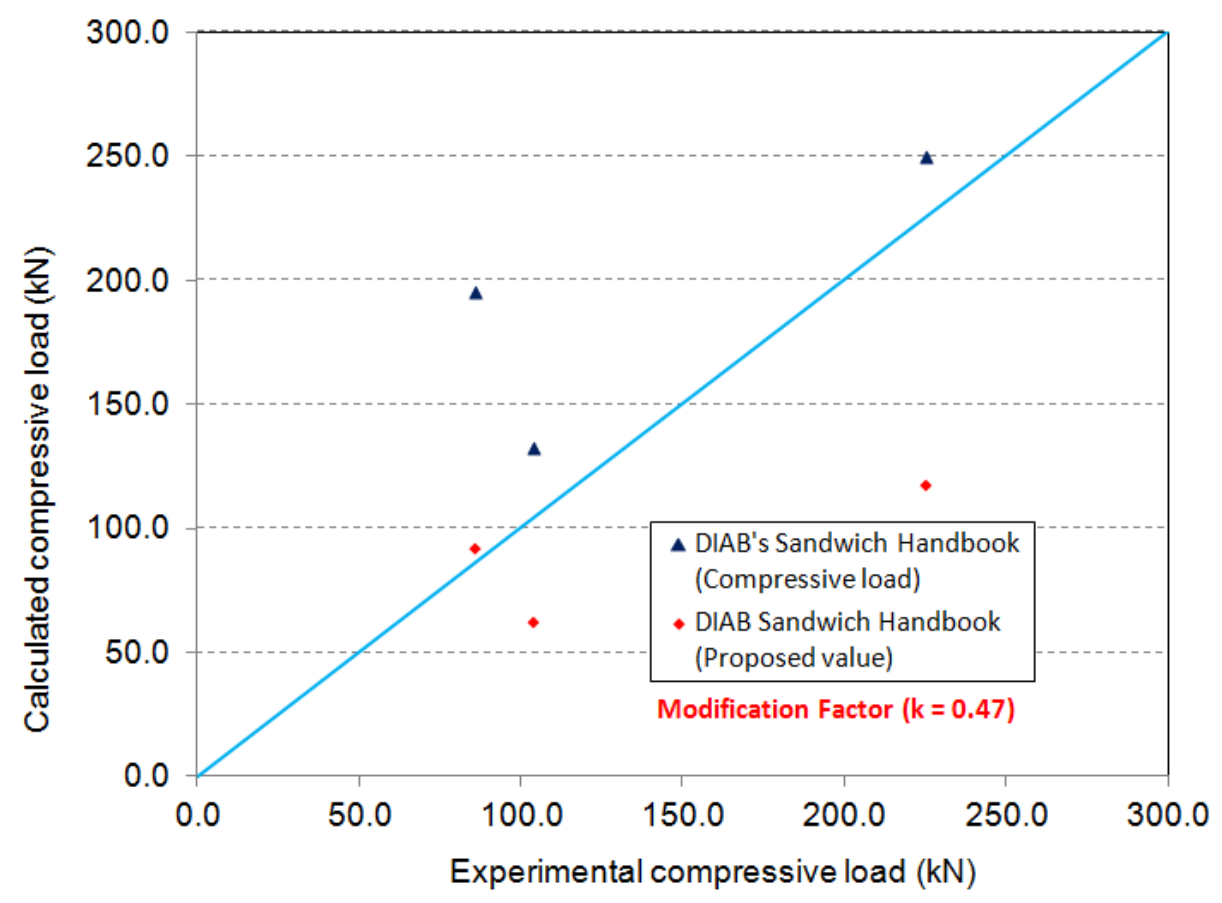

Figure 6.14 - Correlation between the axial compressive resistance obtained experimentally and that obtained using DIAB's Handbook along with the values using the proposed modification factor for compressive loading on SIP walls with $\mathrm{t} / 6$ eccentricity 


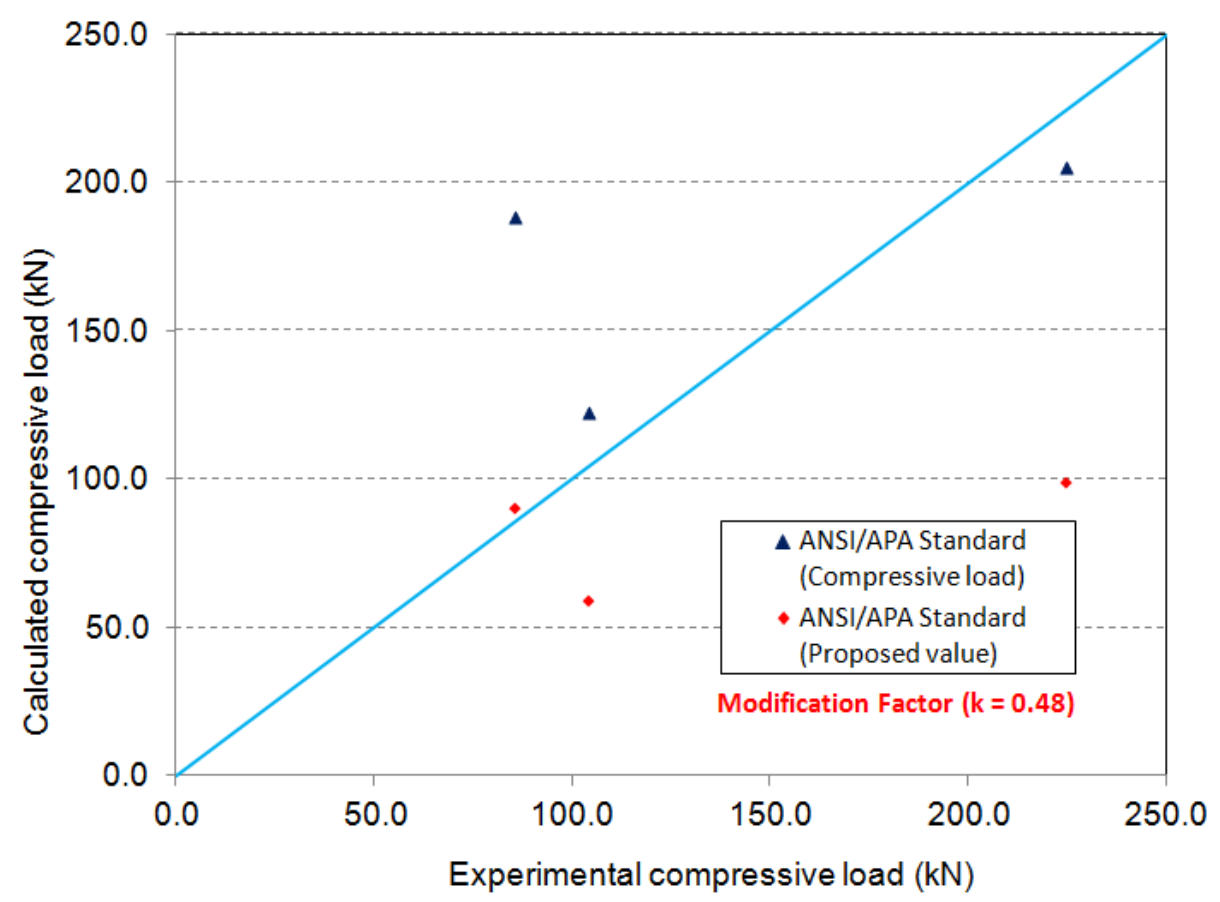

Figure 6.15 - Correlation between the axial compressive resistance obtained experimentally and that obtained using ANSI/APA along with the values using the proposed modification factor for compressive loading on SIP walls with $\mathrm{t} / 6$ eccentricity

Table 6.24 - Proposed modification factors for compressive resistance of SIP panels with $t / 6$ eccentricity

\begin{tabular}{|c|c|c|c|}
\hline $\begin{array}{c}\text { Modification } \\
\text { factor }\end{array}$ & CSA-086-09 & $\begin{array}{c}\text { DIAB's } \\
\text { Handbook }\end{array}$ & $\begin{array}{c}\text { APA } \\
\text { Standard }\end{array}$ \\
\hline $\mathbf{k}$ & 0.39 & 0.47 & 0.48 \\
\hline
\end{tabular}

\subsubsection{Racking load on SIP walls}

Manual calculations were performed to determine the racking load capacity and associated lateral displacement at the top of the SIP walls when subjected to lateral loading at the top of the wall. Table 6.29 shows the racking resistances obtained for the tested SIP walls SIP-R-1 through SIP-R-6 based on BS 5268-6.2, along with the corresponding lateral displacements obtained using UBC Section 1612.2 and CSA-O86-09 Standards. Table 6.25 also shows the ratios between the racking resistance and corresponding lateral displacements obtained experimentally and from available equations. It can be observed that BS 5268-6.2 code equation provides ratios of 0.37 and 0.30 for the $8^{\prime}$ and the 12' long SIP panels, respectively, indication an 
underestimation of predicating the racking resistance of the SIP wall. As such, a modification factor of 3.19 was proposed in Table 6.30 to increase value obtained from BS 5268-6.2 code equation. Table 6.25 shows that the ratios between the code equations and experimental data for lateral displacements obtained are always greater than 1, indicating and overestimation of the prediction of the racking displacement of the SIP wall. As such, modification factors of 0.41 and 0.70 were proposed in Table 6.26 for lateral displacement values obtained from UBC Section 1612.2 and CSA-O86-09 Standards, respectively, for SIP panels. Figure 6.16 shows the correlation between the monotonic racking resistance obtained experimentally and that obtained using BS 5268-6.2 along with the values using the proposed modification factor for racking loading on SIP walls. Figures 6.17 and 6.18 provide the correlation between the monotonic lateral displacement obtained experimentally and that obtained using available equations along with the values using the proposed modification factor for racking loading on SIP walls. This would help in demonstrating confidence on the proposed modification factors. 
Table 6.25 - Comparison of the results from different codes and experimental data for monotonic lateral load on SIP walls

\begin{tabular}{|c|c|c|c|c|c|c|c|c|c|}
\hline \multirow{2}{*}{ Specimen } & \multirow{2}{*}{$\begin{array}{c}\text { Exp. } \\
\text { ultim. } \\
\text { lateral } \\
\text { load, } \\
\text { L }_{\text {r(Exp.) }} \\
\text { (kN) }\end{array}$} & \multirow{2}{*}{$\begin{array}{c}\text { Exp. } \\
\text { ultimate } \\
\text { lateral } \\
\text { Disp., } \\
\text { Disp(Exp.) } \\
\text { (mm) }\end{array}$} & \multirow{2}{*}{$\begin{array}{c}\text { Overturn. } \\
\text { Moment } \\
\text { (kN.m) }\end{array}$} & \multirow{2}{*}{$\begin{array}{c}\begin{array}{c}\text { Racking } \\
\text { resist. of } \\
\text { wall, } \mathrm{L}_{\mathrm{r}(\mathrm{Code})} \\
(\mathrm{kN})\end{array} \\
\text { BS 5268-6.2 }\end{array}$} & \multirow{2}{*}{$\begin{array}{c}\text { Ratio } \\
\mathbf{L}_{\mathbf{r} \text { (Code) }} / \mathbf{L}_{\mathbf{r}(\text { Exp.) }} \\
\\
\text { BS 5268-6.2 }\end{array}$} & \multicolumn{2}{|c|}{$\begin{array}{c}\text { Lateral } \\
\text { displacement, } \\
\text { Disp (Code) }_{(\mathbf{m m})}\end{array}$} & \multicolumn{2}{|c|}{$\begin{array}{c}\text { Ratio } \\
\left(\text { (Disp }_{\text {(Code) }} / \text { Disp }_{(\text {Exp. })}\right)\end{array}$} \\
\hline & & & & & & $\begin{array}{c}\text { UBC } \\
\text { Section } \\
1612.2\end{array}$ & $\begin{array}{c}\text { CSA- } \\
\text { O86-09 }\end{array}$ & $\begin{array}{c}\text { UBC } \\
\text { Section } \\
1612.2\end{array}$ & $\begin{array}{c}\text { CSA-O86- } \\
09\end{array}$ \\
\hline $\begin{array}{c}\text { SIP-R-1 } \\
2440 \times 2743 \times 165 \mathrm{~mm} \\
\left(8^{\prime} \times 9 ' \times 6.5^{\prime \prime}\right)\end{array}$ & 25.33 & 104.25 & 92.65 & \multirow{3}{*}{5.80} & \multirow{3}{*}{0.37} & \multirow{3}{*}{122.45} & \multirow{3}{*}{117.00} & \multirow{3}{*}{1.57} & \multirow{3}{*}{1.50} \\
\hline $\begin{array}{c}\text { SIP-R-2 } \\
2440 \times 2743 \times 165 \mathrm{~mm} \\
\left(8 ' \times 9 ' \times 6.5^{\prime \prime}\right)\end{array}$ & 31.00 & 129.86 & 113.39 & & & & & & \\
\hline $\begin{array}{c}\text { SIP-R-3 } \\
2440 \times 2743 \times 165 \mathrm{~mm} \\
\left(8^{\prime} \times 9 ' \times 6.5^{\prime \prime}\right)\end{array}$ & 22.44 & 107.33 & 82.08 & & & & & & \\
\hline
\end{tabular}


Table 6.25 - Comparison of the results from different codes and experimental data for monotonic lateral load on SIP walls (continued)

\begin{tabular}{|c|c|c|c|c|c|c|c|c|c|}
\hline \multirow{2}{*}{ Specimen } & \multirow{2}{*}{$\begin{array}{c}\text { Exp. } \\
\text { ultim. } \\
\text { lateral } \\
\text { load, } \\
\text { L }_{\text {r(Exp.) }} \\
\text { (kN) }\end{array}$} & \multirow{2}{*}{$\begin{array}{c}\text { Exp. } \\
\text { ultimate } \\
\text { lateral } \\
\text { Disp., } \\
\text { Disp(Exp.) } \\
\text { (mm) }\end{array}$} & \multirow{2}{*}{$\begin{array}{c}\text { Overturn. } \\
\text { Moment } \\
\text { (kN.m) }\end{array}$} & \multirow{2}{*}{$\begin{array}{c}\begin{array}{c}\text { Racking } \\
\text { resist. of } \\
\text { wall, } \mathrm{L}_{\mathrm{r}(\mathrm{Code})} \\
(\mathrm{kN})\end{array} \\
\text { BS 5268-6.2 }\end{array}$} & \multirow{2}{*}{$\begin{array}{c}\text { Ratio } \\
\mathbf{L}_{\mathbf{r}(\text { Code) }} / \mathbf{L}_{\mathbf{r}(\text { Exp.) }} \\
\\
\text { BS 5268-6.2 }\end{array}$} & \multicolumn{2}{|c|}{$\begin{array}{c}\text { Lateral } \\
\text { displacement, } \\
\text { Disp(Code) }_{(\mathbf{m m})}\end{array}$} & \multicolumn{2}{|c|}{$\begin{array}{c}\text { Ratio } \\
\left(\text { Disp }_{(\text {(Code })} / \text { Disp }_{(\text {Exp. })}\right)\end{array}$} \\
\hline & & & & & & $\begin{array}{c}\text { UBC } \\
\text { Section } \\
1612.2\end{array}$ & $\begin{array}{l}\text { CSA- } \\
\text { O86-09 }\end{array}$ & $\begin{array}{c}\text { UBC } \\
\text { Section } \\
1612.2\end{array}$ & $\begin{array}{c}\text { CSA-O86- } \\
09\end{array}$ \\
\hline $\begin{array}{c}\text { SIP-R-4 } \\
3657 \times 2743 \times 165 \mathrm{~mm} \\
\left(12^{\prime} \times 9 ' \times 6.5^{\prime \prime}\right)\end{array}$ & 40.86 & 162.90 & 149.45 & \multirow{3}{*}{11.10} & \multirow{3}{*}{0.30} & \multirow{3}{*}{298.92} & \multirow{3}{*}{153.46} & \multirow{3}{*}{2.62} & \multirow{3}{*}{1.35} \\
\hline $\begin{array}{c}\text { SIP-R-5 } \\
3657 \times 2743 \times 165 \mathrm{~mm} \\
\left(12^{\prime} \times 9 ' \times 6.5^{\prime \prime}\right)\end{array}$ & 38.94 & 197.67 & 142.43 & & & & & & \\
\hline $\begin{array}{c}\text { SIP-R-6 } \\
3657 \times 2743 \times 165 \mathrm{~mm} \\
\left(12^{\prime} \times 9 ' \times 6.5^{\prime \prime}\right)\end{array}$ & 40.65 & 155.02 & 148.68 & & & & & & \\
\hline
\end{tabular}




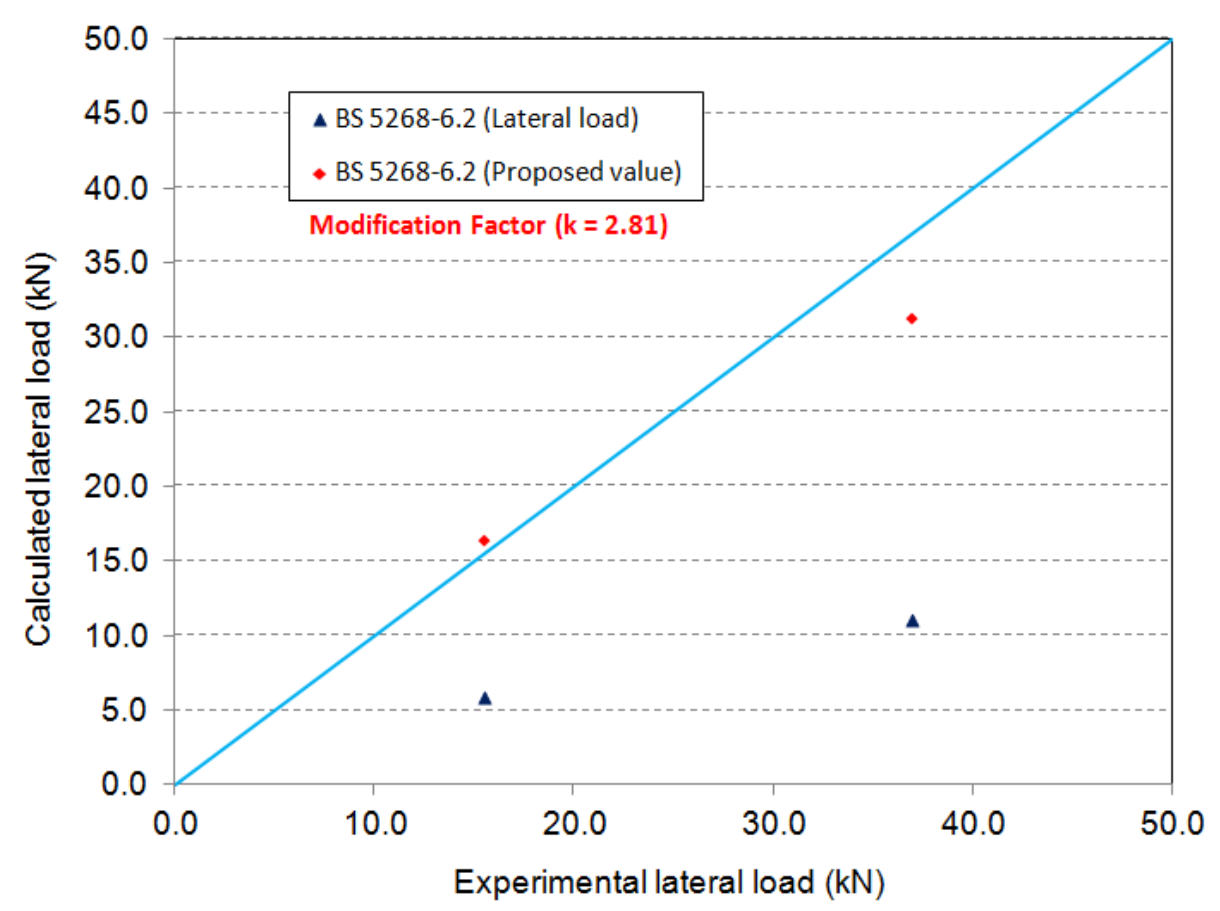

Figure 6.16 - Correlation between the monotonic racking resistance obtained experimentally and that obtained using BS 5268-6.2 along with the values using the proposed modification factor for racking loading on SIP walls

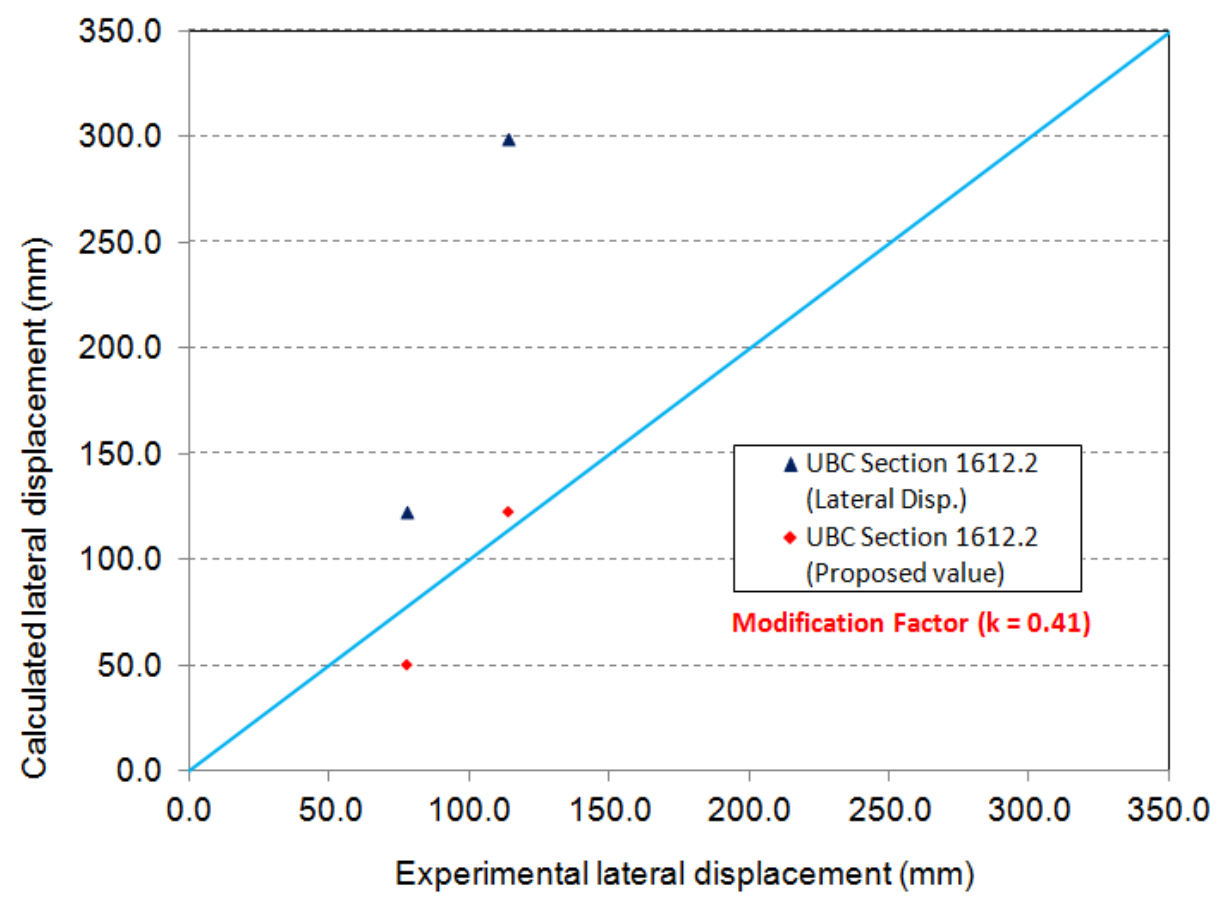

Figure 6.17 - Correlation between the monotonic lateral displacement obtained experimentally and that obtained using UBC Section 1612.2 along with the values using the proposed modification factor for racking loading on SIP walls 


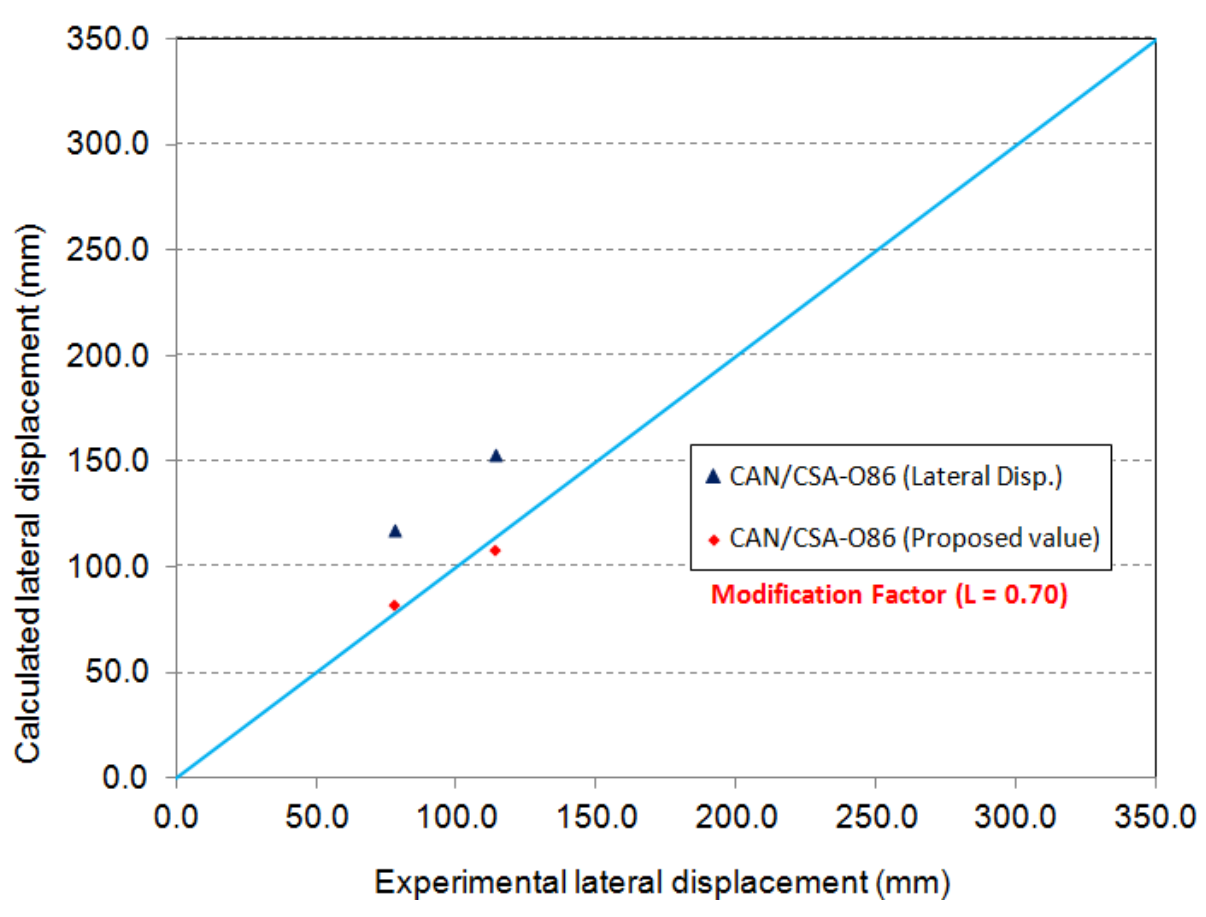

Figure 6.18 - Correlation between the monotonic lateral displacement obtained experimentally and that obtained using CSA-O86-09 along with the values using the proposed modification factor for racking loading on SIP walls

Table 6.26 - Proposed modification factors for racking resistance and corresponding lateral displacement for SIP walls subjected to racking loading

\begin{tabular}{|c|c|c|c|}
\hline \multirow{2}{*}{$\begin{array}{c}\text { Modification } \\
\text { factor }\end{array}$} & Racking load resistance & \multicolumn{2}{|c|}{ Lateral displacement } \\
\cline { 2 - 4 } & BS 5268-6.2 & $\begin{array}{c}\text { UBC Section } \\
\mathbf{1 6 1 2 . 2}\end{array}$ & CSA-O86-09 \\
\hline $\mathbf{k}$ & 2.81 & 0.41 & 0.70 \\
\hline
\end{tabular}

\subsubsection{Cyclic lateral load on SIP walls}

Manual calculations were performed to determine the cyclic lateral load capacity and associated lateral displacement at the top of the SIP walls when subjected to cyclic lateral loading at the top of the wall. Table 6.27 shows the cyclic lateral resistances obtained for the tested SIP walls SIPC-1 through SIP-C-6 based on BS 5268-6.2, along with the corresponding lateral displacements obtained using UBC Section 1612.2 and CSA-O86-09 Standards. Table 6.27 also shows the ratios between the cyclic lateral resistance and corresponding lateral displacements obtained experimentally and from available equations. It can be observed that BS 5268-6.2 code equation 
provides ratios of 0.21 and 0.47 for the $8^{\prime}$ and the 12' long SIP panels, respectively, indication an underestimation of predicating the racking resistance of the SIP wall. As such, a modification factor of 2.68 was proposed in Table 6.28 to increase value obtained from BS 5268-6.2 code equation. Table 6.28 shows that the ratios between the code equations and experimental data for lateral displacements obtained are always greater than 1, indicating and overestimation of the prediction of the cyclic lateral displacement of the SIP wall. As such, modification factors of 0.16 and 0.31 were proposed in Table 6.28 for lateral displacement values obtained from UBC Section 1612.2 and CSA-O86-09 Standards, respectively, for SIP panels. Figure 6.19 shows the correlation between the cyclic racking resistance obtained experimentally and that obtained using BS 5268-6.2 along with the values using the proposed modification factor for cyclic racking loading on SIP walls. Figures 6.20 and 6.21 provide the correlation between the cyclic lateral displacement obtained experimentally and that obtained using available equations along with the values using the proposed modification factor for cyclic loading on SIP walls. This would help in demonstrating confidence on the proposed modification factors. 
Table 6.27 - Comparison of the results from different codes and experimental data for cyclic lateral load on SIP walls

\begin{tabular}{|c|c|c|c|c|c|c|c|c|c|}
\hline \multirow{2}{*}{ Specimen } & \multirow{2}{*}{$\begin{array}{c}\text { Exp. } \\
\text { ultim. } \\
\text { lateral } \\
\text { load, } \\
\text { L }_{\text {r(Exp.) }} \\
\text { (kN) }\end{array}$} & \multirow{2}{*}{$\begin{array}{c}\text { Exp. } \\
\text { ultimate } \\
\text { lateral } \\
\text { Disp., } \\
\text { Disp (Exp.) } \\
\text { (mm) }\end{array}$} & \multirow{2}{*}{$\begin{array}{c}\text { Overturn. } \\
\text { Moment } \\
\text { (kN.m) }\end{array}$} & $\begin{array}{c}\text { Racking } \\
\text { resistance of } \\
\text { Wall, } \mathrm{L}_{\mathrm{r}(\mathrm{Code})} \\
(\mathrm{kN})\end{array}$ & $\begin{array}{c}\text { Ratio } \\
\mathbf{L}_{\mathbf{r}(\text { Code })} / \mathbf{L}_{\mathbf{r}(\text { Exp. })}\end{array}$ & \multicolumn{2}{|c|}{$\begin{array}{c}\text { Lateral } \\
\text { displacement, } \\
\text { Disp(Code) }(\mathrm{mm})\end{array}$} & \multicolumn{2}{|c|}{$\begin{array}{c}\text { Ratio } \\
{\left(\text { Disp }_{(\text {Code })} / \operatorname{Disp}_{\left(\text {Exp. }_{)}\right)}\right)}\end{array}$} \\
\hline & & & & BS 5268-6.2 & BS 5268-6.2 & $\begin{array}{c}\text { UBC } \\
\text { Section } \\
1612.2\end{array}$ & $\begin{array}{c}\text { CSA- } \\
\text { O86-09 }\end{array}$ & $\begin{array}{c}\text { UBC } \\
\text { Section } \\
1612.2\end{array}$ & $\begin{array}{l}\text { CSA- } \\
\text { O86-09 }\end{array}$ \\
\hline $\begin{array}{c}\text { SIP-C-1 } \\
2440 \times 2743 \times 165 \mathrm{~mm} \\
\left(8^{\prime} \times 9 ' \times 6.5^{\prime \prime}\right)\end{array}$ & $\begin{array}{l}+18.52 \\
-40.03\end{array}$ & $\begin{array}{l}+136.62 \\
-131.59\end{array}$ & 109.81 & \multirow{3}{*}{5.80} & \multirow{3}{*}{0.21} & \multirow{3}{*}{122.45} & \multirow{3}{*}{117.00} & \multirow{3}{*}{1.13} & \multirow{3}{*}{1.08} \\
\hline $\begin{array}{c}\text { SIP-C-2 } \\
2440 \times 2743 \times 165 \mathrm{~mm} \\
\left(8^{\prime} \times 9 ' \times 6.5^{\prime \prime}\right)\end{array}$ & $\begin{array}{l}+22.14 \\
-33.40\end{array}$ & $\begin{array}{l}+98.76 \\
-155.49\end{array}$ & 91.62 & & & & & & \\
\hline $\begin{array}{c}\text { SIP-C-3 } \\
2440 \times 2743 \times 165 \mathrm{~mm} \\
\left(8^{\prime} \times 99^{\prime} \times 6.5^{\prime \prime}\right)\end{array}$ & $\begin{array}{l}+22.40 \\
-39.37\end{array}$ & $\begin{array}{l}+40.44 \\
-139.39\end{array}$ & 108.00 & & & & & & \\
\hline
\end{tabular}


Table 6.27 - Comparison of the results from different codes and experimental data for cyclic lateral load on SIP walls (continued)

\begin{tabular}{|c|c|c|c|c|c|c|c|c|c|}
\hline \multirow{2}{*}{ Specimen } & \multirow{2}{*}{$\begin{array}{c}\text { Exp. } \\
\text { ultim. } \\
\text { lateral } \\
\text { load, } \\
\text { L }_{\text {r(Exp.) }} \\
\text { (kN) }\end{array}$} & \multirow{2}{*}{$\begin{array}{c}\text { Exp. } \\
\text { ultimate } \\
\text { lateral } \\
\text { Disp., } \\
\text { Disp(Exp.) } \\
(\mathbf{m m})\end{array}$} & \multirow{2}{*}{$\begin{array}{c}\text { Overturn. } \\
\text { Moment } \\
\text { (kN.m) }\end{array}$} & \multirow{2}{*}{$\begin{array}{c}\text { Racking } \\
\text { resistance of } \\
\text { Wall, } \mathrm{L}_{\mathrm{r}(\mathrm{Code})} \\
(\mathrm{kN}) \\
\\
\text { BS 5268-6.2 }\end{array}$} & \multirow{2}{*}{$\begin{array}{c}\text { Ratio } \\
\mathbf{L}_{\mathbf{r}(\text { Code) }} / \mathbf{L}_{\mathbf{r}(\text { Exp.) }} \\
\text { BS 5268-6.2 }\end{array}$} & \multicolumn{2}{|c|}{$\begin{array}{c}\text { Lateral } \\
\text { displacement, } \\
\text { Disp (Code) }_{(\mathrm{mm})}\end{array}$} & \multicolumn{2}{|c|}{$\begin{array}{c}\text { Ratio } \\
\left(\text { Disp }_{(\text {Code })} / \text { Disp(Exp.) }_{(\text {Exp }}\right)\end{array}$} \\
\hline & & & & & & $\begin{array}{c}\text { UBC } \\
\text { Section } \\
1612.2\end{array}$ & $\begin{array}{c}\text { CSA- } \\
\text { O86-09 }\end{array}$ & $\begin{array}{c}\text { UBC } \\
\text { Section } \\
1612.2\end{array}$ & $\begin{array}{l}\text { CSA- } \\
\text { O86-09 }\end{array}$ \\
\hline $\begin{array}{c}\text { SIP-C-4 } \\
3657 \times 2743 \times 165 \mathrm{~mm} \\
\left(12^{\prime} \times 9 ' \times 6.5^{\prime \prime}\right)\end{array}$ & $\begin{array}{l}+32.01 \\
-25.40\end{array}$ & $\begin{array}{l}+199.54 \\
-132.10\end{array}$ & 69.68 & \multirow{3}{*}{11.10} & \multirow{3}{*}{0.47} & \multirow{3}{*}{298.92} & \multirow{3}{*}{153.46} & \multirow{3}{*}{6.66} & \multirow{3}{*}{3.42} \\
\hline $\begin{array}{c}\text { SIP-C-5 } \\
3657 \times 2743 \times 165 \mathrm{~mm} \\
\left(12^{\prime} \times 9 ' \times 6.5^{\prime \prime}\right)\end{array}$ & $\begin{array}{l}+27.57 \\
-28.82\end{array}$ & $\begin{array}{l}+79.76 \\
-80.92\end{array}$ & 79.06 & & & & & & \\
\hline $\begin{array}{c}\text { SIP-C-6 } \\
3657 \times 2743 \times 165 \mathrm{~mm} \\
\left(12^{\prime} \times 9 \text { 'x } 6.5^{\prime \prime}\right)\end{array}$ & $\begin{array}{l}+30.79 \\
-36.07\end{array}$ & $\begin{array}{l}+92.88 \\
-92.54\end{array}$ & 98.95 & & & & & & \\
\hline
\end{tabular}




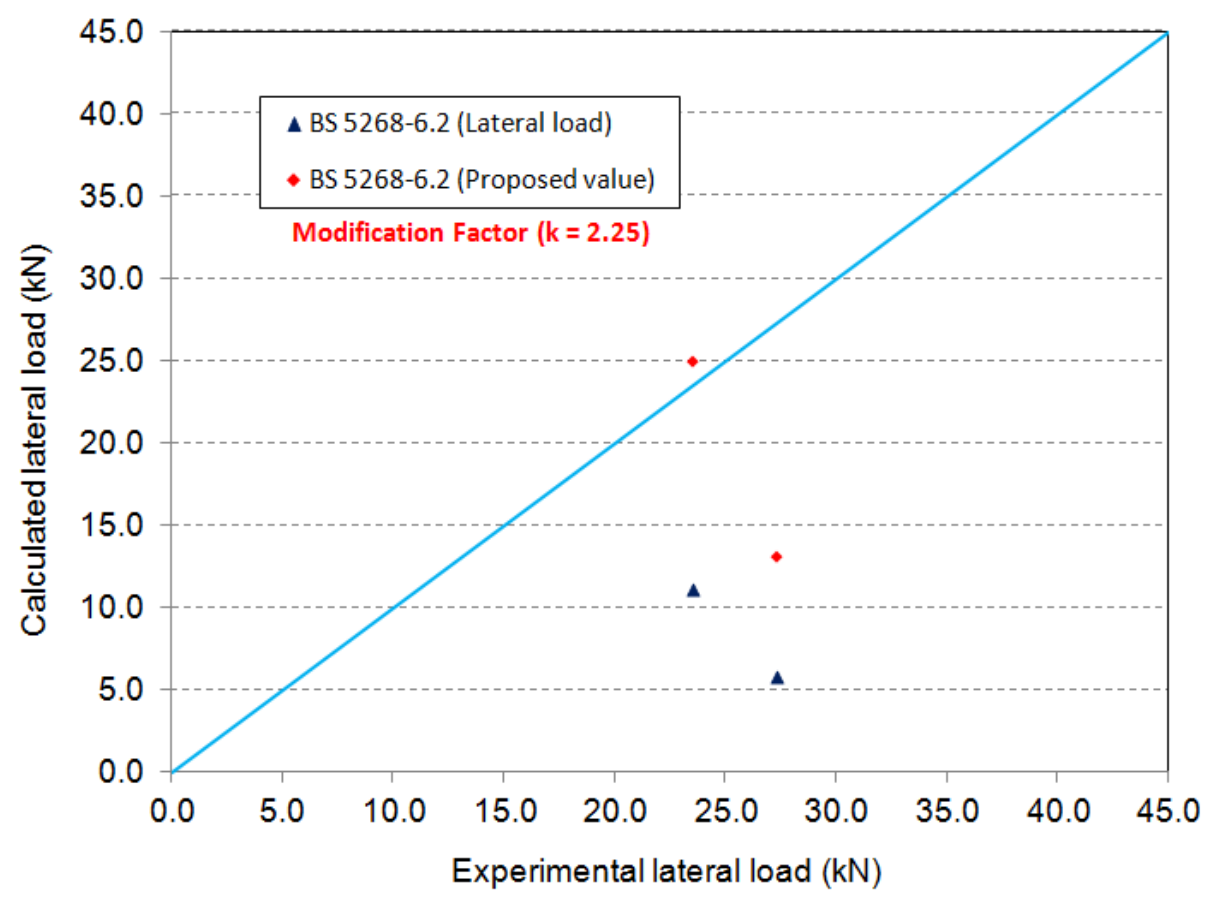

Figure 6.19-Correlation between the cyclic racking resistance obtained experimentally and that obtained using BS 5268-6.2 along with the values using the proposed modification factor for cyclic racking loading on SIP walls

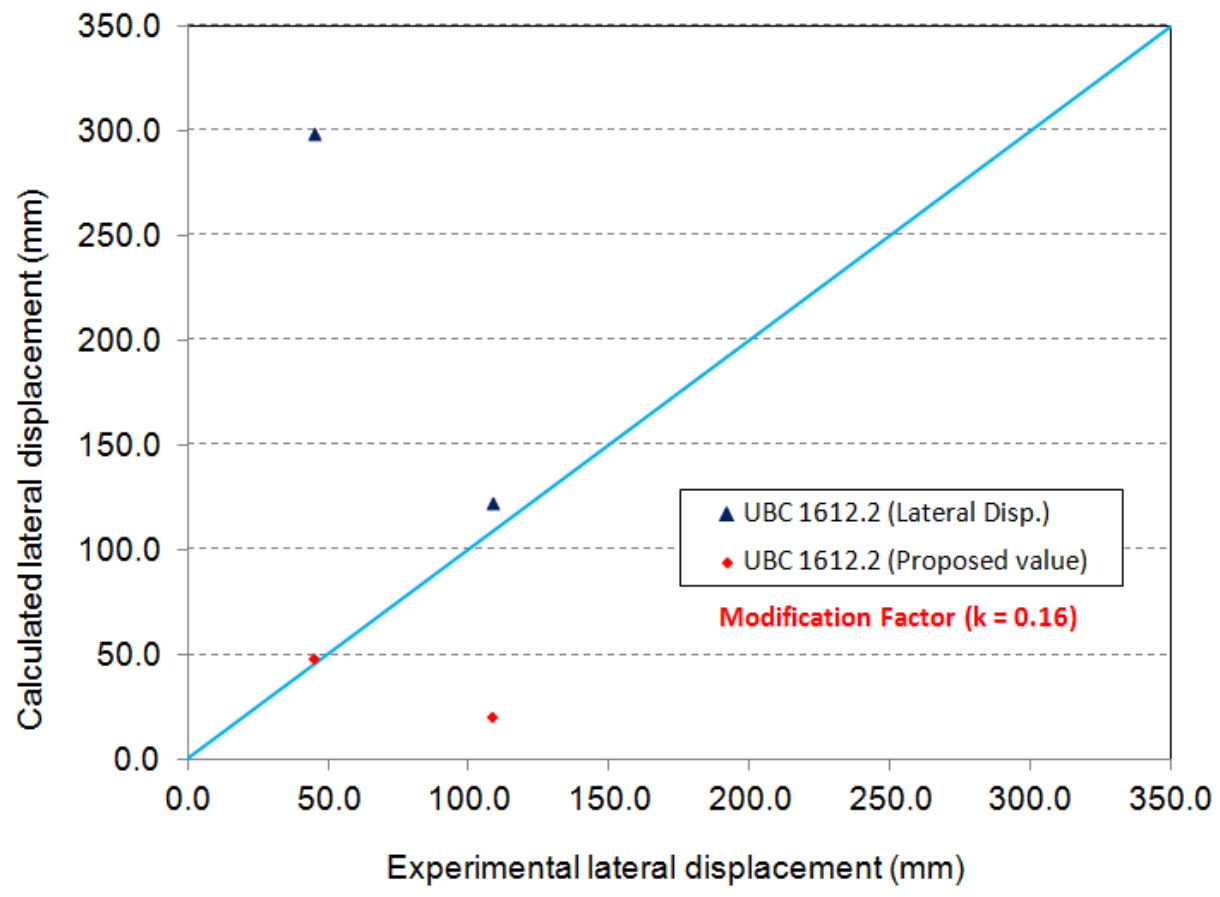

Figure 6.20 - Correlation between the cyclic lateral displacement obtained experimentally and that obtained using UBC Section 1612.2 along with the values using the proposed modification factor for cyclic racking loading on SIP walls 


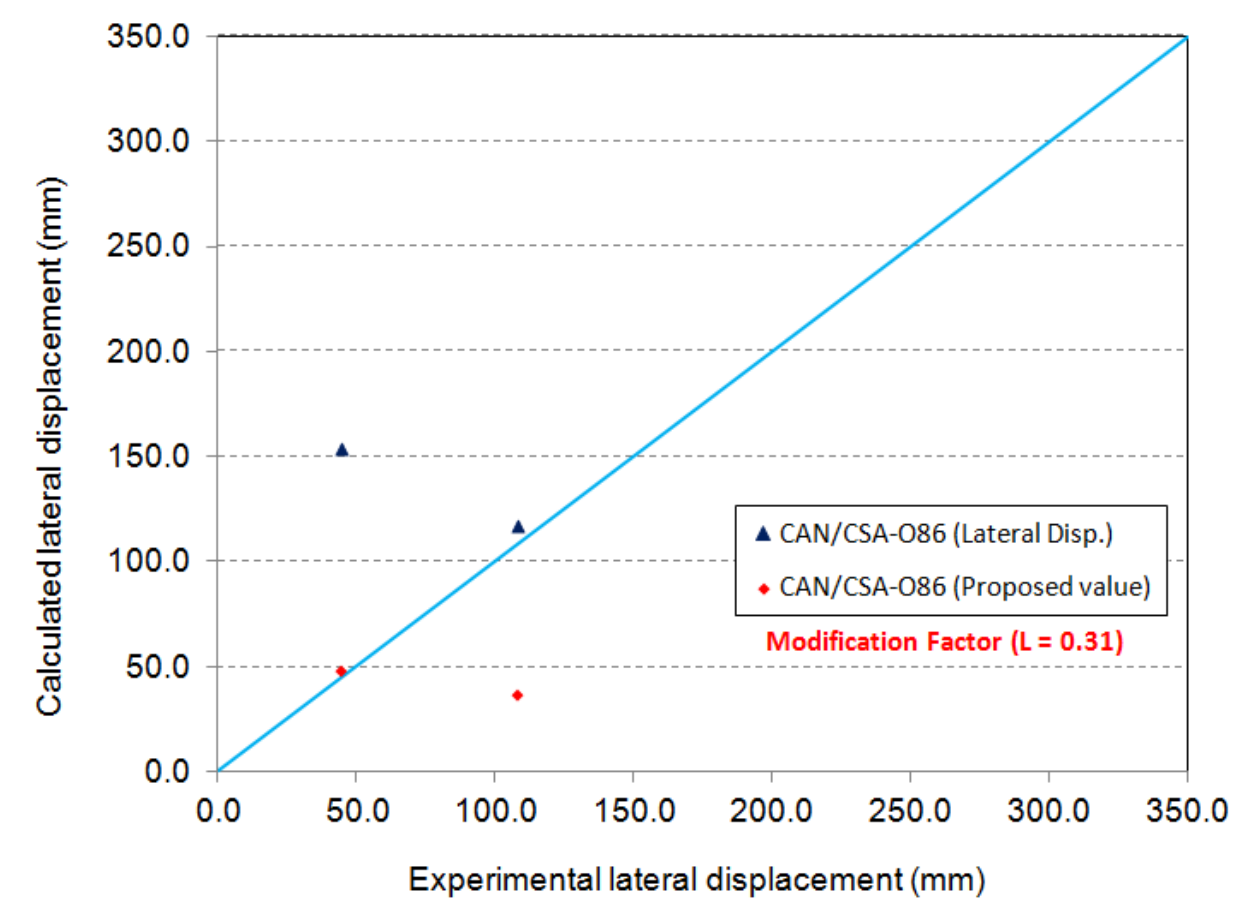

Figure 6.21 - Correlation between the cyclic lateral displacement obtained experimentally and that obtained using CSA-O86-09 along with the values using the proposed modification factor for cyclic racking loading on SIP walls

Table 6.28 - Proposed modification factors for cyclic racking resistance and corresponding lateral displacement for cyclic lateral load on SIP walls

\begin{tabular}{|c|l|c|c|}
\hline \multirow{2}{*}{$\begin{array}{c}\text { Modification } \\
\text { factor }\end{array}$} & $\begin{array}{l}\text { Cyclic racking } \\
\text { resistance }\end{array}$ & \multicolumn{2}{|c|}{ Lateral displacement } \\
\cline { 2 - 4 } & BS 5268-6.2 & $\begin{array}{c}\text { UBC Section } \\
\mathbf{1 6 1 2 . 2}\end{array}$ & CSA-086-09 \\
\hline $\mathbf{k}$ & 2.25 & 0.16 & 0.31 \\
\hline
\end{tabular}

\subsection{Revised equations for stud and SIP design}

As a summary of the findings of sections 6.2 and 6.3 in this chapter, Tables 6.29 through 6.45 summarize the code equation used to conduct the correlations with the experimental findings, along with the proposed modification factors to match the experimental data. 
Table 6.29 - Proposed resisting moment for stud panels under flexural load

\begin{tabular}{|c|c|}
\hline Standard/Code & Resisting moment \\
\hline CAN/CSA-O86-09 & $M_{r}=0.89 \phi P_{p} X_{J} X_{G} \frac{(E I)_{e}}{B_{a} K_{S} c_{c}}$ \\
\hline ANSI/APA & $M_{r}=0.87 b . t_{1} . t \cdot \frac{8 F_{c}\left(E I_{n}\right)}{48 c L^{2} E}$ \\
\hline
\end{tabular}

Table 6.30 - Proposed resisting shear forces for stud panels under flexural load

\begin{tabular}{|c|c|}
\hline Standard/Code & Resisting shear force \\
\hline CAN/CSA-O86-09 & $V_{r}=1.19 \phi F_{v} K_{N} K_{Z v} \frac{(E I)_{e} \sum b_{g}}{E K_{S E} \sum Q_{w}+B_{a} K_{S} b_{f} y}$ \\
\hline ANSI/APA & $V_{r}=1.01$ b.t. $\frac{2\left(E I_{g}\right) \sum F_{s t} t}{4 Q_{s t} L E}$ \\
\hline
\end{tabular}

Table 6.31 - Proposed equations for calculations of stud panel deflection under flexural load

\begin{tabular}{|c|c|}
\hline Standard/Code & Deflection \\
\hline CAN/CSA-O86-09 & $\Delta_{\text {max }}=0.82\left(\frac{P a}{E I}\left(\frac{l^{2}}{8}-\frac{a^{2}}{6}\right)+\frac{P a}{G A^{\prime}}\right)$ \\
\hline ANSI/APA & $\Delta_{\text {max }}=0.46\left(\frac{P a}{E I}\left(\frac{l^{2}}{8}-\frac{a^{2}}{6}\right)+\frac{1.8 P L}{A_{s t} G}\right)$ \\
\hline
\end{tabular}


Table 6.32 - Proposed resisting moment of SIP panels under flexural load

\begin{tabular}{|c|c|}
\hline Standard/Code & Resisting moment \\
\hline CAN/CSA-O86-09 & $M_{r}=0.46 \phi P_{p} X_{J} X_{G} \frac{(E I)_{e}}{B_{a} K_{S} c_{c}}$ \\
\hline DIAB Handbook & $M=\frac{0.27 \sigma_{f} D}{z E_{f}} \quad$ for $\quad \frac{c}{2} \leq z \geq \frac{h}{2} ; \quad-\frac{h}{2} \leq z \leq-\frac{c}{2}$ \\
& $M=\frac{0.27 \sigma_{c} D}{z E_{c}} \quad$ for $\quad-\frac{c}{2} \leq z \geq \frac{c}{2}$ \\
\hline ANSI/APA & $M_{r}=0.27 \frac{P . a}{12}$ \\
\hline
\end{tabular}

Table 6.33 - Proposed resisting shear force for SIP panels under flexural load

\begin{tabular}{|c|c|}
\hline Standard/Code & Resisting shear froce \\
\hline CAN/CSA-O86-09 & $V_{r}=0.26 \phi F_{v} K_{N} K_{Z v} \frac{(E I)_{e} \sum b_{g}}{E K_{S E} \sum Q_{w}+B_{a} K_{S} b_{f} y}$ \\
\hline DIAB Handbook & $V_{r}=\frac{0.61 \tau D}{E_{f} \frac{t d}{2}+\frac{E_{c}}{2} \frac{c^{2}}{4}}$ \\
\hline ANSI/APA & $V_{r}=0.53 f_{v} . b . t \quad ; \quad f_{v}=\frac{w L}{(h+c) 12} \leq F_{V}$ \\
\hline
\end{tabular}

Table 6.34 - Proposed deflection of SIP panels under flexural load

\begin{tabular}{|c|c|}
\hline Standard/Code & Deflection \\
\hline CAN/CSA-O86-09 & $\Delta_{\text {max }}=0.55\left(\frac{P a}{E I}\left(\frac{l^{2}}{8}-\frac{a^{2}}{6}\right)+\frac{P a}{G A^{\prime}}\right)$ \\
\hline DIAB Handbook & $\Delta_{\text {max }}=0.36\left(\frac{P a}{D}\left(\frac{l^{2}}{8}-\frac{a^{2}}{6}\right)+\frac{P a}{G A^{\prime}}\right)$ \\
\hline ANSI/APA & $\Delta_{\text {max }}=0.67\left(\frac{P a}{E I}\left(\frac{l^{2}}{8}-\frac{a^{2}}{6}\right)+\frac{1.8 P L}{A_{s t} G}\right)$ \\
\hline
\end{tabular}


Table 6.35 - Proposed axial compressive resistance of SIP walls under gravity loading

\begin{tabular}{|c|c|}
\hline Standard/Code & Axial compressive resistance \\
\hline NTA IM14 & $P_{r}=0.33 \frac{\pi^{2} E_{b} I}{3 \times(12 L)^{2}\left[1+\frac{\pi^{2} E_{b} I}{(12 L)^{2} \times A_{v} G}\right]}$ \\
\hline DIAB & $P_{r}=0.79 \frac{P_{E}}{1+P_{E} / V} ; \quad V=A G$ \\
\hline Handbook & $P_{r}=0.51\left(f_{c \text { max }}-f_{b \max }\right) \cdot\left(A_{1}+A_{2}\right) \quad ; \quad f_{c}<F_{c} \quad$ and $\quad f_{c}<\frac{1}{3} C_{c r}$ \\
\hline
\end{tabular}

Table 6.36 - Proposed compressive resistance of stud walls subjected to compressive loading at $\mathrm{t} / 6$ eccentricity

\begin{tabular}{|c|c|}
\hline Standard/Code & Compressive resistance \\
\hline CSA-O86-09 & $P_{f}{ }^{2}=0.27\left(\left[1-\left(\frac{M_{f}}{M_{r}}\right)\right] P_{r}{ }^{2}\right)$ \\
\hline ANSI/APA & $P_{r}=0.56\left[\left(1-\frac{M / S}{F_{c}}\right) \cdot P_{a}\right]$ \\
\hline
\end{tabular}

Table 6.37 - Proposed compressive resistance of SIP walls subjected to compressive loading at $\mathrm{t} / 6$ eccentricity

\begin{tabular}{|c|c|}
\hline Standard/Code & Compressive resistance \\
\hline CSA-O86-09 & $P_{f}{ }^{2}=0.39\left(\left[1-\left(\frac{M_{f}}{M_{r}}\right)\right] P_{r}{ }^{2}\right)$ \\
\hline DIAB Handbook & $P_{r}=0.47 \frac{P_{E}}{1+P_{E} / V} ; \quad V=A G$ \\
\hline ANSI/APA & $P_{r}=0.48\left(f_{c \text { max }}-f_{b \max }\right) \cdot\left(A_{1}+A_{2}\right) \quad ; f_{c}<F_{c} \quad$ and $\quad f_{c}<\frac{1}{3} C_{c r}$ \\
\hline
\end{tabular}


Table 6.38 - Proposed lateral load resistance of stud walls under monotonic lateral loading

\begin{tabular}{|c|c|}
\hline Standard/Code & Lateral load resistance \\
\hline BS 5268-6.2 & $L_{r}=2\left(1.25 \times B R C \times L \times K_{m} \times K_{w}\right)$ \\
\hline
\end{tabular}

Table 6.39 - Proposed lateral displacement of stud walls under monotonic lateral loading

\begin{tabular}{|c|c|}
\hline Standard/Code & Lateral displacement \\
\hline UBC Section 1612.2 & $\Delta_{\max }=1.28\left[5.5\left(\Delta_{b}+\Delta_{v}+\Delta_{n}+\Delta_{a}\right)\right]$ \\
\hline CSA-O86-09 & $\Delta_{s w}=0.84\left(\frac{2 v H_{s}^{3}}{3 E A L_{s}}+\frac{v H_{s}}{B_{v}}+0.0025 H_{s} e_{n}+\frac{H_{s}}{L_{s}} d_{a}\right)$ \\
\hline
\end{tabular}

Table 6.40 - Proposed lateral load resistance of SIP walls under monotonic lateral loading

\begin{tabular}{|c|c|}
\hline Standard/Code & Lateral load resistance \\
\hline BS 5268-6.2 & $L_{r}=2.81\left(1.25 \times B R C \times L \times K_{m} \times K_{w}\right)$ \\
\hline
\end{tabular}

Table 6.41 - Proposed lateral displacement of SIP walls under monotonic lateral loading

\begin{tabular}{|c|c|}
\hline Standard/Code & Lateral Displacement \\
\hline UBC Section 1612.2 & $\Delta_{\max }=0.41\left[5.5\left(\Delta_{b}+\Delta_{v}+\Delta_{n}+\Delta_{a}\right)\right]$ \\
\hline CSA-O86-09 & $\Delta_{s w}=0.70\left(\frac{2 v H_{s}^{3}}{3 E A L_{s}}+\frac{v H_{s}}{B_{v}}+0.0025 H_{s} e_{n}+\frac{H_{s}}{L_{s}} d_{a}\right)$ \\
\hline
\end{tabular}

Table 6.42 - Proposed lateral load resistance of stud walls under cyclic lateral loading

\begin{tabular}{|c|c|}
\hline Standard/Code & Lateral load resistance \\
\hline BS 5268-6.2 & $L_{r}=2.3\left(1.25 \times B R C \times L \times K_{m} \times K_{w}\right)$ \\
\hline
\end{tabular}


Table 6.43 - Proposed lateral displacement of stud walls under cyclic lateral loading

\begin{tabular}{|c|c|}
\hline Standard/Code & Lateral displacement \\
\hline UBC Section 1612.2 & $\Delta_{\max }=1.36\left[5.5\left(\Delta_{b}+\Delta_{v}+\Delta_{n}+\Delta_{a}\right)\right]$ \\
\hline CSA-O86-09 & $\Delta_{s w}=0.93\left(\frac{2 v H_{s}{ }^{3}}{3 E A L_{s}}+\frac{v H_{s}}{B_{v}}+0.0025 H_{s} e_{n}+\frac{H_{s}}{L_{s}} d_{a}\right)$ \\
\hline
\end{tabular}

Table 6.44 - Proposed lateral load resistance of SIP walls under cyclic lateral loading

\begin{tabular}{|c|c|}
\hline Standard/Code & Cyclic lateral load resistance \\
\hline BS 5268-6.2 & $L_{r}=2.25\left(1.25 \times B R C \times L \times K_{m} \times K_{w}\right)$ \\
\hline
\end{tabular}

Table 6.45 - Proposed lateral displacement SIP walls under cyclic lateral loading

\begin{tabular}{|c|c|}
\hline Standard/Code & Lateral displacement \\
\hline UBC Section 1612.2 & $\Delta_{\max }=0.16\left[5.5\left(\Delta_{b}+\Delta_{v}+\Delta_{n}+\Delta_{a}\right)\right]$ \\
\hline CSA-O86-09 & $\Delta_{s w}=0.31\left(\frac{2 v H_{s}^{3}}{3 E A L_{s}}+\frac{v H_{s}}{B_{v}}+0.0025 H_{s} e_{n}+\frac{H_{s}}{L_{s}} d_{a}\right)$ \\
\hline
\end{tabular}

\subsection{Design Table for SIP under different load}

For the sake of simplifying the design of SIP panels to be the same as timber joists and stud wall system covered by Part 9 of the National Building Code of Canada (NBBC, 2005), Table 6.46 presents the SIP panel configurations and the corresponding maximum uniform loading that can be carried at ultimate limit state (ULS) and serviceability limit state (SLS) designs. These values were calculated based on the experimental findings for moment, shear and deflection values. However, Table 6.47 shows the maximum uniform loading that can be carried by each SIP panel to satisfy both the ultimate and serviceability limit state designs. 
Table 6.46 - Maximum uniform load based on limiting shear, moment, and deflection obtained experimentally

\begin{tabular}{|c|c|c|c|c|c|c|}
\hline \multirow{2}{*}{$\begin{array}{c}\text { Connection } \\
\text { type }\end{array}$} & \multirow{2}{*}{$\begin{array}{c}\text { Dimensions } \\
\text { (ft-inch) }\end{array}$} & \multirow{2}{*}{$\begin{array}{r}\text { Thick. } \\
\text { (mm) }\end{array}$} & \multirow{2}{*}{$\begin{array}{l}\text { width } \\
\text { (mm) }\end{array}$} & \multirow{2}{*}{$\begin{array}{c}\text { Length } \\
(\mathrm{mm})\end{array}$} & \multicolumn{2}{|c|}{$\begin{array}{l}\text { Max. uniform load (w) } \\
\qquad\left(\mathrm{kN} / \mathrm{m}^{2}\right)\end{array}$} \\
\hline & & & & & $\begin{array}{c}\text { Moment } \\
\text { and shear }\end{array}$ & Deflection \\
\hline Foam Spline & $\left(4^{\prime} \times 8 ' x 6.5^{\prime \prime}\right)$ & 165 & 1220 & 2440 & 17.66 & 24.39 \\
\hline \multirow{2}{*}{ Lumber Spline } & (4'x10'x 10.25") & \multirow{2}{*}{260} & \multirow{2}{*}{1220} & 3048 & 20.53 & 19.42 \\
\hline & (4'x9'x 10.25") & & & 2743 & 33.49 & 15.54 \\
\hline \multirow{6}{*}{ Foam Spline } & (2'x8'x 6.5") & \multirow{2}{*}{165} & \multirow{2}{*}{609} & 2440 & 13.23 & 16.53 \\
\hline & (2'x16'x 6.5") & & & 4876 & 15.98 & 13.97 \\
\hline & $\left(4^{\prime} \times 99^{\prime} x 6.5^{\prime \prime}\right)$ & 209 & \multirow{4}{*}{1220} & 2743 & 17.04 & 17.08 \\
\hline & (4'x9'x 8.25") & 209 & & 2743 & 39.85 & 31.84 \\
\hline & (4'x10'x 6.5") & \multirow{2}{*}{165} & & 3048 & 22.03 & 15.28 \\
\hline & (4'x12'x 6.5") & & & 3657 & 12.38 & 11.32 \\
\hline Lumber Spline & (4'x10'x 8.25") & 209 & 1220 & 3048 & 13.97 & 22.35 \\
\hline Foam Spline & (4'x12'x 8.25") & 209 & 1220 & 3657 & 17.52 & 14.05 \\
\hline
\end{tabular}


Table 6.46 - Maximum uniform load based on limiting shear, moment, and deflection obtained experimentally (continued)

\begin{tabular}{|c|c|c|c|c|c|c|}
\hline \multirow{2}{*}{ Connection type } & \multirow{2}{*}{$\begin{array}{l}\text { Dimensions } \\
\text { (mm/ft-inch) }\end{array}$} & \multirow{2}{*}{$\begin{array}{l}\text { Thick. } \\
\text { (mm) }\end{array}$} & \multirow{2}{*}{$\begin{array}{l}\text { width } \\
\text { (mm) }\end{array}$} & \multirow{2}{*}{$\begin{array}{l}\text { Length } \\
(\mathrm{mm})\end{array}$} & \multicolumn{2}{|c|}{$\begin{array}{l}\text { Max. uniform load (w) } \\
\qquad\left(\mathrm{kN} / \mathrm{m}^{2}\right)\end{array}$} \\
\hline & & & & & $\begin{array}{l}\text { Moment } \\
\text { and shear }\end{array}$ & Disp. \\
\hline Foam Spline & (4'x14'x 8.25") & 209 & 1220 & 4267 & 14.33 & 11.17 \\
\hline Foam Spline & (4'x12'x 10.25") & 260 & 1220 & 3657 & 18.75 & 17.86 \\
\hline Lumber Spline & (4'x14'x 10.25") & 260 & 1220 & 4267 & 15.12 & 12.76 \\
\hline Foam Spline & (4'x16'x 10.25") & 260 & 1220 & 4876 & 14.09 & 10.19 \\
\hline Lumber Spline & (4'x8'x 6.5") & 165 & 1220 & 2440 & 43.84 & 20.37 \\
\hline Foam Spline & (4'x10'x 8.25") & 209 & 1220 & 3048 & 13.61 & 8.77 \\
\hline \multirow{2}{*}{ Lumber Spline } & (4'x12'x 8.25") & \multirow{2}{*}{209} & \multirow{2}{*}{1220} & 3657 & 23.74 & 17.13 \\
\hline & (4'x14'x 8.25") & & & 4267 & 19.94 & 11.87 \\
\hline Foam Spline & (4'x14'x 10.25") & 260 & 1220 & 4267 & 15.12 & 11.94 \\
\hline Lumber Spline & (4'x16'x 10.25") & 260 & 1220 & 4876 & 22.93 & 9.88 \\
\hline
\end{tabular}


Table 6.47 - Maximum uniform load based on limiting shear, moment, and deflection obtained experimentally

\begin{tabular}{|c|c|c|c|c|c|c|c|c|}
\hline \multirow{3}{*}{ Connection type } & \multirow{3}{*}{ Thickness } & \multirow{3}{*}{$\begin{array}{c}\text { Uniform } \\
\text { load } \\
\left(\mathbf{k N} / \mathbf{m}^{2}\right)\end{array}$} & \multicolumn{6}{|c|}{ Panel length } \\
\hline & & & $\begin{array}{c}8 \\
\text { (feet) }\end{array}$ & $\begin{array}{c}9 \\
\text { (feet) }\end{array}$ & $\begin{array}{c}10 \\
\text { (feet) }\end{array}$ & $\begin{array}{c}12 \\
\text { (feet) }\end{array}$ & $\begin{array}{c}14 \\
\text { (feet) }\end{array}$ & $\begin{array}{c}16 \\
\text { (feet) }\end{array}$ \\
\hline & & & $\begin{array}{l}2440 \\
(\mathrm{~mm})\end{array}$ & $\begin{array}{l}2743 \\
(\mathrm{~mm})\end{array}$ & $\begin{array}{l}3048 \\
(\mathrm{~mm})\end{array}$ & $\begin{array}{l}3657 \\
(\mathrm{~mm})\end{array}$ & $\begin{array}{l}4267 \\
(\mathrm{~mm})\end{array}$ & $\begin{array}{l}4876 \\
(\mathrm{~mm})\end{array}$ \\
\hline \multirow{2}{*}{ Foam Spline } & \multirow{2}{*}{$\begin{array}{c}165 \text { (mm) } \\
6.5 \text { (in.) }\end{array}$} & W(ULS) & 17.66 & 17.04 & 22.03 & 12.38 & 11.91 & 15.98 \\
\hline & & W(SLS) & 24.39 & 17.08 & 15.28 & 11.32 & 6.85 & 13.97 \\
\hline \multirow{2}{*}{ Lumber Spline } & \multirow{2}{*}{$\begin{array}{c}165 \text { (mm) } \\
6.5 \text { (in.) }\end{array}$} & W(ULS) & 43.84 & 30.85 & 25.91 & 15.69 & 11.91 & 6.39 \\
\hline & & W(SLS) & 20.37 & 32.31 & 12.21 & 10.50 & 6.85 & 4.40 \\
\hline \multirow{2}{*}{ Foam Spline } & \multirow{2}{*}{$\begin{array}{l}209 \text { (mm) } \\
8.25 \text { (in.) }\end{array}$} & W(ULS) & 26.20 & 17.32 & 13.61 & 17.52 & 14.32 & 14.52 \\
\hline & & W(SLS) & 30.69 & 21.72 & 8.77 & 14.05 & 11.17 & 15.97 \\
\hline \multirow{2}{*}{ Lumber Spline } & \multirow{2}{*}{$\begin{array}{l}209 \text { (mm) } \\
8.25 \text { (in.) }\end{array}$} & W(ULS) & 24.86 & 39.85 & 13.97 & 23.74 & 19.94 & 15.97 \\
\hline & & W(SLS) & 61.39 & 31.84 & 22.35 & 17.13 & 11.87 & 8.73 \\
\hline \multirow{2}{*}{ Foam Spline } & \multirow{2}{*}{$\begin{array}{l}260 \text { (mm) } \\
10.25 \text { (in.) }\end{array}$} & W(ULS) & 21.59 & 19.41 & 13.00 & 18.75 & 15.12 & 14.09 \\
\hline & & W(SLS) & 20.18 & 17.63 & 12.26 & 17.86 & 11.94 & 10.19 \\
\hline \multirow{2}{*}{ Lumber Spline } & \multirow{2}{*}{$\begin{array}{l}260 \text { (mm) } \\
10.25 \text { (in.) }\end{array}$} & W(ULS) & 21.82 & 33.49 & 20.53 & 15.85 & 15.12 & 22.93 \\
\hline & & W(SLS) & 25.83 & 15.54 & 9.42 & 8.80 & 12.76 & 9.88 \\
\hline
\end{tabular}

Note: ULS is calculated based on shear and bending moment. SLS is calculated based on deflection.

As for the load carrying capacity of SIP walls, three building configurations were considered herein, namely: (i) a wall carrying single flat roof, (ii) a wall carrying a flat roof and a floor; and (iii) a wall carrying a flat roof and two floors. For the first case, the factored combined load would be $1.25 \mathrm{D}$ and $1.5 \mathrm{~S}$, where $\mathrm{D}$ and $\mathrm{S}$ are dead load and snow load on the roof, respectively. Assuming that $\mathrm{D}$ is $0.5 \mathrm{kPa}$ for the roof and $0.4 \mathrm{kPa}$ for the wall, the served joist span is calculated as 11.48, 8.93, 7.30, 6.18 and 5.36 meter for specified snow load of 1.0, 1.5, 2.0, 2.5 and $3.0 \mathrm{kPa}$. In case of a wall carrying a roof and a floor, two load combinations were considered as follows: 
Case (1): $1.25 \mathrm{D}+0.5 \mathrm{~S}$ for the roof and $1.25 \mathrm{D}+1.5 \mathrm{~L}$ for the floor, where $\mathrm{L}$ is the floor live load.

Case (2): $1.25 \mathrm{D}+1.5 \mathrm{~S}$ for the roof and $1.25 \mathrm{D}+0.5 \mathrm{~L}$ for the floor, where $\mathrm{L}$ is the floor live load.

Considering the floor live load in residential construction as $1.9 \mathrm{kPa}$, the served span for the first and second load combination cases are 6.41, 5.53, 4.86, 4.34 and 3.91 for case (1) and 8.14, 7.63, 7.17, 6.77 and 6.41 for case (2). Similar procedure was performed for the wall case carrying a roof and two floors, leading to served joist spans of 4.45, 4.00, 3.64, 3.34, and 3.08 meter for snow loads of 1.0, 1.5, 2.0, 2.5 and $3.0 \mathrm{kPa}$, respectively. Final results are reported in Table 6.48.

Table 6.48 - Design table for SIP wall under compressive loading with $t / 6$ eccentricity

\begin{tabular}{|c|c|c|c|c|c|c|c|c|}
\hline \multirow{3}{*}{$\begin{array}{c}\text { Panel size: } \\
\mathbf{I} \times \mathbf{w} \times \mathbf{t} \\
(\mathbf{m m}, \mathbf{f t}-\mathbf{i n c h})\end{array}$} & \multirow{3}{*}{$\begin{array}{c}\text { Resist. } \\
\text { ultimate } \\
\text { jacking } \\
\text { load (kN) }\end{array}$} & \multirow{3}{*}{$\begin{array}{c}\text { Resisting } \\
\text { ultimate } \\
\text { uniform load } \\
\text { capacity = } \\
\text { design load } \\
\text { /1.2 } \mathrm{m}(\mathrm{kN} / \mathrm{m})\end{array}$} & \multirow{3}{*}{$\begin{array}{c}\text { Building } \\
\text { storey }\end{array}$} & \multirow{2}{*}{\multicolumn{5}{|c|}{$\begin{array}{c}\text { Maximum supported joist } \\
\text { length (1), (2), based on ultimate } \\
\text { strength, } \mathrm{m} \\
\text { Specified snow load, kPa }\end{array}$}} \\
\hline & & & & & & & & \\
\hline & & & & 1.0 & 1.5 & 2.0 & 2.5 & 3.0 \\
\hline \multirow{3}{*}{$\begin{array}{c}1220 \times 2750 \times 165 \mathrm{~mm} \\
\left(4^{\prime} \times 9^{\prime} \times 6.5^{\prime \prime}\right)\end{array}$} & \multirow{3}{*}{36.16} & \multirow{3}{*}{30.13} & $\begin{array}{l}\text { Roof } \\
\text { only }\end{array}$ & 11.48 & 8.93 & 7.30 & 6.18 & 5.36 \\
\hline & & & $\begin{array}{l}\text { Roof and } \\
\text { floor }\end{array}$ & 6.41 & 5.53 & 4.86 & 4.34 & 3.91 \\
\hline & & & $\begin{array}{c}\text { Roof and } \\
\text { two } \\
\text { floors }\end{array}$ & 4.45 & 4.00 & 3.64 & 3.34 & 3.08 \\
\hline
\end{tabular}

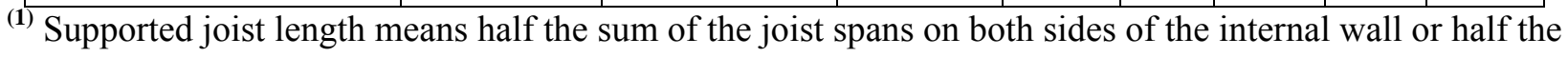
joist span of the exterior wall.

(2) Maximum supported length of roof is based on $0.5 \mathrm{kPa}$ dead load, $1.9 \mathrm{kPa}$ live load for floors and a specified snow load as shown on flat roofs. Wall (with siding, stucco) weight of $0.4 \mathrm{kPa}$ is considered as dead load. 


\section{CHAPTER 7}

\section{CONCLUSIONS AND RECOMMENDATIONS}

\subsection{Introduction}

This research summarizes an investigation of the monotonic and cyclic behavior of structural insulated panel (SIP) conducted using experimental testing and the finite-element modeling. The conclusions of this investigation are presented in this chapter based on experimental testing results on stud and SIP panels when subjected to flexural, compressive, racking and cyclic lateral loading. Also, the conclusions of the finite-element modeling are presented. Moreover, the conclusions of the correlations between experimental data and design equations available in the literature are summarized, followed by recommendations for further research.

\subsection{Conclusions}

Based on the experimental testing and numerical simulation of stud and SIP panels, the following conclusions are drawn:

\subsubsection{Flexural Loading}

- The failure mode of SIP floor was due to horizontal shear failure at the interface between the foam and top OSB facing. This failure occurred between the top surface of the foam and the adhesive over a panel length between the support and the quarter-point line, causing top foam-OSB delamination (debonding) over the supports. Shear failure was sudden causing a sudden drop in the applied jacking load as depicted in the load-deflection history. It has been noted that noise was heard when approaching failure load and the shear failure was abrupt causing a sudden drop in the applied jacking load. It was observed that linear elastic behaviour was maintained along a significant portion of the load history before failure.

- The failure mode of stud panel floor was due to pure flexural at the mid-span region. Tensile fracture in both the stud and the bottom drywall occurred suddenly at failure. 
- Results showed that SIP panel floor is being "as good as" the conventional stud woodframing floor on identical size, with respect to characteristic ultimate resisting moment and limiting deflection.

- It is concluded that the flexural stiffness of SIP panel is more than that of the stud panel, while the ductility ratio of the stud panel is more than that of the SIP panel of the same size under flexural loading.

- Based on the data generated from flexural loading on SIP panels, a design table was developed to provide designers with maximum uniform load that can be carried by the floor for ultimate limit state design for moment and shear and serviceability limit state design for deflection.

\subsubsection{Compressive Loading}

- The failure of the SIP wall was abrupt causing a sudden drop in the applied jacking load. It was observed that linear elastic behaviour was maintained till failure. The change on lateral deflection of the wall at its mid-height was observed with increase in applied load. Given the nature of the load-deformation relationship and the observed failure modes, one may conclude that the traditional Euler buckling failure mode did not exist in this case.

- It was observed that the failure mode in stud wall was due to global bending of the studs. This is shown through the bending deformation of the wall from the drywall side and the widening of the gap between OSB segments, which were oriented horizontally, near the top of the wall. Failure of the stud wall was considered reached with the specimen did not absorb any more loading while deformation was increasing.

- It should be noted that the tested SIP panels in this study had a 2x6 bottom plate connected to the bottom of the OSB sheet with one row of nails. However, Mohamed (2009) tested identical SIP panels with two 2x6 bottom plates connected to the bottom of the OSB sheet with two rows of nails. When compared with the current study, the later SIP walls showed almost double the compressive load carrying capacities that those with one bottom plate along with one row of nails. Failure mode of SIP walls included excessive bearing on the nails at the bottom of the OSB sheet, debonding of foam and OSB sheet at the bottom plate location and in some cases extending in the foam core in the form of diagonal crack. 
- Results showed that SIP walls are being "as good as" the conventional stud wood-framing of identical size, with respect to compressive resistance and deformation per AC04 design criteria irrespective whether one or two plates were used at the bottom of the SIP wall. As for the characteristic ultimate compressive strength, SIP walls with two bottom plates and two rows of nails are being "as good as" the stud wall system. However, SIPs with one bottom plate and one row of nails underestimates the stud wall capacity by $7 \%$.

- Based on the data generated from compressive loading tests with $t / 6$ eccentricity on the 2440x2743x165 mm (8'x9'x6.5”) SIP wall, a design table was developed to provide designers with the maximum served joist span when this wall size is used in residential building.

\subsubsection{Racking Loading}

- Damage of the gypsum board in stud wall was observed at the top of the wall on the back side of the load location. Separation and crushing of OSB sheet at spline connection and movement of OSB sheet in horizontal direction was observed at failure. No wall uplift was observed at failure of stud walls which means that the provided wall anchorage to the base was adequate. It can be observed that the wall behaved elastically along a significant portion of the failure load.

- Failure of SIP walls was mainly due to relative shear deformation between adjacent OSB sheets that appear the spline connection, excessive bearing on the OSB sheet at the nail locations and crushing of OSB sheet at its connection with the bottom plate.

- Results show that the amount of energy dissipated by SIP wall was greater than that of the stud wall of the same size. Therefore, SIP walls can provide better performance than stud walls in energy efficiency in wood building construction.

- Based on racking test results, the average value of ductility ratio was calculated as 6.92 for the stud wall system, 6.38 for the short SIP wall ( $8^{\prime}$ long) and 5.27 for the long SIP wall (12' long). These values show that the ductility of the stud wall is slightly greater than that for SIP wall of the same size ( $8.4 \%$ difference). Also, it can be observed that with increase in length of SIP wall, ductility ratio decreases.

- Based on racking load test results, the average value of elastic stiffness was calculated as $0.77 \mathrm{kN} / \mathrm{mm}$ for the stud wall, $0.79 \mathrm{kN} / \mathrm{mm}$ for the short SIP wall and $0.99 \mathrm{kN} / \mathrm{mm}$ for the 
long SIP wall. These values show that the elastic stiffness of the stud wall is slightly less than that for SIP wall of the same size ( $2 \%$ difference). Also, it can be observed that with increase in length of SIP wall, elastic stiffness increases.

\subsubsection{Cyclic Lateral Loading}

- The failure mode of stud walls under cyclic lateral loading was somewhat similar to that observed for monotonic racking testing. This included OSB sheet and gypsum board at the stud locations, bending of the nail and excessive bearing on the facings at nail locations and OSB crushing at its connection with bottom plates. No wall uplift was observed at failure which means that the provided anchorage was adequate.

- In case of SIP wall, global relative shear deformation occurred at the spline connection. Also, uplift at the bottom of wall panel in one side of the panel happened, while OSB crushing at the same location but on the other side of the wall occurred. Nail bending and OSB crushing at the top and bottom of the wall were observed due to the rotation of OSB sheathing against the top and bottom plates nailed to. It should be noted that at the uplift location, the bottom of OSB sheet tear away from the bottom plate at the nail locations. The separation of the OSB sheet from the top plate was observed due to shear rotation. The shear rotation of the OSB sheets against each other, crushing of the front side of the back OSB sheet and uplift and separation of the back side of the front OSB sheet from the bottom plate were also observed. Moreover, few of the nails at the spline connection and connection to bottom and top plates were pulled out totally and partially from their location.

- The load vs. deformation graph of cyclic test was used to determine the parameters identifying the EEEP bilinear curve. Based on this relationship, the average energies dissipated in the negative portion of the cyclic load history were $0.55,6.37$ and $4.23 \mathrm{kN}$.m for the stud wall, the short SIP wall and the long SIP wall, respectively. Results show that SIP's ability to dissipate energy during cyclic loading was in the range of 6 to 11 times that of the stud wall system.

- In this research, the ratio of the displacement at failure $\left(\Delta_{\text {failure }}\right)$ to the displacement at yield $\left(\Delta_{\text {yield }}\right)$ was presented as the ductility of the system. The values of ductility showed that the structure would not fail suddenly and the failure would not be brittle. However, the ductility factor cannot be a direct indication of the structure subjected to seismic loads. 
- It was found that walls with no hold-down resistant system and with large ductility have large deformation due to large rigid body rotation. Thus, $\left(\Delta_{\text {failure }}\right)$ intensifies while $\left(\Delta_{\text {yield }}\right)$ has a low rate, leading to an increase in the ductility factor of the system.

- Based on cyclic load test results, the average values of ductility ratio were calculated as 4.19 for the stud wall, 4.35 for the short SIP wall and 4.14 for the long SIP wall. These values were calculated based on the ultimate load reached experimentally under cyclic loading. These values show that the ductility ratio of the stud wall is slightly less than that for the SIP wall of the same size (3.8\% difference). Also, it can be observed that with increase in length of SIP wall, ductility ratio decreases.

- Results showed SIP walls have a great ability to dissipate energy under cyclic loading compared to the stud wall system. For example, the maximum values of energy dissipated per cycle was about $550 \mathrm{kN} . \mathrm{mm}$ for the stud wall, $7200 \mathrm{kN}$.mm for the short SIP wall and $7500 \mathrm{kN} . \mathrm{mm}$ for the long SIP wall, respectively. This indicates that the energy dissipated by SIP wall in a seismic zone was about 12 times greater than that for the stud wall of identical size. Therefore, SIP wall can be used so efficient in seismic zones.

- The verification of NBCC-2010 requirements for ductility-related force modification factor $\left(\mathrm{R}_{\mathrm{d}}\right)$ and the over-strength-related force modification factor $\left(\mathrm{R}_{\mathrm{o}}\right)$ was done with ductility ratios, maximum capacities and yield loads. The values of $R_{d}$ of 3.0 and $R_{o}$ of 1.7 for nailed shear walls with wood based panels are recommended in Table 4.1.8.9 of NBCC (NBCC, 2010). Based on cyclic lateral load test results, the values of $R_{d}$ for tested stud wall, short SIP wall and long SIP wall were calculated as $8 \%, 22 \%$ and $14 \%$ lower than the NBCC required value for anchored wall $\left(\mathrm{R}_{\mathrm{d}}=3.0\right)$, respectively. In addition cyclic lateral load test results showed that the values of over-strength-related force modification factor $\left(\mathrm{R}_{\mathrm{o}}\right)$ for tested stud wall, short SIP wall and long SIP wall were observed to be $17 \%, 20 \%$ and $14 \%$ higher than the recommended value of NBCC $\left(\mathrm{R}_{\mathrm{o}}=1.7\right)$ for anchored wall, respectively. So, it is concluded that the over-strength factor indicates a confident reserve of resistance in interconnected wall segments.

- Cyclic load test results showed that the flexural stiffness of SIP is more than that of the stud panel, while the ductility ratio of the stud panel is more than that of the SIP panel of the same size under cyclic loading. 
- Comparisons of the dissipated energy per cycle revealed a higher capacity (about 14 times greater) for SIP walls compared to the conventional stud wall system. Also, the amount of energy dissipated by SIP wall was greater than that of the stud wall of the same size. Therefore, SIP walls can provide better performance than stud walls in energy efficiency in wood building construction. The comparison of cyclic stiffness energy of for SIP and stud walls under cyclic loading showed that the cyclic stiffness of SIP wall was about $20 \%$ greater than that for the stud wall with the same size.

\subsubsection{Finite-Element Modeling}

- Finite-element models of SIPs and conventional wall system were developed and verified by the experimental findings. The comparison between FEA approach and the experimental results showed that the FEA model is sufficient to fairly predict the behavior of the SIP panels under flexural, compressive, monotonic racking and cyclic lateral loads.

- FEA results showed a change in racking capacity with increase in the intensity of gravity loading applied on top of the wall. It was observed that the racking capacity as well as the cyclic lateral load capacity of the wall decreases with increase in the intensity of the gravity loading.

- In FEA modeling, nails were used to connect the ends of the OSB sheets of SIP panel segments to each other through the foam-spline connections. Also, nails were used to connect the ends of the OSB sheets forming SIP panels to the top and bottom lumber plates. The nails were defined as oriented springs at the nail location. The distances between nails along the connection lines were considered 165, 330 and $660 \mathrm{~mm}$ to study the effect of nail spacing. Results show that the short SIP wall racking capacity as well as the cyclic lateral load capacity increases with decrease in nail spacing. However, such effect is less in case of the long SIP panels.

\subsubsection{Proposed Design Equations}

- Correlations between the flexural, compressive, racking and cyclic lateral loading resistance and displacements obtained experimentally and using design equations available in NTA IM14, BS 5268, UBC 1612-2 and CSA-O86-09, and ANSI/APA standards along with DIAB Handbook were performed. Results showed discrepancies between available design 
equations and experimental findings. Therefore, modification factors for these design equations were developed to produce results matching those obtained experimentally. With these equations, designers can design houses and low-rise buildings incorporating SIP construction more reliably and economically.

\subsection{Recommendations for Future Research}

The following recommendations for further research can be made based on the results of this investigation:

- Extend the finite-element modeling to the whole building to study the three-dimensional effect on the racking and cyclic (seismic) responses of SIP panels.

- Develop design tables of walls under combined bending and compressive forces.

- Study the effect of lumber spline connection of the racking and cyclic capacities of SIP walls.

- Study the effect of utility holes in foam core on the structural response of SIP panels.

- Study the use of SIP foam-timber panels as cladding in building construction; subjected to combined wind and gravity loading.

- Study light impact loading on SIP floors, including vibration response to human activities (i.e. walking excitation, rhythmic activities such as aerobics and high-impact dancing, equipment such as generators, exercise equipment).

- Develop a mechanistic or mathematical approach for the design of SIPs for strength and serviceability. 


\section{APPENDIX A}

\section{ABAQUS INPUT FILE}

\section{A.1. SIP Flexural Input File}

*Heading

Applying $30 \mathrm{~mm}$ displacement on middle of the wall

** Job name: SIP-F-01 Model name: Model-1

** Generated by: Abaqus/CAE 6.10-1

$*$ Preprint, echo $=\mathrm{NO}$, model $=\mathrm{NO}$, history $=\mathrm{NO}$, contact $=\mathrm{NO}^{* *}$

** PARTS**

*Part, name $=$ EPS

*End Part**

*Part, name $=\mathrm{H}-$ Lumber

*End Part**

*Part, name $=$ OSB

*End Part**

*Part, name="Ref Point"

*End Part**

*Part, name $=\mathrm{V}$-Lumber

*End Part**

**

** ASSEMBLY**

*Assembly, name $=$ Assembly**

*Instance, name="Ref Point-1", part="Ref Point"

*Node

1, 1220., 1335.39001, 150.149994

*Nset, nset="Ref Point-1-RefPt_", internal

1 ,

*End Instance**

$*$ Instance, name $=$ EPS- 1 , part $=$ EPS

*Node

1, 600.474976, 706.27002, -73 .

2, 600.474976, 668.169983, -73 .

3, 38.0999985, 668.169983, -73 . 
4, 38.0999985, 706.27002, -73 .

5, 600.474976, 668.169983, 73.

6, 38.0999985, 668.169983, 73.

7, 38.0999985, 706.27002, 73.

$8,600.474976, \quad 706.27002, \quad 73$.

9, 600.474976, 2042.60999, -73 .

10, 600.474976, 2004.51001, -73.

2874, 1138.46387, 2443.56372, 0 .

2875, 1138.46387, 2386.28442, 0.

2876, 1138.46387, 2329.00537, 0 .

2877, 1138.46387, 2271.72632, 0.

2878, 1138.46387, 2214.44727, 0.

2879, 1138.46387, 2157.16821, 0 .

2880, 1138.46387, 2099.88916, 0.

*Element, type $=\mathrm{C} 3 \mathrm{D} 8 \mathrm{I}$

$1,98,100,681,673,1,2,65,80$

$2,673,681,682,674,80,65,66,79$

$3,674,682,683,675,79,66,67,78$

$4,675,683,684,676,78,67,68,77$

$5,676,684,685,677,77,68,69,76$

$6,677,685,686,678,76,69,70,75$

$7,678,686,687,679,75,70,71,74$

$8,679,687,688,680,74,71,72,73$

$9,680,688,99,97,73,72,3,4$

$10,8,5,88,89,98,100,681,673$

1780, 2216, 2217, 658, 657, 2874, 2875, 2235, 2234

$1781,2217,2218,659,658,2875,2876,2236,2235$

1782, 2218, 2219, 660, 659, 2876, 2877, 2237, 2236

1783, 2219, 2220, 661, 660, 2877, 2878, 2238, 2237

1784, 2220, 2221, 662, 661, 2878, 2879, 2239, 2238

$1785,2221,2222,663,662,2879,2880,2240,2239$ 
$1786,2222,634,62,663,2880,2062,635,2240$

*Nset, nset $=$ _PickedSet2, internal, generate

$1,2880, \quad 1$

*Elset, elset=_PickedSet2, internal, generate

1, 1786, 1

** Section: EPS

*Solid Section, elset=_PickedSet2, material=EPS

*End Instance**

*Instance, name=H-Lumber-1, part=H-Lumber

*Node

1, 638.575012, 2710.78003, 73.

2, 638.575012, 2672.67993, 73.

3, 1200.94995, 2672.67993, 73.

4, 1200.94995, 2710.78003, 73.

$5,638.575012,2672.67993, \quad-73$.

6, 1200.94995, 2672.67993, -73.

7, 1200.94995, 2710.78003, -73.

8, 638.575012, 2710.78003, -73.

9, 600.474976, 2710.78003, -73.

10, 600.474976, 2672.67993, -73 .

249, 2151.95557, 2672.67993, 0.

$250,2214.44165,2672.67993, \quad 0$.

251, 2276.92773, 2672.67993, 0.

252, 2339.41382, 2672.67993, 0 .

*Element, type $=\mathrm{C} 3 \mathrm{D} 8 \mathrm{I}$

1, 74, 76, 197, 189, 1, 2, 41, 56

2, 189, 197, 198, 190, 56, 41, 42, 55

$3,190,198,199,191,55,42,43,54$

4, 191, 199, 200, 192, 54, 43, 44, 53

5, 192, 200, 201, 193, 53, 44, 45, 52

6, 193, 201, 202, 194, 52, 45, 46, 51

7, 194, 202, 203, 195, 51, 46, 47, 50

8, 195, 203, 204, 196, 50, 47, 48, 49 
$9,196,204,75,73,49,48,3,4$

$10,8,5,64,65,74,76,197,189$

$75,147,145,73,75,22,23,4,3$

$76,19,20,7,6,147,145,73,75$

$77,76,74,110,112,2,1,16,13$

$78,5,8,9,10,76,74,110,112$

$79,111,109,186,185,14,15,33,34$

$80,11,12,35,36,111,109,186,185$

$81,31,181,183,30,37,187,188,40$

$82,181,28,27,183,187,38,39,188$

*Nset, nset=_PickedSet2, internal, generate

$1,252,1$

*Elset, elset=_PickedSet2, internal, generate

$1,82,1$

** Section: Lumber

*Solid Section, elset=_PickedSet2, material=Lumber

*End Instance**

*Instance, name $=$ OSB-1, part $=$ OSB

*Node

1, $1239.05005,2004.51001,84.1500015$

2, 2401.8999, 2004.51001, 84.1500015

3, 2401.8999, 2042.60999, 84.1500015

4, 1239.05005, 2042.60999, 84.1500015

5, 2401.8999, 2004.51001, 73.

6, 2401.8999, 2042.60999, 73.

7, 1239.05005, 2042.60999, 73.

8, 1239.05005, 2004.51001, 73.

9, 1839.52502, 2672.67993, 73.

10, 1801.42505, 2672.67993, 73.

4277, 537.05542, 95.3790894, 73. 
4278, 474.686005, 95.3790894, 73.

4279, 412.316589, 95.3790894, 73.

4280, 349.947144, 95.3790894, 73.

4281, 287.577728, 95.3790894, 73.

4282, 225.208298, 95.3790894, 73.

4283, 162.838867, 95.3790894, 73.

4284, 100.469429, 95.3790894, 73.

*Element, type $=\mathrm{C} 3 \mathrm{D} 8 \mathrm{I}$

$1,8,226,227,7,1,173,208,4$

2, 226, 225, 228, 227, 173, 174, 207, 208

3, 225, 224, 229, 228, 174, 175, 206, 207

4, 224, 223, 230, 229, 175, 176, 205, 206

5, 223, 222, 231, 230, 176, 177, 204, 205

$6,222,221,232,231,177,178,203,204$

7, 221, 220, 233, 232, 178, 179, 202, 203

$8,220,219,234,233,179,180,201,202$

$9,219,218,235,234,180,181,200,201$

$10,218,217,236,235,181,182,199,200$

2040, 1233, 1257, 1256, 1232, 1240, 1248, 1249, 1241

2041, 1232, 1256, 1255, 1231, 1241, 1249, 1250, 1242

$2042,1231,1255,1254,1230,1242,1250,1251,1243$

2043, 1230, 1254, 1253, 1229, 1243, 1251, 1252, 1244

2044, 1229, 1253, 161, 164, 1244, 1252, 158, 157

$2045,71,155,166,99,72,156,165,100$

2046, 92, 2, 3, 108, 91, 5, 6, 105

2047, 34, 37, 38, 35, 94, 93, 102, 101

2048, 98, 97, 154, 153, 96, 95, 167, 168

$2049,126,124,123,125,169,170,171,172$

2050, 14, 12, 124, 126, 107, 106, 170, 169

*Nset, nset=_PickedSet2, internal, generate

1, 4284, 1

*Elset, elset=_PickedSet2, internal, generate

1, 2050, 1

** Section: OSB 


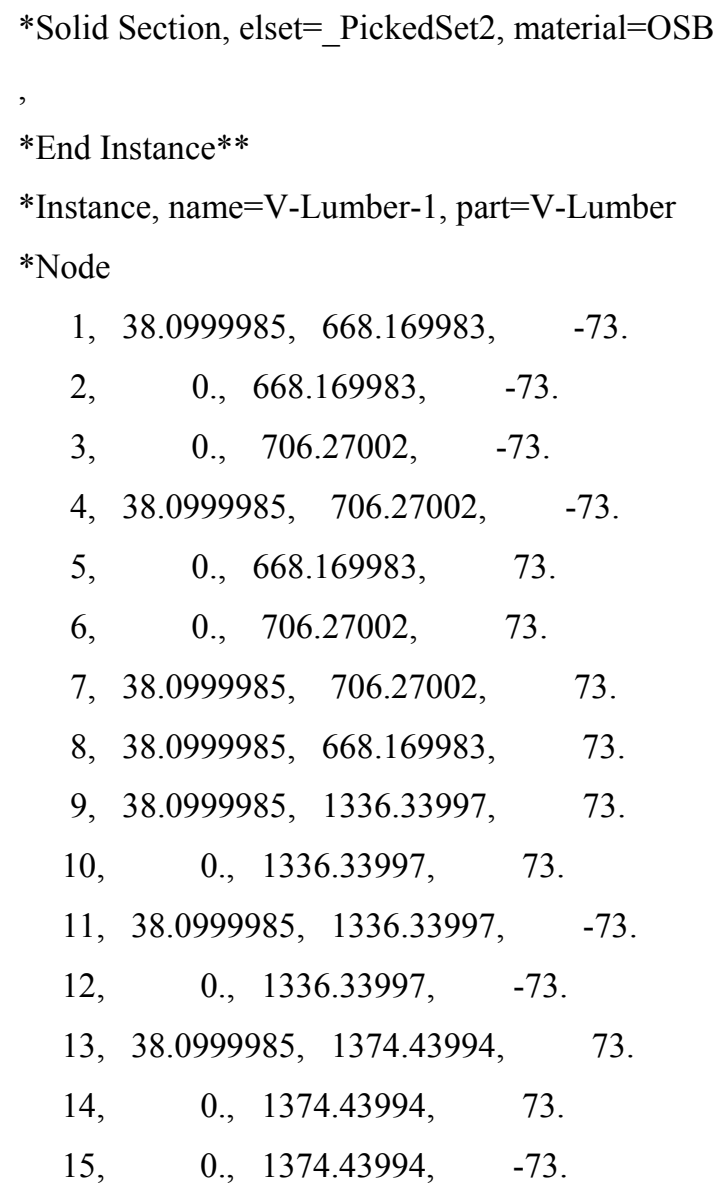

$*$ Element, type $=\mathrm{C} 3 \mathrm{D} 8 \mathrm{I}$

$1,34,36,35,33,1,2,3,4$

$2,8,5,6,7,34,36,35,33$

$3,35,33,219,209,6,7,37,56$

4, 209, 219, 220,210, 56, 37, 38, 55 
$5,210,220,221,211,55,38,39,54$

$6,211,221,222,212,54,39,40,53$

$7,212,222,223,213,53,40,41,52$

$8,213,223,224,214,52,41,42,51$

$9,214,224,225,215,51,42,43,50$

$10,215,225,226,216,50,43,44,49$

90, 204, 193, 192, 205, 274, 284, 285, 275

91, 205, 192, 191, 206, 275, 285, 286, 276

92, 206, 191, 190, 207, 276, 286, 287, 277

93, 207, 190, 189, 208, 277, 287, 288, 278

94, 208, 189, 23, 24, 278, 288, 125, 123

*Nset, nset=_PickedSet2, internal, generate

$1,288,1$

*Elset, elset=_PickedSet2, internal, generate

1, 94, 1

** Section: Lumber

*Solid Section, elset=_PickedSet2, material=Lumber

*End Instance**

*Instance, name=H-Lumber-2, part=H-Lumber
0.
38.1 ,
0.

*Node

1, 638.575012, 2710.78003, 73.

2, 638.575012, 2672.67993, 73.

3, 1200.94995, 2672.67993, 73.

4, 1200.94995, 2710.78003, 73.

$5,638.575012,2672.67993,-73$.

6, 1200.94995, 2672.67993, -73.

$7,1200.94995,2710.78003, \quad-73$.

$8,638.575012,2710.78003, \quad-73$.

9, 600.474976, 2710.78003, -73.

$10,600.474976,2672.67993,-73$.

$11,38.0999985,2672.67993, \quad-73$.

12, 38.0999985, 2710.78003, -73. 
13, 600.474976, 2672.67993, 73.

14, 38.0999985, 2672.67993, 73.

15, 38.0999985, 2710.78003, 73.

240, 2089.46948, 2710.78003, 0 .

241, 2151.95557, 2710.78003, 0 .

242, 2214.44165, 2710.78003, 0.

243, 2276.92773, 2710.78003, 0.

244, 2339.41382, 2710.78003, 0 .

245, 1902.01111, 2672.67993, 0 .

246, 1964.49719, 2672.67993, 0.

247, 2026.98328, 2672.67993, 0.

248, 2089.46948, 2672.67993, 0 .

249, 2151.95557, 2672.67993, 0 .

250, 2214.44165, 2672.67993, 0.

251, 2276.92773, 2672.67993, 0.

252, 2339.41382, 2672.67993, 0.

*Element, type $=\mathrm{C} 3 \mathrm{D} 8 \mathrm{I}$

1, 74, 76, 197, 189, 1, 2, 41, 56

2, 189, 197, 198, 190, 56, 41, 42, 55

$3,190,198,199,191,55,42,43,54$

4, 191, 199, 200, 192, 54, 43, 44, 53

5, 192, 200, 201, 193, 53, 44, 45, 52

6, 193, 201, 202, 194, 52, 45, 46, 51

7, 194, 202, 203, 195, 51, 46, 47, 50

8, 195, 203, 204, 196, 50, 47, 48, 49

$9,196,204,75,73,49,48,3,4$

$10,8,5,64,65,74,76,197,189$

$75,147,145,73,75,22,23,4,3$

$76,19,20,7,6,147,145,73,75$

$77,76,74,110,112,2,1,16,13$

$78,5,8,9,10,76,74,110,112$ 
$79,111,109,186,185,14,15,33,34$

$80,11,12,35,36,111,109,186,185$

$81,31,181,183,30,37,187,188,40$

$82,181,28,27,183,187,38,39,188$

*Nset, nset=_PickedSet2, internal, generate

$1,252,1$

*Elset, elset=_PickedSet2, internal, generate

$1,82,1$

** Section: Lumber

*Solid Section, elset=_PickedSet2, material=Lumber

*End Instance**

*Instance, name $=\mathrm{V}$-Lumber-2, part=V-Lumber

$1200.95, \quad 0 ., \quad 0$.

*Node

1, 38.0999985, 668.169983, -73.

$2, \quad 0 ., 668.169983, \quad-73$.

$3, \quad 0 ., \quad 706.27002, \quad-73$.

4, 38.0999985, 706.27002, -73 .

5, $\quad 0 ., \quad 668.169983, \quad 73$.

6, $\quad 0 ., \quad 706.27002, \quad 73$.

7, 38.0999985, 706.27002, 73.

8, 38.0999985, 668.169983, 73.

9, 38.0999985, 1336.33997, 73.

$10, \quad 0 ., 1336.33997, \quad 73$.

11, 38.0999985, 1336.33997, -73.

$12, \quad 0 ., 1336.33997, \quad-73$.

13, 38.0999985, 1374.43994, 73.

14, 0., 1374.43994, 73.

15, 38.0999985, 1374.43994, -73 .

280, 0., 152.658188, 0.

281, 0., 209.937271, 0.

282, $\quad 0 ., \quad 267.21637, \quad 0$.

$283, \quad 0 ., 324.495453, \quad 0$. 


$\begin{array}{llll}284, & 0 ., & 381.774536, & 0 . \\ 285, & 0 ., & 439.05365, & 0 . \\ 286, & 0 ., & 496.332733, & 0 . \\ 287, & 0 ., & 553.611816, & 0 . \\ 288, & 0 ., & 610.89093, & 0 .\end{array}$

*Element, type $=\mathrm{C} 3 \mathrm{D} 8 \mathrm{I}$

$1,34,36,35,33,1,2,3,4$

$2,8,5,6,7,34,36,35,33$

$3,35,33,219,209,6,7,37,56$

$4,209,219,220,210,56,37,38,55$

$5,210,220,221,211,55,38,39,54$

$6,211,221,222,212,54,39,40,53$

7, 212, 222, 223, 213, 53, 40, 41, 52

$8,213,223,224,214,52,41,42,51$

$9,214,224,225,215,51,42,43,50$

$10,215,225,226,216,50,43,44,49$

90, 277, 207, 190, 287, 276, 206, 191, 286

$91,179,278,288,178,180,277,287,177$

92, 278, 208, 189, 288, 277, 207, 190, 287

93, 1, 34, 36, 2, 179, 278, 288, 178

94, 34, 8, 5, 36, 278, 208, 189, 288

*Nset, nset=_PickedSet2, internal, generate

$1,288,1$

*Elset, elset=_PickedSet2, internal, generate

1, 94, 1

** Section: Lumber

*Solid Section, elset=_PickedSet2, material=LLumber

,

*End Instance**

*Instance, name $=\mathrm{V}$-Lumber-3, part=V-Lumber 2401.9, $\quad 0 ., \quad 0$.

*Node

1, 38.0999985, 668.169983, -73 .

$2, \quad 0 ., 668.169983, \quad-73$. 
$3, \quad 0 ., \quad 706.27002, \quad-73$.

4, 38.0999985, 706.27002, -73 .

$5, \quad 0 ., 668.169983, \quad 73$.

$6, \quad 0 ., \quad 706.27002, \quad 73$.

7, 38.0999985, 706.27002, 73.

8, 38.0999985, 668.169983, 73.

9, 38.0999985, 1336.33997, 73.

$10, \quad 0 ., 1336.33997, \quad 73$.

11, 38.0999985, 1336.33997, -73.

$12, \quad 0 ., \quad 1336.33997, \quad-73$.

13, 38.0999985, 1374.43994, 73.

14, $\quad 0 ., \quad 1374.43994, \quad 73$.

15, 38.0999985, 1374.43994, -73 .

284, 0., 381.774536, 0.

285, $\quad 0 ., \quad 439.05365, \quad 0$.

286, 0., 496.332733, 0.

287, 0., 553.611816, 0 .

288, 0., 610.89093, 0.

*Element, type $=$ C3D8I

$1,34,36,35,33,1,2,3,4$

$2,8,5,6,7,34,36,35,33$

$3,35,33,219,209,6,7,37,56$

4, 209, 219, 220, 210, 56, 37, 38, 55

$5,210,220,221,211,55,38,39,54$

$6,211,221,222,212,54,39,40,53$

7, 212, 222, 223, 213, 53, 40, 41, 52

$8,213,223,224,214,52,41,42,51$

$9,214,224,225,215,51,42,43,50$

$10,215,225,226,216,50,43,44,49$

$11,216,226,227,217,49,44,45,48$

$12,217,227,228,218,48,45,46,47$

$13,218,228,78,77,47,46,9,10$

$14,3,4,66,67,35,33,219,209$

$15,67,66,65,68,209,219,220,210$ 
90, 277, 207, 190, 287, 276, 206, 191, 286

$91,179,278,288,178,180,277,287,177$

92, 278, 208, 189, 288, 277, 207, 190, 287

$93,1,34,36,2,179,278,288,178$

94, 34, 8, 5, 36, 278, 208, 189, 288

*Nset, nset=_PickedSet2, internal, generate

$1,288,1$

*Elset, elset=_PickedSet2, internal, generate

$1,94,1$

** Section: Lumber

*Solid Section, elset=_PickedSet2, material=Lumber

*End Instance**

*Instance, name $=\mathrm{H}-$ Lumber-3, part=H-Lumber

$$
0 ., \quad-2672.68, \quad 0 \text {. }
$$

*Node

1, 638.575012, 2672.67993, -73.

2, 638.575012, 2710.78003, -73.

3, 1200.94995, 2710.78003, -73.

4, 1200.94995, 2672.67993, -73.

5, 638.575012, 2691.72998, 73.

6, 638.575012, 2710.78003, 73.

7, 1200.94995, 2710.78003, 73.

8, 1200.94995, 2672.67993, 73.

9, 638.575012, 2672.67993, 73.

10, 600.474976, 2710.78003, 73.

370, 1301.53613, 2691.72998, 0.

371, 537.988892, 2691.72998, 0.

372, 475.502777, 2691.72998, 0.

373, 413.016663, 2691.72998, 0.

374, 350.530548, 2691.72998, 0. 
375, 288.044434, 2691.72998, 0.

$376,225.558334,2691.72998, \quad 0$.

377, 163.07222, 2691.72998, 0.

378, 100.586113, 2691.72998, 0.

*Element, type $=\mathrm{C} 3 \mathrm{D} 8 \mathrm{I}$

$1,81,241,347,225,1,45,209,62$

2, 241, 83, 233, 347, 45, 2, 46, 209

$3,225,347,348,226,62,209,210,61$

4, 347, 233, 234, 348, 209, 46, 47, 210

$5,226,348,349,227,61,210,211,60$

6, 348, 234, 235, 349, 210, 47, 48, 211

7, 227, 349, 350, 228, 60, 211, 212, 59

$8,349,235,236,350,211,48,49,212$

$9,228,350,351,229,59,212,213,58$

10, 350, 236, 237, 351, 212, 49, 50, 213

160, 190, 35, 39, 202, 344, 200, 204, 345

161, 25, 128, 278, 101, 41, 205, 346, 208

$162,128,27,118,278,205,42,206,346$

$163,101,278,127,26,208,346,207,44$

164, 278, 118, 28, 127, 346, 206, 43, 207

*Nset, nset=_PickedSet2, internal, generate

$1,378,1$

*Elset, elset=_PickedSet2, internal, generate

$1,164,1$

** Section: Lumber

*Solid Section, elset=_PickedSet2, material=Lumber

*End Instance**

*Instance, name=EPS-2, part=EPS

$1200.95, \quad 0 ., \quad 0$.

*Node

1, 638.575012, 1374.43994, -73 .

2, 638.575012, 1336.33997, -73.

3, 600.474976, 1336.33997, -73. 
4, 600.474976, 1374.43994, -73.

5, 638.575012, 1336.33997, 73.

6, 600.474976, 1336.33997, 73.

7, 600.474976, 1374.43994, 73.

8, 638.575012, 1374.43994, 73.

$9,638.575012,668.169983, \quad-73$.

$10, \quad 638.575012, \quad 706.27002, \quad-73$.

11, 1200.94995, 706.27002, -73.

12, 1200.94995, 668.169983, -73 .

13, 638.575012, 706.27002, 73.

14, 1200.94995, 706.27002, 73.

15, 1200.94995, 668.169983, 73.

16, 638.575012, 668.169983, 73.

17, 638.575012, 2042.60999, 73.

18, 638.575012, 2004.51001, 73.

19, 1200.94995, 2004.51001, 73.

20, 1200.94995, 2042.60999, 73.

2870, 163.07222, 763.549072, 0.

$2871,100.586113,1279.06091, \quad 0$.

$2872,100.586113,1221.78186, \quad 0$.

$2873,100.586113,1164.50269, \quad 0$.

2874, 100.586113, 1107.22363, 0.

$2875,100.586113,1049.94458, \quad 0$.

2876, 100.586113, 992.665466, 0 .

2877, 100.586113, 935.386353, 0.

$2878, \quad 100.586113, \quad 878.1073, \quad 0$.

$2879,100.586113,820.828186, \quad 0$.

2880, 100.586113, 763.549072, 0.

*Element, type $=\mathrm{C} 3 \mathrm{D} 8 \mathrm{I}$

$1,66,68,67,65,1,2,3,4$

$2,8,5,6,7,66,68,67,65$

$3,102,104,681,673,9,10,69,84$

$4,673,681,682,674,84,69,70,83$

$5,674,682,683,675,83,70,71,82$ 
$6,675,683,684,676,82,71,72,81$

$7,676,684,685,677,81,72,73,80$

$8,677,685,686,678,80,73,74,79$

$9,678,686,687,679,79,74,75,78$

$10,679,687,688,680,78,75,76,77$

1780, 244, 245, 415, 416, 911, 910, 1334, 1335

1781, 245, 246, 414, 415, 910, 909, 1333, 1334

1782, 246, 247, 413, 414, 909, 908, 1332, 1333

1783, 247, 248, 412, 413, 908, 907, 1331, 1332

1784, 248, 249, 411, 412, 907, 906, 1330, 1331

$1785,249,250,410,411,906,905,1329,1330$

1786, 250, 17, 29, 410, 905, 138, 176, 1329

*Nset, nset=_PickedSet2, internal, generate

1, 2880, 1

*Elset, elset=_PickedSet2, internal, generate

1, 1786, 1

** Section: EPS

*Solid Section, elset=_PickedSet2, material=EPS

,

*End Instance**

*Instance, name $=\mathrm{OSB}-2$, part $=\mathrm{OSB}$

$0 ., \quad 0 ., \quad-157.15$

*Node

$1, \quad 0 ., 668.169983, \quad 73$.

$2, \quad 0 ., \quad 668.169983,84.1500015$

$3, \quad 0 ., \quad 706.27002,84.1500015$

4, $\quad 0 ., \quad 706.27002, \quad 73$.

$5, \quad 38.0999985,668.169983, \quad 73$.

$6, \quad 38.0999985,668.169983,84.1500015$

7, 38.0999985, 706.27002, 84.1500015

8, 38.0999985, 706.27002, 73.

$9, \quad 1200.94995,668.169983, \quad 73$.

10, 1239.05005, 668.169983, 73. 
4270, 1301.53613, 1832.67273, 73.

4271, 1301.53613, 1889.95178, 73.

4272, 1301.53613, 1947.23096, 73.

4273, 1301.53613, 2004.51001, 73.

4274, 1301.53613, 2042.60999, 73.

4275, 1301.53613, 2099.88916, 73.

4276, 1301.53613, 2157.16821, 73.

4277, 1301.53613, 2214.44727, 73.

4278, 1301.53613, 2271.72632, 73.

4279, 1301.53613, 2329.00537, 73.

4280, 1301.53613, 2386.28442, 73.

4281, 1301.53613, 2443.56372, 73.

4282, 1301.53613, 2500.84277, 73.

4283, 1301.53613, 2558.12183, 73.

4284, 1301.53613, 2615.40088, 73.

*Element, type $=\mathrm{C} 3 \mathrm{D} 8 \mathrm{I}$

$1,5,6,7,8,1,2,3,4$

2, 16, 13, 202, 203, 9, 10, 173, 192

3, 203, 202, 201, 204, 192, 173, 174, 191

4, 204, 201, 200, 205, 191, 174, 175, 190

5, 205, 200, 199, 206, 190, 175, 176, 189

6, 206, 199, 198, 207, 189, 176, 177, 188

7, 207, 198, 197, 208, 188, 177, 178, 187

8, 208, 197, 196, 209, 187, 178, 179, 186

9, 209, 196, 195, 210, 186, 179, 180, 185

10, 210, 195, 194, 211, 185, 180, 181, 184

2040, 844, 693, 694, 843, 863, 738, 737, 864

2041, 843, 694, 695, 842, 864, 737, 736, 865

$2042,842,695,696,841,865,736,735,866$

2043, 841, 696, 697, 840, 866, 735, 734, 867

$2044,840,697,698,839,867,734,733,868$

2045, 839, 698, 699, 838, 868, 733, 732, 869 
$2046,838,699,700,837,869,732,731,870$

$2047,837,700,701,836,870,731,730,871$

$2048,836,701,702,835,871,730,729,872$

$2049,835,702,147,138,872,729,148,137$

$2050,105,107,108,106,154,153,171,172$

*Nset, nset=_PickedSet2, internal, generate

$1,4284, \quad 1$

*Elset, elset=_PickedSet2, internal, generate

$1,2050, \quad 1$

** Section: OSB

*Solid Section, elset=_PickedSet2, material=OSB

*End Instance**

*Nset, nset $=$ _PickedSet535, internal, instance $=$ OSB-2

$11,12,39,40,86,87,99,100,105,111,119,120,127,128,133,150$

$154,156,162,164,533,534,535,536,537,538,539,540,581,582,583,584$

$585,586,587,588,613,614,615,616,617,618,619,620,677,678,679,680$

$681,682,683,684,757,758,759,760,761,762,763,764,765,766,767,768$

$769,770,771,772,881,882,883,884,885,886,887,888,913,914,915,916$

917, 918, 919, 920

*Elset, elset=_PickedSet535, internal, instance $=\mathrm{OSB}-2$

$12,46,109,110,111,112,113,114,115,116,117,118,119,120,121,122$

$123,124,125,126,127,128,129,130,131,132,133,134,135,136,137,138$

$139,140,141,142,143,144,145,146,147,148,149,150,151,152,153,154$

$155,156,157,158,159,160,161,162,163,164,165,166,167,168,169,170$

$171,218,265,312,359,406,453,500,547,594,641,688,735,782,829,876$

923, 970, 1017, 1064, 1065, 1066, 1067, 1068, 1069, 1070, 1071, 1072, 1073, 1074, 1085, 1086

1087, 1134, 1181, 1228, 1275, 1322, 1369, 1416, 1463, 1510, 1557, 1604, 1651, 1698, 1745, 1792

1839, 1886, 1933, 1980, 1981, 1982, 1983, 1984, 1985, 1986, 1987, 1988, 1989, 1990, 1991, 1992

1993, 1994, 1995, 1996, 1997, 1998, 1999, 2000, 2001, 2002, 2003, 2004, 2005, 2006, 2007, 2008

2020, 2021, 2022, 2023, 2024, 2025, 2026, 2027, 2028, 2029, 2030, 2031, 2032, 2033, 2034, 2035

2036, 2037, 2038, 2050

*Nset, nset=_PickedSet536, internal, instance="Ref Point-1"

1 ,

*Nset, nset=_PickedSet545, internal, instance="Ref Point-1"

1 ,

*Nset, nset=_PickedSet546, internal, instance $=$ OSB-1 
$1,2,3,4,19,22,29,30,37,38,39,40,57,58,63,64$

$77,78,80,81,92,93,102,108,173,174,175,176,177,178,179,180$

181, 182, 183, 184, 185, 186, 187, 188, 189, 190, 191, 192, 193, 194, 195, 196

197, 198, 199, 200, 201, 202, 203, 204, 205, 206, 207, 208, 337, 338, 339, 340

$341,342,343,344,345,346,347,348,349,350,351,352,353,354,433,434$

$435,436,437,438,439,440,441,442,443,444,445,446,447,448,449,450$

$505,506,507,508,509,510,511,512,513,514,515,516,517,518,519,520$

$521,522,523,524,525,526,527,528,529,530,531,532,533,534,535,536$

$537,538,539,540,691,692,693,694,695,696,697,698,699,700,701,702$

703, 704, 705, 706, 707, 708, 747, 748, 749, 750, 751, 752, 753, 754, 755, 756

$757,758,759,760,761,762,763,764$

*Elset, elset=_PickedSet546, internal, instance $=$ OSB-1

$1,2,3,4,5,6,7,8,9,10,11,12,13,14,15,16$

$17,18,19,666,667,668,669,670,671,672,673,674,675,676,677,678$

$679,680,681,682,683,684,685,686,687,688,689,690,691,692,693,694$

695, 696, 697, 698, 699, 700, 701, 702, 703, 1350, 1351, 1352, 1353, 1354, 1355, 1356

1357, 1358, 1359, 1360, 1361, 1362, 1363, 1364, 1365, 1366, 1367, 1368, 1380, 1624, 1625, 1626

2046, 2047

*Elset, elset $=$ _PickedSurf504_S4, internal, instance=H-Lumber-1, generate

$1,72,1$

*Elset, elset=_PickedSurf504_S4, internal, instance=V-Lumber-1, generate

$3,24,1$

*Elset, elset $=$ _PickedSurf504_S4, internal, instance $=$ V-Lumber-2, generate

$1,72,1$

*Elset, elset=_PickedSurf504_S4, internal, instance=H-Lumber-3

$2,4,6,8,10,12,14,16,18,20,22,24,26,28,30,32$

$34,36,46,48,50,52,54,56,58,60,62,64,66,68,70,72$

$74,76,78,80$

*Elset, elset $=$ _PickedSurf504_S4, internal, instance=V-Lumber-3

$1,2,49,50,51,52,53,54,55,56,57,58,59,60,61,62$

$63,64,65,66,67,68,69,70,71,72$

*Elset, elset $=$ _PickedSurf504_S6, internal, instance=H-Lumber-1

$73,74,77,78$

*Elset, elset=_PickedSurf504_S6, internal, instance=V-Lumber-1

$1,2,25,26,49,50,51,52,53,54,55,56,57,58,59,60$

$61,62,63,64,65,66,67,68,69,70,71,72,73,74,75,76$

$77,78,79,80,81,82,83,84,85,86,87,88,89,90,91,92$ 
93, 94

*Elset, elset $=$ PickedSurf504_S6, internal, instance $=$ V-Lumber-2, generate

$1,72,1$

*Elset, elset=_PickedSurf504_S6, internal, instance=H-Lumber-3

$37,39,41,43,121,123,125,127,129,131,133,135,137,139,141,143$

$145,147,149,151,153,155$

*Elset, elset=_PickedSurf504_S6, internal, instance=V-Lumber-3, generate

$3,48,1$

*Elset, elset=_PickedSurf504_S3, internal, instance=V-Lumber-1, generate

$27,48,1$

*Elset, elset $=$ _PickedSurf504_S3, internal, instance $=$ V-Lumber-2, generate

$73,94,1$

*Elset, elset $=$ _PickedSurf504_S3, internal, instance $=$ H-Lumber-3

$81,82,83,84,85,86,87,88,89,99,100,101,102,103,104,105$

106,107

*Elset, elset=_PickedSurf504_S5, internal, instance=V-Lumber-2, generate

$73,94,1$

*Elset, elset=_PickedSurf504_S5, internal, instance=V-Lumber-3, generate

$73,94,1$

*Surface, type=ELEMENT, name=_PickedSurf504, internal

_PickedSurf504_S4, S4

PickedSurf504_S6, S6

PickedSurf504_S3, S3

_PickedSurf504_S5, S5

*Elset, elset=_PickedSurf505_S6, internal, instance $=$ EPS-1

$281,292,501,512,523,534,545,556,567,578,589,600,611,622,633,644$

$655,666,677,688,1589,1600,1611,1622,1633,1644,1655,1666,1677,1688,1699,1710$

$1721,1732,1743,1754,1765,1776$

*Elset, elset $=$ _PickedSurf505_S6, internal, instance $=$ EPS-2

1111, 1122, 1133, 1144, 1155, 1166, 1177, 1188, 1199, 1210, 1221, 1232, 1243, 1254, 1265, 1276

1287, 1298, 1309, 1320, 1331, 1342, 1353, 1364, 1375, 1386, 1397, 1408, 1419, 1430, 1441, 1452

$1463,1474,1485,1496,1765,1776$

*Elset, elset $=$ _PickedSurf505_S4, internal, instance=EPS-1

65, 76, 929, 940, 951, 962, 973, 984, 995, 1006, 1017, 1028, 1039, 1050, 1061, 1072

1083, 1094, 1105, 1116, 1127, 1138, 1149, 1160, 1171, 1182, 1193, 1204, 1215, 1226, 1237, 1248

$1259,1270,1281,1292,1303,1314$

*Elset, elset $=$ _PickedSurf505_S4, internal, instance $=$ EPS-2 
$87,98,109,120,131,142,153,164,175,186,197,208,219,230,241,252$

$263,274,483,494,527,538,549,560,571,582,593,604,615,626,637,648$

$659,670,681,692,703,714$

*Elset, elset $=$ _PickedSurf505_S5, internal, instance $=$ EPS-1

$9,18,27,36,45,54,171,172,173,174,175,176,177,178,179,180$

$181,270,271,272,273,274,275,276,277,278,279,280,391,392,393,394$

395, 396, 397, 398, 399, 400, 401, 490, 491, 492, 493, 494, 495, 496, 497, 498

$499,500,589,590,591,592,593,594,595,596,597,598,599,688,689,690$

$1726,1727,1728,1729,1730,1731,1732,1733,1734,1735,1736,1737,1738,1739,1740,1741$

$1742,1743,1744,1745,1746,1747,1748,1749,1750,1751,1752,1753,1754,1755,1756,1757$

$1758,1759,1760,1761,1762,1763,1764,1765,1766,1767,1768,1769,1770,1771,1772,1773$

$1774,1775,1776,1777,1778,1779,1780,1781,1782,1783,1784,1785,1786$

*Elset, elset $=$ _PickedSurf506_S1, internal, instance $=$ EPS-2

$2,12,13,14,15,16,17,18,19,20,30,31,32,33,34,35$

$36,37,38,48,49,50,51,52,53,54,55,56,58,68,69,70$

$71,72,73,74,75,76,176,177,178,179,180,181,182,183,184,185$

186, 187, 188, 189, 190, 191, 192, 193, 194, 195, 196, 197, 198, 199, 200, 201

202, 203, 204, 205, 206, 207, 208, 209, 210,211, 212, 213, 214, 215, 216, 217

$218,219,220,221,222,223,224,225,226,227,228,229,230,231,232,233$

$234,235,236,237,238,239,240,241,242,243,244,245,246,247,248,249$

$1586,1587,1588,1589,1590,1591,1592,1593,1594,1595,1596,1597,1598,1599,1600,1601$

$1602,1603,1604,1605,1705,1706,1707,1708,1709,1710,1711,1712,1713,1714,1715,1727$

$1729,1730,1731,1732,1733,1734,1735,1736,1737,1747,1748,1749,1750,1751,1752,1753$

$1754,1755,1765,1766,1767,1768,1769,1770,1771,1772,1773,1774,1775$

*Surface, type=ELEMENT, name=_PickedSurf506, internal

PickedSurf506_S1, S1

_PickedSurf506_S2, S2

*Elset, elset $=$ _PickedSurf507_S1, internal, instance $=$ OSB-1

$1,2,3,4,5,6,7,8,9,10,11,12,13,14,15,16$

$17,18,19,229,230,231,232,233,234,235,236,237,238,239,240,241$

$242,243,244,245,246,247,248,249,250,251,252,253,254,255,256,257$

$258,259,260,261,262,263,264,265,266,267,268,269,270,271,272,273$ 
$274,275,276,277,278,279,280,281,282,283,284,285,286,287,288,289$

290, 291, 292, 293, 294, 295, 296, 297, 298, 299, 300, 301, 302, 303, 304, 305

$306,307,308,309,310,311,312,313,314,315,316,317,318,319,320,321$

1932, 1933, 1934, 1935, 1936, 1937, 1938, 1939, 1940, 1941, 1942, 1943, 1944, 1945, 1946, 1947 1948, 1949, 1950, 1951, 1952, 1953, 1954, 1955, 1956, 1957, 1958, 1959, 1960, 1961, 1962, 1963 1964, 1965, 1966, 1967, 1968, 1969, 1970, 1971, 1972, 1973, 1974, 1975, 1976, 1977, 1978, 1979 1980, 1981, 1982, 1983, 1984, 1985, 1986, 1987, 1988, 1989, 1990, 1991, 1992, 1993, 1994, 1995 1996, 1997, 1998, 1999, 2000, 2001, 2002, 2003, 2004, 2005, 2006, 2007, 2008, 2009, 2010, 2011 2012, 2013, 2014, 2015, 2016, 2017, 2018, 2019, 2020, 2021, 2022, 2023, 2024

*Elset, elset $=$ _PickedSurf507_S2, internal, instance $=$ OSB-1

$20,21,22,23,24,25,26,27,28,29,30,31,32,33,34,35$ $36,37,38,39,40,41,42,43,44,45,46,47,48,49,50,51$ $52,53,54,55,56,57,58,59,60,61,62,63,64,65,66,67$ $68,69,70,71,72,73,74,75,76,77,78,79,80,81,82,83$ $84,85,86,87,88,89,90,91,92,93,94,95,96,97,98,99$

$1515,1516,1517,1518,1519,1520,1521,1522,1523,1524,1525,1526,1527,1528,1529,1530$ $1531,1532,1533,1534,1535,1536,1537,1538,1539,1540,1541,1542,1543,1544,1545,1546$ $1547,1548,1549,1550,1551,1552,1553,1554,1555,1556,1557,1558,1559,1560,1561,1562$ 1563, 1564, 1565, 1566, 1567, 1568, 1569, 1570, 1571, 1572, 1573, 1574, 1575, 1576, 1577, 1578 $1579,1580,1581,1582,1583,1584,1585,1586,1587,1588,1589,1590,1591,1592,1593,1594$ $1595,1596,1597,1598,1599,1600,1601,1602,1603,1604,1605,1606,1607,1608,1609,1610$ $1611,1612,1613,1614,1615,1616,1617,1618,1619,1620,1621,1622,1623$

*Elset, elset $=$ _PickedSurf507_S2, internal, instance $=$ OSB-2

$171,172,173,174,175,176,177,178,179,180,181,182,183,184,185,186$ 187, 188, 189, 190, 191, 192, 193, 194, 195, 196, 197, 198, 199, 200, 201, 202 203, 204, 205, 206, 207, 208, 209,210, 211, 212, 213,214, 215, 216, 217, 218 $219,220,221,222,223,224,225,226,227,228,229,230,231,232,233,234$ $235,236,237,238,239,240,241,242,243,244,245,246,247,248,249,250$ $251,252,253,254,255,256,257,258,259,260,261,262,263,264,265,266$ $267,268,269,270,271,272,273,274,275,276,277,278,279,280,281,282$ 283, 284, 285, 286, 287, 288, 289, 290, 291, 292, 293, 294, 295, 296, 297, 298 
1906, 1907, 1908, 1909, 1910, 1911, 1912, 1913, 1914, 1915, 1916, 1917, 1918, 1919, 1920, 1921

1922, 1923, 1924, 1925, 1926, 1927, 1928, 1929, 1930, 1931, 1932, 1933, 1934, 1935, 1936, 1937 1938, 1939, 1940, 1941, 1942, 1943, 1944, 1945, 1946, 1947, 1948, 1949, 1950, 1951, 1952, 1953

1954, 1955, 1956, 1957, 1958, 1959, 1960, 1961, 1962, 1963, 1964, 1965, 1966, 1967, 1968, 1969

1970, 1971, 1972, 1973, 1974, 1975, 1976, 1977, 1978, 1979

*Surface, type=ELEMENT, name=_PickedSurf507, internal

PickedSurf507_S1, S1

_PickedSurf507_S2, S2

*Elset, elset=_PickedSurf508_S2, internal, instance=H-Lumber-1

$1,2,3,4,5,6,7,8,9,19,20,21,22,23,24,25$

$26,27,37,38,39,40,41,42,43,44,45,55,56,57,58,59$

$60,61,62,63$

*Elset, elset=_PickedSurf508_S2, internal, instance=V-Lumber-1

$3,4,5,6,7,8,9,10,11,12,13,51,52,53,54,55$

$56,57,58,59,60,61,73,74,75,76,77,78,79,80,81,82$

83 ,

*Elset, elset=_PickedSurf508_S2, internal, instance=V-Lumber-2

$3,4,5,6,7,8,9,10,11,12,13,27,28,29,30,31$

$32,33,34,35,36,37,51,52,53,54,55,56,57,58,59,60$

61 ,

*Elset, elset=_PickedSurf508_S2, internal, instance=H-Lumber-3

$1,2,3,4,5,6,7,8,9,10,11,12,13,14,15,16$

$17,18,45,46,47,48,49,50,51,52,53,54,55,56,57,58$

$59,60,61,62,81,82,83,84,85,86,87,88,89,90,91,92$

93, 94, 95, 96, 97, 98, 121, 122, 123, 124, 125, 126, 127, 128, 129, 130

$131,132,133,134,135,136,137,138$

*Elset, elset=_PickedSurf508_S2, internal, instance=H-Lumber-2

$1,2,3,4,5,6,7,8,9,19,20,21,22,23,24,25$

$26,27,37,38,39,40,41,42,43,44,45,55,56,57,58,59$

$60,61,62,63$

*Elset, elset $=$ _PickedSurf508_S2, internal, instance $=\mathrm{V}$-Lumber-3

$3,4,5,6,7,8,9,10,11,12,13,27,28,29,30,31$

$32,33,34,35,36,37,51,52,53,54,55,56,57,58,59,60$

61 , 
*Elset, elset=_PickedSurf508_S1, internal, instance=H-Lumber-1

$10,11,12,13,14,15,16,17,18,28,29,30,31,32,33,34$

$35,36,46,47,48,49,50,51,52,53,54,64,65,66,67,68$

$69,70,71,72$

*Elset, elset $=$ _PickedSurf508_S1, internal, instance $=$ V-Lumber-1

$14,15,16,17,18,19,20,21,22,23,24,62,63,64,65,66$

$67,68,69,70,71,72,84,85,86,87,88,89,90,91,92,93$

94 ,

*Elset, elset=_PickedSurf508_S1, internal, instance=V-Lumber-2

$14,15,16,17,18,19,20,21,22,23,24,38,39,40,41,42$

$43,44,45,46,47,48,62,63,64,65,66,67,68,69,70,71$

72 ,

*Elset, elset $=$ _PickedSurf508_S1, internal, instance $=$ H-Lumber-3

$19,20,21,22,23,24,25,26,27,28,29,30,31,32,33,34$

$35,36,63,64,65,66,67,68,69,70,71,72,73,74,75,76$

$77,78,79,80,99,100,101,102,103,104,105,106,107,108,109,110$

$111,112,113,114,115,116,139,140,141,142,143,144,145,146,147,148$

$149,150,151,152,153,154,155,156$

*Elset, elset $=$ _PickedSurf508_S1, internal, instance $=$ H-Lumber-2

$10,11,12,13,14,15,16,17,18,28,29,30,31,32,33,34$

$35,36,46,47,48,49,50,51,52,53,54,64,65,66,67,68$

$69,70,71,72$

*Elset, elset $=$ PickedSurf508 S1, internal, instance $=$ V-Lumber-3

$14,15,16,17,18,19,20,21,22,23,24,38,39,40,41,42$

$43,44,45,46,47,48,62,63,64,65,66,67,68,69,70,71$

72 ,

*Elset, elset $=$ PickedSurf508 S6, internal, instance $=\mathrm{V}$-Lumber-1, generate

$27,47,2$

*Elset, elset $=$ _PickedSurf508_S6, internal, instance $=\mathrm{V}$-Lumber-2, generate

$73,93,2$

*Elset, elset $=$ _PickedSurf508_S6, internal, instance $=$ V-Lumber-3, generate

$73,93,2$

*Elset, elset $=$ _PickedSurf508_S4, internal, instance $=$ V-Lumber-1, generate

$28,48,2$

*Elset, elset $=$ _PickedSurf508_S4, internal, instance $=$ V-Lumber-2, generate

$74,94,2$

*Elset, elset=_PickedSurf508_S4, internal, instance=V-Lumber-3, generate 
$74,94,2$

*Surface, type $=$ ELEMENT, name $=$ PPickedSurf508, internal

PickedSurf508_S2, S2

_PickedSurf508_S1, S1

PickedSurf508_S6, S6

PickedSurf508_S4, S4

*Elset, elset $=$ _PickedSurf509_S1, internal, instance=OSB-1

$1369,1370,1371,1372,1373,1374,1375,1376,1377,1378,1379,1627,1628,1629,1630,1631$

1632, 1633, 1634, 1635, 1636, 1637, 2036, 2037, 2038, 2039, 2040, 2041, 2042, 2043, 2044

*Elset, elset $=$ _PickedSurf509_S1, internal, instance $=$ OSB-2

$2,3,4,5,6,7,8,9,10,11,12,58,59,60,61,62$

$63,64,65,66,67,68,72,73,74,75,76,77,78,79,80,81$

$82,112,113,114,115,116,117,118,119,120,130,131,132,133,134,135$

$136,137,138,142,143,144,145,146,147,148,149,150,151,152,153,154$

155, 156, 157, 158, 159, 1064, 1065, 1066, 1067, 1068, 1069, 1070, 1071, 1072, 1990, 1991

1992, 1993, 1994, 1995, 1996, 1997, 1998, 2039, 2040, 2041, 2042, 2043, 2044, 2045, 2046, 2047

2048, 2049

*Elset, elset $=$ _PickedSurf509_S4, internal, instance $=$ OSB-1

$1673,1674,1675,1676,1677,1678,1679,1680,1681,1682,1683,1807,1808,1809,1810,1811$

$1812,1813,1814,1815$

*Elset, elset=_PickedSurf509_S4, internal, instance $=$ OSB-2

$14,15,16,17,18,19,20,21,22,23,24,85,86,87,88,89$

$90,91,92,93,94,95$

*Elset, elset $=$ _PickedSurf509_S2, internal, instance $=$ OSB-1

1684, 1685, 1686, 1687, 1688, 1689, 1690, 1691, 1692, 1693, 1694, 2027, 2028, 2029, 2030, 2031

2032, 2033, 2034, 2035

*Elset, elset $=$ _PickedSurf509_S2, internal, instance $=$ OSB-2

$25,26,27,28,29,30,31,32,33,34,35,36,37,38,39,40$

$41,42,43,44,45,46,97,98,99,100,101,102,103,104,105,106$

$107,121,122,123,124,125,126,127,128,129,160,161,162,163,164,165$

166, 167, 168, 1980, 1981, 1982, 1983, 1984, 1985, 1986, 1987, 1988, 1999, 2000, 2001, 2002

2003, 2004, 2005, 2006, 2007, 2009, 2010, 2011, 2012, 2013, 2014, 2015, 2016, 2017, 2018, 2019

2020, 2021, 2022, 2023, 2024, 2025, 2026, 2027, 2028, 2030, 2031, 2032, 2033, 2034, 2035, 2036

2037, 2038

*Elset, elset $=$ PickedSurf509_S6, internal, instance $=$ OSB-1

$1720,1721,1722,1723,1724,1725,1726,1727,1728,1758,1759,1760,1761,1762,1763,1764$

$1765,1766,1798,1799,1800,1801,1802,1803,1804,1805,1806$ 
*Elset, elset $=$ _PickedSurf509_S6, internal, instance $=$ OSB-2

$47,48,49,50,51,52,53,54,55,56,57,1075,1076,1077,1078,1079$

1080, 1081, 1082, 1083, 1084, 1085

*Surface, type=ELEMENT, name=_PickedSurf509, internal

_PickedSurf509_S1, S1

_PickedSurf509_S4, S4

_PickedSurf509_S2, S2

_PickedSurf509_S6, S6

*Elset, elset $=$ _ PickedSurf510_S5, internal, instance $=$ H-Lumber-1

81,82

*Elset, elset $=\_$PickedSurf510_S6, internal, instance $=$H-Lumber-1 $75,76,79,80$

*Elset, elset $=$ _ PickedSurf510_S6, internal, instance $=$ H-Lumber-3

157,159

*Elset, elset $=$ _ PickedSurf510_S3, internal, instance $=$ H-Lumber-3 161,162

*Elset, elset $=$ _ PickedSurf510_S4, internal, instance $=$ H-Lumber-3

118,120

*Surface, type=ELEMENT, name=_PickedSurf510, internal

_PickedSurf510_S5, S5

_PickedSurf510_S6, S6

_PickedSurf510_S4, S4

_PickedSurf510_S3, S3

*Elset, elset $=$ _ PickedSurf511_S5, internal, instance $=$ V-Lumber-1 61,72

*Elset, elset $=$ _ PickedSurf511_S5, internal, instance $=$ V-Lumber-2

61,72

*Elset, elset $=$ _ PickedSurf511_S5, internal, instance $=$ V-Lumber-3 61,72

*Elset, elset $=$ _ PickedSurf511_S2, internal, instance $=$ V-Lumber-1

27,28

*Elset, elset $=$ _ PickedSurf511_S2, internal, instance $=$ V-Lumber-2

73,74

*Elset, elset $=$ _ PickedSurf511_S2, internal, instance $=$ V-Lumber-3

73,74

*Surface, type=ELEMENT, name=_PickedSurf511, internal

_PickedSurf511_S5, S5 
_PickedSurf511_S2, S2

*Elset, elset $=$ _PickedSurf512_S1, internal, instance $=$ H-Lumber-1, generate

$74,80,2$

*Elset, elset=_PickedSurf512_S1, internal, instance=V-Lumber-1, generate

$2,50,24$

*Elset, elset $=$ _PickedSurf512_S1, internal, instance $=$ V-Lumber-2, generate

$2,50,24$

*Elset, elset $=$ _PickedSurf512_S1, internal, instance $=$ H-Lumber-3

39, 40, 43, 44, 119, 120, 159, 160

*Elset, elset $=$ _PickedSurf512_S1, internal, instance $=$ H-Lumber-2, generate

$74,80,2$

*Elset, elset $=$ _PickedSurf512_S1, internal, instance $=$ V-Lumber-3, generate

$2,50,24$

*Elset, elset $=$ _PickedSurf512_S4, internal, instance=H-Lumber-1

82 ,

*Elset, elset $=$ _PickedSurf512_S4, internal, instance=H-Lumber-3

162,164

*Elset, elset=_PickedSurf512_S4, internal, instance=H-Lumber-2

82 ,

*Elset, elset $=$ _PickedSurf512_S2, internal, instance $=$ H-Lumber-1, generate $73,79,2$

*Elset, elset $=$ _PickedSurf512_S2, internal, instance $=$ V-Lumber-1, generate

$1,49,24$

*Elset, elset $=$ _PickedSurf512_S2, internal, instance $=\mathrm{V}$-Lumber-2, generate $1,49,24$

*Elset, elset=_PickedSurf512_S2, internal, instance $=$ H-Lumber-3

$37,38,41,42,117,118,157,158$

*Elset, elset $=$ _PickedSurf512_S2, internal, instance $=$ H-Lumber-2, generate $73,79,2$

*Elset, elset=_PickedSurf512_S2, internal, instance $=$ V-Lumber-3, generate

$1,49,24$

*Elset, elset $=$ _PickedSurf512_S6, internal, instance $=$ H-Lumber-1

81 ,

*Elset, elset=_PickedSurf512_S6, internal, instance=H-Lumber-3

161,163

*Elset, elset $=$ _PickedSurf512_S6, internal, instance $=$ H-Lumber -2

81 , 
*Surface, type=ELEMENT, name=_PickedSurf512, internal

PickedSurf512_S1, S1

_ PickedSurf512_S4, S4

_PickedSurf512_S2, S2

_PickedSurf512_S6, S6

*Elset, elset $=$ _PickedSurf516_S1, internal, instance $=$ OSB-1

1380, 1392, 1624, 1718, 1748, 1796, 1797, 2025, 2045

*Elset, elset $=$ _PickedSurf516_S1, internal, instance $=$ OSB-2

83, 84, 109, 111, 141, 169, 1989, 2008

*Elset, elset $=$ _PickedSurf516_S2, internal, instance $=$ OSB-1

1625, 1649, 1719, 1786, 2026, 2046

*Elset, elset $=$ _PickedSurf516_S2, internal, instance $=$ OSB-2

13, 69, 96, 108, 110, 139, 140, 170, 1086, 2029

*Elset, elset $=$ _PickedSurf516_S6, internal, instance $=$ OSB-1

1626, 1650, 1717, 1747, 1785, 2047

*Elset, elset $=$ _PickedSurf516_S6, internal, instance $=$ OSB-2

1073, 1074

*Elset, elset=_PickedSurf516_S4, internal, instance=OSB-1, generate

2048, 2050, 1

*Elset, elset=_PickedSurf516_S4, internal, instance $=$ OSB-2

$1,70,71,2050$

*Surface, type=ELEMENT, name=_PickedSurf516, internal

_PickedSurf516_S1, S1

_PickedSurf516_S2, S2

_PickedSurf516_S6, S6

_PickedSurf516_S4, S4

*Elset, elset=_PickedSurf517_S4, internal, instance $=$ H-Lumber-2, generate

$1,72,1$

*Elset, elset $=$ _PickedSurf517_S6, internal, instance $=$ H-Lumber-2, generate

$73,80,1$

*Elset, elset $=$ _PickedSurf517_S5, internal, instance $=$ H-Lumber-2

81,82

*Surface, type=ELEMENT, name=_PickedSurf517, internal

_PickedSurf517_S4, S4

_PickedSurf517_S6, S6

_PickedSurf517_S5, S5

*Elset, elset $=$ _PickedSurf518_S4, internal, instance $=$ H-Lumber-1, generate 
$73,80,1$

*Elset, elset $=$ _PickedSurf518_S6, internal, instance $=$ H-Lumber-1, generate

$1,72,1$

*Elset, elset=_PickedSurf518_S3, internal, instance=H-Lumber-1

81,82

*Surface, type=ELEMENT, name=_PickedSurf518, internal

_PickedSurf518_S4, S4

_PickedSurf518_S6, S6

_PickedSurf518_S3, S3

** Constraint: Four Point Loading

*Rigid Body, ref node=_PickedSet545, tie nset=_PickedSet546

** Constraint: Horizontal to Vertical Lumber

*Tie, name="Horizontal to Vertical Lumber", adjust=yes

_PickedSurf511,_PickedSurf510

** Constraint: OSB to Lumber

*Tie, name="OSB to Lumber", adjust=yes

_PickedSurf516,_PickedSurf512

** Constraint: Top Lumbers

*Tie, name="Top Lumbers", adjust=yes

_PickedSurf518,_PickedSurf517

*End Assembly**

** MATERIALS**

** EPS/Urethane

*Material, name=EPS

*Density

1.6e- 06 ,

*Elastic

3.17, 0.165

*Material, name $=$ Lumber

*Density

7.55e-06,

*Elastic

1200., 0.3

*Plastic

$24.15, \quad 0$.

$35.88,0.001$

$46.23,0.002$ 
$57.27,0.003$

$67.62,0.004$

$77.97,0.005$

$88.32,0.006$

99.36, 0.007

$109.02,0.008$

$114.54,0.0087$

$117.3,0.009$

$124.2, \quad 0.01$

*Material, name $=$ OSB

*Density

6.27e-06,

*Elastic

1551., 0.3

*Plastic

$24.15, \quad 0$.

$35.88,0.001$

$46.23,0.002$

$57.27,0.003$

$67.62,0.004$

$77.97,0.005$

$88.32,0.006$

$99.36,0.007$

109.02, 0.008

$114.54,0.0087$

$117.3,0.009$

$124.2,0.01^{* *}$

** INTERACTION PROPERTIES**

*Surface Interaction, name="OSB to EPS (Glue)"

1.

*Friction, slip tolerance $=0.005$, taumax $=200$.

$0.15,0.5$

*Surface Interaction, name="Sheets to Lumbers (Hard)"

1.

*Surface Behavior, pressure-overclosure=HARD**

** BOUNDARY CONDITIONS**

** Name: Bottom-Roller Type: Displacement/Rotation 
*Boundary

_PickedSet535, 1, 1

_PickedSet535, 3, 3

_PickedSet535, 5, 5

PickedSet535, 6, 6

$* *$

** INTERACTIONS**

** Interaction: EPS and Lumber

*Contact Pair, interaction="Sheets to Lumbers (Hard)", type=SURFACE TO SURFACE

_PickedSurf505,_PickedSurf504

** Interaction: OSB and EPS

*Contact Pair, interaction="OSB to EPS (Glue)", type=SURFACE TO SURFACE

_PickedSurf507,_PickedSurf506

** Interaction: OSB and Lumber

*Contact Pair, interaction="Sheets to Lumbers (Hard)", type=SURFACE TO SURFACE

_PickedSurf509,_PickedSurf508

$* *$

$* *$

** STEP: Displacement**

*Step, name $=$ Displacement, nlgeom $=$ YES

Applying $60 \mathrm{~mm}$ Displacement

$*$ Static, stabilize $=0.0002$, allsdtol $=0.05$, continue $=\mathrm{NO}$

$0.1,1 ., 1 \mathrm{e}-05,0.1$

$* *$

** BOUNDARY CONDITIONS**

** Name: Displacment on Floor (Loading) Type: Displacement/Rotation

*Boundary

_PickedSet536, 3, 3, -22.5

$* *$

** OUTPUT REQUESTS**

$*$ Restart, write, frequency $=0$

$* *$

** FIELD OUTPUT: F-Output-1**

*Output, field, variable=PRESELECT $* *$

** HISTORY OUTPUT: H-Output-1**

*Output, history, variable=PRESELECT

*End Step 


\section{A.2. SIP Compressive Input File}

*HEADING

APPLYING 60 MM DISPLACEMENT ON TOP OF THE WALL

** JOB NAME: SIP-A-01 MODEL NAME: MODEL-1

** GENERATED BY: ABAQUS/CAE 6.10-1

$*$ PREPRINT, $\mathrm{ECHO}=\mathrm{NO}, \mathrm{MODEL}=\mathrm{NO}, \mathrm{HISTORY}=\mathrm{NO}, \mathrm{CONTACT}=\mathrm{NO} * *$

** PARTS**

*PART, NAME $=$ EPS

*END PART**

*PART, NAME $=\mathrm{H}-\mathrm{LUMBER}$

*END PART**

*PART, NAME $=\mathrm{OSB}$

*END PART**

*PART, NAME="REF POINT"

*END PART**

*PART, NAME=V-LUMBER

*END PART**

**

** ASSEMBLY**

*ASSEMBLY, NAME=ASSEMBLY**

*INSTANCE, NAME="REF POINT-1", PART="REF POINT"

*NODE

1, 1220., 2888.80005, 25.3999996

*NSET, NSET="REF POINT-1-REFPT_", INTERNAL

1 ,

*END INSTANCE**

$*$ INSTANCE, NAME $=$ EPS -1, PART $=$ EPS

*NODE

$1,600.474976, \quad 706.27002, \quad-73$.

2, 600.474976, 668.169983, -73.

$3,38.0999985,668.169983, \quad-73$.

4, 38.0999985, 706.27002, -73.

5, 600.474976, 668.169983, 73.

6, 38.0999985, 668.169983, 73.

7, 38.0999985, 706.27002, 73.

8, 600.474976, 706.27002, 73. 
9, 600.474976, 2042.60999, -73.

10, 600.474976, 2004.51001, -73.

11, 38.0999985, 2004.51001, -73.

12, 38.0999985, 2042.60999, -73.

13, 600.474976, 2004.51001, 73.

14, 38.0999985, 2004.51001, 73.

15, 38.0999985, 2042.60999, 73.

16, 600.474976, 2042.60999, 73.

$17,600.474976,1374.43994, \quad-73$.

$18,600.474976,1336.33997, \quad-73$.

19, 38.0999985, 1336.33997, -73.

$20,38.0999985,1374.43994, \quad-73$.

$\begin{array}{llll}2875, & 1138.46387, & 2386.28442, & 0 . \\ 2876, & 1138.46387, & 2329.00537, & 0 . \\ 2877, & 1138.46387, & 2271.72632, & 0 . \\ 2878, & 1138.46387, & 2214.44727, & 0 . \\ 2879, & 1138.46387, & 2157.16821, & 0 . \\ 2880, & 1138.46387, & 2099.88916, & 0 .\end{array}$

*ELEMENT, TYPE=C3D8I

$1,98,100,681,673,1,2,65,80$

$2,673,681,682,674,80,65,66,79$

$3,674,682,683,675,79,66,67,78$

$4,675,683,684,676,78,67,68,77$

$5,676,684,685,677,77,68,69,76$

$6,677,685,686,678,76,69,70,75$

$7,678,686,687,679,75,70,71,74$

$8,679,687,688,680,74,71,72,73$

$9,680,688,99,97,73,72,3,4$

$10,8,5,88,89,98,100,681,673$

$11,89,88,87,90,673,681,682,674$

$12,90,87,86,91,674,682,683,675$

$13,91,86,85,92,675,683,684,676$

$14,92,85,84,93,676,684,685,677$

$15,93,84,83,94,677,685,686,678$ 
$16,94,83,82,95,678,686,687,679$

$17,95,82,81,96,679,687,688,680$

$18,96,81,6,7,680,688,99,97$

$19,134,136,697,689,9,10,101,116$

$20,689,697,698,690,116,101,102,115$

1780, 2216, 2217, 658, 657, 2874, 2875, 2235, 2234

$1781,2217,2218,659,658,2875,2876,2236,2235$

1782, 2218, 2219, 660, 659, 2876, 2877, 2237, 2236

1783, 2219, 2220, 661, 660, 2877, 2878, 2238, 2237

$1784,2220,2221,662,661,2878,2879,2239,2238$

$1785,2221,2222,663,662,2879,2880,2240,2239$

1786, 2222, 634, 62, 663, 2880, 2062, 635, 2240

*NSET, NSET=_PICKEDSET2, INTERNAL, GENERATE

1, 2880, 1

*ELSET, ELSET=_PICKEDSET2, INTERNAL, GENERATE

$1,1786,1$

** SECTION: EPS

*SOLID SECTION, ELSET=_PICKEDSET2, MATERIAL=EPS

,

*END INSTANCE**

*INSTANCE, NAME=H-LUMBER-1, PART=H-LUMBER

*NODE

1, 638.575012, 2710.78003, 73.

2, 638.575012, 2672.67993, 73.

3, 1200.94995, 2672.67993, 73.

4, 1200.94995, 2710.78003, 73.

$5,638.575012,2672.67993,-73$.

$6, \quad 1200.94995,2672.67993,-73$.

$7, \quad 1200.94995,2710.78003,-73$.

$8, \quad 638.575012,2710.78003, \quad-73$.

9, 600.474976, 2710.78003, -73.

10, 600.474976, 2672.67993, -73 . 
247, 2026.98328, 2672.67993, 0 .

248, 2089.46948, 2672.67993, 0.

249, 2151.95557, 2672.67993, 0 .

250, 2214.44165, 2672.67993, 0.

251, 2276.92773, 2672.67993, 0 .

252, 2339.41382, 2672.67993, 0 .

*ELEMENT, TYPE=C3D8I

1, 74, 76, 197, 189, 1, 2, 41, 56

$2,189,197,198,190,56,41,42,55$

3, 190, 198, 199, 191, 55, 42, 43, 54

4, 191, 199, 200, 192, 54, 43, 44, 53

$5,192,200,201,193,53,44,45,52$

6, 193, 201, 202, 194, 52, 45, 46, 51

7, 194, 202, 203, 195, 51, 46, 47, 50

$8,195,203,204,196,50,47,48,49$

$9,196,204,75,73,49,48,3,4$

$10,8,5,64,65,74,76,197,189$

$80,11,12,35,36,111,109,186,185$

$81,31,181,183,30,37,187,188,40$

$82,181,28,27,183,187,38,39,188$

*NSET, NSET=_PICKEDSET2, INTERNAL, GENERATE

$1,252,1$

*ELSET, ELSET=_PICKEDSET2, INTERNAL, GENERATE

$1,82,1$

** SECTION: LUMBER

*SOLID SECTION, ELSET=_PICKEDSET2, MATERIAL=LUMBER

,

*END INSTANCE**

*INSTANCE, NAME=OSB-1, PART $=$ OSB

*NODE

$1, \quad 1239.05005,668.169983,84.1500015$

2, $1200.94995,668.169983,84.1500015$

$3, \quad 1200.94995,38.0999985,84.1500015$ 
$4, \quad 1239.05005, \quad 38.0999985,84.1500015$

$5, \quad 1200.94995,668.169983, \quad 73$.

6, 1200.94995, 38.0999985, 73.

$7, \quad 1239.05005,38.0999985, \quad 73$.

$8, \quad 1239.05005,668.169983, \quad 73$.

9, $\quad 1239.05005, \quad 706.27002,84.1500015$

$10, \quad 1200.94995, \quad 706.27002, \quad 84.1500015$

$4280, \quad 100.586113, \quad 324.495453, \quad 84.1500015$

$4281, \quad 100.586113, \quad 267.21637,84.1500015$

4282, 100.586113, 209.937271, 84.1500015

$4283,100.586113,152.658188,84.1500015$

4284, 100.586113, 95.3790894, 84.1500015

*ELEMENT, TYPE=C3D8I

$1,8,5,202,203,1,2,173,192$

2, 203, 202, 201, 204, 192, 173, 174, 191

3, 204, 201, 200, 205, 191, 174, 175, 190

4, 205, 200, 199, 206, 190, 175, 176, 189

5, 206, 199, 198, 207, 189, 176, 177, 188

6, 207, 198, 197, 208, 188, 177, 178, 187

7, 208, 197, 196, 209, 187, 178, 179, 186

$8,209,196,195,210,186,179,180,185$

9, 210, 195, 194, 211, 185, 180, 181, 184

$10,211,194,193,212,184,181,182,183$

$2047,86,85,98,97,84,83,94,93$

$2048,90,89,154,153,88,87,167,168$

$2049,122,120,119,121,169,170,171,172$

2050, 44, 54, 120, 122, 103, 102, 170, 169

*NSET, NSET=_PICKEDSET2, INTERNAL, GENERATE

1, 4284, 1

*ELSET, ELSET=_PICKEDSET2, INTERNAL, GENERATE 1, 2050, 1 


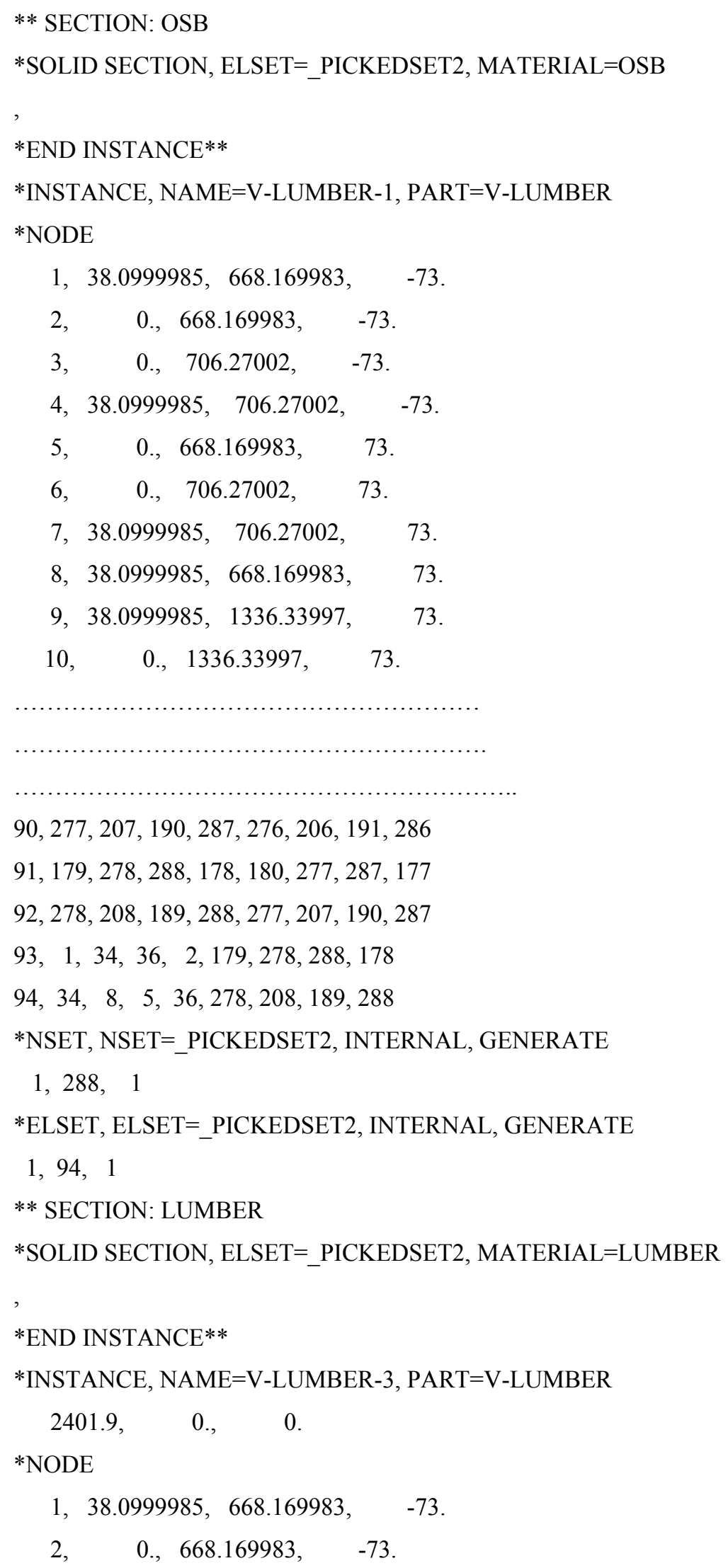


3, $\quad 0 ., \quad 706.27002, \quad-73$.

4, 38.0999985, 706.27002, -73 .

$5, \quad 0 ., 668.169983, \quad 73$.

$6, \quad 0 ., \quad 706.27002, \quad 73$.

7, 38.0999985, 706.27002, 73.

$8,38.0999985,668.169983, \quad 73$.

9, 38.0999985, 1336.33997, 73.

$10, \quad 0 ., 1336.33997, \quad 73$.

4275, 1301.53613, 2099.88916, 73.

4276, 1301.53613, 2157.16821, 73.

4277, 1301.53613, 2214.44727, 73.

4278, 1301.53613, 2271.72632, 73.

4279, 1301.53613, 2329.00537, 73.

4280, 1301.53613, 2386.28442, 73.

4281, 1301.53613, 2443.56372, 73.

4282, 1301.53613, 2500.84277, 73.

4283, 1301.53613, 2558.12183, 73.

4284, 1301.53613, 2615.40088, 73.

\section{*ELEMENT, TYPE $=$ C3D8I}

$1,5,6,7,8,1,2,3,4$

2, 16, 13, 202, 203, 9, 10,173, 192

3, 203, 202, 201, 204, 192, 173, 174, 191

4, 204, 201, 200, 205, 191, 174, 175, 190

5, 205, 200, 199, 206, 190, 175, 176, 189

6, 206, 199, 198, 207, 189, 176, 177, 188

7, 207, 198, 197, 208, 188, 177, 178, 187

8, 208, 197, 196, 209, 187, 178, 179, 186

9, 209, 196, 195, 210, 186, 179, 180, 185

10, 210, 195, 194, 211, 185, 180, 181, 184

2040, 844, 693, 694, 843, 863, 738, 737, 864

$2041,843,694,695,842,864,737,736,865$ 
$2042,842,695,696,841,865,736,735,866$ $2043,841,696,697,840,866,735,734,867$ 2044, 840, 697, 698, 839, 867, 734, 733, 868 $2045,839,698,699,838,868,733,732,869$ $2046,838,699,700,837,869,732,731,870$ $2047,837,700,701,836,870,731,730,871$ $2048,836,701,702,835,871,730,729,872$ $2049,835,702,147,138,872,729,148,137$ 2050, 105, 107, 108, 106, 154, 153, 171, 172

*NSET, NSET=_PICKEDSET2, INTERNAL, GENERATE

$1,4284, \quad 1$

*ELSET, ELSET=_PICKEDSET2, INTERNAL, GENERATE

1, 2050, 1

** SECTION: OSB

*SOLID SECTION, ELSET=_PICKEDSET2, MATERIAL=OSB

*END INSTANCE**

*NSET, NSET=_PICKEDSET521, INTERNAL, INSTANCE=OSB-1

105, 106, 107, 108, 117, 118, 123, 124, 135, 136, 143, 144, 153, 154, 155, 156

$165,166,167,168,717,718,719,720,721,722,723,724,725,726,727,728$

$729,730,731,732,797,798,799,800,801,802,803,804,805,806,807,808$

$809,810,811,812,893,894,895,896,897,898,899,900,901,902,903,904$

905, 906, 907, 908, 909, 910, 911, 912, 913, 914, 915, 916, 917, 918, 919, 920

921, 922, 923, 924

*NSET, NSET=_PICKEDSET521, INTERNAL, INSTANCE=H-LUMBER-3

$1,4,8,9,12,13,17,19,21,22,26,28,30,32,33,35$

$37,39,43,44,55,56,57,58,59,60,61,62,72,73,74,75$

76, 77, 78, 79, 80, 81, 86, 91, 92, 102, 103, 104, 105, 106, 107, 108

$109,119,120,121,122,123,124,125,126,127,138,139,140,141,142,143$

$144,145,155,156,157,158,159,160,161,162,163,165,166,167,168,169$

$170,171,172,182,183,184,185,186,187,188,189,200,204,207,225,226$

$227,228,229,230,231,232,262,263,264,265,266,267,268,269,304,305$

306, 307, 308, 309, 310, 311, 336, 337, 338, 339, 340, 341, 342, 343

*NSET, NSET=_PICKEDSET521, INTERNAL, INSTANCE=OSB-2

94, 95, 97, 98, 110, 112, 114, 115, 117, 118, 139, 140, 141, 142, 143, 144

$145,146,151,152,573,574,575,576,577,578,579,580,589$, 590, 591, 592

$593,594,595,596,669,670,671,672,673,674,675,676,685,686,687,688$ 
$689,690,691,692,773,774,775,776,777,778,779,780,789,790,791,792$

$793,794,795,796,889,890,891,892,893,894,895,896,897,898,899,900$

901, 902, 903, 904

*ELSET, ELSET=_PICKEDSET521, INTERNAL, INSTANCE=OSB-1

1033, 1036, 1037, 1038, 1039, 1040, 1041, 1042, 1043, 1044, 1063, 1074, 1075, 1076, 1077, 1078

1079, 1080, 1081, 1082, 1101, 1114, 1115, 1116, 1117, 1118, 1119, 1120, 1121, 1122, 1123, 1124

$1125,1126,1127,1128,1129,1130,1131,2045,2048$

*ELSET, ELSET=_PICKEDSET521, INTERNAL, INSTANCE=H-LUMBER-3

$1,3,5,7,9,11,13,15,17,19,21,23,25,27,29,31$

$33,35,38,40,42,44,45,47,49,51,53,55,57,59,61,63$

$65,67,69,71,73,75,77,79,90,91,92,93,94,95,96,97$

$98,108,109,110,111,112,113,114,115,116,117,119,122,124,126,128$

$130,132,134,136,138,140,142,144,146,148,150,152,154,156,158,160$

163,164

*ELSET, ELSET=_PICKEDSET521, INTERNAL, INSTANCE=OSB-2

$110,121,122,123,124,125,126,127,128,129,139,160,161,162,163,164$

$165,166,167,168,169,170,1064,1065,1066,1067,1068,1069,1070,1071,1072,1073$

1990, 1991, 1992, 1993, 1994, 1995, 1996, 1997, 1998

*NSET, NSET= PICKEDSET522, INTERNAL, INSTANCE="REF POINT-1"

1 ,

*NSET, NSET=_PICKEDSET535, INTERNAL, INSTANCE="REF POINT-1"

1 ,

*NSET, NSET=_PICKEDSET536, INTERNAL, INSTANCE=OSB-1

$113,114,115,116,119,121,126,127,129,130,137,139,147,149,158,159$

$161,162,171,172,733,734,735,736,737,738,739,740,749,750,751,752$

$753,754,755,756,813,814,815,816,817,818,819,820,829,830,831,832$

$833,834,835,836,861,862,863,864,865,866,867,868,869,870,871,872$

$873,874,875,876,957,958,959,960,961,962,963,964,965,966,967,968$

969, 970, 971, 972

*NSET, NSET=_PICKEDSET536, INTERNAL, INSTANCE=H-LUMBER-2

$1,4,7,8,9,12,15,16,17,20,23,24,25,28,31,32$

$33,35,37,38,49,50,51,52,53,54,55,56,65,66,67,68$

$69,70,71,72,73,74,85,86,87,88,89,90,91,92,101,102$

103, 104, 105, 106, 107, 108, 109, 110, 121, 122, 123, 124, 125, 126, 127, 128

$137,138,139,140,141,142,143,144,145,146,157,158,159,160,161,162$

$163,164,173,174,175,176,177,178,179,180,181,182,186,187,189,190$

191, 192, 193, 194, 195, 196, 205, 206, 207, 208, 209, 210, 211, 212, 221, 222 
$223,224,225,226,227,228,237,238,239,240,241,242,243,244$

*NSET, NSET=_PICKEDSET536, INTERNAL, INSTANCE $=$ OSB-2

$101,102,103,104,106,108,129,130,131,132,134,136,159,160,167,168$

$169,170,171,172,541,542,543,544,545,546,547,548,557,558,559,560$

$561,562,563,564,621,622,623,624,625,626,627,628,637,638,639,640$

$641,642,643,644,941,942,943,944,945,946,947,948,949,950,951,952$

$953,954,955,956,957,958,959,960,961,962,963,964,965,966,967,968$

969, 970, 971, 972

*ELSET, ELSET=_PICKEDSET536, INTERNAL, INSTANCE=OSB-1

1035, 1045, 1046, 1047, 1048, 1049, 1050, 1051, 1052, 1053, 1064, 1083, 1084, 1085, 1086, 1087

1088, 1089, 1090, 1091, 1103, 1104, 1105, 1106, 1107, 1108, 1109, 1110, 1111, 2025, 2036, 2037

2038, 2039, 2040, 2041, 2042, 2043, 2044, 2049

*ELSET, ELSET=_PICKEDSET536, INTERNAL, INSTANCE=H-LUMBER-2, GENERATE

$1,82,1$

*ELSET, ELSET=_PICKEDSET536, INTERNAL, INSTANCE=OSB-2

$112,113,114,115,116,117,118,119,120,141,142,143,144,145,146,147$

148, 149, 150, 1989, 2020, 2021, 2022, 2023, 2024, 2025, 2026, 2027, 2028, 2029, 2030, 2031

2032, 2033, 2034, 2035, 2036, 2037, 2038, 2050

*ELSET, ELSET=_PICKEDSURF504_S4, INTERNAL, INSTANCE=H-LUMBER-1, GENERATE $1,72,1$

*ELSET, ELSET=_PICKEDSURF504_S4, INTERNAL, INSTANCE=V-LUMBER-1, GENERATE $3,24,1$

*ELSET, ELSET=_PICKEDSURF504_S4, INTERNAL, INSTANCE=V-LUMBER-2, GENERATE $1,72,1$

*ELSET, ELSET=_PICKEDSURF504_S4, INTERNAL, INSTANCE=H-LUMBER-3

$2,4,6,8,10,12,14,16,18,20,22,24,26,28,30,32$

$34,36,46,48,50,52,54,56,58,60,62,64,66,68,70,72$

$74,76,78,80$

*ELSET, ELSET=_PICKEDSURF504_S4, INTERNAL, INSTANCE=V-LUMBER-3

$1,2,49,50,51,52,53,54,55,56,57,58,59,60,61,62$

$63,64,65,66,67,68,69,70,71,72$

*ELSET, ELSET=_PICKEDSURF504_S6, INTERNAL, INSTANCE=H-LUMBER-1 $73,74,77,78$

*ELSET, ELSET=_PICKEDSURF504_S6, INTERNAL, INSTANCE=V-LUMBER-1

$1,2,25,26,49,50,51,52,53,54,55,56,57,58,59,60$

$61,62,63,64,65,66,67,68,69,70,71,72,73,74,75,76$

$77,78,79,80,81,82,83,84,85,86,87,88,89,90,91,92$ 
93, 94

*ELSET, ELSET=_PICKEDSURF504_S6, INTERNAL, INSTANCE=V-LUMBER-2, GENERATE $1,72,1$

*ELSET, ELSET=_PICKEDSURF504_S6, INTERNAL, INSTANCE=H-LUMBER-3

$37,39,41,43,121,123,125,127,129,131,133,135,137,139,141,143$

$145,147,149,151,153,155$

*ELSET, ELSET=_PICKEDSURF504_S6, INTERNAL, INSTANCE=V-LUMBER-3, GENERATE $3,48,1$

*ELSET, ELSET=_PICKEDSURF504_S3, INTERNAL, INSTANCE=V-LUMBER-1, GENERATE $27,48,1$

*ELSET, ELSET=_PICKEDSURF504_S3, INTERNAL, INSTANCE=V-LUMBER-2, GENERATE $73,94,1$

*ELSET, ELSET=_PICKEDSURF504_S3, INTERNAL, INSTANCE=H-LUMBER-3

$81,82,83,84,85,86,87,88,89,99,100,101,102,103,104,105$

106,107

*ELSET, ELSET=_PICKEDSURF504_S5, INTERNAL, INSTANCE=V-LUMBER-2, GENERATE $73,94,1$

*ELSET, ELSET=_PICKEDSURF504_S5, INTERNAL, INSTANCE=V-LUMBER-3, GENERATE $73,94,1$

*SURFACE, TYPE=ELEMENT, NAME=_PICKEDSURF504, INTERNAL

_PICKEDSURF504_S4, S4

PICKEDSURF504_S6, S6

PICKEDSURF504 S3, S3

_PICKEDSURF504_S5, S5

*ELSET, ELSET=_PICKEDSURF505_S6, INTERNAL, INSTANCE=EPS-1

281, 292, 501, 512, 523, 534, 545, 556, 567, 578, 589, 600, 611, 622, 633, 644

$655,666,677,688,1589,1600,1611,1622,1633,1644,1655,1666,1677,1688,1699,1710$

$1721,1732,1743,1754,1765,1776$

*ELSET, ELSET=_PICKEDSURF505_S6, INTERNAL, INSTANCE=EPS-2

1111, 1122, 1133, 1144, 1155, 1166, 1177, 1188, 1199, 1210, 1221, 1232, 1243, 1254, 1265, 1276

$1287,1298,1309,1320,1331,1342,1353,1364,1375,1386,1397,1408,1419,1430,1441,1452$

$1463,1474,1485,1496,1765,1776$

*ELSET, ELSET=_PICKEDSURF505_S4, INTERNAL, INSTANCE=EPS-1

65, 76, 929, 940, 951, 962, 973, 984, 995, 1006, 1017, 1028, 1039, 1050, 1061, 1072

1083, 1094, 1105, 1116, 1127, 1138, 1149, 1160, 1171, 1182, 1193, 1204, 1215, 1226, 1237, 1248

$1259,1270,1281,1292,1303,1314$

*ELSET, ELSET=_PICKEDSURF505_S4, INTERNAL, INSTANCE=EPS-2 
$87,98,109,120,131,142,153,164,175,186,197,208,219,230,241,252$

$263,274,483,494,527,538,549,560,571,582,593,604,615,626,637,648$

$659,670,681,692,703,714$

*ELSET, ELSET=_PICKEDSURF505_S5, INTERNAL, INSTANCE=EPS-1

$9,18,27,36,45,54,171,172,173,174,175,176,177,178,179,180$

$181,270,271,272,273,274,275,276,277,278,279,280,391,392,393,394$

395, 396, 397, 398, 399, 400, 401, 490, 491, 492, 493, 494, 495, 496, 497, 498

499, 500, 589, 590, 591, 592, 593, 594, 595, 596, 597, 598, 599, 688, 689, 690

691, 692, 693, 694, 695, 696, 697, 698, 787, 788, 789, 790, 791, 792, 793, 794

$795,796,797,886,887,888,889,890,891,892,893,894,895,896,1007,1008$

1009, 1010, 1011, 1012, 1013, 1014, 1015, 1016, 1017, 1106, 1107, 1108, 1109, 1110, 1111, 1112

1113, 1114, 1115, 1116, 1205, 1206, 1207, 1208, 1209, 1210, 1211, 1212, 1213, 1214, 1215, 1304

1305, 1306, 1307, 1308, 1309, 1310, 1311, 1312, 1313, 1314, 1403, 1404, 1405, 1406, 1407, 1408

$1409,1410,1411,1412,1413,1502,1503,1504,1505,1506,1507,1508,1509,1510,1511,1512$

$1543,1552,1561,1570,1579,1588,1677,1678,1679,1680,1681,1682,1683,1684,1685,1686$

$1687,1776,1777,1778,1779,1780,1781,1782,1783,1784,1785,1786$

*ELSET, ELSET=_PICKEDSURF505_S5, INTERNAL, INSTANCE=EPS-2

$11,20,29,38,47,56,67,76,165,166,167,168,169,170,171,172$

$173,174,175,264,265,266,267,268,269,270,271,272,273,274,363,364$

$365,366,367,368,369,370,371,372,373,462,463,464,465,466,467,468$

$469,470,471,472,605,606,607,608,609,610,611,612,613,614,615,704$

$705,706,707,708,709,710,711,712,713,714,803,804,805,806,807,808$

$809,810,811,812,813,902,903,904,905,906,907,908,909,910,911,912$

1001, 1002, 1003, 1004, 1005, 1006, 1007, 1008, 1009, 1010, 1011, 1100, 1101, 1102, 1103, 1104

1105, 1106, 1107, 1108, 1109, 1110, 1199, 1200, 1201, 1202, 1203, 1204, 1205, 1206, 1207, 1208

1209, 1298, 1299, 1300, 1301, 1302, 1303, 1304, 1305, 1306, 1307, 1308, 1397, 1398, 1399, 1400

1401, 1402, 1403, 1404, 1405, 1406, 1407, 1496, 1497, 1498, 1499, 1500, 1501, 1502, 1503, 1504

$1505,1506,1595,1596,1597,1598,1599,1600,1601,1602,1603,1604,1605,1694,1695,1696$

$1697,1698,1699,1700,1701,1702,1703,1704,1737,1746,1755,1764$

*SURFACE, TYPE=ELEMENT, NAME=_PICKEDSURF505, INTERNAL

PICKEDSURF505_S6, S6

PICKEDSURF505_S4, S4

PICKEDSURF505_S5, S5

*ELSET, ELSET=_PICKEDSURF506_S1, INTERNAL, INSTANCE=EPS-1

$10,11,12,13,14,15,16,17,18,28,29,30,31,32,33,34$

$35,36,46,47,48,49,50,51,52,53,54,66,67,68,69,70$

$71,72,73,74,75,76,78,80,82,182,183,184,185,186,187,188$ 
189, 190, 191, 192, 193, 194, 195, 196, 197, 198, 199, 200, 201, 202, 203, 204 $205,206,207,208,209,210,211,212,213,214,215,216,217,218,219,220$ $221,222,223,224,225,226,227,228,229,230,231,232,233,234,235,236$ $237,238,239,240,241,242,243,244,245,246,247,248,249,250,251,252$ $253,254,255,256,257,258,259,260,261,262,263,264,265,266,267,268$ $269,270,271,272,273,274,275,276,277,278,279,280,292,293,294,295$ 296, 297, 298, 299, 300, 301, 302, 402, 403, 404, 405, 406, 407, 408, 409, 410 $411,412,413,414,415,416,417,418,419,420,421,422,423,424,425,426$ $427,428,429,430,431,432,433,434,435,436,437,438,439,440,441,442$ $443,444,445,446,447,448,449,450,451,452,453,454,455,456,457,458$ 459, 460, 461, 462, 463, 464, 465, 466, 467, 468, 469, 470, 471, 472, 473, 474 $475,476,477,478,479,480,481,482,483,484,485,486,487,488,489,490$ 491, 492, 493, 494, 495, 496, 497, 498, 499, 500, 600, 601, 602, 603, 604, 605 $606,607,608,609,610,611,612,613,614,615,616,617,618,619,620,621$ $622,623,624,625,626,627,628,629,630,631,632,633,634,635,636,637$

1890, 1891, 1892, 1893, 1894, 1895, 1896, 1897, 1898, 1899, 1900, 1901, 1902, 1903, 1904, 1905 1906, 1907, 1908, 1909, 1910, 1911, 1912, 1913, 1914, 1915, 1916, 1917, 1918, 1919, 1920, 1921 1922, 1923, 1924, 1925, 1926, 1927, 1928, 1929, 1930, 1931, 1932, 1933, 1934, 1935, 1936, 1937 1938, 1939, 1940, 1941, 1942, 1943, 1944, 1945, 1946, 1947, 1948, 1949, 1950, 1951, 1952, 1953 1954, 1955, 1956, 1957, 1958, 1959, 1960, 1961, 1962, 1963, 1964, 1965, 1966, 1967, 1968, 1969 1970, 1971, 1972, 1973, 1974, 1975, 1976, 1977, 1978, 1979

*ELSET, ELSET=_PICKEDSURF507_S1, INTERNAL, INSTANCE=OSB-1, GENERATE 47, 939, 1

*SURFACE, TYPE=ELEMENT, NAME=_PICKEDSURF507, INTERNAL PICKEDSURF507_S2, S2 PICKEDSURF507_S1, S1

*ELSET, ELSET=_PICKEDSURF508_S2, INTERNAL, INSTANCE=H-LUMBER-1

$1,2,3,4,5,6,7,8,9,19,20,21,22,23,24,25$

$26,27,37,38,39,40,41,42,43,44,45,55,56,57,58,59$

$60,61,62,63$

*ELSET, ELSET=_PICKEDSURF508_S2, INTERNAL, INSTANCE=V-LUMBER-1

$3,4,5,6,7,8,9,10,11,12,13,51,52,53,54,55$

$56,57,58,59,60,61,73,74,75,76,77,78,79,80,81,82$

83 , 
*ELSET, ELSET=_PICKEDSURF508_S2, INTERNAL, INSTANCE=V-LUMBER-2

$3,4,5,6,7,8,9,10,11,12,13,27,28,29,30,31$

$32,33,34,35,36,37,51,52,53,54,55,56,57,58,59,60$

61 ,

*ELSET, ELSET=_PICKEDSURF508_S2, INTERNAL, INSTANCE=H-LUMBER-3

$1,2,3,4,5,6,7,8,9,10,11,12,13,14,15,16$

$17,18,45,46,47,48,49,50,51,52,53,54,55,56,57,58$

$59,60,61,62,81,82,83,84,85,86,87,88,89,90,91,92$

93, 94, 95, 96, 97, 98, 121, 122, 123, 124, 125, 126, 127, 128, 129, 130

$131,132,133,134,135,136,137,138$

*ELSET, ELSET=_PICKEDSURF508_S2, INTERNAL, INSTANCE=H-LUMBER-2

$1,2,3,4,5,6,7,8,9,19,20,21,22,23,24,25$

$26,27,37,38,39,40,41,42,43,44,45,55,56,57,58,59$

$60,61,62,63$

*ELSET, ELSET $=$ _PICKEDSURF508_S2, INTERNAL, INSTANCE=V-LUMBER-3

$3,4,5,6,7,8,9,10,11,12,13,27,28,29,30,31$

$32,33,34,35,36,37,51,52,53,54,55,56,57,58,59,60$

61 ,

*ELSET, ELSET=_PICKEDSURF508_S1, INTERNAL, INSTANCE=H-LUMBER-1

$10,11,12,13,14,15,16,17,18,28,29,30,31,32,33,34$

$35,36,46,47,48,49,50,51,52,53,54,64,65,66,67,68$

$69,70,71,72$

*ELSET, ELSET= PICKEDSURF508_S1, INTERNAL, INSTANCE=V-LUMBER-1

$14,15,16,17,18,19,20,21,22,23,24,62,63,64,65,66$

$67,68,69,70,71,72,84,85,86,87,88,89,90,91,92,93$

94,

*ELSET, ELSET=_PICKEDSURF508_S1, INTERNAL, INSTANCE=V-LUMBER-2

$14,15,16,17,18,19,20,21,22,23,24,38,39,40,41,42$

$43,44,45,46,47,48,62,63,64,65,66,67,68,69,70,71$

72 ,

*ELSET, ELSET=_PICKEDSURF508_S1, INTERNAL, INSTANCE=H-LUMBER-3

$19,20,21,22,23,24,25,26,27,28,29,30,31,32,33,34$

$35,36,63,64,65,66,67,68,69,70,71,72,73,74,75,76$

$77,78,79,80,99,100,101,102,103,104,105,106,107,108,109,110$

$111,112,113,114,115,116,139,140,141,142,143,144,145,146,147,148$

$149,150,151,152,153,154,155,156$

*ELSET, ELSET=_PICKEDSURF508_S1, INTERNAL, INSTANCE=H-LUMBER-2 
$10,11,12,13,14,15,16,17,18,28,29,30,31,32,33,34$

$35,36,46,47,48,49,50,51,52,53,54,64,65,66,67,68$

$69,70,71,72$

*ELSET, ELSET=_PICKEDSURF508_S1, INTERNAL, INSTANCE=V-LUMBER-3

$14,15,16,17,18,19,20,21,22,23,24,38,39,40,41,42$

$43,44,45,46,47,48,62,63,64,65,66,67,68,69,70,71$

72 ,

*ELSET, ELSET=_PICKEDSURF508_S6, INTERNAL, INSTANCE=V-LUMBER-1, GENERATE $27,47,2$

*ELSET, ELSET=_PICKEDSURF508_S6, INTERNAL, INSTANCE=V-LUMBER-2, GENERATE $73,93,2$

*ELSET, ELSET=_PICKEDSURF508_S6, INTERNAL, INSTANCE=V-LUMBER-3, GENERATE $73,93,2$

*ELSET, ELSET= PICKEDSURF508_S4, INTERNAL, INSTANCE=V-LUMBER-1, GENERATE $28,48,2$

*ELSET, ELSET=_PICKEDSURF508_S4, INTERNAL, INSTANCE=V-LUMBER-2, GENERATE $74,94,2$

*ELSET, ELSET=_PICKEDSURF508_S4, INTERNAL, INSTANCE=V-LUMBER-3, GENERATE $74,94,2$

*SURFACE, TYPE=ELEMENT, NAME=_PICKEDSURF508, INTERNAL

PICKEDSURF508_S2, S2

PICKEDSURF508_S1, S1

PICKEDSURF508_S6, S6

PICKEDSURF508_S4, S4

*ELSET, ELSET=_PICKEDSURF509_S1, INTERNAL, INSTANCE=OSB-1

$1,2,3,4,5,6,7,8,9,10,11,943,944,945,946,947$

948, 949, 950, 951, 952, 953, 2036, 2037, 2038, 2039, 2040, 2041, 2042, 2043, 2044

*ELSET, ELSET=_PICKEDSURF509_S1, INTERNAL, INSTANCE=OSB-2

$2,3,4,5,6,7,8,9,10,11,12,58,59,60,61,62$

$63,64,65,66,67,68,72,73,74,75,76,77,78,79,80,81$

$82,112,113,114,115,116,117,118,119,120,130,131,132,133,134,135$

$136,137,138,142,143,144,145,146,147,148,149,150,151,152,153,154$

155, 156, 157, 158, 159, 1064, 1065, 1066, 1067, 1068, 1069, 1070, 1071, 1072, 1990, 1991

1992, 1993, 1994, 1995, 1996, 1997, 1998, 2039, 2040, 2041, 2042, 2043, 2044, 2045, 2046, 2047

2048, 2049

*ELSET, ELSET=_PICKEDSURF509_S4, INTERNAL, INSTANCE=OSB-1

989, 990, 991, 992, 993, 994, 995, 996, 997, 998, 999, 1123, 1124, 1125, 1126, 1127 
$1128,1129,1130,1131$

*ELSET, ELSET=_PICKEDSURF509_S4, INTERNAL, INSTANCE=OSB-2

$14,15,16,17,18,19,20,21,22,23,24,85,86,87,88,89$

$90,91,92,93,94,95$

*ELSET, ELSET=_PICKEDSURF509_S2, INTERNAL, INSTANCE=OSB-1

1000, 1001, 1002, 1003, 1004, 1005, 1006, 1007, 1008, 1009, 1010, 2027, 2028, 2029, 2030, 2031

2032, 2033, 2034, 2035

*ELSET, ELSET $=$ _PICKEDSURF509_S2, INTERNAL, INSTANCE=OSB-2

$25,26,27,28,29,30,31,32,33,34,35,36,37,38,39,40$

$41,42,43,44,45,46,97,98,99,100,101,102,103,104,105,106$

$107,121,122,123,124,125,126,127,128,129,160,161,162,163,164,165$

166, 167, 168, 1980, 1981, 1982, 1983, 1984, 1985, 1986, 1987, 1988, 1999, 2000, 2001, 2002

2003, 2004, 2005, 2006, 2007, 2009, 2010, 2011, 2012, 2013, 2014, 2015, 2016, 2017, 2018, 2019

2020, 2021, 2022, 2023, 2024, 2025, 2026, 2027, 2028, 2030, 2031, 2032, 2033, 2034, 2035, 2036

2037,2038

*ELSET, ELSET=_PICKEDSURF509_S6, INTERNAL, INSTANCE=OSB-1

1036, 1037, 1038, 1039, 1040, 1041, 1042, 1043, 1044, 1074, 1075, 1076, 1077, 1078, 1079, 1080

$1081,1082,1114,1115,1116,1117,1118,1119,1120,1121,1122$

*ELSET, ELSET = PICKEDSURF509_S6, INTERNAL, INSTANCE=OSB-2

$47,48,49,50,51,52,53,54,55,56,57,1075,1076,1077,1078,1079$

1080, 1081, 1082, 1083, 1084, 1085

*SURFACE, TYPE=ELEMENT, NAME=_PICKEDSURF509, INTERNAL

PICKEDSURF509 S1, S1

_PICKEDSURF509_S4, S4

PICKEDSURF509_S2, S2

PICKEDSURF509_S6, S6

*ELSET, ELSET=_PICKEDSURF510_S5, INTERNAL, INSTANCE=H-LUMBER-1

81,82

*ELSET, ELSET=_PICKEDSURF510_S6, INTERNAL, INSTANCE=H-LUMBER-1

$75,76,79,80$

*ELSET, ELSET=_PICKEDSURF510_S6, INTERNAL, INSTANCE=H-LUMBER-3

157,159

*ELSET, ELSET=_PICKEDSURF510_S3, INTERNAL, INSTANCE=H-LUMBER-3

161,162

*ELSET, ELSET=_PICKEDSURF510_S4, INTERNAL, INSTANCE=H-LUMBER-3

118,120

*SURFACE, TYPE=ELEMENT, NAME=_PICKEDSURF510, INTERNAL 
_PICKEDSURF510_S5, S5

_PICKEDSURF510_S6, S6

_PICKEDSURF510_S4, S4

_PICKEDSURF510_S3, S3

*ELSET, ELSET=_PICKEDSURF511_S5, INTERNAL, INSTANCE=V-LUMBER-1

61,72

*ELSET, ELSET=_PICKEDSURF511_S5, INTERNAL, INSTANCE=V-LUMBER-2

61,72

*ELSET, ELSET=_PICKEDSURF511_S5, INTERNAL, INSTANCE=V-LUMBER-3

61,72

*ELSET, ELSET=_PICKEDSURF511_S2, INTERNAL, INSTANCE=V-LUMBER-1

27,28

*ELSET, ELSET=_PICKEDSURF511_S2, INTERNAL, INSTANCE=V-LUMBER-2

73,74

*ELSET, ELSET=_PICKEDSURF511_S2, INTERNAL, INSTANCE=V-LUMBER-3

73,74

*SURFACE, TYPE=ELEMENT, NAME=_PICKEDSURF511, INTERNAL

_PICKEDSURF511_S5, S5

_PICKEDSURF511_S2, S2

*ELSET, ELSET=_PICKEDSURF512_S1, INTERNAL, INSTANCE=H-LUMBER-1, GENERATE $74,80,2$

*ELSET, ELSET=_PICKEDSURF512_S1, INTERNAL, INSTANCE=V-LUMBER-1, GENERATE

2, 50, 24

*ELSET, ELSET=_PICKEDSURF512_S1, INTERNAL, INSTANCE=V-LUMBER-2, GENERATE 2, 50, 24

*ELSET, ELSET=_PICKEDSURF512_S1, INTERNAL, INSTANCE=H-LUMBER-3

$39,40,43,44,119,120,159,160$

*ELSET, ELSET=_PICKEDSURF512_S1, INTERNAL, INSTANCE=H-LUMBER-2, GENERATE $74,80,2$

*ELSET, ELSET=_PICKEDSURF512_S1, INTERNAL, INSTANCE=V-LUMBER-3, GENERATE

2, 50, 24

*ELSET, ELSET=_PICKEDSURF512_S4, INTERNAL, INSTANCE=H-LUMBER-1

82 ,

*ELSET, ELSET=_PICKEDSURF512_S4, INTERNAL, INSTANCE=H-LUMBER-3

162,164

*ELSET, ELSET=_PICKEDSURF512_S4, INTERNAL, INSTANCE=H-LUMBER-2 82 , 
*ELSET, ELSET=_PICKEDSURF512_S2, INTERNAL, INSTANCE=H-LUMBER-1, GENERATE $73,79,2$

*ELSET, ELSET=_PICKEDSURF512_S2, INTERNAL, INSTANCE=V-LUMBER-1, GENERATE $1,49,24$

*ELSET, ELSET=_PICKEDSURF512_S2, INTERNAL, INSTANCE=V-LUMBER-2, GENERATE $1,49,24$

*ELSET, ELSET=_PICKEDSURF512_S2, INTERNAL, INSTANCE=H-LUMBER-3 $37,38,41,42,117,118,157,158$

*ELSET, ELSET=_PICKEDSURF512_S2, INTERNAL, INSTANCE=H-LUMBER-2, GENERATE $73,79,2$

*ELSET, ELSET=_PICKEDSURF512_S2, INTERNAL, INSTANCE=V-LUMBER-3, GENERATE $1,49,24$

*ELSET, ELSET=_PICKEDSURF512_S6, INTERNAL, INSTANCE=H-LUMBER-1

81 ,

*ELSET, ELSET=_PICKEDSURF512_S6, INTERNAL, INSTANCE=H-LUMBER-3 161,163

*ELSET, ELSET=_PICKEDSURF512_S6, INTERNAL, INSTANCE=H-LUMBER-2

81 ,

*SURFACE, TYPE=ELEMENT, NAME=_PICKEDSURF512, INTERNAL

_PICKEDSURF512_S1, S1

PICKEDSURF512_S4, S4

PICKEDSURF512_S2, S2

_PICKEDSURF512_S6, S6

*ELSET, ELSET=_PICKEDSURF516_S1, INTERNAL, INSTANCE=OSB-1

12, 24, 940, 1034, 1064, 1112, 1113, 2025, 2045

*ELSET, ELSET=_PICKEDSURF516_S1, INTERNAL, INSTANCE=OSB-2

83, 84, 109, 111, 141, 169, 1989, 2008

*ELSET, ELSET=_PICKEDSURF516_S2, INTERNAL, INSTANCE=OSB-1

941, 965, 1035, 1102, 2026, 2046

*ELSET, ELSET=_PICKEDSURF516_S2, INTERNAL, INSTANCE=OSB-2

13, 69, 96, 108, 110, 139, 140, 170, 1086, 2029

*ELSET, ELSET=_PICKEDSURF516_S6, INTERNAL, INSTANCE=OSB-1

942, 966, 1033, 1063, 1101, 2047

*ELSET, ELSET=_PICKEDSURF516_S6, INTERNAL, INSTANCE=OSB-2

1073, 1074

*ELSET, ELSET=_PICKEDSURF516_S4, INTERNAL, INSTANCE=OSB-1, GENERATE $2048,2050, \quad 1$ 
*ELSET, ELSET=_PICKEDSURF516_S4, INTERNAL, INSTANCE=OSB-2

$1,70,71,2050$

*SURFACE, TYPE=ELEMENT, NAME=_PICKEDSURF516, INTERNAL

PICKEDSURF516_S1, S1

PICKEDSURF516_S2, S2

PICKEDSURF516_S6, S6

PICKEDSURF516_S4, S4

*ELSET, ELSET=_PICKEDSURF517_S4, INTERNAL, INSTANCE=H-LUMBER-2, GENERATE $1,72,1$

*ELSET, ELSET=_PICKEDSURF517_S6, INTERNAL, INSTANCE=H-LUMBER-2, GENERATE $73,80,1$

*ELSET, ELSET=_PICKEDSURF517_S5, INTERNAL, INSTANCE=H-LUMBER-2

81,82

*SURFACE, TYPE=ELEMENT, NAME=_PICKEDSURF517, INTERNAL

PICKEDSURF517_S4, S4

_PICKEDSURF517_S6, S6

PICKEDSURF517_S5, S5

*ELSET, ELSET=_PICKEDSURF518_S4, INTERNAL, INSTANCE=H-LUMBER-1, GENERATE $73,80,1$

*ELSET, ELSET=_PICKEDSURF518_S6, INTERNAL, INSTANCE=H-LUMBER-1, GENERATE $1,72,1$

*ELSET, ELSET=_PICKEDSURF518_S3, INTERNAL, INSTANCE=H-LUMBER-1

81,82

*SURFACE, TYPE=ELEMENT, NAME=_PICKEDSURF518, INTERNAL

PICKEDSURF518_S4, S4

PICKEDSURF518_S6, S6

PICKEDSURF518_S3, S3

** CONSTRAINT: HORIZONTAL TO VERTICAL LUMBER

*TIE, NAME="HORIZONTAL TO VERTICAL LUMBER", ADJUST=YES

PICKEDSURF511,_PICKEDSURF510

** CONSTRAINT: OSB TO LUMBER

*TIE, NAME="OSB TO LUMBER", ADJUST=YES

PICKEDSURF516,_PICKEDSURF512

** CONSTRAINT: TOP LUMBERS

*TIE, NAME="TOP LUMBERS", ADJUST=YES

PICKEDSURF518,_PICKEDSURF517

** CONSTRAINT: WALL TOP CORNER (LOADING) 
*RIGID BODY, REF NODE=_PICKEDSET535, TIE NSET=_PICKEDSET536

*END ASSEMBLY**

** MATERIALS**

** EPS/URETHANE

*MATERIAL, NAME=EPS

*ELASTIC

$3.17,0.165$

*MATERIAL, NAME=LUMBER

*ELASTIC

300., 0.3

*PLASTIC

$24.15, \quad 0$.

$35.88,0.003$

$46.23,0.004$

$57.27,0.005$

$67.62,0.006$

$77.97,0.007$

$88.32,0.008$

99.36, 0.009

$109.02,0.01$

$117.3,0.011$

124.2, 0.012

*MATERIAL, NAME=OSB

*ELASTIC

385., 0.3

*PLASTIC

3., 0.

$3.22,0.00039$

3.92, 0.00055

4.65, 0.00074

5.42, 0.00095

$7.03,0.0015$

7.79, 0.00184

$8.61,0.0022$

$9.45,0.0026$

$10.26,0.0031$

$11.06,0.0036$ 
$11.77,0.0044$

$* *$

** INTERACTION PROPERTIES**

*SURFACE INTERACTION, NAME="OSB TO EPS (GLUE)"

1. ,

*FRICTION, SLIP TOLERANCE $=0.005$, TAUMAX=200.

$0.15,0.5$

*SURFACE INTERACTION, NAME="SHEETS TO LUMBERS (HARD)"

1. ,

*SURFACE BEHAVIOR, PRESSURE-OVERCLOSURE=HARD**

** BOUNDARY CONDITIONS**

** NAME: BOTTOM-FIXED TYPE: DISPLACEMENT/ROTATION

*BOUNDARY

PICKEDSET521, 1, 1

PICKEDSET521, 2, 2

PICKEDSET521, 3, 3

PICKEDSET521, 4, 4

PICKEDSET521, 5, 5

PICKEDSET521, 6, 6

**

** INTERACTIONS**

** INTERACTION: EPS AND LUMBER

*CONTACT PAIR, INTERACTION="SHEETS TO LUMBERS (HARD)", TYPE=SURFACE TO SURFACE _PICKEDSURF505,_PICKEDSURF504

** INTERACTION: OSB AND EPS

*CONTACT PAIR, INTERACTION="OSB TO EPS (GLUE)", TYPE=SURFACE TO SURFACE

_PICKEDSURF507,_PICKEDSURF506

** INTERACTION: OSB AND LUMBER

*CONTACT PAIR, INTERACTION="SHEETS TO LUMBERS (HARD)", TYPE=SURFACE TO SURFACE

_PICKEDSURF509,_PICKEDSURF508

**

$* *$

** STEP: DISPALCEMENT**

*STEP, NAME=DISPALCEMENT, NLGEOM=YES

APPLYING 60 MM DISPLACMENT

*STATIC, STABILIZE $=0.0002$, ALLSDTOL $=0.05$, CONTINUE $=$ NO

$0.1,1 ., 1 \mathrm{E}-05,0.1$ 
**

** BOUNDARY CONDITIONS**

** NAME: DISPLACMENT ON TOP TYPE: DISPLACEMENT/ROTATION

*BOUNDARY

_PICKEDSET522, 2, 2, -10.**

** OUTPUT REQUESTS**

*RESTART, WRITE, FREQUENCY=0**

** FIELD OUTPUT: F-OUTPUT-1**

*OUTPUT, FIELD, VARIABLE=PRESELECT**

** HISTORY OUTPUT: H-OUTPUT-1**

*OUTPUT, HISTORY, VARIABLE=PRESELECT

*END STEP 


\section{A.3. SIP Racking Input File}

*HEADING

APPLYING 200 MM DISPLACEMENT ON TOP OF THE WALL

** JOB NAME: SIP-R-01-2D MODEL NAME: MODEL-1

** GENERATED BY: ABAQUS/CAE 6.10-1

$*$ PREPRINT, $\mathrm{ECHO}=\mathrm{NO}, \mathrm{MODEL}=\mathrm{NO}, \mathrm{HISTORY}=\mathrm{NO}, \mathrm{CONTACT}=\mathrm{NO} * *$

** PARTS**

*PART, NAME $=$ EPS

*END PART**

*PART, NAME=H-LUMBER

*END PART**

*PART, NAME $=\mathrm{OSB}$

*END PART**

*PART, NAME="REF POINT"

*END PART**

*PART, NAME=V-LUMBER

*END PART**

**

** ASSEMBLY**

*ASSEMBLY, NAME=ASSEMBLY**

*INSTANCE, NAME="REF POINT-1", PART="REF POINT"

*NODE

1, -200., 2672.67993, 0.

*NSET, NSET="REF POINT-1-REFPT_", INTERNAL

1 ,

*END INSTANCE**

*INSTANCE, NAME=EPS-1, PART=EPS

38.1, $\quad 0 ., \quad 0$.

*NODE

$1, \quad 0 ., \quad 329.32251$

2, 290.712494, 329.32251

3, 290.712494, 658.64502

$4, \quad 0 ., \quad 658.64502$

5, 581.424988, 329.32251

$6,581.424988,658.64502$

7, 872.137512, 329.32251 
8, $872.137512, \quad 658.64502$

9, $1162.84998, \quad 329.32251$

10, 872.137512, 0 .

11, 1162.84998, 0.

$690, \quad 436.068756, \quad 2436.98657$

$691,508.746887,2436.98657$

$692,363.390625,2502.85107$

$693,436.068756,2502.85107$

694, 508.746887, 2502.85107

$695,363.390625,2568.71558$

$696, \quad 436.068756,2568.71558$

$697, \quad 508.746887,2568.71558$

*ELEMENT, TYPE $=$ CPS4I

1, 1, 46, 314, 59

2, 46, 47, 315, 314

3, 47, 48, 316, 315

$4,48,2,49,316$

$5,59,314,317,58$

6, 314, 315, 318, 317

$7,315,316,319,318$

$8,316,49,50,319$

$9,58,317,320,57$

$10,317,318,321,320$

$11,318,319,322,321$

$12,319,50,51,322$

$13,57,320,323,56$

$14,320,321,324,323$

$15,321,322,325,324$

$16,322,51,52,325$

$17,56,323,55,4$

$18,323,324,54,55$

$19,324,325,53,54$

$20,325,52,3,53$ 
$630,689,690,693,692$

$631,690,691,694,693$

$632,691,309,308,694$

$633,295,692,695,296$

$634,692,693,696,695$

$635,693,694,697,696$

$636,694,308,307,697$

$637,296,695,313,43$

$638,695,696,312,313$

$639,696,697,311,312$

640, 697, 307, 45, 311

*NSET, NSET=_PICKEDSET2, INTERNAL, GENERATE

1, 697, 1

*ELSET, ELSET=_PICKEDSET2, INTERNAL, GENERATE

$1,640,1$

** SECTION: EPS

*SOLID SECTION, ELSET=_PICKEDSET2, MATERIAL=EPS

73.,

*END INSTANCE**

*INSTANCE, NAME=H-LUMBER-1, PART=H-LUMBER

*NODE

1, 2111.1875, -38.0999985

2, 2111.1875, -19.0499992

3, $1820.47498,-19.0499992$

4, $1820.47498,-38.0999985$

$5, \quad 2111.1875, \quad 0$.

6, 1820.47498, 0 .

7, 1529.76245, $\quad 0$.

$8, \quad 1529.76245,-19.0499992$

9, $1529.76245,-38.0999985$

$10, \quad 1239.05005,-19.0499992$

$11, \quad 1239.05005,-38.0999985$

12, $2401.8999,-19.0499992$

13, 2401.8999,

0.

14, $328.8125,-38.0999985$ 
$15, \quad 328.8125,-19.0499992$

$16, \quad 38.0999985,-19.0499992$

17, 38.0999985, -38.0999985

$18,910.237488,-19.0499992$

19, $910.237488,-38.0999985$

$20,1200.94995,-38.0999985$

110, 401.490631, 0.

$111, \quad 328.8125, \quad 0$.

112, 256.134369, 0 .

113, 183.456253, 0 .

114, 110.778122, 0 .

115, 1457.08435, 0 .

116, 1384.40625, 0 .

117, 1311.72815, 0 .

*ELEMENT, TYPE=CPS4I

$1,1,2,43,48$

$2,48,43,44,47$

$3,47,44,45,46$

$4,46,45,3,4$

$5,2,5,49,43$

$6,43,49,50,44$

$7,44,50,51,45$

$8,45,51,6,3$

$9,7,8,52,57$

$10,57,52,53,56$

$11,56,53,54,55$

$12,55,54,3,6$

$13,9,8,58,63$

$14,63,58,59,62$

$15,62,59,60,61$

110, 401.490631, 0. 
$111, \quad 328.8125, \quad 0$.

112, 256.134369, 0 .

113, 183.456253, 0.

114, 110.778122, 0 .

115, 1457.08435, 0.

116, 1384.40625, 0.

117, 1311.72815, 0.

*ELEMENT, TYPE=CPS4I

$1,1,2,43,48$

$2,48,43,44,47$

$3,47,44,45,46$

$4,46,45,3,4$

$5,2,5,49,43$

$6,43,49,50,44$

$7,44,50,51,45$

$8,45,51,6,3$

$9,7,8,52,57$

$10,57,52,53,56$

$11,56,53,54,55$

$12,55,54,3,6$

$13,9,8,58,63$

$14,63,58,59,62$

$15,62,59,60,61$

$70,82,21,33,100$

$71,25,24,13,12$

$72,31,30,11,10$

$73,8,7,115,58$

$74,58,115,116,59$

$75,59,116,117,60$

$76,60,117,35,10$

*NSET, NSET=_PICKEDSET2, INTERNAL, GENERATE

$1,117,1$

*ELSET, ELSET=_PICKEDSET2, INTERNAL, GENERATE

$1,76,1$ 


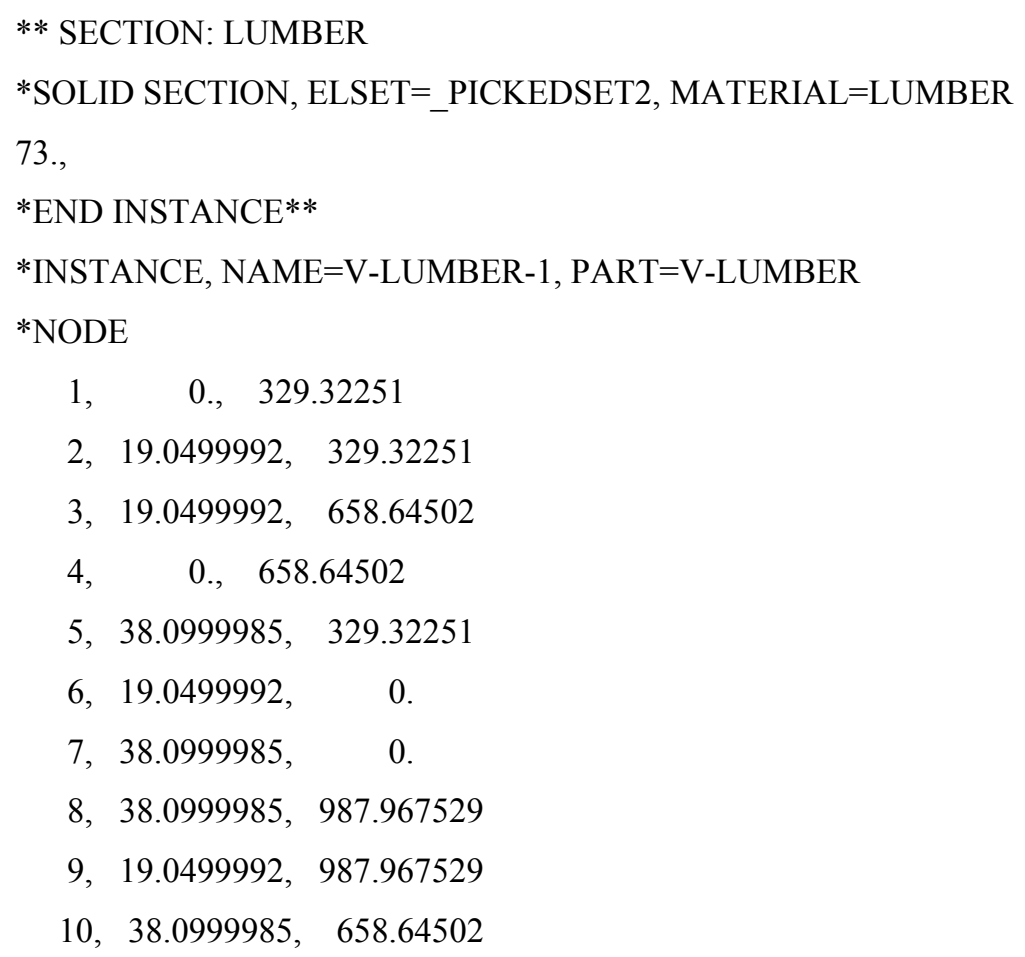

$115, \quad 38.0999985,1251.42554$

116, 38.0999985, 2371.12207

$117,38.0999985,2436.98657$

$118, \quad 38.0999985,2502.85107$

$119,38.0999985,2568.71558$

$120, \quad 0 ., \quad 263.458008$

121, $\quad 0 ., \quad 197.593506$

$122, \quad 0 ., \quad 131.729004$

$123, \quad 0 ., \quad 65.864502$

*ELEMENT, TYPE=CPS4I

$1,1,2,28,35$

$2,35,28,29,34$

$3,34,29,30,33$

$4,33,30,31,32$

$5,32,31,3,4$

$6,5,2,36,43$

$7,43,36,37,42$

$8,42,37,38,41$ 
$9,41,38,39,40$

$10,40,39,6,7$

$11,8,9,44,51$

$12,51,44,45,50$

$13,50,45,46,49$

$14,49,46,47,48$

$15,48,47,3,10$

$75,107,119,26,24$

$76,2,1,120,36$

$77,36,120,121,37$

$78,37,121,122,38$

$79,38,122,123,39$

$80,39,123,27,6$

*NSET, NSET=_PICKEDSET2, INTERNAL, GENERATE

$1,123,1$

*ELSET, ELSET=_PICKEDSET2, INTERNAL, GENERATE

$1,80,1$

** SECTION: LUMBER

*SOLID SECTION, ELSET=_PICKEDSET2, MATERIAL=LUMBER

73.

*END INSTANCE**

*INSTANCE, NAME=V-LUMBER-2, PART=V-LUMBER

1200.95, 0., $\quad 0$.

*NODE

$1, \quad 0 ., \quad 329.32251$

2, $\quad 19.0499992, \quad 329.32251$

$3, \quad 19.0499992, \quad 658.64502$

$4, \quad 0 ., \quad 658.64502$

5, 38.0999985, 329.32251

6, 19.0499992, 0.

7, 38.0999985, $\quad 0$.

$8, \quad 38.0999985,987.967529$

9, 19.0499992, 987.967529

$10, \quad 38.0999985, \quad 658.64502$ 
$115, \quad 19.0499992,2568.71558$

$116, \quad 0 ., \quad 2568.71558$

$117, \quad 0 ., \quad 2502.85107$

$118, \quad 0 ., \quad 2436.98657$

$119, \quad 0 ., \quad 2371.12207$

120, 38.0999985, 2371.12207

$121 ， 38.0999985,2436.98657$

$122, \quad 38.0999985,2502.85107$

$123,38.0999985,2568.71558$

*ELEMENT, TYPE=CPS4I

$1,1,2,28,35$

$2,35,28,29,34$

$3,34,29,30,33$

$4,33,30,31,32$

$5,32,31,3,4$

$6,5,2,36,43$

$7,43,36,37,42$

$8,42,37,38,41$

$9,41,38,39,40$

$10,40,39,6,7$

$70,39,111,24,6$

$71,22,20,112,119$

$72,119,112,113,118$

$73,118,113,114,117$

$74,117,114,115,116$

$75,116,115,25,26$

$76,20,19,120,112$

$77,112,120,121,113$

$78,113,121,122,114$

$79,114,122,123,115$

$80,115,123,27,25$ 


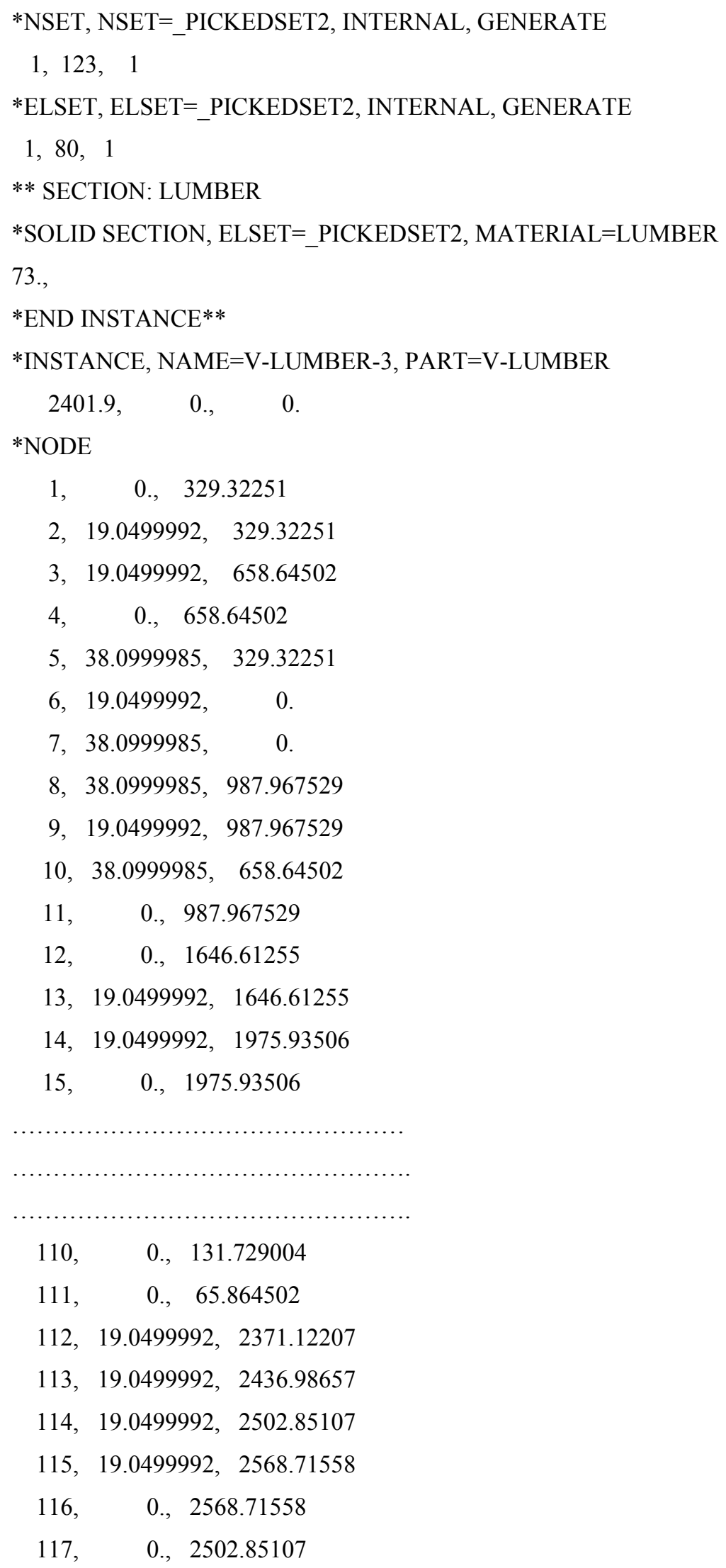


118, $\quad 0 ., \quad 2436.98657$

$119, \quad 0 ., \quad 2371.12207$

$120, \quad 38.0999985, \quad 2371.12207$

121, 38.0999985, 2436.98657

$122, \quad 38.0999985,2502.85107$

123, 38.0999985, 2568.71558

*ELEMENT, TYPE=CPS4I

$1,1,2,28,35$

$2,35,28,29,34$

$3,34,29,30,33$

$4,33,30,31,32$

$5,32,31,3,4$

$6,5,2,36,43$

$7,43,36,37,42$

$8,42,37,38,41$

$9,41,38,39,40$

$10,40,39,6,7$

$11,8,9,44,51$

$12,51,44,45,50$

$13,50,45,46,49$

$14,49,46,47,48$

$15,48,47,3,10$

$70,39,111,24,6$

$71,22,20,112,119$

$72,119,112,113,118$

$73,118,113,114,117$

$74,117,114,115,116$

$75,116,115,25,26$

$76,20,19,120,112$

$77,112,120,121,113$

$78,113,121,122,114$

$79,114,122,123,115$

$80,115,123,27,25$

*NSET, NSET=_PICKEDSET2, INTERNAL, GENERATE 
$1,123,1$

*ELSET, ELSET=_PICKEDSET2, INTERNAL, GENERATE

$1,80,1$

** SECTION: LUMBER

*SOLID SECTION, ELSET=_PICKEDSET2, MATERIAL=LUMBER

73.

*END INSTANCE**

*INSTANCE, NAME=EPS-2, PART=EPS

1239.05, $\quad 0 ., \quad 0$.

*NODE

$1, \quad 0 ., \quad 329.32251$

2, 290.712494, 329.32251

$3, \quad 290.712494,658.64502$

$4, \quad 0 ., \quad 658.64502$

$5, \quad 581.424988, \quad 329.32251$

6, 581.424988, 658.64502

$7, \quad 872.137512, \quad 329.32251$

$8, \quad 872.137512, \quad 658.64502$

9, $1162.84998, \quad 329.32251$

10, 872.137512, 0 .

11, 1162.84998, 0.

12, $1162.84998,987.967529$

13, $872.137512,987.967529$

$14, \quad 1162.84998, \quad 658.64502$

$15, \quad 581.424988,987.967529$

$690, \quad 1017.49377, \quad 2436.98657$

691, $1090.17188,2436.98657$

$692, \quad 944.815613,2502.85107$

693, 1017.49377, 2502.85107

694, 1090.17188, 2502.85107

$695,944.815613,2568.71558$

696, 1017.49377, 2568.71558

697, 1090.17188, 2568.71558

*ELEMENT, TYPE=CPS4I 
$1,1,46,314,59$

2, 46, 47, 315, 314

$3,47,48,316,315$

$4,48,2,49,316$

$5,59,314,317,58$

$6,314,315,318,317$

$7,315,316,319,318$

8, 316, 49, 50, 319

$9,58,317,320,57$

$10,317,318,321,320$

$11,318,319,322,321$

$12,319,50,51,322$

$13,57,320,323,56$

14, 320, 321, 324, 323

$15,321,322,325,324$

$630,689,690,693,692$

631, 690, 691, 694, 693

632, 691, 308, 309, 694

633, 284, 692, 695, 285

634, 692, 693, 696, 695

635, 693, 694, 697, 696

636, 694, 309, 310, 697

637, 285, 695, 313, 41

$638,695,696,312,313$

639, 696, 697, 311, 312

640, 697, 310, 45, 311

*NSET, NSET=_PICKEDSET2, INTERNAL, GENERATE

1, 697, 1

*ELSET, ELSET=_PICKEDSET2, INTERNAL, GENERATE

1, 640, 1

** SECTION: EPS

*SOLID SECTION, ELSET=_PICKEDSET2, MATERIAL=EPS

73.,

*END INSTANCE** 


\section{*INSTANCE, NAME=H-LUMBER-2, PART=H-LUMBER}

0., 2672.68, 0.

*NODE

$1, \quad 2111.1875,-38.0999985$

2, 2111.1875, 0.

3, 1820.47498, 0 .

4, 1820.47498, -38.0999985

$5, \quad 1529.76245,-38.0999985$

6, 1529.76245, 0 .

7, $1239.05005, \quad 0$.

$8, \quad 1239.05005,-38.0999985$

$9, \quad 328.8125,-38.0999985$

$10, \quad 328.8125, \quad 0$.

11, 38.0999985, 0.

12, $38.0999985,-38.0999985$

$13,910.237488,-38.0999985$

14, 910.237488, 0.

$15,619.525024, \quad 0$.

$16, \quad 619.525024,-38.0999985$

$17,2420.94995,-38.0999985$

18, 2420.94995, 0 .

19, 2401.8999, 0.

$20, \quad 2401.8999,-38.0999985$

70, 2329.22192, 0 .

71, 2256.5437, 0 .

$72,2183.86572, \quad 0$.

$73,982.915649,-38.0999985$

$74, \quad 1055.59375,-38.0999985$

$75,1128.27185,-38.0999985$

76, 1128.27185, 0.

77, 1055.59375, 0.

78, 982.915649, 0.

*ELEMENT, TYPE $=$ CPS4I

$1,1,2,31,36$ 
$2,36,31,32,35$

$3,35,32,33,34$

$4,34,33,3,4$

$5,5,6,37,42$

$6,42,37,38,41$

$7,41,38,39,40$

$8,40,39,7,8$

$9,6,5,43,48$

$10,48,43,44,47$

$33,70,69,20,19$

$34,14,13,73,78$

$35,78,73,74,77$

$36,77,74,75,76$

$37,76,75,24,23$

$38,26,25,29,30$

*NSET, NSET=_PICKEDSET2, INTERNAL, GENERATE

$1,78,1$

*ELSET, ELSET=_PICKEDSET2, INTERNAL, GENERATE

$1,38,1$

** SECTION: LUMBER

*SOLID SECTION, ELSET=_PICKEDSET2, MATERIAL=LUMBER

73.,

*END INSTANCE

**

*INSTANCE, NAME=H-LUMBER-3, PART=H-LUMBER

$0 ., \quad 2710.78, \quad 0$.

*NODE

$1, \quad 2111.1875,-38.0999985$

2, $2111.1875,-19.0499992$

3, $1820.47498,-19.0499992$

$4, \quad 1820.47498,-38.0999985$

5, 2111.1875, 0.

6, 1820.47498, 0 .

7, 1529.76245, 0 . 
8, $1529.76245,-19.0499992$

9, $1529.76245,-38.0999985$

10, $1239.05005,-19.0499992$

$110, \quad 1055.59375,-38.0999985$

$111,1128.27185,-38.0999985$

112, 256.134369, 0 .

$113,183.456253, \quad 0$.

114, 110.778122, 0 .

$115,1457.08435, \quad 0$.

116, 1384.40625, 0.

117, 1311.72815, 0.

*ELEMENT, TYPE=CPS4I

$1,1,2,46,51$

$2,51,46,47,50$

$3,50,47,48,49$

$4,49,48,3,4$

$5,2,5,52,46$

$6,46,52,53,47$

$7,47,53,54,48$

$8,48,54,6,3$

$9,7,8,55,60$

$10,60,55,56,59$

$11,59,56,57,58$

$12,58,57,3,6$

$13,9,8,61,66$

$14,66,61,62,65$

$15,65,62,63,64$

70, 84, 114, 40, 19

$71,8,7,115,61$

$72,61,115,116,62$

$73,62,116,117,63$ 
$74,63,117,35,10$

$75,34,33,26,25$

$76,39,41,45,43$

*NSET, NSET=_PICKEDSET2, INTERNAL, GENERATE

$1,117,1$

*ELSET, ELSET=_PICKEDSET2, INTERNAL, GENERATE

$1,76,1$

** SECTION: LUMBER

*SOLID SECTION, ELSET=_PICKEDSET2, MATERIAL=LUMBER

73.,

*END INSTANCE

$* *$

*INSTANCE, NAME $=$ OSB- 1 , PART $=$ OSB

$19.0500000000002, \quad-19.05, \quad 0$.

*NODE

1, 1181.90002, 677.651611

$2, \quad 891.1875,677.651611$

$3, \quad 891.1875, \quad 348.01535$

4, $1181.90002,347.908417$

5, 600.474976, 677.651611

6, 600.474976, 348.122284

7, 309.762512, 677.651611

8, $309.762512, \quad 348.229187$

$9, \quad 0 ., \quad 677.651611$

$10, \quad 0 ., \quad 348.34314$

$695, \quad 154.881256,2581.96973$

$696,232.321869,2581.96973$

697, 77.4406281, 2646.375

$698, \quad 154.881256, \quad 2646.375$

$699, \quad 232.321869, \quad 2646.375$

$700,382.440613,2388.75439$

$701,455.118744,2388.75439$

$702, \quad 527.796875,2388.75439$

$703, \quad 382.440613,2453.15942$ 
704, 455.118744, 2453.15942

$705, \quad 527.796875, \quad 2453.15942$

$706, \quad 382.440613, \quad 2517.5647$

$707,455.118744, \quad 2517.5647$

$708, \quad 527.796875, \quad 2517.5647$

$709, \quad 382.440613,2581.96973$

$710, \quad 455.118744, \quad 2581.96973$

$711, \quad 527.796875, \quad 2581.96973$

$712, \quad 382.440613, \quad 2646.375$

$713, \quad 455.118744, \quad 2646.375$

$714, \quad 527.796875, \quad 2646.375$

*ELEMENT, TYPE=CPS4I

1, 1, 46, 319, 59

2, 46, 47, 320, 319

3, 47, 48, 321, 320

$4,48,2,49,321$

$5,59,319,322,58$

$6,319,320,323,322$

7, 320, 321, 324, 323

$8,321,49,50,324$

$9,58,322,325,57$

$10,322,323,326,325$

$650,709,710,713,712$

$651,710,711,714,713$

$652,711,299,298,714$

653, 307, 712, 318, 44

$654,712,713,317,318$

$655,713,714,316,317$

656, 714, 298, 43, 316

*NSET, NSET=_PICKEDSET2, INTERNAL, GENERATE

$1,714,1$

*ELSET, ELSET=_PICKEDSET2, INTERNAL, GENERATE

$1,656,1$

** SECTION: OSB 
*SOLID SECTION, ELSET=_PICKEDSET2, MATERIAL=OSB

11 .,

*END INSTANCE**

*INSTANCE, NAME=OSB-2, PART=OSB

$1239.05, \quad-19.05, \quad 0$.

*NODE

1, 1181.90002, 677.651611

2, 872.10553, 677.651611

$3, \quad 872.10553,347.573639$

4, $1181.90002, \quad 347.459717$

5, 581.420837, 677.651611

$6, \quad 581.420837,347.680573$

7, 290.709991, 677.651611

$8,290.709991,347.787476$

9, $\quad 0 ., \quad 677.651611$

10, $\quad 0 ., \quad 347.894409$

$710, \quad 1027.00281,2581.96973$

$711, \quad 1104.45142,2581.96973$

$712, \quad 949.554138, \quad 2646.375$

$713, \quad 1027.00281, \quad 2646.375$

$714, \quad 1104.45142, \quad 2646.375$

*NSET, NSET=_PICKEDSET2, INTERNAL, GENERATE

$1,714,1$

*ELSET, ELSET=_PICKEDSET2, INTERNAL, GENERATE

$1,656,1$

** SECTION: OSB

*SOLID SECTION, ELSET=_PICKEDSET2, MATERIAL=OSB

11 .,

*END INSTANCE**

*NSET, NSET=_PICKEDSET578, INTERNAL, INSTANCE="REF POINT-1"

1 ,

*NSET, NSET=_PICKEDSET579, INTERNAL, INSTANCE=H-LUMBER-2

29,30

*NSET, NSET=_PICKEDSET579, INTERNAL, INSTANCE=H-LUMBER-3, GENERATE 
$43,45,1$

*ELSET, ELSET=_PICKEDSET579, INTERNAL, INSTANCE=H-LUMBER-2

38 ,

*ELSET, ELSET=_PICKEDSET579, INTERNAL, INSTANCE=H-LUMBER-3

64,76

*NSET, NSET=_PICKEDSET629, INTERNAL, INSTANCE=H-LUMBER-1

$1,4,9,11,14,17,19,20,22,28,29,30,34,40,42,46$

$47,48,61,62,63,70,71,72,76,77,78,79,80,81,88,89$

90, 91, 92, 93, 94, 95, 96

*ELSET, ELSET=_PICKEDSET629, INTERNAL, INSTANCE=H-LUMBER-1

$1,2,3,4,13,14,15,16,21,22,23,24,25,26,27,28$

$29,30,31,32,33,34,35,36,38,39,41,42,43,44,45,48$

$49,50,51,52,54,72$

*NSET, NSET=_PICKEDSET630, INTERNAL, INSTANCE="REF POINT-1"

1 ,

*NSET, NSET=_PICKEDSET631, INTERNAL, INSTANCE=H-LUMBER-3

$5,6,7,13,14,17,24,26,27,30,33,35,40,41,45,52$

$53,54,58,59,60,70,71,72,79,80,81,94,95,96,100,101$

$102,112,113,114,115,116,117$

*ELSET, ELSET=_PICKEDSET631, INTERNAL, INSTANCE=H-LUMBER-3

$5,6,7,8,9,10,11,12,17,18,19,20,25,26,27,28$

$37,38,39,40,41,42,43,44,49,51,63,66,67,68,69,70$

$71,72,73,74,75,76$

*NSET, NSET=_PICKEDSET663, INTERNAL, INSTANCE=H-LUMBER-1

$13,24,27,32,33,35,37,38,41$

*NSET, NSET=_PICKEDSET663, INTERNAL, INSTANCE=H-LUMBER-2

$8,12,17,20,21,24,26,27,30$

*NSET, NSET=_PICKEDSET664, INTERNAL, INSTANCE=V-LUMBER-1

$6,7,24,25,26,27$

*NSET, NSET=_PICKEDSET664, INTERNAL, INSTANCE=V-LUMBER-2

$6,7,24,25,26,27$

*NSET, NSET=_PICKEDSET664, INTERNAL, INSTANCE=V-LUMBER-3

$6,7,24,25,26,27$

*NSET, NSET=_PICKEDSET665, INTERNAL, INSTANCE=H-LUMBER-3

$1,4,9,11,18,20,21,23,31,32,36,37,38,42,44,49$

$50,51,64,65,66,73,74,75,85,86,87,91,92,93,103,104$

105, 106, 107, 108, 109, 110, 111 
*ELSET, ELSET=_PICKEDSET665, INTERNAL, INSTANCE=H-LUMBER-3

$1,2,3,4,13,14,15,16,21,22,23,24,29,30,31,32$

$33,34,35,36,45,46,47,48,50,52,53,54,55,56,57,58$

$59,60,61,62,64,65$

*NSET, NSET=_PICKEDSET666, INTERNAL, INSTANCE=H-LUMBER-2

$2,3,6,7,10,11,14,15,18,19,22,23,25,28,29,31$

$32,33,37,38,39,46,47,48,49,50,51,55,56,57,64,65$

$66,70,71,72,76,77,78$

*ELSET, ELSET=_PICKEDSET666, INTERNAL, INSTANCE=H-LUMBER-2, GENERATE

$1,38,1$

*ELSET, ELSET=_PICKEDSURF580_S2, INTERNAL, INSTANCE=H-LUMBER-1

$5,6,7,8,73,74,75,76$

*ELSET, ELSET=_PICKEDSURF580_S2, INTERNAL, INSTANCE=V-LUMBER-1

$46,47,48,49,50,51,52,53,54,55,66,67,68,69,70,71$

$72,73,74,75$

*ELSET, ELSET=_PICKEDSURF580_S2, INTERNAL, INSTANCE=V-LUMBER-3

$16,17,18,19,20,36,37,38,39,40,56,57,58,59,60,66$

$67,68,69,70$

*ELSET, ELSET=_PICKEDSURF580_S2, INTERNAL, INSTANCE=H-LUMBER-2

$9,10,11,12,21,22,23,24,30,31,32,33,34,35,36,37$

*ELSET, ELSET=_PICKEDSURF580_S2, INTERNAL, INSTANCE=V-LUMBER-2

$16,17,18,19,20,36,37,38,39,40,46,47,48,49,50,51$

$52,53,54,55,56,57,58,59,60,61,62,63,64,65,66,67$

$68,69,70,76,77,78,79,80$

*ELSET, ELSET=_PICKEDSURF580_S4, INTERNAL, INSTANCE=H-LUMBER-1

$9,10,11,12,17,18,19,20$

*ELSET, ELSET=_PICKEDSURF580_S4, INTERNAL, INSTANCE=V-LUMBER-1

$6,7,8,9,10,11,12,13,14,15,26,27,28,29,30,31$

$32,33,34,35$

*ELSET, ELSET=_PICKEDSURF580_S4, INTERNAL, INSTANCE=V-LUMBER-3

$1,2,3,4,5,21,22,23,24,25,41,42,43,44,45,71$

$72,73,74,75$

*ELSET, ELSET=_PICKEDSURF580_S4, INTERNAL, INSTANCE=H-LUMBER-2

$1,2,3,4,5,6,7,8,13,14,15,16,17,18,19,20$

*ELSET, ELSET =_PICKEDSURF580_S4, INTERNAL, INSTANCE=V-LUMBER-2

$1,2,3,4,5,6,7,8,9,10,11,12,13,14,15,21$

$22,23,24,25,26,27,28,29,30,31,32,33,34,35,41,42$ 
$43,44,45,71,72,73,74,75$

*ELSET, ELSET=_PICKEDSURF580_S3, INTERNAL, INSTANCE=H-LUMBER-1, GENERATE $55,70,1$

*SURFACE, TYPE=ELEMENT, NAME=_PICKEDSURF580, INTERNAL

PICKEDSURF580_S2, S2

PICKEDSURF580_S4, S4

PICKEDSURF580_S3, S3

*ELSET, ELSET=_PICKEDSURF581_S4, INTERNAL, INSTANCE=EPS-1

$1,5,9,13,17,61,65,69,73,77,81,85,89,93,97,161$

$165,169,173,177,221,225,229,233,237,241,245,249,253,257,321,325$

$329,333,337,581,585,589,593,597$

*ELSET, ELSET=_PICKEDSURF581_S4, INTERNAL, INSTANCE=EPS-2

$1,5,9,13,17,61,65,69,73,77,81,85,89,93,97,161$

$165,169,173,177,221,225,229,233,237,241,245,249,253,257,321,325$

$329,333,337,581,585,589,593,597$

*ELSET, ELSET=_PICKEDSURF581_S3, INTERNAL, INSTANCE=EPS-1

$77,78,79,80,517,518,519,520,537,538,539,540,557,558,559,560$

577, 578, 579, 580, 597, 598, 599, 600, 617, 618, 619, 620, 637, 638, 639, 640

*ELSET, ELSET=_PICKEDSURF581_S3, INTERNAL, INSTANCE=EPS-2

$77,78,79,80,517,518,519,520,537,538,539,540,557,558,559,560$

$577,578,579,580,597,598,599,600,617,618,619,620,637,638,639,640$

*ELSET, ELSET=_PICKEDSURF581_S2, INTERNAL, INSTANCE=EPS-1

144, 148, 152, 156, 160, 304, 308, 312, 316, 320, 384, 388, 392, 396, 400, 404

408, 412, 416, 420, 464, 468, 472, 476, 480, 484, 488, 492, 496, 500, 544, 548

$552,556,560,564,568,572,576,580$

*ELSET, ELSET=_PICKEDSURF581_S2, INTERNAL, INSTANCE=EPS-2 144, 148, 152, 156, 160, 304, 308, 312, 316, 320, 384, 388, 392, 396, 400, 404 408, 412, 416, 420, 464, 468, 472, 476, 480, 484, 488, 492, 496, 500, 544, 548 $552,556,560,624,628,632,636,640$

*SURFACE, TYPE=ELEMENT, NAME=_PICKEDSURF581, INTERNAL PICKEDSURF581_S4, S4

_PICKEDSURF581_S3, S3

_PICKEDSURF581_S2, S2

*SURFACE, TYPE=NODE, NAME=_PICKEDSET663_CNS_, INTERNAL

PICKEDSET663, 1.

*SURFACE, TYPE=NODE, NAME=_PICKEDSET664_CNS_, INTERNAL _PICKEDSET664, 1. 
*SURFACE, TYPE=NODE, NAME=_PICKEDSET665_CNS_, INTERNAL

PICKEDSET665, 1.

*SURFACE, TYPE=NODE, NAME=_PICKEDSET666_CNS_, INTERNAL

_PICKEDSET666, 1.

** CONSTRAINT: HORIZONTAL TO VERTICAL LUMBER

*TIE, NAME="HORIZONTAL TO VERTICAL LUMBER", ADJUST=YES

_PICKEDSET664_CNS_,_PICKEDSET663_CNS_

** CONSTRAINT: TOP LUMBERS

*TIE, NAME="TOP LUMBERS", ADJUST=YES

_PICKEDSET666_CNS_,_PICKEDSET665_CNS_

** CONSTRAINT: WALL TOP CORNER (LOADING)

*RIGID BODY, REF NODE=_PICKEDSET578, TIE NSET=_PICKEDSET579, POSITION=CENTER OF MASS

*ELEMENT, TYPE=SPRINGA, ELSET="NAILS ON OSB-SPRING"

1, OSB-1.45, H-LUMBER-3.41

2, OSB-1.45, H-LUMBER-3.43

3, OSB-1.41, H-LUMBER-3.26

4, OSB-1.41, H-LUMBER-3.34

5, OSB-1.40, H-LUMBER-1.39

6, OSB-1.40, H-LUMBER-1.40

7, OSB-1.16, H-LUMBER-1.31

8, OSB-1.16, H-LUMBER-1.20

9, OSB-2.38, H-LUMBER-3.35

10, OSB-2.38, H-LUMBER-3.34

11, OSB-2.45, H-LUMBER-3.29

12, OSB-2.45, H-LUMBER-3.27

13, OSB-2.44, H-LUMBER-1.31

14, OSB-2.44, H-LUMBER-1.11

15, OSB-2.16, H-LUMBER-1.26

16, OSB-2.16, H-LUMBER-1.28

17, OSB-1.44, H-LUMBER-3.14

18, OSB-1.43, H-LUMBER-3.17

19, OSB-1.42, H-LUMBER-3.24

20, OSB-1.39, H-LUMBER-1.14

50, OSB-2.18, V-LUMBER-2.17 
51, OSB-1.21, V-LUMBER-2.9

52, OSB-2.12, V-LUMBER-2.9

53, OSB-1.1, V-LUMBER-2.3

54, OSB-2.9, V-LUMBER-2.3

55, OSB-1.4, V-LUMBER-2.2

56, OSB-2.10, V-LUMBER-2.2

*SPRING, ELSET="NAILS ON OSB-SPRING"

450.

*END ASSEMBLY**

** MATERIALS**

** EPS/URETHANE

*MATERIAL, NAME=EPS

*ELASTIC

5500., 0.165

*PLASTIC

38., 0.

41., 0.04

59., 0.06

72., 0.08

$81 ., 0.1$

86., 0.12

89., 0.14

100., 0.2

*MATERIAL, NAME=LUMBER

*ELASTIC

20800., 0.3

*PLASTIC

$48.3, \quad 0$.

$71.76,0.001$

$92.46,0.002$

$114.54,0.003$

$135.24,0.004$

$155.94,0.005$

176.64, 0.006

$198.72,0.007$

218.04, 0.008 
229.08, 0.0087

234.6, 0.009

$248.4, \quad 0.01$

*MATERIAL, NAME $=$ OSB

*ELASTIC

3850., 0.3

*PLASTIC

20., 0 .

36., 0.0005

55., 0.001

78., 0.002

98., 0.003

115., 0.004

138., 0.005

140., 0.006

140., 0.007

140., 0.008

140., 0.009

**

** INTERACTION PROPERTIES**

*SURFACE INTERACTION, NAME="SHEETS TO LUMBERS (HARD)"

1.

*SURFACE BEHAVIOR, PRESSURE-OVERCLOSURE=HARD

**

** BOUNDARY CONDITIONS**

** NAME: BOTTOM-FIXED TYPE: DISPLACEMENT/ROTATION

*BOUNDARY

_PICKEDSET629, 1, 1

_PICKEDSET629, 2, 2

_PICKEDSET629, 3, 3

_PICKEDSET629, 4, 4

_PICKEDSET629, 5, 5

_PICKEDSET629, 6, 6

** NAME: LATERAL SUPPORT TYPE: DISPLACEMENT/ROTATION

*BOUNDARY

_PICKEDSET631, 2, 2

_PICKEDSET631, 3, 3 


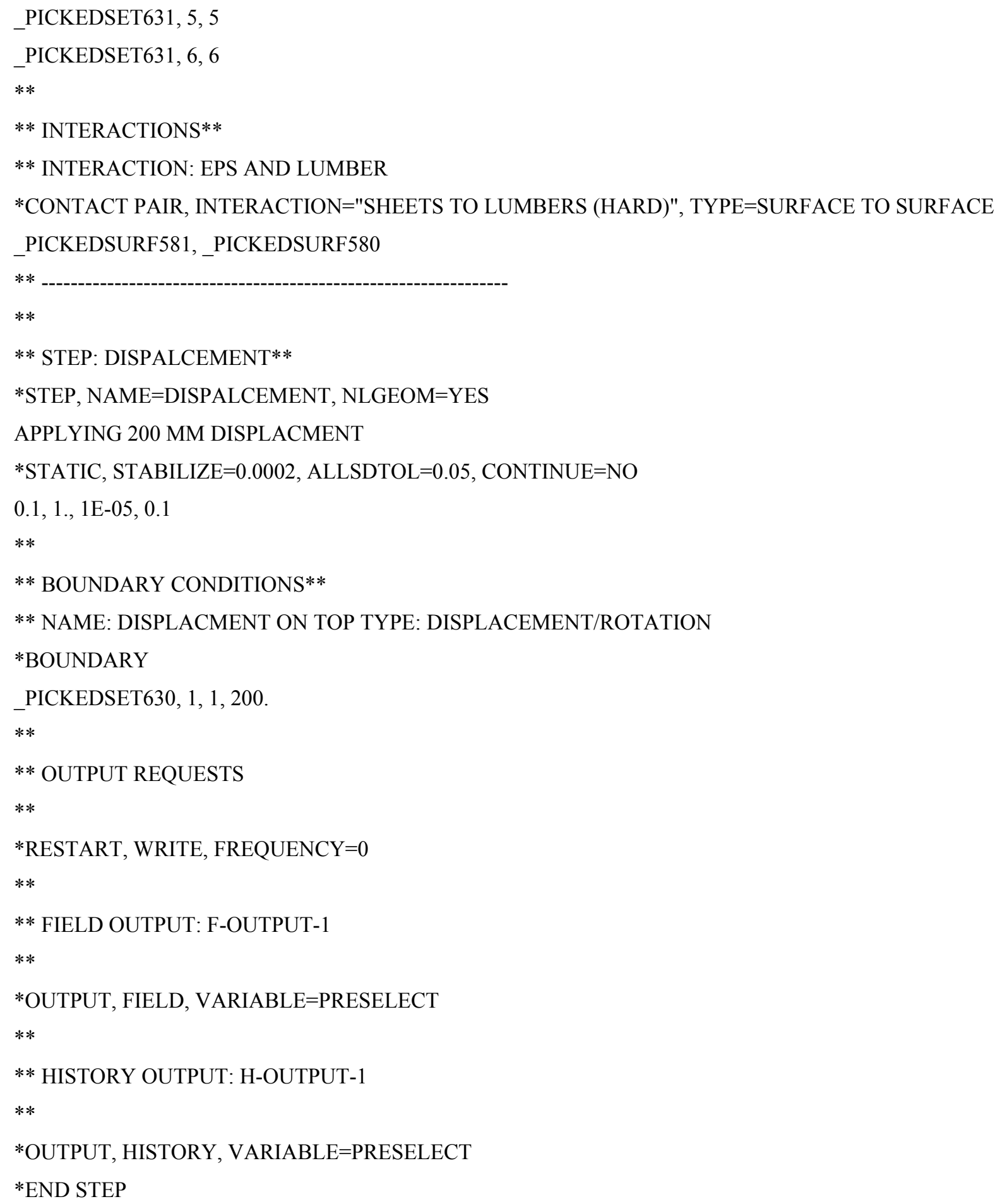




\section{A.4. SIP Cyclic Input File}

*HEADING

CYCLIC DISPLACEMENT $(\mathrm{D}=272.36 \mathrm{MM})$

** JOB NAME: SIP-C-01-2D MODEL NAME: MODEL-1

** GENERATED BY: ABAQUS/CAE 6.10-1

$*$ PREPRINT, $\mathrm{ECHO}=\mathrm{NO}, \mathrm{MODEL}=\mathrm{NO}, \mathrm{HISTORY}=\mathrm{NO}, \mathrm{CONTACT}=\mathrm{NO}$

**

** PARTS**

*PART, NAME=EPS

*END PART**

*PART, NAME=H-LUMBER

*END PART**

*PART, NAME $=\mathrm{OSB}$

*END PART**

*PART, NAME="REF POINT"

*END PART**

*PART, NAME=V-LUMBER

*END PART**

**

** ASSEMBLY**

*ASSEMBLY, NAME=ASSEMBLY**

*INSTANCE, NAME="REF POINT-1", PART="REF POINT"

*NODE

1, -200., 2672.67993, 0.

*NSET, NSET="REF POINT-1-REFPT_", INTERNAL

1 ,

*END INSTANCE**

*INSTANCE, NAME=EPS-1, PART=EPS

$38.1, \quad 0 ., \quad 0$.

*NODE

$1, \quad 0 ., \quad 329.32251$

2, 290.712494, 329.32251

$3,290.712494,658.64502$

$4, \quad 0 ., \quad 658.64502$

$5, \quad 581.424988, \quad 329.32251$

$6, \quad 581.424988,658.64502$ 

7, $872.137512, \quad 329.32251$
$8, \quad 872.137512, \quad 658.64502$
$9, \quad 1162.84998, \quad 329.32251$
10, 872.137512, 0 .
11, 1162.84998, 0.
12, $1162.84998,987.967529$
13, 872.137512, 987.967529
14, $1162.84998, \quad 658.64502$
$15, \quad 581.424988,987.967529$
16, 290.712494, 987.967529
$17, \quad 0 ., 987.967529$
$18, \quad 0 ., \quad 1646.61255$
19, 290.712494, 1646.61255
$20, \quad 290.712494,1975.93506$

690, 436.068756, 2436.98657

$691 ， 508.746887,2436.98657$

$692,363.390625,2502.85107$

693, 436.068756, 2502.85107

694, 508.746887, 2502.85107

$695,363.390625,2568.71558$

696, 436.068756, 2568.71558

697, 508.746887, 2568.71558

*ELEMENT, TYPE=CPS4I

1, 1, 46, 314, 59

2, 46, 47, 315, 314

$3,47,48,316,315$

$4,48,2,49,316$

$5,59,314,317,58$

$6,314,315,318,317$

7, 315, 316, 319, 318

8, 316, 49, 50, 319

$9,58,317,320,57$

$10,317,318,321,320$

$11,318,319,322,321$ 
$12,319,50,51,322$

$13,57,320,323,56$

$14,320,321,324,323$

$15,321,322,325,324$

$635,693,694,697,696$

636, 694, 308, 307, 697

$637,296,695,313,43$

$638,695,696,312,313$

$639,696,697,311,312$

$640,697,307,45,311$

*NSET, NSET=_PICKEDSET2, INTERNAL, GENERATE

1, 697, 1

*ELSET, ELSET=_PICKEDSET2, INTERNAL, GENERATE

$1,640,1$

** SECTION: EPS

*SOLID SECTION, ELSET=_PICKEDSET2, MATERIAL=EPS

73.,

*END INSTANCE**

*INSTANCE, NAME=H-LUMBER-1, PART=H-LUMBER

*NODE

$1, \quad 2111.1875,-38.0999985$

$2, \quad 2111.1875,-19.0499992$

3, $1820.47498,-19.0499992$

4, 1820.47498, -38.0999985

$5, \quad 2111.1875, \quad 0$.

6, 1820.47498, 0 .

7, 1529.76245, 0 .

$8, \quad 1529.76245,-19.0499992$

9, $1529.76245,-38.0999985$

$10, \quad 1239.05005,-19.0499992$

$11,1239.05005,-38.0999985$

$12, \quad 2401.8999,-19.0499992$

13, 2401.8999,

0.

$14, \quad 328.8125,-38.0999985$ 
$15, \quad 328.8125,-19.0499992$

$16, \quad 38.0999985,-19.0499992$

17, 38.0999985, -38.0999985

$18,910.237488,-19.0499992$

19, $910.237488,-38.0999985$

$20, \quad 1200.94995,-38.0999985$

110, 401.490631, 0.

$111, \quad 328.8125, \quad 0$.

112, 256.134369, 0 .

113, 183.456253, 0 .

114, 110.778122, 0 .

115, 1457.08435, 0 .

116, 1384.40625, 0.

117, 1311.72815, 0 .

*ELEMENT, TYPE=CPS4I

$1,1,2,43,48$

$2,48,43,44,47$

$3,47,44,45,46$

$4,46,45,3,4$

$5,2,5,49,43$

$6,43,49,50,44$

$7,44,50,51,45$

$8,45,51,6,3$

$9,7,8,52,57$

$10,57,52,53,56$

$11,56,53,54,55$

$12,55,54,3,6$

$13,9,8,58,63$

$14,63,58,59,62$

$15,62,59,60,61$

$70,82,21,33,100$ 
$71,25,24,13,12$

$72,31,30,11,10$

$73,8,7,115,58$

$74,58,115,116,59$

$75,59,116,117,60$

$76,60,117,35,10$

*NSET, NSET=_PICKEDSET2, INTERNAL, GENERATE

$1,117,1$

*ELSET, ELSET=_PICKEDSET2, INTERNAL, GENERATE

$1,76,1$

** SECTION: LUMBER

*SOLID SECTION, ELSET=_PICKEDSET2, MATERIAL=LUMBER

73.,

*END INSTANCE**

*INSTANCE, NAME=V-LUMBER-1, PART=V-LUMBER

*NODE

$1, \quad 0 ., \quad 329.32251$

$2, \quad 19.0499992, \quad 329.32251$

3, 19.0499992, 658.64502

$4, \quad 0 ., \quad 658.64502$

$5, \quad 38.0999985, \quad 329.32251$

6, 19.0499992, 0 .

7, 38.0999985, 0 .

8, 38.0999985, 987.967529

9, 19.0499992, 987.967529

10, 38.0999985, 658.64502

$11, \quad 0 ., 987.967529$

$12, \quad 0 ., \quad 1646.61255$

13, $19.0499992,1646.61255$

14, 19.0499992, 1975.93506

$15, \quad 0 ., \quad 1975.93506$

$115, \quad 38.0999985,1251.42554$

116, 38.0999985, 2371.12207

$117,38.0999985,2436.98657$ 
$118, \quad 38.0999985, \quad 2502.85107$

$119, \quad 38.0999985, \quad 2568.71558$

$120, \quad 0 ., \quad 263.458008$

$121, \quad 0 ., \quad 197.593506$

$122, \quad 0 ., \quad 131.729004$

$123, \quad 0 ., \quad 65.864502$

*ELEMENT, TYPE=CPS4I

$1,1,2,28,35$

$2,35,28,29,34$

$3,34,29,30,33$

$4,33,30,31,32$

$5,32,31,3,4$

$6,5,2,36,43$

$7,43,36,37,42$

$8,42,37,38,41$

$9,41,38,39,40$

$10,40,39,6,7$

$75,107,119,26,24$

$76,2,1,120,36$

$77,36,120,121,37$

$78,37,121,122,38$

$79,38,122,123,39$

$80,39,123,27,6$

*NSET, NSET=_PICKEDSET2, INTERNAL, GENERATE

$1,123,1$

*ELSET, ELSET=_PICKEDSET2, INTERNAL, GENERATE

$1,80,1$

** SECTION: LUMBER

*SOLID SECTION, ELSET=_PICKEDSET2, MATERIAL=LUMBER

73.,

*END INSTANCE**

*INSTANCE, NAME=V-LUMBER-2, PART=V-LUMBER

1200.95, $\quad 0 ., \quad 0$.

*NODE 

$1, \quad 0 ., \quad 329.32251$
2, $19.0499992, \quad 329.32251$
3, 19.0499992, 658.64502
4, $\quad 0 ., \quad 658.64502$
5, 38.0999985, 329.32251
6, 19.0499992, 0 .
7, 38.0999985, 0 .
8, 38.0999985, 987.967529
9, 19.0499992, 987.967529
$10, \quad 38.0999985, \quad 658.64502$

$115, \quad 19.0499992, \quad 2568.71558$

$116, \quad 0 ., \quad 2568.71558$

$117, \quad 0 ., \quad 2502.85107$

$118, \quad 0 ., \quad 2436.98657$

$119, \quad 0 ., \quad 2371.12207$

120, 38.0999985, 2371.12207

$121 ， 38.0999985,2436.98657$

122, 38.0999985, 2502.85107

$123,38.0999985,2568.71558$

*ELEMENT, TYPE $=$ CPS4I

$1,1,2,28,35$

$2,35,28,29,34$

$3,34,29,30,33$

$4,33,30,31,32$

$5,32,31,3,4$

$6,5,2,36,43$

$7,43,36,37,42$

$8,42,37,38,41$

$9,41,38,39,40$

$10,40,39,6,7$

$70,39,111,24,6$ 
$71,22,20,112,119$

$72,119,112,113,118$

$73,118,113,114,117$

$74,117,114,115,116$

$75,116,115,25,26$

$76,20,19,120,112$

$77,112,120,121,113$

$78,113,121,122,114$

$79,114,122,123,115$

$80,115,123,27,25$

*NSET, NSET=_PICKEDSET2, INTERNAL, GENERATE

$1,123,1$

*ELSET, ELSET=_PICKEDSET2, INTERNAL, GENERATE

$1,80,1$

** SECTION: LUMBER

*SOLID SECTION, ELSET=_PICKEDSET2, MATERIAL=LUMBER

73.,

*END INSTANCE**

*INSTANCE, NAME=V-LUMBER-3, PART=V-LUMBER

2401.9, $\quad 0 ., \quad 0$.

*NODE

$1, \quad 0 ., \quad 329.32251$

2, $\quad 19.0499992, \quad 329.32251$

3, $19.0499992, \quad 658.64502$

$4, \quad 0 ., \quad 658.64502$

$5, \quad 38.0999985, \quad 329.32251$

6, 19.0499992, $\quad 0$.

7, 38.0999985, $\quad 0$.

$8, \quad 38.0999985,987.967529$

9, 19.0499992, 987.967529

10, 38.0999985, 658.64502

$11, \quad 0 ., 987.967529$

$12, \quad 0 ., \quad 1646.61255$

$13,19.0499992,1646.61255$

14, 19.0499992, 1975.93506

$15, \quad 0 ., \quad 1975.93506$ 
$120, \quad 38.0999985, \quad 2371.12207$

$121,38.0999985,2436.98657$

$122, \quad 38.0999985,2502.85107$

123, 38.0999985, 2568.71558

*ELEMENT, TYPE=CPS4I

$1,1,2,28,35$

$2,35,28,29,34$

$3,34,29,30,33$

$4,33,30,31,32$

$5,32,31,3,4$

$6,5,2,36,43$

$7,43,36,37,42$

$8,42,37,38,41$

$9,41,38,39,40$

$10,40,39,6,7$

$75,116,115,25,26$

$76,20,19,120,112$

$77,112,120,121,113$

$78,113,121,122,114$

$79,114,122,123,115$

$80,115,123,27,25$

*NSET, NSET=_PICKEDSET2, INTERNAL, GENERATE

$1,123,1$

*ELSET, ELSET=_PICKEDSET2, INTERNAL, GENERATE

$1,80,1$

** SECTION: LUMBER

*SOLID SECTION, ELSET=_PICKEDSET2, MATERIAL=LUMBER

73.,

*END INSTANCE**

*INSTANCE, NAME=EPS-2, PART=EPS

$1239.05, \quad 0 ., \quad 0$.

*NODE 

1, $\quad 0 ., \quad 329.32251$
2, 290.712494, 329.32251
$3,290.712494,658.64502$
4, $\quad 0 ., \quad 658.64502$
5, 581.424988, 329.32251
$6, \quad 581.424988,658.64502$
7, 872.137512, 329.32251
$8,872.137512, \quad 658.64502$
9, 1162.84998, 329.32251
10, 872.137512, 0 .

690, 1017.49377, 2436.98657

$691, \quad 1090.17188,2436.98657$

692, 944.815613, 2502.85107

693, 1017.49377, 2502.85107

694, 1090.17188, 2502.85107

$695,944.815613,2568.71558$

696, 1017.49377, 2568.71558

697, 1090.17188, 2568.71558

*NSET, NSET=_PICKEDSET2, INTERNAL, GENERATE

$1,697,1$

*ELSET, ELSET=_PICKEDSET2, INTERNAL, GENERATE

$1,640,1$

** SECTION: EPS

*SOLID SECTION, ELSET=_PICKEDSET2, MATERIAL=EPS

73.,

*END INSTANCE**

*INSTANCE, NAME=H-LUMBER-2, PART=H-LUMBER
$0 ., \quad 2672.68$,
0.

*NODE

$1, \quad 2111.1875,-38.0999985$

2, 2111.1875, 0.

3, 1820.47498, 0 .

4, $1820.47498,-38.0999985$

$5, \quad 1529.76245,-38.0999985$ 
6, 1529.76245, 0.

7, 1239.05005, 0 .

$8, \quad 1239.05005,-38.0999985$

$9, \quad 328.8125,-38.0999985$

$10, \quad 328.8125, \quad 0$.

$75, \quad 1128.27185,-38.0999985$

76, 1128.27185, 0 .

77, 1055.59375, 0 .

78, 982.915649, 0 .

*NSET, NSET=_PICKEDSET2, INTERNAL, GENERATE

$1,714,1$

*ELSET, ELSET=_PICKEDSET2, INTERNAL, GENERATE

$1,656,1$

** SECTION: OSB

*SOLID SECTION, ELSET=_PICKEDSET2, MATERIAL=OSB

11.

*END INSTANCE**

*NSET, NSET=_PICKEDSET578, INTERNAL, INSTANCE="REF POINT-1"

1 ,

*NSET, NSET=_PICKEDSET579, INTERNAL, INSTANCE=H-LUMBER-2

29,30

*NSET, NSET=_PICKEDSET579, INTERNAL, INSTANCE=H-LUMBER-3, GENERATE

$43,45,1$

*ELSET, ELSET=_PICKEDSET579, INTERNAL, INSTANCE=H-LUMBER-2

38 ,

*ELSET, ELSET=_PICKEDSET579, INTERNAL, INSTANCE=H-LUMBER-3

64,76

*NSET, NSET=_PICKEDSET629, INTERNAL, INSTANCE=H-LUMBER-1

$1,4,9,11,14,17,19,20,22,28,29,30,34,40,42,46$

$47,48,61,62,63,70,71,72,76,77,78,79,80,81,88,89$

$90,91,92,93,94,95,96$

*ELSET, ELSET=_PICKEDSET629, INTERNAL, INSTANCE=H-LUMBER-1

$1,2,3,4,13,14,15,16,21,22,23,24,25,26,27,28$

$29,30,31,32,33,34,35,36,38,39,41,42,43,44,45,48$ 
$49,50,51,52,54,72$

*NSET, NSET=_PICKEDSET630, INTERNAL, INSTANCE="REF POINT-1"

1 ,

*NSET, NSET=_PICKEDSET631, INTERNAL, INSTANCE=H-LUMBER-3

$5,6,7,13,14,17,24,26,27,30,33,35,40,41,45,52$

$53,54,58,59,60,70,71,72,79,80,81,94,95,96,100,101$

$102,112,113,114,115,116,117$

*ELSET, ELSET=_PICKEDSET631, INTERNAL, INSTANCE=H-LUMBER-3

$5,6,7,8,9,10,11,12,17,18,19,20,25,26,27,28$

$37,38,39,40,41,42,43,44,49,51,63,66,67,68,69,70$

$71,72,73,74,75,76$

*NSET, NSET=_PICKEDSET663, INTERNAL, INSTANCE=H-LUMBER-1

$13,24,27,32,33,35,37,38,41$

*NSET, NSET=_PICKEDSET663, INTERNAL, INSTANCE=H-LUMBER-2

$8,12,17,20,21,24,26,27,30$

*NSET, NSET=_PICKEDSET664, INTERNAL, INSTANCE=V-LUMBER-1

$6,7,24,25,26,27$

*NSET, NSET=_PICKEDSET664, INTERNAL, INSTANCE=V-LUMBER-2

$6,7,24,25,26,27$

*NSET, NSET=_PICKEDSET664, INTERNAL, INSTANCE=V-LUMBER-3

$6,7,24,25,26,27$

*NSET, NSET=_PICKEDSET665, INTERNAL, INSTANCE=H-LUMBER-3

$1,4,9,11,18,20,21,23,31,32,36,37,38,42,44,49$

$50,51,64,65,66,73,74,75,85,86,87,91,92,93,103,104$

105, 106, 107, 108, 109, 110, 111

*ELSET, ELSET=_PICKEDSET665, INTERNAL, INSTANCE=H-LUMBER-3

$1,2,3,4,13,14,15,16,21,22,23,24,29,30,31,32$

$33,34,35,36,45,46,47,48,50,52,53,54,55,56,57,58$

$59,60,61,62,64,65$

*NSET, NSET=_PICKEDSET666, INTERNAL, INSTANCE=H-LUMBER-2

$2,3,6,7,10,11,14,15,18,19,22,23,25,28,29,31$

$32,33,37,38,39,46,47,48,49,50,51,55,56,57,64,65$

$66,70,71,72,76,77,78$

*ELSET, ELSET=_PICKEDSET666, INTERNAL, INSTANCE=H-LUMBER-2, GENERATE

$1,38,1$

*ELSET, ELSET=_PICKEDSURF580_S2, INTERNAL, INSTANCE=H-LUMBER-1

$5,6,7,8,73,74,75,76$ 
*ELSET, ELSET=_PICKEDSURF580_S2, INTERNAL, INSTANCE=V-LUMBER-1 $46,47,48,49,50,51,52,53,54,55,66,67,68,69,70,71$

$72,73,74,75$

*ELSET, ELSET=_PICKEDSURF580_S2, INTERNAL, INSTANCE=V-LUMBER-3

$16,17,18,19,20,36,37,38,39,40,56,57,58,59,60,66$

$67,68,69,70$

*ELSET, ELSET=_PICKEDSURF580_S2, INTERNAL, INSTANCE=H-LUMBER-2

$9,10,11,12,21,22,23,24,30,31,32,33,34,35,36,37$

*ELSET, ELSET=_PICKEDSURF580_S2, INTERNAL, INSTANCE=V-LUMBER-2

$16,17,18,19,20,36,37,38,39,40,46,47,48,49,50,51$

$52,53,54,55,56,57,58,59,60,61,62,63,64,65,66,67$

$68,69,70,76,77,78,79,80$

*ELSET, ELSET=_PICKEDSURF580_S4, INTERNAL, INSTANCE=H-LUMBER-1

$9,10,11,12,17,18,19,20$

*ELSET, ELSET=_PICKEDSURF580_S4, INTERNAL, INSTANCE=V-LUMBER-1

$6,7,8,9,10,11,12,13,14,15,26,27,28,29,30,31$

$32,33,34,35$

*ELSET, ELSET=_PICKEDSURF580_S4, INTERNAL, INSTANCE=V-LUMBER-3

$1,2,3,4,5,21,22,23,24,25,41,42,43,44,45,71$

$72,73,74,75$

*ELSET, ELSET=_PICKEDSURF580_S4, INTERNAL, INSTANCE=H-LUMBER-2

$1,2,3,4,5,6,7,8,13,14,15,16,17,18,19,20$

*ELSET, ELSET=_PICKEDSURF580_S4, INTERNAL, INSTANCE=V-LUMBER-2

$1,2,3,4,5,6,7,8,9,10,11,12,13,14,15,21$

$22,23,24,25,26,27,28,29,30,31,32,33,34,35,41,42$

$43,44,45,71,72,73,74,75$

*ELSET, ELSET=_PICKEDSURF580_S3, INTERNAL, INSTANCE=H-LUMBER-1, GENERATE

$55,70,1$

*SURFACE, TYPE=ELEMENT, NAME=_PICKEDSURF580, INTERNAL

PICKEDSURF580_S2, S2

_PICKEDSURF580_S4, S4

_PICKEDSURF580_S3, S3

*ELSET, ELSET=_PICKEDSURF581_S4, INTERNAL, INSTANCE=EPS-1

$1,5,9,13,17,61,65,69,73,77,81,85,89,93,97,161$

$165,169,173,177,221,225,229,233,237,241,245,249,253,257,321,325$

$329,333,337,581,585,589,593,597$

*ELSET, ELSET=_PICKEDSURF581_S4, INTERNAL, INSTANCE=EPS-2 
$1,5,9,13,17,61,65,69,73,77,81,85,89,93,97,161$

$165,169,173,177,221,225,229,233,237,241,245,249,253,257,321,325$

$329,333,337,581,585,589,593,597$

*ELSET, ELSET=_PICKEDSURF581_S3, INTERNAL, INSTANCE=EPS-1

$77,78,79,80,517,518,519,520,537,538,539,540,557,558,559,560$

$577,578,579,580,597,598,599,600,617,618,619,620,637,638,639,640$

*ELSET, ELSET=_PICKEDSURF581_S3, INTERNAL, INSTANCE=EPS-2

$77,78,79,80,517,518,519,520,537,538,539,540,557,558,559,560$

$577,578,579,580,597,598,599,600,617,618,619,620,637,638,639,640$

*ELSET, ELSET=_PICKEDSURF581_S2, INTERNAL, INSTANCE=EPS-1

$144,148,152,156,160,304,308,312,316,320,384,388,392,396,400,404$

$408,412,416,420,464,468,472,476,480,484,488,492,496,500,544,548$

$552,556,560,564,568,572,576,580$

*ELSET, ELSET=_PICKEDSURF581_S2, INTERNAL, INSTANCE=EPS-2 144, 148, 152, 156, 160, 304, 308, 312, 316, 320, 384, 388, 392, 396, 400, 404 $408,412,416,420,464,468,472,476,480,484,488,492,496,500,544,548$ $552,556,560,624,628,632,636,640$

*SURFACE, TYPE=ELEMENT, NAME=_PICKEDSURF581, INTERNAL PICKEDSURF581_S4, S4

_PICKEDSURF581_S3, S3

PICKEDSURF581_S2, S2

*SURFACE, TYPE=NODE, NAME=_PICKEDSET663_CNS_, INTERNAL PICKEDSET663, 1.

*SURFACE, TYPE=NODE, NAME=_PICKEDSET664_CNS_, INTERNAL PICKEDSET664, 1.

*SURFACE, TYPE=NODE, NAME=_PICKEDSET665_CNS_, INTERNAL PICKEDSET665, 1.

*SURFACE, TYPE=NODE, NAME=_PICKEDSET666_CNS_, INTERNAL PICKEDSET666, 1.

** CONSTRAINT: HORIZONTAL TO VERTICAL LUMBER

*TIE, NAME="HORIZONTAL TO VERTICAL LUMBER", ADJUST=YES _PICKEDSET664_CNS_,_PICKEDSET663_CNS

** CONSTRAINT: TOP LUMBERS

*TIE, NAME="TOP LUMBERS", ADJUST=YES

_PICKEDSET666_CNS_,_PICKEDSET665_CNS

** CONSTRAINT: WALL TOP CORNER (LOADING)

*RIGID BODY, REF NODE=_PICKEDSET578, TIE NSET=_PICKEDSET579, POSITION=CENTER OF MASS 
*ELEMENT, TYPE=SPRINGA, ELSET="NAILS ON OSB-SPRING"

1, OSB-1.45, H-LUMBER-3.41

2, OSB-1.45, H-LUMBER-3.43

3, OSB-1.41, H-LUMBER-3.26

4, OSB-1.41, H-LUMBER-3.34

5, OSB-1.40, H-LUMBER-1.39

6, OSB-1.40, H-LUMBER-1.40

7, OSB-1.16, H-LUMBER-1.31

8, OSB-1.16, H-LUMBER-1.20

9, OSB-2.38, H-LUMBER-3.35

50, OSB-2.18, V-LUMBER-2.17

51, OSB-1.21, V-LUMBER-2.9

52, OSB-2.12, V-LUMBER-2.9

53, OSB-1.1, V-LUMBER-2.3

54, OSB-2.9, V-LUMBER-2.3

55, OSB-1.4, V-LUMBER-2.2

56, OSB-2.10, V-LUMBER-2.2

*SPRING, ELSET="NAILS ON OSB-SPRING"

450.

*END ASSEMBLY**

** MATERIALS**

** EPS/URETHANE

*MATERIAL, NAME=EPS

*ELASTIC

5500., 0.165

*PLASTIC

38., 0.

41., 0.04

59., 0.06

72., 0.08

81., 0.1

86., 0.12

89., 0.14 
100., 0.2

*MATERIAL, NAME=LUMBER

*ELASTIC

20800., 0.3

*PLASTIC

$48.3,0$.

$71.76,0.001$

92.46, 0.002

$114.54,0.003$

$135.24,0.004$

$155.94,0.005$

$176.64,0.006$

$198.72,0.007$

218.04, 0.008

$229.08,0.0087$

234.6, 0.009

$248.4,0.01$

*MATERIAL, NAME $=$ OSB

*ELASTIC

3850., 0.3

*PLASTIC

20., 0.

36., 0.0005

55., 0.001

78., 0.002

98., 0.003

115., 0.004

138., 0.005

140., 0.006

140., 0.007

140., 0.008

140., 0.009

**

** INTERACTION PROPERTIES

**

*SURFACE INTERACTION, NAME="SHEETS TO LUMBERS (HARD)"

$1 .$, 
*SURFACE BEHAVIOR, PRESSURE-OVERCLOSURE=HARD

$* *$

** BOUNDARY CONDITIONS**

** NAME: BOTTOM-FIXED TYPE: DISPLACEMENT/ROTATION

*BOUNDARY

PICKEDSET629, 1, 1

PICKEDSET629, 2, 2

PICKEDSET629, 3, 3

PICKEDSET629, 4, 4

PICKEDSET629, 5, 5

PICKEDSET629, 6, 6

** NAME: LATERAL SUPPORT TYPE: DISPLACEMENT/ROTATION

*BOUNDARY

_PICKEDSET631, 2, 2

PICKEDSET631, 3, 3

PICKEDSET631, 5, 5

PICKEDSET631, 6, 6

**

** INTERACTIONS**

** INTERACTION: EPS AND LUMBER

*CONTACT PAIR, INTERACTION="SHEETS TO LUMBERS (HARD)", TYPE=SURFACE TO SURFACE

_PICKEDSURF581,_PICKEDSURF580

**

$* *$

** STEP: CYCLE-1**

*STEP, NAME=CYCLE-1, NLGEOM=YES

+5.11 MM DISP.

*STATIC

$0.1,1 ., 1 \mathrm{E}-05,0.1$

**

** BOUNDARY CONDITIONS**

** NAME: DISPLACMENT ON TOP TYPE: DISPLACEMENT/ROTATION

*BOUNDARY

_PICKEDSET630, 1, 1, 5.11

**

** OUTPUT REQUESTS**

*RESTART, WRITE, FREQUENCY=0 


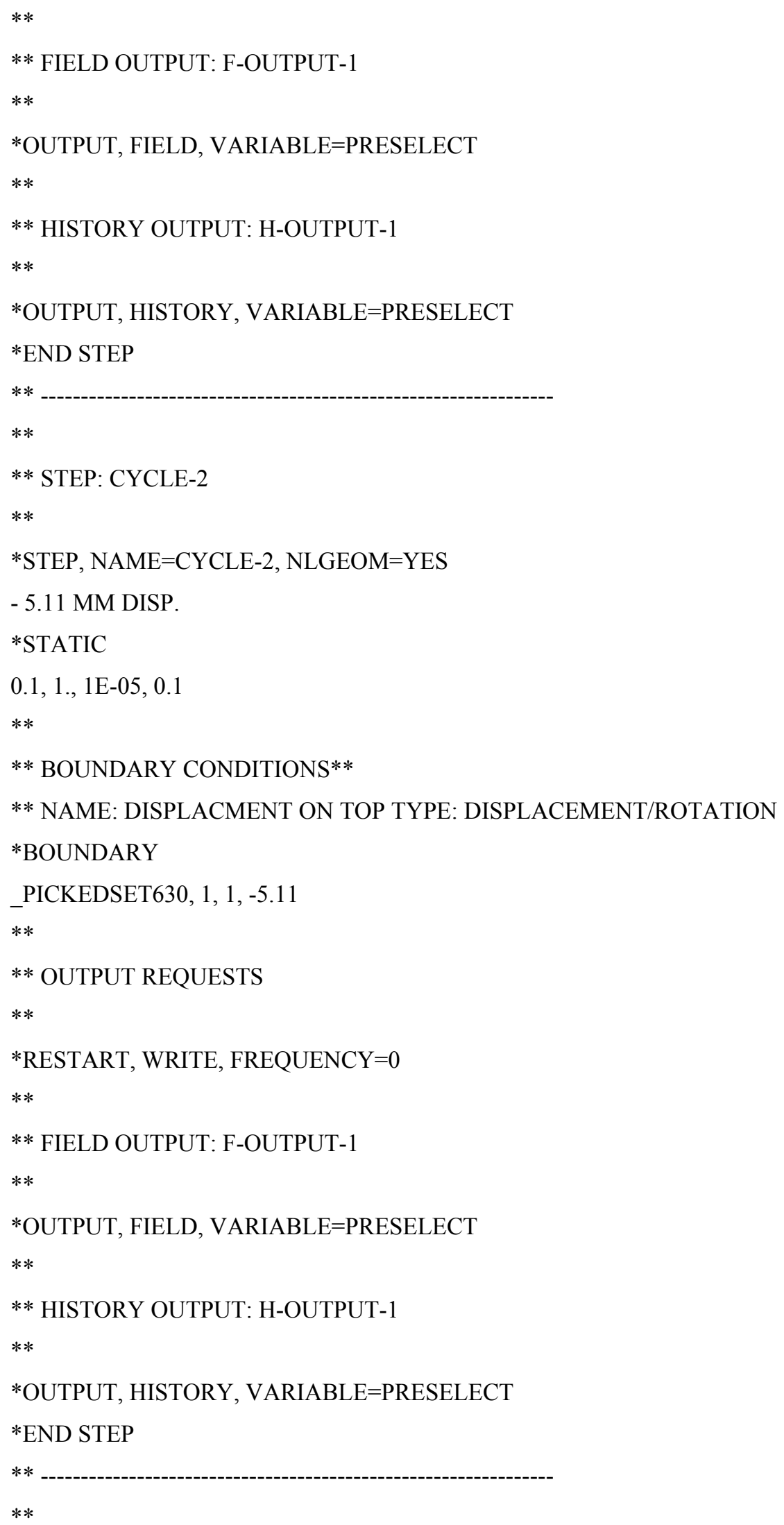


** STEP: CYCLE-3

$* *$

*STEP, NAME=CYCLE-3, NLGEOM=YES

+10.22 MM DISP

*STATIC

$0.1,1 ., 1 \mathrm{E}-05,0.1$

**

** BOUNDARY CONDITIONS**

** NAME: DISPLACMENT ON TOP TYPE: DISPLACEMENT/ROTATION

*BOUNDARY

PICKEDSET630, 1, 1, 10.22**

** OUTPUT REQUESTS**

*RESTART, WRITE, FREQUENCY $=0 * *$

** FIELD OUTPUT: F-OUTPUT-1**

*OUTPUT, FIELD, VARIABLE=PRESELECT $* *$

** HISTORY OUTPUT: H-OUTPUT-1**

*OUTPUT, HISTORY, VARIABLE=PRESELECT

*END STEP

**

**

** STEP: CYCLE-4**

*STEP, NAME $=$ CYCLE-4, NLGEOM=YES

- 10.22 MM DISP

*STATIC

$0.1,1 ., 1 \mathrm{E}-05,0.1$

**

** BOUNDARY CONDITIONS**

** NAME: DISPLACMENT ON TOP TYPE: DISPLACEMENT/ROTATION

*BOUNDARY

_PICKEDSET630, 1, 1, - 10.22

**

** OUTPUT REQUESTS**

*RESTART, WRITE, FREQUENCY $=0 * *$

** FIELD OUTPUT: F-OUTPUT-1**

*OUTPUT, FIELD, VARIABLE=PRESELECT $* *$

** HISTORY OUTPUT: H-OUTPUT-1 ${ }^{* *}$

*OUTPUT, HISTORY, VARIABLE=PRESELECT 
*END STEP

**

$* *$

** STEP: CYCLE-5**

*STEP, NAME=CYCLE-5, NLGEOM=YES

+13.62 MM DISP

*STATIC

$0.1,1 ., 1 \mathrm{E}-05,0.1$

$* *$

** BOUNDARY CONDITIONS**

** NAME: DISPLACMENT ON TOP TYPE: DISPLACEMENT/ROTATION

*BOUNDARY

_PICKEDSET630, 1, 1, 13.62**

** OUTPUT REQUESTS**

*RESTART, WRITE, FREQUENCY $=0$ **

** FIELD OUTPUT: F-OUTPUT-1**

*OUTPUT, FIELD, VARIABLE=PRESELECT**

** HISTORY OUTPUT: H-OUTPUT-1 $* *$

*OUTPUT, HISTORY, VARIABLE=PRESELECT

*END STEP

**

**

** STEP: CYCLE-6**

*STEP, NAME=CYCLE-6, NLGEOM=YES

-13.62 MM DISP

*STATIC

$0.1,1 ., 1 \mathrm{E}-05,0.1$

**

** BOUNDARY CONDITIONS**

** NAME: DISPLACMENT ON TOP TYPE: DISPLACEMENT/ROTATION

*BOUNDARY

_PICKEDSET630, 1, 1, -13.62

**

** OUTPUT REQUESTS**

*RESTART, WRITE, FREQUENCY $=0 * *$

** FIELD OUTPUT: F-OUTPUT-1**

*OUTPUT, FIELD, VARIABLE=PRESELECT** 
** HISTORY OUTPUT: H-OUTPUT-1**

*OUTPUT, HISTORY, VARIABLE=PRESELECT

*END STEP

**

**

** STEP: CYCLE-7**

*STEP, NAME=CYCLE-7, NLGEOM=YES

+27.24 MM DISP

*STATIC

$0.1,1 ., 1 \mathrm{E}-05,0.1$

**

** BOUNDARY CONDITIONS**

** NAME: DISPLACMENT ON TOP TYPE: DISPLACEMENT/ROTATION

*BOUNDARY

PICKEDSET630, 1, 1, 27.24

**

** OUTPUT REQUESTS**

*RESTART, WRITE, FREQUENCY $=0 * *$

** FIELD OUTPUT: F-OUTPUT-1**

*OUTPUT, FIELD, VARIABLE=PRESELECT**

** HISTORY OUTPUT: H-OUTPUT-1 ${ }^{* *}$

*OUTPUT, HISTORY, VARIABLE=PRESELECT

*END STEP

**

**

** STEP: CYCLE- $8 * *$

*STEP, NAME=CYCLE-8, NLGEOM=YES

- 27.24 MM DISP

*STATIC

1., 1., 1E-05, 1.**

** BOUNDARY CONDITIONS**

** NAME: DISPLACMENT ON TOP TYPE: DISPLACEMENT/ROTATION

*BOUNDARY

_PICKEDSET630, 1, 1, -27.24**

** OUTPUT REQUESTS**

*RESTART, WRITE, FREQUENCY $=0 * *$

** FIELD OUTPUT: F-OUTPUT-1** 
*OUTPUT, FIELD, VARIABLE=PRESELECT $* *$

** HISTORY OUTPUT: H-OUTPUT-1 ${ }^{* *}$

*OUTPUT, HISTORY, VARIABLE=PRESELECT

*END STEP

**

** STEP: CYCLE- $9 * *$

*STEP, NAME=CYCLE-9, NLGEOM=YES

+40.86 MM DISP

*STATIC

$0.1,1 ., 1 \mathrm{E}-05,0.1^{* *}$

** BOUNDARY CONDITIONS**

** NAME: DISPLACMENT ON TOP TYPE: DISPLACEMENT/ROTATION

*BOUNDARY

_PICKEDSET630, 1, 1, 40.86**

** OUTPUT REQUESTS**

*RESTART, WRITE, FREQUENCY $=0 * *$

** FIELD OUTPUT: F-OUTPUT-1**

*OUTPUT, FIELD, VARIABLE=PRESELECT**

** HISTORY OUTPUT: H-OUTPUT-1 $* *$

*OUTPUT, HISTORY, VARIABLE=PRESELECT

*END STEP

$* *$

**

** STEP: CYCLE-10**

*STEP, NAME=CYCLE-10, NLGEOM=YES

-40.86 MM DISP

*STATIC

$0.1,1 ., 1 \mathrm{E}-05,0.1^{* *}$

** BOUNDARY CONDITIONS**

** NAME: DISPLACMENT ON TOP TYPE: DISPLACEMENT/ROTATION

*BOUNDARY

PICKEDSET630, $1,1,-40.86^{* *}$

** OUTPUT REQUESTS**

*RESTART, WRITE, FREQUENCY $=0 * *$

** FIELD OUTPUT: F-OUTPUT- $1 * *$

*OUTPUT, FIELD, VARIABLE=PRESELECT $* *$

** HISTORY OUTPUT: H-OUTPUT-1** 
*OUTPUT, HISTORY, VARIABLE=PRESELECT

*END STEP

**

** STEP: CYCLE-1**

*STEP, NAME=CYCLE-11, NLGEOM=YES

+54.48 MM DISP

*STATIC

$0.1,1 ., 1 \mathrm{E}-05,0.1^{* *}$

** BOUNDARY CONDITIONS**

** NAME: DISPLACMENT ON TOP TYPE: DISPLACEMENT/ROTATION

*BOUNDARY

_PICKEDSET630, 1, 1, 54.48**

** OUTPUT REQUESTS**

*RESTART, WRITE, FREQUENCY $=0 * *$

** FIELD OUTPUT: F-OUTPUT-1**

*OUTPUT, FIELD, VARIABLE=PRESELECT**

** HISTORY OUTPUT: H-OUTPUT-1 ${ }^{* *}$

*OUTPUT, HISTORY, VARIABLE=PRESELECT

*END STEP

**

** STEP: CYCLE-12**

*STEP, NAME=CYCLE-12, NLGEOM=YES

-54.48 MM DISP

*STATIC

$0.1,1 ., 1 \mathrm{E}-05,0.1^{* *}$

** BOUNDARY CONDITIONS**

** NAME: DISPLACMENT ON TOP TYPE: DISPLACEMENT/ROTATION

*BOUNDARY

_PICKEDSET630, 1, 1, -54.48**

** OUTPUT REQUESTS**

*RESTART, WRITE, FREQUENCY $=0 * *$

** FIELD OUTPUT: F-OUTPUT-1**

*OUTPUT, FIELD, VARIABLE=PRESELECT**

** HISTORY OUTPUT: H-OUTPUT-1**

*OUTPUT, HISTORY, VARIABLE=PRESELECT

*END STEP

** 
** STEP: CYCLE-13**

*STEP, NAME=CYCLE-13, NLGEOM=YES

+95.32 MM DISP.

*STATIC

$0.1,1 ., 1 \mathrm{E}-05,0.1^{* *}$

** BOUNDARY CONDITIONS**

** NAME: DISPLACMENT ON TOP TYPE: DISPLACEMENT/ROTATION

*BOUNDARY

_PICKEDSET630, 1, 1, 95.32**

** OUTPUT REQUESTS**

*RESTART, WRITE, FREQUENCY $=0 * *$

** FIELD OUTPUT: F-OUTPUT-1**

*OUTPUT, FIELD, VARIABLE=PRESELECT**

** HISTORY OUTPUT: H-OUTPUT-1**

*OUTPUT, HISTORY, VARIABLE=PRESELECT

*END STEP

** -**

** STEP: CYCLE-14**

*STEP, NAME=CYCLE-14, NLGEOM=YES

-95.32 MM DISP.

*STATIC

$0.1,1 ., 1 \mathrm{E}-05,0.1^{* *}$

** BOUNDARY CONDITIONS**

** NAME: DISPLACMENT ON TOP TYPE: DISPLACEMENT/ROTATION

*BOUNDARY

_PICKEDSET630, 1, 1, -95.32**

** OUTPUT REQUESTS**

*RESTART, WRITE, FREQUENCY $=0 * *$

** FIELD OUTPUT: F-OUTPUT-1**

*OUTPUT, FIELD, VARIABLE=PRESELECT**

** HISTORY OUTPUT: H-OUTPUT-1 $* *$

*OUTPUT, HISTORY, VARIABLE=PRESELECT

*END STEP

**

$* *$

** STEP: CYCLE-15** 
*STEP, NAME=CYCLE-15, NLGEOM=YES

+136.18 MM DISP.

*STATIC

$0.1,1 ., 1 \mathrm{E}-05,0.1 * *$

** BOUNDARY CONDITIONS**

** NAME: DISPLACMENT ON TOP TYPE: DISPLACEMENT/ROTATION

*BOUNDARY

_PICKEDSET630, 1, 1, 136.18**

** OUTPUT REQUESTS**

*RESTART, WRITE, FREQUENCY $=0 * *$

** FIELD OUTPUT: F-OUTPUT-1**

*OUTPUT, FIELD, VARIABLE=PRESELECT**

** HISTORY OUTPUT: H-OUTPUT-1**

*OUTPUT, HISTORY, VARIABLE=PRESELECT

*END STEP

**

** STEP: CYCLE-16**

*STEP, NAME=CYCLE-16, NLGEOM=YES

-136.18 MM DISP

*STATIC

$0.1,1 ., 1 \mathrm{E}-05,0.1 * *$

** BOUNDARY CONDITIONS**

** NAME: DISPLACMENT ON TOP TYPE: DISPLACEMENT/ROTATION

*BOUNDARY

_PICKEDSET630, 1, 1, -136.18**

** OUTPUT REQUESTS**

*RESTART, WRITE, FREQUENCY $=0 * *$

** FIELD OUTPUT: F-OUTPUT- $1 * *$

*OUTPUT, FIELD, VARIABLE=PRESELECT**

** HISTORY OUTPUT: H-OUTPUT-1**

*OUTPUT, HISTORY, VARIABLE=PRESELECT

*END STEP

**

** STEP: CYCLE-17**

*STEP, NAME=CYCLE-17, NLGEOM=YES

+204.28 MM DISP

*STATIC 
$0.1,1 ., 1 \mathrm{E}-05,0.1^{* *}$

** BOUNDARY CONDITIONS**

** NAME: DISPLACMENT ON TOP TYPE: DISPLACEMENT/ROTATION

*BOUNDARY

PICKEDSET630, 1, 1, 204.28**

** OUTPUT REQUESTS**

*RESTART, WRITE, FREQUENCY $=0 * *$

** FIELD OUTPUT: F-OUTPUT-1**

*OUTPUT, FIELD, VARIABLE=PRESELECT $* *$

** HISTORY OUTPUT: H-OUTPUT-1**

*OUTPUT, HISTORY, VARIABLE=PRESELECT

*END STEP

**

** STEP: CYCLE-18**

*STEP, NAME=CYCLE-18, NLGEOM=YES

-204.28 MM DISP

*STATIC

$0.1,1 ., 1 \mathrm{E}-05,0.1^{* *}$

** BOUNDARY CONDITIONS**

** NAME: DISPLACMENT ON TOP TYPE: DISPLACEMENT/ROTATION

*BOUNDARY

_PICKEDSET630, 1, 1, -204.28**

** OUTPUT REQUESTS**

*RESTART, WRITE, FREQUENCY $=0 * *$

** FIELD OUTPUT: F-OUTPUT-1**

*OUTPUT, FIELD, VARIABLE=PRESELECT**

** HISTORY OUTPUT: H-OUTPUT-1 ${ }^{* *}$

*OUTPUT, HISTORY, VARIABLE=PRESELECT

*END STEP

**

** STEP: CYCLE-19**

*STEP, NAME=CYCLE-19, NLGEOM=YES

+272.36 MM DISP

*STATIC

$0.1,1 ., 1 \mathrm{E}-05,0.1^{* *}$

** BOUNDARY CONDITIONS**

** NAME: DISPLACMENT ON TOP TYPE: DISPLACEMENT/ROTATION 
*BOUNDARY

_PICKEDSET630, 1, 1, 272.36**

** OUTPUT REQUESTS**

*RESTART, WRITE, FREQUENCY $=0$ **

** FIELD OUTPUT: F-OUTPUT-1**

*OUTPUT, FIELD, VARIABLE=PRESELECT ${ }^{* *}$

** HISTORY OUTPUT: H-OUTPUT-1 ${ }^{* *}$

*OUTPUT, HISTORY, VARIABLE=PRESELECT

*END STEP

**

** STEP: CYCLE-20**

*STEP, NAME=CYCLE-20, NLGEOM=YES

-272.36 MM DISP

*STATIC

$0.1,1 ., 1 \mathrm{E}-05,0.1^{* *}$

** BOUNDARY CONDITIONS**

** NAME: DISPLACMENT ON TOP TYPE: DISPLACEMENT/ROTATION

*BOUNDARY

_PICKEDSET630, 1, 1, -272.36**

** OUTPUT REQUESTS**

*RESTART, WRITE, FREQUENCY $=0$ **

** FIELD OUTPUT: F-OUTPUT-1**

*OUTPUT, FIELD, VARIABLE=PRESELECT $* *$

** HISTORY OUTPUT: H-OUTPUT-1 ${ }^{* *}$

*OUTPUT, HISTORY, VARIABLE=PRESELECT

*END STEP

**

** STEP: CYCLE-21**

*STEP, NAME=CYCLE-21, NLGEOM=YES

+272.36 MM DISP. (END)

*STATIC

$0.1,1 ., 1 \mathrm{E}-05,0.1^{* *}$

** BOUNDARY CONDITIONS**

** NAME: DISPLACMENT ON TOP TYPE: DISPLACEMENT/ROTATION

*BOUNDARY

_PICKEDSET630, 1, 1, 272.36**

** OUTPUT REQUESTS** 
*RESTART, WRITE, FREQUENCY $=0$ **

** FIELD OUTPUT: F-OUTPUT-1**

*OUTPUT, FIELD, VARIABLE=PRESELECT**

** HISTORY OUTPUT: H-OUTPUT-1**

*OUTPUT, HISTORY, VARIABLE=PRESELECT

*END STEP 


\section{REFERENCES}

Allen, H.G., 1969, “Analysis and Design of Structural Sandwich Panels”, Pergaman Press, Oxford, UK.

APA, 1993, "Design and Fabrication of Plywood Sandwich Panels", The Engineering Wood Association, Tacoma, WA, USA.

APA, 1996, "Design and Fabrication of Plywood Stress-skin Panels", The Engineering Wood Association, Tacoma, WA, USA.

APA, 1998, "Design and Fabrication of Glued Plywood-Lumber Beams", The Engineering Wood Association, Tacoma, WA, USA.

APA, 1997, "Plywood Design Specification", The Engineering Wood Association, Tacoma, WA, USA.

APA PRS 610.1, 2013, "Standard for Performance-Rated Structural Insulated Panels in Wall Applications", The Engineering Wood Association, Tacoma, WA, USA, 36 pages.

Applied Technology Council, 1995, ATC-19, Structural Response Modification Factors, Applied Technology Council (ATC), Redwood City, California.

ASCE/SEI 41-06, 2007, "Seismic rehabilitation of existing buildings", American Society of Civil Engineers, Reston, VA, USA.

ASCE/SEI 7, 2010, "Minimum design loads for buildings and other structures", American Society of Civil Engineers, Reston, VA, USA. 
ASTM C203-05a, 2012, "Standard Test Methods for Breaking Load and Flexural Properties of Block-Type Thermal Insulation", American Society for Testing Materials, Philadelphia, PA, USA.

ASTM C273-00, 2000, "Standard Test Method for Shear Properties of Sandwich Core Materials", American Society for Testing Materials, Philadelphia, PA, USA.

ASTM D1037-06a, 2006, "Standard Test Methods for Evaluating Properties of Wood-Base Fiber and Particle Panel Materials", American Society for Testing Materials, Philadelphia, PA, USA.

ASTM E1803-06, 2006, "Standard Test Methods for Determining Strength Capacities of Structural Insulated Panels", American Society for Testing Materials, Philadelphia, PA, USA.

ASTM E2322-03, 2009, "Standard Test Method for Conducting Transverse and Concentrated Load Tests on Panels used in Floor and Roof Construction”, American Society for Testing Materials, Philadelphia, PA, USA.

ASTM E455-04, 2004, "Standard Method for Static Load Testing of Framed Floor or Roof Diaphragm Constructions for Buildings", American Society for Testing Materials, Philadelphia, PA, USA.

ASTM E72-10, 2010, "Standard Test Methods of Conducting Strength Tests of Panels for Building Construction”, American Society for Testing Materials, Philadelphia, PA, USA.

ASTM C 480-62, 1988, "Standard Test Methods of for Flexural Creep of Sandwich Construction”, American Society for Testing Materials, Philadelphia, PA, USA.

Aviles, F. and Carlsson, L., 2006, "Experimental Study of Debonded Sandwich Panels under Compressive loading”, Journal of Sandwich Structures and Materials, Vol 8, pp.7-31. 
Ayoub, A., 2007, "Seismic Analysis of Wood Building Structures," Engineering Structures, Vol. 29, pp. 213-223.

Ba zant, Z.P., 1971, "A correlation study of formulations of incremental deformation and stability of continuous bodies", Transaction ASME Ser. E, J. Appl. Mech. Vol 38(4), pp. 919928.

Ba zant, Z.P., Beghini, A., 2005, "Which formulation allows using a constant shear modulus for small-strain buckling of soft-core sandwich structures", Journal of Appllied Mechanics, Vol 72(5), pp. 785-787.

Ba zant, Z.P., Beghini, A., 2006, "Stability and finite strain of homogenized structures soft in shear: sandwich or fiber composites, and layered bodies", International Journal Solids Structures, Vol. 43(6), pp. 1571-1593.

Beer, J., Johnson, E. R., Dewolf, J., 2012, "Mechanics of Materials", $6^{\text {th }}$ Edition, McGrawHill, New York, USA.

Benadova, D., 2009, "Experimental Study on the Flexural Behavior of Structural Insulated Headers", M.A.Sc. Thesis, Ryerson University, Toronto, Canada.

Bergman, S., and Reissner, H., 1932, "Neuere problems aus der flugzeugstatik. Uber die knickung yon rechteckigen platten bei schubbeansprughung", ZFM Bd. 23, Heft 1:6.

Biezeno, C.B., Hencky, H., 1929, "General theory of elastic stability”, Proceedings Koninklijke Akademie van Wetenschappen te Amsterdam, pp. 444-456.

Biot, M., 1939, "Non-linear theory of elasticity and linearised case for a body under initial stress", Philos. Magazine, Vol. 27, pp. 468-489. 
Boudreault, F.A., C. Blais et C.A. Rogers, 2007, "Seismic force modification factors for lightgauge steel-frame - wood structural shear walls", Canadian Journal of civil Engineering, Vol. 34, pp. 56-65.

BS 5268-6.2, 2001, "Structural use of timber, Code of practice for timber frame walls buildings other than dwellings not exceeding four storeys", London, UK.

BS EN 594, 2011, “ Timber structures. Test methods. Racking strength and stiffness of timber frame wall panels", London, UK.

BS EN 14358, 2006, "Timber structures; Calculation of characteristic 5-percentile values and acceptance criteria for a sample", London, UK.

Butt, A.S., 2008, "Experimental Study on the Flexural Behavior of Structural Insulated Sandwich Timber Panels", M.A.Sc. Thesis, Ryerson University, Toronto, Canada.

CAN/CSA-O86-09, 2010, “Engineering Design of Wood”, Etobicoke, Ontario, Canada.

CAN/CSA-S406-92, 1992, “Construction of Preserved Wood Foundation”, Etobicoke, Ontario, Canada.

Canadian Standard Association, 1997, "Permanent Wood Foundation", Etobicoke, Ontario, Canada.

Canadian Wood Council, 2005, "Wood Design Manual”, Ottawa, Canada.

Council of Forest Industries of British Columbia, 1989, "Design of Glued and Nailed Plywood Beams", Vancouver, Canada.

Cox, H. and Riddell, J., 1945, "Sandwich Construction and Core Materials III: Instability of Sandwich Struts and Beams", ARC Technical Report $R \&$ M 2125, USA. 
CUREE, 2002, “Caltech Wood-Frame Project”, Element 1, USA.

Cylinders Repairs Component (CRC) Inc., 2012, http://www.crconline.com.

Davenne, L., Daudeville, L., Ricahrd, N., Kawai, N. and Yasumura, M. ,1998, "Modeling of Timber Shear Walls with Nailed Joints Under Cyclic Loading," Proceedings of the Fifth World Conference on Timber Engineering, Lausanne, Switzerland, Vol. 1, pp.353-360.

Davies, J. M., 1986, "The Analysis of Sandwich Panels with Profiled Faces", Proceeding of Eighth International Specialty Conference on Cold-Formed Steel Structures, St Louis, pp. 351369.

Davies, J. M., 1987, “Axially Loaded Sandwich Panels”, ASCE, Journal of Structural Engineering, Vol. 113:11, pp. 2212 - 2230.

DIAB AB Group, 2003, “Sandwich Concepts”, Laholm, Sweden.

Dolan, J.D. and Johnson, A.C., 1996, "Performance of Long Shear Walls with Opening", Proceedings of the 1996 International Timber Engineering Conference, Vol. 2, New Orleans, LA, pp. 2.337-2.344.

Dolan, J.D. and Nadsen, B., 1992, "Monotonic and Cyclic Tests of Timber Shear Wall," Can. J. Civ. Eng., 19(4), 415-422.

Dolan, J.D., 1998, “The Dynamic Response of Timber Shear Walls," Doctor of Philosophy Dissertation, University of British Columbia, Vancouver, Canada.

Dolan, J.D., Foschi, R.O., 1991, "Structural Analysis Model for Static Loads on Timber Shear Walls,” J. Structural Engineering, Vol. 117, No. 3, pp. 851-861. 
Enjily V. and Griffiths R.D., 1996, "The Racking of Large Wall Panel", Proceedings of International Wood Engineering Conference 96, vol. 2, pp. 321-328.

European Organization for Technical Approval, 2004, "Prefabricated Wood-based Load Bearing Stressed Skin Panels", EOTA, EU.

Filiatrault A., 1990, "Static and Dynamic Analysis of Timber Shear Walls," Canadian Journal of Civil Engineering, Vol. 17 No. 4, pp. 643-651.

Folz, B. and Filatrault, A., 2001, "Cyclic Analysis of Wood-frame shear walls ." J. Structural Engineering, Vol. 127, No. 4, pp. 433-441.

Folz, B., and Filatrault, A., 2004a, "Seismic Analysis of Wood-frame Structures. I: Model Formulation.” J. Structural Engineering, Vol. 130, No. 8, pp. 1353-1360.

Folz, B., and Filatrault, A., 2004b, "Seismic Analysis of Wood-frame Structures. II: Model Implementation and Verification.” J. Structural Engineering, Vol. 130, No. 8,

Forest Product Laboratory, 1987, "Wood Handbook: Wood as an Engineering Material”, USDA, Madison, Wisconsin, USA.

Foschi, R.O., 1995, Diaphragm Analysis Program 3D--User's Manual. University of British Columbia, Vancouver, British Columbia.

Foschi, R.O., 2000, "Modeling the Hysteretic Response of Mechanical Connections for Wood Structures," World Conference on Timber Engineering, Vancouver, British

Gagnon, M.A. and Admas, R.D., 1999, "A Marketing Profile of the US Structural Insulated Panel Industry", Forest Products Journal, Vol. 49, p 31-35. 
Gatto, K. and Uang, C., 2003, "Effects of Loading Protocol on the Cyclic Response of Woodframe Shearwalls" Journal of Structural Engineering, Vol. 129, No. 10, pp. 1384-1393.

Gerhards C.C., 2000, "Bending Creep and Load Duration of Douglas-fir 2 by 4s Under Constant Load for Up to 12-Plus Years", Wood and Fiber Science, Vol. 32, N 4, pp. 489-501.

Goodier, J. and I. M. Neou, I., 1951, "The Evaluation of Theoretical Critical Compression in Sandwich Plates", Journal of Aeronautical Science, Vol. 18, No. 10, pp. 649-657.

Goodier, J., 1946, "Cylindrical Buckling of Sandwich Plates", Journal of Applied Mechanics, Vol. 13,No. 4, pp. 253-260.

Gramoll, K., 2010, "Enginneeing Solid Mechanics", University of Oklahoma, Norman, OK, USA.

Gupta N., Woldesenbet, E., Hore, K. and Sankaran, S., 2002, "Response of Syntactic Foam Core Sandwich Structured Composites to Three Point Bending”, Journal of Sandwich and materials, Vol. 4, pp. 249-272.

Gupta, A.K. and Kuo, G.P., (1985). "Behavior of Wood-Framed Shear Walls," J. Structural Engineering, Vol. 111, No. 8, pp. 1722-1733.

Gupta, N., Woldesenbet, E. and Hore, K., 2002, "Compressive Fracture Features of Syntactic Foams-microscopic Examination”, Journal of Materials Science, Vol. 37, No. 15, pp. 199-209.

Haung, J.S. and Gibson, L.J., 1990, "Creep of Sandwich Beams with Polymer Foam Cores", Journal of Materials in Civil Engineering, Vol. 2, No. 3, pp. 171-182.

Haung, J.S. and Gibson, L.J., 1991, "Creep of Polymer Foams" Journal of Materials Science, Vol. 26, No. 3, pp. 637-47. 
Heath, W. G., 1960, "Sandwich Construction: Correlation and Extension of Existing Theory of Flat Panels Subjected to Lengthwise Compression", Aircraft Engineering 32, 377 and 378, pp. 186-91 and 230-5.

Hill, R., 1968, "On the constitutive inequalities for simple materials", Journal of Mechanics and Physics Solids, Vol. 15(6), pp. 229-242.

Hossain, K. and Wright, H., 2004, "Flexural and Shear Behavior of Profiled Double Skin Composite Elements", Journal of Steel and Composite Structures, Vol. 4, No. 2, pp.1-20.

Hoyle, R.J. Jr., Griffith, M.C., Itani, R.Y., 1985, "Primary Creep in Douglas-fir Beams of Commercial Size and Quality", Wood and Fibre Science, Vol. 17, No. 3, pp. 300-314.

Huang, J.S. and Gibson, L.J., 1990, "Creep of Sandwich Beams with Polymer Foam Cores", Journal of Materials in Civil Engineering, Vol. 2, No. 3, pp. 171-182.

Huang, J.S. and Gibson, L.J., 1991, "Creep of Polymer Foams", Journal of Materials Science, Vol. 26, No. 3, pp. 637-47.

Huber, M.T., 1922, “Theory of Plates”, Springer, USA.

Huifeng Y., Weiqing L., Weidong L., Shuai Y., 2011, "Experimental Study on Seismic Behaviors of Structural Insulated Panel Shear Walls under Cyclic Loading", Journal of Advanced Materials Research, Volume 413, pages 529-534.

Hunt, M.O., 1975, "Structural Particleboard for Webs of Composite Beams", Forest Product Journal, Vol. 25, No. 2, pp. 55-57.

Ibarra, L.F., Medina, R.A., and Krawinkler, H., 2005, "Hysteretic Models that Incorporate Strength and Stiffness Deterioration." Earthquake Engineering and Structural Dynamics, Vol. 34, pp.1489-1511. 
ICC-AC04, 2004, "Acceptance Criteria for Sandwich Panels", ICC Evaluation Service Inc., USA.

Institute for Research in Construction, 2010,"National Building Code of Canada - NBCC-2010", National Research Council, Ottawa, Ontario, Canada.

Institute for Research in Construction, 2007, "Technical Guide for Stressed Skin Panels (with Lumber 1200 mm o.c. and EPS Core) for Walls and Roofs", National Research Council, Ottawa, Ontario, Canada.

International Code Council (ICC), 2009, “AC 130 - Acceptance Criteria for Prefabricated Wood Shear Panel," ICC Evaluation Service, Inc., Whittier, CA, USA.

International Residential Code (IRC), 2009, International Code Council, USA.

Itani R.Y., Cheung, C.K., 1984, "Nonlinear Analysis of Sheathed Wood Diaphragms," J. Structural Engineering, Vol. 110, No. 9, pp. 2137-2147.

Jamison, J.B., 1997, Monotonic and Cyclic Performance of Structurally Insulated Panel Shear Walls, Master of Science Thesis, Virginia Polytechnic Institute and State University, Blacksburg, Virginia.

Johnston, A.R., Denn, P.K., and Shenton III, H.W., 2006, "Effects of Vertical Load and HoldDown Anchors on the Cyclic Response of Wood-frame Shear Walls," J. Structural Engineering, Vol. 132, No. 9, pp. 1426-1434.

Kasal, B. and Leichti, R.J., 1992, "Nonlinear Finite Element Model for Light-Frame Stud Walls,” J. Structural Engineering, Vol. 118, No. 11, pp.3124-3135. 
Kasal, B., Leichti, R.J., and Itani, R.Y., 1994, "Nonlinear Finite Element Model of Complete Wood-frame Structures,” J. Structural Engineering, Vol. 120, No. 1, pp. 100-119.

Kermani, A. and Hairstans, R., 2006, "Racking Performance of Structural Insulated Panels", Journal of Structural Engineering, Vol. 132, No. 11, p 1806-1812.

Kermani, A., 2006, "Performance of Structural Insulated Panels", Proceeding of the Institution of Civil Engineers, Structures and Buildings, Vol. 159, No. SB1, pp. 13-19.

Kingspan Group Company, 2014, www.kingspanpanels.com

Kosny, J., Christian, J.E., Desjarlais. A.O., Kossecka, E. and Berrenberg, L., 1998, "Performance Check between Whole Building Thermal Performance Criteria and Exterior Wall Measured Clear Wall R-value, Thermal Bridging, Thermal Mass, and Airtightness", ASHRAE Transactions, Vol. 104, No. 2, pp. 1379-1389.

Krawinkler, H., Parisi, F., Ibarra, L., Ayoub, A., and Medina, R., 2001, "Development of a Testing Protocol for Wood-frame Structures," CUREE-Caltech Wood-frame Project Rep. No. W-02, Stanford University, Stanford, California.

Kuhn, R., 1951, "Photo-elastic Partial Solution of Bending Problem of Plates Based on Analogous Conditions of Plate and Disk," Forschung auf dem Gebiete des Ingenieurwesens, Vol. 18, No. 3, pp. 72-80.

Lebeda, D.J., Gupta, R., Rosowsky, D.V., and Dolan, J.D., 2005, "Effect of Hold-Down Misplacement on Strength and Stiffness of Wood-frame shear walls ," Practice Periodical on Structural Design and Construction, Vol. 10, No.2, pp. 79-87.

Lee, S., 1997, “A Fundamental Study of Thermoplastic Foam Extrusion with Physical Blowing Agents", ACS Symposium Series, Vol. 669, pp. 195-205. 
Lewicke, E.T., 1992, "Web Behavior in Wood Composite Box Beams", M.A.Sc. Thesis, University of Alberta, Alberta, Canada.

Li, X., 2005, “Composite Beams of Cold Form Steel Sections and Wood Members", M.A.Sc. Thesis, The University of New Brunswick, Canada.

Liu, Q. and Zhao, Y., 2007, “Effect of soft honeycomb core on flexural vibration of sandwich panel", Collection of Technical Papers - AIAA/ASME/ASCE/AHS/ASC Structures, Structural Dynamics and Materials Conference, Vol. 1, pp. 537-546.

Memari, A. M., Kasal, B., Manbeck, H. B., Adams, A. R., 2009, "Lateral load resistance evaluation of wood- and steel-stud partition shear walls", Journal of Architectural Engineering, Vol. 15, No. 4, pp. 122-130.

Meyer-Piening, H., 2006, "Sandwich Plates: Stresses, Deflections, Buckling and Wrinkling Loads - A Case Study", Journal of Sandwich Structures and Materials, Vol. 8, pp. 381-394.

Mitchell, D., R. Tremblay, E. Karacabeyli, P. Paultre, M. Saatcioglu et D. Anderson, 2003, "Seismic force modification factors for the proposed 2005 edition of the National Building Code of Canada”, Canadian Journal of Civil Engineering, Vol. 30, pp. 308-327.

Milner, H.R., Tan, H.H., 2001, "Modeling deformation in Nailed, Thin-webbed timber Box Beams", Computers and Structures, Vol. 79, No. 29-30, pp. 2541-6.

OMEGA Engineering Inc., 2003, http://www.omega.com

Ontario Building Code, 2012, "Building Code Act, O. Reg 332/12”, Ministry of Municipal Affairs and Housing.

Moghimi, H. , Ronagh, H.R., 2009, "Better connection details for strap-braced CFS stud walls in seismic regions", Thin-Walled Structures, Vol. 47, No. 2, pp 122-35. 
Mohamed, M., 2009, "Experimental Study on the Structural Behavior of Insulated Sandwich Foam-timber under Combined Axial Compression and Bending", M.A.Sc. Thesis, Ryerson University, Toronto, Canada.

Moslam, K.M., Hagerman, J., Kelly, H., 2008, "Seismic evaluation of structural Insulated Panels", ASCE Proceeding of $5^{\text {th }}$ international Engineering and Construction Conference, August 27-29.

Mullens, M.A., Arif, M., 2006, "Structural Insulated Panels: Impact on the Residential Construction Process", Journal of Construction Engineering and Management, Vol. 132, No. 7, p 786-794.

National Forest Production Association, 2005, “The National Design Specification for Wood (NDS)", USA.

NTA-IM 14, 2009, "NTA Design Guide”, www.ntainc.com.

Olsson, R., 2002, "Engineering Method for Prediction of Impact Response and Damage in sandwich Panels", Journal of Sandwich Structures and Materials, Vol. 4, pp. 3-29.

Payne, R.J., 1971, "Plywood Construction Manual”, $2^{\text {nd }}$ Edition, Council of the Forest Industries of British Columbia, Vancouver, Canada.

Percival, D.H., Hunt, M.O., Comus, Q.B., and Suddarth, S.K., 1977, "Pilot Test of Four 16-foot, Wood-based Composite Garage Headers", Forest Products Journal, Vol. 27, No. 9, pp. 45-48.

Porter, M.L. , 1987, “Sequential Phase Displacement (SPD) Procedure for TCCMAR Testing”, 3rd Meeting of the Joint Technical Coordinating Committee on Masonry Research, US-Japan Coordinated Program For Masonry Building Research, Tomamu, Japan, Oct. 15-17. 
Racher, P., Bocquet, J.F. and Bouchair, A., 2005, "Effect of Web Stiffness on the Bending Behaviour of Timber Composite I-Beams", Materials \& Design, Vol. 28, No. 3, pp. 844-9.

Rastra Holding Ltd., 2009, www.rastra.com

Raville, M.E., 1955, "Deflection and stresses in a uniformly loaded, simply supported, rectangular sandwich plate", Forest Product Laboratory Report 1847, USA.

Reissner, E., 1948, "Finite Deflections of Sandwich Plates", Journal of Aeronautical Science, Vol. 15, N 7, pp.435-440.

Sclater, N., 2007, "Mechanisms and Mechanical Devices Sourcebook”, 4th Edition, McGrawHill, USA.

Seaders, J.P., Miller, T. H., Gupta, R., 2009, "Performance of partially and fully anchored woodframe shear walls under earthquake loads", Forest Products Journal, Vol. 59, No. 5, pp. 42-52.

Serrette, R., Nolan, D. P., 2009, "Reversed cyclic performance of shear walls with wood panels attached to cold-formed steel with pins", ASCE Journal of Structural Engineering, Vol. 135, No. 8, pp. 959-967.

Seyad Ahmad, M., 2011, "Flexural Creep Effects on Permanent Wood Foundation Made of Structural Insulated Foam-Timber Panels", M.A.Sc. Thesis, Ryerson University, Toronto, Canada.

Seydel, E., 1933, "Uber das Ausbeulen von rechteckigen isotropen oder orthogonalanisotropen Platten bei Scubbeanspruchung”, lng. Arch., Vol. 4, pp. 169.

Structural Insulated Panel Association, 2003, www.sipa.org 
Songlai, C. , Chengmou, F., Jinglong, P., 2010, "Experimental study on full-scale light frame wood house under lateral load", Journal of Structural Engineering, Vol. 136, No. 7, pp. 805-812.

Southwell, R.V. and Skan, S. W., 1924, "On the stability under shearing forces of a flat elastic strip", Proceeding Royal Society Association, Vol. 105, No. 733:582.

Stewart, W.G., 1987, The Seismic Design of Plywood Sheathed Walls, Doctor of Philosophy Thesis, University of Canterbury, New Zealand.

Stowell, E.Z., 1943, "Critical shear stress of an infinitely long flat plate with equal restraints against rotation along the parallel edges", NACA Report 3K12 or WRL-476.

Structural Board Association, 2004, “OSB Performance by Design Manual: Construction Sheathing and Design Rated Oriented Strand Board”, Markham, Ontario, Canada.

Structural Insulated Panel Association (SIPA), 2007, www.sips.org.

Structural Insulated Panel Association (SIPA), 2011, "Use of Structural Insulated Panels (SIPs) in Seismic Design Categories", Technical Bulletin No.1, Gig Harbor, WA, USA.

Sun Spaces Inc., 2010, www.sun-spaces.com

Talbot, K.C., Reaveley, L.D., and Pantelides, C.P., 2009, "Structural performance of stapled wood shear walls under dynamic cyclic loads”, Earthquake Spectra, Vol. 25, No. 1, pp. 161-183.

Taylor, S.B., 1996, “The Flexural Creep Behavior of Structural Insulated Panel (SIP) Sandwich Beam, PhD Dissertation, The Pennsylvania State University, University Park, USA.

Test \& Measurement World Magazine, 1996, "Decade of Progress Award: Scientific Solutions LabMaster First in PC Data Acquisition", Vol. 11 No. 10, USA. 
Trentiuk, S. and Memari, A. M., 2011, "Seismic Evaluation of Structural Insulated Panels through Full-scale Racking Load Testing," Journal of Architectural Engineering, in press.

Trentiuk, Stefanie, 2009, Parametric Study of Structural Insulated Panels under Monotonic and Cyclic Loading, Submitted in Partial Fulfillment of the Requirements for the Proposal for the Degree of Master of Science, State College, Pennsylvania.

Tham, L.G., Chong, K.P., and Cheung, Y.K., 1982, "Flexural Bending and Axial Compression of Architectural Sandwich Panels by Combined Finite-Prim Strip Method", Journal of Reinforced Plastics and Composites, Vol. 1, pp. 16-28.

The Murus Company, 2008, "Structural Insulated Panel”, www.murus.com.

Thermapan Structural Insulated Panels Inc., 2007, www.thermapan.com.

Timoshenko, S. P. and Gere, J. M., 1961, "Theory of Elastic Stability", 2nd ed., McGrawHill, New York, 1961.

Timoshenko, S.P., 1921, “Über die Stabilität versteifter Platten”, Eisenbau, Vol. 12 , pp. 147163.

Toothman, A.D., 2003, Monotonic and Cyclic Performance of Wood-frame shear Walls with Various Sheathing Materials, Master of Science Degree Thesis, Virginia Polytechnic Institute and State University, Blacksburg, Virginia.

Trayer, G.W. and March, H.W., 1930, "The torsion of members having sections common in aircraft construction", National Advisory Committee for Aeronautics -- Reports, pp. 48.

Trefftz, E., 1933, "Stability of elastic equilibrium”, Zeitschrift fü Angewandte Mathematik Und Mechanik, Vol. 13, pp. 160-165. 
Uniform Building Code, 1997, "Founadtion Plate or Sill Bolting", International Code Council, Vol. 2, Section 1612, Prentice Hall, USA.

Uniform Building Code, 2000, "Structural Engineering Design Provisions", International Code Council, Section 1806.6, Prentice Hall, USA.

Van de Lindt, J.W., 2008, "Experimental investigation of the effect of multiple earthquakes on woodframe structural integrity", Practice Periodical on Structural Design and Construction, Vol. 13, No. 3, pp. 111-117.

Varoglu, E., Karacabeyli, E., 2006, "Midply Wood Shear Wall System: Concept and Performance", ASCE Journal of Structural Engineering, Vol. 132, No. 9, pp. 1417-1425.

Wang, S.M.T., 1994, "Shear Beahviour of OSB Wood Composite I-beams with Web Openings", M.A.Sc. Thesis, University of Alberta, Alberta, Canada.

White, K.B.D., Miller, T. H., Gupta, R., 2009, "Seismic performance testing of partially and fully anchored wood-frame shear walls", Wood and Fiber Science, Vol. 41, No. 4, pp. 396-413.

White, M.W. and Dolan, J.D., 1995, "Nonlinear Shear Wall Analysis," J. Structural Engineering, Vol. 121, No. 11, pp. 1629 - 1635.

Williams D., Leggett, D.M.A., and Hopkins, H.G., 1941, "Flat Sandwich Panels under Compressive End Loads", Report No. A.D. 3174, Royal Aircraft Establishment, Yamborough, England.

Wong, P. C.K., Bach, L. and Cheng, J.J.R., 1988, "Flexural creep behavior of OSB stressed skin panels", Structural Engineering Report, No. 158.

Yoon K.J., Kim, C.K., and Park, H.C., 2002, "Nonlinear Flexural deflection of Thermoplastic Foam Core Sandwich Beam”, Journal of Composite Materials, Vol. 36, pp. 1529-1539. 
Zarghooni, M. H., 2009, "Flexural Creep Behaviour of Structural Insulated Timber Panels", M.A.Sc. Thesis, Ryerson University, Toronto, Canada. 